(ㅅ)(2)

(c) (a)

(d) (2) 


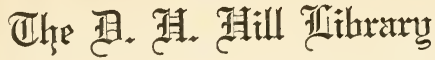

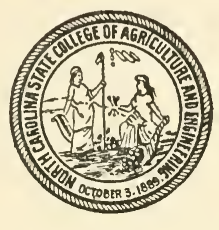

3rorth Uardina \$tate Uallege

QK45

K4

1902

v. 1 


\section{Date Due}

THIS BOOK IS DUE ON THE DATE INDICATED BELOW AND IS SUBJECT TO AN OVERDUE FINE AS POSTED AT THE CIRCULATION DESK.

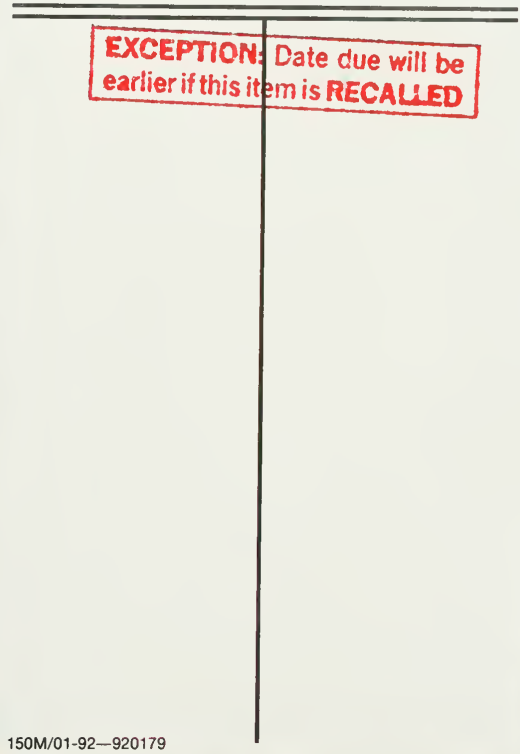





\section{THE}

NATURAL HISTORY OF PLANTS 



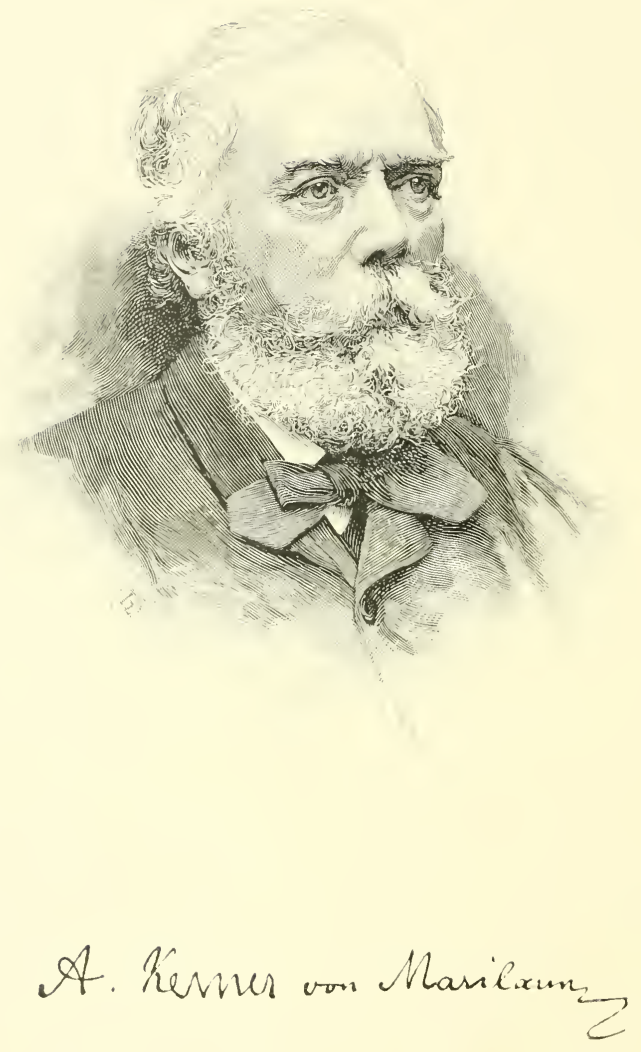


. 



\title{
The Natural History of Plants
}

Their Forms, Growth, Reproduction, and Distribution

From the German of the late

\section{ANTON KERNER VON MARILAUN}

By

F. W. OLIVER, M.A. D.Sc.

With the Assistance of

LADY BUSK, B.Sc. and Mrs. M. F. MACDONALD, B.Sc.

With about Two Thousand Original Woodcut Illustrations

\author{
VOLUME I \\ Biology and Configuration of Plants
}

LONDON

BLACKIE \& SON, Limited, 50 OLD BAILEY, E.C.

GLASGOW AND DUBLIN 


$$
\text { सान4 }
$$




\section{PREFATORY NOTE TO PRESENT ISSUE.}

The present edition of The Natural History of Plants, though it has been revised in minor points, retains the fundamental features of its predecessors. Scientific knowledge is of course continually advancing, but Kerner's methods were so pre-eminent that his work has attained, in a sense, the permanent value and dignity of a classic. It was therefore deemed inadvisable at the present time to make any changes beyond those referred to above, more particularly since the publishers saw their way to bring the book, by means of a reduction in price, within the reach of a larger public.

F. W. O. 



\section{PROFESSOR KERNER'S PREFACE}

TO THE ENGLISH EDITION.

Not long ago two artisans, who had borrowed a copy of The Natural History of Plants from one of the Vienna public libraries and had studied its pages, called upon me, asking me to sbow them under the microscope some of the things there described.

It seems that without any special educational advantages they had availed themselves of leisure moments to extend their knowledge, and had read the work with profit. On leaving, they thanked me in simple words for the pleasure, instruction, and stimulus which they had derived from the perusal of my book.

I confess that these words gave me vastly more pleasure than many of the verbose and flattering reviews that had appeared in newspapers and scientific journals, many of which conveyed the impression of being the result of hasty skimming of copies sent by the publishers.

The satisfaction which the little incident gave me was the greater, in that it was an assurance that I had achieved what had been my intention, namely, to write a book which might serve as a source of knowledge, not only for specialists and scholars, but also for the many who, though compelled to follow some practical calling, still take an interest in science, and who wish, each in his own particular degree, to obtain information of its progress.

Popular treatises on the results of scientific investigation are by no means rare with us Germans; but in too many cases scientific problems involving serious thought are touched superficially, and, like the stone in a sweet fruit, are embedded in picturesque and attractive accounts of things purely of subordinate importance. The reader, gratified by the elegant phraseology, passes by the kernel of fact, and derives little profit from the book. Books such as these have brought the art of popular writing into discredit, and we have arrived at the point when educated people but lightly esteem, or even ignore, the results of careful 
and laborious investigations and the theories based upon them, if they are produced in a popular manner rather than in the conventional language of science.

With the English, however, it is otherwise. I have long regarded with admiration the men of science whom you number amongst your countrymen, who present the results of their studies in words intelligible to all who seriously desire knowledge.

To follow in the path of such men has always been my aim in my work and in my writings; and this was particularly before me in the production of The Natural History of Plants.

\section{A. KERNER VON MARILAUN.}

\section{SOME OPINIONS}

\section{CONCERNING KERNER'S NATURAL HISTORY OF PLANTS}

LORD AVEBURY (Sir Jонм LUввоск) has said, regarding Kerner's Natural History of Plants:

"A man must be dull indeed who can read such a work as Prof. Kerner's Natural Histor" of Plants without deep interest. The adaptation of plants to their circumstances and conditions of life are admirably described, and are, moreover, brought out the more clearly by a great number of instructive, and in some cases beautiful, illustrations.

"It will certainly seem, to anyone who has followed the course of botanical research during recent years, not only a work of supererogation, but I might almost say of impertinence, for me to recommend any work written by Prof. Kerner, and which Prof. Oliver has thought worthy of translation. With regard to the general reader, however, it may not be out of place to assure him that Botany, as revealed in these pages, is a subject of intense interest, which will furnish him with pleasant and stimulating occupation for his leisure moments, and on this ground alone, if there were no other, I welcome with pleasure this edition of Prof. Kerner's Natural History of Plants."

Professor F. O. BOWER, M.A., D.Sc., F.R.S., Professor of Botany, Glasgow University, says:

"Kerner's Matural History of Plants may go far to revolutionize the teaching of Botany in the schools, introducing into it more of biological interest. It is a book to be recommended for the reading of schoolmasters themselves."

F. W. BURBIDGE, M.A., F.L.S., F.R.H.S. (London), Trinity College Botanical Gardens, Dublin, says :

"The Natural History of Plants is a work that should be ntilized by all teachers and lecturers of the various County Councils, and should also be in the hands of all intelligent cultivators of the soil. I could not possibly say too much in praise of such a remarkable work."

WILLIAM CARRUTHERS, F.R.S., F.L.S., British Museum (Natural History), London, says:

"It is certainly a singularly attractive volume for the general reader, and a useful text-book for the student. It deals with recent investigations in a way that one does not meet with in any other popular book." 


\title{
EDITOR'S PREFATORY NOTES
}

\author{
TO THE FIRST EDITION.
}

\section{VOLUME I.}

Professor Kerner has stated very suecinctly, in the preface which he has been good enough to write for the English edition of Pflanzenleben, the main idea which guided him in the writing of that book. Consequently little remains for me to add save a few observations on the book in its present form. On the appearance of the original, the parts as they were issued were widely scanned, and the work soon enjoyed a large circulation. Here was a book at once attractive to the ordinary reader, and retaining unimpaired its value to trained naturalists. The scale of the undertaking was such that it was possible to give a presentment worthy of the subject. Hitherto, though Astronomy, Geology, and other branches of natural knowledge had been long accessible to the ordinary reader in popular books of the greatest value, this service had not been done for Botany. Long before the issue of Pfanzenleben was complete, the idea of an English edition suggested itself to me and to my friend, Mr. Walter Gardiner, of Cambridge. It was my hope that we should, jointly, undertake its preparation. To my great regret, Mr. Gardiner was prevented from co-operating by other duties; thus the whole responsibility of this edition falls to my lot. To my colleagues in this undertaking, Mrs. Busk (Lady Busk) and Miss Ewart (Mrs. M. F. Macdonald), the chief credit is due for this translation. Indeed, without their hearty collaboration, the production of The Natural History of Plants would have been impossible. In the main, the original text has been faithfully adhered to. The translation, though not everywhere precisely literal, never departs from the spirit of the German edition. The Index to the complete work, together with a Glossary, will be appended to the concluding volume.

KEw, November, 1894.

$$
\text { F. IT. O. }
$$

\section{VOLUME II.}

With this, the second and concluding volume of The Natural History of Plunts, a brief statement and explanation of my position as editor is imperative. As stated in my note to Volume I. the English text there followed that of the original with considerable fidelity. In the second volume I have less consistently followed this course. Throughout I have not hesitated to add or substitute new matter, though 
no overt indication of such departure from the original is given either by different type or otherwise. It is needless to explain that these changes are only such as the advance of botanical knowledge has rendered necessary since the original was written, and that I have never desired to depart from the intention of the author. To the specialist these modifications will be from time to time apparent; the general reader will perhaps treat me with indulgence should he think that in this matter my judgment has been at fault. Though changes occur throughout the volume, I have preserved intact the main conclusions of the author and the facts upon which they are based. To have altered these in any way, even had I been so minded, would have been inconsistent with the duties of an editor and translator. But in the purely systematic portion of the work I have been restrained by no such scruples. Professor Kerner himself regarded that portion of his work as but tentative, and as it was difficult to merely modify, the whole of this portion has been written de novo, from the Thallophytes to the end of the Gymnosperms (pp. 616-728), and in part the Monocotyledons. The exigencies of the serial issue of The Nutural History of Plants alone has prevented the re-cast of the Di- . cotyledons, which stand with little modification as in the original. For the portion dealing with the class Gamopleycece up to the end of the Conjugutce (pp. 627-659), I am indebted to my colleague, Mr. A. G. Tansley of University College, who has devoted considerable attention to the group in question. To him I now offer my hearty thanks. The glossary of botanical terms makes claim neither to completeness nor originality. Though a large number of the definitions and explanations have been written specially for this, book, I have never hesitated to lay published sources under contribution. The laborious task of constructing the index has fallen to Mr. George Brebner, and to him is due the gratitude of such as gain through it direct and ready access to the body of the work.

F. IV. O.

KEw, August, 1895. 


\title{
CONTENTS OF VOLUME FIRST.
}

\section{INTRODUCTION.}

\author{
\begin{tabular}{l|l} 
The Study of Plants in Ancient & Page \\
Doctrine of Metamorphosis and Speculations
\end{tabular} \\ ANd in Modern Times. \\ Plants considered from the point of view \\ of Utility, - $\quad$ - $\quad$ - $\quad$ - $\quad$ - $\quad$ - 1 \\ The Description and Classification of Plants, \\ of Nature-Philosophy, - $\quad-\quad$ - 7 \\ Scientific Method based on the History of \\ Development, - - - - $\quad 13$ \\ Objects of Botanical Research at the present \\ day, - - - - - - 15
}

\section{THE LIVING PRINCIPLE IN PLANTS.}

\section{Protoplasts considered as the Seat of LiFE.}

Discovery of the Cell: Researches of Swammerdam, Leeuwenhoek, and Unger, - 21

Discnvery of Protoplasm, - - - - $\quad 25$

\section{Movements of Protoplasts.}

Swimming and Creeping Protoplasts, - - 28

Movements of Protoplasm in Cell-cavities, - 32

Movements of Simple Organisms-Volvocineæ, Diatomaceæ, Oscillariæ, and Bacteria, - - - - - -
3. Secretrons and Constructive Activity of Protoplasts.

Cell-sap: Cell-nucleus: Chlorophyll-bodies : Starch: Crystals, - - - - 41 Construction of the Cell-wall and establishment of Connections between neighbouring Cell-cavities, - - - $\quad$ - 42

4. Communication of Protoplasts with one ANOTHER AND With the OUTER WORLD.

The Transmission of Stimuli and the Specific Constitution of Protoplasm, - - - 47 37 Vital Force, Instinct, and Sensation, - - 51

\section{ABSORPTION OF NUTRIMENT.}

\section{Introduction.}

Classification of Plants, with reference to Nutrition, - $\quad$ - $\quad-\quad-\quad-\quad 55$ Theury of Food-Absorption, - - $\quad-57$

2. Absorption of Inorganic Substances.

Nutrient Gases, - $\quad$ - $\quad$ - $\quad$ - $\quad$ - 60 Nutrient Salts, - $\quad$ - $\quad$ - $\quad$ - $\quad$ - $\quad-66$ Absorption of Food-salts by Water-plants, - 75 Absorption of Food-salts by Lithophytes, - 79 A bsorption of Food-salts by Land-plants, - 82 Relations of the Position of Foliage-leaves to that of Absorbent Roots,

\section{Absorption of Organic Matter from Decaying Plants and Animals.}

Saprophytes and their Relation to Decaving Bodies, - $\quad$ - $\quad$ - $\quad$ - $\quad$ - $\quad$ - 99 Saprophytes in Water, on the Bark of Trees, and on Rocks, - - - - $\quad$ - 104 Saprophytes in the Humus of Woods, Meadows, and Moors, - $\quad$ - $\quad-\quad 109$ Special Relations of Saprophytes to their Nutrient Substratum, - - - $\quad 113$ Plants with Traps and Pitfalls to ensnare Animals, - - $\quad$ - $\quad$ - 119 
Carnivorous Plants with Adhesive Appa-

\section{Absorption of Nutriment by Parasitic Plants.}

Carnivorous Plants which exhibit Movements in the capture of Prey, - - 140 ratus, - $\quad-\quad-\quad-\quad-\quad 153$

Absorption of Rain and Dew by the Foliageleaves, - - $\quad$ - $\quad$ - $\quad$ - $\quad$ - $\quad-225$

Development of Absorption-cells in Special Cavities and Grooves in the Leaves, - 230

\section{Symbiosis.}

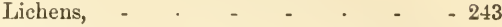

Symbiosis of Green-leaved Phanerogams with Fungal Mycelia destitute of Chlorophyll: Monotropa, - - $\quad$ - $\quad 249$

Animals and Plants considered as a great Symbiotic Community, - $\quad$ - 254

\section{Changes in the Soll incident to the Nutrition of Plants.}

Solution, Displacement, and Accumulation of particular Mineral Constituents of the Soil resulting from the Action of Plants, - - - - - $\quad 257$ Importance of Water to the Life of a Plant, 216 A bsorption of Water by Lichens and Mosses, and by Epiphytes furnished with Aërial Roots, $-217$
Mechanical Changes effected in the ground by Plants, - - • • • 265

\section{CONDUCTION OF FOOD.}

1. Mechanics of the Movement of THE RaW Food-sap.

Capillarity and Root-pressure, - $\quad$ - $\quad$ - 269

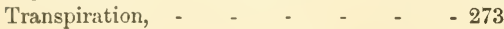

2. Regulation of Transpiration.

Means of accelerating Transpiration, - $\quad 284$

Maintenance of a Free Passage for Aqueous

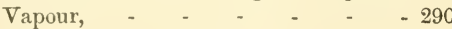

\section{Prevention of Excessive Transpiration.}

Protective Arrangements on the Epidermis, 307 Form and Position of the Transpiring

Leaves and Branches - _ _ - $\quad 325$

\section{Transpiration during various Seasons} of the Year: Transpiration of Lianks.

Old and Young Leaves, $\quad-\quad c_{-} \quad-\quad 347$

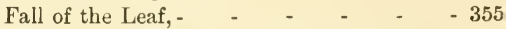

Connection between the Structure of the Vascular Tissues and Transpiration, - 362

5. Conduction of Food-gases to the Places of Consumption.

Transmission of the Food-gases in Land and Water Plants and in Lithophytes: Significance of Aqueous Tissue in the conduction of Food-gases, - $\quad-\quad \ldots \quad-367$

\section{FORMATION OF ORGANIC MATTER FROM THE ABSORBED INORGANIC FOOD.}

\section{Chlorophyll and Chlorophyll- QRANULES.}

Chlorophyll-granules and the Sun's Rays, - 371

Chlorophyll-granules and the Green Tissue under the Influence of various degrees of Illumination,

\section{The Green Leaves.}

Distribution of the Green Leaves on the Stem, 396 Relation between Position and Form of Green Leaves, - - $\quad$ - $\quad$ - 408 Arrangements for retaining the Position assumed, - . - - - 424 Protective Arrangements of Green Leaves against the Attacks of Animals. - $\quad 430$ 


\section{METABOLISM AND TRANSPORT OF MATERIALS.}

1. The Organic Compounds in Plants.

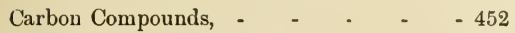

Metabolism in Living Plants, - - $\quad-455$

2. Transport of Substances in Living Plants.

Mechanisms for Conveyance to and fro, - 465 Significance of Anthocyanin in the Trans- portations and Transformations of Ma- Page terials: Autumnal Colouring of Foliage, 483

3. Propelling Forces in the Conversion and Distribution of Materials.

Respiration, - $\quad$ - $\quad$ - $\quad$ - $\quad$ - $\quad$ - 491

Development of Light and Heat, - - 496

Fermentation, - $\quad . \quad$ - $\quad . \quad 504$

\section{GROWTH AND CONSTRUCTION OF PLANTS.}

\section{Theory of Growth.}

Conditions and Mechanics of Growth, - $\quad 510$

Effects of Growing Cells on Environment, - 513

\section{Growth and Heat.}

Sources of Heat: Transformation of Light into Heat, - $\quad$ - $\quad$ - $\quad$ - $\quad$ - $\quad 517$

Influence of Heat on the Configuration and Distribution of Plants,
Measures for protecting Growing Plants from Loss of Heat, - _ $\quad$ - 528

Freezing and Burning, $\quad-\quad 5 \quad-\quad 539$

Estimation of the Heat necessary to Growtl, 557

3. Ultimate Structure of Plants.

Hypotheses as to the Form and Size of the smallest Particles employed in the Construction of Plants, - $\quad$ - $\quad$ - 566 Visible Constructive Activity in Protoplasm, $5: 2$

\section{PLANT-FORMS AS COMPLETED STRUCTURES.}

L. Progressive Stages in Complexity of Structure from Unicellular Plants to Plant-Bodies,

\section{Form of Leaf-structures.}

Definition and Classification of Leaves, - 593

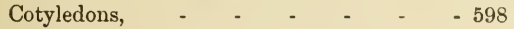
Scale-leaves, Foliage-leaves, Floral-leaves, - 623

\section{Forms of Stem-structures.}

Definition and Classification of Stems: The Hypocotyl: Stems bearing Scale-leaves. 647
Stems bearing Foliage-leaves, - - - $\quad-655$

Procumbent and Floating Stems, $\quad$ - $\quad$ - 661

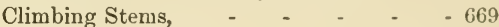

Erect Foliage-stems, - $\quad$ - $\quad$ - $\quad$ - 710

Resistance of Foliage-stems to Strain, Pressure, and Bending, $\quad$ - $\quad$ - $\quad$ - $\quad$ - 724 The Floral-stem, - $\quad$ - $\quad$ - $\quad$ - $\quad$ - 736

\section{Forms of Roots.}

Relation of external and internal structure to Function, - - - - $\quad$ - 748 Definition of the Root, - $\quad$ - $\quad$ - $\quad$ - 764 Remarkable Properties of Roots, _ - 767 


\title{
ILLLSTRATIONS IN VOLUME FIRST.
}

\author{
FROM ORIGINAL DRAWINGS BY E. HEIN, H. v. KÖNIGSBRUNN, E. v. RANSONNET, \\ J. SEELOS, F. TEUCHMANN, O. WINKLER, AND OTHERS.
}

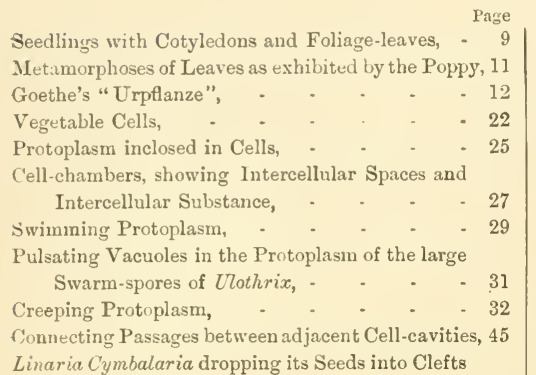
in the Rocks, - - - - - $\quad$ - 53

Absorptive Cells on Root of Penstemon, - $\quad-87$

Centrifugal and Centripetal Transmission of Water, 94

Irrigation of Rain-water in Plants, - - - $\quad 97$

Aerrial Roots of a Tropical Orchid assuming the form of straps, - $\quad$ - $\quad$ - . . . 107

Transverse section through absorption-roots of Saprophytes, - - - . - - 115

Bladderworts, - $\quad$ - $\quad$ - $\quad$ - $\quad$ - 120

Traps of Utricularia neglecta, - . - $\quad$ - 121

Spinous Structures in the Pitfalls of Carnivorous Plants, - - - - . . - 124

Sarracenia purpurea, - . - . 125

Ascidia-bearing and Pitcher-plants, - . 127

Cephalotus follicularis, - $\quad$ - $\quad$ - $\quad$ - 131

Young Nepenthes plants, - . - 132

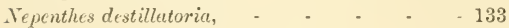

Glandular structures in the Toothwort, Bartsia, and Butterwort, - $\quad . \quad-\quad . \quad-137$

Tentacles on leaf of Sun-dew, - • - - 145

Venus's Fly-trap (Dioncea muscipula), - 148

Capturing apparatus of the leaves of Aldrovandia and Venus's Fly-trap, - . 150

Aldrovandia vesiculos $\alpha$, - $\quad$ - $\quad$ - $\quad$ - $\quad$ - 151

The Fly-catcher (Drosophyllum lusitanicum), - 155

Lonicera ciliosa in South Carolina, . . $\quad$ - 160

Hyphæ of Parasitic Fungi, - - . - 165

Parasites on Hydrophytes, - $\quad$ - $\quad$ - 169

Seedlings of Parasitic Plants, - . . 173

Cuscuta Europra parasitic on a Hop-stem, . - 175

Bastard Toad-flax (Thesium alpinum), . 177
Toothwort (Lathree Squamaria), with Page upon the roots of a Poplar, - . . . 181

Langsdorffia hypogcea, from Central America, - 187 Parasitic Balanophoreæ (Scybalium fungiforme and Balanophora Hildenbrandtii), - 189

Parasitic Balanophoreæ (Rhopalocnemis phalloides and Helosis gujanensis), $\quad-\quad$ - $\quad$ - 191

Parasitic Balanophoreæ (Lophophytum nirabile and Sarcophyte sanguinea), - . - - 195

Cytinus Hypocistus and Cynomorrum coccineum, - 197

Rafflesiaceæ parasitic on trunks and branches, - 201

Parasitic Rafflesiacea upon a Cissus-root, - 202

Rafflesia Padma, parasitic on roots upon the surface of the ground, - $\quad$ - . $\quad$ - 203

The European Mistletoe (Viscum album), - 206

Bushes of Mistletoe upon the Black Poplar in

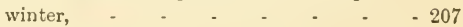

Loranthus Europaus and Mistletoe (Viscum album) -both parasitic on branches of trees, and seen in section. A piece of Fir-tree perforated by the sinkers of a Mistletoe, - 209

Porous Cells of Fork-moss, Bog-moss, and an Orchid root, - $\quad . \quad \ldots \quad \ldots \quad+\quad \cdot 219$

Aërial Roots of an Orchid epiphytic upon bark of the branch of a tree, . . . . 221

Aërial Roots with root-hairs, - . . . 224

Hairs and Leaves which retain Dew and Rain, - 228

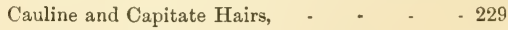

Absorption of Water by Foliage-leaves, - $\quad 232$

Absorptive Cavities and Cups on Foliage-leaves, 233

Water-receptacles in Plants, - . $\quad$ - 239

Gelatinous Lichens, . $\quad$ - $\quad$. $\quad$ - 244

Fruticose and Foliaceous Lichens, - - 245

Roots with Mycelial Mantle; Mycelium entering into the external cells, $\quad$ - $\quad . \quad-250$

Olive Grove on the Shores of Lake Garda, - $\quad 275$

Transpiring Cells, - $\quad . \quad+\quad . \quad 278$

Spongy Tissue of Franciscea eximia and Daphne Laureola, - - • - - - - 279

C'srypha umbraculifera of Ceylon, - - 289

Stomata of Nephrodium Filix-mas and Peperomia arifolia, - - . - . - . 294

Protection of Stomata from Moisture by Papillalike outgrowths of the Surface, - . 295 
Protection of Stomata from Moisture by Cuticular

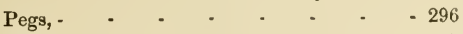

Over-arched Stomata of Australian Proteaceæ, - 297 Stomata in Pit-like Depressions, - - . 298 Stomata in the Furrows of Green Stems, - $\quad 299$ Orchids whose Stomata lie in Hollow Tubercles, - 300 Transverse Sections through Rolled Leaves, - 301 Vertical Section through a Rolled Leaf, - 303 Thickened Stratified Cuticle, - _ - - 310 Caryota propinqua, - - $\quad$ - $\quad$ - $\quad$ - 311

Vertical Section of Leaf of Caryota propinqua, - 312 Edelweiss (Gnaphalium Leontopodium), - $\quad 315$ Covering Hairs of various plants, - - $\quad$ - 321 Covering Hairs of various plants, - - 322 Flinty armour of Rochea falcata, - - - 323 Switch-plants, - - $\quad$ - $\quad$ - $\quad$ - $\quad$ - 331 Switch-shrubs, sections of Stems, - - - 332 Plants with Leaf-like Branches (Cladodes), - 333 Plants with Leaf-like Branches (Cladodes), - 335 Compass Plants, - $\quad$ - $\quad$ - $\quad$ - $\quad$ - $\quad 337$ Folding of Grass-leaves (Sesleria tenuifolia), - 341 Folding of Grass-leaves (Stipa capillata and Festuca alpestris),

Folding of Grass-leaves (Lasiagrostis Calamagrostis and Festuca Porcii),

Folding of Grass-leaves (Festuca punctoria) - 345

Folding of Moss-leaves (Polytrichum commune), - 346

Unfolding of Leaves of various plants, - $\quad 349$

Leaf-unfolding of the Tulip-tree, - - $\quad$ - 352

Unfolding of Beech-leaves, - _ - - 353

Leaf-fall of the Horse-chestnut, • - - - 361

Indian Climbing Palms (Rotang), - - $\quad 363$

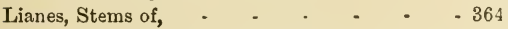

Aroids, with cord-like aërial roots, - - $\quad 365$

Position of the Chlorophyll-granules in the cells of the Ivy-leaved Duckweed (Lemnit trisulca), 382

Plan of Whorled Phyllotaxis, - $\quad$ - $\quad$ - 397

Plan for Spiral Phyllotaxis, - - - - 400

Plan of Five-thirteenths Phyllotaxis, - - . 401

Parastichies of a Pine-cone, - _ _ - - 403

Displacement of the leaf-positions in consequence of torsion of the stem,

Leaf-mosaic, Leaf-rosettes, and Scale-like Leaves, 410

Formation of a Leaf-mosaic, - _ . . 411

Spruce Firs (Abies excelsa), - - - - $\quad$ - 415

Erect Leafy Twig of the Norway Maple, - - 416

Twisting of Internodes and Leaf-stalks, - - 417

Horizontally growing Leafy Twig of the Paper Mulberry-tree (Broussonetia papyrifera), - 418

Leafy Twig projecting laterally from the Stem of the Norway Maple (Acer platanoides), - $\quad$ - 419

Leaf-mosaics of Unsymmetrical Leaves, - - $\quad 420$

Mosaic of Leaves of unequal size, - . $\quad$ - 421

Mosaic of Unsymmetrical Leaves of unequal size, 422

Leaf-mosaic (Ivy), $\quad$ - $\quad$ - $\quad$ - $\quad$ - $\quad$ - $\quad$ - 423

Acantholimon and spiny Tragacanth-shrubs, - 435

Group of Thistles (Cirsium nemorale), - - $\quad 436$

Acanthus spinosissimus, - . . . . 437
Weapons of Plants,

Weapons of Plants,

Chemical Diagrams (three), - - . - 453

Chemical Diagram, - • - . . - 454

Crystals and Crystalloids in Plant-cells, .. $\quad 457$

Various Forms of Starch-grains, - . $\quad 459$

Portion cut from a Branch (diagrammatic), - 469

Organs for Removal of Substances, - - - 471

Rhynchosia phaseoloides, a Liane with ribbon-like Stems,

Transverse sections of Liane Stems, - - $\quad 477$

Leafless Branches of Tecoma radicans, rooted on a wall,

Elevation of a Block of Stone in consequence of the growth in thickness of a Larch Root,

Alpine Willows with stems and branches elinging to the ground,

Periodic bending of Flowers and Inflorescences, - 531

Alteration of Position of Leaflets in Compound Leaves,

Mimosa pudica in day and night positions, - $\quad 537$

Mountain Pines (Pinus humilis) in the Tyrol, - 549

Detachment of special shoots of Potamogeton crispus, for hibernation under water,

Edible Lichen (Lecanora esculenta) in the desert, 555 Changes in the Protoplasm of the Cell-nucleus during its division,

Laminarias in the North Sea, - . . . - 588

Liverworts with Cell-nets, Cell-plates, and Cellrows in various transitional forms,

Cotyledons, various examples shown in detail, - 599

Process of Development-(Rhizophora conjugata), 603 Mangroves on the West Coast of India at ebbtide, -

Germinating Seeds and Seedlings, - - 607

Liberation of the Cotyledons from the cavity of the seed or fruit husk,

Anchoring of the Water-chestnut (Trapa), - - 617

The Boring of Fruits into the Ground, Feathergrass and Stork's-bill,

Cotyledons of various Plants, . _ . - 621

Arrangement of Strands in the blades of Foliage-

leaves. Forms with one main strand, -

Distribution of Strands in the blades of Foliageleaves. Forms with several main strands, - 633

Flowers of the Silver Lime and Arrow-grass, - 646

Cotton Trees of the Brazilian catingas, - $\quad$ - 656

Agaves of the Mexican uplands, - - $\quad 657$

Yucca gloriosa, . . . . . . . . . 659

Vallisneria spiralis, - $\quad$ - $\quad$ - $\quad$ - $\quad .667$

Rotangs in Java, - $\quad$ - $\quad . \quad+\quad-675$

Shoot-apices of three species of Rotang, - . - 676

Branches of the New Zealand Bramble, - $\quad-677$

Palm-stem used as a support by the lattice-forming stems of one of the Clusiacer, $\quad . \quad-681$

Twining Hop (Humulus Lupulus), in detail, - 688 Portion of a Liane stem, twisted like a corkscrew, 
Stipular tendrils of the common Smilax, - $\quad \begin{array}{r}\text { Page } \\ -690\end{array}$

Leaf-stalk tendrils of Atragene alpina, $\quad . \quad 691$

Branch-tendrils of Serjania gramatophora, - $\quad 693$

Tendrils of the Bryony (Bryonia), - _ $\quad .696$

Light-avoiding Tendrils of Vitis inserta and Vitis

inconstans, - . $\quad . \quad+\quad . \quad . \quad 699$

Ivy (Hedera Helix) fastened by climbing roots to the trunk of an Oak, - . - . $\quad-703$

Ficus with girdle-like clasping roots, - - $\quad 705$

Ficus Benjamina with incrusting climbing roots, 707

Bignonia argyro-violacea, from Brazil, - $\quad 709$

Ficus with lattice-forming climbing roots, - $\quad 711$

Bamboos in Java, - $\quad$ - $\quad$ - $\quad$ - $\quad$ - 713

The Oak, - $\quad$ - $\quad$ - $\quad$ - $\quad$ - 716

The Silver Fir (Abies pectinata), - - . 717

Birch Trunks with white membraneous bark, - 721

Eucalyptus trees in Australia, - • - . 723

Diagrammatic representation of various combined girders,
Transverse sections of erect foliage-stems with simple girders not fused together into a tube, 729 Transverse sections of erect foliage-stems with simple girders fused into cylindrical tubes, - 730 Transverse sections of erect foliage-stems with flanges developed as secondary girders, $\quad-731$

Transverse section of the climbing stem of the Atragene (Atragene alpina), . . . 733

Undulations of old ribbon-shaped Liane stems, - 734 Transverse sections of a runner of the Garden Strawberry and of the Water Milfoil, - $\quad 735$ Branch of the Walnut-tree with hanging male catkins, and a small cluster of female flowers, 742

India-rubber Tree (Ficus elastica) and Banyantree (Ficus Indica), - . $\quad$ - $\quad-755$

The Screw Pine (Pandanus utilis), - - 758

Stilt-like and columnar roots of Mangroves, $\quad 759$

Bramble-bush in which the branches have taken root, 769 


\section{THE BIOLOGY}

AND CONFIGURATION OF PLANTS 



\section{THE}

\section{NATURAL HISTORY OF PLANTS.}

\section{INTRODUCTION.}

\section{THE STUDY OF PLANTS IN ANCIENT AND IN MODERN TIMES.}

Plants considered from the point of view of utility.-Description and classification of plants.Doctrine of metamorphosis and speculations of nature-philosophy.-Scientific method based on the history of development.-Objects of botanical research at the present day.

\section{PLANTS CONSIDERED FROM THE POINT OF VIEW OF UTILITY.}

Some years ago I rambled over the mountain district of North Italy in the lovely month of May. In a small seque tered valley, the slopes of which were densely clad with mighty oaks and tall shrubs, I found the flora developed in all its beauty. There, in full bloom, was the laburnum and manna-ash, besides broom and sweet-brier, and countless smaller shrubs and grasses. From every bush came the song of the nightingale; and the whole glorious perfection of a southern spring morning filled me with delight. Speaking, as we rested, to my guide, an Italian peasant, I expressed the pleasure I experienced in this wealth of laburnum blossoms and chorus of nightingales. Imagine the rude shock to my feelings on his replying briefly that the reason why the laburnum was so luxuriant was that its foliage was poisonous, and goats did not eat it; and that though no doubt there were plenty of nightingales, there were scarcely any hares left. For him, and I daresay for thousands of others, this valley clothed with flowers was nothing more than a pasture-ground, and nightingales were merely things to be shot.

This little occurrence, however, seems to me characteristic of the way in which the great majority of people look upon the world of plants and animals. To their minds animals are game, trees are timber and fire-wood, herbs are vegetables (in the limited sense), or perhaps medicine or provender for domestic animals, whilst flowers are pretty for decoration. Turn in what direction I would, in every country where I have travelled for botanical purposes, the questions asked by the inhabitants were always the same. Everywhere I had to explain whether the plants I sought and gathered were poisonous or not; whether they were efficacious as cures for this or that illness; and by what signs the medicinal or otherwise 
useful plants were to be recognized and distinguished from the rest. And the attitude of the great mass of country folk in times past was the same as at the present day. All along anxiety for a livelihood, the need of the individual to satisfy his own hunger, the interests of the family, the provision of food for domestic animals, have been the factors that have first led men to classify plants into the nutritious and the poisonous, into those that are pleasant to the taste and those that are unpleasant, and have induced them to make attempts at cultivation, and to observe the various phenomena of plant-life.

No less powerful as an incentive to the study of herbs, roots, and seeds, and to the minute comparison of similar forms and the determination of their differences, was the hope and belief that the higher powers had endowed particular plants with healing properties. In ancient Greece there was a special guild, the "Rhizotomoi," whose members collected and prepared such roots and herbs as were considered to be curative, and either sold them themselves or caused them to be sold by apothecaries. Through the labours of these Rhizotomoi, added to those of Greek, Roman, and Arabic physicians, and of gardeners, vine-growers, and farmers, a mass of information concerning the plant-world was acquired, which for a long period stood as botanical science. As late as the sixteenth century plants were looked upon from a purely utilitarian point of view, not only by the masses but also by very many professed scholars; and in most of the books of that time we find the medicinal properties, and the general utility of the plants selected for description and discrimination, occupying a conspicuous position and treated in an exhaustive manner. Just as men lived in the firm belief that human destinies depended upon the stars, so they clung to the notion that everything upon the earth was created for the sake of mankind; and, in particular, that in every plant there were forces lying dormant which, if liberated, would conduce either to the welfare or to the injury of man. Points which might serve as bases for the discovery of these secrets of nature were eagerly sought for. People imagined they discerned magic in many plants, and even believed that they were able to trace in the resemblance of certain leaves, flowers, and fruits to parts of the human body, an indication, emanating from supernatural powers, of the manner in which the organ in question was intended to affect the human constitution. The similarity in shape between a particular foliage-leaf and the liver did duty for a sign that the leaf was capable of successful application in cases of hepatic disease, and the fact of a blossom being heart-shaped must mean that it would cure cardiac complaints. Thus arose the so-called doctrine of Signatures, which, brought to its highest development by the Swiss alchemist Bombastus Paracelsus (1493-1541), played a great part in the sixteenth and seventeenth centuries, and still survives at the present day in the mania for nostrums. The inclination of the masses is now, as it was centuries ago, in favour of supernatural and mysterious rather than simple and natural interpretations; and a Bombastus Paracelsus would still find no lack of credulous followers. In truth, the great bulk of mankind regard Botany as subservient to medicine and agriculture, they look at it from the purely 
utilitarian point of view in a manner not essentially different from that of two hundred-or even two thousand-years ago, and it may well be a long time before they rise above this idea.

In addition to the botanical knowledge thus initiated by the necessities of life, a second avenue leading to the same goal was early established by man's sense of beauty. The first effect of this was limited to the employment of wild flowers and foliage for purposes of ornament and decoration. Later on, it led to the cultivation of the more showy plants in gardens, and ultimately to the arts of gardening and horticulture, which at different periods and in different countries have passed through such various phases, corresponding to the standards of the beautiful which have prevailed.

\section{THE DESCRIPTION AND CLASSIFICATION OF PLANTS.}

A third path leading to botanical knowledge springs from the impulse which actuates those who are endowed with a keen perception of form to investigate structural differences down to their most minute characteristics. Workers in this field arrange and classify all distinct forms according to their external resemblances, give them names appropriate to their position and importance, catalogue them, and keep up the register when once it has been started. Many people possess, in addition, the remarkable taste for collecting, which causes them to find pleasure in merely accumulating and possessing enormous numbers of specimens of the particular objects on which their fancy is fixed.

This tendency of the human mind has played a very important part in the history of botany. The first traces of it can be ascribed with certainty to a period long before the commencement of our era; for such descriptions and other notes as are contained in the Natural History of Plants, written by Theophrastus about the year 300 B.C., are founded, for the most part, on the observations and experiments of "Rhizotomoi," physicians and agriculturists, and it is obvious from the text of the book that in some cases those authorities did seek out plants, and learn to distinguish them for their own sakes, and not solely for their economic or medicinal value.

At the time of the Roman Empire and in the Middle Ages, it is true, no one troubled himself about plants other than those known to be in some way useful. But there was a revival of the practice of hunting for plants for the purpose of describing and enumerating all distinguishable forms, at that great epoch when the nations of the West began to study the treasures of Greek thought, endeavouring to adopt the point of view of antiquity, and to harmonize their own circumstances with it. It was at this same period that art too shook itself free from the traditions of the Middle Ages, and became actuated by a new ideal based on the study of the antique; but science, particularly natural science, has as good a claim as art to regard that memorable time as its period of renaissance. Although the ancient Greek writings on natural history, to which people turned with such youthful enthusiasm in the fifteenth century, could not satisfy their thirst for 
knowledge, yet there is no doubt that, as in art, the effect was to stimulate and reform; and that this study led up to the source, so long forgotten, whence the ancients had themselves drawn their knowledge, that is, to the direct investigation of nature, which has invariably given to every branch of human knowledge new and pregnant life.

As regards botanical knowledge in particular, the study of old Greek writings on the part of western nations in both Northern and Southern Europe had the immediate effect of instituting an eager search for all the different kinds of indigenous plants; and, besides arousing a passion for investigation, it evoked untiring industry in this pursuit, the results of which preserved in a number of bulky herbals still excite our wonder and respect. If these folios, dating for the most part from the first half of the sixteenth century, are perused in the hope of their revealing some guiding principle as a basis for the arrangement of the subject, the reader will no doubt be obliged to lay them aside unsatisfied. The plants were described and discussed just as the authors happened to come across them; and it is only here and there that we find a feeble attempt to range together and make groups of nearly-allied species. Only cursory attention was paid to the facts of geographical distribution. Plants native to the soil, herbs which flowered in gardens and had been reared from seed purchased from itinerant vendors of antidotes, and plants whose fruits were brought to Europe as curiosities from the New World recently discovered-all these were jumbled together in a confused medley. The whole endeavour of the time was directed to the enumeration and description of all such things as possess the power of producing green foliage and maturing fruit under the sun's quickening rays.

Owing to the fact that researches were then limited to the native soil of the student, most of the botanical authors of that day had but dark inklings of the extent to which the floras of various latitudes and areas differ. They assumed that plants of the Mediterranean shores, which had been described centuries before by Theophrastus or Dioscorides or Pliny, were necessarily the same as those of their own more inclement countries. The German "Fathers of Botany" (Brunfels, born about 1495, died 1534; Bock, 1498-1554; Fuchs, 1501-1566, are the best known) applied the old Greek and Latin names without scruple to the species growing in their own localities. They were so firmly convinced of the identity of the German, Greek, and Italian floras that even the numerous inconsistencies occurring in the descriptions did not disconcert them, or prevent them from discussing at great length whether a particular name was intended by Theophrastus and Dioscorides to indicate this or that plant. It was by slow degrees that botanists first began to abandon these fruitless debates concerning the Greek and Latin names of plants, with which it had been the custom to fill so many pages of the herbals. Step by step they became conscious that although the yellow pages of the ancient books deserved all gratitude for the stimulating influence they had exercised, yet the green book of nature should be set above them. This led to their devoting themselves entirely to direct researches in the subject of their native floras. The 
herbal of Hieronymus Bock, which appeared in 1546, and in which "the herbs growing in German countries are described from long and sure experience," contains a passage treating of the controversy of the day as to whether the Latin name Erica was applicable to the German Heath or not; and in the midst of the discussion the author expresses the opinion that "the plants we know best were the least known to the Latins;" and at last he exclaims: "Be our heath the same as Erica or not, it is in any case a pretty and sturdy little shrub, beset with numerous brown rounded branches, which are clothed all over with small green leaves; and its appearance is like that of the sweet-smelling Lavender Cotton." And again in a number of other places, after making lengthy philological statements relating to the old names, he ends by losing patience and declaring that the proper thing would be to lay aside all disputes concerning this nomenclature.

At length a Belgian, Charles de l'Ecluse (1526-1609), whose name was latinized into Clusius, emancipated himself entirely from the hair-splitting verbal controversies of the day. He was also the first to abandon the utilitarian standpoint; and in his extensive work, which appeared at the end of the sixteenth century, he was guided solely by the desire to become acquainted with every flowering thing. He therefore endeavoured to distinguish, describe, and where possible to draw the various forms of plants, to cultivate them, and to preserve them in a dried condition. It was just at that time that collections of dried plants began to be made. Such a collection was at first called a "hortus siccus," and later on a "herbarium." All museums of natural history were forthwith furnished with them. Moreover, Clusius, actuated by the wish to see with his own eyes what the vegetation on the other side of the mountains looked like, was the first man to travel for the purpose of botanizing. In order to extend his knowledge of plants he roamed over Europe from the sierras of Spain to the borders of Hungary, and from the sea-coast to the highlands of the Tyrol. Journeys of this kind in pursuit of botanical knowledge were by degrees extended to wider and wider limits, and thus an abundance of material was brought together from all latitudes and from every quarter of the globe.

An immense number of isolated observations were accumulated in this way, till, at length, in the first decades of the eighteenth century, the desirability of sifting and arranging this chaotic mass became urgent. When, therefore, the Swedish naturalist Linnæus (1707-1778), by the exercise of unparalleled industry, mastered in a fabulously short space of time the detailed results of centuries of labour, and afforded a general survey of all this scattered material, he obtained universal recognition. Linnæus introduced short names for the various species in place of the cumbrous older designations, and showed how to distinguish the species by means of concise descriptions. For this purpose he marked out the different parts of a plant as root, stem, leaf, bract, calyx, corolla, stamens, pistil, fruit, and seeds. Again, he distinguished particular forms of those organs, as, for instance, scapes, haulms, and peduncles as forms of stems, and in addition also the parts of each organ, such as filaments, anthers, and pollen in the stamens, and ovary, style, and stigma in the pistil; and to each one of these objects he assigned a technical name 
(terminus). With the help of the botanical terminology thus formulated it became possible not only to abridge the specific descriptions, but also to recognize species from such descriptions, and to determine what name had been given them by botanists, and to what group they belonged.

Linnæus selected as a basis of classification in the "System" established by him the characteristics of the various parts of the flower. In this system the number, relative length, cohesion, and disposition of the stamens formed the ground of division into "Classes." Within each Class, "Orders" were then differentiated according to the nature of the pistil, especially the number of styles; and each Order was again subdivided into more narrowly defined groups, which received the name of "Genera." To the 23 classes of Flowering Plants (Phanerogamia) Linnæas added as a 24th Class Flowerless Plants (Cryptogamia), which were divided into several groups (Ferns, Mosses, Algæ, and Fungi) in respect of their general appearance and mode of occurrence.

This system took immediate possession of the civilized world. Englishmen, Germans, and Italians now worked in unison as faithful disciples of Linnæus. Even laymen studied the Linnæan botany with enthusiasm; and it was recommended, especially to ladies, as a harmless pastime, not overtaxing to the mind. In France Rousseau delivered lectures on botany to a circle of educated ladies; whilst even Goethe experienced a strong attraction to the "loveliest of the sciences," as botany was called in that day. Linnæus had introduced for the first time the name "flora" to signify a catalogue of the plants of a more or less circumscribed district. He had himself written a flora of Lapland and Sweden, and by doing so had stimulated others to undertake the compilation of similar catalogues; so that by the end of the 18th century floras of England, Piedmont, Carniola, Austria, \&c., had been produced. By this means a certain perfection was attained in that field of botany which has only in view the examination of the fully-developed external forms of plants, together with the distinguishing, describing, naming, and grouping them, and the enumeration of species indigenous to particular regions. Later on, unfortunately, botanists lost themselves in a maze of dull systematizing. They either contented themselves with collecting, preparing, and arranging herbaria, or else devoted their energies to endless debates over such questions, for instance, as whether a plant, that some author had distinguished from others and described, deserved to rank as a species, or should be reckoned as a variety dependent on its habitat or on local conditions of temperature, light, and moisture. They took delight in now including a group of forms as varieties of a single species, now dividing some species as described by a particular author into several other species. For this purpose they did not rely upon the only sure method, the determination by cultural experiment of the fact of the constancy or variability of the form in question; nor did they, in general, adhere to any consistent principle to guide them in this amusement.

Aberrations of this kind constituted, however, no serious barrier to progress. On the contrary, the passion for collecting continued to extend its range. The 
vegetation of the remotest corners of the earth was ransacked by travelling botanists without any material advantage being gained, though they not infrequently ran considerable risk to their health, and sometimes sacrificed their lives. As one generation succeeded another thousands of students of the "scientia amabilis" made their appearance in every country. Swept along by the prevailing current of thought they devoted themselves to the examination of native and foreign floras, or to a detailed study of the most insignificant sections of the vegetable kingdom. Those who are not under the spell of this passion cannot conceive the joy experienced by the discoverer of a hitherto unknown moss. To such it is inexplicable how anyone can devote the labour of half a lifetime to a classification of Algæ or Lichens, or to a monograph of the bramble-tribe or orchids. The progress achieved eventually in this department of botany is best appreciated when the wide difference in the numbers of species described in botanical works of different periods is considered. "Theophrastus in his Natural History of Plants (about 300 B.c.) mentions about 500 species, and Pliny (78 A.D.) rather more than 1000; whereas, by the time of Linnæus, about 10,000 were known; and now the number must be all but 200,000. It should be remarked, however, that half the plants described since Linnæus lived fall into the category of Cryptogams, or nonflowering plants, the examination of which was first rendered possible by the widespread use of the microscope in recent times.

The microscope led also to discoveries concerning the internal architecture of plants. A faint attempt in this direction, made 200 years ago, had died away without leaving any trace behind; but at the commencement of this century the "inward construction of plants" was studied all the more eagerly by means of the microscope. In buildings belonging to different styles of architecture it is not only the forms of the wings, stories, rooms, and gables that differ, but also and in no less degree those of the columns, pilasters, and decorations. The same is the case with plants. They possess chambers at different levels, vaults, and passages. They have pipes running through them, and beams and buttresses, some massive and some slender, to support them. The pieces of which they are built vary in size, and their walls are sculptured in all kinds of ways. It was the business of the vegetable anatomist to dissect plants, to look into all these structures under the microscope, to describe the various component parts as well as the ground-plan and elevation of the plant-edifice as a whole; and to name the different forms of structure after the manner of Linnæus when he invented terms for the different forms of stems and leaves, and for the several parts of the flower and fruit.

\section{DOCTRINE OF METAMORPHOSIS AND SPECULATIONS OF} NATURE-PHILOSOPHY.

Side by side with this immense volume of research, which was directed to the separation, description, and synoptical arrangement of mature forms only, there arose about the year 1600 another school which considered vegetable forms from 
the point of view of their life-history, and endeavoured to trace them back to their origin. Tracing the development, from one stage to another, of all the different species, of the multitudinous forms of leaves and flowers, and of the various kinds of cells and tissues, the student of this school has to detect identity in multiplicity, to show that the connection between forms which have arisen from one another is in accordance with fixed laws, and to express those laws in definite formulæ.

The attention of botanists was in the first place directed to the wonderful series of changes in the form of the leaf which occur in all phanerogamic (i.e. flowering) plants as the delicate seedling gradually turns into a flowering shoot. At the circumference of the stem which constitutes the axis of the plant, foliar structures are produced at successive intervals. All these structures are essentially the same; but they exhibit a continuous modification of their shape, arrangement, size, and colour, according to their relative altitudes upon the stem. To discover the causes of this structural variation was an attractive problem, and very diverse theories were suggested for its solution. The earliest explanation, which was given by the Italian botanist Cesalpino in 1583, is founded rather on superficial analogies and remote resemblances existing between tissues than on careful observation. According to this theory the stem is composed of a central medulla highly endowed with vitality, and surrounded by concentric layers of tissue, those namely of the wood, the bast, and the cortex. Each of the foliar structures put forth from the axis is supposed to originate in one of the above-named tissues, the idea being that the green foliageleaf and calyx grew out from the cortical layer, the corolla from the bast, the stamens from the wood, and the carpels from the medulla. It was believed, also, that the outer envelope of a fruit arose from the rind of the fruit-stalk, the seedcoats from the wood, and the central part of the seed from the medulla.

Early in the eighteenth century there came to be connected with this theory the doctrine of so-called "prolepsis," which was founded on more accurate comparative observations. It was thought that the medulla of the stem breaks through the rind at particular spots to form at each a bud, which subsequently grows out into a side branch. Owing to this lateral pressure of the medulla the ascending nutrient sap becomes arrested beneath the rudimentary bud, and, in consequence, the cortex develops under the bud into a foliage-leaf. In the bud the different parts of the future annual shoot are already shadowed forth in stages one above the other; and each is produced always by the one beneath it. As soon as vegetative activity is resumed after the expiration of the winter rest, the bud sprouts. If only that part of it develops which constitutes the first year's rudiment, a shoot furnished with foliage-leaves is produced. But the embryonic structures belonging to succeeding years, which are concealed in the bud, may also be stimulated to development; and when this happens, these premature products do not appear as foliage-leaves, but in more or less altered forms as bracts, sepals, petals, stamens, and carpels. If no such anticipatory activity has been excited, the rudiment which in the previous case would have developed into a bract does not appear till the following year, and then as a foliage-leaf; whilst that which would have formed a calyx in the first 
year lies dormant till the third year, when it too emerges simply as a leaf. This transformation of the leaves, or metamorphosis as Linnæus called it, is, therefore, the result of anticipation; and it was assumed by the Linnæan school that the cause of this metamorphosis or hastened development was a local decrease in the quantity of nutriment. The idea was, that in consequence of the limited supply of sap the incipient leaves were not able to attain to the size of foliage-leaves, but remained

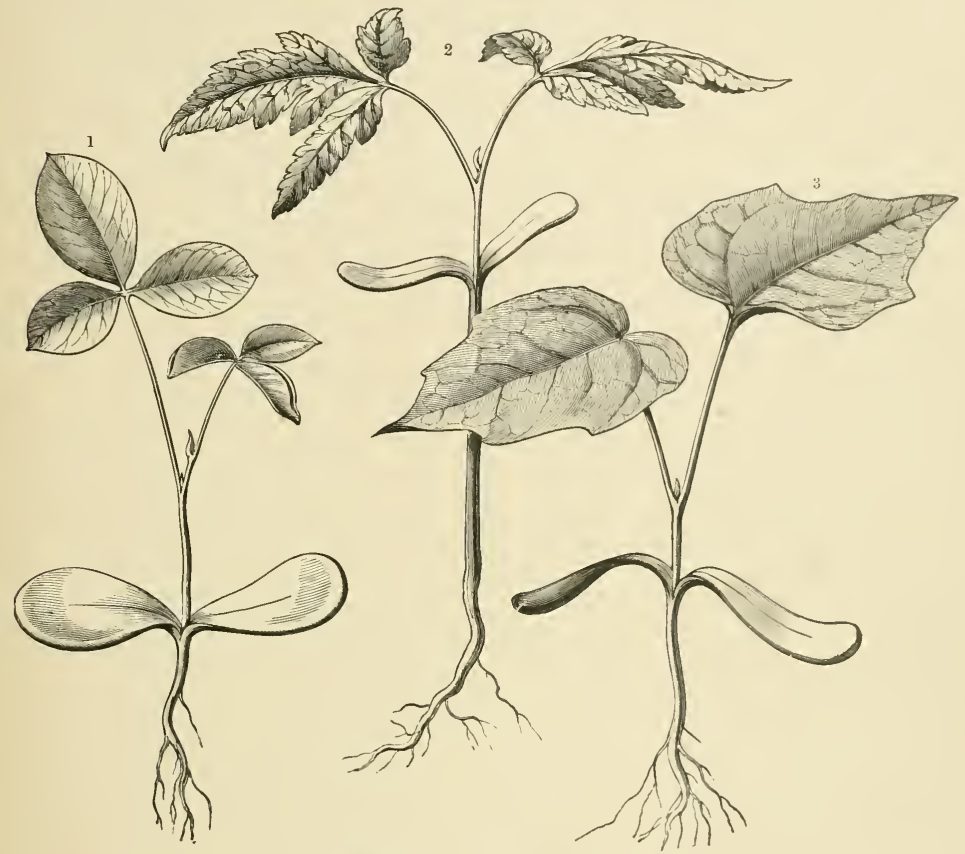

Fig. 1.-Seedlings with Cotyledons and Foliage-leaves.

1 Cytisus Laburnum. 2 Koelreuteria paniculata. 3 Acer platanoides.

rudimentary, as is the case with many bracts; and further, that the axis was no longer capable of elongating, so that the leaves proceeding from it remained close together, became coherent, and thus formed the calyx. The supporters of this explanation relied particularly on the experience of gardeners, that a plant in good soil with a liberal supply of nutriment is apt to produce leafy shoots rather than flowers; whereas, if the same plant is transferred to a poorer soil, where its food is limited, it develops flowers in abundance.

But yet a third attempt was made to explain this process of transformation, by the theory that parts which are identical so far as their origin is concerned, subsequently receive the stamp of distinct foliar organs. The diversity in the development of parts, originally alike, was supposed to depend on a filtration of the nutrient 
sap, the idea being that identical primordial leaves issuing from the axis of a particular plant were fashioned with more and more delicacy as the sap became clarified and refined in its passage through the vessels. This explanation of metamorphosis was first given by Goethe (1790) in a treatise which was much discussed, and which exercised a most important influence in initiating researches of a similar nature. Goethe's interpretation of metamorphosis may be briefly reproduced as follows. A plant is built up gradually from a fundamental organ-the leaf-which issues from the node of a stem. First of all, the organs which are called seed-leaves or cotyledons (fig. 1) develop on the young plant as it germinates from the seed; they proceed from the lowest node of the stem, and are frequently subterranean. They are of comparatively small size, are simple and unsegmented, have no trace of indentation, and appear for the most part as thick, whitish lobes, which are, according to Goethe's expression, closely and uniformly packed with a raw material, and are only coarsely organized. Goethe explains these leaves as being of the lowest grade in the evolutionary scale. After them and above them the foliage leaves develop at the succeeding nodes of the stem; they are more expanded both in length and breadth; their margins are often notched, and their surfaces divided into lobes, or even composed of secondary leaflets; and they are coloured green. "They have attained to a higher degree of development and refinement, for which they are indebted to the light and air." Still further up, there next appears the third stage in foliar evolution. The structure called by Linnæus the calyx is again to be traced back to the leaf. It is a collection of individual organs of the same fundamental type, but modified in a characteristic manner. The close-set leaves, which proceed from nodes of the stem at what is, in a certain sense, the third story of the plant-edifice as a whole, and which constitute the calyx, are contracted, and have but little variety as compared with the outspread foliage-leaves.

On the fourth rung of the ladder by which the leaf ascends in its effort to perfect itself, appears the structure named in the Linnæan terminology the corolla. It consists, like the calyx, only of several leaves grouped round a centre. If a contraction has taken place in the case of the calyx, we have now once more an expansion. The leaves which compose the corolla are usually larger than those of the calyx. They are, besides, more delicate and tender, and are brightly coloured; and Goethe, whose mode of expression is here preserved as far as possible, supposes them to be filled also with purer and more subtle juices. He conceives that these juices are in some manner filtered in the lower leaves and in the vessels of the lower region of the stem, and so reach the upper stories in a more perfect condition. A more refined sap must then, he says, give rise to a softer and more delicate tissue (fig. 2). Above the corolla and at the fifth stage of development there follows the group of stamens, structures which, though not answering to the ordinary conception of leaves, are yet to be regarded again simply as such. In the circle of the corolla the leaves were expanded, and conspicuous owing to their colour; on the other hand, in the stamens they are contracted to an extreme degree, being almost filamentous in part. These leaves appear to have reached a high degree of perfection, 
and in the parts of the stamens termed anthers "pollen-grains" are developed "in which an extremely pure sap is stored." Adjoining these pollen-producing leaves.
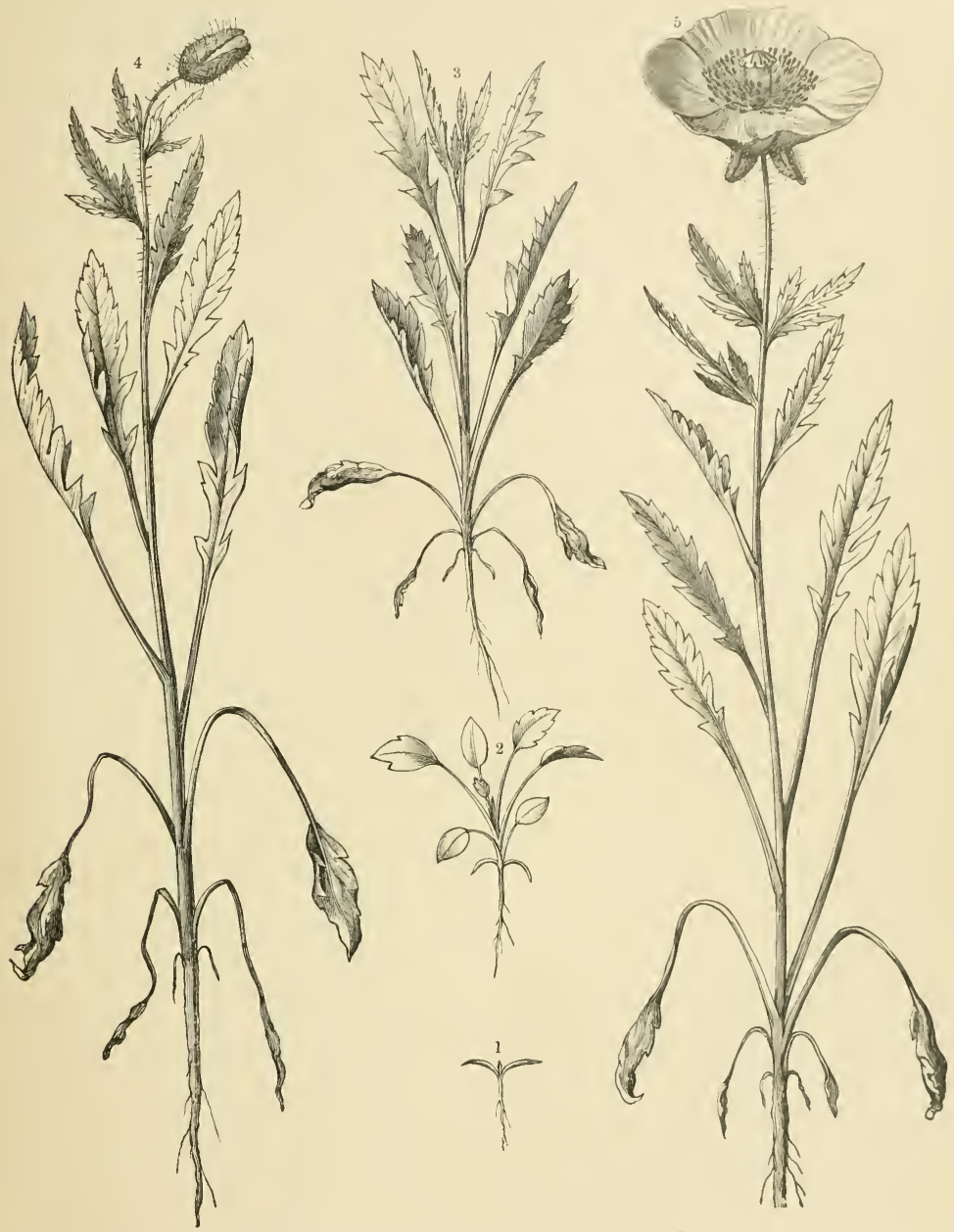

Fig. 2.-Metamorphoaes of Leaves as exhibited by the Poppy.

1 Germinating plant with cotyledons. 2 and 8 The same plant further developer and with foliage-leaves; in $s$ the cotyledons and lowest foliage-leaves are already withered. 4 The same plant with a flower-bud showing the closed sepals. The bud open and with petals, stamens, and carpels (pistil) developed.

where contraction has reached its extreme limit, is the sixth and last story, which is composed of leaves, once more less closely-set, and exhibiting a final expansion on the part of the plant. These are the carpels, which surround the highest part 
of the stem and inclose the seeds, the latter being developed from the tip of the stem. Thus the plant accomplishes its life-history in six stages. It is built up of leaves, the "intrinsic identity" of which cannot be doubted, although they assume extremely various shapes corresponding to the six strides towards perfection. In this process of transformation or metamorphosis of the leaf there are three alter-

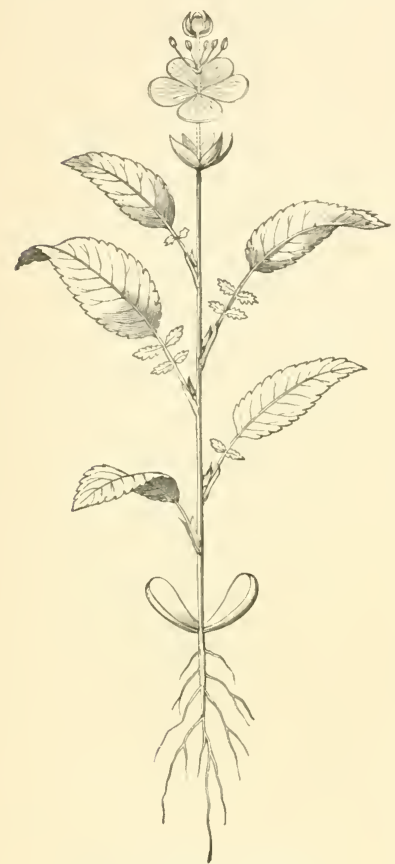

Fig. 3.-Goethe's "Urpflanze." nate contractions and expansions, whilst each stage is more perfect than the one next below it.

Whilst seeking to explain metamorphosis in this manner, and endeavouring, with greater perspicacity than all his predecessors and contemporaries, "to reduce to one simple universal principle all the multifarious phenomena of the glorious garden of the world," Goethe conceived the notion of a typical plant, an ideal, the realization of which is achieved in nature by means of a manifold variation of individual parts. This abstract notion of a plant's development with its six stages corresponding to "three wave-crests" or expansions (Leaf, Petal, Carpel) and "three wavetroughs” or contractions (Cotyledon, Sepal, Stamen) is expressed graphically in figure 3. It still holds its ground at the present day under the name of Goethe's "Urpflanze," and the credit of its invention is entirely his. But it is not quite right to claim for Goethe, in addition, the title of founder of the doctrine of vegetable metamorphosis; for in reality he only offered another interpretation and mode of representation of a phenomenon already included by Linnæus under the term metamorphosis. Linnæus had instituted a comparison between the metamorphosis of plants and that of insects; in particular, he likened the calyx to the ruptured integument of a chrysalis and the internal parts of a flower to the perfect insect (Imago). He also made many different attempts to establish analogies between the development of plants and that of animals; and in so doing he opened up a wide field for the speculations of the "nature philosophers" in the earlier part of the nineteenth century.

An extensive study of this subject now commenced; and writers on naturephilosophy worked indefatigably at the amplification and modification of this theme, first broached by Linnæus.

"A plant is a magnetic needle attracted towards the light from the earth into the air. It is a galvanic bubble, and, as such, is earth, water, and air. The plantbubble possesses two opposite extremities, a single terrestrial end and a dual aërial end; and so plants must be looked upon as being organisms which manifest a 
continual struggle to become earth on the one hand and air on the other, unmixed metal at one end, and dual air at the other. A plant is a radius, which becomes single towards the centre, whilst it divides or unfolds towards the periphery; it is not therefore an entire circle or sphere, but only a segment of one of those figures. The individual animal, on the contrary, constitutes of itself a sphere, and is therefore equivalent to all plants put together. Animals are entire worlds, satellites or moons, which circle independently round the earth; whereas plants are only equal to a heavenly body in their totality. An animal is an infinitude of plants. A blossom which, when severed from the stem, preserves by its own movement the galvanic process or life, is an animal. An animal is a flower-bubble set free from the earth and living alone in air and water by virtue of its own motion."

Page after page of the writings on Nature-philosophy of Oken (1810) and other contemporary naturalists is filled with interminable statements of the same kind. At the present day it seems scarcely credible that such propositions were then received with admiration as profound and ingenious utterances, and that they were even adopted as mottoes for botanical and geological treatises. For example, it is worthy of record that as late as the year 1843 the Austrian botanist Unger made use of the last of the flowers of rhetoric above quoted from Oken's Naturephilosophy as a motto for one of his first works on the history of development, the title of which is Plants at the Moment of their becoming Animals.

The general divisions or systems of the vegetable kingdom which were evolved by adherents of the school of Nature-philosophy were, as may be imagined, just as absurd as the speculations on which they were based. In his Philosophical Systems of Plants Oken develops in the first place the idea that the vegetable kingdom is a single plant taken to pieces. Inasmuch as the ideal highest plant is composed of five organs, there must likewise be five classes: root-plants, stem-plants, leafplants, flower-plants, and fruit-plants. The world is fashioned out of the elements: earth, water, air, and fire. Hereupon is founded a classification of root-plants into earth-plants or lichens, water-plants or fungi, air-plants or mosses, and light-plants or ferns. Proceeding from the assumption that all the groups are parallel and that the principle of classification for each group is always given by the one preceding it, we have next, to take one instance, the second class-that of stem-plantsdivided (in accordance with the subdivision of earth into earths, salts, bronzes, and ores) into earth-plants or grasses, salt-plants or lilies, bronze-plants or spices, and ore-plants or palms.

\section{SCIENTIFIC METHOD BASED ON THE HISTORY OF DEVELOPMENT.}

Though as we see the doctrine of metamorphosis, with its conception of a typical plant, degenerated thus into the most barren of fancies, still from it originated the line of research based on the history of development which has since borne fruit in every department of botany. Observers arrived at the conviction that every living plant undergoes a continuous transformation which follows a definite 
course, and that accordingly every species is constructed on a plan fixed within general limits and exhibiting variation in externals only. These, it is true, are often more conspicuous at first sight than the direction and disposition of the parts which are really fundamental, and secure the stability of the entire structure. But in order to ascertain the plan of construction it was found necessary to go back to the very first visible appearance of each organ; to determine how the original rudiments of the embryo and the beginnings of roots, stems, leaves, and parts of the flower are formed, and to see what rudiments succeed in opening out, branching and dividing, and what remain behind to perish and be displaced by organs growing vigorously in close proximity to them.

These researches into the course of development of the separate parts of flowering plants, and to a still greater extent the observations of the development of cryptogams or spore-plants (rendered possible by improvements in the construction of microscopes), led naturally to a study of the history of the elementary structures of which all plants are composed. Previously three kinds of elementary organs had been supposed to exist, utricles, vessels, and fibres. The observations of Brown and Mohl (1830-1840) resulted, however, in the identification of the cell as the common starting-point of all these elementary organs. This led to the further discoveries that protoplasm is the formative and living part of a cell, and that each cell is differentiated into a protoplasmic cell-body and a cell-membrane. It followed that the envelope of the protoplasmic body, the cell-membrane, which had hitherto been considered the primary formation, was in reality a product of the protoplasm enveloped by it, and this discovery resulted in a complete revolution in the conception of cells generally. Further investigation led to the conclusion that the various modes of growth and multiplication depend on definite laws. That even in the mode of juxtaposition of daughter-cells arising in reproduction, a certain plan of construction may be distinguished in each species which must stand ultimately in some causal relation to the structural system of the whole plant. The progress achieved along these lines in the course of a few decades has been extraordinarily great, no doubt due to the peculiar fascination which the study of the life-histories and transformations of living organisms and the observation of mysterious processes invisible to the naked eye have had for the mind of the inquirer.

In that group of plants which includes the forms classed together by the earlier botanists under the name of Cryptogamia an altogether new world was revealed. An undreamed-of variety was discovered to exist in the processes of propagation and rejuvenescence of these forms of plants by means of single cells or spores. Objects which, having regard to their external form, had been assigned to widely different groups, were found to be connected with one another as stages in the development of one and the same species; and one result of these discoveries was the establishment in this division of the vegetable kingdom of an entirely new system of classification based on life-histories. The systematic arrangement of Flowering-plants or Phanerogams also underwent essential alteration. The Linnæan system, founded on the numerical relations between the different parts of the flower, 
had indeed already been displaced by another method of classification, that of the French observers Jussieu (1789) and De Candolle (1813), who framed systems said to be natural when contrasted with the artificial system of Linnæus. At bottom, however, these classifications only differed from the Linnæan in the fact that they multiplied and widened the grounds of division. The main division of Phanerogamia into those which put forth one cotyledon (or seed-leaf) on germinating (Monocotyledones) and those whose seedlings bear two cotyledons (Dicotyledones) is the only one that could serve as a starting-point for a system based on the history of development; but when we come to the grouping of Dicotyledones into those destitute of corolla (Apetalæ), those with the corolla composed of coherent petals (Monopetalæ), and those with the corolla composed of distinct petals (Dialypetalæ), we have already to admit something forced, and a reliance on characteristics merely external.

The system which is the outcome of the study of development starts with the idea that similarity between adult forms is not always decisive evidence of their belonging to the same group, and that the relationships of different plants is much more surely indicated by the fact of their exhibiting the same laws of growth and the same phenomena of reproduction. Plants exhibiting widely different external forms in the mature state are nevertheless to be looked upon as closely allied if they are constructed according to the same plan, and vice versâ. There can be no question that a system based on these principles means a material advance. At the same time it cannot be overlooked that great difficulties are involved in hitting upon the right selection from among the number of phenomena observed in the course of a plant's development, and in determining which of these phenomena are to be referred to a mode of construction common to a number of plants, and therefore treated as fundamental properties, and which should be esteemed merely as outcomes of the conditions of life affecting the existence of the plant in question.

\section{OBJECTS OF BOTANICAL RESEARCH AT THE PRESENT DAY.}

Descriptive Botany only concerns itself with the configuration of a plant. Comparative Morphologr endeavours to trace back to a single prototype the extremely various forms exhibited by mature plants. The history of development deals with the growth and differentiation of such forms. But all these paths of research shirk the problem of the biological significance of the different forms. The line of investigation starting from the conception of a plant's life as a series of physical and chemical processes, and which attempts to elucidate the configuration of a plant in the light of its environment, could not be developed with the slightest prospect of success until physics, chemistry, and other allied sciences had reached a high degree of perfection, and till botanists had become convinced that the phenomena of life are only to be fathomed by means of experiment.

The earliest attempts to define the biological significance of the several parts of 
a plant do, it is true, take one back as far as Aristotle and his school; but the ideas of vegetable life entertained at that time are scarcely more than fantastic dreams; and the recognition now accorded to them springs rather from a reverence for antiquity than from any intrinsic merit which they possessed. The first experimental investigations into the vital phenomena of plants were published by Stephen Hales in 1718; but it was not till a hundred years later that this kind of research really came into vogue. It brought with it the conception of a cell as a miniature chemical laboratory, and looked for mechanical interpretations of the phenomena of nutrition, sap-circulation, growth, movement-in short, all vital processes-and for some connection between these processes and the external form. Whereas, in the case of descriptive and speculative botany, and in the study of development, the entire plant was first taken into consideration, next its several parts, and lastly the cells and protoplasm; in the new department of inquiry, on the contrary, the complete histories of the ultimate organs were studied first of all, then the significance of the different forms of the several members, and lastly the phenomena occasioned by the aggregate life of all the various kinds of animals and plants.

Modern science, governed as it is by the desire to lay bare the causes of all phenomena, is no longer satisfied with knowledge concerning the existence of cells, the arrangement of the different forms of cell, the development of their contents, and the changes undergone by cell-membranes. At the present day we inquire what are the functions of the various bodies which are formed within the protoplasm? Why is the cell-membrane thickened at a particular spot in a particular manner? What is the meaning of all the tubes and passages which exhibit such great diversity of size and shape? What part is played by the peculiar mouths of these channels, and why do they vary so greatly in shape and distribution in plants which are subject to different external conditions? We are no longer content to determine in what manner the rudimentary organ of a plant is produced, or how it expands in one case and frequently divides, or else is arrested in its growth and shrivels up; but we inquire the reason why one rudiment grows and develops whilst another is obliterated. For us no fact is without significance. Our curiosity extends to the shape, size, and direction of the roots; to the configuration, venation, and insertion of the leaves; to the structure and colour of the flowers; and to the form of the fruit and seeds; and we assume that even each thorn, prickle, or hair has a definite function to fulfil. But efforts are also made to explain the mutual relations of the different organs of a plant, and the relations between different species of plants which grow together. Lastly, this department of research (the rapid growth of which is due to Darwin) includes amongst its objects a solution of the problem of the ultimate grounds of morphological variety, the causes of which can only be sought for in a qualitative variation of protoplasm. Specific relationship is explained by attributing it to similarity in the constitution of the protoplasm of allied species, and the affinities exhibited by living and extinct plants are used as means of unfolding the hereditary connection between the 
thousands of different sorts of forms, and of tracing the history of plants and vegetable life all over the earth.

The various lines of botanical research described in the foregoing pages, with their particular problems and objects, have but slight connection one with another. They run side by side along separate paths, and it is only occasionally that a junction is apparent which establishes a communication between one path and another. The subject-matter, however, is always the same. Whether we have to do with the perfected form or with its growth, whether we try to interpret the processes of life or to trace the genealogy of the vegetable kingdom, we always start from the forms of plants; and the ultimate result is never anything more than a description of the varying impressions which we receive at different times from the objects observed, and which we endeavour to bring into mutual connection. All the different departments of botany are accordingly more or less limited to description; and even when we endeavour to resolve vital phenomena into mechanical processes we can only describe, and not really explain, what happens. The processes which we call life are movements. But the causes of those movements, so-called forces, are purely subjective ideas, and do not involve the conception of any actual fact, so that our passion for causality is only ostensibly gratified by the help of mechanics. Du Bois Reymond is not far wrong when he follows out this train of thought to the conclusion (however paradoxical it may sound) that there is no essential difference between describing the trajectory (or particular kind of curve) in which a projectile moves on the one hand, and describing a beetle or the leaf of a tree on the other.

But even though the ultimate sources of vital phenomena remain unrevealed, the desire to represent all processes as effects, and to demonstrate the causes of such effects-a desire which is at the very root of modern research-finds at least partial gratification in tracing a phenomenon back to its proximate cause. In the mere act of linking ascertained facts together, and in the creation of ideas involving interdependence among the phenomena observed, there lies an irresistible charm which is a continual stimulus to fresh investigations. Even though we be sure that we shall never be able to fathom the truth completely, we shall still go on seeking to approach it. The more imaginative an investigator the more keenly is he goaded to discovery by this craving for an explanation of things and for a solution of the mute riddle which is presented to us by the forms of plants. It is impossible to overrate the value and efficiency of the transcendent gift of imagination when applied to questions of Natural History. Thus when we inquire whether certain characters noted in a plant are hereditary, constant, and inalienable, or are only occasioned by local influences of climate or soil, and hence deduce whether the plant in question is to be looked upon as a species or a variety; when we conclude from the fact of a resemblance between the histories of the development of various species that they are related, and place them together in groups and series; when we unravel the genealogies of different plants by comparing forms still living with others that are extinct; when we try to represent clearly Vor. I. 
the molecular structure of the cell-membrane by arguing from the phenomena manifested by that membrane; when we investigate the meaning of the peculiar thickenings and sculpturings of the walls of cells, or when we discover the strange forms of flowers and fruits to be mechanical contrivances adapted to the forms of certain animals, and judge the extent to which these contrivances are advantageous, or the reverse, to the plants-in all these and similar investigations imagination plays a predominant part. Experiment itself is really a result of the exercise of that faculty. Every experiment is a question addressed to nature. But each interrogation must be preceded by a conjecture as to the probable state of the case; and the object of the experiment is to decide which of the preliminary hypotheses is the right one, or at least which of them approaches nearest to the true solution. The fact that when the imagination has been allowed to soar unrestrained, or without the steadying ballast of actual observations, it has frequently led its followers into error, does not detract at all from its extreme value as an aid to research, notwithstanding the fact that it is responsible for the wonderful fantasies of nature-philosophy of which a few specimens have been given. Nor should we esteem it the less because enlargements of the field of observation and improvements in the instruments employed have again and again led to the substitution of new ideas for those which careful observers and experimentalists had arrived at by collating the facts ascertained through their labours.

For the same reasons it is unfair to regard with contempt the ideas of plantlife formed by our predecessors. It should never be forgotten how much smaller was the number of observations upon which botanists had to rely in former times, and how much less perfect were their instruments of research. Every one of our theories has its history. In the first place a few puzzling facts are observed, and gradually others come to be associated with them. A general survey of the phenomena in question suggests the existence of a definite uniformity underlying them; and attempts are made to grasp the nature of such uniformity and to define it in words. Whilst the question thus raised is in suspense, botanists strive with more or less success to answer it, until a master mind appears. He collates the observed facts, gathers from them the law of their harmony, generalizes it, and announces the solution of the enigma. But observations continue to multiply; scientific instruments become more delicate, and some of the newly-observed facts will not adapt themselves to the scheme of the earlier generalization. At first they are held to be exceptions to the rule. By degrees, however, these exceptions accumulate; the law has lost its universality and must undergo expansion, or else it has become quite obsolete and must be replaced by another. So it has been in all past times, and so will it be in the future. Only a narrow mind is capable of claiming infallibility and permanence for the ideas which the present age lays down as laws of nature.

These remarks on the limitations of our knowledge of nature, the importance of imagination as an aid in research, and the variability of our theories are made with a view to moderate, on the one hand, the exuberant hopes raised by the belief 
that the great questions connected with the phenomenon of life will be solved, and to correct, on the other, the habit of not appreciating impartially the various methods which have been and are still employed by different botanists. In our own time, adhering as we do to the principle of the division of labour, it has become almost universal for each investigator to advance only along a single, very narrow path. But owing to the fact that one-sidedness too often leads to self-conceit, the lines of study followed by others are not infrequently despised, just as overweening confidence in the infallibility of the discoveries of the present day leads to depreciation of the labours of former times.

For the building-up of the science of the Biology of Plants everything relating to the subject has its value, and is capable of being turned to account. Whether the materials are rough or elaborated, massive, fragmentary, or merely connective, howsoever and whensoever they have been acquired, they all are useful. The study of dried plants made by a student in a provincial museum, the discoveries of an amateur regarding the flora of a sequestered valley, the contributions of horticulturalists on subjects of experiment, the facts gleaned by farmers and foresters in fields and woods, the disclosures which have been wrested from living plants in university laboratories, and the observations conducted in the greatest and best of all laboratories-that of Nature herself-all these results should be turned to account. Let us take for the motto of the following pages the text:

"Prove all things; hold fast that which is good." 



\title{
THE LIVING PRINCIPLE IN PLANTS.
}

\author{
1. PROTOPLASTS CONSIDERED AS THE SEAT OF LIFE.
}

Discovery of the Cell.-Discovery of Protoplasm.

\section{DISCOVERY OF THE CELL.}

What is life? This ever-interesting question has seemed to approach nearer solution on the occasion of every great scientific discovery. But never did the hope of being able to penetrate the great secret of life appear better founded than at the time when, among other memorable developments of science, it was discovered that objects could be rendered visible on an enlarged scale by the use of glass lenses, and the microscope was invented. These magnifying glasses were expected to yield, not only an insight into the minute structure of living beings which is invisible to the naked eye, but also revelations concerning the processes which constitute life in plants and animals. The first discoveries made with the microscope, between 1665 and 1700, produced a profound impression on the observers. The Dutch philosopher Swammerdam became almost insane at the marvels revealed by his lenses, and at last destroyed his notes, having come to the conclusion that it was sacrilege to unveil, and thereby profane, what was designed by the Creator to remain hidden from human ken. The observations of Leeuwenhoek (1632-1723) with magnifying glasses formed by melting fine glass threads in a lamp, were for a long time held to be delusions; and it was not till the English observer Robert Hooke had confirmed the fact of the existence of the minute organisms seen by Leeuwenhoek in infusions of pepper, and had exhibited them under his microscope in 1667 at a meeting of the Royal Society in London, that doubts as to their actual existence disappeared. Indeed a special document was then drawn up and signed by all those who were satisfied, on the evidence of their own eyesight, of the accuracy of the observation; and this clearly shows how greatly people were impressed with the importance of these discoveries. Of the different forms of the tiny organisms, amounting to nearly four hundred, which were at that time distinguished, and all included under the name Infusoria, because first seen in infusions of peppercorns, some only are at the present day reckoned as animals. In many cases it has been ascertained that they are the spores of plants, whilst others again belong to the boundary-land where the animal and vegetable kingdoms are merged.

The presence or absence of movement used to be considered as the most decisive mark of the difference between animals and plants, and, accordingly, all the minute 
beings which were seen bustling about in watery media were described and labelled as animals. No movement was found in the higher plants which were studied with the microscope about the same time by Dutch, Italian, and English observers; but, on the other hand, these investigations led to a recognition of the quite special peculiarities of such structures as leaves and stem, wood and pith. These parts of plants appeared under the microscope like honey-combs, which are built up of a
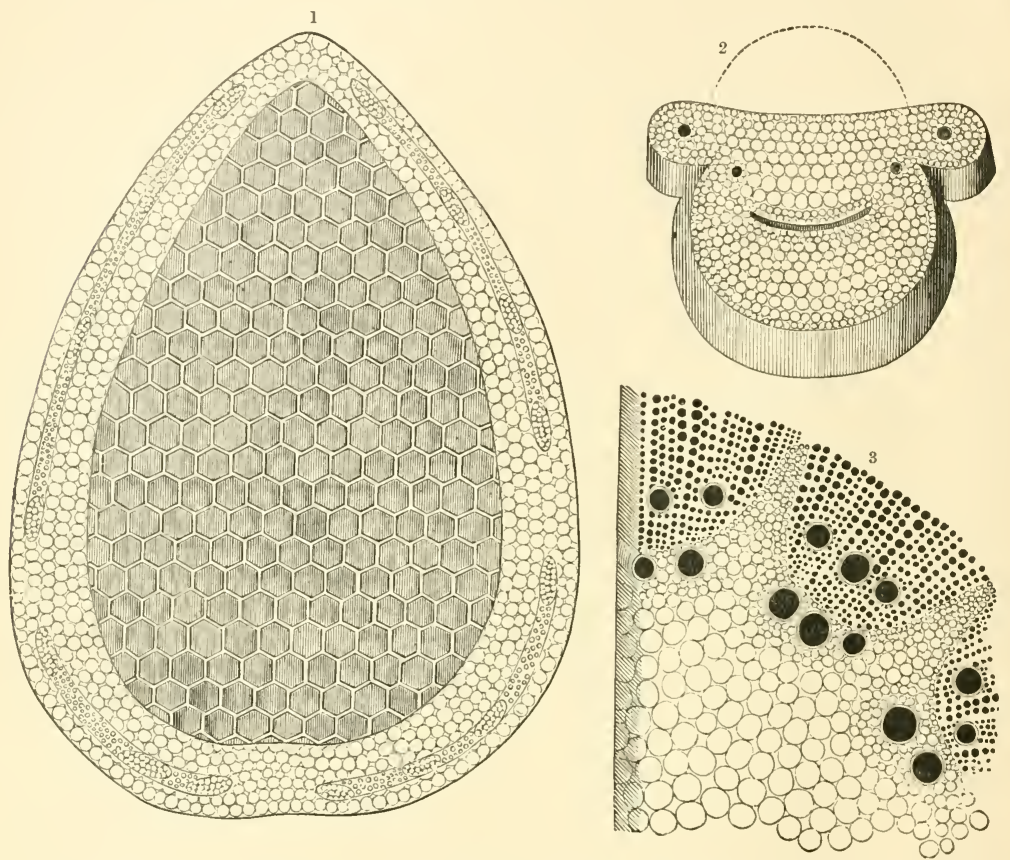

Fig. 4.-Vegetable Cells (from Grew's Anatomy of Plants).

1 Longitudinal section through a young apricot seed. 2 Transverse section of the petiole of the Wild Clary. 8 Transverse section of a pine branch.

great number of cells, some empty and some full of honey. From this similarity the term "cell" arose, which later was to play so important a part in botany. In the drawings of parts of plants as seen under the microscope the resemblance to a honey-comb is very apparent; indeed, it is sometimes rather more striking than when seen in reality, as, for instance, is the case in the above reproduction of three engravings from Nehemiah Grew's fine work published in London, 1672. It was also noticed that, besides the structures which resembled honey-comb, there were little tubes and fibres which were distributed and aggregated in very various ways, and were bound up together into strands and membranes, and into pith and wood; further, all these things were seen to increase in size and number in the growing 
parts of plants. How growth and multiplication took place, and where exactly the seat of a plant's life lay, remained, of course, obscure. It was, however, natural to assume that the walls of these small cells constituted the essential part and living substance of plants, that they drew materials from the fluids which rose by suction in the tubes, and so increased in size and were renewed.

It was as yet hardly suspected that the slimy substance which filled the cells of a plant, like honey in a honey-comb, was the basis of life. The observation made again and again at the beginning of the nineteenth century, that the cell-contents of certain algæ are extruded in the form of globules of jelly, and that each globule moves independently and swims about in the water for a time, but then comes to rest and becomes the starting-point of a new alga, might undoubtedly have led to this conclusion. The accounts of these occurrences were, however, considered incredible by the majority of contemporary observers; and it was not till recently, when Unger established the phenomenon as an indubitable fact, that a proper estimation of its value was accorded. In the year 1826 this botanist investigated under the microscope a water-weed found at Ottakrinn, near Vienna, which had been described by systematic writers as an alga, and named Vaucheria clavata. To the naked eye it appears like a dense plexus of dark-green irregularly branched and matted filaments. These filaments, when magnified, are seen to be tubular cells which wither and die away at the base whilst growing at the apex, and developing sac-like branches laterally. (Fig. 25A.) The free ends of these tubes are blunt and rounded. The substance they contain is slimy, and, though itself colourless, is studded throughout with green granules; whilst near the blunt end of each filament these green particles are so closely packed that the entire contents of that part appear of a dark-green colour.

Now, there comes a time in the life of every one of these filaments when its extremity swells and becomes more or less club-shaped. The moment this occurs, the dark-green contents withdraw somewhat from the extremity, leaving it hyaline and transparent. Almost simultaneously the contents of the swollen part of the tube nearest the apex become transparent, whilst further down the colour becomes very dark. (Figure 25A,a.) Twelve hours after the commencement of this change, that portion of the tube's contents which occupies the club-shaped end separates itself entirely from the rest. A little later, the cell-wall at the apex of the tube suddenly splits, the edges of the slit fold back, and the inclosed mass travels through the aperture (fig. $c$ ). This jelly-like ball, having a greater diameter than the hole, is at first strangulated as it struggles forward, so that it assumes the shape of an hour-glass and looks for an instant as if it would remain stuck fast. There now arises, however, in the entire mass of green jelly an abrupt movement of rotation combined with forward straining, and in another instant it has escaped through the narrow aperture and is swimming freely about in the surrounding water (fig. $d$ ). The entire phenomenon of the escape of these bodies takes place between 8 and 9 A.M., and, in any one case, in less than two minutes. When free, each individual assumes the shape of a perfectly regular ellipsoid (fig. $d$ ), having 
one pole of a lighter green than the other; it moves always in the direction of the former, so that the lighter end may be properly designated the anterior. At first the ball rises to the surface of the water towards the light, but soon after it again sinks deep down, often turning suddenly half-way round and pursues for a time a horizontal course. In all these movements it avoids coming into collision with the stationary objects which lie in its path, and also carefully eludes all the creatures swimming about in the same water with it. The motion is effected by short processes like lashes or "cilia," which protrude all round from the enveloping pellicle of the jelly-like body and are in active vibration. With the help of these cilia, which occasion by their action little eddies in the water, the whole ball of green jelly moves in any given direction with considerable rapidity. But at the same time as it pushes forward, the ellipsoid turns on its longer axis, so that the resultant motion is obviously that of a screw. It is worthy of note that this rotation is invariably from east to west, that is, in the direction opposed to that of the earth. The rate of progress is always about the same: a layer of water of not quite two centimetres $(1.76 \mathrm{~cm}$.) is traversed in one minute. Now and then, it is true, the swimming ellipsoid allows itself a short rest; but it begins again almost immediately, rising and sinking, and resumes its movements of rotation and vibration. Two hours after its escape the movements become perceptibly feebler, and the pauses, during which there is only rotation and no forward motion of the body, become both longer and more frequent.

At length the swimmer attains permanent rest. He lands on some place or other, preferably on the shady side of any object that may be floating or stationary in the water. The axial rotation ceases, the cilia stop their lashing motion and are withdrawn into the substance of the body, and the whole organism, hitherto ellipsoidal and lighter at its anterior end, becomes spherical and of a uniform darkgreen colour. So long as it is in motion the gelatinous body has no definite wall. Its outermost layer is, no doubt, denser than the rest; but no distinct boundary is to be recognized, and we cannot properly speak of a special enveloping coat. No sooner, however, is the ball stranded, no sooner has its movement ceased and its shape become spherical, than a substance is secreted at its periphery; and this substance, even at the moment of secretion, takes the form of a firm, colourless, and transparent membrane. Twenty-six hours afterwards, very short branched tubes begin to push out from the interior, and these become organs of attachment. In the opposite direction the cell stretches into a long tube which divides into branches and floats on the water. After fourteen days the free ends of this tube and of its branches swell once more and become club-shaped; a portion of their slimy contents is, as before, separated from the rest and liberated as a motile body, and the whole performance described above is repeated.

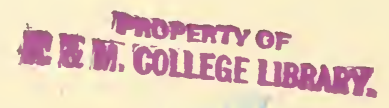




\section{DISCOVERY OF PROTOPLASM.}

The study of Vaucheria led, then, to the discovery that there are plants which, in the course of their development, pass through a motile stage, propelling themselves about the water as tiny halls of jelly with ciliary processes, and giving exactly the same impression as infusoria. Hand in hand with this discovery went the further observation that a portion of the plastic cell-contents in all plants lies, like a lining, in contact with the inner face of the cell-walls, so that we find that these latter, at a certain stage of maturity, are made up of two layers lying close

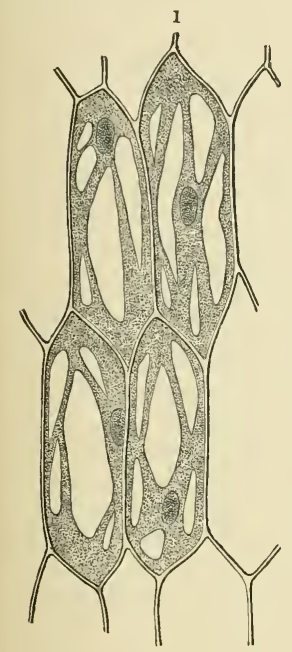

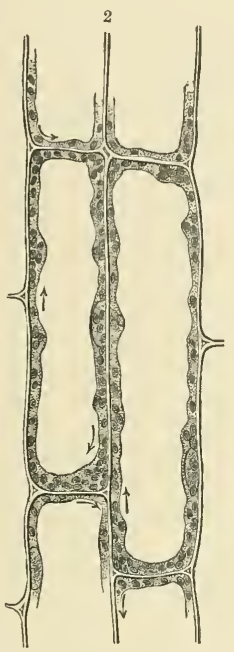

Fig. 6. - Protoplasm inclosed in Cells.

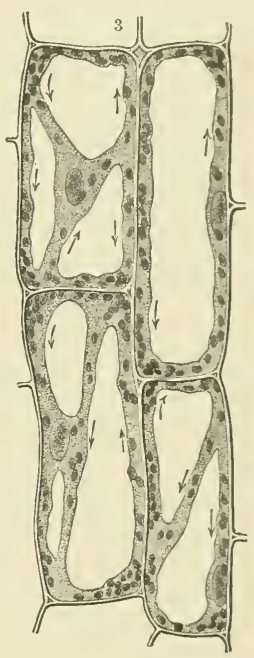

1 Protoplasm in cells of Orobanche. 2 Streaming protoplasm in cells of Vallisneria. I Streaming protoplasm in cells of Elodea.

together, the outer one firm and the inner soft. The name of "primordial utricle" was given to this inner layer. On further investigation it turned out that this primordial utricle belongs to a body of gelatinous, slimy consistency which lives in the cell-cavity like a mussel or a snail in its shell. At first it is shapeless and fills the whole cavity with what appears to be a homogeneous mass; but later on it is differentiated into a number of easily-recognizable parts-i.e. into the abovementioned lining towards the inner surface of the cell-membrane, and into folds, strands, threads, and plates stretching across the interior of the cell. (See fig. 5.) Mohl of Tübingen, the discoverer of these facts, applied in 1846 the name of protoplasm to the substance of which the cell-contents are composed.

It is possible for protoplasm, under certain conditions, to exist for a time without any special protective envelope; but, as a general rule, it secretes at once a firm, 
continuous coat, and, so to speak, builds itself a little chamber wherein to live. We may therefore distinguish naked protoplasm from that kind which inhabits the interior of a cell of its own creation, and compare the former to a shell-less snail, and the latter to a snail that constructs the house in which its life is spent. Still better may we compare the firm and solid cell-membrane with which the protoplasm clothes itself to a protective coat, a garment fitted to the body; and, following out this analogy, the protoplasm must be designated the living entity in the cell, and the secreted envelope must be considered as merely the skin of the cell. Consequently, although this cell-wall was the part which was first revealed by magnifying glasses, and was called a cell on account of its form, this is not the essential formative element, which has the power of nourishing and reproducing itself. It is the body within the cell, the slimy, colourless protoplasm in full activity within the surrounding membrane made by itself, which must be taken to be the essential part of the cell and the basis of life.

The term cell had become so naturalized in the science that protoplasm which had escaped from a cell-cavity was also called a cell, and the unfortunate name of "naked cell" was brought into use to designate it. More recently many of these older designations have been abandoned as unsuitable. We now include under the term "protoplasts" all these individual organisms, consisting of protoplasm, which occupy little chambers made by themselves, living either alone like hermits or side by side in sociable alliance in more or less extensive structures, able under certain circumstances to leave their domiciles, laying aside their envelopes and swimming about as naked globules.

Only when the protoplasts live in innumerable little cavities congregated close together in colonies, and when these cavities are bounded by even walls and are for the most part uniformly developed in all directions, does the part of a plant composed of them look under the microscope like a honey-comb, and each cavity like a cell. But even in these cases of external similarity there is the essential difference that in a honey-comb each of the walls separating individual cells is common to both the adjacent spaces, and, accordingly, the cells of the comb are like excavations in a continuous matrix; whereas, in sections of cellular plants, every cell possesses its own particular and independent wall, so that in them every partition-wall between neighbouring cavities is composed, properly speaking, of two layers (fig. 6). These two layers are scarcely distinguishable in the case of delicate cell-membranes newly secreted by the protoplasts. Later on, however, they are always to be made out clearly (fig. $6^{2}$ ). Frequently the layers separate one from another at certain spots, and thus channels are formed between the cells (fig. $6^{1}$ ); these are called "intercellular spaces." One often sees cells, too, whose entire surfaces are, as it were, glued together with a kind of cement, and then this substance which is stored between the two layers is called "intercellular substance" (fig. $6^{3}$ ).

By loosening the intercellular substance, where present, by mechanical or chemical means, we can easily separate adjacent cells from one another; the two layers of the partitioning cell-walls come asunder, and then each separate cell exhibits a 
complete envelope. The individual cell-cavities are often elongated and shaped like either rigid or flexible tubes; or the wall of such a cavity may become very thick and encroach to such an extent on the cavity that the latter is scarcely recognizable. Cells of this kind look like fibres and threads, groups of them look like bundles and strands, and do not resemble even remotely the cells of a honey-comb. The term "cellular" is hence no longer suitable in the case of these structures.

The expression "cellular tissue" is calculated also to occasion a wrong idea of the grouping and connection of the single cell-cavities. By a tissue one would surely understand a collection of thread-like elements so arranged that some of the threads run parallel to one another in one direction, whilst similar threads crossing
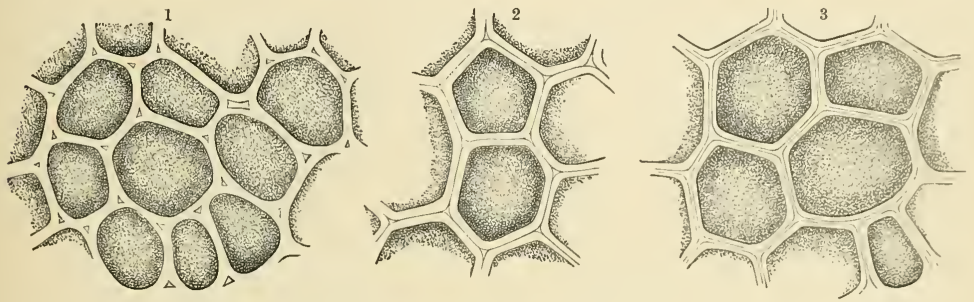

Fig. 6.-Cell-chambers. Showing Intercellular Spaces ( ${ }^{1}$ and ${ }^{2}$ ) and "Intercellular Substance" $\left({ }^{8}\right)$ in the Partition-walls of the Chambers.

the first at right angles are interwoven with them. In such a tissue, as of woven silk or the web of a spider, the threads are held together by intertwining; but this is by no means the case with the collections of cells which have been called celltissues. Even where the parts of a so-called tissue of cells are tubular, thread-like, or fibrous, they lie side by side and are joined as it were by a cement, but are never crossed or twisted together like the threads in a woven fabric.

Again, cells have been compared to the bricks of a building, but this analogy is not exact. The process of formation of a cubical crystal from a solution of common salt may perhaps be compared to the piling up of bricks; but when a leaf grows the process is not for one layer of cells to be superimposed from the outside upon another previously deposited. The development of new cells proceeds in the inside of existing cells and ensues from the activity of the protoplasts inclosed within the cellwalls; and these protoplasts not only provide the building materials, but are themselves the builders. It is in this very fact indeed that we grasp the sole distinction between organic and inorganic structures, and on this account especially the above analogy is inadmissible and should be avoided.

Cells and cell-aggregates may be conceived most clearly by considering their analogy to the shells of living creatures, as we have already done more than once in the foregoing pages. Protoplasts are either solitary, inhabiting isolated cell-cavities; or else they live in associated groups, the cells being crowded close together in great numbers and firmly attached to one another-each cavity being inhabited by one such protoplast. When the latter is the case, division of labour usually takes place 
in a plant, so that, as in every other community, some of the members undertake one function, some another. The older cells in these plants often lose their living protoplasts, and then, for the most part, serve as an uninhabited foundation to the entire edifice, which may thus be penetrated by air and water channels. The protoplasts have meanwhile erected new stories for themselves and their posterity on the old deserted foundations, and are pursuing their indefatigable labours in the little chambers of these upper stories. This work of the living protoplasts consists in absorbing nutriment, increasing their own substance, maturing offspring, searching for the places which offer most favourable conditions with a view to an eventual transmigration and to colonization by their families; and lastly, securing the region where all these tasks are performed against injurious external influences. The sequence of these labours is always governed by conditions of time and place. Many of them are only to be observed with difficulty in their actual performance and are first recognized in their perfected products, while others are attended by very striking phenomena and are easily followed in their progress.

\section{MOVEMENTS OF PROTOPLASTS.}

Swimming and creeping protoplasts.-Movements of protoplasm in cell-cavities.-Movements of Volvocineæ, Diatomaceæ, Oscillariæ, and Bacteria.

\section{SWIMMING AND CREEPING PROTOPLASTS.}

Among the most striking phenomena observed in connection with living protoplasts are, without question, the temporary locomotion of the protoplast as a whole and the displacement and investment of its several particles. The freest motion is of course exhibited by protoplasts which are not inclosed in cell-cavities, but have forsaken their dwelling and are wandering about in liquid media. Their number, as well as the variety of their forms, is extremely great. These naked protoplasts are evolved by several thousands of kinds of cryptogamic plants, at the moment of sexual or asexual reproduction in these plants. The escape from the enveloping cell-wall alone takes place in countless different ways, though the process, as a whole, is conducted in the manner already described in the case of Vaucheria clavata. Sometimes a single comparatively large protoplast glides out of the opened cell by itself; at other times, before the cell opens the protoplasmic body divides into several parts-often into a great number-and then a whole swarm of protoplasts struggle out.

These swarming protoplasts differ considerably in form. Usually their outline is almost ellipsoidal or oval; but pear-shaped, top-shaped, and spindle-shaped forms also occur. Often the body of the protoplast is spirally twisted like a corkscrew, and has in addition one end spatulate or clavate. Thread-like processes, definite in number and dimensions and arranged variously, according to the kind of protoplast, 
project from the surface of its body. In some instances the whole surface is thickly covered with short cilia, as in Vaucheria (fig. $7^{1}$ ); in others the cilia form a close ring behind the conical or beak-like end of the pear-shaped body, as in Edogonium (fig. $7^{2}$ ); and in others again, one or two pairs of long and infinitesimally thin threads, like the antennæ of a butterfly, proceed from some spot, generally the narrow end (fig. $7^{3}$ and $7^{4}$ ). Many forms are provided with a single long lash or flagellum at one extremity (fig. $7^{7}$ ), and yet others are spirally wound and are beset with cilia, thus presenting a bristly or hirsute appearance (fig. $7^{11}$ ).

These ciliary processes have a combined lashing and rotatory motion, and by their means the protoplasts swim about in water. In many cases, however, swim-

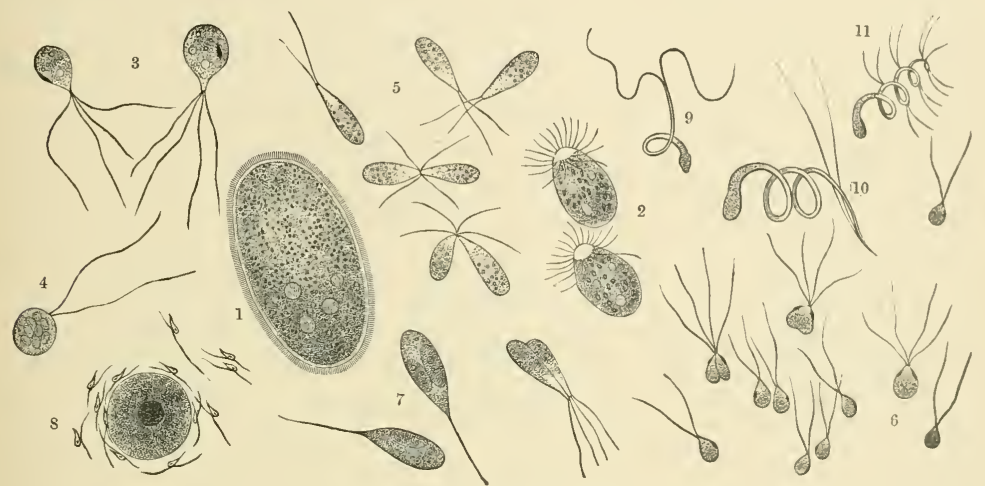

Fig. 7.-Swimming Protoplasm.

1 Vaucheria; ${ }^{2}$ Edogonium; 8 Draparnaldia; 4 Coleochote; 5 and 7 Botrydium; 6 Ulothrix; 8 Fucus; 9 Funaria; 10 Sphagnum; 11 Adiantum.

ming is hardly an appropriate expression; certainly not if one associates the term with the idea of fishes swimming with fins. In point of fact there is, associated with progression in a particular direction, a continuous rotation of the protoplast round its longer axis, and on this account its motion may be compared to that of a rifle-bullet, since in both cases the movement of translation takes place in the direction of the axis round which the whole body spins. The movement in question is not unlike the boring of one body inside another; according to this, the soft protoplasts bore through the yielding water, and by this action make onward progress.

The microscope magnifies not only the moving body, but also the path traversed; and when one contemplates a protoplast in motion, magnified, say, three hundred times, its speed appears to be three hundred times as fast as it really is. As a matter of fact, the motion of protoplasts is rather slow. The swarm-spores of Vaucheria, described above, which traverse a distance of 17 millimeters in a minute are amongst the fastest. The majority accomplish an advance of not more than $5 \mathrm{~m} . \mathrm{m}$., and many only $1 \mathrm{~m} . \mathrm{m}$. per minute. 
As was mentioned in the description of Vaucheria the locomotion of ciliated protoplasts lasts for a comparatively brief period. It gives the impression of being a journey with a purpose: a search, as it were, for favourable spots for settlement and further development; or else a hunt after other protoplasts moving about in the same liquid. Green protoplasts always begin by seeking the light, but after a time they swim back into the shadier depths. Many of these, especially the larger ones, avoid coming into collision, and are careful to give each other a wide berth. If numbers are crowded together in a confined space, and two collide or their cilia come into contact, the motion ceases for an instant, kut in a few seconds they free themselves and retire in opposite directions.

Contrasting with these unsociable protoplasts are others, which have a tendency to seek each other out and to unite; and protoplasm acts in many cases on protoplasm of identical or similar quality, perceptibly attracting it and determining the direction of its motion. It is very curious to watch the tiny pearshaped whirling protoplasts of Draparnaldia, Ulothrix, Botrydium, and many others, as they steer towards one another and, upon their ciliated ends coming into contact, turn over and lay themselves side by side (fig. $7^{5}$ ); or, to see one pursued and seized by another, the foreparts of their bodies brought into lateral contact, and, finally, the two, after swimming about paired for a few minutes, fusing together into a single oval or spherical protoplast (fig. $7^{6}$ ). Even the minute fusiform protoplasts which are moved by cilia proceeding from the sides of their bodies (fig. $7^{8}$ ), as well as the spirally-coiled forms (figs. $7^{9,10,11}$ ) endeavour to unite with some other protoplast. They always move towards larger protoplasmic bodies at rest, cling to them closely, and at last coalesce with them into single masses (fig. $7^{8}$ ).

As a rule no striking change is to be perceived in the inside of motile protoplasmic bodies during the rotatory and progressive motion caused by their cilia; and the granules and chlorophyll-corpuscles dotted about in the body of the protoplast seem to remain, throughout the period of locomotion, almost unchanged as regards both position and shape. It is only in the vicinity of certain little spaces, called "vacuoles," in the substance of the protoplasm, that changes in many instances are observed, which indicate that, during the motion of the whole apparently rigid mass, slight displacements may also occur in the interior, somewhat in the same way as, when a man walks, the heart inside his body is not still (relatively to the body), but continues to pulsate and cause the blood to circulate. The changes observed in vacuoles have, moreover, been described as pulsations, because they are accomplished rhythmically and manifest themselves as alternate expansions and contractions of the vacant space.

In each of the motile protoplasts of Ulothrix (fig. 8) there is found, near the conical end, which is furnished with four cilia, a vacuole which contracts in from 12 to 15 seconds, and dilates again in the succeeding 12 or 15 seconds. In the swarm-spores of Chlamydomonas and those of Draparnaldia two such vacuoles may be observed close together, whose rhythmic action is alternate, so that the 
systole (contraction) of the one always takes place synchronously with the diastole (expansion) of the other. The contraction often continues until the cavity entirely disappears. It must depend, as also does the expansion, on a displacement of that part of the protoplasm which immediately surrounds the vacuole. But such a motion as this in the protoplasmic substance, even if only visible in a small part of the whole body, can scarcely be without its effect on other more distant parts; and it may, therefore, be concluded that the interior of a protoplast, endowed with ciliary motion, rotatory and progressive, does not remain quite at rest relatively, as seems on cursory inspection to be the case.

Protoplasts whose motion is effected by means of cilia have no more need of their vibratile organs when once they have reached their destination. The cilia,
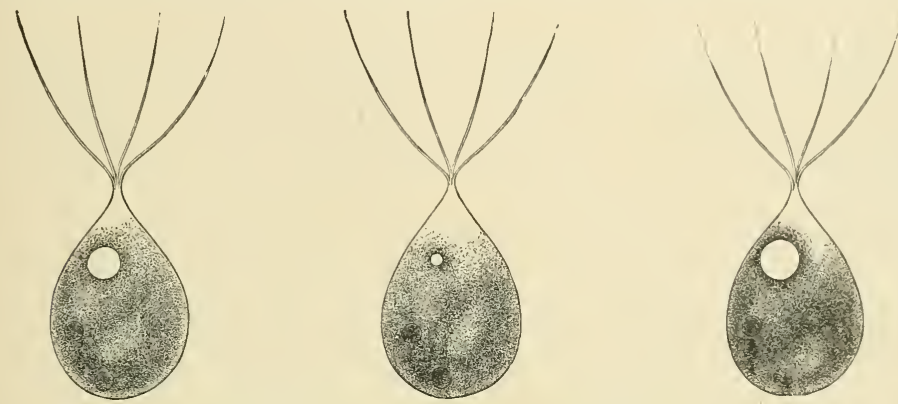

Fig. 8.-Pulsating Vacuoles in the Protoplasm of the large Swarm-spores of Ulothrix.

whether numerous or solitary, whether short or long, first of all become stationary and then suddenly disappear. Either they are drawn in or else they deliquesce into the surrounding liquid. Whether the motile protoplasts have come to rest because they have reached a suitable place for further development, as happens in Vaucheria, or because they have united, like with like, into a single mass, the form taken by the resulting non-motile body is always spherical. The final act is the development around itself of an investing cell-membrane, so that its soft and slimy substance may be protected by a firm covering from external influences.

Essentially different from the motion just described is that of certain protoplasts which are unprovided with cilia, but perpetually change their outlines, thrusting out considerable portions of their gelatinous bodies in one direction or another, and at the same time drawing in other parts. At one moment they appear irregularly angular, shortly afterwards stellate; then, again, they elongate, become fusiform, and gradually almost round (fig. 9). The protruded parts are sometimes delicate, tapering off into mere threads; sometimes they are comparatively thick, and have almost the appearance of arms and feet in relation to the principal mass. The motion is not in this case like boring, but is best described as creeping. As one or a pair of foot-like appendages is thrown out 
in one direction, others on the opposite side are retracted, and the protoplast as a whole glides over the intervening space like a snail without its shell. The analogy is all the more exact since the protoplast, as it glides onward, leaves a slimy trail in its wake, so that the latter is marked by a streak resembling the track of a snail. When two or more of these creeping protoplasts, or plasmodia, meet, they merge into one another, flowing together somewhat in the same way as two oil-drops on water coalesce into one-leaving no distinguishable boundaries between the united bodies. Thus, slimy lumps of protoplasm, which may attain to the dimensions of a closed or open hand, result from the coalescence of great numbers of minute protoplasts. And it is a very remarkable fact that these plasmodia can themselves change their form, putting out lobes and threads, and
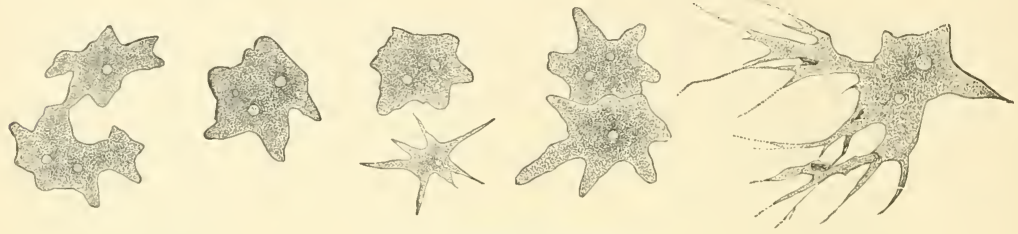

Fig. 9.-Creeping Protoplasm.

creeping about in the same way as the single protoplasts from whose fusion they have arisen.

Creeping masses of jelly sometimes move in the direction of incident light; at other times they avoid light and hide in obscure places, wriggling through the interstices of heaps of bark or into the hollows of rotten trunks; or they may creep up the stems of plants, or glide over the brown earth in a viscous condition. On these occasions they resolve themselves not infrequently into bands, cords, and threads, which surround fixed objects, divide, and combine again, forming a net-work of meshes, or else perhaps frothy lumps like cuckoo-spit. If foreign bodies of small size are enmeshed by the viscous threads of the reticulum, they may be drawn along by the protoplasm as it creeps; and if they contain nutritive material, they may be eaten up and absorbed. Plasmodia are, for the most part, colourless, but some are brightly tinted; in particular may be mentioned the best-known of all plasmoid fungi, the so-called "Flowers of Tan" (Fuligo varians), which are yellow, and Lycogala Epidendron, which comes out on old stumps of pines, and is vermilion in colour.

\section{MOVEMENTS OF PROTOPLASM IN CELLCAVITIES.}

In the case of a protoplast which is not naked, but clothed with an attached cell-membrane, the movements are limited to the space included by the membrane, that is to say to the cell-cavity. Until the protoplasmic cell-body is differentiated into distinct individual portions no very lively motion can in general take place in the coated protoplast; though it is not to be assumed that it abides completely 
at rest at any time, except perhaps during periods of drought in summer and of frost in winter, and in seeds during their time of quiescence. This applies particularly to immature cells. In them the protoplast forms a solid body whose substance entirely fills the cell-cavity. The young cell, however, grows up quickly, its cavity is enlarged, and the space, hitherto filled by the protoplast, becomes two or three times as large as before. But the increase of volume on the part of the protoplast itself does not keep pace with the enlargement of its habitation. It is true that it continues to cling closely to the inner face of the cell-wall, thus forming the primordial utricle; but the more central part of its body relaxes, and in it are formed vacant spaces, the vacuoles above mentioned, wherein collects a watery fluid known as the "cell-sap." The portions of protoplasm which lie between the vacuoles resolve themselves gradually into thin partitions bounding them; and lastly, these partitions split up into bands, bridles, and threads, which stretch across the cell-cavity from one side of the primordial utricle to the other, and are woven together here and there where they intersect. With these protoplasmic strands we have already become acquainted.

But the protoplasm in the interior of a growing cell, whilst relaxing and breaking up, also becomes motile if the liquid attains a certain temperature, and then the appearance presented is like that of a lump of wax melting under the action of heat. These movements may be observed very clearly under the microscope in the case of large cells with thin and very transparent cell-membranes, especially when the colourless, translucent, and gelatinous substance of the protoplasm-not always sharply defined in contour-happens to be studded with minute dark granules, the so-called "microsomata." These granules are driven backwards and forwards with the stream, like particles of mud in turbid water, and their motion reveals that of the protoplasm wherein they are embedded. Seeing particles gliding in all directions through the cell-cavity, arranged irregularly in chains, rows, and clusters in the protoplasmic strands, we are justified in concluding that this motion takes place in the substance of the strands itself. The movement, moreover, is not confined to isolated strands, but occurs in all. Granular currents flow hither and thither, now uniting, now again dividing. They often run in opposite directions even when only a trifling distance apart; sometimes two chains are drifted in this way when actually close together in the same band of protoplasm. The streams pour along the primordial utricle and whilst there divide into a number of arms, meeting and stemming one another and forming little eddies; then they are gathered together again and turn into another strand of the more central protoplasm. The individual granules in the currents are seen to move with unequal rapidity according to their sizes; the smaller particles progress faster than the larger, and the larger are often overtaken by the less, and when this happens the result often is that the entire stream stops. If so, however, the crowded particles are suddenly rolled forward again at a swifter pace, like bits of stone in the bed of a river as it passes from a level valley into a gorge. The course of the

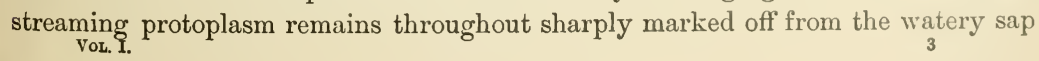


in the vacuoles, and none of the granules ever pass over into the cell-sap from the protoplasm.

Larger bodies, such as the round grains of green colouring-matter or chlorophyll, are in many instances not carried forward, but remain stationary, the protoplasmic stream gliding over them without altering them in any way. Further, the outermost layer of the protoplast, contiguous with the cell-membrane, is not in visible motion in most vegetable cells. On the other hand, occasionally the entire protoplast undoubtedly acquires a morement of rotation, and then the larger bodies imbedded in its substance, i.e. chlorophyll corpuscles, are driven along like driftwood in a mountain torrent (fig. $5^{2}$ and $5^{3}$ ). On these occasions a wonderful circulation and undulation of the entire mass takes place: chlorophyll grains are whirled along one after the other at varying speeds as if trying to overtake one another; and yet another structure, the cell-nucleus presently to be discussed, is dragged along, being unable to withstand the pressure, and, following the various displacements of the net-work of protoplasmic strands in which it is involved, is at one moment pulled alongside of the cell-wall, at another again is taken in tow by a rope of central protoplasm and hauled transversely across the interior of the cell (fig. $5^{3}$ ).

When the rate of the current itself is estimated by the pace at which the granules are driven along, results which vary considerably are obtained, depending chiefly on a qualitative difference in the protoplasm, but secondarily also on temperature and other external conditions. A rise in temperature up to a certain point as a general rule accelerates the rate of the stream. Particles of protoplasm in particularly rapid motion pass over $10 \mathrm{~m} . \mathrm{m}$. in a minute; others in the same time traverse from 1 to $2 \mathrm{~m} . \mathrm{m}$;; and some, in still less haste, advance only about a hundredth part of a millimeter. Larger bodies, especially the bigger chlorophyll grains, move slowest of all. So it is often hours before chlorophyll grains lying near one side of a cell are pushed through the protoplasm over to the other side, a distance only equal to a small fraction of a millimeter.

The minute granules, as well as the larger grains of chlorophyll and the cellnucleus, are entirely surrounded by protoplasm; and the protoplasm, whether in the form of bands or threads, whether a peripheral lining or an indefinite mass, must be conceived as always composed of two layers, the outer "ectoplasm" being tougher and denser than the inner "endoplasm," which is softer and somewhat fluid. The former is homogeneous and non-granular, so that it is the more transparent and has the effect of a skin clothing the inner, softer layer, which is granular and turbid. It would be incorrect, however, to think of this as a very strongly-marked contrast, sufficient to mark off one layer clearly from the other. In reality there are no such sharp boundaries, and the tougher ectoplasm passes gradually into the softer and more mobile endoplasm. Of course the granules and corpuscles which one sees drifting in streaming protoplasm are situated within the more yielding endoplasm. It is true, minute particles often appear to glide from one side to the other upon a delicate protoplasmic strand as if it were a tight-rope; but on closer 
study it is apparent that the granules which seem to be travelling on the protoplasmic thread are covered by a delicate and transparent protoplasmic peilicle. Thus, these granules imbedded in the substance of protoplasts have no independient motion, but are pushed along by the spreading protoplasm.

Each stream of protoplasm is shut off from its environment and limited by a layer tougher than the rest. But this does not prevent the currents, with their crowds of drifting granules, from changing their direction. In fact we have only to follow for a short time the course of one such granular stream to remark a continuous series of changes: a current from being in a straight line bends suddenly to one side, it broadens and contracts again, now it runs close alongside another channel, now breaks away once more, divides into two little arms, and loses itself finally in the primordial utricle. On the other hand, fresh folds start from the primordial utricle, stretch and grow until they have pushed across the cell-cavity to the other side in the form of bands, or the protoplasm may be drawn out into threads, which elongate until they encounter other similar strings and form a junction with them. The same processes then that are observed in free creeping protoplasts take place to some extent here. Imagine a protoplast captured whilst on its travels-creeping along the level ground-and imprisoned in a completely closed vessel; it would spread itself out over the inner surface of the vessel, would branch and creep about and have just the same appearance as the protoplasts, just described, which inhabit cell-cavities from their earliest youth. This is but the converse of the power possessed by a protoplast set free from its cell, which enables it to move, stretch out, and draw in its various parts, and so to effect locomotion.

Another motion, differing from the creeping, gliding, and streaming action of protoplasts, manifests itself in the so-called swarming of granules contained in the protoplasm. It may be best observed in the cells of the genera Penium and Closterium, both of which are shown in figure $25 \mathrm{~A}, i, k$, though the same phenomenon is to be seen in many allied forms, living in lakes and ponds either singly or congregated in colonies, and remarkable for their bright green colour. The above-mentioned genus Closterium includes delicate unicellular forms having a curved or scimitar shape unusual in plants, whence one of its species, in which the semi-lunar form is most striking, has been named Closterium lunula. The cell-membrane in all these little water-plants is clear and quite transparent. The greater part of the cell-contents consists of a dark-green chlorophyll body longitudinally grooved; but the protoplasm which is visible in the two sharply tapering ends of the cell-cavity is colourless, and embecded within it is a swarm of microsomata. These granules or microsomata appear to be in a most curious state of motion so long as the protoplast lives. They are to be seen plainly within the limits of the tiny cavity, jumping up and down, whirling, dancing, and rushing about without really changing their position. One is reminded of the apparently purposeless journeyings to and fro within reach of their homes of ants or bees, and the movement has been called not inaptly 
"swarming." It is difficult to imagine the kind of motion possessed by the protoplasm in which these swarming microsomata are embedded; but however closely it is confined, there must be continual rapid displacements in its sulstance, which is very fluid, and it may be assumed that here again it is not so much the tiny grains that bestir themselves as the protoplasm which holds them. Probably the protoplasmic matter spreads and stretches out and rotates, and individual granules are carried about by it. This, of course, does not exclude the possibility of the granules possessing a vibratory motion of their own within the mass of protoplasm.

Similar, but not identical, is the swarming movement of protoplasm observed in cells of the Water-net (Hydrodictyon utriculatum), and in several other plants allied to it. Hydrodictyon looks like a net in the form of a sac, and composed of green threads. The meshes of this net, which are generally hexagonal, consist, however, not of filaments but of slender cylindrical cells joined together by threes at their extremities, somewhat in the same way as are the leaden frames of the little hexagonal panes of glass in gothic windows. The protoplasmic body of one of these cells in due time breaks up into a great multitude $(7000-20,000)$ of tiny clots, which begin to move and swarm within the cell-cavity in what appears to be a disordered medley. In half an hour, however, the excited mass is again restored to rest: the minute particles take form and arrange themselves in definite order, each having two others at either extremity, making an angle of $120^{\circ}$ with it; and, lastly, all unite to form a single tiny net having exactly the same shape as the one whose component cell constituted the arena of this process of construction. The miniature water-net so formed then slips out of the cell, the latter opening for the purpose, and in from three to four weeks it grows to the same size as the parent plant.

In the above we have an instance of a protoplast producing a whole colony of cells, which are obliged to leave their home for want of space. In cases previously considered we have found the protoplast stretching and elongating in all directions, drawing itself out into bridles and spreading as a delicate lining to walls, and so endeavouring generally to expand and present the greatest surface possible. Again, we have seen it wandering freely, creeping, swimming, and rotating, and by this method also covering as much space as it can. But, conversely, there is a time when a protoplast tends to the other extreme; the expanded mass of its body gathers itself together again, contracts more and more, and at length becomes a resting sphere, that is to say, it assumes the configuration which exposes the least surface to the environment.

This process exhibits itself with particular clearness within the cell-cavities of the green algæ known by the name of Spirogyra, a species of which is represented, magnified three hundred times, in figure $25 \mathrm{~A}, l$. In this alga the protoplasm in each mature cell-cavity forms, as a general rule, a very delicate parietal lining wherein green chlorophyll bodies are embedded, arranged in a spiral band. All of a sudden, however, this lining strips itself off the inner 
face of the cell-wall and shrinks together so as in a short time to present the appearance of a sphere occupying the middle of the cell-cavity. Again, just as this contraction is an instance of a special form of protoplasmic motion, so also the further change which the contracted protoplast in a cell of Spirogyra undergoes is reducible to displacements in its substance, and must be mentioned as a special kind of protoplasmic movement. For the conglomerated protoplast remains but a short time in the middle of the cell-cavity. It leans almost immediately to one side, thrusting itself into a protuberance of the cell-membrane, which is concurrently developed, and which, when further developed, forms a passage leading over into another cell-cavity. Its body becomes longer and narrower, and at last slips through the passage into the next cavity, where a second protoplast awaits it; and the two then unite, fusing together into one mass. It is not premature to remark that all these displacements and investments of the protoplasmic substance in cells of Spirogyra, including the phenomena of contraction, as well as those of pushing forward, escape, and coalescence, are not produced as the results of a shock, impulse, or stimulus from without, but are to be looked upon as movements proper to the protoplasm, and resulting from causes inherent in the protoplasm.

\section{MOVEMENTS OF VOLVOCINEÆ, DIATOMACEÆ, OSCILLARIA AND BACTERIA.}

Very remarkable is the movement of those wonderful organisms which are comprised under the name of Volvocineæ. One species, Volvox globator, was known to so ancient an observer as Leeuwenhoek; but he, and after him Linnæus, took it to be an animal on account of 1ts extraordinary power of locomotion, and it was named the "globe-animalcule." A Volvox-sphere consists of a large number of green protoplasts living together as a family and arranged with great regulärity within their common envelope. They appear to be disposed radially, and to be linked together and held firm by a net-work of tough threads, their poles being directed towards the centre and the periphery of the sphere respectively. From the peripheral extremity, which in each protoplast is marked out by a bright red spot, proceed a pair of cilia, and these protrude through the soft gelatinous envelope of the whole sphere, and move rhythmically in the surrounding water. A Volvox-globe rolls along in the water propelled by regular strokes, like a boat manned by a number of oarsmen, as soon as the protoplasts, which form the crew of this strange vessel, begin to manipulate their propellers. The effect is exceedingly graceful, and has justly filled observers of all periods with astonishment; indeed no one seeing for the first time a Volvox-sphere rolling along can fail to be impressed and delighted.

Another plant allied to the foregoing, the so-called "red-snow," has always excited wonder in no less degree from the remarkable phenomena of motion which it exhibits, but also because of its characteristic occurrence in situations where one 
might suppose all vital functions would be extinguished. It was in the year 1760 that De Saussure first noticed that the snowfields on the mountains of Savoy were tinged with red, and described the phenomenon as "red-snow." Once on the look-out for it, people found this red-snow on the Alps of Switzerland, Tyrol, and the district of Salzburg, on the Pyrenees, the Carpathians, and the northern parts of the Ural Mountains, in aretic Scandinavia, and on the Sierra Nevada in California. But redsnow has been seen on the most magnificent scale in Greenland. When Captain John Ross in 1818 sailed round Cape York on his voyage of discovery to Arctic America, he noticed that all the snow patches lying in the gorges and gullies of the cliff's on the coast were coloured bright crimson; and the appearance was so startling that Ross named that rocky sea-shore the "Crimson Cliffs." On the occasion of later expeditions to the arctic regions, red-snow was observed off the north coast of Spitzbergen, and in Russian Lapland and Eastern Siberia, but never in such surprising luxuriance as on the Crimson Cliffs of Greenland.

If a snow-field coloured by red-snow is examined near at hand it is found that only the most superficial layer, about 50 millimeters in depth, is tinged. It is also present in the greatest quantities in places where the snow has been temporarily melted by the heat of summer, particularly therefore in depressions, whether big or little, and towards the edges of the snow-field, where the so-called snow-dust or Cryoconite extends regularly in the form of dark, graphitic smeary streaks. Examined under the microscope, the matter which causes the redness of the snow appears as a number of spherical cells having a rather substantial colourless cellmembrane and protoplasmic contents permeated by chlorophyll. The green colour of the chlorophyll is, however, so disguised by a blood-red pigment that it is only possible to detect it when the latter has been extracted, or in cases where it is limited to a few definite spots in the cell. These spherical cells do not move, and so long as the snow is frozen they show no sign of life. But as soon as the heat of the summer months melts the snow, these cells acquire vitality, visibly increasing in size and preparing for division and multiplication the moment they have attained a certain volume. The growth, so far as it depends on nutrition, takes place at the expense of carbon dioxide absorbed by the melted snow from the atmosphere and of the inorganic and organic constituent parts of the dust. We shall frequently have occasion to return to this dust, but at present it is only necessary to observe, for the comprehension of the drawing of red-snow as seen under the microscope (figure $25 \mathrm{~A}, e-l h$ ), that in the Alps, amongst the organic materials which constitute the dust, pollen-grains of conifers occur with great frequency, especially those of the fir, arolla, and mountain pine. These pollen-grains have been swept up into the high Alps by storms, and are already partially decayed. In all the material that I investigated I found the red-snow cells mixed with pollen-grains of the above-mentioned conifers. The pollen-grains are oval in crosssection, of a dirty yellow colour, and swollen laterally into two hemispherical wings, as is shown in figure $25 \mathrm{~A}, e-h$.

As has been stated, the red cells are nourished by the constituent elements of 
the dust, which are dissolved in the melted snow. They grow and at last divide so as to form daughter-cells, usually four in number but often six or eight and less frequently two only (figure $25 \mathrm{~A}, f, g$ ). As soon as the division is accomplished, the daughter-cells, so produced, free themselves, assume an oval shape, and display at their narrower extremity two rotating cilia by means of which they move about in snow-water with considerable vivacity. The interstices of the still unmelted, but now granular, snow, are filled with water from the melted parts, and through these the red cells swim away and are thus diffused over the snow-field. At the moment of escape and first assumption of movement the cell-body appears to be uninclosed. But it soon clothes itself with an extremely delicate, though clearly discernible skin, which, curiously enough, does not lie close to the protoplasm, which is withdrawn slightly and inclosed as in a distended sac (see figure $25 \mathrm{~A}, e$ ). Only in front, where the two cilia carry on their whirling motion, does the skin lie close to the body of the cell; and it must be presumed that the cilia, which are simply extensions of the protoplasmic substance, are projected through the envelope. The swarm-spores afford an example of an unusual type of protoplasts, namely of those that move about singly in the water by means of cilia and at the same time carry their self-made cell-membranes with them.

How long the motile stage lasts under natural conditions has not been determined for certain. On the mountains of central and southern Europe, where hot days are followed, even in the height of summer, by bitterly cold nights, causing the melted snow which has not run off to freeze again in the depressions of the snow, the movement no doubt is often interrupted. On the other hand, in high latitudes, where the summer sun does not set for weeks together, such interruption would be exceptional. In any case, however, the locomotion of the red cells with their hyaline cell-membranes is not limited to so short a period as is that of naked ciliated protoplasts. Moreover they have the power of nutrition and growth like the red resting-cells from which they originate, and they have been observed, in a culture, to increase in size fourfold within two days. When at last they come to rest they draw in their cilia, assume a spherical shape, thicken their cell-membrane, which now once more lies close to the protoplasmic body, and divide anew into two, four, or eight cells (figure $25 \mathrm{~A}, f, g$ ). The fusion of the protoplasts of the red cells in pairs, and their sexual propagation, which has been observed in addition to the above-described asexual multiplication, will be the subject of discussion later on. At present we need only add with reference to this remarkable plant that it was named Sphcerella nivalis by the botanist Sommerfelt, and that not only in mode of life, but also in form and colour, it most closely resembles a kind of blood-red alga, which makes its appearance in Central Europe in little hollows temporarily filled with rain-water in flat rocks and slabs of stone, and also inside receptacles exposed to the open. This alga has received the name of Sphcerella pluvialis, and also that of Hamatococcus pluvialis.

Lastly, we have to consider the mysterious movements exhibited by many Diatomacer, and by the filamentous species of Zonotrichia, Oscillaria, and 
Beggiatoa. As regards the Diatoms, some of them are firmly attached to a support, and are not generally capable of locomotion; but others are almost incessantly in motion, and these little unicellular organisms steer themselves about with great precision near the bottom of the pools of water in which they live. Their cell-membrane is transformed into a siliceous coat, and this coat, which is hyaline and transparent, but very hard, consists of two halves shutting together like the valves of a mussel. The entire cell thus coated has the form of a gondola or little boat, with a keel either straight or curved (Pleurosigma, Pinnularia, Navicula), and is provided with various bands, ribs, and sculpturings on its siliceous walls. Driven by inherent forces, these little protected cruisers pursue their way at the bottom of the water or over objects which happen to be in the water. They either glide evenly over the substratum, or else proceed by fits and starts at rather long intervals, and apparently with difficulty. For some time they may hold a straight course, but not infrequently they deviate sideways without apparent cause, and after deviating return again. They double round projecting objects or push them out of the way with one of their hard points, which are often thickened into nodules, and cause the obstructing objects to slip by alongside the keel of the little vessel. Yet no paddles or cilia are to be seen projecting from it, as in the case already described of Volvocineæ; nor does the siliceous coat exhibit any sort of motile processes whereto the movements might be attributed. But the strong analogy between the structure of these Diatomacer and that of mussels seems to justify the assumption that the two siliceous valves, which are fast shut during the period of rest of the Diatoms in question, move a little apart, so that the protoplast living within can push out one edge of its body and creep along over the substratum by means of it.

The movements of the filaments of Beggiatoa, Oscillaria, and Zonotrichia are explained in a similar manner. These filaments are made up of a number of short cylindrical or discoid cells, and are attached by one end, but with the other execute most striking movements. They stretch themselves and then contract again, coil up and straighten out like snakes, and, most characteristic of all, make periodic oscillations in the water. The belief is that the mechanism of this motion is similar to that of the preceding, that infinitesimally fine filaments of protoplasm inserted spirally penetrate the cell-walls, and that these act like the propeller of a ship.

On looking back over the multifarious examples of movement that have been described, the conviction that the capacity for motion is inherent in all living protoplasts is difficult to resist. In many cases, of course, the displacement and replacement of the substance no doubt takes place so slowly that it is scarcely possible to express its amount numerically. Movement may even entirely cease for a time; but, as necessity arises, and under favourable external circumstances, the protoplasmic mass always becomes mobile again-the direction of its motion being determined by inherent forces. There is still much to learn, no doubt, concerning the objects and significance of the different movements of protoplasm; 
but in this connection we are justified in assuming that all these movements have to do with the maintenance and multiplication of the protoplasts. For instance, amongst the objects of the various movements are the search for food, the elimination of useless material, the production of offspring, the discovery of the rays of sunlight necessary to the existence of chlorophyll-bodies and of suitable spots to colonize. This conception has been lirought out frequently in the course of the foregoing description, and will again engage our attention in succeeding pages.

\section{SECRETIONS AND CONSTRUCTIVE ACTIVITY}

\section{OF PROTOPLASTS.}

Cell-sap.-Cell-nucleus.-Chlorophyll-bodies.-Stareh.-Crystals.-Construction of the Cell-wall and Establishment of Communication between Neighbouring Cell-cavities.

\section{CELL-SAP.—CELL-NUCLEUS.-CHLOROPHYLL-BODIES.-STARCH._CRYSTALS.}

In addition to the powers which the living protoplast possesses of shifting its parts, of expanding and contracting, of dividing and of fusing like with like, it has also the properties of adapting different parts of its body to particular functions, of building up various chemical compounds, and of separating them out when necessary. As the protoplast stretches and expands, spaces and depressions arise within it, and these form ultimately, when the protoplast is limited to a peripheral layer lining the walls of the cavity, a single central vacuole. In the spaces there is secreted, in the first instance, the cell-sap, a watery fluid containing a variety of substances either suspended or in solution, of which the chief are sugar, acids, and colouring matters. Moreover, in the interior of the protoplasm itself, structures with quite different forms occur, and are easily recognizable by their contours; these are the cell-nucleus, chlorophyll-bodies, and starchgrains.

The principal feature of the cell-nucleus is that, although the substance of which it is composed is only slightly different from the general protoplasm of the cell, yet it is always clearly marked off from the protoplasm. In the undeveloped protoplast the nucleus is usually situated in the middle, but in mature protoplasts it is either pressed against one wall of the cell or suspended in a sort of pocket of protoplasmic filaments in the interior ( fig. $5^{1}$ and $5^{3}$ ). It may be pushed along by the streaning protoplasm and dragged into the middle of the cell, and in that case its shape is sometimes altered and it becomes for a time somewhat elongated and flattened. The nuclear substance, which, as has been already mentioned, differs but little from ordinary protoplasm, is colourless, and studded with microsomata, and is liable to internal displacements similar to those of the entire cell-body. When a protoplast divides, the nucleus plays a very 
important part in the process, and it will be necessary later on to discuss its significance in this connection.

The chlorophyll-bodies, mentioned already more than once incidentally, are green corpuscles, roundish, ellipsoidal, or lenticular in shape, and grouped in a great variety of ways (figure $25 \mathrm{~A}, i, k, l, m, p$ ). They are produced generally in great numbers by the protoplast in special sac-like excavations in its body, but nowhere except where they are necessary, that is, in those cells wherein the transmutation of inorganic food-stuffs into organic matter takes place. This transformation, so important to the existence of the organic world, will be considered in detail later on. Chlorophyll-corpuscles are not, as regards their material basis, essentially different from the substance of the protoplasm in which they are formed, and in which they remain embedded for life, but their green colour distinguishes them very clearly from their environment. This greenness is due to a colouring matter stored in the protoplasmic substance of the corpuscle; and our ideas of plant-life are so intimately associated with this remarkable pigment, that a plant that is not green seems to us to be almost an anomaly.

Besides the nucleus and the chlorophyll-bodies or corpuscles, protoplasts produce starch-grains, aleurone-grains, crystals of oxalate of lime, and drops of oil, all of which will be dealt with presently in their proper place. They are evolved in accordance with the requirements of the moment and with the position held in the edifice of the plant by the cells concerned. Moreover, the walls of the cells themselves are the work of the protoplasts, and it is not a mere phrase, but a literal fact, that the protoplasts build their abodes themselves, divide and adapt the interiors according to their requirements, store up necessary supplies within them, and, most important of all, provide the wherewithal needful for nutrition, for maintenance, and for reproduction.

\section{CONSTRUCTION OF THE CELL-WALL AND ESTABLISHMENT OF CONNECTIONS BETWEEN NEIGHBOURING CELL-CAVITIES.}

Of all these performances, the construction of the cell-wall shows the greatest variety from the nature of the case. For the envelope with which each individual protoplast surrounds itself serves at once as a protection for the delicate protoplasm, and as a firm support for structural additions; and, at the same time, it must not impede the reciprocal action between the protoplasts and the external world, or the intercourse between those living in adjoining cavities. These cell-walls are accordingly very wonderful structures, and we shall often have occasion to discuss them, especially with reference to the significance of variations in their structure in particular cases. At present it is sufficient to remark that the original envelope which is secreted from the body of a protoplast and which appears at first as a delicate skin, is made of a substance composed of carbon, hydrogen, and oxygen, belonging to the class of carbohydrates.

The name of cell-membrane, usually applied to the original envelope formed by 
the cell-body, is one quite suitable for the purpose. But this earliest covering undergoes many modifications. The protoplast is able to store up in it suberin, lignin, silica, and water in greater or smaller quantities, and by this means it either makes the envelope more flexible than it was in the first instance, or else hard and stiff, converting it into a shell-like case. Even the shape is seldom preserved as it was originally. The solitary protoplast surrounded by its cell-membrane is generally in the form of a roundish ball, and its envelope, which is closely adherent exhibits a corresponding configuration. Young cells, aggregated together, have outlines too which remind one of crystalline forms, such as dodecahedra, cubes, and short six-sided prisms. But when a protoplast has produced its first delicate covering it does not come to rest, but goes on working at the membrane, distending and thickening it, transforming a cavity which was originally spherical or cubical into one of cylindrical, fibrous, or tabular shape, and strengthening its walls with pilasters, borders, ridges, hooks, bands, and panels of various kinds. Where a number of protoplasts work gregariously at one many-chambered edifice, cells of most diverse forms are produced in close proximity to one another. These varieties are, however, never without method and design, but are invariably such as to adequately equip each cell for the position it holds and for the particular task allotted to it in the general domestic economy.

The volume attained by cell-cavities in consequence of the expansion of their walls varies within very wide limits. The smallest cells have a diameter of only one micro-millimeter, i.e. the thousandth part of a millimeter; others, as for example yeast-cells, measure perhaps two or three hundredths of a millimeter; and yet others have outlines perceptible to the naked eye and have a volume amounting to one cubic millimeter. Tubular and fibrous cells often stretch longitudinally to such an extraordinary extent that some with a diameter of scarcely the hundredth part of a millimeter reach a length of one, two, or even as many as five centimeters. An instance may be seen in the filaments of Vaucheria clavata (figure $25 \mathrm{~A}, a-d$ ), and again in the fibrous cells from which our linen and cotton fabrics are manufactured.

The enlargement of a cell-cavity, or, in other words, the growth in area of its walls, ensues in consequence of the intercalation of fresh particles between those which, by their mutual coherence, form the delicate skin of the protoplast - the earliest stage of the cell-wall. When these intercalated particles are situated in the same plane as are those already deposited, the cell-wall resulting from this method of construction will increase in area without adding to its thickness. But when once the cells are full-sized, the constructive activity of the protoplasts has to be directed in many cases to the strengthening and thickening of their walls, so that later on they may be able to perform special duties. From the appearance of this thickening one would judge that a number of layers were deposited on the thin original wall according to requirement, and in many instances no doubt the process corresponds to this appearance; but, as a rule, the thickness of the wall is increased by intercalation, on the part of the protoplasts, of 
additional material between the original particles, a process which has been termed "intussusception."

The appearance of stratification in thickened cell-walls is naturally most striking where substances of different kinds have been deposited alternately in the different parts of the wall, and when successive layers take up unequal quantities of water. The thickening may at length result in such an extreme restriction of the cell-cavity that its diameter is less than that of the inclosing wall. Sometimes nothing remains of the cavity but a narrow passage, and then the cells are like solid fibres. Formerly they would not have been classed with cells at all, but would have been distinguished under the name of fibres, from the forms resembling honey-comb cells. The protoplasts in these contracted cells languish and often die, especially when the walls of the self-made prison are greatly thickened and do not allow of intercourse with the world outside. But generally a protoplast takes care, in constructing its dwelling, not to close itself in entirely, nor to cut itself off permanently from the outer world. It either makes from the very beginning little windows in the walls of its house, leaving them quite open or closed only by thin, easily-permeable, membranes; or else, after constructing a completely closed envelope, it redissolves a piece of it, thus making an aperture through which in due time it is able to effect its escape. The scope of this work does not admit of an exhaustive treatment of the formative power possessed by protoplasts needful for these results; it will be sufficient to give a general description of some of the more important processes which have for their object the establishment of a connection between adjacent cell-cavities and of communication with the external world.

The new particles of material, or cellulose, which are to strengthen the delicate original cell-membrane, are in many instances not deposited or intercalated evenly over the entire surface of the protoplast. Little isolated spots are left unaltered, and these may be compared in a way to the small glazed windows in a living-room, or cabin port-holes closed by thin panes of glass. The part of the thickened wall which immediately surrounds the little window, and which so to speak constitutes its frame, has, besides, often a very characteristic structure, being elevated so as to form first a ring-like border, and eventually a hood, arching over the window and perforated in the middle (see fig. $10^{1}$ ). A comparison of this structure, arched over the thin spots in a cell-wall, to the iris spread in front of the crystalline lens in an eye would be still more appropriate. A similar annular border projects likewise from the window-frame on the other side, facing a neighbouring cell-cavity, so that the window appears symmetrically vaulted on both sides by mouldings with round central apertures (fig. $10^{2}$ ). Supposing someone wanted to pass from one cell-cavity to the other he would have in the first place to go through the hole in the moulding on his side. He would then find himself in a roomy space, which we will call the vestibule, and would next have to break through the little window, which is somewhat thickened in the middle, but elsewhere is as soft and thin as possible. On the further side 
again would be a vestibule, and it would not be until he had emerged from this through the aperture in the second moulding that he would reach the interior of the adjoining cell. Seen from in front, the outline of one of these windows, or rather the outline of the common floor of the vestibules, appears as a circle, whilst the aperture or opening in the moulding-which is exactly in the centre of this circle-is seen as a bright dot or pit encompassed by the circle which defines the limits of the vestibule. Hence these curiously protected window structures are named bordered pits. They are shown in fig. $10^{1}$ and $10^{2}$, and are to be seen in great perfection in the wood-cells of pines and firs.

Whenever bordered pits are formed, the thickening of the cell-membrane is comparatively slight; the frame of the window in the cell-wall is never more than.
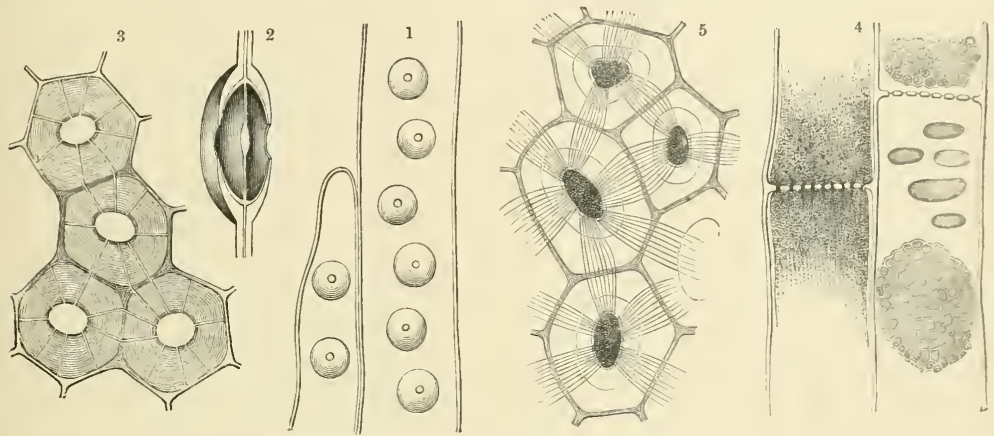

Fig 10.-Connecting Passages between adjacent Cell-cavities.

1, Bordered pits. 2, Section of a bordered pit. 3, Mode of connection of adjacent cells in the bundle-sheath of Scolopendrium. 4 , Sieve-tubes. 5, Group of cells from seed of $N u x$-vomica, the protoplasts of adjoining cell-cavities connected by fine protoplasmic filaments.

five times as thick as the window-pane itself. In other cases, however, the cell-wall becomes twenty or thirty times as thick as it was at first, and the interior of the cell is thereby seriously diminished in size. But even if, little by little, the cell-wall augments in thickness a hundredfold, any spot where thickening has not taken place from the first, and where, accordingly, a little depression occurs, is not subsequently covered with cellulose, but is carefully kept open by the protoplast as it builds. A greatly thickened wall of this kind resembles a fortification provided here and there with deep, narrow loopholes. Where two cells thus provided adjoin one another, the windows in the one occur, normally, exactly opposite those of its neighbour, and the result is the formation of canals, very long relatively, which penetrate through the two adjacent cell-walls and connect the neighbouring cellcavities together (fig. $10^{3}$ ). A canal of this kind is still closed, it is true, in the middle by the original cell-membrane as though by a lock-gate; but this slight obstruction may be removed later by solution, and the contiguous cells have then perfectly open connection through the canal.

Very frequently provision is made in the very first rudiments of a cell-mem- 
brane, destined to constitute a partition-wall, for open communications such as the above. For segments of the wall of various sizes are made from the beginning with sieve-like perforations, as is shown in fig. $10^{4}$, which represents diagrammatically portions of tubular cells called "sieve-tubes." The pores are crowded close together on the perforated areas of the walls of the sieve-tubes, and their dimensions are relatively broad and short. Thus, when two neighbouring protoplasts reach out to one another through these pores, that is to say, when there is continuity of the protoplasm of the two cell-cavities, the connecting filaments, which pass through the pores and which fill them completely, are short and thick and have the appearance of pegs or stoppers.

But in many cases the pores through which adjoining cell-cavities communicate are drawn out to a great length, forming infinitesimally slender passages. They are situated close together in great numbers and penetrate transversely through the thick cell-walls (fig. $10^{5}$ ). Neighbouring protoplasts may be brought equally well into mutual connection by means of these canals, or perhaps it would be better to say that their connection may be equally well maintained. For it is very probably the case that in the first rudimentary partition-wall, which is produced between the products of division of a protoplast, minute spots remain open and are occupied by connecting threads common to both halves of the protoplasm as they draw apart. Then in proportion as the partition-wall between the two protoplasts, produced by the division, becomes thicker, the openings take the form of fine canals, and the connecting filaments are modified into long and exceedingly fine threads which fill the canals. These protoplasmic threads pierce through the thickened cell-wall in the same way as a dozen telegraph-wires might be drawn through a partition from one room into another. Often a number of protoplasts living side by side and one above the other are linked together by filaments of this kind, which radiate in all directions.

This species of connection, of which an intelligible idea is given by fig. $10^{5}$, escaped the notice of observers in former times owing to the extraordinary minuteness of the canals, and delicacy of the protoplasmic filaments. Another method of communication between protoplasts in adjoining cells has, on the other hand, been long known and often described, its phenomena being very striking and visible when only slightly magnified. The connection referred to is that which is afforded by the formation of so-called "vessels." By vessels the older botanists understood tubes or utricles, arising from the dissolution of the partition-walls between a series of cells. Either the partition-walls in a rectilineal row of cells vanish, in which case long straight tubes are produced; or portions of the walls of cells arranged at different angles to one another are dissolved, and then tubes are formed having an irregular course, and sometimes branching or even uniting, so as to make a net-work. In instances of the first kind the lateral walls of the series of cells which are to lose their transverse partitions are previously thickened and made stiff by the protoplasts, which also provide them with various mouldings and panellings, and above all with bordered pits. This task accomplished, the protoplasts forsake the tubes, whose 
function thenceforth it is to serve as passages for air and water; thus the continued presence of the protoplasts is no longer advantageous. On the other hand, in the second class of vessels the lateral walls of the cells, which have coalesced to form them, exhibit no thickening, but are soft and delicate, and resemble flexible tubing. These tubes, moreover, are not deserted by their protoplasts; but, after the coalescence of a number of cells into a single duct has taken place, the protoplasts in the cells are themselves merged together, and the entire tube is then occupied by an uninterrupted mass of protoplasm, which generally persists as a lining to the wall.

As the initiation and construction of cell-walls are the work of the living protoplast, so also is their removal. The home it has made for itself the protoplast can also demolish-either partially or completely. But this demolition is preluded by the importation of particles of water into the portions of the wall which are to be destroyed. The introduction of water brings the wall into a gelatinous condition: the cohesion of its constituent particles is loosened, little by little, and at length completely abolished.

\section{COMMUNICATION OF PROTOPLASTS WITH ONE ANOTHER AND WITH THE OUTER WORLD.}

The transmission of stimuli and the specific constitution of protoplasm.-

Vital Force, Instinct, and Sensation.

\section{THE TRANSMISSION OF STIMULI AND THE SPECIFIC CONSTITUTION OF PROTOPLASM.}

As has been already intimated, the breaking down of individual cell-walls and the formation of the various pits, sieve-pores and fine canals in thickened membranes, in the manner described in preceding pages, are processes of great importance to the life of protoplasts. In the first place, many of the resulting structures are the means of preserving the possibility of intercourse with the outside world. In a space inclosed by evenly thickened walls, the absorption of air, water, and other raw materials from the environment would be very difficult if not impossible; the protoplast inside would soon lack the provisions needful for further development, and would at last die of starvation, drought, and suffocation. But the little windows, whether open or closed by thin permeable membranes, enable it to supply itself with all necessaries of life. Another advantage is derived, in the case of many of these structures, inasmuch as the protoplasts on occasion escape through the open doors and settle down in some other part of the cell-colony, where they are able again to make themselves useful. Lastly, one of the most important benefits of all is due to the fact that mutual intercourse between protoplasts, living together as a commonwealth, is rendered possible by the canals which join them together. And 
such an intercourse must of necessity be presumed to exist. When one considers the unanimous co-operation of protoplasts living together as a colony, and observes how neighbouring individuals, though produced from one and the same mother-cell, yet exercise different functions according to their position; and, further, how universally there is the division of labour most conducive to the well-being of the whole community, it is not easy to deny to a society, which works so harmoniously, the possession of unity of organization. The individual members of the colony must have community of feeling and a mutual understanding, and stimuli must be propagated from one part to another. No more obvious explanation offers than that the protoplasmic filaments, which run like telegraph-wires through the narrow pores and canals in the cell-walls (see fig. $10^{5}$ ), serve to propagate and transmit stimuli from one piutoplast to another. These threads of protoplasm may indeed be likened to nerves which convey impulses determining definite actions from cell to cell.

Imagination takes us further still, and raises the cell-nucleus to the position of the dominant organ of the cell-body For the nucleus not only determines the activity of the individual protoplast within its own cavity, but continues in sympathetic communion with its neighbour by means of all the threads and ligaments which converge upon it. This last idea in particular derives support from indications that the filaments uniting neighbouring protoplasts have their origin in specific transformations in the substance of the nucleus itself. When a protoplast living in a cell-cavity is about to divide into two, the process resulting in division is as follows:- The nucleus places itself in the middle of its cell, and at first characteristic lines and streaks appear in its substance, making it look like a ball made up of threads and little rods pressed together. These threads gradually arrange themselves in positions corresponding to the meridian lines upon a globe; but, at the place where on a globe the equator would lie, there then occurs suddenly a cleavage of the nucleus - a partition-wall of cellulose is interposed in the gap, and from a single cell we now have produced a pair of cells. In this way, from the nucleus, and from the protoplast of which the nucleus is the centre, two protoplasts have been produced, each having a nucleus of its own, and they thenceforth live side by side, each in its own chamber. It has been proved that in this process of division the substance of the nucleus is not completely sundered by the partition as it grows, but that, as we have already mentioned, minute pores are kept open in the cellulose wall, and that the pair of protoplasts continue joined together by threads running through these pores.

When we realize that every plant was once only a single minute lump of protoplasm, inasmuch as the biggest tree, like the smallest moss, has its origin in the protoplasm of an egg-cell or a spore; and when we consider how, by growth and repeated bipartition, thousands of cells are evolved, step by step, from a single one, whilst their protoplastic bodies still remain united by fine filaments, we arrive of necessity at the conclusion that the whole mass of protoplasm, living in all the myriads of cells whose aggregation constitutes a tree, really is, and 
continues to be, a single individual, whose parts are only separated by perforated sieve-like partitions. Every member of this community occupies a particular compartment or cavity, and is governed by a central organ, the cell-nucleus; but being linked to its fellows by connecting threads of protoplasm, a mutual understanding is thus established among them.

The physical basis of such an understanding may in this manner be represented with tolerable certainty. But it is extremely difficult to throw light upon the process of this mutual intelligence, the actual method whereby the cell-nuclei not only govern within their own narrow spheres, but also co-operate harmoniously for the good of the whole. And yet the problem involved in this unanimity of action, with a view to a systematic development of the plant in its entirety, is of such extreme importance that we cannot evade it even if, in the endeavour to solve it, we have to move altogether in the region of hypothesis.

In every attempt at explanation of the kind we must, at all events, bear in mind that the agreement in question, as well as the processes which take place in pursuance of this agreement, such as the nutrition, growth, and the organization of the entire plant, are reducible to the subtlest atomic agencies in the living protoplasm. They may be resolved into the motion of minute particles, into attractions and repulsions, oscillations and vibrations of atoms, and into re-arrangements of the atomic groups called molecules. Again, these movements are the result of the action of forces, especially of gravity, light, and heat. As regards gravity and light, experiment shows, however, that, when acting on living protoplasm, they give rise to varying effects even under the same conditions; and this fact, which will be discussed frequently later on, indicates that these forces are at any rate only to be conceived as stimulative and not coercive, and that they have no power to determine the kind of form. It is characteristic of the processes set up by gravity and light, especially when they take place in the continuous protoplasm of a great cell-community, that the coarser movements visible to the naked eye are often manifested in members comparatively remote from the part immediately affected by the stimulus. We cannot well represent this to ourselves except by supposing that the stimulus, which is the cause of the movement, is propagated through the threads of protoplasm from atom to atom, and from nucleus to nucleus. But the great puzzle lies, as already remarked, in the circumstance that the atomic and molecular disturbances occasioned by such stimuli and transmitted through the connecting filaments are not only different in the protoplasm of different kinds of plants, but even in the same plant they are of such a nature, according to the temporary requirement, that each one of the aggregated protoplasts in a community of cells undertakes the particular avocation which is most useful to the whole, the effect of this joint labour conveying the impression of the presence of a single governing power of definite design and of methodical action.

That a stimulus causes different occurrences in different species of plants, and, more especially, that cell-communities arising from different egg-cells develop into VoL. I. 
different forms, though under identical conditions and subjected to the same stimuli, are phenomena which have parallels in the inanimate world. A different sound is produced by striking the key of a piano which is connected to an A-string from that resulting from the transmission of a similar impulse to an F-string; and the difference depends on a difference of structure and an inequality of tension in the strings. Again, solutions of the sulphate and of the hyposulphite of sodium in similar glass vessels are indistinguishable at sight, both being colourless and transparent. These solutions will preserve their liquid condition when cooled down gradually to below freezing-point if they are kept absolutely still; but the moment the vessels are touched and a vibration thereby transmitted to the contents, they freeze. Crystals are formed in the apparently identical liquids, but crystals of different kinds, Glauber's salts in the one case, hyposulphite of sodium in the other. The variety of form depends simply on the sort of atoms, and on their number and mode of grouping.

In a similar manner must be explained the variety of forms in many plantspecies developed under the same conditions and affected by the same stimuli. Dozens of kinds of unicellular Desmids and Diatoms are often developed at the same time in a single drop of water in close proximity to one another. Although the protoplasm in the spores of these different species is absolutely identical to our vision, aided by the best microseopes, yet the mature cells exhibit a multiplicity of form which is quite astonishing to the observer on first inspection. One cell is semi-lunar, another cylindrical, a third stellate, a fourth lozenge-shaped, and a fifth acieular. In one specimen the cell-membrane is smooth, in another it is beaded; some are provided with siliceous coats, whilst others have flexible envelopes.

The same thing holds good with respect to the vegetable structures, which are composed of myriads of cells, and develop into huge shrubs or tall trees. The protoplasm in the egg-cell of an oleander is produced close to that of a poplar on the same river-bank, and under exactly the same external conditions. The cells divide, and partition-walls are introduced in the proper direction in either case, according to a plan of structure which is adhered to with marvellous precision by the protoplasts engaged in the work of construction. In each species, stem, branches, foliage, and blossoms have invariably a particular form and arrangement, have the same colour and smell, and contain the same substances. How utterly different are the mature leaf, the opened flower, and ripe fruit of the oleander from the corresponding parts of a poplar. Yet both were nourished by the same earth, were surrounded by the same atmosphere, and encountered the same rays of sunshine. We cannot otherwise explain it than by the supposition that, in a case like this, the difference of form in the perfected state is based upon a difference in the self-developing protoplasm, and that the atoms and molecules of this protoplasm, which appears to us to be uniform, vary in kind, number, and grouping in the two species of plants. Consequently, we must assume that every vegetable organism, every species of plant that appears invariably in the same external form when mature, and develops according to an invariable plan, has a protoplasm 
of its own of a certain specific constitution. And, further, we must assume that this specific protoplasmic constitution is transmitted from one generation to another, so that the protoplasm of the oleander, for example, had exactly the same constitution thousands of years ago as it has to-day. Lastly, we must assume that each special kind of protoplasm has the power to reproduce its like, ever anew, from the raw materials occurring in its environment.

\section{VITAL FORCE, INSTINCT, AND SENSATION.}

The phenomena observed in living protoplasm, as it grows and takes definite form, cannot in their entirety be explained by the assumption of a specific constitution of protoplasm for every distinct kind of plant; though this hypothesis will again prove very useful when we inquire into the origin of new species. What it does not account for is the appropriate manner in which various functions are distributed amongst the protoplasts of a cell-community; nor does it explain the purposeful sequence of different operations in the same protoplasm without any change in the external stimuli, the thorough use made of external advantages, the resistance to injurious influences, the avoidance or encompassing of insuperable obstacles, the punctuality with which all the functions are performed, the periodicity which occurs with the greatest regularity under constant conditions of the environment, nor, above all, the fact that the power of discharging all the operations requisite for growth, nutrition, renovation, and multiplication is liable to be lost. We call the loss of this power the death of the protoplasm. It ensues upon assaults from without if they succeed in destroying the molecular structure so entirely as to render reconstruction impossible; but, furthermore, death may take place without external cause.

If cells of the blood-red alga, previously mentioned as allied to the red-snow, are collected from hollows in stones, casually full of rain-water, and are kept dry for weeks and then again moistened, the water is found to have a very powerful effect. The protoplasm becomes mobile, and swarm-spores are formed which put forth vibratile cilia, propel themselves about for a short time in the water, and then settle down in some favoured spot, draw in their cilia, come to rest and divide, producing offspring which again are motile. This alga may be kept dry for months, nay even over a year, and still its cells exhibit the movements above described when put into water. But if a mass of it is preserved under these same conditions for many years and then moistened, the little cells will, it is true, take up additional water, but motile cells are no longer formed. The cells do not move, nor grow, nor divide, but gradually become discoloured; are first disintegrated and then dissolved. We say then that in them life could no longer be recalled, and we describe them as dead.

The same thing is observed in great cell-communities. The seeds of many species of plants preserve the capacity for germination for an incredibly long period, especially when kept in a dry place. If after ten years such seeds are transferred into 
moist earth, the protoplasm in the majority of cases begins to bestir itself and to move, and the embryo grows out into a seedling. After twenty years, perhaps, only about five per cent of the seeds preserved would germinate. The rest are not stimulated by damp earth to further development; their protoplasm no longer possesses the power of augmenting its volume by absorption of matter from the environment, or of developing a definite form, but is disintegrated by the influx of air and water and breaks up into simpler compounds. After thirty years hardly one of the seeds would spront. Yet all these seeds were kept throughout the time at one place and under precisely the same external conditions; nor can the slightest change in their appearance be detected. Gardeners express the fact by saying that the capacity for germination becomes extinct in from twenty to thirty years. But what kind of a force is this which may perish without a physical change of the substance concerned affording the basis of the extinction? In former times a special force was assumed, the force of life. More recently, when many phenomena of plant life had been successfully reduced to simple chemical and mechanical processes, this vital force was derided and effaced from the list of natural agencies. But by what name shall we now designate that force in nature which is liable to perish whilst the protoplasm suffers no physical alteration and in the absence of any extrinsic cause; and which yet, so long as it is not extinct, causes the protoplasm to move, to inclose itself, to assimilate certain kinds of fresh matter coming within the sphere of its activity and to reject others, and which, when in full action, makes the protoplasm adapt its movements under external stimulation to existing conditions in the manner which is most expedient?

This force in nature is not electricity nor magnetism; it is not identical with any other natural force, for it manifests a series of characteristic effects which differ from those of all other forms of energy. Therefore, I do not hesitate again to designate as vital force this natural agency, not to be identified with any other, whose immediate instrument is the protoplasm, and whose peculiar effects we call life. The atoms and molecules of protoplasm only fulfil the functions which constitute life so long as they are swayed by this vital force. If its dominion ceases, they yield to the operations of other forces. The recognition of a special natural force of this kind is not inconsistent with the fact that living bodies may at the same time be subject to other natural forces. Many phenomena of plant life may, as has been already frequently remarked, be conceived as simple chemical and mechanical processes, without the introduction of a special vital force; but the effects of these other forces are observed in lifeless bodies as well, and indeed act upon them in a precisely similar manner, and this cannot be said of the force of life.

Were we to designate as instinctive those actions of the vital force which are manifested by movements purposely adapted in some manner advantageous to the whole organism, nothing could be urged against it. For what is instinct but an unconscious and purposeful action on the part of a living organism? Plants, then, possess instinct. We have instances of its operation in every swarm-spore 
in search of the best place to settle in, and in every pollen-tube as it grows down through the entrance to an ovary and applies itself to one definite spot of an ovule, never failing in its object. The water-crowfoot, in deep water, fashions its leaves with finely divided tips, large air-passages, and no stomata; whilst, growing above the surface of the water, its leaves have broad lobes, contracted intercellular spaces and numerous stomata. Linaria Cymbalaria (see fig. 11) raises its flower-stalks from the stone wall over which it creeps towards the light, but as soon as fertilization has taken place, these same stalks, in that very place and amidst unchanged external conditions, curve in the opposite direction, so as

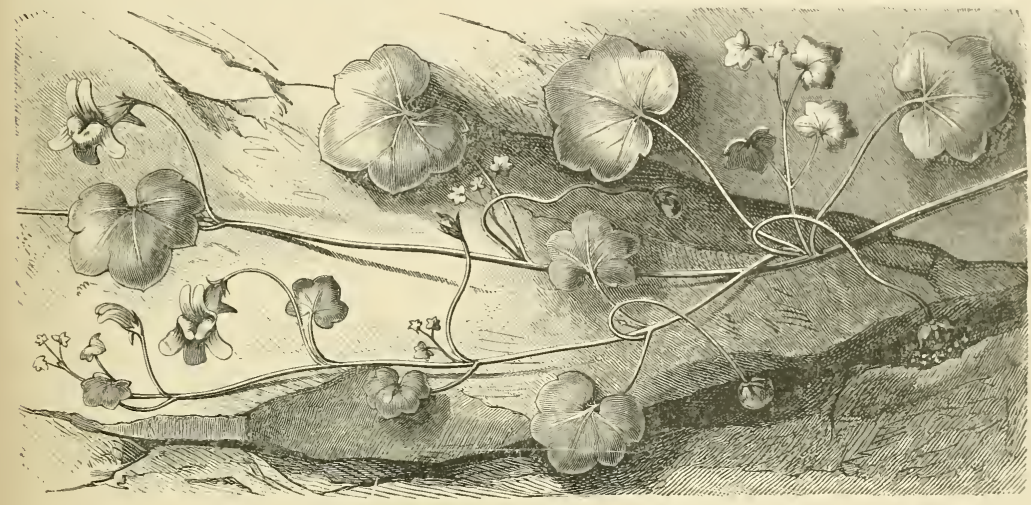

Fig. 11.-Linaria Cymbalaria dropping its Seeds into Clefts in the Rocks.

to deposit their seeds in a dark crevice. The flower-stalk of Vallisneria twists itself tightly into a screw and draws the flowers, which previously it had borne upon the surface of the water, down to the bottom when their stigmas have been covered with pollen-dust at the surface. These are all cases of unconscious action for a definite object, that is to say, they are the result of instinct.

If, however, we attribute instinct to living plants, it is but a step further to consider them as endowed with sensation also. Feeling in animals is the concomitant of a condition of disturbance in nerves and brain caused by a stimulus, which acts on the organs of sense, and is conveyed by nerves to the central organ. The transmission of the stimulus and the excited state of the brain and nerves are only molecular movements of the nervous substance, or, let us say, of the protoplasm, for nerve-fibres and nerve-cells are simply protoplasm developed in a particular manner. But the state induced by the stimulation of protoplasm, which is what we call sensation, cannot be essentially different in vegetable protoplasm from what it is in animal protoplasm, since the protoplasm itself, the physical basis of life in both plant and animal, is not different. In isolated plant-cells, indeed, it may amount to such a concentration of the condition of stimulation as to be called sensation, for the cell-nucleus is to all appearance 
a central organ in relation to the protoplast that lives in a solitary cell. It is not of course to be supposed that within a whole plant-structure, that is in the community of live protoplasts which constitutes an individual plant, such a concentration of stimulation could occur as is the case with individual animals which have nerve-fibres all converging into the brain; but between the sensation of animals without nerves and that of plants no essential difference can exist.

Hence we infer that there is no barrier between plants and animals. The attempt to establish a boundary-line where the realm of plants ceases and the animal world begins is a vain one. If we naturalists, all the same, agree to separate plants and animals, we do so only because experience shows that a division of labour conduces to a speedier attainment of our object. On the intermediate ground where animals and plants meet, zoologists and botanists encounter one another, not, however, as hostile rivals with a view to exclusive possession of the field, but as colleagues with a common interest in the administration and cultivation of this jointly tenanted region. 


\section{ABSORPTION OF NUTRIMENT.}

\section{INTRODUCTION.}

Classification of plants with reference to nutrition.-Theory of food-absorption.

\section{CLASSIFICATION OF PLANTS WITH REFERENCE TO NUTRITION.}

The object of a plant's vital energy, next in importance to the resistance of such influences as are likely to bring about the death of the protoplasm, is growth, i.e. the addition of substance to its body, or, in other words, the absorption of nutriment. A living plant, whether consisting of a single cell or of a vast community of cells, takes up food from its environment in quantities varying according to the needs of the moment. But its method of action-how it sets about acquiring possession of this raw material, how it manages to incorporate the substances absorbed from without, how it contrives to retain only such part as is useful to it, and to reject and get rid of, like ballast, what does not subserve its own growth-is infinitely varied. This variety in the processes of food-absorption corresponds, on the one hand, to differences in the habitat of plants, and, on the other, to the requirements of particular species, which requirements in their turn depend upon a specific constitution of the protoplasm in each species concerned. The difference must be very great between this process as manifested in plants which are immersed in water during their whole lives and the same as it occurs in plants which live in desert sands and are not supplied with water for months together. And again, absorption in those fungi which grow luxuriantly on damp timber in the deep obscurity of a mine must take place very differently from the corresponding process in the delicate alpine plants which on our mountain slopes are exposed periodically to the most intense sunlight, and then, for weeks at a time, are wreathed in sombre mists. So, also, the reciprocal action between plants and their environment must have a character of its own in the case of parasitic growths which absorb their food from other living organisms, and in those remarkable plants, too, which catch and devour small insects, and in such minute organisms as yeast, the vinegar ferment, and others, which play so important a part in our daily life, and lastly, in the gigantic trees which form our forests.

To acquire a general notion of these forms, with reference to their varieties as regards nutrition, it is best to classify them in the first place in groups according to their habitat, viz.: into water-plants or hydrophytes, stone-plants or lithophytes, land-plants, and epiphytes. But here again it is necessary to remark that no sharp 
line of demarcation exists between these groups; all are connected by numerous intermediate links, and there are forms which belong to one group at one stage of development and to another at another stage.

The distinctive property of aquatic plants is that they derive their nourishment either entirely or principally from the surrounding water. Some preserve their freedom, floating or swimming about in the liquid medium; but the majority are fixed somewhere under the water by special organs of attachment. Many plants that are rooted in the mud at the bottom of pools are able to derive their food from the water when it is high, and when it is low, from the atmosphere as well: such amphibious organisms form a transitional group between water-plants and landplants. The number of lithophytes is comparatively very small. They include those lichens and mosses which eling in immediate contact to the surface of stones and derive their food in a fluid state direct from the atmosphere. All lithophytes are so constituted that they can, without injury, dry up and suspend their vitality for a time when there is a failure of atmospheric precipitation lasting over a long period or when the air itself is very dry. But not every plant which grows upon rocks is to be regarded as a lithophyte in the narrower acceptation of the term. Those that are rooted in earth in the cracks and crevices of the rock must be classed amongst land-plants. To this class indeed more than half the plants now in existence belong. Though surrounded by air as regards a part of their structure they have another part sunk in the soil, and from the soil they take up water and inorganic compounds in aqueous solution. Plants which grow attached to other plants or to animals are called epiphytes.

The majority of plants are during the period of food-absorption connected with the foster-earth and are not capable of locomotion. The plant being fixed to one spot must therefore sooner or later exhaust the ground in its neighbourhood, and must require a further supply of nutritive substances. The parts specially devoted to food-absorption often lengthen out in these circumstances beyond the impoverished region, and thus endeavour to bring areas more and more distant within the range of absorption. Many plants possess the faculty, to which reference has already been made, of alluring animals and of killing and sucking their juices. Not only amongst saprophytes and parasites, but also amongst aquatic plants, instances occur in which certain movements are performed involving the whole body of the organism, with a view to promoting the absorption of nutriment. Particularly striking in this respect are many plasmoid fungi (which we may well refer to here, not on this account alone, but also for the additional reason that they take in nourishment without the intervention of a cell-membrane). The naked protoplasm in these cases, which include in particular the class of Amœbæ, crawls in its search for food over the nourishing substratum, and derives from it immediately the materials needful for growth. Loose bodies are liable to be seized by the radiating processes of the protoplasm, which then closes round them and drains them completely of their juices (see fig. 9, the last figure to the right). These bodies encompassed by the protoplasm, if small, are drawn inwards from the periphery and are regularly digested in the 
interior. Such parts of foreign bodies as are not serviceable for nutrition are subsequently eliminated or are left behind by the protoplast as it creeps onward. But this method of food-absorption is limited to amceboid forms belonging to the boundary-land of animal and vegetable life. The movements of other naked protoplasts, such as those which are carried about in the water by vibratile cilia, have nothing to do with the search for food or with its absorption, but are connected rather with the processes of distribution and propagation.

\section{THEORY OF FOOD-ABSORPTION.}

In the case of protoplasts inclosed in cell-membranes the food necessary for nourishment must always pass through the cell-membrane and peripheral protoplasmic layer (ectoplasm) into the interior of the protoplasmic bodies. And so, conversely, such of the substances absorbed as are of no use in the construction of the organism or for any other purpose, must be separated and passed out through these envelopes. The cell-membranes of those protoplasts which are employed in absorbing food must accordingly have a special structure: the ultimate particles must be so arranged as to allow of the passage of nutritious material inwards, and of rejected matter outwards, without prejudice to their own stability. The passages in cell-walls used for this purpose are very minute, much smaller at all events than the pore-canals described above as being occupied by fine protoplasmic filaments; the dimensions are in fact so trifling as to be invisible even with the best microscopes. Still we are forced to conclude that they exist by a posteriori reasoning from a series of phenomena, and to assume that the cellmembrane, like almost every other kind of body, consists not of continuous matter, but of minute particles, which are termed atoms, and are separated from one another by infinitesimally small spaces. Various processes and appearances have also led physicists and chemists to the conclusion that these atoms are not aggregated in disorder, but are always combined together in groups of two or more, even in the case where all the atoms in a body are of the same kind, i.e. are the same element. If a body contains different elements they are not mixed together indiscriminately, but are grouped in conformity to a definite law: every group includes atoms of all the different elements concerned, arranged in a certain invariable manner, not only as regards number, but also as regards relative position. Groups of atoms of this kind are called "molecules," and the spaces between them are supposed to be larger than those between single atoms. Further, it is not improbable that the molecules themselves form groups, each group consisting of molecules conglomerated in a definite manner, and that the passages separiting these molecular groups are larger again than those separating the single molecules within each group. These groups of molecules have been called "micellie" or Tagmata, and they also are supposed to be aggregated together in definite order.

According to this theory the cell-membrane is analogous to a sieve, the pores of which are grouped in a definite manner, the broadest perforations being between 
the micellæ or groups of molecules, narrower apertures between the molecules or groups of atoms in each micella, and lastly the finest pores between the atoms themselves in each molecule. These interspaces are liable to contraction and expansion, for the union of the molecules is affected by two forces, one of which manifests itself as a mutual attraction between atoms and atomic groups, whilst the other tends to drive atoms and molecules asunder. Of these forces the former, i.e. the attractive force existing in all material particles, is called chemical affinity when it causes atoms of different kinds to unite to form a molecule; and it is called cohesion when applied to the mutual attraction of similar molecules, and adhesion where it holds together masses of molecular groups with their surfaces in contact. The action of heat is opposed to this attractive force, which is only effective at infinitesimal distances. Bodies are all caused to expand by heat, their atoms, molecules, and micellæ being forced apart. Heat is believed to be a vibratory motion of these ultimate particles, and it is supposed that the greater the vibrations the greater is the separation of atoms and atomic groups, the interspaces expanding and the heated body increasing consequently in volume. As is well known, the atoms and molecules may be forced so far apart by increase of temperature that cohesion is entirely overcome, and solids are converted, first into liquids and at last into gases.

The interspaces or passages between the molecules and molecular groups composing a cell-membrane are penetrable by molecules of other substances, provided always, firstly, that the admitted molecules are not larger than the passages; and secondly, that there exists between the molecules of the cell-wall and those of the penetrating body that sort of attractive force which has been designated chemical affinity. Both premises are satisfied in the case of aqueous molecules, and experiment proves that they are admitted into the inter-molecular spaces of a cellmembrane with great ease and readiness. The cell-membrane saturates itself with water, or, to use the technical phrase, it has the tendency and ability to "imbibe" water. The force of attraction between molecules of a cell-membrane and watermolecules is indeed so intense that the cohesion of the molecules in the membrane is partially neutralized, and the imbibed water causes them to move apart. In consequence of this, the cell-membrane swells up and its dimensions are increased.

It is also supposed that the micellæ of a cell-membrane attract and admit watermolecules to such an extent as to surround themselves with watery envelopes. Such a condition would no doubt be nothing but beneficial, promoting, as it would, the interchange of materials through the cell-membrane, and the mixing of fluid substances situated on either side of the porous membrane. At all events this mixing process must ensue in the interspaces of the cell-membrane; and, in the particular case out of which this discussion has arisen, viz. food-absorption, the interacting substances are, on the one hand, the compounds in the soil outside the cell-membrane, and, on the other, the organic compounds under the control of the live protoplast within the cell-membrane. Both the outgoing and the incoming substances must be soluble in water, and must, therefore, have an attraction 
for water. But the power of a substance in aqueous solution, whether without or within the cell-membrane, to permeate the saturated pores, and to mix thoroughly there, certainly depends also on the degree of chemical affinity and of adhesion existing between the molecules and micellæ of the cell-membrane on the one hand, and these infiltrating substances on the other. A very complex interaction of forces takes place which we cannot here investigate any further, as it would take us much too far afield.

Returning to the explanation of food-absorption, attention must be drawn to the fact that the mixing or diffusion which takes place through the cell-membrane differs from the free diffusion which would occur if the cell-membrane were not present. Experiment has proved that if one side of a cell-membrane is steeperl in a saline solution and the other in an equal volume of pure water, the number of saline particles which pass through into the water are many fewer than the number of water-particles which pass into the solution of salt; and, moreover, if an organic compound, such as albumen or dextrin, is on one side, and water on the other, water transfuses to the organic compound, whereas no trace of the albumen or dextrin (as the case may be) passes through to the water. Now this phenomenon, which is called "osmosis" ("endosmosis and exosmosis"), is of great importance for the conception we have to form of food-absorption. It is clear that, whilst water and substances dissolved in water are brought under the control of the protoplast within a cell through the cell-membrane, as a consequence of the action of albuminous and other compounds constituting the body of the protoplast, and of the salts dissolved in the so-called cell-sap in the vacuoles, there is no necessity for any part of the cell-content to pass out through the cell-membrane. Thus the protoplasm is able to exercise an absorptive action on aqueous solutions outside the cell-membrane, and to continue to absorb until the cell is filled. Indeed, the chemical affinity for water possessed by the substances in a cell may occasion so great an absorption of water that, in consequence, the volume of the cell is enlarged and the cell-membrane is subjected to pressure from within. The cellmembrane is able to yield to this pressure to the extent permitted by its elasticity; but excessive stretching of the cell-membrane is at length counteracted by cohesion, and thus a condition is attained in which the cell-contents and the cell-membrane are subjected to mutual pressure, a state which is called "turgidity."

The process just described, of the absorption of water in large quantities into the precincts of the protoplasm without any simultaneous transmission of matter to the outside, is certainly in no respect an exchange. But it obviously does not exclude the possibility of a real exchange taking place between substances on either side of a cell-membrane, i.e. between solutions in the soil and those in the cellsap contained in lacunæ of the protoplasm. Certain phenomena in fact put it beyond doubt that on occasion a real exchange of this kind does occur. But it is complicated by the circumstance that substances in process of being exchanged have to pass not only through the cell-membrane but also through the primordial utricle; and the primordial utricle consists of molecules of a kind other thru 
those of the cell-wall, having different chemical affinities, and these molecules again are differently grouped; nor are the passages for aqueous solutions the same. All this cannot but have an important bearing on the permeating capacity of the substances that are being interchanged.

Although all these ideas concerning the molecular structure of cell-membranes and of protoplasm, concerning the intermixture and exchange of materials and the absorption on the part of cells and their swelling up, have only the value of theories, still we have good ground for assuming that they are fairly near the truth. They give us, at all events, an intelligible representation of the interaction which takes place between living protoplasts, with their need for food, and the environment, which supplies the nutriment.

\section{ABSORPTION OF INORGANIC SUBSTANCES.}

Nutrient Gases.-Nutrient Salts.-Absorption of Nutrient Salts by Water-plants, Stone-plants, and Land-plants.-Relations between the position of Foliage-leaves and Absorption-roots.

\section{NUTRIENT GASES.}

One of the most important sources of the nourishment of plants is carbonic acid. The living protoplasts appropriate it from water and from air, in the latter case chiefly by attracting the carbon-dioxide. ${ }^{1}$ This gas penetrates a cell-wall saturated with water more readily than the other constituent gases of the atmosphere (nitrogen and oxygen). In the wall it is converted into carbonic acid, and it then passes on into the cell-sap contained in the cavities of the protoplast. Apart from the effects of temperature and atmospheric pressure, the quantity of carbonic acid absorbed is chiefly determined by the requirements of the cells whose nourisiment is in question. These requirements, however, vary considerably according to the specific constitution of the protoplasm and with the time of day. During daylight the need of carbon is very great in all green plants. As soon as the carbonic acid reaches the cell-sap it is decomposed and reduced by the action of sunlight, and from it are formed compounds known as carbo-hydrates. The oxygen thus set free is, however, removed from the cell precincts, and expelled into the surrounding air or water. In this way the gas when barely absorbed is withdrawn, as such, from the cell-sap, the carbon alone being retained and the oxygen eliminated, and a renewed attraction of carbon-dioxide from the surrounding medium ensues. The fresh supply again is immediately worked up in the green chlorophyll-bodies, so that there is a constant influx of carbon-dioxide, and therefore indirectly of carbonic acid, from the environment into the interior of green cells to the part where its consumption takes place. Were it possible to see

\footnotetext{
1 The atmosphere contains free carbon-dioxide and not carbonic acid. But carbonic acid is formed when the
} dioxide is absorbed into water. 
the molecules of carbon-dioxide in the air, we should observe how much faster they are impelled towards the leaves and other green parts of plants, where the intense craving for carbon is localized, than are the other constituent particles of the air. This impulsion and influx lasts so long as the green cells are under the influence of daylight. The first thing in the morning when the first ray of sunshine falls upon a plant the protoplasts begin work in their little laboratories decomposing carbonic acid, and producing from it sugar, starch, and other similar organic compounds. And it is not till the sun sets that this work is suspended, and the influx of carbondioxide stopped till the following morning.

The green plants that spend all their lives under water are supplied with carbonic acid by the water surrounding their cells, which always contains some of that material. In the case of unicellular plants of this class, absorption of carbonic acid takes place through the whole surface of the cell-membrane. Multicellular plants, with their cells arranged in filaments or plates, only take in carbonic acid through those parts of the walls of their cells which are in immediate contact with the water. This applies also to submerged plants composed of several layers of cells and of considerable dimensions. Thus, in plants of this kind, the cells in contact with the water constitute the skin. They are always pressed closely together and squeezed flat, are not thickened on the side exposed to the water, and are united everywhere edge to edge leaving no gaps. But in the interior of these water-plants large lacunæ and cavities are formed from earliest youth, owing to the detachment of single rows of cells; and the spaces so formed are filled with a quantity of nitrogen, oxygen, and carbon-dioxide, that is to say, with a gaseous mixture not essentially different from atmospheric air. Although this organization may have as its primary object the reduction of the plant's weight as a whole, it cannot be without a further importance inasmuch as carbonic acid can be taken up from the air-spaces into adjacent cells. But there is no doubt that, even in this case (of water-plants provided with large internal air-cavities), the chief absorption of carbonic acid is through the epidermis, or more precisely through those walls of the epidermal cells which are in immediate contact with the water.

The carbonic acid taken up by cells, wholly or partially immersed in water, is either contained as such dissolved in the watery medium, or occurs in combination with calcium as bicarbonate of lime. Part of the carbonic acid in this bicarbonate in aqueous solution is susceptible of being withdrawn by water-plants, mono-carbonate of lime, which is insoluble in water, being then precipitated on the cell-wall through which the rest of the carbonic acid has passed into the cell-interior. Accordingly, a large number of water-plants are found incrusted with lime in both fresh and salt water. We shall return to this important phenomenon when we treat of the influence of living plants on that part of the environment which comes within their sphere of action for purposes of nutrition.

Lithophytes obtain carbonic acid from the moisture deposited upon them from the aqueous vapour in the atmosphere, and attract carbon-dioxide direct from the 
air around them. The chief members of this class are those mosses, liverworts, and lichens which, though clinging to dry rocks, behave just like water-plants as regards the absorption of carbonic acid. There is no reason to think that these plants absorb carbonic acid in dry weather; for under the influence of dry air they lose water fast, and meanwhile receive no compensation from the rock to which they are attached, and in a short time they become so dry that they crumble into powder when rubbed between the fingers. Vitality is suspended for a time, and it is out of the question that there should be any absorption of carbon-dioxide from the atmosphere under such circumstances. But the moment the plant is moistened by rain or dew, the cell-walls directly exposed to the air become saturated, and are enabled to admit water into the interior. Then the lithophytes suck up water very fast; the dry, apparently dead, incrustations swell up again, and, together with the rain and dew, carbonic acid is absorbed, it being contained in all depositions of atmospheric moisture. A tumescent moss tuft can, in addition, absorb carbon-dioxide direct from the atmosphere through its saturated superficial cells; but the quantity of carbonic acid thus acquired by a plant is in any case only secondary. Many mosses, as for example the widely-distributed Grimmia apocarpa, are also able to live just as well under water as in air; nor is any alteration of their leaves necessary in either condition, nor any special contrivance for the absorption of carbonic acid and water. These substances reach the interior by similar passage through cell-walls of identical construction, whether the Grimmia spends its life attached to submerged rocks or in the open air at the top of a mountain; whence we may infer that there is a greater resemblance between lithophytes and water-plants as regards nutrition than between lithophytes and land-plants.

Land-plants satisfy their need of carbon almost exclusively by withdrawing the dioxide from atmospheric air. For the purpose of this direct appropriation, specially adapted structures are found in them. Seeing that these plants are not able to endure periodic desiccation in times of drought, as lithophytes are, it is necessary for them to be secured against excessive loss of water. Accordingly, the cell-walls in immediate contact with the air, that is to say, the outer walls of the epidermis, are thickened by a layer (cuticle) which is impermeable by air or water, and, in general, they are so organized that water cannot readily escape from the interior of the cells. Obviously, however, a cell-wall which opposes a strong resistance to the extravasation of water will not give easy admittance to an influx either, and the conditions for the passage of gases through a cell-membrane, thickened and cuticularized in this way, would be far from favourable. As a matter of fact many of the constituent gases of the atmosphere permeate these thickened walls of the epidermal cells only with great difficulty, and others not at all. Carbon-dioxide alone has the power of penetrating, but even in the case of this gas the quantity is not always sufficient to satisfy the demand. To ensure that so important a form of plant-food should reach in proper amount those cells lying under the epidermis, which are occupied by protoplasts engaged in the regu- 
lation of nutrition, there is an adaptation of structure of the following nature. Among the firmly connected epidermal cells with their thickened outer walls almost impervious to air, other cells are interspersed at intervals. They are always in pairs, are generally rather smaller than the rest, and have a little cleft open between them. Inasmuch as these apertures (stomata) always exist where passages and canals, the so-called intercellular spaces, have arisen from the separation of individual cells of the sub-epidermal tissues, each stoma constitutes the mouth of a system of channels ramifying between the thin-walled cells of the interior. The components of the atmosphere, especially carbon-dioxide, are able to reach these internal passages through the stomata, and in them they travel to the chlorophyllcontaining cells. Through the thin, saturated walls of these cells they are able to penetrate with ease, and so they reach the living protoplasts, with their equipment of chlorophyll, whose daily work it is, as already mentioned, to decompose-under the transforming power of light-the carbonic acid as it reaches the chlorophyllbodies, to work up the carbon and expel by the same path as they entered not only the oxygen but also all other aerial constituents which may have penetrated and for the moinent find no employment.

These ventilation-canals, with stomata as orifices at the epidermis, have other uses besides the importation of carbon-dioxide (and therefore of carbonic acid) and the exportation of oxygen. For the same pores, passages, and lacunæ, as serve for the influx and exit of carbon-dioxide and oxygen respectively, are the channels of a plant's respiration. Moreover, they play a very important part also in the escape of aqueous vapour, the process known as "transpiration;" and as the variety in their structure is to be interpreted chiefly as an adaptation to the different conditions under which transpiration occurs, it cannot be profitably discussed until we treat of that process.

Those saprophytes and parasites which contain no chlorophyll or practically none, do not absorb any free carbon-dioxide from the atmosphere, but supply themselves with carbon from the organic compounds in the nutrient substratum on which they grow. But saprophytes and parasites, abundantly furnished with chlorophyll, doubtless do attract free carbon-dioxide in addition. They may do so either after the manner of water-plants and lithophytes, as is the case with Euglenæ, and with mosses growing on the dung of mammalia; or else after the manner of land-plants, as instances of which the cow-wheat, yellow-rattle, and eye-bright may be quoted.

It is a very remarkable fact that no plant is known which takes up carbondioxide or carbonic acid from the earth. One might expect that the roots of landplants at any rate, ramifying as they do in a stratum of earth saturated with water containing carbonic acid in solution, would suck up to some extent so important a food, and that it would be from them conducted to the green-foliage leaves. But so far as experiments have gone, they indicate that this is not the case.

Equally curious is the circumstance that nitrogen, which is an indispensable constituent of protoplasm, and therefore a very important means of subsistence, is 
not absorbed from the surrounding air, although, as is well known, the atmosphere contains nitrogen to the amount of 79 per cent of its volume. There can be no doubt that though nitrogen permeates the cell-walls of an air-encompassed plant much less readily and quickly than carbon-dioxide, yet it is carried from the atmosphere into the ventilation-spaces of green foliage-leaves, and further through the thin cell-walls into the laboratories of the protoplasts, where one would expect it to be worked up in the same way as carbonic acid. The most careful experiments have determined, however, that it is not turned to account in this form by the protoplasts, but that on the contrary it is given back unused to the air, and only such nitrogen as reaches the interior of plants in combination with other substances is of any service there.

The principal sources of the nitrogen required by plants are nitrates and ammoniacal compounds absorbed from the ground; but nitric acid and ammonia themselves, of which there are traces in the atmosphere and in water, must not be overlooked. The quantity of nitric acid in air is, it is true, even less than that of carbon-dioxide; but just as the small amount of carbon-dioxide can be absorbed from the air with highly productive results, so may also the still smaller proportion of nitric acid be turned to account. The sources of nitric acid are dead organic bodies as they decompose and become oxidized. In many ways the process of formation of nitric acid from decaying bodies may take place so as to produce ammonia in the first place and from it nitric acid. It would seem possible, though it is an unproved assumption, that in places where dead bodies of plants and animals vegetable mould, manure, and such things are undergoing oxidation, that is to say, in woods and fields, the small quantities of nitric acid that are given off are immediately taken up by the plants growing there. It must be borne in mind that plants behave with reference to what is necessary or useful to them like a chancellor of the exchequer preparing his budget; they take these things where they find them.

The question has been raised, too, as to the source from which the first plants that appeared on the earth were able to obtain nitric acid. We are obliged to assume that, at that time before the existence of nitrogenous organisms to supply nitric acid by oxidation of their dead bodies, all nitric acid, and therefore all the nitrogen used in the nourishment of plants, was generated by thunder-storms. We know that nitric acid is formed in the air on occasion of electric discharges and is deposited on the earth together with rain and dew. This source of nitric acid is not yet exhausted, and even at the present day it no doubt plays the same part as in the ages long past at the commencement of all vegetable life.

If nitric acid is used by protoplasts, in the building up of the highly important albuminous compounds, it is broken up in a manner similar to the decomposition of carbonic acid to form carbohydrates, that is to say, oxygen is separated out. In this case, however, sunlight and, therefore, chiorophyll are not immediately concerned. Moreover, the oxygen that is set free is not eliminated, but is used in the manufacture of other compounds in process of formation in the plant, probably in that of vegetable acids. 
Ammonia behaves in relation to plants just in the same way as carbon-dioxide and nitric acid. It is disengaged from dead decomposing organic bodies, anr is found in traces, either alone or with equally minute quantities of carbon-dioxide and carbonic and nitric acids in the air, in atmospheric deposits, and in all water wherein animals and plants reproduce their kind, the old individuals dying and making way for the young. Water-plants are all limited to this source for acquisition of nitrogen. As regard lithophytes, it stands to reason that they must derive their nitrogen from the ammonia contained in the air, in atmospheric deposits, and from nitric acid. Whence otherwise could a crustaceous lichen attached to a quartz rock on a mountain supply itself with the nitrogen essential for the growth of its protoplasm? Horeover, some of the larger lithophytes, especially mosses, seem to be capable of absorbing ammonia direct from the air. An observation made in the Tyrolese Alps has some bearing on this question:-The ridges of the Hammerspitze, a peak rising to 2600 meters between the Stubaithal and the Gschnitzthal, is, in favourable weather in the summer, the resting-place of hundreds of sheep, and is consequently covered with an entire crust of the excrements of these animals. A highly offensive and pungent smell of ammonia is evolved, and renders a prolonged stay on this spot anything but pleasant, notwithstanding the beauty of the view. Now, it is worthy of note that the mosses, which are produced in abundance on the rocks above this richly-manured ground, but are not themselves actually amongst the sheep-droppings, exhibit a luxuriance unparalleled on any of the neighbouring summits belonging to the same formation but unfrequented by sheep. The gaily-coloured green carpet extends as far as the ammoniacal odour is perceptible, and it is natural to suppose that this luxuriant growth is stimulated by the absorption of ammonia direct from the air.

Land-plants also can take up ammonia from the air. It has been shown that the glandular hairs of many plants, for instance those on the leaves of Pelargonium and of the Chinese Primrose, have the power of absorbing traces of ammonia, and of sucking up carbonate and nitrate of ammonia in water with rapidity. When we consider that a single one of these primroses (Primula sinensis) possesses two and a half millions of absorbent glandular hairs so placed as to be able to take up the ammonia brought to the plant by rain, we are unable to look upon this process as of altogether trifling importance. It is highly probable that almost all ammonia, after its formation from decaying substances in the ground, is at once absorbed by the plants growing in the immediate neighbourhood, and that the relatively small quantity of ammonia in the upper atmospheric strata is referrible to this cause. The splendid luxuriance of the pelargoniums, thickly studded with glandular hairs, which one sees in front of cottage windows in mountain villages where a dung heap is close by, and in the windows of stables, frequently excites admiration and surprise. Whether it is due to the fact that in these situations there is the possibility of absorbing an unusually large quantity of ammonia is a question which we will leave undecided. 


\section{NUTRIENT SALTS.}

If wood, leaves, seeds, or any other parts of plants are subjected to a high temperature with free access of air, the first changes that occur are in the compounds of nitrogen and of carbon contained in the heated matter. They turn black, are charred and burnt, and ultimately the products of combustion pass into the atmosphere in gaseous condition. The incombustible part which remains behind is called the "ash." The quantity of this ash, as well as its composition, varies very much in different species of plants, and even in different parts of the same plant. Generally the weight of ash is only one or two per cent of the entire weight of the plant in a dry state before burning. The greatest relative proportion of ash is that which is obtained from the combustion of those hydrophytes which live in the sea; and next in quantity is the ash of the family of Oraches which abound on salt-steppes. On the other hand, the smallest quantity is that afforded by fungi and mosses, by Sphagnum in particular, and with these must be mentioned the tropical orchids living on the barks of trees. Seeds and wood yield relatively much less ash than leaves. But, as above remarked, some ash is formed upon the combustion of any part of a plant or even of a single cell, and this residue of ash sometimes allows of our recognizing exactly the size, form, and outline of the cells. The universal distribution of ash-forming constituents permits us to conclude with certainty that they do not exist fortuitously in plants, but are essential to them. That these constituents are indispensable may also be proved directly. If an attempt is made to nourish a plant on filtered air and distilled water exclusively, the plant soon dies; but if a small quantity of the constituents of its ash are added to the distilled water in which the roots are immersed, the plant grows visibly in the solution, and develops leaves and flowers and even seeds capable of germination.

Experiments of this kind with cultures have been the means of almost completely establishing the division between those constituents which are indispensable for all plants, and those which are only necessary under certain conditions and to particular species, or, still less, only beneficial. Those elements must be regarded as essential, which are used by plants for the process of construction, and enter into the composition of the protoplasm or of the cell-membrane-such, for instance as are essential constituents of proteid substances, or are in some way necessary to the formation of these products. Amongst these must be included sulphur, phosphorus, potassium, calcium, and magnesium. Some plants, especially those that live in the sea, require sodium, iodine and chlorine, and, for green plants, iron is necessary. Silicon is also very important for most plants in helping them to flourish in the wild state. Most of these elements are taken into a plant, in the colrse of nutrition, in a condition of extreme oxidation, that is to say in combination with a quantity of oxygen; in fact, as a general rule, they are absorbed in the form of salts, and we may for the sake of brevity include all the mineral foorstuft's under the name of nutrient salts or food-salts. 
It is obvious that food-salts can only pass through cell-membranes and reach the interior of a plant in a state of solution. On this account the soluble sulphates, phosphates, nitrates and chlorides of calcium, magnesium, potassium and iron, may pre-eminently be called food-salts. Whether an essential element is absorbed by a plant in the form of one of these compounds or another appears to be unimportant; phosphorus, for example, may be proffered by the soil in the form either of potassium phosphate or of sodium phosphate, with like results. As regards the importance of sulphur to plants, it is at any rate established that it is necessary for the production of proteid substances. Phosphorus appears to be indispensable in the transformation of certain compounds of nitrogen. Potassium is supposed to play a part in the formation of starch. Calcium is introduced into plants in combination with sulphuric acid as calcium sulphate. This salt is decomposed, the lime combining with oxalic acid to form insoluble calcium oxalate, and the sulphur going to form the sulphuric acid which is used in the construction of albuminous substances or proteids. Lime is therefore important, inasmuch as it is a medium of transport for sulphur. Iron certainly participates in the formation of chlorophyll, even if it does not enter into its composition, as was formerly supposed. For, it has been proved, by means of artificial cultures, that plants reared in solutions free from iron were white instead of green, and died at last; whereas, after the addition of a small quantity of a soluble iron salt, such plants became green in a very short time, and were able to continue their development. The utility of most of these elements does not therefore appear to consist necessarily in their entering into the composition of organic compounds, but in the promotion and regulation of the constructive and destructive chemical processes.

Silicic acid, which occurs so plentifully in the ash of many plants as to constitute often more than 50 per cent, has a different function. If the minute unicellular water-plants known as Diatoms are incinerated, or if stems of Equisetum, Juniper-needles, or leaves of grasses, \&c., are subjected to a red heat, white skeletons remain behind which consist almost entirely of silicic acid, and exhibit not only the forms of the cells, but even the finest sculpturing of the cell-walls. In particular, the stiff hairs on the leaves of grasses are preserved, and better still the cell-membranes of diatoms. The latter present very beautiful forms with their outlines quite distinct, and many structural properties of the cell-membranes, especially their moulding, striation, and the dots and other excrescences are to be seen much more clearly after than before ignition, when the transparency was less owing to the protoplast occupying the interior of each cell. In order to describe exactly the very varied form of Diatomaceæ, specimens are carefully and thoroughly ignited, and the descriptions and illustrations of these microscopic plants are for the most part made from siliceous skeletons prepared in this way. These skeletons show clearly that silicic acid occurs only in the cell-membrane, and plays no part as constituent of any chemical compound in the protoplasm; nor does it appear to be instrumental in the formation of any such compound. The molecules of silicic acid are so closely packed and so evenly distributed amongst the mole- 
cules of cellulose that, even after the removal of the latter, the entire structure is preserved in outline and in detail. They form, therefore, a regular coat of mail which may be looked upon as a means of protection against certain injurious external influences.

For a large number of plants living in the sea, sodium, iodine, and bromine also are of especial importance as food-stuffs. How far fluorine, manganese, lithium, and various other metals, which have been detected in the ash of some plants, are of use is not determined, for our knowledge is particularly incomplete with respect to the various uses subserved in nutrition and growth by the different mineral food-stuffs. It is worthy of note that alumina, which is so widely distributed and easily accessible to plants, is only very rarely absorbed. The ash of Lycopodium is the only kind in which this substance has been identified with certainty in any considerable quantities.

Lastly, amongst the sources of elements contained in the food-salts, we must consider the solid crust of the earth. But it is only in the case of comparatively few vegetable organisms that this earth-crust forms the immediate foster-soil. The majority derive the salts that nourish them from the products of the weathering of rocks, from refuse and the decaying remains of dead animals and plants, which, in decomposing, give back their mineral substances to the ground, from underground waters that filter through fissures in rocks and through the interstices of sandy or clayey soils soaking with lye, the adjacent parts of the earth's crust: and, lastly, from the water of springs, streams, ponds, and lakes, which have come to the surface holding salts in solution, as also from sea-water with its rich supply of salts.

The very salts that are needed by most plants are amongst the most widely distributed on the earth's surface. The sulphates of calcium and of magnesium, for example, and salts of iron, potassium, \&c., are found almost everywhere in the earth, and in water, whether subterranean or superficial. At the same time it is very striking that these mineral food-salts are not introduced into plants by any means in proportion to the quantity in which they are contained in the soil, but that, on the contrary, plants possess the power of selecting from the abundance of provisions at their disposal only those that are good for them and in such quantity as is serviceable. This selective capacity of plants is manifested in many ways, and we will now briefly consider some of the most important of them.

In the first place we have the fact that plants reared close together in the same soil or medium may yet exhibit an altogether different composition of ash. This is particularly striking in water and bog-plants, which, though rooted in close proximity and immersed in the same water, show very considerable differences in respect of mineral food absorbed. The result, for instance, of testing specimens of the Water-soldier (Stratiotes aloides), the White Water-lily (Nymphaca alba), a species of Stone-wort (Chara fotida), and the Reed (Phragmites communis), all growing close together in a swamp, was as follows as regarded the potash, soda, lime, and silicic acid, held by them respectively:- 


\begin{tabular}{|c|c|c|c|c|}
\hline & Water-soldier. & Water-lily. & Stone-wort. & Reed. \\
\hline 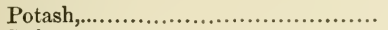 & $30 \cdot 82$ & $14: 4$ & 0.2 & $8 \cdot 6$ \\
\hline Soda,.................................... & $2 \cdot 7$ & $29 \cdot 66$ & $0 \cdot 1$ & 0.4 \\
\hline Lime, ................................... & $10 \cdot 7$ & $18 \cdot 9$ & $54 \cdot 8$ & $5 \cdot 9$ \\
\hline Silicic Acid, ........................... & $1 \cdot 8$ & 0.5 & 0.3 & $71 \cdot 5$ \\
\hline
\end{tabular}

The other constituents of the ash of these plants, in particular iron oxide, magnesia, and phosphoric and sulphuric acids, exhibited less marked differences; but the inequality in the amounts of potash, soda, lime and silicic acid are so great, as only to be explicable on the assumption of a power of selection on the part of these plants. Various species of brown and red sea-weeds, which had been attached to the same rock and developed in the same sea-water, showed similar variations in the composition of their ash.

On the mountains of serpentine rock near Gurhof, in Lower Austria, specimens of Biscutella lavigata and Dorycnium decumbens were collected from plants growing together, and one above the other, upon a declivity which they clothed. Their roots, interlaced here and there, were fixed in the same ground, and drew nourishment from the same store. The following table gives the composition of the ash in these two species:-

\begin{tabular}{|c|c|c|c|c|c|}
\hline & $\begin{array}{l}\text { Biscutella } \\
\text { lævigata. }\end{array}$ & $\begin{array}{l}\text { Dorycnium } \\
\text { decumbens. }\end{array}$ & & $\begin{array}{l}\text { Biscutella } \\
\text { lævigata. }\end{array}$ & $\begin{array}{l}\text { Doryenium } \\
\text { decumbens. }\end{array}$ \\
\hline Potash,............... & $9 \cdot 6$ & $16 \cdot 7$ & Silicic Acid,........... & $13 \cdot 0$ & $6 \cdot 3$ \\
\hline Lime, ................ & $14 \cdot 7$ & $20 \cdot 9$ & Sulphur, .............. & $5 \cdot 2$ & $1 \cdot 6$ \\
\hline Magnesia,........... & $28 \cdot 0$ & $19 \cdot 6$ & Phosphorus,........... & $15 \cdot \overline{9}$ & $22 \cdot 3$ \\
\hline Iron Oxide, .......... & $7 \cdot 8$ & $2 \cdot 8$ & Carbonic Acid,....... & $5 \cdot 4$ & $9 \cdot 7$ \\
\hline
\end{tabular}

The differences here seem to be not so great as in the case of the water-plants previousiy given, but they are sufficient to prevent our regarding them as merely the result of chance.

If, on the other hand, we compare the composition of the ash of different specimens of the same species, which have been reared on similar soils, but at great distances from one another, the discrepancies are comparatively slight. Foliage from beech-trees growing on the limestone mountains near Regensburg yielded an ash practically identical with that obtained from leaves of beeches on the Bakonyer-Wald hills in Hungary. The ash of different individuals of a single species even exhibits the same constitution, in the main, when those individual plants have obtained their nutriment from soils differing greatly in chemical composition. Only in cases where the quantity of a substance in one soil is more abundant than in the other there is generally a greater or less amount of it to be found in the ash.

That under these circumstances certain substances may replace one another is not improbable. But such substitution must be confined to those nearly allied compounds whose molecules are capable of being used indifferently by the formative 
protoplasm in construction, and in the storage of materials. The annexed table, which gives side by side analyses of the ash of branches of the Yew (Tuxus baccata) with their leaves attached, illustrates the replacement of calcium by magnesium :-

\begin{tabular}{|c|c|c|c|}
\hline & \multicolumn{3}{|c|}{ Ash from branches and leaves of the Yew from } \\
\hline & Serpentine. & Limestone. & Gneiss. \\
\hline 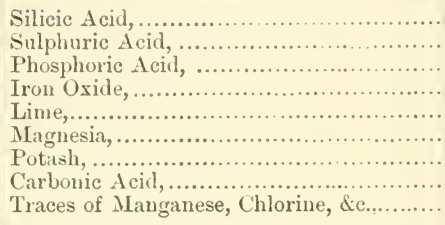 & 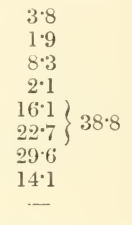 & 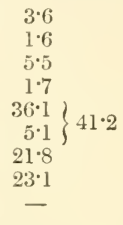 & 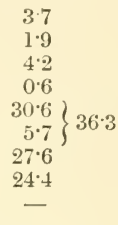 \\
\hline Totals, ...... & $99 \cdot 6$ & $98 \cdot 5$ & $98 \cdot 7$ \\
\hline
\end{tabular}

The Yew occurs in Central Europe on very various mountain formations, chiefly on limestone, but not infrequently on gneiss, and occasionally on serpentine rocks. On comparing the quantities of calcium and of magnesium in the ash of yews, grown on lime and on gneiss respectively, with those yielded in the case of serpentine formation, we find that magnesia preponderates considerably in weight over lime in a yew from serpentine rocks (which are in the main a compound of magnesia and silicic acid), whilst the proportion between these two salts is reversed in a yew grown upon limestone. The obvious inference from the table is that, in plants from a serpentine ground, lime is to a great extent replaced by magnesia. This is further supported by the circumstance that if lime and magnesia are counted together the resulting numbers are very near one another, namely 41.2 per cent of the ash for limestone, 38.8 per cent for serpentine rock, and 36.3 per cent for gneiss.

But all these phenomena observed in connection with the selection of food-salts are not nearly so surprising as the fact that plants are also capable of singling out from an abundance of other matter particular substances, which are of importance to them, even from a soil containing them in barely perceptible quantities, and of concentrating them to a certain extent. As has been shown above, nearly a third of the ash of the white water-lily is composed of common salt. One might, therefore, suppose that the water in which water-lilies flourish contains a particularly large quantity of common salt. But nothing of the kind is the case. The bog water which bathed the stem and leaves of this specimen only contained 0.335 per cent of common salt, and the mud through which the roots straggled contained only 0.010 per cent.

No less astonishing is it to find Diatomacer, with cell-membranes, as above mentioned, sheathed in silicic acid, existing in water which contains no trace of silicic acid. Above the Arzler Alp, in the Solstein chain near Innsbruck, there is a spring of cold water which falls in little cascades between blocks of rock. The 
water of this spring is hard, and it deposits lime at a little distance from the source. Exactly at the spot where it wells out of a fissure in the rock its bed is entirely filled by a dark-brown flocculent mass which consists of millions of cells of the beautiful Odontidium hiemale, a species of diatom with siliceous coating. These cells are ranged together in long rows, and are present in numbers and luxuriance such as are scarcely ever to be observed in other situations. Yet the spring water flowing round contains so little silicic acid that no trace of this substance could be discovered in the residue from the evaporation of 10 litres.

An instance similar to this of silicic acid, is afforded by the iodine in the sea. Most of the sea-wracks inhabiting the North Sea contain iodine, many indeed in considerable quantity, and yet we have not hitherto succeeded in detecting iodine in the water of the North Sea. Similar phenomena, sometimes quite baffling explanation, are exhibited by land-plants. The clefts in the rocks of quartziferous slate in the Central Aips are, in many places, overgrown by saxifrages (Saxifraga Sturmiana and Saxifraga oppositifolia) with leaves aggregated together in closely-crowded rosettes, which are conspicuous from afar, owing to their pale colouring. On closer inspection one finds that the apices and edges of these rosulate leaves are covered with little incrustations of carbonate of lime, a substance which will be frequently referred to in connection with its importance to plants. But one seeks in vain for any lime compound in the earth which fills the clefts, and the only traces of lime contained in the adjacent rock itself are those occurring in the little scales of mica scattered about, and these are not readily decomposable. Yet the lime incrusting the saxifrage leaves can only be derived from the underlying rock, just as in former instances the silicic acid in the cell-membranes of diatoms must be secreted from the spring described, the iodine in sea-weeds from the sea, and the common salt in water-lilies from the pond where they grow, although in each case the substance concerned is only to be found, if at all, in scarcely ponderable traces in the soil or liquid serving as medium. Facts of this kind have a special interest, because they prove that plants have the power of appropriating a substance, if it is important to them, even when it is only present in extremely minute quantities. Where a plant is surrounded by liquid, we can well imagine that fresh portions of the medium are constantly coming into contact with its surface; for, even in water apparently still, compensating currents are continually being caused by changes of temperature. Thus, in the course of a day, thousands of litres of sea-water may flow over a sea-weed with a surface of one square meter, and, even if only a small portion of the substance, traces of which we are supposing to exist in the water, is wrested from each litre, still, the absorbing plant might collect quite a profitable quantity in a number of days. The volume of water flowing over a plant situated in the source of a spring is still greater, and it is readily conceivable that even the most minute trace of silicic acid may become of account in course of time. There is more difficulty in understanding how plants with roots in the earth set about atilizing substances contained in the soil in scarcely appreciable quantities. These plants 
must at all events come into contact with as great a mass of nutrient soil as possible, and this is effected by means of a widely-ramifying system of roots; and, in addition, they must assist in making available desirable matter in the soil by the elimination from themselves of certain substances.

In order to explain the remarkable power that plants possess of exercising a choice in the absorption of certain food-stuffs from amongst the whole number presented to them, we must in the first place assume a special structure to exist in the cells which are in immediate contact with the nutrient medium. To reach the interior of a cell, the salts must pass through the cell-membrane and the so-called ectoplasm. We may look upon these walls, that are to be penetrated, as filters, or, to abide by our previous simile, as sieves, which allow only certain kinds of molecules to pass and arrest others. Moreover, just as the structure of a sieve, especially the size and shape of its pores, has its effect in the separation of the particles of the matter sifted, so also may the structure of a cell-wall have a discriminating influence in the absorption of food-salts. It may be supposed that the cell-wall in one species of plant acts as a sieve capable of letting through molecules of potash but none of alumina, whilst the cell-wall in a second species allows molecules of alumina to pass as well, but is impervious to those of chloride of sodium. This hypothesis would also explain why the absorption of food-stuffs by plants generally takes place through cell-walls, and why absorption into the organs concerned by means of open tubes, which would be at all events a much simpler method, is not preferred. It is, however, necessary to investigate first the nature of the force which causes molecules of the various salts to move from the soil to the cell-membranes, which we suppose to be like sieves, and through them into the interior of a plant. A force acting in this sense from without is inconceivable, and we must therefore look for the motive stimulus in the plant itself.

As has been already stated in connection with the absorption of carbonic acid, it is believed that the cause of this movement is the disturbance of the molecular equilibrium in the growing vegetable organism. If at one spot in the protoplasm of a cell a particular substance is altered, and, let us say, converted into an insoluble compound, the previous grouping of molecules appears to be altered, or in other words, the molecular equilibrium is disturbed. To restore equilibrium, there must be a re-introduction of molecules of the material that has been removed; and the attraction of them from the quarter where they occur in a fluid, that is to say in a mobile condition, is the more energetic. Supposing, for instance, gypsum (i.e. sulphate of lime) is being decomposed within a cell, and the lime combines with the oxalic acid (set free in the same cell) to form insoluble oxalate of lime, whilst the sulphur combines with other elements to form insoluble albuminoids, this use of the gypsum occasions a violent attraction of that substance from the environment, or, to put it another way, it causes a movement of gypsum towards the place of consumption. If this latter place is a cell in immediate contact with the nutrient substratum, the absorption of the substance 
attracted is direct; but if the cell in which the material is used up is separated from the substratum by intervening cells, the attraction must act through all those cells upon it. The substance consumed must be taken in the first place from the cell adjoining the consuming cell on the side towards the periphery; this cell again must take it from its neighbour, which is still nearer the periphery, and so on until the external cells themselves exercise their influence upon the nutrient substratum. Thus, one may regard the growing cells in which substances are used up, as centres of attraction with respect to those substances. This also explains why it is that the influx of food-salts takes place only so long as the plant is growing; and we see, too, that the direction of the current must vary according to the position of the growing cells, and according to the degree of their constructive activity.

But that one plant prefers one substance and another another-that one species attracts iodine, a second sodium, and a third iron-can only be interpreted as a result of the specific constitution of the protoplasm. The protoplasm of a growing cell which contains no iodine does not require that substance either, for the processes of transmutation and storage. A protoplast of this kind will not therefore be a centre of attraction for iodine, but will draw from the environment with great force substances which are its essential constituents. Having 'gained this conception of the absorption and selection of food-salts, we are able to imagine the possibility of a substance being sought after by one species whilst acting as poison on another. Iodine itself exercises a prejudicial effect on many plants, even when present in very small quantities. Cell-membranes in immediate contact with a medium containing iodine are modified as regards their structure by the iodine: their pores are enlarged, lose their value as orifices adapted to the admittance of certain food-salts in limited quantities, and they no longer prevent the influx of injurious substances. Ultimately they die, and by so doing the entire plant suffers. On the other hand, plants to which iodine is an indispensable constituent are not hurt in any way by the presence of small quantities of this substance in the nutrient medium: their cell-membranes are neither paralysed nor destroyed, and suction is able to take place through them in a perfectly normal manner. But we must in this case specially emphasize the condition of the amount being small, for a larger quantity of this substance is positively injurious even to plants which require iodine.

The general rule for a great number of plants is that they thrive best when the food-salts necessary to them are supplied in very dilute solutions. An increase in the quantity of the salts administered not only fails to promote development, but, on the contrary, arrests it. This is the result even if the salts are such as are absolutely necessary in small quantities to the plants in question. A very minute amount of an iron salt is indispensable to all green plants; but, if a certain measure is exceeded, iron salts have a destructive effect on the cell-membranes and protoplasm, and cause the plant to die. But at what point the boundary lies between salubrious effects and the reverse, where the beneficial action of particular 
substances ceases and detrimental action begins, is not known more precisely than has been stated. We only know that different plants behave very differently in this respect. Suppose, for example, that we scatter wood-ash over a field which is overgrown by grasses, mosses, and various herbs and shrubs. The result is that the mosses die; in the case of the grasses growth is somewhat increased; whilst some of the herbs and shrubs, notably polygonaceous and cruciferous plants, exhibit a strikingly luxuriant growth. If we scatter gypsum instead, the development of clover is enhanced, and, on the other hand, there are certain ferns and grasses that die earlier when gypsum is supplied, or, at least, are considerably stunted in their growth.

The fact that certain plants predominate on calcareous and others on siliceous ground has been the subject of very thorough investigation; and these researches were regarded as justifying the assumption that particular species require a more or less considerable quantity of lime for food, whilst others require similarly silicic acid. Hereupon was founded a division of plants into those which required and were tolerant of lime, and into such as required and tolerated silica. The explanation given of these facts does not seem, however, to be satisfactory, at any rate in the case of siliceous plants. It is much more probable that the so-called silicaloving plants are produced on ground composed of quartz, granite, or slate, not by reason of the abundance of silicic acid, but because of the absence of lime in any large quantity, such as would be liable to injure plants of the kind; for only traces of lime are found, and its presence to this extent is absolutely necessary for every plant. This is not of course inconsistent with the fact that individual species require larger quantities of particular food-salts and only flourish luxuriantly when these nutritive salts are not meted out too sparingly. In the case of oraches, thrifts, wormwood species, and cruciferous plants, alkalies, in comparatively large quantities, are necessary for hardy development. The proper habitat for these plants, therefore, is on soils which contain an abundance of easily soluble alkaline compounds, in places where the ground is regularly saturated by saline solutions, and where crystals of salt effloresce on the drying surface. Such places are the sea-shore, the salt steppes, and the neighbourhood of salt-mines. The above plants not only flourish in these localities in great abundance and perfection, but they supplant all other species on which the excessive provision of soluble alkaline salts is not beneficial. If the seeds of such plants happen to fall upon the salt ground they germinate, but only drag out a miserable existence for a short time, and in the end are crowded out by the luxuriant oraches and crucifers. Plants which only flourish abundantly on soils rich in alkaline salts are called halophytes. The same name has also been applied to plants which only thrive in sea-water. Most of the species used by us as edible vegetables, as, for instance, cabbages, turnips, cress, \&c., are really descesıded from halophytes, and accordingly require a soil that contains a comparatively rich supply of alkalies. An opportunity will occur, later on, of returning to the question as to how far agriculture has gained by all these discoveries, and of considering what processes, based upon 
the results of scientific research, have been introduced into practice. Amongst these processes may be mentioned the rotation of crops, the artificial application of manure to exhausted land, and the restitution of the mineral food-salts which the particular plants last cultivated have withdrawn from the land under tillage.

\section{ABSORPTION OF FOOD-SALTS BY WATER-PLANTS.}

It is usual to designate all plants that grow in water as hydrophytes or waterplants. But in their narrower sense these names are only applicable to those plants which, during their entire lives, vegetate under water and derive their nutriment, especially carbonic acid, direct from the water. A number of plants have widely ramifying roots fixed in the earth at the bottom of water, and the lower parts of their stems, either temporarily or throughout life, immersed in water, whilst the upper parts of their stems and their upper leaves are exposed to the air and take carbonic acid direct from the atmosphere, and these should be regarded as marshplants and classed with land-plants so far as regards food-absorption. Reeds and rushes, water-fennel and water-plantain, the yellow water-lily, even the amphibious Polygonum and the white water-lily, are marsh-plants and not true hydrophytes. It is characteristic of all these marsh-plants, that if they are entirely submerged for any length of time they die, whereas they are not injured if the water's level at the place where they grow sinks so as to expose the lower portions of the stem. In places formerly submerged, but from which, in course of time, the water has retreated, so that they have been turned into meadows, one may come across not only clumps of reeds and rushes but even yellow and white water-lilies, flourishing perfectly on the moist earth.

Water-plants, or hydrophytes in the proper acceptation of the term, perish if they are kept for a length of time out of their proper medium and exposed to the air. In most of them death ensues quickly, for their delicate cell-membranes are not able to prevent the exhalation of water from the interior of their cells; and, there being no provision for a replacement of the evaporated fluid, the whole plant dries up. If one supplies aquatic plants, thus desiccated, with water, though it is indeed absorbed it no longer has the power of reviving them. Those hydrophytes which occur in the sea, near the shore, are able to stand exposure to the air for a comparatively long time, and they are regularly subject to it during ebb-tide. Sea-wracks which at high-tide were floating in the water are then seen lying on the dry rocks or sand of the shore. But the membranes of the cells forming the outermost layer in all these sea-wracks is very thick. They retain water staunchly and prevent the plants from drying up, at least until high-tide occurs again, when they are once more submerged.

Amphibious plants in which the lower leaves are like those of aquaties and the upper like those of land-plants so far as desiccation is concerned (e.g. several kinds of pond-weed-Potamogeton heterophyllus and P.natans-and a few white-flowered Raunculi-Ranunculus aquatilis and R.hololeucus), exhibit a transition stage from 
aquatic plants to land-plants. When the water sinks and they are finally left lying exposed on the mud or wet sand, to which they appear to be firmly attached by their abundant roots, it is only the previously submerged leaves that dry up. That part of the foliage which floated on the surface and was consequently always in contact with the air continues to thrive, and any fresh leaves that may be developed adapt themselves completely to the new environment. Similar behaviour is observed in many of the plants which float freely on the surface of water. Such, for instance, is the ease with some species of duckweed (Lemna minor and $L$. polyrrhiza), with Azolla, Pontederia and Pistia; they do not die when the water sinks, leaving them stranded, but absorb food-stuff's from the wet earth through their roots, and in this condition are not to be distinguished from land-plants.

Hydrophytes in the narrow sense, i.e. plants which are entirely submerged and die if they are surrounded by air instead of water for any length of time, are for the most part fixed to some support beneath the water. In many cases the characteristic method of reproduction consists in the separation of special cells, which then swim about for a time in the water. Sooner or later, however, they re-attach themselves to some seemingly suitable spot, and the further phases of their development are again stationary. Comparatively few permanently submerged species are freely suspended in the liquid medium in every stage of development. Such free plants are liable to be shifted by currents in the water, but the extent of their displacement is never very great, owing to the fact that submerged species of this kind occur almost exclusively in still water. As instances may be mentioned the ivy-leaved duckweed (Lemna trisulca), the water-violet (Hottonia palustris), the various species of hornwort (Ceratophyllum), in all of which roots are absent; and in addition amongst the lower or cryptoganic plants Riccia fluitans, and many of the Desmidiacere, Spirogyras and Nostocinex.

Some of these aquatic plants periodically rest on the bottom of the pond or lake in which they live. An example is afforded by the remarkable plant known as the water-soldier (Stratiotes aloides), which, as is indicated by its Latin name, is not unlike an aloe in appearance. During the winter, this plant rests at the bottom of the pond it inhabits. As April draws near, the individual plants rise almost to the surface and remain floating there, producing fresh sword-shaped leaves and bunches of roots which arise from the abbreviated axis, and finally flowers which, when the summer is at its height, float upon the surface. When the time of flowering is over, the plant sinks again to mature its fruit and seeds, and develop buds for the production of young daughter-plants. Towards the end of August, it rises for the second time in one year. The young plants that have meantime grown up resemble their parent completely, except that their size is smaller. They grow at the end of long stalks springing from amongst the whorled leaves, and the stately mother-plant is now surrounded by them like a hen by her chickens. During the autumn, the shoots connecting the daughter-plants with their parent rot away, and, thus isolated, each little rosette, as well as the mother-plant, sinks once more to the bottom of the pond and there hibernates. 
Altogether the number of submerged plants which live suspended in water is very small. As has been said before, by far the greater number are attached somewhere. Seed-bearing plants or Phanerogamia, such as Vallisneria, Ouvirandra, Myriophyllum, Najas, Zannichellia, Ruppia, Zostera, Elodea, Hydrilla, and several species of Potamogeton ( $P$. pectinatus, P. pusillus, P. lucens, P. densus, P. crispus); as also Cryptogams, such as the various species of Isoetes and Pilularia and sulmerged mosses, are fastened in the mud under water by means of attachment-roots or of rhizoids, whilst the almost illimitable host of brown and red sea-weeds are fixed by special cells or groups of cells, which are often root-like in appearance. The sea-weeds choose rocks and stones, by preference, for their support, but they also make use of animals and plants. The shells of mussels and snails are often completely overgrown by brown and red sea-weeds. Larger kinds of Fucaceæ, especially the species of Sargassum and Cystosira, which form regular submarine forests, bear upon their branches numerous other small epiphytes, chiefly Floridere, and these again are themselves covered by minute Diatomaceæ. Many of the huge and lofty brown sea-weeds which raise themselves from the bottom of the sea, remind one forcibly of tropical trees covered with Orchider and Bromeliacer, whilst the latter are themselves overgrown by Mosses and Lichens. These epiphytes are for the most part, however, neither parasitic nor saprophytic. In general hydrophytes attached by means of single cells or groups of cells derive no nutriment, i.e. no food-salts, from the support they rest upon. When loosened from the substratum they continue to live in the water for a long time; they increase in size, and if they come into contact with a solid body are apt to attach themselves to it. In this connection it is well worthy of remark that certain Crustacea have their carapaces entirely covered by hydrophytes of this kind, and that it takes a very short time for the plants to establish themselves upon them. For instance, some species of crabs, such as Maja verrucosa, Pisa tetraodon and P. armata, Inachus scorpioides and Stenorrhyncus longirostris, cut off bits of Wracks, Florider, Ulve, \&c., with their claws, and place them on the top of their carapaces, securing them on peculiar spiky or hooked hairs. 'The fragments grow firmly to the crabs' chitinous coats, and far from being harmful to the animals are, on the contrary, an important means of protection. The crabs in question escape pursuit in consequence of this disguise, and it is to be observed that each species chooses the very material which makes it most unrecognizable to plant upon the exterior of its body: those species which live chiefly in regions where Cystosiras are indigenous deck themselves in Cystosiras, whilst those which inhabit the same places as Ulva, carry Ulvæ on their backs. This phenomenon has for us a special interest in that it shows that the water-plants we are discussing draw no food-salts from their place of attachment, and that accordingly the chemical composition of the support is a matter of utter indifference to all these Fucaceæ, Florideæ, Ulvæ, \&c.

There is no doubt that food-salts are absorbed by these hydrophytes from the surrounding water through their whole surface. Accordingly the structure of their peripheral cells is much simpler than is the case in land-plants. In the latter very 
complicated adaptations are necessary for the extraction of food-salts from the earth. In particular, the portions which are exposed to the air above ground exhibit a number of special structures connected with this extraction. These structures (cuticle, stomata, \&c.) are superfluous in the case of aquatic plants, for there is with them no necessity for raising and conducting food-salts into the parts where they can be used up. Moreover the absorption of nutritious matter is much simpler, inasmuch as it is not necessary for the absorbent parts to search for a perpetual source of the requisite substances. The roots of land-plants have often to range over a wide area in order to find sufficient nourishment in the earth, and frequently they have then to liberate it, i.e. bring it into a state of solution. This is not the case with water-plants. They are completely surrounded by a medium which is itself to a large extent a solution of food-salts, and no sooner are substances withdrawn by the absorbent cells from the layers of water immediately bounding them than those substances are again supplied from the more remote environment. Constant compensating currents occur in water, and there is, therefore, scarcely an aquatic plant towards which there is not a perpetual flow of the foodsalts it requires in a form suitable for absorption. In connection with this kind of food-absorption there is also the fact that the parts by which hydrophytes attach themselves to a support are relatively small in area. Fucoids, as large as hazel trees in height and girth, are fixed to submerged rocks by groups of cells perhaps only $1 \mathrm{~cm}$. in diameter.

The quantity of food-salts absorbed by hydrophytes is very considerable compared with the amounts absorbed by other plants. As has been mentioned before, soda and iodine play a very important part in the thousands of different varieties which live in the sea. If Florideæ are transferred from the sea into pure distilled water, common salt and other saline compounds diffuse out of the interior of the cells through the cell-membranes into the fresh water around. The red colouring matter of these Florideæ also passes through the cell-walls into the water, proving that the molecular structure of the membrane is adapted to the agency of salt water in the osmotic processes of food-absorption.

Plants living in fresh, or in brackish water, likewise absorb relatively large quantities of food-salts; and this accounts for the fact that water which is very poorly provided with nutriment of the kind contains only very few vegetable species.

One would expect that exceedingly abundant vegetation would be evolved in running water, provided the latter contained food-salts in solution, however small they might be in quantity. For, in such a situation, it is not necessary to wait for the salts withdrawn by the plants from their immediate environment to be restored by the slow processes of mixture and equilibration; the water which has been drained of nutriment is replaced the next moment by other water bearing fresh food-salts. Experience shows, however, that flowing water is not so favourable to the development of hydrophytes as is the still water of pools, ponds, and lakes. This may partly depend on the fact that running water is always poorer in food-salts, and 
partly also on the circumstance that mechanical difficulties are opposed to the taking up of saline molecules from water in rapid motion. There are only a few plants that are able to absorb under these conditions, and these choose, by preference, the very spots where they are most exposed to the dash of the water. Thus, certain Nostocineæ (Zonotrichia, Scytonema) are to be found constantly in waterfalls at the parts where the most violent fall occurs. Lemanea, Hydrurus, and many mosses and liverworts, grow by preference in the foaming cascades of rapid torrents. Amongst flowering plants we only know of the Podostemaceæ as choosing a habitat of this kind. Podostemaceæ are exceedingly curious little plants, which at first glance one would take for mosses or liverworts without roots. Some of them, e.g. the Brazilian species of the genus Lophogyne and the various species of Terniola growing in Ceylon, exhibit no differentiation into stem and leaves, but are only represented by green fissured and indented lobes attached to stones. They belong without exception to the tropical zone, and occur there in the beds of streams, attached to rocks, over which the foaming water rushes.

\section{ABSORPTION OF FOOD-SALTS BY LITHOPHYTES.}

Nothing would seem more natural, as to the absorption of mineral salts by lithophytes, than that the stone which constitutes their support should yield the salts, and that the attached plants should suck them up; but, generally speaking, the case is not so simple. There are mosses and lichens which cling to the surfaces of rocks on mountain tops. These rocks are sometimes composed of perfectly pure quartz, and yet the plants in question contain very little silica; they contain, on the other hand, a number of substances entirely wanting in the composition of the underlying rock, and which could not, therefore, have been derived from that source. For many of these lithophytes the rock is, in the main, only a substratum for attachment, and in no way a nutrient soil; just as, in the case of many aquatic plants, the stones to which they cling by their discs of attachment are anything but sources of nourishment.

From what source, then, do stone-plants of this kind derive the food-salts which are wanting in their substratum? It may sound paradoxical, but it is nevertheless the fact, that they obtain those salts from the air through the medium of atmospheric precipitation. Rain and snow not only absorb carbon dioxide, sulphuric acid, and ammonia-which occur in air universally, although in extremely minute quantities -but they also collect, as they fall, floating particles of dust. The opinion is widely entertained that although the atmosphere is full of dust in the neighbourhood of cities and human settlements generally, where the soil is laid bare and ploughed up, and roads and paths have been made for purposes of traffic, and perhaps also over steppes and deserts where large areas of ground are destitute of regetation, yet that there is no dust in the air over land remote from places of that kind or in the air of marshes, lakes, or seas. This notion has certainly some warrant if we regard as dust only the coarser particles which are raised from loose eartl $l_{\perp}$ and 
whirled into the air by the wind. Moreover, the quality of the dust will no doubt be characteristically affected by the vicinity of areas of industry. One has only to look at the sooty leaves and branches of trees in parks near manufactories to convince oneself of the reality of this influence. But it would be quite erroneous to suppose that the air in regions far from land that has been cultivated or otherwise opened up is free from dust. It contains dust everywhere. There is dust in the air of the extensive ice-fields of arctic regions and of high mountain glaciers, and there is dust in the air of great forests and over the boundless sea.

If the rays of the setting sun fall obliquely through a gap between two peaks in a wood-clad mountain valley, sun-motes may be seen floating up and down and in circles, just as they do in a room when the last rays before sunset fall through the window. These motes are of course not usually visible, and they are moreover much smaller than the particles of dust which are raised by the wind from roads and then again deposited. Now, when rain falls, it takes the sun-motes from the air and brings them down to earth, and the air is thus washed to a certain degree of purity. This mappens still more completely in the event of snow. The latter acts not unlike a mass of gelatine used to purify cloudy liquids, its effect being to drag down with it all the particles to which the turbidity is due, leaving the upper part of the liquid quite clear. Similarly, falling snow-flakes filter the air; and, mixed with fallen snow, there are accordingly innumerable particles of dust. If afterwards the snow gradually melts, it dissolves some of the dust, which then drains away into chinks and depressions; but a portion remains behind undissolved. This portion is gradually consolidated, and then appears lying on the parts of the snow that are still unmelted in the form of dark patches, streaks, and bands; often also it forms a smeary graphitic covering so widely spreading over the last remnants of melting snow that the latter resemble lumps of mud rather than snow. Accordingly we find it everywhere - in regions cultivated and uncultivated, in tilled lowlands and on high grassy plains above forest limits, where no tilled land is to be seen in any direction, and lastly in arctic regions in the middle of glaciers several miles across.

All this snow dust is not invariably deposited as a result of the filtering of the air by falling snow-flakes; an additional supply is brought by the winds which blow across the snow-fields. It is not of rare occurrence in the Alps for snowfields to exhibit suddenly, after violent storms, an orange-red coloration. On closer inspection one finds that the surface of the snow is strewn with a layer of powder, infinitesimally fine and for the most part brick-red, which has been brought by the gales. Investigation of this "meteoric dust" shows that it is composed chiefly of minute fragments of ferruginous quartz, felspar, and various other minerals. Mixed with these there are, however, sornetimes remnants of organic bodies, such as bits of dead insects, siliceous skeletons of diatoms, spores, pollen-grains, tiny fragments of stems, leaves, and fruits, and the like. Once, after a south wind had prevailed for several days, the snow-fields of the Solstein range near Innsbruck were covered, at a height of from two to three thousand meters above the sea-level, 
with millions of a species of Micrococcus, which lent a rosy hue to vast expanses of snow.

Most of the dust in the atmosphere originates, doubtless, from our earth. The air that blows in waves over the earth can carry along with it not only dead and detached portions of plants, but also loose particles of rock, sand, earth, and dried mud. If one draws one's palm across the weather side of a dry rock composed of dolomitic limestone, gneiss, trachyte, or mica-schist, the surface of the stone always feels dusty, and the slightest movement of the hand is sufficient to detach a number of particles which were already separate from the rock and only held in loose connection with it. This dust is liable to be detached and carried away by any strong gust of wind. Larger and heavier particles are not, it is true, lifted much above the ground; they are rolled and pounded along and thereby reduced to a still finer powder. This finer dust may then be scattered afar by gales blowing horizontally, or even ascend into higher atmospheric strata. The finest dust in particular, however, is carried up into the higher layers of the air by the currents which ascend from the earth in calm weather; and this applies not only to the tropies but to the temperate zones as well, and even to the frigid regions of the arctic zone. When, therefore, this dust is brought back by rain or snow from the upper aerial strata to the earth, it but completes a circuit. Indeed it is highly probable that the particles of dust restored to earth by means of atmospheric deposits recommence their aerial travels as soon as they are thoroughly dry again, and that there is thus a circulation of dust analogous to that of water.

There is of course no inconsistency in the fact that meteoric dust, which is often drifted along in surprisingly large quantities, may originate quite suddenly during volcanic eruptions; nay, it is even possible that cosmic dust reaches our atmosphere and thence falls to the earth. Chemical investigation of aerial dust has, no doubt, yielded in most cases only sulphuric and phosphoric acids, lime, magnesia, oxide of iron, alumina, silica, and traces of potash and soda, that is to say, the most widely distributed constituents of the solid crust of our earth; but cobalt and copper have also been found in it, over and over again, and it has hence been inferred that the dust in these cases was of cosmic origin.

In relation to the question which we have here to answer the above is, after all, almost a matter of indifference. The only important facts are that dust in a state of extremely fine division is blown about in the air, that this dust contains the salts required by plants for their food, that it is carried for the most part mechanically by drops of water and flakes of snow, condensed in the atmosphere, and is partially dissolved, that the atmospheric deposits supply lithophytic plants with a sufficient quantity of nutrient salts, and that the aqueous solution so supplied is rapidly absorbed by the whole surface of the plants in question. We must not omit to mention here that the demand of lithophytes for mineral food-salts is not very great. In particular the protonemæ and even the leafy shoots of Grimmice, Rhacomitrice, Andreceacece and other rock mosses, and the Collemacece and most crustaceous lichens only contain very minute quantities of these substances. Water containing vou. I. 
the usual mineral salts in about such proportion as is necessary for the cultivation of cereals in fields has actually an injurious effect on these lithophytes and soon kills them.

At the end of this section we shall consider what happens to dust which is brought to earth from the air by rain and snow but is not dissolved, and the important part it plays in clothing the naked ground and in ehanges of vegetation. Here, however, it must be noted that most lithophytes are true dust-catchers, that is to say, they are able to retain, mechanically, dust conveyed to them by wind, rain, and snow, and to use it in later stages of development by extracting nutriment from it. Many mosses are completely lithophytic in early stages of development whilst later they figure as land-plants.

\section{ABSORPTION OF FOOD-SALTS BY LAND-PLANTS.}

In no class of plants is the absorption of mineral food-salts accomplished in so complicated a manner as in land-plants. Moreover, this absorption is by no means uniform in different forms of plants, and we must beware of generalizing with regard to processes which have only been traced and studied in isolated groups-perhaps only in the commonly distributed cultivated plants. On the other hand, with a view to synoptical representation, it is not desirable to enter into too great detail or to attempt to describe all the various differences minutely.

At the outset, it is difficult to give an accurate account of the soil which constitutes the source of nutriment in the case of land-plants. From the dark graphitic mass composed of sun-motes, which is deposited in the place of a melted layer of snow, to coarse gravel, there is an unbroken chain of transition stages; loam, sand and gravel are only specially-marked members of this chain. Again, just as earth varies in respect of the size of its component parts, so also it varies in the mineral salts it contains, in the amount of admixture of decaying vegetable and animal remains, in the nature of the union of its constituents, and in its capacity to absorb, to retain, or to yield up water. Compare the sand composed of quartz on the bank of a mountain stream with that of calcareous origin which is found impregnated with salt on the sea-shore, or with the sand at the foot of mountains of trachyte, which has an efflorescence of soda-salts. Or compare the granite bed of a desert, bare of soil, with the loam on the granitic plateaus of northern regions where there is an intermixture of the remains of a vegetation for centuries active. How great is the difference in each case! But whatever the kind of earth, it is only of value as a source of nutriment for a plant when the interstices of its various particles are filled with watery fluid for the time during which the plant is engaged in the construction of organic substances.

But how is the earth supplied with water?

"Das hat nicht Rast bei Tag und Nacht, Ist stets auf Wanderschaft bedacht." 
Streams fall into lakes, rivers into the sea, and hence the water ascends into the atmosphere in the form of vapour, and returns once more to earth as snow, rain, and dew. Through porous earth it percolates until it has filled all the interspaces. If its further descent be impeded by impervious strata, it spreads literally as subterranean water, or else comes up at some special spot as a spring. Earth which is richly endowed with decaying vegetable remains is able to absorb vapour in addition from the atmosphere. When this occurs, carbonic and nitric acids are always absorbed along with the aqueous vapour. These are contained, as has been mentioned before, in atmospheric deposits, and another source of these acids is afforded by the decay of dead parts of plants. Water precipitated from the atmosphere, and containing carbonic and nitric acids, is able by their means to decompose the compounds in all the rocks which come in its way as it percolates through the ground, especially when its action is long continued. The siliceous compounds or so-called silicatesfelspars, mica, hornblende, and augite in particular-and quartz, the anhydride of silicic acid, which form the preponderant mass of the rocks of the solid crust of our earth, either contain a great quantity of silica, alumina, and alkalies, or if they are relatively poor in silica they may be rich in iron. The former are found chiefly in granite, gneiss, mica-schist, and argillaceous slate; the latter preponderate in serpentine, syenite, melaphyr, dolerite, trachyte and basalt. First the felspars are decomposed by the acid water. Their alkalies combine with the carbonic and nitric acids forming soluble salts, and the alumina and silica remain behind as clay. Iron is also converted into soluble salts. The most difficult substances to decompose are the mica and quartz, and it is on that account that they so often appear in the form of glittering scales and angular nodules mixed with the clay produced from the decomposition of felspar. But, ultimately, even they are unable to withstand the continuous action of the acidulated water. The result of these chemical changes is an earth, which, according to the nature of the parent rock, contains a preponderating amount of clay, of quartzose sand or of mica, which is coloured in various ways by iron compounds. Of substances useful to plants these earths yield generally on analysis the following: potash, soda, lime, magnesia, alumina, ferrous and ferric oxides, manganese, chlorine, sulphuric acid, phosphoric acid, silica, and carbonic acid, sometimes one sometimes another in greater proportion relatively, and traces of many substances often so slight as hardly to be detected.

It is true that limestone and dolomite, which, next to the above-mentioned rocks, enter most largely into the composition of the solid crust of the earth, consist chiefly of carbonate of lime and magnesium carbonate respectively; but wherever they occur in extensive strata and piles, they always contain in addition an admixture of alumina, silicic acid, ferrous oxide, manganese, traces of alkalies in combination with phosphoric and sulphuric acids, \&c. Of the carbonates of lime and magnesia a great part is gradually dissolved and carried away upon the invasion of water containing carbonic and nitric acids, and a proportion also of the substances mixed with them, as above mentioned, is lixiviated. What remains 
behinl then consists of an argillaceous, loamy mass, variously coloured by iron and very similar in appearance to the elay formed from the decomposition of felspar. Acerrling to the yuantity of the substances mixed with the earbonate of lime in the rock, the loamy earth formed from limestone is either abundant or only in matricted layers, binds and pockets lying on, or interealated within, the undecomprosed dibris of the stone. Chemical analysis has resulted in the diseovery that thre are, as a rule, in loamy earth of this kind the same ingredients available for flunts as have been ilentified in earth produced from silicates; and we are leal to belicve that earths, collected in widely different places and covering rocks of most various kinds, are much more uniform qualitatively than has been supposel. Only, the relative proportions of the substances forming the mixture ure usually ditferent. Silica and the alkalies are less conspicuous in earth derived from limestone, and earbonate of lime in that which is formed from silicates. This diflerence is particularly striking in instances where the rock consisted aluost entirely either of quartz and mica or of nearly pure carbonates of lime and mignesium. In these cases the earth formed is not argillaceous, but of loose consistence, very abundant, and composed, according to the kind of rock, of quartzose sand and mica scales or calcareous and dolomitic sand.

The conversion of rocks into earths by the action of water from the atmosphere containing carbonic and nitric acids is, besides, materially modified by the disruptions which ensue from changes of temperature, more particularly by the freezing of water within the pores of rocks. It is also affected, though more remotely, by the mechanieal action of water and air in motion, and, lastly, by the plants themselves, which penetrate with their roots into the narrowest crevices and mingle their dearl remains with the portions of the rock that are decomposed, broken up, or abraled by ehemical and mechanical agencies. The substance produced from a rock in the manner explained is called earth-mould, or simply earth. The matter resulting from the decomposition of plants and animals is designated by the term "humus." Earth which includes an abundance of decomposed fragments of plants, i.e. has a large admixture of humus, is ealled vegetable mould.

Every kind of earth, but especially earth rich in humus and clay, has the power of retaining gases, and especially water and salts. When water containing salts in solution is pourer over a layer of dry vegetable mould, it percolates into the spaces between the particles of earth, and speedily drives out of them the air which has but slight adhesion, and which then ascends in bubbles. It is not till all the interspaces are full of water, whilst a fresh supply is constantly maintained from above, that any of the liquid oozes out from beneath the stratum of earth. The water remaining in the interstices is held there by adhesion to the particles of earth, and we must conceive each of these particles as surrounded by an adherent film of water. The inorganie salts, infiltrating with the water, are held with still greater energy. The water which trickles from the bottom of the earth always contains a much smaller proportion of salts in solution than that which was poured on above, whence we conclude that the latter are in part absorbed by the earth. 
The salts are to be regarded as forming an extremely delicate coating round minute particles of earth where they are forcibly retained. If a plant rooted in the earth is to take in these salts it has to overcome the force by which their molecules are detained. This is effected, however, by means of a very powerful attraction exerted by the protoplasts of the plant as they grow, carry on the work of construction, and use up material. What actually happens is an energetic suction by the cells that are in close contact with particles of earth. This suction depends, however, upon the chemical affinity between the substances in the interior of the cells and the salts adhering to the earth-particles, as well as upon the consumption of food-salts for the manufacture of organic compounds within the green cells. It is supposed that whenever salts are abstracted from soil-particles by suction, a restitution of like salts immediately takes place, particles still unresolved in the immediate neighbourhood being dissolved, and a fresh influx taking place from the environment. Consequently the concentration of the solution retained by the earth is always approximately the same, or, at any rate, equilibrium is very quickly restored. One advantage of this is that the cells in immediate contact with particles of earth, and their adherent liquid, can only meet with a saline solution of constant weak concentration, and are therefore secure from injury such as would result in the case of most plants, from contact with a very concentrated solution. In other words, the absorptive power of earth acts as a regulator of the process of absorption of food-salts by plants, and is the means of keeping the saline solution in the earth always at the degree of strength best suited to the plants concerned.

Naturally, the passage of salts from the earth to the interior of a plant is dependent on the aid of water containing both the substances composing cellcontents and the food-salts in solution. The cell-membranes, through which absorption takes place, are saturated with this solution. The aqueous films adhering to the particles of earth, the water saturating the cell-membrane, and the liquid inside the cells are really in unbroken connection, and along this continuous waterway the passage of salt molecules in and out can take place easily.

The absorption of food-salts directly from the earth by green cells occurs very rarely. The protonema of Polytrichum, which spreads its threads over loamy earth and wraps it in a delicate green felt, and that of the famous Cavern Moss (Sclisstostega), whose long tubular lower cells penetrate the earth in the recesses of caves, do undoubtedly suck up their necessary food-salts by means of cells containing chlorophyll. A drawing of the latter is given in figure $25 \mathrm{~A}, p$.

The majority of land-plants have, however, special absorptive cells for the taking-up of salts in solution. These cells are imbedded amongst or lodged upon the earth-particles, and are usually in intimate connection with portions of them. Any part of a plant that penetrates into the earth or lies upon it, may, if it performs the function of absorption, be equipped with cells of the kind. Plagiothecium nekeroideum, a delicate moss belonging to the flora of Germany, and growing on earth under overhanging rocks, where it is not exposed to rain, and therefore caunot receive any food-salts through that agency, develops absorption-cells on the apices 
of its green leatlets. So also does Leucobryum javense, a species native to Java. Sereral delicate ferns of the family of the Hymenophyllacece exhibit them on their subterranean stems. Many liverworts and the prothalli of ferns bear them on the muler surlices of their flat thalli which lie outspread on damp earth. But most commonly of all are they to be fomil close behind the growing tips of roots. Their form ilues nut vary very much. On the roots of plants fringing the sources of cold unmut:in-springs, as on those of many marsh-plants in low-lying land, they are in the form of comparatively large, oblong, flattened, elosely united cells, with thin walli am colourless contents. In some conifers, whilst having in the main the shape just deseribed, they differ in that they are arehed outwards so as to form papillie; but in most other phaneroganis the external cell-wall projects outwards, and the whole absorptive cell develops into a slender tube, set perpendieularly to the lon ritulinal axis of the root (fig. $12^{4}$ ).

Seen with the naked eye, or but slightly magnified, these delicate tubes look like fine lairs, and have reeeived the name of "root-hairs." The end of a root often aplears to be covered with velvety pile, and the absorptive cells are then very elosely packel; more than four hundred per square millimeter have been occasionally counted. In other eases, however, there are hardly more than ten on a syuare millimeter. When in such small numbers they are usually elongated and clearly visible to the naked eye. Their length, for the most part, varies from the fraction of a millimeter to three millimeters, and their thickness between $0.00 \mathrm{~m}$ m.m. and $0.14 \mathrm{~m} . \mathrm{m}$. It is only exceptionally that one meets with plants, rooted in mud, possessing root-hairs $5 \mathrm{~m} . \mathrm{m}$. or more in length. The absorptive cells of phanerogims are almost always simple epidermal cells of the particular part of the plant that bears them, and are not partitioned by any transverse walls. In mosses and ferm prothalli, on the other hand, the absorption-cells are generally segmented by transverse septa and are usually greatly elongated. In those liverworts which belong to the genus Marchantia they form a thick felt on the under side of the leaf-like plant, or rather, on sueh part of it as is turned away from the light, and sone of these tangled rhizoids attain a length of nearly 2 c.m. The stems of many mosies also are wrapped in a regular felt. This property is rendered very striking in the species of Barbula, Dicranum, and Inium, and especially in such forms as have bright green leaves, by the reddish-brown colour of the cells in question. Sometimes the long eapillary cells of which the felt is composed are twisted tomether spirally like the strands of a rope. A good instance of this is Polytrichum. These fine, hair-like, segmented and branched structures, found on mosses, variously matted and intertwisted, are ealled rhizoids. But only those cells which come into contact with the earth-particles are truly absorbent. The rest do not serve to imbibe from the ground, but to conduct the aqueous solution of food-salts, after it has been taken up by the absorptive cells, to the stem and to the leaves.

The tubular cells resulting from the development of a root's epidermis are placed, as before observerl, at right angles to its longitudinal axis. They only grow, however, in earth that is very lamp, and even then their course is not always a straight 
line, for as a rule they describe a spiral as they elongate. Their movement seems as though it were for discovering the most favourable parts of the earth for absorption and attachment. In this manner they penetrate into the interspaces in the earth which are filled with air and water. They also have the power of thrusting aside minute particles of earth, especially if the latter consists of loose sand or mud. If they strike perpendicularly a solid immovable bit of earth, they bend aside and grow round it with their surfaces closely adpressed to that of the obstacle until they reach the opposite point on the other side, when they once more resume their original direction (fig. $12^{3}$ ). When they encounter large grains of earth they

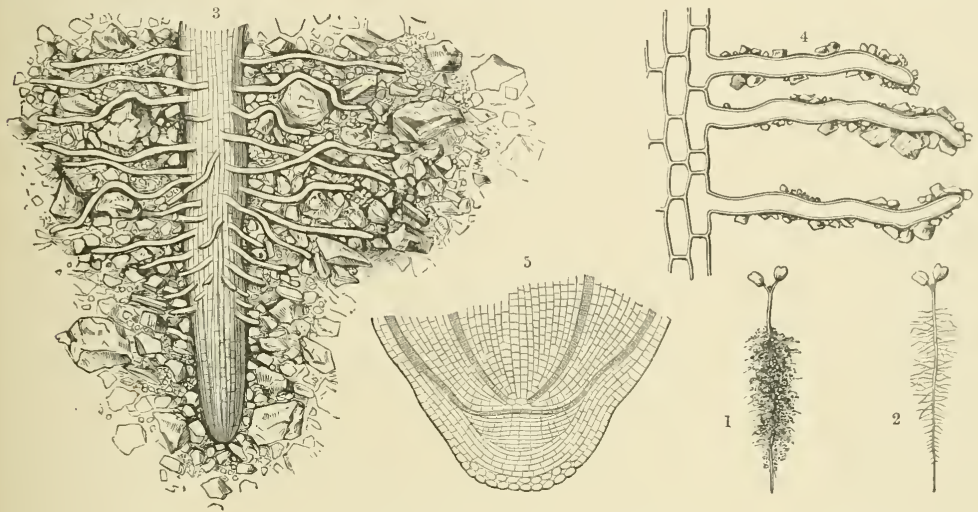

Fig. 12.-Absorptive Cells on Root of Penstemon.

1 Seedling with the long absorptive cells of its root ("root-hairs") with sand attached. 2 The same seedling; the sand removed by washing. ${ }^{3}$ Root-tip with absorptive cells; $\times 10$. 4 Absorptive cells with adherent particles of earth. 5 Section through the root-tip; $\times 60$.

sometimes stop and swell up to the shape of a club. The club divides into two or more arms, which grasp and cling to the granule like the fingers of a hand. Many fragments of earth remain thus in the grasp of finger-like processes, whilst others are held fast in the knots and spirals of corkserew-shaped root-hairs which are often found tangled together. But the retention of most of the earth-particles which adhere to a plant, including fragments of lime, quartz, mica, felspar, \&c., as well as plant-residues, is due to the fact that the outermost layer of the absorptive cells is sticky, it being altered into a swollen gelatinous mass which envelops the particles. When this sticky layer becomes dry it contracts and stiffens, and the granules partially imbedded in it are thereby cemented so tightly to the absorptive cells that even violent shaking will not dislodge them.

In the case of most seedlings, and in that of grasses, the absorptive cells which proceed from the roots and which are especially numerous in the latter, are generally thickly covered with particles of earth (see fig. $12^{4}$ ). If such a root is pulled out of sandy soil it appears to be completely encased in a regular cylinder of sand (fig. $12^{1}$ ). A root of Clusia alba, taken from coarse gravel, had its root-hairs so tightly 
adherent to bits of gravel that several little stones, weighing 1.8 grms., were found clinging to it when it was lifted. The gelatinous mass, resulting from the swellingup of the external coat of the cell, does not in any way hinder absorption or the passage of food-salts in solution. Nor does the inner coat, the thickness of which varies between $0.0006 \mathrm{~m} . \mathrm{m}$. and $0.01 \mathrm{~m} . \mathrm{m}$., constitute any impediment to imbibition.

In addition to the absorption of nutritive salts by root-hairs, there is also, in many cases, an interchange of materials; that is to say, not only do substances infiltrate from the earth into the absorption-cells, and so onward into the tissues of a plant, but others pass out of the plant through the absorptive cells into the earth. Amongst these eliminated substances, carbonic acid, in particular, plays an important part. A portion of the earth-particles adhering to root-hairs is decomposed by it, and food-salts in immediate proximity to those cells are hereby rendered available and pass into the plant by the shortest way.

Having now seen that land-plants take in food-salts by means of special absorptive cells, it is natural to find that each of these plants develops its absorption-cells, projects them, and sets them to work at a place where there is a source of nutritive matter. The parts that bear absorptive cells will accordingly grow where there are food-salts and water, which is so necessary for their absorption. The Marchantias and fern prothalli spread themselves flat upon the ground, moulding themselves to its contour. From their under-surfaces they send down rhizoids with absorptive cells into the interstices of the soil. Roots provided with root-hairs behave similarly. If a foliage-leaf of the Pepper-plant or of a Begonia be cut up, and the pieces laid flat on damp earth, roots are formed from them in a very short time. The roots on each piece of leaf proceed from veins near the edge, which is turned away from the incident light, and grow vertically downwards into the ground.

It is matter of common knowledge that roots which arise upon subterranean parts of stems, like those formed on parts above-ground, grow downward with a force not to be accounted for by their weight alone. This phenomenon, which is called positive geotropism, is looked upon as an effect of gravitation. The idea is that an impetus to growth is given by gravity to the root-tip, and that a transmission of this stimulus ensues to the zone behind the tip where the growth of the root takes place. It is noteworthy that if bits of willow twigs are inserted upside down in the earth, or in damp moss, the roots formed from them, chiefly on the shady side, after bursting through the bark, grow downwards in the moist ground, pushing aside with considerable force the grains of earth which they encounter. The appearance of a willow branch thus reversed in the ground is all the more curious inasmuch as the shoots, which are developed simultaneously with roots from the leaf-buds, do not grow in the general direction of the buds and branches, but turn away immediately and bend upwards. Thus the direction of growth of roots and shoots produced on willow-cuttings remains always the same, whether the base or the top of the twig used as a cutting is inserted in the earth. A similar phenomenun is observed if the leafy rootless shoot of a succulent herb (e.g. Sedum reflexum) is cut 
off and suspended in the air by a string. Whether it hangs with the apex uppermost, i.e. in the position in which it grew naturally, or with the apex towards the ground, it always, in a short space of time, produces roots which spring from the axis between the fleshy foliage-leaves and bending sharply grow to the earth. Thus in the former case their direction is contrary to the apex of the shoot; in the latter, curiously enough, it is in the same direction. If the height at which the shoot is suspended is only $2 \mathrm{c.m}$. above the earth, the roots growing towards the ground develop their root-hairs $2 \mathrm{c.m}$. from their place of origin. But if the shoot is at a distance of 10 c.m., the roots only develop their root-hairs when they have attained a length of $10 \mathrm{c.m}$. The rule is, therefore, for the roots to grow until they reach the nutrient soil without developing absorption-cells, and only to provide themselves with them when they are in the earth. It is to be observed that these roots are produced on the suspended shoot at places where, under normal conditions (i.e., if the shoot were not cut off and hung up), no roots would be developed. Subject to abnormal conditions and liable to starvation, the plant sends out these roots for self-preservation.

Phenomena of this kind force one to conclude that a plant discerns places which offer a supply of nutriment, and then throws out anchors for safety to those places. This power of detection may, undoubtedly, be explained by the influence which conditions of moisture, in addition to the action of gravitation, have on the direction taken by growing roots. The root-hairs can only obtain food-salts when the ground is thoroughly moist; and whenever roots, or rather their branches, have to choose between two regions, one of which is dry and the other wet, they invariably turn towards the latter. If seeds of the garden-cress are placed on the face of a wall of clay which is kept moist, the rootlets, after bursting out of the seeds, grow at first downwards, but later they enter the wall in a lateral direction. The longitudinal growth of the roots is greater on the dry side than on the wet side, and this results in a bending of the whole towards the source of moisture, in this instance the damp wall. It has been established that the tip of a rootlet is very sensitive to the presence of moisture in the environment. Where there is a moist stratum on one side and a dry stratum on the other, a root-tip receives a stimulus from the unequal conditions in respect of moisture; the stimulus is propagated to the growing part of the root, which lies behind the tip, and the result is a curvature of the root towards the moist side. Thus, the presence of absorbable nutriment, or rather of moisture, in the ground explains the divergence of roots from the direction prescribed by gravity.

The extent to which the direction taken by roots in their search for food is dependent upon the presence of that food, and the fact that roots grow towards places that afford supplies of nutritious material, are strikingly exhibited, also, by epiphytes growing on the bark of trees, such as tropical orchids and Bromeliacece; and again by plants parasitic on the branches of trees, of which the Nistletoe and other members of the Loranthacece afford examples. Although the absorption of food by these plants will not be thoroughly discussed till a later 
stage, this is the proper place to mention the fact that in them positive geotropism appears to be completely neutralized. The growing rootlets which spring from the seed, and the absorptive cells produced from minute tubercles, grow upwards if placed on the under surface of a branch, horizontally if placed on the side, and downwards if on the upper surface. Thus, whatever the direction, they grow towards the moist bark which affords them nourishment.

Positive geotropism seems to be quite abolished also in those marsh-plants which live under water. When, for instance, the seed of the Watcr-chestnut (Trapa natans) germinates under water in a pond, the main root emerges first from the little aperture of the nut and begins by growing upwards. Soon the smaller scale-like cotyledon is put forth, whilst the other, which is much larger, remains within the nut. The whole plant so far is standing on its head, as it were, and is growing upwards with its principal root directed towards the surface of the water. Gradually the leafy stem emerges from the bud between the two cotyledons, and likewise curves upwards and grows towards the surface, whilst an abundance of secondary roots is developed at the same time from the main root. Their function is to absorb nutritive substances from the water around, now that the materials for growth stored in the seed are exhausted. Finding an aqueous solution of food-salts everywhere these roots grow in all directions, upwards, downwards, or horizontally to right or left, forwards or backwards, only they carefully avoid touching one another or interfering with each other's sphere of absorption. It is not till much later that the main root changes the direction of its apex and bends downward. New roots are then produced from the stem; but this subject has no further bearing on the problems at present before us.

The movements of roots, as they grow in earth, suggest that they are seeking for nutriment. The root-tip traces, as it progresses, a spiral course, and this revolving motion has been compared to a constant palpitation or feeling. Spots in the earth which are found to be unfavourable to progression are avoided with care. If the root sustains injury, a stimulus is immediately transmitted to the growing part, and the root bends away from the quarter where the wound was inflicted. When the exploring root-tip comes near a spot where water occurs with food-salts in solution, it at once turns in that direction, and, when it reaches the place, develops such absorptive cells as are adapted to the circumstances.

As has been mentioned before, the roots of most land-plants bear root-hairs on a comparatively restricted zone behind the growing point (see fig. $12^{3}$ ), and these hairs have only an ephemeral existence. As the root grows and elongates, new hairs arise (always at the same distance behind the tip), whilst the older ones collapse, turn brown, and perish. In ground which contains on every side food-salts in quantities adequate to the demand, and sufficient water to act as solvent and as medium for the transmission of the salts, the absorptive cells are rarely tubular, but exhibit themselves, as already described, in the form of flat cells destitute of outward curvature. This is the case, for instance, with those Alpine plants which grow in 
ever-moist hollows and depressions in proximity to springs (e.g. Saxifraga aizoides and many others). But wherever the substances to be absorbed are not so easily obtained, the surfaces of the absorptive cells are increased by means of a protrusion of the outer cell-wall, the whole cell being converted into a tube. These tubular absorptive cells are most elongated in mossy forests, where rather large gaps occur not infrequently in the soil. When a root in the course of growth reaches one of these lacunæ, filled with moist air, its root-hairs often lengthon out to an cxtraordinary extent, and sometimes attain to twice the length of those which are in compact soil. The absorptive cells on the roots of the Water-hemlock (Cicutce virosa) and the Sweet Flag (Acorus Calamus) do not project at all if the earth in which they grow is muddy; whilst, if the earth is only slightly damp, and an increase of surface is therefore advantageous, the absorptive cells become tubular. Plants which grow in ground liable to periodic drought, and which at these times must secure all the moisture retained by the earth to save their aerial portions from death by desiccation, endeavour to obtain as great an area of absorption as possible by the development of long tubular cells.

The fact must not be overlooked, however, that the form and development of absorptive cells depend partly on the quantity of water that is given off from the aerial parts of the plant, that is to say, by the transpiration of the foliage-leaves. Plants which lose a great deal of water in this way must provide for abundant restitution. They must absorb from as large an area as possible, and enlarge their absorptive surfaces adequately by pushing out the cells into long tubes. For this reason all plants with very thin, delicate, expanded foliage-leaves, which transpire readily and abundantly, have numerous long tubular root-hairs. Examples are afforded by Viola biflora and the various species of Impatiens. On the other hand, plants with stiff, leathery leaves, being protected by a thick epidermis from excessive transpiration, as, for instance, the Date-palm, exhibit flat, non-protuberant absorptive cells, because there is a very limited amount of evaporation from these plants, and the quantity of water to be absorbed to replace what is lost is therefore small. The same thing holds in the case of evergreen Conifers, in which, owing to the structure of the stiff needles and to the peculiar formation of the wood, water is conducted very slowly from the roots to the transpiring green organs. It has been ascertained that they exhale from six to ten times iess vapour than do ashes, birches, maples, and other flat-leaved trees growing on the same ground.

We shall presently return to the question of the substitution for absorptive cells in many coniferous and angiospermic trees and in evergreen Daphnacece, Ericacece, Pyrolacece, Epacridece, \&c., of the mycelium of fungi, and shall treat also of the importance of the form of the absorptive cells, and of the roots which bear them, in relation to the mechanism of striking root in the ground. 


\section{RELATIONS OF THE POSITION OF FOLIAGE-LEAVES TO THAT OF}

\section{ABSORBENT ROOTS}

Anyone who has ever taken refuge from a sudden shower under a tree will remember that the canopy of foliage afforded protection for a considerable time, and that the ground underneath was either not wet at all, or only slightly so. No doubt some of the rain flows down the bark of the trunk, and in many species, as, for instance, the Yew and the Plane-tree, the volume of water conducted down the trunk is considerable; but in the case of most trees the rain-water which reaches the earth in this manner is not abundant, and in comparison with that which drips from the peripheral parts of the foliage its quantity is negligeable. This phenomenon is dependent upon the position of the foliage-leaves relatively to the horizon. In almost all our foliage-trees-in limes and birches, apple and pear trees, planes and maples, ashes, horse-chestnuts, poplars, and alders-these organs slope outwards, and are so placed one above the other that rain falling upon a leaf un one of the highest branches flows along the slanting surface to the apex, collects there in drops, and then falls on to a lower leaf whose surface is also inclined outwards. Here it coalesces with the water fallen directly upon this leaf; and so it goes from one tier to another, lower and lower, and at the same time further and further from the axis, till a number of little cascades are formed all round the tree. From the under and outermost leaves of the entire mass of foliage the water falls in great drops to the ground, and after every shower of rain the dry area at the foot of the tree is surrounded by a circular zone of very wet earth It is only necessary to dig at these places to convince one's self that the tree's absorptive roots penetrate the earth precisely to the wet zone. When a tree is young, its roots lie in a small circle, and the crown too is not extensive, so that the damp zone is proportionately restricted. But as the latter is enlarged there is a corresponding elongation of the roots in their search for moisture, and thus roots and foliage progress pari passu in peripheral increase. It seems not improbable that the custom amongst gardeners and foresters of trimming the foliage and roots of trees when the latter are transplanted is to be attributed to the phenomenon above described. For the rule is observed that the branches of the trunk and those of the root must be about equally shortened, and accordingly the suction - roots, as they develop, reach the zone of drip of the growing crown.

A similar method of carrying off water is to be observed in coniferous trees. Take, for example, the Common Pine. The lateral branches are horizontal near the main trunk; the secondary branches curve upwards like bows The needles near the tip of each of the latter slant obliquely upwards from the axis, whilst the older needles, situated on the under side of the part of the branch which is almost horizontal and at some distance from its extremity, are directed obliquely downwards and outwards. Rain-drops striking the upturned needles glide down them to the bark of the branch in question, and thence to other needles whose 
inclination is downwards and outwards. On their apices great drops are gradually formed, which finally detach themselves and fall on to the mass of needles belonging to a lower branch. Thus transmitted, the rain-water travels through the foliage lower and lower and at the same time further from the axis. This is also the case with larches. The drops of rain which fall upon the erect needles of the tufted "short branches" collect and gradually descend to the needles of the drooping "long branches" on lower boughs. Large drops are always to be seen on their drooping apices, whence they drip to the earth. Owing to the pyramidal form of larches, and to the circumstance that the long shoots on each branch are terminal, almost all the water which falls upon one of these trees reaches the long shoots hanging down from the lowest branches, which discharge most of all. Although larches with their tender needles do not look at all as though they would be any protection against rain, the ground underneath them keeps dry nevertheless, the principal part of the water falling upon them being conducted to the periphery. Indeed, the larch belongs to the number of trees which conduct almost all the rain that falls upon them to a certain distance from the axis where the absorbent roots lie, and only allow a little to trickle down the bark of the main trunk.

Many shrubs and perennial herbs also transmit the water, which falls on their upturned laminæ, to parts of the ground where their absorbent roots are embedded; or, rather, the roots send forth their branches bearing absorptive cells to the area which is kept moist by drippings from the leaves. Particularly striking in this respect are the species of the two genera of Aroids Colocasia and Caladium. A specimen of the latter is figured below (fig. $13^{1}$ ). If one digs about individuals of this genus cultivated on open ground, one invariably finds that the tips of the lateral roots, which proceed in a horizontal direction from the bulbous root-stock, are buried under the point of the great leaves which slope obliquely outwards. We must not omit to mention, in addition, that the stalks of leaves which conduct the rain centrifugally are not channelled on the upper surface; they are round, and comparable to wires supporting at their upper extremities the laminæ in an outward and downward direction. As instances we may quote the Horse-chestnut, Maple, and Lime, and many shrubby, suffruticose, and herbaceous plants, such as Sparmannia, Spircea, Aruncus, and Corydulis, and also climbing and trailing plants (e.g. Menispermum, Banisteria, Aristolochia, Hoya, Zanonia, and Tropocolum). Whenever a system of grooves is developed on the surface of an outward sloping leaf, the channels run along the veins and terminate at the apex of the leaf, or at the apices of the leaf's lobes, and invariably caus the water to travel, not to the basal part, but to a spot on the margin whence it will detach itself in the form of a drop, and fall upon the leaves situated immediately below and at a greater distance from the axis.

A striking contrast to these trees and shrubs, climbing and trailing plants, and suffruticose and herbaceous species, with their absorptive roots lying in one plane, and usually spreading at but little depth, is afforded by plants which possess 
bulbs or short root-stocks with deep-reaching suction-roots, and those which have tap-roots descending vertically in continuation of the main stem, and whose secondary roots are short and travel only a little distance from their places of origin. This other extreme in root-structure, which is represented in fig. $13^{2}$, has its counterpart above-ground in the form and direction of the laminæ upon which the rain falls. In all these plants the surfaces of the leaves are not directed

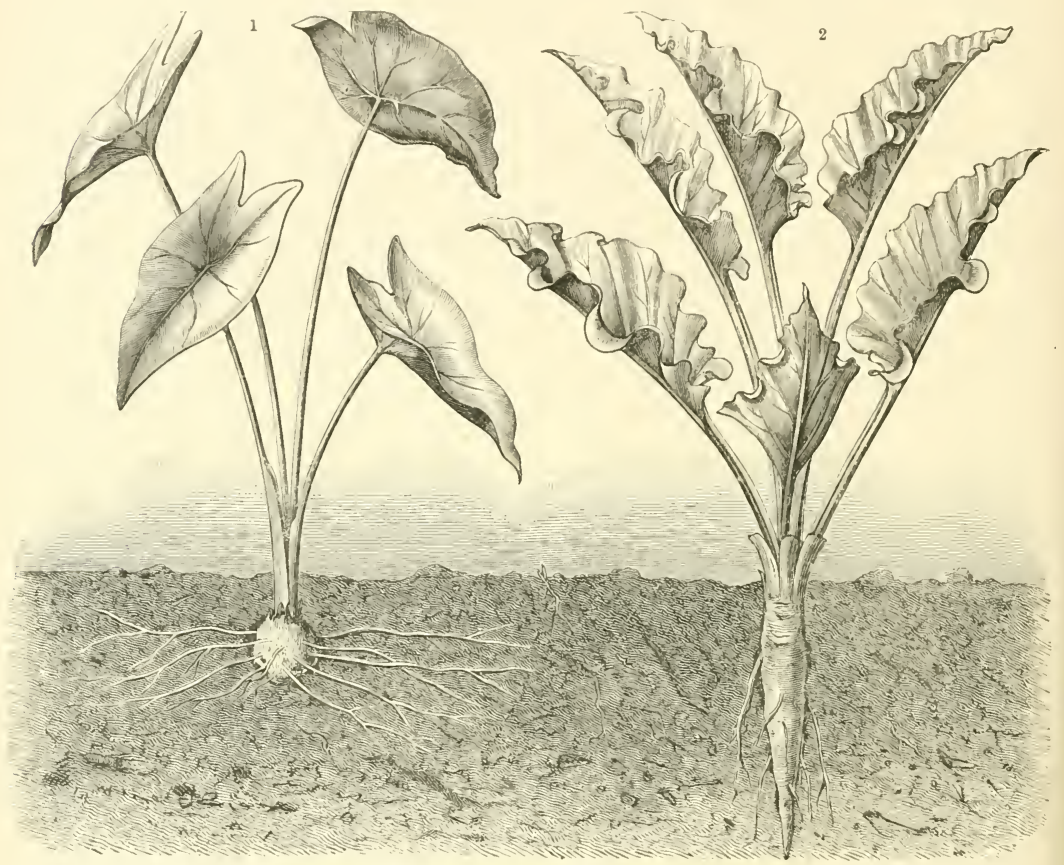

Fig. 13.-Centrifugal and Centripetal Transmission of Water.

1 By a Caladium, 2 By a Rhubarb plant.

outwards, but slope obliquely towards the central axis. Their upper sides, moreover, are concave and exhibit a system of grooves, which conveys the water collected by the leaf towards the stem, and therefore also, towards the tap-root and suctionroots. The leaves of bulbous plants, such as the Hyacinth and Tulip, all stand up obliquely, and their upper surfaces are concave and often deeply channelled. Along the grooves the rain flows centripetally downwards, and so directly reaches the part of the earth where the bulbs and suction-roots, which proceed in a tuft from underneath the bulbs, are situated. The young leaves of Cannacex and of the Lily-of-the-valley are coiled up like a trumpet; and rain, falling from above upon the expanded portion, is led along the coiled surface, describing a helix as 
it goes, to the earth in the neighbourhood of the absorptive roots, which proceed from the short root-stock. When the leaves of plants furnished with tap-roots are arranged in whorls, and are without internodes, and the rosette rests upon the ground, as is the case in the Mandrake, the Dandelion, and several species of Plantain (Mandragora officinalis, Taraxacum officinale, Plantago media), there are always one or more main grooves on the upper surfaces of the leaves, and the leaves have always such form and position as compel the rain which falls upon them to flow centripetally, i.e. towards the tap-root growing vertically bereath the centre. Plants with petiolate leaves, which conduct rain centripetally, always have on the upper side of each leaf-stalk an obvious groove, the depth of which is frequently increased by the development of green or (in many cases) membranous ridges on the two lateral edges. Grooves of this kind are to be seen particularly well on the petioles of the radical leaves of the Rhubarb (see fig. $13^{2}$ ), Beet-root, Funkias, and most Violets.

Far more complicated in structure than the radical leaves just described, are cauline leave3. Leaves proceeding from the stem high above the ground, and forming receptacles for rain-water, like those of the Rhubarb, are best fitted to preserve their proper direction when they have no stalks and the base fits directly on to the stem or passes into it. Cup-shaped laminæ, if borne on long erect petioles, necessitate a great expenditure on supporting-cells, and they are, therefore, on the whole, rare. Of the plants we know, only certain Stork's-bills, Pelargonium zonale, $P$. heterogamum, \&c., afford examples of cup-shaped, cauline leaves of the kind, borne on long, rigid petioles. In most cases, therefore, cauline leaves which conduct water centripetally are either sessile or very shortly petiolate, have their bases close to the stem, and even extend their edges down it more or less in the form of wings and ridges, or surround it in the form of collars, lobes, and auricles, as in the case of so-called amplexicaul leaves.

When the leaves are in pairs opposite one another and the alternate pairs at right angles, an arrangement known as decussate, the surplus water is usually conveyed through two grooves, which run down the intervening piece of stem from one pair of leaves to the next. Each of these grooves begins in an indentation between the margins of the bases of a pair of leaves, and terminates above the midrib of one of the leaves belonging to the next pair. Now, water trickling down such a groove falls precisely on that part of a lower leaf where the rain retained by the surface of that leaf is collected; and so the stream of water becomes more and more copious as it approaches the ground. These grooves may be seen in many species of ringent Labiata, Scrophulariacex, Primulacece, Gentianacece, Rubiacece, and Willow-herbs; the best-marked instances are found in the Knotty Fig-wort (Scrophularia nodosa), the Yellow-rattle (Rhinanthus), the meadow-gentians (Gentiana germanica, Rhatica, \&c.), and the Centaury (Erythrcea). The grooves always possess the property of being wetted by water, whereas the ungrooved parts of the same stem are not wetted. Sometimes the grooves are fringed with hairs which absorb the water like the threads of a 
wick. By means of both contrivances advantage is ensured in that the water only oozes quite gradually down the moistened grooves, or else is conducted by the hairy fringes to the base of the stem, and does not rebound at any spot in the form of drops. Irregularly bounding drops would be liable to fall on the ground at spots where no absorptive organs awaited them.

In cases where foliage-leaves, adapted to a centripetal conduction of rain, are arranged upon a spiral line down the stem, instead of in pairs opposite one another, the water leaks away along the spiral from one leaf to the next, and finally to the bottom. Then, again, there are often grooves in the stem along which the water trickles, as, for instance, in the Common Whortleberry (Vaceinium Myrtillus). The erect leaves of this plant conduct the drops as they fall to the branches, which are deeply furrowed. The water travels through the furrows into those of lower branches, and finally along those of the main stem of the whole bush down to the earth. In Veratrum album each of the concave cauline leaves has, on the upper surface, a number of deep longitudinal grooves, which all discharge together at the base of the leaf. The water collected there at length overflows and runs down the round stem in no particular channel.

The descent of rain-water along a spiral line may be very clearly traced in many plants of the Thistle tribe. If tiny shot-grains are substituted for raindrops in a stiff-leaved plant, the course designed for the drops in that particular species may be followed with ease. When strewn on a mature plant of the Safflower (Carthamus tinctorius) or of Alfredia cernua (fig. $14^{1}$ ), the grains of shot roll down the somewhat channelled surface of the highest cauline leaf, which stands up obliquely, and dash against the stem. The latter is half encompassed by the leaf-base, and the shot then roll over one of the basal lobes of the leaf and travel out of the range of that leaf, falling on to the middle of the one next below. For the amplexicaul foliar bases are so placed that each leaf has one of its basal lobes above a concave part of the next lower leaf. In precisely the same way the shot descend from the second leaf to the third, and so on until they reach the earth quite close to the stem. The descent reminds one of the game in which a little ball is made to roll along a spiral groove on to a board furnished with numbered holes. Rain-drops falling upon thistle-like plants of this kind naturally follow the same course as the shot. Only, the additional fact must be taken into account that not only the highest but all the leaves are adapted as receptacles for the rain as it falls, and that consequently the drops falling from leaf to leaf are augmented by new tributaries, and become greater and greater as they descend.

A somewhat different method of water-conduction from that which occurs in the Safflower and in the nodding Alfredia is observed in the Milk Thistle (Silybum Marianum), in the Cotton Thistle (Onopordon), and in the Mullein (Verbascum phlomoides). The upper leaves, which have two semi-amplexicaul lobes, are as nearly erect as those of the Safflower and the nodding Alfredia, and lead the rain off in exactly the same way. But the leaves in the middle 
of the stem are only erect for about three-quarters of their length; the uppermost third, including the apex, is bent obliquely outwards and downwards. Drops of rain falling on this upper third of a leaf would flow in a centrifugal direction, and do, as a matter of fact, drip down from the apex. Now the leaves in all
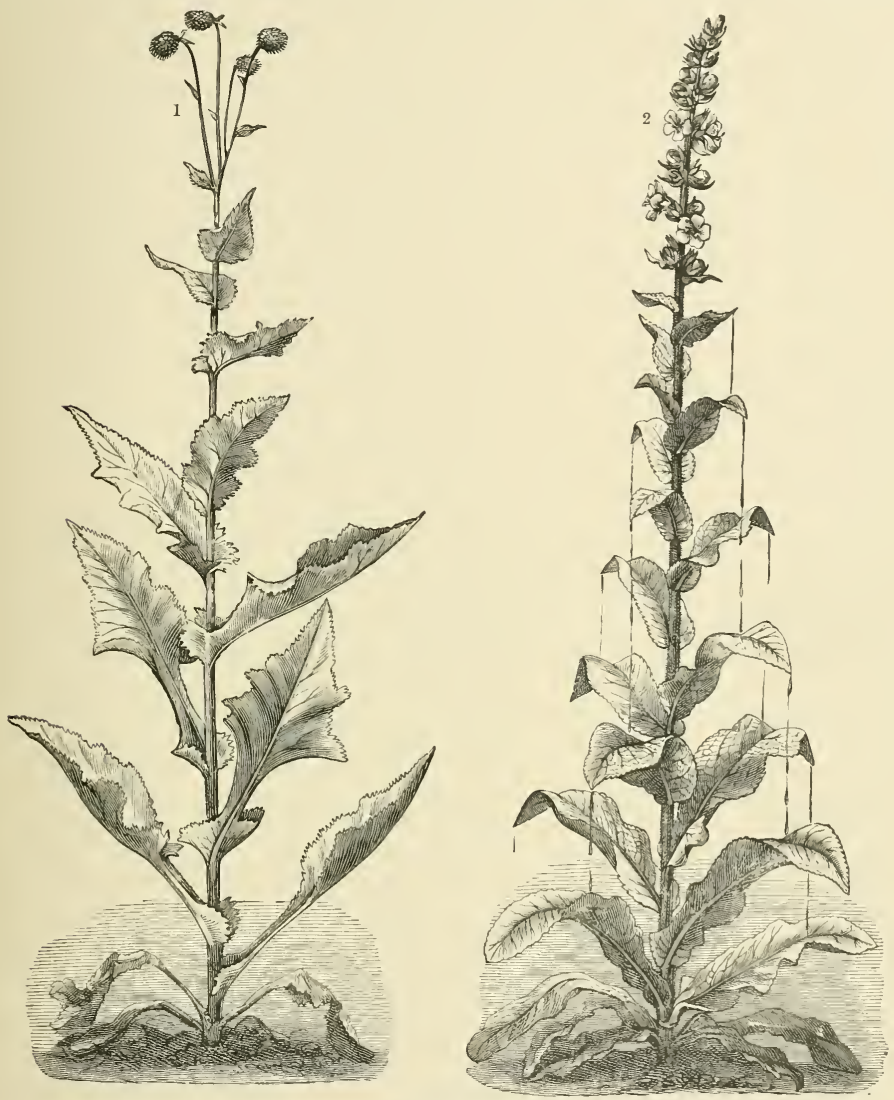

Fig. 14-Irrigation of Rain-water.

1 In Alfredia cernua. 2 In a Mullein (Verbascum phlomoides).

these plants are shorter the higher their position upon the stem, so that the total contour of the plant may be described as a slender pyramid. In consequence of this, water dropping from the outward-bent and drooping apices of superior leaves is arrested by that part of an inferior leaf which shelves towards the stem, and is thereby conducted centripetally. Thus all the rain-water received by a plant of this kind at last reaches the immediate neighbourhood of the tap-root, and is VoL. I. 
a source of nutriment to the absorption-roots which proceed from it. In the Milk Thistle (Silybum Marianum) the margins of the cauline leaves are very much waved, and, in consequence of this undulation, three or four depressions exist on each side, through which part of the rain, when there is a heavy downpour, flows off sideways. But even this water, falling laterally, drops upon parts of lower leaves, which conduct centripetally, and so coalesces with the streamlets otherwise produced.

It is very rare for plants which convey water centripetally to have their leaves arranged in two rows. The most striking example of this class is the Japanese Tricyrtis pilosa. Its leaves are situated on the fully-developed stem very regularly, one above the other, in two series. Each leaf has two lobes embracing the stem, but the base is fixed somewhat obliquely, so that one of the lobes is fixed higher than the other. Moreover, the higher lobe is closely adpressed to the stem, whilst the lower forms a channel which discharges exactly above the concave surface of the next lower leaf belonging to the other side. When rain falls on this plant, the water, collected by one leaf, flows through the broad exit-channel on to the leaf below on the other side. Thence a somewhat augmented stream falls upon a leaf of the first series, and so on, a peculiar cascade resulting, which falls in a zigzag, from leaf to leaf, until it reaches the bottom, close to the stem.

It would, however, be wrong to suppose that the above explanation sets forth the only significance to be assigned to the various arrangements described. To many plants it is a matter of indifference in what direction rain-water falls from the leaves. Such, for instance, is the case with all marsh-plants with roots buried in mud under water, inasmuch as the rain, as it drops, only goes into the water in the pond or marsh, and could not be conveyed to a definite spot for the sake of the absorbent roots. In the Water-plantain, the Flowering-rush, and the Arrow-head (Alisma, Butomus, Sagittaria), accordingly, no relationship between the form and direction of the leaves and the position of the absorbent roots is to be discovered.

On the other hand, in arundinaceous plants (Arundo, Phragmites, Phalaris) an arrangement has been hit upon which is obviously designed to prevent rainwater from collecting between the haulm and the leaf. As is the general rule with grasses, so also in the above-named kinds of reeds, the stem or haulm is furnished with nodes, and from each node proceeds a leaf the lower part of which encases the haulm in the form of a tube or sheath, whilst the upper part is expanded and presents a flat, strap-shaped or concave surface, standing well away from the stem. The leaves may be folded round the haulm like banners. At the place where the sheath passes into the part of the leaf which stands away from the axis at an obtuse angle, one observes on the edge of the leaf close to the angle, two distinct depressions which represent conduits and convey part of the rain from the lamina. There is also a very neat contrivance here in the form of an erect dry membrane which acts as a dam, the so-called "ligule." This membrane, inserted upon the leaf-sheath, is, like the sheath, in contact with the haulm. When rain- 
water flows down to this place it is stemmed by the membrane, as by a dam, and diverted right and left into the two grooves. In this way water is prevented from accumulating between the leaf-sheath and haulm, where it might do damage. In many reeds the contrivances for irrigation are even more complete than this. Sometimes hairs depend from the margin of the membrane in the direction of the grooves and, like a wick, lead the water in the proper direction.

An opportunity will occur later on of showing how the conduction of rain to particular spots has an important bearing on the phenomenon of absorption by aerial parts of plants: and also in the regulation of transpiration; and how, by means of the apparatus for water-irrigation, not only absorptive cells at the extremities of roots in the earth, but special organs on the foliage-leaves as well, are often supplied with water.

\section{ABSORPTION OF ORGANIC MATTER FROM DECAYING PLANTS AND ANIMALS.}

Saprophytes and their relation to decaying bodies. - Saprophytes in water, on the bark of trees, and on rocks.-Saprophytes in the humus of woods, meadows, and moors.-Special relations between Saprophytes and the nutrient substratum.-Plants with traps or pitfalls for animals.-Insectivorous plants which perform movements for the capture of prey.-Insectivorous plants with adhesive apparatus.

\section{SAPROPHYTES AND THEIR RELATION TO DECAYING BODIES.}

Whenever plants which take up organic compounds formed in the process of decay are the subject of discussion, the first examples that occur to everyone are members of the great family of Fungi, specimens of which make their appearance wherever dead animals or plants are undergoing decompositicn. We recall the moulds, plasmodia, puff-balls, and mushrooms, which grow from dead organic bodies, and are associated with the unpleasant mouldy and cadaverous smell always perceptible in their neighbourhood.

Many of these organisms do, in fact, belong to the class of Saprophytes. Indeed, one group of them is itself the cause of the chemical decomposition of dead plants and animals called decay. Their elongated thin-walled cells, the so-called "hyphæe", thread themselves through dead bodies, and unite to form strands, bundles, networks, and membranes, the whole constituting a structure to which the term "mycelium" is applied. These mycelia are often to be seen, with the naked eye, covering large areas. For instance, in damp cellars, mines, and railway-tunnels, any old rotten wood-work is clothed with delicate, whitish reticula and membranes. The heaps of grape-skins, stalks, and other refuse piled up in the open air by the side of vineyards after a vintage, are usually so completely overgrown by mycelia 
that their colour is quite altered. The so-called "mushroom-spawn", used in the cultivation of mushrooms, is also nothing but a mycelium, which entirely invests the manure employed in the cultivation of that fungus, and gives it a white mottled appearance.

In addition to Fungi, however, a number of Mosses, Liverworts, Ferns, Lycopods, and Phanerogams take up organic compounds from the products of decay to serve as their food.

In deciding whether a plant takes up only the mineral substances rendered soluble by the decomposition of the soil, or only organic substances disengaged by the decay of dead plants and animals, we depend generally on the condition and appearance of the nutrient substratum, and, in particular, on its composition, i.e. whether it is exclusively or predominantly organic. But such observations give a very uncertain indication. For, on the one hand, it is possible for plants rooted in a substratum of decaying matter to take nothing but mineral salts (i.e. inorganic compounds) from it; and, on the other hand, it frequently happens that sand or clay, apparently uncontaminated with organic matter, is saturated by water which oozes from a layer of humus in the vicinity, and brings with it organic compounds in solution. The following facts are instructive with reference to the former of these two phenomena. Maize, barley, and other cereals may be reared in fluids, so prepared as to contain a small quantity of mineral food-salts dissolved in distilled water (12 mg. potassium phosphate, $12 \mathrm{mg}$. sodium phosphate, $27 \mathrm{mg}$. calcium chloride, $40 \mathrm{mg}$. potassium chloride, $20 \mathrm{mg}$. magnesium sulphate, $10 \mathrm{mg}$. ammonium sulphate, and a few drops of iron chloride in a litre of distilled water), all organic compounds being carefully excluded. When the plants germinate, they develop roots which descend in the liquid and absorb from it mineral salts according to their requirements. They produce stems, leaves, flowers, and, ultimately, seeds capable of germination. Other plants of maize or barley reared simultaneously in richly-manured ground develop likewise leaves, flowers, and fruit. Moreover, analysis of the ash in both cases reveals the fact that the plants which took their nutriment from the manure contain the same salts as those reared in the made-up solution of salts free from organic compounds. Hence, the conclusion may be drawn that a plant of this kind is capable of obtaining an adequate supply of food-salts equally well, either from earth free from humus and manure, or from humus or manure themselves. The experiment further shows that, in the latter case, organic compounds need not necessarily be absorbed, in addition to the mineral constituents of humus or manure which are disengaged during decomposition.

We must next refer to a fact in connection with the second point above mentioned, viz. that plants rooted in sand or loam devoid of humus may yet have organic compounds brought to them by water filtering through a stratum of humus near at hand. The fact in question is, that the very water which one would least expect should contain organic compounds, that, for instance, of cold mountain streams, does very generally include traces of such compounds. On looking through analyses of mineral springs, one finds for the most part, amongst their constituents, 
combustible bodies arising from the dissolution of organic matter. Even the acid formerly designated by Berzelius by the name of "spring-acid", is doubtless a product of the decay of fragments of plants in the place where the water of the spring collects. So also is humic acid, a compound produced by decay. The nature of this acid is not yet, it is true, thoroughly known, and it may be a mixture of several acids. We know, however, that it is easily soluble in water, and that it forms soluble compounds with alkalies. Brooks running through woods or meadows, small mountain lakes adjoining peat-beds, and pools in actual peat, consist of water, brown in colour, which gives an acid reaction, and contains invariably organic substances in solution.

The following observations are of great interest in connection with this subject. In the salt-mine at Hallstatt (Upper Austria) one of the galleries, which is hewn through rock and contains no wood-work of any kind, exhibited (spread out upon its smooth limestone roof) the mycelium of a fungus (an Omphalia), which certainly required organic nutriment. There were no decaying animal or vegetable remains anywhere in the gallery, and the mycelium derived nourishment solely from water oozing from above through a few narrow cracks in the stone whereby the surface of the latter was kept moist. This water came from a meadow lying high above the mine. Between the two was a thick stratum of limestone with a deep layer of earth resting upon it. The water was clear and colourless, and contained a certain amount of lime, but no perceptible trace of organic substances. Yet this water must have brought organic matter from the meadow above into the mine, and the minute quantity so introduced sufficed to enable the fungus mycelium to grow luxuriantly.

In the Volderthal, near Hall, in Tyrol, there is a spring of cold clear water rising out of slate at a height of 1000 metres above the sea-level, which is filled at its source with a dark thick felt. The felt may be lifted out in pieces the size of one's hand, and it is the mycelium of a fungus, probably a Peziza. It clings to slabs of slate, between which the water trickles abundantly, and its nutriment can only be derived from this water. There are pine-woods and meadows in the neighbourhood, but no greater amount of vegetation, humus, or rotten timber than is found near other springs.

These instances satisfactorily prove that even the clearest mountain springs contain organic substances in quantities sufficient, however minute, to nourish fungi. When the origin of springs is taken into account, this result is not really surprising. They are fed by deposition from the atmosphere. The water thus deposited percolates into the ground, passing, in the first place, through a layer of earth-mould which is covered by vegetation, and contains more or less humus in its upper strata. A small quantity of the products of decay is inevitably absorbed, and even if they are partially withdrawn again in lower strata of the earth, traces are still retained by the water in its descent to greater depths, and re-ascent to the surface in the form of springs. The characteristics of the great veins of water which ascend in this way are no doubt common to the smaller veins which originate 
in the regetable mould saturated by snow and rain on the ground of forests or in the humus covering meadows, and which percolate through into the sand or loam beneath. Plants whose roots ramify in this deeper layer of earth derive thence the organic compounds conveyed by the water, and have the additional advantage of being able to satisfy at the same time their requirements as regards mineral substances. This circumstance is of importance not only to flowering-plants but also to many fungi, as, for instance, to all species of Phallus, they having need of a great deal of lime. An explanation is thus afforded of the fact, formerly difficult to understand, that in forests and meadows not only the upper black or brown humus layer, but also the underlying yellow loam, or pale sand, neither of which latter contains any humus, has mycelia of fungi running through it in every direction, and weaving their threads over little fragments of rock. Indeed, it sometimes happens that the lower layer of earth is more abundantly penetrated with plexuses of hyphæ than is the upper layer, consisting of vegetable mould. The greatest number of saprophytes is to be found therefore at places where the humus layer is not too thick and loam or sand occurs at no great depth; but where decaying vegetable remains are piled metres high, as on moors, for example, instead of fungi being produced in extraordinary abundance, as one might expect, only a few occur. Pure peat is by no means a favourable soil for fungi, a circumstance which may be partly due to the antiseptic action of certain compounds developed in it.

It follows from the foregoing observations that a sure conclusion as to the nature of plants rooted in a particular substratum cannot possibly be derived from the mere appearance of the substratum. Moreover, the conditions necessary for the growth of plants requiring organic products of decay as nutriment appear to be of much wider occurrence than one would suppose upon a cursory observation of the conditions existing in fields and forests, or, if one considers exclusively instances of cultivated plants reared on arable land, which is manured and constantly turned over. The great variety of plants produced on a limited area is also now intelligible. From the same soil some absorb organic compounds, others mineral substances only; whilst others again take some organic and some mineral foodsalts. The determining factor is not the amount of a given substance present in the substratum, but rather the special needs of each species, and ultimately the specific constitution of the protoplasm in each one of the plants which thus, side by side, nourish themselves in totally different ways.

If, then, neither the appearance of the ground nor its richness in respect of humus affords any certain indication as to whether a particular plant lives on organic products of decay or not, the question may perhaps be solved by the fact of the plant's containing or not containing green chlorophyll-corpuscles. We may take it as proved by many results of investigation, that the decomposition of the carbon-dioxide absorbed by a plant from the air, and the formation of the organic compounds of carbon, hydrogen, and oxygen known as carbohydrates (which play so important a part in vegetable economy), only take place in organs possessing the green pigment known as chlorophyll. We shall return to a discussion of these 
processes in detail later on, but the fact must be taken into consideration here. One would suppose, accordingly, that plants able to obtain ready-made organic compounds from a nutrient substratum could spare themselves the trouble of building them up, so that the presence of chlorophyll would be superfluous. This conjecture is in fact supported by the absence of chlorophyll in fungi, which are typical instances of saprophytes. But, on the other hand, some plants appear to negative this assumption, or at any rate to deprive it of general application. In mountain districts, where cattle continually pass to and from the meadows and alps, one notices on their halting grounds, and along their tracks, moss of a conspicuous green colour growing on circumscribed spots. On closer examination we find that we have here an example of the remarkable group of the Splachnacece, and that it has selected the cow-dung to be its nutrient substratum. Each growth of emerald green, Splachnum ampullaceum, is strictly limited to the area of a lump of dung; no trace of it is to be seen elsewhere. All the stages of development of this moss follow one another upon the same substratum. First of all the lumps of dirt which are kept moist by rain or by standing water, become enveloped in a web of protonemæ, and their surfaces acquire thereby a characteristic greenish lustre. Later, hundreds of little green stems, thickly clothed with leaves, emerge, and the spore-cases, which resemble tiny antique jars, and are amongst the prettiest exhibited by the world of mosses, become visible as well. Just as Splachnum ampullaceum is produced on the dung of cattle, so is Tetraplodon angustatus on that of carnivorous animals, and there can be no doubt that these, and in general all Splachnacece, are true saprophytes. A similar remark holds with regard to the green Euglence which escape from Hormidium-cells, and fill the foul-smelling liquor in dung-pits and puddles near cattle-stalls in mountain villages, and which multiply to such an extent that in a few days the liquid changes colour from brown to green.

Thus plants do exist containing chlorophyll although absorbing from the substratum organic compounds alone, and containing it, indeed, in such quantities that its presence cannot be looked upon as accidental. It follows, firstly, that absence of chlorophyll is not the distinguishing mark of saprcphytic plants; and, secondly, that the organic nutriment of the plants above mentioned cannot be used forthwith unaltered in the building up and extension of their structures, but, like inorganic material, must undergo various changes, that is, must be to a certain extent digested before being used for construction. The probability is that green saprophytes take carbon from their substratum in a form unfitted for the manufacture of cellulose and other carbohydrates. Saprophytes that are not green must obtain carbon from the substratum in the form of a compound, the direct absorption of which could be dispensed with if chlorophyll were present; but it does not necessarily follow that all the organic compounds absorbed by non-green saprophytes are capable of immediate service as materials for construction without any preliminary alteration.

Impartial consideration of the above facts forces us to conclude that there is no 
well-marked boundary line between plants which absorb organic compounds and those which absorb inorganic compounds from their respective substrata; and that there undoubtedly exist plants capable of taking up both kinds of material at the same time. This conviction is strengthened still further by the circumstance, which has been repeatedly confirmed by experiment, that plants susceptible of being successfully reared in artificial solutions of mineral salts-to the exclusion of organic compounds-do not entirely reject organic compounds when the latter are tendered to them, but unquestionably assimilate some of them (urea, uric acid, glycocoll, \&c.) and work them up into constituents of their own frames.

But, in spite of the impossibility of drawing a sharp line of demarcation between the two groups, it is convenient to treat of the absorption of organic compounds separately, because this division of the subject affords the best opportunity of inspecting in detail, and of surveying generally, the conditions of food-absorption, the comprehension of which is otherwise difficult. In order to determine in each individual case whether a given plant lives either exclusively or principally upon organic food, derived from decaying animal or vegetable remains, reliance must be placed on experiments with cultures; and, in the absence of better vantage-ground, the results of the rougher experiments made by gardeners should not be neglected, always providing that they are accepted subject to possible correction by subsequent exact experiment.

\section{SAPROPHYTES IN WATER, ON THE BARK OF TREES, AND ON ROCKS.}

Of the special cases of absorption of organic compounds from decaying bodies, we have first of all to consider those occurring amongst water-plants. In the sea, wherever there is an abundance of animal and vegetable life there is also plenty of refuse, for there death and decay hold a rich harvest. The quantity of organic matter dissolved in the water is naturally greater in these places than where vegetation and animal life are less conspicuous. There is a much more varied flora and fauna to be met with in the sea near its coasts, especially in shallow inlets, than at a greater distance from the shore; and the number of dead organisms is also greater near the coast. A mass of organic remains is thrown up by the tide, and by waves in stormy weather. This mass rots during the ebb. Part of it is dragged out to sea again by the next high tide, and then flung up once more; so that the beach is always strewn with dead remains, and the sea near the shore contains more products of decomposition than in the open.

In the immediate neighbourhood of seaports, moreover, or wherever people live, the volume of refuse is considerably increased, and the water in harbours and stagnant inlets behind breakwaters, and at the mouths of canals and sewers, contains such a large quantity of organic refuse in a state of decomposition that its presence is revealed by the odour emitted. Now it is just at these places that an abundant vegetation of hydrophytes is developed. Not only the bottom of shallows, but stones, stakes, quays, buoys, and even the keels and planks of boats long anchored 
in harbour, are overgrown by Ulvœ, wracks, filamentous algæ, and Floridece. Not a few, as, for instance, the so-called sea-lettuce (Ulva lactuca), several species of Gelidium, Bangia, and Ceramium, and the great Cystosira barbuta, thrive best and in greatest abundance in polluted water of the kind; and there can be no doubt that this is to be accounted for by the presence of a greater quantity of organic compounds in that water.

It is not only in contaminated sea-water, but also in other collections of water which contain products of putrefaction in solution, that we find a characteristic vegetation. We have already alluded to the presence of Euglence in the liquor of manure-pits. They occur also at the foot of shady walls, in dirty back streets in towns, in the puddles, and on ground which is saturated with urine and impurities of every kind. These places are the home of a number of other minute plants, which stain the polluted ground after rain with the gayest colours. There, side by side with black patches of Oscillaria antliaria and verdigris-coloured films of Oscillaria tenuis, are blood-red patches of Palmella cruenta, and brick-red patches of Chroococcus cinnamomeus. Equally characteristic is the vegetation which covers the earth at the mouths of drains, and is bathed by the trickling sewage. Large areas here are overgrown by the green Hormidium murale, which weaves itself over the mire, and by the dark, actively-oscillating Oscillaria limosa; and, above all, the curious Beggiatoa versatilis makes itself conspicuous, sending out from a whitish gelatinous ground mass long oscillating filaments, which emerge after sundown, and next day split up into innumerable little bacteria-rods. The red-snow alga, too (represented in fig. 25A), lives at the expense of the pollengrains, bodies of insects, and other decaying matter blown on to snow-fields; whilst the nearly allied blood-red alga (Homatococcus pluvialis or Sphcerella pluvialis) lives in the water in hollow stones where all sorts of animal and vegetable remains collect. Leaves blown into deep pools, and lying rotting at the bottom, are everywhere overgrown by green Edogonium, by Pleurococcus angulosus, and by the anethyst-coloured Protococcus roseo-persicinus. The bottoms of ditches on peat-bogs, which are full of brownish water containing an abundance of compounds of humic acid in solution, are covered with this amethyst Protococcus, whilst a profusion of small filamentous algæ, Oscillariæ and so forth (Bulbochate parvula, S'chizochlamys gelatinosa, Sphcerozosma vertebrata, Microcystis ichthyloba, \&c.), as well as a group of dusky mosses (Hypnum giganteum, $H$. sarmentosum, H. cordifolium), all have their home exclusively in still water richly supplied with organic compounds. When we include also the curious mould-like Saprolegnia produced on dead bodies floating in water-Saprolegnia ferax and Achlya prolifera on flies and fishes-some idea is obtained of the great variety of saprophytes living in fresh water, as well as of those inhabiting the sea.

A much more agreeable and attractive picture than that of these aquatic saprophytes is afforded by plants whose sole habitat is the bark of trees. The dead bark does not constitute the nutrient base of all the plants which grow from trunks and branches, or climb up them in the form of clinging and twining lianas. 
Often the trees only serve as supports, by means of which the plants in question raise themselves out of darkness into light. Such food-salts as they require they take, not from their support, but from the earth, into which they send absorptive roots. As years go by, a quantity of inorganic dust collects in the forks of branches and in the little rents and fissures in the bark of old trees, and this dust gets mixed with crumbled particles of bark. The clefts, therefore, are more or less full of vegetable mould, and this forms an excellent foster-soil for a large number of plants. But it is not necessarily the case that all plants rooting in this mould take up organic compounds from it. Thus, one finds not infrequently in the angles of bifurcation of the trunks of old limes and other trees, little gooseberry and elder bushes, and bitter-sweet plants, which have germinated there from fruits brought by black-birds, thrushes, and other frugivora. These shrubs, in the forks of limes and poplars hardly take any organic compounds from the mould in which they are rooted, but confine themselves to the absorption of such mineral salts as they may require.

But, with the exception of instances of that kind, the great majority of plants, nestling in the mould in crevices of bark, do take nutriment from this their substratum in the form of organic compounds. In cold regions the plants living in the mould of bark are for the most part mosses and liverworts. They cover trunks and branches of old ashes, poplars, and oaks, with a thick green mantle, and grow especially on the weather-side of the trees. In the tropics, on the other hand, the fissured bark of trees is a rallying ground not only for delicate mosses and moss-like Lycopodia, but also for a whole host of ferns and vivid flowering plants. The number of small ferns which develop and unroll their fronds from chinks in the bark of trees is so great that old trunks appear wrapped in a regular foliage of fern-fronds. Of Phanerogams, in particular, the Aroidece, Orchidacece, Bromeliacece, Dorstenice Begoniacece, and even Cactacece (species of the genera Cereus and Rlipsalis) bury their roots in the mould of bark. It is to be remarked that the rosettes of Bromeliacea ornament chiefly the forks of trunks, whilst Dorstenice, Orchidece, and the various species of Rhipsalis grow on the upper side of branches that ramify horizontally; whilst, lastly, Aroidece and Begonice take root, for the most part, on the surfaces of huge erect trunks.

Besides the mould collected in crevices and fissures of bark, the bark itself, that is, the cortical layer, dead but not yet crumbled and mouldered into dust, forms a nutrient substratum for a whole series of plants of most various affinity. Many fungi and lichens penetrate deeply the compact bark, and their hyphal filaments ramify between its dead cells. Other plants, instead of piercing through the substance of the bark, lay themselves flat upon its surface, and grow to it so firmly that if one tries to lift them away from the substratum, either part of the latter breaks off, or the adnate cell-strata are rent, but there is no separation of the one from the other. If a tuft of moss (e.g. Orthotrichum fallax, O. tenellum, or O. pallens), growing on bark, or a liverwort (e.g. Frullania dilatata) closely adherent to a similar basis, is forcibly removed, little fragments of the bark may be 
always seen torn off with the rhizoids at the places where they issue from the stemlets. The same thing occurs in the case of the roots of tropical orchicls growing to the tree-trunks which constitute their habitat. The majority of these tree-orchids nestle, no doubt, in mould-filled crevices of the bark, and nourish them-

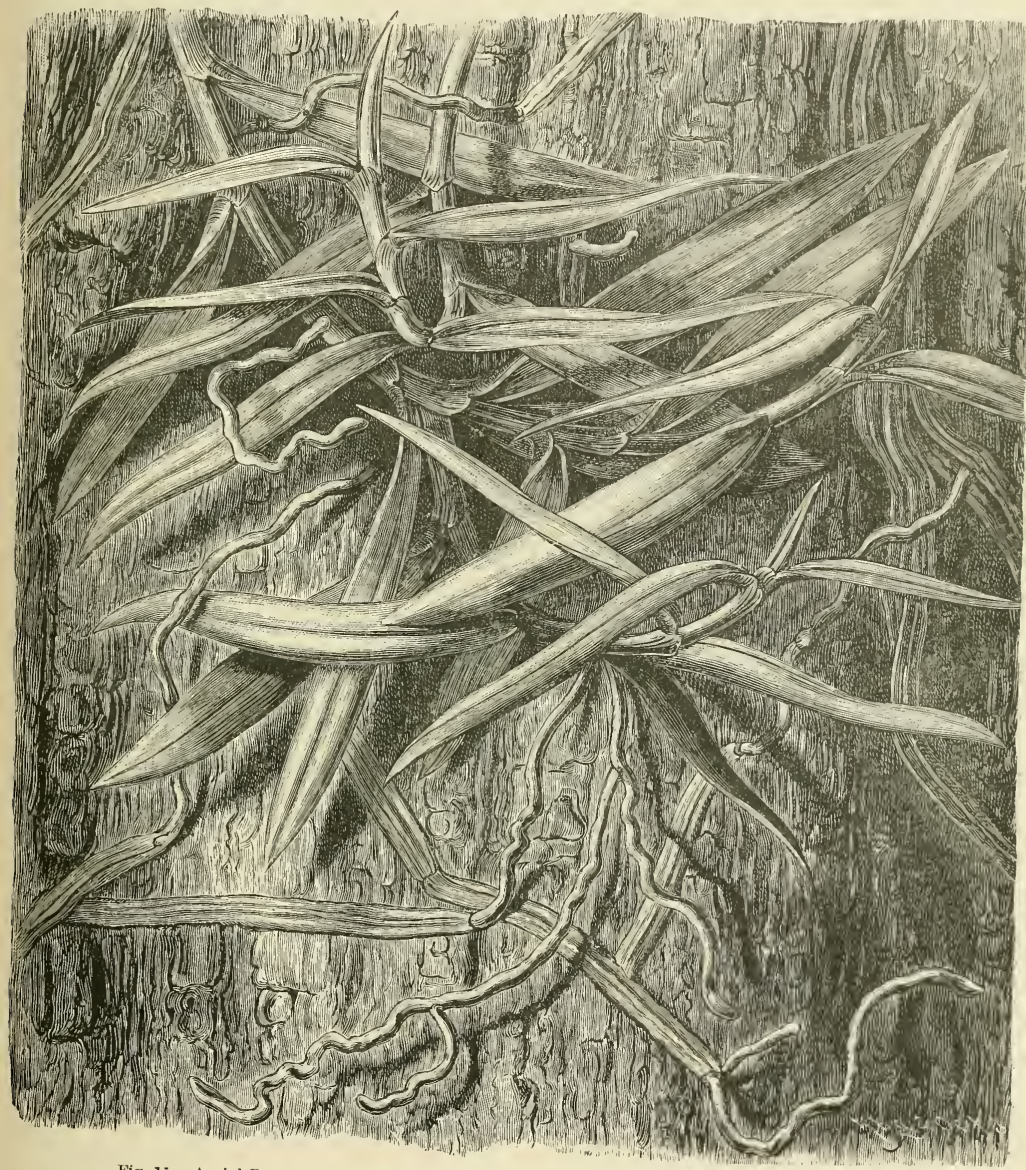

Fig. 15.-Aerial Roots of a Tropical Orchid (Sarcanthus rostratus) assuming the form of straps.

elves, besides, by means of special aerial roots which hang down in white ropes ind threads, like a mane, from the places where the plants are situated upon the rees, and which will presently be described in detail. But a small section develops trap-shaped roots as well, which adhere firmly to the bark with their flat surfaces. his phenomenon is most strikingly exhibited by the splendid Phalcenopsis 
Schilleriana, a native of the Philippine Islands; its roots are rigid, compressed, and about $1 \mathrm{c.m}$. in breadth; the surface turned away from the trunk is slightly convex, and has a granular structure and metallic glitter like a lizard's or chameleon's tail. The surface towards the trunk is flat and without metallic glitter, and upon it, close behind the growing point, there is a whitish fur consisting of short, thickly packed, absorptive cells. When the tip of one of these roots comes into contact with the bark it grows so firmly to the substratum by means of the absorption-cells, that it is easier to detach superficial bits of the bark itself than the root. The latter, once fixed, flattens out still more and becomes strap-shaped, whilst creeping outgrowths proceed from it, forming strips which may ultimately attain a length of $1 \frac{1}{2}$ metres. The sight of a trunk covered with these long metallic bands is one that never fails to excite wonder even in the midst of the world of orchids, wherein, as is well known, there is much to marvel at.

In other species of tropical orchids, e.g. in Sarcanthus rostratus (fig. 15), the roots are not flat from the beginning, but become so when they come into contact with the bark. A root is often to be seen which arises as a cylindrical cord from the axis, then lays itself upon the bark in the form of a band, and further on lifts itself once more, resuming at the same time the rope form, as is shown in the illustration. Here also complete coalescence takes place between the bands and the bark, and the union is extremely close. Similar conditions have been observed to hold in many Aroidece living on the bark of trees. The plants in question lie with their stems, leaves, and roots flat against the trunks, so that they suggest a covering of drapery. Taking, for instance, the Marcgravice (Marcgravia paradoxa, M. umbellata), one might at first sight suppose that they adhere to the bark not only by the roots, but also by the large discoid leaves, which are arranged in two rows. A very remarkable fact also, in connection with these plants, is that they only grow on very smooth and firm bark. When transferred to a soft substratum, such as mould or moss, they languish, because their roots are unable to enter into close union with a support of such loose texture. This is also true of most tropical orchids living on bark. When their seeds are transferred to loose earth devoid of humus, they do indeed germinate, but then perish; whereas when sown on the bark of a tree, they not only germinate, but grow up with ease into hardy plants.

Where steep rocks occur near clumps of trees it is not uncommon for the same species of plants to grow on both. Allusion is not here made to kinds which, like ivy, have their roots in the earth at the foot of rocks and trees, and creep up the one or the other indifferently, using both merely for support and not as sources of nutriment, and clinging to them by means of special attachment-roots. The remark is applicable also to plants which live on the products of the decay of organic bodies, for example many tropical Orchidece, Dorstenice, Begonice, and Ferns; and in cooler parts a number of Mosses and Liverworts. It is not difficult to explain this phenomenon in the case of species which derive their food from vegetable mould. The crannied wall of rock is, in a certain way, analogous to the rugged bark of a tree. The holes in the rock are filled in course of time with black vegetable mould, 
ind plants with foliage, flowers, and fruit of a form adaptable to cracks and holes re able to establish themselves in the mould there, just as well as in that collected n crevices of bark. In one respect, indeed, they are even more favourably situated. For the humus in bark gets quite dry in long periods of drought, because no water 8 yielded to the bark by the wood of a tree, even though the latter be abundantly upplied with sap; whereas, in the case of rocks the probability is, the clefts being rery deep, that even when the top layers of humus filling them yield up their water ;o the air, a certain restitution of moisture takes place from the deeper parts, which ure never quite dry. Moreover, plants growing in the mould of rock crevices are uble to send their roots down to much deeper strata than is possible in the case of ark. This is another reason why deep cracks in rocks, filled with humus, exhibit richer flora, as a rule, than do the much shallower crevices in the bark of trees, lthough, as has been said before, the two habitats have many plants in common.

It is more difficult to explain how it happens that plants which derive their ustenance, not from the mould in crevices, but from the substance of the bark tself, and which lie flat against its surface, are also found adhering to walls of ock. As an example take Frullania tamarisci, a Liverwort with small brown jifurcating stems, which bear double rows of leaves and are of dendritic appearance. This plant grows equally well on the bark of pines or on the face of adjacent gneiss ocks. At first sight it would seem scarcely possible that a plant of this kind, linging to the unfissured surface of rock, should be in a position to obtain organic compounds from its substratum. This is nevertheless the ease. Closer inspection eveals the fact that the Liverwort does not adhere to blank rock, but to a part ormerly clothed by rock-lichens. This inconspicuous incrustation of dead lichens s a complete substitute for the superficial layer of bark, and it is into it that the Frullania tamarisci sinks its roots. Another way by which food is supplied to olants adherent, like the above, to vertical and unfissured rocks will be discussed ater on.

\section{SAPROPHYTES IN THE HUMUS OF WOODS, MEADOWS, AND MOORS.}

Damp shady woods, especially pine woods, are particularly well furnished with aprophytes. Here again we find representatives of the same families as choose the ark of trees for their habitat. On the ground of woods, the most characteristic orms are mosses, fungi, lycopods, ferns, aroids, and orchids. The dark-brown iumus, produced from dropped and decaying needles, is first of all covered by a ich carpet of mosses, such as the widely distributed Hylocomium splendens, Hypnum triquetrum, and Hypnum Crista-castrensis. The mouldered dust of lead trees has a clothing of Tetraphis pellucida and of Webera nutans, and lecaying trunks are overgrown by the cushions of species of Dicranum (Dicranum coparium, D. congestum, Dicranodontium longirostre), pale feathery mosses Hypnum uncinatum and H. reptile) and various liverworts. Everywhere above he soft, ever-moist carpet of moss rise green frnnds belonging to broad-leaved ferns. 
Woods are also the special abode of fungi, and the damp ground is covered towards autumn by innumerable quantities of their curious fructifications. Dropped needles and cones, leaves and sticks strewn upon the ground, fallen trunks, and even the dark amorphous dust arising from the mouldering of these bodies and of the numerous roots ramifying in the ground, appear to be perforated by and wrapped in the protoplasmic threads of plasmoid fungi, or similarly invested by a plexus of filaments, the so-called mycelia of other forms of fungi. Amongst the scaly fragments of bark, peeling from the trees, they appear in the form of slimy strings, or as a dark trellis and net-work, inserted between the bark and wood of the rotting tree; on the stripped white trunk they are in dark zigzag lines like those of forked lightning; and between, the white mycelia of huge toadstools and tremellas are woven in all directions. Here and there large areas of the brown decaying soil are flecked and speckled by these mycelia, and even the dead stems of the mosses on the ground are festooned with white fleece, and wrapped round by hyphæ.

It is worth while to glance too at the reciprocal relations of these woodland plants. We find mosses, lycopods, and various ferns and phanerogams living upon the fallen twigs and needles, and on the mouldering roots of pines and firtrees. The dead remains of those plants afford sustenance to the fungi, which lift their fructification above the bed of moss. In their turn the rotting fructifications of the larger fungi form a nutrient substratum for smaller fungi, which cover the decaying caps and stalks with a dark-green velvet. Lastly, these little fungi, too, fall a prey to corrupting bacteria, and are resolved into the same simple inorganic compounds as were absorbed from the air and earth, in the first instance, by the pines and fir-trees. In the depths of forests there is going on, for the most part unseen by us, a mysterious stir and strife, accompanied by an uninterrupted process of exchange between the living and the dead, and a marvellous transformation of those very substances whose secret we have only partially succeeded in solving.

The results of cultivation have proved that in the group of flowering-plants belonging to the woodlands of Central and Northern Europe, which derive sustenance partially or entirely from the organic compounds afforded by the humus, are to be included, amongst others, the various species of coral-wort (Dentaria bulbifera, D. digitata, D. enneaphyllos), Circaa alpina, Galium rotundifolium, and Linnoca borealis, and above all a large number of orchids. Of these, Dentaria prefers mould produced from the beech leaves, and Circae, Galium, and Linnaa appertain to the mould of pine-woods. Of the orchids some are provided with green leaves, as, for instance, the delicate little Listera cordata, Goodyera repens remarkable for its villous petals, and the various species of Cephalanthera, Epipactis, and Platanthera; others, such as Limodorum abortivum, the bird's-nest orchis, the coral-root, and Epipogium aphyllum have none. Limodorum abortivum belongs rather to the warmer districts of Central Europe. It has fleshy rootfibres, twisted and twined into an inextricable ball, and a slender steel-blue stem, over half a metre in height, bearing a lax spike of fairly large flowers, which 
subsequently become paler in colour. The bird's-nest orchis (Neottia Nidus-avis) is of wide distribution both in forests of pines and in those composed of angiospermous trees. Its stem and flowers are of a light-brown colour, unusual in plants, but somewhat like that of oak-wood. The flowers have no scent, and the numerous roots, issuing from the subterranean part of the stem and imbedded in humus, remind one in form and colour of earth-worms, and together constitute a strange tangled mass as large as a fist. The latter has been thought to resemble a bird's nest, and to this is due the name of the plant. The coral-root (Corallorhiza innata), unlike the bird's-nest orchis, has no root at all; but, on the other hand, the subterranean portion of the stem, the so-called rhizome, possesses a distant resemblance to the root-tangle of Neottia. Pale-brownish branches of this rhizome, which bifurcate repeatedly at their obtuse and whitish extremities, looking as if they had been subjected to pressure for a time, and all the short lobe-shaped branchlets thereby spread out into one plane, lie closely crowded together, sometimes crossing one another, and so form a body which vividly recalls the appearance of a piece of coral. This underground coral-like stem-structure develops each year pale greenish shoots which rise above the ground and bear small flowers speckled with yellow, white, and violet, and exhaling a scent of vanilla; later, green fruits of a comparatively large size develop, turning brown when they ripen.

The fourth mentioned of these pale wood-orchids, the Epipogium aphyllum, is at once the rarest and most curious of them all. Like the coral-root it has no true roots. Its rhizome so closely resembles the latter's that it is easy to mistake the one for the other; but they may be distinguished by the fact that in the case of Epipogium the rhizome sends out long filiform shoots, which swell up like tubers at their tips, and may be regarded as subterranean runners. The swollen extremity becomes the point of origin of a new coral-like structure, which develops at about the distance of a span from the old one; whilst the latter, usually exhausted after flowering, gradually perishes. This coral-like stem lives of course underground, and is not visible till one lifts away the moss from the mould on the ground. It is often completely imbedded in sandy loam, lying immediately beneath the black mould. Many years frequently go by without the Epipogium producing flowers. The plant meanwhile lives entirely underground. In the course of a summer in which it has not flowered, anyone not having previous exact knowledge of its whereabouts might pass by without dreaming that the bed of moss and humus on his path concealed this strange growth. The flowering stems which at length emerge, when there is a warm summer, are right above the place where they branch off from the subterranean rhizome. They are thickened in a fusiform manner, and have, for the most part on one side, a reddish or purplish tinge. Everything connected with them is tense, smooth, full of sap, and almost opalescent. The few flowers that are borne by the stem are comparatively large, and emit a strong perfume resembling that of the Brazilian genus of orchids Stanhopea. The colouring, too, a dull yellowish white with touches of pale red and violet, reminds one of these tropical orchids. 
The sight of the pale-coloured plants lifting their heads, at flowering time, from the tumid carpet of moss has all the stranger effect because, as a rule, no other flowering plants are visible in any direction. The flowers are suspended by delicate drooping pedicels, and owing to their peculiar colour, fleshy consistence, and form - the erect concave petal like a Phrygian cap or helmet, and the others stretched out like prehensile limbs-remind one of the opalescent medusæ which float on the blue sea waves. The propriety of the analogy is enhanced by the fact that the form and colour of other saprophytes produced near Epipogium in woods have a striking resemblance to the animals and wracks which inhabit the sea-bottom. The fungi, known by the name of club-tops, much-branched, fleshcoloured, yellow or white Clavarice, which often adorn whole tracts of ground in a wood, imitate the structure of corals; Hydnece are like sea-urchins, and Geaster like a star-fish, whilst the various species of Tremella, Exidia, and Guepinia, which are flesh-pink, orange, or brownish in colour, and the white translucent Tremellodon gelatinosum, resemble gelatinous sponges. The small stiff toad-stools (Marasmius), which raise their slender stalks on fallen pine-needles, remind one of the rigid Acetabularice. Other toad-stools, with flat or convex caps exhibiting concentric bands and stripes, such as the different species of Craterellus, have an appearance similar to the salt-water alga known by the name of Padina. Dark species of Geoglossum imitate the brown Fucoidece; and one may fancy the red warts of Lycogala Epidendron, a plasmoid fungus inhabiting the rotten wood of dead weather-beaten trees, to be red sea-anemones with their tentacles drawn in, clinging to gray rocks. However far-fetched this comparison between the two localities may seem at first sight, everyone who has had an opportunity of thoroughly observing the characteristic forms of vegetable and animal life in woods, and at the bottom of the sea, will inevitably be convinced of its accuracy.

Meadow-land, rich in humus, is much more sparsely occupied by saprophytes than the soil of woods. There is no lack of the strange forms of toad-stools and puff-balls, whose fructifications often spring up in thousands, especially in the autumn, in company with the meadow-saffron; but in numbers they are not to be compared with those which occur in the mould of woods. Amongst ferns and phanerogams, the following spesies are dependent upon the organic compounds arising from the decomposition of the humus: Moonwort (Botrychium Lunaria), numerous orchids, blue and violet-flowered gentians, the famous Arnica, Polygalaceæ, and more especially several grasses, chiefly the Matweed (Nardus stricta) which, when once it has struck root in the humus, extends in dense masses over large areas. Several plants, too, adorning alpine pastures, and belonging for the most part to the same families as the species mentioned above, are to be regarded as humus-plants. Such are the Alpine Club-moss (Lycopodium alpinum), the dark-flowered Nigritella nigra, and several other sub-alpine orchids; a number of small, sometimes tiny, gentians (Gentiana nivalis, G. prostrata, G. glacialis, G. nana, Lomatogonium Carinthiacum), Valeriana celtica, the Scottish asphodel (Tofieldia borealis) of the north, a few grasses, sedges, and rushes (e.g. Agrostis 
alpina, Carex curvula, Juncus trifidus), various anemones, campions, umbelliferous plants, violets and campanulas (e.g. Anemone alpina, Silene Pumilio, Meum Mutellina, Viola alpina, Campanula alpina) and several mosses (e.g. Dicranum elongatum and Polytrichum strictum) which clothe the humus on stretches of turf and in inclosures.

Many of the plants also that are native on the black graphitic soil in hollows of high mountain ridges take up organic food from their substratum. These include Meesia alpina and various other mosses produced exclusively in places of the kind; and, above all, numerous Primulaceæ and Gentianeæ (Primula glutinosa, Soldanella pusilla, Gentiana Bavarica). It seems, moreover, to be by no means a matter of indifference to these plants at what temperature, and in what state of the air, in respect of moisture, the decomposition of humus takes place. If species which grow abundantly in these localities are dug up and transferred, together with the black earth in which their roots are imbedded, into a garden, and are there cultivated in such a way that the external conditions are as nearly as possible those of the original habitat; or if young plants are reared from seed in the same black humus-filled earth, they thrive only for a short time, soon begin to fade, and within the space of a year are dead; whereas, alpine plants belonging to the same altitude above the sea, but rooted in loamy or sandy earth, flourish excellently in gardens as well. Various moor-plants (e.g. Lycopodium inundatum, Eriophorum vaginatum, Trientalis Europcea) only live a short time in a garden even though the clods of peat, in which their roots are imbedded, are transplanted with them. This fact can scarcely be explained except by supposing that the organic compounds, produced by the decay of vegetable remains on alpine heights and moors, are essentially different from those evolved by similar matter under the changed conditions of temperature and moisture occurring in a garden at a lower level. Gardeners say that the peat and black graphitic soil from the slopes of snowy mountains turn sour in gardens, and they may be to this extent right, that in all probability the humic acids produced under altered circumstances are different.

\section{SPECIAL RELATIONS OF SAPROPHYTES TO THEIR NUTRIENT SUBSTRATUM.}

In the plants under discussion, the cells which absorb organic compounds are, taken all in all, very similar to those which absorb mineral food-salts. Where there is no cell-membrane, as in the case of Plasmodia and Euglenæ, the food diffuses through the so-called ectoplasm, or outer layer of the protoplasm, into the interior of the cell. Saprophytic marine and fresh-water algæ are able to absorb the products of decay in the water around by means of their superficial layers of cells. The mycelia of fungi have the power of taking in nourishment with special rapidity. Each hypha, or more accurately, each long, delicate-walled cell of a mycelium is, to a certain extent, an absorptive cell; its entire surface is capable of exercising the function of suction and of withdrawing from the environment, along with water, very substances which are needed. The coral-like underground stem of
vou. 
Epipogium aphyllum, as well as that of the "Coral-root", which is entirely destitute of roots, develop fascicles of absorptive cells on their ramifications, and on special little swellings; and the white subterranean stem structures of Bartsia alpina are also provided with long absorptive cells. The white, fusiform, tuberously thickened, underground stems of the Alpine Enchanter's Nightshade (Circaea alpina) exhibit no roots during autumn and winter, nor until such time as new leafy stems sprout from them and lift themselves into the daylight; they only have seattered clubshaped absurptive cells. Yet it is inconceivable that the few absorptive cells meet the entire requirements of these plants at the season of the development of their aërial stems. Food is absorbed in these cases also by the epidermal cells of the entire tuber, underground stem, or coral-like rhizome, as the case may be. The epidermal cells of these subterranean caulomes which lie immediately in contact with the black mould or humus on the ground of forests, have such thin and tender walls that they are quite as well adapted to the absorption of nutriment as are the projecting absorptive cells; indeed the club-shaped absorptive cells on the small tubers of Enchanter's Nightshade exhibit somewhat thicker walls than those forming the general epidermis of these tubers.

We may compare food-absorption as performed by these coral-like and tuberous structures, imbedded in decaying plant residues, with the action of tape-worms in process of sucking in through their entire epidermis the fluid filling the intestines they inhabit. The epidermal cells of the thick tortuous root-fibres of Neottia Nidus-avis are all capable of absorbing nutriment, though they do not project as tubes, but are tabular, and have their outer walls, which are in immediate contact with the nutrient soil, only slightly arched outwards (see fig. $16^{2}$ ). The green leafy orchids rooted in the vegetable mould of woods and meadows are, on the contrary, furnished with very long tubular absorption cells; and these cells do not wither and collapse forthwith when the root elongates, but long retain their vigour and activity. Whereas in the case of land plants adapted to mineral food-salts, the tubular absorption cells ("root-hairs") are limited to a narrow zone behind the growing point of the root and always die comparatively soon; in the case of orchids, having cylindrical roots imbedded in vegetable mould, these structures appear to be beset from end to end with long scattered tubular absorption cells, which are retained even through the drought of summer or the frost of winter right into the next period of vegetative activity; and these cells occur most abundantly in parts of the ground where there happens to be a bed of humus or mouldering remains particularly amenable to their purpose. Similar relations are found to exist in the case of the dichotomously-branched roots of the Club-moss. They are twisted in spirals and bore into the vegetable mould like corkscrews, and their absorption cells form in some places regular tassels, which are completely cemented over with fine black mould. The roots of grasses which, like the Mat-grass, live on the decomposition-products of vegetable mould, are also distinguished by strikingly long absorption cells, which grow in black or brown humus and there undergo the strangest bends and contortions. When, for instance, a fragment of a dead root or 
underground stem, peculiarly suitable for absorption, is encountered, it is regularly embraced by the suction cells, and as great an absorbent surface as possible is thus brought into contact with the nutritious fragment. Indeed, the development of suction cells on the roots of many gentians (viz. Gentiana ciliata, G. germanica, G. Austriaca, and G. Rhatica) is confined to the parts of the root-branches, which, in the course of their passage through the vegetable mould, have come into contact with a particularly nutritious portion of it. Wherever there is contact, the root is thickened, and absorption cells project unilaterally from the epidermis and grow into the decaying fragment of wood or bark which is to be drained of its nutrient
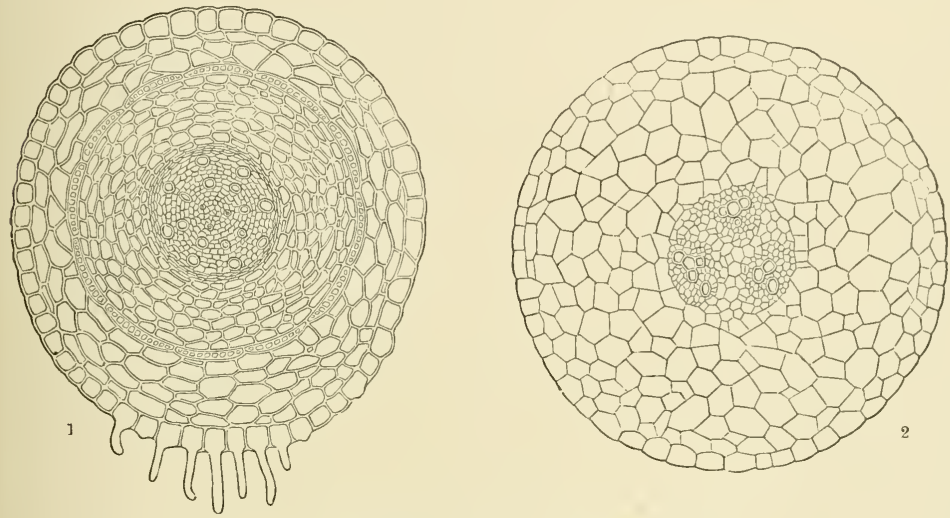

Fig. 16.-Transverse section through absorption-roots of Saprophytes.

1 Gentiana Rhotica.

2 The Bird's Nest Orchis (Neottra Nidus-avis).

material (see fig. $16^{1}$ ). Roots of this kind remind one of the root-structures of parasites which are furnished with so-called "haustoria", and which will be discussed more in detail in subsequent pages. But they are different in that they absorb food not from living but from decaying parts of the nutrient substratum.

Most plants that grow on the vegetable mould of alpine meadows, and the black earth deposited by snow-drifts in mountainous regions, develop flat instead of tubular epidermal cells as suction cells, and in this resemble marsh-plants. In many of these cases the roots are so abundantly and minutely ramified that they form a plexus investing the humus. This is likewise true of the absorptive cells on the rhizoids of mosses.

Plants which lie flat against the bark of trees and have no connection with the ground, so that they are unable to derive nutriment from it, have a very peculiar method of maintaining themselves. Their roots, rhizoids, or hyphæ, as the case may be, either grow straight into the bark or are merely adnate to its surface. In the latter case they are exposed on one side to the open air, and form more or less projecting lines and ridges ramifying in all directions, often constituting a regular trellis-work cemented to the bark. Sometimes, too, they are represented 
by thicker ropes or bands which run longitudinally down or encircle the trunk. These structures certainly serve as instruments of attachment, but at the same time they also absorb nutriment from the substratum, the decaying bark upon which the plant is epiphytic. In periods of drought the absorption of food by plants of this kind is, in general, interrupted and suspended. But when the rainy season commences and there is a long duration of wet weather, water trickling over the surface of boughs and trunks washes the bark, cleanses it as it were, and, falling lower and lower, brings down not only tiny loosened particles of bark but mineral and organic dust which has been blown into it by the wind; it dissolves all the soluble matter it finds on its way, and so reaches the roots, rhizoids, and hyphæ which adhere to the bark, in the form of a solution of mineral and organic compounds, chiefly the latter. The trickling water is in some measure stopped by the projecting ridges of these adnate structures; here and there also it deposits particles mechanically suspended in it, and so it conveys to these curious epiphytes the requisite nourishment.

In the same way, no doubt, epiphytes which grow upon other epiphytes are nourished. In more inclement regions, the green bark, stem, and, less frequently, the green leaves of the mistletoe are found to be beset by rosses and lichens; and, in the tropics it is a common phenomenon for mosses, liverworts, and even small kinds of Bromeliaceæ to settle on the green and still living leaves of Bromeliacex, Orchideæ, and Loranthaceæ, although they are certainly not properly parasitic, and only use their absorption cells for the purpose of clinging to the thick epidermis of the living leaves or stems which support them. The principal part of the liquid substances absorbed by these plants is conveyed to them by the rain-water that washes over the substratum.

The species of plants also which have been mentioned as sometimes growing on smooth vertical faces of rock, though the bark of trees is their usual habitat, are able to obtain their food-materials in a similar way. If the summit of a cliff is covered by a continuous carpet of plants, or if ledges and terraces projecting somewhat from its face support sods of grass, tufts of moss, and various small kinds of bushes, it must inevitably happen when there is an abundant fall of rain that the water flowing down the declivity conveys with it organic compounds in solution. First the sods of grass and moss on the ledges and on the top of the cliff are wetted, then the humus, which is their substratum, becomes saturated, and such part of the water as cannot be retained by this humus, or does not percolate into the cracks and crevices of the rock, trickles down from the ledges and moistens the face of the rock as it soaks down to the bottom. A rocky declivity is thus washed in the same way as is the bark of trees, and small fragments of organic and inorganic bodies must of necessity be rinsed out and carried down by the trickling water, and then again be deposited in heaps where projecting obstacles are encountered. It is just in the tracks along which the water flows down steep rocks of the kind that the plants of which we have made mention are situated.

Associated with the above are generally a number of other plants, for the most 
part microscopic, all of which cannot be classed as saprophytes, but which, in order to be able to thrive in the tracks of trickling water, must have the capacity of surviving desiccation for weeks, and even months, on the barren rock after having been previously supplied with copious moisture for a time. In the case of lichengrowths in particular these are very favourite sites; and when the lichens corer a large area they attract one's attention from afar. In limestone ranges, the light-gray rock of steep declivities, interrupted by ledges covered with grass and low brushwood, is extensively coloured by dark vertical bands and streaks, and the effect is the same as if a dye had flowed from the ledges over the face of the rock. These dark streaks indicate the course of the water which oozes from the humus and renders possible the existence of numberless minute plants on the precipitous face, in particular several dark crustaceous lichens (Acarospora glaucocarpa, Aspicilia flavida, Lecidea fuscorubens, Opegrapha lithyrga, \&c.).

The quantity of organic compounds brought down in solution by the water which filters from the layers of humus on rocky ledges, and that which trickles down the bark of trees, is, however, very small. Still, it is amply sufficient to meet the requirements of the plants occurring at the spots in question. The claims made by them upon their nutrient source are very moderate. We may here recall the instances previously mentioned of mycelia of fungi which have been found satisfied with the scarcely perceptible quantities of organic compounds in water filtering into the shaft of a mine, and in the pure water of a mountain spring respectively. To these instances must here be added the production of mycelia in the wooden pipes through which the clear water of mountain springs is conveyed. After these pipes, which are made from the trunks of pines, have been used as conduits for years, and their inner layers of wood have long since been washed out, the mycelium of the fungus Lenzites sepiaria is not infrequently developed within them, and in such luxuriance, indeed, that it forms great yellowishgray flocculent masses, which issue from the pipe's inner surface, and float in the stream of running water. In time these flocculent masses increase in the clear spring-water to such a degree that the pipes become completely blocked, and the flow of water is arrested. And yet the water conducted through the pipes is so pure, where it enters into and issues from them, that the residue obtained by the evaporation of hundreds of litres afforded no trace of any organic matter.

Seeing that most saprophytes absorb only such a comparatively small amount of organic matter, one is all the more surprised to notice that a large number of them fall suddenly, at certain times, into the opposite extreme. People speak of things rapidly produced in abundance as "mushroom-growths", and as "shooting up like fungi". The fructifications of many fungi are in fact developed with a rapidity which borders on the miraculous. The various species of Coprinus living on dung produce their long-stalked, cap-shaped fructifications during the night, and by the evening of the next day the caps have already fallen to pieces, and are in a state of decomposition, and nothing is to be seen in their place but a black deliquescent mass like a blot of ink. The weight of this fructification, thus maturerl within 
twenty-four hours, is certainly many times as great as that of the entire mycelium which produced it; and it is quite incomprehensible how this mycelium, which for weeks only achieves a moderate development, and adds but little to its dimensions, is in a position suddenly, and in so short a time, to supply the amount of water and organic compounds requisite for the building up of the fructification. Epipogium aphyllum exhibits a similar property. After producing nothing for two years excepting a few branches on its subterranean stem, it develops all at once and in a very short space of time fleshy stems with large flowers, and one asks with astonishment how the relatively small coral-shaped stock sets about obtaining the quantity of nutrient materials necessary for the construction of these flowering stems. We are here confronted again with the great mystery of periodicity, the solution of which we must for the present forego.

Saprophytes are much more fastidious as regards the quality of their nutriment than one might expect. It is true that certain fungi are producel wherever there are plants in a state of decomposition, and to them it is quite indifferent whether the mouldered dust, which serves as a nutrient soil for their mycelia, has arisen from one species or another. Also in the case of orchids imbedded in vegetable mould, and in that of most of the mosses and liverworts adherent to the barks of trees, it is, as a rule, of no consequence whether the tree constituting the substratum is a conifer or a dicotyledon. But a large number of species are associated with the decaying remains of particular plants or animals only. For example, certain small species of Marasmius, belonging to the group of the Agarici, occur only on mouldering pine-needles; another small fungus, Antennatula pinophila, is found exclusively on fallen needles of the Silver Fir; Hypoderma Lauri, which resembles small black type on rotting laurel leaves, and the tiny Septoria Menyanthis on leaves of the Bog-bean (Menyanthes trifoliata) lying under water in a state of decay. The cinnamon-coloured receptacles of Lenzites sepiaria only grow from prostrate trunks of conifers, and the black fuliginous fructifications of Bulgaria polymorpha only on those of oaks. A small discoid fungus named Poronia punctata, white with black spots on the top, is only found on cow-dung; another fungus, Gymnoascus uncinatus on that of mice, and Ctenomyces serratus on decaying goose feathers.

That many mosses are also very fastidious in the selection of their substratum has already been intimated. Just as in the Alps Splachnum ampullaceum is only found growing on the putrefying dung of cattle, so in arctic regions the splendid, large-fruited Splachnum luteum and S. rubrum occur exclusively on that of reindeer. Tetraplodon urceolatus is met with on mountains always with decaying excrements of chamois, goats, or sheep for a substratum, whilst Tetraplodon angustatus chooses the excrements of carnivorous animals, and T'ayloria serrata is only seen near cow-chalets on decomposing human frees. The circumstances of the occurrence of another moss belonging to the Splachnaceæ, i.e. Tayloria Rudolfiana is also very interesting. It grows usually on the branches of old trees, especially maples in sub-alpine regions, and one is tempted to believe that in respect of its nutrient substratum it is an exception to the rule of the rest of the 
Splachnaceæ. But on closer examination there is convincing evidence that this moss also lives only on animal dung undergoing putrefaction. For remains of broken mouse and bird bones are invariably to be discovered in the sulustratum, and there can be no doubt that the Tayloria chooses for its site boughs of old trees upon which birds of prey have dropped their excrements. Of the mosses living on the bark itself, one instance is also worth mentioning. Whereas in the case of most species of the genus Dicranum, the mouldering residues of conifers constitute the favourite substratum; there is one species, viz. Dicranum Sauteri, which is found only on the bark of the beech. The weather-worn bark of this tree is seen, in sub-alpine districts, covered with the most brilliant emerald-green films of the above-named moss; whilst on adjacent pines and fir-trees no trace of it can be found.

\section{PLANTS WITH TRAPS AND PITFALLS TO ENSNARE ANIMALS.}

A number of plants exhibit contrivances which obviously have for their object the capture and retention of such small creatures as may fly or creep on to their leaves; and it has been ascertained by searching experiments that the majority of these plants use the animals they capture, in one way or another, as sources of nutriment. For the most part the animals that are caught are insects, and hence the term "insectivorous plants" has been applied to the class in question. The flesh of the insect being the part of it principally serviceable for food, the name "carnivorous" or "flesh-eating", or better, perhaps, "flesh-consuming" plants has also been used; and seeing that the most important part of the whole process is really the digestion, or taking in of organic compounds from the captured animals after they are dead, we might call those plants which are furnished with organs for the absorption of the dissolved flesh of animals ensnared by them, "flesh-digesting" plants as well. As will appear from the following discussion of the subject, no one of these names completely covers the wonderful phenomena in question, and it is scarcely possible to find a short and not too cumbrous expression which shall henceforward exclude all misconceptions.

In round numbers we may estimate the plants which capture animals and demolish them for food at five hundred. Within this comparatively small range, however, the variety of the mechanism for seizure and absorption of nutritive matter is so great that in order to give a general picture of them it is necessary to classify them into several sections and groups. In the first section we have a series of plant-forms wherein chambers are developed, which admit of the entrance of small animals, but not of their escape. The organs of capture and digestion of the plants belonging to this section exhibit no external movements of any kind, and are thereby differentiated from the forms belonging to the second section, which perform definite movements, in response to a stimulus caused by the contact of the animals, with the object of covering the prey with as great a quantity of digestive fluid as possible. Lastly, there is a third section wherein the individual forms are 
neither provided with pitfalls nor capable of performing special movements, but have leaves converted into lime-twigs and on them animals stick and are also digested.

The first and most extensive group included in the first section is that of Utriculariæ or Bladderworts. Their capturing apparatus consists of little bladders with orifices closed in each case by a valve, which permits objects to penetrate into

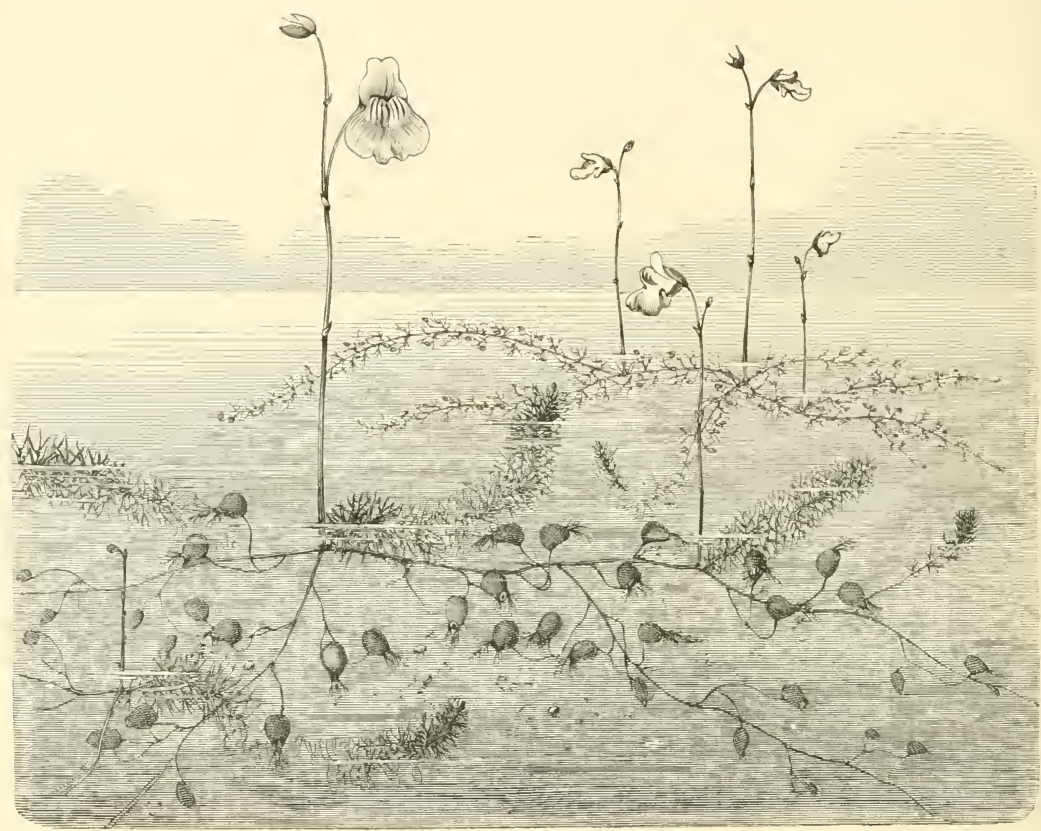

Fig. 17.-Bladderworts.

In the foreground Utricularia Grafiana; in the background Utricularia minor.

the cavity of the bladder, but not to issue out of it. The Utriculariæ are rootless plants which live suspended in water, and, according to the season of the year, either sink down to the bottom or ascend to just below the surface. Upon the approach of winter, when animal life is gradually disappearing in the chilled and freezing upper layers of water, the leaves at the extremities of the floating stems are enlarged and form spherical winter buds; the older parts of the stems together with the leaves die, their cavities hitherto occupied by air are filled with water, and they sink to the bottom drawing down with them the winter buds. After the winter these buds elongate, detach themselves from the old stems and ascend near the surface, where innumerable little aquatic animals are swimming to and fro, and there develop two rows of lateral branches in rapid succession. Either all of these are thickly covered with leaves which are divided into thread-like, repeatedly 
bifurcating, segments, or else only half of them are thus elothed with leaves whilst the other half bear the before-mentioned bladders. The former is the case in Utricularia minor, the plant represented in the background of the figure on p. 120 and the latter in Utricularia Grafiana, which is drawn in the foreground. In instances of the former kind obliquely ellipsoidal bladders are to be seen on short stalks on the principal segments of the leaves, usually quite near their angles of bifurcation. In the smaller species, such as Utricularia minor, they have a diameter of about $2 \mathrm{~mm}$. In individuals of the latter kind the bladders have longer stalks, and are about $5 \mathrm{~mm}$. in diameter. They are always pale-green and partially transparent. Each bladder is somewhat flattened at the sides and exhibits a markedly convex dorsal surface and slightly curved lateral surface. An orifice, whose border is fringed with peculiar stiff tapering bristles, leads into the interior of each of these stalked bladders. The aperture has four rounded angles and is framed as it were, by a pair of lips. The under lip is strongly thickened, and is furnished with a solid cushion projecting into the interior of the bladder. From the upper lip hangs a thin transparent, obliquely-

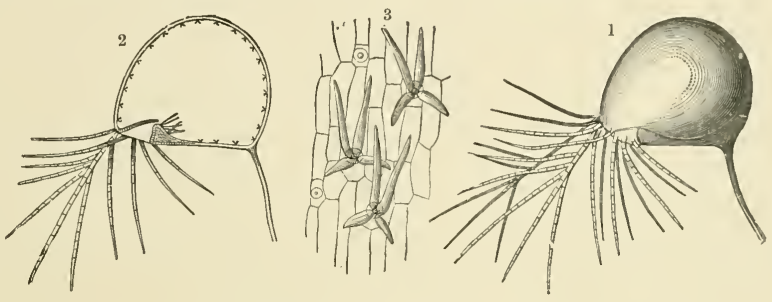

Fig. 18. - Traps of tricularia neglecta.

1 A bladder magnifled $(x 4)$. 2 Section of a blatler. 3 Absorption-cells on the internal surface of the bladder $(\times 250)$. placed valve (see fig. $18^{2}$ ), the free edge of which rests upon the inner surface of the cushion before referred to, and closes the entire orifice. This valve is very elastic and yields easily to any pressure from outside. A tiny animal is able, by pressing against it, to force a way without difficulty from the nether lip into the interior of the bladder, and to slip in through the opening thus made. But as soon as the animal has got inside, and ceases to press upon the valve, its elasticity brings it back upon the under lip again. It cannot be opened by pressure from within; for, resting as it does upon the projecting cushion, it is impossible for the little prisoner to force it over the latter in an outward direction.

The whole apparatus forms a trap for small aquatic animals, they being able, as before observed, to slip into the bladder but not to get out again. Most animals that enter make, it is true, efforts to escape, but they are all in vain. Many perish in a short time-about twenty-four hours-others live from two to three, or, in some cases, even as much as six days. But in the end they must suffer death by suffocation or starvation, and they then decay, and the products of their decomposition are sucked in by special absorption cells developed within the bladder. These absorption cells (see fig. $18^{3}$ ) are linear-oblong and somewhat like little rods in shape, and they line the whole internal surface of the cavity of the bladder. They are arranged in fours, each group of four forming a cross and being united by a 
common basal cell. The basal cells themselves are intercalated amongst the cells lining the bladder. The organic substances from the decaying bodies of captured animals are sucked up by these stellate groups of cells, and from them pass into the basal cells, and later, into the other adjacent cells of the bladder and those of the plant at large.

The majority of the animals caught by the bladders are crustaceans. It is principally larvæ and adult individuals of small species of Cypris, Daphnia, and Cyclops that fall into the trap; but larvæ of gnats, and various other small insects, little worms, and infusoria, are also not infrequently met with imprisoned in the bladders. The number of animals captured is comparatively large. In single bladders the remnants of no less than twenty-four small crustaceans have been observed. The prey secured by Utricularia minor (fig. 17), which lives in little pools of still water in peat-bogs, is very abundant. The North American Utricularia clandestina seems also to use its capturing apparatus with great success.

What it is that induces the animals to press upon the valves and so fall into the trap is not fully explained. We may suppose that they expect to find food in the bladder-cavity, or that they hope it will afford a shelter where they can rest for a time and be protected from their pursuers. The last suggestion is especially supported by the circumstance that the approach to the valve-covered orifice of the bladder is guarded against the intrusion of larger animals by stiff sharp bristles which stick out from it (fig. $18^{1}$ ). Only very small animals, which can easily slip in between the relatively large bristles, reach the inside of the bladder, whilst larger creatures, which would injure the whole apparatus, are prevented from coming near it. Thus, the most probable explanation is that lesser animals pursued by greater take refuge in the hiding-places behind the bristles, and so fall into the trap. Another very striking fact is that the bladders of Utriculariæ, living in still water, look delusively like certain Ostracoda, especiaily species of the genus Daphnia. The bladder itself resembles the shell-covered body in size and form, and the bristles the antennæ and swimmerets of one of these crustaceans. Whether there is any significance in this curious similarity of outward appearance must be left undecided.

The majority of Utriculariæ live in pools of water beside foot-tracks on moors and in the little collections of water between clumps of reeds in peat-bogs; and these are precisely the haunts of the little creatures that are to fall into the traps. Every handful of water that one scoops up contains hundreds of midge-larvæ, water-fleas, Ostracoda, and one-eyed Cyclops, which rush about promiscuously, pursuing and seizing one another. One species of these plants lives in the mountains of Brazil in the rain-filled receptacles of Tillandsia plants. The Tillandsia is allied to the pine-apple, and has rosettes of concave leaves, the latter resting one upon the other in such a way as to form a niche or cavity in front of each leaf which fills with rain like a cistern. Many different kinds of small animals are always swimming about in these little cisterns, and almost every one of the latter 
is the sphere of activity of an individual Utricularia nelumbifolia. This plant is remarkable also from the fact that long runners are thrown out from its stems, which grow across, in wide arches, from its cistern to a neighbouring Tillandsia, where it selects one of the reservoirs in the rosettes as a new site and dips down into the water-a fantastic method of propagation of which we shall speak again on another occasion.

A few Utriculariæ do not live in water at all, but grow amongst mosses, liverworts, and lycopods, in the vegetable mould filling the clefts and crevices of rocks, and the bark-fissures of old trees. Of this habit, for example, is the pretty Brazilian Utricularia montana, which, in spite of the difference of its habitat, is provided with an apparatus for capturing animals agreeing in all essential respects with the description already given. The bladders used by these plants for purposes of prey are produced on subterranean filiform stems which thread their way in the vegetable mould and wefts of decaying moss-stems, and here and there swell into tubers. The bladders are hyaline and transparent, and are filled with watery liquid, sometimes also with air. They are only 1 millimeter in diameter, but are present in large numbers. The entrance into these bladders is much more concealed than in the species that live in water. The dorsal surface of the bladder being still more strongly curved, the position of the orifice is altered so as to be quite close to the little stalk of the bladder. In addition, the orifice is, as it were, roofed over, and thereby protected against the possibility of being stopped up by particles of earth, and the passage leading to it is very narrow. That, in spite of the difficulty of entrance, a number of minute animals do seek a hiding-place here is proved by the circumstance that, besides various infusoria, rhizopoda, and creatures of that kind inhabiting damp earth, species of Acarus and larvæ of other animals have been found, both dead and alive, in the bladders.

With this first group of insectivorous plants, wherein the capturing apparatus includes a valve to prevent the egress of such animals as fall into the trap, is associated in the first section a second group, viz. that of the ascidia-bearing or pitcher-plants, in which the foliage-leaves are converted into pitfalls, and the escape of the captured prey prevented by a number of points lining the inner wall of the cavity, and directed from the aperture towards the closed bottom. There is an extraordinary variety in the form of the pitfalls. Sometimes they are tubular, utricular, or funnel-shaped cavities, sometimes mug or pitcher-shaped, or urceolate; in some cases these are straight, in others bowed like sickles, or spirally twisted. They always arise from the part of the petiole upon which the lamina immediately rests. The lamina is always relatively small, being represented in the majority of the traps by a scale or lobe, and it only appears to be an appendage of the large expanded and hollowed-out petiole. In many pitcherplants the little lamina looks like a lid placed over the orifice to the pitfall, as, for instance, is shown in the illustration (fig. $21^{4}$ ), whilst in others (Nepenthes ampullaria and $N$. vittata) it has the form of a handle or stalk, and serves as a place for animals visiting the pitchers to alight upon. 
In each pitfall there are always three kinds of contrivance to be distinguished: first, a device for the allurement of animals; secondly, an arrangement for entrapping the prey enticed, which at the same time prevents individuals once imprisoned from returning and escaping through the entrance hole; and thirdly, a structure for causing the decay or dissolution of the dead animals at the bottom of the pitfalls, and for rendering possible the absorption of the products of decomposition as nutriment. The means of allurement are similar to those which cause the visits of small creatures to flowers, that is to say, principally honey and bright and varied coloration, whereby the nectar-secreting spots are recognized from afar, especially by flying insects. The escape of animals when they have once entered the cavity of a petiole is prevented, as has been already mentioned, by a fringe of sharp hairs pointed downwards, or by various spinous structures on the inner
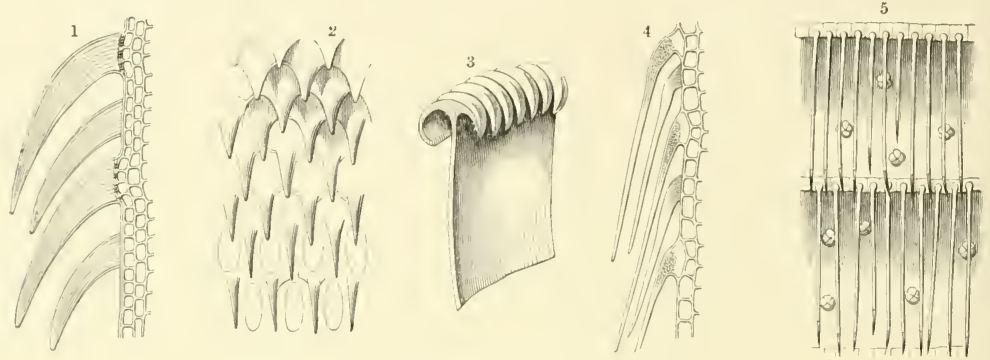

Fig. 19.--Spinous Structures in the Pitfalls of Carnivorous Plants

1 Genlisea; a piece of the tube seen from inside. 2 IIeliamphora nutans; spines on the walls of pitfalls. 3 Sarracenia purpurea; a piece of the lining of the pitcher near the orifice seen from insicle. 4 Sarracenia purpurea; longitudinal section throngh the membrane covered with spinous bristles in the lower part of the pitcher. ${ }^{5}$ Nepenthes hybrida; fringe of spines at the orifice of the pitcher. 1, 2, 4, 5 greatly magnified; 8 slightly magnifled.

surface of the cavity. The decomposition and dissolution of the prey are effected by fluids secreted by special cells at the bottom of the utricles and pitchers.

But although in respect of the consecutive and co-ordinate operation of these three kinds of contrivance, all ascidia-bearing and pitcher-plants resemble one another, there are considerable individual divergences as to structure and function that it is well worth while to study in some detail the most noticeable of them.

One of the most noteworthy is the genus Genlisea, which is nearly related to Utriculariaceæ in the structure of its flowers and fruit. It is composed of a dozen species growing in water and marshy places. Of these one is a native of tropical and southern Africa, whilst others are found in Brazil and the West Indies. In addition to ordinary leaves, which in them are spatulate, most of the Genlisex possess leaf-structures metamorphosed so as to constitute pitfalls. Each pitfall consists of a long, narrow, cylindrical utricle, which at its blind end is enlarged into a bladder, whilst at the narrow orifice at the opposite end are placed two peculiar ribbon-shaped processes twisted spirally. The orifice of the utricle is set with very small sharp teeth bent inwards; and the tubular part of the utricle has its inner surface lined throughout with innumerable little bristles, which arise from rows 
of cells forming inwardly projecting ridges, and have their sharply-pointed tips directed downwards (see fig. $19^{1}$ ). Amongst these needles are also found, scattered over the whole internal surface, roundish wart-like glands or papillæ, composed of four or eight cells. The bottom of the bladder-like cavity in which the utricle terminates is destitute of bristles, and provided only with glands arranged in rows. Small worms, mites, and other segmented animals which enter through the orifice of the utricle can easily reach the enlarged base. But as soon as they try to com-

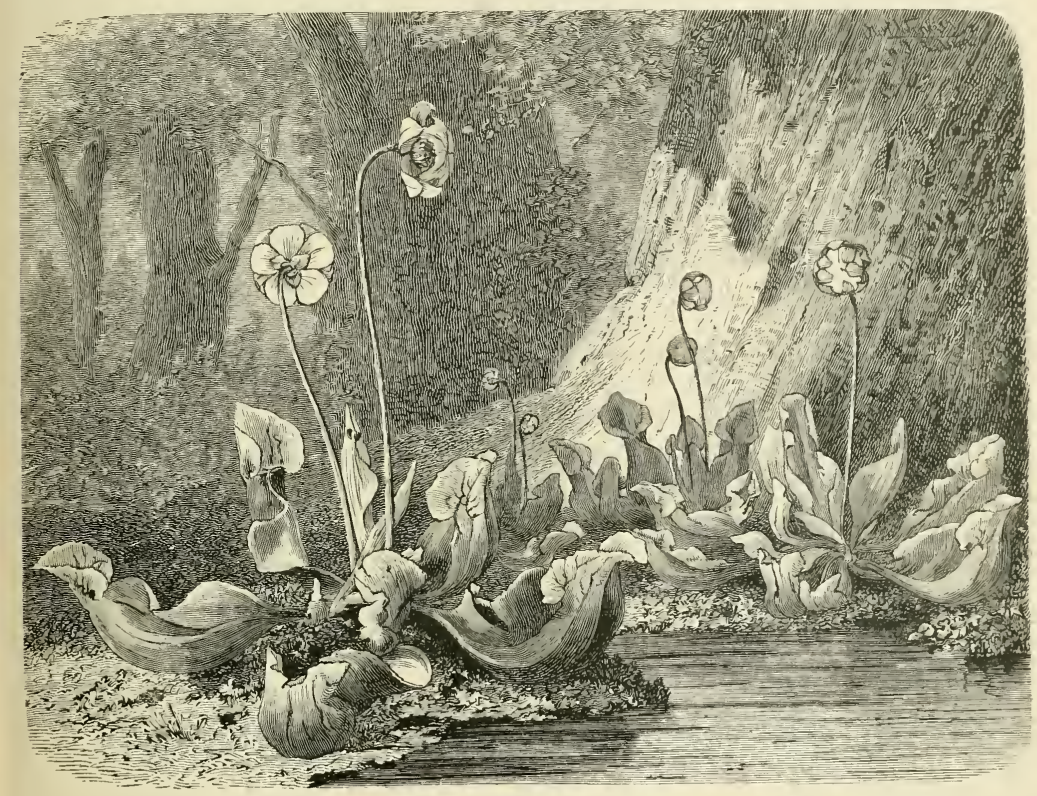

Fig. 20.-Sarracenia purpurea.

mence the return journey they are opposed by the points of a thousand bristles. Thus caught they die, and the products arising from the decay of their bodies are absorbed by the glands situated, as above mentioned, at the bottom of the bladder and on the walls of the utricle.

As types of a second series of carnivorous plants belonging to the group of pitcher-plants may be taken Heliamphora nutans, a native of moorlands on the mountains of Roraima, on the borders of British Guiana, and Sarracenia purpurea (see fig. 20), which is widely distributed in the marshes of eastern North America from Hudson's Bay to Florida. In both instances the leaves metamorphosed into ascidia are arranged in rosettes, rest their bases on damp earth and thence curve upwards. They are somewhat inflated, like bladders, at about their middle, but contract again at the orifice where they pass into the relatively small laminæ. The latter are threaded by red streaks like blood-vessels, have the form of valves, 
and turn their concave surfaces towards falling rain. They serve, moreover, at least in Sarracenia purpurea, to eatch the drops of rain, which then flow down into the bottom of the ascidia and fill them more or less with water. There is very little evaporation from the hollow pitchers; and even when there has been no rain for a week, one always finds some of the previously-collected water at the bottom. The inner surface of a pitcher is lined by cells arranged like the scales of enamel on a pike's back (see fig. $19^{2}$ ). The internally-projecting wall of each of these scales is transformed into a stiff decurved point, and the lower the position of the cells the longer do the points become. The shell-like lamina again, above the contracted orifice, bears glandular hairs which exude honey, so that the parts surrounding the aperture are covered by a thin film of sweet juice.

Many animals are attracted by this honey. Some are winged and alight from flying; others, being wingless, make use of a peculiar ridge, which projects on the concave side of the utricle, to help them to creep up the latter. If these honeyeaters happen to travel away from the lamina to that part of the pitcher which is lined with the smooth and slippery decurved cells, they are as good as lost. They slip down over the brink, every attempt to climb up again being rendered futile by the downwardly-pointing needles which clothe the lower part of the wall; and ultimately they fall into the water collected at the bottom, where they are drowned and their bodies putrefy. The products of decay are absorbed as nutriment by the epidermal cells in this region. The number of animals meeting with this fate is often so great that an offensive odour, arising from the decaying bodies, is emitted by the utricles and is noticeable at a considerable distance. In the wild state, the ascidiform utricles are often half-full of drowned animals and it is stated that in these circumstances birds also put in an appearance and pick some of the dead remains out of the utricles.

Whether the liquid filling the bottom of the pitchers consists simply of rainwater, or whether the latter is modified by a secretion originating in the glandlike groups of cells there (see fig. $28^{7}$ ), is still uncertain. A centipede over 4. centimeters long having fallen into a utricle of Sarracenia purpurea in the night was found only half immersed in the water. The upper half of the creature projected above the liquid, and made violent efforts to escape; but the lower part had, after a few hours, not only become motionless but had turned white from the effect of the surrounding liquid; it appeared to be macerated, and exhibited alterations which are not produced in so short a time in centipedes immersed in ordinary rain-water. When a number of captured animals are undergoing putrefaction at the same time in a pitfall, the liquid turns brown and has the appearance of manure-liquor.

There is a great difference between the utricles of Sarracenia purpurea and the apparatus adapted to the capture of prey in the plants of which we have chosen as examples, Sarracenia variolaris, a native of the marshes of Alabama, Florida, and Carolina, and the Darlingtonia Californica, found growing at a height of from 300 to 1000 meters above the sea on Californian uplands from the borders of 
Oregon to Mount Shasta. In both of these the liquid with an acid reaction, which fills the bottom of each utricle, is certainly only secreted by the cells in the interior of the cavity itself, and it is quite impossible that a single drop of the rain or dew deposited upon the plant should reach the interior of the eavity. The hollow petiole is in both plants, above mentioned, utricular or tubular, and only slightly

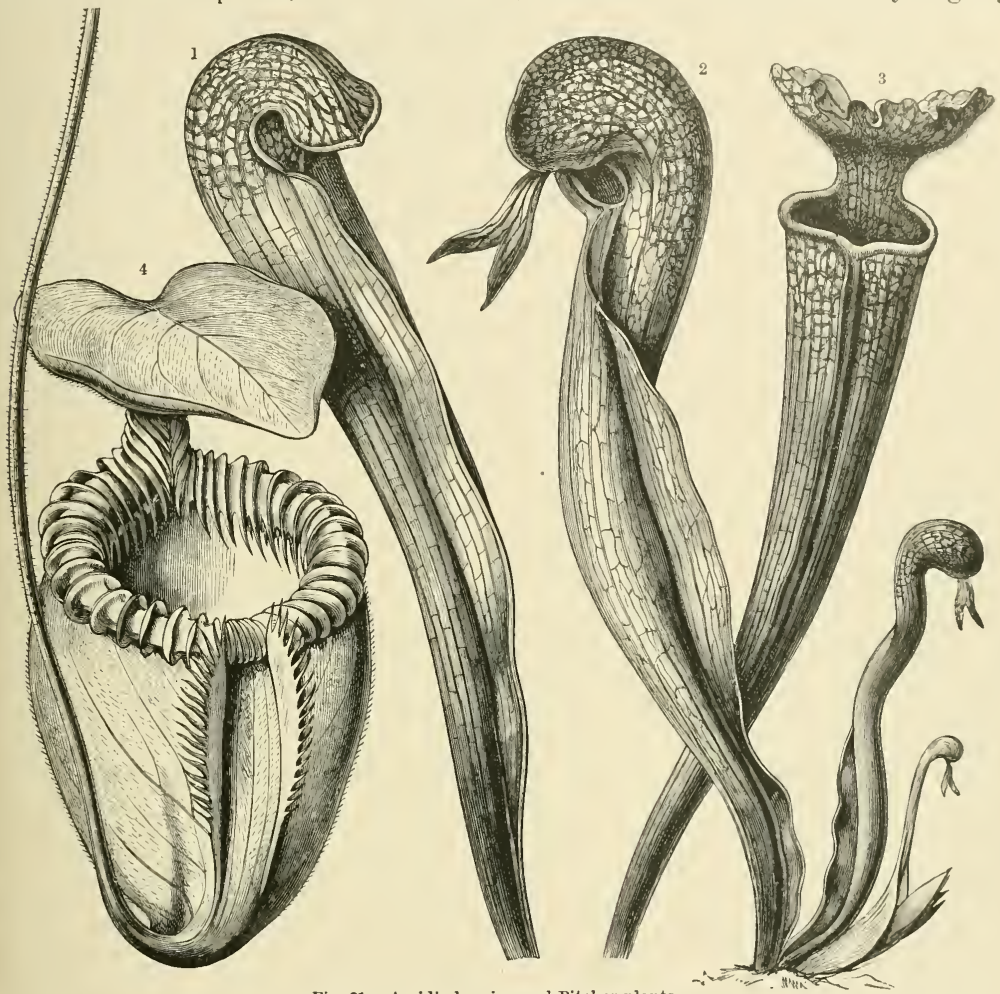

Fig. 21.-Ascidia-bearing and Pitcher-plants.

I Sarracenia variolaris. '2 Darlingtonia Californica. ' Sarracenia laciniata. \& Nepenthes villosa, reduced to one-half natnral size.

enlarged towards the top. The dorsal side of each leaf is, however, at its upper end hollowed out like a cowl or a helmet, and forms a cupola as is shown in fig. $21^{1}$ and $21^{2}$. The orifice or entrance into the utricle is consequently covered over and is reduced to a slit or hole under the hood. The lamina is transformed into a lobe, which in Sarracenia variolaris is small and roofs over the orifice of the utricle, and in Darlingtonia is shaped like the tail of a fish, and hangs down in front of the aperture. The lower part of the utricle is of a uniform green colour, but the upper part (i.e. the cupola and lobe-like appendage) has red ribs and veins, and here and there is quite purple. Between the veins the 
leaf is thin, translucent, and pale-green or whitish; and these clear translucent patches, framed by purple or green ribs, look as if they were little windows, especially when seen from within the utricle. The mixture of green, red, and white gives the upper parts of the leaves such a gay appearance that, from a distance, they might be mistaken for flowers.

Insects are doubtless attracted by these bright colours, and both round the orifice, and on the inner surface of the cupola, they find exudations of honey which they suck or lick up with avidity. In Sarracenia variolaris, honey is to be seen besides, on the edge of a broad free border which is decurrent along the utricle, and extends from the ground to the orifice. This border forms a favourite path for wingless insects, especially ants, which are particularly eager in their quest for honey. For them it is a sure way to destruction, for when they, gradually following the honey-baited pathway, arrive at the orifice to the utricle and pass through it, they inevitably get upon the smooth decurved points of the epidermal cells, constructed just like those in Sarracenia purpurea, and then, unable to stop themselves, slip down to the bottom of the pitcher. When small winged insects alight from flying and fall down the slide into the interior, they make use of their wings in the hope of saving themselves, but they never succeed in finding the aperture by which they entered, as it slants downwards and is situated in shadow. They invariably try to escape through the cupola, mistaking the thin portions, through which the light penetrates into the interior, for gaps permitting egress. But just as flies in rooms dash against the windows hoping to pass through them into the open air, so the small insects in the utricles of Sarracenia variolaris and Darlingtonia Californica knock against these windowed cupolas, in their desire to save themselves by flying through. They always fall down again to the bottom of the utricle as though into a cistern. If they are immersed in the liquid there secreted, or only in partial contact with it, they are stupefied, but not immediately killed. They often live incarcerated for two days, and it would therefore be erroneous to suppose that the fluid in the pitchers acts on the prey as a deadly poison. But it assists the decay and dissolution of the eaptives as they die of starvation and suffocation, and, as in the case of the utricle-plants previously described, a brown liquor of very unpleasant odour is produced, and there is a residue of solid pieces of skeleton difficult to decompose, such as the wing-cases, claws, and thoraces of various beetles, lice, ants, and other small insects which have shared the same unlucky fate.

The number of animals captured is very considerable. The pitchers of Surracenia variolaris, which attain to a length of $30 \mathrm{~cm}$., are usually found, when growing in their natural habitat, filled to a height of from 8 to $10 \mathrm{~cm}$. with animal remains, and even a heap $15 \mathrm{~cm}$. high has been observed. We must here remark that in the ascidia of Sarracenia variolaris, wingless insects, which creep about the earth, are found to predominate, whilst in Darlingtonia, on the contrary, most of the insects are winged. The cause of this is easily understood. The former plant has honey exuding on the flap or ridge running down from the orifice to the 
ground, and many wingless insects are thus induced to elimb up the alluring path and to enter the cavity of the pitcher. Darlingtonia, on the other hand, is destitute of honey on its decurrent ridge, and only provides the sweet meal at the top in the vicinity of the orifice, where it is available for flying insects, which, as a rule, only visit nectar-secreting flowers. The purplish-red scale, shaped like a fish's tail, and hung out like the sign-board of an inn in front of the entrance to the pitcher, constitutes an instrument for the attraction, from afar, of these winged creatures, which are endowed with a vivid sense of colour; and, as experience shows, it does not fail in its object.

What significance is to be attributed to the spiral torsion of Darlingtonia leaves (see fig. $21^{2}$ ) it is difficult to say. Perhaps the escape of animals once imprisoned in the depths of a pitfall is hereby rendered more remote. It would at all events be much more difficult for an insect trying to escape by the use of its wings to ascend a canal which, in addition to being lined with decurved points, was spirally wound, than a similar canal, straight and widened towards the top. We must not omit to mention that a few flies and a small moth have selected as their ordinary habitat the pitchers of both the plants just described, in spite of their being so fatal to most insects. The grubs of a blow-fly (Sarcophaga Sarracenice), in particular, live in large numbers amidst the heaps of decaying insect bodies at the bottom of the pitchers, and are there nourished just as are the grubs of allied species in the rotten flesh of birds and mammals. When mature, the grubs quit the environment of dead remains, passing through holes which they bore in the side wall of the pitcher, and turn into chrysalises in the earth. But the fly itself can without danger pass in and out of the pitfalls, which are so perilous in the case of other insects, and it is enabled to do this by means of the special structure of its feet. On the last joint of each foot it has a long claw and sole-like attachment-lobe, and it is able to push these appendages between the sharp, slippery, decurved hairs lining the inner surface of the pitcher, and so to hook itself to the deeper strata of the wall. This apparatus may be likened to the grapple-like climbing irons of Tyrolese mountaineers, and, thus armed, the fly is in a position to ascend the inner wall of a pitcher unscaleable by other insects. The case of the small moth Xanthoptera semicrocea is similar. The tibiæ of this insect are armed with long, sharp spurs, one pair on each of the two middle legs, and two pairs on each of the two hindermost legs; and, by the help of these spurs it likewise is able to tread uninjured over the dangerous surface of the wall. Its caterpillars, too, cover the sharp slippery hairs with a web, and so render them harmless.

The presence of these animals in the death-traps of Sarracenias is of special interest, inasmuch as it shows that the animals which perish at the bottom of the pitchers are not exactly digested. If maggoty flesh enters the stomach of a carnivorous animal, not only the flesh itself but the maggots as well (which, indeed, immediately die on reaching the stomach) are speedily dissolved by the action of the gastric juice. Such is also the case with several animal-capturing plants to be described in the next pages. But the fluid secreted in the pitchers of Darlingtonia VOL. I. 
and Sarracenia variolaris cannot exercise this digestive action, for if it did the maggots in the heap of rotting insects could not remain alive and well. Its action is limited to the promotion of decay and the formation of a foul liquor, in other words, a liquid manure, which is absorbed as nutriment by the epidermal cells at the bottom of the pitchers.

Another series of pitcher-plants comprises forms in which the petioles are converted into symmetrical sacs with apertures at the top, and the laminæ spread out over them like lids for protection. Most frequently the pitfalls in plants of this kind are shaped like pitchers, jars, urns, cups, or funnels; and the lid over the orifice of each cavity is, for the most part, so placed as to prevent rain-drops from falling in, but not to hinder in any way the entrance of animals. In this series are included, firstly, a few species of Sarracenia, viz. Sarracenia Drummondii and S. undulata, next, the Australian Cephalotus follicularis, and lastly, the numerous species of the genus Nepenthes, which are designated by gardeners by the name of "pitcher-plants" in the narrow sense.

The leaves in both the Sarracenias just named are heteromorphic. Some of them have acute linear-lanceolate petioles of a uniform green colour, and not hollowed out; and it is only in the case of from three to five leaves in each individual plant that the petioles are transformed into tubes with infundibuliform enlargements at the top. The rim round the mouth of the funnel is somewhat swollen and doubled down externally; but above the orifice the lamina is arched so as to form a cover to the pitcher. The margin of the leaf of Sarracenia laciniata, which is shown in fig. $21^{3}$, is crinkled and sinuously folded. The cover and also the upper funnel-shaped enlargement of the pitcher are very conspicuous on account of the contrast of the colours displayed upon them. The green of the lower part of the pitcher gets paler and paler above, and merges into a pure white, whilst dark-red veins stand out from the green and white ground tints, having the effect of a net-work of bloodvessels. At the mouth of the pitcher, and on the under side of the lid, honey is secreted in such abundance that little drops of it are not infrequently to be seen on the swollen rim, and some oozes down into the infundibuliform portion of the pitcher. But at the very spots where the honey occurs there are also innumerable smooth conical cells with their solid apices directed downwards; and these cells become longer the lower their position in the pitcher. When insects, attracted by the gay-coloured lid, and lured on by the honey, come to the mouth of the pitcher and tread upon the parts covered with the sharp slippery papillæ, they are drawn into the depths as though by an invisible power. After they have once alighted on the perilous area, every movement and every effort to climb up against the points causes them to slide further and further down towards the bottom of the pitcher, where they are hopelessly lost, being killed within a short time and ultimately decomposed.

An instance of an exactly similar kind is afforded by Cephalotus follicularis, which has long been known as a plant native on moorlands in eastern Australia. It is allied to saxifrages and currants, and is represented on a scale of half the 
natural size in fig. 22. This Cephalotus also has two kinds of leaves, which are closely crowded in a rosette round the erect flower-stalk. Only the lower leaves of the rosette are transformed into traps for animals, and these are pre-eminently adapted for wingless creatures creeping upon the earth. The tankard-shaped traps all rest on the damp earth, and are furnished externally with borders or winged ridges, which facilitate the ascent of crawling animals to the mouth of the tankard. Flying insects are of course not excluded, and here again they are made aware from afar of the feast of honey provided by the presence of bright colours. The half-open lid is very prettily adorned with white patches and brilliant purple veins, and at a distance is readily mistaken for a flower.

When small animals, whether with or without wings, approach to take the honey, they are so eager in their search that they get upon the inner surface of the mouth of the tankard-pitcher, which, though fluted, is also very smooth and slippery, and thence they easily slide into the interior of the cavity. The pitchers being half-full of liquid, most of the unlucky creatures die there in a short time by drowning. But even if this were not the case, they would never succeed in working their way up to the light of day. For every animal that wishes to save itself from a Cephalotus pitcher has three obstacles to overcome: first, a circular ridge projecting inside the pitcher; secondly, a bit of wall thickly covered with

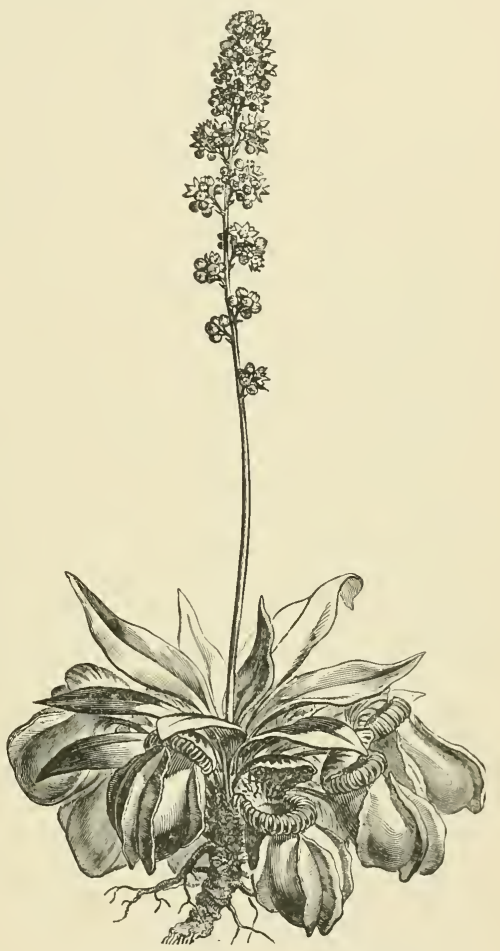

Fig. 22,-Cephalotus follicularis.

little papillæ, sharp, ridged, and pointed downward, the whole being comparable to a flax-comb; and, lastly, on the involute rim round the mouth of the pitcher, another fringe composed of hooked, decurved spines which bristle like an impenetrable row of bayonets in front of such animals as may have surmounted the other difficulties. The abundance of the booty found at the bottom of Ceplaclotus pitchers shows how efficiently these contrivances serve to prevent escape. Ants, for instance, sacrifice themselves recklessly in their pursuit of honey, and one often finds great numbers of them drowned in the liquid in the pitchers. The prey is not in this case converted into a putrid liquor, but is partially dissolved by a secretion having an acid reaction. This secretion is separated out by special 
glandular cells situated on the lining of the pitcher; and the whole process, wherein they are concerned, corresponds to that which obtains in the pitchers of Nepenthes, and which will be more thoroughly discussed in the case of these latter plants.

The species of the genus Nepenthes, of which we know at the present time thirty-six, are all confined to the tropics. Their area of distribution extends from New Caledonia and New Guinea over tropical Australia to the Seychelles Islands and Madagasear, and over the Sunda Islands, the Philippines, Ceylon, Bengal, and Cochin-China. They only flourish on marshy ground on the margin of small collections of water in damp primeval forests. There the seeds germinate in shallow water. The young plants (see fig. 23), which spring from the boggy

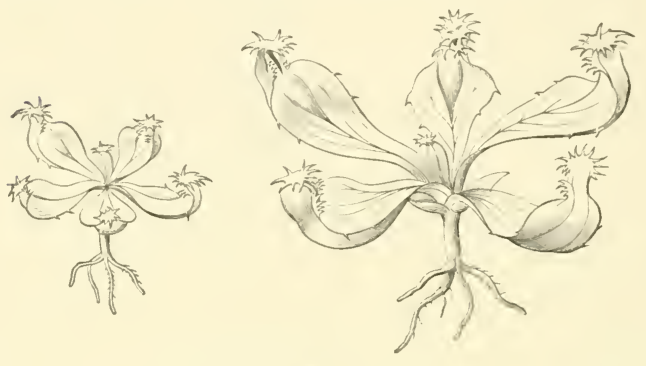

Fig. 23.-Young Nepenthes plants. ground, have their leaves arranged in rosettes just like those of Sarracenias (see fig. 20). They are, too, so nearly identical in form with the latter that anyone seeing a young Nepenthes plant for the first time, and not knowing the history of its development, would take it for a Sarracenia. The leaves, succeeding the cotyledons and forming a circle above them, rest their lower portions upon the mud, but their upper parts are curved upwards, and each carries at its extremity a scale resembling a cock's comb, which is, strict speaking, the lamina. This seale roofs over a slit-like aperture, the entrance to a cavity within the swollen petiole. In addition a green lobe with a few coarse projecting points is to be seen on either side of the orifice.

Altogether different from the rosettes of young Nepenthes plants are the foliar structures clothing the stems which subsequently arise from the rosettes (see fig. 24). In these leaves the lower part of the petiole is winged and flat, has a linear or lanceolate outline, and resembles the leaf-blade of Draccena; its functions, too, are those of a green lamina. This expanded section of the leaf-stalk passes next into a part which is terete and coiled like a snake, and acts as a tendril. Every stem or branch belonging to a plant, whether living or dead, with which this part of the petiole comes into contact, is seized and encircled by it; and the third portion of the petiole, i.e. the pitcher, being situated at the extremity of this clasping portion, is thus slung upon the branch of some other plant growing at the edge of a pool of water. Meanwhile the Nepenthes plant rises higher and higher above the wet soil where its seeds germinated and the young rosette rested, becomes entangled with the ramifications of the underwood and with prostrate branches of trees of the primeval forest; in a word, with everything available as a support, and so not infrequently climbs, as a true liane, to the tops of trees of moderate height.

The pitcher must be looked upon as an excavated portion of the petiole, and 


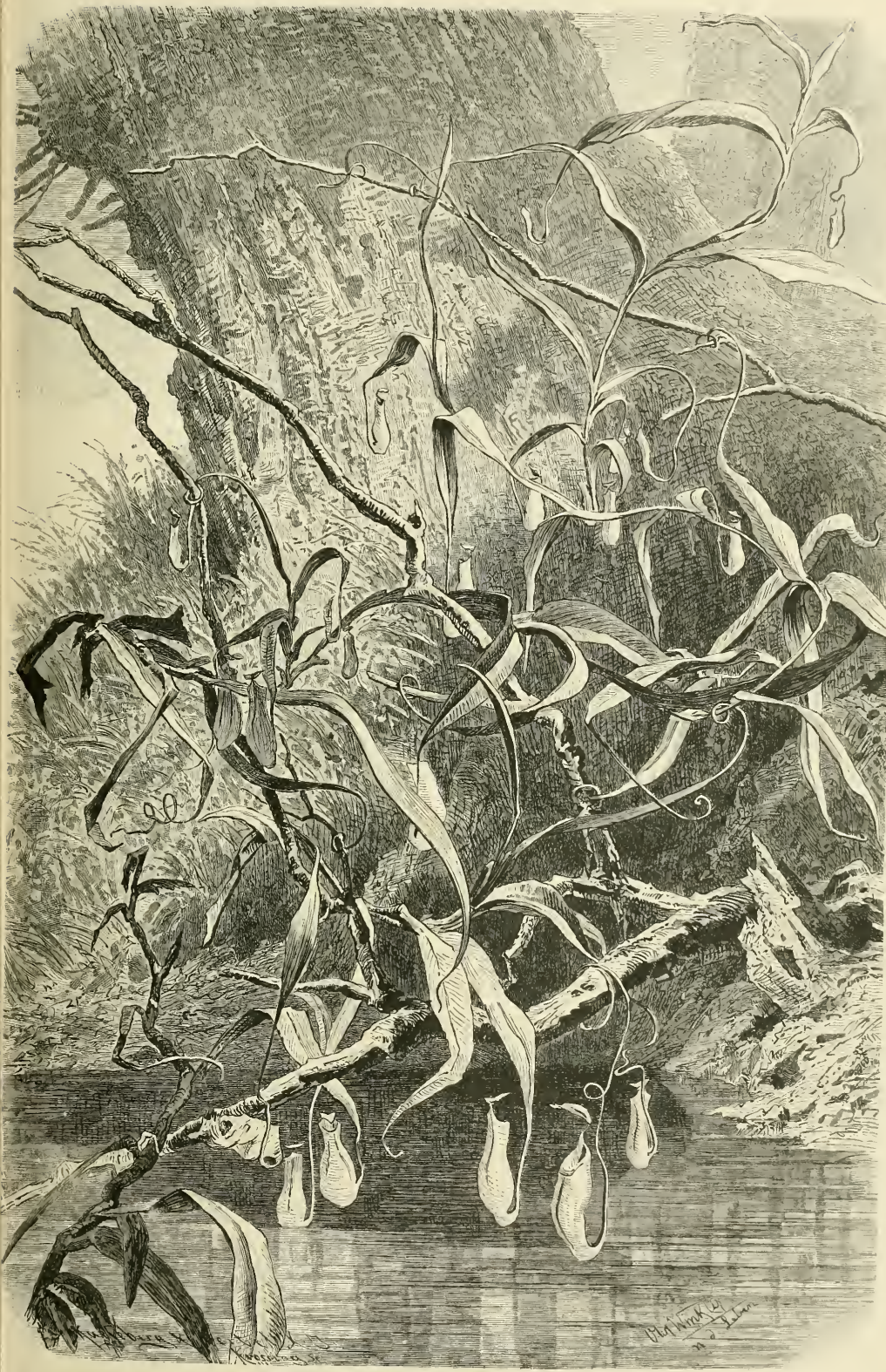

Fig. 24.-Nepenthes destillatoria. 
what appears to be the lid of the pitcher is the lamina, as it is in Cephalotus and the Sarracenias. In this case also the lamina seems to be but little developed in comparison with the wonderfully metamorphosed petiole. In the majority of the species of Nepenthes, the mature pitchers are from $10 \mathrm{~cm}$. to $15 \mathrm{~cm}$. in height. In the graceful Nepenthes ampullaria they are only from $4 \mathrm{~cm}$. to $6 \mathrm{~cm}$. high; but, on the other hand, in the species indigenous to the primeval forests of Borneo they reach a height of $30 \mathrm{~cm}$. or even more. The pitchers of Nepenthes Rajah have a height of $50 \mathrm{~cm}$., and their orifices are $10 \mathrm{~cm}$. in diameter, whilst below the orifice they expand to $16 \mathrm{~cm}$; so that if a pigeon were to $\mathrm{fly}$ into a pitcher of this kind it would be completely hidden in it. Immature pitchers are still closed by their covers. Often they are hairy outside; and, according to the colour and lustre of the hairs, they may be rusty in tone or glittering like gold; not rarely they look as if they were powdered with flour (e.g. N. albo-marginata), and sometimes are even snow-white. Subsequently the lid is raised, and the downy coat disappears either partially or entirely. Having thus become glabrous, the pitchers display a yellowishgreen ground colour, for the most part flecked and veined with purple; and many are of a bluish, violet, or rose tint near the orifice, or dark-red as though saturated with blood. The lid is similarly gaily coloured; and the variety of the tints is increased by the fact that a pale-blue zone is visible in the interior, beneath the swollen involute rim of the opening, which is itself brownish, yellowish, or orangered. Gaily-coloured pitchers of this kind look at a distance just like flowers, and remind one, in particular, of the most brilliant floral forms of the liane-like Aristolochias indigenous to tropical forests. This fact is the more noteworthy, because the genus Nepenthes is closely allied to the genus Aristolochia in respect of systematic relations.

The bright pitchers of Nepenthes, visible from afar, are sought, just as flowers are, by insects, and probably by other winged creatures as well; and this occurs all the more because there is a copious secretion of honey by the epidermal cells upon the under surface of the lid, and on the rim round the mouth of each pitcher. The swollen and often delicately-fluted rim, in particular, drips and glitters with the sugary juice; and it would be permissible in this connection to speak of a honeyed mouth and sweet lips in the most literal sense of the words. Animals which suck honey from the lips of Nepenthes pitchers wander, as they do so, only too readily upon the interior surface of the orifice. But the inner face is smooth and precipitous, and rendered so slippery by a bluish coating of wax that not a few of the alighted guests slip down to the bottom of the pitcher and fall into the liquid there collected. Many of them perish in a short time; others try to save themselves by climbing up the internal face of the pitcher, but they always slip again on the polished, wax-coated zone, and tumble back once more to the bottom. In large pitchers the involute rim of the aperture is in addition armed with sharp teeth, which are pointed downwards and bristle in front of such of the unlucky victims in the pitfall as try to emerge (see fig. $19^{3}$ ). In a number of species (N. Rafflesiana, N. echinostoma, N. Rajah, N. Edwardsiana, and N. Veitchii, all 
natives of Borneo) this fringe of sharp teeth looks like the set of teeth of a beast of prey; and in Nepenthes villosa, of which a pitcher is represented in fig. $21^{4}$, a double row of bigger and smaller teeth directed towards the bottom of the pitcher is developed, and renders the escape of prey, once caught in the trap, impossible.

Most of the creatures that fall into the pitchers are, however, speedily drowned in the large quantity of liquid at the bottom. For a third part or even a half of the cavity is filled with liquid. This liquid originates from special gland-cells on the inner surface of the pitcher, consists mainly of water, and so long as there are no animals in the pitfall, gives only a very weak acid reaction. But as soon as the body of an animal reaches the bottom, more fluid is secreted. This has a distinctly acid taste, possesses the power of dissolving albuminous substances, such as flesh and coagulated blood, and corresponds, not only in respect of this action but also in chemical composition, to the gastric juice. For, in addition to organic acids (malic, citric, and formic acids), an organic body like pepsin has been detected in it, and nitrogenous organic compounds have been brought into solution in it artificially as well. If the liquid from a Nepenthes pitcher, which has not yet captured any animal, is poured into a glass vessel containing a small piece of meat, the flesh is at first but little affected; but, if a few drops of formic acid are added, the flesh is dissolved and undergoes the very same changes as it does in the stomach of a mammal. The process going on in the pitchers of Nepenthes when animals fall into them is therefore not only analogous to digestion, but may be properly designated digestion.

The digested portions of the bodies are afterwards absorbed by special cells at the bottom, and on the lower parts of the lining wall of the Nepenthes pitchers.

Another series of plants was at one time regarded as belonging to our present section of carnivorous plants. These include forms possessing subterranean stem structures, bearing hollow, scale-like leaves, or leaves so arranged that chink-like spaces exist between them. Into these chambers or spaces it was supposed that minute animals, Infusoria, Rhizopods, Aphides, and the like found their way, and that here they met their death, their bodies being digested through the agency of peculiar glands which line the walls of these chinks and spaces. Though this view of the carnivorous function of these subterranean organs has failed to become established on a solid basis of fact, the plants in question are of considerable interest and may be conveniently treated here.

One of the most remarkable of the plants belonging to this group is the Toothwort (Lathrcea Squamaria), of which we shall repeatedly have occasion to speak. It is nearly allied to the Yellow-Rattle and Cow-wheat, but it is destitute of chlorophyll, and lives underground, parasitic on the roots of arborescent Angiosperms, except during a brief period annually when it sends up above-ground a few short shoots covered with flowers. The subterranean stems are white, have a fleshy, solid, and elastic appearance, and are covered throughout their entire length with thick squamous leaves placed closely one above the other (see fig. $25^{1}$ and fig. 37). In colour and consistence these leaves are like the stem; in outline they 
are broadly cordate, and they give the impression of being mounted fairly and squarely upon the stem by means of the highly swollen and notehed basal portion. But it is only necessary to detach one of the seales from the stem to convince one's self that this is not the case, and that the part taken at first sight to be the underside or back of the leaf is only a portion of the superior surface. In reality each of these thick squamiform leaves is rolled back, and in it the following parts may be distinguished: first, the place of insertion on the stem (fig. $25^{3}$ ), which is relatively small; secondly, the portion taken on cursory examination to be the whole upper surface of the leaf, and consisting of an obliquely ascending blade limited by a sharp border; next, starting from this sharp border, the part which, owing to its being suddenly bent down at an acute angle and falling away steeply, is usually taken for the dorsal or inferior surface of the leaf, but which belongs, in point of fact, to the front of the lamina; fourthly, the free extremity of the leaf in the form of an involute limb; and fifthly, the true dorsal part, which is very small relatively and is not visible until the involute tip is removed. Owing to the involution of the apex, a canal or rather a recess is formed and runs across beneath the leaf, close under the place where the latter is joined to the stem (see fig. $25^{2}$ ). From five to thirteen (usually ten) chambers open into these recesses through a series of little holes. They are excavations in the thickness of the scales and are probably, in this form at any rate, unique in the realm of plants. These extraordinary chambers must be described as deep excavations in the foliar substance proceeding from the back of the leaf. With a view to elucidating their function in relation to the life of the plant, their structure must be more particularly considered.

The chambers radiate as it were from the orifice at the base of the leaf. Though closely adjoining one another, they are not in lateral connection by means of passages or canals. Their walls are irregular and undulating (see fig. $25^{3}$ ), and are characterized by the peculiar structures which are borne on the lining-raised up above the ordinary epidermal cells and projecting into the cavity. These structures, of two sorts, are shown in fig. $25^{4}$, under a considerable magnification. One sort, and these are by far the more numerous, are of the nature of short capitate hairs. The head is formed of a pair of cells, and they are supported on a short cylindrical cell which serves as a stalk. The other sort is sparsely scattered amongst these capitate hairs. They are oval in outline and but slightly raised above the ordinary epidermal cells. Each consists of a tabular cell upon which rests a slightly convex cushion composed of not more than four cells all lying in the same plane. One such sessile gland is shown in the centre of fig. $25^{4}$. In this case the cushion consists of three cells. A further peculiarity has been observed in these sessile glands. The summit of each is marked by a tiny pore (not shown in the figure), an actual hole in the wall at the geometrical centre of the convex surface.

In the wall of the chamber, just below the lining epidermis, run the vascular strands (fig. $25^{3}$ ). The vessels of which they are composed form a considerable plexus or net-work in this region. Now it is known that the ground in which 
Lathroea passes its existence is often drenched through with moisture in the immediate neighbourhood of the plant. A water-excreting function has long been attributed to the chambered leaves of the rhizomes. That such is the case has been demonstrated by forcing water under pressure through the cut ends of the rhizomes, when streams gush forth from the basal orifices of the leaves. In this

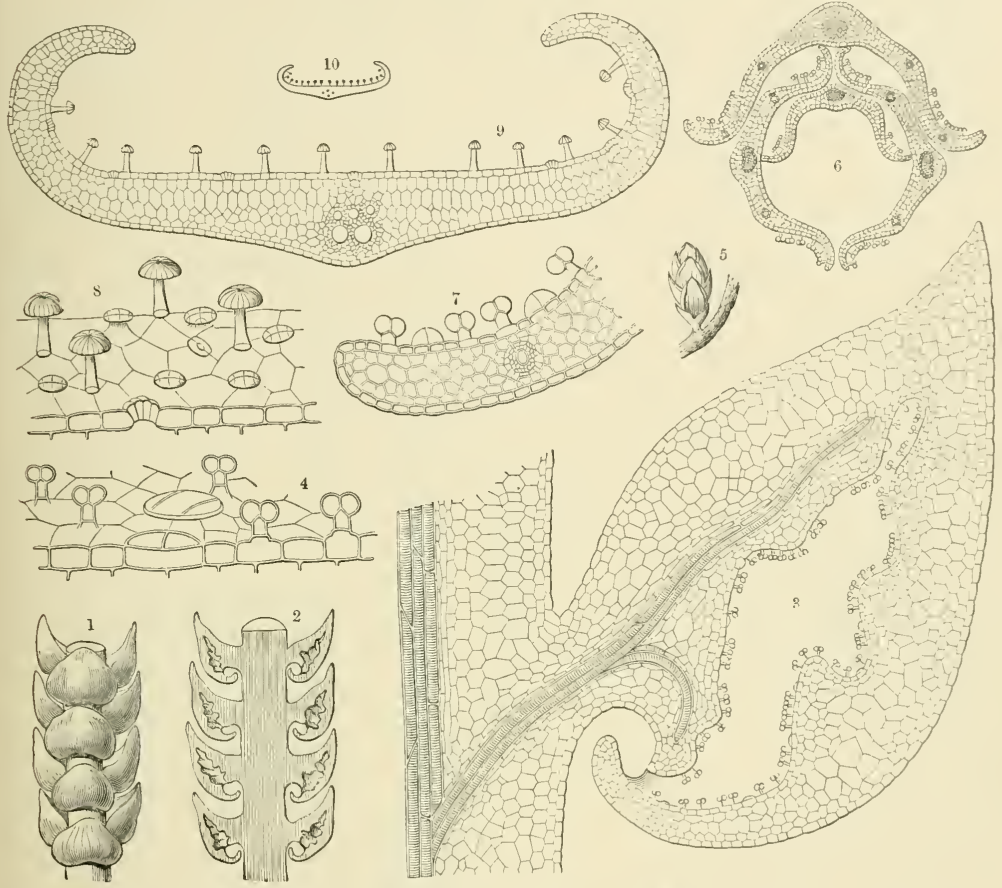

Fig. 25.-Glandular structures in the Toothwort, Bartsia, and Butterwort.

1 Piece of an underground leaf-shoot of the Toothwort. ${ }^{2}$ Longitudinal section through the same; $\times 2 .{ }^{3}$ Longitudinal section throngh one of these underground leaves; $\times 60$. ${ }^{4}$ Piece of the wall of a cavity; $\times 200 .{ }^{5}$ Subterranean bul of bartsio: natural size. 6 Cross-section through part of this bud; $\times 60$. 7 The margin of a bud-scale in section; $\times 200$. 8 Piece of the epidermis of a leaf of Butterwort; $\times 180 .{ }^{9}$ Transverse sectiou through the leaf of a Butterwort (Pinguicula alpina); $\times 50$. ${ }_{10}$ Transverse section through Butterwort leaf; natural size.

instance it is uncertain whether the stalked or the cushion glands assist in this excretion, though from the minute details of their structure it would seem probable that it is the latter. On any other hypothesis it is difficult to understand the meaning of the pore on the summit. The matter has, however, been placed beyond doubt by experiments on other allied plants, as, for instance, the Lousewort (Pedicularis palustris), in which the glands are more easily kept under observation. We have apparently in these gland-bearing chambers of Lathrcea a water-excreting mechanism for the elimination of the surplus moisture, which in most plants is transpired or evaporated into the air. Lathroea being almost wholly subterranean 
is unable to do this, as the air in the chinks and crannies of its matrix of soil is generally saturated. The water is therefore excreted in liquid form by a special mechanism.

This view of the function of the scales is confirmed by reference to other allied types with subterranean scales. An instance in point is afforded by Bartsia alpina. This remarkable plant is distributed in the Arctic region and amongst the high mountain flora throughout almost the whole of Europe, and is very striking owing to the colour of its foliage being a mixture of black, violet, and green. The flower; too, is of a sombre dark-violet hue, and the entire plant, by reason of this peculiar colouring, gives a truly funereal impression. We may remark incidentally that the name Bartsia was chosen by Linnæus for this sad-hued plant as an expression of his own grief at the death of the zealous naturalist and physician, Bartsch, who was his intimate friend, and who succumbed at a comparatively early age to the climate of Guiana. Damp black earth in the neighbourhood of springs constitutes the favourite habitat of these plants. Upon digging in summer time down to their roots, one sees that a few suckers proceed from them, and fasten upon the sedges and other plants growing in the vicinity; but one also discovers subterranean shoots having "root-hairs" developed near the nodes, at which are inserted the paired white scales; and these "root-hairs" have the function of absorption-cells. Towards the autumn, oval buds, likewise subterranean, are matured, in form not unlike horse-chestnut buds (see fig. $25^{5}$ ), and composed of etiolated scales arranged in four rows and overlapping one another like tiles, so that only the back of the upper part of each scale is visible, the lower part being covered by the scale next beneath it.

On the visible part of each scale's convex under surface three sharply projecting ribs are noticeable near the middle, whilst the two margins are rolled back so as to form a recess in each case. But, as may be seen in the cross-section of a Burtsia bud (see fig. $25^{6}$ ), one pair of scales lies over the next higher pair in such a way as to convert the recesses into ducts. Owing to this construction the interior of the bud is perforated by twice as many ducts as there are covered leaf-scales, and the orifices of each pair of ducts occur at the spots where the evolute margins of one scale begin to be covered by the middle of the next lower scale. On one wall of the ducts, i.e. in the recesses, structures like those which occur in the cavities of Latlercea are developed, i.e. stalked glands, each composed of two cells borne upon a basal cell; secondly, pairs of hemispherical domed cells; and, lastly, ordinary flat epidermal cells (see fig. $25^{7}$ ). There can be little doubt that the whole apparatus acts in the same way as in Lathrcea, and is adapted to the excretion of water. The cavities and spaces between the scales of the buds serve the same purpose as the chambers in the leaves of Lathrcea, viz., that of affording cover to the delicate excretory glands and of protecting them from immediate contact with the soil.

Mechanisms of this sort are not restricted to subterranean organs, but are found likewise on the aerial leaves of many plants. Indeed such arrangements, supplementing ordinary transpiration, are common, especially amongst tropical plants. 


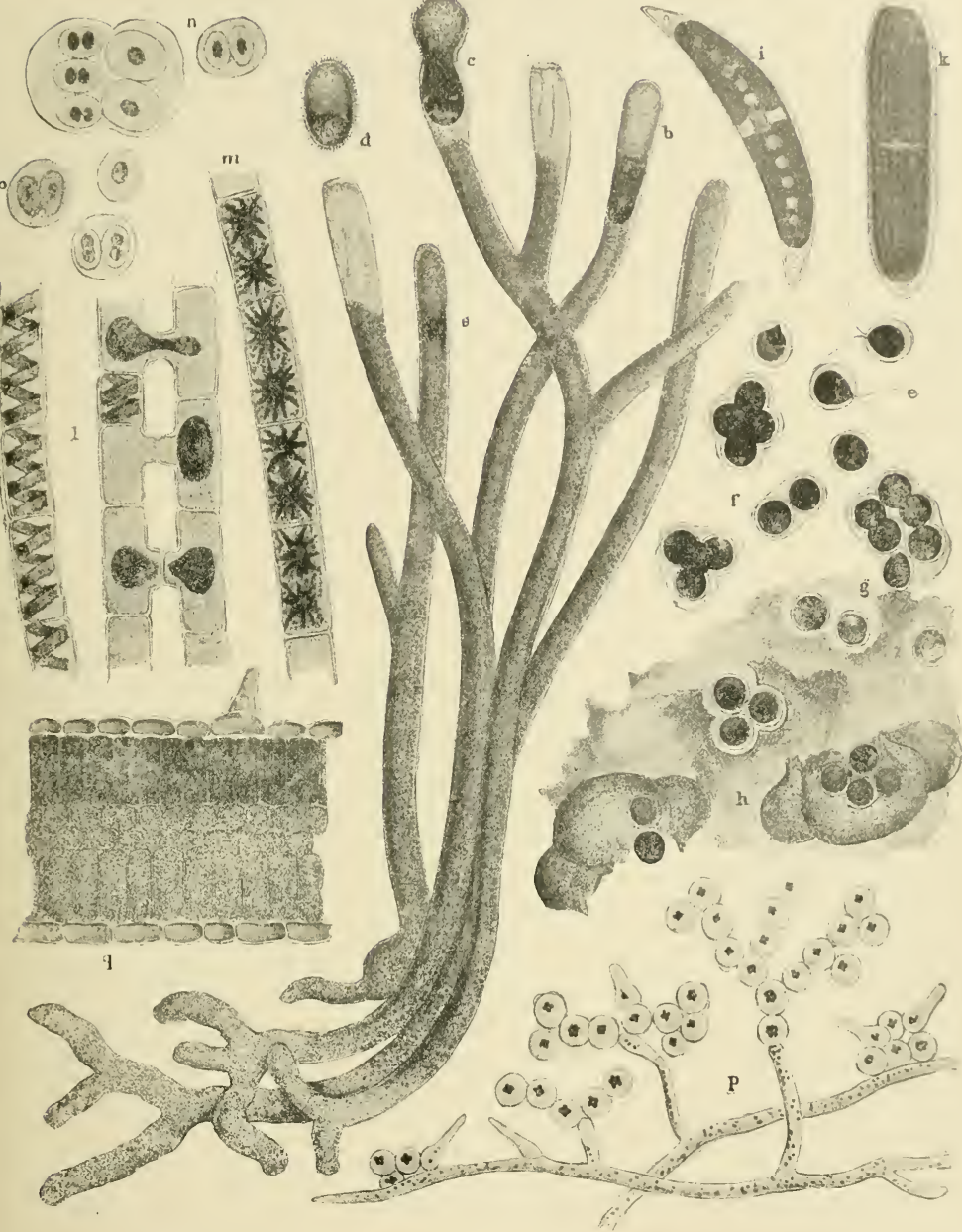

Fig. 25A.-Swarmspores, Zygospores, and Chlorophyll-bodies.

a-d, Development of Swarmspores in the tubular cells of Vaucheria clavata. e-I, Swarmspores and Resting-cells of "redsnow" (Sphaerella nivalis), mixed with pollen-grains of Pines. i-k, Forms of Chlorophyll in cells of Desmidiez (i, Closterium Leibleinii; k, Penium interruptum). 1, Formation of Zygospores and spiral arrangement of Chlorophyll-bodies in cells of Spirogyra arcta. $\mathrm{m}$, Star-shaped Chlorophyll-bodies in cells of Zugnema pectinatum. n-o, Gloeocapsa sanguinea. p, Protonema of Schistostega osmundacea. q, Transverse section of the foliage-leaf of Satureja hortensis. All figs. enlarged.

Restricting ourselves to a consideration of other members of the family Scrophulariaceæ allied to Lathraa and Bartsia, we find in the Lousewort (Pediculuris.) 
a similar mechanism. Here the glands occur on the under surface of the aerial leaves, the cushion glands being by far the most numerous. Shoots of this plant if injected with water at the cut end, readily pass it out by their leaves, and in particular by those portions which abound in cushion glands. As the water percolates through these areas it gushes from the leaves with great rapidity. The younger leaves drip with moisture and water drops from the leaf-tips and wells up in the leaf-axils, running in cascades down the stem.

Somewhat similar is the Yellow-Rattle (Rhinanthus Christa-galli). Here, too, under pressure, water is forced from the leaves, but less rapidly than in the last instance. It is thus possible to observe its excretion from the edges of the under surfaces of the leaves, to see the water drawn round by capillarity on to the upper surface, whence it runs down the vein furrows, as in irrigation canals, to the base of the leaf.

In these and other cases like them we are dealing with plants which live in moist or even marshy situations. When this is understood, it is not surprising that they should exhibit supplementary mechanisms for eliminating their excess of water.

\section{CARNIVOROUS PLANTS WHICH EXHIBIT MOVEMENTS IN THE CAPTURE} OF PREY.

We have taken Nepenthes, Sarracenia, and other forms as types of that section of carnivorous plants which manifest no external visible movement in the pitfalls for the purpose of capture or digestion. The second section, now to be discussed, includes plants in which movements of the leaves, or parts of leaves, modified as organs of seizure and digestion, take place as a result of the contact of animal bodies-movements which have the common object of bringing about the digestion of the animals, whilst the retention of the latter is effected in very various ways.

Whilst in the forms hitherto considered the mechanism of capture is wholly passive, the plant with its pitfall attractively coloured or cunningly baited with honey merely awaiting the moment when the insect slips on the treacherous surface, in those which we are now about to review, a series of movements simple or complex is set up by the stimulus received when the insect alights. In some cases the whole leaf suddenly changes its form, going off like a rat-trap, in others it is merely the digestive tentacles which change their position. In general, when the movement is slow the organ is sticky, but when instantaneous, adhesiveness is not met with.

The first group of carnivorous plants which perform movements for the capture of prey is composed of the various species of the genus Pinguicula (Butterwort). Of this stock nearly forty species are known; and they are all much alike. Scarcely any difference would be detected by an ordinary person between Pinguicula calyptrata from the mountains of New Granada and Pinguicula vulgaris from our own hills. In respect of habitat, too, they exhibit close conformity. In both the Old World and the New they only thrive on damp spots, the neighbourhood of 
springs, banks of brooks, moorlands, and black peat-bogs. In the equatorial zone they have retired into the cool regions of the higher mountains. The mountain ranges of Mexico are particularly rich in species of Pinguicula, but all the forms existing there occupy a circumscribed area. Southern and western Europe also harbour a few native species whose area of distribution is surprisingly limited. The species occurring in the arctic and sub-arctic zones are, on the contrary, exceedingly widely distributed. One species has been found in antarctic regions at the Straits of Magellan.

The species best known and most available for study is Pinguicula vulgaris. The area of its distribution extends over the whole of the aretic and sub-arctic regions, over the part of North America which lies to the north of the Mackenzie River, over Labrador, Greenland, Iceland, and Lapland, throughout Siberia down to the Baikal Mountains, and through Europe to the Balkans, Southern Alps, and Pyrenees. This graceful plant, generally referred to the family Lentibulariaceæ, is nearly allied to the group of scrophulariaceous genera of our last section. It has bilabiate flowers of a violet-blue colour, with palates covered with velvety-white hairs, and with a sharp spur at the back. The flowers are borne singly on slender stalks which rear themselves in an elegant curve from the centre of a rosette of leaves that rests upon the ground. The leaves of the rosette in Pinguicula vulgaris, as in all other species of Butterwort, are oblong-ovate or ligulate and of a yellowish-green colour, and rest their under-surfaces upon the wet ground, whilst their upper faces are exposed to the sky and rain. Owing to the lateral margins being somewhat upturned, each leaf is converted into a broad flatbottomed trough (cf. the section taken right across a leaf in fig. $25^{10}$ and $2.5^{11}$ ). The trough is covered with a colourless sticky mucilage which is secreted by glands distributed in large numbers over the entire upper surface of the leaf.

The glands are of two kinds. One variety is distinguishable by the naked eye as consisting of a stalked head, and looks under the microscope like a tiny mushroom (see fig. $25^{9}$ ). Its parts are a swollen disc composed of from eight to sixteen cells grouped radially, and a stalk, consisting of an erect tubular cell supporting this disc. A gland of the other sort is made up of eight cells grouped in the form of a wart or knob supported by a very short stalk-cell, and only slightly raised above the surface of the leaf. For the rest, ordinary flat epidermal cells make up the epidermis, with here and there interspersed the guard-cells of stomata.

It has been calculated that there are 25,000 mucilage-secreting glands on a square centimeter of a butterwort leaf, and that a rosette composed of from six to nine leaves bears about half a million of them. Momentary contact, whether due to rapid brushing by a solid body or to the incidence of drops of rain, causes no kind of movement in them. The long-continued pressure of grains of sand or of solid insoluble bodies in general stimulates the glandular cells to an inconsiderable augmentation of the quantity of mucilage discharged, but does not cause secretion of any acid digestive fluid. But as soon as a nitrogenous organic body is brought into continuous contact with the glands, they are forthwith stimulated not only to 
a more profuse elimination of mucilage, but also to the secretion of an acid liquid, which has the power of dissolving all bodies of the kind, namely, such as clotted blood, milk, albumen, and even cartilage. It has been experimentally established (for example) that small solid bits of cartilage placed on a leaf of Pinguicula vulgaris, whose mucilage shows no sign of an acid reaction, cause, after ten or eleven hours, the secretion of an acid liquid, and after forty-eight hours are almost entirely dissolved by it. At the end of eighty-two hours the bits of cartilage used in the experiment were completely liquefied, the whole secretion was reabsorbed, and the glands had become dry. When small insects such as midges alight from flight on a leaf of Pinguicula they remain glued by the mucilage, and their struggles to extricate themselves only cause them to sink deeper into it. Thus they generally perish in a very short time, are digested by the acid juice poured from the glands in response to the stimulus, and are absorbed with the exception of the wings, claws, and other parts of the skeleton.

The acid liquid secreted by the glands is viscous, and when a number of glands are irritated it may exude so copiously as to fill the whole trough of the leaf. If the margin of the leaf alone is stimulated, as when a small creeping insect, or a midge alighting from above, gets upon the slightly up-curved margin of the leaf, not only do the marginal glands, which are comparatively infrequent, discharge their secretion, but in addition the edge curls over; the object of this movement being to cover, if possible, the prey whilst it is held fast by the sticky mucilage, or to push it into the middle of the flat channel, and so, in one way or another, to bring it into contact with as many glands as possible. The marginal glands alone could not produce the requisite quantity of acid liquid to effect solution, and, on this account, the glands on a wider area are summoned to assist in the manner described. The involution of the margin takes place very slowly; it is usually some hours before the animal sticking to the edge is enfolded, or, in the case of the larger specimens, is pushed into the middle of the leaf. After solution and absorption are accomplished, usually by the end of twenty-four hours, the leaf expands again, and its margins assume the position which they had before their involution.

Besides small insects, pieces of plants, such as spores and pollen-grains brought by the wind, not infrequently fall on the viscid surfaces of Pinguicula leaves. These are subjected to the same fate as animal organisms, their protoplasts being dissolved and absorbed like the flesh and blood of insects.

The action of the acid juice secreted by the glands of butterwort leaves upon albuminous bodies is identical with that of the gastric juice of animals. We may presume therefore that there are in it, as in the gastric juice, two kinds of substance: firstly, a free acid, and, secondly, a ferment completely analogous to pepsin in its action; for, as is well known, it is by means of this combination that the juice of the animal stomach effects the solution of albuminoid compounds. Inasmuch as the gland-cells of Pinguicula absorb all the soluble part of the prey, and re-absorb the solvent previously discharged by them, the action of this plant's leaves 
is exceedingly like that of the animal stomach, and the process may, as in the case of Nepenthes, be fairly regarded as digestion. Whether, in carrying out this process, the different forms of glands have also different functions, whether those of one kind serve principally to secrete and those of the other to absorb, or whether, perhaps, the one variety only discharges viscid mucilage to capture the prey, and the other only a liquid containing acid and pepsin, are questions not yet determined with certainty, although such a division of labour is in itself highly probable.

The similarity existing between the leaf of Pinguicula and the animal stomach in respect of their action on albuminous substances was turned to a practical application in dairy-farming long before the discovery of the relationship by men of science. The very same changes as are brought about in milk by the addition of the rennet from a calf's stomach can be induced by means of butterwort leaves. If fresh milk, warm from the cow, is poured over these leaves, a peculiar tough mass of close consistence is formed, the "Tätmiölk" or "Sätmiölk" of Laplanders, mentioned by Linnæus a hundred and fifty years ago as constituting a very favourite dish in northern Scandinavia. In particular, the fact that by means of a trifling quantity of Tätmiölk, produced in the manner described, a large amount of fresh sweet milk may be also converted into Tätmiölk is specially worthy of emphasis, for we learn from it that the substance generated by Pinguicula behaves in this respect too, like other ferments. The immemorial use of Pinguicula leaves by shepherds in the Alps as a cure for sores on the udders of milch cows is also interesting, inasmuch as the curative effect on the sores is to be explained by the antiseptic action of the secretion of the leaves in question; and a method of healing, used empirically two centuries ago, thus finds confirmation and a scientific explanation at the present day.

Since the curling up and unrolling of the leaf-margin in butterwort is accomplished but slowly, the process above described is not at all conspicuous. Moreover, the margin of a young leaf is always incurved, and that of a mature leaf is also somewhat turned up before stimulation has taken place; so that, strictly speaking, we only have to do with a greater or smaller degree of involution, and its nature can only be determined by careful observation.

In the plants which form the second group in this section of carnivorous plants, and of which the best known representatives are the various species of the genus Sun-dew (Drosera), the movements, whereby the capture and digestion of small animals is effected, occur much more rapidly and obviously. These species are usually rooted in the damp dark soil of moors. They have also the same habitats as Pinguiculæ, and often enough sun-dew and butterwort are to be seen flourishing close together on a patch of boggy ground no larger than a pockethandkerchief. Hunting thus in couples these two bloodthirsty organisms, quite unrelated as regards family, alike only in their common object, seem to thrive amazingly. The thing that strikes one most at sight of the round-leaved sun-dew as it grows in its natural marshy habitat, and in general of all the forty known 
species of Drosera, is the presence of the delicate wine-red filaments, clavate at their free ends and each supporting a glistening droplet of fluid, which stand out from the leaves, and whose function is essentially the same as that of the glands, stalked and sessile, on the leaf of Pinguicula. These filaments only proceed from the upper surface and margin of the sun-dew leaf. The under surface is smooth and hairless, and in many species, including the Drosera rotundifolia of our indigenous flora, it rests upon the damp mossy ground. In this particular, and also in the circumstance that all the leaves of each individual are adpressed to the ground and grouped in a rosette or radially around the central slender flowering-stem, there exists a very obvious analogy between Drosera, and not Pinguicula alone, but many other carnivorous plants, such as Sarracenia, Heliamphora, Cephalotus, and Dioncea, the fly-trap presently to be described.

The filaments or tentacles projecting from the upper surface and margin of the leaf look like pins inserted in a flat cushion and are of unequal size. Those which stand up perpendicularly from the middle are the shortest, and those which radiate from the outermost edge are the longest (sce fig. $26^{4}$ ). Between these extremes are intermediate lengths gradually leading from the one to the other. There are on a leaf, in round numbers, about two hundred of these tentacles. The clavate head at the free extremity of each tentacle is really a gland. It secrets a clear, thick, sticky matter which is readily drawn out into threads, and which shines and glitters in the sunlight like a drop of dew, whence the plant has derived its name of sun-dew. Shocks occasioned by wind or the dropping of rain do not excite any kind of movement in the tentacles. If grains of sand are blown upon them by the wind, or if little bits of glass, coal, gum, or sugar, or minute quantities of paste, wine, tea, or any other non-nitrogenous substance are brought by artificial means into contact with the enlarged extremities of the tentacles, the exudation of liquid at the places in question is augmented, and the secretion also becomes acid, but there is no elimination of pepsin, and no change of importance ensues in the direction of the tentacles, or the attitude of the leaf-margin. But the moment a small insect, mistaking the glittering drops on the tentacles for honey as it flies by, alights on the leaf and so touches the glands, or upon the artificial placing of particles of nitrogenous organic matter, such as flesh or albumen, on the tentacle-heads, there ensues, as in the case of Pinguicula, an increase in the discharge of acid juice, as well as the addition of a ferment to its composition. The action of this ferment on albuminous compounds is entirely similar to that of pepsin, and we may even go so far as to speak of it as pepsin.

The insects that fly on to the leaves and are caught by the sticky juice try to disencumber themselves by stroking the viscous matter off with their legs, but they only besmear themselves still more, and are soon plastered all over the body, and have their movements greatly impeded by the secretion. Their efforts to save themselves soon cease, the orifices of their respiratory organs are covered with the juice and choked, and after a brief interval they die from suffocation. All these phenomena correspond, in the main, to those occasioned by identical causes in the 
case of Pinguicula. But the leaves of the sun-dew are especially characterized by the movements performed by the tentacles in response to stimulation by animal matter. These movements are exhibited most conspicuously by the longest tentacles, which stand out radially from the edge of a leaf. A few minutes after the gland of one of these marginal tentacles has been excited by a living or dead animal becoming glued to it, a systematic disturbance is set up in the whole fringe of tentacles. First, the tentacle bearing the gland originally irritated with the animal's body attached to it, bends inwards, performing a movement similar to that
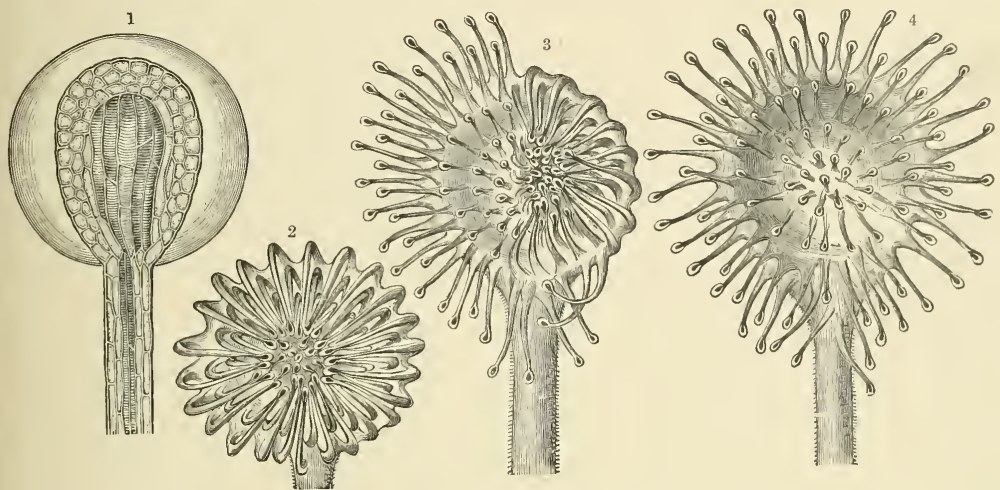

Fig. 26.-Tentacles on leaf of Sun-dew.

1 Glands at the extremity of a tentacle; $\times 30 . \quad 2$ Leaf with all its tentacles inflexed towards the middle. ${ }^{3}$ Leaf with half the tentacles inflected over a captured insect. 4 Leaf with all the tentacles extended. Figs, ${ }^{2}, 3$, and $4 \times 4$.

of the hand of a watch. Under peculiarly favourable circumstances it describes an angle of $45^{\circ}$ in from two to three minutes, and an angle of $90^{\circ}$ in ten minutes. A still more intelligible comparison than that of the hand of a watch is afforded by the human hand. Supposing that the foreign body is glued to the tip of a finger it would be moved by the curvature of the finger to the palm in the course of ten minutes. About ten minutes after the first tentacle has been set in motion, those standing near it begin to bend also (see fig. $26^{3}$ ). After another ten minutes, tentacles situated further off follow suit; and in the course of from one to three hours all the tentacles are inflected and converge upon the body in question.

We must not omit to mention that this object does not always occupy the same place on the surface of the leaf. Often, no doubt, the prey is exactly in the middle, and the tentacles then swoop down one after the other to that spot; but often also the place is elsewhere and yet the movements never fail in their aim. It may happen that a median tentacle, on repeated excitation, may have to bend now to the right, now to the left. When little bits of meat are placed simultaneously on the right and left halves of the same sun-dew leaf, the two hundred tentacles divide into two groups, and each one of the groups directs its aim to one of the bits of meat. This happens also if two small insects alight at the same moment on a leaf, voL I 
one on one side and the other on the other. The movement of the tentacles is often accompanier? by an inflection of the whole surface of the leaf, the lamina becoming concave like a hollow palm, and when, under these circumstances, the tentacles have converged from the margin on to the concave central part, the leaf resembles a closed fist (see fig. $26^{2}$ ).

All these movements vary from one case to another and supplement one another according to the needs of the moment and with a view to immediate advantage. The one result that is always attained by the combined action is the covering of the prey with a copious supply of the secretion poured from a number of glands, so that it is dissolved and rendered fit for absorption and for the purposes of nourishment. When an insect is caught by one of the marginal tentacles, the secretion there discharged would not suffice for these purposes. The prey is accordingly transported as far as possible towards the middle of the lamina, where it comes into contact with the digestive juice exuded from a maximum number of glands. It is only when the size of the animal is rather large that the leaf becomes hollow in the middle like a spoon, with the juice of more than fifty glands concentrated in the depression. In a case of this kind the tentacles remain inflected much longer, because the solution of the prey requires more time. If the captive is very small, its solution and absorption are completed in a couple of days. Afterwards, the tentacles lift, straighten themselves, and resume their original positions. The jaws, wings, compound eyes, leg-bones, claws, \&c., of the captured animals are left behind undigested; but the flesh and blood are totally absorbed, and the liquid poured out by the glands to effect solution is also re-imbibed by them. The undigested remnants being now suspended on dry tentacles are easily blown away from the sun-dew leaves by the wind. After an interval of a day or two the glands at the ends of the tentacles, now occupying their original positions, again separate out a viscid fluid in the form of tiny dewdrops, and the leaf is once more furnished with the means of securing insects, and is able to repeat the movements above described.

Amongst the animals which fall victims to the sun-dew the most predominant are little midges; but rather larger flies, too, ants both with and without wings, beetles, small butterflies, and even dragon-flies, as they run, creep, or fly past, adhere to the extended gland-bearing tentacles as though they were limed-twigs. The larger animals, such as dragon-flies, are secured by the co-operation of two or three adjacent leaves. Some idea of the large number of captives made by a sun-dew is given by the fact that once upon a single leaf were found the remains of thirteen different insects.

In order to place in a true light the vast significance of the movements of the tentacles belonging to Drosera leaves in relation, not only to the nourishment of that plant, but to plant-life in general, it is necessary to direct attention to the facts that these movements are accomplished not in the cell directly excited, but in others, i.e. in adjacent cells belonging to the same community; that a propagation of the stimulus takes place from one protoplast to a second, thence to a third, fourth, 
tenth, and so on, to a hundredth, and that the speed of transmission is susceptible of measurement. The movements occasioned in protoplasts situated at a distance from the seat of irritation by the stimulus propagated from its vicinity are, according to the position of the stimulating object, sometimes in one direction, sometimes in another, but in every case they are purposeful and for the benefit of the whole organism.

Investigations with a view to determining the degree of sensitiveness of Drosera leaves yielded the following results. A particle of a woman's hair, $0.2 \mathrm{~mm}$. long and weighing $0.000822 \mathrm{mg}$., when placed upon a gland of Drosera rotundifolia, caused a movement of the tentacle belonging to the excited gland, which manifested itself externally as an inflection. If so minute a body of the kind is placed on the human tongue, its presence is not perceived, so that the sensitiveness of the protoplasts in the glands of the sun-dew is greater than that of the nerve extremities in the tip of the tongue, though the latter are well known to be the most sensitive in the human body. A four-thousandth part of a milligram of ammonium carbonate sufficed to induce motion, as also did $\frac{1}{30000} \mathrm{mg}$. of ammonium phosphate, It would lead us too far to consider all the experiments in detail, but they point to the conclusion that liquid substances stimulate more strongly than solid bodies, and that the more nutritious to the plant the material placed upon the gland, the more quickly does the inflection of the tentacles ensue.

The propagation or conduction of a stimulus from cell to cell, as it takes place in the cell-community constituting a sun-dew leaf, may be compared to the conduction of stimulus by nerves from a sense-organ to the central organ, and of the force of will from the brain to the muscles. This transmission is conceived to be a progressive movement affecting the ultimate particles of the nerves, and comparable to the conduction of sound, light, and electricity; but no one has yet succeeded in making these movements visible. So much the more interesting is it to be able to see and follow in the glands and tentacles, by the aid of very slight magnifying power or even with the naked eye, the material change which occurs in the protoplasts of the sun-dew leaf when they are receiving or transmitting a stimulus. The pedicel of a tentacle is penetrated by one or two vessels with fine spiral sculpturing on the inner surface, and around these are parenchymatous cells. The gland has in the middle a group of oblong cells sculptured internally with very delicate spiral thickenings ("spiroids"), and the vessel or pair of vessels running down the middle of the tentacle (see fig. $26^{1}$ ) merge into these spiroids. A parenchyma composed of two or three layers surrounds the median group of spiroids. In each parenchymatous cell the protoplast is discerned forming a thick lining to the wall, and having a continuous streaming motion: whilst within the vacuole is contained a homogeneous liquid of a purple colour. If the minutest fragment of animal matter, such as flesh or albumen, be placed on these cells it acts as a stimulant on the contents of the cell-cavities, and the impulse manifests itself in a division of the hitherto homogeneous purple liquid into dark, roundish, clubshaped and vermiform lumps, cloudy spheres, and an almost colourless liquid. 
This change, known as "aggregation", is propagated from the spot irritated down from one cell to another through the tentacle, across the leaf surface to adjoining tentacles, up to the heads of these, and so further and further radiating, so to speak, in all directions. Accompanying this visible sign of conduction, we have the bending of all tentacles in which the purple fluid is altered in the way described. When the source of excitation, the piece of flesh, is dissolved and digested, and the tentacles resume their original position, the dark lumps and spheres in the cavities

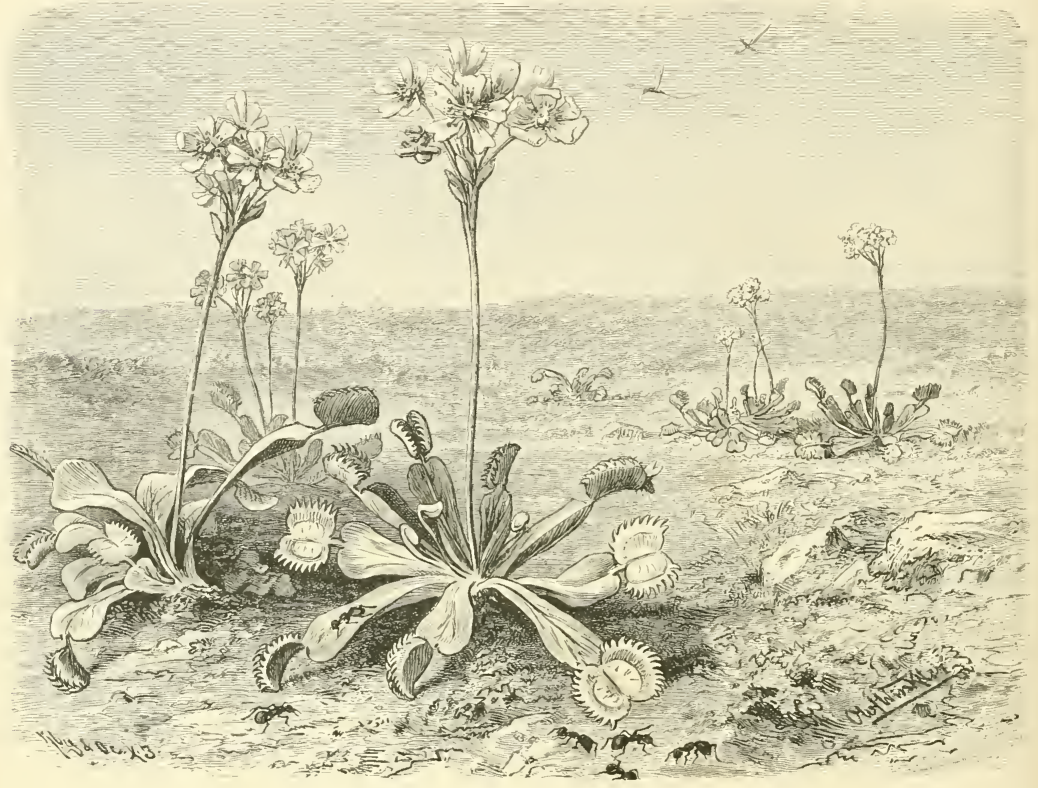

Fig. 27.-Venus's Fly-trap (Dioncea muscipula).

of the protoplasts disappear, and the homogeneous purple colour is restored as it existed before the stimulation.

The various species of the Sun-dew genus are distributed over all parts of the world, and are more numerous than those of any other genus of the family of Droseraceæ. Most of the other genera belonging to this order (Dioncka, Aldrovandia, Byblis, Roridula, Drosophyllum) are by no means rich in members. Each is represented merely by a single or few species, and is found exclusively in a very limited district. Like Drosera, they are all insectivorous plants, and all have the power of dissolving, absorbing, and using as supplementary nutriment, nitrogenous compounds from dead animals. The most striking of them are Dioncea and Aldrovandia. They form the very small third group of animal-captors, in which movements are performed for the purpose of prey, and their apparatus for 
seizure and digestion is one of the most curious adaptations displayed by the vegetable world.

The Venus's Fly-trap (Dioncea muscipula), represented opposite (fig. 27), in half its natural size, grows wild only in a narrow strip of country in the east of North America (from Long Island to Florida) in the vicinity of peat-bogs. The leaves, like those of many other carnivorous plants, are grouped in rosettes round the flowering axes, and for the most part rest their under surfaces either entirely or partially upon the ground. Each leaf consists, first, of a flat, spatulate petiole, which is, as it were, truncated in front and suddenly contracted to the midrib, and, secondly, of a roundish lamina. The latter is divided by the midrib into two symmetrical halves, inclined to one another at an angle of from $60^{\circ}$ to $90^{\circ}$ like the leaves of a half-open book. Both margins of the lamina run out into from twelve to twenty long, sharp teeth, which, however, do not earry either glands or any other special structures on their tips.

On the central part of each half of the leaf there are three very stiff and sharp spines, which are always shorter than the marginal teeth, and which stand up obliquely. They are composed of elongated cells whose protoplasm throughout life is in very active circulation. At the base of each spinous process is a short cylindrical pad of tissue formed of small parenchymatous cells, and this pad allows the spine to be deflected. The spines themselves are rigid and do not bend in response to pressure; they are forced down on to the surface of the leaf, the pad of tissue referred to acting as a hinge. In addition to these processes, glands are seattered over the whole upper surface of the lamina. They look like the shortly-stalked glands of a butterwort leaf, are composed of some twenty-eight small cells, are purple in colour, and capable of secreting a mucilaginous liquid. Little trichomes, stellate hairs, are also borne on the edge of the leaf between the sharp teeth, and also on the under-surface.

No visible change is produced by a blow or shock or by pressure affecting the whole plant or leaf, as might be caused by wind or falling drops of rain, nor even by injuries to the petiole or back of the lamina. But as soon as the upper surface of the lamina is touched, the two lobes, hitherto at right angles, approach one another until the sharp marginal teeth are interlocked, and the body touching the leaf is inclosed within two walls (fig. $2 S^{2}$ ). When the places beset with purple glands are alone excited by contact with the object, this inflection and closing follows very slowly; but if one of the six spines projecting in trios from the two foliar lobes is ever so lightly touched, the leaf shuts up within 10-30 seconds, i.e. quickly and steadily; an action best compared to the closing of a half-open book. The teeth standing at the edge of the leaf lock into one another on these occasions like the fingers of clasped hands. The lobes, however, whose surfaces were hitherto plane, become at the moment of closing somewhat concave, so that when contracted they do not lie flat against one another but inclose a cavity, the contour of which nearly corresponds with that of a bean.

The further changes and processes now ensuing depend upon whether the 
sensitive part of the leaf was subjected to prolonged or only momentary contact, and also upon the nature of the body touching it, whether inorganic or organic. non-nitrogenous or nitrogenous. When rapidly touched or stroked, the leaf folds together, but only remains closed for a short time. The lobes soon begin to reopen, and can be stimulated afresh immediately and caused to shut again. This is also the case when the disturbance was due to the impact of a grain of sand or any other inorganic body, and likewise when the stimulus proceeded from an organic but non-nitrogenous object. But if, on the other hand, the body upon the upper surface of the lamina was nitrogenous and the contact not too hasty, the two lobes of the leaf remain closed over the object for a longer period. They also
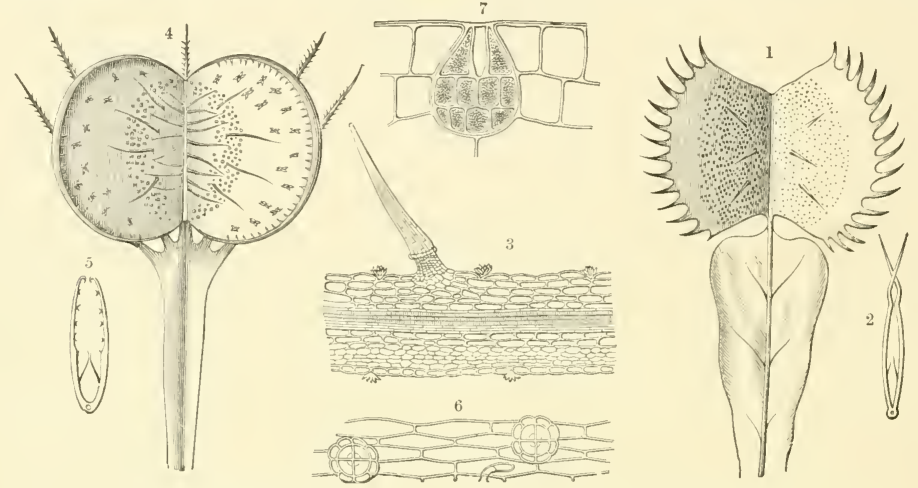

Fig. 28.-Capturing apparatus of the leaves of Aldrovandia and Venus's Fly-trap.

1 Expanded leaf of a Venus's Fly-trap. 2 Section of a closed leaf. $s$ one of the sensitive bristles on the surface of the leaf. 4 Expanded leaf of Aldrovandia. 5 Section of a closed leaf. 6 Glands on the surface of leaf of Aldrovandia. 7 Gland from the wall of a Sarracenia pitcher.

become flat and even again, and are pressed together so tightly that intervening bodies, if soft, are squeezed and crushed to pieces. In addition, the glands, dry till then, begin to secrete a slimy, colourless, highly acid juice; and this is true even of those glands which are not at all in contact with the nitrogenous bodies inclosed. The secretion flows so copiously that it can be seen in the form of drops if the shut lobes be forcibly separated. It covers the imprisoned body and gradually dissolves the albuminous compounds therein contained. Afterwards, the secretion and the matter dissolved in it are re-absorbed by the same glands as previously discharged the acid liquid, containing pepsin, in response to the stimulus; and when the trap reopens, the glands are dry. The soluble part of the prey has now vanished: the six little spinous processes, which were bent in the closed leaf like the blades of a pocket-knife and lay pressed down upon the surface, stand up; and the leaf is once more equipped for making fresh captures.

The time requisite for the digestion of a nitrogenous body resting upon the surface of a leaf varies according to the size of the body. The leaf usually remains closed for from eight to fourteen days, but often even for twenty days. Although 
the larger live articulated animals-earwigs, millipedes, and dragon-flies-caught upon the upper surface of the leaf, cause the lobes to slam together, they are able to slip out if part of their bodies projects beyond the toothed margin, for the teeth are flexible and yield to strong pressure. But small creatures are hopelessly lost when the lobes have closed over them. They are at once suffocated in the liquid which is poured out copiously by the glands and are then dissolved and absorber with the exception of their claws, leg-bones, chitinous rings, \&c., which are incapable of being digested.

In spite of the identity of aim and of result, the mechanism of a Dioncea leaf differs very materially from that of the sun-dew leaf described above. Division of labour is carried much further in the Fly-trap. The pre-eminently sensitive structures, viz., the six filaments situated upon the upper surface of the leaf,

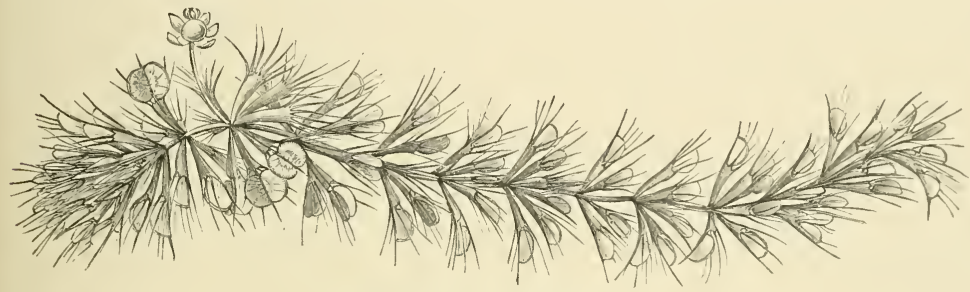

Fig. 29.-Aldrovandia vesiculosa.

do not act also as digestive glands. Again, the long sharp teeth at the edge of the leaf, which correspond in position to the marginal tentacles of a sun-dew leaf, carry no glands, and only serve to close the trap securely when an animal has been caught. Accoraingly in Dioncea there exist special structures for three different functions, namely, stimulation, seizure, and digestion, whilst in the case of Drosera all these functions belong to the gland-bearing tentacles alone. The stimulus acting on the sensitive filaments on the leaf of the Fly-trap is liberated in the form of a rapid motion of the lobes and a discharge of digestive fluid from the glands, and this discharge of secretion ensues therefore through the mediation of cells which have not themselves been directly excited. The process here again is much more striking than in the sun-dew leaf. The transmission of stimulus, though as a fact identical in the two plants we are comparing, proceeds at any rate with much greater rapidity in Dioncea than in Drosera.

The analogy existing between these processes, especially the conduction and liberation of stimulus, and similar phenomena of the muscles and nerves in an animal organism, has already been brought out in discussing the sun-dew. It is a noteworthy fact that, in the fly-traps, actual electric currents have been observed, which shows that the greatest resemblance exists to muscles and nerves as regards electro-motor action also. A positive current runs from the base to the apex of the larnina; another current running in the opposite direction is demonstrable in the petiole; and the upper layers of cells in the lamina and the midrib are ascertained 
to be the seat of origin of this phenomenon. A great alteration in the intensity of the current ensues upon each excitation of the leaf; and, inasmuch as this fluctuation of the electric current precedes the movement of the leaf caused by the stimulus, it is natural to assume that it depends upon the conduction and liberation of the stimulus.

Aldrovandia, the plant nearest allied to the Fly-trap in the structure of its leaf, is a water-plant, which occurs scattered over the southern and central parts of Europe. It only flourishes in shallow ditches, pools, and small ponds inclosed by banks of reeds and rushes, where the plants are immersed in clear, so-called soft water, attaining in summer to a temperature of $30^{\circ} \mathrm{C}$., and are exempt from any incrustation of carbonate of lime, whereby the tender parts of the leaves might be hindered in their movements. On cursory inspection, one might take Aldrovandia vesiculosa, which is represented in fig. 29 full size and in its natural position, for a Utricularia (cf. fig. 17). It lives, like the latter, floating in water; is destitute of roots, and has a slender filiform stem with leaves arranged in whorls and terminating in bristles. In proportion as it grows at the apex, the hinder part dies away and decays. The development of hibernating buds takes place also in precisely the same manner as in Utricularia. Towards autumn, the stem ceases to elongate, and the two hundred small and young leaves, which adorn the extremity of the stem and whose cells are quite full of starch, remain lying closely wrapped one upon another and form a dark, oval, bristly ball, which sinks at the commencement of winter to the bottom of the pool or pond and hibernates there lying upon the mud.

It is not till very late in the following spring, when little midge-larvæ and other animals begin to move about in the water, that fresh life is awakened in these structures. The starch-grains in the leaves are brought into solution and used for building-material; the axis elongates, and lacunæ filled with air are developed, whereupon the plant becomes lighter, ascends, and remains throughout the summer and autumn floating just below the surface of the water. Although the little leaves of the winter-buds generally admit of the recognition of their future form, the apparatus adapted to the capture of animals is but little developed on them. But when once the leaves are mature, they bear laminæ, which are extremely like those of Dioncea in shape, and serve, as do the latter, for the capture of small animals. Each leaf is differentiated, as in Dioncea, into a strong, dark-green petiole expanded and anteriorly clavate, and into a roundish lamina with a delicate epidermis and with two lobes connected by the midrib and inclined nearly at right angles to one another (see fig. $28^{4}$ ). The midrib projects beyond the apex of the delicate lamina in the form of a bristle. In addition, comparatively long, rigid bristles, tipped with extremely fine spines, proceed from the petiole close to where the latter is joined to the lamina; and these bristles, which are directed forwards, give the whole leaf-structure a spiky appearance and prevent the approach of such animals as are not suitable for prey. The two margins of the lamina are bent inwards, and their rims are studded with small conical points. On the surface of 
the lamina, especially along the midrib, there are pointed hairs, whilst a great number of glands, some larger and some smaller, occur from the midrib to nearly the middle of each lobe. The larger glands are discoid, and not unlike the sessile glands on the leaves of Pinguicula. They consist of four median cells with twelve others grouped round about them, and are borne upon a very short stalk. The small glands are few-celled, being usually composed simply of a capitate-cell resting upon a short foot-cell (see fig. $28^{6}$ ). Towards the incurved margin of the lamina are displayed scattered stellate hairs, i.e. groups of cells so arranged as to present the appearance of a St. Andrew's cross when seen from above.

If minute animals or Diatomaceæ, especially species of Navicula, whilst swimming about in the water, touch the upper surfaces of the lobes set at rightangles-in particular, if the hairs in the middle are stroked as they creep by - the two lobes shut together quickly in the same way as those of Dioncea, and the animal or Navicula, as the case may be, is then enclosed in a cage between two somewhat inflated walls. The possibility of an attempt on the part of the captive to escape by the place where the margins of the lamina meet is met by the circumstance that the edges of the incurved margins are furnished with sharp indentations turned towards the interior of the cavity enclosed between the lobes (see fig. $28^{5}$ ).

Amongst the prisoners we find the same company as in the traps of Utricularia, namely, small species of Cyclops, Daphnia, and Cypris, larvæ of aquatic insects, and not infrequently also species of Navicula and other free and solitary Diatomaceæ.

How the prey is killed and digested has not yet been ascertained. It dues not in any case take place so quickly as in Dionoea, for instances have been seen of animals still living in their prison six days after being caught. But, at last, movements and vital actions cease, and if after a couple of weeks the two lobes of the lamina are pulled apart, the only contents to be found are shells, bristles, rings, and siliceous skeletons, whilst everything soluble has vanished, having evidently been absorbed.

Very similar to the species distributed through Southern and Central Europe are Aldrovandia australis, a native of Australia, and Aldrovandia verticillata, inhabiting tropical India. The fact that the remains of small aquatic beetles and other creatures have been found within their closed laminæ, leads us to the conclusion that they act as entrappers of animals in the same way as Aldrovandia vesiculosa.

\section{CARNIVOROUS PLANTS WITH ADHESIVE APPARATUS.}

The forms constituting the third section of carnivorous plants neither have pitfalls nor move in response to the contact of animal matter, but the leaves act as motionless limed twigs, their glands having the power of pouring out sticky substances to capture prey and juices to digest it, being able besides to re-absorb the albuminoid compounds dissolved. The most striking representative of this section, 
and the one most accurately studied, is the Fly-catcher (Drosophyllum lusitanicum), which is indigenous to Portugal and Morocco, and is shown in the illustration on p. 155. This plant differs from all the carnivorous kinds hitherto discussed in respect of habitat, inasmuch as it does not grow under water or even in swampy places but on sandy ground and dry rocky mountains. The stem in robust specimens is nearly 9 inches high, and bears, on a few short branches at the top, flowers from 2 to $3 \mathrm{~cm}$. in diameter. The leaves are very numerous and particularly crowded round the base of the stem. Their shape is linear and much attenuated towards the filiform tip, whilst the upper surface is somewhat hollowed so as to form a groove. With the exception of these grooves, the leaves are entirely covered with beads, which glisten in the sunshine like dewdrops; and it is to this circumstance that the plant owes its name of Drosophyllum, i.e. Dew-leaf. The glittering drops are the secretion of glands, which in form remind one in some respects of the long-stalked glands of the butterwort, and in others of those of the Sun-dew (Drosera). They resemble the latter in their red coloration, in the fact that the pedicel bearing the gland contains vessels whilst the glands themselves have oblong cells with internal walls thickened by fine spiral ridges, and further, in the circumstance that the secretion covers the gland with a colourless film in the form of a drop. But in shape they especially resemble the glands of the butterwort, being just like little mushrooms.

Besides these glands, which are borne on stalks of unequal lengths and are plainly to be distinguished with the naked eye, there are also very small sessile glands. These latter are colourless, and in particular differ from the stalked variety in the fact that they discharge an acid liquid only when they come into contact with nitrogenous animal matter, whereas the production of drops on the stalked glands is accomplished without any such contact. This secretion is acid and extremely viscid. It has the property of adhering immediately to foreign bodies coming into contact with it, though it is readily withdrawn from the gland itself. When an insect alights on the leaf, its legs, abdomen, and wings instantly stick to the drop touched by them. The insect, however, is not held fast by the gland which secreted that drop, but, being able to move, drags the drop off the gland. Its movements bring it into contact with other drops, which thereupon are similarly detached from the glands; and so, in a very short time, the insect is smeared with the secretion from a number of glands. Thus clogged and overwhelmed, it is no longer able to crawl along, but, suffocating, sinks down to the sessile glands which cover the surface of the leaf at a lower level. All the soluble parts of its body are then dissolved by means of the secretion of these glands and are afterwards absorbcd.

The glands renew the drops of secretion of which they are despoiled with great rapidity. The quantity of acid liquid secreted is, in general, very great, so that it is not surprising to find Drosophyllum covered at the same time with remains of besmeared dead bodies drained of their juices, and with still struggling insects which have recently alighted and become clogged. The number of animals caught by the leaves of a single plant is very great; and even people who are not 
otherwise interested in the vegetable world are impressed by the sight of a plant with its leaves covered with a number of insects adhering to them as though they were limed twigs. In the neighbourhood of Oporto, where Drosophyllum grows abundantly, the peasants use these plants instead of limed twigs, hanging them up

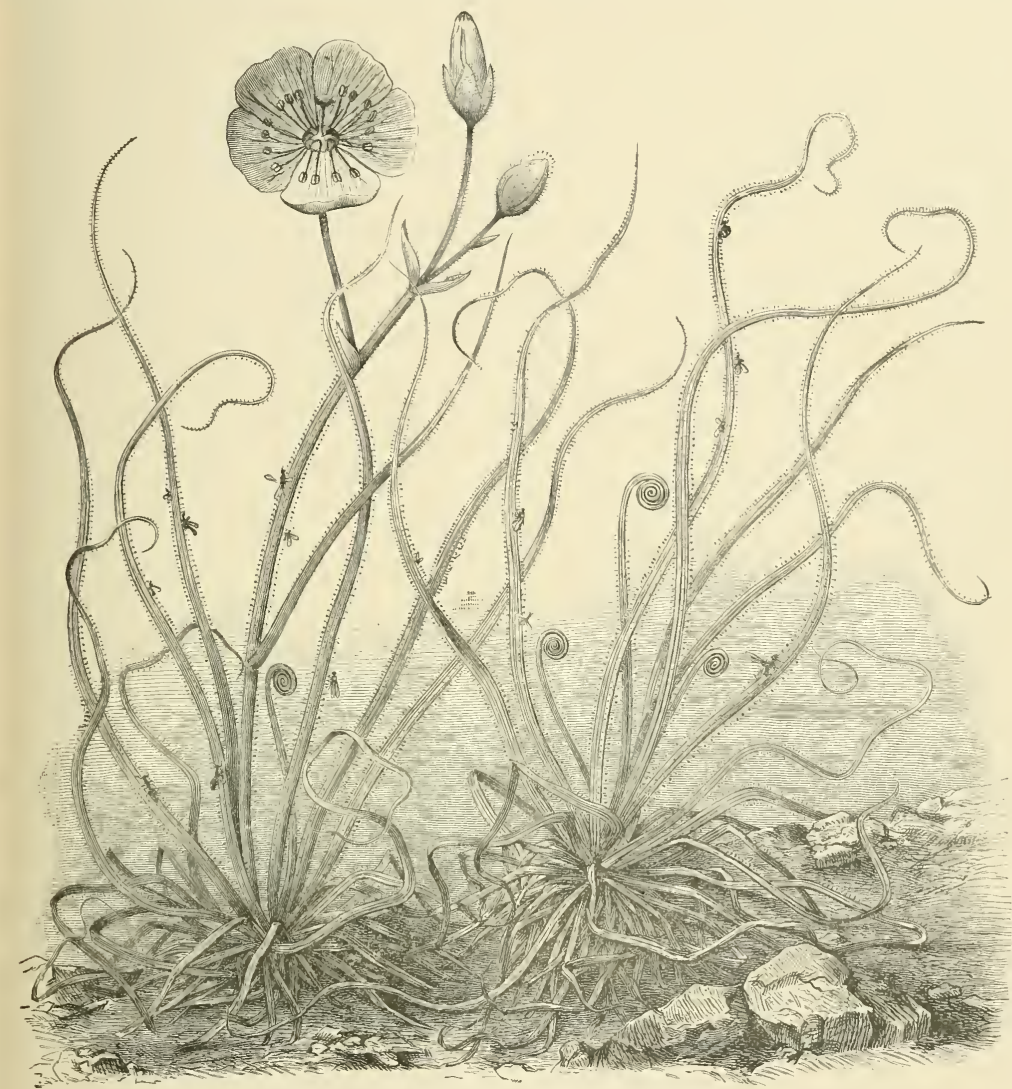

Fig. 30.-The Fly-catcher (Drosophyllum lusitanicum).

in their rooms, and so getting rid of numbers of troublesome flies which stick to them and are killed.

A number of other plants have the power, though in a less conspicuous degree than Drosophyllum, of obtaining additional nitrogenous food out of adherent animals by means of secretory and absorptive glands. Such are many species of primulas, saxifrages, and house-leeks, which bury their roots in cracks and crevices of rock (e.g. Primula viscosa, P. villosa, P. hirsuta, Saxifraga luteo-viridis, S. bulbifera, S. tridactylites, Sempervivum montanum), secondly, caryophyllaceous 
plants and species of the caper order (e.g. Saponaria viscosa, Silene viscosa, Cleome ornithopodioides, Bonchea cohiteoides), and lastly, a series of plants which flourish in peat-bogs and upon deep beds of humus, such as Sedum villosum, Roridula dentata, Byblis gigantea, and many others besides.

It would, however, be erroneous to suppose that in all cases where a sticky coating occurs on leaves and stem a solution and digestion of the insects adhering to the viscid parts is necessarilv denoted. In many instances structures of this kind, which are analogous to limed twigs, are a means of protecting honey-bearing flowers against unwelcome guests belonging to the world of insects, as will be explained in greater detail later on. Glands secreting a viscid substance may, no doubt, often possess two kinds of function - they may, on the one hand, prevent unbidden animals from approaching the honey, and, on the other, by dissolving their flesh and blood with the aid of the secretion and then absorbing them, turn to advantage such insects as are tempted by immoderate craving to step upon the perilous path leading to the honey-receptacles and adhere there and die.

Many plants have structures on the epidermis of their leaves corresponding in form to the glands of insectivorous plants, but which do not discharge secretions either spontaneously or when irritated. On the other hand, these structures have the power of imbibing water, and are, in this relation, of the greatest importance to the plants in question. Although the more detailed treatment of them is postponed until we have occasion to deal with the absorption of water by aërial organs, it is advisable to refer now to the fact that chemically pure water only very rarely reaches the interior of a plant by means of the absorptive organs mentioned. Sulphuric acid is almost always introduced with atmospheric water, and in some circumstances ammonia also. However trivial the amount of the nitrogen conveyed to plants in this way, it must not be undervalued, at all events in the case of those which are only able to acquire small quantities of nitrogenous compounds from the ground by means of their roots. Now, it is very probable that plants of this kind do not reject even other nitrogenous compounds which are brought with the water from the atmosphere to their aërial leaves. The foliage-leaves of many plants display contrivances whereby rain-water is often retained for a considerable time in special hollows. In these depressions there is invariably a collection of dust-particles, small dead animals, pollen-grains, \&c., which have been blown in by the wind, whilst rain trickling down the stem brings very various objects with it from higher up and washes them into these reservoirs in the leaves. Sometimes too a few animals are drowned in the water-receptacles. As a matter of fact, the water in the hollows of the leaves of the Peltate Saxifrage and of Bromeliads, in the inflated vaginæ of many umbelliferous plants, and in the cups formed by the coalescence of opposite leaves in many Gentianeæ, Compositæ, and Dipsaceæ, is always brown-coloured, and contains nitrogenous compounds in solution, derived from the decaying bodies of dead animals which have fallen into these receptacles.

If absorbent organs are present in the reservoirs in question, the water, together 
with the nitrogenous compounds dissolved therein, is absorbed without delay. Hollows of this kind occurring in foliage-leaves only differ from those above described as developed on sarracenias in being destitute of special contrivances for decoying animals into the traps, and for rendering their escape from the latter impossible. It cannot be denied that through forms of this kind a gradual transition has been proved to exist between plants which absorb nearly pure water by means of their foliage-leaves and those which capture animals. And, further, amongst the latter we find ali gradations of mechanism from Drosophyllum and the Primulas with their epiphyllous secretory glands up to the Fly-trap (Dioncea), which exhibits the most complex apparatus of all for capturing and digesting prey, and in which division of labour is carried to its highest development by the communities of cells constituting the foliage-leaves.

It is not surprising that the first apparatus for capturing and digesting insects to be noticed, to have its functions recognized and to be described, was that of Dioncea. But it strikes one as all the more strange that of late the question has repeatedly been mooted in the very case of Dionoea, as to whether the capture and digestion of insects is not injurious instead of beneficial to these plants. Gardeners, who have cultivated Dionoe in greenhouses, have made the observation that individuals protected from the visits of insects thrived at least as well as those whose leaves were covered with bits of meat, \&c., or, to employ the usual phrase, were fed with meat. It has also been found that a leaf cannot stand more than three meals; indeed, it often happens that even after the first occasion of digesting a bit of meat, the leaf concerned shows signs of having been injured by the repast. That is to say, a long time elapses before leaves which have digested a largish albuminoid mass regain their normal irritability; and often they wither and die. If cheese is placed on Dioncea, it is true the leaf closes over it, and there is a commencement of the process of solution, but before this is accomplished the leaf turns brown and perishes. Yet if Dionoea were obliged to lose a leaf after every meal, the result would be very disadvantageous.

As against these considerations, we have first of all to remark that the absorption of nutriment takes place in nature in a manner differing materially from the phenomenon in greenhouses. A leaf of Dioncea in the wild state is protected against the possibility of receiving too plentiful a dose of albuminoid substances at a time. Insects so large as not to allow the lobes to close together over them slip out again, and only small ones are caught and retained. When, in the latter case, one deducts the chitinous coat, and in general all parts not susceptible of being digested, such a small quantity of albuminoid compounds is left that, compared with it, the little cubes of meat used in the experiments made in greenhouses must be looked upon as an exceedingly sumptuous repast. But that so small an amount of nitrogenous food as is to be derived from a tiny captured insect does not act injuriously, follows from the fact that dionæas growing wild flourish excellently, and do not exhibit the brown discoloration of the leaves which is caused in a greenhouse by placing bits of cheese upon them. If the absorption of nitrogenous. 
aliment from prey were injurious to Dioncea, the plant would certainly have died out long ago. If, therefore, cultivated specimens of Dioncea have suffered from being fed with meat, fibrin, cheese, and other such materials, only this much is proved, that the nutriment in question was not beneficial to them owing to its quality or to its being too concentrated.

As regards the other point, that Dioncea thrives well under cultivation, even when all visits from insects are excluded, we must, on the other hand, bear in mind that the successful growth of Dioncea, like that of Drosera, Pinguicula, \&c., is not conceivable unless in some way or another the nitrogen indispensable for the construction of the protoplasm is conveyed to the individuals in question. The source from which it is taken varies according to the site. If the roots are buried in deep sods of bog-moss upon a flat expanse of moorland, the supply of nitrogen from the ground, and also from the air, will be extremely limited, and probably insufficient; under these circumstances the nutriment derived from the dead bodies of captured insects would be not only useful and beneficial, but may be even essential. If, on the contrary, the place where the plants have been reared or have grown up spontaneously is such that they can obtain the requisite nitrogen from the ground or air, they are able without harm to dispense with the available source of nitrogen afforded by the capture of insects. It is worthy of notice that insectivorous plants always grow wild only in places that are poorly supplied with nitrogenous food. The majority occur in pools fed by subterranean water, whose course lies through layers of peat, or in the spongy peat itself, or in the sods of Sphagnum. Others are rooted in deep chinks in the stone on the declivities of rocky mountains, whilst yet others occur in the sand of steppes. The water available in such situations for absorption by the suction-cells is, to say the least, very poorly furnished with nitrogenous compounds; and the quantity of these compounds passing from the ground into the air at the places mentioned is extremely minute and inconstant. Under these circumstances, the acquirement of nitrogen from the albuminoid compounds of dead animals is certainly of benefit, and all the various pitfalls, traps, and limed twigs are explained as contrivances by means of which this advantage is secured. 


\section{ABSORPTION OF NUTRIMENT BY PARASITIC PLANTS.}

Classification of parasites.-Bacteria.-Fungi.-Twining parasites.-Green-leaved parasites.-Toothwort.-Broom-rapes, Balanophoreæ and Rafflesiaceæ.-Mistletoe and Loranthus.-Grafting and budding.

\section{CLASSIFICATION OF PARASITES.}

The ancients understood by parasites people who intruded uninvited into the houses of the rich in order to obtain a free meal. The designation was first applied to plants by an eighteenth - century botanist, named Micheli, in his work "De Orobanche" (1720) wherein are described amongst others, many kinds of "plantæ secundariæ aut parasiticæ". Micheli included under this term plants which withdraw organic compounds from living plants or animals, thus sparing themselves the labour of forming those compounds out of water, salts, and constituents of the air. For a long time all epiphytes, including mosses and lichens growing on the bark of trees, and indeed even many climbing plants, were held to be parasites. Thus, it is not long ago that Clusia rosea, which occurs in the Antilles, was described as a regular vampire, in whose embraces other plants met their death; and it has been asserted respecting a whole series of other plants of the tropical zone, including, for instance, several species of fig, that they attach their stems and branches to other trees, divest themselves of their bark, and cause the death of that of the neighbour attacked as a consequence of the pressure which they exert. The young wood of the invader would then come into direct connection with the young wood of the plant assailed, and the possibility would thus be afforded of draining the latter of all its juices.

These assumptions, at least as regards the exhaustion of juices, have not been confirmed. When individuals of species of Clusia or Ficus, which have roots buried in the earth, and are themselves already grown up into stately leaf-bearing plants, attach their flattened stems and branches to other plants, investing them so completely as to interfere with the process of respiration, this constitutes, at all events, an invasion of one of the most important of the vital functions of the plant attacked, and may ultimately cause its death; but the killing is not under these circumstances due to drainage of juices, but is brought about by suffocation. Lichens, too, when they cover the bark of trees with a close-fitting mantle, may possibly restrict the process of respiration through particular parts of the cortex, and thereby injure the development of the tree in question; but they are not on that account to be looked upon as parasites any more than the fructifications of the species of Telephora, and other Basidiomycetes, which grow up rapidly from the ground, and, spreading out like plastic doughy masses, envelop all objects which come in their way, and ultimately stifle such as are living, namely, grass haulms, bilberry bushes, \&c. Even creepers, which impose woody stems upon the trunks of young trees, winding round them like serpents, and restricting their circumferential growth at the parts in contact with the coils, so that ultimately the latter lie 
imbedded in regular grooves in the cortex, ought not to be considered as parasites. The Lonicera ciliosa of North America, represented in fig. 31, may be taken as an example of creepers of this kind. They only interfere with the conduction of the constructive materials generated in the green foliage, preventing, in particular, the

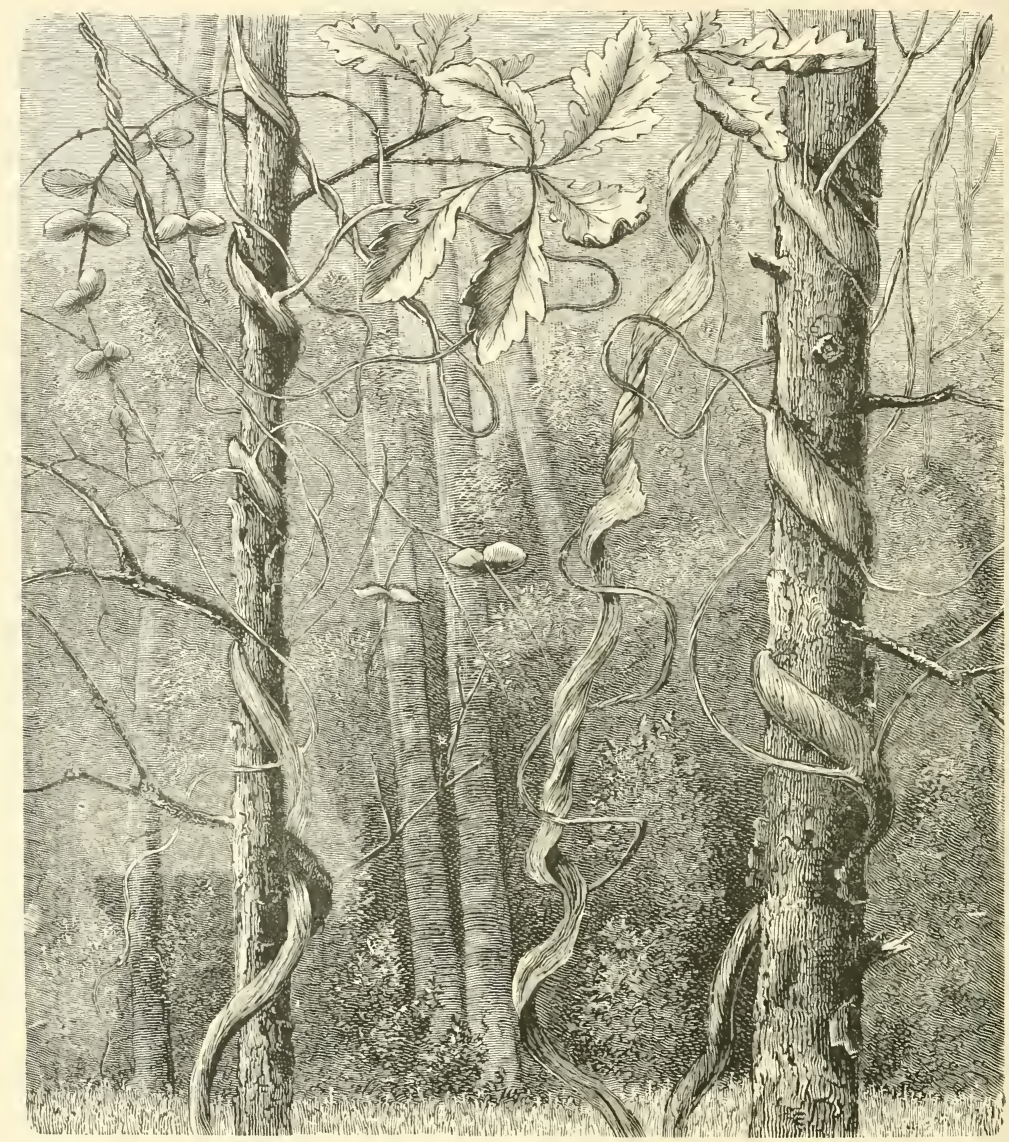

Fig. 31.-Lonicera ciliosa in South Carolina.

part of the axis below the strangulating coils from being supplied with those materials; and so at last they cause the whole trunk, which serves as their support, to dry up. The assertion may then be made that the young tree assailed has been strangled or throttled by the creeper, but not that the latter has drained it of juices and adapted them to its own use. Still less would the statement be applicable to the numerous brown and red sea-weeds, which settle upon the ramifications of the great species of Sargassum, or of the innumerable Diatomaceæ, which often entirely 
cover both fresh and salt-water plants. In still inlets of the sea it is not rare to see the larger sea-wracks with smaller specimens clinging to them, whilst Floridero are fastened to the latter, and minute siliceous-coated diatoms to the Floridex. Even in fresh water, e.g. in cold and rapid mountain streams, we find little tufts of Chantransia or Batrachospermum developed as epiphytes upon the black-green filaments of Lemanea, and on the former, again, Diatomaceæ. One of these Diatomaceæ, which, from its resemblance to a scale insect, has received the name of Cocconeis Pediculus, is especially conspicuous, and is often found by the score upon the green filaments of Algæ. Such a connection does, no doubt, suggest the idea that the Cocconeis drains the green algal cells of nutriment; nevertheless, such an assumption is not well founded, and if algæ, beset by Cocconeis, derive injury at all from their presence, it is chiefly owing to a restriction of their absorption of nutrient substances from the surrounding water and to interference with their respiration.

The distinctive property of true parasites does not lie, therefore, in the habit of growing upon other plants and animals, or even in the fact of killing their living supports, but resides exclusively in the withdrawal of nutrient substances from the living vegetable or animal bodies which they invest.

The plants and animals attacked and drained of their juices by parasites are called hosts.

From the point of view of food absorption, true parasites may be classified in three groups. The first group includes generally all microscopic forms which live in the interior of human beings and animals, chiefly in the blood; the second comprehends fungi possessing mycelia, which have the power of withdrawing by the entire surface of their filamentous cells, or by clavate outgrowths of the same, nutritive material from the tissues of the host invaded by them; and the third group comprises flowering plants wherein the seedling, upon emerging from the seed, penetrates into the host, by means of suction-roots or some other part which subserves the function of a suction-root, so as to absorb juices from the host.

\section{BACTERIA. FUNGI.}

In treating of parasites of the first group, we must, in the first place, refer to several of the unwelcome visitors known by the name of Bacteria. They appear to be invariably unicellular, sometimes spherical, sometimes shortly cylindrical or rod-shaped; some are straight, and others curved in ares or spirals; a few are nonmotile, whilst some are actively motile. The largest forms have a diameter of $\frac{1}{500} \mathrm{~mm}$; the smallest do not measure more than $\frac{1}{2000} \mathrm{~mm}$., and are reckoned amongst the minutest organisms hitherto revealed by the aid of the best microscopes. In liquids of suitable chemical composition and temperature, they multiply with extraordinary rapidity, reproduction being effected by division. The rodshaped cells elongate somewhat and divide into two equal halves, each half, when grown to a certain size, divides once more into two, and so on without limit. VoL. I. 
The process is of the nature of a repeated splitting of the cells, and this is the origin of the name of Fission-fungi (Schizomycetes) used to designate these organisms. It has been observed that within 20 minutes a bacterium-cell grows enough to be able to divide or split into two, and hence it has been calculated that from a single cell, under favourable external conditions, upwards of 16 millions of similar cells are produced in 8 hours; and in 24 hours many millions of millions.

It is this very capacity for rapid multiplication that gives so great an importance to Bacteria as parasites. For multiplication can only take place at the expense of the juices and nutrient substratum in which they live. If this nutrient substratum is to afford materials for constructing the millions of millions of cells produced within two periods of 24 hours, a far-reaching transformation is inevitable. Now, for certain bacteria, the blood, with its albuminoid compounds and carbohydrates, is an extremely favourable medium of nutrition; moreover, the temperature of the blood of men and other mammals $\left(35^{\circ}-37^{\circ} \mathrm{C}\right.$.) could not be more suitable for the development of bacteria. Hence, it is readily intelligible that if a single parasitic bacterium-cell gets into the blood, it may be the origin of innumerable other cells, and that these are in a position, in a comparatively short time, to alter and decompose the whole mass of the blood. Owing to their extraordinary minuteness, bacteria are able to penetrate from outside into the channels of the blood by a number of spots; every abrasion, pin-prick, and sore place, may become an entrance-door; so, too, through all the external orifices of the various canals in the bodies of men and animals, the bacteria can enter, especially through the passages to the respiratory organs - and it becomes more and more probable that bacteria, diffused in the air, are in the main introduced into the respiratory organs by the process of breathing, thence penetrating into the finest blood-vessels, the so-called capillaries, and so pass into the current of the blood.

As regards the parasitic action of bacteria when they have penetrated into the bodies of men and animals, the supposition is that the protoplasm of each bacterium works as a ferment upon the environment, splitting up the chemical compounds in immediate proximity to it, and attracting and incorporating such products of the decomposition as are necessary for its own growth. Parasites with this method of operation act, at all events, much more destructively than those which, although they too absorb part of the host's juices, yet do not enter upon the necessary decompositions until the juices have passed into the cavities of their own bodies, and, therefore, do not alter the constitution of the unabsorbed residue. When the component parts of the blood are split up and resolved by bacteria, the nutrition of the host must be especially disturbed, and so must all the functions of the organs through which the blood perpetually circulates. Ultimately it may culminate in the organs ceasing to exercise their functions, and in the death of the host. When one remembers how fast the blood is pumped by the heart's action into every part of the body, it becomes intelligible how bacteria, possessing the power of decomposing the blood, may also cause the death of the host at very short notice, as we have occasion to observe whenever there is an epidemic of cholera. 
That numerous diseases affecting men and animals are caused by bacteria is established beyond question. Indeed, the conviction is gradually gaining ground that all infectious illnesses are occasioned by bacteria, and that the contagious matter which used to be called virus or miasma, but as to the nature of which people formerly had only very confused notions, consists of parasitic bacteria. Different phenomena in organisms in which illness has been induced by infection point to differences in the decompositions effected by the bacteria. But a particular kind of parasitic cell can only set up the same decomposition in any given liquid. If, therefore, the products of separation or decomposition vary in one and the same liquid, this can only be attributed to a difference in the impetus causing decomposition, and therefore to a difference in the parasitic cells; in other words, we are justified in assuming that every distinct infectious disease is due to a special kind of parasitic bacterium. This assumption is believed to be warranted even when no difference in the form of the bacteria is to be discovered which is discernible to sight or demonstrable by the expedients of research.

Most of the parasitic bacteria regarded as causes of diseases in man and beast are moreover capable of being very clearly distinguished from one another by the shape of their cells. The bacterium supposed to be the cause of diphtheria (Micrococcus diphthericus) presents itself in the form of minute spherical cells crowded together in close masses. The bacterium which causes anthrax in cattle (Bacterium Anthracis) has straight rod-like stationary cells. In the blood of people suffering from relapsing typhus, infinitesimally fine spiral filaments (Spirochaete Obermeieri) are found during the fever, whilst in the intestines of cholera patients, the commabacilli, so frequently described, occur; and in these cases, likewise, the organisms are brought into causal connection with the illnesses mentioned respectively. The answer to the question as to whether parasitic bacteria are developed and propagated in dead bodies also, thus becoming saprophytic, and, in general, the detailed description of the organisms, which are so important a factor for the weal or woe of humanity, are reserved for another section.

The second group of parasitic plants, according to the classification above given, includes several thousands of different kinds of moulds, toad-stools, and Discomycetes, which, notwithstanding great diversity in the conditions of life, dissimilarity in the history of their development, and endless variety in the form of their fructifications, yet exhibit great uniformity in respect of food-absorption and in their methods of attacking and draining their hosts. Spores, conveyed by currents of air or carried by animals, germinate under the influence of atmospheric moisture wherever they happen to come to rest. Tubular thin-walled cells, called hyphæ, emerge from them and endeavour to grow into the stems, branches, leaves, or fruits of the host, sometimes horizontally, sometimes from above downward, sometimes up in the opposite direction. Many select spots where the resistance offered is nil or only very weak: they grope about on the surface of the host until they find a stoma, and then use it as an entrance, and so enter the passages and lacunæ, of which the stomata are the orifices. Others seek out places where the 
surface of the plant serving as host has become broken-wounds occasioned by animals, violent wind, hailstones, or the weight of superincumbent snow-and use these as means of ingress. Yet others adopt the shortest route by breaking through the wall and so effecting an entrance for themselves. The tips of the hyphæ and also of the outgrowths developed by them have the power of decomposing and destroying the membrane of cells in the living plant serving as their host. At the spots to which they apply themselves, little gaps are shortly produced in the cellmembranes, and through them the hyphæ penetrate, either in their entirety or by means of special processes, into the interior of the cells attacked. In this operation it does not matter whether the hypha concerned has just emerged from a germinating spore or is a ramification of a mycelium several years old, which has been quiescent for a time and then begun to germinate again vigorously; the power of perforating cell-walls is a property possessed by the one as much as the other.

The aspect of the host's epidermal cells at the places where the hypha comes into contact with its victim is, on the other hand, not quite such a matter of indifference. For plants liable to become hosts are not without contrivances for protecting themselves against intruders. Thus their epidermal cells have their external walls greatly thickened and invested with cuticle. Although the main object of this is merely to afford protection against excessive transpiration and desiccation of cells filled with sap, a thickening of the kind constitutes also a coat of armour which is not liable to be broken through by every hypha. Still greater security is afforded by a double or triple layer of thick-walled cells destitute of sap, such as a solid corky bark. Coats of this kind are not penetrated even by the most vigorous hyphæ. In order to gain admittance notwithstanding, many force their conical tips into the fissures and crannies of the bark, push the peeling scales apart or even burst them, and so succeed ultimately in reaching parts which are susceptible of being pierced and allow the hyphæ to conduct their mining operations with effect. In the majority of cases the parasite is not content with perforating and exhausting the superficial cells alone of the host; its hyphæ grow faster as they penetrate deeper, a process generally accomplished irrespective of the number or direction of the partition walls in their way. Thus the hyphæ of Polyporeæ, which are parasitic in the wood of living trees, penetrate whole series of cells, now growing through a bordered-pit, now piercing the uniformly thickened part of the wall of a wood-cell (see fig. $32^{3}$ ). Others, as, for instance, the Peronosporeæ, prefer to bury themselves in the passages between individual cells, i.e. in the so-called intercellular spaces. The hyphr imbedded in this way then develop lateral outgrowths which perforate the walls of the cells adjoining the intercellular space, and upon entering the interior of the cells swell up to the shape of a club (see fig. $32^{1}$ ). By means of these clavate or almost spherical excrescences, which are named haustoria, the parasite sucks the substances required for its own nourishment from the living substance of the penetrated cells.

The hyphæ of the above-mentioned parasitic fungi have the peculiarity that in 
proportion as the one end elongates the other dies away. Hence the same effect is produced as if the progressive motion of these hyphæ were like that of ship-worms. This impression is particularly strong in cases wherein one part of the mass of wood attacked exhibits hyphæ occupied with their mining operations and growing through partition walls, whilst the other part has been the scene of past activity, and exhibits numbers of drilled holes, but no longer any trace of hyphæ. The fact that a plant is thus invaded internally by the parasitic mycelia of fungi is not always betrayed by its external appearance. Sometimes the hosts remain somewhat backward in development, but this circumstance might be just as well due to other causes, perhaps to unsuitability of situation. It is not till the mycelia need once
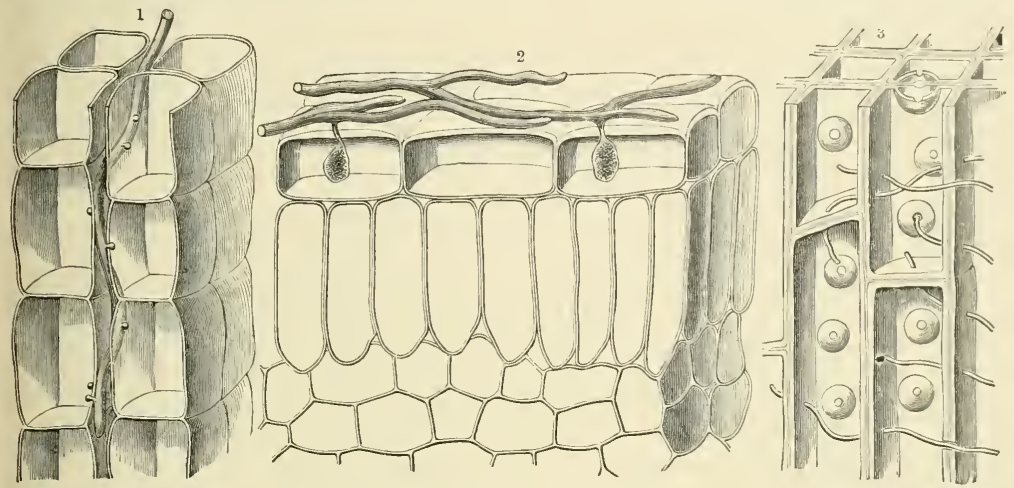

Fig. 32. - Hyphæ of Parasitic Fungl.

1 Of one of the Peronosporeæ. 2 Of a Mildew. ${ }^{3}$ Of one of the Polyporeæ.

more to multiply and distribute their kind that they emerge partially from the host; they then lift their spore-forming hyphr above the surface, leaving it to the wind to distribute the spores as they are detached.

This process vividly recalls the similar behaviour of those water-plants which, in a similar manner, vegetate submerged for months, and only come to the surface at the flowering and fruiting seasons, in order to expose their flowers to insects, and their seeds to the breeze. We are also reminded of the saprophytic orchids already described, which nourish themselves and grow for years imbedded in the humus of woods, and then seize the opportunity afforded by a favourable summer to raise up in a few weeks flowering stems above the bed of the forest. As a rule the spore-bearing hyphæ, emerging from the hosts of parasitic fungi, are highly conspicuous both in form and colour. As well-known instances we may here mention the powdery, rust-coloured, chocolate-brown, or coal-black masses of spores, known by the names of rust and smut; the mealy, orange-coloured masses which make their appearance on the green stems and fruits of roses (Ecidium stage of Phragmidium subcorticum), and the discomycetous Peziza Willkommii, which is parasitic in the branches of green larches, and exposes its fructifications beyond 
the bark in the form of small scarlet shields. Again, we have the yellow Polyporus sulfureus with its immense yolk-coloured, bracket-like fructifications, which in the space of a week grow out from the trunks of larches, although the outward appearance of the host gives no indication of its being completely occupied internally by a mycelium. Polyporus betulinus and $P$. fomentarius likewise grow to a considerable size, and in both cases it is specially deserving of notice that the colour and structure of the surface of the fructification is surprisingly like the bark of the trees upon which they are respectively parasitic; that is to say, the fructification of Polyporus betulinus strongly resembles the whitish bark of the birch, and that of Polyporus fomentarius, parasitic on old beech-trees, exhibits the same pale gray as does the trunk of a beech.

Mildews form in some respects a contrast to these parasites whose hyphæ penetrate into the interior of their hosts. They attack tender green leaves, stems, and young fruits, and accomplish their entire development upon the epidermal cells of the hosts. At first sight the parts assailed appear to be strewn with flour or dust from the road. But on closer inspection a delicate weft is to be distinguished, composed of filaments ramifying extensively upon the green substratum, intersecting one another, uniting to form reticula, and in parts a regular felt-work covered at certain spots with the small dark spheres of the sporocarps. Individual hyphæ of this weft adhere closely to the epidermal cells of the host, dissolve the outer walls of these cells at the points of contact, so as to make little apertures, and then develop processes which grow into the interior of the epidermal cells in question, assume a club-like form, and exhaust the cell-contents. The mycelia of mildews do not penetrate into the host beyond the epidermal cells. Fig. $32^{2}$ shows a piece of a leaf of Acanthus mollis attacked by mildew, with hyphal suckers penetrating into the epidermal cells of the leaf. One of the best-known mildew fungi is the Vine-mildew (Erysiphe Tuckeri), which weaves itself over the epidermis of still green and unripe grapes, and has frequently manifested itself through the districts where the vine is cultivated in southern and central Europe in the form of a ravaging disease.

The protuberances sent by the hyphæ, in the form of clavate swellings, or more rarely winding tubes, into the cells of the host-plants, correspond to the absorptioncells of land plants, and the conditions under which suction takes place are essentially analogous in the two cases. The absorption-cells on the roots of land plants do not take in all the substances in their nutrient substratum, and similarly the hyphr only appropriate by means of their organs of suction a portion of the contents of the cells invaded. They begin by dissolving and breaking up for this purpose the substances in the infested cells of the host. What compounds they then select from among the pioducts of decomposition, and what they leave behind, cannot certainly be specified in detail. It is believed that, in many cases, tannin is appropriated first of all by parasites. The wood of a healthy oak, for instance, has a characteristic smell due to the abundance of tannin it contains, whereas this odour is not emitted by wood attacked by the mycelia of fungi, and this decayed 
wood is destitute of tannin. It is natural to suppose, therefore, that the mycelium takes away and uses up the tannin. It has also been observed that wherever the hyphre of the Pine-blister (Peridermium Pini) ensconce themselves, the nitrogenous parts of the protoplasm and the starch vanish, whilst turpentine remains behind, clinging in drops to the inner walls of the cells. These are, to be sure, very sparse data; but they show that the entire cell-contents are not absorbed by the parasite unaltered, or used in that condition as material for the building up of its own body.

Not only the contents of the cells preyed upon, but the walls as well, are partially used as food by the hyphæ which penetrate into the woody axes of arborescent angiosperms and gymnosperms. The mycelium of several species of Polyporus and Trametes begins by bringing the lignin in the cell-walls into solution, leaving nothing but a pale-coloured cellulose wall. Soon afterwards, the so-called middle lamella, which connects adjoining wood-cells, is also dissolved, and the colourless wood-cells, now almost like asbestos-fibres in appearance, fall apart at the slightest touch. When the wood of a larch has been infested by the mycelium of Polyporus sulfureus, there are always deep furrows running obliquely on the internal walls of the wood-cells; this loss of substance, too, can only arise from the solution, and absorption as nutriment, of parts of the walls by the action of the hyphr.

All decompositions and alterations of structure of the above kind within the precincts of the host's cells are naturally followed by a disturbance of function, and ultimately by death. The entire plant is, however, but rarely killed by parasites belonging to this group. The decomposition by bacteria of a mammal's blood, though at first confined to a particular part of the body, spreads in a moment throughout the whole organism, owing to the heart's action and the circulation of the blood. But the decomposition taking place in the manner just described, through the intervention of hyphæ, propagates itself, on the contrary, only very gradually from the cells immediately attacked to their neighbours, and it gets weaker and weaker as the distance from the site of the invasion increases, a circumstance to which we shall recur later on when discussing the phenomena of fermentation and decay. The nature of the parasite and the power of resistance of the host have an undoubted influence on the rate of distribution. In many cases alteration is limited to the cells attacked and those immediately adjoining, so that the area destroyed is circumscribed. It is manifested on fresh green leaves, often merely in the form of small, isolated, yellow, brown, or black spots and patches, which only slightly interfere with the activity of the leaf, and do not cause it to change colour, wither, or fall off any earlier. In other instances, however, the entire leaves and stem do undoubtedly become flaccid and shrivelled and dried up into a black mass, looking as though they had been carbonized; or else putrefaction, such as that which is excited by bacteria, invades the whole mass.

As above stated, when the wood in the trunks of trees is perforated and consumed by hyphæ it is resolved into fragments. It becomes rotten, takes the form of an asbestos-like or crumbling and pulverulent mass, and is then obviously no longer capable of fulfilling its various functions in the living plant. If the 
invasion is limited in extent, and the host succeeds in surrounding the area of infection with a rampart of cells capable of resistance, and not liable to be pierced by the hyphæ, then the tree may live for years although its trunk is infested, and in parts rotten. Such is also the case when particular branches of a tree are alone attacked by the mycelium of a fungus. When, for example, the branch of a larch is assailed by the mycelium of the Discomycete, Peziza Willkommii, the fact is first manifested externally by the fascicles of needles on the branch in question becoming discoloured in the summer, and acquiring, prematurely, an autumnal appearance, so that, among the fresh green shoots, individual branches are to be seen bearing goldenyellow needles. Towards autumn, scarlet cup-shaped fructifications make their appearance upon the surface of the bark on the branch; in the course of the next few years the whole branch as a rule dries up, withers, and dies. It is then broken by the first violent shock of wind and falls to the ground; but the tree, disembarrassed of the dead bough, continues to grow unharmed, and to put forth green shoots. It is only when almost all the branches of the larch are infested by the mycelium of this fungus that the whole tree perishes as a result of the invasion.

Certain groups of plants are specially liable to be attacked by parasitic fungi, and there are some conifers and angiospermous trees in which the same stem is colonized by three, four, or five kinds of parasite. The green foliage leaves of large numbers of flowering plants are also apt to be selected by parasites, as also are their roots, tubers, and bulbous structures. Many parasites only attack the anthers in Howers; others, as for instance the ergot, only the young ovaries. Parasitic fungi are rarely found on mosses or ferns; whereas a considerable number of parasites settle upon lichens and even on the fructifications of fungi, moulds even being infested by other fungi; for example, a fungus named Piptocephalis Freseniana is parasitic upon the very common mould, Mucor Mucedo.

A fungus known by the name of Cordyceps militaris is parasitic in the caterpillars and pupæ of butterflies and other insects, and its relatively very large fructification at length bursts out of the body infested by the mycelium in the form of a club nearly $6 \mathrm{~cm}$. long. This clavate structure, built up at the expense of the insect's flesh and blood, produces tubular cells in special receptacles, and, inside these, little rod-like spores, which afterwards fall out and infect other caterpillars, developing within the bodies of these animals into a hoary mycelium and ultimately causing their death. The disease of silk-worms, known as muscardine, is likewise occasioned by a species of Cordyceps. We must also refer here to the widelydistributed Empusa Musco, a mould which attacks flies and causes every autumn a regular epidemic amongst house-flies. The flies so often seen at that season adhering stiff and dead to window-panes are surrounded by a whitish halo, and this is composed of a conglomerate of spores thrown off by the mould which is parasitic upon the flies and causes their death. Parasitic fungi have also been observed in the human skin, and recognized as the causes of skin-diseases. For instance, to the mould Achorion Schoenleinii is due the disease of the skin popularly known as 
"honey-combed ringworm", and named Favus by doctors; dandruff (Pityriasis versicolor) is produced by Microsporon furfur, and Herpes tonsurans by Trichophyton tonsurans. The latter has a remarkable effect on the hair, causing it to fall out and leave the part of the skin affected bald.

Water-plants are attacked by parasitic fungi comparatively rarely, which is the more noteworthy because such large numbers of non-parasitic epiphytes settle upon the filaments of green algæ, and on the brown Fucoideæ, and red Florideæ. Minute
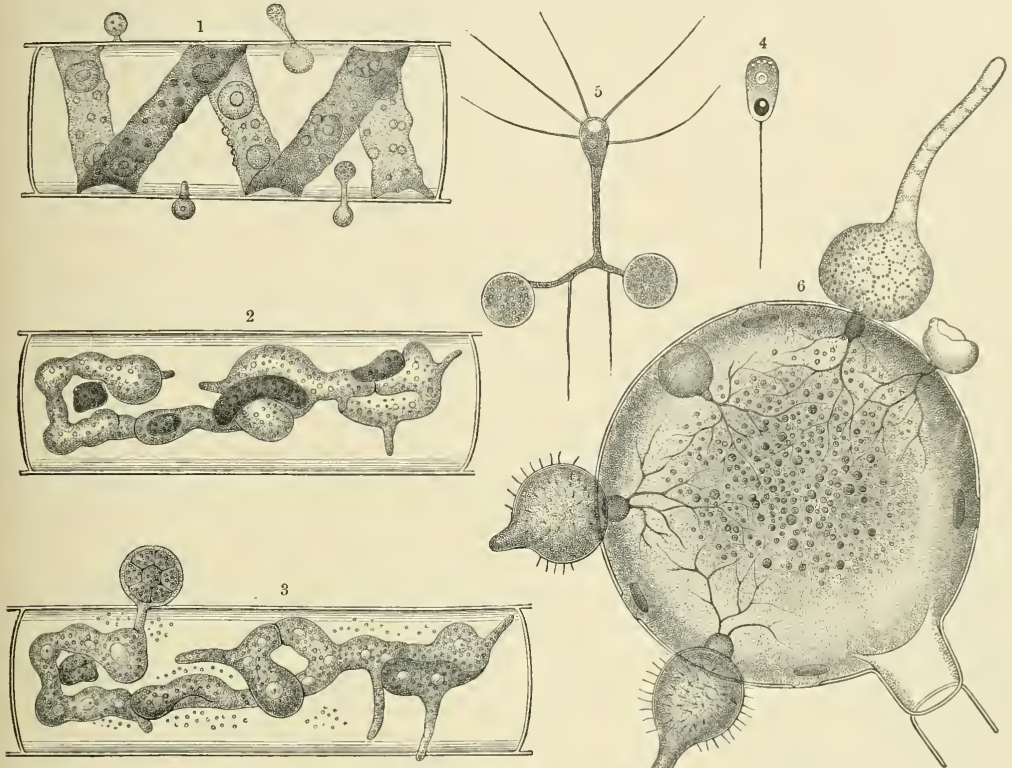

Fig. 33-Parasites on Hydrophytes.

1, 2, and 3 Lagenidium Rabenhorstii. 4, 5 Polyphagus Euglence. '6 Rhizidiomyces apophysatus.

forms of fungi, invisible to the naked eye, and belonging to the Chytrider and Saprolegniæ, are parasitic upon green algal filaments, especially on the fresh-water species of the genera Edogonium, Spirogyra, and Mesocarpus. One of these microscopic parasites is represented in fig. $33^{1,2,3}$, and bears the name Lagenidium Rabenhorstii. It develops non-ciliated, spherical swarm-spores, which lay themselves upon the walls of Spirogyra-cells, perforate them, and insert a club-like process. The protuberance forthwith becomes a tube, which increases rapidly in size in the interior of the cell, ramifying and completely destroying the bands of chlorophyll. The branched tubes of Lagenidium reproduce themselves in two ways at the expense of the host's cells infested by them: they form on the one hand so-called oospores by means of fertilization, and on the other sporangia. The latter process is clearly shown in fig. $33^{1,2,3}$. In this case, one of the tubular 
processes of the parasite fungus pushes out of the cell-cavity of the invaded Spirogyra into the surrounding water again and there swells up into a spherical vesicle, within which the protoplasm divides into eight spores. These spores are then set free as swarm-spores and attack new healthy Spirogyra-cells.

Materially different is the behaviour of the parasite Chytridium Ola, which attacks the green cells of fresh-water Edogoniæ. Its roundish swarm-spores are furnished each with one long cilium, and swim, searching about in the water until they meet with an Edogonium-cell to their taste just occupied in the formation of oospores. When they find one, they fasten upon it and send infinitesimally fine hair-like tubes (which have been called rhizoids) into the interior. By means of these tubes they derive their nutriment from the host. The body of the parasite, which remains outside the invaded cell, increases in size, and at length grows out into a sporangium; the latter opens at the top by a lid and once more sets free swarm-spores into the surrounding water.

Polyphagus Euglence, a member of the Chytrides, is parasitic on the green cells of Euglenæ living in water. The swarm spores of this microscopic fungus (see fig. $33^{4}$ ) are oval and furnished, like those of Chytridium Ola, with a long cilium. They swim about the water with the non-ciliate extremity leading, so that the cilium appears to be a tail at the posterior end. As soon as these swarm-spores have come to rest, they assume a spherical form and send out in all directions thin, hair-like tubes, which search for a host. When a tube reaches an Euglena-cell, it penetrates into the body of the latter, drains it, and, continuing to grow, produces fresh hair-like tubes, which attack other green Euglenæ, often linking together dozens of them (see fig. $33^{5}$ ). In this way the Polyphagus grows apace and becomes a comparative large oblong vesicle, whilst the protoplasm within it divides into a number of parts. These, again, turn into swarm spores, with long ciliary filaments, and they slip out of the vesicle and may attack fresh Euglenæ.

Curiously enough, even saprophytic water-plants destitute of chlorophyll are sometimes attacked by parasites, and that, indeed, by species belonging to the same group. Thus, for instance, the species of Achlya growing on the dead bodies of fishes and other animals which have perished in the water, are themselves infested by small parasitic Saprolegniaceæ and Chytrideæ. The example of these minute parasites represented in fig. $33^{6}$ is named Rhizidiomyces apophysatus, and its host is Achlya racemosa. The swarming spores of the parasite lay themselves, in the manner described in previous instances, upon the spherical oogonia of Achlya, and insert extremely fine hair-like tubes into the interior of the cells attacked. These ramify like roots in the Achlya-cells, exhaust them of nutriment, grow perceptibly, and at length form spherical swellings, which, after reaching a certain size, break through the walls of the host-cells, project from the opening, and, lastly, push out in each case a sporangium. The latter produces a number of swarm-spores, which escape into the water and are able to seek fresh prey.

We cannot here enter into details respecting the other kinds of reproduction occurring in the minute fungi parasitic upon hydrophytes. This is the right place, 
however, to mention the fact that the various species of Chytrider and Saprolegniacer do not content themselves with plants that are second-rate hosts, but exercise a selection amongst the different green algæ living in the water. It is astonishing to find that the swarm-spores invariably swim to cells whose protoplasm affords the most suitable nutrient basis for them, and attach themselves to those cells only, and never on other species unadapted to their requirements.

\section{CLIMBING PARASITES. GREEN-LEAVED PARASITES. TOOTHWORT.}

The third group into which parasites were divided at the beginning of this chapter is composed of flowering plants throughout. According to their method of attacking the host for the purpose of absorbing nutriment from it, they range themselves in six series. In the following pages we shall discuss the characteristics of each series as manifested in the most remarkable forms belonging to it.

The first series includes plants destitute of green leaves and of chlorophyll in general, whose seeds germinate on the ground and send forth each a filiform stem, which brings itself, by means of peculiar movements, into contact with the hostplant, coils round it, and develops organs of suction whereby it takes nutriment from the plant assailed.

To this series belong the genera Cassytha and Cuscuta. The former includes some thirty species, all of which appertain to warm climates. Most of the Cassythæ inhabit Australia, where they attack, in particular, the copses of Casuarinæ and Melaleucæ, fastening their wart-shaped, or, in many cases, shield-like or discoid suckers upon the young green shoots of those plants. Several species also are indigenous to New Zealand, others to Borneo, Java, Ceylon, the Philippines, and the Moluccas. South Africa, too, is the home of a few Cassythæ, and one species. (C. Americana) is distributed over the West Indies, Mexico, and Brazil. A European, seeing these parasites with their twining, thread-like, leafless stems, and their flowers aggregated in capitula, umbels, or spikes, takes them at first to be species of the genus Cuscuta, popularly called Dodder. That these plants should be most nearly related to laurel-trees is the last thing one would expect. Examination of the flowers and fruit reveals, it is true, a close resemblance to those of laurel and cinnamon trees, and, therefore, these Cassythæ are rightly placed by systematic botanists among the Lauraceæ. But in respect of food-absorption, as in general aspect, they are entirely analogous to the various species of the genus. Cuscuta, which belong to the family of Bindweeds (Convolvulacer). The lastnamed genus is even more variously differentiated than the genus Cassytha, and includes about fifty species dispersed pretty evenly over the whole world. Every part of the world has its own characteristic forms. One group occurs in California, Carolina, Indiana, Missouri, and Mexico, another in the West Indies, Brazil, Peru, and Chili, a third at the Cape of Good Hope. Other species are natives of China, the East Indies, the steppes of Central Asia, Persia, Syria, the Caucasus, and Egypt. 
A comparatively large number of species, i.e. twenty-five, are distributed through central and southern Europe. A few have been introduced recently for the first time with seeds from the New World, as, for instance, C. corymbosa, which was accidentally conveyed with lucerne seeds from South America to Belgium, and has latterly begun to range over central Europe.

The various species of Cuscuta attack chiefly small herbaceous, suffruticose, and shrubby plants; but a few American species coil themselves round branches growing at the top of the highest trees. Notice has been especially drawn to certain European species on account of their disastrous effects upon cultivated plants. The most famous is Cuscuta Trifolii, known as the Clover-Dodder, the appearance of which in clover-fields causes so much anxiety to farmers, and which is so difficult to exterminate. Another unwelcome visitor is Cuscuta Epilinum, which coils round flax stems and hinders their growth, and a third species, Cuscuta Europcea, sometimes ravages hop-plantations. This last is, indeed, the most widely distributed of all the Cuscutas, and extends from England over central Europe and Asia to Japan, and southwards as far as Algiers. It is parasitic not only on hops, but also on elder, ash, and various other shrubs and herbs; in particular it exhibits a preference for nettles.

The seeds of this species, and of Dodders in general, germinate on damp earth, on wet foliage undergoing putrefaction, or on the weathered bark of old trunks. The seedling, which in the seed lies imbedded in a cellular mass full of reservefood, is filiform and spirally coiled. It is twisted once, or once and a half, and is thickened at one end like a club. In true Cuscutas, no trace of cotyledons is to be perceived, nor does one find vessels in the interior of the seedling; but chains of cells arranged with great regularity are noticed in the axis of the filiform body, and are easily distinguished from the surrounding cells. In nature, the seeds, after falling to the ground and lying there through the winter, do not germinate till very late in the following year, i.e. at least a month later than the majority of the other seeds reaching the same ground simultaneously with them. Perennial herbs, also, have, by the time that germination takes place, already developed shoots from their subterranean roots or rhizomes above the surface of the ground, later a circumstance of great importance to the parasites. If a Cuscuta were to germinate early in the spring, it would not readily find close by a support up which to twine; whereas later, there is seldom any lack of annual stems or shoots of perennial plants in the immediate neighbourhood.

When the twisted embryo germinates, it stretches and at the same time revolves from right to left, assuming the shape of a screw and pushing its lower clavate extremity out beyond the coat of the seed (see fig. $34^{1,2,3,4,5,6}$ ). This extremity forthwith grows into the earth and fastens tightly on to particles of the soil, withered foliage, and other objects of the sort. The other, attenuated extremity of the filiform seedling, which is still wrapped in the seed-coat and the mass of reservefood, lifts itself up in the opposite direction, avoiding such solid bodies as it may happen to encounter, and grows in a curve round them. Further growth does 
not take place at either extremity, but always in the median part of the filament. It is so rapid that by the fifth day after the commencement of germination the entire seedling has increased fourfold in length. As early as the third day after the emergence of the tip that fastens itself in the earth, the integument of the seed, which until then continues to envelop the opposite extremity, is thrown off and the seedling's apex is exposed. The reserve-food, given by the parentplant to the seedling as provision for the journey, has meanwhile been absorbed and consumed, so that the seedling is now thrown entirely upon its own resources, and depends for sustenance upon the earth, to which it is firmly attached, and upon the surrounding air. Having no chlorophyll, it is not in a position to take up

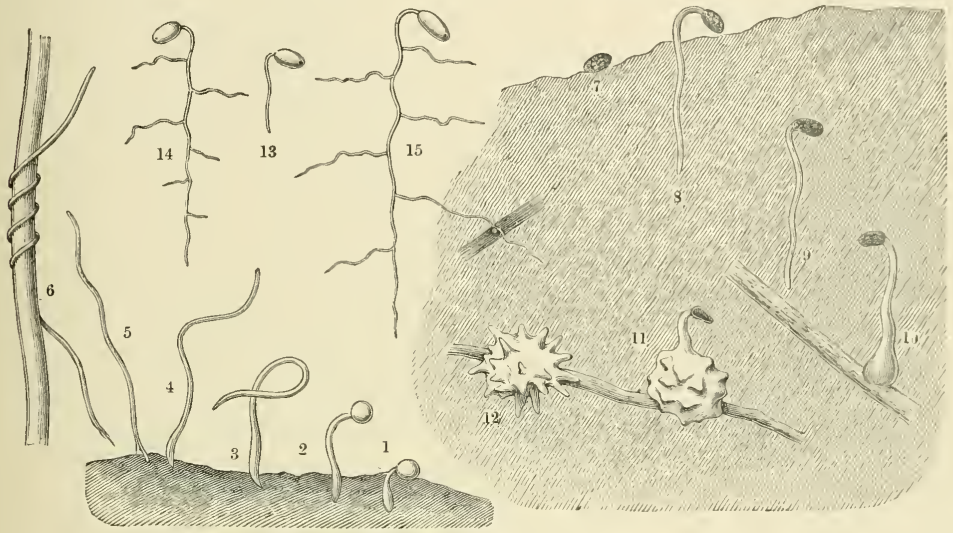

Fig. 34.-Seedlings of Parasitic Plants.

1. 2, 3, 4, 5, 6 The Great Dodder (Cuscuta Europoea). 3, 8, 9, 10,11. 12 A Broom-rape (Orobanche Epithymum). 13, 14, 15 Wood Cow-wheat (Melampyrum sylvaticum).

materials from the air; nor can it derive sufficient nutriment from the earth, even supposing that water is imbibed by the cells of the clavate extremity. There is no doubt that it now grows at the expense of the substances contained in the cells of this club-shaped end. The latter at once begins to shrivel and soon dies, whilst the upper part of the filament elongates conspicuously. Should this portion of the seedling meantime come into contact with a neighbouring plant, a rigid haulm, or anything else that will serve as a support, it straightway coils itself round the object in question, and its future is then, as a rule, assured.

Failing such a support, the seedling, after the death of the clavate extremity, falls down and sinks to the ground. In doing so, it almost invariably touches an adjoining object, whereupon it immediately winds tendril-like round the support thus afforded. But if there is nothing anywhere around to serve as a prop, and the young seedling, by this time from 1 to 2 centimeters long, comes to rest upon the bare earth, all further growth is stopped. It preserves its vitality, however, for a surprisingly long time, and may remain almost unal- 
tered, lying on the damp earth for four or five weeks waiting for something to turn up. Not infrequently something of the sort happens, for another plant may germinate close by or extend a growing shoot from the vicinity and touch the Cuscuta seedling. In this event, the latter at once seizes the anchor thus thrown out, and winds round it. But if no support of the kind is to be had, the seedling must ultimately perish. It is, to say the least, a very remarkable thing that a filament, capable of developing suckers when adherent to a living plant, is not able in damp earth to produce any absorbent organs whatsoever.

If the thread-like Dodder plantlet succeeds in seizing a support of any kind, either during the existence of the swollen extremity, or later, after it has been absorbed, it makes a single, or from two to three, coils round the prop, raises its growing point from the substratum, and moves it round in a circle like the hand of a watch. By means of these manœuvres, which look exactly like a process of feeling or seeking, the filament is brought into contact with fresh haulms, twigs, and petioles belonging to other plants. To these it adheres, making once more two or three tight coils round them. Throughout, it is obvious that the growing point of the young Dodder rejects dead props, as far as is practicable, and shows a striking preference for living parts of plants.

At each place where the Dodder is pressed in a coil against the support, the filament becomes somewhat swollen, and wart-like suckers are developed, which are usually situated close together in rows of three, four, or five (see fig. $35^{1}$ ).

A piece of stem thus furnished with suckers or haustoria resembles a small caterpillar creeping up the supporting stem. These haustoria, arranged close together in rows, and corresponding in origin entirely to rudimentary roots, are at first smooth, but acquire soon a finely-granulated aspect owing to the walls of the epidermal cells projecting outwards. With the help of the papillæ thus formed, and especially through the action of a juice secreted by them, the suckers fasten themselves to the host. If the plant has been obliged to clasp a dead object for support, the wart-like processes flatten themselves against it and assume the form of a kind of disc, which exhibits no further development, and only serves as an organ of attachment; but, if the substratum is a living plant, a bundle of cells forces its way out from the middle of the haustorium and grows into the substratum direct. The phenomenon here manifested is altogether characteristic. Each sucker from the time of its production exhibits a kind of core composed of cells arranged in regular rows, which, together with a few spirally-thickened vessels, constitute a bundle standing at right angles to the axis of the Dodder's stem. This bundle now breaks through the coat formed by the rest of the cells of the sucker and penetrates into the living tissue of the plant attacked (see fig. $35^{2}$ ). Great force is exerted in the penetrating process. The closely-joined cells of the epidermis, and not infrequently a cortex of considerable density are pierced, and the bundle of cells often penetrates right into the body of the wood. Having once reached the interior of the host, the cells, till then bound together in a bundle, diverge a little, insert themselves singly between the cells of the host, 
and energetically absorb food-materials. They withdraw organic compounds from the host and convey them by a short route to the strands developed meantime in the axis of the Cuscuta-stem. When once a union of this kind between the parasite and the host has been established, the portion of the Cuscuta situated below the first haustorium gradually dies. The lowest extremity, i.e. the clavate tip, has already perished, so that the Cuscuta-plant is now no longer in any connection with the ground whereon it germinated, but only remains rooted to its living host by means of the suckers. If it has had the good fortune to cling to a host with green foliage, which generates an abundance of organic compounds, such as the luxuriant juicy stems of the Hop, or the Nettle, with its
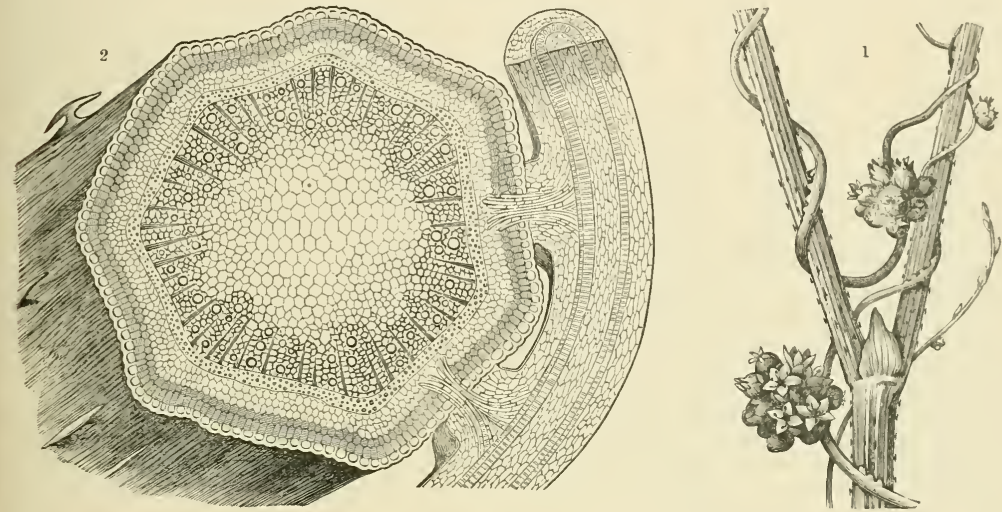

Fig. 35.-Cuscuta Europoea parasitic on a Hop-stem.

1 Natural size. 2 Section; $\times 40$.

plentiful dark green leaves, which are shunned and spared by grazing animals on account of their unpalatable stinging hairs, the parasite continues to grow with extraordinary rapidity, and puts forth a number of branches immediately above the lowest group of haustoria. All these again feel around with their tips, develop tendrils and suckers, sometimes intertwining and becoming entangled together, cover an ever-increasing area of the host with their network, and in this condition fully deserve the name of "Hell-bind", sometimes popularly applied to this plant Little spheres of rose-coloured flowers are then formed on individual threads of this tangle, and from them balls of small capsular fruits, which dehisce by means of lids and have their seeds shaken out by the wind.

The European species of Cuscuta are all annuals. Even when their haustoria are attached to perennial plants, as, for instance, on young branches of woody plants, they wither after the seeds have ripened, and nothing is to be seen of them in the following spring except a few dried tendrils coiled round branches of ash or willow. But under a tropical sun, perennial species flourish as well. The suckers of Cuscuta verrucosa, for example, continue to exercise their function 
throughout the year wherever they have once attacked the host. If the woody branches of the host, with haustoria fastened in them, grow in thickness and superimpose new wood-cells upon the wood, down to which the absorbent cells of the haustoria have penetrated, these suction-cells of the Dodder are likewise inclosed by the wood-cells, and, in proportion to the augmentation of the circumference of the wood in the branch in question, they also lengthen out so that the bundle of absorption-cells proceeding from a sucker may, in such cases, be seen imbedded in the wood to a depth of several annual rings.

The Cassythæ, referred to above, behave exactly like the Dodders. In them also the seedling which issues from the seed is filiform, and lives originally at the expense of reserve-food stored up within the coat of the seed. So, too, it grows upward, ramifies, and endeavours, by means of revolving movements of the apex, to reach a living support, coils round the latter when found, and uses it as a nutrient substratum. Here, again, at the parts where the tendrils of the filiform stem are firmly appressed to the living support, rows of wart-like suckers are developed, and a bundle of absorption-cells grows from eash into the host. As in the Dodder, the lower extremity of the filiform stem then dries up at once, and connection with the earth is thus cut off. The parasite, once attached by its haustoria to the host, is able to branch repeatedly, to weave its thread-like stems over all the branches and to climb to the top of the host, even should the latter be a tall bush. At some spots everything is entangled to such an extent that one would think there were birds' nests amongst the boughs.

The second series of parasitic Phanerogams consists of herbs bearing green foliage-leaves, whilst the seed contains an embryo furnished with seed-leaves (cotyledons) and root. The seeds germinate in the earth and there develop seedlings without the support of a host; it is branches of the root that first attach themselves by means of suckers upon the roots of other plants. To this series belong about a hundred Santalaceæ, mainly of the genus Thesium, and many more than two hundred Rhinanthaceæ besides. The chief examples of this latter family are the various species of the Eyebright (Euphrasia), the Yellow-rattle (Rhinanthus), Cow-wheat (Melampyrum) and Lousewort (Pedicularis), and also Bartsia, Tozzia, Trixago, and Odontites. The most extensive genera are Euphrasia and Pedicularis, the species of which, with few exceptions, are found in the northern hemisphere, adorning grassy meadows with their pretty flowers, especially in the arctic zone, and the high mountain regions of the Himalaya, the Altai and Caucasus, the Alps and the Pyrenees.

Little suggestion of parasitic habit is given in the first stages of development of any of these plants. A seedling of the Cow-wheat within a week puts forth a primary root $4 \mathrm{~cm}$. long, from which half a dozen lateral roots ramify at right angles without there being any attachment to a host to be noted (see fig. $34{ }^{13,14,15}$ ). Suckers are never developed until the secondary roots have attained a length of from 12 to $24 \mathrm{~mm}$., and then only if the latter come into contact with other living plants to their taste, a circumstance which doubtless is almost certain to happen, 
seeing that the lateral roots are numerous and are sent out in all directions from the main root, and therefore must inevitably come across the root-systens of other plants.

The seedling in perennial species of Thesium develops comparatively slowly. It reaches a length of from 3 to $4 \mathrm{~cm}$. in the first year, sends a tap-root into the earth, and puts forth a few branchlets, which do not fasten upon the roots of other plants by means of suckers until several weeks after germination. These suckers are relatively large in all species of Thesium, and they catch one's eye the moment the roots of a plant are carefully divested of earth. They are then recognized, as may be seen in fig. $36^{1}$, as little white knobs, which stand out clearly from the dark earth and are always inserted laterally upon the secondary roots. They are
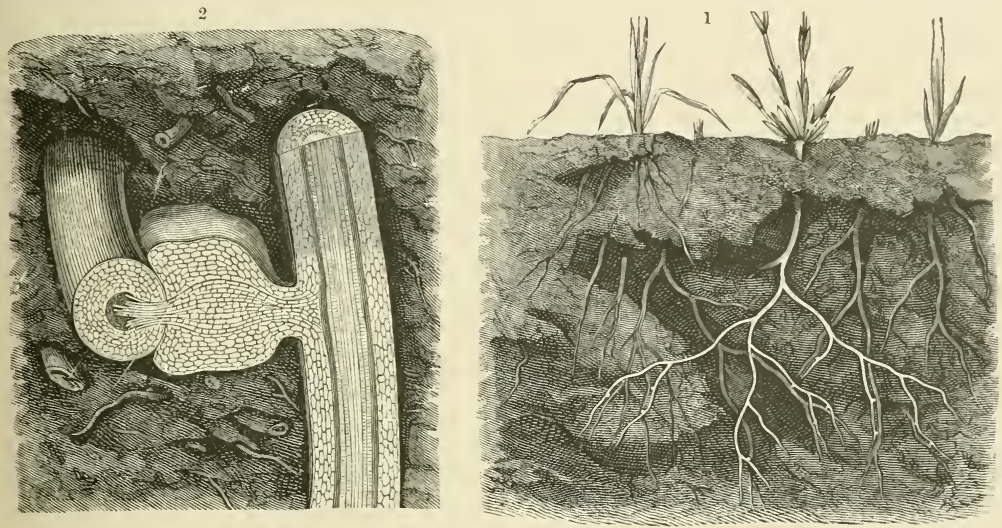

Fig. 36. -Bastard Toad-flax (Thesium alpinum).

1 Poot with suckers; natural size. 2 Piece of a root with sucker in section; $\times 35$.

constricted near their insertion, and the strangulated portion often gives the impression of being a pedicel upon which the knob is seated. This knob is differentiated into a central core and a multicellular, cortical coat enveloping it. The cellular coat rests upon the root of the host attacked, and does not merely adhere to one limited spot, but spreads itself out over the root like a plastic mass, and forms a cushion surrounding about a fourth or fifth part of the eircumference (see fig. $36^{2}$ ) without, however, penetrating into the substance of the root. There are in the core two strands or bundles of vessels, and between them small cells arranged in rows, from which absorption-cells arise at the spot where the sucker first applied itself to the nutrient root. These absorption-cells grow out beyond the rind-like envelope round the core, perforate the cortex of the host, penetrate into the wood at the centre of the invaded root, and there diverge like the hairs of a dry paint-brush.

The suckers of the green-leaved Rhinanthaceæ are on the whole similarly constructed; only they are relatively smaller and more delicate, being sometimes almost translucent, and they are either not at all or only slightly constricted at the VoL. I. 
base. Whereas in Thesium they never issue otherwise than laterally from the ramifications of the roots, in Rhinanthaceæ they are often terminal. A differentiation into core and rind-like envelope is never clearly marked; a vascular bundle runs through the middle of the sucker and is surrounded by thick-walled cells. The absorbent cells are, moreover, shorter than in the Santalacea. The individual genera of the Rhinanthacer exhibit amongst themselves only very slight differences in respect of their suckers. On the roots of Eyebright (Euphrasia), the haustoria are tiny roundish nodules which rest upon the host's root without encompassing it. The absorption-cells are very short, and only just penetrate into the host. The vascular bundle is either entirely wanting within the sucker, or its place is taken by a single, comparatively large vessel. On the roots of the Yellow-rattle (Rhinanthus) the suckers are spherical and of considerable size (up to $3 \mathrm{~mm}$. in diameter); their margins are swollen and often encompass more than half the circumference of the roots attacked. The absorbent cells are short but very numerous. In the Cow-wheat (Melampyrum) the suckers resemble those of the Yellow-rattle in size and shape and in the shortness of the absorption-cells; but in the former the margins of the suckers not only embrace the roots of the host, but cling to them in such a way as to penetrate their substance and form circular grooves upon them.

All the Rhinanthaceæ mentioned are herbaceous annuals. Their suckers are few in number, and therefore easily escape observation. By the time these plants ripen their seeds any piece of a root that has been attacked has for the most part already turned brown and been killed, and is in a state of decay. But shortly aftcrwards the parasite itself withers. The comparatively large seeds, wellfurnished with reserve-material for the nourishment of the embryo, fall out of the dry capsules, and generally reach the ground at no great distance from the motherplant and germinate there. In the autumn, close to Cow-wheat plants, which are still green but have already let fall the seeds from their lowest capsules, individual examples of those seeds may be seen already sprouting in the damp moss and mould on the ground of woods. If they fall to earth not very far from the parent-plant, the seedlings may happen to attack the host which has already had one of the branches of its root sucked and killed by the latter in the previous summer.

Nearly all these annual green-leaved parasites make their appearance in numbers close together. If, for instance, a species of Cow-wheat has taken up its quarters in a particular part of a wood, there are always collections of hundreds and thousands of specimens to be found together. The small-flowered Yellowrattle often grows so abundantly in damp meadows that one might suppose it to have been sown by the bushel. The large-flowered, hairy Yellow-rattle is similarly exuberant in ploughed fields, and the Eyebright, with its large number of species, is produced in such abundance in mountainous districts that, at the season when its little milk-white flowers are open, regular milky ways seem to stretch across the green meadows. Millions of them are situated together rooted in the grass-covered ground, and one would suppose that in course of time the growth of 
grass at such places would be injured. This conclusion appears to be supported by the assertion of the country folk that after the season when the Eyebright is in full bloom, the cows yield less milk, a fact which explains the German name of "Milchdieb" (milk-thief) popularly given to the plant. The diminution in the quantity of milk yielded is, however, certainly connected with other circumstances. It depends especially upon the universal abatement of the growth of grasses in early autumn and the consequent curtailment of the food afforded by the pastures. The injury done by the Eyebright to its hosts by the withdrawal of nutriment and destruction of rootlets cannot be very considerable, for the appearance of the grasses and other host-plants, which are affected, is not noticeably different from that of the plants of the same kind which escape invasion.

The same statement is true in the case of the various species of Lousewort (Pedicularis), almost all of which are meadow-plants; that is to say, they are present in great abundance in upland and alpine pastures without apparently injuring the species growing in their company and used by them as hosts. Unlike the species of Cow-wheat, Yellow-rattle, and Eyebright, however, nearly all the Louseworts are perennial, and accordingly differ from them also in the construction of their suckers. There is, it is true, no difference in shape between the suckers of the Cow-wheat and those of Pedicularis, but they are dissimilar in respect of size and place of origin. The suckers of the perennial Louseworts are barely more than half the size, and are only developed near the attenuated extremity of a rootlet. They are very few in number; each of the long, thick, fleshy rootlets, proceeding from the base of the stem usually produces a single sucker only which settles upon the root of a suitable host-plant in the same way as the suckers of Cow-wheat. By the time that the parasite's fruit ripens, the piece of root which has been invaded has usually already turned brown and fallen into decay. Now in the case of Cow-wheat it may undoubtedly be immaterial whether the piece of root attacked by it is living or not when its fruit is ripening, inasmuch as its own annual root rots as soon as the seeds have been produced from the flowers above ground. But with Pedicularis it is different. The perennial roots of this plant require a host to nourish them next year, and when the piece of a host's root which has been attacked and sucked as a nutrient substratum one year dies, the sucker belonging to the root parasitic upon it is no longer in a position to fulfil its function by continuing to absorb fresh juices. Suckers thus reduced to a state of quiescence soon perish, and only leave little scars to indicate the places where they existed. The perennial root of the Pedicularis has now to seek a new source of nutriment, and this is effected by the elongation of its tip, which continues to grow until it reaches the living root of another plant suitable as host, whereupon it develops a fresh sucker upon that root. This elongation doubtless requires a large quantity of plastic materials; but these are found stored in abundance in the older parts of the parasitic root.

These circumstances explain, at anyrate in part, the characteristic structure and disproportionate length of the roots of Pedicularis. From all round the short erect 
root-stock, which is generally only from $\frac{1}{2} \mathrm{~cm}$. to $2 \mathrm{~cm}$. long, issue fleshy rootlets of the thickness of a quill, but, in many species, as long and thick as a little finger. These rootlets are abundantly supplied with starch, and, in course of time, elongate till they measure $20 \mathrm{~cm}$. They radiate in all directions in the black soil of the meadow, wherein are buried the root-systems of grasses, sedges, and various other plants, and fasten on to suitable hosts by means of one or two suckers yearly, and repeating this process until at length their tips travel into earth devoid of roots, where no more prey is to be found, and there growth ceases. This explains also why these long Pedicularis-roots never descend vertically in the earth, but remain only in the upper strata of soil on a meadow, where a number of other roots are interwoven together, and where it is most likely that the tapering growing-point will meet with the root of some new host or other.

The Alpine Bartsia (Bartsia alpina), one of the perennial Rhinanthaceæ prevailing in the arctic regions as well as in mountainous parts of Europe on damp, marshy, grass-covered spots, is distinguished by the sombre dusky violet colouring of its leaves, and has already been noticed amongst carnivorous plants. On the secondary roots are suckers exactly like those of the Yellow-rattle (Rhinanthus), and by means of these organs it clings to the fibrous roots of sedges and grasses, and sucks their juices. The long, subterranean, runner-like stems, which are covered with small, whitish scales, also bear, however, elongated absorption-cells (root-hairs), which are distinctly differentiated, and take up nutriment from the vegetable mould around. This Bartsia is, therefore, half-parasitic and half-saprophytic, and it is not improbable that many other perennial Rhinanthaceæ behave in the same way.

The species of Pedicularis which constitute the most extensive group of perennial green-leaved and parasitic Rhinanthaceæ are, it is true, destitute of tubular absorption-cells (root-hairs) whether on the subterranean stem-structures or on the root-tip, with the exception of those which develop in the middle of the suckers. But the construction of the epidermal cells on the roots, and the circumstance that these epidermal cells are always in intimate connection with dark particles of humus, would favour the idea that these plants are capable of taking up organic compounds from the mould of meadows in addition to the food acquired by means of suckers from their hosts. This supposition is further supported by the fact that I succeeded in rearing a species belonging to the Rhinanthaceæ, namely, Odontites lutea, from a soil composed of a mixture of sand and humus, in which no other plants were rooted, so that the possibility of a withdrawal of nutritive matter from hosts was excluded. It is true that the plants thus reared remained comparatively small and poor, and only developed few flowers and fruits. But at anyrate they may be considered to prove that plants exist, which, though normally parasitic, are yet on occasion able to subsist in vegetable mould without the assistance of hosts.

The third series of parasitic flowering-plants is very restricted, contrasting in this respect with the second series, composed of the numerous green-leaved Santalaceæ and Rhinanthaceæ. The species belonging to it differ from those of the 
second series chiefly in their lack of chlorophyll. They all live underground on the roots of trees and shrubs, develop deep down in the earth a number of flowerless perennial shoots thickly covered with scales, and, in addition, push up annually into the light temporary axes bearing flowers, which ripen their fruits and die after the fall of the seed.

As the best known representative of this series, we may take the Toothwort (Lathraa Squamaria), which is represented in fig. 37, and has been already

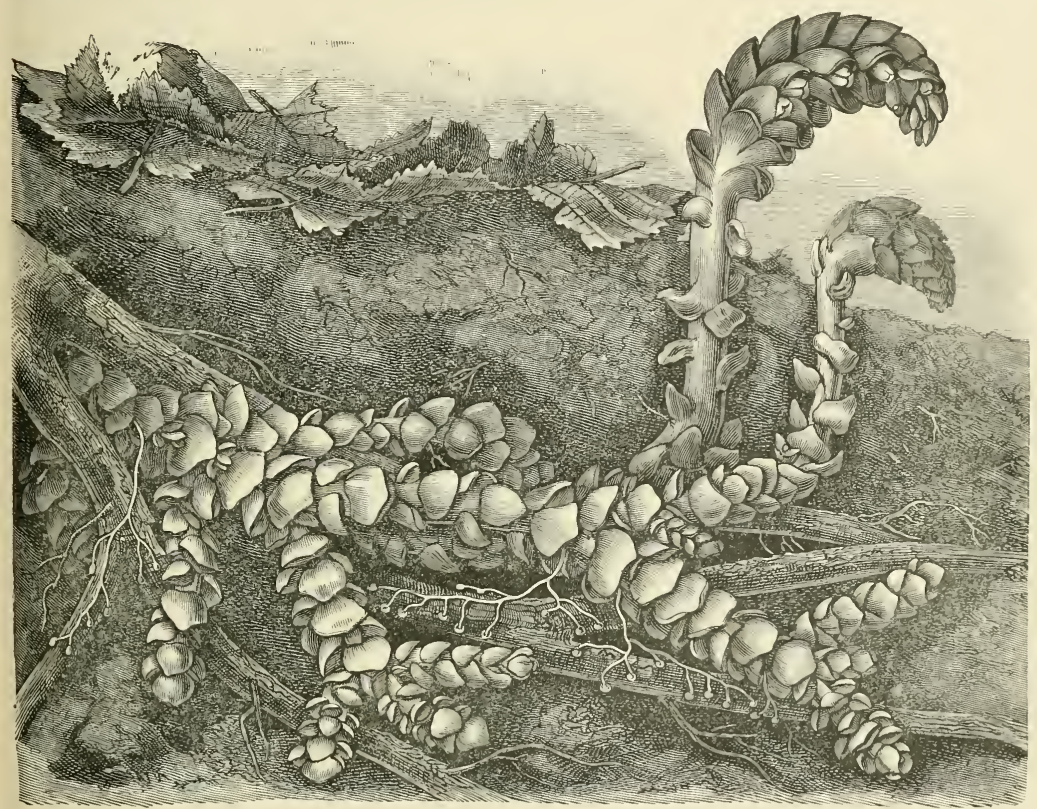

Fig. 37.-Toothwort (Lathroe Squamaria) with suckers upon the roots of a Poplar.

described on a previous occasion as an instance of a plant possessing in the seclusion of its curious hollowed scale-leaves a special mechanism for the elimination of water from its system quite supplementary to the normal method of surface transpiration. Formerly, the Toothwort used to be included in the family of Broom-rapes (Orobancher) on account of the structure of its capsules, but it is entirely different as regards the form of its seedling. For, whereas the seedling of a Broom-rape is a thread without any trace of cotyledons, as will be seen when we study its development and mode of attachment to the host in the next few pages, that of the Toothwort is clearly differentiated into radicle, cotyledons, and rudimentary stem, corresponding in this respect entirely with the Rhinanthaceæ. Moreover, the Toothwort resembles Rhinanthaceæ much more than Broom-rapes in the manner in which it attacks its hosts and withdraws nutriment from them. 
The seed of Lathroea germinates on damp earth. The young root of the seedling grows at first at the expense of reserve material stored in the seed, penetrates vertically into the earth and sends out lateral branches, which, like the main root, follow a serpentine course and search in the loose damp earth for a suitable nutrient substratum. If one of these meets with a living root belonging to an ash, poplar, hormbeam, hazel, or other angiospermous tree, it fastens on to it at once and develops suckers at the points of contact; these suckers are at first shaped like spherical buttons, but soon aequire, as their size increases, the form of discs adherent to the host's root by the flattened side and with the convex hemispherical side turned towards the rootlet of the parasite. These discoid suckers cling to the root attacked by means of a viscid substance produced by the outermost layer of cells. As in the case of the parasites already described, a bundle of absorption-cells grows out of the core of each sucker into the root of the plant serving as host, and the tips of the absorbent cells reach to the wood of the root. The shoot extremity of the seedling, thus nourished by the juices of the host, now develops very quickly, elongating and producing thick, white, fleshy, scale-like leaves which overlap one another closely, the whole thus acquiring the appearance of an open fir-cone. The scaly stems also branch underground, and thus a curious structure is gradually produced, consisting of crossed and entangled cone-like shoots covered with white scales, and this structure fills entirely the nooks and corners between the woody roots on which it preys. Individual plants extending over a square meter and weighing 5 kilograms are by no means rare. Later on, inflorescences raise themselves above the surface from the extremities of the scaly subterranean shoots. Their axes are at first curved like crooks, but straighten themselves out by the time the fruit ripens. Whereas the subterranean portions are white as ivory, the flowers and bracts pushed up above the earth are of a purplish tinge. The roots, which issued originally from the seedling, and their suckers have long since ceased to meet the requirements in respect of nourishment of so greatly augmented a structure, and therefore additional adventitious roots are produced every year, springing from the stem and growing towards living woody branches of the thickness of a finger, belonging to the root of the tree or shrub that serves as host. When there, they bifurcate, forming numerous thickish filiform arms, which lay themselves upon the bark of the nutrient root and weave a regular web over it. Sometimes two or three of these root-filaments of the parasite coalesce, forming tendrils, and the resemblance to a lace-work or braid is then all the more pronounced. Suckers such as have been described are developed by these rootfilaments laterally, and more especially on the ends of the branches.

Lathree is interesting in so many different connections that we shall again return to this plant later on. As has been stated before, it affords a type of a series of parasites which resembles the species of Cassytha and Cuscuta in the absence of chlorophyll, Rhinanthaces in the shape and development of the seedling and the form of the suckers, and the Balanophoreæ, presently to be described, in being parasitic upon the roots of woody plants. Lathrae Squamaria, the species repre- 
sented in fig. 37, is indigenous to Europe and Asia, its area of distribution extending from England eastwards to the Himalayas, and from Sweden southwards to Sicily. Two species are confined to the East, the Crimea and the Balkans, and another Toothwort (Lathroea clandestina), distinguished by large flowers, but slightly raised above the earth, extends in western and southern Europe from Flanders over France to Spain and Italy. This last has the distinctive feature that the discoid suckers developed on its yellow roots, which latter are of the thickness of a quill, are as large as lentils and the biggest hitherto discovered on any plant.

\section{BROOM-RAPES, BALANOPHOREA, RAFFLESIACEA.}

The fourth series of parasitic Phanerogamia is composed of plants destitute of chlorophyll, whose seed contains an amorphous embryo without cotyledons or radicle. The seed germinates on the earth, and the embryo grows as a filiform body into the ground and there fastens upon the root of a host-plant, penetrates into and coalesces with it in growth, forming a tuberous stock, from which, later on, flowering stems are projected above the earth.

To this series belong the Broom-rapes or Orobancheæ and the Balanophoreæ. Of the genus Orobanche about 180 species are recognized, which, exhibiting great uniformity in floral structure and in their general development, can only be distinguished by minute characteristics. The flowering stem growing up from the subterranean tuber is, in all the species, rigid, erect, thick, fleshy, and covered at the top with dry scales. The open flowers, ringent in shape, are crowded together in a terminal spike, and often emit a strong scent like that of pinks or sometimes of violets. The colour of the flowers is in one group (Phelypcea) mostly blue or violet; in the rest it is waxen yellow, yellowish-brown, dark-brown, rose-red, flesh-tint, or whitish. Orobancle violacea and 0 . lutea, both natives of Northern Africa, have stems which grow to a height of half a meter and become almost as thick as an arm. The best-known species is the Branched Broom-rape (Orobanche ramosa), which is parasitic on the roots of hemp and tobacco plants, and is very widely distributed. The greatest number of species belong to the East and to Southern Europe. The extreme north of America harbours one species which bears a single flower at the end of its stem. In all the species the stem projects only partially above the earth. The subterranean portion, adherent to the root of a host, is often greatly swollen and thickened above the place of attachment; in the case of Striga orobanchoides, which is prevalent in the Nile basin, it is irregularly lobed above the host's root. The root of the nutrient plant also is usually somewhat swollen wherever a parasitic Orobanche has settled upon it, and sometimes it exhibits an irregular outgrowth inclosing the spot whereto the Orobanche is adnate like a cup. Beyond the place of attachment of the parasite the root has often the appearance of having been bitten off, and this is owing to the fact that the particular piece of root has been killed and demolished by the attack of the parasite. From the base of the stem, near the point of adhesion to the host, spring short, thick, fleshy fibres, and 
one or other of these bends its tip towards the root of the foster-plant and clings to it. These fibres are, in many species, very numerous, and are interlaced and entangled so as to form a reticulate mass, which vividly recalls that of the Bird'snest, and is an instance of the general resemblance existing between Orobancheæ and the Orchideæ destitute of green leaves (Neottia, Corallorhiza, Epipogum, Limodorum), which have already been discussed.

The establishment of parasitic Orobancheæ upon the roots of host-plants takes place in the following manner. The embryo imbedded in the small seed shows no trace of differentiation into root and stem, possesses no cotyledons, and indeed consists only of a group of cells; it is surrounded by other cells filled with reservenutriment. When this embryo grows forth from the seed, during which process it consumes the reserve-food, it exhibits no distinction between root, stem, and leaf, but is a spiral filament consisting of delicate cells. One extremity, the shoot end, of this filiform seedling, remains covered by the seed-coat, which looks like a dark cap (fig. $34^{8}$ ); the opposite extremity is the root.

The seedling Broom-rape stretches downwards just as the Dodder (Cuscuta) extends upwards. In so doing the descending tip traces a spiral line, and so, as it were, seeks in the earth for the root of a plant suitable as host. If the search is fruitless, and if the reserve-material in the seed has meantime been altogether consumed, the seedling begins to wither and gradually shrivels, turns brown, and dries up. It lacks the power of nourishing itself by means of the surrounding earth. But, if the lower, foraging extremity of the seedling succeeds in finding a live root belonging to a plant able to serve as host, it not only adheres closely to it, but swells in such a way as to give the young plantlet a flask-shaped appearance (fig. $34^{9}$ and fig. $34^{10}$ ). The upper end is still inclosed by the seedcoat, but in proportion as the lower part thickens, the upper shrivels till no trace of it is left. The thickened part, on the other hand, which has become attached to the root of the host, becomes nodulated and papillose. Some of the papillæ develop into elongated conical pegs, and the young Broom-rape now rests upon the nutrient root in the shape of the head of a fighting-club (see fig. $34^{12}$ ). At the place of attachment one of the conical pegs has meanwhile penetrated the cortex of the root, and there it continues to grow energetically, forcing the cortical tissue apart, until it reaches the wood. Vessels now arise in the body of the young clublike plant, and, passing through the middle of the plug, wedged in the nutrient root, are brought into connection with the vessels of the latter. At the point of union between host and parasite, a bud is formed, clothed with abundant scales, which may best be likened to the bulb of the Martagon Lily. Lastly, out of this bud grows a strong, thick stem, which breaks through the earth and lifts a spike of flowers into the sunlight.

That portion of the Broom-rape which is buried in the root of the host-plant is so intimately associated with the separate parts of that root in the development of a tuber that it is usually difficult to determine which cells belong to the parasite and which to the host. The degree of union is such that one cannot even state with 
certainty where the epidermis of the nutrient root ceases, and that of the Broomrape begins. The latter looks as if it were a branch growing out of the root it preys upon, and this apparent fusion gave some colour to the view of the earlier botanists, who, ignorant of the life-history of these parasites, believed that they did not arise from seeds, but were pathological outgrowths of the roots, produced from their tainted juices; in other words, that they were "pseudomorphs" sprouting from diseased roots in the place of leafy branches.

It is also deserving of mention that some of the thick, fleshy fibres issuing laterally from the nodulated seedlings curve towards the host's root, bury their tips in the cortex, and thenceforth behave exactly like the peg which was inserted at the point where the seedling first became attached. We must leave undecided the questions as to whether the other fibres, which terminate freely in the earth, are capable of taking up food-materials from that source, whether these fibres are only present in perennial species and become the starting-points of new individuals, and lastly, whether they should be looked upon as root-structures or as stem-structures.

In addition, it is noteworthy that in many Orobancheæ only those embryos continue to develop which meet with a plant suitable to be their host. Although it is not the case that every species of Orobanche adopts one particular species of plant as foster-parent, yet thus much is certain, that most of them only thrive on members of a limited circle of species; one lives exclusively on kinds of Wormwood, a second on species of Butter-bur, and a third on those of Germander. For example, Orobanche Teucrii prevails on Teucrium Chamcedrys, Teucrium montanum, \&c., the hosts being invariably species of the genus Teucrium. Suppose a hill thickly covered with plants comprising Teucrium montanum growing in company with thyme, rock-roses, globe-flowers, sedges, and grasses, but no great abundance of the Teucrium, a plant belonging to the species named occurring only here and there, and let Orobanche Teucrii have established itself at one particular spot, have attained to flowering and developed fruits, the tiny seeds of which have been shaken by the wind out of the ripe capsules. Owing to the exceptional minuteness and lightness of its seeds, every gust of wind will scatter them in innumerable quantities over the entire hillside and beyond it. The next step is germination. Filiform embryos emerge from the seeds, in the manner described above, and penetrate into the earth. Teucrium montanum being only sparsely present on the hill in question, comparatively few seedlings will meet with the roots of that plant, whereas thousands will fall in with the roots of the thymes, rockroses, globe-flowers, sedges, and grasses. But, curious to relate, only those seedlings of Orobanche Teucrii which come into contact with the roots of Teucrium montanum establish themselves firmly, penetrate into them, and continue their development; whilst the numerous individuals which touch the roots of the thyme and other plants perish. This phenomenon can scarcely be explained in any other way than by the supposition that the roots of Teucrium montanum alone, by virtue of their special structure and quality, afford a suitable nutrient substratum, and therefore constitute centres of attraction for seedlings of Orobanche Teucrii; 
and that the roots of the thyme, rock-roses, and other plants growing upon the hill side by side with Teucrium montanum do not share this property.

Whereas the Broom-rapes constitute a family of plants, the species of which, though very numerous, are so similar in the structure of flowers and fruit, in the history of their development and in the general impression they convey, that it is necessary to discover minute distinctive marks in order to be able to classify them with tolerable completeness, the Balanophoree, which, together with these Orobancher, belong to the fourth series of parasitic Phanerogams, are related to one another in a manner quite the reverse. Only forty species of them are known, but they are so various that, on the basis of the obvious differences, no less than fourteen genera have been distinguished, among which the forty species are fairly equally divided. In respect of distribution and occurrence they also contrast strikingly with both Broom-rapes and Rhinanthaceæ. The Orobancheæ belong in particular to the Mediterranean flora, and to the East, and the Rhinanthaceæ, as has been already stated, adorn chiefly sunny pastures in arctic regions and in mountain districts of the northern hemisphere. Balanophoreæ, on the other hand, are only found within a belt encircling the Old and New Worlds, which stretches little beyond the equatorial zone to the north or south, and they almost all inhabit the dark bed of primeval forests, where they are parasitic on the roots of woody plants, beneath a covering of vegetable mould.

The genus of Balanophoreæ named Langsdorffia is confined exclusively to tropical America. One of its species (Langsdorffia Moritziana) is found native in the damp forests of Venezuela and New Granada, where it is parasitic on the roots of palms and fig-trees; a second species (Langsdorffia rubiginosa) occurs in Guiana and Brazil in the region of the sources of the Orinoco, and a third, the most common of all (Langsdorffia hypogcea) represented in fig. 38, has an area of distribution extending from Mexico to the south of Brazil. They all avoid the hottest districts, remaining rather in cool regions; indeed the species first named has been found at an elevation of from 2000 to 3000 meters. Unlike all the rest of the Balanophoreæ, Langsdorffica exhibits a branched, cylindrical stock ascending from the place of attachment to the nutrient root, more or less felted externally, and before putting forth any flowers has a remote resemblance to a doe's antlers with their winter covering of downy skin. These stems are almost as thick as a little finger, have a fleshy consistence, and exhibit a clavate expansion at the base where they rest upon the root of the host. Many of those stems which bear the male flowers are $30 \mathrm{~cm}$. long; those which bear the female flowers are usually somewhat shorter. They are all of a pale-yellowish colour; the thickly tomentose Langsdorffia rubiginosa looks as if it were covered with a yellowish velvet. At the extremity of each of the ramifications of the stem, which are often extremely short, having then the form of lobes or knobs, a bud is developed sooner or later in the lower cortical layer. This bud swells, bursts the outer layer of cortex, uplifts itself and grows out as an inflorescence between the four lobes formed by the cruciform rupture of the bark. The inflorescence is surrounded, like 
the capitulum of a composite, by a whorl of imbricating scales, of which the lower are shorter and broader, and the upper longer, narrower, and pointed at the apex. These scales being stiff, somewhat shiny, and varying in colour from a waxen yellow to orange or red-in the case of Langsdorffia Moritziana brown-red,- the whole inflorescence has a vivid resemblance to certain immortelles, namely, the large species of Helichrysum occurring at the Cape. The inflorescences bearing male flowers are elongated and egg-shaped, those possessing only female flowers are shorter and capitulate. The seeds dropped from the nut-like fruits, which are pulpy internally, have no special integument. The embryo exhibits no trace of

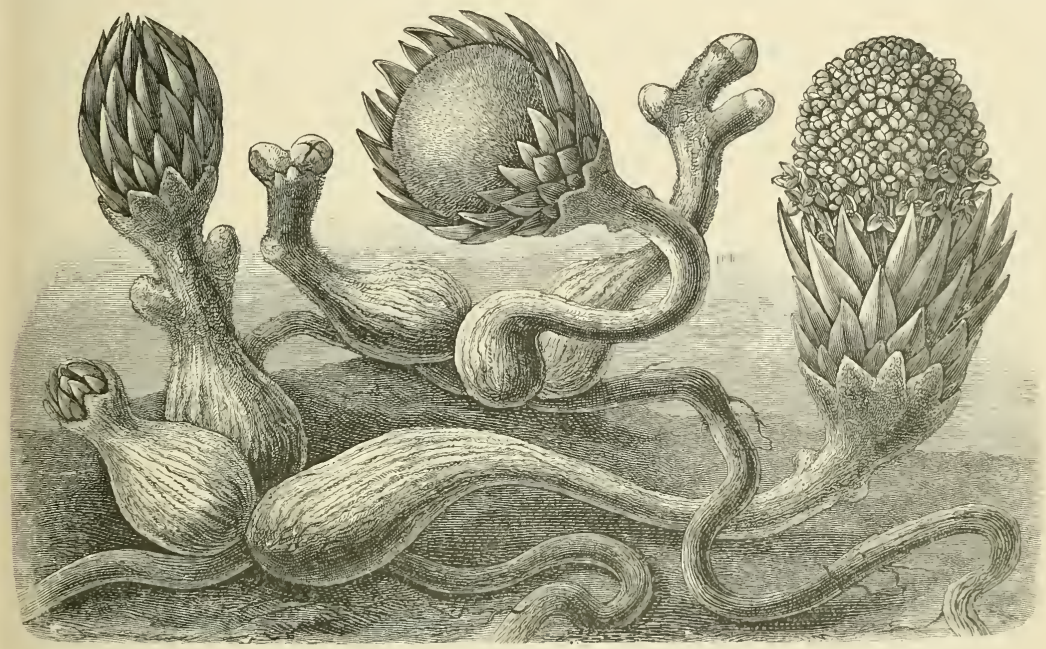

Fig. 38.-Laingsdorffia hypogcea, from Central America.

cotyledons or radicle, but consists of an undifferentiated group of cells which may be likened to a tiny bulbil.

Seeds of this kind germinate like those of Lathrcea, and upon meeting with the root of a tree or shrub suitable for prey, develop into larger tubercles and have a remarkable effect upon the substratum. The cortex of the host-root is destroyed at the place of adhesion of the tubercle, and its wood is laid open, lacerated, and unravelled. The woody bundles are diverted from their previous direction, ascend towards the parasitic tubercle, which meantime has grown into a full-sized tuber, and spread out like fans. The cells and vessels of the parasite penetrate between the ascending wood-fibres, and this results in the formation of a zone at the place of union of the parasite and root, where cells and vessels belonging to both interlace, traverse, and join one another, coalescing completely in exactly the same way as happens in the case of the species of Toothwort. A similar phenomenon occurs also when one of the wavy stems of Langsdorffia comes into contact with a root adapted to the purpose. The cortex of the root is demolished at the place of 
contact; the wood is exposed, split open, and unravelled, whilst the tissue of the parasitic stem fills up all the interspaces in the upcurved and sundered woody bundles and fibres, and so intimate is the union thus effected that the stem of the Langsdorffia might be taken to be a branch of the root of the host-plant which sustains it. At the point of connection of an already adult Langsdorffia stem, the hypertrophy of the tissue is not very striking; but the base of each stem of an individual produced from a seed presents a highly swollen and clavate appearance. At first the parasite is only fastened by one side of this thickened base to the nutrient root, but later on it wraps both sides round the root, and rests upon the latter like a saddle on the back of a horse.

Between the bundles of a Langsdorffia stem there are passages filled with a peculiar wax-like matter named balanophorin. The quantity of this substance is so great that if one end of a stem of Langsdorffia is lighted, it burns like a waxtaper, and in the region of the Bogota these Langsdorffias are collected and sold under the name of "siejos", and are used for illuminating purposes on festive occasions. In New Granada they have also been employed in the making of candles; and, although this source of wax is not sufficiently abundant for us to be able to believe in its consumption and conversion on a large scale, the fact of its application in this manner shows that the parasite we are discussing must occur in great exuberance in many tracts of country in Central America.

Much rarer than the parasitic Langsdorffias are the species belonging to the genus Scybalium. Like the former these are confined to the equatorial zone of America. Two species, viz. Scybalium Glaziovii and S. depressum, flourish in mountainous districts, one of them indeed occurring only on the mountains of New Granada; two other species (Scybalium jamaicense and S. fungiforme) live in the woods and savannahs of lower-lying regions. The aspect of the last-named species when seen growing on the ground of a primeval forest, tempts one to suppose it to be a fungus, and it is easily understood why the first discoverer selected the term fungiforme to apply to it. Figure $39^{\mathrm{I}}$, representing this rare and marvellous plant, is taken from the original specimens discovered in the year 1820 by Schott in the Sierra d'Estrella of Brazil, and brought thence by him to Vienna. We see that, in this case, instead of the elongated, wavy, branched stem characteristic of Langsdorffias, a lumpy, tuberous mass rests upon the root of the host-plant. This tuber is sometimes rounded and sometimes compressed and discoid; it is nodulated and often irregularly lobed also, and grows to the size of a fist. It is developed from a seed which, as is the case in all Balanophoreæ, is a cellular structure without integument containing an embryo destitute of cotyledons and radicle, and is best described as a minute tubercle. The embryo, after emerging from the seed and finding the living root of a woody plant, increases in volume, and, in the form of a little knob the size of a pea, exercises the same influence on the plant preyed upon as has been noted in the case of Langsclorffia. The root attacked is stripped of bark at the place where the tubercle is attached; the wood is then resolved into a fringe of fibres which stand straight up, and, diverging like the spokes of a fan, 
distribute themselves in the tissue of the parasite, the latter having in the meantime developed into a tuberous stock as large as a nut. These radiating bundles, issuing from the wood of the nutrient root, come then into such intimate connection with the vessels formed in the tuber of the parasite, that the one appears to be a continuation of the other. They are, besides, entangled together, and between them is intercalated a mass of small parenchymatous cells which also adheres to the yet unfrayed portion of the foster-root's wood, and coalesces with it. The tuberous body of the parasite, which in the first instance is only adnate to the host on one

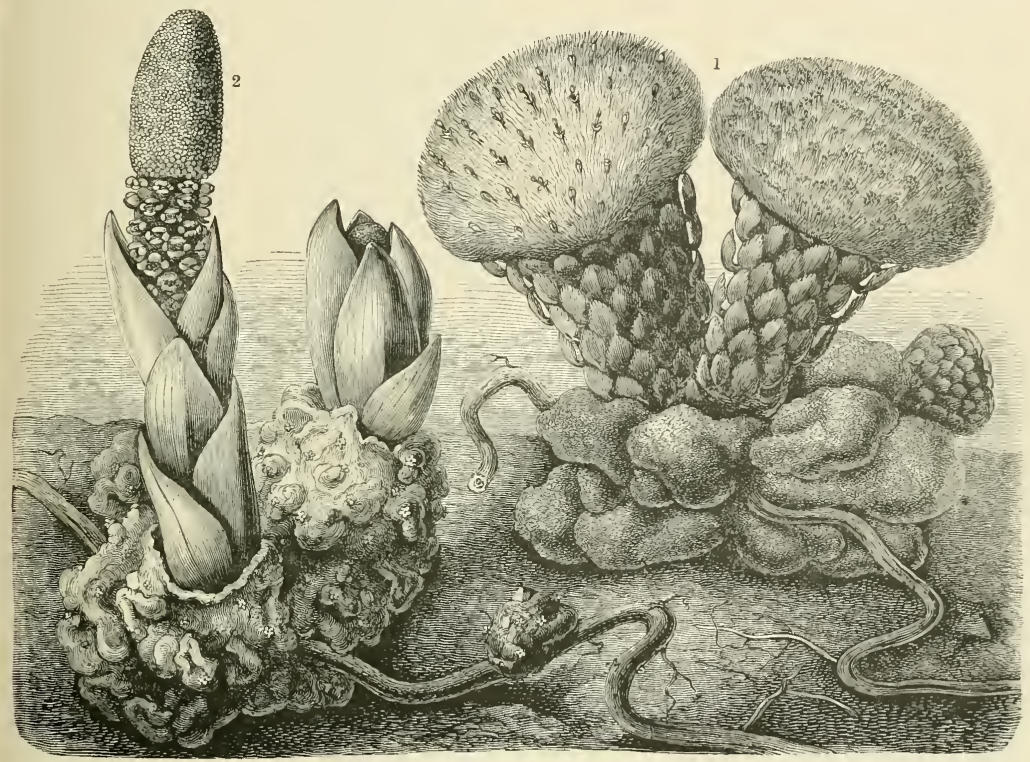

Fig. 39. - Parasitic Balanophoreæ.

1 Scybalium fungiforme, from Brazil.

2 Balanophora Hildenbrandtii, from the Comoro 1slands.

side, gradually encompasses it entirely, and the nutritive root then appears to perforate this irregular tuber. The inflorescences are produced direct from buds, which are formed under the bark at projecting spots of the brown tuberous stem, the cortex bursting open and allowing a thick flesh-coloured shoot, closely beset by ovoid pointed scales, to emerge and grow up into a form resembling a mortar-pestle. At the summit this shoot expands into a disc, and upon this are borne little capitulate groups of flowers, which are inserted amongst innumerable quantities of scales and hairs. The pistillate and staminate flowers are separated in different inflorescences, whilst the entire structure has an undeniable resemblance when in bloom to the inflorescence of an artichoke gone to seed, and later on to a toad-stool.

In the eastern hemisphere we find the various species of the genus Bulanophor $a$ replacing the Langsdorffias and Scybalia. One of these, Balanophora Hilden- 
brandtii, which is represented on the left side of the figure 39 , occurs in the Comoro Islands off the east coast of Africa; seven species inhabit the islands of Java, Ceylon, Borneo, Hong-Kong, and the Philippines, and three species the East Indies. Bulanophora fungosa, first discovered by Forster, is parasitic on the roots of Eucalyptus and Ficus, and is indigenous to Australia and the New Hebrides. The more elevated regions of Java and the Himalaya abound especially in these singular organisms. Balanophora elongata is so prevalent in Java on mountains of between 2000 and 3000 metres, that it is collected in quantities for the sake of the wax-like matter obtained from it. In that island candles are made from Balanophoras as they are from Langsdorffias in New Granada, or else rods of bamboo are smeared with the viscid substance, as they are then found to burn quite quietly and slowly. In the Himalaya, Balanophora dioica or B. polyandra are the commonest and most widely distributed species, and Balanophora involucrata is there met with upon the roots of oaks, maples, and araliads even at a height of from 2300 to 2500 metres above the sea-level. They possess in almost all cases very vivid and conspicuous colouring-deep-yellow, purple, red-brown or flesh-tint, thus resembling the Gastromycetes, Clavarieæ, and Toad-stools, in whose company they grow, and with which they manifest an additional uniformity in being all of fleshy consistence and containing no trace of chlorophyll. At a certain distance, moreover, the inflorescences rising from the dark ground in a wood, have the appearance of fungi, and all the early observers describe these Balanophorea with one accord as truly abnormal growths, viz. as fungi which by some marvellous accident bear flowers. They were also the object of the boldest speculations and most exuberant imagery on the part of the botanists belonging to the school of the "nature philosophers" of the first decades of this century. Even as late as the forties a famous German botanist says of them: "They are in the position of a hieroglyphic key between two worlds, which intercept and evade one another in an infinite variety of ways, like dreaming and waking moments", and the worthy Junghuhn, who discovered several of these plants in Java, writes: "Those are words which we may hope will be rightly interpreted thousands of years hence. Their sublime truth affected me deeply. There, flowerless and leafless, stood the mysterious plants which afford an instance of the combination of special vessels in a stalk like that of Balanophoreæ with the fructification of imperfect Hyphoinycetes!"

A young Balanophora not in flower is not unlike a Scybalium in appearance at the corresponding stage of its development. It consists of an irregular tuberous stem, which rests upon the creeping root of a tree or shrub. The exterior of this structure, which sometimes attains to the size of a man's head, is uneven, and in some cases convoluted like the human brain, or it may project in humps and knobs, or be divided into lobes or short branches like a coral-stem. The resemblance to the latter is heightened by the fact that the surface is covered by little papillæ shaped like stars or forget-me-nots, which distinguish the genus Balanophora from all allied genera. 
The seeds settle upon the roots of trees, develop into tuberous axes, and unite with the nutrient root in the same manner as the Balanophoreæ already described. Also the inception of the rudimentary inflorescence beneath the cortex of the tuber and its eruption are similarly accomplished. In this genus the cortical layer thus broken through and forced outward always forms a large cup-shaped or crateriform sheath with an irregularly-lobed margin surrounding the base of the inflorescence.

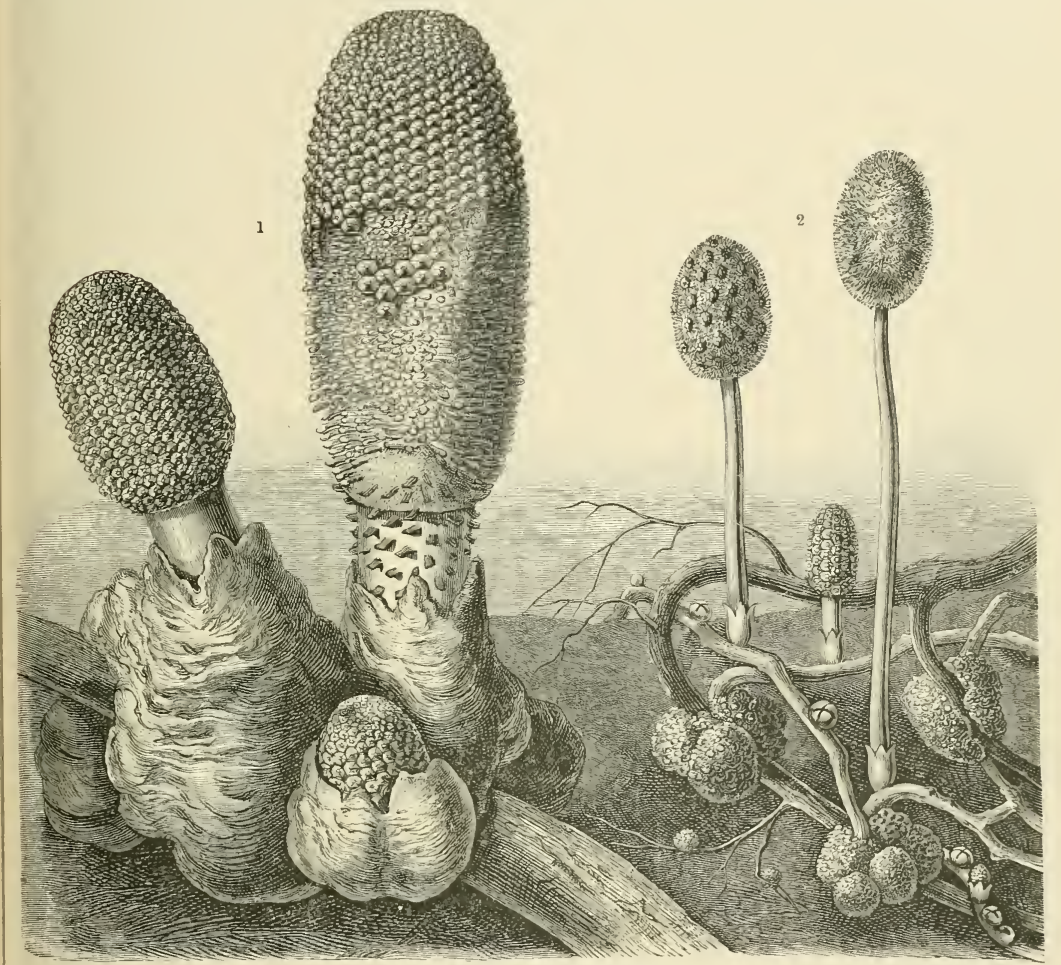

Fig. 40.-Parasitic Balanophoreæ.

1 Rhopalocnemis phalloides, from Java.

${ }^{2}$ Helosis gujanensis, from Mexico.

The inflorescence itself is spadiciform, and is borne by a thick shaft beset with large squamous leaves. The spadices growing from a tuber-stock are, for the most part, only as long as a little finger, but occasionally they reach a height of $30 \mathrm{~cm}$., as, for example, is the case in the Balanophora elongata of Java, which is parasitic on the roots of Thibaudia.

The species of the American genus Helosis, whereof the most common (Helosis gujanensis) is represented above, resemble those of the genus Balanophora in the shape of the inflorescence. There is, however, considerable difference in the method adopted by these Helosis species of settling upon the roots of host-plants and in 
the whole mode of growth. The phenomena of the swelling of the embryo into a tubercle after it has chanced upon a nutritive root, the destruction of the cortex, the exposure of the wood at that part of the root where the tubercle is adnate, and the derangement of the course of the woody bundles ensue, it is true, in the same manner as in the other Balanophoreæ; but the frayed wood-burdles of the fosterroot only form quite short lobules which penetrate but a short distance into the parasitic tuber-stock, whilst the vascular bundles, formed meantime in the latter, adhere to them in such a manner that they might be mistaken for direct continuations of them.

When once the parasitic tubers have thus become adnate to a root, and by means of this union are provided with food, they grow round the nutrient roots in such a way that the latter appear to perforate or actually to issue from the tubers. They are always roundish, brown outside, and warty, but without scales, and they never produce inflorescences directly, but put forth in the first place several whitish or yellowish runners varying in thickness from a quill to a finger, which creep along horizontally under the ground, bifurcating, and becoming interlaced with other ramifications. At the places of contact they coalesce, and so occasionally form a net-work which is almost inextricably entangled with the rootsystem of the plant preyed upon. Whenever a runner of this kind comes into contact with a living root belonging to the host-plant, the surface of contact at once swells up. The part affected is converted into a tuberous mass and becomes adnate to the root, the process being the same as occurs in the case of the tubercle produced from seed. A net-work of runners thus connected with the root-system of the nutrient plant at several spots by means of tubers as large as peas might be compared to the reticulum woven by Lathroea round the roots of its hosts; but, apart from the size, there is the essential difference that inflorescences are never produced from the white threads of the ramifying and sucker-bearing roots of Lathrcea, whereas the runners of Helosis afford points of origin for new inflorescences. Warts are produced on the surfaces of the thicker cylindrical runners, and within these are developed the buds of the inflorescences. The outer coat of the warts is then rent open at the top and constitutes a little cup, out of which grows a naked, scaleless shaft terminated by an oval spadix. Seeing that the runners rest horizontally under the earth whilst the shafts ascend bolt upright from the ground, the latter are always at right angles to the runners, of which they are to be regarded as branches.

The flowers are grouped in capitula, presenting in the spadix a dense mass. They are protected by peculiar bract-scales, each of which by itself is like a nail with a facetted head. These heads are in close contact with one another, so that the young inflorescence seems to be inclosed in a panelled coat of mail, and resembles to a certain extent a closed fir-cone. By degrees, however, these bractscales detach themselves and fall off, and thus the flowers, till then roofed over by them, become visible. When the seeds are mature, the whole runner concerned in the production of the inflorescence, and usually also the tuber which served as the 
starting-point of that runner, perishes, and another tuber belonging to the net-work above described, or rather the system of runners proceeding from it, becomes the basis for the development of new inflorescences. To this extent we may regard these Helosis species as perennial plants, whereas the majority of the other Balanophorex can lay no claim to this distinction, inasmuch as in their case the whole plant dies after it has flowered and ripened its seeds. The floral spadices in Helosis have a purple or blood-red colour, and in Brazil are called "Espigo de sangue". Only three species of Helosis have been discovered up to the present time, and those are distributed over equatorial America, in the Antilles, and from Mexico to Brazil.

Nearly allied to Helosis is the genus Corynoea, which resembles it in having facetted bract-scales like nails and a cone-like inflorescence, but differs eatirely in other respects in its mode of growth, especially in being without runners. Four species of this genus have been discovered in the Andes of South America, in Peru, Ecuador, and New Granada, where they are parasitic, like the rest of the Balanophoreæ, upon the roots of trees. One of them, Corynoea Turdiei, is worthy of notice as living on the roots of Peruvian-bark trees, and is rendered conspicuous by its purple spadix, borne on a white shaft. Rhopalocnemis phalloides (see fig. $40^{1}$ ) is another root-parasite related to Helosis, and the single representative in Asia of these pre-eminently American groups. It is found preying upon the roots of fig-trees, oaks, and various lianes, in mountainous parts of Java and the eastern Himalayas, and is one of the biggest of all the Balanophoreæ. The fleshy, yellowish or reddish-brown tuber-stock attains to the size of a man's head; the inflorescences, which burst from the protuberances of this lumpy mass and are from two to six in number, are over $30 \mathrm{~cm}$. long and from 4 to $6 \mathrm{~cm}$. thick. The protuberances are light-brown in colour, and resemble in form a cycad-cone. Rhopalocnemis, a drawing of which is given in fig. $40^{1}$ on a scale of one-half the natural size, is distinguished, like Corynoea, from Helosis by having no runners issuing from the tuberous axes.

The Lophophyteæ are set apart as a further group of parasitic Balanophorea, and differ from all the groups hitherto described in having their flowers arranged in separate roundish capitula upon a fleshy rachis springing from the tuberous-stock. They, again, belong to Central America, and are divided into three genera (Lophophytum, Ombrophytum, and Lathrophytum) into particulars of which we cannot enter without exceeding our limits. Only the genus Lophophytum, which is in many respects different from other Balanophoreæ, and in particular has been more thoroughly studied with reference to its peculiar mode of connection with the host-plant, demands special consideration. The Lophophytum mirabile (see fig. $41^{1}$ ) found in the primeval forests of Brazil adhering to the roots of Mimoseæ, to those of Inga-trees especially, occurs at some places in such profusion that areas of ground, occupied by Inga-roots, from twenty to thirty paces in circumference appear to be entirely overgrown by the parasite. Hundreds of tubers, some large, some small, rest upon the roots of the trees, covered by fallen leaves and a light VOL. I. 
stratum of vegetable mould. Most of them are the size of a fist, but a few are as big as a head, and then weigh 15 kilogr. and more. The tubercles formed directly by the germinating seeds which chance upon the roots are, by the time they attain to about the size of a pea, already in connection with the wood of the attacked root. The cortex and a portion of the wood at the place where the parasite is adnate are absorbed by this root. The tissue of the small tuber-stock is squarely and firmly inserted into the superficial notch thus made in the root, and short, pegshaped bundles, isolated by the loosening of the wood of the nutrient root, appear to grow into the substance of the parasite. As the tuber increases in size vascular bundles are developed in it also, and these grow towards the said bundles of the host and unite with them.

No boundary can then any longer be certainly recognized between host and parasite, and the strangest fact of all is that we find, in these bundles, cells concerning which we are not able to decide, even by reference to their shape, whether they belong to the one or to the other. The cells which belong undoubtedly to the wood of the nutrient root have dotted walls; the bundles unquestionably developed in the parasitic tuber exhibit, on the other hand, cells with reticulate thickening, which, when slightly magnified, look as if they were transversely striated. Wherever these pitted and reticulate cells meet, cells are intercalated which do not altogether correspond either to the pitted variety belonging to the host or to the reticulate cells of the parasite, but display a form intermediate between the two. Here and there, too, cell-groups belonging to the parasite are entirely buried in the wood of the foster-root in its growth, and in the older tubers the cellular elements of the two plants there bound together are so involved that it is, as has been stated, impossible to establish any line of demarcation between the two.

By the time the tubers have reached the size of a fist their cortical layer is always solid, corky, and areolated; each of the areas being more or less uniformly angled, as is shown in the illustration below. Some of the more protuberant portions elongate and grow out into short, thick stumps bearing scales all round, each of the little areas having a triangular-pointed scale situated in the middle of it. At this stage of development the entire Lophophytum plant has an extraordinary resemblance to the squamigerous rhizome of a fern, or to a dwarf cycad-tree, stripped of its green leaves; and this likeness is enhanced by the fact that the bark and scales of Lophophytum are dark-brown in colour. From the centre of each of these thick stumps, which often reach a height of $15 \mathrm{~cm}$., there now arises a spadiciform inflorescence. At first it is so thickly covered with ovate lanceolate scales possessing dark-brown, quasi-horny tips, overlapping one another like tiles, that the spadix as a whole looks extremely like an erect cycad-cone. Imagine the surprise of a traveller, who chances upon a spot in the depths of a primeval forest where the ground is occupied by Lophophytum, upon seeing hundreds of these brown, scaly cones grow up suddenly, in the course of a night following some days of rain, from the subterranean roots of the trees. A day or two later, this garden 
of Lophophyta presents an altogether different picture. The brown scales have detached themselves from the rachis, first those at the base of the cone, then also those on the upper parts. They fall off almost simultaneously, and with them the envelope which up to that time has concealed the flowers. The erect, fleshy, white, or reddish rachis bearing the flowers then becomes visible. The female flowers are

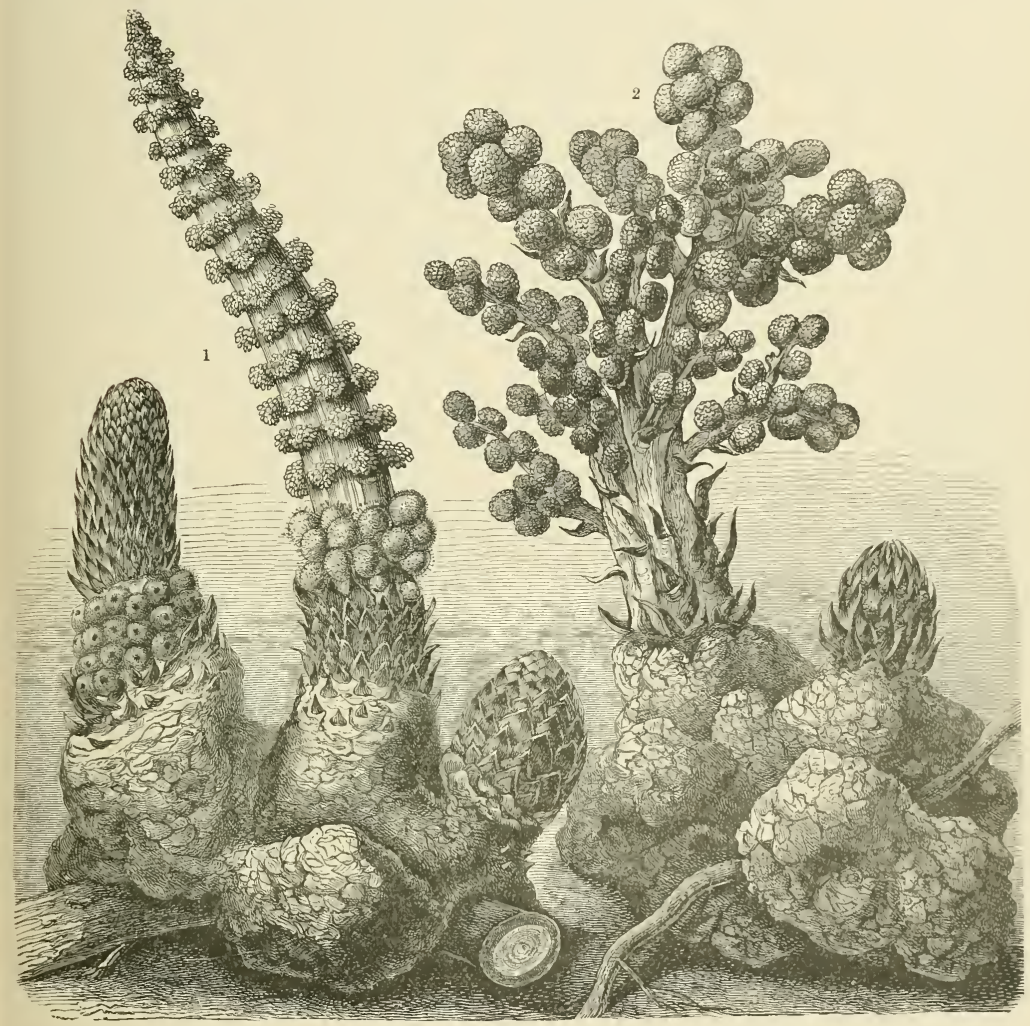

Fig. 41.-Parasitic Balanophoreæ.

1 Lophophytum mirabile, from Brazil.

2 Sarcophyte sanguinea, from the Cape of Good Hope.

on the lower part, and arranged in spherical, deep yellow or orange-coloured capitula which are packed close together; the male flowers are situated above the lowermost third of the spadix, and are arranged in looser and less crowded capitula of a pale yellow colour.

However striking the phenomenon presented by these flowering cones of Lophophytum mirabile, it is surpassed by another native of Brazilian forests, the Lophophytum Leandri. The colouring of the inflorescence in this species cannot 
be exceeded in variety, its rachis being pale reddish-violet, the bract-scales gamboge, the ovaries yellowish, the styles red, and the stigmas white. It is not surprising that even in Brazil, where there is certainly no lack of curious plantforms, they have attracted attention, and that they are used there, as is the case with all rare plants, for purposes of healing and magic. The tubers of Lophophytum mirabile, which have a disagreeable, bitter, resinous taste, and bear the popular name of "Fel de terra", or earth-gall, are employed by quacks against jaundice, and a belief also prevails that by secretly eating the blossoms youths are enabled to win the affection of the maidens they admire. The same may be said of Lophophytum Leandri, and, in addition, there is a tradition that the eating of it brings luck and agility in hunting, fishing, fighting, and dancing, and for this reason the Indian youth collect the plants secretly and eat them on particular days.

Of the other parasitic Balanophoreæ most nearly allied to Lophopliytum we will here only mention in passing the species of Ombrophytum, known in Peru by the name of "Mays del monte", which has a yellowish inflorescence over $30 \mathrm{~cm}$. high, and from 6 to $7 \mathrm{~cm}$ thick, somewhat resembling a spike of maize, and lastly, the Lathrophytum Peckoltii of Brazil, to which a special interest attaches inasmuch as it is the sole instance of a flowering plant entirely destitute of all structures of the nature of leaves, with the exception of the stamens and ovaries. Langsdorffia, Scybalium, Lophophytum, and even Balanophora, Helosis, and Rhopalocnemis exhibit scales, which, though transformed in various ways, are yet always in point of position and form recognizable as leaves; but neither on the tuber, shaft, nor spadix of this Lathrophytum is any trace of a scale to be seen, nor even a swelling or rim that might be looked upon as a degenerate leaf.

In comparison with equatorial America with its wealth of parasitic Balanophoreæ the corresponding zone of Africa must be called poor so far as these plants are concerned. Possibly further explorations may bring to light a few more of these wonderful vegetable parasites, but it is hardly to be expected that such a variety as is presented in Brazil, the Peruvian Andes, New Granada, and Bolivia will be found Only three Balanophoreæ have been discovered in the Cape regions, where the flora is well known. One of these, which is represented on the righthand side of fig, 41, bears the name of Sarcophyte sanguinea (i.e. blood-red fleshplant), whilst the name of Icthyosoma (i.e. fish-carcase) has also been applied to it because it smells of rotten fish. These names imply that the plant resembles an animal rather than a vegetable organism. The host-plants adapted to this Sarcophyte are various Mimoseæ, especially Acacia caffra, Acacia capensis, \&c. In the first place, as is the case with all Balanophoreæ, small tubers are formed on the roots of the above-mentioned woody hosts, and enter into connection with the wood of the nutrient roots in the manner already described more than once. An inflorescence then emerges from a bud originating beneath the cortex of the tuber, and rapidly grows up from out of the cortex, which is rent and pushed up in the process. The axis of this inflorescence resolves itself into a number of thick, repeatedly ramifying, fleshy branches, differing in this respect from every other 


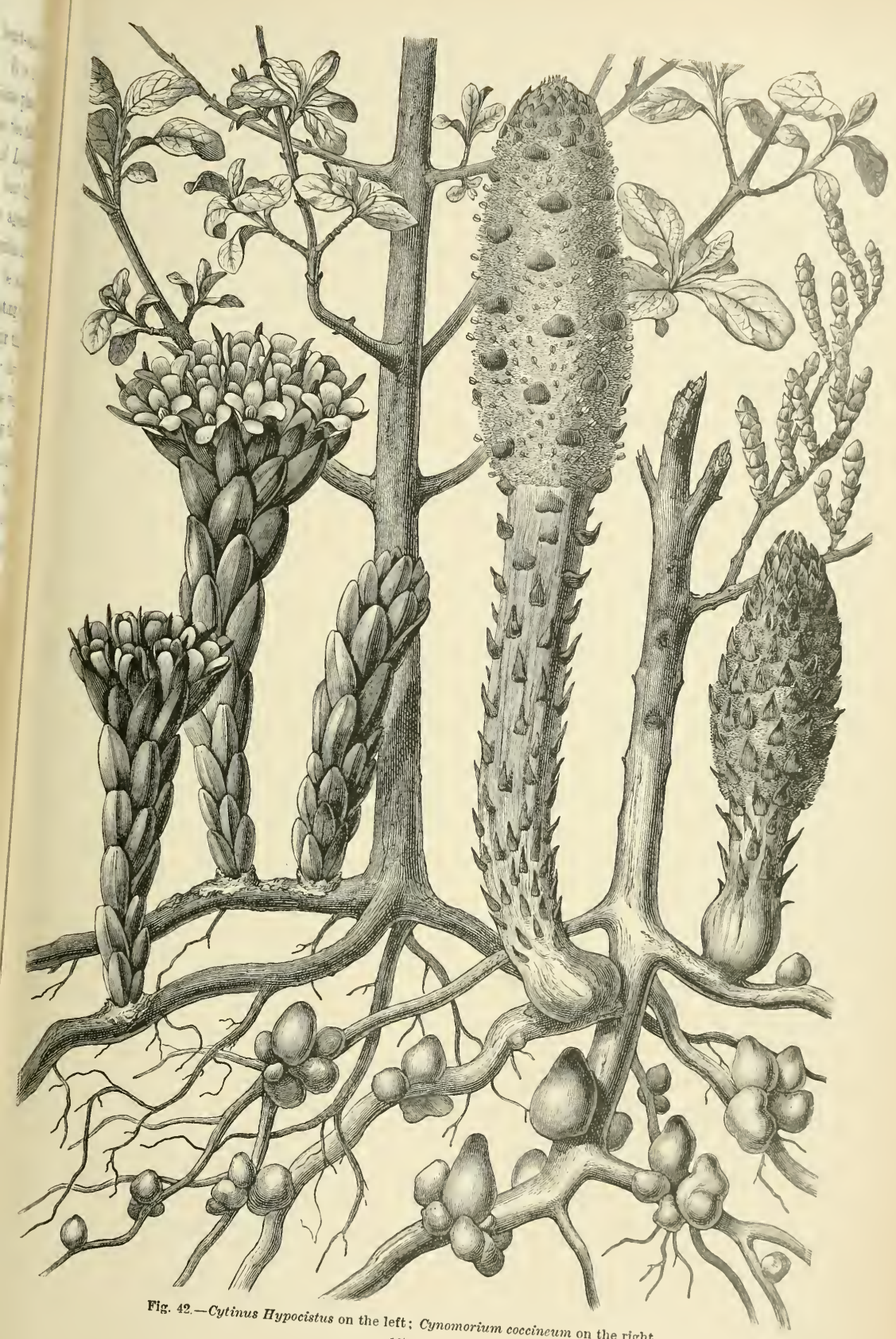


example of the Balanophorex. The flowers are arranged side by side on the branches, staminate flowers on one plant, and pistillate flowers on another, the latter always grouped in spherical eapitula, as is shown in fig. $41^{2}$. Reddish-brown scale-like leaves are situated at the points of origin of the branches, and also at the base of the entire inflorescence. The general aspect is that of a bunch of verucose grapes ascending from the root, or of the fruiting axis of Ricinus, and is very striking owing to the blood-red colouring of all the parts.

As a final instance of the Balanophorer we may take the genus Cynomorium, which was so highly valued in olden times, and is the sole species belonging to this family of plants indigenous in the south of Europe. A drawing of it is given on the right-hand side of fig. 42.

Whilst other Balanophoreæ are parasitic on the roots of trees and lianes in the shade of lofty woods, this Cynomorium thrives most luxuriantly upon plants near the sea-coast, on the roots of Pistacias and Myrtles, and even on actual salt-loving maritime plants, the various Tamarisks, Salicorniæ, Salsolaceæ, and Oraches, which are sprinkled with foam whenever the breakers are high. The seed is like that of other Balanophoreæ and those of the Orobanche species, and germinates in the same way as they do. From the group of cells in the seed which represent the embryo, a filiform body emerges, and then grows downwards, its upper part remaining for some time in connection with the other cells in the seed, which are richly furnished with food-materials. The filiform embryo continues to grow deeper and deeper at the expense of this nutritive store, and as soon as it reaches a living root, swells into an oval or irregularly-lobed tubercle, which unites with the wood of the nutrient root in the manner already described. These tubercles swell, and from the summit of each a spadix is produced, as in Lophophytum, which is raised above the surface of the earth. The spadix is clothed with pointed scales, and is clearly differentiated into a lower stalk-like support, and a fleshy inflorescence resembling a cone. The small scales are separated from one another by the process of elongation of the spadix, and some fall off. Others of them, situated about the middle of the inflorescence, persist, however, until the time when the entire spadix dries up. The whole of the structure standing above the ground has a blood-red colour, and when it is injured a red fluid exudes, which was at one time supposed to be blood. At an age when the peculiar properties of extraordinary plants were looked upon as an indication given by higher powers that they were to be used for curative purposes, it was believed that the spadices of Cynomorium, being blood-red in colour, and bleeding when wounded, had styptic properties. In those days they were even collected for the sake of this property, and sold in apothecaries' shops under the name of the Maltese fungus (Fungus melitensis). Various miraculous virtues were also attributed to this plant, and the demand for it was so great that it became a regular article of commerce, its main source being the Island of Malta, whence is derived the name above referred to.

Of the Hydnoreæ, which are most properly included in the same series as 
Balanophoreæ in consideration of their coalescence with the roots of their hosts, only three species are known. Two of them (Hydnora Africana and H.triceps) belong to South Africa, the third (Hydnora Americana=Prosopanche Burmeisteri) to South Brazil. The tuber is represented by a prismatic body with from four to six angles furnished with papillæ along the edges. The flower-buds which burst from it have at first the form of spherical Gasteromycetes, but gradually elongate and assume the form of a large fig or upright club. This structure opens at the thickened upper extremity by three stout fleshy valves representing petals. At the base of this curious flower no appendage is to be seen that could be interpreted as a bract or leaf. The fleshy mass of flowers evolves a disagreeable putrid odour, and in this property the Hydnoreæ resemble the Rafflesiæ, which belong to the next group of parasitic Phanerogams.

The fifth series of flowering parasites is composed of the Raflesiaceæ, plants connected with Balanophoreæ and Hydnoreæ by their general aspect, the absence of chlorophyll, and the undifferentiated embryo which consists merely of a group of cells. They used all to be classed together under the name of Rhizanther; but the Rafflesiaceæ are now treated as a separate family on account of the characteristic structure of their flowers and fruit. The formation of these organs will again come up for discussion later on when we treat of the wonderful structure of the famous giant-flower Rafflesia; at present we are only concerned with the relationship of the parasite to the food-providing host-plant. This is, if possible, even more remarkable than in the case of Balanophoreæ and Hydnoreæ. In the latter the union is effected within a structure like a tuber or a rhizome, the vessels and cells of the parasite coalescing with the exfoliated and disordered wood-cells belonging to the root or stem of the host-plant; whereas in Rafflesiaceæ the embryo, having penetrated beneath the cortex of the host, produces a more or less definite hollow cylinder which surrounds the wood of the host's root or stem (as the case may be), and constitutes a sort of vestment intercalated between the wood and the cortex of the host. There is no production of tuberous enlargements as in the Balanophoreæ. The stem or root attacked by the parasite only exhibits a moderate thickening at the place where the parasite dwells beneath the cortex, and the cortex itself is only destroyed at the spot where the embryo pierces through it, and where subsequently the flowers emerge. When roots constitute the substratum whereupon the parasite has established itself, they are always of a kind that run throughout upon the surface of the ground; when stems are chosen for attack, they are either the branches of trees or shrubs, shoots elothed with dead foliage belonging to dwarf suffruticose bushes, or else woody lianes of tropical forests. The seeds are conveyed to the host-plants through the intervention of animals.

Rafflesias are found in the haunts of elephants and along the tracks followed by those beasts. The Rafflesia-fruits are accordingly no doubt trampled upon and crushed, and the little seeds imbedded in the pulpy mass of the fruit thus have an opportunity of adhering to the elephants' feet. The seeds are afterwards rubbed oft by projecting roots at places more or less remote from the original locality, and if 
the root upon which they are detained belongs to a Cissus plant, they germinate. Dn the other hand, such Rafflesiaceæ as occur on the woody branches of trees, shrubs, and undergrowths, or on lianes, develop succulent fruits, which are eaten by animals. Their seeds are protected by a horny coat, and preserve their power of germination unimpaired as they pass through the animals' alimentary canals and are deposited with the excrements on the stems of fresh host-plants; or the seeds may stick to some part of an animal that happens to rub against them, and be brushed off later on as being an uncomfortable appendage, and in this way also they may fall upon the stem of a host-plant. Those Rafflesiaceæ which occur in Venezuela on the woody lianes (Caulotretus), known by the name of "monkey-ladders", owe their dispersion for the most part probably to monkeys.

Now, if a seed has been deposited in one way or another upon a woody root, creeping along the surface of the ground, or upon the stem of a woody plant, the filiform embryo emerging from the seed finds a suitable nutrient substratum present and it pierces the cortex of the root, and develops beneath it a tissue, which incloses the wood like a sheath. In Rafflesia and in the Pilostyles parasitic on the suffruticose shrubs of Tragacanth (P. Haussknechtii, see fig. $43^{1}$ ), this tissue consists of rows of cells, which to the naked eye look like threads. Some are simple and greatly elongated, others branched, and they are united together to form a net-work, so closely resembling the mycelium of a fungus as to be readily mistaken for one. The most complete similarity to these vegetative bodies living beneath the cortex of a host-plant is exhibited by the mycelia of the toad-stools which spread themselves in the form of nets and webs between the wood and the cortex of old trunks of trees. The vegetative bodies of the other species of Pilostyles consist, in each case, of a tissue composed of many layers of cells forming a parenchyma imbedded between wood and cortex in the host-plant and including some vessels and rows of cells capable of being interpreted as vascular bundles. Only in rare instances does this tissue of the parasite form an unbroken hollow cylinder encompassing the wood of the host; usually the elements of the host's tissues penetrate into it and permeate and split up the cylindrical soma (vegetative body) in the form of bands, ribs, and fibres. Many elements of the tissues, which the imbedded parasite has displaced from the living wood, and carries, as it were, on its back, perish; but sometimes these discarded layers remain in connection with other living tissues and so preserve their own vitality and power of expansion, and develop layers of woodcells covering the parasite. There is then a general confusion and entanglement, and it is difficult to say what part belongs to the parasite and what to the host.

When the somatic tissue of the parasite has accomplished its connections with the host-plant in the manner just described, the latter is unable to rid itself of its occupant. A portion of the juices of the host-plant passes into the parasite's cells and the unwelcome guest augments in volume, and endeavours forthwith to reproduce and distribute its kind by the formation of fruit and seeds. For this purpose buds are developed at suitable spots in the reticular body of the parasitc, each of which is manifested as a parenchyma of pulvinate appearance, and is 
termed a floral cushion. The cells in this cushion, however, now group themselves in a definite way; ducts and vessels are produced, and, at the same time, a differentiation into axis and flowers is exhibited. These members continue their development, increase in size, and finally the enlarged bud breaks through the cortex of the host-plant under shelter of which it has been evolved.

In the genus Cytinus alone do we find a stem richly furnished with leaves and bearing at the top a flattened symmetrical tuft of flowers (see fig. 42, left-hand side) developed from this bud; in the rest of the Rafflesiacex, the bud, which has

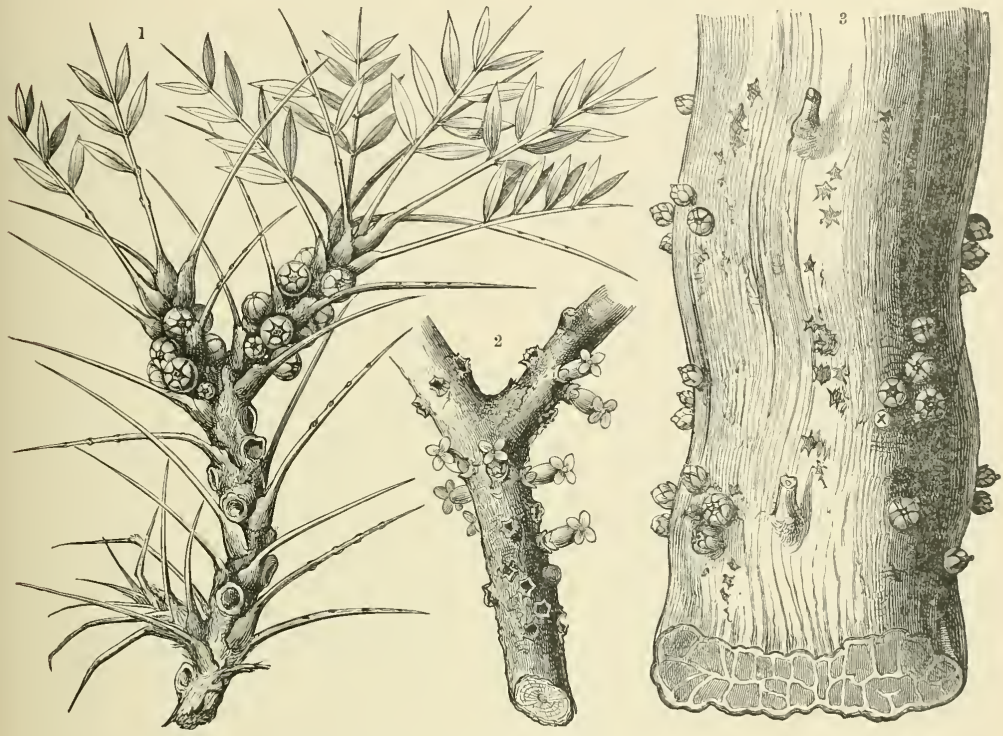

Fig. 43.-Rafflesiaceæ parasitic on trunks and branches.

1 Pilostyles Haussknechtii.

2 Apodanthes Flacourtiana.

3 Pilostyles Caulotreti.

emerged from beneath the cortex of the host, is the flower-bud itself. The axis supporting the bud is extremely abbreviated and clothed merely by a few scales, and the flowers are sessile directly upon the root or stem of the host (see fig. 43). In the case of roots creeping upon the ground, the buds always emerge vnly on the side turned towards the light; on lianes, also, they are only formed on the side more exposed to light where subsequently the opened flowers are easily accessible to flying insects (see fig. $43^{3}$ ); on upright shrubs and under-shrubs, on the other hand, they burst forth on all sides upon the branches. Branches of this kind bearing ubiquitously extruded flowers of a parasite such as Apodanthes Flacourtiana (see fig. $43^{2}$ ) look delusively like the Mezereon (Daphne Mezereum) when the latter is in bloom in the early spring before the development of foliage-leaves, its wcody branches being similarly studded all round with flowers, which stand out horizontally 
from them; but, in the one case the flowers belong to a foreign parasite living under the cortex and have broken through it, whereas in Mezereon it is the flowers of the plant itself that have unfolded. In the case of Pilostyles Haussknecltii, which is parasitic on the low bushy tragacanth shrubs of the Persian plateaus, the buds are formed regularly on both sides of the leaf-bases of the host, so that at the insertion of every one of the older foliage-leaves, one finds a pair of buds, which subsequently expand into flowers (see fig. $43^{1}$ ).

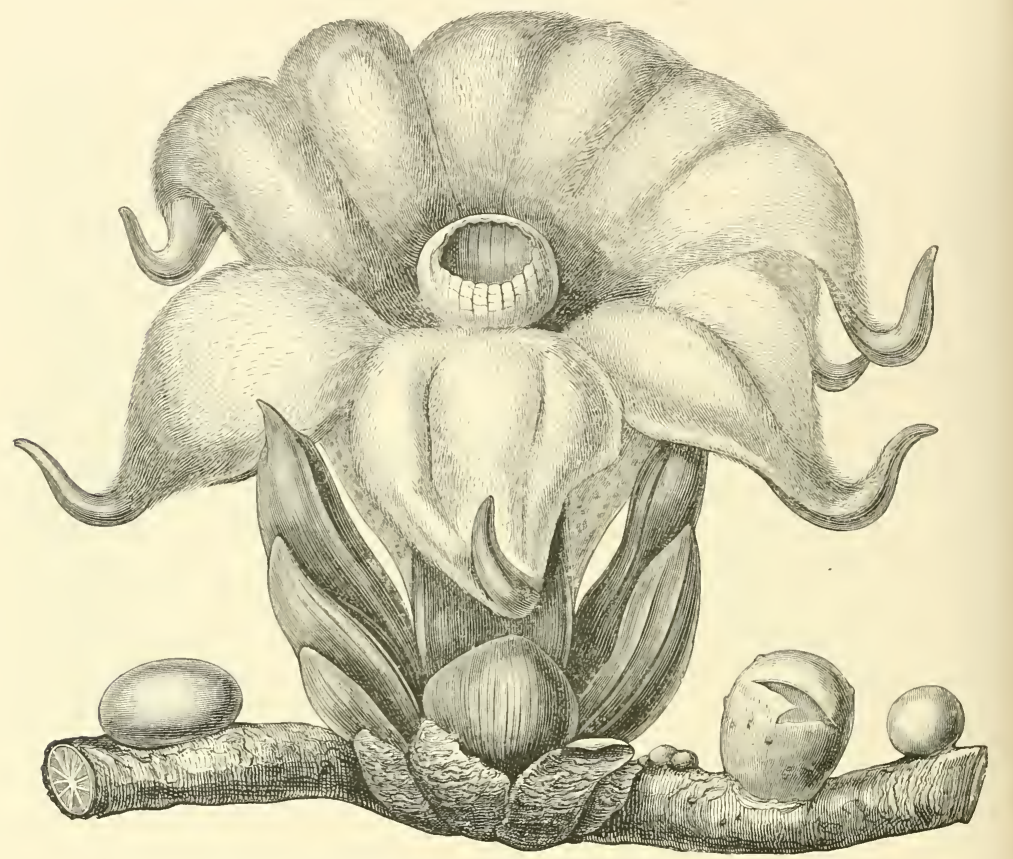

Fig. 44.-Parasitic Raftlesiacea (Brugmansia Zipellii) upon a Cissus-root.

Throughout the species of Apodanthes and Pilostyles the flowers are smallabout the size of elder, jasmine, or winter-green blossoms-and by no means conspicuous. But this is not the case in the genera Brugmansia and Raflesia. The Brugmansias, indigenous to Borneo and Java, have very handsome flowers, as may be seen in the above drawing, which represents on the natural scale Brugmansia Zipellii parasitic upon the root of a Cissus. But in magnitude they are far surpassed by the flowers of the Rafflesiæ, one of which, viz.: Rafflesia Arnoldii, may be described as actually the largest flower in the world. When open it has a diameter of 1 meter, a dimension exceeding even that of the gigantic blooms of Scruth American aristolochias. At the period of emergence of the buds of Rafflesia Arnoldii from the roots of the vines which serve them as hosts, they 
are only as large as a walnut and give scarcely any indication of their future magnitude; but they gradually increase in size, and before opening are currously like a cabbage. Up to this time the bracts still inclose the flower proper, and to them is due the above-mentioned resemblance. They now open back, and the flower, which, to the last, grows rapidly, unfolds and displays five immense lobes around a central bowl or cup-shaped portion. The form of the giant-flower when open is best likened to that of a forget-me-not blossom. The semicircular outline of the lobes, at least, is similar, and the very short throat of the flower also exhibits a distant resemblance. At the part where the bowl-shaped centre, which

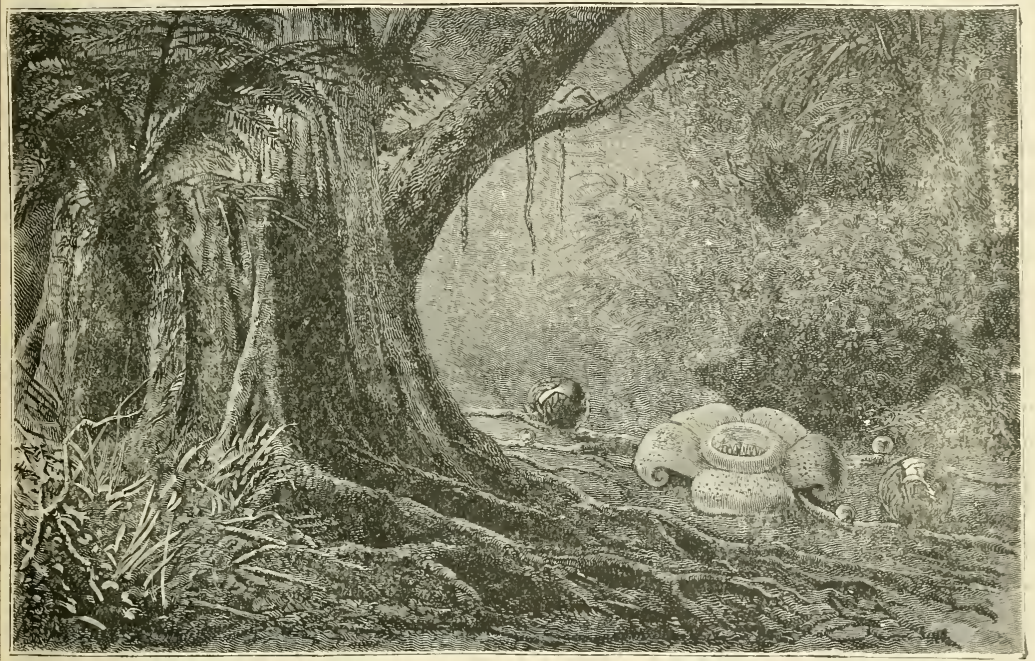

Fig. 45,--Raflesia Padina, parasitic on roots upon the surface of the ground.

has the stamens and styles inserted in it, passes into the lobes there is a thick, fleshy ring like a corona. The upper surface of the lobes is covered with numbers of papillæ. The lobes themselves, the hollow central bowl, and the ring, are all fleshy, and the flower, as a whole, emits an unpleasant putrescent smell. This floral prodigy was first discovered in the year 1818 in the interior of Sumatra at Pulo Lebbas on the river Manna, where it occurs parasitic on the roots of wild vines in places where the ground is strewn with the dung of elephants. It has never yet been seen anywhere outside Sumatra. Four other Rafflesiæe have, however, been discovered, but all in the islands of the Indian Ocean-Java, Borneo, and the Philippines. In mode of growth, as also in the form of the flowers, they resemble the species above described, but their flowers are rather smaller. Rafflesia Padma, which occurs in Java, and is represented in fig. 45, possesses flowers with a diameter of half a meter. The hollow, somewhat ventricose centre and the ring bordering the floral receptacle are in this Raflesia of a dirty 
blood-red, whilst the verrucose lobes have almost the colour of the human skin. The flowers are sessile upon roots which wind about upon the dark forest ground, and a cadaverous smell, anything but pleasant, issues from them. All these peculiarities explain the uncanny impression made by the organisms in question upon their original discoverers and upon all subsequent observers.

Whilst the Rafflesiæ, as well as the genera Brugmansia and Sapria, belong to the tropical and sub-tropical regions of Asia, and to the world of islands adjacent thereto on the south side, the genus Apodanthes is confined to tropical America. Most of the species of Pilostyles also appertain to tropical America, especially to Brazil, Chili, Venezuela, and New Granada. One species alone-Pilostyles Ethiopica-has been observed in the mountains of Angola, and another, as has been mentioned before, in Persia.

The only European representative of the remarkable group of Rafflesiacer is Cytinus Hypocistus, represented on the left side of fig. 42, but its distribution is coincident with the entire range of the Mediterranean flora. The roots of cistus shrubs, plants which are characteristic of the vegetation belonging to the basin of the Mediterranean, constitute the nutrient substratum in the case of Cytinus. It is especially where the layer of earth-mould is not deep, and consequently the roots of the shrubs in question are exposed, that Cytinus is met with growing in abundance amongst the under-wood of the cistus plants. The squamous leaves clothing the stem of this parasite being scarlet, and the plants not solitary but in large numbers, one sees here and there a flaming red colour glowing in the gaps in the cistusgroves, and one is thus from far off made aware of the presence of the parasite. The flowers themselves, which open between the red scale-like bracts, are yellow. 'The combination of colour thus afforded is a rare phenomenon in the vegetable world, and gives a very strange appearance to the plant. Besides the species of Cytinus distributed over the area of the Mediterranean flora, there are two other species in Mexico, and one also at the Cape, which, although not parasitic on Cistus shrubs but on other woody plants, especially Eriocephalus, yet do not differ from Cytinus Hypocistus in floral structure or in mode of connection with their host.

\section{MISTLETOES AND LORANTHUSES.}

The sixth and last series of parasitic phanerogams includes epiphytes of bushy appearance with much bifurcated branches, green cortex, green leaves, and berries containing large seeds, which germinate whilst resting immediately upon the branches of such trees as are adapted to act as host-plants, and will surrender to the invader a portion of their nutriment. To this series belong a dozen different species of the genus Henslowia, belonging to the family of Santalacer, and indigenous to the South of Asia-chiefly the East Indian Archipelago-and, in addition, upwards of 300 species included in the family Loranthaceæ. Amongst the latter, the plant that is best known and most widely distributed is the European Mistletoe (Viscum album) represented in fig. 46, and as it is also fitted, in 
respect of its life-history, to serve as type of the entire series, we will describe it first of all.

As is well known, the Mistletoe is parasitic upon trees, and these may be either Angiosperms or Gymnosperms. Most frequently it establishes itself upon trees the branches of which are coated by a soft sappy cortex-an extremely delicate and tender cork-tissue in particular - as is the case with silver-firs, apple-trees, and poplars. The Mistletoe's favourite tree is certainly the Black Poplar (Populus nigra). It flourishes with astonishing luxuriance on the branches of that tree, and wherever there is a small plantation of Black Poplars, the Mistletoe takes up its abode.

Along the shores of the Baltic and by the Danube near Vienna-especially in the celebrated Prater from which fig. 47 is taken, one finds, on many of the Black Poplars, tufts of Mistletoe measuring 4 meters in circumference, and with axes of a thickness of $5 \mathrm{~cm}$. Birds use their most crowded branches, by preference, to nest in. In the forests of Karst, in Carniola, and in the Black Forest, where poplar trees play merely a subordinate part, whilst on the other hand, quantities of silver firs shade the ground, large numbers of these conifers have their tops covered with Mistletoe; and in the Rhine districts and the valley of the Inn in Tyrol, the same parasite occurs as a troublesome visitor upon apple-trees in the neighbourhood of the peasants' farms. In localities destitute of these three kinds of trees, which are pre-eminently the Mistletoe's favourite host-plants, it puts up with other trees, and is then usually found on whatever species happens to be the most common in each particular country. Thus, in the Black Pine district of the Wiener Wald, it occurs upon the Corsican Pine, whilst on the heaths of the sandy lowlands of the March, it settles upon the Scotch Pine. Much less frequently it has been observed on walnut-trees, limes, elms, robinias, willows, ashes, whitethorns, pear-trees, medlars, damsons, almond-trees, and on the various species of Sorbus. Mistletoe has also been found by way of exception upon the oak and the maple, and upon old vines. On one occasion, in the district of Verona, it has been seen established upon the parasitic shrubs of Loranthus Europaus, that is to say, one member of the Loranthaceæ was found parasitic upon another. The birch, the beech, and the plane, are avoided by the Mistletoe, a fact which no doubt depends upon the special structure of the cortex in those trees.

The dissemination of the European Mistletoe is effected, as in all the other Loranthaceæ, through the agency of birds-thrushes in particular-which feed upon the berries and deposit the undigested seeds with their excrement upon the branches of trees. That a preliminary passage through the alimentary canal of birds is essential to the germination of these seeds is no doubt a delusion, this assumption of former times being easily refuted by the fact that one can readily induce the seeds of berries, taken fresh from a tree, and stuck into fissures in the bark of moderately suitable trees, to germinate; it is, however, true, that in nature, mistletoe-seeds are dispersed exclusively by birds in the manner above mentioned. To this method of dissemination must be attributed the phenomenon, which, at first 
sight, is surprising, that Mistletoe-plants are rarely seated upon the upper surface of branches, but very frequently on the sides. For the dung of thrushes, which live upon Mistletoe-berries, is in the form of a semi-fluid, highly viscid mass, ductile like bird-lime; and, even when it is deposited upon the upper surface of slanting branches, it immediately runs down the sides, sometimes extending in ropes 20 or 30 centimeters in length. Owing to the viscous mass thus following the law of gravity, the Mistletoe-seeds imbedded in it are conveyed to the sides, and even to the under surface of the bark, and there remain cemented.

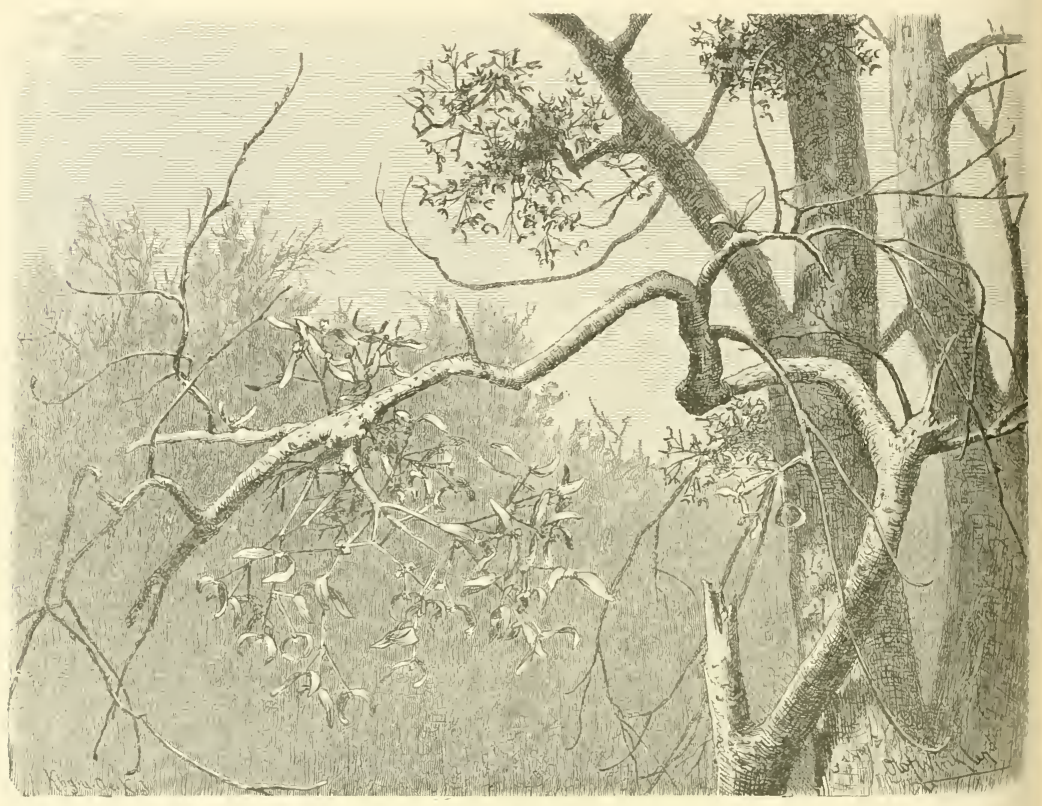

Fig. 46. -The European Mlistletoe (Viscum album).

It may be a long time before a seed of the kind germinates, especially if it does not become attached until the autumn. The embryo is completely surrounded in the seed by reserve food. It is club-shaped and comparatively large, and is distinguished by the fact that the two oblong cotyledons, which are closely pressed together, but often somewhat wavy at the margins, are coloured dark green by chlorophyll, like the environing cellular mass filled with reserve materials. In the process of germination the axis of the embryo, especially the part lying beneath the cotyledons, and passing into the hemispherical radiele, lengthens out; the white seed-coat is pierced, and the radicle makes its appearance through the breach. Under all circumstances the emergent radicle is directed towards the bark of the branch to which the seed is adherent. This is the case even when the seed chances 
to stick with the radicle of the seedling pointing away trom the branch; the whole axis of the embryo curving towards the surface of the bark in a very striking manner. Thus the radicle always reaches the bark, and having done so it becomes adpressed and cemented to its surface, spreads itself out in the form of a doughy mass, and so develops into a regular attachment-dise. From its centre a slender process now grows into the bark of the host-plant, piercing the latter and penetrating as far as the wood, but not growing into that tissue. This penetrating process has been termed a "sinker", and must be looked upon as a specially modified root.

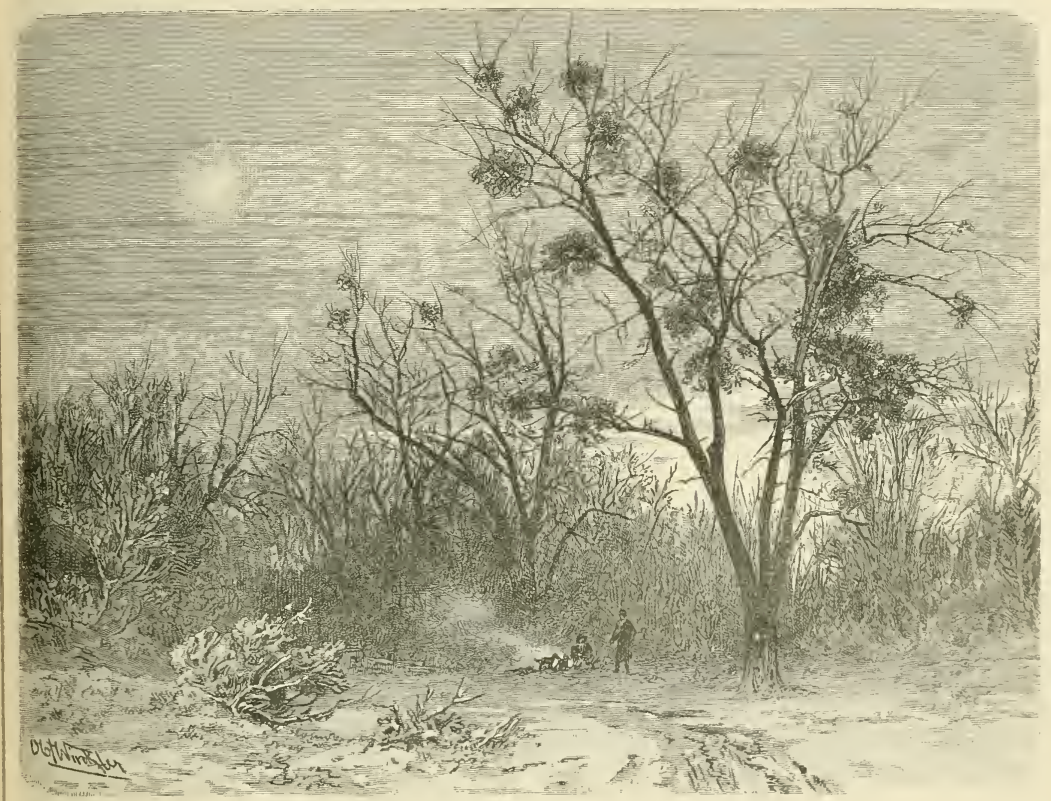

Fig. 47.-Bushes of Mistletoe upon the Black Poplar in winter.

The development of the first year ends with the formation of this sinker. When the winter is over, the branch, into which the sinker is inserted so as just to reach the wood with its point, grows in thickness, a new layer of wood-cells-a so-called annual ring-being superimposed upon the wood of the previous year. The increasing mass of wood first surrounds the tip of the sinker with wood-cells, then forms a rampart all round it, pushing the cortical tissue, wherein that organ has hitherto been wedged, in front of it in an outward direction, and in this way the sinker is at length fixed deep within the woody eylinder. The process of inclosure by the wood-layers, as they are built up, may be compared to the gradual surrounding of a stake on the sea-shore by the rising tide; the lowermost extremity is first immersed and then higher and higher parts until the whole is enveloped. The 
sinker itself remains, strictly speaking, stationary; it does not grow into the wood, but the wood overgrows it. But what happens in the following season when a fresh annual ring is once more added to the wood? If the sinker had entirely ceased growing it would of necessity be ultimately completely closed by the layers of wood, as they develop with ever-increasing energy and add to the thickness of the branch, and at last it would be quite buried. To prevent this result, which would be fatal to the Mistletoe, a zone of cells is provided near the base of the sinker, which zone, at the time when the rampart of wood is being raised, adds in an equal degree to its own height, and causes, of course, an elongation of the sinker in a peripheral direction. The length of the piece thus intercalated in the haustorium is exactly equal to the thickness of the corresponding annual ring in the surrounding wood of the branch. Thus at length the Mistletoe-sinker is found imbedded in a number of annual rings, although it has not grown into the latter, but has been banked up by them year by year.

That zone of the sinker which possesses the capacity for growth, and which is always to be sought, in accordance with what has been said above, at the outside limit of the wood of the branch, in the so-called "bast" layer situated on the inner face of the cortex, produces, in the second year after the adhesion of the Mistletoeembryo, lateral ramifications which are called cortical roots. They are thick, cylindrical, or somewhat compressed filaments, and all run close together under the cortex in the bast layer of the invaded branch. These rootlets issuing from the sinkers pursue a course parallel to the longitudinal axis of the branch, whilst the sinkers themselves are at right angles to the axis (see fig. $48^{3}$ ). If a rootlet springs from the sinker in a direction transverse to the longitudinal axis it bends immediately afterwards so as to be parallel to the long axis, and adopts the same direction as the rest, or else it bifurcates just above its place of origin into two branches which separate suddenly, and in their further course follow the axis of the branch. Thus it comes to pass that all the rootlets of a Mistletoe run up and down in the infested branch of the host-plant in the form of thick green parallel strands, but that none of them ever encircle the branch in the form of an annulas coil. Each of these cortical roots may now develop from behind the growing-point new sinkers, which are formed in the same way as the first one above described as proceeding from the actual seedling. They, too, penetrate into the branch perpendicularly to the axis, and as far as the solid wood are then encompassed by the growing mass of wood, but maintain the power of growth in the part close to their insertions, and in their growth keep pace with the thickening of the wood of the branch. The fact of the yearly recurrence of this formation of sinkers explains how it is that those situated nearest the growing-points of the cortical roots are the shortest, they being the youngest, whilst those which arise near the first sinker are the longest and oldest. It also accounts for the former being only inclosed by one annual ring of the host's wood, and the others being surrounded by an increasing number of rings the nearer they are to the spot where the Mistletoe-plant first struck root. 
The root-system of the Mistletoe taken as a whole may be described as like a jaw-bone in shape, or, still better, a rake. The cross-beam of the rake corresponds to the cortical root, whilst the teeth are analogous to the sinkers; the cross-piece must be supposed to be parallel to the axis of the branch and lying under the bark, and the spokes must be thought of as perpendicular to the axis and driven into the wood.

Whilst the roots of the Mistletoe-plant are spreading in the interior of the branch in the manner described, the stem is developed outside. At the time when the process, subsequently to be the first sinker, emerges from the attachment-disc of
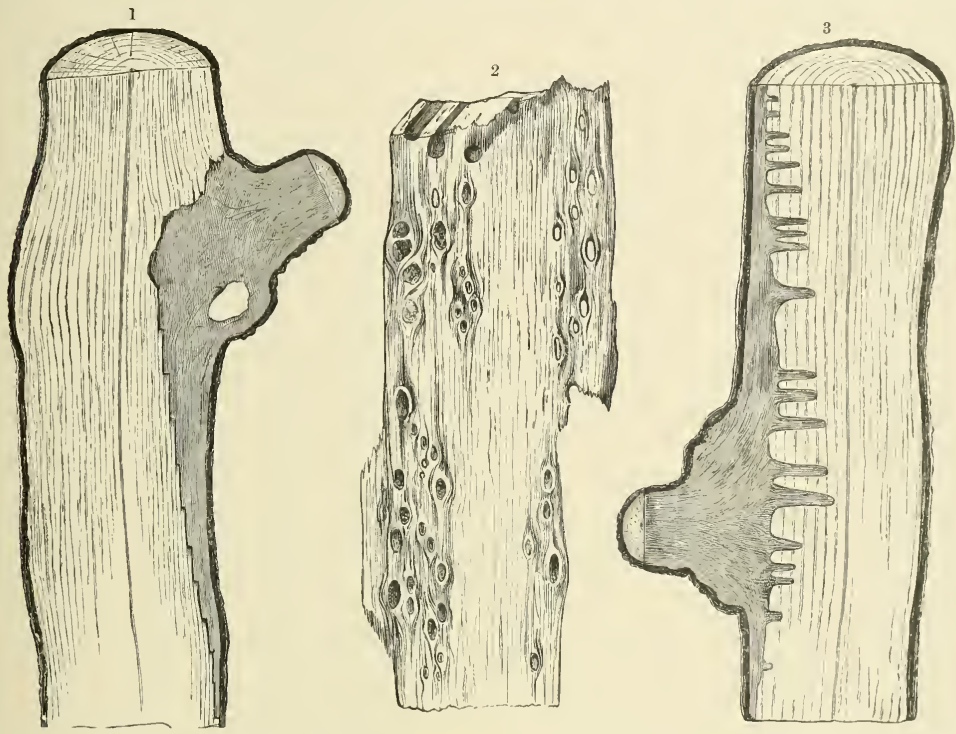

Fig. 48.-1 Loranthus Europaens, and 3 Mistletoe (Viscum album)-both parasitic ou branches of trees, and seen in section. ${ }^{2} \mathrm{~A}$ piece of the wood of a Fir-tree perforated by the sinkers of a Mistletoe.

the embryo and pierces through the bark, the cotyledons are still covered by the white seed-coat, which rests upon them like a cap. But when once this first sinker is firmly fixed and in a position to take up nutritive juices from the wood of the host, the seed-coat is thrown off; the apex of the stem, which is still very short, is raised; the cotyledons are detached, whilst close above them is produced a pair of green leaves. Thenceforward the development of the visible portion of the Mistletoeplant outside the bark keeps pace with that of the roots underneath the cortex, and is moreover dependent upon the quantity of food taken up by the sinkers from the wood. Where there is an abundant supply of nutriment, as in the case of poplars, the growth of the Mistletoe is correspondingly exuberant; where the flow of juices is scarce, the parasite is stunted in its growth, and often develops only small yellowish sickly-looking tufts. If the foster-plant is of a lavish nature, advenVoL. I. 
titious buds are produced regularly by the cortical roots to which the absorbed nutriment is first of all conveyed from the sinkers. These buds occur on the side of the rootlets nearest the exterior of the bark, and later they burst through the rind, and develop into new Mistletoe-plants.

These outgrowths are analogous to the adventitious shoots produced from the subterranean roots of the Aspen, and this comparison is rendered all the more appropriate by the fact that the removal of the tuft of Mistletoe encourages the sprouting of adrentitious root-buds just as in the case of the Aspen, the growth of shoots from the roots is promoted by the felling of the trees to which those roots belong. If a large Mistletoe-bush, growing in solitude on a Black Poplar, is removed from the tree with the intention of freeing the latter from its parasite, the hopes entertained by the operator are disappointed; for, an outgrowth of shoots from the cortical roots ensues at a number of different spots, and in a few years' time the poplar in question is the prey of a dozen Mistletoe-bushes instead of one. Inasmuch as these bushes, produced from offishoots, are able, under favourable conditions, to send out fresh roots, and these again may develop shoots, a good host of the kind will at last have all its boughs from top to bottom overgrown by Mistletoes. In the Prater at Vienna there are poplars beset by at least thirty large Mistletoeshrubs, and double that number of small ones, and if one catches sight of such a tree at some distance in winter-time when the branches have lost their leaves, one takes it to be a Mistletoe-tree, for almost the entire system of branches is mantled in a continuous tangle of evergreen bushes of Mistletoe, which are in a state of parasitism upon it.

Sinkers of the Mistletoe, $10 \mathrm{~cm}$. in length, and inclosed in forty annual rings, have been found in the wood of the Silver Fir, whence we may conclude that the Mistletoe may live for forty years. A greater age could scarcely be attained by one and the same bush of the parasite. If the Mistletoe dies, the rootlets and haustoria survive for a time, but at length moulder and fall to pieces, whilst the wood in which they were imbedded remains unaltered. The affected parts of the wood exhibit in that case numerous perforations, and look just like the wood of a target which has been fired at and struck by shot or small bullets (see fig. $48^{2}$ ).

A small plant belonging to the Loranthaceæ and named Juniper-Mistletoe ( $V i s$ cum Oxycedri or Arceuthobium Oxycedri) occurs on the red-berried juniper bushes (Juniperus Oxycedrus) of the Mediterranean flora. It is very different from the common European Mistletoe, as is obvious at first sight, its foliage-leaves being reduced to little scales, which gives a characteristic jointed appearance to the ramifications. A whole series of leafless forms allied to this species is found to exist in India, Japan, Java, Bourbon, Mexico, Brazil, and at the Cape. They are nearly all small bushes which project from the boughs of host-plants and sometimes clothe the latter so thickly that the boughs in question serving as nutrient substratum are entirely enshrouded by the parasitic growth. The Juniper-Mistletoe is only from $3 \mathrm{~cm}$. to $5 \mathrm{~cm}$. tall, and the branchlets are not woody, but soft and herbaceous; the fruits are blue oblong berries, almost destitute of succulence. The latter are 
dispersed by birds like the berries of the common Mistletoe, and the way in which the parasite settles upon and clings to branches of the host-plant is the same as in that species. It also develops sinkers and cortical roots, but these root-structures are not by any means so regularly arranged as in Viscum album, but form an inextricable web of strands and filaments pervading the internal layers of cortex, and resolving itself into finer and finer groups of cells, which end by looking not unlike a mycelium, and also remind one of the suction-apparatus possessed by Rafflesiacex. Such of these strands and cellular filaments as are imbedded in the wood of the juniper do undoubtedly play the part of suction-organs. They are present in large numbers, and some of them are occasionally encompassed by several annual rings. They possess no special zone of growth. The elongation necessary to prevent their being enveloped and overwhelmed by the wood, as it adds to its thickness, is effected by the division of individual cells and groups of cells. The outgrowth of shoots from the root is much more exuberant than in the common Mistletoe; but the death of the original plant takes place much earlier, and close to yellowish-green bushes of various degrees of smallness, one finds very regularly dead or dying shrublets already turned brown, all growing promiscuously over the somewhat swollen branches of the red-berried Juniper.

The behaviour of Loranthus Europaus, which is parasitic on oaks and chestnuts in the east and south of Europe, is altogether unique. The mode of its attack upon the branches of oaks is, it is true, similar to that of the two other Loranthaceæ just described. The yellow berries, which are grouped in graceful biseriate racemes, are eaten with avidity by thrushes in the autumn and winter, and the undigested seeds are deposited with the dung of those birds upon the branches of trees. The embryo, on emerging from the seed, bends towards the bark and sticks to it, at the bottom of little rifts and crevices, for the most part, by means of the radicle, which becomes an attachment-disc. A process now arises from the centre of the attachment-disc, and pierces through all the cortical layers of the oak-branch as far as to the zone of young wood, just as if it were a small nail driven in. This process increases in thickness at the expense of the nutriment it withdraws from the young wood, and from it are developed one, two, or three branches, which, however, invariably run downwards beneath the bark, that is to say, in the direction opposed to that of the stream of sap ascending in the oak-wood, and never produce the sinkers so characteristic of the Mistletoe. Each of these roots is shaped like a wedge, even from the rudimentary stage, and acts, too, in the manner of a wedge, penetrating between the yet soft and delicate cells of the cambium, which were formed in the spring at the periphery of the solid older wood of the previous year, and were destined to constitute a new annual ring, splitting and tearing in the process that cell-tissue. Such of these tender cells as lie outside the wedge die, those situated within become lignified and altered into solid wood, to which the wedgeshaped root firmly adheres. Beneath the apex of the wedge, the lignification of cambium cells naturally extends much further towards the exterior, because there it is not at all broken or dead. In front of the apex of the wedge, therefore, there 
is, presently, solid resisting wood. The root being no longer able to split the tissue with its point, is stopped in its growth at this spot. But there is nothing to prevent its continuing to grow along a course somewhat nearer the periphery, and outside the limit of the new annual ring of solid wood, where a fresh development of soft and tender cells has taken place in the cambium, and this indeed actually happens.

Thus, every addition to the length of the Loranthus-root, as it grows onward between the wood and the cortex of the oak-branch, is further removed from the axis of the branch; or, in other words, the surface of contact between root and wool has the conformation of a flight of stairs, of which the lowest step constitutes the base, and the uppermost the apex of the root (see fig. $49^{1}$ ). These steps are very small, their height varying from about $5 \mathrm{~mm}$. to $7 \mathrm{~mm}$., but they may be distinguished quite elearly in longitudinal sections, on account of the darker colour of these roots contrasting with the lighter oak-wood. Nutritive fluids are imbibed by the Loranthus-root from the wood of the oak at the surface of contact, and it is probable that this absorption takes place especially at the notches forming the steps. The root can only elongate, naturally, during the period when there is a young and fragile cell-layer superimposed upon the solid wood, whence it follows that in Loranthus the continuation of the root's growth is more dependent upon a particular season and upon the annual progress of development of the host than is the case with the Mistletoe. There may be some connection between this circumstance and the fact that the Mistletoe possesses evergreen leaves, whilst Loranthus is green only in summer, acquiring fresh green foliage in the spring in the very same week as the oak does, and easting its leaves in the autumn simultaneously with the tree it infests.

The stem which issues from the embryo of a Loranthus-seed grows away from the oak-branch into the open air, and develops with great rapidity at the expense of the nutriment absorbed from the host's wood, and conveyed to it by the root above described, into a dense, dichotomously-branched bush. In summer it is not unlike a Mistletoe-bush, but in autumn, when it has cast its leaves, it acquires a totally different aspect owing to the dark-brown branches and the conspicuous yellow clusters of berries.

Bushes of Loranthus grow to a greater size even than those of the Mistletoe; their stems attain not infrequently a thickness of $4 \mathrm{~cm}$., and clothe themselves with a blackish, rugged bark, the older stems of this kind being then usually studded by an abundance of lichens. At the spots where stems of Loranthus spring from an oak-branch they are always surrounded by a great rampart of wood belonging to the oak, and the base of the stem is often fixed in a deep symmetrically-rounded bowl reminding one vividly of the similar structures out of which the stems of Balanophoreæ arise. But whereas in Balanophoreæ this bowl-shaped rampart appertains to the parasite, in Loranthus it is formed from the wood of the hostplant, i.e. the oak. It must, in the case we are considering, be interpreted as an exuberant growth of wood-cells and compared to the hypertrophies called galls, 
which will be treated of in detail in a subsequent part of this book. On old oaks in the east of Europe these growths round the bases of Loranthus-plants sometimes reach the size of a man's head. In the case of a bush of Loranthus nearly 100 years old, from the Ernstbrunner Wald, in Lower Austria, which had reached a height of $1.2 \mathrm{~m}$. and a circumference of $5.5 \mathrm{~m}$., the hypertrophy in question measured $70 \mathrm{~cm}$. round. It is not only the base of a bush that is overgrown by wood-cells, but the older portions of the roots described above are frequently walled in and partially inclosed by the wood of the branch as it becomes thicker. They may often be seen fixed deep in the wood, yet still preserving their freshness and vitality, and this is to be explained by the fact that they retain connection with other parts of the roots by means of isolated ledges and bridges. Indeed an adventitious shoot may develop from a piece of a root thus deeply wedged in the wood of the oak, and this shoot then grows so outwards and breaks through all the layers lying above it and originates a young bush, which pushes roots under the host's bark and afterwards behaves in exactly the same manner as a plant produced from a seed cemented to the oak-branch.

The Loranthus chosen here for description (L. Europceus) has only small inconspicuous yellowish flowers; on the other hand, under the tropical sun of Africa, Asia, and, above all, Central America, the parasitic species of this genus are amongst the most splendid-flowered of plants. There are species in the tropicse.g. Loranthus formosus, L. grandiflorus, and L. Mutisii-whose flowers attain a diameter of 10, 15, or even 20 centimeters, and are besides clothed in the most gorgeous purple and orange colours. Many Loranthi are like small trees grafted upon other trees. The host-plants of these Loranthi are principally angiospermous trees; members of the genus have also repeatedly been met with parasitic upon one another-as, for instance, Loranthus buxifolius upon L. tetrandrus in Chili. The fact has been already mentioned that the European Mistletoe has been observed near Verona parasitic upon Loranthus. It is also worth noticing, in order to complete the account of the complex relationships between parasites, that one species of $V$ iscum has been found in India parasitic upon another, viz.:-Viscum moniliforme on $V$. orientale.

\section{GRAFTING AND BUDDING.}

Parasitism of one woody plant upon another, such as occurs in the case of Loranthaceæ, calls to mind certain modes of organic union between woody plants that are artificially effected by gardeners. From ancient times gardeners have performed special operations which are known as processes of "ennobling", and consist in the transference of the branch or bud of one plant on to another plant as substratum, and the inducement of organic union between the two. The plant from which the branch or bud is taken is perhaps a valuable variety of fruit-tree, or a handsorne specimen of an ornamental shrub, whilst for the purpose of a substratum a robustly-growing individual belonging to a wild species of shrub or tree is selected 
as a rule, and constitutes the so-called wild "stoek". The branch which yields tho bud for the operation or which is itself transferred in its entirety to the wild stock is named, in the terminology of hortieulture, the noble "scion".

The process of ennobling is effected either by grafting or by budding. In grafting the stem of the stoek is cut off transversely, an excision is made at the periphery of the surface of the seetion and the scion is inserted in this opening. The scion must be previously trimmed to fit; in preparing it care must be taken that it bears a pair of healthy buds, and that the end to be inserted is cut so as to correspond to the form of the fissure made in the stock. In inserting it one must see that, as far as possible, the bark, bast, and wood of the one eome into contact with the corresponding parts of the other. The wounds of the stock caused by the operation are then covered by a mass of putty, wax, or some other protective medium, and the chances are that the braneh thus introduced will contract an organic union with the substratum, that nutritive matter will be supplied it by the substratum, and that new branches will sprout from its buds. In this case therefore the nutriment taken from the ground by the stock passes into the grafted scion, and the scion, which develops branches from its buds, and ultimately may beeome a densely ramifying tree-top, behaves as a parasite, whilst the stock plays the part of host.

It not infrequently happens that a substratum supporting at its summit the branehes of a grafted scion develops subsequently branehes of its own lower down as well, and the curious sight is then afforded of a tree or shrub bearing different foliage, flowers, and fruit on its inferior parts from those of its upper regions. If, for example, the stem of a Quince is used as substratum, and Medlar branches are grafted upon it, the result may be a bush or tree which exhibits below branches with the round leaves, rose-coloured flowers, and golden "pomes" of the Quince, and above branches with the oblong leaves, white flowers, and brown fruit of the Medlar. Gardeners, of eourse, do not willingly allow this to happen, but carefully remove the branches belonging to the stock in order that all the food materials may fall to the lot of the grafted plant, and the latter thrive as vigorously and luxuriantly as possible.

The same result is obtained by budding as by grafting; but here a single bud of the scion, instead of an entire branch, is transferred to the stock. This is accom. plished in the following manner:-Two incisions at right angles forming a $T$, are made in a branch of not too great age belonging to the plant employed as substratum. These cuts are carried through the bark as far as the wood. The two lobes of bark, formed by the $T$-shaped incision, are then carefully raised from the wood, and the bud to be transplanted is pushed in under them. The bud which has previously been taken away from the scion must have retained in that process a portion of bark, and usually the bit of bark peeled off is given the shape of a little shield. This shield, carrying the bud that is to be transferred upon it, is now introdueed between the two lobes above mentioned, and the lobes are folded over it in such a manner as to allow the bud to project freely from the slit between the 
lobes. Besides this, the whole is held together by a bandage, the shield in particular with its bud being pressed firmly on to the new substratum, and thereupon, as a rule, coalescence takes place at once, and the inserted bud grows out into a branch which stands in exactly the same relation to the stock as a Loranthus to the oak whereon it is parasitic. All the branches belonging to the substratum, that is to say, to the wild stock, may then be removed, leaving only the one branch that has sprung from the stranger-bud, the result being that all the juices absorbed from the ground by the substratum are concentrated in this branch and cause it to grow with the greatest exuberance.

There is between this process of budding and the settling of a parasite a further resemblance in that shrubs and trees cannot all be made to unite at pleasure one with the other. A successful result of grafting or budding can only be counted upon when nearly allied species, belonging to the same genus or family, are employed for the purpose. Almonds, peaches, apricots, and plums can be grafted the one upon the other; so also can quinces, apples, pears, medlars, and whitethorns. But we must relegate to the realms of fiction such assertions as that peaches might be successfully grafted upon willow stocks, or that the Siberian Cral, (Pyrus salicifolia) has sprung from the grafting of branches of the Pear upon the Willow and other tales of the sort. Whether it is possible by grafting or budding to produce new forms, or at least hybrids, is a question which will claim our attention in connection with the problem of the origin of new species. The only additional remark to be made here is that notwithstanding the undeniable similarity between grafted or budded plants and the parasitic Loranthacer, a very essential difference exists in the circumstance that the latter develops roots which continue to grow year by year, and are always penetrating into new layers of the host's tissues, whereas this is never observed in the case of grafted or budded plants. When the branch of a Peach is grafted on an Almond-tree, there is, it is true, an organic union of the two at the place of contact, and the juices from the wood of the Almond stock are conducted direct into the grafted Peach-branch; but neither roots nor sinkers ever arise from the base of the adnate branch or penetrate into the stem of the Almond-tree. 


\section{ABSORPTION OF TVAER.}

Importance of water to the life of a plant-Absorption of water by Lichens and Mosses, and by Epiphytes furnished with aërial roots-Absorption of rain and dew by foliage-leaves-Development of absorptive cells in special cavities and grooves in the leaves.

\section{IMPORTANCE OF WATER TO THE LIFE OF A PLANT.}

In the building up of the molecules of sugar, starch, cellulose, fats, and acids, of proteids, and, in short, of all the important substances of which a plant is composed, atoms of water have to be incorporated as constructive material, and without water no growth or addition to the mass of a plant whatsoever could take place. From this point of view water must be considered just as indispensable an item in the food of plants as the carbon-dioxide of the air. But water plays, in addition, another important part in plant-life. The mineral salts which serve to nourish hydrophytes, land-plants, and lithophytes, as also the organic compounds which are the food of saprophytes and parasites, can only reach the interior of plants in the form of aqueous solutions. They can only pass through a cell-wall when it is saturated with water, and, having reached the interior of a plant, they can only be conveyed to the places where they are worked up through the medium of water. In connection with the discharge of these functions in a living plant, water must be regarded as a dynamic agent. Just as a mill on a stream only works so long as its wheels are kept in motion by the water, and stops at once if the latter fails, or flows by in insufficient quantity, so the living plant, as it nourishes itself, grows and multiplies, needs a continuous and abundant supply of available water to render possible the performance of the complicated vital processes within it. This available or organizing water is not in chemical combination like that which is present as food-material, and is, in general, not permanently retained. On the contrary, we must conceive it as perpetually streaming through the living plant. In the course of a summer, quantities of water, weighing many times as much as the plant itself, pass through it. The total amount of water in chemical combination in the organic compounds of a plant is very trifling compared with this, though it often happens that the weight of the latter in a particular plant is greater than that of all the other substances put together.

Inasmuch as this water evaporates from plants in dry air, and that it may also easily be withdrawn by alcohol or other means, very simple experiments suffice to give an idea of the great bulk of free water in any plant. Berries, fleshy fungi, succulent leaves, and things of that kind, if left in alcohol, are reduced in a short time to barely half their size in the fresh state. The Nostocineæ, which are gelatinous when alive, and many fungi (e.g. Guepinia, Phallus, Spathularia, Dacryomyces) shrivel up so stringently in drying, that a piece possessing an area of 1 square centimeter when fresh leaves only a dry crumbling mass covering scarcely 3 square millimeters. A Nostoc, which weighed 2.224 grms. in the fresh state only 
weighed 0.126 grm. after desiccation, so that when alive it must have contained 94 per cent. of water. Bog-moss, weighing $25.067 \mathrm{grms}$. before the abstraction of the water was reduced to 2.535 grms. afterwards, showing that the percentage of water was 90. Similar results are obtained in the cases of succulent leaves and stems of flowering plants, Cucurbita, and other fruits. The least proportion of water is contained by mature seeds, solid stony seed-coats, wood, and bark; but even in these an average proportion of 10 per cent of water has been detected. We shall not go wrong in assuming, on the evidence of the weights determined, that most parts of plants, when fresh, consist of dry substance only as regards a third, and as regards two-thirds, of water of imbibition, which passes over into the surrounding air in the form of vapour when desiccation takes place.

From all this it follows that water is absolutely necessary to plants as foodmaterial, that it is indispensable as a medium of transport of other substances, and that the demand for water on the part of all plants is very great. Further, we may infer that the importation and exportation of water must be regulated with exactitude if the nutrition is not to be disturbed and development hindered.

Water-absorption is at its simplest in hydrophytes. In this case it coincides with the absorption of the rest of the food-materials, and there is therefore nothing material to add to the statements already made on that subject.

As regards land-plants, lithophytes, and epiphytes, we may likewise refer to what has been already said in so far as these plants suck up water at the same time as food-salts, by means of absorption-cells, from the substratum to which they are attached, or the earth in which they are rooted; but to the extent that they take also water direct from the atmosphere, and have the power of absorbing that water immediately they require it, must be discussed in the following pages.

\section{ABSORPTION OF WATER BY LICHENS AND MOSSES, AND BY EPIPHYTES FURNISHED WITH AËRIAL ROOTS.}

The plants which absorb water direct from the atmosphere may be classified in several groups with reference to the contrivances adapted to the purpose. Of all plants lichens are most dependent on atmospheric moisture. Many of them, especially the Old Man's Beard Lichens, which hang down from dried branches of trees, and the gelatinous, crustaceous, and fruticose lichens, which cling to dead wood, and on the surface of rocks and blocks of stone, do in fact derive their necessary supply of water entirely from the atmosphere, and that by absorbing it, not in a liquid but in a gaseous form. The latter circumstance is of the greatest importance to those species in particular which occur on receding rocks, or on the under face of overhanging slabs of stone. Rain and dew cannot reach such places directly, but only by some of the water trickling down from the wet top and sides of the rocks on to the receding wall, and this happens but seldom. Accordingly, lichens occurring in situations of the kind are entirely dependent upon the water contained in the air in the form of vapour. Lichens, however, are also, of all plants, 
the best adapted for the absorption of aqueous vapour from the air. If living lichens, which have become dry in the air, are left in a place saturated with moisture, they take up 35 per cent of water in two days, and as much as 56 per cent in six days. Water in the liquid form is naturally absorbed much more rapidly still. When Gyrophoras, which project in the form of cups after a long continuance of dry weather, are moistened by a fall of rain, they swell up completely within ten minutes, and spread themselves flat upon the rocks, having in that short space of time absorbed 50 per cent of water. The saying, "Light come, light go," is no doubt true in these cases. When dry weather sets in, evaporation from the masses of lichens goes on at a pace corresponding to the previous absorption. In the Tundra, the lichens, which form a soft tumid carpet when moistened by rain, are liable to be so powerfully desiccated in the course of a few hours of sunshine, that they split and crackle under one's feet, so that every step is accompanied by a crunching noise.

In the power of condensing and absorbing the aqueous vapour of the atmosphere, lichens are most analogous to mosses and liverworts, and to those preeminently which live on the bark of dry branches of trees or on surfaces of rock, covering places of the kind with a carpet which is often enough interspersed and interworen with lichens. Like the latter these mosses and liverworts are able to remain as though dead in a state of desiccation for weeks together, but as soon as rain or dew falls upon them they resume their vitality; and similarly if the air is so damp as to enable them to derive sufficient water of imbibition from that source. A specimen of Hypnum molluscum, a moss which covers blocks of limestone in the form of soft sods, was after a few rainless days detached from the dry rock and placed in a chamber saturated with vapour, and it was found that after two days it had absorbed water from the air to the extent of 20 per cent, after six days 38 per cent, and after ten days 44 per cent. Many mosses condense and absorb water with the whole surfaces of their leaflets, others-as, for example, the gray rockmosses clinging to slate formations (Rhacomitriæ and Grimmiæ)-do so especially with the long hair-like cells at the apices of the leaflets, whilst others again only use the cells situated on the upper saucer-shaped or canaliculate leaf-surface.

In some bearded mosses (Barbula aloides, B. rigida, and B. ambigua) chains of barrel-shaped cells occur closely packed together upon the upper surface of the leaf and at right angles to it, which to the naked eye have the appearance of a spongy dark-green pad. The terminal cells of these short moniliform chains have their upturned walls strongly thickened, but the other cells have very thin walls and take up water rapidly. It is the same with the various species of Polytrichum, which are provided on their upper leaf-surfaces with parallel longitudinal ridges likewise composed of thin-walled, highly-absorbent cells. The rhizoids also play an important part in these processes. These brown, elongated, thin-walled cells entirely clothe the moss stems, usually in the form of a dense felt, and often project from the under surface of the leaves, whilst in a few tropical species they make their appearance, strangely enough, in the form of little tufts at the apices of the 
leaflets. In many instances this felt of rhizoids does not come into contact at all with the soil, rock, or bark (as the case may be), but is surrounded by air alone, and is able to condense or attract, to use a common expression, the aqueous vapour of the air like a piece of cloth or blotting-paper. In dry weather, it is true, mosses, like lichens, lose their water, but they part with it much more slowly than the latter. This is chiefly due to the fact that the moss-leaflets at the commencement of a drought wrinkle, curl up, become concave, and lay themselves one above the other, so that the water is retained at the bottom for a longer period.

A very remarkable contrivance for the absorption of water from the atmosphere is also exhibited by the white-leaved Fork-mosses (Leucolryum) and Bogmosses (Sphagnaceæ). Although they possess chlorophyll, and assimilate under the
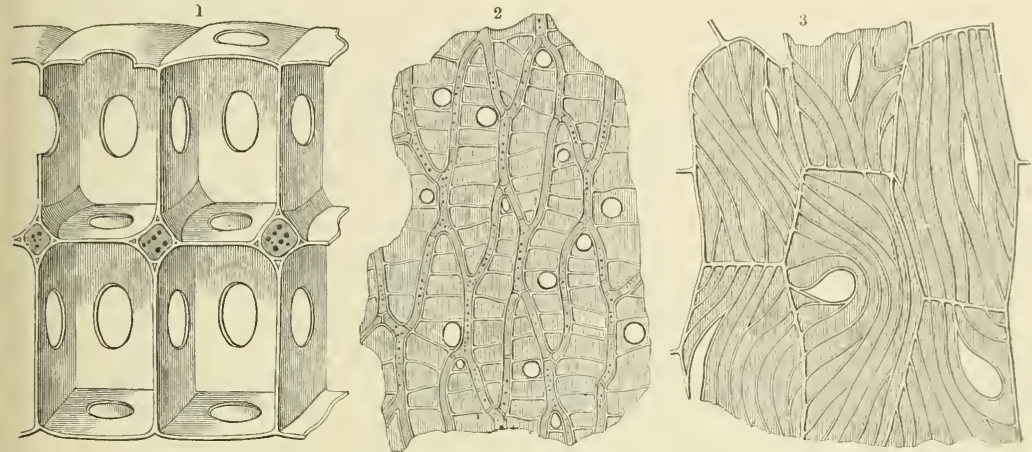

Fig. 49.-Porous Cells.

1 Of the white-leaved Fork-moss (Leucobryum); $\times 550.2$ Of the Bog-moss (Sphagnum); $\times 230.3$ of the root of an Orchid (Lotia gracilis); $\times 310$.

influence of sunlight, yet they look like parasitic and saprophytic plants destitute of chlorophyll. They are of a whitish colour and always grow in great cushionlike sods, so that the spots where they grow are deficient in verdure, and stand out conspicuously from their surroundings in consequence of their pale tint. Microscopic investigation at once explains this appearance. The cells containing chlorophyll and living active protoplasts are relatively small, and, as it were, wedged and hidden between other cells many times as great, which have entirely lost their protoplasm by the time they are mature, and then cause the paleness of colour appertaining to the plant as a whole. The walls of these large colourless cells are very thin, and in the Bog-mosses have spiral thickening-bands running round them, being thus secured against collapse. After remaining for a time in a dry environment they are full of air only; but the moment they are moistened they fill with water. If there were an actively absorbent protoplast at work in the interior, the water would be able to pass into the cell-cavity through this easily moistened wall, as in the case of other mosses, owing to the delicacy of the cellmembrane. But the air which fills the cells is not absorptive, and in the case of Leucobryum and Bog-mosses the water reaches the interior, not in consequence of 
a chemical affinity on the part of the cell-contents, but solely by capillary action. All the cell-walls are perforated and furnished with pores, and through these the water rushes into the interior with lightning rapidity.

This extremely rapid influx of water into an air-filled cavity leads us necessarily to the conclusion that each cell has a number of pores in its walls, and that in proportion as water enters through one of the small apertures the air can escape equally fast through another. This is in fact the case. The large cells not only have pores on their external walls, but communicate one with another by similar holes, and the water soaks in from the one side as it does into a bath-sponge, whilst the air is at the same time forced out on the other. This absorptive apparatus is exceptionally elegant in Leucobryum, which grows abundantly in many woods. In it, as is shown in the illustration above (fig. $49^{1}$ ), the adjacent prismatic cells communicate by highly symmetrical, circular gaps made in the middle of the partition-walls, whilst in the Bog-mosses (the various species of Sphagnum), they are to be seen scattered here and there between the thickening bands on the cellwalls (see fig. $49^{2}$ ). Now these porous groups of cells possess not only the power of taking up water in the liquid state, but also that of condensing it when in the form of vapour. There is no need of any more proximate proof of the fact that the cells previously mentioned as containing chlorophyll, and lying imbedded between the large perforated cells, take up water supplied by the latter, or perhaps it is better to say that the large perforated cells suck in the water for the living green cells. We have only to ask why it is, then, that these small green cells do not absorb water themselves direct from the environment, as is done in the case of so many other mosses and liverworts. It is difficult to answer this quite satisfactorily, but thus much seems certain, that the large porous cells, when full of air, afford a means of protecting the small living cells from too excessive desiccation, and that they are in addition preservative of the chlorophyll in the small cells, a matter to which we shall return presently.

A certain resemblance to these Leucobryums and Sphagnums, in respect of waterabsorption, is exhibited by a few Aroideæ, and more especially by a whole host of Orchidaceæ. Of the $\$ 000$ different orchids hitherto discovered, a good proportion, it is true, are rooted in the earth. But more than half these wonderful plants flourish only on the bark of old trees, and most of them would quickly perish if they were detached from that substratum and planted with their roots buried in earth. A double function appertains to the roots of these Orchideæ which inhabit trees. On the one hand they have to fix the entire orchid-plant to the bark, and, on the other, to supply it with nutriment. When the growing tip of an orchid's root comes into contact with a solid body, it adheres closely to it, flatiens out more or less, sometimes even becoming strap-shaped (see fig. 15), and develops papilliform or tubular cells, which grow into organic union with the substratum, and might conveniently be termed clamp-cells. In many cases these cells creep over the bark, divide, interlace, and form regular wefts. The organic connection with the substratum is so intimate that an attempt to separate the two usually results 
in a detachment of the most superficial parts of the bark, but not of the tubular cells. Now, if a root, after having sent out cells of this kind which contract an organic union with the substratum, reaches into the open, beyond the limit of the

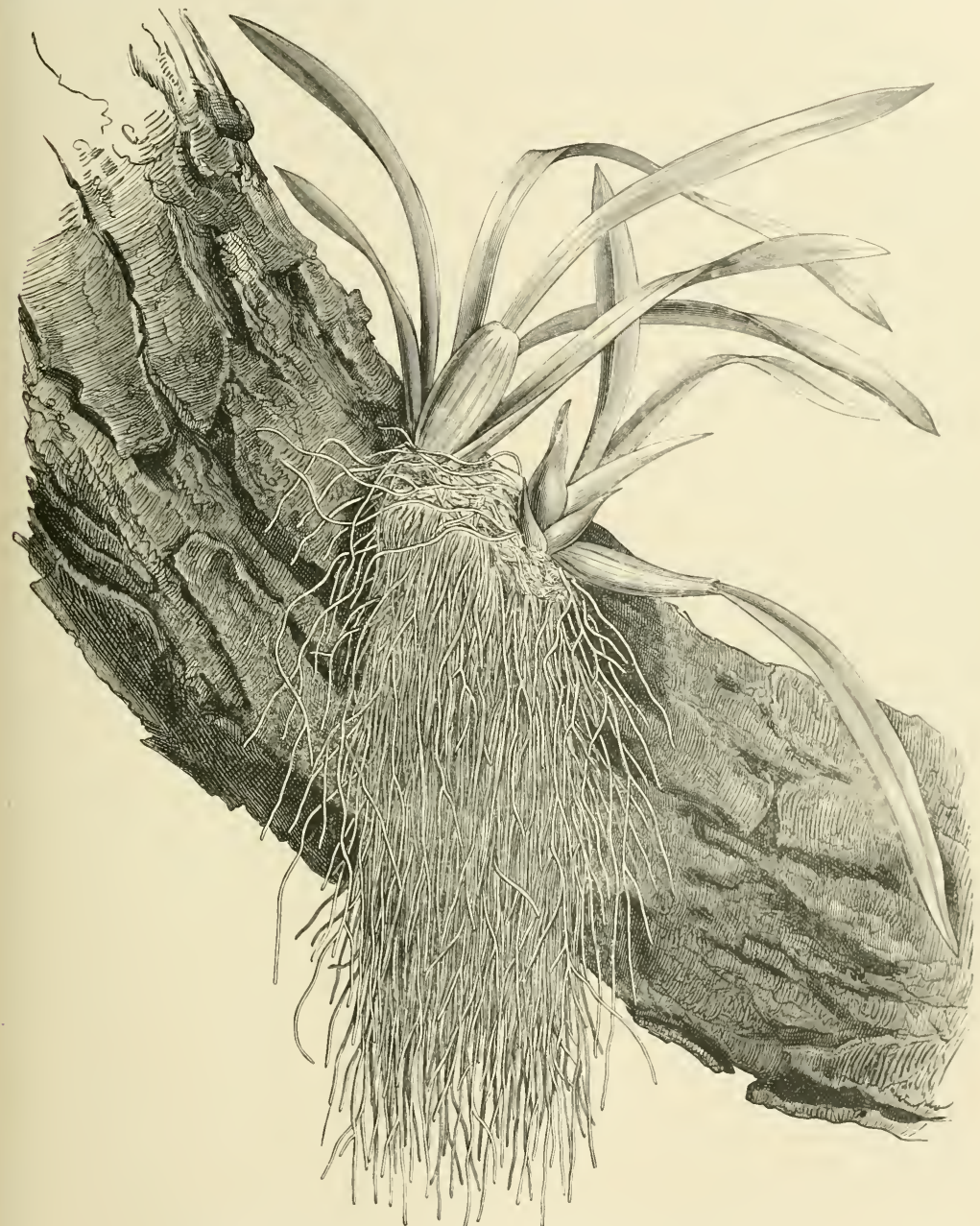

Fig. 50.-Aërial Roots of an Orchid epipłıtic upon the bark of the branch of a tree.

substratum, it immediately ceases to develop clamp-cells, loses its ligulate shape, and hangs down from the tree in the form of a sinuous white filament. A few root-fibres are as a rule sufficient to fix the plant to its substratum, the bark of the tree, and the rest of the roots put forth by the orchid grow from beginning to end. 
freely in the air. They are not infrequently to be seen crowded together in great number's at the base of the plant, forming regular tassels suspended from the dark bark of the branches as may be seen in fig. 50, where an Oncidium is represented.

Each of these aërial roots is invested externally by a white membranous or papery envelope, and it is the cells of this covering that own the resemblance, above referred to, to the cells of Leucobryum and Bog-mosses. Their walls are furnished with narrow, projecting spiral thickenings and therefore do not collapse, notwithstanding their delicacy or the circumstance of their inclosing at times an air-filled carity; they are further abundantly perforated, two kinds of apertures indeed being found. The one variety arises in consequence of the tearing of the portions of the cell-wall situated between the rib-like projections and consisting of extremely thin and delicate membranes (see fig. $49^{3}$ ); the existence of the other variety is due to the detachment of the cells which protrude in the form of papillæ, the result being, in this latter case, the formation of circular holes very similar to those already described as occurring in Leucobryum. The cells resembling papillæ have the peculiarity that they roll off when they get old in the form of spiral bands. The holes, of course, can only occur on the external walls of the outermost cells which border upon the open air, whilst in the interior the communication between the cells themselves is established by means of the rents previously referred to. The entire covering thus composed of perforated cells may be compared to an orlinary sponge, and, indeed, acts after the manner of a sponge. When it comes into contact with water in the liquid state, or more especially when it is moistened by atmospheric deposits, it imbibes instantaneously its fill of water. The deeperlying living green cells of the root are then surrounded by a fluid envelope and are able to obtain from it as much water as they require.

But these roots also possess the power of condensing the aqueous vapour contained in the air. They act upon the moist air in which they are immersed in exactly the same way as spongy platinum or any other porous body. If the aërial roots of Oncidium sphacelatum are transferred from a chamber full of dry air to one full of moist air, they take up in 24 hours somewhat more than 8 per cent of their weight of water, those of Epidendron elongatum absorb 11 per cent, whilst in the ease of many other tropical orchids the amount thus imbibed is doubtless much more considerable still.

The power of condensing aqueous vapour, and other gases as well, is of the greatest importance to these plants. The tree-bark serving as their substratum, to which they are fastened merely by a few fibres, is anything but a permanent source of water. Such water as the bark does contain reaches it, not from the interior of the trunk and indirectly from the soil in which the trunk has its roots, but from the atmosphere; that is to say, from the very source whence the epiphytes upon the bark must also derive their supply. Now, when on the occasion of a long-enduring uniform aërial temperature, there is a failure of atmospheric deposits, which is a regularly recurring circumstance in the habitat of the orchids in question, the sole source of water left is the vapour in the air, and the 
only possible method of acquiring that vapour is the condensation of it by the porous tissue investing the roots. In the event of the air around the orchid-plant containing temporarily but very little moisture, the porous tissue dries up, it is true, very quickly; its cells fill with air and their function as condensers is interrupter. But these air-filled cellular layers then form a medium of protection against excessive evaporation from the deeper strata of the root's tissues, which might be very dangerous in the case of this kind of epiphyte. There is a wide-spread impression that the tropical orchids grow in a perpetually moist atmosphere in the dark shade of primeval forests, and this preconception is fostered by pictures of tropical orchids representing these plants as living in the most obscure depths of woods. In reality, however, the orchids of the tropies are children of light. They thrive best in sunny spots in open country. Those species in particular which have their aërial roots invested each by a thick, white, papery, porous covering belong to regions where a long period of drought occurs regularly every year, and where, in consequence, vegetative activity is subject to periodical interruption, as it is in the cold winter season of the more inclement zones.

For epiphytes inhabiting these regions of the tropics a more expedient structure of root cannot easily be imagined. In the dry season the papery covering reinforces the safeguards against too profuse transpiration on the part of the living cells in the interior of the root, and in the wet season it provides for the continuous supply of the requisite quantity of water. In this sense the porous layer is to a certain extent a substitute for wet soil, or, in other words, the concealment of the living part of an aërial root in the saturated envelope is analogous to that of the rootfibres of land-plants in the damp earth. The manner in which the water reaches the inner cells of an aërial root from the saturated envelope is also quite characteristic. Under the porous tissue lies a layer composed of two kinds of cells of different sizes. The larger cells are elongated and have their external walls, which are adjacent to the porous tissue, thickened and hardly permeable by water. Between these lie smaller, thin-walled, succulent cells, which admit the water from the porous envelope, and should therefore be regarded as absorption-cells. It is also noteworthy that the porous, paper-like covering is discarded as soon as an aërial root is placed in earth. Most orchids with aerial roots perish, it is true, when they are treated like land-plants and planted in soil; but a few species, on occasion, bury their aërial roots spontaneously in the earth and push off their envelopes, and then the imbedded parts exercise the same functions as in the case of land-plants.

We have already mentioned that, in addition to thousands of orchids, several Aroideæ exhibit the porous, papery covering on their aërial roots. But still more frequently the air-roots of Aroids, which live as epiphytes upon trees, are furnished with a dense fringe of so-called root-hairs in a broad zone behind the growingpoint. The hairs project on all sides from the roots, which are surrounded by air; they are crowded very closely together and give the parts affected a velvety appearance. Besides several Aroideæ, one of which (Philodendron Lindeni) is drawn on the left side of fig. 51, many other epiphytes, such as the South 
American Campelia Zanonia, belonging to the Commelynaceæ, represented on the right side of the same figure, and also several tree-ferns, display this velvety

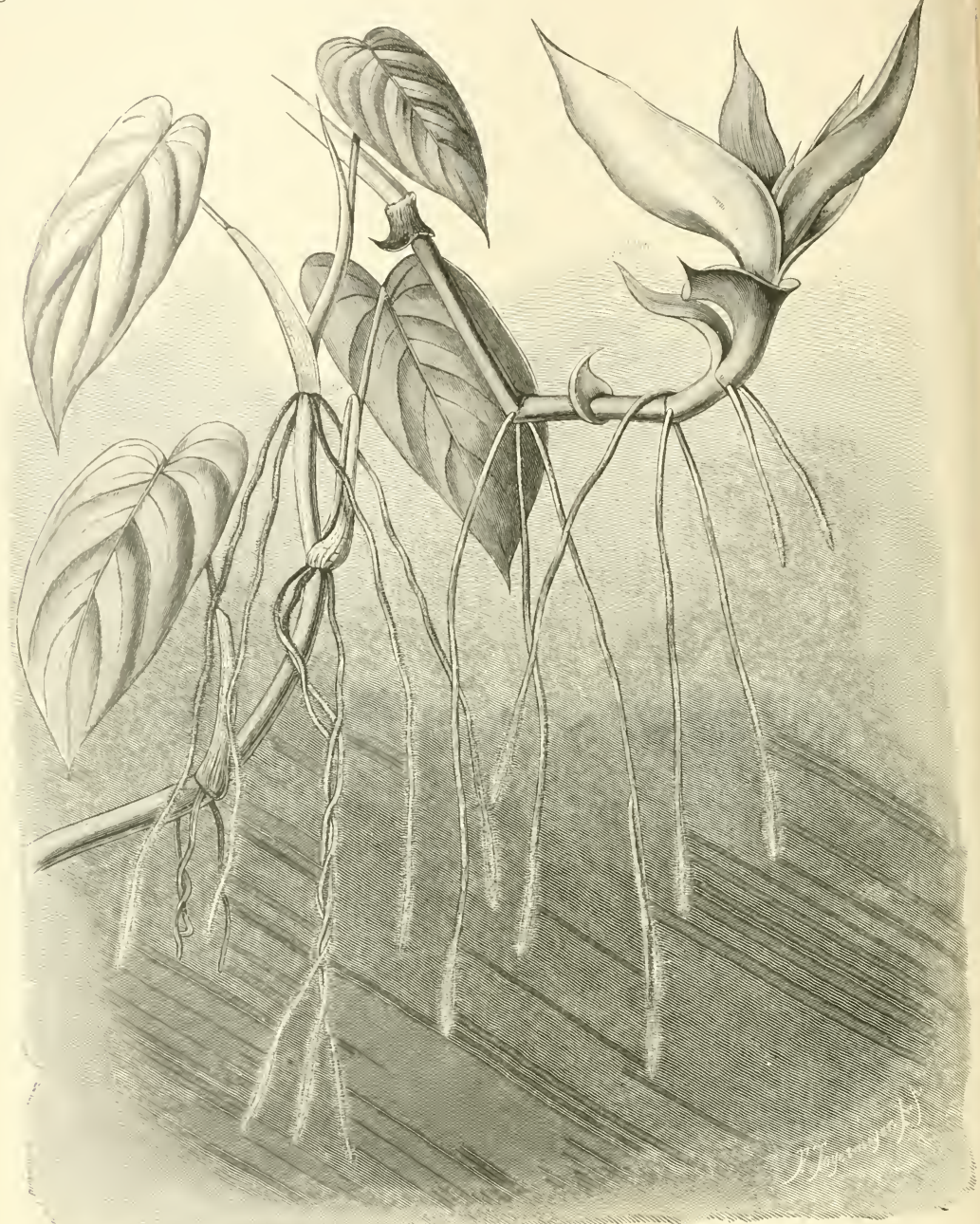

Fig. 51.-Aërial Roots with root-hairs; on the left Philodendron Lindeni, on the right Campelia Zanonia.

coating on their aerial roots. The roots of the tree-ferns are short, but spring in thousands from the thick stem, and are so closely packed that the whole surface is clothed as it were by a woven mantle of rootlets. After some time these aërial roots turn deep brown, whilst the hairs collapse and die, and both are converted 
into a mouldering mass. But as soon as they perish other new air-roots, covered with golden-brown velvet, make their appearance and take their place. These aërial roots never reach the ground or adhere to any substratum, so that their hairs cannot contract an organic connection with a solid body. It is consequently also impossible in this case for the root-hairs to draw moisture from the soil in the capacity of absorption-cells.

These root-hairs, however, are scarcely ever in a position to take up even the atmospheric deposits. The various species of Philodendron and the other epiphytes referred to, have large leaves which cover the air-roots hanging from the stem like umbrellas, and every tree-fern also bears at the top of its stem a tuft of great fronds, which prevents falling rain from wetting the aërial roots. Moreover, the very plants whose air-roots exhibit a velvety coating occur in woods where the tops of the trees arch over the ground in lofty domes, and form a sheltering roof against deposits from the atmosphere. On the other hand, the air within these forests is saturated with aqueous vapour, and it is certain that the velvety roots have the power of condensing vapour, and that the root-hairs instantly suck up the condensed water and convey it to the deeper-lying layers of cells. The truth of this has been established by the results of repeated experiments. Thus, air-roots of the tree-fern Todea barbata, after being transferred from moderately damp air into a chamber full of vapour, condensed and absorbed in the space of twenty-four hours water amounting to 6.4 per cent of their weight. There is, therefore, no doubt that water may be acquired in this way also by plants, even though the instances may not be very numerous. All plants in which this kind of water-absorption has been hitherto observed grow in places where the air is very moist the whole year round, and where there is also no risk of the temperature falling below freezingpoint. Under other conditions, especially in places where the air is periodically very dry, these plants would not be able to survive; for, although they possess organs for the condensation and absorption of water, they have no means of protection against the desiccation of these organs.

\section{ABSORPTION OF RAIN AND DEW BY THE FOLIAGE-LEAVES.}

The idea that plants absorb with their roots such water as they require is so intimately associated with our whole conception of plant-life, that this process is commonly adduced for the purpose of analogies of the most various kinds, and one looks upon the water-absorption effected by aërial roots in the manner just described really as a thing to be expected, notwithstanding the fact that in this case, as the above account shows, the phenomenon is not so simple as is usually supposed. We now turn to the consideration of land-plants. If the leaves of plants cultivated in pots become flaccid, water is poured as quickly as possible upon the dry soil with a view of supplying the roots which ramify in it with moisture. Nor does the result fail to be produced. In a short time the foliage becomes fresh and elastic again, the roots having discharged their function. Even in the open air, it is especially 
the soil in which the roots are imbedded that a gardener waters on dry days, although incidentally he may pour the water over the aërial parts of the plants. He sees, however, that the water which falls in the form of rain or dew upon the foliage and stems normally runs off them at once, or else collects in drops, which trickle down whenever the plant is shaken by the wind, and are sucked up by the thirsty ground. This phenomenon must be due to the possession by the leaves of special contrivances to prevent their being wetted. It does not in any case support the idea that foliage is as well adapted for the absorption of water as experience has proved subterranean roots to be. This train of thought, which forces itself upon every unbiassed observer of the processes as they take place in nature, is certainly warranted in the majority of cases. Each absorption-cell on the roots buried in the earth has an easily permeable membrane, and, as is well known, water passes from damp earth through the cell-membranes into the interior of a plant with great rapidity. The water in the interior of the plant would be equally easily withdrawn through these cell-membranes by dry surroundings, but, as it is, this scarcely ever happens, in consequence of the roots being situated underground. In the case of aërial parts, especially the foliage-leaves, the circumstances are quite different. The leaves have to yield up to the air a portion at least of the water conducted from the roots, because, as will be more thoroughly explained later on, it is only by means of this evaporation that the entire machinery in the interior of the plant can be kept in motion. But this evaporation must not go too far; it must be in proper relation to the absorption of water by the subterranean roots, and be regulated to that end if the plant is not to run the risk of drying up altogether at times-an occurrence which flowering plants are unable to survive, although the mosses described in former pages have that power. Accordingly, in the case of the foliage-leaves of flowering plants, evaporation is confined to certain cells and groups of cells, and these, in addition, have contrivances by means of which evaporation can be entirely stopped on occasion of great drought. It stands to reason that all contrivances which make it impossible for water to pass from the interior of the leaves through the walls of the superficial cells into the surrounding air also hinder the entrance of water into the leaves from the atmosphere.

It would be altogether inconsistent with the system of arrangement of the subject adopted in this book if we were to discuss here all the contrivances serving to regulate the exhalation of water by leaves, and we must, therefore, confine ourselves to referring, by way of introduction, quite briefly, to the following facts, namely, that those pores on the surface of leaves which are known by the name of stomata, and are used as doors of egress by the exhaled water, do not admit rain or dew, or in general, any water in the liquid state; that the so-called cuticle covering the external walls of the epidermal cells in leaves is an additional barrier to both egress and ingress of water; that when, in particular, this cuticle is furnished with a wax-like coating, water does not adhere to the surface of cells so protected; and, lastly, that atmospheric moisture can only penetrate into the interior of the plant at parts of the leaves where the waxen incrustations are absent, where water remains adherent 
to the leaf-surfaces, and they are distinctly wetted. But even cells and groups of cells of this kind usually act but for a short time as absorption-cells, and only when the necessity and craving for water is very great, or when there is an opportunity of acquiring nitrogenous compounds at the same time as the water; and here, again, special contrivances are always present which regulate this kind of waterabsorption, and render it impossible whenever it is not truly advantageous.

At first one would suppose that amongst the cells composing the epidermis of foliage-leaves, those are best adapted to the absorption of water from the atmosphere which take the form of hairs. The superficial area being as great as possible, and the contained matter relatively little, one can scarcely in fact conceive a conformation better suited to the purpose of water-absorption. As, moreover, the area of contact between the cells of the leaf and of a hair is small, there would afterwards be but very little evaporation through the surface of the hair of the water once sucked up by it and conducted into the interior of the leaf. In a word, these hairs on the surface of a leaf appear to be peculiarly adapted to the taking up of water, and not at all favourable to its exhalation. The hypothesis based on these observations is indeed entirely applicable to the case of hairs occurring on the leaflets of mosses, as has been already stated. But it does not hold in the case of the hair-like structures which spring from the leaf-surfaces of flowering plants. These are frequently not wetted at all by water; rain and dew roll off them in drops, and cannot, therefore, be absorbed by them. This is true even of many soft trichomes (hair-structures) which form investments upon leaves, and which seem to be more than any fitted for the absorption of water. For instance, experiments upon the woolly leaves of the Great Mullein (Verbascum Thapsus) have shown that they neither condense aqueous vapour nor take up water in liquid drops. Small importance must be attributed to the thickness of the cuticle, for sometimes it is the very cells which are equipped with a cuticle of considerable stoutness that are adapted to admit water, under certain circumstances, through their walls. On the other hand, much depends upon the presence of wax in the cuticle and upon the contents of the cells; that is to say, upon whether those contents in particular have a strong or weak affinity for water. If the cells of the hairs are full of air they are not adapted to the absorption of water.

If a hair is septate, i.e. consists of a simple series of cells, only the undermost or else only the uppermost cells of the series absorb water. Instances wherein it has been observed that the lowest cells alone in hairs of the kind become absorptioncells are afforded by the Alfredia, represented in fig. 14, by Salvia argentea, and several other steppe-plants. The same statement is made concerning the widelydistributed Stellaria media, the common Chickweed. This last has hairs on the internodes of the stem, running down in ridges from node to node. Usually only one side of the stem exhibits a ridge of hairs of the kind, and the ridge always terminates at the thickened node, whence springs a pair of opposite leaves. The stalks of these leaves are somewhat hollowed out and have their edges beset with hairs like lashes. The hairy ridges on the segments of the stem are readily wetted 
by rain and retain a considerable quantity of water. The water that they cannot hold they conduct downwards to the ciliate axils of the next lower pair of leaves, where it is drawn through the lash-like hairs in due course and collected into a ring of water surrounding the node (see fig. $52^{3}$ ). If this accumulation of water becomes so voluminous and heavy that it cannot any longer be retained by the fringe of lashes, the surplus glides on to the unilateral ridge of hairs on the adjacent internode down to the pair of leaves below. Accordingly, after a shower every node from which leaves arise is seen to be inclosed in a water-bath, and the hairy

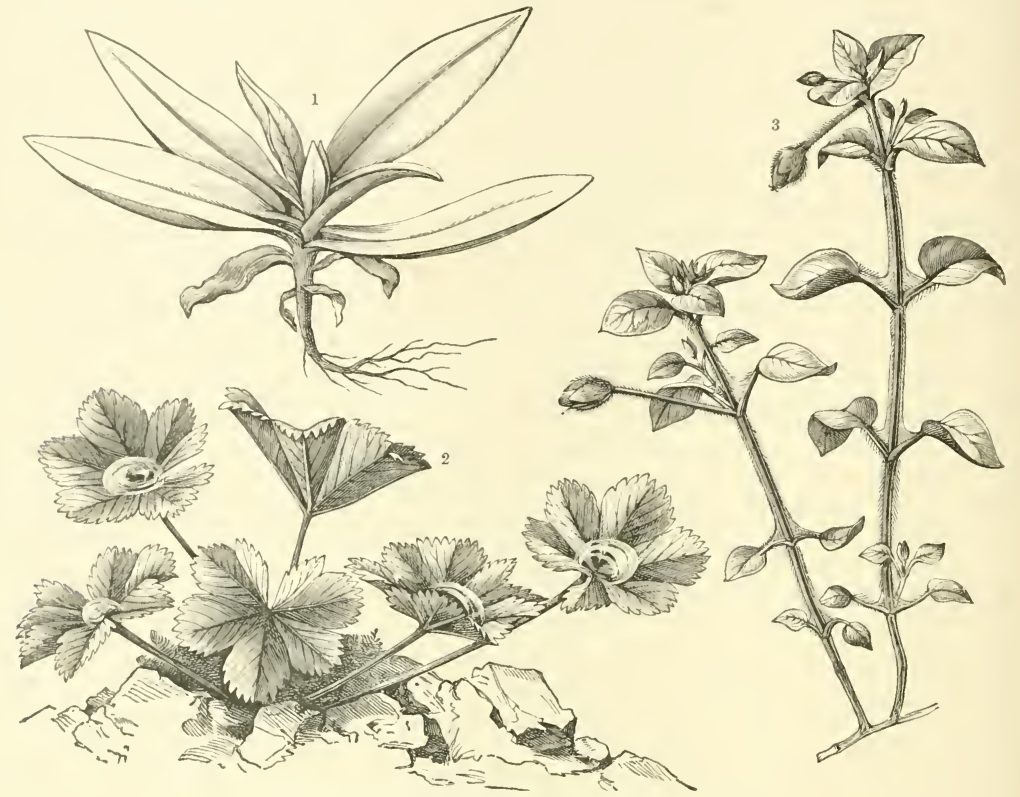

Fig. 52.-Hairs and Leaves which retain Dew and Rain.

1 Dwarf Gentian (Gentiana acaulis). $\quad 2$ Lady's Mantle (Alchemilla vulgaris). $\quad 3$ Chickweed (Stellaria media).

ridges also are so soaked with water that they look like edgings of glass. All the individual cells in each of the hairs are full of protoplasm and cell-sap, but only the lowest, which are very short, really act as absorption-cells. When these cells become at all relaxed in dry air, the fact is indicated by the appearance on the external cell-wall of fine strix (see fig. $53^{1}$ and $53^{2}$ ). The protoplasts inhabiting them attract water, and after being relaxed in the manner referred to the cells regain their turgidity on being wetted, whilst the fine wrinkles on the outer membrane are in consequence immediately smoothed out. Although the upper cells of the hair possess a less thick cuticle, they, on the other hand, seem not to absorb any water, but to serve rather to conduct it by their surfaces.

This case is, as we have said, comparatively rare, and the corresponding absorp- 
tion of water is not very considerable. But it often happens that the upperinost cells of a septate hair are developed into absorption-cells. The terminal cell is then usually spherical or ellipsoidal and larger than the rest, or else this cell is divided into two, four, or a greater number of cells, which together form a little head, whilst the lower cells constitute a stalk supporting it (see fig. $53^{3}$ and $53^{4}$ ). In botanical terminology structures of this kind are named capitate or glandular hairs. The protoplasm in the cells of the head is, for the most part, of a dark colour, and the

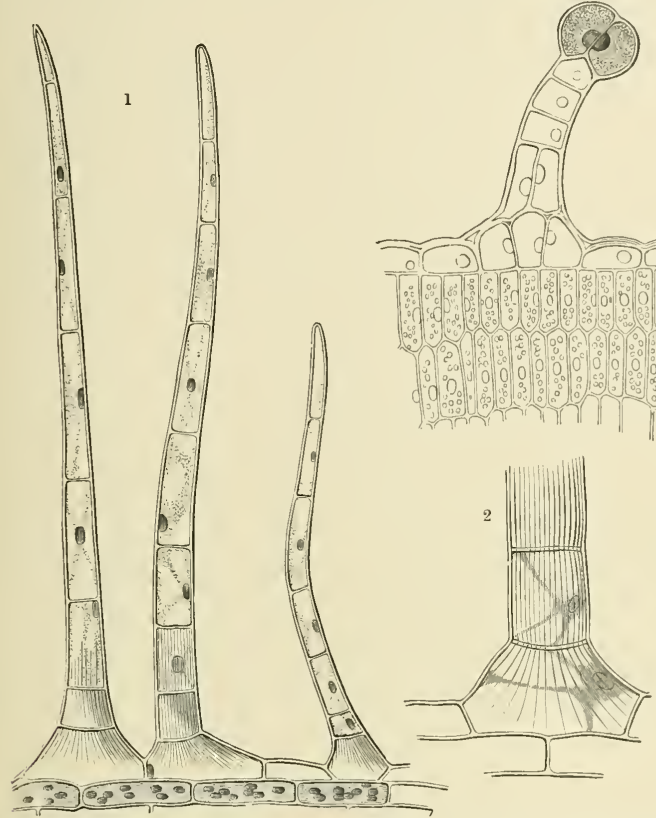

Fig. 53. - 1 Hairs from stem of Stellaria media; $\times 110 . \quad 2$ Lowest cells of the same hairs; $\times 200 . \quad 3$ Capitate hairs of Centaurea Balsamita; $\times 150$. Capitate hairs of Pelargonium lividum; $\times 150$.

cell-membranes are readily permeable by water, which is attracted with great energy by the cell-contents. The cell-membrane is often very thick, it is true, but as soon as water comes into contact with it the outer layer is discarded, the inner layers swell up and the water passes through these swollen layers into the interior of the cell. This happens, for instance, in many pelargoniums and geraniums, wherein the capitate cells go through a process of excoriation on every occasion of the imbibition of water (see fig. $53^{4}$ ). In other plants the walls of the capitate cells are everywhere thin, and not only do the cell-contents consist of a viscid gumlike mass, but the external surface of the wall is also covered by a layer of viscid excretion. In many cases the viscid matter excreted by the glands spreads over the entire surface of the leaf, so that the latter feels sticky and looks as if it were 
coated with varnish. Many plants which have their ioots buried in crevices of rock and no small number of herbaceous steppe-plants are quite thickly covered with glandular hairs of the kind. Centaurea Balsamita (see fig. $53^{3}$ ), a plant occurring on the elevated steppes of Persia, may be selected as an example of the latter group. The advantage of the structure of capitate hairs is not far to seek. In dry weather the thick cuticle (Pelargonium) or the varnish coating (Centaurea Balsumita), as the case may be, prevents desiceation of the cells and groups of cells in question. But as soon as rain or dew falls, the cuticle and the coat of varnish take up water, and it is by their instrumentality that water reaches the interior of the cells. Thus, whilst the exhalation of water is hindered, its absorption is not.

Other epidermal cells of foliage-leaves besides trichomes are capable of acting as absorption-cells, although this action, for reasons already given, is very restricted, and is only had recourse to when the turgidity of the cells of the foliage-leaves has diminished, and the water exhaled by those cells is not being restored by the ordinary apparatus of conduction from the roots. If branches are cut from plants which bear no glandular or other form of hair on their leaves or stems-as, for instance, the leafy stem of Thesium alpinum - and the cut ends are closed with sealing-wax, and the branches left to wither, and, when quite withered, are immersed in water, they freshen up speedily and the leaves become tense again, the cells having recovered their turgidity. Here, then, decidedly absorption has taken place through the ordinary cuticularized epidermal cells. Certainly these epidermal cells in Thesium are not protected against wetting. Wherever the epidermal cells are not susceptible of being wetted owing to a coating of wax or any other contrivance there could naturally be no question of water being absorbed. This very circumstance, however, leads to the supposition that an important part in water absorption is to be attributed to the alternation of wettable and non-wettable parts on one and the same leaf. In the case of many foliage-leaves one can see that only those cells of the epidermis which lie above the veins of the leaf retain the water which comes upon them, that is to say, are wetted by it, whilst the water rolls off the intervening areas of the lamina. Indeed, there are in many instances contrivances obviously designed for the purpose of conducting water from parts of the epidermis not liable to be wetted to parts that can be moistened.

\section{DEVELOPMENT OF ABSORPTION-CELLS IN SPECIAL CAVITIES AND GROOVES IN THE LEAVES.}

The contrivances last described are all only adapted to rather a casual appropriation of water from the atmosphere. But besides these we find a number of other contrivances, which render it possible for every rolling dewdrop and every passing shower to be made of use to the utmost extent. These contrivances consist of a variety of depressions and excavations, in which rain and dew are collected and protected against rapid evaporation. Some species have deep hollows or channels, others little pits, whilst others again have basins, vesicular or bowl- 
shaped structures, to collect and absorb the water; and the construction of the protective apparatus, which prevents too rapid evaporation into the air of water that has once flowed into the depressions, is as various as the form of the depressions themselves. A short account of the most striking of these structures will now be given.

Such water-collecting grooves as are closed, so as to form ducts, occur principally in petioles and in the rachises of compound leaves. For instance, in the Ash the leaf rachis, from which the leaflets arise, is furnished with a groove on its upper surface. Owing to the fact that the edges of this groove, which are strengthened by a so-called collenchymatous tissue, are bent up and curved over the groove, a duct or conduit pipe is produced, and this duct only gapes open at the places where the leaflets are inserted upon the rachis, and where, therefore, the drops of rain to which the leaflets are exposed flow off into the groove (see fig. $54^{1}{ }^{1}$ ). The simple hairs and peltate groups of cells developed in the grooves and ducts (fig. $54^{2}$ and $54^{3}$ ) are not merely transiently moistened, but inasmuch as the water is retained there for several days after a fall of rain, they are during that time immersed in a regular bath of water, and are able to absorb the moisture very gradually.

In many Gentianeæ-most conspicuously in the large-flowered Dwarf Gentian (Gentiana acaulis) - the decussate pairs of radical leaves form a loose rosette (see fig. $52^{1}$ ). The larger dark-green blade of each leaf is flat and even, and only the pale-coloured base is fashioned into a groove. This groove is made deeper by the tissue of the leaf being puffed up round it, and as all the leaves of the rosette arise close together, the groove of each leaf is covered by the lamina above it. The rain or dew accumulated from the blade remains standing in this concealed nook for some time without evaporating, so that absorptive apparatus with the power of taking up water has plenty of time for the purpose. In this case the absorptive apparatus is in the hindmost extremity of the groove, and consists of long, club-shaped structures composed of extremely thin-walled cells (see fig. $54^{4}$ ), and these act so energetically that if leaves are cut off and left to fade, and if the cut surfaces are stopped with sealing-wax, and the whole then bathed with rainwater, they take up in twenty-four hours about 40 per cent of their weight of water. A similar phenomenon occurs in the case of a number of Bromeliaceæ which adhere by a few roots to the bark of trees in the tropics, and have grooved rosetted leaves, the latter covering one another, and being arranged in such a manner as to form a regular system of cisterns. At the bottom of each cistern there are special groups of thin-walled cells which suck up any water that flows in when rain falls.

On the under surface of the leaves of the Cow-berry (Vaccinium Vitis-Idoca) little depressions are formed, and in the middle of each depression there is a clubshaped structure composed of small thin-walled cells, which contain slimy, viscid substances and act as absorbent organs. The rain which falls upon the upper surface of the leaf gets drawn over the edges on to the under surface, fills the small depressions occurring there, and is taken up by the absorptive apparatus. A 
similar contrivance is also exhibited by the leaves of alpine roses and those of the American Bacharis. For instance, on the under surface of the leaves of the Alpine Rose (Rhododendron hirsutum) there is a large number of discoid glands (fig. $54^{5}$ ), each of which is supported on a short stalk and sunk in a little hollow (fig. 54 ${ }^{e}$ ) The cells composing the gland are arranged radially, and contain slimy, resinous matters capable of swelling up. These contents are also excreted, and then cover the entire glandular disc, and often even the whole surface of the leaf in the form

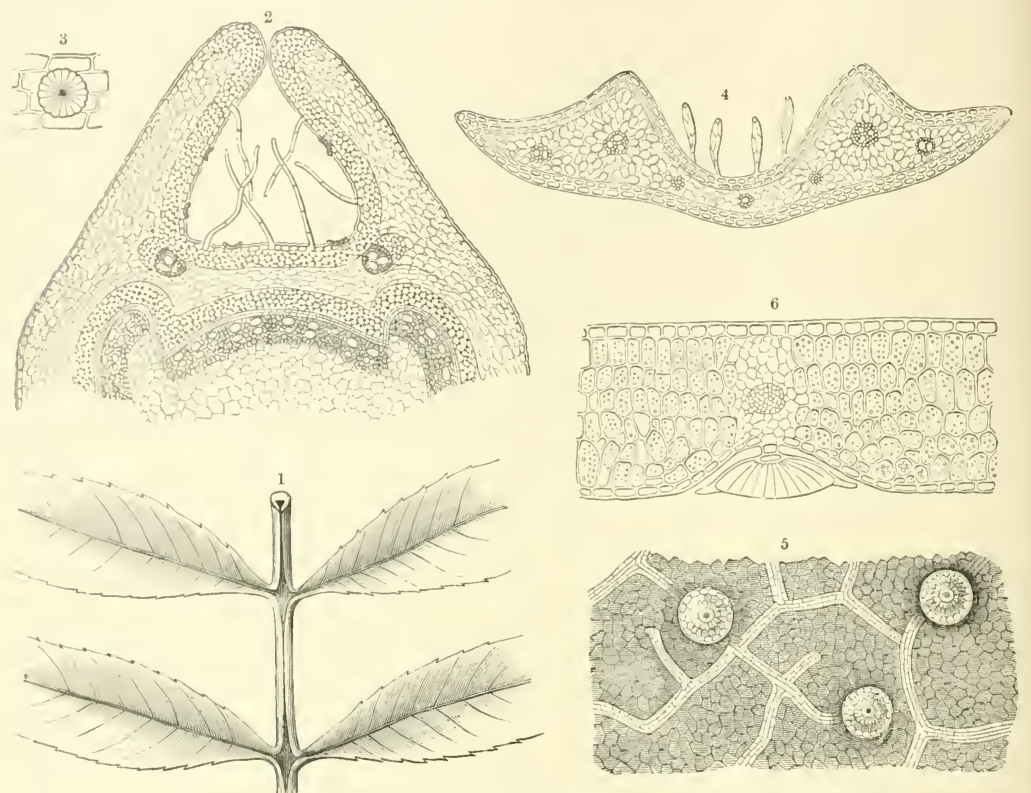

Fig. 54.-Absorption of Water ly Foliage-leaves.

1 Grooved rachis of the ash-leaf. 2 Section through the same; $\times 30$. 3 Peltate group of cells from the groove. 4 Section through the base of a leaf of the Dwarf Gentian; $\times 20$. ${ }^{5}$ Under side of a leaf of Rhododendron hirsutum; $\times 30$. ${ }^{6}$ Section through a leaf of Rhododendron hirsutum.

of a light-brown crumbly crust. When drops of rain fall upon Alpine Rose leaves, the whole of the upper surfaces, in each case, is in the first place moistened; but without delay, and partly through the action of the hairs fringing the margin, the water soaks on to the under side of the leaf. As soon as it reaches the glands it is taken up by the crumbly incrustation mentioned above, which swells up in consequence. The little cavities in which the glands are situated also fill with water, and each gland is then immersed, as it were, in a bath, and able to absorb as much moisture as is required. Owing to the glands being invariably developed above the vascular bundles of the leaf (see fig. $54^{6}$ ), the water that is absorbed can be conducted without delay by them to the places where it is required. As soon as the leaves of alpine roses become dry again, the mass of resinous mucilage again 
forms a dry crust over the glands and protects their tender-walled cells from too great evaporation.

Very remarkable also are the structures adapted to absorption on the leaves of saxifrages belonging to the group Aizoon, and on those of a large proportion of the Plumbagineæ. The saxifrages in question have little depressions visible to the naked eye upon the upper surface of the leaves behind the apex, and along the margins. When the margin is dentate or crenate, as, for instance, in Saxifraga

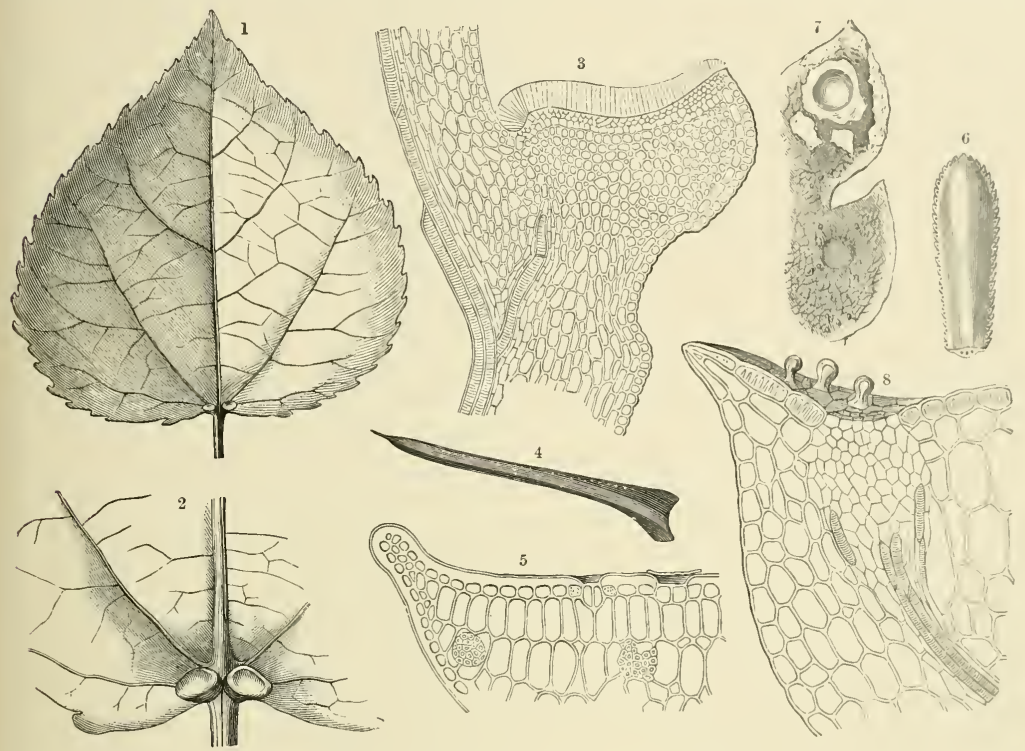

Fig. 55.-Absorptive Cavities and Cups on Foliage-leaves.

1 Leaf from a shoot of the Aspen. 2 The hase of this leaf; $\times 3$. 3 Section through an absorption-cup; $\times 25$. 4Leaf of Acantholimon Senganense. 5 Section through part of this leaf; $\times 110$. ${ }^{6}$ Leaf of the Evergreen Saxifrage (Saxifraga A izoon). 7 Two teeth from the margin of this leaf. The absorptive cavity in the upper tooth incrusted with lime; the lower one with the incrustation removed. ${ }^{8}$ Section throngh a tooth from the leaf and its absorptive cavity; $\times 110$.

Aizoon (see fig. $55^{6}$ ), one of these cavities occurs in the middle of each tooth. The cells forming the outer edge of the tooth or scallop are always much thickened, firm, and rigid; but the median portion of the leaf as a whole is fleshy, and composed of a bulky large-celled parenchyma. The vascular bundle, after entering the leaf at its base, divides into a number of lateral bundles which either run towards the margin without further ramification (as in Saxiccesia), or else form a net-work by uniting one with another in their course (as in Saxifraga Aizoon). These lateral bundles terminate in the marginal teeth of the leaf and immediately beneath the little cavities which occur there, whilst the extremity of each bundle swells into a knob or pear-shaped enlargement strongly resembling the roundish groups of spirally-thickened cells in the tentacles of the Sun-dew 
(cf. fig. $26^{1}$ ). The bottom of each depression is made up of cells with very thin external walls, and the function of these cells is to suck up the water that flows into the cavity. It is obvious that the absorbed water passes thence into the enlarged extremities of the branches of the vascular bundles, and may then be conlucted to other parts of the leaf. Seeing that all these saxifrages have their habitat in crevices of rocks on sunny declivities, they are much exposed to desiccation in times of drought. The epidermal cells of the medial area and those of the extreme edge are no doubt protected by a very thick cuticle (see fig. $55^{8}$ ); but in the case of the thin-walled cells at the bottom of the depression there is the danger of as much or even more water escaping through them, in the form of vapour, than has been previously taken in during the prevalence of rain.

In order to prevent this loss of moisture recourse is had to a very remarkable contrivance for elosing the cavity, viz., an incrustation of carbonate of lime. In many saxifrages this crust covers the whole face of the leaf, in others only the margin, or the spot where the depression occurs. In the latter case it looks like a lid over the cavity. At that spot the crust is always thickened, and sometimes it forms a regular stopper which fills up the entire cavity. It rests upon the epidermis of the leaf, but is not adnate thereto, and may be removed with a needle. When a leaf is bent the crust is ruptured and breaks up into irregular plates and scales, and a strong gust of wind would then easily strip off the fragments and blow them away. In species subject to this danger, as, for instance, Saxifraga Aizoon, in which the rosetted leaves curl strongly upwards and inwards in dry weather, the crust of lime is held fast by peculiar plugs which arise from individual epidermal cells projecting above the rest in the form of papillæ (see fig. $55^{8}$ ). These plugs are found principally on the side walls of the cavities, but are also scattered everywhere on the epidermis of the margin of the leaf. They are so incrusted with the lime that the latter cannot easily fall off, and a comparatively strong pressure must be applied with the needle to detach it from the substratum. The calcium carbonate of which these crusts consist is excreted in solution by the plant from pores occurring at the bottom of the depressions. The pores are constructed like ordinary stomata, but are, as a rule, somewhat bigger, and it is not improbable that, when once the lime crust has formed from the excreted solution, they take part in the function of transpiration.

There is scarcely any need for further explanation of the manner in which the apparatus here described acts. When rain or dew falls on a saxifrage leaf the whole upper surface is moistened directly, whilst the water soaks under the crust of lime, and, diffusing itself there, fills in a moment the depressions, and is taken up by the absorption-cells situated at the bottom of the latter. The calcareous stopper imbedded in each cavity is only upheaved by this process to a trifling extent. In dry weather the crust is appressed closely to the epidermal cells, and the stopper descends again and impedes the evaporation of water from the thinwalled cells within the cavities.

The absorptive organs on the leaves of Acantholimon, Goniolimon, and a few 
other Plumbagineæ, resemble in an extraordinary degree those pertaining to suxifrages. The depressions are here found uniformly distributed over the entire surface of a leaf, and when they are closed by a crust or scale composed of calciun carbonate, the leaves are dotted with white spots, as may be seen in the drawing of a leaf of Acantholimon Senganense given in fig. $55^{4}$. Upon the calcareous scale being removed, a little cavity is revealed beneath, and one observes that the floor of this cavity is composed of from four to eight cells, separated by radial partitionwalls, and with exceedingly thin and delicate outer walls. The other epidermal cells adjoining the cavity are, on the contrary, always furnished with a thick cuticle (see fig. $55^{5}$ ). Whenever water is being copiously supplied to the roots, and the turgidity of the cells in the leaves is great, the cells forming the floor of the cavity excrete bicarbonate of lime in solution. Part of the carbonic acid escapes into the air, and the insoluble mono-carbonate of lime in the water then forms a crust, which fills and covers the cavity, and often even spreads over the whole leaf, constituting a coherent calcareous coat.

All Plumbagineæ which exhibit this contrivance-that is to say, the various species of Acantholimon, Goniolimon, and Statice-inhabit steppes and deserts, where in summer no rain falls for months together, and the soil becomes dry to a considerable depth, so that extremely little water is available for the roots. Although the rigid leaves are protected by a thick cuticle, and by crusts and scales of lime against excessive evaporation of their aqueous contents, still it is difficult to avoid some slight loss of water, especially when the noon-day sun beats down upon the steppe, and, owing to the extremely arid nature of the soil, it is scarcely possible to replace this loss, however small it may be, by absorption from the earth on the part of the suction-cells on the roots. All the more welcome to plants of the kind is the dew which sometimes falls copiously on steppes and in deserts in the course of the night; it wets the rigid leaves, and, soaking immediately underneath the crusts and scales of lime to the thin-walled cells at the bottom of the cavities, is absorbed with avidity by them. When drought returns with the day, the scales of lime close tightly down like lids on the epidermis beneath, and, so far as possible, prevent evaporation. In particular, they impede the exhalation of water from the thinwalled cells at the bottom of the cavities-a loss which would otherwise be quite inevitable, and would be followed by a rapid desiccation of the entire plant. To prevent the calcareous lids from dropping off, there are either, as in Suxifraga Aizoon, papilliform or conical projections from cells in the immediate vicinity of the cavities, which projections often have hooked ends and confine the crust of lime, or else each cavity is somewhat contracted at the top and enlarged below, so that the lime stopper, being shaped according to the contour of the cavity, cannot fall out.

A significance similar to that attributed to calcium carbonate excretions belongs also to the saline crusts which are found covering the leaves of a few plants growing on the arid ground of steppes and deserts in the neighbourhood of salt lakes and on the dry tracts of land near the seashore. Owing to the fact that in these 
situations crystals of salt are sometimes to be seen separated out from the soil, and lying as a white efflorescence upon the ground, it used formerly to be believed that the salt incrusting leaves and stems was derived, not from the plants in question, but from the soil around, and had only spread from there over the various plantmembers. But this is not the case. As a matter of fact, the salt observed on the leaves and stems of Frankenia, Reaumuria, Hypericopsis persica, and a few species of Tamarix and Statice, is produced from the substance of the leaves. It is excreted in just the same way as the crust of lime, above described, is from the leaves of saxifrages. To the naked eye the surfaces of the leaves in all the plants enumerated have a punctate appearance. On closer inspection, it is evident that, corresponding to each dot, there is a little cavity, the deepest part of which is constructed of cells with extremely delicate external walls. In quite young leaves only a single thinwalled cell of the kind is to be seen at the bottom of each shallow depression. But this divides, and, by the time the leaf is full-grown, from two to four cells are seen to have arisen by division of the one cell. Stomata are, in addition, intercalated in the membrane in the neighbourhood of these thin-walled cells, and, in the rainy season, when there is no lack of water in the habitats of the plants in question, a watery juice, containing a large amount of salts in solution, exudes from these stomata. The saline solution soaks over the whole surface of the leaf, and in a dry atmosphere crystals form from it and adhere to the leaf in the form of little glandlike patches or continuous crusts.

If these tamarisks, frankenias, and reaumurias are observed during a rainless season, the crystals of salt are seen under the noon-day sun glittering on the leaves and stems, and may be detached in the form of a fine crystalline powder. But if the same place is visited after a clear night, no trace of crystals is to be seen; the little leaflets have a green appearance, but they are covered with a liquid with a bitter salt taste, ${ }^{1}$ and are damp and greasy to the touch. The crystals have attracted moisture from the air during the night, and have deliquesced, and the saline solution not only covers the whole of the leaf, but also fills the little cavities visible as dots to the naked eye. The thin-walled cells at the bottom of the cavities differ from the rest of the epidermal cells and the guard-cells of the stomata, in that they are susceptible of being wetted, and they may act as absorption-cells, and allow the water, attracted by the salts from the air, to pass through their thin walls into the interior of the leaves.

When the air dries under the rising sun, crystals are again formed from the solution of salts, and, covering the leaves once more in the form of crusts, fill up the depressions and protect the plants during the hot hours of the day from excessive evaporation. Whilst, therefore, in the dewy night these plants are indebted to their salt crusts for water, they are in the day-time preserved from desiccation by the action of the same contrivance.

\footnotetext{
${ }^{1}$ The salt incrustations which were removed from plants of Frankenia hispida, collected on a Persian salt-steppe, consisted principally of common salt (chloride of sodium). They contained in smaller quantities, gypsum, magnesium sulphate, calcium chloride, and magnesium chloride.
} 
It is also worthy of mention that papillæ are developed near the absorptioncells, with a view to the retention of the salt crystals, similar to those which hold the calcareous incrustations on the leaves of saxifrages and Acantholimon. The leaves of plants covered with crystals of salt are also for the most part furnished with little bristles, to which the salt adheres so firmly that it is not readily detached, even by violent shaking.

But however striking the analogy may be between the development and significance of lime crusts and salt crusts, there is the essential difference that the former have not, like the latter, the power of attracting moisture from the air. And on this particular stress must be laid. In the broken and hillocky tracts on the shores of salt-lakes or of the sea, where tamarisks and frankenias are especially wont to live, the sandy ground dries up to such an extent in the height of summer that it is scarcely conceivable how plants growing in it are able to preserve their vitality. The proximity of the sea has no immediate effect on the moisture of the ground in such situations. The sea-water does not penetrate into the ground far beyond the high-water line, and it is out of the question that the layers of soil serving as substratum to the frankenias and tamarisks should be irrigated by subterranean water. When in summer there is an absence of rain for months together, these plants-even though in close proximity to the sea-would necessarily perish of drought. Only the circumstance that they turn to account the moisture of the atmosphere by means of the excreted salts renders it possible for them to flourish in these most inhospitable of all inhospitable sites.

Many plants which are periodically exposed to great dryness have the tips of the teeth on the leaf-margins thickened into little cones or warts. They also glitter somewhat and at times are sticky. The glitter and viscidity are due to a resinous slimy substance, which often contains sugar and tastes sweet. This substance covers the teeth and sometimes spreads from the teeth inwards to a great distance over the face of the leaf in the form of a delicate film-like varnish. The greatest resemblance exists between this varnish (sometimes known as "balsam") and the secretions of the glands on the leaves of the Alpine Rose and of the glandular hairs on those of Centaurea Balsamita. It is excreted by special cells, which are intercalated in the epidermis of the foliar teeth, and are at once marked out from the other cells of the epidermis by the facts that their protoplasm is of a brownish colour and that their external walls are easily permeable by water. The excretion of the varnish-like layer takes place at a time when the entire plant is distended with sap, chiefly, therefore, in the spring. When summer is at its height the varnish dries and thenceforward affords an excellent preservative from the risk of too much evaporation from the cells it covers, and especially from those situated on the teeth of the leaves by which it was excreted. But if this dried film of varnish is wetted it saturates itself quickly with water and renders moisture accessible to the cells beneath it. Thus its value is similar to that of the crusts of lime and salt on the leaves of the plants above described. When moist it effects the absorption of water, when dry it guards against desiccation. 
The reason for the contrivance just described being exhibited especially by the marginal teeth of the leaf, lics in the fact that dew is deposited particularly at those spots. If one looks at the leaves of the dwarf almond and plum trees in the steppe-districts, after clear summer nights, one finds a dewdrop suspended to every tooth on the margins; but by noon all the teeth are dry again and protected from loss of water by the coat of varnish. Moreover, not steppe-plants alone, but very many plants which grow in poor sandy soil on the banks of streams and rivers, exhibit this contrivance for the direct absorption of water from the atmosphere. Instances are afforcted by the Sweet Willow, the Crack-willow, Poplars, the Guelderrose, the Bird-cherry, and many others. It is at once evident that this contrivance is observed chiefly on the leaves of trees, shrubs, and tall herbs, whilst incrustations of lime occur only on shorter plants with rosulate leaves spread out on the ground, or with rigid acicular leaf-structures. The grounds of this distinction may well reside in the fact that the weight of a crust of lime is many times as great as that of the dry film of varnish. A load capable of being borne without hazard by the leaves of a Statice plant, they being spread out on the ground, or by the rosettes of Saxifraga Aizoon, would be unfit for the leaves of a Cherry or Apricot tree, or for those of the Sweet Willow, or the Crack-willow; indeed the branches of these trees would break down under the burden if their leaves were incrusted with lime.

In many cases only a few of the marginal teeth of the leaf are transformed into absorbent apparatus, and special contrivances then always exist to convey rain and dew to those teeth. The Aspen (Populus tremula) serves as a very good example of this. This tree has, as is generally known, two kinds of leaves. Those arising from the branches of the crown have long petioles and laminæ of roundish outline and with somewhat sinuate margins; those which are borne by the radical shoots have shorter stalks and larger sub-triangular laminæ sloping outwards; and the whole leaf is so placed and its margin so curved as to oblige the rain which strikes the upper surface in its deseent to flow down towards the petiole (see fig. $55^{1}$ ). Now, situated exactly on the boundary of lamina and petiole are two cup-shaped structures (fig. $55^{2}$ ) originating from the lowest teeth of the leaf, and so arranged that every drop of rain descending from the lamina must encounter their shallow cavities and fill them with water. These cups are brown in colour and the size of a grain of millet; and the cells of their epidermis are furnished with a thick cuticle. Only the cells lining the shallow depression of each cup have thin walls, and they excrete a swcet-tasting, slimy, resinous substance which in dry weather films over the cavity like a varnish, and protects, at all events, the cells lying beneath it against an injurious desiccation. When, however, this coat is itself in contact with water it swells up, and the moisture is then absorbed by the cells in the pit-like depression and is transmitted to the vessels running underneath the cups (see fig. $55^{3}$ ).

A number of tall herbs, principally of the group of Compositæ, have, like the Aspen, leaf-teeth which are developed at the part where petiole and lamina join and act as organs of absorption. In some, besides, the margin of the green lamina extends in the form of a narrow ridge down the pale canaliculate petiole; and, when 
this is the case, teeth of the kind are found on this narrow green ridge which runs along the groove. In Telekia, a handsome herbaceous plant of wide distribution in the south-east of Europe, these teeth-conical or club-shaped-springing from the margin of the petiole-groove are incurved, and are in general so placed that their blunt apices prcject into the groove. But precisely on these obtuse tips of the teeth are situated cells with very thin outer walls easily permeable to water, and having contents with a strong attraction for it. Thus, as soon as the groove of the
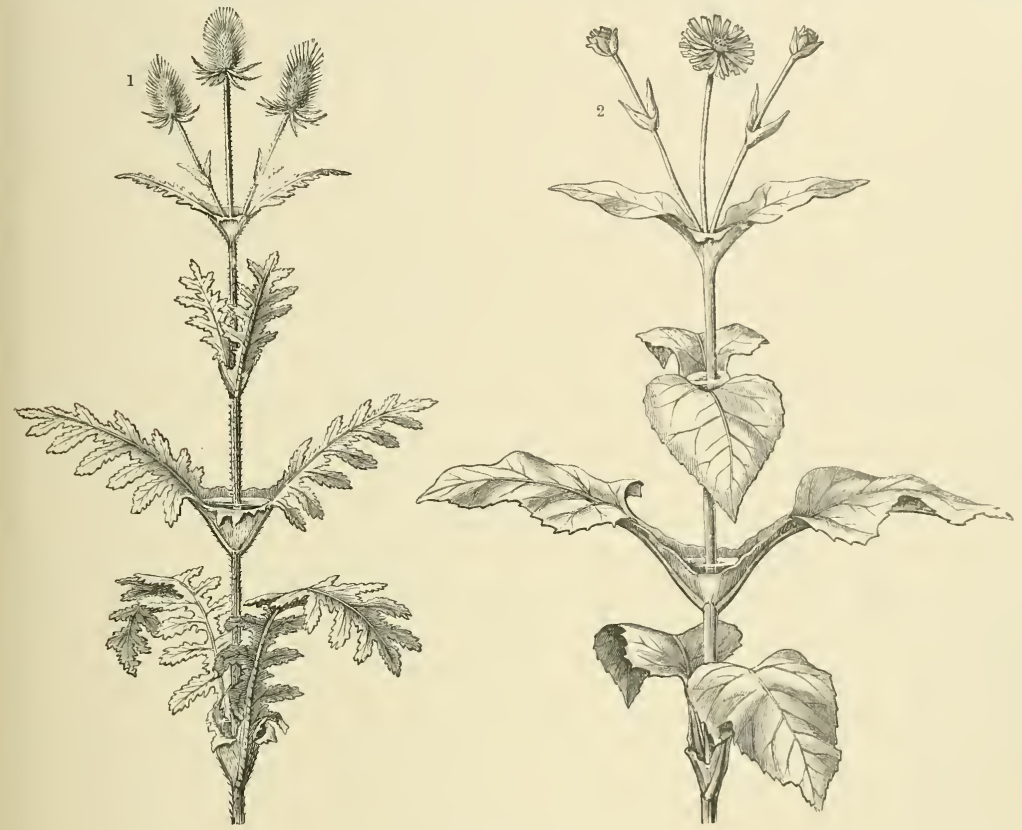

Fig. 56.-Water-receptacles.

1 In a Teasel, Dipsacus laciniatus.

2 In the American Silphium perfoliatum.

petiole is filled with rain, collected from the surface of the leaf, the tips of the conical teeth are moistened, and they suck up the water.

Lastly, we have to mention the curious receptacles appertaining to foliageleaves in which water from the atmosphere accumulates and continues to stand for weeks without being protected from evaporation by the excretion of special substances. Any region or portion of the leaf may participate in their construction. In Saxifraga peltata the lamina is shaped like a shield and forms a shallow plate with the concave surface turned to the sky. In the Cloud-berry (Rubus Chamamorus) the formation of basins is brought about by the margins of the reniform lamina being superimposed over one another as if to make a spathe. In the various species of Winter-green, especially in Pyrola uniflora, the pale cauline leaves, 
inserted above to the green leaves, are metamorphosed into little saucers. In one species of Teasel, Dipsucus laciniatus (see fig. $56^{1}$ ), and in the North American Silphium perfoliatum (fig. $56^{2}$ ) the two sheathing portions (vaginæ) of every pair of opposite leaves are connate and form comparatively large and deep funnel-shaped basins, from the middle of which rises the next higher internode of the stem. In several Meadow-rues (Thalictrum galioides and T. simplex) the secondary leaflets, which are opposite one another and shut close, almost like the valves of a mussel, are moulded so as to form cavities for the retention of water, and in many Umbelliferæ, such as Heracleum and Angelica, the vagina of each individual leaf is ventricose or inflated, thus forming a sac enveloping the segment of the stem which stands above it.

These basins, saucers, and dishes are always so placed, relatively to their surroundings, that the water derived from rain and dew is directed into them from the surfaces of the leaves, or by the segment of the stem which rises from their centres, and thus it is that the depressions are filled. Whether in all cases much of the water accumulated is absorbed is certainly open to doubt. In the case of the leaves of the Alchemilla (fig. $52^{2}$ ), which exhibit the phenomenon so conspicuously that the plant has received the popular name of Dew-cup; the absorption of water is, at anyrate, very inconsiderable, and here the retention of the dew secures advantages of a different kind to which we shall presently have occasion to return. On the other hand, it is established that in the case of basins belonging to tall herbaceous plants, particularly such as grow on steppes and prairies where often no rain falls for a long interval, the water collected is absorbed by the glandular hairs and thin-walled epidermal cells developed within them. The fact of this absorption may be proved by a very simple experiment. Let a stem of the Silphium, represented in fig. $56^{2}$, be cut off beneath the pair of connate leaves, which form a basin by their union, and let the cut surface l e closed with sealing-wax, so that no water can be taken up by the stem from below. If the water accumulated in the basin is now emptied out, the leaves shortly become flaccid and droop; but if the basin is left full of water, the leaves preserve their freshness a long while and do not begin to wither until all the water has evaporated and disappeared from the basin. If oil is poured upon the collection of water in the basin, so that evaporation from the latter is impeded, a constant diminution of the water in the basin is observed notwithstanding; this leads to the conclusion that the water in question is really taken up by the absorption-cells at the bottom of the basin and conveyed to the tissue of the leaf.

The first thing that strikes one on surveying once more all the plants possessing on their aërial organs special contrivances for water-absorption is that a large proportion of them have taken up their abode in swamps and on the banks of rivers and streams, or if not there, at all events in situations where no danger exists of the ground being thoroughly dried up. No doubt this appears to be inconsistent. How are we to explain the fact that Gentianeæ, ashes, willows, alpine roses, bog-mosses, \&c., are still in need of water from the atmosphere, when they all 
grow either in damp meadows, peat-bogs, on the borders of never-failing springs, or in ever-moist ravines, where their requirements in respect of nutrient water and imbibitious water can be supplied all around by means of the roots? A glance at the company in which these plants occur may perhaps lead to a solution of the problem. In the damp meadows and along the margins of springs where gentians, the Sweet-willow, and plants of that kind are found, the Butterwort (Pinguicula), which has been described in earlier pages amongst earnivorous plants, is never absent; whilst wherever the pale cushions of the Bog-moss spring, there also the Sun-dew is certain to spread out its tentacles for the capture of prey.

With reference to community of site the assumption is warranted that all these plants which flourish under identical conditions of life endeavour to acquire the same material by means of their aërial parts. Now, this material cannot well be other than nitrogen, of which they do not find a sufficient store in the substratum. What then is more natural than that those plants, which are not adapted to the capture of animals, should use their aërial organs, when these are moistened with rain or dew, to take up direct nitric acid and ammonia, which are containedthough in small traces only-in the atmospheric deposits, instead of waiting till compounds of such great importance to them penetrate into the ground where they may chance to be detained at spots whence the roots could only obtain them after long delay and by a highly complicated process? When one considers that plants, growing amid the sand and detritus of steppes, on ledges, and in crevices of steep rocks, or epiphytic on the bark of trees, are also able to acquire little or no nitrogenous food from the substratum by means of their roots, their especial equipment with apparatus for the absorption of atmospheric water becomes explicable on the ground of the latter being the medium of solution and transport of nitrogenous compounds. In the case of epiphytes and of plants growing on steppes or rocks, there is the additional consideration that a supply of pure water, supplemental to that which can be withdrawn from the substratum, must be very weleome to them in dry weather, and that at such times it is a great advantage for the atmospheric water to be absorbed directly by the aërial organs instead of reaching them in a roundabout manner through the substratum.

If this idea is justified, the atmospheric moisture taken up by the aërial organs with the help of the above-described contrivances, would be of value to the plant chiefly in being a carrier of nitrogenous compounds, and in this acceptation would have to be looked upon as water of imbibition. Whether it is also used, at least in part, as food-material can neither be asserted nor controverted. A separate absorption of water which serves only for motive power, and of that which is in addition employed in the construction of organic compounds does not take place in a plant, it is not possible to make any a priori statement concerning the moisture taken up, as to which part it has to play in the plant. Most probably the allotment of functions is not at all uniform, but varies considerably according to conditions of time, place, and requirement.

On a former occasion it has been mentioned that small animals are not vor. I. 
infrequently killed accidentally in the water filling the larger kinds of basins formed as parts of foliage-leaves, that pollen, spores, and particles of earth also are blown by the wind into these basins, and that, after the ensuing solution and decomposition of the organic and mineral bodies in question, the water exhibits a brownish colour and contains organic compounds as well as food-salts in solution. It is not necessary to repeat that these compounds are able to pass into the interior of the plant with the water through the action of the absorption-cells which are never absent from the bottom of the basins; but it seems proper to consider specially in this connection the most conspicuous cases of the phenomenon which have been observed. The greatest quantity of matter, dissolved and undissolved, is found in the flat, saucer-shaped laminæ of Saxifraga peltata, which grows on the sites of springs in the Sierra Nevada of North America. The water in these saucers is sometimes coloured quite a dark brown by the presence of decayed beetles, wasps, centipedes, fallen leaves, and animal excreta; and when it evaporates a regular crust is left behind at the bottom of the reservoir. Three days after rain I still found in the inflated vagina of Heracleum palmatum, a species of cow-parsnip, a pool of brown water $2 \mathrm{~cm}$. deep, and at the bottom a deposit of blackish, oily mud in which the remains of decayed earwigs, beetles, and spiders, were still recognizable. The same thing is observed in the cisterns of Bromeliaceæ and in the water-basins of Dipsacus laciniatus and Silphium perfoliatum (fig. 56), and it is interesting to find there are cells also at the bottom of the basins of the Dipsacus in question from which protoplasmic threads radiate forth, as in the case of the chambers of the Toothwort, and that numberless putrefactive bacteria always make their appearance in the water in these basins. The quantity of organic residue is less considerable in the saucer-shaped leaves of pelargoniums, but, on the other hand, earthy particles are frequently met with in them to such an extent that, when the water has evaporated, the concave surface of the leaf is covered with an ashen-gray layer of earth.

Observations of this nature establish the conviction that no sharp line of demarcation exists in respect of the absorption of water either between carnivorous plants and land plants, or between land plants and saprophytes, or between saprophytes and carnivorous plants; and they lead further to the conclusion that water, mineral food-salts, and organic compounds are susceptible of being taken up not only by subterranean but also by aërial absorptive apparatus 


\section{SYMBIOSIS.}

Lichens.-Cases of symbiosis of Flowering Plants having green leaves with the mycelia of Fungi destitute of chlorophyll.-Monotropa.-Plants and Animals considered as a vast symbiotic community.

\section{LICHENS.}

In describing the vegetation of a limited area botanical writers are apt to designate the various species of plants as "denizens" of the country in question. The conditions under which the plants live are likened to political institutions, and the relations existing amongst the plants themselves are compared to the life and strife of human society. By no means the least important factor in the suggestion of these analogies is the circumstance that often as a matter of fact one has opportunities of seeing how the species of plants which live together in a locality are dependent in various ways upon one another; how they exist in continual conflict for the food, the ground, for light and air; how some are preyed upon and oppressed by others, whilst others are supported and protected by their neighbours; and how, not infrequently, quite different species join together in order to attain some mutual advantage.

As regards the preying of one upon another the subject has been treated in detail in a previous chapter, and it was also stated then that the term parasite can only be applied to those plants which withdraw materials from the living parts of other organisms without rendering a reciprocal service in return. The host attacked by a parasite supplies food and drink without being in any way compensated. One might suppose that nothing would be simpler and easier than to ascertain the existence of this relationship, and yet many difficulties are encountered in the determination of parasitism in individual cases. The main difficulty is rue to the fact that one cannot always say with certainty whether the host does not perhaps get some advantage from the parasite which drains its juices. Should this be the case, however, the latter would be no longer a parasite, and the relationship between the two would rather be that of simple commerce and mutual assistance, an amicable association for the benefit of both.

Whilst discussing the second series of parasites, the fact was mentioned that the plants upon which the various species of Eyebright fasten their suckers suffer no apparent injury as a consequence of this connection. The rootlet organically united to the suckers does, it is true, die away in the autumn; but the Eyebright also withers at that season, and it is not inconceivable that the useful substances existing in the green leaves of the Eyebright may be transferred, shortly before the latter withers, to the host-plant and deposited there at a convenient time in the permanent part of the root as reserve-material, and that in this way the host-plant ultimately derives benefit from the so-called parasite. The idea here suggested as a possibility for the case of Eyebright and the grasses connected with it is an ascertained fact in the case of some other plants. For plants are known which unite to 
form a single organism and thenceforward so co-operate in their functions that ultimately both derive advantage from the arrangement. The one takes food-stuffs from the substratum and from the air and transmits them to the other; whilst, in the green cells of the other, the raw material is worked up, under the influence of sunlight, into organic compounds. The organic compounds thus created are used by both for the further production of organs, and therefore a connection such as this must be looked upon as a true case of symbiosis, i.e. associated existence for purposes of nutrition.

The first place amongst social communities of the kind must be assigned to Lichens, a section of Cryptogams possessing an extraordinarily large number of species and differentiated into thousands of forms, representatives of which are
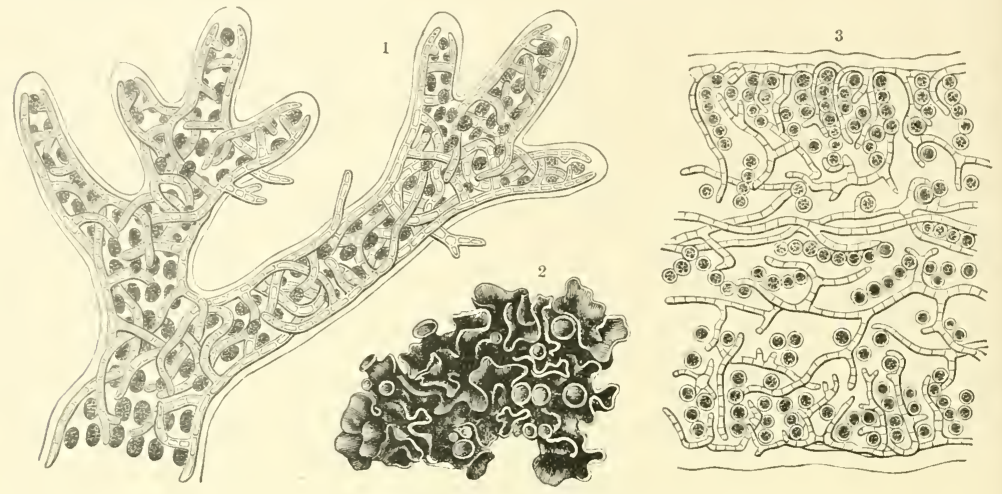

Fig. 57.-Gelatinous Lichens.

${ }^{1}$ Ephebe Kerneri; $\times 450.2$ Collema pulposum; natural size. 3 Section through Collema pulposum; $\times 450$.

everywhere distributed, from the sea-shore to the highest mountain peaks yet scaled by man, and from the tropics to the arctic and antarctic zones.

The partners in the Lichen communities appear to be, on the one hand, groups and filaments of round, ellipsoidal, or discoid green cells belonging to plant species included under the general name of Algæ; and, on the other hand, pale, tubular cells or hyphæ, which are destitute of chlorophyll, and pertain to species of plants comprised under the general name of Fungi (see fig. 58).

The form assumed by a large proportion of these lichens is that of incrustations on stones, earth, bark, or old wood-work; the entire structure of the lichen is either ensconced and imbedded in the depressions of weathered surfaces of stone, or else between the cell-walls of dead fragments of wood and bark, so that it often happens that attention is only drawn to its presence by the altered colour of the substratum, or by the fructifications which lift their heads above the substratum.

Lichens of the kind are termed Crustaceous Lichens, and the wide-spread Graphic Lichen (Lecidea geographica) may serve as an example. A second great group nearly allied to the first is that of Foliaceous Lichens. The form of the 
vegetative body in these is best compared to the foliage-leaves of the Curled Mint, with their corrugated or sinuate margins, or to those of Malva rotundifolia. It may also be described as a number of lobes radiating irregularly and bifurcating repeatedly, and only lightly joined to the substratum by root-like fringes, and therefore capable of being readily loosened and detached. The light-grey Parmelia saxatilis, which bear brown saucer-shaped fructifications, may be taken as a representative of these Foliaceous Lichens. The Fruticose Lichens are distinguished as a third group in which the thallus rises from the ground in the shape of a shrub, whilst the cylindrical, fistular, and ligulate stemlets, which ramify profusely, are only adherent to the substratum by a very small surface at the base. With these are associated the Beard Lichens, which hang down from the bark of old trees in the form of pale, copiously-branched filaments. Lastly, there is a fifth group, the
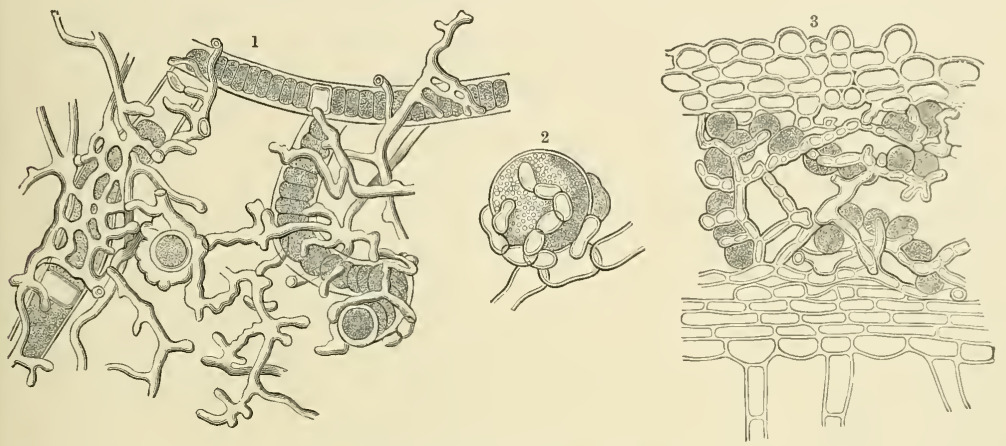

Fig. 58.-Fruticose and Foliaceous Lichens.

1 Stereocaulon ramulosum in conjunction with Scytonema; $\times 650,2$ Cladonia furcata with Protococcus; $\times 950$ 3 Coccocarpia molybdae; section, $\times 650$ (after Bornet).

Gelatinous Lichens, which when moistened look like dark, olive-green, or almost black lumps of wrinkled and wavy jelly or as if composed of variously-divided bands and strips packed together into little cushions.

In the gelatinous expansions last mentioned the algal cells are arranged in moniliform rows and are interwoven with the hyphal filaments of the fungus throughout the entire thickness of the thallus, as in Collema pulposum (see fig. $57^{2}$ and $57^{3}$ ), or else they form regular ribbon-shaped double rows, interwoven with few hyphæ, as in Ephebe Kerneri (see fig. $57^{1}$ ). In crustaceous, foliaceous, and fruticose lichens, the algal cells constitute a disorderly heap and are crowded together in the middle stratum of the thallus, where they are imbedded between an upper and a lower layer of densely felted hyphal threads, as in Coccocarpia molybdaea (fig. $58^{3}$ ).

Seeing the wide distribution of lichens it must be assumed that both partners occurring in the lichen-thallus are able to range about with extraordinary ease and latitude. When one observes how patches of the most various lichens are produccd in a few years after a lardslip on the freshly-broken surfaces of the stones which 
have fallen down into the valley beneath, one can only explain the phenomenon by supposing that the algal and fungal cells concerned have been blown together, and that the opportunity has been afforded them on the blocks of stone of contracting a union. Now, so far as regards one of the two partners, viz.: the one devoid of chlorophyll, and known as a fungus - the idea that everywhere in the air spores of fungi are swarming about is so familiar to us that the supposition of an occasional stranding of individual spores, which are being blown about by the wind, upon the moist broken surfaces of stones can encounter no opposition. Respecting those spores in particular which are ejected from the aërial fructifications of lichens, the discussion of their life-history and distribution must of course be reserved for a later section; but it is necessary to make here the one statement that provision exists for the most profuse and distant dissemination of these spores.

Thus, in the case of one of the partners, there is no difficulty in realizing its ubiquity. But when one comes to the Algæ, the name at first calls up to mind the green filaments which occupy our pools and ponds, or the brown wracks and red Florider of the sea-shore, and we ask ourselves how it can be possible for these plants to occur on fractured surfaces of stone, especially on the débris of mountain sides. Indeed, it is certainly not Algæ of these kinds that take part in the construction of Lichens. The name Algæ is properly only a general name for all Thallophytes containing chlorophyll, and it is applied to many small organisms besides those mentioned above, namely, to numbers of Nostocineæ, Scytonemeæ, Palmellaceæ, Chroolepideæ, and these are the kinds which fall in with the cells of fungi and form lichens in conjunction with them. Owing to their minute size, they are apt to escape observation, and, in general, only attract attention when myriads of them clothe the bark of trees, cliffs, stones, or earth. In these situations they need but little moisture, and it is not necessary for any of them to live under water like other algæ; they become desiccated without sustaining the slightest injury and make their appearance on the substratum occupied by them at the first stage of their development, as powdery coats, and, in this condition being extremely light, are liable to be blown away by a wind of moderate strength, and so distributed over mountain and valley.

That this dissemination is not merely hypothetical but an actual fact has been susceptible of easy proof by the following experiment, made in a mountain-valley in the Tyrol. A plane surface covered with white filter-paper, which was kept moist, was exposed to a south wind; in the course of a few hours numerous particles, like dust, adhered to the paper, and amongst them cell-groups of Nostocineæ and others of the above-mentioned algæ occurred regularly, in addition to organic fragments of the most various kinds, such as pollen-grains and spores of all sorts of mosses and fungi. All these bodies were deposited in the little depressions on the sheet of paper, and in the same way they rest in the grooves, cavities, and cracks in the surfaces of stone, bark, and old wood-work, where they succeed in reaching a further development as soon as the requisite quantity of water is provided. Now, if at these places the little algal cell-groups meet with hyphæ belonging to the 
other potential partner, the latter embrace and enmesh them, as is shown in the above figures, and thus is produced the confederacy called a Lichen. The member destitute of chlorophyll takes up nutriment from the external environment; it possesses, in particular, the property of condensing aqueous vapour, and has, besides, the power of bringing the solid substratum partially into solution by means of excreted substances; it effects adhesion to the substratum, and, in a majority of cases, determines the form and colour of the lichen-thallus as a whole. The second member, whose cells contain chlorophyll, undertakes the task of producing organic matter, under the influence of sunlight, from the materials conveyed to it; by this means it multiplies the number of its cells and increases in volume, whilst, at the same time, it yields to its mate so much as is necessary in order to enable the latter to keep pace with it in growth.

The number of algæ which enters into a partnership of this kind is, in any case, much less considerable than that of the fungi, and it must be assumed that one species of alga may unite with the hyphr of different lichen-fungi. The extreme variety, moreover, in the combinations of the two sorts of confederate occurring on a very small area is obvious from the circumstance that it is not rare for half a dozen different species of lichen to spring up side by side on a patch of rock no bigger than one's hand. Whether they all achieve an equally hardy development, or whether some perchance are not crowded out and overgrown by others depends on various external conditions-on the chemical composition of the substratum, and particularly on the conditions of moisture and illumination of the site in question. Lichens are very sensitive in this respect, and the different sides of a single rock often exhibit quite different growths of lichens. A very instructive example of this is afforded by a marble column near the famous castle of Ambras in Tyrol. This column is octagonal, and has been standing in its place for more than two hundred years, with all its sides exposed to wind and weather. Lichens have settled on all the eight faces, and, indeed, are present in such abundance that the stone is quite covered by patches the size of a man's hand. Many of these growths are but poorly developed, and not susceptible of being identified with certainty; but altogether on this column there must be over a dozen different species, the germs of which can only have been brought by winds. These species are, however, by no means uniformly disposed; some prevail on one side, some on another, and a few are confined exclusively to one of the eight faces. Of three species of Amphiloma, the one named A. elegans is restricted to the warmest side, i.e. the face exposed to the south-west; a second, Amphiloma murorum, is to be seen on the upper part of the southern face; whilst Amphiloma decipiens occurs on the same face, but only near the ground. On the side with a northern aspect Endocarpon miniatum predominates, and on the north-west face Calopisma citrinum and Lecidea are the prevailing forms.

What thousands of spores and algal cells must have been blown on to this pillar to enable all these combinations to arise! What complex processes must have gone on before the selection of lichens best adapted to each different quarter 
of the compass was effected on this little marble column! It is necessary to add, however, that lichens growing on stone, bark, or any situation of the kind do not in all cases owe their original appearance on the substratum to a fresh union of Algre and Fungi, but that there is a second mode of distribution of lichens. This method consists in the transportation by air-currents of already completed social colonies to places often situated at a great distance from the spots where the initial union between Alga and Fungus was contracted. The process is as follows: -in the interior of an old, large, and fully developed lichen-thallus certain groups of cells separate from the rest, each group consisting of one or more green algal cells enmeshed in a dense weft of hyphæ. When a sufficient number of these daughter-associations has been formed the thallus of the parent lichen is ruptured and the little miniature social-groups, which are termed "soredia", come to the surface. To the naked eye a single soredium is only visible as a bright dot, but all together they have the appearance of a mass of powder or meal lying loosely upon the old lichen-thallus. In dry weather this mealy efflorescence is easily blown away with other organic particles. If, then, a soredium thus removed comes to rest in the crack of a rock or on any suitable substratum, the alga and hyphæ composing it continue to develop, and the organism grows into a larger lichen-thallus, which is able to repeat the process just described. In regions where lichens abound, soredia of the kind are found regularly amongst the elements of the organic dust, and occur, indeed, mixed with fungal spores and algal cells, so that it certainly happens not infrequently that two spots close together in the same cranny of stone exhibit both sorts of lichen-growth, the one newly produced by the concurrence and union of algal and fungal cells, the other a daughterassociation which has arisen from an old lichen, as a soredium, and is continuing its development.

Another case of symbiosis allied to that of lichens is manifested by certain Cryptogams which live socially together under water and have received the systematic names of Mastichonema, Dasyactis, Enactis, \&c. In them also a plant containing ehlorophyll, and belonging to the group of Nostocineæ, appears as one member of the partnership; whilst the second is some species of Leptothrix or Hypheothrix. The green moniliform rows of cells of Nostocineæ are enmeshed and wrapped round by the delicate, filamentous cells devoid of chlorophyll of the Leptothrix or Hypheothrix; and later, by repeated processes of division, whole colonies of green cell-filaments ensheathed in this manner are produced, which to the naked eye appear as small soft tufts, usually clinging to porous limestone in the spray of waterfalls. In many cases the filaments destitute of chlorophyll rest upun the moderately thickened cell-membranes of the green algæ, whilst in other cases they insinuate themselves into the thick cell-membranes, permeate them with their webs, and form in conjunction with them the sheathing envelope. 


\section{SYMBIOSIS OF GREEN-LEAVED PHANEROGAMS WITH FUNGAL MYCELTA DESTITUTE OF CHLOROPHYLL-MONOTROPA.}

Another instance of symbiosis is observed to exist between certain flowering plants and mycelia of fungi. The division of labour consists in the fungus-mycelium providing the green-leaved Phanerogam with water and food-stuffs from the ground, whilst receiving in return from its partner such organic compounds as have been produced in the green leaves.

The union of the two partners always takes place underground, the absorbent roots of the Phanerogams being woven over by the filaments of a mycelium. The first root that emerges from the germinating seed of the phanerogamic plant destined to take part in the association descends into the mould still free from hyphæ; but the lateral roots and, to a still greater extent, the further ramifications, become entangled by the mycelial filaments already existing in the mould or proceeding from spore-germs buried there. Thenceforward the connection continues until death. As the root grows onward, the mycelium grows with it, accompanying it like a shadow whatever its course, whether the root descends vertically or obliquely, or runs horizontally, or re-ascends, as is sometimes necessary when it happens to be turned aside by a stone. The ultimate ramifications of roots of trees a hundred years old, and the suction-roots of year-old seedlings, are woven over by mycelial filaments in precisely the same manner. These mycelial filaments are always in sinuous curves and intertwined in various ways, so that they form a felt-like tissue, which looks, in transverse section, delusively like a parenchyma. As regards colour the cell-filaments are mostly brown, sometimes they are almost black, and it is rare for them to be colourless. The epidermis of many roots is covered as if by a spider's web, whilst the hyphæ form a complex tangle of bundles and strands broken here and there by open meshes through which the root is visible. In other cases an evenly woven but very thin layer is wrapped round the root; and in others, again, the fungus-mantle forms a thick layer which envelops uniformly the entire root (see fig. 59). Here and there the hyphæ insinuate themselves also inside the walls of the epidermal cells, and the latter are permeated by an extremely fine small-meshed mycelial net (see fig. $59^{3}$ ). Externally the mantle is either fairly smooth and clearly marked off from the environment, or else single hyphæ and bundles of hyphæ proceed from it and thread their way through the earth. When these branching hyphæ are pretty equal in length they look very much like ordinary root-hairs. And they not only resemble them, but assume the function of root-hairs. The epidermal cells of the root, which would in an ordinary way act as absorption-cells, being inclosed in the mycelial mantle cannot exercise this function, and have relegated the business of sucking in liquid from the ground to the mycelium. The latter undoubtedly acts as an absorptive apparatus for the partner on whose roots it has established itself; and the water in the soil, together with all the mineral salts and other compounds 
dissolved in that water, are caused by the mycelial mantle to pass from the surrounding ground into the epidermal cells of the root in question, and thence onward, ascending into axis, branches, and foliage.

Thus the fungus-mycelium not only inflicts no injury on the green-leaved plant by entering into connection with its roots, but confers a positive benefit, and it is even questionable whether a number of green-leaved plants could flourish at all without the assistance of mycelia. The experience gained in the cultivation of those trees, shrubs, and herbs, which exhibit mycelial mantles on their roots, does not, at any rate, lead to that conclusion. Every gardener knows that attempts to rear the various species of winter-green, the bog-whortleberry, broom, heath, bilberries, cranberries, rhododendrons, the spurge-laurel, and even the silver-fir and
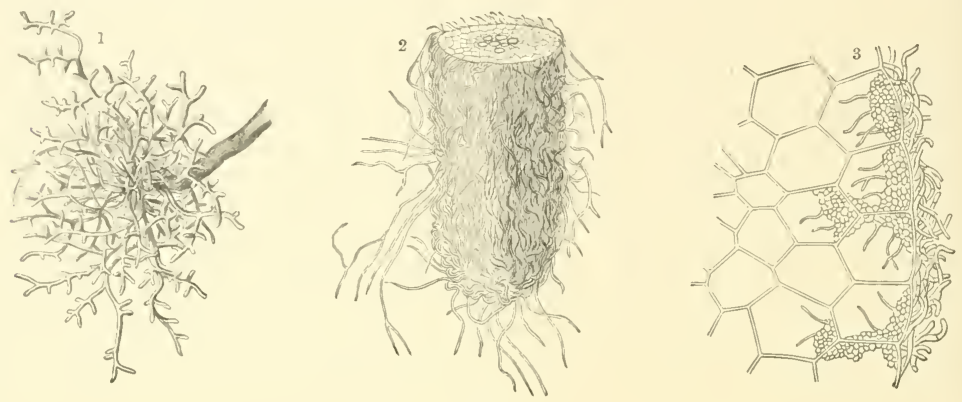

Fig. 59.

1 Roots of the White Poplar with mycelial mantle. 2 Tip of a root of the Beech with closely adherent mycelial mantle; $\times 100$ (after Frank). 3 Section through a piece of root of the White Poplar with the mycelium entering into the external cells; $\times 480$.

the beech, in ordinary garden soil are not attended with uniform success. Therefore, as is well known, soil consisting of vegetable mould from the top layer of earth in woods or on heath is chosen for the cultivation of species of the genera Erica, Daphne, and Rhododendron. But it is not even every kind of forest- or heathmould that can be made use of. When earth of that nature has been quite dry for a long time it is no longer fit for this purpose. On the other hand, it is known that the above-mentioned plants should be transplanted from their forest-home with the soil still clinging to the roots, and it is also laid down as an axiom that the roots of these plants should not be exposed and should be cut as little as possible. The following reasons account for all this. Firstly, fresh earth from a heath, or mould recently dug from the ground in a wood, contains the mycelia still alive, whereas in dry humus they are already dead; secondly, the mycelia woven round the roots are transferred together with the balls of earthy matter suspended to them into the garden; and, lastly, any considerable clipping of the roots would remove the ultimate ramifications which are furnished with the absorbent mycelial mantle.

The failure of all attempts to propagate the oak, the beech, heath, rhododendron, winter-green, broom, or spurge-laurel, by slips or cuttings, if the shoot which is cut 
off and used for the purpose is put into pure sand, is explicable in the same way. Limes, roses, ivy, and pinks, the roots of which possess no mycelial mantle, are notoriously propagated very easily by putting branches cut from them into damp sand. Rootlets are at once produced on those parts of the branches which are buried in the sand, and their absorption-cells carry on the task of taking up nutriment from the ground. But though cuttings of oak, rhododendron, wintergreen, bog-whortleberry, and broom strike root, no progress in their development is to be observed, because the superficial cells of the rootlets, in these cases, have not the power of absorbing food when they are not associated with a mycelium. It is only when the slips from these plants are put into sand with a rich admixture of humus, the latter having just been taken from a wood or heath and containing the germs of mycelia, that some few are successfully brought to further development. The result is even then often not assured, and the cuttings of several of the plants enumerated die even in sand mixed with humus before they have produced rootlets.

Seeing also that the result of attempts to rear seedlings of the beech and the fir in so-called nutrient solutions, where there could be no question of any union with a mycelium, has been that the plantlets dragged on a miserable vegetative existence for a short time and ultimately died, we have good grounds for assuming that the envelope of mycelial filaments is indispensable for the Phanerogams in question, and that the prosperity of both is only assured when they are in social alliance.

The facts ascertained in cases of analogous relationship lead one to expect that the fungus-mycelia also derive some advantage from the flowering-plants, the roots of which they clothe, and to which they render the service of acting as absorptioncells. The benefit in question is undoubtedly the same as that derived by the hyphæ of a lichen-thallus from the enwoven green cells. The mycelial mantles withdraw from the roots of the Phanerogams the organic compounds which have been elaborated by the green leaves in the sunshine above-ground, and which are conducted thence to all growing parts, that is to say, downwards as well as in other directions, to the tips of the swelling and elongating roots. According to this, therefore, the division of labour between the members of the alliance for joint nutrition consists in the mycelium supplying the green-leaved plant with materials from the ground, and the green-leaved plant supplying the mycelium with substances which have been worked up above-ground in the sunlight.

The range of species which live in a social union such as is here described is certainly very large. All Pyrolaceæ, Vaccineæ, and Arbuteæ, most, if not all, Ericaceæ, Rhododendrons, Daphnoideæ, and species of Empetrum, Epacris, and Genista, a great number of Conifers, and apparently all the Cupuliferæ as well as several Willows and Poplars are dependent for nutrition on the assistance of mycelia. We find, too, that this condition recurs in every zone and in every region. The roots of the Arbutus on the shores of the Mediterranean are equipped with a mycelial mantle in precisely the same manner as those of the low-growing Whortleberry of the High Alps. 
Special importance is given to the social life by the fact that the chief species of Phanerogams participating in it are of gregarious growth and cover whole tracts of country, forming boundless heaths and measurcless forests, as, for instance, the various heaths, the oak, the beech, the fir, and the poplar. The conception of this subterranean life affecting every moorland and vast timbered tract is one full of wonder and interest.

We can now see why it is that the ground in woods is the abode of such a profusion of fungi. No doubt some of these fungi draw their nutriment exclusively from the store of dead plant-organs accumulated there; but others, as certainly, are in social connection with the living roots of green-leaved plants. It is true we cannot yet state precisely what are the species of fungi which contract this sort of union, or whether generally a definite elective affinity exists between certain fungi and certain green-leaved plants. There is much in favour of this supposition in a few cases: but, on the other hand, it is very unlikely that each of the various Phanerogams occupying a limited area of ground in a pine-forest, where a few square meters of earth contain so many tangled roots belonging to pines, spurge laurels, bilberries, cranberries, heath, and winter-green, that they can only be separated with difficulty, should select from the great host of fungi growing in the forest a different partner. In instances of this kind it seems just to suppose that the mycelium of one and the same species of fungus enters simultaneously into connection with all or several of the plants growing close together; it is similarly probable that the mycelia of different species of fungi render to one and the same flowering-plant the service of absorption according to the locality in which it occurs. This surmise is supported by the fact that when certain species, brought from distant parts and regularly exhibiting mycelial mantles on the ends of their roots, are reared in our gardens and greenhouses from seed, they unite in these abodes with fungus-mycelia, which certainly do not exist in the regions where the Phanerogams in question grow wild. Thus, for instance, the roots of the Japanese tree, Sophora Japonica, and those of the Epacrideæ of Australia, are found in European gardens in social union with fungi, which with us are native, but which certainly do not occur in Japan or Australia; and it is therefore scarcely open to doubt that the Sophora Japonica, to take one example, associates itself with different fungi in different regions.

Now that the symbiosis of fungi devoid of chlorophyll with green-leaved Phanerogams has been discussed, we are for the first time in a position to deal with that most remarkable of all cases of food-absorption wherein the subterranean roots of a flowering-plant are completely wrapped in a mycelial mantle, whilst the parts which shoot up above ground bear no green leaves, and, in general, possess no trace of chlorophyll. Such is the case of Monotropa, the various species of which are intimately allied in the structure of flowers and fruit with the Primrose and Wintergreen, and are met with scattered everywhere in shady woods. Their stems, which are from 10 to 20 centimeters in height and emerge from the mould of the forestground in summer time, are thick, fleshy, succulent, and profusely beset with 
membranous and transparent scales, and the extremity of each is bent back like a hook. The cylindrical flowers are developed at the top of the stem with their open ends turned to the ground, and are half-covered by the scales. Everything about this plant (stem, leaf-scales, and flowers) is of a pale waxen-yellow colour, and the general impression it produces is much more that of a Toothwort, or one of the colourless forest orchids, than of a species of primula or winter-green. Towards autumn, when ripe fruits have been produced from the flowers, the hitherto drooping extremity of the stem lifts itself into an upright position, whilst the entire aërial portion of the plant turns brown and dries up. Every disturbance caused by the wind, however slight, shakes out of the spherical fruits many thousands of tiny seeds as fine as dust, which, like the winter-green seeds, consist of only a few cells, and do not admit of the recognition of any differentiated embryo within them. Moreover, underground, the rhizomes, from which the small group of pale stems have arisen in summer, continue to live through the winter, and a number of new buds are developed on them. On digging down to the hibernating plant and removing the mould which conceals it, one finds at a depth of from 10 to 40 centimeters bodies like coral-stems consisting of dense masses of roots crowded together and ramifying multifariously. All the root-branches are short, thick, fleshy, and brittle, and are matted together to form turf-like masses, which are not infrequently interwoven with the rootlets of pines, firs, and beeches, and have all their interstices filled with humus. Each rootlet is enveloped, right up to the growing apex, in a thick mycelial mantle. The hyphal filaments of this mycelium do not penetrate into the tissue of the root of Monotropa, nor do they send any haustoria into the superficial cells of these roots. The hyphæ and the epidermal cells of the root are, however, in such close and continuous contact that sections exhibit a complete continuity of the tissues.

Monotropa is therefore only able to withdraw nutriment from the hyphal weft of the mycelium so far as its subterranean parts are concerned, and, seeing that it is quite destitute of chlorophyll, and its aërial stem and leaves display no trace of stomata, the possibility of creating organic matter and of adding in general to its substance by means of its aërial parts is excluded. It therefore receives all the materials of which it is constructed from the mycelium of the fungus, whilst it is not in a position to render anything in return to this mycelium that it has not previously derived from the latter. If the mycelium subsequently withdraws any materials whatever from the still living or decaying Monotropa, the process is only one of restitution and not of exchange. Thus, in this case, there can be no talk of reciprocity in the processes of nutrition or division of labour such as occurs when there is symbiosis. The Monotropa grows in height and in circumference entirely at the expense of the mycelium in which it is imbedded, so that we have here the remarkable phenomenon of a Phanerogam parasitic in the mycelium of a Fungus. We so often come across the converse process in our experience that we cannot easily familiarize ourselves with the idea of a flowering-plant draining the mycelium of a fungus of nutriment: nevertheless there is scarcely any other inter- 
pretation possible in this case, for all the other hypotheses,- - such as that Monotropa enters into connection with the roots of trees, or that it is parasitic in the first stages of development, but subsequently detaches itself from its host and becomes a saprophyte,-rest on inaccurate observations, and have long been disproved. As a parasite Monotropa ought to have been discussed at the same time as others in earlier pages, but it was not without intention that the description of this plant was reserved for this place, for it would have been difficult to state and explain the method of nutrition exhibited by it before some previous knowledge of the curious phenomena of union of the mycelia of fungi with the roots of green-leaved Phanerogams had been acquired.

\section{ANIMALS AND PLANTS CONSIDERED AS A GREAT SYMBIOTIC COMMUNITY.}

If we look back at the cases of symbiosis already discussed and inquire what is their value, we find it consists in an integration of the functions of plants possessing chlorophyll and plants not possessing it. The reciprocity here implied is, however, at bottom, but a copy of the complementary interaction of plants and animals which takes place on a grand scale in the organic world. The associated plant, destitute of chlorophyll, in which capacity fungi are always the organisms concerned, really plays the same part in the social life as is taken by animals in the great economy of nature, and this is in harmony with the fact that in other respects as well fungi exhibit so many similarities to animals that in many instances one looks in vain for a line of division to separate them from animal organisms. Hence there is no need for surprise when cases come under observation wherein a quite unmistakably animal organism enters, instead of a fungus, as one of the partners in a symbiotic community. Certain Radiolariæ have small yellowish spots upon them, which were formerly held to be pigment-cells, but have proved to be little algæ, with cells furnished with true chlorophyll. Similar properties are exhibited by the fresh-water polyp, Hydra, and by the marine sea-anemones. Small algæ occur in social union with these also in the shape of cells with membranes made of cellulose and containing chlorophyll and starch-grains in their protoplasmic bodies. These algæ are in no wise injurious to the animals with which they are associated; on the contrary, their presence is beneficial, their partners reaping an advantage from the fact that the green constituents split up carbonic acid under the influence of the sun's rays, and in so doing liberate oxygen which may be again taken in by the animals direct, and serve a useful purpose in their respiration and all the processes connected therewith. Conversely, the alga, in association with the animal's body, will derive a further advantage from the latter, inasmuch as it receives at first hand the carbonic acid exhaled by the animal in breathing. The small algæ living socially with animals cannot be reckoned as parasites in any case, nor can the animals be looked upon as parasites of the algæ, but we have here the phenomenon of mutual assistance and of a bond serving for the benefit of both 
parties, precisely similar to that noticed in the case of lichens and in the others which have been described above.

Several of the liverworts which live as epiphytes on the bark of trees exhibit on the under surface of their leaflets (which are inserted on the stem in two rows, and are pressed flat against the bark) little auricular structures, and in species of the genus Frullania, these take the form of definite hoods or pitchers. The rain that trickles down the trunks of the trees, washing the bark and wetting the liverworts in its course, fills the hooded receptacles referred to with water, and is retained longer in these protected cavities than anywhere else, if a period of drought ensues and the liverwort becomes dry again. Now these cowls are the abode of tiny rotifers (Callidina symbiotica and $C$. Leitgebii), which live on the organic dust brought thither with the water. In return for the peaceful home thus afforded them in the hooded chambers of the leaves, the rotifers supply the liverworts in question with nitrogenous food. For as such must serve the matter excreted by the rotifers in the interior of the cowls. Without the intervention of the rotifers, the living organisms (Infusoria, Nostocineæ, and spores) contained in the water could not be converted into food by the liverworts, whereas the liquid manure arising from the Infusoria, Nostocineæ, and spores, digested in the bodies of the rotifers, contains highly nitrogenous compounds, which are of great value to the liverworts in question, as indeed they are to all epiphytes living on the bark of trees. It stands to reason that the symbiotic liverworts and rotifers derive also a mutual advantage from the fact that the oxygen set free by the former comes into the possession of the rotifers and the carbonic acid emitted by the rotifers into that of the liverworts by the most direct method.

Moreover, these cases of partnerships further remind us of other analogous relations existing between plants and animals, which it is necessary to refer to now, although they cannot be treated in detail till later on. A great number of floweringplants excrete honey into their flowers, and so attract flying insects to them, which supply themselves plentifully, and in their turn render to the plants they visit the service of transferring the pollen from flower to flower, thus making possible the development of fruits and fertile seeds. Certain small moths which visit the flowers of $Y$ ucca bring the pollen to the stigmas, and force it into the stigmatic orifices in order that mature fruits and seeds may be produced from the rudimentary fruits, a result which is indeed a matter of vital importance to these moths. For the moths lay their eggs in the carpels of $Y u c c a$, and from the eggs larvæ are developed which live exclusively on the seeds of this plant. If the Yucca were not fertilized, and did not develop any fruit, the larvæ would die of hunger. A similar phenomenon occurs in many other cases of the kind, where both plant and animal reap some benefit. On the other hand, in the formation of galls, which are produced by animals laying their eggs in particular parts of plants, the advantage (with few exceptions) is all on the side of the animals, and these gallstructures might most justly be placed by the side of parasitic structures.

It is obvious from all this that such of the mutual relations of plants and of 
their relations to animals as are occasioned by the endeavour to acquire nutriment are extremely various and often linked together and complicated or deranged by one another in the most curious manner. Cases occur of a particular plant being socially connected with another, and at the same time also beset by vegetable and animal parasites. The absorption-roots of the Black Poplar are covered with a dense mycelial mantle, so that this tree is associated for purposes of nutrition with the fungus to which the mycelium belongs. Such parts of the roots of the Black Poplar as are left free from the mycelium are fastened upon by suckers sent forth by Toothwort plants, which withdraw from the roots the juices absorbed by the latter from the earth through the instrumentality of the mycelial mantles clothing them. Meantine, in the cavities in the leaves of the Toothwort various small animals are caught and made use of as nitrogenous food. Again, the poplar-tree bears Mistletoe on its boughs, and its presence there is due to the missel-thrush. The thrush takes the Mistletoe-berries for food, and, in return, renders the plant the service of dispersing the seeds and establishing them on other trees. The parasitic Mistletoe takes its liquid nutriment from the wood of the poplar-tree; but, on the other hand, its own stems are covered with lichens, and these lichens are themselves a symbiotic community of algæ and fungi. Within the wood of the poplarstems spread the mycelia of certain Basidiomycetes (Panus conchatus and Polyporus populinus), whilst the foliage-leaves are covered with a little orange-coloured fungus, Melampsora populina. In addition, no less than three gall-creating species of Pemphigus live on the leaves and branches of the Poplar, and a number of beetles and butterflies are nourished by them. Certain lichens, mosses, and liverworts regularly settle on the bark of old trunks, and included amongst these may be the species of liverwort which is inhabited by rotifers. If all the plants and animals which live upon the poplar-tree, within it or in association with it, are counted, the number turns out to be not much fewer than fifty. 


\section{CHANGES IN THE SOIL INCIDENT TO THE NUTRITION OF PLANTS.}

Solution, displacement, and accumulation of particular mineral constituents of the soil owing to the action of living plants.-Accumulation and decomposition of dead plants.-Mechanical changes effected in the soil by plants.

SOLUTION, DISPLACEMENT, AND ACCUMULATION OF PARTICULAR MINERAL CONSTITUENTS OF THE SOIL RESULTING FROM THE ACTION OF PLANTS.

Reference was made in the preceding section to a marble pillar on the faces of which a dozen different lichens have settled in the course of centuries. I again introduce to the reader's notice this unobtrusive monument in order to demonstrate in its case the changes to which stone is subjected by the plants clinging to it or nestling in its crevices. It may be premised, as a matter of course, that when the marble column was erected two hundred years ago the eight sides were polished, and presented perfectly even surfaces. But what is its appearance to-day? The whole is rough and uneven; in parts it is as though corroded, and there are little pits clustered together in places. The idea might arise that depressions have been formed in course of time by the impact of drops of rain, but nearer inspection shows that there can be no question that the inequalities have been produced in this way; on the contrary, it is by the influence of the lichens adherent to the stone. Especially on the two sides of the pillar facing south and south-west, one sees clearly how each pit corresponds exactly in size to a species of grey lichen there ensconced, and how this lichen, as it continues to grow and extends radially, corrodes and etches the marble it touches in ever-widening circles. The expression "to etch" may here be taken literally, for there is no doubt that the process, the result of which is manifested in the formation of little pits, is mainly caused by the excretion of carbonic acid from the lichen's hyphæ, whereby the calcium carbonate is converted into bicarbonate. The latter, being soluble in water, is, in part, taken up by the lichen as nutriment, whilst part is washed away by the rain.

In addition to this chemical action, the hyphal filaments exercise also a purely mechanical influence. A growing hypha penetrates wherever the merest particle of carbonate of lime has been dissolved and accomplishes regular mining operations at the spot. Projecting particles of the carbonate not yet dissolved are separated by mechanical pressure from the main mass; and at the places in question where a lichen is in a state of energetic growth, tiny loose rhombohedral fragments of the lime are to be seen, which are washed away by the next shower or else carried off as dust by the wind. The same process as that which may be so clearly traced on the marble pillar at Ambras takes place, of course, also on the limestone that has not been carved or polished, in every locality where lichens exist at all. We notice it in the case of other kinds of stone as well-in dolomite, felspar, and even in pure quartz rock-for even quartz is not able to withstand the long-continued action of VOL. I. 
carbonic acid and the mechanical operations above referred to in the performance of which the hyphæ act like levers. Some of the powerful iron bands belonging to the great suspension bridge across the Danube at Budapest afford us the opportunity of observing the mining operations of lichens on a substratum of pure iron. Of course in these cases the decomposition and solution initiated by the carbonic acid varies according to the nature of the substratum; the result is, however, invariably the same; there is always a loss of substance on the part of the substratum, and a part of the dissolved matter is always taken up by the adherent plant, whilst another part is carried away either in solution or mechanically by wind or rain.

Mosses act in precisely the same manner as lichens. If a tuft of Grimmia apocarpa is lifted away from the side of a block of limestone, it becomes evident that in the neighbourhood of the place where all the stemlets of the little mosscolony meet, the underlying stone is threaded through and through, and rendered friable. There lie the rhizoids imbedded between isolated particles of lime, which are as fine as dust, and have been disintegrated by the chemical and mechanical activity of the organs in question. At spots where plants of Grimmia have died, the limestone always exhibits an obvious loss of substance in the form of unevenly corroded depressions.

The fact that the roots of Phanerogams also alter the subjacent stone in a similar manner may be proved by the following experiment. A polished slab of marble is covered with a layer of sand, and seeds of plants caused to germinate in this sand. The roots of the seedlings as they grow downwards come almost immediately upon the marble slab, and, turning round, creep onward in close contact with the stone. After a short time the parts of the slab against which the roots are pressed become rough as though they had been etched; a solution of individual particles of the carbonate of lime takes place under the influence of the acid juice saturating the cell-walls of the root's cells, and this circumstance reveals itself to the naked eye as a roughness which is readily perceptible.

Whereas the loss of substance affecting the solid substratum of plants may thus be at once detected by sight, the removal of constituents of the air and of water eludes direct observation. The ingredients withdrawn by plants are instantly replaced in water and still more in the air by influx from the environment, and obviously no holes or pits are the outcome as in the case of a surface of limestone rock.

In the discussions that follow it is important to retain the conception that in the process of vegetable nutrition certain substances may undergo local displacement, accumulation, and aggregation, and temporary consignment to a state of quiescence. Ingredients of the earth's crust are borne upwards into atmospheric regions, and constituent parts of the air are carried deep down into the ground. Lime, potash, silicic acid, iron, \&c., pass from disintegrated rocks into the realms above ground-into stems and leaves, and to the tops of the highest trees, whilst carbon and nitrogen pass from the aërial shoots and from the foliage spread out in the sunshine into the deepest shafts which the roots have bored for themselves in 
the ground. If one were to mark out the space of ground from which the lime, potash, and other nutrient salts used in the construction of a birch-tree were derived, its bulk would certainly be found to be much larger than that of the birch; and, if we were to try to estimate the volume of air through which the carbon, which has been converted into organic compounds in the tree, was previously distributed in the form of carbon dioxide, it would turn out to exceed the volume of the birch a thousandfold. In this sense, every plant may justly be considered as an accumulator of those substances which serve for its nutriment. Every plant continues, so long as it lives, to store them up in ever-increasing quantities in its own body, and in the case of long-lived plants there is thus collected ultimately quite a considerable quantity. When the life of an accumulator of the kind is extinguished, those materials which were taken from the atmosphere are able to return into the atmosphere; but such mineral food as has been derived from the ground and lifted into the upper parts of the plant-particularly those above the ground-and has there been amassed in a confined space, does not return to its original place. A dead tree breaks down on the first provocation, and the trunk lies on the ground and rots. Such part of its substance as can pass into the atmosphere in gaseous form escapes; but the salts accumulated within it, which it raised from deep under ground during its lifetime, are retained by the surface-layers of the soil. Even though some of them are washed out of the trunk by the lixiviating action of rain-water, the superficial layers of earth operate as a filter, and do not allow any part to return to the underlying strata. So, too, the nutrient salts which reach the foliage of plants are added to the top layers of the soil; for fallen leaves go through much the same process as the trunk which is broken by storms and undergoes decay as it lies prostrate upon the ground.

Thus, wherever men do not interfere by clearing away the accumulative agents in question (i.e. plants), where there is no removal of the haulms of cereals from fields, or of mown grass and herbs from meadows to serve as hay, or of timber from the forest-wherever, in a word, the vegetable world is left to itself and the natural progress of evolution is not frustrated by any disturbing element-the food-salts which have been amassed will accumulate in the uppermost layers of the earth. Moreover, seeing that, as has been already pointed out, every plant has the power of possessing itself of substances of value to it, even when they are only present in the environment of the roots in scarcely appreciable quantities, it is possible for the top layers of soil to contain a considerable amount of a substance which only occurs in the subjacent rock in such small measure as to be detected with difficulty. The percentage of lime yielded by the subsoil on the Blöckenstein, a granitic mountain 1383 meters high, on the borders of Bavaria and Upper Austria, was $2 \cdot 7$, whilst that of the top layer was $19 \cdot 7$; the percentage on Mount Lusen, situated to the north of the Blöckenstein, was 1.9 for the subsoil and 8.6 for the superficial layer. When one considers that fresh plants strike root in the ground near the surface and these again act as accumulators, and remembers in addition that snails make their appearance in abundance wherever vegetable food containing 
lime is to be found, that these snails again are to be reckoned as accumulators, and that their shells, which consist almost entirely of lime, remain after the animals' deaths in the top layer of soil, it is not surprising to find that the earth-mould on a granite plateau contains a proportion of lime not much less than that yielded by mould resting on argillaceous limestone.

Still more striking than the influence of rock plants and land plants in transposing and accumulating lime is the agency of hydrophytes in causing the same results. In the trickling springs of mountainous regions as well as in the standing pools of level country and no less in the depths of the sea, plants occur which obtain part of the carbonic acid they require by the decomposition of the bicarbonate of lime dissolved in the surrounding water. The monocarbonate of lime, which is insoluble in water, is then precipitated in the form of incrustations upon the leaves and stems of the plants in question. Many of these hydrophytes take up carbonate of lime into the substance of their cell-membranes; and in other cases both phenomena occur, that is to say, not only are they incrusted externally with calcium carbonate, but the cell-walls are also thoroughly impregnated by the same salt. In the streams arising from springs loaded with bicarbonate of lime in solution derived from the heart of a mountain, a number of mosses regularly occur-Gymnostomum curvirostre, Trichostomum tophaceum, Hypnum falcatum, and others besides. These mosses and also several species of Nostocineæ belonging to the genera Dasyactis and Euactis become completely incrusted with lime, in the manner referred to, but go on growing at the apical end as the older and lower parts imbedded in lime die off. In consequence, the bed of the stream itself becomes calcified and elevated, and, in course of time, banks of calcareous tufa are formed, which may attain to considerable dimensions. Banks raised in this manner are known which are no less than 16 meters in height; to construct them mosses must have worked for more than 2000 years.

Numerous Stoneworts (species of Chara or Nitella), the Water-milfoil and Hornwort (Myriophyllum and Ceratophyllum), Water-crowfoots (Ranunculus divaricatus and $R$. aquatilis), and more especially many Pond-weeds (Potamogeton), which grow in continuous masses in still, inland waters, incrust their delicate stems and leaves with lime during the summer, but in autumn shrink away, that is to say, their stems and leaves fall and decay, leaving scarcely any trace of the mass of vegetation till the advent of the following spring. The calcareous deposits, however, are preserved, and, sinking to the bottom of the water where the incrusted plants lived, form a layer which year by year increases in thickness. Anyone who undertakes the investigation of the sequestered wastes of water in the shallow lakes of lowland districts will be convinced of the magnitude of the scale on which this kind of accumulation must take place. As one's boat glides over places where there is a luxuriant growth of the lime-incrusted Chara rudis and C. ceratophylla, there is a crepitating sound in the water like the snapping of dry sticks of birchwood. Great numbers of stoneworts are fractured by the boat as it strikes against them, and if one takes hold of the fragments they feel like a heap of brittle glass 
fibres. What a quantity of carbonate of lime must be deposited yearly at the bottom of these lakes and ponds! Amongst pond-weeds, Potamogeton lucens, in particular, clothes its large shining leaves with a very stout, uniform crust, which drops off in scales as the plant dries, the weight of which can be exactly determined in the case of each separate leaf. The result of careful weighing showed that a single leaf equal in weight to 0.492 grm. was covered with a calcareous crust weighing $1.040 \mathrm{grm}$. Now, supposing one shoot of this pond-weed, having five leaves, and covering an area of 1 square decimeter, decays in the autumn, and lets its lime sink to the bottom of the pond, the approximate weight of lime deposited each year on a square decimeter of the ground at the bottom is 5 grms., and, if this process is repeated every year, a layer is deposited in ten years which weighs 50 grms., and consists of calcium carbonate and traces of iron, manganese, and silicic acid. ${ }^{1}$

There is no doubt that it is possible for calcareous strata of great depth to be produced in this way in fresh water. That also in times past lacustrine deposits of lime have had a similar origin is inferred from the fact that the spore-fruits of stoneworts (Characeæ) and the nutlets of pond-weeds have been found over and over again inclosed in these formations of lime. Calcareous deposits originating in this manner are, at present at least, less frequent in the sea. Still, the Acetabulariæ undergo similar changes there, and may be the cause of an elevation of the sea bottom and of an accumulation of lime. On the other hand, in the sea, the Lithothamnia and Corallinas play a predominant part, and form-just like true corals, and often indeed in conjunction with these and other marine animalslime reefs of great magnitude.

The agency of plants may occasion accumulations of iron hydroxide, silicic acid, and salts of potassium and sodium at particular places besides lime. The formation of meadow iron-ore, spring iron-ore, and bog iron-ore, the construction of tripoli, agate, and flint, by the conglomeration of siliceous-coated Diatomacer, and the accumulation of potassium and sodium salts in the superficial strata of salt steppes are processes which take place essentially in the same manner as the piling up of carbonate of lime, although upon a more modest scale.

The question now arises, why it is that the substances which are stored in preponderant quantities in the vegetable frame, which are the main constituents of the living part of plants, and represent the alpha and omega of plant life, are not preserved as well as the mineral food-salts in question. Why do not carbon and nitrogen, materials so eagerly appropriated by the living plant, compounded by it with the elements of water, secured in some measure in organic compounds, and constituting the fundamental mass of the vegetable structure, remain behind in the same condition after the death of the plant? When autumn comes and the limeladen pond-weed dies, only the calcareous crust falls to the ground, and, at the bottom of the pond, enters upon a period of quiescence. The tissue of the plant

\footnotetext{
${ }^{1}$ In the case investigated 96 per cent calcium carbonate, 0.28 per cent iron oxide, 1.51 manganese oxide, and 1.51
} per cent silicic acid; the last, from the Diatomaceæ, settled on the calcareous crust. 
itself-all its carbohydrates and albuminoid compounds-cannot remain dormant, but are split up without delay into those simpler compounds of which they were compounded in the summer; and, by the following spring there is nothing more to be seen of any of the pond-weed's stems and leaves. Certainly this is only to such a conspicuous extent true of plants living under water; dead plants buried in earth or exposed to the atmosphere are resolved less rapidly, and under certain circumstances deposits of organic remains on limited areas are preserved even almost unaltered through boundless ages.

Let us try to obtain a somewhat closer knowledge of these various degrees of preservation. Thoroughly dried wood, leaves, and fruit, if protected from all but transient moisture, are capable of being preserved unaltered for long periods of time. When wood is exposed in a dry place to the sun, it turns brown, and in the course of years becomes quite black outside, the most superficial layers being regularly carbonized, as may be seen particularly well in the case of woodwork situated under the projecting roofs of old mountain chalets. This wood exhibits no sign of crumbling, mouldering, or rotting. In the dry chambers of old Egyptian graves fruits, foliage, and flowers have been found which were laid by the side of the dead 3000 years ago, and they had not undergone a greater change than if they had been dried but a few days. Even the colours of flowers of the Larkspur, the Safflower, and other plants of the kind, were still apparent, and the separate stamens in Poppy flowers were in a state of complete preservation. Dryness therefore may be looked upon par excellence as one of the preventives of the decomposition of organic matter.

The same result as is secured by dryness in the cases cited is brought about in the ground of moors by humous acids. The dead plants saturated with these acids are not resolved into carbonic acid, water and ammonia, but preserve their form and weight almost unaltered, and are converted into peat. Above the mass of peat new generations of plants continue to spring up and produce ever fresh organic matter, which, in its turn, becomes peat, and is added to the mass beneath, so that gradually a very deep bed of organic matter may be accumulated in this manner. In the low country lying between East Friesland and the Hümmling, from the river Hunte to the marshes on the Dollart, there is a stretch of nearly 3000 sq. kilometers covered with a layer of peat which has an average depth of 10 meters.

Of minor importance is the preservation of dead plants and parts of plants in snow and ice. The leaves, twigs, and seeds, which are carried by the wind on to the snow-fields of the high mountains, remain there a long time almost without alteration in respect of form or size; they only turn brown under the influence of the intense sunlight, and at last become quite black as though they were carbonized, which, in fact, they are. So also such insects as meet their death on the snow-fields are converted there into a black, cindery mass. Indeed, even all the minutest organic fragments lying on a glacier become carbonized, and this explains the fact that the so-called cryokone, or snow-dust, which we have already had occasion to allude to, has a graphitic appearance. 
Dead leaves, haulms, branches, and tree-trunks, when they rest upon damp ground, as also lifeless roots, rhizomes, bulbs, and tubers, buried in moist earth, pass into a state of putrefaction, provided that their temperature does not fall below freezing-point, that is to say, they are resolved into carbonic acid, water, and ammonia, the rapidity of the process varying directly as the supply of water and the degree of temperature to which the dead matter is exposed, and inversely as the quantity of compounds of humous acid present. If more dead fragments of plants accumulate within a particular interval of time on one spot than decay, a formation of vegetable mould takes place there; on the other hand, the ground remains destitute of humus when the entire accretion of organic matter is quickly decomposed as soon as it is dead. The general fact turns out to be that the decomposition of organic bodies is prevented, or at least limited, by a dry condition, and is promoted by moisture, and that it can only be prevented in moist surroundings by the presence of large quantities of humous acids, or by the temperature being low enough to turn water into ice.

This result directs attention to those inconceivably small animate beings, which, as has been proved by experience, are arrested in their activity by scarcity of water and are killed by the antiseptic substances referred to. That they are the cause of the resolution of dead plants is corroborated by the facts that they are always present where vegetable putrefaction is in progress, and that, on the other hand, decomposition can be prevented by rendering the access of these minute organisms impossible. First in importance in this respect of course are bacteria, the causal connection of which with processes of dissolution, and especially with those decompositions, which are known by the name of putrefaction, is established. Of these bacteria, Bacterium Termo, and several micrococci, bacilli, vibriones, and spirilla, are the commonest. Their multiplication and the withdrawal for this purpose of substances from dead plants cause a splitting up of the organic compounds in the latter. The albuminoid compounds are first of all peptonized; next, tyrosin, leucin, volatile fatty acids, ammonia, carbon-dioxide, sulphuretted hydrogen, and water are formed, this stage of the process being accompanied by the evolution of an offensive odour of decomposition, and later, nitrous and nitric acids are produced by further oxidation. The carbohydrates, too, chiefly cellulose and starch, are split up, and the products of this analysis, in so far as they are not used up by the bacteria for their growth and reproduction, pass in a gaseous condition into the atmosphere, or into the water surrounding the dead plants. Moreover, the bacteria themselves do not remain at the spots where they have been battening on vegetable matter, but swarm away through the water, or else come to rest in a short time, in which case if the seat of their activity dries up they are blown away by currents of air, and so conveyed to other dead plants. Similar decompositions can be induced by moulds (Eurotium, Mucor, Botrytis cinerea, Penicillium glaucum) as well as by bacteria, and, in addition, the disintegration of wood occasioned by the mycelium of Dry-rot (Merulius lacrymans), the green-rot of trunks of oaks, and beeches, caused by Peziza ceruginosa, the mouldering of wood induced by the mycelium of Polyporus 
sulfureus and various other fungi, the red-rot, \&c., all depend on similar disruptions of the organic compounds in dead plants, and result in the ultimate dispersal of these in the air in the form of carbon-dioxide, ammonia, nitric acid, and water.

Thus, ultimately, the exercise of this destructive activity only effects a return of the compounds just enumerated - the most important to plant-life-to the regions whence they had previously been withdrawn by the plants when living. Carbon and nitrogen, in particular, are set free from their bonds and given back to the atmosphere in the form and combination in which they are capable of being appropriated anew by living plants as food-material.

Considered from this point of view the phenomena of putrefaction and rotting appear as important and even necessary incidents in the history of the substances which are of the greatest importance to plants. Abhorrence of putrefaction is innate in us all, and everything connected with it_-in particular, the entire race of bacteria-is looked upon with aversion. To estimate these processes according to their deserts requires a sort of self-abnegation. But when we overcome our repugnance and weigh the whole subject impartially, we come to the conclusion that the continued existence of vegetable life and of life in general depends upon the occurrence of putrefaction. If the untold numbers of plants which die in the course of a year did not rot sooner or later, but remained unchanged as lifeless forms, a certain quantity of carbon and nitrogen would be idle, being withdrawn from the sphere of activity and locked up, so to speak. Now, assuming this to be repeated year by year, a time must come when all the carbon and nitrogen would be imprisoned in dead plants. Thereupon, all life would cease, and the whole earth would be one great bed of corpses.

Not only putrefaction, but also the minute organisms which excite putrefaction appear in a more favourable light when viewed from this standpoint. Let such bacteria as act in the capacity of foes to the human race, ravaging town and village in the form of infectious diseases, be exterminated if possible; but annihilation of putrefactive bacteria would mean a disastrous interference with the cycle of life upon the earth. These latter are not to be reckoned as enemies but friends to human beings. The effect of their invasion of dead plants and animals is certainly first made manifest, not in the most agreeable manner, for some of the substances mentioned as being evolved in the early stages of the onslaught, viz.: various ammoniacal compounds, sulphuretted hydrogen, and the volatile fatty acids, are disgusting to us; but as decomposition advances these phenomena, which are so unpleasant to our senses, abate, and the action of putrefactive bacteria becomes ultimately a beneficent process of purification of the last remnants of dead organisms. The final result of the decomposition of organic bodies by bacteria has been termed mineralization. It is a fact that nothing is ultimately left behind, in the ground or water, of bodies decomposed by the indefatigable exertions of bacteria excepting some nitric acid and the small quantity of mineral food-salts which has been taken up by the living organism in its time and are now in the form of dust and ash. 
By filling with water a glass which contains vegetable and animal remains in a state of putrefaction and swarming with bacteria, one is enabled to follow this process of mineralization from day to day. First, a decrease of the organic matter clouding the liquid, accompanied by simultaneous increase of ammonia and nitrous and nitric acids, is observed; then, after about two months, a complete clearing up of the liquid. The water is now colourless and odourless, but a precipitate has formed at the bottom, which contains, in addition to insoluble food-salts, bacteria in a state of temporary quiescence on the termination of their task and waiting till fresh prey becomes accessible. No doubt these processes occur in nature in just the same manner as in the glass of water, and the so-called self-purification of rivers, for example, has been rightly attributed to mineralization. It was long ago noticed that the water of such rivers as flow through great towns and consequently take up considerable quantities of animal and vegetable refuse contains no discoverable trace of all these impurities a few miles below the mouths of the drainage pipes and sewers. The water of the Elbe, which receives the refuse of the towns of Prague, Dresden, and Magdeburg, is so pure at Hamburg that it is there used for drinking purposes without protest ${ }^{1}$. The Seine, after taking up masses of rubbish in Paris, is already by the time it reaches Meulan, a distance of 70 kilometers, clear and pure again, and does not even exhibit there any traces of the organic matter received in the great city. Were it not for the activity of the putrefactive bacteria, this purification would never take place; and although the statement that putrefactive bacteria are the best of purifiers sounds at first like a paradox, it must be acknowledged to be consistent and based on experience.

\section{MECHANICAL CHANGES EFFECTED IN THE GROUND BY PLANTS.}

All the alterations hitherto spoken of as being brought about in earth and under the influence of vegetation subsisting therein are reducible to chemical transpositions. Added to these, there are always certain purely mechanical changes. The penetration of the rhizoids of a rock-moss or the hyphæ of a crustaceous lichen into limestone is accompanied, as has been already stated, by a solution of part of the substratum and a mechanical separation of another part; the rhizoids or hyphæ, as the case may be, becoming imbedded amongst tiny detached fragments of the underlying stone. When the hyphæ and rhizoids die, the corresponding piece of the substratum is left porous, and admits air and water, whilst other plants are enabled to settle on it, although they may not perhaps possess the power of eating into stone and pulverizing it in the same degree as their predecessors. This is also true of the roots of Phanerogams. The food-seeking root-tips and their absorptioncells displace particles of earth as they insinuate themselves, and when they decay later on, the soil at those particular places is intersected by passages of varying size. No doubt these passages mostly collapse like the abandoned shafts and galleries of a mine, but some trace of root-action always remains behind in the shape of an

1 This was written before the last outbreak of cholera. 
increased looseness of the soil in the locality, a result of the greatest importance, inasmuch as it enables air and water to permeate to a depth much more easily and quickly by the ways that the roots have previously opened up. Dead roots rotting underground constitute, moreover, the source of the carbonic and nitric acids which help to render available the mineral constituents, and so serve the turn of subsequent generations of plant-settlers on the same spots, whilst they accomplish fresh disintegration of the substance of the soil.

If, however, the subterranean parts of plants are continually engaged in mining, and so change in various ways the position of the component particles of soil, the organs above ground exert an influence in some measure opposed thereto, in that they retain and bring to rest particles of earth which are set in motion by currents of air or water. In the section that treats of the absorption of nutrient salts by lithophytes, attention was directed to the fact that the dust pervading the atmosphere, and blown from place to place by the wind, is arrested to a remarkable extent by mosses and lichens. One need only detach a small tuft of the common Barbula muralis, which everywhere occurs on walls by roadsides, to convince oneself of the extent to which dust from the road is lodged amongst the leaves and stemlets, and of the tenacity of its adhesion. Moreover, not only such dust as rises from roads, but also that variety which, though not easily observed, yet fills the air of remote mountain-valleys, of arctic ice-fields, and of the most elevated parts of the earth's crust, is arrested in those localities by mosses and liverworts, and by many Phanerogams besides, the growth of which is similar to that of mosses. There is not much less dust clinging amongst the stemlets of the dark Grimmias, Andreæas, and other rock-mosses, which grow in small cushion-like tufts on weather-beaten mountain crags, than is attached to the Barbula living by the dusty roadside. If one of the tufts in question is detached from its substratum, a fine powder composed of micascales, granules of quartz, chips of felspar, and a number of minute organic fragments pours out from between the moss-stems, whilst another portion of this finely powdered earth is left clinging to the leaves and stemlets, and is found to be regularly adnate to them.

It is never, however, the still fresh and living upper parts of these leafy mossstems that arrest and carry dust, but always the older dead parts below. The lower dead half of the moss, whether still in a state of preservation or already rotting, is alone capable (in consequence of characteristic alterations in the lifeless cell-tissue) of holding fast the atmospheric dust. The under part of moderate-sized cushions of moss constitutes a compact mass composed half of imprisoned dust and half of brown lifeless moss-stems. These little cushions, clothing rocky crags, become a favourable site for the germination of a whole host of seeds, which are conveyed thither by the wind and detained in the same manner as the dust. The seedlings arising from these seeds send their rootlets into the subjacent portion of the ked of moss, where the interstices are full of dust or finely-divided earth. Here they find all the conditions prevailing necessary for their nourishment, and they expand, and, little by little, crowd out the mosses which received them so hospitably, 
forming ultimately a bed of flowering plants, including in especial abundance representatives of the orders of grasses, pinks, and composites.

Many water-plants-in particular, aquatic mosses and algæ-possess, in an almost greater degree than lithophytes or land plants, the power of laying hold of inorganic particles, and thus exercise a far-reaching influence as mud-collectors on the conformation of the ground. It is wonderful how plants are able to arrest large quantities of the fine sand hurried along by a flood, although they are exposed to the violent rush of the water. The tufts of the dark green alga Lemanea fluviatilis and of the aquatic moss Cinclidotus riparius, which cling to rocks in the cascades of clear and rapid mountain torrents, are so conglomerated by mud and sand that they cannot be freed therefrom until the tissue has become dry and shrivelled. Limnobium molle, which grows in the turbid waters from glaciers, has such an abundance of earthy particles adhering to it that only the green tips of the leaf-bearing stems are visible above the grey-coloured cushions imbedded in the mud. The felted masses of Vaucheria clavata, filling the channels of apparently clear, gently-flowing streams, are so mixed with mud that if a lump of this alga is fished out, the weight of mud clinging to it exceeds that of the alga itself a hundredfold. In these cases of submerged plants, it is, again, not the living but the dead parts which serve to arrest the mud. On lifting up a lump one sees clearly that only the uppermost and youngest prolongations of the filaments-those situated at the periphery of the algal cushion as a whole-are living and filled with chlorophyll; the fundamental mass has become colourless and lifeless. But these dead parts, which form a thick felt of interwoven filaments, alone retain in their meshes the finely-divided mud and sand in such surprising quantities; these particles slip off the green living parts without adhering to them. An important consideration in this connection is the fact that the dead cell-membranes swell up and become slightly mucilaginous, so that fine particles of mud lodge more easily in the soft swollen substratum thus formed. Wooden stakes stripped of bark and fixed in a strong current show this very clearly, as do also the trunks of trees that are thrown up by floods and lie stranded on the shore with their bared boughs projecting into the stream. However strong the current to which wood in that condition is exposed, it covers itself in a short time with a grey coat consisting of earthy particles brought down by the water. If a piece is cut off and exposed to the air, the earthy deposit does not become detached until the wood-cells have dried up and shrunk. As long as they are moist the particles of mud continue to adhere to them.

This mechanical retention and storage of dust by rock-plants and of mud by aquatic plants is of the greatest importance in determining the development of the earth's covering of vegetation. The first settlers on the bare ground are crustaceous lichens, minute mosses and algæ. On the substratum prepared by them, larger lichens, mosses, and algæ are able to gain a footing. The dead filaments, stems, and leaves pertaining to this second generation arrest dust in the air and mud in the water, and thus prepare a soft bed for the germs of a third generation, which on rocks consists of grasses, composites, pinks, and other small herbs, and in water 
of pond-weeds, water-crowfoots, hornwort, and various plants of the kind. The second generation is produced in greater abundance than the first, and the third develops more luxuriantly than the second. The third may be followed by a fourth, fifth, and sixth. Each successive generation crushes out and supplants the one preceding it.

As on the rocky heights and in the roaring torrents of mountains, so also on the sandy plain and in the depths of the sea, a perpetual variation in the nature of the vegetation is taking place. At all times and in all places we see younger generations displacing the older and building upon the foundations laid by their predecessors. The first settlers have a hard fight with uncompromising elements to seize possession of the lifeless ground. Years go by before a second generation is enabled to develop in greater luxuriance upon the earth prepared by the first occupiers; but there is no cessation in the productive and regulative effects of vegetable life, and its energy and aptitude in the work result in the erection of its green edifices over wider and wider areas. New germs are established upon the mouldered dust of dead races, and others on the plant forms adapted to the altered substratum, and so, for hundreds and thousands of years, the changes go on, until at length the tops of forest-trees wave above a black and deep soil, the battle-field of a number of bygone generations. Thus, the life of plants, like that of the human race, has its epochs and its history: as in the one so in the other a continual struggle prevails; processes of ousting and of renovation are always in progress, and there are ever new arrivals upon and departures from the scene. 


\section{CONDUCTION OF FOOD.}

\section{MECHANICS OF THE MOVEMENT OF THE RAW FOOD-SAP.}

Capillarity and root-pressure.-Transpiration.

\section{CAPILLARITY AND ROOT-PRESSURE.}

Unicellular plants make use individually of the food material which they absorb from their surroundings, and work it up into the organic substances which they require for their structure and increase in bulk, and also for the production of future generations. In all plants composed, on the other hand, of aggregates of cells, there is a division of labour. Of the protoplasts occupying the cell-cavities of such larger plant-structures, one part provides for the absorption of the water and food-salts, another for the taking in of the gases which are used as food, and yet another part works up this food into organic substances for constructive purposes. The centres in which these various industries are carried on are frequently situated at some distance from one another, and it is obvious that there must not only be some communication between the various regions of activity, but that active forces must come into play which will effect the transport of the food from the cells whose function it is to receive it, to those in which it is to be elaborated into building material. It is evident that the greater the distance is between the various centres of the plant in question, the more difficult will be the performance of this task. In aquatic plants and lithophytes, all of whose superficial cells have the power of taking in nourishment from their environment, these distances are proportionately small, while they attain their greatest dimensions in land-plants whose roots are embedded in the earth, and whose leaves are surrounded by air. In trees the food materials which are taken up by the absorbing roots beneath the ground must frequently travel far more than 100 metres before reaching the topmost leaves. The path to the summit is very steep, and the fluid in rising must be able to overcome the force of gravitation, which has no inconsiderable significance at heights such as these.

Naturally, desire for knowledge has at all times directed attention to this phenomenon, and the most diverse attempts have been made to explain by what means the food-sap taken in by the roots of trees is enabled to reach their summits. It was first considered to be in virtue of capillarity; that just as oil, alcohol, or water, is drawn up the wick of a lamp, the liquid food can rise in the delicate tubular cell-formations called vessels, which, united together in groups or 
bundles, traverse the stems and leaves of plants. But the vessels are closed in above and below, and therefore it is impossible that capillarity should be sufficiently developed in them. At best it could only raise the sap a trifling distance, and could never convey fluid to a height of many metres. It is a striking fact that in many plants the ascent of the sap is most vigorous after the evaporation from the superficial parts exposed to the air has been weakest. The so-called "weeping" of vines, i.e. the outflow of sap from the flat surface of a cut vine-branch, does not take place in summer and autumn, immediately after the branch has been fully adorned with foliage, and when its extensive leaf-surfaces have given up large quantities of moisture to the surrounding air; it occurs at the end of the winter sleep of the plants, when the brown branches rising above the ground are still in a bare and leafless condition. The cause of the ascent, or at least of the ascent in the lower leafless branches, must therefore be sought for in the absorbent roots, and it may be assumed that here the same causes are at work which induce the fluid food materials of the surrounding earth to enter the superficial cells at the root-tips.

It has already been shown that the contents of these cells suck up the water of the nutritive ground with great force in consequence of the chemical affinity they have for it, or in other words, that the fluid reaches the interior of plant-cells by endosmosis; it has also been mentioned that in conscquence of the taking in of water the volume of the cell-contents increases, producing pressure from within outwards on the cell-wall, and the cell swells and becomes turgid. From this one of three cases might be deduced:- first, suppose that the cell-wall is so composed throughout that it allows the entrance of water into the cell, but not its exit, and that consequently the cell-contents absorb water, but that a filtration of the same towards the exterior cannot take place. Granted this hypothesis, the cell-wall by virtue of its elasticity would yield to the pressure of the cell-contents, but only within the limits of that elasticity; hence a condition of tension would be produced, in which the reciprocal pressures of the cell-wall and cell-contents would be in equilibrium. In the second case, suppose that the pressure of the cell-contents is greater than the force of cohesion between the molecules of the cell-wall, this consequently ruptures, and the cell-contents issue from the rent which is formed. This phenomenon is seen in certain pollen grains when placed in water. In half a second the cells absorb so much water that they double their volume; the cellcontents still absorb the fluid, and the cell-wall can at length no longer withstand the pressure; it bursts, and the contents, from which the pressure is now removed, pour through the opening, and are diffused in the surrounding water.

There is a third case possible. Suppose that in a given cell the opposite walls are not of identical structure; that the wall which is in contact with the damp earth is so organized as to allow the entrance of water, but not its filtration to the exterior, while the opposite wall offers only a slight resistance to such filtration; then by the increasing pressure of the cell-contents fluid will be forced through that wall which offers least resistance, and the greater the affinity of the cell- 
contents for the fluids in the nutritive earth, the more abundantly and energetically will this be carried on. The phenomenon can be well seen in some moulds, especially Mucor Mucedo, which makes its appearance in such quantity on succulent fruits; and in the mycelium of the so-called Dry-rot, Merulius lacrymans. Fluids are sucked up by the lower portions of the tubular cells which cover the nutritive substratum, and expelled again through the walls of upper parts of the same cells, which project freely into the air. These upper portions of the mycelium cells appear as though ornamented with tiny dewdrops, which in the case of the Dry-rot coalesce and attain to a considerable size. Damp woodwork in cellars, where this fungus has established itself, is often thickly besprinkled with the drops which have been excreted on the surface, and if a lamp is brought into the darkness, and the infected places illuminated, hundreds of these tiny drops sparkle and glitter like the "jewels" in a cave of stalactites. Suppose then that such a cell, one wall of which allows fluid to enter, is attached by the wall opposite to that through which the fluid enters, to another cell; then this second cell will absorb the liquid, and, if tubular, the sap may rise higher and higher in it, and by the pressure of the liquid continually arising from below, even be forced through other higher cells which are capable of filtration. Naturally the rising current of sap thus generated follows the line of the least resistance; if then the cell-tissue where this action terminates is perforated by canals ending in pores on the surface, the fluid will emerge from these pores in the form of drops. This actually happens not only in many large-leaved Aroids, but also in plants growing in the open country if the air which passes over the leafy parts above the ground be very humid, and the soil in which the roots are buried proportionately warm. In many plants with succulent foliage, drops of water may be seen issuing from the thinwalled cells and pores of the leaves when the almost saturated air becomes cooled after sunset, while the soil, round about the absorbent roots, having been exposed all the day to the sun's rays, retains its higher temperature. Young blades of corn have rows of such drops, which look exactly like dewdrops, and have often been mistaken for them. This extrusion of water from the leaves can easily be produced artificially by placing the plants in a saturated atmosphere, and at the same time slightly warming the earth round the roots. There is no doubt that the sap which exudes from the leaf-pores originates in the nutritive soil, and is taken up by the absorbent cells of the root; from these the vessels and cells of the main root and stem, through which the sap can filter, carry it up to the leaves. If, therefore, we cut across a stem a little distance above the ground, we shall see the sap, which has already accomplished half its journey, welling up as drops on the cut surface; i.e. we shall see the remarkable phenomenon called "weeping", of which mention has already been made. The quantity of sap which flows from such a cut surface is in many cases astoundingly great. In Java certain Cissus plants, belonging to the family of lianes and living in damp woods, are actually made use of as vegetable springs. The watery sap flows so abundantly from a cut branch that in a very short time it will fill a glass, and forms a cool and 
refreshing beverage. Many Araliaceæ also furnish a sap fit for drinking. Some native Indian genera which are used as vegetable wells have on this account received the name of "plant springs" (Phytocrene, e.g. P. gigantea and bracteata). If the young flower-stalk of Agave americana, an American plant which is cultivated in European gardens under the name of the "hundred years' aloe", be cut across, in twenty-four hours about 365 grammes, and in a week more than 2500 grammes of sap will flow out. This exudation continues for four to five months, and a vigorous Agave will produce in this time as much as 50 kilogrammes of sap, which will ferment, since it contains both sugar and albuminous substances, and is indeed used by the Americans in the preparation of an intoxicating drink called "pulque". The quantity of sap which exudes from vines is also very great. A branch $2 \frac{1}{2} \mathrm{~cm}$. thick, cut across $1 \frac{1}{2} \mathrm{~m}$. above the ground, produced within a week over 5 kil. of sap. In a week, from the cut stem of a rose, more than 1 kil. was exuded. From maples and birches a proportionately large amount of sap can be obtained, when the trunks are cut about a metre above the ground. The sap which flows from species of maple contains pure crystallizable sugar, and in some North American species this is present in such abundance that it was found to be worth while to collect the sap, at least in former times.

It should be noticed that the volume of the exuded sap is in all these cases greater than the volume of the root together with that of the stump of the stem from which the sap is forced out, and this is a proof that it does not consist only of the water which was contained in the root and stem stump at the time of cutting, but that there is a continual upward current of sap, and that the absorbent cells of the roots, for a long time after the operation, continue to draw up water from their environment.

An ingenious experiment was performed at the beginning of last century in order to ascertain the amount of pressure by means of which the sap is forced from the cut surface of the vine and other stems. A vine stem without branches and about the thickness of one's finger was cut across in the spring at a height of about $80 \mathrm{cms}$. above the ground, and on the root-stock was fixed a glass tube with a double bend, in such a way that one end fitted exactly over the cut surface of the stump, and the tube was then filled with mercury. By the pressure of the sap which welled from the cut surface the mercury was forced up the tube, and in a few days it actually reached a height of $856 \mathrm{~mm}$. The weight of a column of mercury $760 \mathrm{~mm}$. high is equal to that of a column of air as high as the atmosphere of the earth, or of a column of water about $10.3 \mathrm{~m}$. high, and consequently the pressure by which the sap is forced out of the vine is considerably greater than the weight of one atmosphere, or of a column of water of the height mentioned. From these data it has been estimated that the sap can be raised through $11.6 \mathrm{~m}$. by the pressure originating in the absorbent cells of the root. The pressure is naturally greatest in the lower portions of a stem, and gradually diminishes towards the higher regions; the ascending current of sap to which it gives rise is also not uniform, but shows daily, and even hourly, fluctuations. Moreover, the quantity of 
sap exuded, neglecting these said fluctuations, is greatest soon after the stem is cut. and then becomes gradually less until finally the outflow ceases entirely with the death of the stump.

The magnitude of the pressure, and the quantity of the sap forced up by the absorptive power of the cells, vary with the circumstances of the plants considered. The pressure appears to be greatest in species of vine, and in the vine stem, as already remarked, it will support the weight of a column of mercury $856 \mathrm{~mm}$. high. In the stem of the Foxglove it equals the pressure of a column of mercury $461 \mathrm{~mm}$. high; in the stem of the nettle the column is $354 \mathrm{~mm}$; in the poppy stem $212 \mathrm{~mm}$; in the stem of a bean $159 \mathrm{~mm}$; and in the trunk of the White Mulberry tree 12 $\mathrm{mm}$. high. In the majority of herbaceous plants this pressure is quite sufficient to drive the sap from the root-tips up to the leaves and top of the stem; but this is not the case with leafy trees and pines, with palms and creeping and climbing plants. Although watery fluid can be raised according to the above calculation to a height of $11.6 \mathrm{~m}$. by root-pressure, there is still a great distance between this level and the leaves of such trees and climbing plants, which may be as much as $160 \mathrm{~m}$. high; and the question which presents itself is this: By what means is the sap carried to the higher regions from this level to which it is raised by root-pressure?

It may be supposed that cells are present at the various heights in the stem to which the water is driven, which act in a manner similar to those of the root; i.e. cells which actively absorb, whose cell-wall on one side only slightly resists filtration, and which therefore are able to force up the sap a little higher. The results of the following experiments seem to support such a supposition. If a piece of a branch be cut from the middle portion of a tree, and the lower end be peeled and placed in water, sap will flow out from the upper cut surface with considerable force. The same thing occurs when a leafy branch is placed in water so that its leaves are submerged, while the upper cut piece of the branch projects a good way out of the water. In this case the cells of the leaves must function as the absorptive cells. However, even if, as is probable, parenchymatous cells are to be found at all levels of the plant stem behaving exactly like the absorptive cells of the root, this arrangement would scarcely suffice in all cases to carry the sap to its destination. Atmospheric pressure as well as the rarefaction of the air observed in the vessels of the stem during the summer have been made use of in explaining the upward current of the sap, and this rôle may actually belong to these factors; but all these mechanical powers are quite overshadowed by that one which has been termed by botanists "Transpiration".

\section{TRANSPIRATION.}

By transpiration of plants we mean the act of giving off aqueous vapour to the surrounding air-briefly and in plain terms, the perspiring of plants. Vapour escapes from the cells of the plant which are in contact with the air, the formation of these cells being specially adapted to the process of evaporation, just as it is given vor. I. 
off from moist inorganic bodies and exposed liquids. Of the materials which are held in solution in the sap of plants, only those which have the property of passing from the fluid to the gaseous condition, at the same temperature which transforms water into water-vapour, can evaporate with this fluid. All the others remain behind, and the natural consequence is that the sap in the transpiring cells becomes more concentrated. If water, which contains in solution extremely small quantities of sugar, organic acids, nitric, sulphuric and phosphoric acids, and salts of potassium, calcium, and iron, be set to evaporate slowly in a shallow dish, it will gradually come about that only a thin layer of fluid is left on the bottom of the dish; but this now is seen to consist of a very concentrated solution of the substances mentioned; i.e. of the sugar, organic acids, and the various salts. It has also all the properties of such a concentrated solution, i.e. it has the power of sucking in water in the liquid condition from its surroundings. In the same way the contents of a cell in contact with the air become more concentrated by evaporation, and thus obtain the power of abstracting water from the environment of the cell, that is to say, of sucking it up. If two adjacent cells contain sap of the same density, whilst only one of them has the power of exhaling water, the condition of equilibrium between them will be destroyed. However, the balance naturally tends to be restored, and the cell whose sap has become more concentrated by the evaporation of water, takes up watery fluid from the neighbouring cell. Now picture a chain of cells containing abundance of sap connected with one another by cell-walls through which fluid can filter, and let them be so arranged that only the uppermost member of the chain is in contact with the atmospheric air. The sap of this uppermost cell having become concentrated by evaporation will first of all exert a suction on the cell immediately below. As fluid is withdrawn from this second cell, its sap also undergoes concentration, and in consequence produces suction on the third cell, the third in like manner on the fourth, the fourth on the fifth, \&c., passing from above downwards. In this way innumerable compensating currents are set up between the adjoining cells, which, however, never lead to true equilibrium as long as evaporation continues in the cell in contact with the air, but combine together to form a single ascending stream.

Such a current actually exists in all living plants which evaporate from the portions above the ground and in contact with the air, while their lower extremities are embedded in a damp nutritious soil. This has been termed the Transpiration Current. Its source is the fluid which has been drawn from the earth by the absorptive cells and brought within the sphere of the living cells of the plant; we may retain for this fluid the old and very appropriate name "crude" or "raw sup". Its direction and destination are determined by the position of the evaporating cells, and its path is through the wood, which in tree-trunks is inserted as a huge layer between the bark and the pith; in lesser stems it passes through the bundles and strands of woody cells and vessels which traverse them, being connected, deep under the ground, by groups of parenchymatous cells, with the absorptive cells of the young rootlets, or with the hyphæ of the mycelial mantle, which replace the 


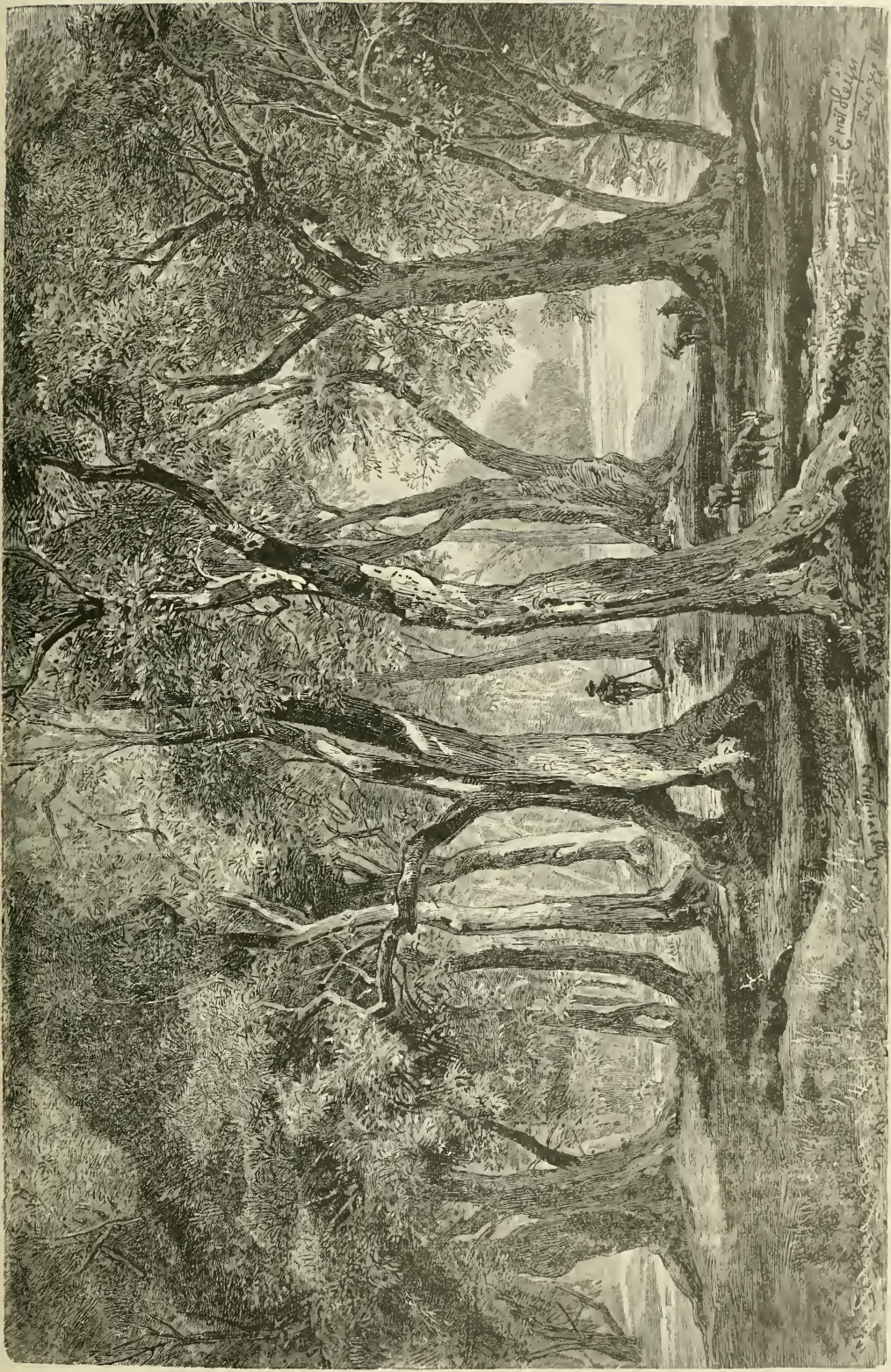

Fig. 60.-Olive Grove on the Shores of Lake Garda. 
absorptive cells (beech, \&c.). These bundles pass above into the leaves, forming there the "veins" of the leaf-blade, which spread out into an extremely fine network of tiny strands, and terminate quite close to the evaporating cells on the surface. That the wood actually forms the conducting tissue of the transpiration current is satisfactorily demonstrated by the existence of old trees whose trunks have long been hollow, whose pith is disintegrated and fallen away, and which have also been deprived of bark around their base. In the olive plantations at Lake Garda, one of which is reproduced in figure 60, many trees are to be seen in which the lower part of the trunk is not only hollow and without bark, but is also often tunnelled and split, so that the upper part of the tree looks as if it were raised on stilts. The only communication between the soil and the upper part of the tree is by means of these props, which are continuous with the roots below and are composed entirely of woody cells and vessels. And yet these olive-trees are still vigorous, putting out new branches and leaves every year, and blossoming and producing fruit; and they derive their necessary food from the ground by supplies which have no other upward path than the wood of these props.

Moreover, by repeated experiments it has been proved that the bundles of woody cells and vessels which are united together into a woody cylinder, inserted between the pith and the cortex in the trunks and stems of trees and shrubs, serve as conductors of the transpiration current. If a ring of cortex is removed from the stem of a leafy plant, whose leaves are transpiring in dry air, and are supplied with water from below by the transpiration current, this flow of sap to the leaves will not be interrupted, and the leaves remain firm and tense. But as soon as a piece of the wood is removed or the above-mentioned strands are cut through, even though the cortex be left entire, the flow to the leaves stops immediately, and they become flaccid and hang down in a withered condition.

The cellular formations of the wood and strands, which function as the conductors of the crude nutritive sap to the leaves, are-as already mentioned-woodcells and wood-vessels. Formerly the idea was held that these structures served for the passage of air, and it was believed that they were analogous to the respiratory organs-the so-called tracheæ-of insects; therefore these wood-vessels were also called "tracher", and the wood-cells "tracheides". The wood-cells are elongated chambers, on an average $1 \mathrm{~mm}$. long and 0.05-0.1 $\mathrm{mm}$. broad, and their walls are unequally thickened, either by reticulate or annular bands, or spiral threads projecting slightly from the inner wall into the lumen, or by so-called bordered pits, which are represented in fig. $10^{1}$ and fig. $10^{2}$. The wood-vessels are tubular, and very long in proportion to their width, which is never more than a fraction of a millimetre; they extend uninterruptedly through stalks, branches, leaves, perhaps even through the entire plant from the root-tip to the crown. They are composed of rows of cells whose separation walls have been broken down. The walls of the wood-vessels exhibit similar thickenings to those of the wood-cells or tracheides. When the chambers and tubes of the wood, with their bordered pits and projecting bands, are fully developed, the living protoplasm which carried on the building forsakes the 
scenes of its activity, and consequently in fully formed wood-cells and vessels living protoplasmic contents are wanting. They must be regarded in a certain sense as dead structures, for they have no further power of growth, and the reciprocal pressure of wall and contents observable in absorptive cells and other cell-cavities occupied by living protoplasm, which has been termed "turgescence", is never seen in them.

In the walls of the wood-cells as well as of the vessels, woody material (Lignin) is deposited. It appears to be in consequence of this that they are much less capable of swelling than are cell-walls which consist chiefly of cellulose. The amount of sap which presses its way in between the groups of molecules of the lignified walls, and with which these walls are saturated, is also comparatively very small. On the other hand, of course, this imbibed sap is conducted much more quickly through the lignified walls of the cell chambers and tubes than through nonlignified walls. More fluid is carried up by the intermolecular stream through the woody walls of the cells and vessels than by the ascension of the raw nutritive sap in the interior of the wood-cells and tubes. If no evaporation is going on from the leaves, or if this is only very slight, the vessels and cells become filled with sap. As soon as transpiration becomes active, part of the sap is taken up, and if fresh supplies do not arrive quickly enough a limited amount of air can get in temporarily, which of course must be in a very rarefied condition on account of the obstacles which oppose its entrance. The passage of the sap is quicker through the nonseptate vessels than through the much shorter woody cells. The sap on its way through the latter, to the transpiring leaves, must filter through innumerable transverse walls. This filtration will of course be materially helped by the bordered pits with which the wood-cells are so regularly provided; for the extremely delicate membrane which is stretched between the two cavities of such an apparatus at any rate allows the sap to pass through very easily. The bordered pits are exactly like clack-valves, and they also appear to regulate the sap-stream, though the way in which they do this is not yet completely understood. The nearer the path of the raw sap approaches to the spots in which evaporation is being carried on, the greater is the number of cells in the sap-conducting strands, while the vessels in the same become fewer and fewer. The termination of the whole sap-conducting apparatus consists entirely of cells whose walls are stiffened by spiral bands on the inside. Between this termination and the transpiring cells some parenchymatous cells with living protoplasmic cell-contents are interposed, whereas, it must again be insisted, the tubes and chambers composing the sap-conducting apparatus have no living protoplasm in their interior.

The whole mechanism for the transmission of the raw nutritive sap may be considered as a system of tubes and chambers provided with clack-valves, into which the fluid taken up by the absorbent root-cells is forced, and through which it is conducted to the transpiring cells of the green leaves or of the green cortex, which takes the place of the green leaves in leafless branches. This does not exclude the activity of cells at certain levels, as it were at intermediate stages of the road traversed by 
the current, which have the power of invigorating the stream, of hastening it if necessary, and also of lessening it under certain circumstances. Also it is arranged that in case of need fluid nourishment in the higher regions of the stem may reach the leaves by side paths.

The cells which by means of the exhalation of aqueous vapour into the atmosphere originate the transpiration-current are, as already mentioned, not far from the terminations of the sap-conducting apparatus. In some mosses they are freely exposed to the air. In the Polytrichaceæ and several other mosses (Barbula aloides, ambigua, rigida) they form short chains of cells like strings of pearls, or bands projecting from the grooved concave upper surface of the tiny leaves (see fig. $61^{2}$ ). Again, among the liverworts are forms, e.g. Marchantia polymorpha, which contain large characteristic air-chambers in the body of their green leaf-like thallus (fig. $61^{1}$ ). On the floor of this chamber are green cells which are so grouped together

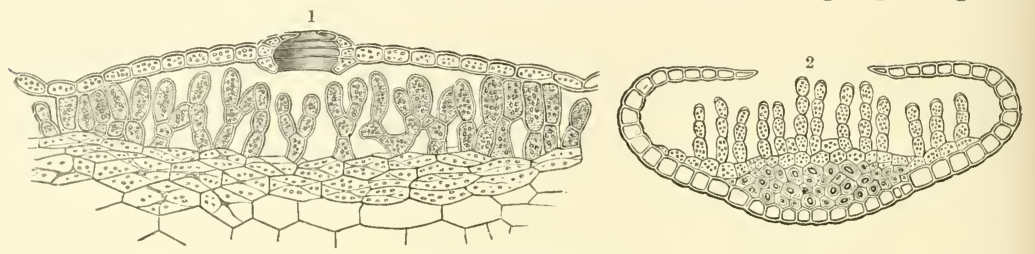

Fig. 61.-Transpiring Cells.

1 Vertical section through an air-chamber of the Liverwort Marchantia polymorpha; $\times 300.2$ Vertical section through a leaf of the Moss Barbula aloides; $\times 380$.

as to remind one of the shape of the Prickly Pear (Opuntia). These green cells are thin-walled, and it is from them that water is evaporated. They are not quite freely exposed, like those of the mosses mentioned above, since the roof of the chamber, composed of transparent cells, is extended over them; a chimney-shaped passage, however, is left open through the roof of each chamber by which the watervapour given off from the opuntia-like cells can escape. These Marchantias furnish a transitional form between the freely exposed transpiring cells on the upper surface of the leaf of the moss and those of flowering plants. In flowering plants the transpiring cells are situated as a rule in the interior of the green leaves, and also in the green cortex of leafless branches, forming a part of that green tissue which has been termed chlorenchyma, or when in the leaves, mesophyll.

Leaves may be described as consisting of cells filled with leaf-green, or chlorophyll, placed closely together and joined into layers above one another so as to form a soft mass of tissue containing abundance of sap; this green tissue pierced by the branched water-conducting strands whose ultimate divisions terminate in the tissue mass; the whole surrounded and shut in by a firm cuticle which is perforated in many places by stomata. Cellular passages are also regularly arranged for the purpose of conducting away the organic materials manufactured in the green cells, whilst groups of cells for the support of the whole, serving as beams, strengthening props, and the like, are placed at definite points. 
In most thin membraneous leaves the upper and under sides are differently constructed, and the difference is not confined only to the cuticle, but is also plainly recognizable in the green tissue. The green cells below the epidermis on the upper side of the leaf have the form of prisms, cylinders, or short tubes, and are arranged very regularly in ranks and files. In the leaves of plants belonging to the lily tribe, they lie with their long axes parallel to the surface; but in most other plants these cylindrical cells have their smaller side directed to the surface, and stand side by side like palisades, with only very narrow air-passages between them. Bclow these palisade-cells, and bordering on the epidermis of the under side of the leaf, is another stratum of cells of a much looser texture (see fig. $62^{1}$ ). The cells of this under layer are not so crammed with chlorophyll, and therefore appear a lighter
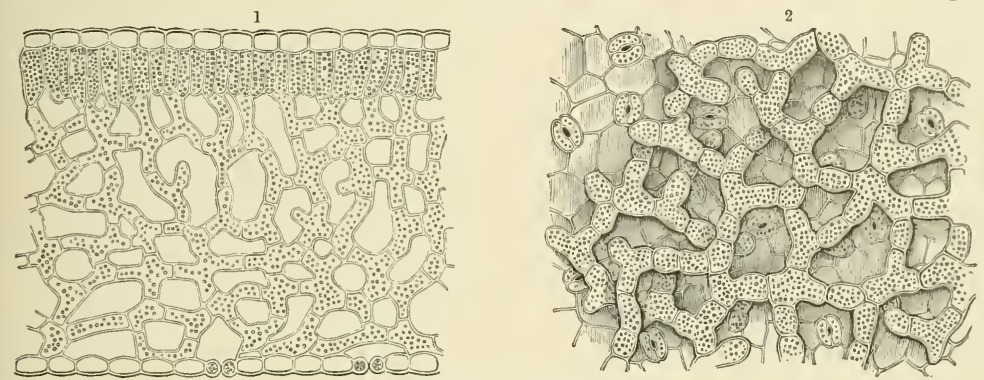

Fig. 62.-Spongy Tissue.

1 Vertical section through leaf of Franciscea eximia. 2 Spongy tissue in leaf of Daphne Laureola.-The epidermis and palisade cells of the upper side of the leaf are removed. The epidermis of the under side of the leaf, with its stomata, can be seen through the spaces in the spongy tissue; $\times 320$.

green than the palisade-cells. In shape they are elliptical, rounded, angular, sinuous, or generally very irregular; usually they possess protuberances which project in various directions, and they are so arranged that the outgrowths of adjoining cells come into contact with one another. It looks as if the neighbouring cells were stretching out their arms and extending their hands to one another, and consequently these cells have been called "many-armed cells". When several adjoining stellate cells are connected together in the manner just described so as to form a tissue, lacunæ and passages are seen in the tissue, which are broken through by the joined arms of neighbouring cells as if by pillars, couplings, and bridges. The whole tissue has the loose perforated appearance of a bath sponge, and is called accordingly spongy tissue, or spongy parenchyma (see fig. $62^{2}$ ).

This spongy tissue is the proper place for transpiration. Nowhere else in the plant are the conditions governing this process so well fulfilled as just here. The surfaces of the cells are rendered large in proportion to their size by their outgrowths; and they impinge as far as possible on the larger or smaller lacunæ, gaps, and passages filled with air, which all communicate with one another, thus constituting an unmistakable ventilating system.

Since the spongy parenchyma in the leaves described does not lie freely exposed, 
but is shut off from the atmosphere by a firm cuticle through which water-vapour can only penetrate with great difficulty, the aqueous vapour which is exhaled by the branched and other cells of this parenchyma would saturate the lacunæ, and further evaporation would be thereby prevented. There must, therefore, be a direct communication with the outer air surrounding the leaf; the epidermis of the leaf must possess apertures through which the water-vapour can escape. The already repeatedly mentioned stomata are to be looked upon as such apertures.

Stomata arise in this way; in a particular epidermal cell a partition wall first of all divides it into two cells. This cell-wall splits, and the cleft widens, forming a short canal which pierces the epidermis, and constitutes a connection between the outer air and the air-containing lacunæ in the interior of the leaf. This short canal is called the pore of the stoma, and the two cells which border it are termed guard cells. These two cells regulate the outrush of aqueous vapour, i.e. of that vapour which has been excreted by the thin-walled cells of the spongy parenchyma, and passed into the adjoining passages in the interior of the leaf. That cavity which is placed immediately beneath the narrow, short canal of the stoma, and is connected by passages with other spaces further within the green tissue of the leaf, is termed the respiratory cavity.

The number of the stomata or transpiration-pores which pierce the epidermis of the leaf varies very considerably. In the leaves of cabbages (Brassica oleracea) on 1 sq. mm. of the upper surface there are nearly 400, and on the under side over 700 . In the leaves of the olive-tree, on the same extent of surface of the under side, over 600. Succulent plants have remarkably few stomata. On 1 sq. mm. of the leaves of the House-leek (Sempervivum tectorum) and of the yellow Stone-crop (Sedum acre) only 10-20 are to be met with. In the majority of cases, on a similar extent of surface, between 200 and 300 stomata are to be found. The under side of an oak leaf, 50 sq. cms. in area, showed over two million stomata. They are in most cases scattered fairly uniformly over the surface of the leaf; on the leaves of grasses and pines, as well as on the green stalks of the horsetails, they form straight regular rows which run longitudinally; on the leaves of some species of saxifrage Saxifraga sarmentosa, japonica, \&c.) they appear crowded together in small solated groups; and on the leaves of the Begonia they are generally to be seen side by side in pairs. Obviously they are principally developed just where the epidermis overlies spongy parenchyma, and as in the majority of cases this parenchyma is situated towards the under side of the leaf, the greater number of stomata are to be found on this side.

In most flat membraneous leaves, which have one side directed towards the sky and one towards the earth, stomata are entirely wanting on the upper surface, being restricted to the under side. An exception to this is afforded by the orbicular flat leaves which float on the surface of water, e.g. those of the floating Pond-weed (Potamogeton natans), of the Frogbit (Hydrocharis morsus-rance), and of the water-lilies (Nymphaea, Nuphar, Victoria). These are covered with stomata on the upper side, while on the lower side, which is in contact with the water, stomata are 
entirely absent. On the upright leaves of flags, asphodels, amaryllis, and various other bulbous plants, and on the vertical leaf-like structures (phyllodes) of the Australian acacias, as well as on some of the needle-like leaves of conifers, the stomata occur on both sides in almost equal number. In the mimosas and various other plants, having, in common with the mimosas, the characteristic faculty of altering the position of their leaflets when stimulated externally, numerous stomata are found on both sides of the leaf.

Most stomata are elliptical when open; rarely circular or linear. The length of stomates varies between 0.02 and $0.08 \mathrm{~mm}$, the breadth between 0.01 and $0.08 \mathrm{~mm}$. Pines, orchids, lilies, and grasses have the largest stomata; water-lilies, olives, and some fig-trees, the smallest.

The stomata in the epidermis, the passages and cavities below them into which the thin-walled cells of the green tissue evaporate water, and the strands through which the sap is conducted from the roots to the green tissue, all work in connection with one another like the various parts of a machine. Each portion of the mechanism helps and depends upon the others, the immediate result of the common work being always the elevation of that nutritive fluid which is brought by the absorptive roots into the plant. In the main, therefore, the result obtained by transpiration is the same as that which root-pressure aims at, and it might be thought (taking for granted the truth of the above statement) that either rootpressure or transpiration is superfluous. Or perhaps transpiration and root-pressure work in a complementary manner together. Perhaps the conditions between the two forces are so arranged that the fluid taken in by the absorptive cells from the nutritive soil is forced up to a certain level by root-pressure, and from thence is promoted to still higher levels by means of transpiration? This would suggest a comparison with the raising of water from a spring situated in a valley-basin surrounded and shut in by mountains. In the depth of the basin exists underground water which is fed by the subterranean supply coming from the mountains. According to the pressure of this supply, the water in the lower earth-strata of the basin rises to a certain height. This pressure is not strong enough, however, to drive the water to the surface of the basin, and in order that it may reach this, it is necessary to employ a pump, which will reach down to that stratum of earth which is saturated by the underground water. But the level of this water differs in summer and winter. It depends also upon the amount of rainfall on the neighbouring mountains, which may undergo great fluctuations. In some years the underground water in the spring has almost risen to the upper opening; at other times only the deepest strata of the valley-basin contain water. The pump, by which the water has to be raised, must be constructed with all these possibilities in view, and must be so regulated that the absorbent action is felt as far down as the deepest position which the underground water is known to take.

Transpiration behaves in like manner in the portions of a plant above ground, and its action on the fluid food taken in by the roots may be compared with that of a suction-pump. It would be a quite inadequate arrangement if the sucking action 
produced by transpiration could only reach down to the highest level attained by the water which has been forced up by root-pressure, and precautions must be taken that, in case of the abatement of the root-pressure, water would be raised from the lower positions up to the transpiring cells, and that under certain conditions the action of transpiration should reach even to the absorbent cells at the root-tips. It has been shown by experiments that plants with large leaves lose in the summer more water by transpiration than is forced up into the stem by rootpressure, and yet the leaves do not become faded. The conclusion drawn from this is that at certain times the effect of transpiration makes itself felt down from the leaves through the stem as far as the root-tips. It has also been shown that in many plants, just when the most active evaporation is taking place in the leaves, none, or only very little sap is forced into the stem by root-pressure. If the stem of a vine be cut across in the height of summer, when the green leaves have been unfolded some time and are transpiring actively, no "tears" are seen on the cut surface of the stump, no drops are pressed out. The vessels contain rarefied air but no sap, and water can be sucked through the stump by the vessels even in the direction of the root.

Let us pause here in order to get a clear idea of the relations between transpiration and root-pressure. Given the conditions for an abundant evaporation from the aërial portions of a plant-i.e. a fairly dry air, water, and an appropriate development of transpiring surface-then the action of root-pressure is diminished, while that of transpiration is increased, and governs the whole of the movement of the sap. If, on the other hand, the conditions for evaporation from the aërial portions of the plant are unfavourable-if the air is very damp, or if the branches of the plant are not yet in leaf-then root-pressure comes into play, and, supported by cells with absorbent contents which occur in the higher regions of the plant, can force up the sap to the tops of trees and to the highest shoots of vine-branches which remain leafless all the winter. So far, therefore, root-pressure can supersede and replace transpiration, a fact of great importance in places where the air is sometimes very damp, and in countries where the trees and lianes shed their leaves in autumn; at the commencement of the next period of vegetation they have not yet put out their new foliage, and therefore do not possess a sufficiently large transpiring surface. It is very probable that in the autumn, when preparing for the winter, certain cells in trees and lianes provide themselves with materials by means of which in the coming spring they may exercise a very strong sucking action. This would also partly explain how it comes about that in the spring there is such a strong upward current of sap in the still leafless trees and vine branches, and that the water is conducted up even to the topmost shoots of lianes 100 metres long, which have shed all their leaves in the previous autumn.

A perfect substitute for transpiration in the form of the pressure produced by the absorbent cells is seen in moulds, in the already-mentioned dry-rot fungus, and generally in leafless cryptogams: possibly also in those orchids possessing neither green leaves nor stomata, and in other humus plants (saprophytes) such as the 
Monotropa, mentioned earlier on, which stands in such a peculiar relation to the mycelium of fungi. On the other hand, in most green flowering plants which bear leaves, a complete replacement of transpiration, continuing for a long time, is not an advantage. Experience has shown that green leafy plants, when kept for a long while in an atmosphere saturated with vapour, cease to grow and become unhealthy; they lose their leaves, and at length succumb altogether. This happens even if the amount of light, the temperature of the atmosphere and of the earth, the composition and humidity of the soil, in short, if all the other conditions of life are the most favourable that can be imagined for the plants in question. It follows from this that it is not immaterial to leafy plants how the sap reaches the leaves, whether it is drawn up by transpiration, or forced up by root-pressure. If the leaf transpires, water, in the form of vapour only, is given off to the atmosphere; all the materials which have been brought in solution from below to the leaves remain behind in the cells of the leaf. If, on the other hand, fluid water is pressed from the pores of the leaves by root-pressure, salts, sugar, and other compounds are always to be found in the exuded drops, having passed through the cell-wall in solution in the water. When it is a question of secreting sugar as a means of alluring insects, or salts for a protective covering, such an exudation cannot advantageously be given up, but is on the contrary a fundamental part of the economy of the whole plant. If this is not the case, and if materials which have a part to perform in the leaf by the formation of organic substances are exuded with the drops of water, and the drops falling from the surface of the plant trickle to the ground, there is loss of material, which does not contribute to the advantage, but rather to the detriment, of the organism.

The signification of transpiration may be explained in this way. By transpiration not only is water brought from below to the more highly situated parts of the plant, but nutritive salts in solution are also conducted to the green tissue of those branches and leaves which are exposed to light and air. The greater part of the ascended water is only used as a medium for the transmission of mineral salts, which have been taken from the soil into the plant. When it has reached the leaves, most of the water evaporates into the atmosphere, while the salts conducted by it into the green tissue remain behind, in order to take part in the chemical changes by which organic compounds are manufactured out of the raw materials. These salts are indispensable here, and transpiration is therefore also necessary in a corresponding degree. Without transpiration, it would be impossible that plants, whose green branches and leaves are surrounded by air, or that trees, which rank before all other plants on account of their superior size, could be properly nourished; consequently transpiration must be regarded as one of the most important lifeprocesses of terrestrial plants. 


\section{REGULATION OF TRANSPIRATION.}

Means of accelerating transpiration.-Maintenance of a free passage for aqueous vapour.

\section{MEANS OF ACCELERATING TRANSPIRATION.}

Aquatic plants do not transpire; therefore they do not require either vascular bundles or stomata. Neither trees nor shrubs grow under water, and even the largest Florideæ and the most gigantic sea-wracks have no wood nor stomata. These structures are on the other hand very important for land plants, and in these they are developed in extraordinary variety. When one considers how much the humidity and temperature of the air, those very conditions which influence the transpiration of plants, are continually changing, this diversity is not really surprising. What endless series of gradations there are between the damp air of a tropical estuary, and the arid wastes in the interior of large continents! What varieties of temperature in the different zones and regions of the earth, and in the changing seasons; what differences, even in a narrow space in a single small valley, between the conditions of moisture of the air and ground in the depths of a shady glen, and on the sunny, rocky slopes! In the one place the air is so saturated with water-vapour that even evaporation cannot take place from exposed pieces of water, much less then from plants; in the other it is so dry and the sun is so strong that plants can hardly suck up enough from the ground to compensate for the water evaporated from their surface. In the former case contrivances must be devised which will promote transpiration as much as possible; in the latter, however, it is important that too much evaporation, which would cause the drying up and death of the plant, should be prevented.

One of the most important ways of increasing transpiration consists in the development of many cells whose surface is in contact to the greatest possible extent with the atmospheric air, and which are so organized that water in the form of vapour can be exhaled from them. Further, it is of importance that the access of air to these cells is not rendered difficult, and that as great a portion as possible of these cell-groups, which help in transpiration, are reached by the rays of the sun. It is only in the delicate-leaved mosses, which have no stomata, that the whole of the cells of a leaf, in contact with the air, give off unlimited water, in the form of vapour, directly to the atmosphere. In plants possessing leaves provided with stomata, the outer walls of the epidermal cells, which are directly in contact with the air, are almost always rather thicker than the inner and side walls; moreover, the outer wall is overlaid by the already repeatedly mentioned covering, termed "cuticle", through which water-vapour can pass only with difficulty. In tropical ferns, especially in the tree-ferns, which grow in narrow wind-sheltered ravines, traversed by streams of water, and which spread out their frouds in the still, damp, warm air, the outer walls are so thin and delicate, and are covered by a cuticle of 
such tenuity, that if the humidity of the air sinks only a few degrees below saturation point, or if a transient sunbeam enters the ravine even for a short time, they immediately give off water-vapour.

Apart from such cases, the exhalation of water-vapour from the superficial cells is scarcely worth noticing; it is almost entirely restricted to the cells of the spongy parenchyma. Here are to be found, indeed, very striking arrangements, which must be regarded as contrivances for increasing transpiration. First of all, where transpiration is to be accelerated, the green, spongy tissue is very strongly developed, the air-containing lacunæ and passages, which penetrate the net-work of branched cells like a maze, are enlarged and numerous, and the collective free surface of all the air-bordered cells in the interior of the leaf has a much greater extent than the mere outer surface of the epidermis. In the leaves of many tropical plants which are always surrounded by damp warm air, e.g. in those of the Brazilian Franciscea eximia, of which a section is represented in fig. $62^{1}$, almost the entire thickness is made up of loose wide-meshed spongy parenchyma, and it is evident that water will be exhaled from the cells of this tissue as soon as the temperature of the leaf is raised even to the extent of a few degrees above that of the moist surrounding air by the sunbeams falling upon it.

In many such plants which urgently require a help to transpiration on account. of their situation, the cavities of the spongy parenchyma are extraordinarily enlarged and widened at certain points where the greatest number of stomata are developecl. The difference in appearance between such places and other parts of the leaf having dense spongy parenchyma can indeed be recognized by the unaided vision. In such a leaf looked at from above, the large-meshed portions of the spongy parenchyma. appear as lighter spots in the dark-green grounding; the leaf is flecked and marked with white. This is not only the case with many plants of damp, tropical forests, but also in those of temperate zonos, such as species of the genus Cyclamen, Galeobdolon luteum, the Lungwort (Pulmonaria officinalis), and frequently also in Hepatica triloba, if they grow in very shady places on the damp ground of a forest. It must, of course, not be forgotten that all the white spots and markings of green leaves, which have been named collectively "variegations", are not due to this cause. In those nettle-like plants, known as Bochmerias, the white spots on the central part of the leaf lamina are caused by peculiar groups of crystals in the epidermal cells, the so-called cystoliths, which reflect the light; in some Piperaccs they are due to groups of epidermal cells which are filled with air, and below which the palisade cells are absent; in other plants, again, they may be caused by the formation of aqueous tissue, a structure which will be discussed later. In many of those plants with variegated leaves, which are so extensively cultivated for purposes of decoration, the variegation is not normal, but must be considered as pathological, and is in no way connected with transpiration.

Since, as we know by experience, transpiration of green leaves is increased by light and warmth, it is evidently an advantage for all those plants to which only a restricted number of sunbeams can obtain access, if their leaf-blades are very large 
and have such a form and position that the small supply of light can be utilized to the full. The resultant action is just the same whether 1000 green cells are only moderately illuminated, or if 500 cells are illuminated by a light twice as strong. If this argument will not apply to all plants, it certainly fully applies to some, and it is a fact that plants growing in damp, shady places are characterized by their comparatively large, thin, delicate leaves. These leaves are also spread out horizontally in such localities; they are smooth and not wrinkled; neither rolled back nor bent up. Suppose we enter a thick wood in the north temperate zone, perhaps in S. Germany. By the side of delicate-leaved ferns grow species of Corydalis (Corydalis fabacea, solida, cava), together with species of Dentaria (D. bulbifera, digitata, enneaphyllos), dog's mercury (Mercurialis perennis), Isopyrum thalictroides, bitter vetch (Orobus vernus), woodruff (Asperula odorata), Lunaria rediviva, herb Paris (Paris quadrifolia), cuckoo-pint (Arum maculatum), spurge-laurel (Daphne Mezereum), and many other species belonging to very different families, but all having the common characteristic of possessing flattened leaves and no covering of hairs. If a brook ripples through the shady wood, growing on its banks will be found the yellow balsam (Impatiens nolitangere), the broad-leaved garlic (Allium ursinum), Streptopus amplexifolius, and the butter-burr (Petasites officinalis), with its huge foliage, all again characterized by their large, smooth, flat leaves. In such places in S. Germany are generally to be found the largest leaves. Those of the butter-burr attain to a length of over a metre, and are almost a metre broad. The fronds of the common bracken-fern (Pteris aquilina) are equally large in such situations; and on the ground in damp, shady alder woods, growing in comparatively cold mountain glens, another fern (Polypodium alpestre) is to be met with, whose frond is $1 \frac{1}{2}$ metres long. But they only possess these extended leaves when growing in the situations described, in the damp air of cool and shady woods. One would expect that under similar conditions outside the wood, the leaves would exhibit a more luxuriant growth, and would attain to a still larger size in consequence of the influence of a higher temperature; but this is not the case. In the drier air and sunshine on the unshaded banks of a rivulet, the leaves of the butter-burr are scarcely half as large as those growing in the neighbouring cold shady glen, from whose dim light the brooklet flows out into the open country; and on sunny ground neither of the two above-named ferns will even approximately reach that size to which they grow when surrounded by the cold, damp air in the depth of the alder wood.

This difference in the relative size of the leaves of one and the same species, according as to whether they grow in sunny places in dry air, or in shady spots in damp air, is sometimes carried so far that the whole physiognomy of the plants becomes altered, and they might easily be thought to belong to distinct species. Thus plants of Convallaria Polygonatum, growing in shady meadows watered by rivulets, show leaves at least three times as large as those which grow on the rich damp earth on the steep sides of rocks down which water rushes, where they are warmed by the sun all the day. This comparison might be illustrated by 
numerous other plants of the flora of Central Europe, which are sometimes to be found in damp, shady woods, sometimes in sunny fields; but the above examples will suffice to demonstrate the fact that in shady places and dainp air, in spite of the smaller amount of heat, and even when the humidity of the soil is less, the leaves will, notwithstanding, have a greater size than in sunny places where they are surrounded by a drier air.

An apparent exception is to be found only when these plants are situated above the tree-line in Alpine regions. On the sunny slopes of Monte Baldo, in Venetia, far above the wood-line, Corydalis fabacea grows with the same luxuriance as in the shady forests of the lower hilly regions; and on one place on the Solstein chain, in the Tyrol, at a height of 1800 metres above the sea, dog's mercury and Galeobdolon luteum, species of valerian, spurge-laurel, and ferns can be seen rising above the boulders with leaves as large as those growing in the shade of the woods below. But this exception, as stated, is only an apparent one. Where these plants flourisn on Alpine heights flooded with light, the air is just as damp as in the depth of the woods 1000 metres lower in the valley. For weeks the mist sways like drapery around the heights, and the air, consequently, is certainly not drier than in the woods down in the valley. Indeed, the fact that plants, which one is accustomed to see inhabiting the shady woods in the depth of the valley, grow in Alpine regions on unshaded places with leaves of the same size and shape as before, is a proof that the large size of their leaves in the dark woods of these lower places is not due to the absence of light, but to the very moist condition of the air which prevails there. Plants, whether in the shade of the forests, or on the illuminated heights of the mountains, endeavour to compensate for the detrimental influence of the greater humidity of the air by the formation of an extensive transpiring surface.

So far the increase of leaf surface may be considered absolutely as a means of helping transpiration. This method of increasing transpiration comes into action in the tropics in a much more striking way than even in the temperate zones. Especially in the most characteristic plant-structures of the tropics may it be observed how intimately the size of the leaves corresponds to the conditions of moisture of the air, and how it is that palms develop the largest leaves just in those districts where, in consequence of the air being saturated with aqueous vapour, plants can only transpire with difficulty. In the dampest parts of Ceylon grows the gigantic Corypha umbraculifera. A copy of a drawing of this tree, sketched on the spot by Ransonnet, is given in fig. 63. It towers above the tops of all other plants, and its leaf-blades are from 7 to 8 metres long, and 5 to 6 metres broad. In similar situations in Brazil the palm Raphia toedigera spreads out its fronds like gigantic feathers. The petiole of this leaf alone is 4 to 5 metres long, and the green feather-like blade is from 19 to 22 metres long and 12 metres broad-the greatest extent which has hitherto been observed in any leaf. Other palms besides these giants, whose fronds wave all the year round in a damp atmosphere, are but little inferior to them. Under one leaf of the Talipot ten persons can easily find room and shelter, and if the pinnate leaves of the Sago-palm be imagined 
propped up against the houses in the streets of our towns, their tops would reach to the second story, and it would be possible to climb up to the windows by them as if by the rungs of a ladder. Many of these palm leaves if placed in an upright position would be equal in height to our forest trees. In all these leaves the epidermis is only slightly thickened, the spongy parenchyma is well developed, stomata are present in large numbers, and the surfaces of the leaves are so directed towards the incident sunbeams that they are abundantly illumined and warmed throughout. The leaves become decidedly heated by the sun's rays, and thus, even in the saturated air of the tropics, the necessary amount of transpiration becomes possible. Arrangements similar to those of the palms may be observed in the Aroids and Bananas. These also develop their most extended leaves in the saturated or almost saturated atmosphere on the banks of still or flowing water, and in the moist heavy air of tropical primeval forests.

It is obvious that means of increasing transpiration are required in those waterplants whose roots are in the wet mud at the bottom of lakes and ponds, whose stems and leaf-stalks are directly surrounded by water, and whose leaf-blades float on the surface of the water, as for example the water-lilies (Nymphaca, Victoria), the Frogbit (Hydrocharis morsus-rance), and the Nymphæa-like Villarsia (V. nymphoides). The blade of the leaf is disc-shaped in all these plants, and the discs lie side by side flat on the surface of the water. Frequently large areas of lakes and ponds are covered with the floating leaves of these plants. The whole of the upper side of such a leaf can receive the rays of the sun, and the leaf is thus warmed and illuminated throughout. The under side of the leaf is coloured violet by a pigment called anthocyanin, which we will consider more in detail later, and of which it need only be mentioned now that it changes light into heat, and thereby materially helps to warm the leaves.

The aqueous vapour which is in consequence developed cannot escape below from the large air-spaces which permeate the leaf, because the under side, which floats on and is wetted by the water, possesses no stomata. The upper side is so richly furnished with stomata that on $1 \mathrm{sq} . \mathrm{mm} .460$ are to be seen, and on a single water-lily leaf about $2 \frac{1}{2} \mathrm{sq}$. dms. in area, about $11 \frac{1}{2}$ millions. This upper side alone provides a means of exit, and it is therefore important that the passage should not be obstructed at the time of transpiration. If the rain should fall unrestrainedly on the upper side of the floating leaves, the collected rain-water might remain there for a long time, even while the sunbeams breaking through the clouds after the shower are warming the floating leaves and inciting them to transpire. In order to avoid this an arrangement is made by which it is rendered an impossibility to wet the upper side of the floating leaves. The falling rain is formed into round drops on reaching them, and does not spread over the leaf-surface so as to wet it. But in order that the drops should not remain long on the leaves in many of these forms, such as in the wideiy distributed water-lily (Nymphoca alba), the leaf, where it joins the stalk, is somewbat raised, and the edges are bent a little up and down in an undulating manner. This gives rise to very shallow depressions 
round the edge of the disc, on account of which the drops of water roll down from the middle of the leaf to the edge on the slightest rocking movement, and there coalesce with the water on which the leaves float.

This puckering of the margin of the leaf is attended in the water-lilies by a phenomenon which, although not directly associated with the matter in hand, is so full of interest that it cannot be passed without notice. If we take a boat in the bright sunshine at midday, and float over the calm inlet of a lake, whose surface is overspread with the leaves of waterlilies, and if the water is clear to the bottom, we shall see the shadows of the leaves which float on the surface sketched out on the ground below. But we can scarcely believe our eyes-these do not look like the shadows of the leaves of waterlilies, but rather

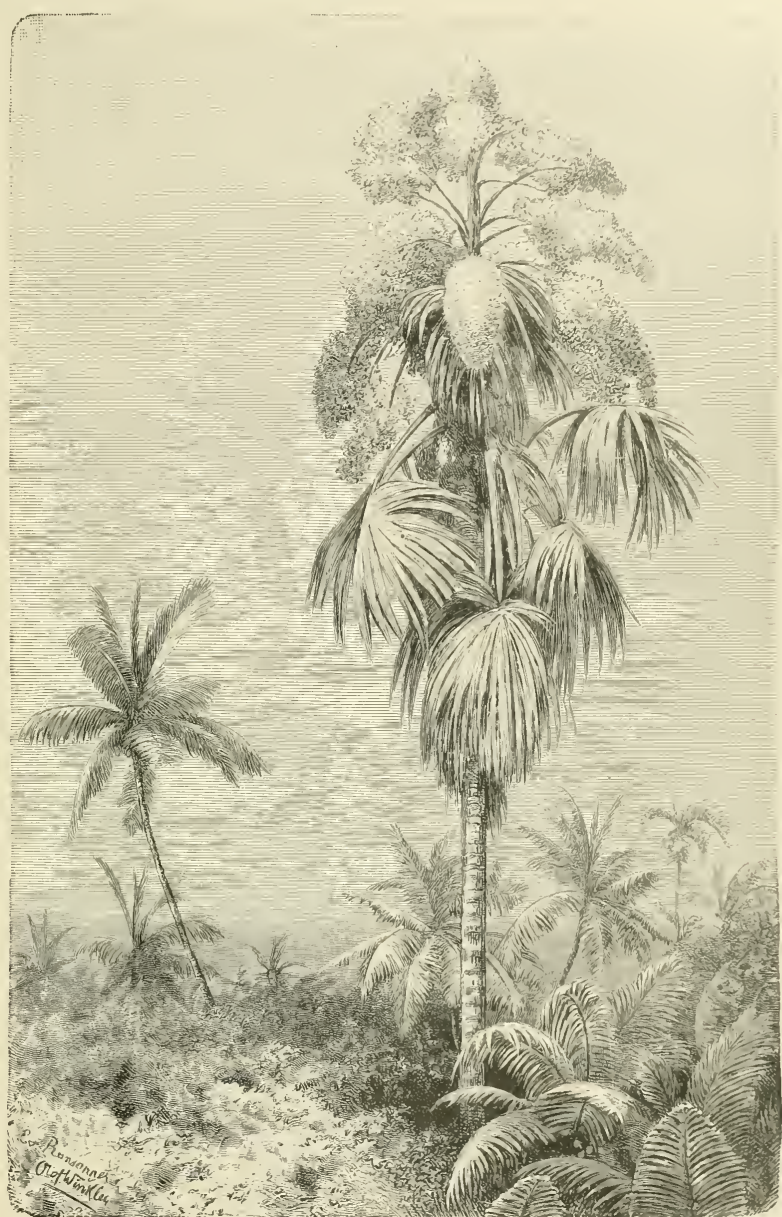

Fig. 63.-Corypha umbraculifera of Ceylon (after Ransonnet). of the fronds of huge fan-palms. From a dark central portion raaiate out long dark strips which are separated from each other by as many light bands. The cause of this peculiar form of shadow is to be found in the undulating margin of the floating leaves. The water of the lake adheres to the whole of the under surface of the dise as far as the edge, and is drawn up by capillarity to the arched portions VOL. I. 
of the undulating margin. The sun's rays are refracted as through a lens by this raised water, and so a light stripe corresponding to each convex division of the curved margin is formed on the bed of the lake, and a dark stripe corresponding to each concave part. These are arranged in a radiating manner round the dark central portion of the shadow.

\section{MAINTENANCE OF A FREE PASSAGE FOR AQUEOUS VAPOUR.}

Special arrangements are met with in all plants which possess stomata, in order that the giving off of aqueous vapour may continue without hindrance. Water falling on the upper side of the leaf, in the form of rain and dew, threatens to cause the greatest obstacle to this free passage should it be able to collect directly in the stomata. The width of an open stomate does not render the entrance of water by capillarity impossible. As long as light and warmth exercise their power, as long as the temperature in the neighbourhood of the spongy parenchyma is higher than that of the surrounding air, and water-vapour in consequence is produced in the spongy tissue and driven out with force from the stomata, such an entrance is indeed inconceivable. It is impossible for aqueous vapour to pass out and at the same time for fluid water to enter by the same passage and through the same gate. But should the leaf become cooled by radiation after sunset, and dew be deposited upon it, or should a cold rain trickle down over the leaves, and the stomata have been unable to close quickly enough, it is quite possible that water might enter, just as it enters a retort (whose narrow mouth dips into water, and whose contents have been vaporized by placing a lamp under them), when the lamp is removed, and the bulb of the retort with its contents becomes cooled. But putting aside the possibility of water thus pressing its way in, this much is certain, that the formation of a layer of water over the cells in the immediate neighbourhood of the stomata would cause great injury to the plants; and this, not only as affecting transpiration, but also the free entrance and exit of gases. Therefore, the immediate surroundings of the stomata must be kept open as a path for aqueous vapour, and no water must be allowed to collect and take up a position there.

Stomata are much too small to be seen with the naked eye. However, it can be ascertained by a very simple experiment whereabouts, on a leaf or green branch, stomata are to be found. A twig or a leaf is dipped in water, and then withdrawn after a short time and lightly shaken; some spots will be found wet, while other places remain dry. Where water remains and spreads out to form an adhering film, no stomata will be found in the epidermis; but where the twig or leaf is dry, one can be sure of finding them. In 80 per cent of cases experimented upon in this way, only the upper leaf-surface became wetted, while the under side kept dry; in 10 per cent both sides remained ary; and in the other 10 per cent the upper side kept dry, while it was the under side which was wetted. With this corresponds the actual fact that in far the greater number of instances the under side possesses most stomata, while the upper side is free from them. It seems as if 
this circumstance could be explained thus, that the upper side is usually turned towards the rain, and that the stomata are on this account collected together on the under side, which is sheltered from it. This explanation, however, which at first sight seems so plausible, does not quite correspond to the true state of the case. The consideration of the reasons for believing that it is an advantage for the plant to have the upper side of the leaf free from stomata will indeed come later, but one thing must be noted here,- that the side of the leaf turned towards the ground, which in most cases contains all the stomata, remains anything but dry. Of course the rain-water only reaches the surface of the horizontal leaf-blade when the margin is so formed that the adherent layer of water which wets the surface is drawn over gradually from the upper to the under side, and that is very seldom the case; but the wetting of this surface by mist and dew is all the more important on this account. On taking a stroll through fields and meadows on a dewy morning, as a rule only the upper surfaces of the leaves come into view, and one might easily be led to think that the dew is deposited only on this side. We constantly use the expression that the dew "falls". Underlying this is the idea that the dew comes down like rain, and that only the upper leaf-surface becomes covered with dewdrops. But one has only to turn the leaf over to convince oneself that the lower surface is likewise bedewed, and on a closer examination it will even be seen that dew is of more importance in connection with the lower than the upper side, because it remains there so much longer. When the sun is already high in the heaven, and the dewdrops have long disappeared from the upper surface, and transpiration is in full force, the under side may still be found studded with dewdrops. If in the majority of cases the stomata lie on the under side, and this side is exposed to the danger of being covered with water as much as the upper one, it is evident why contrivances for hindering the access of water to the stomata are to be found much more abundantly on the under than on the upper side of the leaf.

The most important of these arrangements are the following:-

First the coating of wax. This is either in the form of a granular covering; or as a fine crust which fits closely to the epidermis; or, most commonly, as a continuous thin layer which is easily rubbed off, forming a delicate film popularly known as "bloom". A group of primulas, belonging to mountainous districts and to the moors of low countries, of which Primula farinosa may be taken as the most widely distributed and best known representative, have a rosette of leaves spreading over the damp ground, and on the lower side of these leaves is a white coat, which under the microscope is seen to consist of a collection of short rods and knobs of a waxy nature. If such a leaf is plucked and placed in water for a short time, and then withdrawn, the upper side, which is quite free from stomata, will be moistened by a layer of water, while the under side, on which are the stomata protected by the granular coating of wax, remains quite dry. The lower surface of the leaves is covered with a fine closely adherent wax layer, in many of the willows growing in damp misty places near rivers (Salix amygdalina, purpurea, pruinosa), as well as 
in a great number of rushes, bulrushes, and reed-like grasses. If when the dew falls heaviest one roams through a thicket of willows, or across a moor, one may see plenty of drops hanging from the under side of the leaves, but they do not actually wet this surface, and on the slightest movement of the leaves they roll off and fall down. It is, indeed, in consequence of this that one is more likely to get thoroughly wet by walking through meadows and dwarf willows than by an excursion through country overgrown with ordinary herbs. The two white stripes, so well known on the under side of fir leaves, are also formed by a waxy coat, which prevents the stomata below from being wetted. In species of juniper (e.g. J. communis, nana, Sabina) the two white stripes occur on the upper side of the leaf, and it is interesting to see how the distribution of the stomata again corresponds; for junipers belong to that group of plants whose under leaf-surface is free from stomata, these being present only on the wax-coated region of the upper side of the leaves. Many grasses, to which we shall refer later for other reasons (e.g. Festuca punctoria), only possess stomata on the upper side of the leaf, and again only where the strips of wax are situated. Generally speaking, wax is a protection from moisture, and is most frequently formed when the stomata make their appearance on the upper side of the leaf. The leaves of peas, nasturtiums, woodbine, poppies, fumitory, many pinks, cabbages, woad, and many other cruciferous plants, which have stomata on the upper surface, also produce a covering of wax there. Water poured on the upper surface of a cabbage-leaf rolls off in the form of drops, exactly as it runs off a duck's back, without wetting the surface. In the fronds of ferns (e.g. Polypodium glaucophyllum and sporodocarpum), on the upright leaves of Irises (Iris germanica, pumila, pallida), as well as on the vertical leaves and leaf-like branches of many Australian acacias and myrtles, and lastly in the erect whiplike, leafless or scantily-leaved papilionaceous plants (Retama, Spartium), the stomata are protected from the wet by a coat of wax.

The formation of hairs furnishes another barrier to the entrance of water into the stomata. We shall come back again to these structures, which serve so many different purposes in the plant economy, but here only those hairy and felted coverings whose task is to hinder the wetting of the stomata will be considered. Examples of these are furnished by many Malvaceæ which grow in marshes and ditches (e.g. Althcea officinalis), and also by some mulleins (e.g. Verbascum Thapsus, phlomoides), whose leaves are provided with stomata on both surfaces, and with hairy coverings which it is impossible to moisten. In the damp meadows of the valleys of the Lower Alps grows Centaurea Pseudophrygea, whose large leaves, hairy on both sides, are very rough and much wrinkled. The stomata are only situated in the hollows between the ridges. When rain falls, or the leaf becomes bedewed, the water remains in the form of drops on the hairs of the elevated portions, and the cells in the hollows are not wetted. In many alpine plants, for example the Hairy Hawkweed (Hieracium villosum), after a fall of rain or dew the long projecting hairs of the leaves are thickly beset with drops of water, none of which can reach the stomata on the epidermis beneath. 
It should be particularly noticed here that plants with two-coloured leaves, such as those whose upper surfaces are green, smooth, free from stomata and easily wetted, while their under surfaces, covered with gray or white hairs, and rich in stomata, which cannot be wetted, are generally to be found on the banks of rivers and streams.

In the open woods which skirt the banks of rivers in the valleys of mountainous districts, i.e. in places where mist rises on summer evenings, and all the twigs, leaves, and stalks are covered with drops of water, the most characteristic plants are the Gray Alder (Alnus incana) and the Gray Willow (Salix incana), and as undergrowth everywhere the Raspberry-all plants adorned with the twocoloured leaves just described. Leaving the region of woods growing on river banks for the neighbouring meadows, through which ripples fresh water from a spring, and where everything drips with dew from evening until the middle of the following day, we come to the natural home of herbs and shrubs with leaves green on the upper and white on the under sides. There Fuller's Thistles (Cirsium heterophyllum and canum) grow luxuriantly, and the Meadow-sweet, with its large two-coloured leaves; whilst the whole course of the brook is bordered by the Colt's-foot (Tussilago Farfara) with leaves which may be considered typical of this group.

What a contrast does this present to the lofty vaults of a dense forest, perhaps only a thousand paces away, where on the shady ground little or no dew is formed, and where the leaves which canopy the brown soil are never exposed to a thorough wetting! No parti-coloured leaf is to be found there, no leaves whose upper surface is green and smooth, while the under side is covered with white hairs; and plants which exhibit a thick coating of wax on their under surface, like the Primula farinosa of the moors, are also absent. On the other hand ferns are here, as for example the Hard Fern (Blechnum Spicant), whose leaves are furnished with stomata which open quite without protection on the tops of projecting undulations. This contrast between the leaves of plants in the open marshy country and in the interior of forests is found, not only in the colder territories of the north, but also in tropical districts. Moreover, plants whose leaves are covered with white hairs on the under surface are never to be found under the close leafy roof of huge trees which prevent nocturnal radiation and the formation of dew. Here occur, rather, plants having totally unprotected stomata opening on slightly raised areas of the surface, as for example in Pomaderis phylicifolia, and on the leaves of the Pepper family, e.g. Peperomia arifolia (see fig. $64^{3}$ and $64^{4}$ ).

A very remarkable contrivance by which stomata are protected from moisture consists in providing the stomata of the upper surface with countless papillæ and cone-shaped projections; between them, of course, being innumerable hollows and depressions. Falling water-drops roll off such surfaces; the water cannot displace the atmospheric air in the depressions, and therefore the leaves and stems, in so far as their epidermis presents the aforesaid irregularities, appear covered with a thin layer of air. As the stomata are situated in the small hollows, they always remain 
dry, and even if that particular part of the plant is wholly immersed, they do not come into contact with the water. There are two causes for the unevenness of the leaves: first, the outer walls of a portion of the superficial cells may become strongly arched outwards; or secondly, solid peg-like projections may arise from the cuticle, and to these projections the air adheres so firmly that it cannot be displaced even by a considerable pressure of water. This protection of stomata against moisture by papilla-like outgrowths is to be found especially in marsh plants which are exposed to a changing water-level. On the banks of streams and rivers, and
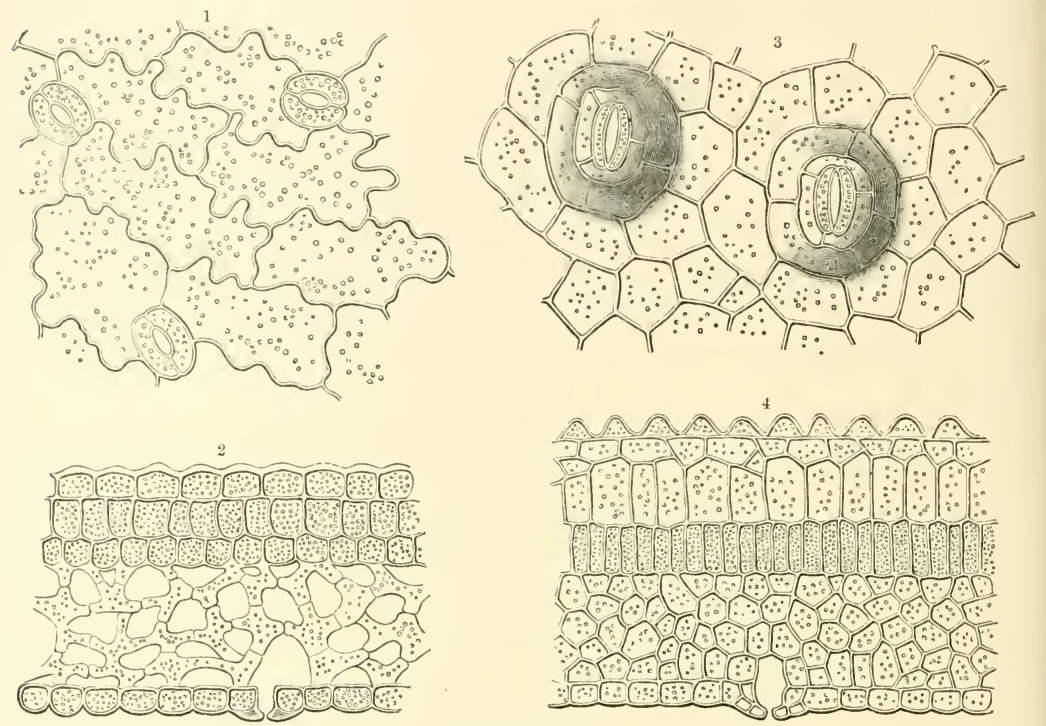

Fig. 64. - Stomata.

1 Surface view of a portion of the frond of the fern Nephrodium Filix-mas. 2 Vertical section through this portion. 3 Surface view of a portion of the leaf of Peperomia arifolia. \& Vertical section through this portion; $\times 350$.

where water welling up from below forms pools and ponds, it may happen that plants are submerged for a week at a time, and then again remain dry for some months.

Most of the plants growing in such situations, particularly the sedges (e.g. Carex stricta and paludosa), the rushes (e.g. Scirpus lacustris), most of the tall fistular grasses (Glyceria spectabilis, Phalaris arundinacea, Eulalia japonica), the plants which grow with the sedges (e.g. Lysimachia thyrsiflora, Polygonum amphibium), and many other marsh plants, are all saved from the danger of having their stomata wetted during their submersion by the papilla-like outgrowths of some of the epidermal cells, near the stomata, as shown in the figures on next page.

Bamboos, and the grasses Arundinaria glaucescens and Phyllostachys bam- 
busoides, which so much resemble the bamboos, besides some sedges (e.g. Carex pendula), exhibit on the other hand the above-named peg-like projections of the cuticle; these are shown in the section of a bamboo leaf in fig. $66^{2}$ ). On plunging such a bamboo leaf in water, a surprising sight presents itself. The upper side, covered by a dark green, smooth, flat epidermis, with no stomata, becomes wet all over, and retains its dark colour and dull appearance; but the under surface, bluegreen in colour, and beset with stomata and thousands of cuticular pegs, does not allow the air to be displaced; and this layer of air, spread thin over the surface, glistens under water like polished silver! The leaf may be shaken under water to any extent, and may even be left submerged for a week, but the silvery glistening air-stratum is not dislodged. If such a leaf is now taken out of the water, the upper surface is quite wet, but the under surface is dry, like a hand which has been dipped in mercury and then withdrawn, and not the smallest drop of water
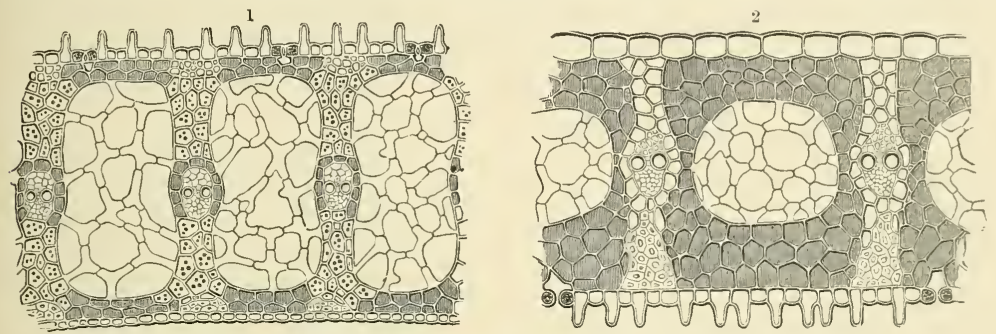

Fig. 65.-Protection of Stomata from Moisture by Papilla-like outgrowths of the Surface.

1 Vertical section through a portion of the leaf of Glyceria spectabilis. 3 Vertical section through a portion of the leaf of Carex paludosa; $\times 200$.

adheres to it. On placing a vessel of water, in which some bamboo leaves are half immersed, under the receiver of an air-pump, and then pumping out the air, numerous small air-bubbles are at once given off from the submerged portions of the leaves. At length the silvery lustre disappears, and the air between the cuticular pegs is replaced by water. If now the leaves be completely submerged, the silver lustre is only shown on those parts which were not previously immersed, and where water could not replace the exhausted air;-the spaces round the pegs in this region having been again supplied with air on the opening of the stop-cock of the pump in order to submerge the leaves. It may be imagined from this experiment how much the stomata would be damaged by water if the plants mentioned were not protected from moisture by the pegs to which the air adheres so strongly.

In many plants which grow in the sunshine, and particularly in those whose foliage is evergreen and only exposed to moisture at the time of the greatest activity of the sap (while later it is exposed for months to dry air), the stomata are to be found surrounded by an embankment, or sunk in special pits and furrows. Even in the leaves of many indigenous plants, which are green in the summer, e.g. those of the Carrot (Daucus Carota), the guard-cells of the stomata are so 
over-arched by the neighbouring epidermal cells that a sort of vestibule is formed in front of the true pore. It can easily be imagined that drops of water which come to such places are not able to press out the air from this vestibule, and therefore cannot penetrate to the guard-cells of the stomata. In Hakea florida and Protea mellifera, two Australian shrubs (see fig. 67), similar arrangements are met with, but here the stomata are still more over-arched, so that they are only visible to anyone looking at the surface of the leaf through small holes at the top of the dome. The stomata on the green branches of various species of Ephedra are surrounded by mound-like projections from the cuticle of neighbouring epidermal cells, and are at the same time somewhat sunken, so that an urn-shaped space is
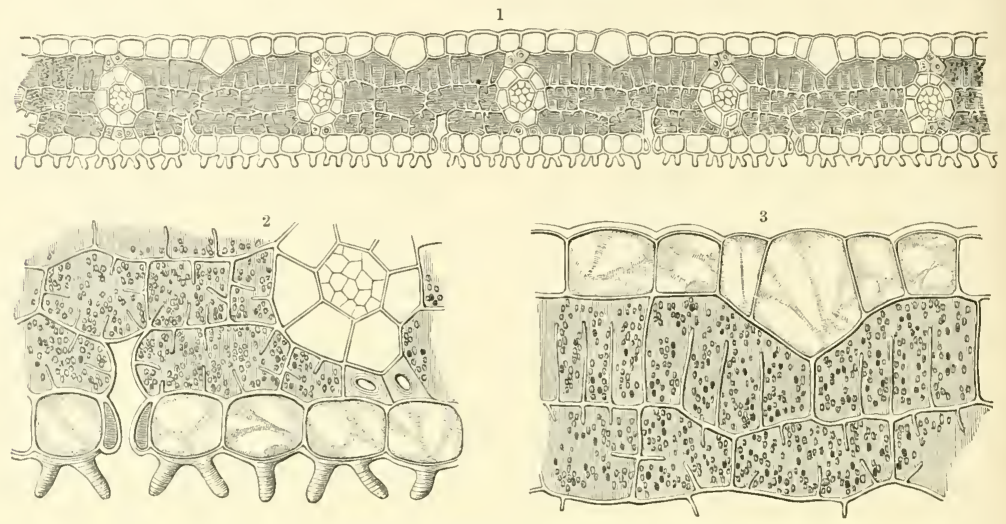

Fig. 66. -Protection of Stomata from Moisture by Cuticular Pegs.

1 Vertical section of a Bamboo leaf; $\times 180$. 2 Part of the lower portion of the section; $\times 460$. $\quad 3$ Part of the upper portion of the section; $\times 460$.

formed above each stoma, from which water cannot dislodge the air. On the leaves of Dryandra floribunda, one of the Proteaceæ which grows in the thick Australian bush, several stomata occur at the bottom of small pits on the under side of the leaf, and from the side walls of the depression spring hairlike structures which interlace and form a loose felt-work, easily penetrated by gases but not by fluids (fig. 68). The stomata on the leaves of the Oleander (Nerium Oleander) are similarly situated. These also are at the bottom of deep pits on the lower side of the leaf, and the entrance to them is beset with extremely delicate hair-like structures (see fig. $73^{3}$ ). The oleander fringes the banks of streams in the sunny open country of Southern Europe and the East, and in its natural position it is most exposed to wetting by rain, mist, and dew, just when transpiration is an absolute necessity for it. But even when the leaves are covered on both sides with a layer of moisture, none can force its way into the hairlined depressions which conceal the stomata, and consequently transpiration is not hindered even in the wettest season of the year. 
Stomata, which are spread over the green tissue of stems and flattened shoots, are frequently sunk in furrows, channels, and pits, in plants whose greatest activity occurs in the short rainy season, and they are saved from wetting in this position by the most varied contrivances. On the rocky shores of Lake Garda, and up over the mountain slopes to the heights of Monte Baldo, grows Cytisus radiatus, a shrub of unusual appearance (see fig. $69^{1}$ ). Its branches only possess rudimentary green leaves, and are themselves furnished with green tissue, which plays the same rôle as that assigned to the mesophyll of the leaf-lamina in normal foliaceous plants.
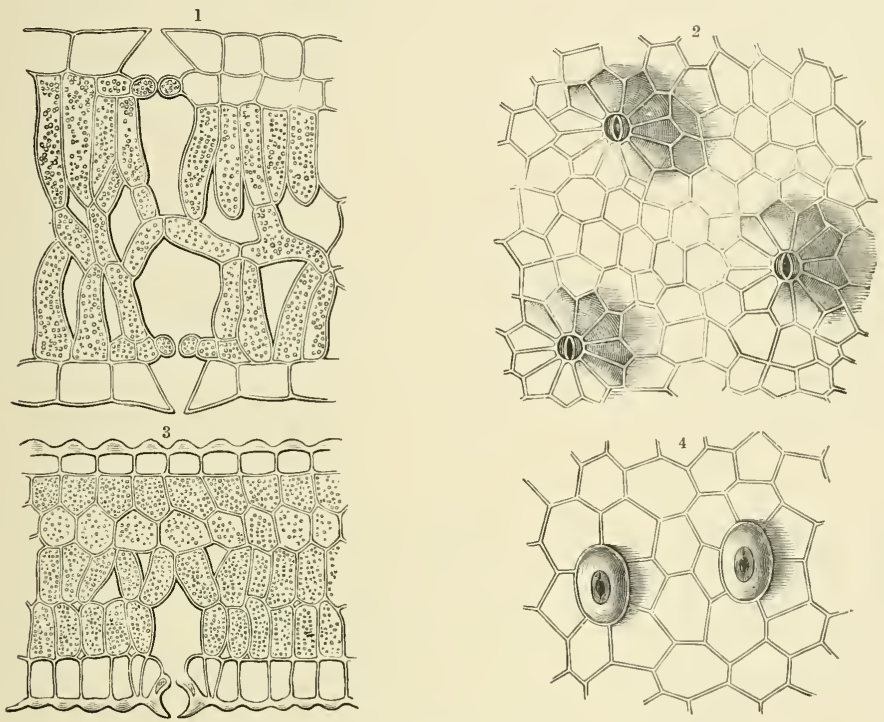

Fig. 67.-Over-arched Stomata of Australian Proteaceæ.

I Vertical section through a leaf of Hakea florida. 2 Surface view of the same leaf; $\times 320 . \quad 3$ Vertical section of a leaf of Protea mellifera. 4 Surface view of the same leaf; $\times 360$.

These green branches bear very numerous secondary branches inserted in decussating pairs. On the secondary branches new shoots develop every spring exactly similar in form, and arranged in the same manner. At the period when this development is taking place, the humidity in that part of the Southern Alps, to which Monte Baldo belungs, is very great. In dull weather, rain and mist, or dew in fine weather, deposit large quantities of water on the soil, and on the plants covering it, particularly in the alpine region of the above-named mountains, on the westerly slopes leading down to the lake, which are thickly clothed with the shruls in question. It is therefore important that the rod-like branches of this Cytisus should be able to breathe and transpire without hindrance, and that the short time during which the conditions for these vital transactions are favourable, should be fully and wholly taken advantage of. Here again the point above all others to be 
aimed at is to keep a free passage for the water-vapour which must escape from the stomata. To bring this about, the stomata are situated in grooves filled with air which are sunk in the green tissue, and which give a striped appearance to the branches. Water cannot force out the air from these narrow furrows which run along the green branches and twigs, eight of them to each branch. The branches may remain submerged in water for an hour without a trace of moisture entering the furrow. Moreover hairs are present in the furrows as a guard against moisture. These cannot be wetted, and the air adheres to them just as to the cuticular pegs of the bamboo leaf. A clear idea of this arrangement is given in the transverse section of the stem shown in fig. $69^{3}$ and $69^{4}$. The adjacent section of the green branch of the Australian Casuarina quadrivalvis shows that these curious plants also have exactly the same arrangements, that the stomata lie at the bottom of
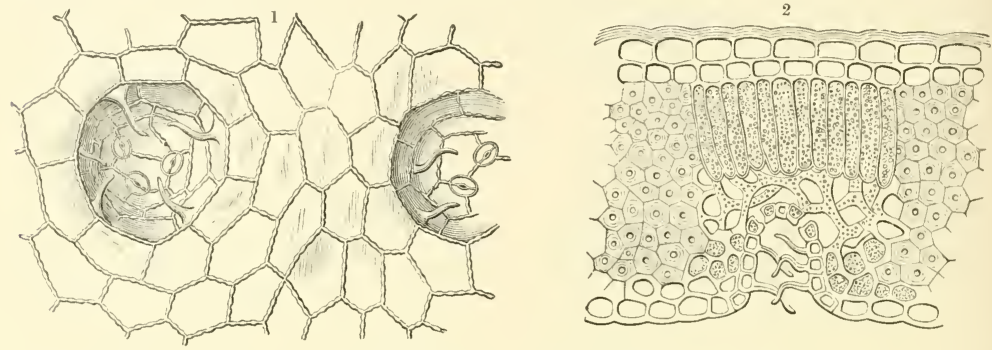

Fig. 68.-Stomata in Pit-like Depressions.

1 Surface view of a leaf of Dryandra floribunda. A portion of the hairs which fill the pit is removed, in order to show the stomata; $\times 350$. 2 Vertical section through a leaf of Dryandra floribunda; $\times 300$.

narrow furrows which run along the green leafless branches, and that peculiar hairstructures are present in the furrows, to which the air adheres, forming a barrier against water, exactly as in those of the Cytisus. The Casuarinæ, which must finish their work for the year during the very short rainy period of their native country, require during this time arrangements providing for unhindered transpiration no less than does the Cytisus in the Southern Alps. Altogether this contrivance is found to be present in only a limited number of cases; in perhaps only twenty papilionaceous shrubs, most of which belong to the Spanish flora, of the genera Retama, Genista, Ulex, and Sarothamnus, in addition to the Australian Casuarinas, and in allied species of Cytisus (holopetalus, purgans, ephedroides, equisetiformis, candicans, albus, \&c.). Most remarkably also this arrangement occurs in a small species of Broom (Genista pilosa), which is distributed over the mountains of Central Europe, over the heaths of the Baltic Lowlands, Denmark, Belgium, and England. And the presence of this contrivance here is the more strange, from the fact that the green branches with their furrows, in which lie stomata, are not leafless, but, on the contrary, are provided with a comparatively well-developed foliage.

Among the most peculiar plants whose stomata are concealed in hidden nooks, 
impenetrable by water, are two very small orchids, of which one, Bolbophyllum minutissimum, grows in company with mosses on blocks of sandstone and on the bark of trees in the rocky ravines near Port Jackson, and on the Richmond River on the east coast of Australia; the other, Bolbophyllum Odoardi, lives in similar

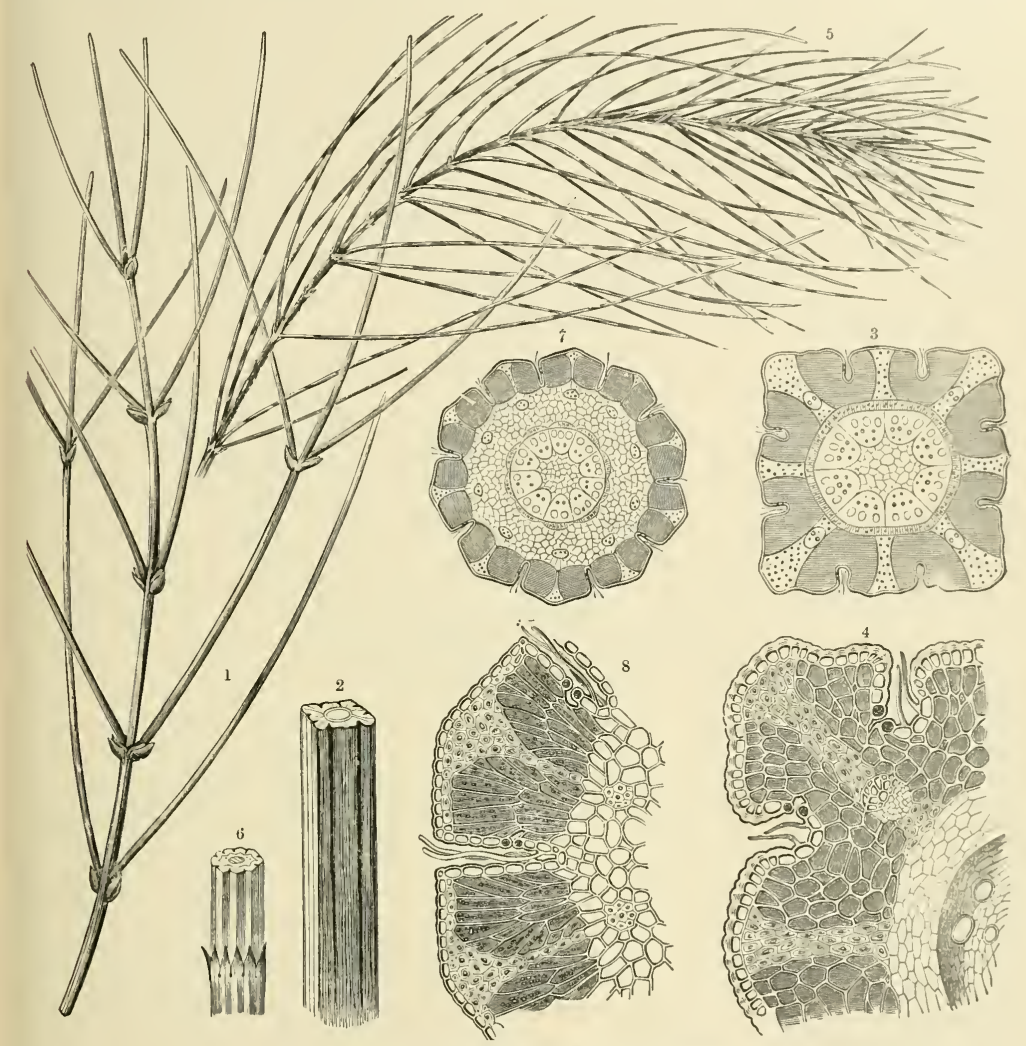

Fig. 69. - Stomata in the Furrows of Green Stems.

1 Branch of Cytisus radiatus; natural size. 2 Portion of a branch; $\times 10$. 3 Cross section of this branch; $\times 30$. I Part of the same section; $\times 150.5$ Branch of Casuarina quadrivalvis; natural size. 8 Portion of a branch; $\times 8$. 7 Cross section of this branch; $\times 30$. 8 Part of the cross section; $\times 130$.

situations in Borneo. Both have a filamentous rhizome from which spring rootlets (from 2 to $5 \mathrm{~mm}$. long and $0.3 \mathrm{~mm}$. thick), arranged in pairs, by which they attach themselves to the stone and the bark of trees. Above the origin of each pair of rootlets is a little disc-shaped tuber, from $1 \frac{1}{2}$ to $3 \mathrm{~mm}$. in diameter, and $\frac{1}{2} \mathrm{~mm}$. thick, with an aperture on the upper surface, scarcely $\frac{1}{10} \mathrm{~mm}$. broad, leading into a hollow chamber within the disc-shaped tubers, about $0.5 \mathrm{~mm}$. broad and $0.1 \mathrm{~mm}$. high (see figure 70). The leaves of Bolbophyllum minutissimum are reduced 
to tiny pointed seales about $\frac{1}{2} \mathrm{~mm}$. in length; two of them are situated at the mouth of each cavity, and are infleeted towards one another across it. In Bolbophyllum Odoardi, each of the small tubers bears only one small green leaf, which is about $1 \frac{1}{2} \mathrm{~mm}$. long and $1 \mathrm{~mm}$. broad, and is placed close to the opening of the chamber (see fig. $70^{4,5,6}$ ). Stomata are found exclusively in the interior of the hollow tubers. Water cannot enter through the narrow mouth into the air-containing chamber, and even when, in the rainy season, the whole of the mossy carpet, in which these smallest of all orchids are interwoven, is saturated with water, their transpiration continues unhindered, provided that the other conditions on which it depends are fulfilled. It is obvious that these structures which prevent moisture reaching the stomata during the wet season of the year ean take on another function
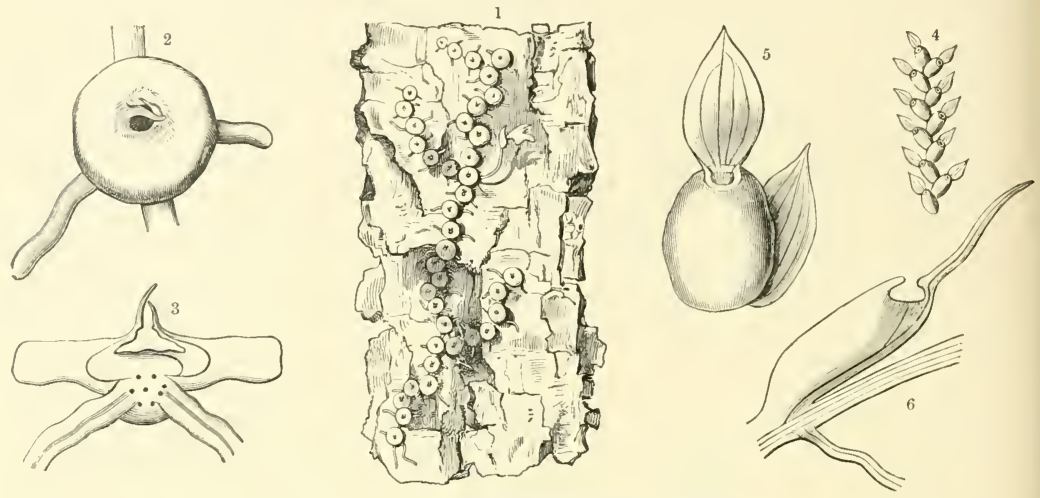

Fig. 70.-Orchids whose Stomata lie in Hollow Tubercles.

Bolbophyllum minutissimum. ${ }^{2} \mathrm{~A}$ tuber seen from above; $\times 8$. 3 Vertical section through this tuber; $\times 15$. 4 Bolbophyllum Odoardi. ${ }^{5} \mathrm{~A}$ tuber; $\times 6$. ${ }^{6}$ Longitudinal section through this tuber; $\times 6$.

in a succeeding dry period, which may follow immediately; but this must he spoken of again later.

The occurrence of "rolled leaves", which are observed in so many plants of widely different affinity, is also connected with the keeping of water from the stomata. The rolled leaf is always undivided, of small area, generally linear, but sometimes ovate-linear, elliptical, or even circular in outline; always stiff, and usually evergreen, and therefore living through two or three periods of vegetation. Its edges are bent down and more or less rolled back, even whilst still hidden in the bud. In eonsequence of this, the lower side which faces the soil is hollowed to a greater or less extent, while the upper side, turned skyward, is arched. Frequently the leaf is rolled so as to inclose an aetual chamber, which only communieates with the outer world by a very narrow fissure, as is the case, for example, in the Crowberry (Empetrum). The rolled-back margins of the leaves in this plant almost touch one another, and the epidermis of the lower side of the leaf forms the actual lining of the cavity which resulted from the rolling of the leaf (see fig. $71^{2}$ ). 
If the bent-back margins do not fit so closely together, a groove appears on the under side of the leaf, which is more or less sunken according to the extent of the rolling, as for example in the Heaths (Erica caffra, vestita, \&c., see fig. $71^{1}$ ). Occasionally a groove is developed which divides into two side furrows running
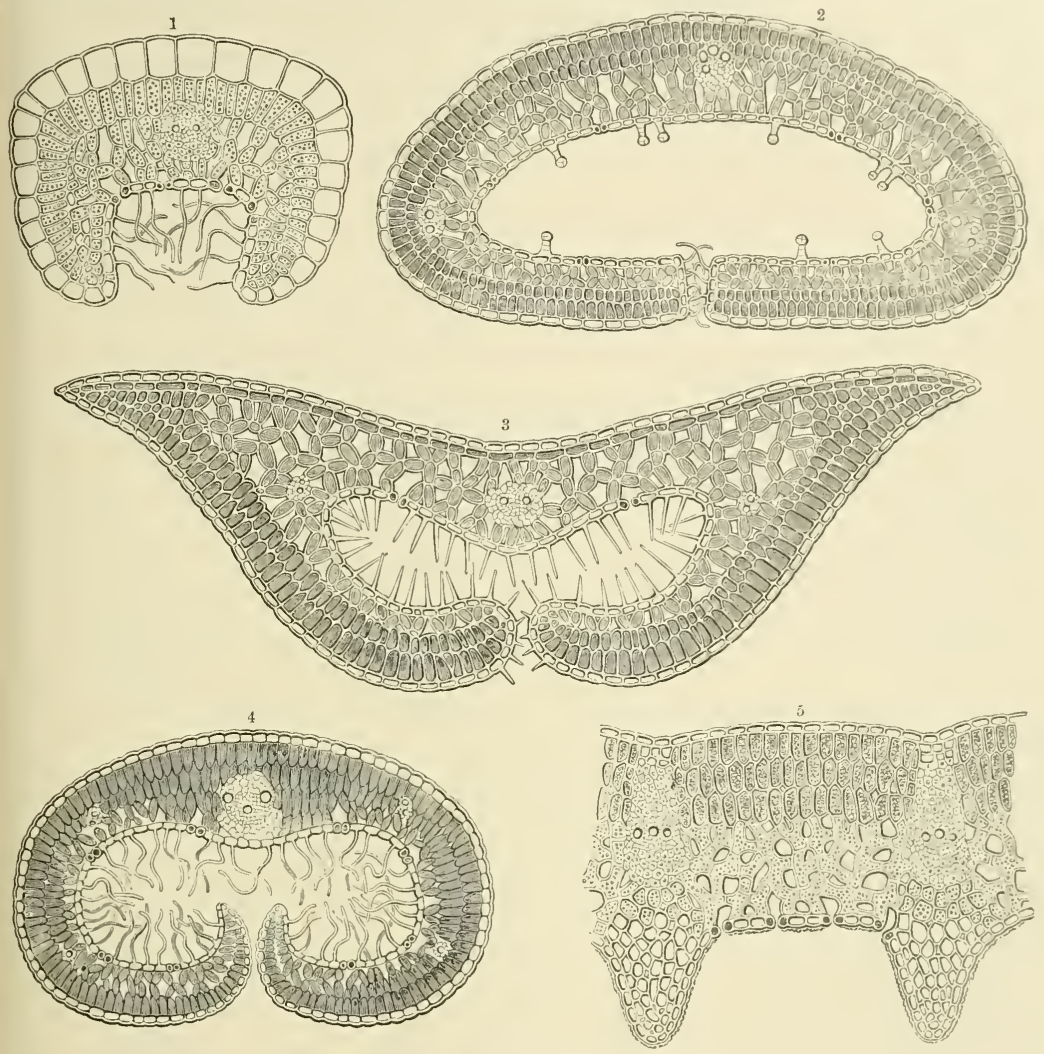

Fig. 71.-Transverse Sections through Rolled Leaves.

1 Erica caffra; $\times 280.2$ Empetrum nigrum; $\times 160 . \quad 3$ Andromeda tetragona; $\times 150 . \quad$ Tylanthus ericoides; $\times 130$. 5 Salix reticulata; $\times 200$.

beneath the rolled edges, as for example in the leaves of Andromeda tetragona (fig. $71^{3}$ ), and in those of the Cape Rhamnea, Tylanthus ericoides (fig. $71^{4}$ ). The central portion of the space framed in by the rolled-back leaves is also frequently divided into two longitudinal grooves, and in such a manner that the tissue below the midrib of the leaf may project as a broad strong band. On the under side of the leaf, therefore, are three longitudinally elongated parallel projections, a central one under the midrib, and two lateral, which have been formed by the rolled-back margins 
of the leaf. On the right and left of the middle ridge lie two deep grooves, which are apparent to the naked eye as light stripes between the dark green projecting portions. This is the case, for example, in the leaves of the Azalea procumbens, also in one of the Ericacer known by the name of Loiseleuria, which covers the soil with a close-matted carpet wherever it makes its appearance, and is widely distributed through Labrador, Greenland, Iceland, Lappland, and generally through the whole Arctic region, as well as over the high mountains of Scandinavia, the Pyrenees, Alps, and Carpathians. The annexed figure 72 represents a transverse section through a single rolled leaf of Azalea, a hundred and forty times its natural size.

Occasionally several strong anastomosing ribs project from the under side of the rolled leaf, inclosing small pits and depressions in whose depth stomata are situated, as may be seen in the leaves of the widely distributed Willow, Salix reticulata (see fig. $71^{5}$ ).

Although all these rolled leaves have an appearance of firmness and solidity, and frequently remind one of the needle-like leaves of the conifers, they are, unlike these, filled up with a very loose spongy parenchyma, which takes up far more room than the palisade tissue lying beneath the epidermis of the upper side. The upper epidermis of all rolled leaves is easily wetted, frequently uneven and finely wrinkled, destitute of any waxy covering; the cells strongly thickened on their outer walls, and pressed closely together, so as to leave no spaces between them. On the under side it is very different. Here stomata are present in great number, and the epidermis is either covered with wax, as in the Marsh Andromeda, the Whortleberry, and the Reticulate Willow (Andromeda polifolia, Oxycoccos palustris, and Salix reticulata), or it is clothed with a fine felt-work, as, for example, in Ledum palustre. Very often peculiar rod-shaped or filamentous projections of the cuticle are present, which at first sight might be taken for hairs, but which differ from hairs in being solid, not hollow. Figs. 72 and $71^{1,2,3}$ show these structures (which may be considered as counterparts of the cuticular pegs on the bamboo leaf) on the under side of Azalea procumbens, Erica caffra, and Andromeda tetragona, as well as on the edges of the fissure which leads into the hollow leaf of the Crowberry (Empetrum nigrum). These structures are to be found almost without exception in the heathers of the northern moors as well as in the Mediterranean and Cape flora. The importance of this continuous delicate coat lies chiefly in the fact that air adheres to it as to the cuticular pegs of the bamboo leaf, and indeed so firmly that even water, under considerable pressure, is not able to displace it. On placing a leaf of Azalea procumbens under water, two elongated air-bubbles are seen along the two longitudinal furrows, which glisten like two strips of silver. Even shaking the leaf to and fro will not dislodge these air-vesicles, and even if the branch has been left submerged for a week, this air will still cling to the depressions in whose depths the stomata occur. If the branch be removed from the water it will be seen that the upper side of the leaves is wet, while water has been kept away from the stomata of the under side. And as with Azalea procumbens, so is 
it with all other rolled leaves, whether they belong to Cape plants or to heath plants of the Baltic lowlands.

It cannot be doubted that the mechanism of rolled leaves, as just described, furnishes a protection for the stomata against moisture, and keeps open a passage for aqueous vapour and excreted gases. The question is now only how it comes about that this arrangement is to be met with in plants of such widely distant countries and under such differences of climate?

In order to understand this clearly, let us imagine ourselves in some of the regions which are specially characterized by the abundance of plants with rolled leaves. First, on one of the high ridges of the Central Alps, where the low-lying Azalea spreads a thick covering over the soil, where Erica carnea in great quantity

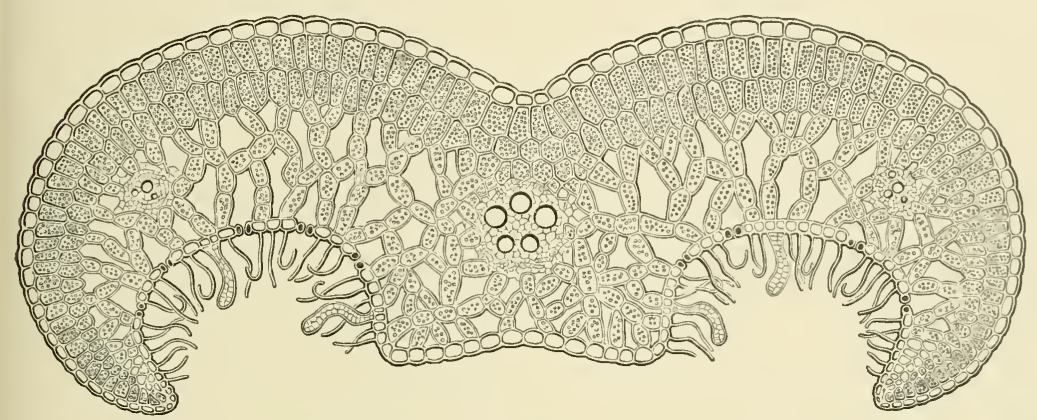

Fig. 72. -Vertical Section through a Rolled Leaf of the Trailing Azalea (Azalea procumbens); $\times 140$.

covers broad slopes, where Dryas octopetala, Salix reticulata, Homogyne discolor, Saxifraga casia, and many other plants which possess evergreen rolled leaves weave their carpet over the stony earth. The ground in which all these plants are rooted, and from which they draw their fluid nourishment, has many natural dikes and retains a large quantity of water, not only from the melting of the heavy winter mantle of snow, but also from the abundant rain of summer. For weeks together the heights are wrapped in a cold mist which saturates everything with moisture, and drops of water hang from the stems and leaves, unable to evaporate as long as the air remains so supercharged with vapour. At length the sky clears again, and the water on the plants begins to disappear. But even during the fine night following, all the plants become covered with a very heavy dew in consequence of their rapid cooling and radiation, and this not unfrequently remains until the middle of the next day. Transpiration at last occurs when the sun shines, and particularly if dry winds sweep over the heights. But who knows how long this state of things will continue? Each moment is precious, and every hindrance to the evaporation, so important for the plants, would be a distinct disadvantage. The outlets for aqueous vapour on the under side of the leaves especially should not be obstructed, and the above described contrivance is arranged with this end in 
view. It can hardly be doubted that the earlier mentioned plants of high mountainous regions cease to transpire for weeks at a time in the wet seasons, when a thick unbroken mist cover's the slopes, and earth, stones, and vegetation are dripping with moisture; and of course the conduction of food-salts to the green leaves is interrupted to a corresponding extent. If one considers how short a period is afforded to plants of high mountain districts in which to perform their year's work, it will be understood how the most active means for promoting transpiration must be brought into play, and how everything which might interrupt or limit this process, so important to the welfare of the plant, must be avoided. A few months after the last snow has melted on the heights, fresh snow again falls, and entirely prevents growth and nourishment during the long winter.

These climatic conditions account for the fact that so many Alpine plants, almost all those having rolled leaves, are evergreen. It is necessary that cvery sunbeam during the short vegetative period should be utilized, and that the leaves retained from the previous year should be able to transpire and to form organic materials on the first sunny day after the winter snow has melted, although the soil may have become only slightly warmed. It may perhaps be urged against this explanation that though, in the steppes the period of vegetation is restricted to the brief space of three months, nevertheless evergreen plants with rolled leaves are completely absent. But the conditions of moisture on the steppes during this three months' vegretative period are essentially different from those of the high mountain region. In the steppes, transpiration is never brought to a temporary standstill by too much moisture; evaporation can take place uninterruptedly from the leaves, and they have to be protected not from moisture, but from over-transpiration. With the exception of the halophytes and a few other growths which are particularly well protected, no plants, on account of the extreme dryness of the air, can retain their green foliage in the height of summer on the steppes.

Some of the plants which adorn the high mountains of southern regions make their appearance in the lower plains of the extreme north. The same carpet of Trailing Azalea, Dwarf Willows, and Dryas (Azalea procumbens, Salix reticulata, Dryas octopetala) is found on the soil underfoot. In addition are other small plants which remain green during the winter (e.g. Cassiope tetragona), which are similarly provided with rolled leaves. Even if we were not informed by Arctic explorers that the number of foggy days in the course of the short Arctic summer is much greater than on the mountain heights of the south, and that therefore a help instead of a hindrance to transpiration is required, the utmost use being made of the short time in which it is possible to draw food-salts from the soil, we might infer this to be the case from the frequent appearance of these small carpet-forming plants with their evergreen rolled leaves. Apart from other considerations, and disregarding the devclopment of the various floral areas in point of time, the above signification of the evergreen rolled leaves explains the similarity and partial identity of the arctic flora with that of the heights mentioned.

Let us turn now to the low-lying country along the North and Baltic Seas, and 
to the lowlands, which extend as far as the northern slopes of the Alps. Where man has not transformed the ground into arable soil, only moor and heath, heath and moor, are seen in wearisome monotony. On the moors especially are always the same plants-various Heaths (Calluna vulgaris, Erica Tetralix, Erica cinerea), Black Crowberry (Empetrum nigrum), Whortleberry (Oxycoccos palustris), Marsh Andromeda (Andromeda polifolia), Wild Rosemary (Ledum palustre)-all plants with evergreen rolled leaves, just as on the mountain heights. Some of these small evergreen bushes, viz. the Crowberry and the common Ling (Calluna vulgaris), may be traced in an unbroken range from the plains up to a height of 2450 metres on the slopes of the Alps. Strange to say, these plants do not blossom much earlier on the lowlands than on the high Alpine regions, and it has actually been shown that Calluna blooms rather sooner at a height of 2000 metres than in the northern portion of the Baltic lowlands. How is this? The winter snow has long disappeared from the lowlands, while the hill-sides above are still concealed under their cold white covering. The winter snow has gone, to be sure, but not the winter! While everything around is already in blossom, while the ear is already visible on the stalks of rye, the neighbouring moor is still dismal, waste, and lifeless. A month or so later there is a stir on the dry soil of the cold moor, and the absorbent roots of the plants which have evergreen rolled leaves commence their activity. When the warm days of midsummer arrive and the sun sends down its powerful rays, the temperature of the soil quickly increases, and indeed rises far more than would be thought possible. The damp cushion of bog-moss at mid-day feels quite warm; and a thermometer placed $3 \mathrm{cms}$. below the surface in the uppermost mossy layer of a moor on a cloudless summer day (22nd June) showed a temperature of $31^{\circ} \mathrm{C}$. while the temperature of the air in the shade was $13^{\circ}$ ! An unpleasant vapour rises from the damp earth, which settles on the surface, and makes a walk over the moor particularly disagreeable. Scarcely has the sun set in glowing red on the horizon when this vapour condenses into patches of mist which settle over the dark expanse; stems, branches, and leaves are covered with drops of water, and next morning everything is as thoroughly soaked as if it had rained throughout the night. This process, which is regularly repeated during the fine weather, is only interrupted when a damp wind from the sea blows, driving masses of cloud over the heath, or when copious rain saturates the soil. It needs no further showing that under such conditions an abundant and continuous transpiration from plants is impossible, and tlat in the short intervals which are allowed to the leaves for transpiration, the outlets from the wide-meshed spongy parenchyma must not be obstructed; and it does not need further proof that the evergreen rolled leaf is the form most suited and adapted to these conditions.

The flora of the Cape of Good Hope may not unjustly be compared with that of the Baltic lowlands-countless low bushes which are very like Heaths, Wild Rosemary, and Crowberry in appearanee-all with small rigid evergreen leaves, and entire rolled-back margins; the upper side of the leaf usually dark green, the under side having the same arrangements as shown in the rolled leaves of plants growing on
VoL, I. 
moors which border the Baltic Sea, and in the cold Arctic tundra. This shrubby evergreen vegetation of the Cape belongs indeed in part to the same families as these. Heaths especially are to be found in abundant variety; as many as 400 species can be counted-many more than are furnished by the whole of the rest of the world taken together. But a great number of species from other families, viz. Rhamneæ, Proteaceæ, Epacrideæ, and Santalaceæ, possess an exactly similar foliage, and without blossom and fruit are often indistinguishable from the heaths. 'This low evergreen bush vegetation is not distributed all over the Cape, but is restricted to the neighbourhood of the coast, to the country which slopes in terraces down to the south-west, and to the celebrated Table Mountain, rising abruptly above Cape Town. The aqueous vapour brought by the sea-winds condenses directly over these regions, and for five months, from May till the beginning of October, the soil is not only soaked by abundant rain, but what is perhaps of even greater moment, all the evergreen bushes are kept in a damp condition by the falling mist, and often are dripping with water just like the heaths on the moors of the Baltic lowlands. When the development of vegetation on the lower terraces of the south-west coast is at a standstill on account of the increasing dryness, the summit of the Table Mountain is still enveloped in the celebrated mass of cloud known as the "tablecloth", and the plants growing on the ridges and plateaus are during this time as much saturated as the Trailing Azalea, which robs the south wind of its moisture on the mountain ridges of the Central Alps. It is, however, in this damp period that the growth of the plants in question takes place. Most of the plants on the heights of the Table Mountain blossom and put forth new shoots in February, March, and April; on the lower terraces from May to September. In the northern regions and on mountain heights the beginning and end of the year's work in plants is limited by the cold, but in the Cape the dryness of the soil is the cause which brings the upward current of the sap in vegetation to a standstill for so long a time. At the coast, however, this dryness is never so severe that the plants are exposed to the danger of withering up altogether, as on the steppes.

As on the south-west coasts of the Cape, so is it round about the Mediterranean Sea and in the west of Europe, which is swept by sea-winds laden with vapour from the Atlantic; for example, Portugal and the south-west of France, which are in like case, characterized by an abundance of low bushes, with evergreen rolled leaves, and especially by some gregarious heaths. Here also the year's growth takes place in the wettest season, and arrangements must be made that during this period the formation of organic materials, the withdrawal of food-salts from the soil, and consequently unhindered transpiration may be carried on. Here, too, dryness interrupts the activity of the absorbent roots, and the evergreen vegetation of the coast-line extends inland as far as the damp sea-winds make themselves felt; while still further inland a steppe-like vegetation preponderates. The analogy presented by the Mediterranean flora goes so far that, on the southern point of Istria, for example, which may be compared as to shape with the south point of Africa, quantities of the evergreen Erica arborea are only to be found on the south-west 
coast district on a comparatively narrow strip of land; while in the interior of Istria the waste dry terraces of the Tschitscherboden (which might be compared with the arid plains of the Cape) show no trace of a heath vegetation.

Why the plants with evergreen leaves which grow in the far north, on the heights of the Alps, on the Baltic lowlands, on the shores of the Atlantic Ocean, on the borders of the Mediterranean basin, and at the Cape of Good Hope are not all of the same species, is a question which cannot be answered here; yet it seems proper to point out that all plants furnished with evergreen rolled leaves, whose year's work is stopped by dryness, would freeze in countries where the earth in winter is covered with snow, i.e. the molecular structure of their protoplasm would be entirely altered by the frost, which would kill it; while the protoplasm of the analogous northern forms would suffer no harm from the cold. It is well worthy of remark in this connection that some of the last-mentioned plants have an extraordinarily wide distribution; that they may actually be found, quite similar in appearance, in the bleak north, and in the southern districts, if only those conditions of moisture which we have shown to account for the form of the leaves obtain in the places mentioned. Thus the Irish Heath (Dabeocia polifolia) may be found along the Atlantic coast as far as Portugal, and the common Ling (Calluna vulgaris) grows just as well at a height of 2450 metres above the sea beside the glaciers of the CEtzthal in the Central Alps, as further south on the Abazzia, surrounded by laurel groves on the sea-coast of Istria.

\section{PREVENTION OF EXCESSIVE TRANSPIRATION.}

Protective arrangements on the Epidermis.-Form and Position of Transpiring Leaves and Branches.

\section{PROTECTIVE ARRANGEMENTS ON THE EPIDERMIS.}

The relation of the form of the evergreen rolled leaf to transpiration is anything but exhausted in the foregoing account. The part played by this form of leaf, in particular during the dry season of the year, yet remains to be discussed. If it is necessary during the wet period that transpiration should be increased as much as possible, and that everything which might restrict the exhalation of aqueous vapour from the stomata should be kept away, it is also of importance that on the appearance of the dry season the equilibrium between the water taken from the soil and the water excreted by the leaves should not be destroyed, and that an excessive evaporation from the portions of the plant above ground should be hindered. New seasons bring new problems to be solved. At the time when the water-current begins to ascend from the soil saturated by the winter rains, we have an aid to transpiration; later on, in the dry period, we have a protection against the dangers which might attend excessive evaporation. It is certainly of great interest to see 
how a whole group of the arrangements discussed above, among which the rolled leaf is not the least noticeable, serve, at different seasons of the year, and often at different times of the same day, two distinct purposes, as indicated.

First, the stomata themselves. While the green tissue has need of food-salts from the soil for the manufacture of organic materials, they cannot be too widely opened; everything is welcome, then, which promotes transpiration, and consequently assists in the elevation of fluid nourishment from the saturated soil. But if the temperature and dryness of the air increase after the green parenchyma has finished its yearly task, or if the soil from which the absorbent roots have hitherto derived their supply of fluid become so dry that the water exhaled from the aërial positions can no longer be replaced, it is of the greatest importance that the stomata should be closed. This is brought about by the two cells bounding the stoma, which have been termed the "guard" cells.

In order to clearly understand the mechanism of the opening and closing of stomata, it is necessary to examine the structure of these guard-cells more in detail. Both are bean-shaped in outline, their concave surfaces being turned towards the stoma; they are only connected with one another at their extremities. By their convex sides they are in contact with ordinary epidermal cells; their outer walls are in contact with the atmospheric air, and their inner walls with the spongy parenchyma. Both the innermost and outermost walls of the guard-cell are strongly thickened, but the wall by which they are connected with neighbouring epidermal cells, as well as that portion which directly borders the stoma, is relatively thin, elastic, and extensible. If the figure of two such guard-cells be imitated in croutchouc, and they be fitted together like an actual closed stomate-water being forced into them under considerable pressure-the curvature of the thin and elastic portions of the walls will be most altered. The side wall in contact with the neighbouring epidermal cell bulges out, and at the same time the whole cell becomes elongated in a direction perpendicular to the surface. By this means the two guard-cells are forced apart. When the water is allowed to flow out of the swollen caoutchouc cells, they again fall back into position, the two portions of the walls which border the stoma coming into contact with each other and closing the opening. The same thing occurs in the actual guard-cells of the living plant. As soon as they become distended, they separate from one another; when they relax and resume their original position, they come closely into contact again. This process bears a strong resemblance to the changes in the cells of the pulvini at the base of the sensitive leaves of Mimosa, which will be described later, and it is highly probable that it may be traced back to a similar stimulation. That the guard-cells actually senarate from one another by swelling up, i.e. by absorbing fluid, and then close together again in consequence of the loss of water, can be shown by first supplying water and then withdrawing it by a solution of sugar. In the former case the stomata open, in the latter they close, and it may therefore be considered an established fact that a closing movement is brought about by the extra loss of water in dry air. But if these pores, through which water vapour escapes when the plants 
are full of sap, close as soon as there is a danger of too much evaporation, the mechanism must be considered as excellently regulating transpiration, and as providing a true preventative against over-evaporation.

This closure of the exhalent chambers in the interior of the leaf, important as it is, would alone be sufficient in but a very few cases to ward off this threatened danger. If the epidermis which stretches over the thin-walled transpiring cells of the spongy parenchyma is itself thin-walled and succulent, water will be exhaled from it also into the dry atmosphere; this loss of water from the epidermal cells is compensated for by water drawn from the neighbouring parenchymatous cells in the interior of the leaf, and ultimately the leaves would wither up if the supply of water from the roots were stopped or became insufficient. Therefore the epidermal cells must be adequately prevented from exhaling. When this is the case, and when the stomata are closed, the spongy parenchyma, and, generally speaking, all the succulent cells in the interior, are securely protected.

The walls of the epidermal cells in the first stages of their development are composed mainly of cellulose, and are uniformly thin and delicate on all sides. The outer wall, which is in contact with the air, then becomes thickened and divided into an inner and an outer layer. The inner retains its original character, but the outer -the so-called "cuticle"-undergoes great modifications. The cellulose becomes changed, and is replaced by a mixture of stearin and the glyceride of a fatty acid (suberic acid), forming a tallow-like fat which is termed cutin or suberin. In consequence of this metamorphosis the cell-wall becomes less and less permeable to water, and when it has attained a considerable thickness it becomes at length almost entirely impervious to water and aqueous vapour. Frequently, between the inner cellulose and the outer corky layer, other so-called "cuticularized layers" are formed, whose chief constituent is again suberin, and which often attain to a considerable bulk.

Aquatic plants, which are not exposed to the danger of excessive evaporation, of course do not require this protection. Plants whose leaves are surrounded by air, on the other hand, can never entirely dispense with it. The thickness of these corky layers is extremely variable according to the condition of humidity of the air. Where the air is very damp throughout the year, the outer wall of the epidermal leaf-cells appears to be only slightly thicker than the inner, and the cuticle only forms a thin continuous layer. On the other hand, plants which are temporarily exposed to dry air possess very highly developed cuticular strata. Especially when the leaves are evergreen and remain several years on the branches, as, for example, in the Holly (Ilex Aquifolium, see fig. $73^{2}$ ), and in the Oleander (Nerium Oleander, fig. $73^{3}$ ), the cuticular layers are so strongly developed that the outer wall of the epidermal cells is many times thicker than the inner wall. Evergreen parasites, as, for example, the Mistletoe (fig. $73^{1}$ ), those tropical orehids and Bromehacer which live epiphytically on the bark of trees and are often exposed to great dryness in the hot season of the year, cactiform plants, and generally the majority of succulent plants, possess epidermal cells with very strongly thickened outer walls. This is so 
also in the case of the pines with evergreen needle-shaped leaves, where, as a rule, the water compensating for that exhaled by the leaves cannot come quickly through open channels, but only slowly through the woody cells. Usually the cuticle and cuticular layers are of equal thickness over the whole leaf surface; this is so especially in smooth, shiny, leathery evergreen leaves. Not infrequently, however, an irregular thickening is seen, particularly in the neighbourhood of stomata, where circular ramparts are raised, as in Protea mellifera (see fig. $67^{3}$ ), or peg-shaped projections are formed, as in the Bamboo (see fig. 66), or elongated hair-like filaments arise, as in the rolled leaves of Azalea and many Heaths (see figs. 71 and 72).

It would, however, be erroneous to suppose that this formation of a thick cuticle on the epidermis is a peculiarity of evergreen leaves. Plants which are surrounded
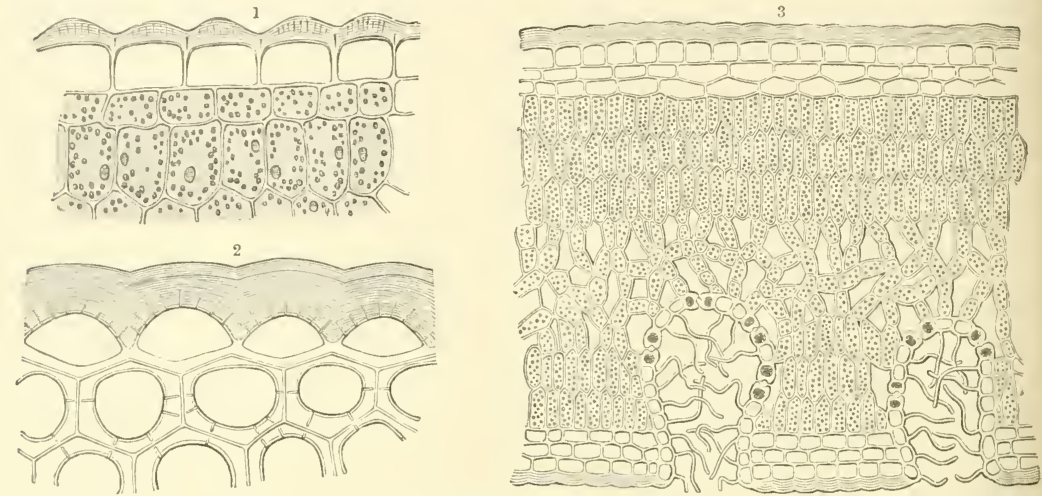

Fig. 73.-Thickened Stratifled Cuticle.

1 Vertical section of a portion of the leaf of Mistletoe (Viscum album); $\times 420.2$ Vertical section of a portion of the leaf of Holly (Ilex Aquifolium); $\times 500$. 3 Vertical section of leaf of Oleander (Nerium Oleander); $\times 320$.

all the year by a damp atmosphere, and are never exposed in their natural condition to the danger of too much evaporation, very often have evergreen leaves, and yet the outer wall of the epidermal cells is not at all, or only very slightly, thicker than the inner; and conversely, plants with apparently thin delicate leaves, which are green only in the summer, have quite conspicuous thickening-layers. A knowledge of these conditions is of the utmost importance in plant culture, and gardeners know very well that many plants, although they appear to be capable of resistance, can never be removed from the damp air of the greenhouses, because the leaves then become desiccated like those of aquatic plants which have been taken out of water and exposed to the air. A species of palm, Caryote propinqua, which is represented in its native habitat in fig. 74 opposite, was grown in the botanical gardens at Vienna, and it developed in the damp air a magnificent stem with fine large leaves. On a summer day, when the temperature of the open air coincided with that of the greenhouse, this Caryota, together with the tub in which it was rooted, was carried into the open and placed in a somewhat shady place, but partly exposed 


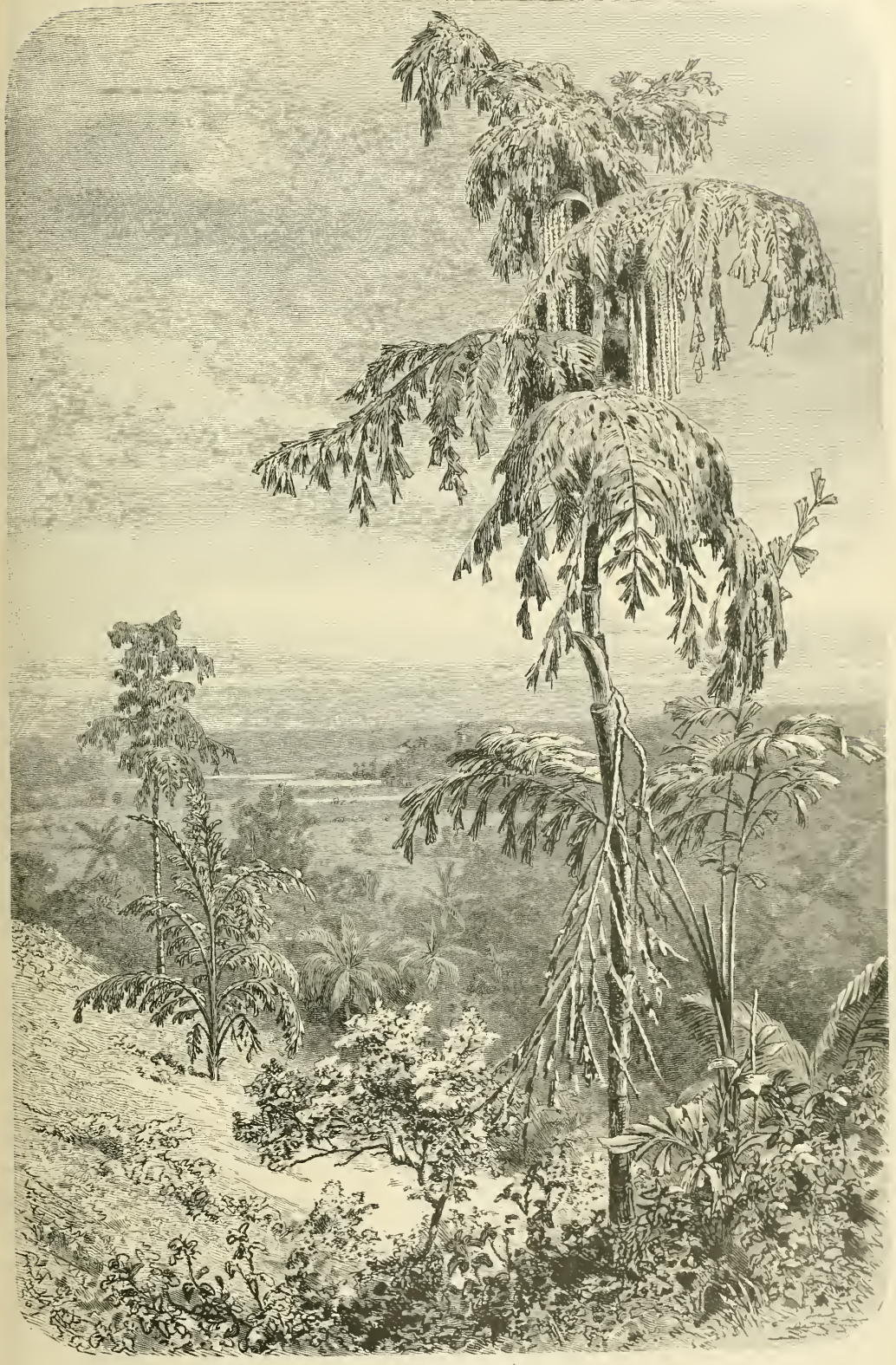

Fig. 74--Caryota propinqua. 
to the sun's heat. One day, after a warm dry east wind had swept for only a short time over the foliage, it became quite brown, and in the evening all the leaves were entirely dried up and dead. And yet leaf-segments of this palm appear to be firm, leathery, and dry, and one would have supposed them to be particularly well protected against drying up. The section of part of a leaf which is represented in fig. 75, however, corrects this impression. This shows that the epidermal cells are certainly very compact, by which the firmness of the leaf is materially increased, but that their walls are not thickened, being only like those of a delicate fern in this respect. Under these small thin-walled epidermal cells lie large succulent cells which form the so-called aqueous tissue, the structure of whose walls likewise cannot limit evaporation; below these are the large succulent cells of the green tissue. A glance at this leaf section will make it clear that this palm is well

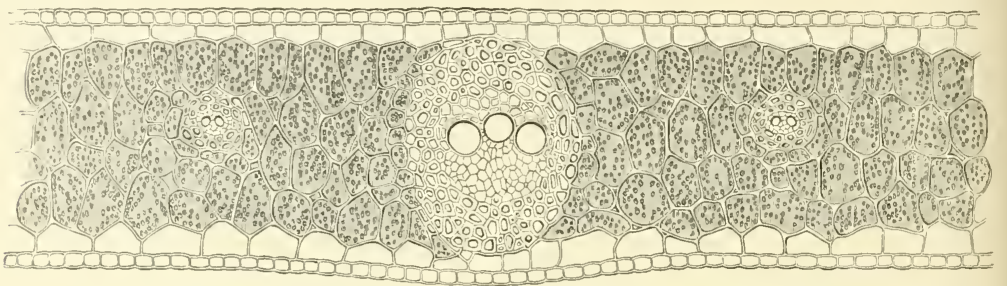

Fig. 75. -Vertical section of a portion of the leaf of Caryota propinqua; $\times 260$.

adapted to its warm damp habitat, where it is never exposed to a strong evaporization, but not to the dry, even if warm, air of a Continental climate.

To the wax-like excretions of the cell-wall which form a delicate bloom, easily rubbed off, on both sides of the leaf, frequently colouring it pale blue, grey, or white instead of dark green, it has already been stated that the rôle is assigned of protecting the stomata from moisture. From what has been said, one would expect that these waxy coverings, which are especially to be met with in the Cruciferæ and Rutaceæ of steppes, in many acacias and Myrtaceæ of Australia, and in the pinks and spurges of the Mediterranean flora, would also be able to limit transpiration in the epidermis - that is, in the structures over which the bloom-like covering extends. Experiments specially undertaken, have also shown that in the same space of time, and under otherwise similar conditions, leaves whose bloom had been carefully rubbed off lost almost a third more water than others whose waxy covering had been left intact.

That the varnish-like covering of the epidermis, composed of a mixture of mucilage and resin ("balsam"), which is excreted from capitate hairs and other glandular structures, is able to restrict transpiration has also been pointed out. These coverings are especially developed in many plants of the Mediterranean flora, particularly in a whole group of Cistus (C. laurifolius, populifolius, Clusii, ladaniferus, monspeliensis, \&c.); further in shrubby plants which develop late, in the height of summer-as, for example, in Inula viscosa, which is so abundant on the 
coast. Plants of steppes and prairies (e.g. Centaurea Balsamita of the Persian steppes and Grindelia squarrosa in the prairies of North America) are likewise protected throughout life from over-vaporization by varnish-like coverings of this kind; while the foliage of Cherry, Apricot, and Peach trees, as well as of Birches, Sweet Willows, Balsam and Pyramidal Poplars, and the Black Alder, is only covered with such a varnish while young, when it has just burst from the buds, and the outer walls of the epidermal cells have not yet become sufficiently thickened; later on, however, when the cuticularized layers have become fully formed, this covering which limits transpiration disappears. Only on those places of the epidermis, where the outer walls of the cells remain very thin and permeable by fluids and gases, is this coat of balsam retained until the leaf is to be thrown off; but in this case it probably regulates the absorption of atmospheric water.

How far the incrustations of lime and salt excretions take part in the absorption of atmospheric water by organs situated above the ground has likewise already been considered in the section on water absorption. It is obvious that these concretions and coverings of the epidermis must be capable of restricting transpiration. Incrustations of lime are principally found in plants which grow in the clefts and crevices of rocks; excretions of salt are only observed in shore-plants and those of steppes and wastes, but then always on low bushes and shrubs with small narrow leaves, and herbs whose foliage rests on the soil. The reason for this is again easily found. High trees could not support the weight of leaves loaded with incrustations of lime and salt, even if their trunks and branches possessed the greatest strength imaginable.

It has been observed that plants whose leaves are covered by incrustations of lime and salt, or whose epidermal cells are strongly thickened on their outer walls by corky layers, are almost always destitute of hairs; while plants, on the other hand, whose epidermal cells possess delicate outer walls, if they are not surrounded by a damp atmosphere throughout the year, nor submerged in water, are usually furnished with structures known as plant-hairs (trichomes); from which it may be inferred that the hairy covering of the leaf or stalk in question is able to protect it from drying up in just the same way as the corky layers. Of course only those hairs are meant whose protoplasmic contents have disappeared, and which have become sapless and filled with air; for those hair-structures, which consist of cells rich in sap and usmotic contents, would not help in preventing evaporation from the deeper tissue; they are themselves in need of protection, and special protective arrangements exist for them, as already set forth in the discussion on the absorption of water by aërial portions of the plant. Such structures would, if unprotected, give off water to the surrounding air, and continually absorb fluid from adjacent cells below them. This action does not take place in air-containing cells, and if their dry membranes, and the air which they inclose, are interpolated between the dry atmosphere and the succulent tissue below, this latter will be protected from evaporation, like damp earth covered with a layer of dry straw or reeds, or the fluid at the bottom of a bottle whose neck is closed with a plug of cotton-wool. 
The importance of air-containing cells as a covering for succulent tissue must also be considered in another relation. It is well known that evaporation from the surface of fluid or a damp body is much increased by the warmth of the sun's rays. On the other hand, if the heating is restricted, so also is the evaporation. If we use a dry cloth to shade from the sun, we lower not only the temperature, but also the amount of evaporation from the shaded body. The covering of air-containing hairs on leaves may be compared to such dry screens, and its action may be demonstrated by the following experiment:-Take two of the bi-coloured leaves of a Bramble bush, which are smooth on the upper side, but covered with a white felt-work of hairs on the lower, and which are exactly similar in size and position with regard to the sun, being situated very near each other on the stem. If these leaves are wrapped round thermometers, in such a way that the leaf which covers one thermometer bulb has its white felted side turned towards the sun, that covering the other, the green hairless side, it will be found that the temperature in the leaf whose smooth green side is directed towards the sun will in less than five minutes rise $2^{\circ}-5^{\circ}$ above that of the leaf whose white felted side is so directed. If such leaves are plucked and exposed to the sun, some with the white felted side, others with the smooth green side uppermost, the latter always shrivel and dry up much sooner than the former. There can be no doubt, after this, that a dry coat of hair over succulent plant tissue, which is exposed to the sun's rays, considerably restricts the heating of, and exhalation from this tissue.

The significance of the coverings of hair on portions of plants turned away from the sun, particularly on the under sides of flat and rolled leaves, has already been discussed. These coverings are only of slight importance as a means of protection against over-transpiration. In rare cases, indeed, it happens that the hairy covering on the side of the leaf turned from the sun, the lining of the leaf, so to speak, must act as a protection, since the flat leaf-lamina is so twisted and turned that the sun's rays strike not on the upper but on the under surface. There are certain ferns of Southern Europe (Ceterach officinarum, Cheilanthes odora, Notochlana Maranto), which, contrary to the habits of most of this shade-loving group, grow on blocks and walls which are exposed to the burning sun. In these ferns the upper surface of the leaf is smooth, but the under, on the other hand, is thickly covered with dry hair-scales. In wet weather the leaves are spread out flat, with the smooth surface uppermost; in dry weather they become rolled up, and the under cottony side is then exposed to the sun and to dry winds. Among the low herbaceous growths of the Mediterranean flora, a like behaviour is shown by the widely distributed Hawkweed, Hieracium Pilosella, whose radical leaves, forming a rosette on the soil, appear green on the upper and white on the under side, by reason of a feltwork of star-shaped hairs. In places where the ground easily dries up, and when there have been no showers for a long while, it is usually seen that first the margins of the leaves turn up, and then by degrees the whole leaf becomes bent and rolled, so that the lower side is turned towards the sun's rays, and the white felt of hairs functions as a protective screen to the whole leaf. 
The relations between the hairy covering on the upper side of the leaf and transpiration stand out, most strikingly, in those districts where plants during their vegetation period are, as a rule, exposed to dry air only for a few hours each day, and where their activity is not interrupted by a long warm dry period, but by frost and cold-as is the case, for example, in the Alpine region of mountain heights. On the Alps, the drying up of flowering plants by the sun only occurs in a very few

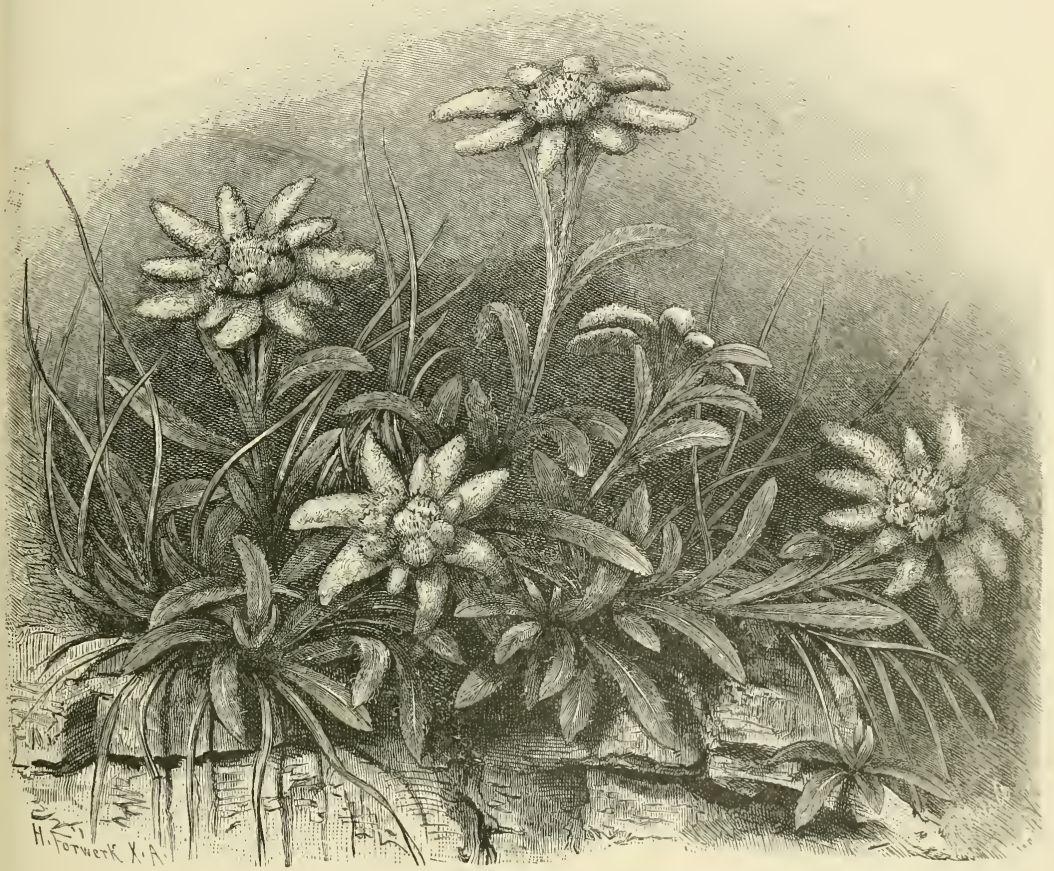

Fig. 76.-Edelweiss (Gnaphalium Leontopodium).

cases, viz., where the scanty soil on the narrow ledges of steep projecting rocks, and crags, and on rocky slopes, \&c., is only watered by rain, mist, and dew. If no showers fall for several successive days, and the south wind blows over the heights with a clear sky day and night, these scanty layers of soil may dry up to such an extent that they are unable to supply the necessary fluid food to the plants rooted in them. Under these circumstances plants growing there have most pressing need of means of lessening transpiration in the leaves. In places such as these are to be found, almost without exception, plants whose leaves and stems are thickly covered on all sides with hairs, together with succulent plants and saxifrages incrusted with lime. This is the habitat of the felted Whitlow-grass (Draba 
tomentosa, stellata), of the grey-leaved Ragwort (Senecio incanus and Carniolicus), of the magnificent silky Cinquefoil (Potentilla nitida), and of the white-leaved bitter Milfoil (Achillea Clavennoe); especially is this the habitat of the most celebrated Alpine plants, of the scented Edelraut and the beautiful Edelweiss-the former (Artemisia Mutellina) with a grey shimmering silky coat, the latter (Gnaphalium Leontopodium) wrapped in dull white flannel. On looking at the vertical section of the Edelweiss leaf (see fig. $77^{1}$ ), one sees that the epidermal cells with their thin outer walls would be unable to regulate exhalation and drying in the sun, and that a powerful protection is afforded against too rapid evaporation, in case of extraordinary dryness, by the possession of a layer of sapless, air-filled, interwoven hair-structures. The Edelraut, Ragwort, and the other plants named, which grow on the sunny rocks of the Alps, show these same characters of leaf structure, and what has just been said about the Edelweiss applies fully to them also. It should be mentioned that on the heights of the Pyrenees, Abruzzi, and Carpathians, as well as on the Caucasus and Himalayas, the plants growing on sunny ridges of rock, where they are exposed to the wind, are covered with silk and wool exactly after the model of the Edelraut and Edelweiss, and that there is on the Himalayas an Edelweiss which is wonderfully similar to that of the European Alps. In the far north, on the other hand, where the flora in other respects has so much in common with that of the Alps, these plants are absent, and generally a search over the rocky crags for herbs and shrubs, whose leaves are furnished with silky or feltlike coverings on the upper surface, is futile. The genera which grow on these places and form a characteristic feature of the vegetation in consequence of their great abundance-as, for example, Diapensia Lapponica, Andromeda hypnoides, Mertensia maritima, Draba alpina, and others, possess remarkably smooth green leaves. When hairy coverings are present, they are restricted to the under leafsurface, especially to that of rolled leaves. They are never found on the plants of rocky slopes, but only on those of damp marshy ground, or by the side of water which is for a short time free from ice. Here, however, they certainly do not help to lessen transpiration, but function in the way described above in the discussion on rolled leaves. It is indeed not too much to connect these facts with the conditions of the climate, and especially to explain the absence of plants whose foliage is silky or felt-like on the upper surface, by saying that a drying up of the soil and a limiting of the water supply never occurs on the narrow terraces of steep rocky declivities in Arctic regions, and that therefore there is no danger of over-evaporation to plants growing in those regions.

It is in keeping with this explanation that on Central and South European mountains, on whose heights an Alpine vegetation is to be found, the number of forms having silky and felted foliage increases as these mountains are situated further south, and the more they are exposed to temporary dryness. Plants of the Edelweiss type are still wholly foreign to the Riesen-Gebirge; in the Northern Alps their number is comparatively small, in the Southern Alps they increase in a surprising manner, and the summits of the Magellastock, the ridges of 
the Sierra Nevada, and the mountains of Greece are unusually rich in such forms.

If plants growing in such situations are protected against the dangers of too rapid and too abundant evaporation, how much more must this be the case in those regions where, with the increasing warmth of summer, the number of showers steadily diminishes; and where the soil becomes dried more and more deeply, so that all the plants whose roots are near the surface are unable to derive a drop more water from it? All plants which are to survive the dry period in such places must during this time entirely cease transpiring-they must, as it were, turn into a chrysalis and sleep during the summer. They actually do this in all sorts of different ways, and by the most diverse means. One of the commonest and most widely spread methods is, without doubt, by having the transpiring organs clothed with a thick covering of dry air-containing hairs. Plenty of examples of this are furnished by the flora of the Cape, Australia, Mexico, the savannahs and prairies of the New World, and the steppes and deserts of the Old. In the dry elevated plains of Brazil, Quito, and Mexico, there are large tracts covered with gregarious spurgelike growths and grey-haired species of Croton, and when the wind blows, moving these bushes to and fro, undulations are set up over wide extents of country, the whole appearing like a billowy sea of grey foliage. A similar picture is presented by the Painciras belonging to the Compositæ, or by the Lychnophora, on the high plains of Minas Geraes in Brazil. Nowhere in the whole world, however, does the presence of hairs on foliage, as a protection against exhalation, appear in such an abundant and varied manner as in the floral region surrounding the Mediterranean, known as the Mediterranean district. The trees have foliage with grey hairs; the low undergrowth of sage and various other bushes and semi-shrubs (for which the name "Phrygian undergrowth", used by Theophrastus, may be retained), as well as the perennial shrubs and herbs growing on sunny hills and mountain slopes, are grey or white, and the preponderance of plants coloured thus to restrict evaporation has a noticeable influence on the character of the landscape. He who has only heard from books of the evergreen plants of the Greek, Spanish, and Italian floras, feels at the first sight of this grey vegetation that he has been in some degree deceived, and is tempted to alter the expression "evergreen" into "ever grey". Every conceivable sort of hair structure is to be met with in these parts-coarse felt-work, thick velvet, and white wool mixed in endless variety. Here is a leaf looking as if covered with a cobweb; there another as if bestrewn with ashes or clay; here a leaf surface, covered with closely pressed hairs or scutiform scales, glistens like a piece of satin; and here again is a plant with such a long flock of hair that one might imagine that sheep in passing had left pieces of their fleece hanging on it. There is; hardly a family in the flora of the Mediterranean district which does not possess members richly provided in this way. The Composites are the most remarkable in this respect, especially the genera Andryala, Artemisia, Evax, Filago, Inula, and Santolina; then come the Labiates of the genera Phlomis, Salvia, Teucrium, Marrubium, Stachys, Sideritis, and Lavandula; rock-roses, bindweeds, scabious, 
plantains, papilionaceous plants, and plants of the Spurge-laurel family-just those plants which constitute the main part of the vegetation on the shores of the Mediterranean Sea, and which possess a thickly-woven covering of hair. Indeed representatives of families such as the Grasses, whose members are usually bare, here appear to be quite shaggy with hair. It is also very interesting to see that so many species, which have a wide range of distribution, and which, from Scandinavia to the coasts of the Mediterranean, have bare foliage, can in the South protect themselves from drying up, by developing hairs on their epidermis. For instance, from Northern and Central Europe as far as the Alps, the epidermis of the stems and foliage of Silene inflata, Campanula Speculum, Galium rotundifolium, and Mentha Pulegium is smooth and bare; in the South,-_particularly in Calabria,- the leaves and stems of these species are covered with thick down.

Next to the Mediterranean flora, the neighbouring Egyptian and Arabian desert regions, the elevated steppes of Persia and Kurdistan, as well as the lowlands of Southern Russia and the plains of Hungary, show a comparatively large number of species whose leaves are thickly coated with hairs on both surfaces. Their number is less than that of the flora of the Mediterranean district, because in the steppes and deserts the dryness of the summer is greater than in that region, and even thick hairy coverings are not always a sufficient protection against this dryness, and also because in some of these districts the dry period passes directly into a severe winter, and the hairs would offer but a poor protection against the cold. Since on the coasts of the Mediterranean Sea the winter temperature never falls below freezing point, evergreen and grey leaves remain there unmolested, and recommence their activity at the beginning of the next season.

The successive developments of certain plant forms are very instructive with regard to the relations existing between whole floral regions and transpiration. In the steppes, Mediterranean district, and at the Cape, bulbous plants and annuals first make their appearance; then follow the perennial grasses and woody plants; and finally succulent plants and thickly-haired immortelles. The numerous tulips, narcissi, crocuses, stars of Bethlehem, asphodels, amaryllises, and all the other bulbous growths, which begin to sprout immediately after the first winter or spring rain, always have bare foliage. Their transpiration is very active in consequence of the rapidly-increasing temperature of the air, but the saturated soil provides a sufficient substitute for the evaporating water, and also has ready in a free state the food-salts which are required for rapid growth. The shrubs which sprout at the same time, the peonies and hellebores, as well as the host of annuals which spring up, blossom, and fructify in an inconceivably short time, almost all possess bare foliage, especially in the steppes. Towards midsummer, when the drought commences, all these plants are already in fruit; their foliage, which until now has been actively at work, begins to turn yellow and to dry up; their succulent tubers and bulbs are imbedded below the surface in soil which is now as hard as a stone; and the seeds which have fallen from the annual plants are easily able to survive the aridity of the summer and the severity of the winter, since they are inclosed in 
protective coverings of great variety. Any plants which are still to retain their activity during the summer on the steppes or in the Mediterranean floral district would succeed very badly if only furnished with the bare foliage of the spring vegetation. If such a plant is to be protected from drying up, its transpiration must be lessened. This is effected by various protective arrangements, but best of all by a thick coating of hair. The papilionaceous plants and species of Orache, above all the immortelles and wormwoods (Helichrysum, Xeranthemum, Artemisia), which are still in bloom in the height of the summer and can bear the strongest heat of the sun, are, as a rule, thickly covered with hair, and regions, which perhaps only a month before were clothed in fresh green, are now shrouded in dismal gray. With the transition from the wet period of the spring and winter rains to the dryness of midsummer, there is a corresponding gradual transition from the green of the bare, succulent hyacinth leaf to the grey of the rigid felt-covered leaf of the immortelle.

A peculiar appearance is shown in Mediterranean floral districts by many biennial and perennial plants which one spring give rise to a rosette of leaves close to the soil, and in the spring following to a stem bearing both leaves and blossom, which arises from the centre of the rosette. This rosette formed in the first spring has to live through the dry hot summer, and is therefore covered with felted grey hairs; the stem formed in the second year which gives rise to the blossom, since it is formed during the wet period, has no need of the protective hairs, and is therefore furnished with green foliage. The Salvia lavandulafolia and Scabiosa pulsatilloides of Granada, the Hieracium gymnocephalum of Dalmatia, and in the Mediterranean flora the wide-spread Helianthemum Tuberaria may be mentioned as examples of such plants. Their appearance is so strange that one involuntarily asks whether this green leafy stem really belongs to the grey rosette of leaves, or whether some one has not been playing a joke by putting together the stem and rosette of two different kinds of plants.

These hair-like structures, called "covering hairs", whose function is a protection against excessive exhalation, exhibit a very great variety with regard to form. Notwithstanding this diversity, however, a certain degree of uniformity must not be overlooked, inasmuch as in individual species the same kind of hairs are always present. The coat of hair contributes not a little to the characteristic appearance of the species, and therefore has always been considered of especial value in description and discrimination. As a help to description the older botanists introduced a series of expressions into botanical terminology by which to denote shortly and tersely the most pronounced varieties, and this seems to be the most suitable place for explaining these terms-i.e., the forms of covering hairs which are signified by them.

First, those covering hairs consisting of a single epidermal cell, which grows out beyond the other epidermal cells, must be distinguished and set apart from those which have become multicellular by the formation of separation walls.

Unicellular clothing hairs in many cases only project slightly above the surface 
of the leaf to which they belong; they bend down nearly at a right angle almost immediately above their place of insertion, so that the long tapering part of the hair cell lies on the leaf-surface, as shown in fig. $77^{3}$. When such hair-forms, in great numbers and parallel to one another, entirely cover the surface of the leaf, light is strongly reflected from them, and the surface looks just like a piece of silk. Such a covering of hair, which is seen particularly well on the shining foliage of the South Furopean bindweeds (Convolvulus Cneorum, nitidus, olecefolius, tenuissimus, \&c.), is termed "silky" (sericeus). Two varieties of this may be distinguished, viz. the more usual case in which all the hairs of the leaf lie parallel with the midrib, and the rarer case where the hairs assume a different position on the right and left of the midrib, the whole of those on either side being respectively parallel to the lateral ribs of their respective sides. The reflected light then only meets the eye of the observer, in any one position, from one half of the leaf, the other half therefore appearing dull. In such a case the whole leaf has that peculiar shimmer, changing on the slightest movement, which we admire on the wings of certain butterflies, and which is also shown by that variety of silken material known as satin. When the unicellular hairs do not lie on the surface, but rise up from it, the shimmer is altogether absent, or is only present to a small extent. If the hairs are short, very numerous, and closely pressed together, they are said to be "velvety" (holosericeus); if they are of greater length and situated further apart, the expression "shaggy" (villosus) is used. Hairs which consist of single elongated air-containing cells, much twisted and bent, with thin and pliant walls, are called wool-hairs, and the covering formed by them is said to be "woolly" or "tomentose" (lanuginosus). Woolly hairs are always twisted spirally, sometimes loosely, sometimes tightlyfrequently almost like a corkscrew. As a rule the spiral is in the opposite direction to the movement of the hand of a watch, whose direction is said to be to the left. It should also be noticed whether the elongated twisted cells of the wool-hairs are circular in cross section, as in the South European Centaurea Ragusina (see fig. $77^{5}$ ), or whether they are compressed like a ribbon, as in Gnaphalium tomentosum (fig. $77^{4}$ ). The latter case is by far the most common.

Multicellular clothing hairs originate by the repeated division of certain epidermal cells caused by the formation of separation walls. These dividing walls are either all parallel to the surface of the leaf or stem, or some of them are perpendicular to the plane of the leaf. In the first case the cells are usually arranged like the links of a chain, and are termed jointed or articulated hairs. When such articulated hairs are short and not interwoven-as, for example, is the case in the beautiful gloxinias (see fig. $77^{2}$ ), the surfaces clothed with them appear like velvet; when they are elongated, curved, and twisted and entwined, the leaf appears to be covered with wool (see fig. $77^{1}$ ), and to the naked eye this form of covering is the same as that already stated to be shown by unicellular covering hairs. Silky coats are also produced by multicellular hairs, even by such a peculiar form as is represented in fig. $78^{3}$. These hairs are developed in the following manner. A superficial cell by the formation of a septum parallel to the leaf-surface divides into two 
daughter-cells; the division is repeated and gives rise to a small chain of three, fuur, or five short cells which project slightly above the surface of the leaf. The top cell does not divide further, but enlarges in a striking manner, not, oddly enough, lengthening in an upward direction, but transversely, parallel to the leaf-surface, forming a lancet-shaped, rod-like structure, which shades the leaf, and is supported by its sister cells as if on a pedestal. Thousands of such curious hair-structures,

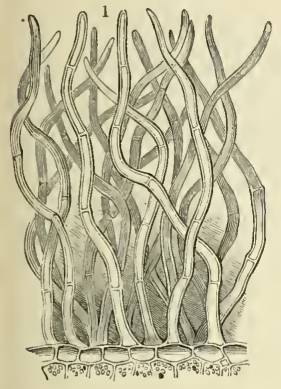

5
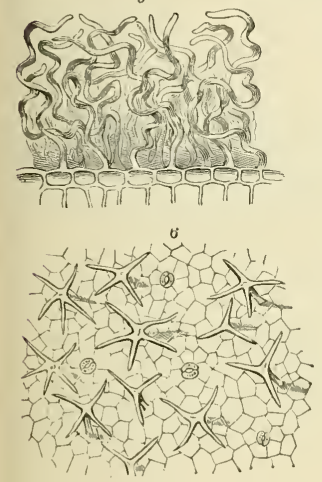
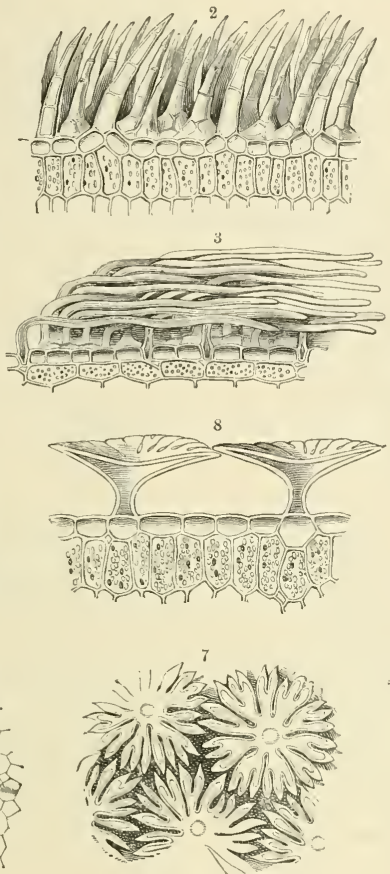

Fig. 77.-Covering Hairs.
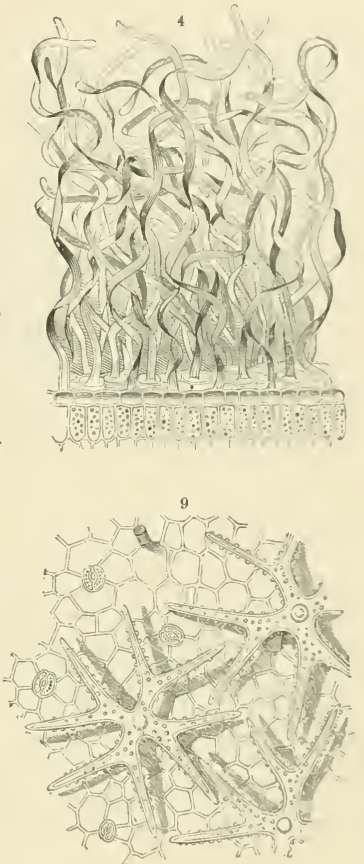

1 Articulated woolly hairs of Gnaphalium Leontopodium. 2 Articulated velvety hairs of Gloxinia speciosa. s Silky hairs of Convolvulus Cneorum. 4 Ribbon-like flattened woolly hairs of Gnaphalium tomentosum. 5 Spiral woolly hairs of Centaurea Ragusina. 6 Stellate hairs of Alyssum Wierzbickii. 7 Umbrella-shaped hairs of Koniga spinosa; surface view. 8 Vertical section of the same hairs. ${ }^{9}$ Stellate hairs of Draba Thomasii. $\times$ about 50.

which may best be compared to compass-needles, clothe the surface of the leaf in close proximity to each other, and when they are arranged in a regular manner, they reflect the light uniformly, and produce a distinctly silky lustre. If they are twisted, this lustre is lessened to a greater or less extent. This variety of hairs, called T-shaped, is distributed in a remarkable way. Numerous species of Astragalus, the scabious of the Mediterranean flora (Scabiosa cretica, hymeltia, graminifolia), several Crucifers (Syrenia, Erysimum), native on the steppes of Southern Russia, the magnificent Aster argophyllus of Australia, and particularly numerous Vol. I. 
species of wormwoods; the South European Artemisia arborescens and argentea, the Artemisia sericea and laciniata belonging to the steppes and Siberian flora, the Common Wormwood, Artemisia Absynthium, and the frequently-mentioned Edelraut, Artemisia Mutellina, growing on the rocky crags of mountain heights-all owe their silky appearance to these $\mathrm{T}$-shaped hair-structures.

It may also happen that the cell which is elongated transversely (i.e. parallel to
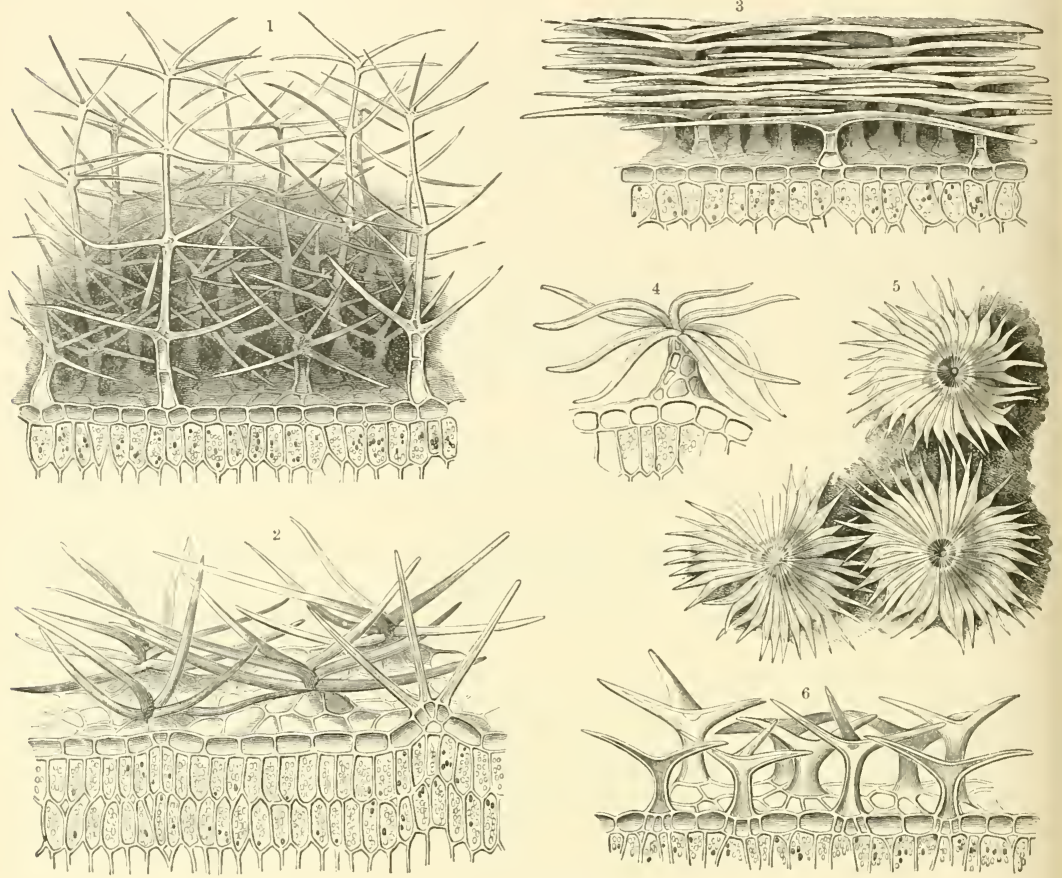

Fig. 73. - Covering Hairs.

${ }^{1}$ Floccose hairs of Verbascum thapsiforme. 2 Tufted hairs of Potentilla cinerea. 3 T-shaped hairs of Artemisia mutellina. - Actinia-like hairs of Correa speciosa. 5 Scutiform scales of Elocagnus angustifolia. 6 Stellate hairs of Aubretia deltoidea. $\times$ about 50 .

the leaf-surface), and which is the uppermost of the small group of cells projecting above the epidermis, is prolonged in three, four, or even more directions, so as to have a stellate appearance. Thus the covering of the leaf is seen to consist of three, four, or many-rayed stars, each supported on a short stalk (see figs. $78^{6}$ and $77^{6}$ ). The rays of the stellate cells are frequently forked, as in Draba Thomasii (see figs. $77^{9}$ ). In rare cases they have a comparatively large central portion, and are only divided at their circumference into short rays; they then look exactly like small sunshades spread out over the leaf-surface. This elegant form, which is represented in figs. $77^{7}$ and $77^{8}$, has a particularly beautiful appearance in 
Koniga spinosa, a member of the Mediterranean flora. All these clothing hairs, with star-shaped indented upper cells, are grouped together under the name of "stellate hairs" (pili stellati). In Cruciferæ and Malvaceæ they occur in endless variety.

When the uppermost cell of the group forming the stellate hair is divided by separation walls, which in part are placed perpendicularly to the leaf-surface, branched hairs are the result. In branched hairs the branches, which are almost
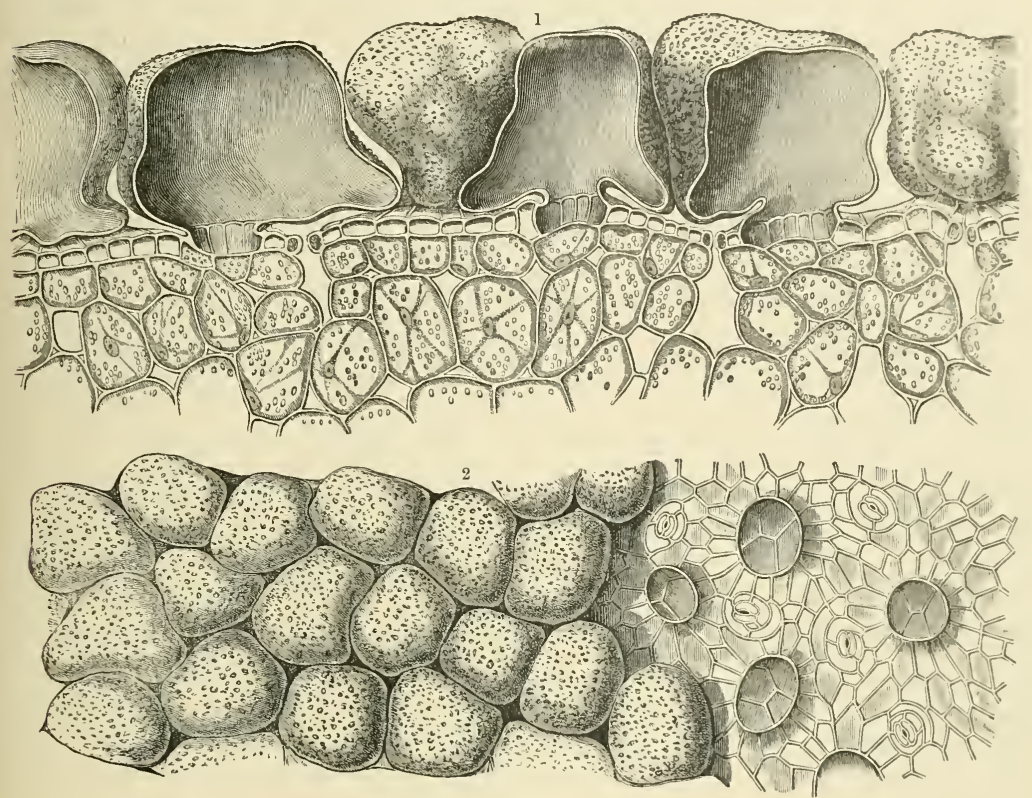

Fig. 79.-Flinty armour of Rochea falcata.

1 Section perpendicular to the leaf-surface. 2 Surface view; on the right hand the vesicular distended portion of a few superficial cells is removed and the stomata are brought into view ; $\times 350$.

always arranged in a stellate manner and are usually unicellular, can be distinguished from the part which supports the branches. This portion usually looks like a pedestal, and is sometimes multicellular, sometimes formed from a single cell. When the pedestal is very short, and the cell supported by it is divided by several radiating divergent septa, which are either oblique or perpendicular to the leafsurface, tufted hairs (pili fasciculati) are formed. These look like sea-urchins lying on the surface in close proximity to each other; they vary very much in the size, number, length, and direction of their branches, and they are particularly abundant on the cinquefoils (Potentilla cinerea and arenaria), cistus and rockroses (Cistus and Helianthemum). A common form is represented in fig. $7 \mathrm{~S}^{2}$. When the foot-stalk is very short, and the radiating branch-cells borme by it are 
joined to one another, a star-shaped, ribbed, multicellular plate, indented at the margin, is produced (see fig. $78^{5}$ ). These plates are generally flat, lie level on the surface of the leaf or stem, overlap one another with their indented margins, and cover the green surface of the leaf so completely that it appears to be white instead of green, and invest it with a bright, almost metallic, lustre. Such leaves are said to be "scaly" (lepidotus). The best known examples of such leaves, covered with shining silvery hair-scales, are those of Elccagnus and of the Sea Buckthorns (Hippophä̈). If the plates are bent, irregularly fringed, and lustreless, the leaf covered with them looks just as if it were strewn with bits of clay, and is said to be "clayey" (furfuraceous). Examples of this are well shown by the leafcoverings of many plants allied to the Pine-apple (Bromeliaceæ). When the top cell of the hair is supported on a moderately high pedestal, and is divided into numerous radiating daughter-cells which diverge from one another, a structure is produced which is somewhat like a knout, or, if the radiating cells are short, like a sea-anemone (Actinia). This form of hair is seen, for example, in the Southern and Eastern European Phlomis, in many mulleins (Verbascum Olympicum), and, with multicellular pedicels, on the leaves of Correa speciosa, an Australian shrub (see fig. $78^{4}$ ). Occasionally a branched hair produces several whorls of branches above one another, and then hair-structures are formed which resemble stoneworts (Characeæ) or miniature fir-trees under the microscope. When many such tiny tree-like hairs are placed close together with interwoven branches, they look under a magnifying-glass like a small plantation, and the analogy is heightened if onestoried tree-shaped hairs, like the undergrowth in a high forest, occur under the higher many-storied ones. This is the case in the Torchweed, Verbascum thapsiforme, whose hairs are represented in fig. $78^{1}$. Hair-structures like these appear to the naked eye like flock, and are described as "floccose" hairs (pili floccosi). Many of these have the peculiar habit of rolling themselves together into small balls, which make the leaf-surface look as if it were bestrewn with coarse white powder. This is the case, for example, in the mullein known as Verbascum verulentum.

In the crowded condition of stellate and tufted hairs, of branched floccose and unbranched woolly hairs, it is unavoidable that the neighbouring hair-cells should cross one another, intertwine, and be more or less interwoven; and thus arises a felted mass which covers the surface of the organ in question. Such hair-masses are termed "felt" (tomentum), and the varieties are distinguished as "felted" (or "tomentose") stellate or woolly hairs, \&c. Often the felt only forms a thin loose layer, through which the green of the leaf-surface can be seen: hut occasionally it is so thick that the leaf uppears snow-white.

While in all these cases the covering which protects the leaves and stem of the plant from over-transpiration is woven from air-containing cells, cylindrical and elongated-usually, indeed, very much elongated-in some thick-leaved plants, especially in species of the genus Rochea, a native of the Cape, these cells become vesicular and distended; they are arranged in rank and file adjoining one 
another, so that taken together they form a layer which spreads over the other epidermal cells like a coat of mail. The ordinary epidermal cells are small and ouly slightly thickened on their outer walls, as shown in the illustration above. The cells which are placed together to form the armour, however, are enlarged in quite an unusual way; their stalk-like base, looking as if wedged in between the ordinary epidermal cells, is indeed comparatively large, but the bladder-like swollen portion exhibits dimensions which are about six hundred times greater than those of the ordinary epidermal cells. The vesicles are closely packed together, and become almost cubical by the mutual pressure they exert on each other. Where a space might occur, the bladders form protuberances and bulgings at the side which fit in together in such a way that a completely closed coat of mail is the result. The expression "coat of mail" is the more justified here since the swollen bladder-like cells of Rochea are as hard as pebbles. Large quantities of silica are present in the cell-walls, and by burning them a complete skeleton in silica can be obtained, as in the case of the silica-coated Diatomaceæ. It needs no further explanation that in the dry season such a coat of armour affords to the succulent cells it covers an excellent protection against evaporation.

There is, however, still another point to be considered. The vesicular swollen cells on fully-grown leaves are still occupied by protoplasm, which forms a thin layer round the walls, while in the centre is a large cavity filled with cell-sap; it is only in older leaves that the bladder-like cells become filled with air. As long as they contain watery cell-sap they serve as reservoirs of water from which the green chlorophyll-bearing cells below can obtain supplies at the periods of greatest drought, when all other sources are exhausted. This fact, that the water-reservoirs are situated on the exterior of the plants, where there exist so many aids to exhalation, shows how well the silicified walls of these bladders function. They may be compared to glass vessels whose mouths are directed towards the green tissue, and whose walls allow absolutely no water to pass through.

\section{FORM AND POSITION OF THE TRANSPIRING LEAVES AND BRANCHES.}

The enlargement of the green leaf-surface has been already explained as a means of increasing transpiration, which is of special importance when the plants considered grow in damp air. Similarly a diminution of the green surface signifies a restriction of transpiration. This relation is illustrated by the fact that in all floral areas, in which the activity of the vegetation is restricted or entirely stopped by increasing dryness, the foliage of the plants is not so widely outspread, i.e. it undergoes a diminution. It is also a well-known fact that one and the same species, if grown in a dry sunny position, will exhibit smaller, and in particular, narrower leaves than when it has been grown in a damp situation. This is well seen in passing from the mountainous districts bordering the Hungarian lowlands to the plains of the lower regions. A number of shrubs and herbs, Anchusa officinalis, Linum hirsutum, Alyssum montanum, Thymus Marschallianus, \&c., exhibit on 
the dry sands of the plains much narrower leaves than in the valleys of the mountainous regions. In conjunction with the narrowing of the foliage, the wrinkling of the leaves has to be considered, i.e. the formation of grooved depressions on the surface. Strictly speaking, there is no lessening of the whole surface of the leaf, but only of that portion of the surface which is exposed to sun and wind. This is the point with which we are concerned. With regard to the exhalation of water, only the extent of the surface direetly influenced by the agents for increasing transpiration is to be considered; whilst the extent of the grooved depressions, which are not exposed to the sun's rays, nor to dry currents of air, may be in a certain measure neglected. On the whole, plants with wrinkled and grooved foliage are not very abundant. For the most part the crumpling is to be seen on quite young leaves when first they break through the bud-scales, and when their epidermal cells are not yet sufficiently thickened with cuticular material. Later, when the formation of the cuticle is advanced, the wrinkles gradually become smooth, and the leaf becomes flat.

It has already been pointed out that those pit-like depressions, on the floor of which stomata are concealed (cf. figs. 68 and 73), may also serve to restrict transpiration. There is no contradiction in the statement that the same structure at one time hinders the entrance of water and the wetting of the stomata at the bottom of the pit, and at another time prevents direct contact with dry winds and consequent over-transpiration. Each has its turn. When the foliage of the Australian Proteaceæ, during the summer sleep, is exposed for months to the scorching rays of the sun and to the warm dry air, and when all supplies of water from the soil have ceased, evaporation from the leaves must be restricted as much as possible; it is then that the pit-like depressions perform their duty in this respect. When, later on, the plants are aroused from their long sleep, and have to provide themselves with food, to grow, blossom, and fructify in an extremely short space of time, while violent showers of rain are pouring down from the clouded sky, and all the leaves are dripping with wet; it is then very important that, in spite of these exceedingly unfavourable conditions for evaporation, an abundant transpiration should nevertheless take place, and that the function of the stomata should be in no way impaired by the moisture. These pit-like depressions, which in the dry period prevented evaporation, now have to keep moisture away from the stomata.

In many plants evaporation from the superficial tissue is restricted by the close contact of the leaves to their supports, like the seales on the back of a fish. The upper side of a leaf in contact with the stem, and frequently adhering to it, is thus deprived of the means of exhalation, and transpiration can only take place on the somewhat arched or keeled under side of the green scale-like leaf. This occurs, for example, in the Tree of Life (Arbor vitce), in several species of Juniper, in Thujopsis, Libocedrus, and various other Conifers. It is not without interest to notice that in several of these Conifers the little green scale-like leaves only become close pressed to the stem when they are exposed to the sun, whilst they project from it if the branches in question are shaded. 
A further reduction of the evaporating surface is brought about by the development of thickened or fleshy leaves. In order to render the points under. consideration as clear as possible, it is perhaps well to insert here the following observations. By altering the form of a sheet of lead $8 \mathrm{cms}$. square and $1 \mathrm{~mm}$. thick into a solid cylinder, the diameter of this cylinder is seen to be only $1 \mathrm{~cm}$., and the whole surface of the cylinder is only one-fifth of that of the previous flat sheet. The application of these figures to the tissue of a leaf demonstrates how much smaller is the transpiring surface of a thick cylindrical leaf than of a thin flattened one. Such thickened leaves, which approach more or less to the cylindrical shape, are to be found regularly where transpiration has to be reduced for a considerable time-as, for example, in the mountainous districts of Central and Southern Europe, in the genus Sedum, growing on sandy soil which easily dries up, and on stone walls and battlements (Sedum, album, reflexum, dasyphyllum, atratum, Boloniense, Hispanicum, \&c.). They also occur in a striking manner in many tropical orchids which grow on rocks, or epiphytically on the bark of trees in the East Indies, Mexico, and Brazil, exposed for more than six months to great aridity (Brasavola cordata and tuberculata, Dendrobium junceum, Leptotes bicolor, Oncidium Cavendishianum and longifolium, Sarcanthus rostratus, Vanda teres, and many others); but especially are they found in aloes and stapelias and species of Cotyledon, Crassula, and Mesembryanthemum, whose habitat is in the dryest districts of the Cape. Several Umbelliferæ, Compositæ, and Portulaceæ (Inula crithmoides, Crithmum maritimum, Talinum fruticosum) growing on stony places of the sea-shore in the burning sun, and many salsolas of the deserts and salt steppes, as well as finally some Proteaceæ, which for two-thirds of the year are exposed to the droughts of Australia-all are characterized by their development of fleshy leaves.

Just as thick-leaved plants have acquired their succulence by a modification of their foliage, similarly, in the so-called cactiform plants, it is the stems which become thick and fleshy, and take on the functions of leaves. Here the green tissue is situated in the cortex of the stem, the epidermis covering it contains stomata just like the epidermis of foliage-leaves, and the green cortex transpires, and functions on the whole exactly as the green leaves do. When the stems of the cactiform plants are richly branched and the branches are short, they sometimes much resemble thick-leaved plants. Frequently also the separate portions of the stem and branches take the form of fleshy leaf-like discs, as in the genus of the Prickly-pear (Opuntia), and such stem-structures are usually mistaken by the uninitiated for thick leaves. Gardeners, as a rule, group the thickleaved and cactiform plants together under the single term "succulent plants". To the cactiform plants belong the opuntias and cacti, species of Cereus, Eclinocactus, Melocactus, and Mammillaria, which are distributed from Chili and South Brazil over Peru, Columbia, the Antilles, and Guatemala. These are, however, especially developed on the high plains of Mexico in astonishing variety of form. To the cactiform plants belong also the leafless candelabra-like tree-shaped spurges 
of Africa and the East Indies. These plants are exposed, far more than the thick-leaved plants, for the greater portion of the year to extraordinary dryness. Their usual habitats are dry sandy and stony plains, waste rocky plateaus, and crevices of rocks which are almost completely wanting in soil. They always inhabit regions where no rain falls for about three-fourths of the year, and which usually belong to the driest parts of the earth. The whole organization of these plants corresponds to these conditions of their habitat. Dry scales and hairs are produced instead of foliage-leaves, or these are often metamorphosed into thorns which project in great numbers from the thick stem-structures, and efficiently protect them from the attacks of thirsty animals. The epidermis of the pillar-like, discshaped, or spherical stem-portions is thickened on the outer wall, so as to almost resemble cartilage, and frequently it forms a coat of mail round the deeper-lying green tissue by the abundant deposition of oxalate of lime (as much as 85 per cent). Most of the succulent plants, whose cell-walls, which are in contact with the air, are fortified by oxalate of lime, silicic acid, or suberin, have in their tissue peculiar aggregates of cells which apparently serve for the storing-up of water for the dry season, and which have been termed "aqueous tissue". The water in these reservoirs is always so apportioned that it lasts from one rainy season to another; that is to say, the adjoining green tissue which exhales the stored-up water does not suffer from drought during the dry season. Also, it is contrived in these plants that, immediately after the fall of the first rain, the reservoirs are again filled with water, and that the emptying and filling of the cells and the decrease and increase of their volume exercise no harmful influence on the adjoining tissue. Succulent plants have been not inaptly compared to camels, the "ships of the desert", which provide themselves with a large quantity of water, and are then able to dispense with further supplies for a long time without injury. The cells of the aqueous tissue are comparatively large and their walls thin; the active protoplasm within forms a delicate layer round the walls-that is to say, a sac whose cavity is filled with watery, often somewhat mucilaginous, fluid. In the cactuses the aqueous tissue is hidden as much as possible in the interior of the thick rod-shaped or spherical stem; also in many thick-leaved plants, such as some of the European species of the genus Sedum (e.g. Sedum album, dasyphyllum, glaucum); in South African species of the genera Aloë and Mesembryanthemum (e.g. Mesembryanthemum blandum, foliosum, sublacerum), the aqueous tissue is concealed in the interior of the leaf, and is usually composed of cells surrounding vascular bundles there situated. In Sedum Telephium, known by the name of Orpine, as well as in species of House-leek (Sempervivum), and many salsolas growing on steppes, the ramifications of the vascular bundles are enveloped in a mantle of green tissue, and the bundles, which are, as it were, overlaid with green cells, are so arranged with regard to the colourless aqueous tissue, that to the naked eye they look like green strands in a transparent matrix which is as clear as water. In the Mexican Echeverias the aqueous tissue is inserted as broad stripes in the green tissue, and in the thick-leaved orchids it appears as if sprinkled between the green 
cells. The epidermis in numerous other thick-leaved plants serves as a store-house for water in a marvellous way. Individual epidermal cells are then greatly enlarged and project beyond the others in the form of sacs, clubs, or bladders, as shown in the picture of Rochea (fig. 79). These bladders either fit together into a one-layered extended coat of armour, or they are frequently placed irregularly side by side or above one another. In some instances they form isolated groups or occur singly, and appear then to the naked eye like protuberances on the green stems and leaves, where they glitter and sparkle in the sunshine like an embroidery of dewdrops. Many leaves and branches-as, for example, those of the widely-distributed Ice-plant (Mesembryanthemum cristallinum)-have the greatest resemblance to candied fruit covered with clear, colourless, sparkling sugar crystals.

When the walls of the enormously-distended vesicular or bladder-shaped cells of the epidermis are silicified, as are those of the repeatedly-mentioned Rochea, it is easily understood that the watery cell-sap which they contain is not exhaled into the air; the fluid is, so to speak, inclosed in a glass bottle and can only be given off in the direction of the green tissue. But when the walls of the bladder-like giant cells are not silicified, and not even particularly thickened, what is the result? From the aspect of the Ice-plant one would think that a single warm dry day would suffice to shrivel and dry up the watery vesicles. But this is certainly not the case. Leafy twigs cut from the Ice-plant may be left all day on the dry ground in dry air and sunshine, and the large bladder-like cells on the surface will not lose their aqueous contents. After a week they become collapsed, having given up their water, not to the atmosphere, but to the green tissue covered by this swollen coat. Without doubt this phenomenon is to be associated with a peculiar formation of the cell-wall; but it is as certain that the constituents of the cell-sap, which fills the vesicles are also important, and it must be assumed that substances are dissolved in this aqueous fluid which restrict the evaporation of the water.

These substances, which hold water with great energy, and thereby enable the plants in question to survive through periods of the greatest dryness, are partly viscous, gummy, and resinous fluids, partly salts. It is well known that the sticky, watery pulp of crushed mistletoe berries, used in the manufacture of "bird-lime", may be exposed to the air for months without quite drying up, and the mucilaginous juices of many cactuses and thick-leaved plants behave in a similar manner, especially those of the Cape aloes, which exhale no water, and enable the plants possessing them to withstand the drought for months. In the thick-leaved plants of the salt steppes and deserts, the fluids are rarely resinous or gummy, but they frequently contain a surprising quantity of salts dissolved in water, such as common salt, chloride of magnesium, and the like; and these also obstinately retain water in proportionately large quantities. It is one of the most surprising of phenomena to see the thick-leaved salsolas rising above the soil of salt steppes, green and succulent, when the ground is at its driest in the height of summer, when for months no clouds have tempered the sun's rays and not a drop of rain has fallen, and when almost all other plants have long ago turned yellow and faded. The large amount 
of salts contained in the sap of these plants renders them capable of a resistance which is almost greater than that afforded by mucilaginous materials and gum-resins.

It must, however, be remarked here that not all green leaf- or stem-cells containing abundant water have the function of storing it up for a dry season, and that the aqueous cell-groups and strands adjoining the green tissue, especially the so-called outer aqueous tissue, in very many cases, has another important function, viz. the conducting of carbonic acid to places where it can be assimilated, but this will be described in the next chapter.

An extreme reduction of the leaf-surface, combined with a formation of green transpiring tissue in the cortex of the stem, is also shown in another group of plants known by the name of "Switch" plants. They are characterized by thin rodshaped stems and branches, while the cactiform plants, on the contrary, always have their axes but little branched, and massive, thickened, fleshy and rigid stem-structures which are unaffected by the wind. The switch-plants may be subdivided into those which are flexible, hollow, and only slightly branched-as, for example, the horse-tails (Equisetum), reeds (Scirpus), rushes (Juncus), bog-rushes (Schcenus), and several cyperuses (Cyperus); and into broom-like shrubs with rigid woody boughs breaking up into innumerable branches and twigs. The former are distributed over the whole world; the latter are principally to be found in Australia and in districts bordering on the Mediterranean Sea. In Australia it is chiefly Casuarinas and some genera of Papilionaceæ and Santalaceæ (Sphcerolobium, Viminaria, Leptomeria, Exocarpus) which take on this odd form, and some of them even attain to the size of trees. In the Mediterranean flora isolated species and groups from the families of Asparagineæ, Polygalaceæ, and Resedaceæ are seen with thin, stiff, rodshaped, leafless branches, which project stiffly into the air with green cortex; but again, most of these plants belong to the Papilionaceæ and Santalaceæ. Several switch-plants of the papilionaceous genera Retama, Genista, Cytisus, and Spartium, growing together, often cover wide tracts of country in densely-crowded masses, and thus contribute not a little to the scenic peculiarity of the district. Many small rocky islands off the coast of Istria are entirely overgrown by Spartium scoparium, which is represented in the illustration opposite. In May large golden flowers, scented like acacias, appear on the green rods of the Broom, and then for a short time the dark green of the switch-plant is changed into a brilliant yellow. On passing near the coast, just at this time, the remarkable phenomenon is seen of golden yellow islands rising above the dark blue sea. This floral adornment is, however, but transitory, and nothing more monotonous and desolate than such a dry unwatered islet, covered with these shrubs, can be imagined

The Spartium belongs to those switch-plants which are not entirely leafless, but which develop little green lancet-shaped leaves at intervals on their long twigs. But these are of such secondary importance that their green tissue can only form the smallest portion of the organic substances necessary to the further growth of the plants, and this duty chiefly falls to the share of the cortex of the switch-like branches. The cortex is also characteristically formed in accordance with this fact. 
Under the epidermis, whose outer walls are much thickened and coated with wax, is the green transpiring tissue or "chlorenchyma", consisting of from five to seven rows of cells. This green tissue does not form a continuous mantle round the stem, but is divided into from ten to fifteen thick strands by strips of hard bast (see fig. 81). Below this cortex of alternating green tissue and strips of bast are soft bast, cambium, wood, and a very large pith; but these have no further interest for us here. It is, however, worthy of remark that in the green strands of the cortex

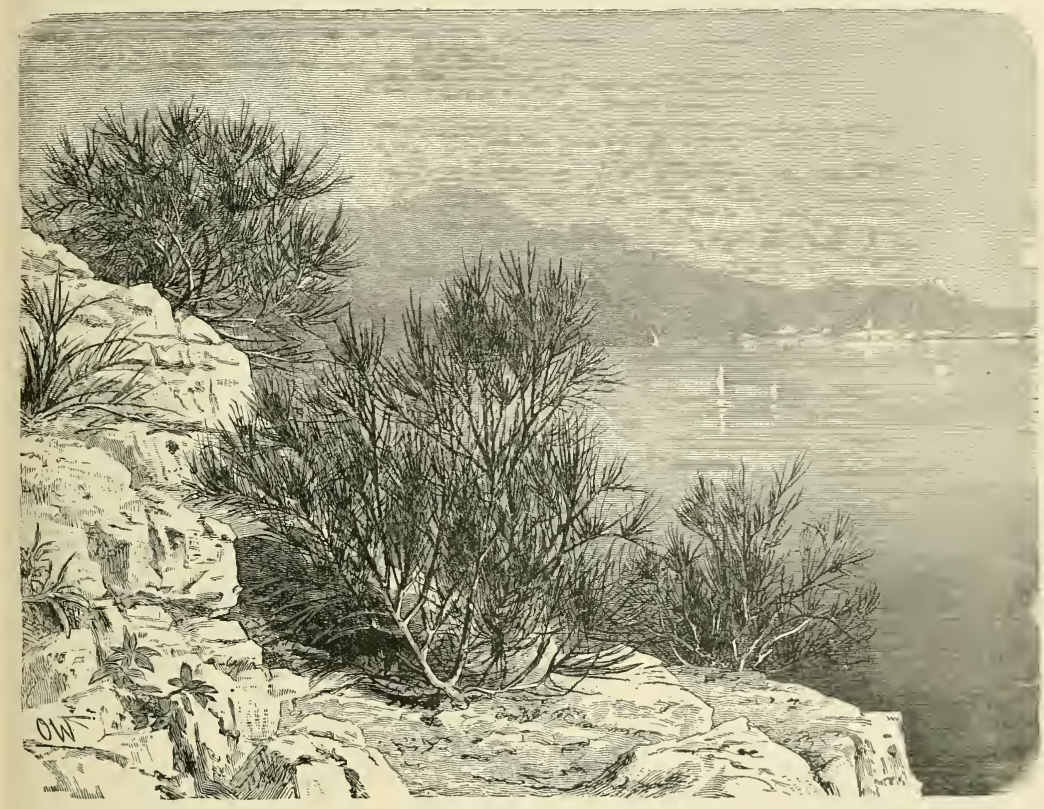

Fig. 80. - Switch-plants.

Bushes of Spartium scoparium near Rovigno in Istria.

of the Spartium, the crowded green chlorophyll-containing cells of the chlorenchyma closely adjoin one another, and that only very narrow air-passages ramify between them, so that here there is no formation of a spongy parenchyma penetrated by wide canals and passages. On the other hand, large cavities occur where the green tissue touches the epidermis, and these act as substitutes for the wide ramifying canals. Over each of the cavities a stoma is to be seen in the epidermis through which the water vapour, exhaled chiefly from the green cells, can escape (see fig. $81^{2}$ ). The stomata are proportionately small, but their number is very great. Since the guard-cells are not so strongly thickened on their outer walls as are the other epidermal cells, the stomata appear to be somewhat sunken. By this arrangement, and also by the epidermal coating of wax, they are protected from moisture. 
In the Casuarineæ and in Cytisus radiatus (see fig. 69), the green tissue is distributed in the cortex of the branches exactly as in the case just described; but the strips of green tissue traversing the stem are deeply cut into by longitudinal furrows. In some other leafless switch-shrubs, such as species of the genus Ephedra, the chlorenchyma forms a continuous and uniform mantle round the stem, uninterrupted by strips of bast. But in this case the stomata are distributed uniformly over the whole surface of the rod-shaped branches, while in the brooms, Casuarineæ, and in Cytisus radiatus they are absent from those portions of the epidermis which cover the strips of hard bast.

Plants with leaf-like branches or cladodes are distinguished from switch-plants
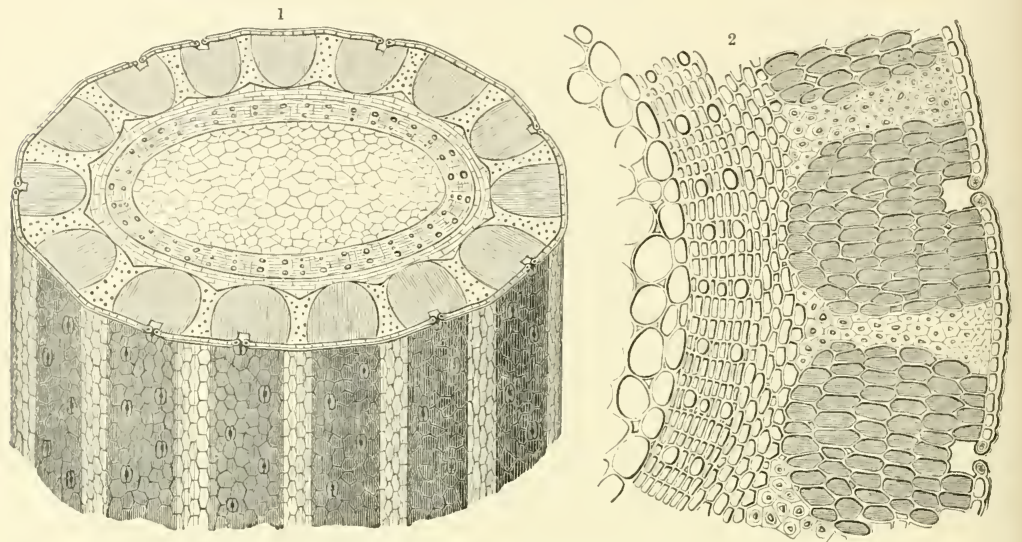

Fig. 81.-Switch-shrubs.

1 Part of stem of Spartium scoparium cut transversely; $\times 30.2$ Part of the transverse section; $\times 240$.

by the fact that all their shoots are not circular in section, but some are flattened, looking as though they had been pressed out. When this flattening is restricted to the so-called "short branches", i.e. when on a stem only the ultimate, comparatively short branches are flattened, the main axes remaining cylindrical, like ordinary stalks, these structures have quite the appearance of leaves which are sessile on the rounded stems. This explanation of them, however, given by botanists, is not at first sight satisfactory to the uninitiated. Why should these flat green structures be branches, and not leaves? The illustration opposite at once makes the matter clear. It represents two cladode-bearing plants, viz. two species of Butcher's-broom (Ruscus Hypoglossum and aculeatus), each at an early stage of development and also when fully grown. On the young shoots, which have just made their way out of the soil (see figs. $82^{1}$ and $82^{3}$ ), the true leaves can be seen in the shape of small sessile pale scales on the long, rounded, finely-ridged axis; and from the angles which these scales make with the long axis arise darker, much thicker organs which rapidly increase in size, while the supporting covering-scales become dry, shrivel up, and finally 
disappear, leaving no traces. Since the members which arise from the axils of leaves (whether these are small clothing-scales, or large green laminæe does not matter) are not considered to be leaves, but shoots, the flat leaf-like structures of the Butcher's-broom are also regarded as shoots, and are named "flattened shoots" (cladodes) -or, considering their similarity to leaves, "leaf-branches" (phylloclades).

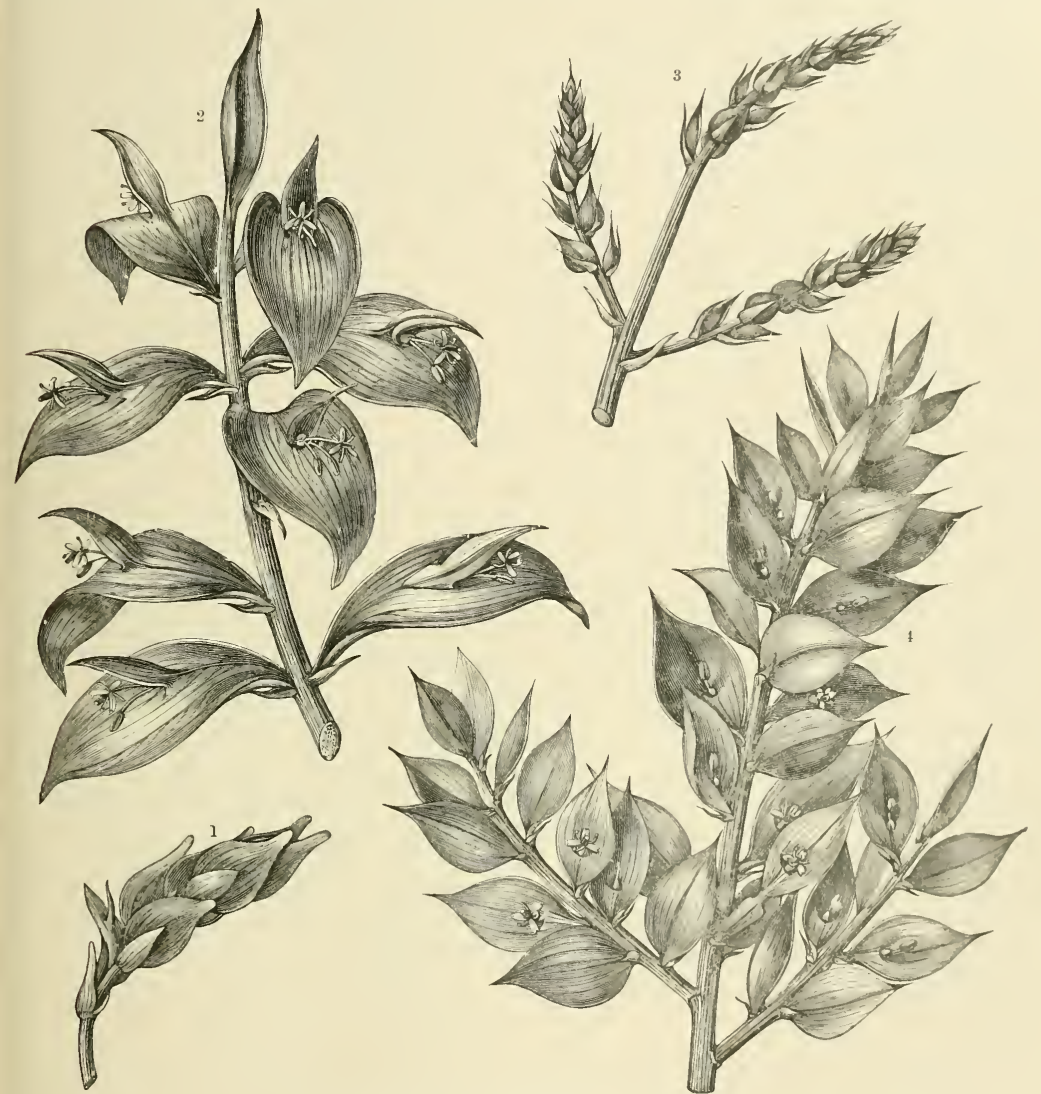

Fig. 82.-Plants with Leaf-like Branches (Cladodes).

1 Young shoot of Rusens Hypoglossum. 2 The same branch fully grown, with flowers on the cladndes 8 Ynune shnot. of Ruscus aculeatus ; The game braweh witb flowers ou the cladedes.

This view is strengthened materially by the fact that these leaf-like structures, $m$ their further development, and in the production of shoots, behave exactly like ordinary cylindrical axes. That is to say, small scale-like leaves spring from them. and from the axils of these scales arise stalked flowers (see figs. $82^{2}$ and $82^{4}$ ) which ultimately fructify. Plants possessing such phylloclades are not very numerous on 
the whole. The Butcher's-brooms, chosen above as examples, belong to Southern Europe, and occur in large quantities on the soil of dry woods, where everything is wrapped in deep sleep during the height of summer. In the Antilles, and in the prairies of the East Indies, are about twenty shrub-like species, belonging to the genus Phyllanthus of the Spurge-family. New Zealand also possesses one of these peculiar phyllocladous plants, belonging to the papilionaceous genus Carmichelia. In the species of both these genera (see fig. 83) the flattened shoots are exceedingly like lancet-shaped foliage-leaves, and the true leaves are transformed into small pale scales. These tiny scales are situated on the margins of the phylloclades, and from their axils arise stalks bearing the flowers and fruit. On the Andes of South America occur the remarkable colletias, of which a species, Colletia cruciata, is represented in fig. 83 . The leaflets on these extraordinary shrubs are diminutive, but not pale and scale-like; whilst the green phylloclades, which play the part of the foliage-leaves, form very strong flattened organs, tapering to a point, and placed opposite one another in pairs, so that each pair is always at right angles to the couple next above or below. Yet another arrangement is seen in Coccoloba platyclada (Polygonacex), a native of the Salomon Islands, and in Cocculus Balfourii, growing in the island of Socotra. But it is impossible here to enter into all these variations in detail; it is enough to have brought forward the most striking forms of phyllocladous plants which are represented in figs. 82 and 83.

If in all these peculiar plants the branches are flattened and spread out, it cannot indeed be asserted that the surface of their transpiring tissue has undergone diminution, and thus far of course this strange development has nothing to do with the restriction of transpiration. The arrangement by which this is brought about must be sought for elsewhere. It consists in this: the leaf-like shoots are so directed that their surfaces are vertical and not horizontal. Contrary to most flat leaves, which turn their broad surfaces fully to the incident light, the flattened shoots are placed vertically so that at mid-day they only cast a very narrow shadow, and do not stop the sunbeams on their way to the soil. It is obvious, however, that such a leaf-like structure placed vertically, as it were on edge, will exhale much less than a foliage-leaf whose surface is opposed to the mid-day sunbeams. The work carried on in the green cells, under the influence of light, is not hindered by this position of the leaf-like organs. If the vertical green surfaces are not so well illuminated by the sun's rays during the warmest part of the day, this is abundantly compensated for by the fact that their broad surfaces are exposed to the light both of the morning and evening sun. On the other hand, when the sun rises and sets, the heat is not so powerful, and consequently there is no such rapid exhalation to be feared as when the sun is in the zenith. To put the matter shortly, transpiration alone-not illumination-is restricted by the vertical position of the green laminæ, and therefore this metamorphosis has rightly been considered a protective measure against excessive transpiration.

This arrangement is only found in plants of dry regions, where transpiration 
requires no assistance, but where, on the contrary, the danger is often imminent that water cannot be drawn from the soil in sufficient quantity to replace that lost by exhalation.

The phylloclades, moreover, are only a type of a large number of organs which, in a word, all agree in this; the edge or narrow side of the flattened exhaling structure, not the broad surface, is turned towards the zenith. In many of the



Fig. 83.-Plants with Leaf-like Branches (Cladodes).

1 Colletia cruciata. 2 Carmichelia australis. 8 Phyllanthus speciosus.

vetches of the Southern European flora (Lathyrus Nissolia, Ochrus), but especially in a large number of Australian shrubs and trees, principally acacias (Acacia longifolia, falcata, myrtifolia, armata, cultrata, Melanoxylon, decipiens, \&c.), it is the leaf-stalks which are extended like leaves placed vertically, and then the development of the leaf-lamina is either entirely arrested, or has the appearance of an appendage at the apex of the flat green leaf-stalk, or "phyllode", as it is called. In many Myrtaceæ and Proteaceæ, especially in species of the genera Eucalyptus, Leucaden- 
dron, Melaleuca, Protea, Banksia, and Grevillea, the leaf-blades themselves are not placed horizontally like those of our inaples, elms, beeches, and oaks, but vertically on edge, like the phylloclades and phyllodes. Imagine now an entire wood of such eucalypti and acacias, on which the mid-day sun is pouring down its rays. If it is not exactly literally true to say that each vertical leaf only casts a linear shadow at noon, it is at least certain that there is not much shade on the ground of such a wood. The sunbeams find their way everywhere between the erect leaf-blades, penetrating the depths below, and it is impossible to speak of the dim forest-light under such circumstances. The Casuarineæ, which grow with eucalyptus, acacias, and Proteaceæ do not help to make such woods shady: and thus one is quite justified in speaking of the shadowless forests of Australia.

Although Australia stands alone in the variety and abundance of its plants possessing vertical leaf-blades, other floral areas furnish numerous and remarkable examples of this arrangement. One has only to think of the curious shape of the so-called "equitant" leaves belonging to many plants of the Lily family (Tofieldia, Narthecium), numerous Irideæ, and the closely-related genera, Gladiolus, Ferraria, Witsenia, Montbretia, \&c., chiefly natives of the Cape. The leaves exhibit the peculiarity of being folded together lengthwise, and the sides thus brought into contact become fused with one another. Only at the point where they join the stem do the two halves remain distinct, forming a groove in which is inserted the base of an upper leaf. The formation of such equitant leaves from ordinary leaf-blades may perhaps be illustrated by taking a strip of paper smeared on one side with paste and folding it longitudinally so that the pasted sides are in contact and become joined together. Such equitant leaves are so directed that their broad surfaces are much less exposed to the perpendicular rays of the mid-day than to those of the rising and setting sun.

In the Mediterranean flora, and on many steppes, plants are not seldom to be met with whose leaves look as if they had not been able to free themselves from the stem. In such plants the projecting portion of the foliage-leaf is very small, but the margins are continued for some way down the stem as projecting strips and wings. Leaves of this kind are termed "decurrent". They are particularly abundant amongst Composites, viz. in the genera Centaurea, Inula, Helichrysum; but they also occur in many Papilionaceous plants and Labiates. The position of these vertical wings, which traverse the stem, is exactly the same, with regard to the sun, as that of the phyllodes, phylloclades, and equitant leaves, and they behave in respect to transpiration in exactly the same way.

In many plants the blarles of the foliage-leaves when young have not a vertical position, but graclually assune it during development, i.e. the blades at first are turned so that the flattened surfaces are horizontal and face upwards and downwards. Later they twist round at the point where they are inserted on the stem, so that their margins become directed upwards and downwards. As already stated, this peculiarity is observed in many eucalypti and various other Australian trees and shrubs. But plants in sunny situations in other regions also exhibit this 
peculiarity. In the Spanish flora, for example, is an Umbellifer (Bupleurum verticale) whose leaves are so twisted with regard to the sun that they remind one forcibly of the Australian acacias. Many Composites, especially the widely-distributed Wild Lettuce (Lactuca Scariola), growing on dry soil in Central Lurope,
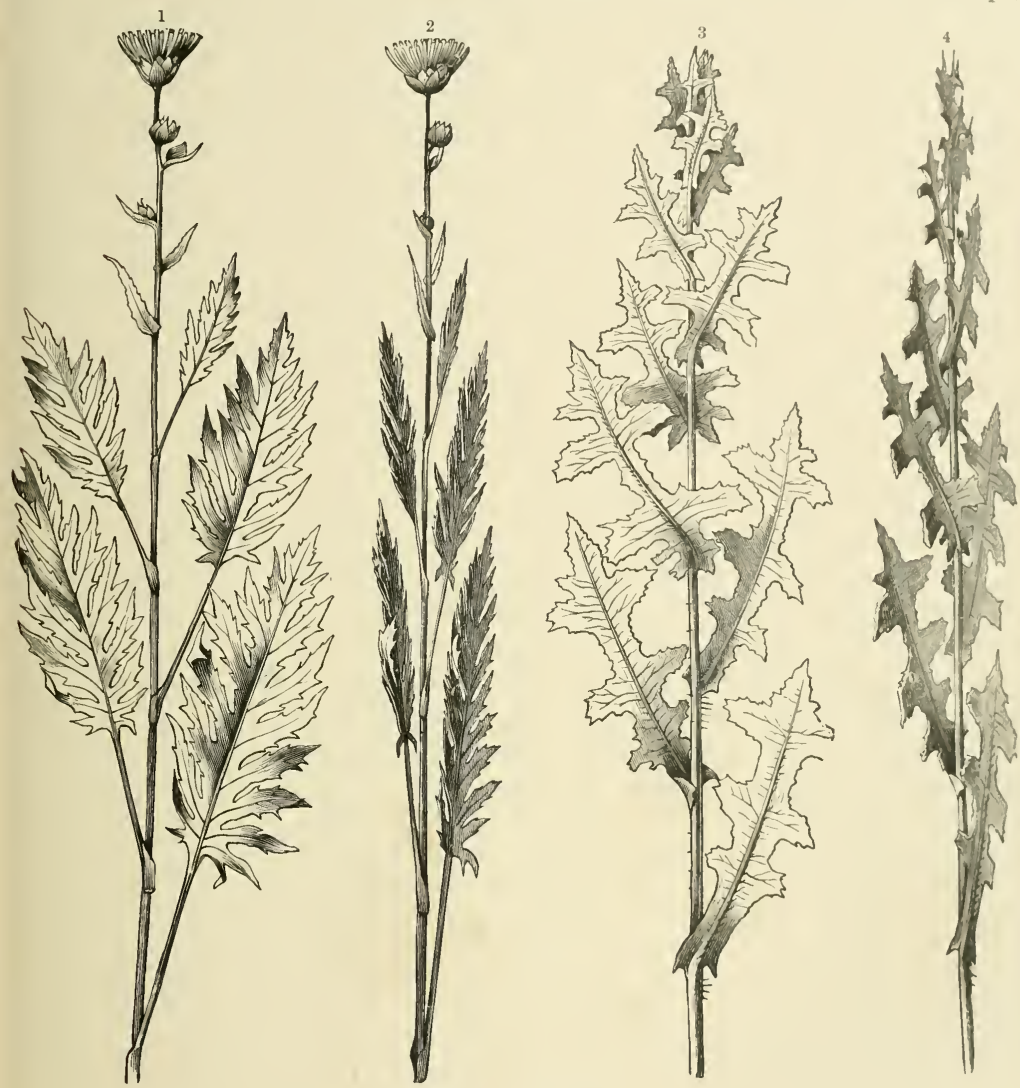

Fig. 84.-Compass Plants.

${ }^{1}$ Silphium laciniatum, seen from the east. 2 The same plant seen from the south. 8 Lactuca Scariola, seen from the east. 4 The same plant from the south. Both species are considerably reduced.

exhibit this contrivance in a striking manner. A Composite shrub, Silphium laciniatum, to be found in the prairies of North America, from Michigan and Wisconsin as far south as Alabama and Texas, has obtained a certain renown by reason of the remarkable twisting of its leaf-blades. It long astonished hunters in the prairies that in these plants (represented in fig. 84) the leaf-laminæ, especially thnse springing from the lowest portions of the stem, not only assumed a vertical voL.:. 
position, but that the broad surfaces of each leaf always faced the rising and setting sun. Healthy living plants as they grow in the sunny meadows look as though they had been laid between two gigantic sheets of paper, somewhat pressed, and dried for some time in the way plants are prepared for herbariums, and had then been removed from the press and set up so that the apex and profile of the vertical leaf-blades point north and south, i.e. in the meridian; while their surfaces face the east and west. This inclination is so well and regularly observed by the living plants on the prairies, that hunters are enabled to guide themselves over such regions, even under a clouded sky, by means of these plants; for this reason Silphium laciniatum has been called a "compass" plant. The life of the compass plant is assisted by this placing of the vertical leaves in the meridian, in that the broad surfaces are placed almost at right angles to the incident sunbeams which illuminate them in the cool and relatively damp morning and evening, while at the same time they are not too strongly heated nor stimulated to excessive transpiration. At mid-day, on the other hand, when the sun's rays only fall on the profile of the leaves, the heating and transpiration are proportionately slight. It is of interest that the leaves of these compass plants, as well as those of the above-mentioned Lettuce represented with the compass plant in fig. 84, show this inclination and position when they grow on level, moderately dry, unshaded ground, and that in damp shady places, where there is no danger of over-transpiration from the powerful rays of the noon-tide sun, the twisting of the leaves does not take place, and they are not brought into the meridian.

The placing of their leaf-blades parallel to the ground when in the shade, but vertically when in dry sunny places, is, generally speaking, a phenomenon which may be seen in very many plants, including shrubs and trees. A species of lime, a native of Southern Europe, viz. the Silver Lime (Tilia argentea), is particularly noticeable in this respect. On dry hot summer days the leaves assume an almost vertical position, but only on those boughs and twigs which are exposed to the sun. If the tree stands at the foot of a wall of rock, or on the edge of a thick wood, so that a portion of it is shaded, the leaves on this shaded part remain extended horizontally. Such a tree then presents a strange aspect, as the leaves are of two colours-dark green on the upper side, and white on the under surface by reason of a fine felt-work of white stellate hairs-and it is scarcely credible at first sight that the shaded and sunny portions of the tree belong to one another.

In the compass plants and also in the Silver Lime the alterations in the direction of the leaves are brought about by alterations in the turgidity of certain groups of cells in the leaf-stalk. It is exactly the same cause which produces the periodic movements of numberless plants with pinnate or palmate leaves, and the leaf-folding of many grasses; and it is natural to conjecture that these phenomena of movement are also connected with transpiration. This is in part actually the case. In consequence of alterations in turgidity of the pulvini, the pinnate leaflets of the Gleditschias and some Mimosas rise up after sunset, while those of the Amorphas fall down, and assume a vertical position during the night; but this is con- 
nected with the nocturnal radiation of heat (as will be explained later) and not with exhalation. It is, however, equally certain that the placing together and folding up of leaves and leaflets in many other plants is brought about in order to prevent over-transpiration and consequent withering up. Many shrubby, thorny mimosas of Brazil and Mexico, when in their native habitat and position, extend their leaflets horizontally when evening approaches, contrary to the behaviour of the well-known Sensitive Plant (Mimosa pudica), and they remain in this position throughout the night. Next morning they are still widely outspread. As soon as the sun has risen, and its beams fall on the foliage, the leaflets shut together; the menacing thorns, which until now have been hidden by the extended leaves, become apparent; and the leaflets remain in the vertical position during the hottest and driest hours of the day. Towards sunset they again rise and are extended horizontally. There is but one exception to this cycle of changes-if the opened leaf is shaken by the wind, and if the sky has been gray and clouded all day. In the former case, under the influence of the wind, a rapid closure occurs; in the latter ease, when the weather is bad, they remain open all day. One of the Rutaceæ, Porliera hygrometrica, behaves like these mimosas. In Peru, the native country of these plants, where they abound, the opening and closing of the leaves has even been made use of for weather predictions, for when the vertical leaves are closed, dry hot weather can be reckoned upon; when they are open, damp cool weather. In the cultivated Bean (Phaseolus), moreover, alterations of position in parts of the leaflets may be observed to take place during the day. When the sun is powerful, the leaflets assume a vertical position, so that at noon the sun's rays only reach a small portion of the blade.

In several species of Wood-sorrel belonging to the South African flora, and also in the widely-distributed Common Wood-sorrel (Oxalis Acetosella), it may be noticed that the leaflets, as soon as they are directly struck by the sun's rays, sink down, so that their under surfaces-on which the stomata are situated-face one another, the three leaflets together forming a pyramid; while these same leaflets in damp shady places remain extended. The leaflets of the water fern, Marsilea quadrifolia, which grows in marshes and is distributed through Central and Southern Europe, temperate Asia, and North America, are very similar to those of the Wood-sorrel, but carry their stomata on the upper surface. As long as they remain floating on the surface of water, these leaflets are extended, but as soon as the water-level sinks and the leaflets become surrounded by air, they fold together above in the sunshine, and their position becomes vertical, precisely as in the compass plants.

As another phenomenon of this kind the periodic folding or closing of the leaves of grasses must be specially mentioned. It has long been noticed that certain grasses exhibit a very different aspect according as they are observed on a dewy morning or in the noon-day sunshine. In the morning their long linear leaves are fluted on the upper surface, or spread ouv quite flat. As soon as the humidity of the air diminishes, in eonsequence of the higher position of the sun, they fold 
together lengthwise; again after sunset they widen and become flat or fluted. This process may be repeated twice on a summer's day within twenty-four hours, if a storm intervenes at mid-day and is followed by a sunny afternoon. How much this depends upon the conditions of humidity of the air, is demonstrated by the fact that such grasses, when grown in pots, can be easily made to open and close their leaves by alternately sprinkling them with water and placing in damp air, and then for a short time exposing them to dry air. The leaf-folding in various species of Sesleria is exceedingly quick and also very interesting. The species of this genus grow principally on the Alps, Carpathians, and Balkans. They always grow together and often cover wide stretches of hilly and elevated districts with thick grassy turf. One species (Sesleria corulea) is distributed over Northern Europe in Finland, Sweden, and England. The closing of the leaves of these moorgrasses reminds one strongly of the Venus Fly-trap (Dioncea muscipula), which has already been fully described. It is indeed an actual shutting together of the two halves of the leaf. As in the leaf of the "Fly-trap", the midrib of the leaf of the Sesleria remains in its original position unaltered; also the two halves of the leaf do not come flatly in contact, but rise up obliquely so as to leave between them a deep, narrow, groove-like cavity, widest at its lowest part (see fig. $85^{2}$ ). While the open leaf turns its upper surface, rich in stomata, towards the sky, the two raised halves of the folded leaf are parallel with the incident sunbeams, and the folded leaf of the moor-grass may then be compared to the equitant leaf of an iris. In the cavity produced by the closing up of the leaf are the stomata, however, and thus the green tissue next them is excellently protected from the sun's rays as well as from the direct action of the wind. The epidermis of the lower surface, which is exposed on the folded leaf to all the agencies which excite transpiration, possesses no stomata, but is provided with a thick cuticle.

A leaf-folding similar to that of Sesleria, along the midrib, has been observed in the leaves of Avena planiculmis, which grows in sunny fields on the Sudetics and Carpathians. It also occurs in Avena compressa, and many others related to these species. The folding or closing of the leaves in the large section of fescue-grasses (Festuca) is carried on somewhat differently. In Sesleria, the opened upper surface of the leaf forms only a single shallow groove, and the folding only occurs at the midrib; but on the upper side of the fescue-grass leaf several parallel grooves are to be seen, and the green tissue is divided up by these grooves into several projecting ridges, exhibiting a very remarkable structure. In each ridge can be distinguished the base which forms a part of the under side of the whole leaf; then the apex opposite the base, belonging to the upper surface of the entire leaf; and finally, the two side portions forming the sloping sides of the grooves which run between the ridges (see figs. 87 and 88 ).

The greater part of each ridge consists of green tissue. The stomata on the ridge only open on the sloping sides facing the grooves. Neither the crests of the ridges nor the lower surface of the leaf exhibit a single stomate. The apex is without chlorophyll, and almost always has a border of elongated cells with strong elastic 
walls under the epidermis; the same thing occurs on the under side of the leaf (२.e. at the base of the ridges), which is formed of one or several layers of cells without chlorophyll, but furnished with thickened walls. The closing of the leaf is not so simple here as in the Seslerias. There the leaf-folding only produced a single deep channel, widened at its base; in the fescue-grasses all the small grooves between the ridges become narrowed by the closing, i.e. by the upward inclination of the right and left halves of the leaf, those adjoining the central ridge to the greatest
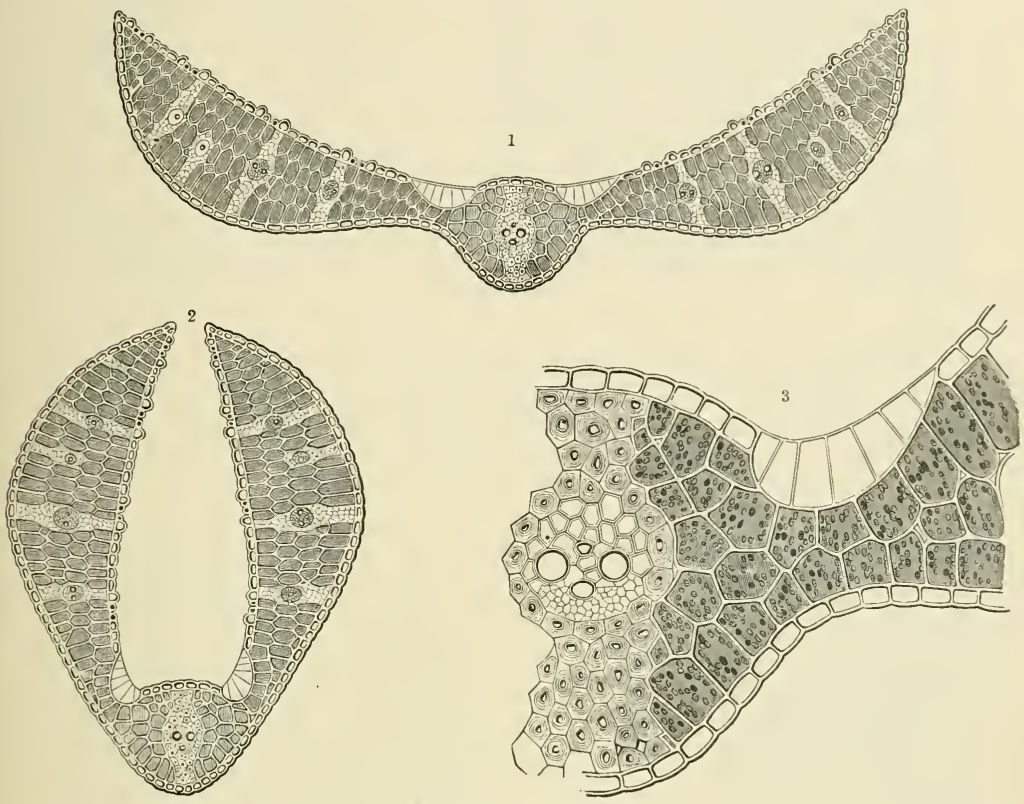

Fig. 85. - Folding of Grass-leaves.

1 Vertical section through an open leaf of the thin-leaved Moor-grass (Sesleria tenuifolia). 2 Vertical section through a closed leaf; $\times 40$. 8 Portion from the centre of an open leaf; $\times 300$.

extent, those in the neighbourhood of the approximated margins in a lesser degree (see fig. $88^{2}$ ). Since the stomata lie on the sides of the ridges, it is obvious that transpiration is checked to the utmost by the closing and consequent approximation of the opposite sides of each groove.

In individual cases among various fescue-grasses are to be found manifold differences in the number and shape of the ridges, also with respect to the formation of the under surface of the leaf, and most of all in the form assumed by the leaf in its expanded condition. There is a large group of festucas which are said to be poisonous by the shepherds in the mountain regions of Spain, and in the Alps, the Taurus, and the Elbruz. These will be spoken of again later. When open in damp weather they form only a moderately narrow main furrow, with several 
narrow secondary grooves leading from it, as can be seen in a vertical section of an open leaf of Festuca alpestris, a plant very abundant in the Southern Alps (see fig. $86^{5}$ ). In Festuca alpestris, the blunt apex of each ridge has a border, three layers deep, of cells destitute of chlorophyll, and the lower side of the leaf is provided with an actual armour of thick-walled bast cells, covered by an epidermis,
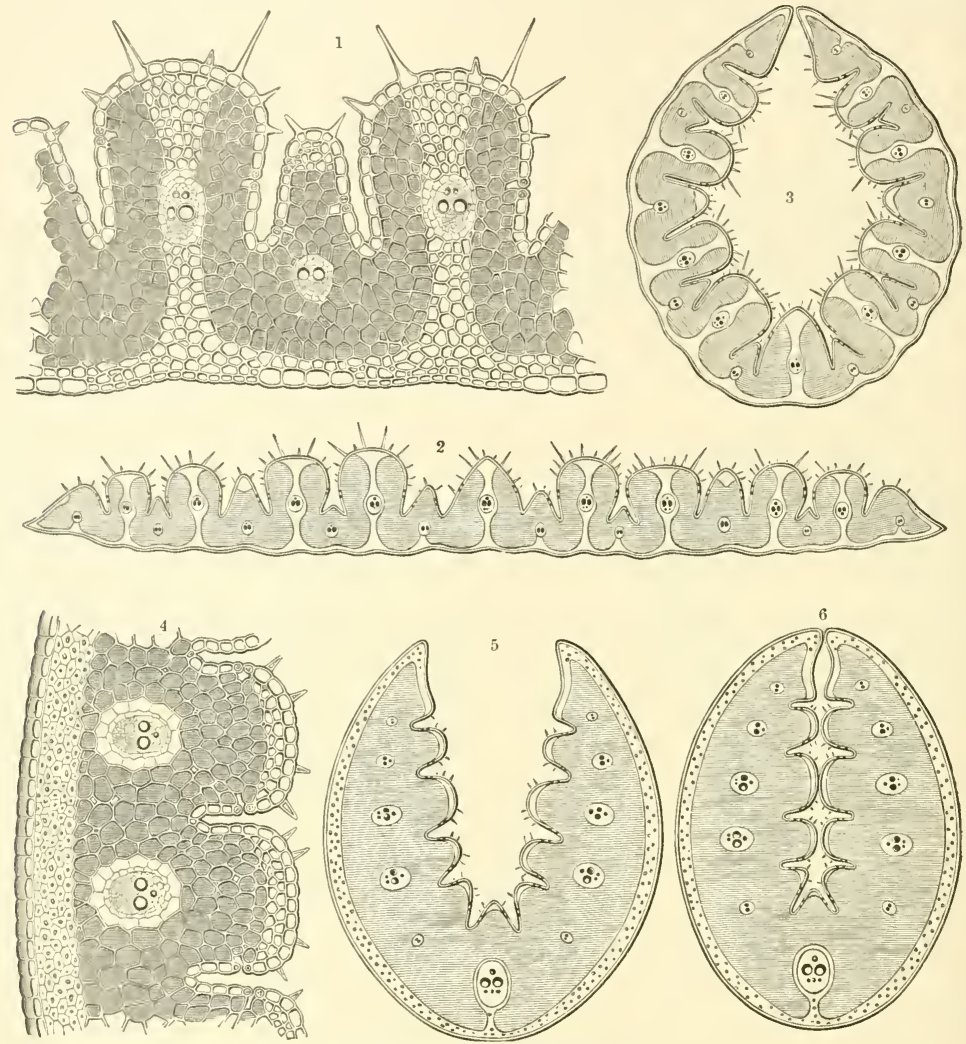

Fig. 86. - Folding of Grass-leaves.

1 Vertical section through part of the open leaf of Stipa capillata; $\times 240$. 2 Vertical section through an entire open leaf. ${ }^{3}$ Vertical section through a closed leaf; $\times 30$. 4 Vertical section through a portion of the leaf of Festuca alpestris; $\times 210$. ${ }^{5}$ Vertical section through an entire open leaf. $\quad$ Vertical section through a closed leaf; $\times 30$.

whose outer walls are much thickened. A vertical section through the leaf of Festuca punctoria, a native of the Taurus, is represented in fig. 88 . In this plant, the leaves, when open, present a fairly shallow depression; the under surface is clothed with a protective mantle of five layers of strong cells devoid of chlorophyll; the ridges are rounded off and possess only a single layer of covering cells, provided with an extremely strong wax-like coat. The open leaves of Festuca Porcii, 
a native of the Carpathians, are relatively thin (see figs. $87^{4}$ and $87^{5}$ ). Below the epidermis of the under side is no mantle of bast cells as in the species already described, but only isolated strands of bast; however, the crest of each ridge is furnished with a strand of bast cells; the ridges themselves project very much, and the whole leaf is traversed by six deep narrow grooves.

In the three fescue-grasses cited here as examples, and in all species of the genus Festuca, forming the main part of the turf of our fields, a vascular bundle
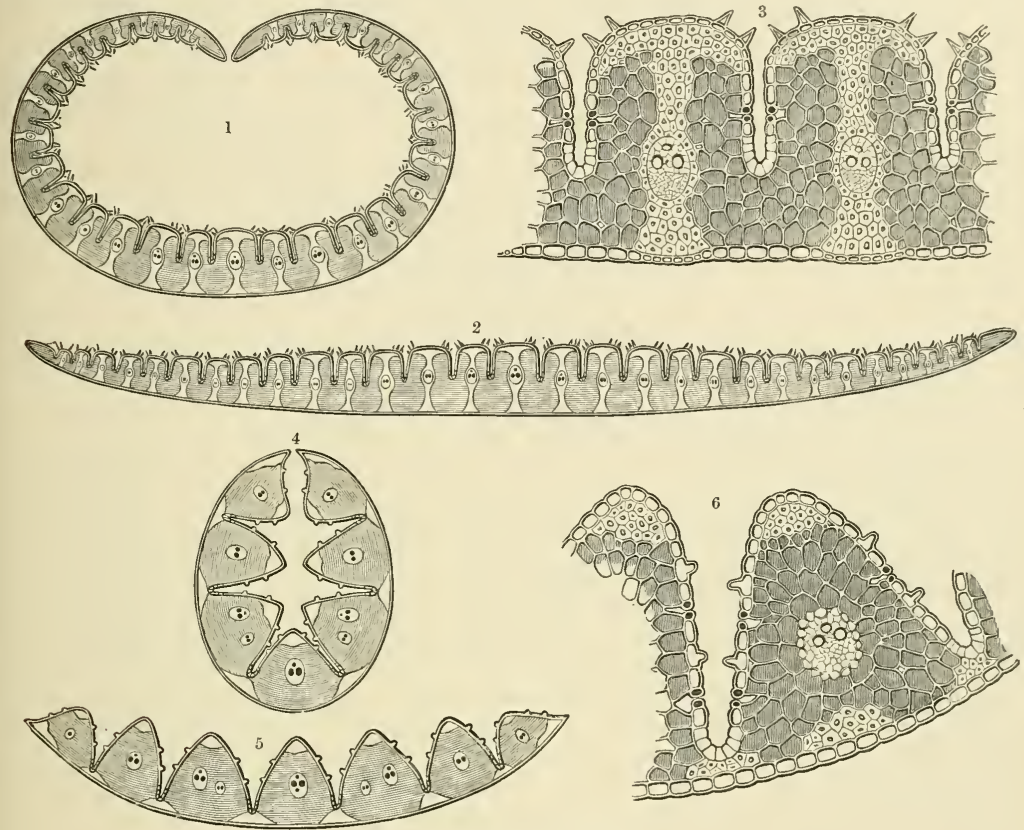

Fig. 87.-Folding of Grass-leaves.

1 Vertical section through a closed leaf of Lasiagrostis Calamagrostis. 2 Vertical section through an open leaf; $\times 24$. 3 Vertical section through a portion of the open leaf; $\times 210$. 4 Vertical section through a closed leaf of Festuca Porcii.

5 Vertical section through an open leaf; $\times 24,6$ Vertical section through a portion of the open leaf; $\times 210$.

surrounded by green tissue traverses each ridge. In the hinged leaves of many other grasses, the green tissue of each ridge is divided into two portions. The vascular bundle is bordered above and below by strands of thick-walled cells devoid of chlorophyll, and thus arises a strong septum in the green parenchyma, beautifully shown in the transverse section of a leaf of Lasiagrostis Calamagrostis, illustrated in fig. 87. In the leaves of the Feather-grass (Stipa capillata) are alternating higher and lower ridges; a vertical section is shown in fig. $86^{1,2,3}$. In the higher ridges nccur septa similar to those in Lasiagrostis, but in the lower there is only a vascular bundle surrounded by green tissue as in the fescue-grasses. No less than 
twenty-nine ridges can be counted on the leaf of the above-mentioned Lasiagrostis, a plant widely distributed in the valleys of the Western and Southern Alps, where it clothes the sunny slopes in thick masses. When the leaf folds up, the twentyeight grooves between the ridges, on whose sides are the stomata, become narrowed, and the entire leaf assumes a tubular form, so that transpiration is almost completely suspended. In Stipa capillata, which is very abundant on clay steppes, the same thing occurs (see fig. $86^{3}$ ). In both grasses the closure of the grooves on whose sides are the stomata, is completed by short stiff hairs on the summit of the ridges, which interlock when the ridges approach one another, and so block up access to the grooves (fig. $86^{3}$ ). It would take us much too far to describe the numerous other modifications which are to be met with in the structure of hinged grass-leaves. The examples given suffice to make it evident that the danger of over-transpiration is avoided by the folding of the leaf, and that amongst the grasses very many arrangements obtain in order, sometimes, to expose those green parts of the leaf whose epidermis is supplied with stomata to the rays of the sun, and at other times to withdraw them, according to the humidity of the soil and of the surrounding air, thus suitably regulating transpiration to the existing circumstances.

The mechanism by which grass-leaves open and close may be explained in two ways-either the process is due to hygroscopic changes, as in the opening and closing of the "Rose of Jericho", or to alterations in the turgidity of certain groups of cells, as in the mimosas. If the former alone were the case, a dry, dead grass-leaf should be still capable of opening and closing in accordance with its damp or dry condition; but a leaf of any of these when cut off and dried no longer opens, even after being moistened for a considerable time, and therefore the first explanation cannot be accepted, at any rate for most of the grasses. Apparently, the mechanism consists of alterations in the turgescence of those groups of cells situated in the angle of the grooves. Since the floor of the grooves was frequently found to consist of peculiar thin-walled cells destitute of chlorophyll, and filled with colourless watery sap, it was concluded that the opening and closing of the grass-leaves was due to the change in turgidity of these cells. However, this was going too far. These cells in most instances, for example, in Festuca punctoria (see fig. $88^{2}$ ), would be much too delicate to effect, unaided, the closure of the leaf by their loss of turgidity, or to open it by their increasing turgescence. In many grasses these cells are completely wanting (e.g. in Festuca alpestris and Stipa capillata, fig. 86). Moreover, it is observed that the opening and closing of the leaf is still carried on when the thin-walled cells at the bottom of the grooves are destroyed, artificially, by puncturing with fine needles. The cause of the movement must therefore be looked for in the alteration of turgescence of other cells below the grooves. When a mantle of several layers of thick-walled cells is present on the under side of the leaf, their walls are seen to swell up simultaneously with the alterations of turgescence of the parenchymatous cells. Of course the inner cell-layers of the mantle must be capable of swelling up to a greater extent than the outer, and this has 
actually been shown to be the case in some species. Moreover, although the thinwalled cells at the bottom of the furrows are not considered strong enough to bring about the opening and closing by changes in their turgidity alone, it is by no means asserted that they have no other part to play. When they are constructed as in the leaves of the moor-grasses and in the fescue-grass of the Taurus (Festuca punctoria, figs. 85 and 88 ), they certainly are not without a purpose. Their advantage to the plant lies in the fact that they can be much compressed without harm by the closure of the leaf, whereby the neighbouring parenchymatous cells are pro-

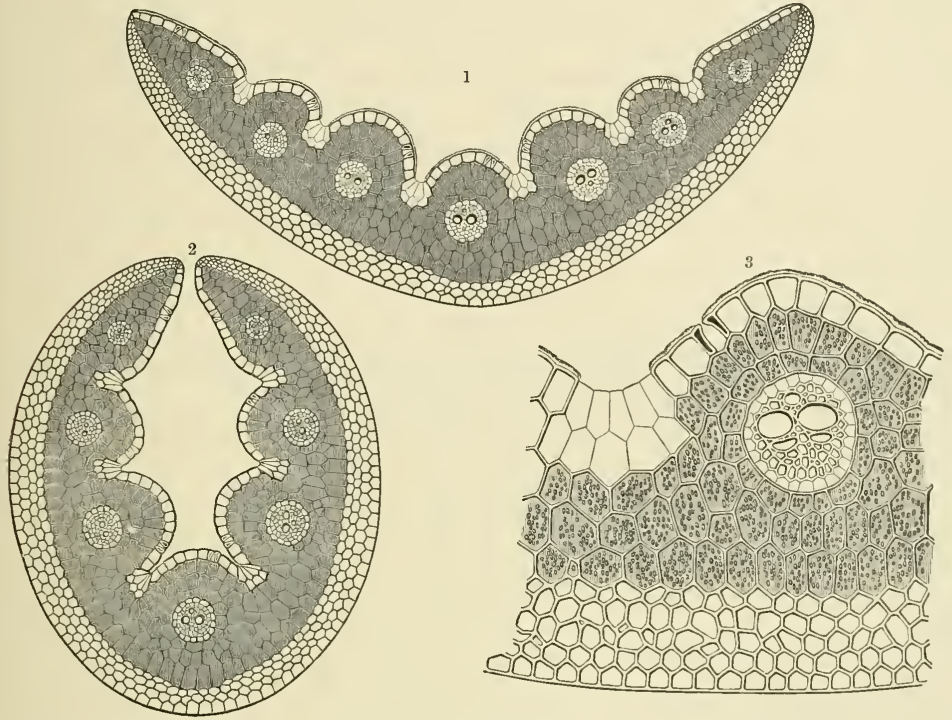

Fig. 88.-Folding of Grass-leaves.

${ }^{1}$ Vertical section through an open leaf of Festuca punctoria, of the Taurus. 2 Vertical section through a closed leaf; $\times 40$. 3 Vertical section through a portion of the open leaf; $\times 280$.

tected from injury; also that by means of these cells, which are filled with watery sap, carbonic acid from the atmosphere is conducted to the underlying green tissue; and lastly, that in case of necessity, water can be absorbed from the air. They remind one strongly of the thin-walled groups of cells of foliage-leaves used for the direct absorption of moisture, and possibly they can function in this way. If, in places where these grasses grow naturally, a slight shower of rain falls after a long period of drought, or if dew falls during clear nights, little or none of the water reaches the roots, since it is retained by leaves overspreading the soil. But the water easily runs into the furrows of the folded leaves of grass, and since the large thin-walled cells at the bottom of the grooves can be wetted, they offer to the water which can pass through them the shortest path to the green cells in the interior of the leaf. 
A process, very similar to the opening and closing of grass-leaves, is also to be observed in the true mosses, in all species of the genus Polytrichum, and in some of the Barbulas. The peculiar structure of the leaves of these mosses has been already treated of. In addition to the description there given, it may be mentioned that the ridges of thin-walled green cells, which are present on the upper surface of such a leaf (see fig. 89), only remain exposed to currents of air as long as this air possesses the requisite degree of humidity; that is to say, the blade of the leaf from whose upper surface the bands project only remains expanded while that is the case (fig. $89^{2}$ ).

As soon as the air becomes dry, the lateral portions of the leaf-blade bend upwards, and envelop the green ridges like a mantle (fig. $89^{1}$ ). These are then
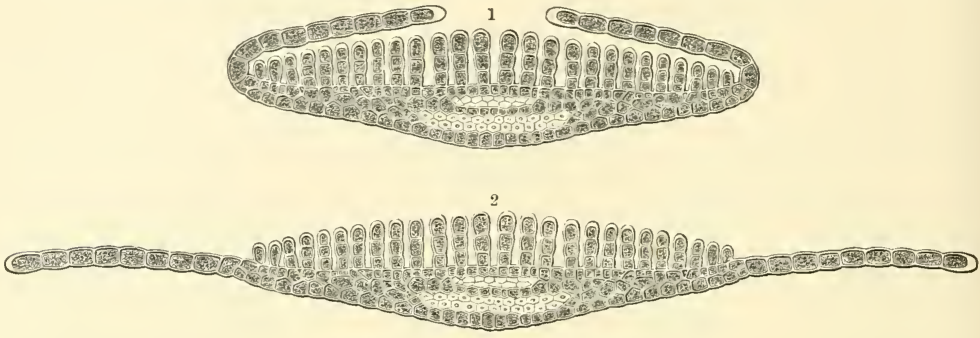

Fig. 89.-Folding of Moss-leaves.

Transverse sections through the leaf of a Polytrichum (Polytrichum commune). 1 The leaf dry and folded. 2 The leaf damp and open; $\times 85$.

inclosed in a hollow chamber, and only retain communication with the surrounding air by a narrow slit above, which is left open between the inflected leaf-margins. But here again it should be noticed that the highest cells in each ridge are strongly thickened on the part turned towards the opening, which doubtless helps to lessen transpiration. The opening and closing of the Polytrichum takes place very rapidly. By repeated hygrometric changes in the air, the process may be performed naturally several times in a single day. In Polytrichaceæ, which have been plucked while their leaves were open, the closure is seen to be completed, in dry air, in a few minutes. Dead and withered leaves are always closed, and never reopen, even when kept damp for a long time-from which it may be concluded that the mechanism of the opening and closing cannot be due to a simple hygroscopic phenomenon. Probably, the same mechanical forces come into action as produce the folding of leaves of grasses; but the process in moss-leaves is much more complicated, since it consists, not merely in the upward inclination of the leaf-edges, but also in an upward curvature and spiral twisting of the whole leaf. 


\section{4. 'TRANSPIRATION DURING VARIOUS SEASONS OF THE YEAR. TRANSPIRATION OF LIANES.}

Old and Young Leaves.-Fall of the Leaf.-Connection between the structure of the Vascular Tissues and Transpiration.

\section{OLD AND YOUNG LEAVES.}

The various regulators of transpiration, hitherto described, either persist in the plant-organs in question throughout life, or only remain for a comparatively short time. They are present throughout life in evergreen leaves, particularly in regions where wet and dry seasons alternate during the year. In this case the plants require powerful aids to transpiration in the rainy season, and in the dry season abundance of protective measures against excessive loss of water. Evergreen leaves cannot afford to dispense with either the promoting or inhibiting arrangements after the expiration of the first year, because for several years they still have to pass through both these seasons. It is otherwise with those leaves whose activity only lasts for a single summer. These burst from the buds at the beginning of the vegetative period, and then unfold, transpire and respire for a few months, producing organic materials, and conduct them towards the places where they are required. At the commencement of the drought, however, or on the appearance of frost, they turn yellow and fade, are detached from the stems and branches which bear them, and die. In leaves of this kind, an arrangement which is very necessary during the first season may become superfluous later-it may even become disadvantageous under changed external influences, and the leaf would then be benefited by freeing itself entirely from the contrivance. It would often be useful to the plant to substitute in the place of a protective contrivance, which is only beneficial at the commencement of the vegetative period, another arrangement fitted to the new and altered conditions. In the so-called deciduous leaves, i.e. in those which throughout the year are only active in the summer, often only for two months, it is a fact that an alternation of this kind may be regularly observed in the mechanisms which govern transpiration.

It will be noticed in a young foliage-leaf which has just pierced through the ground, or in one which is still half-hidden between the cotyledons of a seedling, or surrounded by the loosening scales of a winter bud, that the development of that portion whose duty will later on be to transpire and assimilate, is very backward. The leaf-veins are already very prominent, but the green tissue is in quite a rudimentary condition. It is not only that the extent of the surface is very small, but that the epidermis which covers it is not yet properly developed; the outer walls of the epidermal cells are not yet fortified with a cuticle, and are consequently neither water-tight nor impermeable to aqueous vapour. If exposed to sun or wind, the green tissue would at once dry up. When the young foliage-leaf has 
forced its way out of the bud above the soil, or from between the cotyledons, the conditions are still the same, and therefore particularly efficacious protective arrangements are required that the leaves just merging from the bud, and thus exposed to the vicissitudes of the weather, may grow up properly, i.e. that their green transpiring tissue may be normally developed.

Some of these protective contrivances belong exclusively to the developing period of the leaves, and are lost when they become fully grown. Others may be seen in the adult leaves. The most striking instances are perhaps the diminution of the surfaces directly exposed to the sun and wind, the vertical inclination of the leaf-blades, and the conccalment of the green tissue under a protective mantle.

The diminution of the surface directly exposed to the sun and wind is caused by the position which the foliage-leaf takes up within the bud. Space is very limited here, and the youngest and smallest leaves appear to be fitted into the space by the rolling, or folding, or crumpling of their blades. This diminution is obviously of great advantage when the leaves open out into the daylight: it constitutes a special protection against the drying up of the green tissues, and is, therefore, retained until other protective measures are developed, and in some cases even throughout life. In many Polygonacer (e.g. Polygonum viviparum and Bistorta), in species of Butter-bur (Petasites), in some Primulacer, and especially in many bulbous plants, the green portions of the leaf are rolled. The midrib, and frequently a fairly broad central strip of the leaf in addition, remains flat, and the right and left halves are rolled up from the margins, sometimes towards the upper, sometimes towards the lower surface. The stomata are chiefly, or wholly, to be found on the concave side, beneath which lies the soft green tissue with its ramifying air-passages. In the Crocus, the two halves of the leaf are rolled outwards; they are connected togethe1 by a broad, white, central stripe which is not rolled, and is devoid of chlorophyll; in the Star of Bethlehem (Ornithogalum), whose leaves are traversed by a similar white stripe, the leaf margins are rolled inwards. In species of Crocus the stomata are placed in the two grooves on the under surface; in the Star of Bethlehem, in the grooves on the upper surface. The central stripe of the young leaves in the plants mentioned always remains flat, but in young fern-leaves, which are also rolled, the strongly-developed midrib is curled spirally inwards like a watch-spring, and thus the green feather-like pinnæ, springing from the rachis, are placed one above the other. Most ferns in their native habitat rarely require special protection against over-transpiration during the first stages of development; but when this is necessary, it is afforded in every case by the form assumed by the young leaf just described. Moreover, in such instances special protective envelopes are, as a rule, to be found, which will be spoken of later.

Leaves are not so often crumpled as rolled on first emerging from the bud. In crumpled leaves the net-work of anastomosing veins forms a strong lattice-work, and the green leaf-substance, fitted into the interstices of the lattice, is swollen up like bubbles or sunken into pits, giving the whole leaf the appearance of a crumpled sheet of paper or cloth. The vernation (or position occupied by the leaf in the bud) is 
therefore aptly termed "crumpled". Leaves specially noticeable in this respect are those of the many species of dock (Rumex), rhubarb (Rheum), and also of several spring primulas (Primula acaulis, elatior, denticulata, \&c.). Frequently the crumpling and rolling occur together, leaves with crumpled vernation having their lateral margins also somewhat rolled inwards.

Young leaves which have just burst from the bud, and still retain the form they possessed there, are very often seen to be "plaited". The veins of the leaf form,

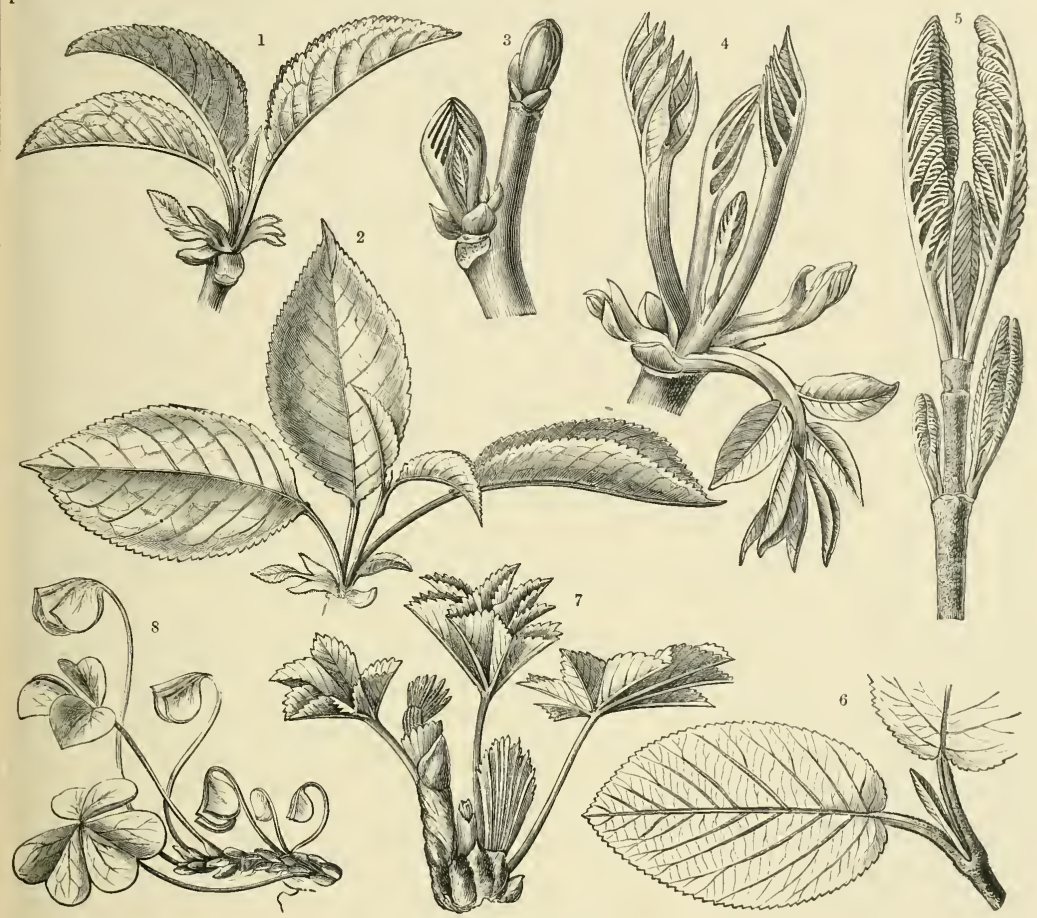

Fig. 90.-Unfolding of Leaves.

1, 2 Wild Cherry (Prunus avium). 3, 4 Walnut (Juglans regia). 5, 6 Wayfaring Tree (Viburnum Lantana).

7 Lady's-mantle (Alchemilla vulgaris). 8 Wood-sorrel (Oxalis Acetosella).

as it were, the fixed framework, and it is only the green portions between which are laid in folds. From the multiplicity in form and division of the leaf-veins, the kind and manner of folding is also very varied. When the leaf-blade is traversed, by radiating veins, as, for example, in the Lady's-mantle (Alchemilla vulgaris), shown in fig. $90^{7}$, the leaf is folded in vernation just like a fan; the veins which radiate out in the adult leaf are as yet parallel to one another, and the green portions which in the fully-formed leaf are stretched between the veins, form deep folds, which are closely packed together. The same arrangement occurs when each of the radiating: 
veins becomes the midrib of a leaflet, as in the cinquefoils, and species of clover and Wood-sorrel. Each leaflet is folded up along the midrib like a sheet of paper, and the folded leaflets are placed side by side in the same way as folded leaves in a book.

When the leaves are pinnate, and the leaflets are arranged in pairs on a common rachis, the latter are folded together along their midribs, and placed side by side, so as to resemble the pages of a book. This vernation occurs in roses, Mountain Ash (Sorbus aucuparia) and Walnut (Juglans regia), see figs. $90^{3}$ and $90^{4}$. In the roses the rachis is so short in the bud that the leaflets springing from it appear to originate from one point, as in the cinquefoils. In most maple-leaves and those of Saxifraga peltata, the folding takes place not along the radiating veins alone, but along the short lateral veins which spring from the larger radiating ribs. In this way small folds are inserted between the larger, and this vernation leads up to that which was described before as "crumpled". The leaf-folding exhibited by the foliage of the Beech (Fagus silvatica, see fig. 92), the Hornbeam and the Hophornbeam (Carpinus and Ostrya), the Oak (Quercus), and many other plants, whilst in the bud, is very characteristic. Each foliage-leaf possesses a midrib and numerous strong lateral veins, which run right and left from the midrib like the bony processes from the spinal column of a fish. The green portions of the leaf form deep folds between these lateral veins, which are as yet very close to one another, and the folds are thus arranged exactly as in a fan. Yet another method of folding occurs in the Cherry (Prunus avium). Each leaf, while in the bud, and for some time after it has burst from it, is folded along the midrib only (see figs. $90^{1}$ and $90^{2}$ ). The right and left halves are so flatly folded together, and fit over one another so completely, that at first sight they appear to form only a simple leaf-blade. Moreover, the two halves which are in contact are actually joined by means of a balsamlike secretion. At this stage of development they are always erect; and this brings us to another protective contrivance to be observed in young undeveloped leaves.

It may be stated that, with the exception of a few "crumpled" forms, all young foliage-leaves when they emerge from the bud-scales, or from between the cotyledons, or as they force their way through the soil into the light of day, are so directed that their blades are not horizontal. In this first stage of development, indeed, the green transpiring, but still delicate, portions of the leaf have always a vertical position. Their blades usually exhibit the direction observed in phylloclades and phyllodes, in the equitant leaves of irises and tofieldias, in the leaves of the compass plants during their greatest activity, and in the leaves of grasses when folded together in dry air. Sometimes the entire extended or rolled blade is erect, as in most bulbous plants and grasses; or the midrib is inclined towards the horizon, in which case the halves of the leaf are folded together and the two margins come into contact, forming a sharp edge which is turned towards the sun at noon. This is seen in some grasses (Glyceria, Poa), and in the Cherry (Prunus avium). If the blade is not erect, the stalk of the leaf is perpendicular while the 
still delicate blade hangs from it like a closed parasol, as in Podophyllum, Cortusa, Hydrophyllum, and several Ranunculaceæ. In the Horse-chestnut (Asculus Hippocastanum) the folded leaflets are erect when they emerge from the bud; they then sink down so that their apices point to the ground; and later, when the epidermis has become more thickened, they again rise until they are almost horizontal. Leaves of limes (Tilia grandifolia and parvifolia) are vertical when they first break through the bud, the apex directed towards the ground; it is only later that they become almost parallel with its surface. The upright leaf-stalk is often bent like a hook at the end, and the vertical folded leaflets depend from the hooked portion. This arrangement is shown in the common Wood-sorrel, and many other plants (see fig. $90^{8}$ ).

A third method of protecting these delicate undeveloped green portions of young leaves consists in the formation of screens and coverings, which exhibit the greatest variety. The envelope is frequently furnished by the so-called stipules. In many plants two lobes arise on the right and left of the leaf-stalk at the point of junction of the leaf and stem, and these have been termed "stipules" (stipulce). In figs, oaks, beeches, limes, magnolias, and numerous other plants, the stipules are membraneous, pale, usually without chlorophyll, and they appear like scales placed as screens in front of the small, tender green leaflets when they burst through the bud, and in any case must be considered to protect them from the sun's rays (see fig. 92). When once the young leaf has grown beyond the top of these screens and no longer needs them, they shrivel up, are detached, and fall to the ground. Millions of such fallen scales, called in botanical terminology "deciduous stipules", are to be seen on the ground in oak and beech forests shortly after the leaves have attained their normal size. The stipules of magnolias, particularly of the Tuliptree (Liriodendron tulipifera), a native of North America, but now cultivated all over Europe, are very remarkable (see fig. 91). They are comparatively large and boat-shaped, and are always so arranged in pairs as to form a closed cup. Shut up within this membraneous, slightly transparent cup can be seen the young leaf, its stalk being bent into a hook, and the two halves of the blade folded together along the midrib like those of the Cherry. In this position the leaf grows gradually as if in a small greenhouse; it enlarges, and as soon as the epidermal cells are so much thickened that there is no further danger of it drying up, the cup opens and the two boat-shaped stipules separate from one another, shrivel up, and at length fall off. Only two scars at the base of the leaf remind one that two stipules were situated here in the spring, whose function was to protect the delicate young leaf from desiccation.

One of the most noticeable arrangements for the protection of the tender, undeveloped green tissue consists in the peculiar grouping of the leaf-veins. This may be best observed in foliage-leaves which are folded along the lateral veins in vernation. Each individual leaf is erect, usually a little bent at the apex and margins, and slightly hollowed so that the upper surface is concave, and the lower side, which is turned towards the incident light, convex. Since the midrib of the 
leaf is still comparatively short, while the numerous lateral veins, on the contrary, are already strongly developed, the latter must lie so close to one another that they actually come into contact. Consequently on the under surface of the erect leaf, which is turned towards the sun, nothing can be seen of the delicate green tissue;

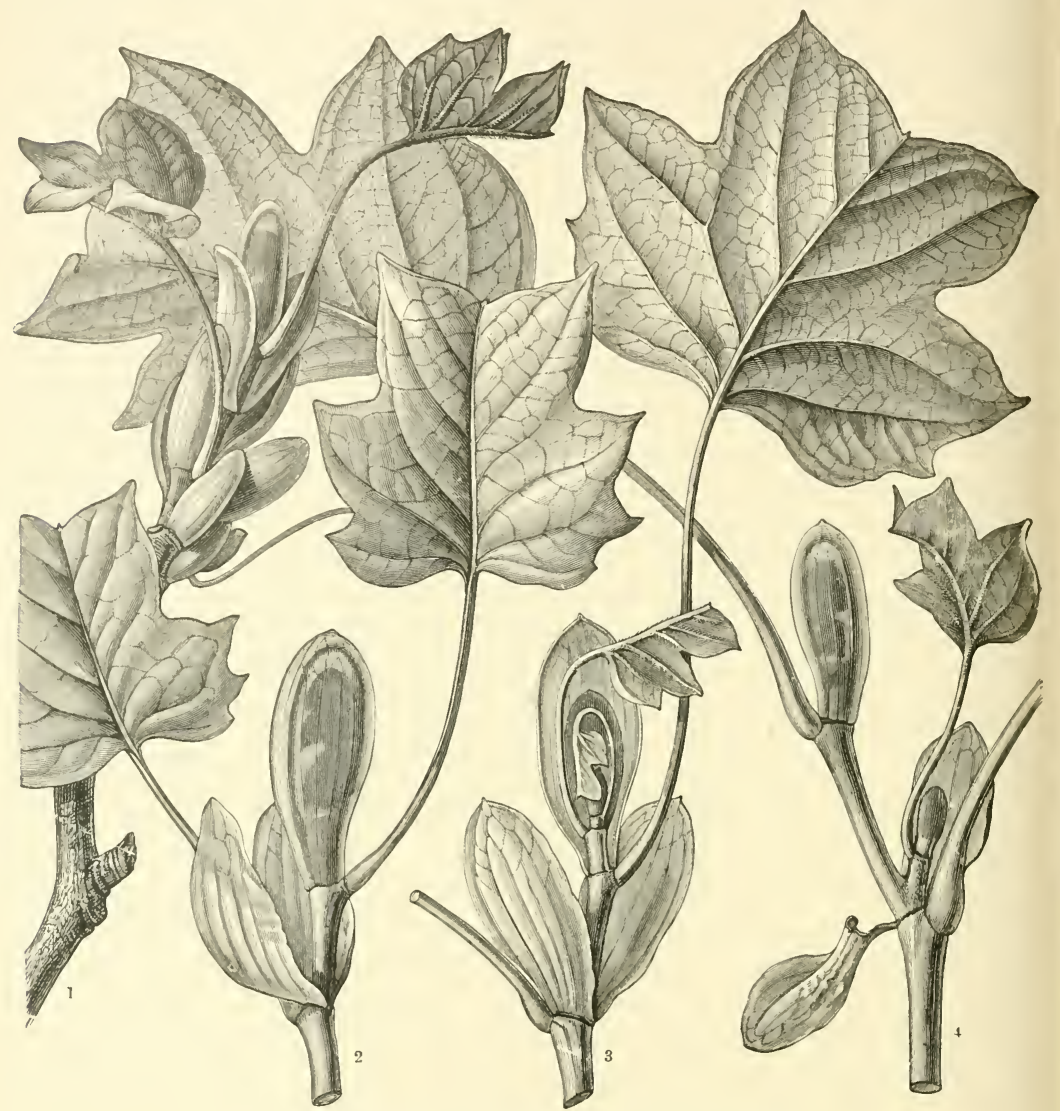

Fig. 91.-Leaf-unfolding of the Tulip-tree (Liriodendron tulipifera).

1 A twig at the end of which the leaves are beginning to unfold. 2 End of the same twig, the leaves being further expanded. 3 The anterior boat-shaped stipule artificially removed from the upper bud. One of the stipules about to fall off.

only the thick lateral veins, devoid of chlorophyll, stand out side by side like the supporting framework of a rush mat. The green portions of the leaf, which extend between the veins, form projecting folds on the concave surface, i.e. on the surface which is turned from the sun. They are thus hidden behind the close-pressed layer of ribs as if by a roof, and are consequently protected as efficiently as possible from 
the sun and wind. The ribs themselves are composed of cellular structures which are not open to the danger of over-transpiration, and the epidermis which covers them is entirely devoid of stomata. When the leaves at the ends of the young twigs are opposite, erect, and concave, and their margins are in contact, they form an actual capsule round the apex of the shoot. This occurs in the Wayfaring Tree (Viburnum Lantana), illustrated in fig. $90^{5}$. The small folds of green tissue project into the interior of the capsule, and the still closely-pressed lateral veins form the outer wall, and at the same time furnish a protective covering for the enlarging green portions of the leaf. As soon as these are fully developed, and the
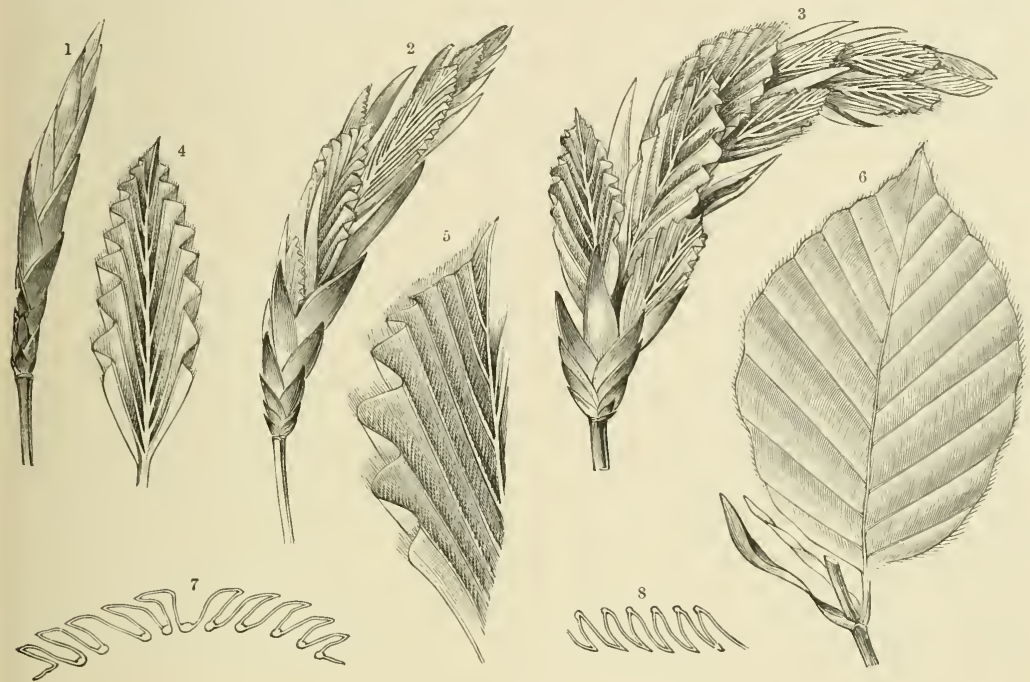

Fig. 92.-Unfolding of Beech-leaves.

1 The brown bud-scales have been loosened, and the membraneous stipules surrounding the foliage-leaves are visible above. 2 Further stage of development, the folded foliage-leaves being visible between the stipules. 3 The same twig further developed. 4 Lower surface of a young folded leaf. 5 Portion of the same leaf; the depressions caused by the folding are bridged over by silky hairs. BSurface view of an unfolded leaf; the stipules are withered and about to fall. 7 Vertical section of a leaf at right angles to the midrib. 8 Vertical section parallel with the midrib.

epidermal cells are correspondingly thickened, the projecting folds become smooth, the veins separate from one another, and the whole leaf becomes flat, assumes a horizontal instead of a vertical position, and turns the upper instead of the lower surface to the incident light (see fig. $90^{6}$ ).

It has already been repeatedly stated that coats of varnish as protective coverings are especially to be met with on young leaves, which they guard from over-transpiration and desiccation during their development, and that when the leaf-laminæ become provided with a cuticularized epidermis, these coats disappear. It has also been pointed out, incidentally, that coats of hairs are of great use as protections and screens to the young foliage-leaves when they first emerge from the VoL, I. 
buds. The leaves of a great number of plants are only hairy during the commencement of development. Long hair-cells may be seen inserted by their narrow bases between the flattened epidermal cells; these at an early stage shrink up close to their origin, and then break off. They may remain hanging to the leaf for a little while, but afterwards are thrown or pushed off by the enlargement and expansion of the leaf-blade, or are frequently blown away by the wind. The leaflets, which were originally quite thickly clothed with hairs, then appear partially or entirely smooth and green on both sides. A remarkable instance of this is furnished by A melanchier vulgaris, whose foliage, early in the spring, is folded along the midrib and covered with snow-white wool, reminding one strongly of the Edelweiss, while in the summer no trace of the covering remains. The White Poplar (Populus alba), pear-trees, and mountain-ashes behave in like manner. Horse-chestnut leaves, when they make their way through the brown, loosened bud-scales, are thickly covered with wool, but during the spring they lose this so completely that only here and there on the fully-formed leaves can remnants still be observed clinging to the leaf. It is, however, not only woolly coverings that are later either partially or wholly thrown off as superfluous. On the foliage-leaves of the alreadymentioned Wayfaring Tree (Viburnum Lantana) appear felted stellate hairs which fall off as soon as the epidermis is sufficiently thickened. In a species of Rhubarb (Rheum Ribes) brittle, candelabra-like, short-branched trichomes are situated on the edge of the leaf, which is much crumpled at an early stage, and later, when of no further use, they break away in pieces and fall off. Again, in many mulleins (e.g. Verbascum pulverulentum and granatense), there are branched, shrub-like hair-structures which become detached from the surface of the fullydeveloped leaves, and are carried away in loose flakes by the wind.

The covering of the young leaves of the Beech (Fagus silvatica) consists of silky hairs, and the way in which these are arranged and utilized is so peculiar that it is worth while to inquire further into the details. At first sight, the under surface of the young beech-leaf appears to be entirely covered with silky hair; on a closer examination, however, it is seen that the hairs are only inserted on the margins and on lateral veins, and that the green portions of the leaf are in reality perfectly smooth and free from hairs. Since the green portions of the leaf are thrown into deep folds (see figs. $92^{4}$ and $92^{5}$ ), and the veins are still close to one another, while the tops of the silky hairs springing from these veins reach far beyond the vein next to them, all the furrowed depressions caused by the folding are completely covered over. Each groove is bridged over by the hairs, which are regularly arranged, side by side, parallel to one another; thus the leaf appears to be clothed completely in a delicate silken coat. There can be no doubt as to the function of these hairs. The green tissue overspanned by them is protected from the sun until its epidermis is sufficiently thickened, and when this is the case the folds flatten out (fig. $92^{6}$ ) and the leaf assumes a horizontal instead of a vertical position, thus turning the lower surface away from the sun, and rendering the hairs of no further use. They have become 
superfluous, and usually fall off-or, if they still remain on the lateral veins, they are shrivelled, insignificant, and meaningless.

The dry membraneous scales seen on young fern-leaves should be mentioned here. Let us examine a frond of the first wild fern we meet-say of Nephrodium Filix-mas. The young frond is still spirally rolled, although it has forced its way through the soil, and is now exposed to the wind. Moreover, nothing is to be seen of the fresh green which later adorns this fern; the lower part of the midrib and lateral veins appear to be strewn with chaff, being entirely covered with dry membraneous brown scales and shreds. Later, as the leaf unrolls more and more, its green fronds also become expanded, but by this time the cell-walls are sufficiently strengthened, and no longer require the chaffy coat. In ferns which grow in sunny, rocky situations, and as epiphytes on the fissured bark of old trees in tropical regions, this coat of chaffy scales is even more noticeable, and, as stated earlier, in such plants it persists throughout life.

\section{FALL OF THE LEAF.}

Just as many phenomena of the sprouting and unfolding of foliage are dependent upon transpiration at the beginning of the vegetative period, so many processes, but chiefly that of the fall of the leaf, stand in causal connection with transpiration at the close of that period. Sooner or later, of course, the activity of each leaf entirely ceases; it dies, becomes detached from the stem to which it has rendered service, and falls to the ground, where it decays. In districts where the vegetation can continue its activity uninterruptedly throughout the year, there is nothing very noticeable about the fall of the leaf. As a rule, as the new leaves arise below the growing apex of the shoot, the lower, older leaves wither up and decay; the fall is quite gradual, and takes place, like the development of new leaves, all through the year. In neighbourhoods, however, where the changes of climate prevent the uninterrupted activity of plants throughout the year, it is essentially different. Trees and shrubs, and many smaller plants, shed the whole of their foliage in a few days at certain annually-recurring periods, and then remain with bare branches for a considerable time, apparently quite lifeless. This is the case in regions where a long, hot, dry period follows the short rainy season, and also in very cold districts where the long-continued frost causes an icy winter, and the plants are locked in a deep sleep. In tropical and sub-tropical regions, where no showers occur for many months at a time, the branches become stripped of their leaves. Even at the beginning of the dry hot season, they remain apparently dead for months, but again break out into leaf at the commencement of the cooler rainy season, when invigorating moisture is supplied to the parched ground. On the other hand, in those regions of the temperate zone in which there is no sharp distinction between the rainy and dry seasons, and rain falls every month, the foliage is stripped from the trees at the beginning of the cold period, and after the winter is over, fresh green leaves once more burst from the buds on the branches. 
It certainly appears strange that the leaf-fall should be sometimes connected with the approach of cold, and sometimes with that of hot weather. And yet this is the fact. Heat and cold are only the indirect causes; the primary cause of the fall of the leat is the danger threatened to the plant by the continuance of transpiration when either heat or cold is excessive. The danger of transpiration during continued dryness of soil and air scarcely requires much explanation. The conditions may be summed up in a few words: the throwing off of the transpiring surfaces when the drought commences, and the temporary stoppage of the sap-current-i.e. the socalled "summer sleep"-furnish one of the best protective measures in plants surrounded by air against excessive transpiration and withering. It is more difficult to explain the connection between the fall of the leaf and the commencement of the cold period. This is best indicated by some culture experiments which illustrate these relations. When the soil, in which are cultivated plants with actively transpiring leaves (melons, tobacco, and the like), is cooled down to a few degrees above zero, the leaves after a short time become faded, even although the temperature of the air and the humidity of both soil and air are entirely favourable. By the lowering of temperature in the soil, the absorbing activity of the roots buried therein is so reduced that the water which is lost by transpiration from the foliageleaves can no longer be replaced. The leaves wither, dry up, turn brown or black, and appear to be burnt or charred. In the ordinary language of gardeners they are said to be "frozen"-frozen at a temperature above the freezing point, which phenomenon is said to be due to the peculiar sensitiveness of these plants. It is incorrect to speak of freezing in this case, however. The plants are in reality dried up by reason of the low temperature of the soil and consequent lessening of the stream of fluid up to the transpiring foliage-leaves. In regions which annually pass through a long period of cold, the leaves of the plants are as liable to be dried up by the cooling of the soil round their roots when winter approaches, as are the trees in the catingas of Brazil when the hot dry season commences. They also denude themselves of their leafy raiment as these do, since otherwise they would be unable to make good the water exhaled by the leaves. When the temperature of the air sinks below zero, frost ensues, and the water in the plant stiffens into ice, this hastens the fall of the leaf, but it was already partially accomplished before the frost set in, and where the leaves still cling to the branches, preparations are already made for their detachment, which is brought about by the limitation of transpira tion. It must not be concluded from this that plants foresee the approach of winter, and that the preparations for the fall of the leaves result from such an intelligent foresight; the phenomenon is much more easily explained on the assumption that in a climate which renders necessary a long cessation of transpiration, those plants flourish and multiply best whose natural characteristic is to follow a period of energetic work by a long season of rest. The ultimate cause of this instinctively adaptive periodicity is certainly not yet explained; it is as mysterious as those life processes and phenomena which regularly recur at certain periods, which are perhaps hastened or retarded by favourable or unfavourable external conditions, but cannot 
be stopped by them, and which the plant carries out, or endeavours to carry out, without immediate external stimulus.

It is highly interesting, with respect to the acceleration or retardation of the leaffall, to observe how the same species of plant will behave under various favourable or retarding external influences; or how, in each region and locality, a selection has been made to a certain extent of the plants best adapted to the given conditions. First it is to be noticed that, under otherwise similar circumstances, the foliage remains green for a longer time, and is retained longer on the branches in places where the soil and air are more humid. In damp, shady, wooded glens, not only ferns, but the leaves of birches, beeches, and aspens are still green while on the sunny hillocks close at hand the brown leaves flutter down on to the withered fronds of the Bracken Fern.

The most remarkable fact, however, is that in elevated mountain regions a plant loses its leaves much earlier than does the same species growing in the lowlands. From the fact that in the Alps, the larches and whortleberry bushes, on the upper limits of the woods, put forth their green needles and leaves about a month later than in the valleys at a height of 600 metres above the sea, it would naturally be expected that this considerable delay would be compensated for by a corresponding postponement of the ending of the year's work, and that the fall of the foliage on the upper limits of the wood would also be postponed for about a month. But this is far from being the case. The same species of larch which becomes green a month later, up on the mountain slopes, also turns yellow a month earlier in the autumn. While the whortleberry bushes in the depths of the valley are still adorned with dark-green leaves, the same species growing in the glades on the upper limit of the wood, already, from the valley, appear to be shrouded in deep crimson. Their leaves are becoming discoloured above, and are withering and dropping from the twigs. The explanation of this phenomenon follows naturally from what has just been said. In the high mountain regions where tall trees find their uppermost limit, the ground is frequently covered with frost at the end of August; snow falls regularly in the first half of September, and although this may be melted in sunny places, the soil is nevertheless thoroughly cooled by the water so produced. The days rapidly become shorter, and the sunbeams can no longer replace the heat lost by radiation in the lengthened nights. The temperature of the soil in which the plants are rooted consequently falls rapidly, and the immediate results are that the absorbent roots stop working, the decolorization progresses, and the foliage-leaves, which are no longer able to repair the loss caused by transpiration, wither and fall away. Accordingly, on this upper tree limit, only those larches and whortleberry-bushes can thrive which are organized to commence their year's work a month later, and to finish it a month earlier, than those which have taken up their position 1400 metres below.

This obviously applies not only to the larches and whortleberries, cited here as examples, but to all other plants whose range of distribution extends from the lowlands up to the wood limit on the slopes of the mountains. It also applies 
further to those plants which have a wide horizontal distribution; for example, to those which grow wild or are cultivated from the lowlands at the northern foot of the Alps to South Italy, and even further south, on the further side of the Mediterranean. By journeying southwards, it will be seen that the beeches and elms which, on the northern foot of the Alps near Vienna, lose their colour in the beginning of October, are never discoloured before November on the mountains of Madeira, and that whilst the planes already show leafless branches in the North Tyrolese valleys at Innsbruck, they retain their leaves (although these are turning yellow) on the mild shores of Lake Garda at the southern foot of the Alps. In Palermo they are still adorned with dark-green foliage. Planes, indeed, in certain instances remain green all winter in Greece, and thus far it was no myth when Pliny spoke of evergreen planes. The Elder, which in the north is a deciduous plant, in Poti, on the Black Sea, retains its green leaves through the whole winter. In the oases of the North African deserts the Peach-tree keeps its foliage fresh and green from one vegetative period to another, and while the blossom of this tree in Central and South Europe unfolds on branches which have lost their foliage in the previous autumn, in the oases the flowers are situated amongst the still green leaves of the last period of vegetation. It may be confidently assumed that here also the cause is the temperature and humidity of the ground, and that the planes and peaches, whose roots at the end of autumn and winter are buried in a damp and relatively warm soil, are the last to throw off their foliage.

From all these considerations it cannot be doubted that the stripping of the foliage depends upon the stoppage of transpiration, and primarily upon the drying-up of those sources from which the transpiring leaves derive their water. Plants which denude themselves of their foliage of course lose with it much organic material, for whose production they have toiled for months; but this loss will stand no comparison with the advantages gained by the abscission of the leaves. In reality, it is only a framework of empty cells-the dead envelopes of the living portion of the plant-which is thrown away. The protoplasm has opportunely withdrawn, the plastids which carried on their activity in the cells of the foliage have migrated thence and taken up winter quarters in other sheltered parts of the plant-in the stem, roots, or tuhers, and have there deposited everything which will be of use in the following year, such as starch, sugar, \&c. The empty cells can thus be easily sacrificed to the common weal. The leaves fall to the ground, where they decay and help to form natural mould, of which the posterity of the deciduous plants reap the benefit. Since, by the formation of albuminous compounds in the leaves, an abundance of calcium oxalate arises which is of no further use to the plant, and is consequently stored up in such quantity at the end of summer that it at last becomes burdensome to the plants, the throwing off of the foliage must really be regarded as a method of removing waste materials, and may be compared to the excretion of waste which occurs in animals.

Finally, it should be noted that only plants whose foliage lies flat on the ground, or whose branches and twigs are very elastic and bear needle-shaped leaves; are 
unharmed by the pressure of snow. Trees, bushes, and shrubs with broad outspread leaves, such as planes, maples, limes, beeches, and elms, are not capable of supporting the weight of snow lying on their large leaf-surfaces. When, as occasionally happens, mountain and valley are covered in snow in the autumn before the leaf-fall has commenced, or when, late in the spring, to the terror of the farmer, snow falls on wood and meadow after the young leaves have attained to a considerable size, the devastation produced is fearful. The large-leaved shrubs are pressed down and their stems broken. Branches as thick as one's arm and huge tree-trunks are shattered, and in the woods quantities of maples and beeches are felled, or even uprooted. Such devastation would recur every year in regions with snowy winters if the leafy trees did not strip off their foliage in time, and it can easily be imagined what would happen to the woods after a series of such catastrophes.

There is, consequently, a widespread idea that the autumnal leaf-fall is brought about by frost. This idea is founded on the observation that when the temperature in October and November falls below zero, quantities of leaves drop from the branches in the early hours following the cold bright nights. Though it can scarcely be denied that the fall of the leaf is in some measure connected with frost, still that it is not always the immediate cause, is demonstrated by the fact that when plants with leafy branches are exposed at the end of August or beginning of September to a temperature below zero the leaves do not fall immediately; while, on the other hand, the foliage of limes, elms, maples, cherry-trees, \&c., is at last stripped off in the autumn even though no frost has occurred. It can only be said, therefore, as already stated, that frost is favourable to the fall of the leaf, and that it hastens the commencement of the process; but not that the detachment of the foliage is brought about by its sole agency.

The detachment of the leaves from the branches is brought about by the formation of a peculiar layer of cells, from the co-operation of a special tissue, which has been termed the layer of separation. As a rule, leaves cannot detach themselves without the previous formation of this tissue, not even if they are exposed for a long time to a very low temperature, and the sap in their cells and vessels is stiffened into ice. That portion of the leaf in which the separation is to take place is made up of a strong tough tissue, and the mechanical alterations produced by the frost would not suffice to complete the rupture. The separation-layer, on the other hand, which is formed within this tissue in one or several definite places, consists of succulent parenchymatous cells, whose walls are so constructed that they are easily separated by mechanical or chemical agencies, thus rendering possible a disintegration of the cell-tissue. The incitement to the construction of a layer of separation is indeed usually the limitation of transpiration by the gradual cooling of the ground, and the cessation of the absorbing power of the roots in those regions which experience a cold winter. As soon as this restriction of transpiration commences-and it varies very much, as shown in the previous discussion, with the latitude and altitude of the region in question-thin-walled cells arise in the lower 
portion of the leaves and leaflets, which rapidly increase by division, and in a short time form a zone, readily to be distinguished from the thick older tissue by its lighter tint and by the fact that it is somewhat transparent. Usually this zone is formed in the petiole, and at those places where the vascular bundles become narrowed in passing from the twig to the leaf-blade, there to divide up into the ribs and veins. The growing tissue is inserted just at this place; it actually presses and tears the other older cells apart, and even causes a rupture between them. As soon as the separation-layer has attained its proper thickness, its thin-walled cells separate from one another, but so as not to injure or burst their membranes in any way. It seems that the so-called middle lamella of the cell-wall is dissolved by organic acids, and that thus the continuity between the cells of the separation-layer is destroyed. The most trifling cause will now effect a splitting in the loose tissue and a fracture between the cells of the separation-layer; and when no other external shock follows, the detachment ul imately takes place of itself, the weight of the leaf helping to bring about a complete severance. As a rule, however, the fall of the leaf is hastened by external influences. Every gust of wind brings down the leaves; the alterations in volume dependent on the frost and chill and the subsequent thawing of the cell-sap, aid the severance and also hasten the tearing of vascular bundles which are still entire; and thus it happens that thousands of leaves fall to the ground even in the absence of wind, especially when, after a frosty night, the rising sun illuminates the autumn-tinted leaves, and dissolves the frozen sap.

The region where the separation is effected is usually sharply marked off, and it looks as if the leaves and leaflets had been cut through with a knife. The severed surfaces present a variety of contours, according to the shape of the leaf-stalk. Sometimes it is horseshoe-shaped, sometimes triangular or rounded, or it reminds one of a trefoil-leaf, and sometimes it has an annular shape. The stalk of the plane-tree lewt has at the base a conical swelling which incloses a bud; when the leaf falls a fissure is formed entirely going round it. Many of the separation surfaces of the leaf-stalks are like the articular surfaces of the long bones in the human skeleton (of the radius, tibia, and at the elbow). Vine leaves form two layers of separation, one close to the stem at the base of the leaf-stalkthe other at the upper end of the leaf-stalk immediately below the blade. In the palmate leaves of the Horse-chestnut and Virginian Creeper (Ampelopsis), in the compound leaves of Spircea Aruncus, in the pinnate leaves of the Chinese Tree of Heaven (Ailanthus glandulosa), and in the bipinnate leaf of the North American Gymnocladus Canadensis, a small separation layer arises below each leaflet, and a larger one, in addition, at the base of the leaf-stalk. Such leaves, consisting of several leaflets, collapse like houses built of cards when touched, and under the trees late in the autumn lies a confused heap of leaflets and leaf-stalks, the latter sometimes looking like long rods (as, for example, in the Ailanthus and Gymnocladus), sometimes almost like long bones (as in the Horse-chestnut, fig. 93).

Frequently the layer of separation is so situated on the leaf-stalk that after the 
detachment a small portion of the stalk remains on the branch. This is the case in the so-called Syringa, or Mock Orange (Philadelphus), where the scale-like part which is left has to protect the bud situated just above the leaf-stalk.

In some trees and shrubs defoliation is very rapid, in others only gradual. In the Japanese Maidenhair Tree (Ginkgo biloba), the formation of the separation-layer and the detachment of the leaves is completed in a few days; in hornbeams and vaks the stripping of the foliage continues for weeks, and frequently only a portion

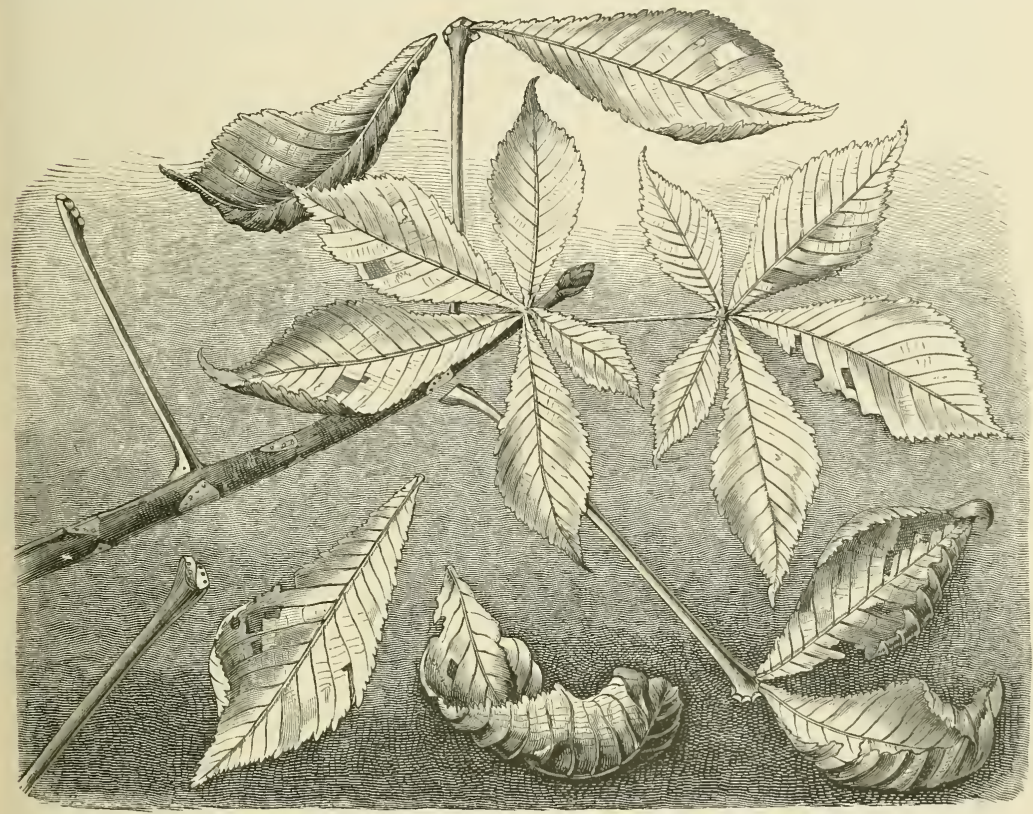

Fig. 93. -Leaf-fall of the Horse-chestnut (Esculus Hippocastanum).

of the dead leaves is thrown off in the autumn, the remainder not until the close of the winter.

It is also worthy of remark that in some trees the leaf-fall begins at the end of the branches and gradually proceeds towards the base, while in others the contrary is the case. In ashes, beeches, hazels, and hornbeams, the apices of the branches are leafless when the lower parts still bear firmly-fixed foliage; in limes, willows, poplars, and pear-trees, on the other hand, the lower portions of the branches are seen to lose their leaves early in the autumn, the denudation gradually extending upwards; on the extreme ends of the branches some leaves, as a rule, obstinately remain for a long time, until they also are at length whirled away by the first snowstorm. 


\section{CONNECTION BETWEEN THE STRUCTURE OF THE VASCULAR TISSUES AND TRANSPIRATION}

It is naturally to be expected that between the contrivances regulating transpiration in the immediate neighbourhood of the green tissue, and those mechanisms which effect the transport of the crude sap from the roots, through the stem and branches, up to the region of this transpiring tissue, a mutual co-operation will exist.

Where much water is exhaled from the surface, much water must be supplied, and in tracts leading to extensive and strongly-transpiring leaf-blades, the fluid moves more quickly than in a conducting apparatus leading to green tissue, which transpires but slowly and to a small extent. In pines, whose stiff acicular leaves transpire but little, the raw food-sap moves much more sluggishly than is the case with maples, whose flat leaves give off large quantities of water in the form of vapour. The quickest movement, however, is to be found in twining and climbing plants, whose stems, a few centimetres in thickness, may attain to a length exceeding 100 metres. This is the case in those peculiar climbing palms, which at first wind over the ground in numerous snake-like coils, and then rise to the tops of the highest trees, and unfold their leaves there in the sunshine. Climbing palms (Rotang) are known whose stems actually attain a length of 180 metres, and which, when they have reached the summit of the trees after numerous windings, become erect and extend their larger pinnate leaves just like the straight-stemmed palms. The illustration opposite (fig. 94) depicts in the background the edge of a wood up whose trees have climbed examples of such a species of Rotang.

Many hours of the day may pass, when, on account of a clouded sky and the great humidity of the air, the transpiration in the wide-spreading leaves above the tops of the trees will be extremely little; but when the sun shines brightly and the leaves become thoroughly warmed, a large quantity of water vapour must be exhaled in a very short time. This quantity of water must be replaced, and very quickly, but by means of a stem 180 metres long and only some centimetres thick. In order to render the replacement possible, everything which might hinder the rapid movement of the water and its dissolved food-stuffs on its long journey, especially the resistance of the conducting tubes, must be minimized as much as possible. The forward movement of fluids in a channel is, however, rendered more difficult as the tube narrows, because in a narrower tube a relatively larger amount of the fluid adheres to the inner surface, and therefore it is necessary, in order to obtain a rapid movement, that this adhesion be reduced as far as possible. This is most simply effected by widening the channel, since the adherent surface is thus diminished in comparison with the large amount of the fluid passing through. As a matter of fact, in the stems of climbing palms relatively very wide tubes are to be seen, through which a large quantity of fluid can be brought from the roots to the transpiring leaf-surfaces in a very short time, and this actually occurs. The climbing palm, Calamus angustifolius, has conducting tubes 
of more than $\frac{1}{2} \mathrm{~mm}$. diameter, and in the species of Rotang illustrated in fig. 94 . they are almost as wide.

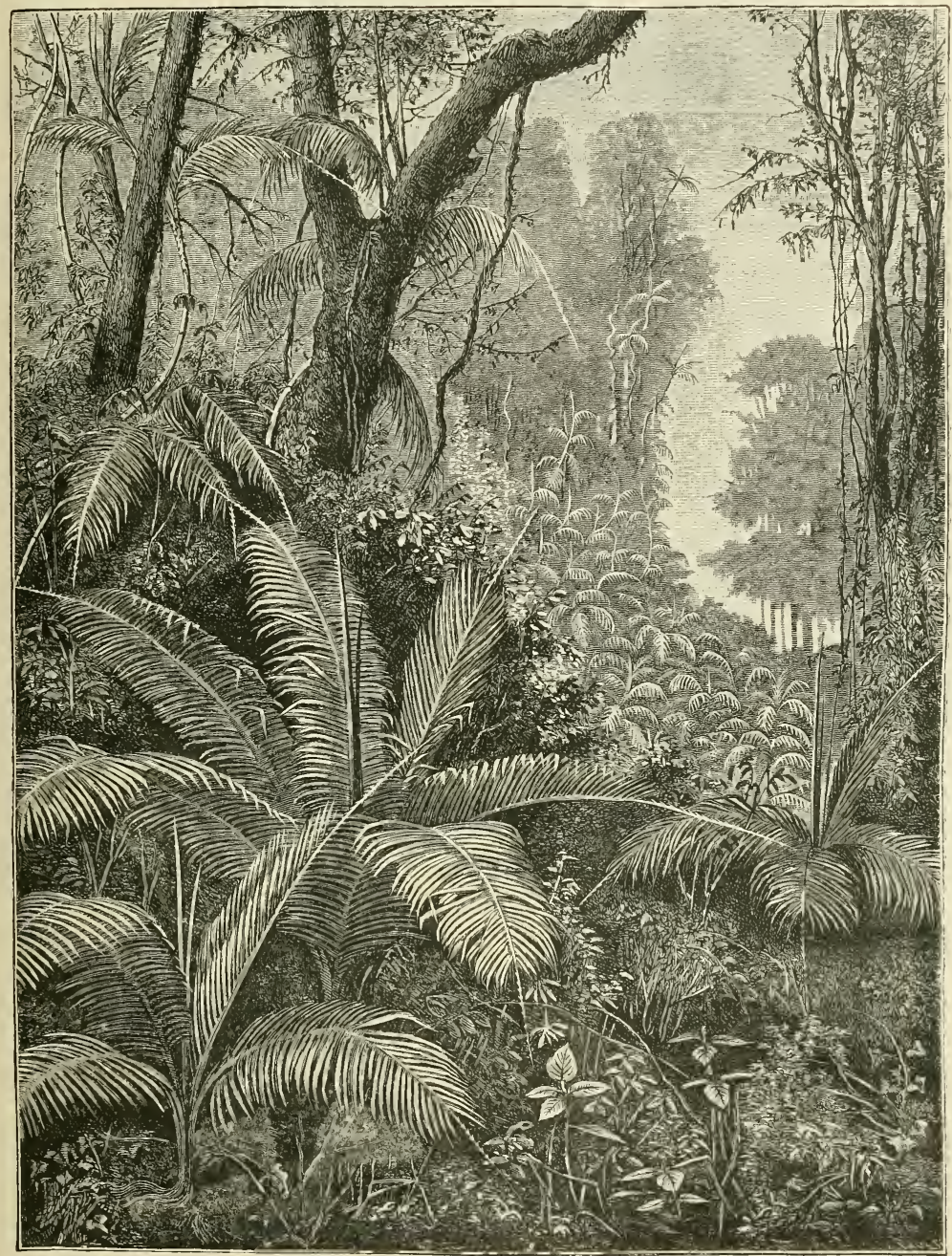

Fig. 94.-Indian Climbing Palms (Rotang). From a photograph.

What has been stated here with especial regard to the Rotang or Climbing Palm applies also to all other twining and climbing plants known by the name of lianes, and their sap-conducting tubes are the wider, the longer their stems and the larger 
their transpiring leaves. In very many lianes the cavities of the conducting vessels can be plainly seen with the naked eye. This is the case, for example, in the crosssection of the liane represented in natural size in fig. $95^{5}$. A diameter of $\frac{1}{3} \mathrm{~mm}$. is

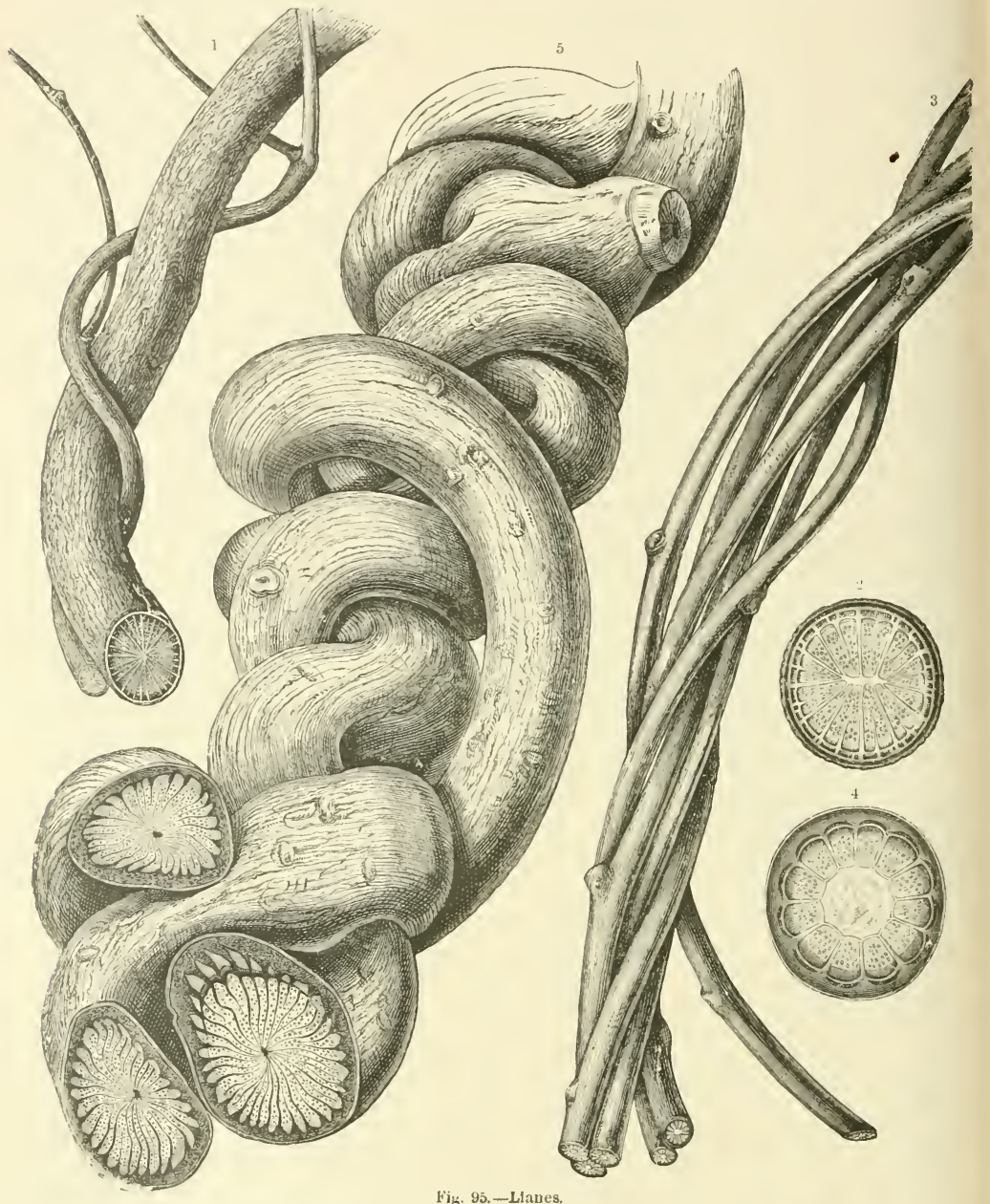

1 Portion of tne suem of a tropical Aristolochia. 2 Cross section of a liane-like Aristolochia. 3 Menispermum Carolinianum. 4 Cross section of the twining stem of Menispermun (nagnified). 5 Portion of a liane (probably an Asclepiad) gathered in a tropical forest; nat. size.

not at all rare in passion-flowers and aristolochias, and, generally speaking, in most twining and climbing plants; whilst in many lianes the conducting tubes have even been observed to be $0.7 \mathrm{~mm}$. in diameter. 


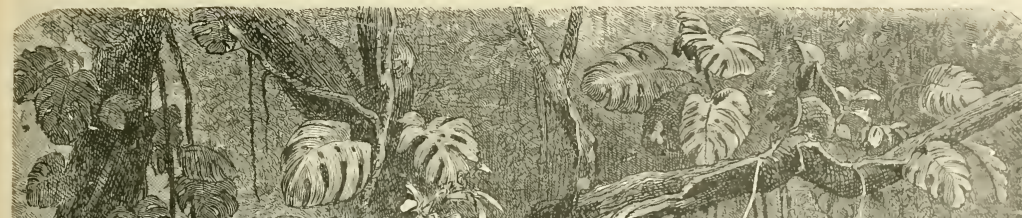

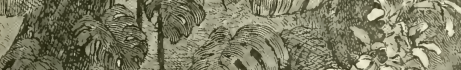
(3)
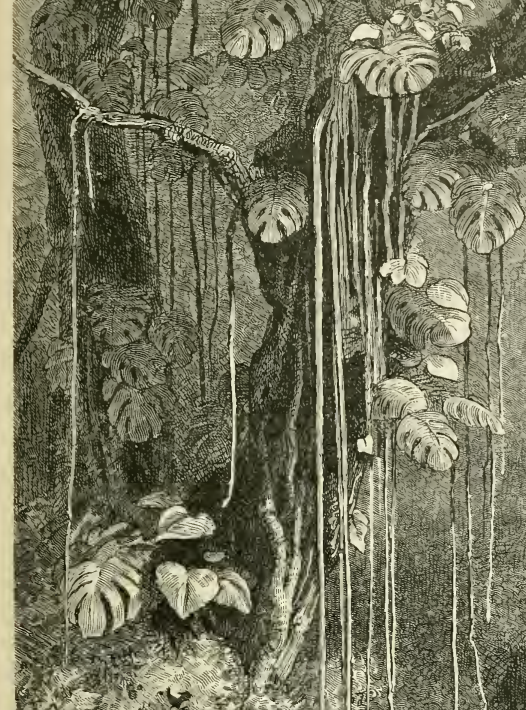

\section{(1)}

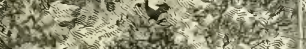

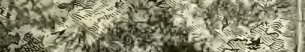

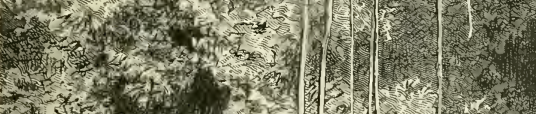

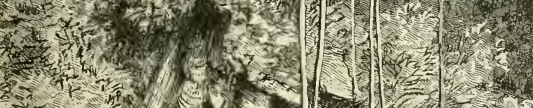

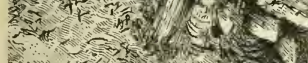

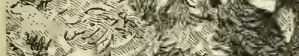

ind

-

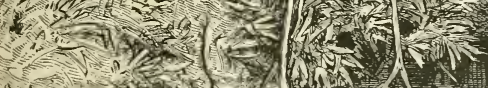

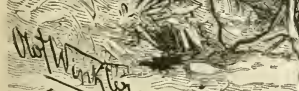

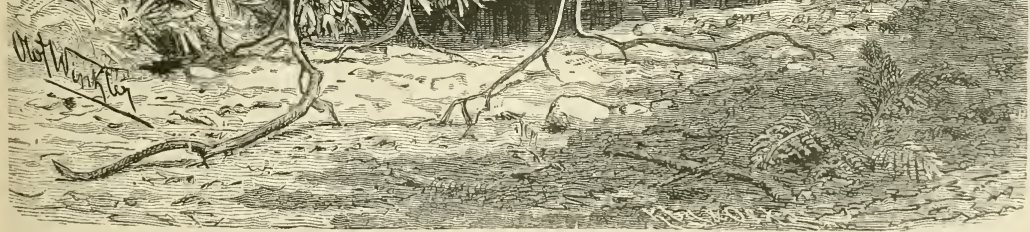

Fig. 96. - Aroids (Philodendron pertusum and Philudendron Imbe) with cord-like aerial roots. 
A particularly noticeable method of conducting water from the soil to the green leaf-blades is exhibited by some large-leaved tropical Aroids which climb up troes, and are provided with aërial roots. These plants have really two kinds of aërial roots, viz.: shorter ones, which are at right angles to the stem, by means of which they climb up their support, usually old tree-trunks; and longer ones, passing down perpendicularly to the ground like ropes or strings. In the Mexican Tornelia fragrans (Philodendron pertusum) represented in fig. 96, these latter roots attain a length of 4-6 metres and a diameter of 1-2 cm. They are of uniform thickness, brown, smooth, unbranched, and quite straight. As soon as they reach the ground, the tip bends round almost at a right angle, and sends a number of lateral roots which are covered with an actual fur of root-hairs into the soil. The bent end then enters the soil for a short distance, and thus the entire aërial root is rendered fairly tense. As a rule, two such cord-like aërial roots originate below each new leaf, and it seems as if this arrangement was specially adapted to transport the necessary food-sap from the soil to the large luxuriant leaf above by the shortest path. But it not only seems so, for this is actually the case, and it is especially remarkable that root-pressure takes a prominent part in the transport. On cutting through one of these cord-like aërial roots about a span above the ground, watery fluid is immediately seen to ooze from the middle of the cut surface. The woody portion of the root, which here forms a central strand, contains very wide conducting tubes, like those in the stems of lianes, and the quantity of fluid exuded in thirty-six hours amounts to as much as 17 grms. It is noteworthy that the root-pressure here, according to all appearances, acts with the same force all through the year. In the vine this is not the case. Vines which are cut through in the summer, it is well known, no longer weep; the cord-like aërial roots of tropical aroids, on the other hand, weep at all seasons of the year when cut across. Indeed, the vegetative activity is never entirely interrupted in these plants all the year, and it should be remembered, in connection with this fact, that they grow in places where the air and soil are always warm, and where their humidity is only subject to slight variations. It may happen that in damp, warm places transpiration from the leaves ceases for a time entirely, and then it is very necessary that the amount of food-sap should be forced up to the leaves by rootpressure in order that they may be supplied with the food-salts they require. The water, which contained dissolved food-salts, is of no use when it has given these up, and it is therefore forced out of the stomata, these in consequence being transformed into water pores.

The aërial roots, which form the shortest and straightest channels for conducting the raw food-sap to the leaves, are, moreover, of great importance to these tropical aroids, since it not infrequently happens that the lower portion of the stem in an old plant dies off, leaving the upper part, which is fastened to the trunk of a tree by the earlier-mentioned short supporting roots, and therefore in no direct connection with the ground. The supporting roots would not be sufficient to supply the fluid food required, and the whole plant is therefore provided 
with this food only through these cord-like aërial roots which are sent down into the soil.

These few examples are enough to show that the construction of the stem and roots stands most intimately related to transpiration, inasmuch as the transpiring green tissue is effected by the structure. But since the construction of these plant members, i.e. the architecture of the stem, is also dependent upon various other vital processes to be described later, it would not be fitting to discuss their relations here in detail, and their treatment must be postponed until a later section.

\section{CONDUCTION OF FOOD-GASES TO THE PLACES OF CONSUMPTION.}

Transmission of the food-gases in land and water plants and in lithophytes.-Significance of aqueous tissue in the conduction of food-gases.

It has been repeatedly pointed out that a division of labour occurs in all large plants, so that one portion of the cells provides for the reception of water and foodsalts, another for that of food-gases, and yet another for the conduction and transmission of fluid and gaseous nourishment to the places where they are consumed.

How the aqueous food-salt solutions derived from the soil are brought to the green tissue, what contrivances are thereby brought into action, and what phenomena of plant-life are related to this conduction have been discussed, as far as practicable, in the previous pages, and it now only remains to describe the transmission of the gaseous food-materials. This is far more simple than the conduction of the solutions of food-salts. The most important of the food-gases in question are carbonic acid and nitric acid. Carbonic acid is continually being conducted by means of water to the green tissues. The shortest passage is to be found in aquatic plants whose protoplasm, provided with green chlorophyll and in need of carbonic acid, is only separated from the surrounding water by a thin cell-wall, while this water always contains carbonic acid, though perhaps only in small quantity. Under the influence of sunlight, the groups of green cells in hydrophytes form a centre of attraction to the carbonic acid, which is sucked up with great energy from the surrounding water, passes easily through the cell-wall, and so comes directly into the neighbourhood of the green protoplasm, i.e. that place where its decomposition is effected. The green cells of water plants therefore furnish an apparatus for both absorbing and decomposing carbonic acid, and usually no further means and no special conduction through other cells are required.

In lithophytes it is otherwise. Here we have the remarkable fact that they are only active at times; only, that is to say, when they are sufficiently moistened by rain, dew, and mist, and are to some extent submerged for a time by heavy downpours. In dry air their vital activity is suspended; they then adhere to the rocks like 
withered turf and dry scales, as if dead. But as soon as they are moistened, or can condense moisture from the air, they are aroused to renewed vitality, and then suck up with great eagerness atmospheric water, which always contains small quantities of carbonic acid gas, and also traces of nitric acid. In the rock-inhabiting mosses the cells, which absorb water from the atmosphere containing carbonic acid, are also those in which the decomposition of carbonic acid takes place. In this respect these mosses behave exactly like aquatic plants; nor is it perhaps superfluous here again to point out the interesting fact already mentioned, that there are mosses which permanently live under water, and there behave like true water plants, though they are able equally to live on rocks, where they remain dried up for weeks together, and only resume their activity when wetted by rain. It is to be taken for granted that such damp, water-saturated mosses have the capacity of absorbing carbon dioxide from the surrounding atmosphere. The carbon dioxide is changed into carbonic acid by its passage through the cell-wall saturated with water. Probably it is only when carbonic acid is dissolved in water that it reaches the active protoplasm in the cells in question. In lichens the carbonic acid which reaches the protoplasm provided with chlorophyll is also dissolved in water; however, in most lichens the green cells do not come in contact with the atmosphere, but are separated from it by a layer of hyphal threads. Thus the conduction to the green cells takes place by means of the hyphal layer destitute of chlorophyll.

In land plants also the cells which are filled with chlorophyll-bearing protoplasm seldom come directly into contact with the atmosphere; usually the green tissue is surrounded with an actual mantle of water. That is to say, the cavity of each epidermal cell contains very watery fluid, or, in other words, in the fully-formed epidermal cells the protoplasm constitutes merely the parietal layers without chlorophyll, their large cavities being filled with water. These epidermal cells fit closely to each other, and on the upper side of the leaf are only rarely interrupted by stomata. Usually the epidermis on the upper side of the leaf gives rise to a layer of cells with clear watery contents, directly bordering on the green palisade tissue; and as the carbon dioxide of the atmosphere has to pass from the upper side to this green tissue, it must first of all pass through this watery cell-layer of the epidermis. There it becomes changed into carbonic acid, and passes from this epidermal sphere of activity, not in the form of gas, but dissolved in water, to the cells of the palisade tissue below. Since the green palisade tissue under the influence of sunlight uses up the carbonic acid in the manufacture of organic material, it becomes a centre of attraction for this acid as long as the illumination continues. At first the carbonic-acid-bearing contents of the contiguous cells are eagerly absorbed, and indirectly carbon dioxide also is drawn from the surrounding air and made to force its way into the epidermal cells. The cell-wall offers no great resistance to this entrance. It has been proved that carbonic acid, or rather carbon dioxide, passes very easily through the cell-wall. According to all this, it is evident that the small quantity of carbon dioxide is drawn from the air by the green illumimated tissue of the leaves and stem, that carbon dioxide streams 
rapidly towards these parts, penetrates into the epidermal cells, is changed into carbonic acid, and reaches the green tissue by means of the aqueous contents of the epidermal cells.

According to the previous statement, which has been discussed in detail, the epidermis has also to provide for the transmission of the carbonic acid to the places of consumption, viz. to the green tissue.

In accordance with climatic and other local conditions, and corresponding to the individuality of separate species, the epidermis presents, as is well known, endless variations in structure. This variety of formation is concerned chiefly with the part which it has to play as a protective covering, as strengthener, and the like. As a conducting apparatus for carbonic acid, that is, in the form of a water mantle or outer aqueous tissue, it exhibits comparatively little variation. In evergreen plants which grow in warm, damp situations where transpiration is limited, and where the water of the soil is often conducted by root-pressure to the large transpiring leaf-surfaces, as, for examples, in tropical bananas, palms, mangroves, figs, and peppers, the aqueous cells which lie above the green palisade tissue are always arranged in several layers. In all those plants also whose outermost cells in contact with the air have much thickened walls, and consequently a restricted lumen, as, for example, in the Oleander, which grows on the sides of brooks (see fig. $73^{3}$ ), and in the proteaceous Dryandra floribunda growing in the Australian bush (see fig. 68), the water mantle consists of a double layer of cells. When the green tissue is penetrated by vascular bundles and groups of strengthening cells without chlorophyll, the aqueous epidermal layer is also interrupted, and is usually only co-extensive with the palisade cells. In the leaves of grasses the colourless aqueous cells form rows which are placed above the green assimilating tissue, and surround this tissue as an actual mantle.

The demand of the green tissue for carbonic acid regulates itself to the consumption in the formation of organic substances. But the consumption is at a maximum at the time of strongest illumination and greatest warming of the green tissue, and therefore coincides with the most abundant transpiration. At such a time the carbonic-acid-bearing sap is drawn by the active protoplasm in the green tissue with great eagerness from the epidermal cells lying above, often so abundantly that a quick replacement is impossible. But in consequence of this the epidermal cells lose their turgescence; they collapse, and the hitherto tense epidermis presents a flaccid appearance. In order that this collapse may take place without injury, the following contrivance has been devised. The side-walls of those cells which form the epidermis, i.e. the outer aqueous tissue, are delicate, thin, and flexible, and as these cells give up a portion of their sap, their side-walls are folded together just like a bellows from which the air has been expelled. When the cells become again filled with fluid, the folds are straightened out as in a bcllows filled with air, and the cells regain their former tenseness.

In the course of the foregoing representation we have only described the transmission of carbonic acid through the epidermal cells rich in watery cell-sap on
vol. 1 . 
the upper side of the leaf. But it must not be forgotten that the same process also takes place on the under side of the leaf, particularly when the green tissue is not divided into palisade cells and spongy parenchyma, and also when the epidermis is provided with stomata both on the upper and under sides of the leaf. In certainly 70 per cent of all leafy plants the arrangement is such that palisade tissue occurs beneath the succulent epidermis of the upper side, under this again spongy parenchyma, and again under this the epidermis of the lower side, which is abundantly pierced by stomata. It can therefore be asserted, for the majority of plants with green foliage, that the epidermis of the upper side chiefly regulates the transmission of carbonic acid to the palisade cells, and that transpiration is chiefly regulated by the epidermis of the lower side.

It is hardly probable that carbonic acid finds entrance to the green tissue through the stomata. At the time when the demand for carbonic acid is at a maximum in the green tissue, a considerable quantity of food-salts must be delivered to the green cells, and the water which provides for the transport of the food-salts from the soil up to the small chemical laboratories, as the palisade cells may be called, is rapidly expelled from the stomata in the form of vapour. But while water-vapour is streaming out of the stomata, the carbon dioxide of the air can hardly stream in through the same avenues at the same time, and it may be concluded that when, generally speaking, this gas is absorbed through the stomata, the occurrence is exceptional.

Concerning the filling of the epidermal cells with water and carbonic acid, it should be here again pointed out that in not a few plants the absorption of rain and dew takes place directly through the foliage-leaves. Since rain and dew always contain small quantities of carbonic acid and traces of nitric acid, this method of filling the epidermal cells is so much the less to be undervalued. In very many green foliage-leaves the continuous epidermis above the palisade cells is capable of being moistened, while the lower epidermis, rich in stomata, on the other hand, is kept dry by the most varied contrivances; and it is very probable that in such cases the water of rain and dew is taken up by the whole epidermis of the upper leafsurface, especially when these epidermal cells have a short time previously given up a portion of their contents to the green tissue, and have become consequently somewhat collapsed. In many cases it must be concluded, from their shape and position, that the filling of the epidermal cells is only caused by the watery sap brought from the roots, and indeed only by means of the green palisade tissue, i.e. of the same tissue which, on occasion, again receives watery fluid from the epidermal cells. This periodic alternation of absorption and expulsion may be explained in the following manner. The water arriving from the soil is given off by the palisade tissue to the epidermal cells at certain times, i.e. when no carbonic acid is required, in order that carbon dioxide may there be drawn from the air and changed into carbonic acid. When this has happened, and a demand for carbonic acid is set up in the palisade tissue, this tissue takes back the water it had previously given off, now of course accompanied by the absorbed carbonic acid. 


\title{
FORMATION OF ORGANIC MATTER FROM THE ABSORBED INORGANIC FOOD.
}

\section{CHLOROPHYLL AND CHLOROPHYLL-GRANULES.}

\begin{abstract}
Chlorophyll-granules and the sun's rays.-Chlorophyll-granules and the green tissue under the influence of various degrees of illumination.
\end{abstract}

\section{CHLOROPHYLL-GRANULES AND THE SUN'S RAYS.}

In the former section of this book it has been described how everything which serves as food for plants is conducted to the green tissues. Food-salts, food-gases, and water arrive at the same goal by the most diverse contrivances-to the green cells as those places where the raw material is worked up and organic substances prepared from it; to the place of need where the materials for further building and development, for rejuvenescence, multiplication, and reproduction of the plants in question have to be provided. The question how living plants manufacture organic substances in the green cells from the raw materials which stream to them, particularly from the raw food-sap and carbonic acid, must now be discussed.

First, it should be remembered that the formation of organic materials always commences with the decomposition of the absorbed carbonic acid. This decomposition, however, is only carried on by that protoplasm in which are imbedded chlorophyll-granules. The protoplasm in question can only accomplish the indicated task by the help of these structures, and the chlorophyll-granules are therefore really the organs on which everything depends. It is in them that those remarkable processes are carried on, upon which depends the renewal, and ultimately the existence, of all life. The description of these organs must, therefore, precede all further discussion.

Having regard to the importance of their function, the structure of the chlorophyll-granules appears to be simple enough. It is possible that later researches, with instruments and methods of observation more perfect than those now at our disposal, will furnish more accurate details about their minute structure, and particularly as to their dissimilarity from the protoplasm in which they are imbedded. In the meantime, only this much is known-that the ground-work of the chlorophyll-granules differs but little in its structure and composition from the surrounding protoplasm. Like all sharply-defined protoplasmic bodies, chlorophyllgranules exhibit a pellicle-like thickened outer layer; the inner portion, on the 
other hand, is formed of a porous mass of reticular or scaffold-like strands, which may best be compared to a bath sponge. The holes and meshes of this spongy colourless ground substance contains a green colouring matter, which is dissolved in an oily material, and clothes the continuous small spaces in the form of a parietal layer. This green colouring matter of the chlorophyll-granules, which has been called clilorophyll, is easily soluble in alcohol, ether, and chloroform. If green leaves are steeped in an alcoholic solution, they become blanched in a short time, and the colouring matter passes entirely into the fluid. The alcohol assumes the beautiful green colour which the leaves formerly possessed, and the previously green leaves are now to be seen floating in the green alcohol. In transmitted light the solution appears a beautiful green; but when observed in reflected light it appears blood-red, and therefore the colouring matter displays a marked fluorescence. If a fatty oil is added to the green-tinted alcohol, and the two are shaken up together, the green colour passes into the added medium, while in the alcohol a yellow substance remains, which has been termed xanthophyll. The chemical composition of chlorophyll is not yet so clearly understood as we could wish. It is asserted that it is possible to obtain chlorophyll in a crystallized form. The crystals obtained form green transparent rhomboids, which, when exposed to the light, slowly decompose again. This chlorophyll behaves like a weak acid; contrary to earlier belief, it is free from iron, but leaves behind almost 2 per cent of ash, consisting of alkalies, magnesia, some calcium, phosphoric and sulphuric acids. The fact that the production of these crystals must be preceded by a series of longcontinued operations, together with the fact that chlorophyll is extremely delicate and easily decomposed, always allows us to suppose that the crystals mentioned are only a product of decomposition, and do not belong to that chlorophyll which colours the chlorophyll-granules in living cells. It was previously thought that chlorophyll was a mixture of two colouring matters, viz. a blue and a yellow, until later researches demonstrated that this supposition was unfounded, and that a false impression had been received through observation of the process of decomposition. A characteristic absorption spectrum has been obtained for chlorophyll, which is especially useful in all cases where it is a question of demonstrating the presence of very small quantities of the colouring matter in any parts of the plant. With respect to this it is enough to say that the whole of the violet and blue and the ultra-violet rays are cut off from the spectrum, and that it exhibits seven characteristically distributed absorption-bands. It may be further remarked here that after treating the chlorophyll with hydrochloric acid tiny crystals arise, which have been called hypochlorin. The results of all these researches have thrown but little light upon the part which chlorophyll plays in those processes which commence with the decomposition of the absorbed carbonic acid in the chlorophyll-granules.

Compared with the size of the whole mass, chlorophyll forms only an extremely small fraction of the granules it colours green, and when it is withdrawn by the addition of alcohol, only the colour and not the size of the granules in question is found to be altered. 
Chlorophyll-granules appear to be imbedded in protoplasm from their origin until their disappearance. When the protoplasm is situated round the wall-or, in other words, when the central cavity of the protoplasm is large and filled with watery cell-sap, and the plasma which surrounds the sap-cavity is sac-like and only forms a thin covering to the cell chamber, then the chlorophyll-granules are usually imbedded in the middle layer of the parietal plasma, so that they are separated from the sap-filled central cavity, as also from the cell-wall, by a layer of colourless protoplasm. The same thing occurs when the chlorophyll-granules are imbedded in the plasma strands which are stretched across the cell-cavity (see figs. $5^{2}$ and $5^{3}$ ). Frequently the chlorophyll-granules project like warts, and thus give a knotty appearance to the protoplasmic strands; but even then they are always covered by a thin colourless layer of protoplasm.

In spite of this close connection, chlorophyll-granules always appear to be sharply defined, and exhibit in their entire development a certain separateness from the protoplasm in which they may reasonably be supposed to take their origin. They enlarge, divide, and multiply, and occasionally in the course of their life alter their form. With respect to their shape there is little variety in the green tissue of the stem and leaves of higher plants. The chlorophyll-granules almost always appear there as rounded or occasionally angular, sometimes even as lenticular or many-sided grains. A much greater diversity is observed in those simple green plants which live in water, and have been classed together under the name of Algæ. In the cells of the green filaments of Zygnema, which are represented in figure $25 \mathrm{~A}, m$, the chlorophyll bodies are stellate, and are so arranged in each cell that there are usually two stars side by side. In species of the genus Spirogyra (fig. $25 \mathrm{~A}, l$ ) they form spirally wound, usually knotty, bands, and in most species of the genus only one band in each cell; but in some species there are two bands, whose spirals cross one another, whereby very ornamental structures come into view under the microscope. In species of the unicellular Penium (figure $25 \mathrm{~A}, k$ ), the chlorophyll bodies form plates or bands parallel to the long axis of the cell, projecting against the cell-wall in all directions. In Mesocarpus a single green plate is observable, which divides the cavity of the cell into two almost similar halves; Edogonium exhibits a latticed plate; species of the genus Ulva have plate-shaped chlorophyll bodies which lie close to the wall; in the cells of Podosira are seen disc-shaped chlorophyll bodies which jut out in all directions; and in the liverwort Anthoceros the chlorophyll bodies are in the form of hollow spheres surrounding the centres of the cells.

The number of chlorophyll-granules in the protoplasm of the cell varies from one to several hundreds. In the cells of selaginellas there are usually $2-4$; in those of the luminous moss, Schistostega osmundacea, to be described later more in detail, 4-12 (fig. 25A, $p$ ). The green cells of most leafy flowering plants contain $20-100$, many even 200. In the cells of Vaucheria (figure 25,$a-d$ ), the protaplasm is so crowded with thickly-pressed small green granules as to make one think that the whole cell-body contained but a single chlorophyll mass. Foliage- 
leaves, in which a distinct separation between the palisade and spongy parenchyma is completed, always show many more chlorophyll-granules in the former than in the latter. Careful countings have shown that the palisade cells usually contain three or four times-occasionally even six times-as many chlorophyll-granules as the adjoining cells of the spongy parenchyma. When the chlorophyll-granules in a cell are so many that the whole inner wall of the cell can be covered with them, they arrange and distribute themselves very equally in this manner, and such cells appear uniformly green. It then seems as if the whole cell-chamber were entirely filled with chlorophyll-granules, but this is not really the case. The central cavity of the protoplasm filled with cell-sap never contains a single chlorophyll body.

The chlorophyll-granules imbedded in the parietal protoplasm can also undergo the most remarkable displacements, which we will forthwith describe.

With regard to shape, cells with active protoplasm, containing chlorophyllgranules, exhibit the widest variety. Especially are all imaginable cell shapes to be found in the group of Desmids which live in water: rod-shaped, cylindrical (fig. $25 \mathrm{~A}, k$ ), crescent-shaped (fig. $25 \mathrm{~A}, i$ ), tabular, stellate, tetrahedral, and many others for which it would be hard to find short and suitable names. The Algæ, which to the naked eye seem composed of green threads, are built up of cells which are, for the most part, tubular and cylindrical (fig. 25A, $a, b$, and $l, m$ ). In Lichens and Nostocaceæ the cells which form the tissues are spherical; in Mosses and Liverworts they are pentagonal and hexagonal.

As already mentioned in former sections, the green tissue in the foliage of Phanerogams is formed, in the majority of instances, of two kinds of cells-of branched cells forming the spongy parenchyma, and of cylindrical cells which constitute the palisade tissue (fig. 62, p. 279). The latter are often short, their length being not much greater than their width, but usually they are five or six times, and occasionally even ten or twelve times, longer than broad. In bulbous plants the palisade-shaped cells are arranged parallel to the upper leaf-surface, but in the majority of seed-bearing plants they are at right angles to the upper surface of the foliage-leaf, as shown in the cross-section of a leaf of Salix reticulata, fig. $71^{5}$, p. 301. The green cells below the epidermis of pines and various firs exhibit a very peculiar form. In contour they appear angular and tabular, and are fitted closely to one another without intercellular spaces. From the cell-walls parallel to the upper surface of the leaf trabeculæ project into the interior, by means of which each cell is divided up into niches usually of equal size. Such cells remind one of stables in which the stalls of the different horses are separated by boarded partitions. The projecting trabeculæ are always so arranged that the entire cell-chamber appears like a group of palisade cells whose side walls separating one from another have been interrupted. These partitions, which, as stated, are to be found in many firs, but also in grasses and many Ranunculaceæ-especially in the Monkshood (Aconitum), Peony (Pceonia), and Marsh Marigold (Caltha)-increase the internal surface of the chamber, and this appears to be advantageous, inasmuch as by this means many more parietal chlorophyll-granules can find a place than wouli; 
be possible in a single cell of equal dimensions, but devoid of such projecting trabeculæ.

It is shown by very accurate investigations that the quantity of organic substances formed in a cell, by the decomposition of carbonic acid, is greater the greater the number of chlorophyll-granules, provided that all of them are so arranged within its protoplasm that they can discharge their functions. A heap of chlorophyll-granules filling the cell irregularly would be little suited to effect this result. The small, green chlorophyll-granules must, on the contrary, be so arranged that no one deprives another of light, and this is most easily possible, especially in a many-storied plant-structure, composed of numerous cells, when the chlorophyllgranules are grouped together like the stones in a mosaic, and are arranged along the walls in this order. When, moreover, the light falls unhindered through certain portions of wall, as through a window into the cell-cavity, all the chlorophyllgranules there situated are almost equally illuminated. The larger the extent of wall surface, the more chlorophyll-granules can be accommodated on it, and therefore the more abundantly can the decomposition of carbonic acid be carried on in such cells. For such green multicellular tissue, whose most important function is the decomposition of carbonic acid and the formation of organic substances, the parietal grouping of the chlorophyll-granules, the above-mentioned infolding of the inner surface of the cells, generally the increase of the inner surface of the cellwalls clothed with chlorophyll, is accordingly the most advantageous arrangement for the best possible utilization of the available space.

When one speaks of the "green" of plants one thinks first of all of the foliageleaves, in which that colour is especially noticeable. The name "chlorophyll" translated by "leaf-green" might lead to the idea that cells and tissues provided with chlorophyll are only to be found in the leaves; but this would not at all correspond to the true state of the case. Those plants which are not differentiated into stem and leaves, especially the many kinds of green water-plants classed under the name of Algæ, generally consist entirely of chlorophyll-bearing cells. In those mutually-nourishing combinations named Lichens, one of the partners is without, while the other is provided with, chlorophyll.

When stem and foliage-leaves are clearly differentiated, a portion of the tissue is deprived of chlorophyll while the other portion is more or less rich in the same. Chlorophyll-containing tissue is found in all the members of these stem-plants, in roots, in stems, in foliage, in floral leaves, in fruits, and seeds. In tropical orchids the aërial roots when dry appear white and are seemingly quite devoid of chlorophyll; but when moistened their green colour is seen, because when the outer porous covering is filled with water, and its cells become transparent, the colour of the green tissue-layer below shines through. There are even orchids, e.g. Angraecum globulosum, funale, and Sallei, which, when not flowering, have no other green tissue than that in the aërial roots, and in which not only the absorption of foodmaterials, but also the working up of the absorbed nourishment, particularly the decomposition of carbonic acid and the formation of organic substances, is carried 
on by the green tissue of the aërial roots. Green tissue is much more frequently to be met with in stem-structures than in roots. Hundreds of rushes, bulrushes, cyperuses, and horse-tails, as well the Casuarineæ and species of Ephedra, included under the switch plants, many papilionaceous plants of the genera Retama, Genista, and Spartium, a number of Salicornias, tropical orchids, and cactiform plants, the Duckweed (Lemna), and all the plants possessing flattened shoots (see fig. 82), contain green tissue, without exception, in the cortex of their stem and branches. Also ovaries and fruits which are not yet fully ripe are so universally coloured green that in popular language green fruit and unripe fruit are synonymous. Chlorophyll is more rarely observed in seeds. Those whose embryos are differentiated into axis and leaf only seldom-as, for example, in the pines-show green tissue in the cotyledons. The seeds of orchids, especially those living epiphytically on the bark of trees, behave in a peculiar manner. These are marvellously small, consist of only a single group of parenchymatous cells, and no trace is to be seen in them of a radicle or cotyledon. They only retain the capacity of germinating a short time, and it is important to these seeds, which are poorly supplied with reserve food, that immediately after leaving the fruit-capsule they may be able to provide themselves with nourishment from their surroundings, and to manufacture organic substances from this food. This they can naturally only do by the help of chlorophyll, and it is interesting to notice that they also are actually endowed with this substance. Even when they are still inclosed in the capsule of the parent plant these seeds become green, and when they are carried by the wind into some cleft on the bark of an old tree-trunk the chlorophyll is able at once to function. After a short time a small green tubercle grows out of the green seed, and fixes itself by absorbent cells to the substratum, then very gradually it grows up to form a large plant-stem.

Large flowers whose petals, from the commencement to the end of the flowering period, exhibit a green colour are esteemed rarities. On the other hand, small floral leaves, rich in chlorophyll, are of very common occurrence. The change of the floral colour also, during the flowering period, from white, red, violet, and brown, to green, has been frequently observed in small as well as in fairly large flowers. A very striking example of this is the Black Hellebore (Helleborus niger). When its flowers open, the outer large leaves situated below the petals (which are transformed into small nectaries), are snow-white, and show up conspicuously from their darker surroundings. From afar they attract the attention of honey-collecting insects, by whom they are eagerly sought out. When, by means of these honey-sucking insects, pollination is brought about, the small nectaries, as well as the large dazzling-white outer floral leaves called sepals, become superfluous. The nectaries forthwith fall off, but the large sepals remain and take up another function. Chlorophyll is abundantly developed in their cells; the white colour disappears; fresh green appears in its stead, and the same floral leaves which previously had attracted insects by their conspicuous colour now function as green leaves exactly like foliage-leaves. A similar alteration of colour, which also has the same 
significance, is observed in many orchids and liliaceous plants, but on the whole such a change of function in floral leaves is not common. These cursory observations must show that chlorophyll may appear in all the members of a plant, although it is also true that foliage-leaves chiefly contain the green tissue, so that certainly in 90 per cent of all chlorophyll-bearing plants the decomposition of carbonic acid is carried on in the foliage-leaves.

If, now, after the description of the arrangement, form, and distribution of chlorophyll-granules, we would also learn something as to how organic substances are formed in the cell-chambers by means of these structures, we find ourselves in the position of an inquirer who visits a chemical laboratory without a guide, and wishes to ascertain in what way some material-for example, a pigment-is manufactured. He notices apparatus set up there, sees the raw materials heaped together, and also finds the finished product. If the manufacture is actually proceeding, he can also observe whether warmth or cold and greater or less pressure are brought into action as propelling forces, and he can, if intrusted with the manipulation necessary to the production of such pigment, imagine the relation of the different parts to the whole. Of the details, indeed, much must remain incomprehensible, or quite unknown. Especially with reference to the quantity of the transformed raw material, and with regard to the propelling forces, must the visitor's knowledge remain incomplete.

It is not otherwise with us when we would inspect the processes carried on in the cells where chlorophyll-granules develop their activity. We see the effective apparatus, we recognize the food-gases and food-salts collected for working up, we know that the sun's rays act as the motive force, and we also identify the products which appear completed in the chlorophyll-granules. By careful comparison of various cells containing chlorophyll, on the ground of observations which establish the conditions under which the manufacture of organic substances succeeds best and worst, having found by experience that under certain external conditions the whole apparatus becomes disintegrated and destroyed; it is indeed permissible to hazard a conclusion about the character of the propelling forces. But what is altogether puzzling is how the active forces work, how the sun's rays are able to bring it about that the atoms of the raw material abandon their previous grouping, become displaced, intermix one with another, and shortly appear in stable combinations under a wholly different arrangement. It is the more difficult to gain a clear idea of these processes, because it is not a question of that displacement of the atoms called decomposition, but of that process which is known as combination or synthesis. Decompositions and analyses, even of the most complicated compounds into simple combinations are well understood, but not so the converse. It is always considered a fortunate occurrence when a chemist succeeds in producing from its fundamental elements, or from the simplest combination of these, one of those complicated bodies, which are, nevertheless, formed with such ease in plant cells. When sugar is "made" in a manufactory, it is not that carbon and the elements of water are used, although these are so abundantly 
at disposal, but only that the sugar is isolated which has been formed synthetically from these substances in those tiny chemical laboratories, the vegetable cells. Consequently it is really incorrect to say that sugar is "made" in our manufactories; we should only say that there the sugar manufactured by the plants is separated from other substances and prepared for further use.

Although it is not possible to represent the processes concerned in the synthesis of organic materials in plant cells as a matter beyond all doubt, one is justified in taking refuge in hypotheses. And it must be looked upon as an hypothesis when we consider the movement by which the atoms of the food-gases and food-salts are displaced by the sun's rays in the vegetable cells as a transmission of the vital force of the sun. The atoms have arranged themselves by this movement in a different order, they hold and support one another, they are stable, and a condition of mutual tension has been set up. The vital force of the sun has become the hidden spring of all these changes. The now stable organic material formed by synthesis is thus equipped with an adequate supply of energy, designated in other words as latent heat. If the atoms of the organic material from whatever cause again break loose, abandoning their combination and arrangement, they perhaps so displace and rearrange themselves that those groups which previously existed are formed again, and thus the potential energy is changed to vital force, the latent heat to sensible warmth. When a tree-trunk is consumed, the vital force of the sun, which had been changed by the formation of cellulose and the other organic materials composing the wood of that time into latent force, is again transformed into active energy; and when we burn coals, the sun's rays, which thousands of years ago brought about the formation of organic vegetable substances and were imprisoned in the coal, will again be set free, will warm our rooms, drive our machines, or propel our steamships and locomotives. Keeping this idea in view, it is at least possible to imagine the mechanical significance of the sun's rays in the formation of organic substances in plants, and it may be reckoned that the quantity of organic substance produced stands in a fixed proportion (which may be expressed in figures) to the store of energy in the same.

One circumstance on which particular stress must here be laid is that the various rays of which sunlight is composed, the rays with various wave-lengths and refrangibility, which, some of them at least, appear to our eyes as the different coloured bands in rainbows, play each a very distinct part in the formation of organic materials in plant cells. Under the influence of the blue and violet rays, i.e. of those which are most highly refrangible and have the smallest wave-lengths, the oxidation of the organic materials called carbohydrates is assisted, that is to say, not the formation but the decomposition and transformation of these compounds are favoured. The red, orange, and yellow, i.e. those rays which are less refrangible and have a greater wave-length, behave quite otherwise. These favour the reduction of carbonic acid, assist the formation of carbohydrates from raw materials, and are therefore chiefly concerned in the originating of such organic substances. When a sunbeam passes through a colourless glass prism a 
continuous spectrum is produced-violet, dark blue, light blue, green, yellow, orange, and red. If the same sunbeam passes through a transparent but coloured body, which may be either solid or fluid, whole groups of colour absent themselves from the spectrum. Dark bands appear in the corresponding places, and we say that the light in question has been absorbed by the coloured body. Now, if chlorophyll has the property of absorbing those colours of the spectrum which are not advantageous in the formation of organic substances from raw material, the rôle of this chlorophyll cannot be too highly estimated. It must not be overlooked, moreover, that many bodies have the capacity of absorbing light rays of shorter wave-length, and, on the other hand, of giving out rays of greater wave-length. It is precisely those pigments which are distributed in plants, again above all, chlorophyll, which possesses this property called fluorescence; and we must therefore also assign this significance to chlorophyll, that it can transform rays of light which are useless in the synthesis of organic materials into those which show the best possible action in this respect. If the fluorescing pigments of plants (chlorophyll, anthocyanin, phycoërythrin) can transform the violet and blue rays into yellow and red, it is to be supposed that their activity goes further, and that they will be able to change rays of small wave-length and higher refrangibility into rays which are found beyond the red, which are imperceptible to our eyes, and which possess very great heat-giving powers, or, in other words, that they will be able to transform light into heat. From all this it may be seen that the significance of chlorophyll in the formation of organic materials would be threefold. First, a retention or extinction of those rays which might hinder the formation of those compounds known by the name of carbohydrates; further, the transformation of rays with short wave-length into those of longer wave-length, which, according to experience, most favourably effect the production of sugar and starch; and, finally, the conversion of light into heat, and ultimately into latent heat.

\section{CHLOROPHYLL-GRANULES AND THE GREEN TISSUE UNDER THE INFLUENCE OF VARIOUS DEGREES OF ILLUMINATION.}

If it is beyond question that organic materials can only be formed from the absorbed carbonic acid in the presence of chlorophyll, it is, on the other hand, equally certain that the sun creates and works through these formative processes by its rays, and thus, as the propelling force, becomes the fountain of all organic life. The sun rises and sets, day follows night, and during the night the process just mentioned, upon which the existence of the living world depends, is interrupted. But even in the daytime also, the strength of the sun is very unequal; it is one thing at mid-day, when the source of light is in the zenith and the rays fall perpendicularly on the earth, and quite another in the evening, as the illuminating orb sinks below the horizon and the last rays spread almost horizontally over the surface. Clearly it is anything but a matter of indifference to the organs possessing a certain 
amount of chlorophyll in what manner the sun's rays light upon them, or what quantity of vital force is transmitted to them in a given time. Various species of plants may make very different demands for sunlight, but for each individual species the need of propelling force fluctuates only within very narrow limits, which cannot be exceeded without injury. The greatest possible equality in the supply of propelling force is an indispensable condition of a successful career. In order to meet the inequality in the flow of light on bright and dull days, and also during various parts of the day, it is arranged that the green organs can turn towards the sun, and that according to the hour of the day and the strength of the sun's rays at that particular time, they can take up a definite position, and again alter this position with ease. And, indeed, the green chlorophyll-granules in the interior of the cells also show this capability of accommodating themselves in accordance with the demand for light as well as the entire cells, and, finally, even the green leaves, together with the stems and branches which bear them.

If one would obtain a clear idea of the withdrawal of the chlorophyll-granules from the sunlight, one must remember, first of all, that these green bodies, whatever may be their form, are imbedded in the protoplasm of the cell, and that the protoplasm is mobile and easily capable of displacement-or, in other words, that the protoplasm which contains the green chlorophyll-granules twists and rotates within the cell it inhabits, and can transport the granules hither and thither. Still more. Chlorophyll-granules can be temporarily heaped up and crowded together in definite places; they may again be separated from one another, and distributed equally throughout the whole cell-body. In the tubular cells of Vaucheria clavata, represented in figure $25 \mathrm{~A}, a$, the protoplasm forms a lining layer on the inner side of the colourless transparent cell-wall, and is so thickly studded with round chlorophyll-granules that the cell appears of a uniform dark green. But this is only the case with light of moderate intensity. When strongly illuminated the chlorophyll-granules move apart from one another, arrange themselves in isolated balls, and in a very short time, in each tubular cell, dark-green spots and zones may be seen corresponding to the crowded granules, and light, irregular bands appearing in those places from which the chlorophyll has been withdrawn. If the intensity of the light diminishes, the green clusters dissolve, and the former equal distribution and colouring is resumed. In another filamentous green alga, which lives in water and belongs to the genus Mesocarpus, each of the long cylindrical cells contains a plate-like chlorophyll body, which in weak diffuse light turns itself at right angles to the incident rays. In this position the broad side, i.e. the larger surface of the chlorophyll body, is turned to the sun, and the incident light is in this way utilized to the utmost possible extent. As the plate-like chlorophyll body usually extends right across the cell, under the conditions indicated, the cell appears of a uniform green colour. If the full rays of the sun fall on such Mesocarpus cells, the plate-like chlorophyll bodies begin to turn so that the plane of the plate is parallel to the direction of the rays. Now the narrow side, i.e. the smaller surface of the chlorophyll body, is turned to the rays, and only a dark-green 
stripe is to be seen. This turning movement of the chlorophyll body is very quickly performed, and can be repeatedly effected by darkening and illuminating the cells of the Mesocarpus filament.

In cells, too, which are joined together to form tissues, this displacement and movement of the chlorophyll-granules often appears. It has been noticed for a long time that in the prothallium of ferns, in the leaf-like liverworts, in the leaflets of many mosses, and even in the large, delicate foliage-leaves of flowering plants, the green tissue appears to be coloured a lighter or darker green according to the intensity of the incident light; that under the influence of intense sunlight they become blanched and yellowish-green, but in weak light assume a darker tint. If a strip of black paper be placed on a foliage-leaf, illuminated by the sun, so that only a portion of the leaf-surface is covered by it, and if the paper be removed after a short time, the portion left uncovered and illuminated by the uninterrupted rays of the sun appears light green, while that part on which lay the strip of paper, and from which the sun's rays were withheld, is dark green. Careful investigations have shown that this change of colour is due to displacement of the chlorophyllgranules. In diffuse light the chlorophyll-granules group themselves on those cellwalls on whose surface the light falls perpendicularly, and consequently in the cylindrical palisade cells of the foliage-leaf on the small walls parallel to its upper surface, and it is clear that such cells (and therefore the tissue formed by them) have a dark-green appearance when looked at in the direction of the incident light. As soon as they are illuminated by direct sunlight, the chlorophyll-granules retire from these walls and take up their position on the cell-walls which are parallel to the direction of the incident light. In palisade cells the chlorophyll-granules group themselves by the side of the long lateral walls, while the short walls, which are at right angles to the rays, are colourless and free from chlorophyll. In the branched cells of the spongy parenchyma the chlorophyll-granules, which in diffuse light were equally distributed in the cell, heap themselves together in groups in the branches, while the central portions of the cells become clear and free from chlorophyll. The whole tissue, however, in which this displacement has been completed appears much paler than before, and displays usually a decidedly yellowish-green tint. This change of position of the granules, according to the intensity of illumination, may be particularly well seen in the very simply constructed leaf-like duckweed, especially in Lemna trisulca. Three sections of the green tissue of this plant, vertical to the surface, are shown in fig. 97.

With these phenomena is indeed also connected the alteration of shape which is observed under varied illumination in chlorophyll-granules. In the leaflets of Funaria hygrometrica, a moss very common on piles of charcoal, damp walls, and rocks, the chlorophyll-granules, which are close to the outer walls of the cells, are flattened out, angular, and comparable to small polygonal tablets, in diffuse light. They are also so arranged that the entire wall covered by them appears an uniform green, and only narrow, colourless lines remain between them. As soon as direct sunlight falls on them they quickly alter their shape, the tablets becoming hemi- 
spherical or spherical bodies, which project towards the centre of the cell-cavity. By this means the area of the chlorophyll-granules attached to the cell-wall is contracted, and consequently the green of the leaf-surface in question is diminished. In the leaves of many flowering plants, also, the chlorophyll-granules which are distributed in the palisade-cells along the elongated side-walls appear, in diffuse light, hemispherical or even conical, and project towards the centre of the cells so that they are illuminated to the greatest possible extent by the light rays passing through. Under the influence of direct sunlight they flatten out, become discshaped, and withdraw to some extent from the bright rays passing through the centre of the cells. The significance of all these processes, the changes of shape as well as the displacements of the chlorophyll-granules, is evident when it is considered that an over-abundance as well as a deficiency of light would be prejudicial, and that for every species the quantity of the sun's rays absorbed by the chlorophyll-granules is definite. Protoplasm, provided with chlorophyll,
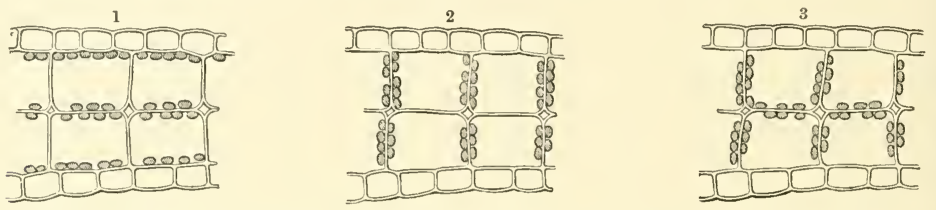

Fig. 97.-Position of the Chlorophyll-granules in the cells of the Ivy-Ieaved Duckweed (Lemna trisulca).

1 In darkness. ${ }^{2}$ In direct sunlight. 3 In diffuse light.

tries under all circumstances to obtain this definite amount. When weakly illuminated, chlorophyll-granules maintain a shape and position in consequence of which they present the largest possible surface to the light; when strongly illuminated, they assume a shape and position by which the smallest possible surface is so exposed. These processes, especially the displacement of the chlorophyll-granules, obtain a heightened interest from the fact that they can only be brought about by the streaming movements of the irritable protoplasm. It must be borne in mind that it is really living protoplasm which displaces the chlorophyll-granules imbedded in it in order to bring them to the places best suited to the illumination then existing, and to place them in sunlight or shade; so that it always happens that the displaced green bodies are neither too much nor too little illuminated.

Many unicellular water-plants, especially zoospores, attain the same result not by displacement of the chlorophyll-granules in the interior, but by movements of the entire cells. These green unicellular organisms may be seen swimming towards the light by means of their cilia, and in this way they take up the position always best adapted to the given conditions. If many swarm-spores are collected together in a limited area, it may happen that they all travel to one particular place; there they swarm about in the water and appear to the naked eye like a little green cloud. Or they may settle on the bottom of the pool, there arranging themselves side by side, so that no one deprives another of light, and they then appear 
to the naked eye as green stripes and patches. If swarm-cells of Sphcerella pluvialis are cultivated in a flat white china dish filled with rain-water, and onehalf of the dish is darkened by means of an opaque body while the other half remains illuminated, the whole of the swarm-cells swim from the darkened to the illuminated water in order to take up a position as favourable as possible with regard to the light. If now the china dish is turned round so that the hitherto illumined portion becomes darkened and light falls on the part previously obscured, the swarm-cells forsake the position which they had recently taken up, swim from the now darkened place to the illuminated side opposite, and arrange themselves there according as the illuminating conditions are favourable.

If, instead of the Sphcerella pluvialis discussed above, clumps of Vaucheria clavata are cultivated in a china dish filled with water, and the water is again partially darkened, together with the green tufts growing in it, it will be seen that the cells, which are elongated and fixed at one end, seek with the other end those places where they can find the best light. Vaucheria clavata, which has been repeatedly cited as an example, and which is represented in the middle figure on page 139, consists of long tubular cells, frequently bulging and branched, whose blunt growing ends appear dark green, while the lower dead portions are branched and coloured yellowish-white. The protoplasn is so richly studded with chlorophyllgranules that the entire inner wall of the tubular cells appears covered with a green lining. At the bottom of shallow pools, which is the natural habitat of these plants, they form hemispherical clumps, and all the tubular cells which compose the clumps have their green ends directed upwards towards the source of light. The same thing occurs when the Vaucheria cultivated in the china dish is uniformly illumined from above; but, if partially obscured, those filaments over which the darkening shadow is thrown very quickly alter their position. They bend towards the light side, and then the clump looks just as if its filaments had been combed in this direction. Moreover, the same thing is also seen when the china dish containing clumps of Vaucheria (on which until now diffused light has fallen uniformly from above) is placed at the further end of a one-windowed room, so that the light can only reach it from one side. Here, again, all the filaments, or rather, tubular cells of the clump, bend towards the source of light, and if they continue to grow, the increase in length is universally in a line with the direction of the incident rays. After a few days these Vaucheria clumps also look as if they had been combed out.

The green tissues of thallophytes, and the green leaves and stems of ferns, and phanerogams, i.e. those extensive combinations of green cells whose function is to work in a harmonious manner, and to manufacture organic substances for the plant to which they belong from carbonic acid with the help of other foodmaterials; these behave in the same way as the individual green cells which swim freely in water, and as the tubular cells of Vaucheria, which are attached at one end. Arrangements are necessary for these likewise, by which they can always be placed in the most favourable light. Of course, in these plants where 
division of labour has been so far developed, the conditions are not so simple as in those plants which consist only of single cells, and it is naturally to be expected that, according to the character of the individual species and the places which they inhabit, the arrangements would be very varied. The fact must also be kept in mind that each spot on which a plant has settled itself in the course of time may undergo alterations in consequence of which the amount and strength of the light affecting that part varies considerably. Long-lived plants, which grow vigorously in height and breadth, alter in their relation to the sun in various stages of growth, and must also alter their form in a corresponding manner, or, at least, must alter the direction and position of their green tissues. All this requires a multiplicity of contrivances which are, as a matter of fact, innumerable, and the exhaustive treatment of which is scarcely possible. In order to obtain a general view, it will be better to pick out some of the most remarkable of the long series of arrangements whose significance lies in this, that each species of plant receives for its green organs neither too much nor too little light, and to describe them in their relations to light as types of smaller or larger groups.

We will begin with those arrangements which are rendered necessary by a peculiar habitat, and, first of all, we will investigate those plants which have taken up their quarters in caves or grottoes, and there pass through all their stages of development. In deep excavations shut off entirely from the light, as well as in those which have been formed without human interference, and those which have been dug in order to obtain metal ore, coal, salt, and water, plants with chlorophyll-bearing cells and tissues are completely wanting. The plants which we find there consist only of pale fungi, which live on the scanty organic compounds which the infiltrating rain-water brings with it into the depths from the surface of the sunny land above, or which have established themselves on organic decaying bodies brought there by chance or intentionally by animals and men. It is otherwise in caves, mines, grottoes, pits, and wells, where light is able to penetrate from above or from the sides, even if only through a comparatively small aperture. Truly the vegetation developed there is not very luxuriant, but it is a very remarkable circumstance that there, as a rule, the plants are still green. What actually astonishes one at first sight of this vegetation, flourishing in caves illuminated only from one side, is the fact that they exhibit the most beautiful and vigorous green, a green much fresher, indeed, and more pronounced than that displayed by the plants outside. Thus the Hart's Tongue (Scolopendrium officinarum), widely distributed in Southern Europe, when adorning the deep shady walls of rocky ravines is coloured a much brighter green than when it grows on stony places in the open country where light can reach it from all sides. Also the liverworts which cover the damp stones with their leaf-like thallus, in grottoes through which waters ripple, show there in the half-light a distinctly richer green than when outside the grotto. But this phenomenon is most striking in the prothallia of some ferns belonging to the section of the Hymenophyllaceæ, and in many mosses.

A tiny moss, called popularly the Luminous Moss, but which has received trom 
botanists the name Schistostega osmundacea, has even attained a certain celebrity on this account. It is found distributed throughout the Central European granite and slate mountains, but is only to be met with in clefts of the rocks, caves and similar spots. As a rule it covers the yellow, clayey earth and the decayed and disintegrated pieces of stone which form the soil of these caverns and small grottoes. On looking into the interior of the cave, the background appears quite dark, and an ill-defined twilight only appears to fall from the centre on to the side walls; but on the level floor of the cave innumerable golden-green points of light sparkle and gleam, so that it might be imagined that small emeralds had been scattered over the ground. If we reach curiously into the depth of the grotto to snatch a specimen of the shining objects, and examine the prize in our hand under a bright light, we can scarcely believe our eyes, for there is nothing else but dull lustreless earth and damp, mouldering bits of stone of a yellowish-grey colour. Only on looking closer will it be noticed that the soil and stones are studded and spun over in parts with dull green dots and delicate threads, and that, moreover, there appears a delicate filigree of tiny moss-plants rising star-like, pale bluishgreen in colour, and resembling a small arched feather stuck in the ground. This phenomenon, that an object should only shine in dark rocky clefts, and immediately lose its brilliance when it is brought into the bright daylight, is so surprising that one can easily understand how the legends have arisen of fantastic gnomes, and cave-inhabiting goblins who allow the covetous sons of earth to gaze on the gold and precious stones, but prepare the bitter disappointment for the seeker of the enchanted treasure; that, when he empties out the treasure which he has hastily raked together in the cave, he sees roll out of the sacks, not glittering jewels, but only common earth.

It has been mentioned that on the floor of rocky caves one may discern by careful examination two kinds of insignificant-looking plant-structures, one a web of threads studded with small crumbling bodies, and the other bluish-green mossplants resembling tiny feathers. The threads form the so-called protonema, and the green moss-plants grow up as a second generation from this protonema. How this comes about will be described in another place; here it only interests us that the gleams do not issue from the green moss-plants, but only from their protonema. If this is viewed under the microscope a sight is presented like that depicted in fig. $25 \mathrm{~A}, p$. From the much-branched threads, composed of tubular cells, which spread horizontally over the ground, numerous twigs rise up vertically, bearing groups of spherical cells arranged like bunches of grapes. All the cells of a group lie in one plane, and each of these planes is at right angles to the rays of light entering through the aperture of the rocky cleft. The grape-like groups of cells have longer or shorter stalks, but they always appear in rows side by side and behind one another, placed cup-like, that the anterior groups do not deprive those behind them of too much of the light which enters the cavity. Each of the spherical cells contains chlorophyll-granules, but in small number; usually four, $\underset{\text { six, eight, or ten and they are always collected together on those sides of the }}{25}$ 
cells which are turned towards the dark background of the cave. There they are grouped like a mosaic, and usually so that one green granule forms the centre, while the others surround it very regularly in a circle. Such groups remind one of the arrangement of the floral-leaves in Forget-me-not flowers, and give a very ornamental appearance to the cells. Taken together, these chlorophyll-granules form a layer, which, under a low power of the microscope, appears as a round green spot. With the exception of these chlorophyll-granules the contents of the cell are colourless and transparent, and share these characteristics with the unusually delicate cell-wall. The light which falls on such cells through the opening of a rocky cleft behaves like the light which reaches a glass globe at the further end of a dark room. The parallel incident rays which arrive at the globe are so refracted that they form a cone of light, and since the hinder surface of the globe is within this cone, a bright disc appears on it. If this disc, on which the refracted rays of light fall, is furnished with a lining, this also will be comparatively stronglyilluminated by the light concentrated on it, and will stand out from the darker surroundings as a bright circular patch. This lining has the power of manufacturing organic substances in the spherical cells of the protonema of the Luminous Moss, and in this way the scanty incident light is turned to the greatest possible advantage; it is refracted and concentrated on those places where the chlorophyllgranules are situated, and consequently these receive in the dark recesses an amount of light which amply suffices for their special functions. It is well worthy of notice that the patch of green chlorophyll-granules on the hinder side of the spherical cell extends exactly so far as it is illumined by the refracted rays, while beyond this region, where there is no illumination, no chlorophyllgranules are to be seen. The refracted rays which fall on the round green spot are, moreover, only partially absorbed; in part they are reflected back as from a concave mirror, and these reflected rays give the cells of the protonema a luminous appearance. This phenomenon, therefore, has the greatest resemblance to the appearance of light which the eyes of cats and other animals display in half-dark places, only illumined from one side, and so does not depend upon a chemical process, an oxidation, as perhaps does the light of the glow-worm or of the mycelium of fungi which grows on decrying wood. Since the reflected light-rays take the same path as the incident rays had taken, it is clear that the gleams of the Schistostega can only be seen when the eye is in the line of the incident rays of light. In consequence of the small extent of the aperture through which the light penetrates into the rock cleft, it is not always easy to get a good view of the phenomenon described. If we hold the head close to the opening, we thereby prevent the entrance of the light, and obviously in that case no light can be reflected. It is, therefore, better when looking into the cave to place one's self so that some light at anyrate may reach its depths. Then the spectacle has indeed an indescribable charm. What has just been said about the isolated cells is exemplified in groups of cells placed behind one another, of which usually many thousands are found in a very small area. 
Among the mosses which find their home in deep shady places, principally in hollow tree-trunks, and are noticeable there for their glossy green, Hookeria splendens is especially worthy of attention. To be sure, its leaves do not shine as brightly as the protonema of Schistostega, but the appearance is, on the whole, much the same, and here also a similar development is the eause. The leaves of Hookeria are comparatively large, but at the same time very thin and delicate. They are composed of a single layer of rhombic cells, very convex above and below, so that the whole leaf may be compared to some extent to a window with very small so-called "bull's eyes" in the glass. The chlorophyll-granules are here arranged with far less regularity than in the protonema of the Luminous Moss, but they are heaped together just as in that plant on the side of the leaf facing the ground, that is to say, which is turned from the light. The side which is turned in the direction of the scanty incident light has no chlorophyll layer. The hemisphericallyconvex cells, opposed to this scanty light which falls on one side of the leaf, act like glass lenses; they concentrate the weak light on the chlorophyll-granules heaped up on the other side; but, on the other hand, light is also reflected, and this gives rise to the green lustre with which the Hookeria shines forth from its dim surroundings.

Like those plants which inhabit rocks, grottoes, and stony clefts, and the shady obscurity of hollow trunks, plants whose habitat is at the bottom of the sea, and in the depths of lakes and ponds, are only visited by weakened sunbeams. The illumination becomes the dimmer the deeper the habitat in question lies below the surface of the water, since the intensity of the light penetrating the water diminishes with the increasing length of the distance travelled. At a depth of 200 metres under the sea complete darkness reigns; at 170 metres the intensity of illumination is like that observed above the water on a moonlight night; such an illumination is insufficient to enable chlorophyll-bearing plants to manufacture organic substances from the absorbed raw materials, even although the plants were provided with all possible aids for the collection of this exceedingly weak light. It is only at a depth of not more than 90 metres that light is sufficient for the chlorophyll cells to decompose carbonic acid, and this depth is ascertained to be the lowest limit of chlorophyll-bearing plants. Moreover, these figures are only applicable in the most favourable circumstances in broad daylight, and only when the water is very clear and transparent, which really only seldom occurs, we might even say exceptionally. The substratum on which the submerged plants are situated, whether sand, mud, or rock, is usually sloping, and is most visited by the oblique rays of the sun. Frequently also small solid particles are suspended in the water, even in water which in the aggregate appears to be quite clear, and so the light is again considerably weakened. This happens especially in the neighbourhood of steep coasts, where the seething of the waves works uninterruptedly at the destruction of the solid shore, and consequently at a depth of 60 metres on such steep declivities, plants possessing chlorophyll are seldom met with.

Generally speaking, the vegetation in the sea is limited to a zone of about 30 metres 
in depth, whose width varies with the steepness of the shore. Below this narrow girdle, vegetation is practically extinguished, and the depths of the ocean are in all parts of the globe a plantless waste. This statement is not contradicted by the fact that sea-wracks have been found showing a length of 100, it is alleged even of 200 and 300 metres, as, for example, the celebrated Macrocystis pyrifera, between New Zealand and Tierra del Fuego. These sea-wracks do not rise perpendicularly from the bottom to the surface of the sea, but proceed from steep declivities, and grow at an angle to the surface, on which account they often take the direction of the current. One must imagine their position in the water to be almost like that of the Floating Pondweed, or the Water Crowfoot (Potamogeton fluitans and Ranunculus fluitans), which occur in brooks only a few decimetres deep, and nevertheless may attain a length of more than a metre.

It is naturally to be expected that plants which grow in the dim light, deep under the water on a rocky reef, would behave exactly like the grotto-inhabiting Luminous Moss; and if the barrel-shaped and spherical cell-structures connected into chains, the cyst-like and berry-shaped outgrowths of the unicellular Caulerpas and Halimedas, as well as the facetted cell-walls of those diatoms living in the abysses of the sea in dim twilight, are accepted as contrivances by which light is collected and focussed on those places within the cells where the chlorophyll-bodies are heaped up, then no mistake will be made. Several of the sea-inhabiting Floridex and sea-wracks belonging to the genera Phylocladia, Polysiphonia, Wrangelia, and Cystosira, even exhibit under the water a peculiar luminosity which may be compared with that of the Luminous Moss, although the optical apparatus is here essentially different. In the superficial cells of the luminous Phylocladias are to be found plates segregated out of the protoplasm and closely adhering to the outer walls, which contain a large number of small crowded lenticular bodies. From these minute lenses the blue and green rays are chiefly reflected, and thus the peculiar iridescence is produced. But, on the other hand, yellow and red rays are refracted on to the chlorophyll-granules, and consequently these plates must be regarded as an apparatus for focussing the light, which, by its passage through the thick layers of water, has undergone a considerable diminution.

In the depths of the sea, however, another optical phenomenon must be taken account of, by which the illumination of chlorophyll-granules in the plants growing there becomes in the end a favourable one, and that is the fluorescence of erythrophyll, the fluorescence of that red pigment to which the Floridex owe their characteristic colour. In order to make this phenomenon clear, it seems necessary first of ali, to rectify a wide-spread error with regard to the colour of water generally, and particularly of sea-water. In the very attractively-written work by Schleiden, The Plant and its Life, the seventh chapter, which treats of the sea and its inhabitants, begins with the following lines:- "O learn to know them, the horrible deeps, which are concealed beneath the shining treacherous surface. You descend, the blue of the sky vanishes, the light of day is gone, a fiery yellow surrounds you, then a flaming red, as if you were plunged into a watery sea-hell, without 
glow and without warmth. The red becomes darker, purple, finally black, and impenetrable night holds you enchained". This description is founded doubtless on the account of divers of the olden time, according to which red light should predominate in the abysses of the ocean. These accounts must, however, be retained only to the following extent. The cliffs and the rocky bottom to which the divers descended might have been richly carpeted with red Florider, possibly also just then the strata of water above were filled with those unicellular red algæ, which cause the so-called "flowers of the sea". In the neighbourhood of the mouth of the Tejo at times a superficial area of sixty million of square metres is coloured scarlet by Protococcus Atlanticus, a unicellular alga, 40,000 of which cover only a square millimetre; and Trichodesmium Erythrceum, another microscopic alga consisting of bundles of delicate articulated threads in innumerable milliards, fills the watery strata in the Red Sea as well as in the Indian and Pacific Oceans, so that there immeasurable stretches of water receive a dingy red colouring. When these algæ make their appearance the sea is said to blossom, and at those times the depths may appear to the diver as shrouded in a reddish-yellow twilight. At times the same colour has even been observed in the Lake of Geneva when its waters had been disturbed; it is due to the fact that the blue rays of the incident light are weakened by the fine atoms suspended in the water. With respect to this occurrence, we may consider that the above-mentioned accounts of divers are not the results of intentional deception, but only refer to particular cases. They cannot be applied universally. As a matter of fact, the colour of sea-water, in direct as well as in reflected light, is blue, and the diver who carries on his work at the bottom of the untroubled and non-blossoming sea, is not surrounded there by red, but by blue light. The greater the quantity of salt contained in the water, the deeper the blue. This blue nowhere appears so beautiful and so deep in tint as in the Dead Sea, and in the region of the Gulf Stream and of the Kurosiur, where the water is particularly rich in dissolved salts, and also has in the upper strata a comparatively high temperature. The blue colour of the water is explained thus: from among the rays which are characterized by different wave-lengths and different refrangibility (which, taken together, form colourless daylight, and which we admire separated in the colours of the rainbow), the red, orange, and yellow are absorbed in their passage through the water, and only those rays which are characterized by high refrangibility, viz. the blue, are allowed to pass through. The rays on the further side of the red, not perceptible to our eyes, the so-called dark heat-rays, are likewise absorbed in their passage through the water, and an object at some depth under water would therefore only be reached by rays of high refrangibility, particularly blue rays. The conditions of illumination for plants growing in the depths of the ocean are consequently in reality quite unfavourable. It is not only that a portion of the light falling on the surface of the water is reflected, and the other portion is weakened by its passage through the water, but besides, those rays which are necessary to the formation of organic matter by the chlorophyll-granules in the 
plant cells are abstracted from the light which passes through; for the chlorophyllgranules need just the red, yellow, and orange rays if they are to perform their functions; only under the influence of these rays can the decomposition of carbonic acid, the separation of oxygen, and the formation of carbohydrates, take place. The blue rays do not assist at all in this respect; they are even hurtful to these processes, since they assist the oxidation, that is, the decomposition of organic substance. Consequently, phycoërythrin, the red pigment of the Florideæ, now appears, and indeed so abundantly, that the chlorophyll-granules in the interior are quite hidden by it. This colouring-matter displays a very marked fluorescence, that is to say, it absorbs a large portion of the light rays falling on it, and gives out other rays of greater wave-length. The blue rays are to some extent changed by it to yellow, orange, and red, and thus the chlorophyll-granules finally receive those rays which act as the propelling force in the decomposition of carbonic acid. But this also affords an explanation of the remarkable phenomenon that seaplants are only coloured green close to the shore, and only in the most superficial layers of water, while lower down they appear red. Only quite on the surface the emerald-like Ulvaceæ and Enteromorphas sway hither and thither, forming thus a light-green belt; these algæ are to be sought for in vain in the depths beneath; of the plants which flourish below this region it can no longer be said that they grow green; this mark of vegetation has entirely vanished. Green has given place to red. All the innumerable Florideæ are reddened-sometimes a delicate carmine, sometimes a deep purple; then again a light brownish-red and a dull, dark crimson, and as we admire in the bush the innumerable gradations of green colour, so is the eye delighted in the manifold shades of red, in which the different variegated species of Florideæ, intermixing with one another, display themselves.

Let us now leave the blue twilight of the sea-depths, and set foot on the strand lapped by the blue waves sparkling with white foam, and climb up one of the rocky crags rising there above the seething waters. Around us is the bright daylight, and broad terraces of rock thickly overgrown with plants, all brilliantly illumined by the unclouded sun. But where is that fresh green which we expect to find up here according to the foregoing definitions in herbs and bushes? Here are not green, but grey foliage and branches, white-haired stems and leaves, and the whole woven and bound together into a carpet, which looks as if it had been strewn with ashes, or as if the wind had for a week brought hither the dust from the neighbouring streets and deposited it. The plants here on the sunny rocks have provided themselves with silky, woolly, and felted coverings for the purpose of softening the too glaring light. In the depths of the sea and in the grottoes of the slate rocks, the light was too weak; here, however, it is too strong. The chlorophyllgranules tolerate neither the one nor the other; they require light of a definite intensity. If the limit, which in this matter is exactly defined for each species, is overstepped, the chlorophyll is destroyed. Too much light may be no less injurious to the plants than if the chlorophyll-granules are condemned to inactivity on account of the want of light. 
How quickly a glaring light is able to destroy the chlorophyll can be well seen in the green Sea-lettuce on the shore below. In a high sea a violent wave tears fragments of the Ulvaceæ, known under the name of Sea-lettuce, from the coastrocks; a second wave as it rushes up washes the leaf-like structures on to the shingle of the shore, and there it remains with other débris lying amongst the stones. The sea now becomes calm, the sky has cleared, the sun's rays are again burning with undiminished strength on the shadeless strand. As long as the Sealettuce adhered closely to the rocks below the surface of the water it displayed a brilliant emerald green; the water in which it was submerged to some little depth, even at a low tide, sufficed to somewhat temper the sunlight; but the stranded Ulva is deprived of this light-regulating covering of water, and in a few hours its chlorophyll is destroyed. It is turned yellow, and looks like a lettuce-leaf which has lain for a week in a dark cellar. A similar appearance is also seen in confervas and spirogyras which fill stagnant pools of water with their masses of united filaments. Two decimetres below the water they display a beautiful dark-green colour, while close to the surface they appear a yellowish-green, and if the pool dries up so that the masses of filament come to lie on the damp slime, in two days they are quite bleached; the undimmed sunlight has completely destroyed the chlorophyll in the cells. In the depth of beech-groves the Woodruff (Asperula odorata) raises its leaves arranged in whorls on the stem; over it the thickly-leaved branches of the beeches bend together, forming a roof through whose interstices only here and there a weak sunbeam finds its way into the depths. In the dim light the leaf-stars of the Woodruff appear of a deep, dark-green tint. Now the axe of the woodeutter resounds through the forest-the beeches are felled, the shading roof of foliage is demolished, and the floor of the wood is exposed to the glaring sunbeams. Within two weeks the Woodruff can no longer be recognized; it has become sickly and pale; the leaf-stars have lost their dark green, and the chlorophyll has been destroyed by the glaring light. The same thing occurs with ferns as with the Woodruff. In the dimness of the floor of the forest, between steep-walled rocks, and on shady northern declivities they are tinted dark green; in sunny situations they become pale, and then are noticeably retarded in growth. All these plants are not organized to adapt themselves, in the case of an alteration of the illumination of their habitat, to the new conditions and to protect themselves from the undimmed rays falling on them. They are only fitted for the shady floor of the wood, and an over-abundance of light is their death.

But how is the vegetation protected in a habitat where during the whole of the vegetative period full light predominates, where the sun makes itself felt from rise to setting with uninterrupted power? It has already been pointed out that the plants on the broad ridges and terraces of the rocky shores of the Mediterranean are shrouded in dull grey, clothed in silk or wool, or else overstrewn with chaff-like scales, and consequently have lost their fresh green colour. In reality it is not quite correct to say that they have "lost" the green, for their parenchymatous cells, especially those of the palisade and spongy tissues in the foliage-leaves, are no less 
rich in chlorophyll-granules than those of shaded plants, only they have developed from their epidermal cells those structures which have been previously described as covering hairs. These cellular structures, devoid of chlorophyll, cover over the green tissue, and thus give to the leaf in question a grey or white colour. They play the part of awnings and light-extinguishers, and when they are removed the leaf appears just as green as one that has been plucked from the shade of the wood.

Silky, velvety, and woolly coats may thus doubtless take on the function of extinguishers. We meet, therefore, the same contrivances apparently which already on a previous occasion have been treated of, viz. when describing the protective measures against excessive transpiration. Thus through these structures two birds are killed with one stone. All contrivances which keep off too glaring sunbeams, and thereby hinder the destruction of chlorophyll, at the same time diminish transpiration; and inasmuch as these contrivances perform two such important functions for the life of plants, their wide distribution and great diversity is accounted for. Suited to the conditions, adapted to the habitat and season of the year, and in harmony with other developments, they change in a thousand ways, and thus display a diversity which can scarcely be treated exhaustively. Besides the covering hairs which are placed above the green tissue, as a protection and shade against too intense light, and at the same time against excessive transpiration, obviously all the other contrivances previously described are to be taken into account. The development of one or several layers of cells, filled with watery cell-sap, above the tissue exposed to the sun's rays, the thickening of the cuticular layers, the waxy and varnish-like coatings, the lime incrustations and salt excretions, the diminution of the illuminated portion of the leaf-surface, the formation of wrinkles, folds, pits, and grooves on the illumined surface of the foliage-all these are able to interrupt and diminish the rays and to reduce their intensity to the right degree.

The number of the special contrivances which simply secure chlorophyll from destruction by too glaring light, without at the same time protecting the green tissue from excessive transpiration, must indeed be very small. First of all, we may mention the dry thin-skinned scales which in many plants are inserted between the green leaves. These are seen, for example, in species of the genus Paronychia, which in masses have their habitat in sunny places, and produce silver-glittering transparent scales, devoid of chlorophyll, close to that portion of the stem from which the small green leaves originate. These scales, which are designated stipules, and which, here, are usually as large, occasionally even larger, than the green leaves, take up naturally such a position in the plants growing on shadeless hillocks that the sun's rays first of all fall on them, and only reach the green leaflets in a weakened state.

Another arrangement, which indeed is able to restrict the destruction of the chlorophyll by the sun's rays, without affecting transpiration, consists in the development of a blue or violet colouring-matter in those cells which compose the superficial covering of the leaves and stem which is directly illuminated by the sur's 
rays. Such an arrangement is found, for example, in the leaves of the aromatic Satureja hortensis, originally growing wild in the Mediterranean floral district, and cultivated in gardens under the name of Summer Savory, of which leaves a small portion is represented in cross section on page 139, figure $25 \mathrm{~A}, q$. Before the sunbeam reaches the chlorophyll-granules of the green cells in the middle of the leaf, it must pass through these epidermal cells filled with violet sap, and here it becomes so weakened and also so changed that an injurious influence on the chlorophyll-granules is out of the question. We must not omit to notice here that the violet light-reducing colouring-matter in the epidermal cells is more abundantly developed the intenser the light to which the plants in question are exposed. If plants of the Summer Savory grow in shady places, their leaves remain green on the upper sides, and scarcely any traces of the violet colouring-matter are to be discovered in the epidermal cells. If, on the other hand, they have germinated in shadeless districts, both stem and leaves are coloured dark violet, and the cell-sap in the epidermal cells is then of a deep tint (see fig. $25 \mathrm{~A}, q$ on page 139). Some years ago I cultivated seeds of the Summer Savory in my experimental garden at a height of 2195 metres above the sea-level in the Tyrol. As is known, the sun's rays are much more powerful in the Alpine heights than in the valley, and it was therefore, indeed, to be expected that the leaves of the germinating plants would be of a much darker tint than in the shadeless gardens of the valley below. In fact, the colouringmatter developed in extraordinary abundance; even the stems and leaves actually became a dark brownish violet. It is, therefore, beyond question that the quantity of colouring-matter in the epidermal cells directly exposed to the sun increases with the increase of the intensity of the light. Obviously this protection of the chlorophyll can only occur when the plants possess the materials for forming the pink colouring-matter in their green organs. When this is not possible, when the characteristic constitution of the protoplasm does not permit the development of the colouring-matter named in the foliage-leaves, the chlorophyll must be protected against the glaring light in another way, and if the plant species is not able to ward off the over-abundance of sunlight in the new position, it perishes entirely. Flax (Linum usitatissimum) was sown next to the Summer Savory in the Alpine experimentil garden-a plant which bears the direct sunlight quite well, and flourishes in the valley as well as in the plains in sunny situations. However, the light of the Alpine region was too brilliant for the germinating flaxplants; the leaves turned yellow, their chlorophyll was destroyed, and the seedlings became pale and perished. Flax has not the capacity of manufacturing the colouring-matter in its superficial cells, and it is also not organized to produce covering hairs on the leaves and stem, or to thicken its cuticular strata suitablyin a word, to adapt itself to the position and to provide itself, under the increased light intensity, with corresponding sun-shades and light-extinguishers. While close at hand; the Summer Savory, which requires just as much warmth, and an equally long vegetative period as flax, reached the flowering stage, and even produced ripe fruits capable of germinating, the flax died before the development of its flowers. 
From these culture experiments two things may be learned: first, that a very brilliant light is able to influence the distribution of plants and to set up an impassable barrier for many of them; and secondly, that many plants have the capacity of adapting themselves to various degrees of light intensity; but in consequence of this they occasionally develop such a varying character that they might be mistaken for wholly cifferent species. But I shall return again later when speaking of the origin of new species to this result of cultivation. Here we shall only discuss, in order to prove and make clear the connection between certain plant characteristics and the conditions of illumination, how it happens that the surface of foliage exposed to the direct rays of the sun is so frequently coloured violet or red, or is completely covered over with hairs, while the leaves of the same species if they have been developed on shady soil in dispersed light are coloured green, and remain almost bare; how it happens that plants of one and the same species in the deep valleys possess but few hairs, or are provided with but thin cuticular layers but on the sunny slopes of high mountains are shrouded in thick grey or white fur, or appear thick and almost leathery in consequence of strongly-developed cuticular layers. In order to prevent misconception, it must indeed be pointed out here that all this only refers to the epidermis over the green tissue which is exposed to direct or diffuse sunlight, chiefly, therefore, to the upper side of the foliage-leaf, and that when the blue colouring-matter and also the covering hairs are developed on the under side of the leaf, or in floral leaves devoid of chlorophyll, they have then an essentially different significance, which will be described in the next section.

When describing the protective measures of the green tissues against the dangers of over-transpiration, the vertical direction of branches, flattened shoots, phyllodes, and especially of the green leaf-surfaces, was pointed out. The leaves of irises, and of the so-called compass-plants, the flattened outspread petioles, with their edge directed towards the zenith, in so many Australian trees and slirubs, were there more especially described, and finally it was pointed out that the leaflets of many papilionaceous plants, and the leaves of numerous grasses, temporarily take up a position by sinking, rising, and folding together, in which not the broad side, but the narrow edge, is exposed to the vertical rays of the mid-day sun.

A leaf-surface which assumes one of these positions with regard to the sun will transpire much less than a foliage-leaf on whose broad surface the mid-day sun falls vertically, or almost vertically; but by such a position the leaf is also afforded a protection against the too vivid light of noon. The rays which reach a vertical leaf-surface at morning and evening are not so intense as to be able to destroy chlorophyll; they have rather just that intensity which the chlorophyllgranules require for their activity. Therefore, by this arrangement the function of the chlorophyll-granules is not restricted, but is actually assisted, and in this sense the vertical direction of the green surfaces is to be looked upon also as an arrangement for regulating the activity of the chlorophyll-granules.

It is evident after this explanation that herbs with vertically-directed leaf- 
surfaces are never to be met with in shady places. On the floor of a thick wood grow no irises and no compass-plants; these are at home on the ridges of rocky mountains, and on treeless prairies, and if it happens that a seed of such a plant falls into the shade of a wood and germinates there, developing foliage-leaves, then the leaf-surfaces do not assume a vertical position, and twist and bend themselves until their broad surface is turned towards the scantily-penetrating diffuse light. If the light falls from above through the interstices of the leafy covering, the leaf-surface becomes horizontal and parallel to the ground; if the crests of the trees close together to form a thick, opaque canopy, and the diffuse light penetrates from the side between the trunks of the trees, the leaf-laminæ bend and turn to the openings of the wood, giving the impression that they are looking out longingly to the sunny country which borders the dense, deep-shaded forest.

The same thing is seen under every small shady bush, and, generally speaking, in all places where dissimilar tall plants overlap one another, and where the leaves of the lower are arched over by those of the higher plants. If they belong to different species, they cannot be said to have any consideration for one another. Each looks out only for itself, and the lofty species do not trouble themselves about the inferior stuff which arises from the soil under their leaves. If in the depths below there are plants which find all they require in the diffuse light and the green rays passing through the leafy roof, very well; if not, these lower plants must perish in the shade. But it is otherwise if the leaves overlapping each other belong to one and the same branch, to one and the same plant; where they must co-operate for the weal of the whole plant, and the whole can only maintain itself in the struggle for existence by harmonious division of labour. Therefore care must be taken that. no leaf shall take too much light away from another; that one shall protect and support the other; that neighbours shall not jostle if one or the other has to bend, turn, and extend itself in order to best adapt itself to the incident light.

And this foresight actually occurs. It is exhibited, first of all, in the position of the leaves on the stem, or in other words, in the regulation of the intervals between the places of origin of neighbouring leaves; secondly, by the fact that the stalks of the green leaf-blades have the capacity of rising and sinking, twisting and bending, and also of elongating if required; and thirdly, through the form which the leaf-surfaces possess. 


\section{THE GREEN LEAVES.}

Distribution of the green leaves on the stem.-Relation between position and form of green leaves. - Arrangements for retaining the position taken up.-Protective arrangements of green leaves agaiust the attacks of auimals.

\section{DISTRIBUTION OF THE GREEN LEAVES ON THE STEM.}

Landscape painters tell us how difficult it is to treat foliage correctly, and at the same time artistically; how hard, for instance, so to reproduce the leafy crown of maples, beeches, elms, limes, and oaks that they shall immediately be recognized for that which they are intended to represent, and at the same time that that effect and tone shall be produced which is aimed at in the picture. The variety of the foliage is caused not least by the distribution of the green leaves on the branches, and by the branching dependent upon this; things as definite as possible for each species of tree, and, generally speaking, for every plant.

On cutting various leafy branches and observing the distribution of the leaves on them, the following differences first strike the eye. In numerous plants it is seen that two or more leaves originate at the same height on a branch, while in many other plants, at a particular level of the stem or branch, only a single leaf is produced. In order to be able to understand these circumstances, it is advisable to imagine the leaf-bearing shoot or stem as a cone. The apex of the cone corresponds to the upper end, and the base of the cone to the lower portion, i.e. to the oldest part of the shoot. The whole shoot is not at any time in a completed state; it continues to grow at the apex, and at the upper part is not only younger, but is also less bulky than the older portions lying nearer to the base. It can, therefore, indeed be quite well compared to a cone, although this form is only seldom so noticeably to be met with as in the following diagrammatic figures.

That which applies to the age of the various portions of the shoot naturally applies also to the leaves projecting from the shoot. That is to say, the lower leaves of the shoot are the older, the upper leaves are the younger. On looking at the top of the cone (see fig. 98), the places of insertion of the older leaves appear to arise, first of all, from the circular disc which forms the base of the cone, while the younger leaves originate close to the apex, therefore close to the centre. The stem is to a certain extent divided up by the leaves into sections one above another. Usually it is somewhat thickened or knotted at those places where the leaves project from it, and therefore the places of origin of the leaves are designated as nodes. Each portion of the stem lying between two successive nodes is called an internode. When two leaves project at the same height from the stem, they are inserted opposite one another, not unlike the two extended arms of a human body, and they appear on the cone-shaped stem (whose cross section at all heights forms a circle) at a distance from one another of exactly half the circumference of the circle $\left(180^{\circ}\right)$, (fig. $\left.98^{1}\right)$. If three leaves spring together from the stem, 
as, for example, in the Oleander, these are separated from one another in a horizontal direction by one-third of the circumference of the circle $\left(120^{\circ}\right)$. Several leaves springing from the same height form together a whorl, and the distance of the individual members of a whorl from one another is called the horizontal distance, or the divergence. The divergence amounts to $\frac{1}{2}$ in fig. $98^{1}$, and $\frac{1}{3}$ in fig. $98^{2}$, of the circumference of the circle, and can be thus shortly expressed by means of these fractions.

It is very remarkable that the whorls which follow after and above one another according to their age on one and the same shoot do not originate at corresponding places of the circumference, but are displaced regularly with regard to one another. Thus the point of origin of the second two-membered whorl in
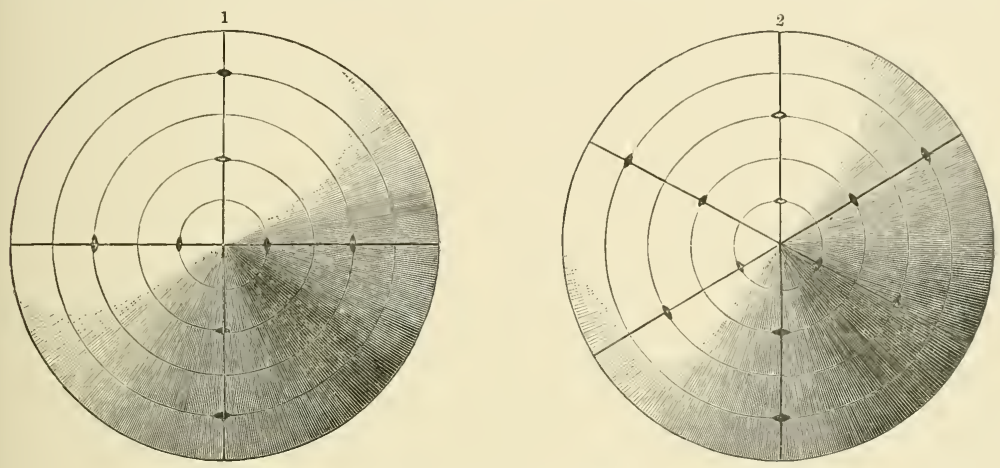

Fig. 98.-Plan of Whorled Phyllotaxis.

1 Two-membered whorl. 2 Three-membered Whorl.

fig. $98^{1}$ is shifted through a quarter of the circumference (i.e. through $90^{\circ}$, a right angle) from the point of origin of the first, oldest, and lowest two-membered whorl. The third whorl is again shifted through a right angle with regard to the second, and so it continues up the stem as far, generally speaking, as foliage-leaves are to be found on it. If the stem is elongated in the case described, four rectilineal lines (orthostichies) appear to be developed on it (fig. $98^{1}$ ). If a whorl is composed of three leaves, and if the successive whorls be displaced through one-sixth of the circumference, as, for example, in the Oleander (see fig. $98^{2}$ ), six rectilineal series of leaves or orthostichies originate, running parallel to one another down the stem.

The leafy stem can also be imagined as divided into stories, each of which displays the same number, position, and distribution of the leaves, and agrees completely in the plan of its construction with the adjoining story. In one such case (fig. $98^{1}$ ), each story possesses four leaves in the form of a cross; in another case (fig. $98^{2}$ ), it possesses two sets of three leaves separated from one another by a distance of $60^{\circ}$. If the stories standing above one another are separated, they would be so alike in arrangement as to be easily mistaken for one another. Each 
originates below and ends above exactly like the one over and the one under it, and the only difference rests in the fact that the sections closer to the summit of the branch have smaller diameters, and often also a somewhat different outline of their members. The plan of construction is, however, as stated, exactly the same in the successive stories.

In those instances where each story consists of two whorls of leaves, which are displaced with regard to one another through a certain angle, especially in the very common case where the whorl is two-membered, i.e. where the leaves are opposite one another in pairs, and where the successive pairs of leaves are alternately displaced through a right angle from one another, appearing thus like a cross, the leaves are said to be decussate. This arrangement is seen especially in maples and ashes, in lilac and olive-trees, in elder and honeysuckle, in labiates, gentians, Apocynaceæ, and numerous other families of plants.

Still more common than this arrangement of the leaves is that which has been called the spiral. Here at one and the same height only a single leaf originates from the stem, and therefore all the leaves of a stem are not only shifted with respect to one another in a horizontal, but also in a vertical direction. If one imagines the nodes of a stem with decussate leaves to be so arranged longitudinally that the leaves are inserted no longer at the same heights, but at definite intervals above one another, then from the decussating, i.e. whorled, arrangement a spiral is produced. In many willows (e.g. Salix purpurea), and very regularly also in some buckthorns (e.g. Rhamnus cathartica), in the speedwells (e.g. Veronica spicata and longifolia), and also in many composites leaves arranged partly in whorls and partly in spirals occur on the same axis, and doubtless the one merges into the other, but for the sake of clearness it is better to keep them distinct, and to draw a line between them, even though it be an imaginary one.

It may be observed that stems with spirally-arranged leaves are constructed exactly like those which bear leaf-whorls, and that they consist of many stories each displaying a similar plan of construction, so that the number, position, and distribution of the leaves is repeated in each story, and as a matter of fact the following plans of construction are actually to be found very frequently.

First case. In each story only two leaves arise from the circumference of the stem. These two leaves are displaced with regard to one another in a horizontal as well as vertical direction, and their horizontal divergence amounts to half the circumference of the circle $\left(180^{\circ}\right)$ as shown in the plan in fig. $99^{1}$. If a continuous line be drawn from the point of insertion of each lower older leaf to the younger one next above it on the surface of the stem, this will display the form of a spiral. It has been called the genetic spiral. In the first case here discussed it forms in each story only a single spiral band. This arrangement is repeated in the second, third, and perhaps in many other stories which follow successively on the same axis. In this way the lower leaf of the second, third, or fourth story always lies exactly above the lower leaf of the first story. The same applies to the upper leaves of all the stories. Thus two rectilineal lines or orthostichies are formed on 
the circumference of the stem by the leaves situated vertically above one another. The two lines are opposite, or, what comes to the same thing, they are separated from one another by half the circumference of the stem. This arrangement of the leaves, which may be observed, for example, on the branches of elms (Ulmus) and limes (Tilia), is called the one-half phyllotaxis.

Second case. Three leaves are developed in one story, each at a definite height, an under, a middle, and an upper leaf. In a horizontal direction two of the leaves following one another in age are always shifted from one another through a third part of the circumference (see fig. $99^{2}$ ). If the point of insertion of the lower leaf is connected with that of the middle leaf, and this again with that of the upper leaf by a line, and this line is continued to the beginning of the next story, a single spiral is thus formed surrounding the stem. Now above the story just described, which we will call the lowest, a second follows, which is again provided with three leaves arranged in exactly the same way. The lower leaf of the second story is situated vertically above the lower leaf of the first story, the middle above the middle, and the upper above the upper leaf, and the same arrangement is continued through all the stories. In this manner three rectilineal lines, or orthostichies, arise on the circumference of the stem from the leaves situated above one another, and each of the lines is separated from the other two by $\frac{1}{3}$ of the circumference. This arrangement, which is to be found on the upright branches of alders, hazels, and beeches, is called the one-third phyllotaxis.

Third case. Five leaves originate in each story, which are designated according to age as the first, second, third, fourth, and fifth, the lowest being the oldest, the highest the youngest. These five leaves give place to one another in a horizontal direction, and the shifting, i.e. the horizontal distance between two leaves next in age, amounts to $\frac{2}{5}$ of the circumference of the circle (see the plan, fig. $99^{3}$ ). If the five leaves are joined together in succession according to their age, a spiral line is obtained consisting of two revolutions, and the "genetic spiral" consequently forms two circuits round the stem. If a stem, whose leaves are arranged in this manner, is built up of two or several stories, then the similarly numbered leaves are situated in straight lines above one another, the first (lowest) leaves of all the stories form together one straight line (orthostichy); in the same way the second, the third, \&c. Thus five lines are developed on the circumference of the stem by the leaves situated one above the other, and each line is separated from another by $\frac{1}{5}$ of the circumference. This arrangement, which is found in oaks, round-leaved willows, and in many buckthorns, is designated the two-fifths phyllotaxis.

Fourth case. Eight leaves are to be found in each story, which may again be numbered from one to eight according to their age. Any two successive leaves are separated from one another horizontally by $\frac{3}{8}$ of the circumference (see fig. $99^{4}$ ). If a line be drawn starting from the first and lowest leaf, joining all the eight leaves of the story in the order of their ages, this forms a spiral line, or "genetic spiral", which traverses the stem three times. In a stem consisting of several such stories, the leaves named by the same numbers are placed in straight lines above one 
another, and accordingly eight rectilineal lines (orthostichies) run down the stem. Each line is separated from its neighbour by $\frac{1}{8}$ of the circumference. This arrangement, which occurs in roses, raspberries, pears, and poplars, in laburnuns, and in the barberry, is called the three-eighths phyllotaxis.

Yet a fifth case is very often to be found in trees and bushes with narrow leaves, viz. in the Almond-tree, in the Goat's-thorn, in the Sweet Willow, in the Sea Buckthorn, and many Spiræa bushes. Each story contains thirteen leaves.
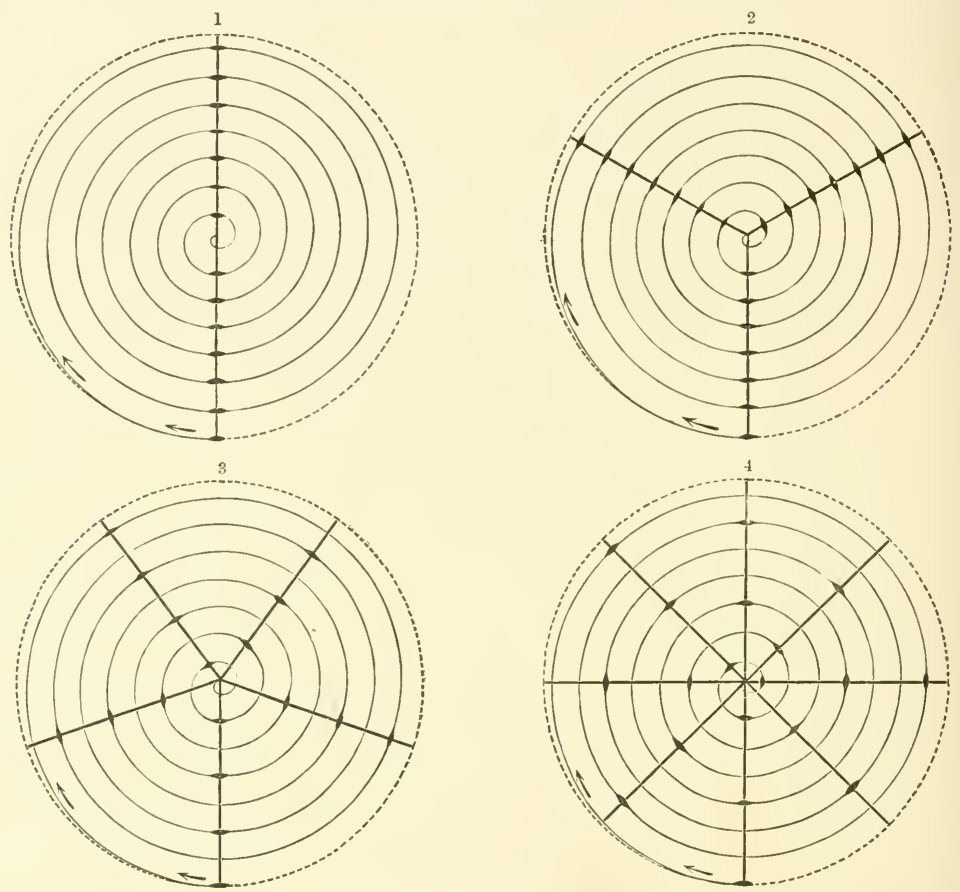

Fig. 99.-Plan for Spiral Phyllotaxis.

1 One-half Phyllotaxis. 2 One-third Phyllotaxis. 8 Two-ffths Phyllotaxis. 4 Three-eighths Phyllotaxis. The conical stem horizontally projected; the points of insertion of the leaves on the circumference of the stem marked by a dot.

which may be connected by a spiral line, i.e. a "genetic spiral", with five revolutions. The number of the straight lines here amounts to thirteen, and the distance between two leaves following one another in age is $\frac{5}{13}$ of the circumference, i.e. $138^{\circ}$ (see fig. 100).

Not so common, or rather not demonstrable with the same precision, are instances in which one story shows twenty-one leaves which are connected by a genetic spiral with eight revolutions; and where a story includes thirty-four leaves which are connected by a genetic spiral with thirteen revolutions. In the one case any two leaves next one another in age in a story are separated from one 
another $\frac{8}{21}$ of the circumference; in the other case by $\frac{13}{34}$; and from this it follows that in the one instance there are twenty-one, and in theother thirty-four orthostichies.

If we place these actually-observed instances together, we have the series $\frac{1}{2}, \frac{1}{3}, \frac{2}{5}, \frac{3}{8}, \frac{5}{13}, \frac{8}{21}, \frac{13}{34} \ldots \ldots$

But the variety of the conditions on which the leaves are arranged is not exhausted by a long way. Although but seldom, still cases have been observed which can be placed together in the series $\frac{1}{4}, \frac{1}{5}, \frac{2}{9}, \frac{3}{14}, \frac{5}{23} \ldots .$. , and also in the series $\frac{1}{6}, \frac{2}{7}, \frac{3}{11}, \frac{5}{18} \ldots .$. . In all these series this very remarkable peculiarity occurs, that

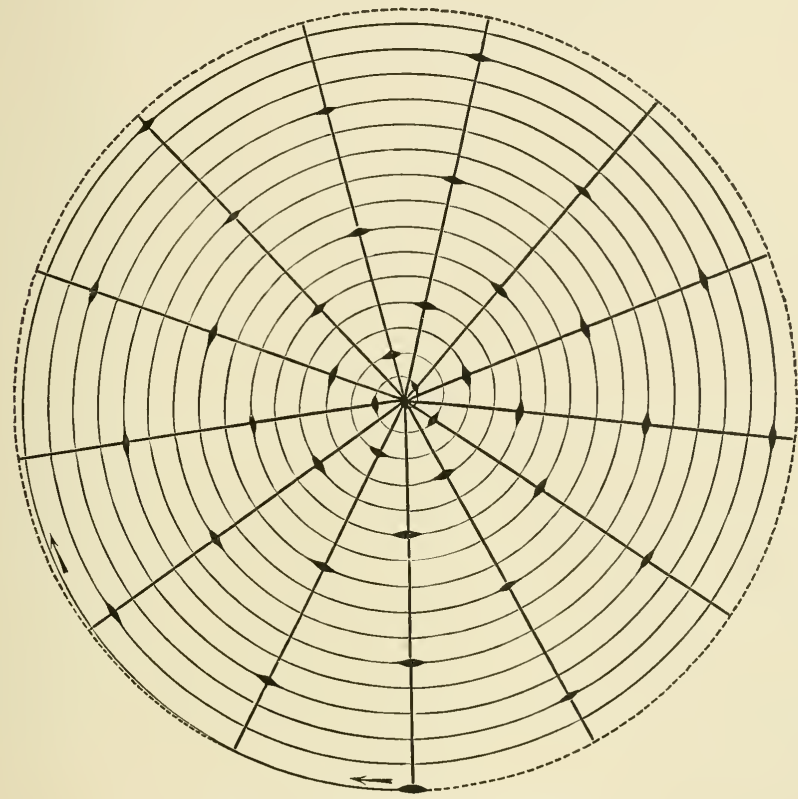

Fig. 100.-Plan of Five-thirteenths Phyllotaxıs.

in each individual fraction the denominator is equal to the sum of the denominators, and the numerator is equal to the sum of the numerators of the two preceding fractions.

Moreover it must be here particularly mentioned that the divergence, by which the leaves following one another in age are separated in a horizontal direction, is the more difficult to establish the smaller it becomes. The one-third, two-fifths, and three-eighths arrangements are the most easily demonstrable on the full-grown shoots, although occasionally doubt arises as to whether the three, five, and eight orthostichies represent completely straight lines. But the demonstration of $\frac{8}{21}$ and the $\frac{13}{34}$ arrangements, especially in green herbaceous stems, is very difficult and uncertain. 
There are only few plants on whose branches or axes several stories occur with twenty-one or thirty-four successive leaves in each. On the other hand, it happens that on many shoots, not even one story is completely formed, or in other words, that in more than a hundred leaves which project from the axis, no two are to be found situated quite vertically above one another, and consequently, in these cases, rectilineal orthostichies are out of the question. In many fir-cones, for example, rectilineal lines are sought for in vain, and it is impossible, even approximately, to estimate how many leaves are included in one story. It has been also conjectured that in such cases the leaves of a story are innumerable, and if so, the fraction by which such a system of leaf-insertion would be represented would be an absurd figure.

In such shoots it is anything but easy to establish the successive ages of the leaves, that is, to number them in their proper order of development, especially when the leaves are thickly crowded together. This becomes the more difficult when the leaves on such very crowded axes arrange themselves in spiral series, or lines which are much more apparent to the eye than the lines of development or genetic spirals. These spiral series, which are seen on shoots of many succulent plants (Sedum, Sempervivum), on species of Pandanus and Yucca, on the branches of lycopodiums and conifers, and especially also in the inflorescence of crucifers and the cones of many firs, of which a pine-cone, represented in fig. 101, may be taken as an example-these series are called parastichies. They may be utilized in order to ascertain which leaves succeed one another in age, thus-by first of all ascertaining how many such parallel spiral lines ascend to the right, and how many to the left on the axis examined. In a pine-cone, for example (see illustration below), eight such lines or parastichies are seen to ascend in a somewhat sharply oblique direction to the left, and five to the right in a rather less sharply oblique direction. In order to find out which leaves succeed one another in age, the lowest leaf is called 1 , and the numbers 8 and 5 are used in the following manner. The leaves of those steep parastichies, on the left adjoining 1 , are numbered by additions of 8 respectively, $9,17,25,33,41$, \&c. The leaves of the less steep parastichies on the right, which adjoin 1 , are numbered, on the other hand, by additions of 5 respectively, $6,11,16,21,26, \&$ c. The numbering of the other parastichies is then easily completed by subtractions and additions of the numbers 8 and 5 , and the numbers so obtained represent the successive ages of the leaves on the cone. This somewhat complicated arrangement may be best demonstrated by imagining the surface of a leafy, almost cylindrical axis, e.g. of a pine-cons, to be slit up longitudinally, rolled out flat, and extended so that all the leaf-scales lie in one plane, as represented in the plan illustrated in the right-hand figure opposite.

Naturally the most lively interest has been aroused at all times by the geometrical ratios of phyllotaxis here generally reviewed, and it could not fail to follow that the most diverse speculations should have been connected with them. This is not the place to consider these in detail, but in so far as the remarkable and actually existing conditions of the geometric arrangement of the leaves have a 
significance in the life of the plant, the attempts to explain them must not be passed over. First of all, it must be pointed out that the number of orthostichies, i.e. of the leaf-members of a story, as well as the number representing the circuits made by the genetic spiral in each story, is connected with the extent of the horizontal divergence between consecutive leaves. In order to make this clear, let us draw a spiral line on the surface of a cone, as shown in fig. 99, and let us place dots on this line at regularly recurring intervals. The length of the interval between the dots is quite immaterial, it is only of importance that the successive dots shall remain separated from each other by the distance originally fixed upon.
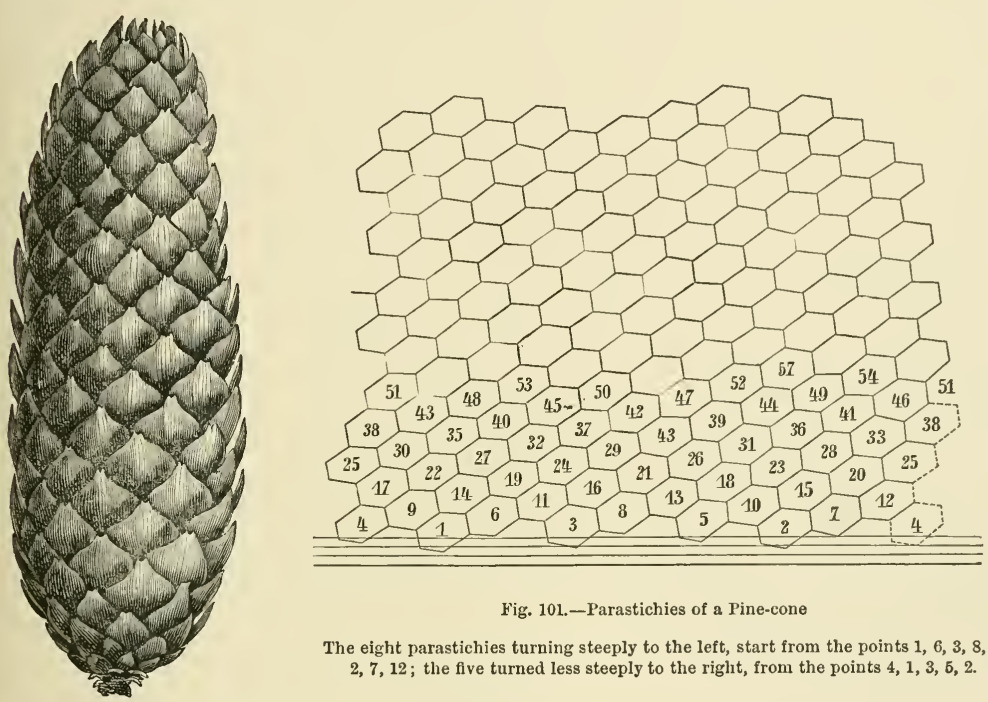

Fig. 101.-Parastichies of a Pine-cone

The eight parastichies turning steeply to the left, start from the points $1,6,3,8,5$, $2,7,12$; the five turned less steeply to the right, from the points $4,1,3,5,2$.

Suppose that the dots are placed on the spiral line at intervals of $\frac{1}{10}$ of the circumference of the circle $\left(36^{\circ}\right)$, then in each revolution of the spiral there will be 10 dots, separated by equal distances from one another. With the tenth $\frac{1}{10}$, however, the spiral line has completed the circuit of the cone, i.e. of the axis. The eleventh dot lies vertically above the first dot, and with it begins a new revolution and a new story. On such a stem ten orthostichies would necessarily be produced, and if we substitute actual leaves for the dots, the phyllotaxis will be represented by $\frac{1}{10}$. As another example, let us place the dots on the spiral line at horizontal distances of $\frac{2}{7}$ of the circumference. How will the dots then be arranged? Dot 2 is $\frac{2}{7}$ of the circumference of the circle from $\operatorname{dot} 1 ; \operatorname{dot} 3, \frac{2}{7}+\frac{2}{7}=\frac{4}{7} ; \operatorname{dot} 4, \frac{2}{7}+\frac{2}{7}+\frac{2}{7}=\frac{6}{7} ; \operatorname{dot} 5$, $\frac{2}{7}+\frac{7}{7}+\frac{2}{7}+\frac{2}{7}=\frac{8}{7}$ of the circumference from dot 1 , measured along the genetic spiral. Dot 4 is not quite vertically above $\operatorname{dot} 1$, and $\operatorname{dot} 5$ lies beyond it, neither of the two, therefore, coming exactly above 1. More dots are now placed at the same intervals on the second revolution of the spiral line; first dot 6 , which is $\frac{10}{7}$, then 
$\operatorname{dot} 7$, which is $\frac{12}{7}$, and, finally, $\operatorname{dot} 8$, which is $\frac{14}{7}$ of the circumference from dot 1 along the genetic spiral. Dot 8 is found to lie exactly above dot 1 , and here the second revolution of the spiral line is completed. This is the termination of the first story, and with dot 8 a new one commences. On a stem whose leaves are distributed in the same way as the dots in the example just described-any two of which are always separated from one another by $\frac{2}{7}$ of the circumference in a horizontal direction-seven orthostichies will be produced, and the genetic spiral, i.e. the line which connects the leaves consecutively following one another according to their age, will make two revolutions round the stem. Such an arrangement would be designated as a two-sevenths phyllotaxis. From these examples it follows that a definite phyllotaxis corresponds to each horizontal divergence between leaves following one another in age, whatever this may be, as long as it only remains constant. The divergence measured along the circumference of the stem may be large or small. Finally, there will be an equal distribution of leaves around the stem, and they will project at equal horizontal distances in as many directions as are given by the denominator of the fraction representing the divergence. But the spiral line which connects all the leaves represented by the denominator with one another will make as many circuits round the stem as the number constituting the numerator of the fraction. In other words, the extent of the horizontal divergence always gives us the phyllotaxis. The denominator of the fraction is equal to the number of orthostichies, and the numerator is equal to the number of revolutions made by the genetic spiral in each story.

The observation already alluded to above, according to which those fractions by which the phyllotaxes actually found in plants may be expressed as members of a definite series, must now be considered further. It has been found that the horizontal divergences between consecutive leaves respectively form part of a continued fraction of the form

$$
\frac{1}{z}+\frac{1}{1}+\frac{1}{1} \ldots \ldots,
$$

In which $z$ is a whole number. If for $z$ we substitute the number 1 , the successive parts of the fraction will give us the series $\frac{1}{2}, \frac{2}{3}, \frac{3}{5}, \frac{5}{8}, \frac{8}{13}, \frac{13}{21}, \ldots \ldots$. If $z=2$, the series $\frac{1}{2}, \frac{1}{3}, \frac{2}{5}, \frac{3}{8}, \frac{5}{13}, \frac{8}{21} \ldots \ldots$ is obtained. If $z=3$, the series $\frac{1}{3}, \frac{7}{4}, \frac{2}{7}, \frac{3}{11}, \frac{5}{18}, \frac{8}{29} \ldots \ldots$, and if $z=4$, the series becomes $\frac{1}{4}, \frac{1}{5}, \frac{2}{9}, \frac{3}{14}, \frac{5}{23}, \frac{8}{37} \ldots \ldots$. It is remarkable here that among all the phyllotaxes, those represented by the numbers $\frac{1}{2}, \frac{2}{3}, \frac{2}{5}, \frac{3}{8}, \frac{5}{13} \ldots \ldots$. occur most frequently, while phyllotaxes belonging to the other above-quoted series are only occasionally met with. Thus, as a matter of fact, the series occurs oftenest in which 2 is substituted for $z$. The advantage offered by the series produced from this number has been explained in this way: by it, on the one hand, phyllotaxes are produced by which an equal distribution of the leaves is obtained by the smallest possible number in each story; and, on the other hand, phyllotaxes again in which leaves may project from the stem in very many different directions.

The reason why each species of plant arranges its leaves, even while in the 
bud, in the most advantageous manner, quite independently of external influences, without the knowledge, so to speak, of the conditions to which its foliage-leaves will be exposed in the future, can only be explained by the specific constitution of its protoplasm. Just as crystals are formed from the aqueous solution of a salt which, according to the nature of the salt, are sometimes six-sided, sometimes three-sided, whose surfaces are always the same in outline, and whose edges always form exactly the same angles, so bands, bars, and partition-walls arise in the growing cells, by which these cells become articulated and divided; and the shape and position of these intercalated walls and their geometrical ratios are no less definite in the most diverse plant species than are the surfaces of the crystals arising from the salt solution. But that which applies to the plan of construction of the individual cells must also apply to the plan according to which a group of cells-a tissue, a growing shoot, a stem with its leaves, even the entire plantis constructed. The position on the circumference of the stem at which a leaf originates is certainly not determined by chance, but is based upon the molecular constitution and composition of the protoplasm of the species of plant in question; and if the leaves on an oak-branch always arrange themselves in $\frac{2}{5}$ phyllotaxis, the constancy of the arrangement is neither more nor less remarkable than the constancy of the size of the angles in an alum octahedron.

It should be noted here, in this connection, that the geometrical arrangement of the cells in simple elongated tissues, easily accessible to observation, is exactly similar to the arrangement of the leaves on stems. For example, the cells on the hair-like stigmas of grasses follow the one-third arrangement very beautifully. A connection between the geometrical arrangement of the cells at the apex of a growing stem, and the geometrical arrangement of the leaves on the same, may now also be considered. A group of cells is formed out of each cell at the growing point of the stem by the repeated intercalation of division-walls. If the position of these dividing cells is geometrically defined, and if the partition-walls resulting from their division assume definite directions in each species of plant, then the arrangement of the cell-groups produced from these cells which build up the stem must also be geometrically defined. Supposing now that from each of these groups of cells which build up the stem a leaf arises, then the distribution of the leaves on the circumference of the stem will be only a repetition of the distribution of the cells at the growing point of the stem. In the simplest of all leafy stems, in that of a moss-plant, this relation is noticeable enough; but in plants of more complicated construction it is not so easily demonstrated. In these the constancy of the geometric ratios of the cells at the growing point is beset with many difficulties, and the groups of cells produced from them are also much displaced and distorted. Nevertheless in each form of plant a uniform plan of construction very probably exists; and it may be taken for granted that in each species the arrangement of the atoms in the protoplasm, the arrangement of the cells, and the arrangement of the leaves, are based upon the same symmetrical construction.

Indeed, even the displacements and torsions of the cells which occur in leafy 
stems without doubt take place according to rule, although they may be in part due to external causes. Numerous comparative observations have shown that the building, and especially the lengthening of the growing stem, does not always follow the direction of a straight line; that, rather, a spiral torsion of the cells and tissues not infrequently occurs, so that the idea that such a stem by its growth bores its way through the air is quite justified. This does not, indeed, refer to the twining of the stem, which will be discussed later, but to the torsion of the tissue mass of a straight stem which remains straight after the torsion has been effected, and which may best be compared to the twisting of a bundle of rectilineal strands to form a string. In every bud from which a leafy branch arises, the points of origin of the leaves may be seen on the periphery of the still very short conical axis; frequently, also, the shape and outline of the leaves are perceptible, and the position and divergence of the leaf-insertions can be geometrically established. If the axis has elongated, and an extended branch been produced from the bud, the arrangement displayed by the fully-formed, displaced leaves does not always coincide with that in the bud. The phyllotaxis has become altered by reason of the pressure which the individual groups of cells exercise on one another in their increase in length and breadth, and in consequence of displacements connected with these pressures, i.e. torsions arise. If the torsion is restricted to one portion of the stem only, an actual transition of one phyllotaxis into another is seen, and occasionally it is very pronounced.

In order to make clear the alterations arising in this way, it is only necessary to remove the leaves from a herbaceous leafy stem, to hold it by the two ends, and to twist it as a bundle of threads might be twisted into a string. The points of insertion of the leaves are thus mutually displaced, parastichies are formed from the orthostichies, and new, often very complicated, leaf-arrangements come into view. The alterations produced by the torsion of the stem may also be rendered evident by a consideration of fig. 102. Let us suppose that the black dots on the three thick lines of the young conical stem, horizontally projected in this illustration, indicate leaf-positions which are separated from one another by a distance of $\frac{1}{3}$ of the circumference of the circle $\left(120^{\circ}\right)$. Suppose now that the stem has undergone a torsion as it lengthened, which is quite definite and equally distributed over all portions of the stem. Each portion of the stem between two consecutive leaves, following one another in age, is twisted through, say $\frac{1}{15}$ of the circumference $\left(24^{\circ}\right)$, and in consequence of this the divergence of the leaves is no longer $\frac{1}{3}$ of the circumference, i.e. $120^{\circ}$, but $120^{\circ}+24^{\circ}=144^{\circ}$, or, as much as $\frac{2}{5}$ of the circumference. By reason of this the points of origin of the leaves come to lie in the positions indicated by the thinner lines, and a two-fifths is produced from a one-third phyllotaxis. In the same way the three-eighths arises from the one-third phyllotaxis if the consecutive dots are displaced $\frac{1}{24}$ of the circumference $\left(15^{\circ}\right)$ by the torsion, and the horizontal divergence no longer amounts to $\frac{1}{3}$ of the circumference, but to $\frac{3}{8}$. The one-third becomes changed into the one-half phyllotaxis if the second leaf of a story, which in the bud was separated 
from the first by $\frac{1}{3}$ the circumference, in consequence of the torsion of the growing stem, is displaced about $\frac{1}{6}$ the circumference $\left(60^{\circ}\right)$; that is to say, exactly so much that it is now separated from the first by half the circumference $\left(180^{\circ}\right)$. This particular alteration can be very well seen in the developing branches of beeches, hornbeams, hazels, and many other trees and shrubs. In the buds the leaves have a one-third arrangement, in the fully formed, now woody branches the phyllotaxis appears to be one-half. Since, as a rule, amongst buds, the simplest cases, especially

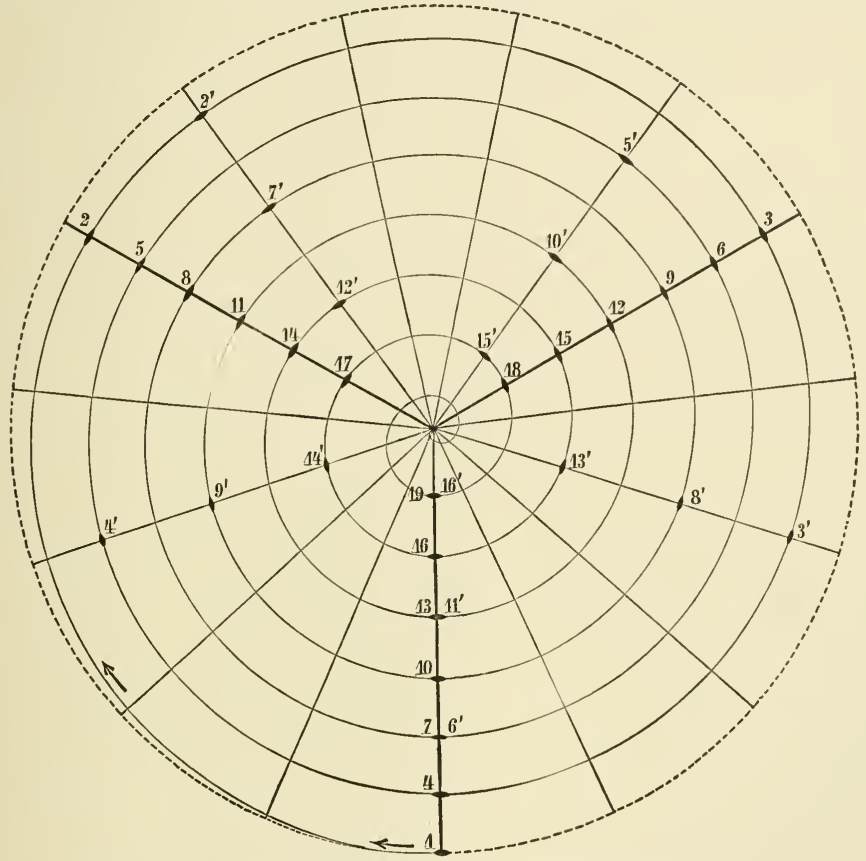

Fig. 102.-Displacement of the leaf-positions in consequence of torsion of the stem.

Transformation of the one-third into the two-flfths phyllotaxis. Dot 2 is displaced by torsion to 2'; dot 3 to $3^{\prime}$, \&c.

the one-third arrangement, are most frequently observed, it appears probable that the number of original phyllotaxes is really only very small, and that complicated leaf arrangements, which are represented by fractions whose numerator consists of two figures, frequently are produced by torsion of the individual parts of the stem during their growth. It still remains to point out here that the phyllotaxis becomes the more complicated, the less the amount of torsion undergone by an internode, which is, indeed, evident from the preceding representation. It is also worthy of note, that in plants whose foliage-leaves originate 2,3 , or more together at the same height on the stem (which therefore possess whorled leaves), such torsions of the internodes, and alterations of the phyllotaxis dependent upon them, very frequently occur. 


\section{RELATION BETWEEN POSITION AND FORM OF GREEN LEAVES.}

Now that the distribution of the green leaves on the surface of the stem has been generally described, it is possible to discuss the relation of the phyllotaxis to the length and breadth, as well as to the shape and direction, of the leaf-blades.

If a small leafy moss-plant, or a huge densely-leaved tree be examined, it will always be found that the number of orthostichies on the straight stems becomes smaller as the leaf-blades become broader. If the leaf-blades are circular, like those of the Judas Tree (Cercis Siliquastrum), or if they are broadly ovate or cordate, being broadest at the base, like those of limes and elms, or if they are not perhaps borne on very long petioles, i.e. like those of the Aspen (Populus tremula), then they pass down the stem in two lines, thus displaying a one-half phyllotaxis. If the leaf-blades are broadly elliptical, and therefore broadest about the middle, and also have but short stalks, like those of beeches, alders, and hazels, then they are arranged regularly in three rows on the erect branches and display a one-third phyllotaxis. If the leaves are obovate, i.e. broader at the top than at the base, and at the same time have only short stalks, as, for example, those of oaks, then they are arranged in five lines, according to the two-fifths phyllotaxis. If they are lanceolate or oval, like those of the Almond-tree, they usually have the threeeighths phyllotaxis; and finally, the narrow linear leaves on the twigs of the Genista tinctoria, as well as the long narrow leaves on the stems of the Goldenrod (Solidago), are regularly arranged in a five-thirteenths phyllotaxis. In the mosses the same relations hold good; the broad leaves of the Mnium species display the one-third; the elliptical and oval leaves of many earth-mosses (Barbula) the two-fifths; and the narrow linear leaflets of polytrichums the three-eighths, five-thirteenths, and more complex phyllotaxis. This connection between the breadth of the leaf-blade and the number of rectilineal leaf-rows on the erect stem is very noticeable even in members of the same genus, and in this respect perhaps no genus is so instructive as the willow. There are willows with circular, elliptical, oval, and narrow linear leaves, and in these it can be plainly seen that the number of orthostichies increases in proportion as the leaves become narrower. Salix herbacea with circular leaves has a one-third, Salix Caprea with elliptical foliage a two-fifths, Salix pentandra with lanceolate foliage a three-eighths, and Salix incana with linear leaves a five-thirteenths phyllotaxis.

If we take erect branches from each of these willows, placing them all together, and look down upon them from above, we see how the three, five, eight, and thirteen rows of leaves radiate out from their respective axes. But it is also plainly evident that in each case the neighbouring rows so adjoin one another as to leave no gaps between them, so that the space round the stem may be utilized to the greatest possible extent. In one case, therefore, we have three rows of very broad leaves, in other cases five or eight rows of moderately broad leaves, and again in another instance thirteen rows of very narrow leaves. 
All the rows of leaves, whether there be three, five, eight, or thirteen of them, are equally illumined by the sunbeams which strike them from above in the direction of the axis of the branch; no row throws another into the shade, and only the upper individual members of a row standing above one another can deprive the lower members of light. But even this shading is avoided, chiefly by the adaptation of the length and direction of the foliage-leaves to the height of a story.

If the stories are low, so that the consecutive leaves of a rectilineal row are separated only by short distances, then the leaves are short; if the stories are high, then the leaves are long; the length is always so arranged that the sunbeams can penetrate into the space between every two leaves of a row, and can, so to speak, illuminate the interior of the story.

It should be remembered here that the sun does not shine down vertically upon branches having an upward direction, that its rays, even at the equator, fall obliquely in the morning and evening, and at these times illuminate the space, bounded above and below by two consecutive leaves of a row, just like the rays of the rising and setting sun which enter a room through the window. But this does not say that no leaf is thrown into the shade throughout the entire day. This would be impossible, from the fact that the sun's rays at each hour of the day fall at a different angle on the plants which remain firmly fixed and immovable in the soil. The leaves of one side are partially shaded in the morning, and those of the other side in the afternoon; or they are only illuminated by diffuse light; and the upright stem, which is set round about with projecting leaves, must necessarily shade a portion of them for a short time during the day. But these shadows, like the dark lines thrown by the gnomon of a sun-dial, must continually move forward with the sun, and only remain in one place for a little while.

The entrance of the sun's rays between the leaves situated above one another is, moreover, materially influenced by the direction of the leaf-blades. A leaf projecting obliquely upwards from the stem, with its midrib in the plane of the incident rays, will not at any hour of the day deprive its lower-placed neighbour of too much light, or at any rate to a much less extent than will a leaf whose blade is extended horizontally or sloped a little in an outward direction, and which presents its broad side to the incident sunbeams. This explains a phenomenon which is seen very often in annual and biennial composites and crucifers with straight, erect stems. The lowest leaves of these plants form a right angle with the axis of the stem, and lie with their broad surfaces on the soil, completely covering a larger or smaller area. These can obviously not take away the light from any other leaves of the same plant. The leaves inserted higher up the stem are, on the other hand, no longer extended horizontally, but rather in an upward direction, and form an angle with the stem which is less than a right angle; and the highest leaves even approach the upright, their midribs lying in the plane of the incident noonday rays.

In accordance with this adjustment, an alteration of the dimensions, particularly of the length of its leaves, may be observed at different heights of an erect thickly- 
leaved stem. The lowest leaves originating next the soil are the longest; the leaves next above these are, on the other hand, visibly shorter, and often in the region of the flowers are changed into insignificant scales closely applied to the stem. It can easily be seen in every plant of the Shepherd's Purse (Capsella Bursa pastoris), on every mullein (Verbascum), and every hawkweed (Hieracium), that such small upwardly-directed leaves cannot injure by overshadowing the leaves growing below them either in the same or in adjacent rows.

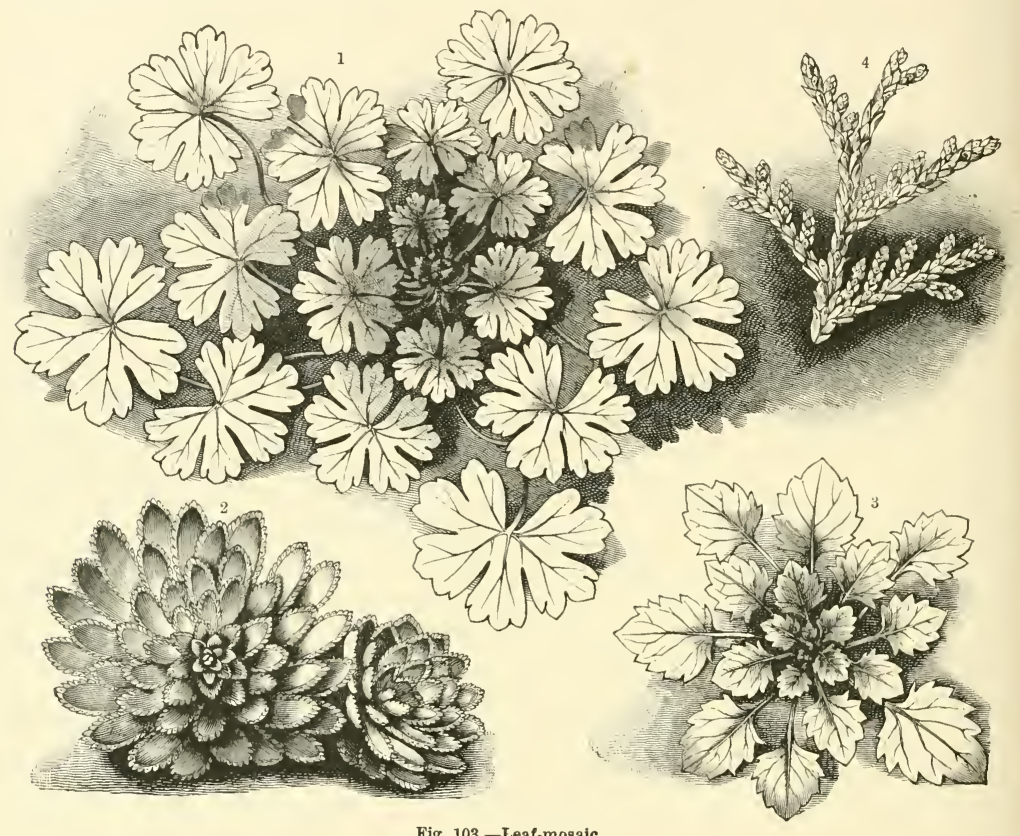

Fig. 103.-Leaf-mosaic.

1 Leaf-rosettes of a Crane's-bill (Geranium Pyrenaicum) seen from above. ${ }^{2}$ Leaf-rosettes of a Saxifrage (Saxifraga Aizoon). ${ }^{3}$ Leaf-rosette of a Bell-flower (Campanula pusilla) seen from above. 4 Adpressed scaie-like leaves on the twig of an Arbor Vitæ (Thuja).

Many plants produce within a year, at the ends of their upright shoots, a large number of leaves which radiate out from the stem with very small horizontal divergencies, standing close above one another, and forming a so-called rosette. In order that all the leaves of such a rosette may receive an equal proportion of light, it is absolutely necessary that the upper leaves should be considerably shorter than the lower. And in all rosettes this is actually the case. However, some very interesting modifications are to be seen. In rosette-forming succulent plants (e.g. Echeveria and Sempervivum), and in many saxifrages (Saxifraga), of which a species (Saxifraga Aizoon) is represented in fig. $103^{2}$, the leaves are tongue-shaped or spatulate, and about twice as broad near the further end than at the point of 
insertion upon the abbreviated axis. It is unavoidable that the narrower, proximal halves of most of the leaves should be covered by the leaves above and fail to receive sufficient light. But these covered portions are always destitute of chlorophyll, and so have no need of direct sunlight. The distal halves, on the other hand, which display green tissue, can by this arrangement be all well illumined simultaneously by the sun. In many other instances the increase in length is only found in the leaf-stalks of the lower leaves of the rosette. These increase in length, that is to say, until the blades borne by them are moved out of the shadow of the

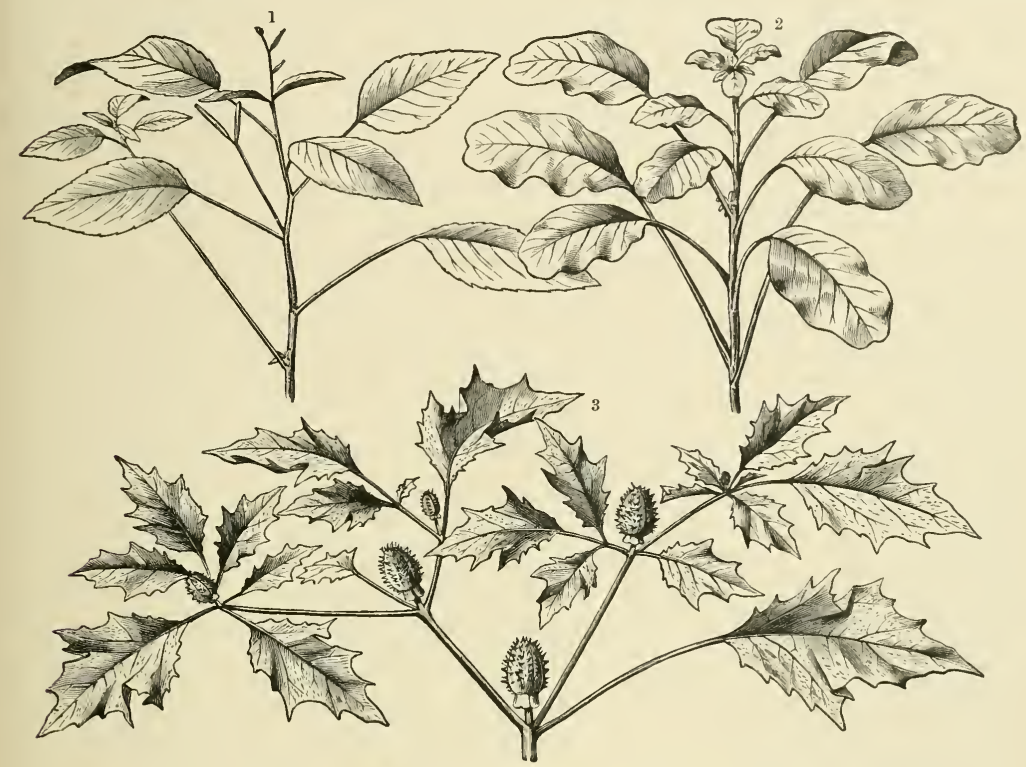

Fig. 104.-Formation of a Leaf-Mfosaic by the lengthening (relative shortening) of the Leaf-stalks.

1 Small-leaved Balsam (Impatiens parviflora), 2 Green Amaranth (Amarantus Blitum). 3 Thorn-apple (Datura Stramonium).

leaves above. This is the case, for example, in the leaf-rosettes of Geranium Pyrenaicum, represented in fig. $103^{1}$, and in the leaf-rosettes of the dainty little bell-flower (Campanula pusilla, fig. $103^{3}$ ) growing on the débris-covered slopes of the sub-alpine regions. In these bell-flowers the great difference in shape between the rosette-leaves and those clothing the flower-stalk is worthy of remark. The latter, which spring at an acute angle from the stem, are narrowly lanceolate, and have very short stalks, while the lower rosette-leaves, extended flatly over the soil, have long stalks, and possess a broad, ovate blade. It is no disadvantage to the leaf-stalks, which have no chlorophyll, if they are placed in the shade. But by this arrangement all the broad, green leaf-blades are well illumined, and this applies also to the more loosely-arranged, upwardly-directed, narrow leaves of the stem. 
The leaves of many plants with elongated, erect stems, though at a moderate distance from one another, are often arranged in a kind of rosette, and this is effected by the stalks of the lower leaves becoming considerably longer than those of the leaves situated near the apex. This condition is especially seen in the marsh-plants, whose flat leaves lie on the surface of the water, viz. in Villarsia, Hydrocharis, Polygonum amphibium, many species of the genus Callitriche, and many water-inhabiting Ranunculaceæ. Among terrestial plants this grouping of the leaves is displayed particularly by many Amarantaceæ. In the erect shoots of Amarantus Blitum, illustrated in fig. $104{ }^{2}$, the stalks of the lower leaves of a row are six, seven, or eight times as long as those of the upper leaves. In this way the whole of the green foliage of the plant can be spread out almost at the same level without any one overshadowing another.

In plants with elongated stems, the mutual encroachment of the numerous leaves situated one above another is also prevented by a further arrangement. We mean the development of the leaves in the form of green scales adpressed to the stem, as observed in so many conifers, e.g. in the twigs of a Thuja, as represented in fig. $103^{4}$. It is true that only the under surface of the small leaflets can meet the sun's rays, but the effect is the same as if only the upper side had been illumined, as, for example, in those leaves projecting from the erect stems at a right angle, or inclined with their apex towards the soil. Since the small green leaflets clothing the stem are arranged side by side, like the tiles on a roof, and the greater portion of the under surfaces remains uncovered by the adjoining leaves, no mutual withdrawal of light can be said to occur, in spite of the crowded arrangement.

The arrangements of green leaves as just described relate exclusively to instances in which the blade of the leaf is neither lobed nor compound, but entire. A leaf can deprive another, originating a little below it from the erect stem, having the same shape and size, and the same inclination, either entirely or almost entirely of the sun's rays, only when entire. A leaf whose green lamina is sinuous, lobed, divided, or incised, will always allow abundant sunlight to pass between the lobes and segments on to the leaves below; and the deeper, wider, and more numerous the incisions producing the separation into lobes and segments, the more will be the light passing through. Of course strips of shadow will be formed, but they move their position during the day, remaining in one spot only for a short time; and it would appear that such a rapidly passing shadow has anything but an injurious effect on the green tissue. From this it follows that in plants with divided foliage, the adjustment described previously for the case of entire leaves is superfluous. As a matter of fact, in plants whose foliage-leaves have a much-divided blade, the fully-grown upper and lower leaves are of equal length; they all project from the erect stem at the same angle, and the stem is, generally speaking, never clothed with lobed or pinnate leaves closely covering it like scales. In the Fennel and Dill, in Chamomile, Larkspur, and species of the genus Adonis, the lower and upper leaves of the stem are so alike that it is hardly possible to say whether an isolated 
single leaf had been plucked from the lower or upper part of the stem. Only the lowest leaves of all, whose shadow falls on the ground and not on neighbouring leaves, are divided into broader sections; the others are equally divided and project at equal intervals round the stem. While the Mullein, with its entire foliageleaves, rapidly diminishing in size towards the summit, presents a pyramidal appearance from a distance; the Fennel and Larkspur, whose finely-divided leaves are similar all along the stem, rise up like cylindrical columns. In other words, if the extreme outer point of all the leaves of the last-named plants are connected together in a surface, this will take the form of a cylinder. Only when projecting, divided leaves are crowded above one another on a very short stem, as, for example, in ferns, and where the plants are growing in shady places where the light is very scanty, it happens that the lower leaves are raised above the upper in order not to miss too much of the enjoyment of the light.

The perforation of the leaf-blades, which is observed, though but seldom, in many aroids, has now to be considered. The best known in this respect are the Brazilian Monstera egregia, and the Tornelia fragrans, illustrated in fig. 96, which has also been called by gardeners, in consequence of the gaps in the leaves, Philodendron pertusum. The circular or elliptical holes do not originate late on in the leaf-blade, but can actually be seen when the small and undeveloped leaves are yet folded. They are always formed on the upper leaves of older plants; the leaves of younger, shorter specimens do not possess them. This circumstance suggests that the holes have the same significance as that previously assigned to the deep incisions and clefts between the leaf-lobes. They are chinks in the broad upper leaves whose shadow extends over a large area, through which a portion of the obliquely falling rays of light can reach the more deeply situated leaves. The peculiar notches in the blades of certain leaves of the Black Mulberry-tree (Morus nigra), as well as of the Japanese Paper Mulberry (Broussonetia papyrifera), may be explained in like manner. They are only found on the upper leaves of a branch, and are best seen on the erect slender shoots which spring from the base of old trunks. Sometimes, in these highest. leaves, only one half has an incision extending almost as far as the midrib; sometimes again both halves are provided with deep clefts; in the highest shootleaves of the Black Mulberry-tree the blade is often divided up into fairly narrow segments by several incisions on both sides. If such developing shoots, crowded closely together, are observed at mid-day, when they are directly illumined by the sun, the shadow of the upper leaves can be seen sketched out on the leaves below, but to each incision and indentation of a leaf at the apex, a patch of light corresponds on the leaf-surfaces in the stories next below. Suppose now that the holes above had been closed; immediately it would become darkened underneath, the spots of light which continually move according to the position of the sun from place to place and from leaf to leaf would be wanting, and the activity of the green tissue in the leaves of the lower region would be to some extent impaired. 
It was not without reason that in each separate instance hitherto described, emphasis has been laid on the fact that the foliage-leaves in question were situated on erect stems, and this must again be particularly pointed out here. The conditions on horizontal branches are entirely different, and what is suitable for one is not always fitted to the other. It is easy to make this evident. It is only needful to bend down an erect leafy maple-branch until it becomes horizontal, and it will immediately be seen that the surfaces of the leaves on the branch assume a position and direction very different from their previous attitude. The narrow side, instead of the broad side as previously, is directed towards the incident light, and the leaves now stand above one another which formerly stood opposite at the same height from the ground. If the arrangement of the foliageleaves on the erect branch was previously suitable and beneficial, the contrary is now the case. Such alterations in the position of the foliage-leaves or shoots and branches of plants, however, occur not only by way of exception, but very frequently. It signifies the less that strong winds bend and incline the leafstalks and twigs, since this alteration of position is, as a rule, only of short duration, and when the storm is past, the former position is again taken up. The pressure which snow exerts on plants in regions where in winter the fall is heavy, is, indeed, of more importance, and can produce alterations in the position of the twigs and branches which are of longer duration. But most important of all is the fact that perennial plants add a new portion to the end of their shoots every year, that they always develop each year new sprouts above those already existing, and not only at the apex, but also from buds which arise laterally on the branches. Let us observe a young maple whose topmost branch terminates in three buds. Twigs issue from the three buds with the renewal of activity in the spring; the central bud grows directly upwards, the two lateral rise obliquely; all three are thickly leaved, and the foliage of the three twigs covers over and shades three, four, perhaps ten times as large a space as the pair of leaves from whose base the buds had developed in the previous summer.

Now, above the centre of the maple as it was in the previous year, what may be termed a new richly-leaved and thickly overshadowing little maple-tree grows up. That mutual consideration, which is otherwise observed by members of the same plant, and which was described earlier, here ceases. The leaves of the topmost shoot are, of course, so arranged that no mutual injury is done; but very little attention appears to be paid to the leaves below, as little perhaps as to the lower grasses and herbs which grow under the maple-tree on the ground.

But what are the branches to do which spring from the buds in the centre of the maple-tree under consideration? If they take the same direction as the branches at the extreme summit, they will come into the area of the dark shadows thrown by the numerous broad leaves of the top branches. They are, therefore, compelled to take up another direction if their leaves are not to perish from want of light. And, as a matter of fact, this is what they do. They arrange 


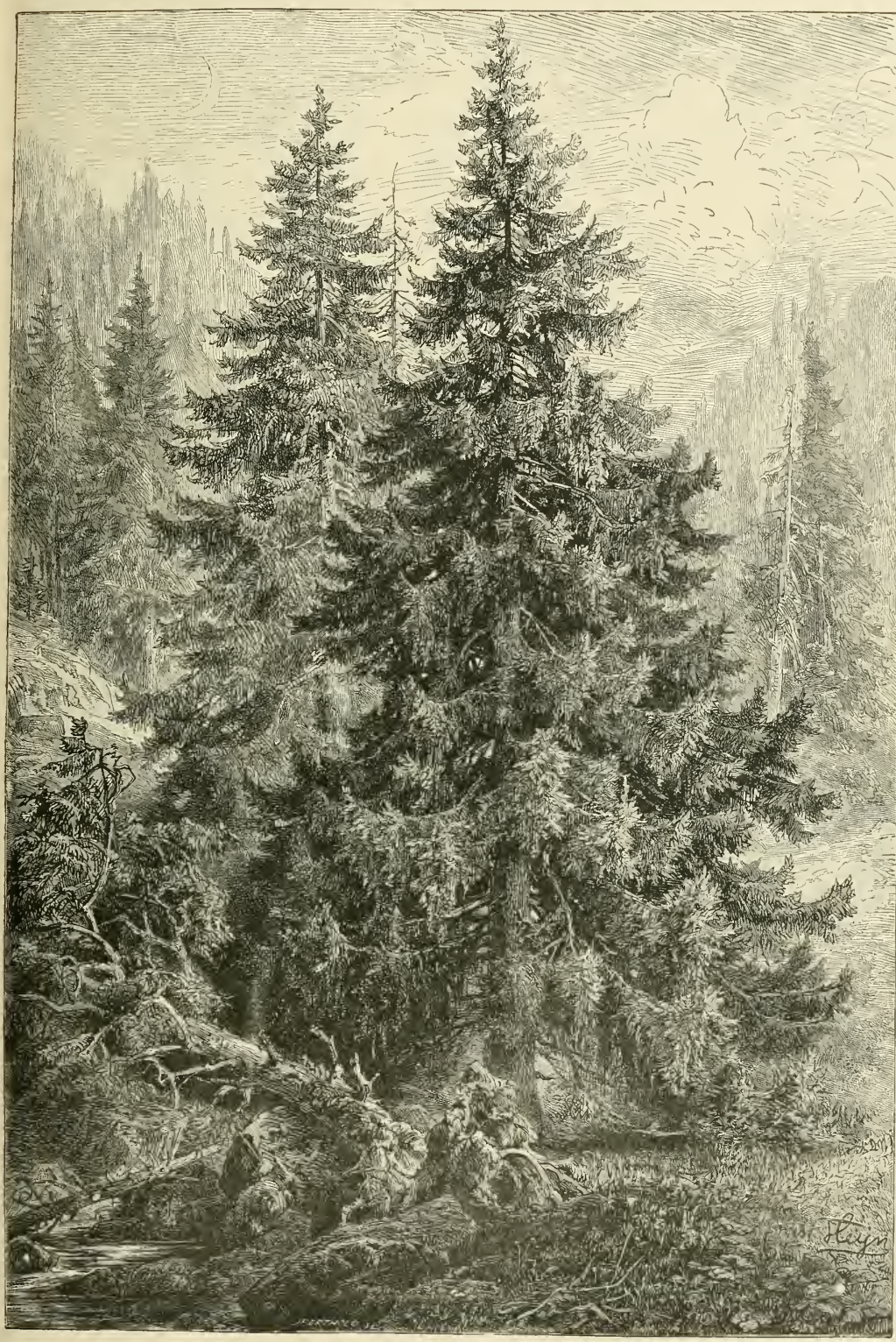

Fig. 105.-Spruce-Fir Trees. 
themselves, that is to say, more or less horizontally, and increase in length in this direction until their leaves project outside the shadow of the topmost leafy branches, so that they may be able there to catch the sunlight. All this is observed not only in maples, which have been selected as examples, but in all richly-leaved trees and shrubs; the topmost branches are directed vertically upwards, the next lower rise obliquely, those still lower extend horizontally, and the lowest of all frequently even incline earthwards. The twigs of the

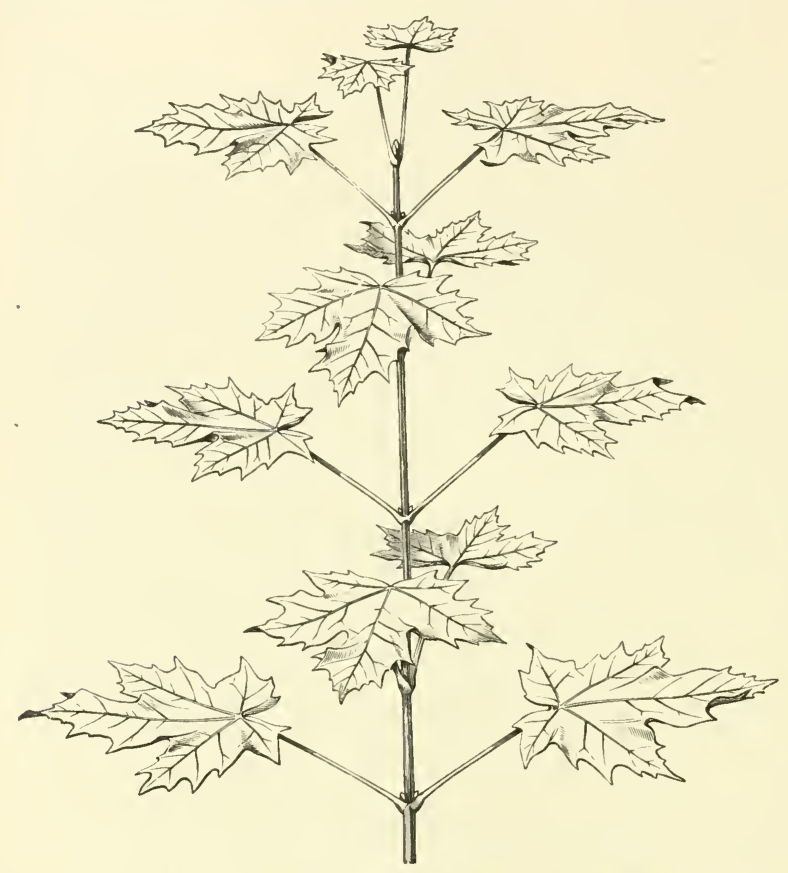

Fig. 106.-Erect leafy Twig of the Norway Maple (Acer platanoides) older,lower branches which have grown out beyond the shaded area often again try to rise, and assume a direction which is almost similar to that of the highest branches at the summit. Such branches and twigs then display a curvature which is like a Roman $N$ lying sideways. Oaks and horse-chestnut trees furnish striking examples of this. The phenomenon is shown still better in firs (see fig. 105), in which the twigs springing from the lowest branches frequently rise almost vertically. This last circumstance is also of interest in so far as it indicates that it is not only the weight of the leaves which brings about the altered direction of the branching, but that it depends also on other conditions, to be discussed later on.

In the terminal twigs of the lowest branches, which are again turned upwards, the same distribution and direction of the leaf-blades as are displayed by the erect twigs of the summit will naturally be resumed; but it is not so in the case of those twigs which retain a horizontal direction, or whose summits are even inclined towards the ground. Suppose that the maple-twig, which is illustrated here, has not grown from a central bud of the summit, and does not rise vertically upwards, but that it has been developed from an older, lower branch, and is extended 
almost horizontally. If the surface of the foliage-leaves on the horizontal twig retains the same direction as those on the erect twig here represented, this will be the most disadvantageous position imaginable with regard to the incident light. It is urgently necessary that they should alter this position and again arrange themselves suitably. This rearrangement of the leaf-surfaces proceeding from the horizontal twigs is carried out, and, indeed, in four different ways. Either an adequate twisting of the internodes is effected; or a twisting of the leaf-stalks occurs; or the leaf-stalks do not undergo actual torsion, but their inclination to the leaf-blade becomes altered; or, finally, individual leaf-stalks lengthen to an extraordinary extent; so that the blades borne by them are carried far beyond

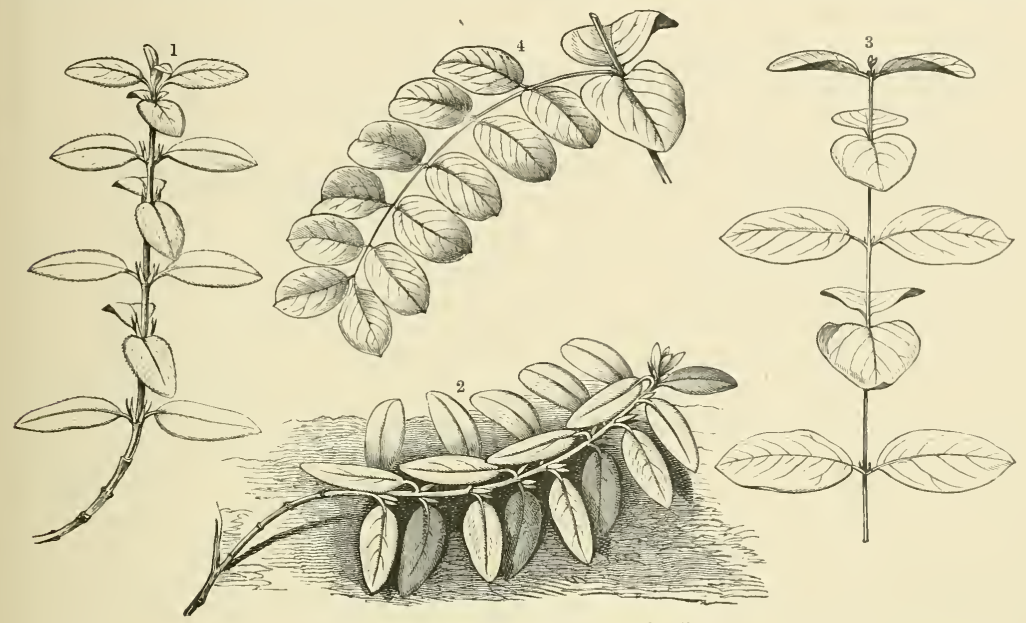

Fig. 107. - Twisting of Internodes and Leaf-stalks.

1 Erect twig of the large-flowered Rock Rose (Helianthemum grandiflorum). 2 Procumbent twig of the same plant. $s$ Erect twig of Diervilla Canadensis. 1 Twig of the same plant, bent downwards.

their neighbours. It naturally very frequently happens that these alterations are also combined in many ways.

The first instance, the twisting of the internodes, may be observed in hazels beeches, and hornbeams, and especially in trees, shrubs, lianes, and bushes with decussating short-stalked leaves, as for example in Cornus and Thunbergia, in Lonicera and Diervilla, in Androscemum and Hypericum, in Thymus and Vinca, Coriaria myrtifolia, Gentiana asclepiadea, and innumerable others. Fig. $107^{3}$ represents an erect twig of Diervilla Canadensis. As soon as such a twig develops no longer upwards, but horizontally, a twisting of $90^{\circ}$ takes place in each internode, and the consequence is that the surfaces of all the pairs of leaves take up the same position towards the sun, as shown in fig. $107^{4}$. The leaves are now no longer arranged in four, but in two, rows.

Very often twisting of the petioles goes hand in hand with that of the intervor. I. 
nodes. The torsion of the leaf-stalks of the Judas Tree (Cercis Siliquastrum), where this alone occurs, i.e. without a simultaneous twisting of the internodes, is particularly striking. The leaves of the plant, as can be seen on the erect twigs, and especially well in the suckers, are arranged in the one-half phyllotaxis, i.e. in two rows. The leaf-blades on the erect branches are parallel with the ground. If a sucker be cut off and held horizontally, all the leaf-laminæ will be directed at right angles to the earth. One might perhaps expect that they would also assume this direction if the twig had grown horizontally. Anything but that, however. The stalks of all the leaves twist round instead, until the laminæ, or blades they bear, are again placed in a direction parallel with the ground on the horizontal branches, and the result is that the leaves on all the branches of

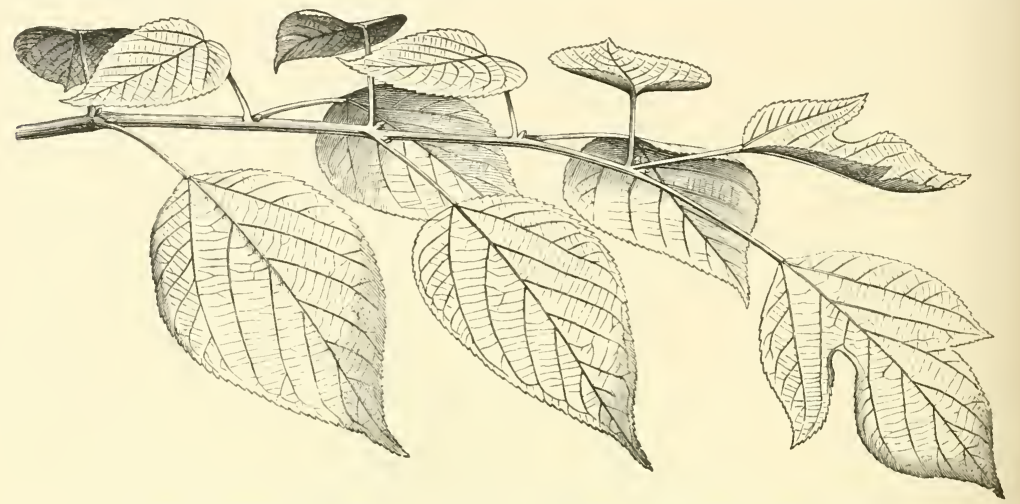

Fig. 108.-Horizontally growing leafy twig of the Paper Mulberry-tree (Broussonetia papyrifera).

the Judas Tree, whether erect, oblique, horizontal, or inclined towards the earth, always present the same attitude to the incident light.

The third case, the alteration in the inclination of the blade to the leaf-stalk, which, on the whole, is but seldom met with, is represented in the best known example, the cursorily mentioned Japanese Paper Mulberry (Broussonetia papyrifera) in fig. 108. In this plant the leaves are decussate, i.e. arranged in four rows, each pair of leaves being inserted at the same level, and the successive pairs alternating at right angles. In erect twigs, therefore, they display the arrangement seen in the twigs of maple (see fig. 106) or of Diervilla (see fig. $107^{3}$ ). The following alteration, however, is seen to occur in the horizontal branches of the lower boughs of the Paper Mulberry. In each pair of leaves the leaf-stalk of one leaf becomes parallel to the surface of the ground, and lies in the plane of the blade it supports, which is also almost horizontally extended, or but slightly inclined. The other leaf-stalk, however, rises vertically from the horizontal twig; the leaf-blade it supports is bent down at right angles from it, and consequently is here again parallel to the surface of the ground. A slight torsion of the internodes, a shortening of the erect leaf-stalks, and a diminution of the leaves 
borne by them, certainly assist in the completion of this peculiar arrangement of the leaves; the above illustration will demonstrate other particulars far better than the most detailed description.

The elevation of individual leaf-stalks above the horizontal branches occurs, somewhat more often in low semi-shrubs and herbs, than in trees and shrubs, whose shoots, furnished with decussate leaves, come to lie flat on the ground, as in some species of speedwell (Veronica officinalis and Chamoedrys), and in many species of Rock Rose (Helianthemum). In the large-flowered Rock Rose (Helianthemum grandiflorum), an erect branch of which is illustrated in fig. 107 ${ }^{1}$,

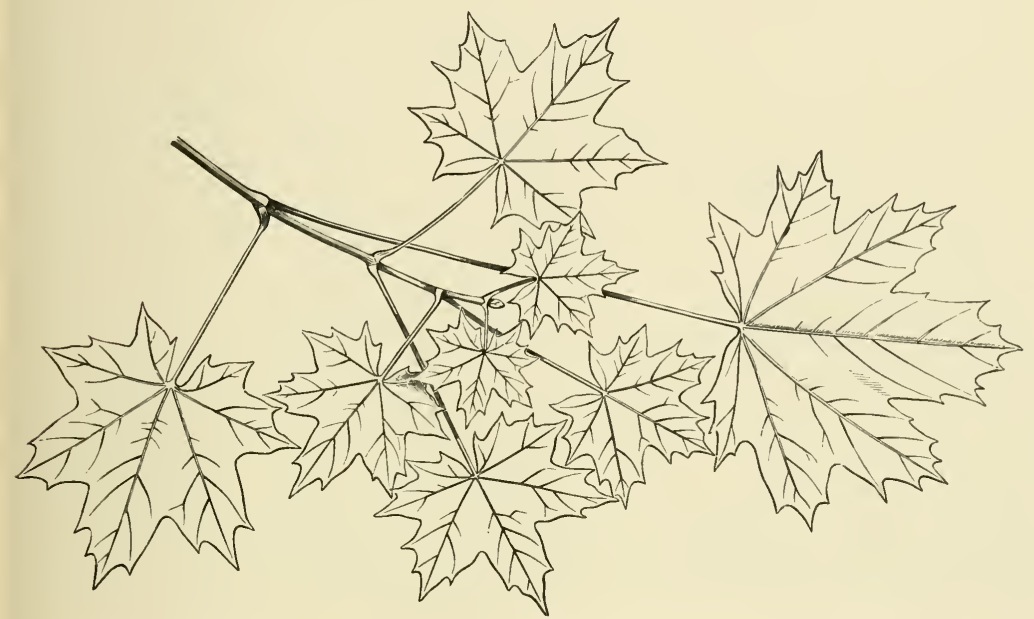

Fig. 109.-Leafy Twig projecting laterally from the Stem of the Norway Maple (Acer platanoides).

the leaves are arranged in pairs and placed crosswise, so that they occur on the stem in four rows. If such a shoot bends down over the ground, a slight twisting of the leaf-stalks occurs first of all, so that their leaf-blades come to lie parallel to the soil; but another alteration is yet to be noticed. In every alternate pair of leaves one of the leaf-stalks rises up, and its blade is bent down almost at a right angle and lies above the horizontal stem as shown in fig. $107^{2}$. In consequence of this alteration of position the leaves no longer form four rows as on the erect shoots, nor two as in Diervilla, but three rows, the middle one, however, consisting of a smaller number than the two side rows.

The fourth case, which still remains to be discussed, is the increase in length of individual leaf-stalks. It may be very well seen in maple-trees, especially in the Norway Maple (Acer platanoides), and this species will therefore serve us as an example. Fig. 106 shows an erect branch of this maple. The stalks of every pair of opposite leaves are of equal length on the erect branch. But how entirely different in respect to length are those leaf-stalks which embellish the horizontally- 
directed branches of this species. Here one of the pair always appears considerably longer than the other; and it is not a rare occurrence for it to be three times as long as its neighbour, as may be seen in figure 109. And why this striking dissimilarity? The reason is again the same as in all the previous cases. If all the leaf-stalks were to retain the same length on the horizontal twigs which they have on the erect branches (see fig. 106), then one of the leaves of every alternate pair would come to be very unfavourably situated in its neighbour's shadow. This detrimental condition must be prevented, and this may be effected most simply by the leaf-stalk increasing in length until the blade it carries is projected beyond the area of the shadow.

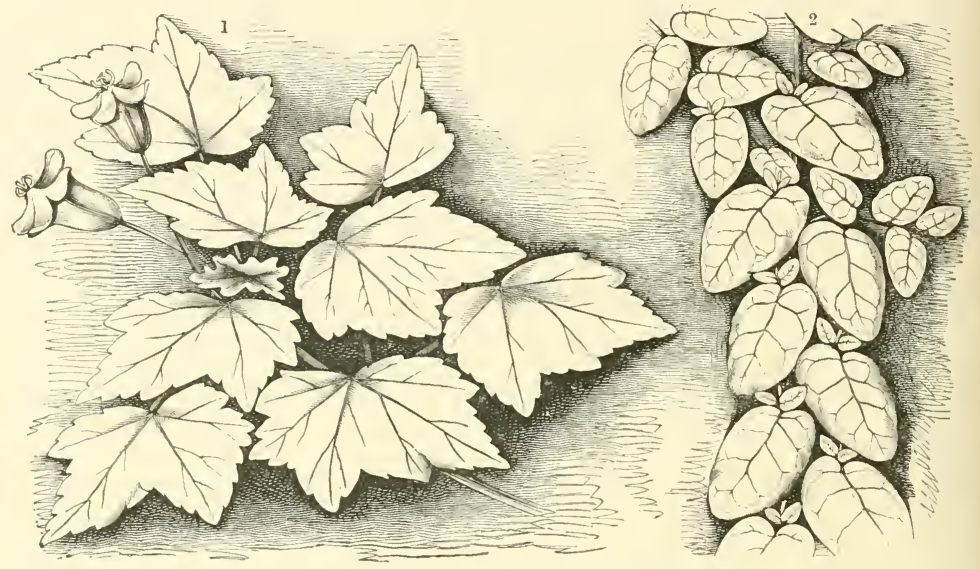

Fig. 110.-Leaf-mosaics of Unsymmetrical Leaves.

1 Begonia Dregei growing in front of a vertical wall. 2 Ficus scandens, growing on a vertical wall.

It may be expected that alterations of direction, shortenings and elongations, similar to those just described in the case of the horizontal leafy twigs of the lower boughs of trees, shrubs, and bushes, will be found on those plants which are attached to a steep face of rock, a vertical wall, or to the bark of an upright treetrunk. As a matter of fact all the instances discussed here are again met with in various climbing and twining growths, as well as in those whose stem is parallel to a vertical wall without being attached to it, e.g. as in Rhamnus pumila, and in many begonias. But here the leaf-blades do not place themselves parallel to the ground, but to that surface on which the plants in question are supported, or which they adjoin. In these plants another peculiarity is often observed which it will be most fitting to speak of here, viz. the want of symmetry of the leaves. While in the majority of plants each foliage-leaf is divided by a midrib, running from the apex to the leaf-stalk, into two similar or almost similar halves, in the begonias, many climbing figs, in Celtis occidentalis, elms, and numerous other plants, the two 
portions of the leaf separated by the midrib are very unlike. The dissimilarity is seen principally at the base of the leaf-it looks as if a piece had been taken out of one side, or as if the leaf had there been cut off obliquely (see fig. 110). The correct explanation of this want of symmetry will perhaps be arrived at most easily by supposing the suppressed portion to be completed, or in other words, let us suppose the smaller half to be just as large and well-developed as the other. It is then evident that the added portions would be covered over by the neighbouring leaves, and consequently they would be deprived of light, and that in these parts, therefore, the chlorophyll-bodies, if present, would not be able to

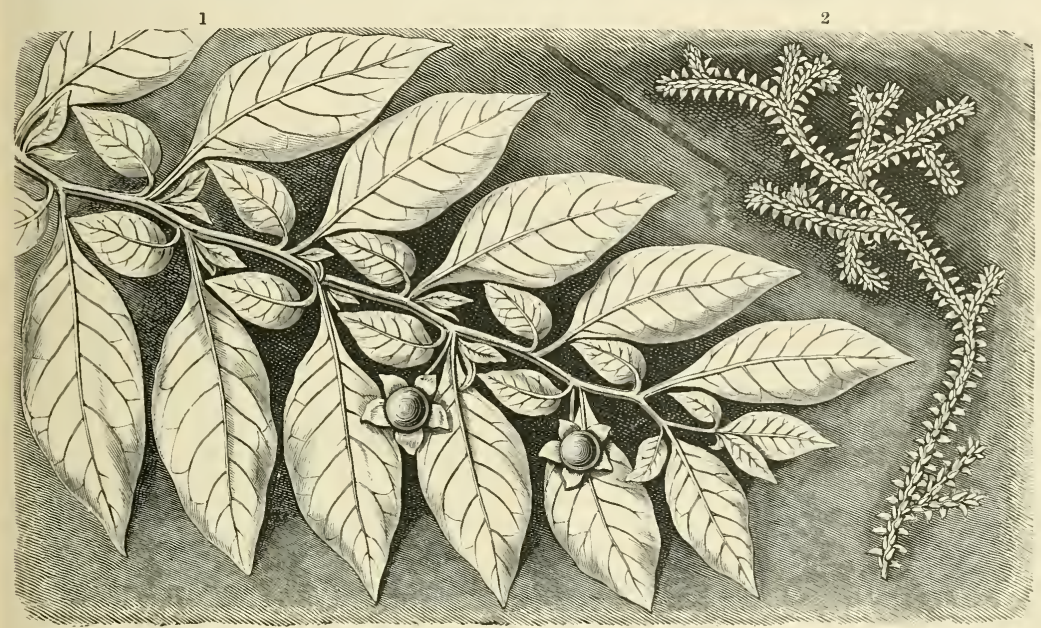

Fig. 111.-Mosaic of Leaves of unequal size.

1 Projecting branch of Deadly Nightshade (Atropa Belladonna) looked at from above. 2 Selaginella Helvetica, seen from above.

carry on their activity. These portions of the foliage-leaves would accordingly be superfluous, and it is foreign to the ways of plants to manufacture so much leaf-tissue for no purpose whatever. Plants never form anything which is superfluous and useless; in the construction of all the organs the principle apparently is to attain the greatest possible result with the least amount of material, and to utilize the given conditions, above all, the existing space, as far as possible.

Yet another phenomenon, viz. the unequal size of adjoining leaves on the same plant, must be considered from this point of view. It must strike everyone who looks down upon a horizontally-projecting branch of the Deadly Nightshade (Atropa Belladonna, see fig. $111^{1}$ ), that larger and smaller leaves are here arranged in quite a peculiar manner. The larger leaves stand in two rows, and in virtue of their shape it happens that, between every two, gaps are left near the stem. 
These cannot be of use as apertures, through which light can pass to leaves situated below, for the simple reason that, as a rule, no other leaves requiring light are to be found under the branches in question. Smaller green leaves are now inserted in these gaps, which serve as protective leaves for the flowers, that is, indirectly for the fruits, but whose function also coincides entirely with that of the large foliage-leaves. The small leaves twist and turn until each comes to lie exactly in the middle of a gap, where they neither encroach upon the large leaves, nor are encroached upon by them. An exactly similar insertion of smaller leaves in the gaps between the larger can also be observed in the Thorn-apple (Datura

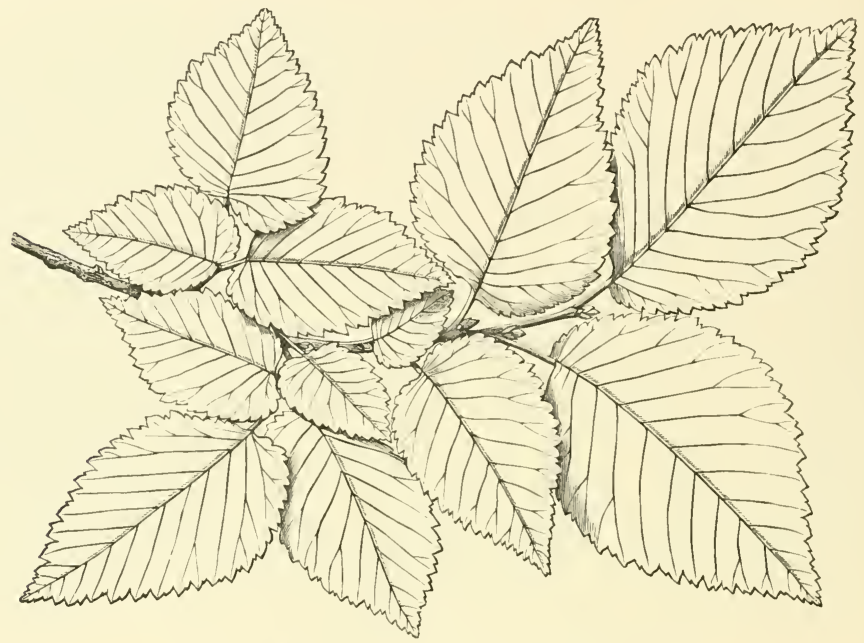

Fig. 112.-Mosaic of Unsymmetrical Leaves of unequal size.

Leafy horizontal Twig of an Elm (Ulmus) seen from above.

Stramonium), and in Impatiens parviflora, illustrated respectively in fig. $104^{3}$, and fig. 104. ${ }^{1}$. This mosaic-like fitting together of larger and smaller blades appears to be combined with the want of symmetry of the leaf-base in shortstalked leaves, as e.g. in the wall-climbing stem of Ficus scandens (see fig. $110^{2}$ ), and on the older horizontal branches of elms (Ulmus), one of which is illustrated in fig. 11\%. It has been already mentioned that the blades with erect petioles, arranged in the central rows on the Paper Mulberry, are considerably smaller than the lateral rows of leaves with horizontal stalks (see fig. 108). This difference in the size of the central and lateral rows of leaves on horizontal stems is very noticeable also in the dainty selaginellas, belonging to the family of Lycopodiacex, of which a species (Selaginella Helvetica) is represented in fig. 111 .

It is worth noticing that the occurrence of leaves of two sizes on the same stem, as well as the masaic-like arrangement and fitting together of the leaves in one 
plane, is observed especially in plants growing in dark or half-shaded places. There they do not require to protect themselves against an over-abundance of light, but on the contrary have to make what use they can of its scanty amount, and this is best effected by the fitting together of all the leaves on a stem in one plane, like the stones of a mosaic. It is, of course, not so easy to produce a mosaic from symmetrically circular or elliptical leaves; but unsymmetrical, or rhomboidal, triangular, pentagonal, and, generally, polygonal blades lend themselves particularly well to this arrangement. Excellent examples of this are furnished in the leafmosaics in fig. 110, as well as in the elm twig represented opposite. The leaf-mosaic formed by the ivy on the ground of shady woods is particularly instructive in this respect. In the picture below, which is a faithful reproduction of a piece of ivy carpeting the ground of a wood, it is seen how the lobed, five-pointed leaves

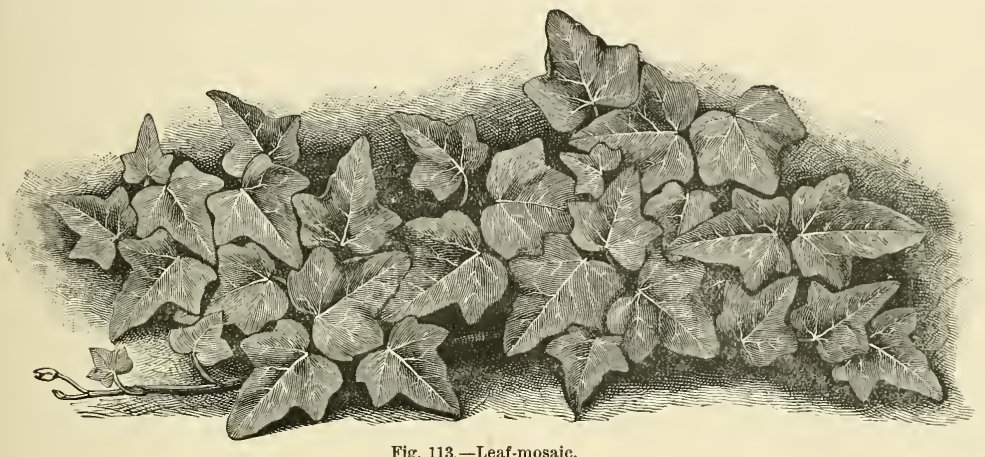

Fig. 113-Leaf-mosaic.

Ivy on the ground of a forest.

have in the course of time fitted into one another. The lobes and points of one fit into the indentations of another, and thus originates a layer of leaves than which one better fitted to the given external conditions could hardly be imagined. In this mosaic, indeed, we no longer see two rows of leaves symmetrically arranged on the horizontal stem. What manifold elevations and depressions, torsions, displacements, and elongations must have taken place in order to produce such a leaf. mosaic from the regular rows of leaves! But we learn from the consideration of all these instances, that not only the arrangement and distribution of the foliage, and the direction and length of the leaf-stalks, but the size and even the shape of the leaf-blades also, and the resultant mosaic-like piecing together, stand in causal relation to the conditions of illumination; and that in dimly-lighted situations plants endeavour to utilize, and turn to account, the sunlight for the green tissue of the foliage-leaves as far as possible by the means at their disposal, and with regard to the given conditions of space. 


\section{ARRANGEMENTS FOR RETAINING THE POSITION ASSUMED.}

When the green tissues of plants have once assumed the position most beneficial to them, they must be kept as long as they can be in that position, and any further alteration must be as far as possible avoided. The displacements, curvatures, and extensions described in the preceding pages, representing a struggle for the best arrangement of the green tissue for light, must not be restricted; whilst distortion, folding, and rupturing of the chlorophyll-containing tissues, which would be synonymous with the destruction of the portion in question, must obviously be warded off.

In the depths of still water, at the bottom of pools, ponds, and lakes, an alteration of the position assumed by the fully-developed plants in consequence of an external stimulus occurs but seldom; and although currents and eddies are set up in the water by passing aquatic animals, and temporary oscillations caused in the water-plants, these quickly subside, and the agitated portions return forthwith to their original position, having suffered no injury. In aquatic plants of this kind there are no special contrivances for strengthening the individual organs, and in particular no contrivances for protecting the green tissue from rupture and crushing. The small amount of strength and elasticity of the cell-walls suffices to withstand the thrusts, and pulls, and the pressures which make themselves felt in the depths of the water, and to restore the temporarily displaced green portions to their right position. Firm woody cells, and strands of elastic bast-fibres, which play such an important part in the aërial portions of plants, are wanting here. Woody plants neither grow in the sea, nor in fresh water. Aquatic plants, indeed, quickly collapse, in consequence of the absence of wood and kast, when brought from the depths into the air; the leaves collapse of their own weight, and sink flaccidly on to the substratum. They are able to retain an erect position in the water, because a portion of their tissue is penetrated by comparatively large air spaces, by which means their specific gravity, compared with that of the water, becomes much diminished. If aquatic plants were not firmly attached to the sand and slime, or submerged rocks, they would rise to the surface and float there. But as they are fixed in the depths, the air spaces within the green tissue of the leaves or stalks bearing the leaves cause these organs to remain erect as if suspended in the water.

Plants growing in running water, and such as are exposed to the lapping of the waves on the shore, are indeed subjected to a severer proof of their firmness and tenacity. Thus many of them, e.g. sea-wracks on the sea-coast, the longleaved pondweeds in the quick-flowing mountain streams, and the Podostemacer in the rushing torrents and waterfalls in tropical regions, are actually swayed hither and thither and continually shaken, and accordingly due allowance must be made in their construction for this circumstance of their habitat. The tissue of these plants is much tougher than that of the Characeæ, of the Naiadaceæ, 
of Water Milfoil, and of various others which lead a peaceful life in the depths of calm waters. Their tissues are not feeble, but elastic and pliant, and many sea-wracks look just like leathern straps and bands. Many of these sea-wracks are periodically left lying on the dry ground at low tide, but they do not in consequence shrivel, or at least not if the water soon returns, but lie with their pliant leaf-like surfaces flat on the dry sand or stone. When the tide returns, they are again gradually raised up, and assume an upright position in the surrounding water; and this is materially assisted in the sea-wracks by the swollen bladder-like cavities, in reality swim-bladders, which they contain in their tissues. Many species of Characeæ, but still more the Lithothamneæ and Corallineæ, acquire an increased capacity of resistance against the force of the waves by the deposition of lime in the cell-membranes; others again so closely apply their large surfaces to the rocky reefs and stones of the shore, that they look like coloured patches on them, so that the crushing or tossing effect of the surging waves is entirely obviated. This applies, for example, to Hildebrandtia rosea and Hildebrandtia Nardi, which cover the stones with blood-red patches.

Many marsh-plants, which are only partially, and often only temporarily, submerged, whose floating leaves are half in contact with water and half with air, or whose leaf-blades are wholly raised above the water, behave just like these water-plants. The alteration of the water-level brings about, of course, a higher or lower position, an elevation and sinking of the floating leaves, but this is effected without the slightest rupture of the parts in question. The stem and the leaf-stalks, which proceed from a stock rooted at the bottom of the water, resemble long strings and threads to whose upper ends the leaf-blades are fastened. At the highest water-level the floating leaf-dises stand perpendicularly above the stock to which they belong, which is rooted in the depths. If the water then sinks, the leaves, floating on its surface, fall with it, and at the same time separate from one another. The stalks and stem proceeding from a stock perform approximately the same movement as that seen in the ribs of an umbrella held downwards and then opened. As soon as the level of the water rises again, the reverse movement naturally occurs. Many of these marsh-plants, as, for example, the Water Chestnut (Trapa), also possess air-bladders in the floating portions of their leaves, having the same function as those of the sea-wracks. Moreover, usually two kinds of green foliage-leaves are noticed in these. Submerged leaves, which are constructed like those of aquatic plants, and floating leaves which display a more or less disc-like form, and whose under side is in contact with the water, the upper with the air, but which under certain circumstances may be entirely surrounded with air without injury. If the marsh should dry up, long thin stems and leaf-stalks would be anything but beneficial; the metre-long leaf-stalks of a water-lily would not be able to support the leaves in an erect position, but would fall and become bent. Stretched out on the ground, also, such long filamentous leaf-stalks would not be advantageous. It is seen, too, that marshplants of this kind immediately become modified when the water recedes. The 
fresh leaves have only short stalks, and these become so strong and elastic that they are well able to support the leaves. Water-lilies are striking examples of this. In Polygonum amphibium, the long stems of the aquatic form, bearing at their upper ends groups of floating leaves, are much thinner than the short stems of the terrestrial forms, which are uniformly beset with leaves from top to bottom.

The green tissues which are surrounded with air are much more exposed to the danger of being torn, bent, and broken up by violent gusts of wind than those which live either wholly or partially submerged in water.

When green tissue is only developed in the cortex of the branches, as in the leafless switch-plants, the branches are always elastic and supple, and in order to produce this quality, bundles and strands of hard bast, i.e. elongated spindleshaped thick-walled cells of fibrous appearance, are inserted at suitable places. The wood in these branches is also very tough, and gusts of wind can consequently do them little harm. They are often prostrated by storms; but when the wind subsides, the branches forthwith rise up, and in consequence of their elasticity, resume their former position towards the light. The bundles of hard bast-cells alternate in many instances regularly with the green tissue, as, for example, in Spartium scoparium, illustrated in fig. 81, and, generally, very manifold contrivances are to be found in the internal construction of the branches for hindering the bending up and crushing of the green tissue.

Leaves, as well as stems and branches, have originally a tendency to grow up perpendicularly in the atmosphere, and there are many plants whose foliage remains throughout life in this position. Obviously these leaves are no less exposed to damage by storms than are the upright branches of the switch-plants. It must be borne in mind that gusts of wind rush over the ground in waves like a powerful torrent, and that the direction of the air-current is usually parallel to the surface of the earth. Plant-organs which grow up from the ground are struck at right angles by such gusts, and are thus exposed to the most violent attacks of the wind. Leaves, especially, whose blades are inclined at right angles to the direction of the storm, are much more easily bent and crushed than those whose blades lie parallel to the current. The effect of the attacks of wind increases in proportion to the extent of the surface exposed to the air-current, and a large upright projecting leaf will be bent much more by the wind than a small leaflet which lies close to the stem like a scale.

In what way can the dangers of rupture be varded off from a green leaf which grows towards the light of heaven, is surrounded by air, and is exposed on all sides to the attack of wind? First of all, at any rate, by the same developments as those mentioned in the case of the upright green branches of switch plants, i.e. a suitable placing of the green tissue between flexible, elastic, fibrous bundles of bast-cells, by support from thick-walled woody cells, and other cellular formations; by these means firmness is given to the whole structure with the least possible expenditure of material; an arrangement which the thin-walled green tissue can, by itself, never have on account of its special function. 
But the whole shape and position of the leaf must also be adapted to the circumstances, for the simple reason that a plant constructed unsuitably with regard to the prevalent winds would suffer injury, perish, and sooner or later be supplanted by other species better adapted to the given conditions. Therefore, it may so far be looked upon as an adaptation of form when a leaf lies with its surface parallel or only slightly inclined to the surface of the earth, and therefore to the direction of the wind, so that the moving currents strike it at a very oblique angle, and rupture of the blade can hardly ensue. Since this position of the green leaves is also very favourable for most plants with regard to light, it is not surprising that it occurs so generally. In such flat leaves, a rising and falling, and occasionally a bending of the blade is unavoidable, especially when the gust of wind comes from that side towards which the tip of the leaf is turned. But such an attack on the leaf-blade, which is parallel to the surface of the ground, or inclined slightly to the horizon, is rendered as little injurious as possible by two arrangements.

One consists in the fact that the moderately stiff leaf-blades can turn like weathercocks on the stem from which they project; this occurs in many reedlike grasses, particularly in Phalaris arundinacea, Eulalia Japonica, and in the widely-distributed Phragmites communis. The latter, which often grows in immense quantity in the marshy lowlands, in the depth of valleys, and on the banks of rivers, develops lofty, slender culms bearing numerous leaves. These leaves, like all grass-leaves, consist of a linear, fairly broad, and tapering blade projecting from the stem; and also of a sheath in the form of a hollow cylinder surrounding the haulm, and from which the portion of the haulm in question proceeds as from a tube. As long as the haulms and leaves are not fully developed, the leaf-blades are strongly directed in a line parallel to the culm; later, they decline, project horizontally, and finally become even somewhat depressed, so that their apices are directed groundwards. They then remain flat, and are so stiff that they cannot be bent by light winds. Moreover, if a stronger gust occurs, they do not bend, but twist round like the weathercock on the roof-gable in the direction of the wind. This is rendered possible only by the fact that the haulm and the tubular leaf-sheath surrounding it are very smooth at the surfaces in contact with each other, and that the leaf-sheath may undergo a slight splitting without. damage.

This development is found in the reed-like grasses mentioned; and in them there is a further contrivance, an interrupted membrane or flap inserted at the boundary between the blade and sheath; this protects the sheath from the entrance of rain-water, and consequent increase of friction, rendering the twisting difficult. The common Reed (Phragmites communis), growing in quantities, presents a characteristic appearance, in consequence of the arrangement here described, every time a breeze passes over such a bed. If the wind blows from the east, all the leaves are directed to the west; if it comes from the west, all their apices are turned to the east. The whole mass looks as if it had been combed, as if all the 
leafy blades had been stroked like the hair of a horse's mane in the direction of the wind.

The second arrangement for protecting broad flat leaves against crushing is observed in fan-palms, in maples, poplars, birches, in pear and apple trees, and in innumerable other woody growths of all regions. It consists in the development of long, elastic leaf-stalks. The Aspen (Populus tremula), which may be regarded as the best example, exhibits leaves on the branches of its crown, whose circular blades are always somewhat shorter than the stalks. At the slightest movement of the air these are seen to tremble and sway hither and thither, and this phenomenon is so striking that it has furnished the nucleus of many sayings, such as to "tremble like an Aspen leaf". But even in the most severe storms it is only the leaf-stalks which bend, having acquired a high degree of elasticity by the development of bast strands. The leaf-blades borne by them remain flatly extended, stiff, and rigid. They are not bent by the wind, and, therefore, these elastic leaf-stalks ward off the danger of fracture from the blades they support.

In many grasses-for example, in the most widely-distributed cereals, wheat, rye, and barley-it is observed that the first green leaves developed by the seedlings are erect, while those developed later, which arise from the slender haulm which springs from their midst, are more or less parallel with the ground. In many other plants with much contracted subterranean stem-structures, viz. in the Reed-mace (Typha) and in many bulbous plants, all the foliage-leaves assume an erect position and remain so until they fade and die. Leaves, when erect, are far more exposed to the wind passing horizontally over the ground, and require much stronger protections against bending than those which are extended flatly over the soil; and in order that they may be able to escape fracture, they must be provided with specially effective contrivances.

The fistular leaf is to be regarded as one of the most striking of these contrivances. Fistular leaves are always ereet at the lower end, where they surround the stem or the neighbouring leaves, like the equitant leaves of irises; they are sheathing and hollow, terminating above in a hollow cone. There is no conspicuous midrib; a shallow groove is frequently seen on the side directed towards the central axis of the whole plant; otherwise the hollow leaf is developed uniformly all round. It has no appearance of special resisting capacity, and those cellular elements which, as a rule, are used to increase strength, are absent; and yet, like all tubes, it possesses a relatively great resistance to flexion, and it is scarcely injured, even in violent storms. On the whole, this striking form of leaf is not common; it is most often seen in bulbous plants, e.g. in Chives and in the Common and Winter Onions (Allium Schœnoprasum, Cepa, and fistulosum). Structures are more often met with which resemble the fistular form to some extent, since their long green blades are rolled up lengthwise, sometimes towards the side facing the central axis of the whole plant, and sometimes away from it. The rolling observed in leaves of crocuses is particularly noticeable. A white central strip runs the whole length of the erect leaf, which is bordered by two 
green bands. At first sight these two green bands appear to be flat, but they are not really so; each is convolute, and thus in the crocus-leaf there are actually two green tubes united to the white central stripe, which is destitute of chlorophyll. This leaf may be distinguished by its erect position from the rolled leaves which have been described in detail, and which are similar in some respects, although they differ from them in significance.

The spiral leaf furnishes another contrivance of this kind. It is frequently seen in the leaves of bulbous plants, bur-reeds, and grasses, principally in young plants-as, for example, the first green leaves of barley and rye. Leaves spirally twisted like this are always long, narrow, and erect. Sometimes but a single spiral revolution is found, or even only a half revolution; sometimes two, three, often even four circuits are described. The leaves of the New Zealand Flax (Phormium tenax), and those of the Asphodel (Asphodelus albus), of narcissus, of many irises, and of some pines, exhibit only a half, or at most only a single spiral twist; those of the Lesser Bulrush (Typha angustifolia), and numerous species of Garlic (Allium senescens, rotundum, obliquum) present two to three, those of Sternbergia Clusiana three to four, and the Persian Sternbergia stipitata five to six revolutions. Leaves of this kind have, consequently, a curled appearance. That such a spiral leaf resembles the fistular leaves in its mechanical significance, and that it possesses a greater resistance to flexion than a flat leaf, is beyond question.

It may also be noticed in the Reed-mace, that in a strong wind the leaves are not only bent, but are also somewhat elongated, i.e. the spiral becomes somewhat looser in the bent leaf. But as soon as the wind subsides, and the leaf returns to its vertical position, the previous form of torsion is resumed. The advantage possessed by an upright spirally-twisted leaf over an erect flat one, with regard to wind, becomes quite obvious when one imagines the two forms exposed side by side to the same wind-pressure. When the gust strikes an erect flat and rigid leaf, the whole of its surface is encountered at right angles, and the leaf undergoes a large amount of bending, and possibly fracture; but when it strikes a spirallytwisted erect leaf, the various portions of the blade are met at different angles; the air current becomes, as it were, diffused into innumerable streams, which, passing along the revolutions of the spiral, effect only a comparatively small curvature, and scarcely ever cause the leaf to be broken. When these spiral leaves are swayed by the wind, from a distance the movement has a very peculiar look, much more like trembling, tossing, and twisting than like bending.

The arched form of leaf is closely allied to the spiral. It, too, is found in long ribbon-shaped leaves. At the commencement of development the arched leaf is erect and lies in one plane, but when fully developed it takes the form of a bow, with the convex side directed upwards. It may spring from the sides of erect lofty stems, or may originate close to the soil. Arched leaves appear very noticeably in those grasses whose habitat is on the ground in, and at the margins of, woods and on steep mountain slopes, e.g. in Milium effusum, Melica altissima, Calamagrostis Halleriana, Brachypodium silvaticum, Avena flavescens, and 
Triticum caninum. When the wind sways the leaves of these plants, the arch formed by them is either narrowed or widened, according as the wind comes from this or that side. In still air the leaf assumes a middle position. Although the arch may be widened or narrowed by the wind, in no case does the bending go so far as to break the blade. Moreover, these leaves are rendered so elastic by a suitable arrangement of bundles of bast, that even violent storms cannot do them much harm. These arched, overhanging, ribbon-like leaves are often further complicated by the fact that all the leaves are turned to the same side so that they present a combed appearance-like those of the reed-although their sheaths cannot twist round the haulm. This is seen especially when the plants are growing on the margin of a wood or on the narrow terraces of a rock face, i.e. on places where they are only illumined on one side. The one-sided direction of the leaves is connected with the illumination, and is due to the fact that a semi-arched leaf turned towards the gloom of a wood, or towards a shady rock-wall, would not obtain sufficient light. This gives rise, indeed, to an inversion of the leaf-blade, so that the originally lower side of the leaf becomes the upper.

It is scarcely necessary to state that the relations with regard to light exercise a no less important influence in the determination of the shape of the spiral and fistular leaves than in the above-mentioned grasses, whose leaves are arched, overhanging, and partially twisted. If these relations are not taken into consideration, it is not because the significance of light in these special instances is not appreciated, but only because a clear view of these extremely complicated conditions can only be obtained by a rather one-sided treatment.

\section{PROTECTIVE ARRANGEMENTS OF GREEN LEAVES AGAINST THE ATTACKS OF ANIMALS.}

The matrix of the chlorophyll-granules is very similar in composition to that of protoplasm, and, like it, consists of nitrogenous compounds; by the activity of the chlorophyll-bearing cells sugar and starch are produced, and the green cells contain not only albuminous compounds, but also carbohydrates, and these, too, in a form in which they are digested with comparative ease. What wonder, then, that these green cells furnish a very desirable food for innumerable animals. Many animals, it is well-known, live exclusively on a vegetable diet, and principally on chlorophyllbearing tissues. On the other hand, the plants in question would perish with the loss of all their green organs, especially if the store of reserve food in them were also exhausted. The animal and vegetable kingdoms in this sense are at war with one another. The instinct of self-preservation forces animals living on green vegetables to seek their food at any cost, to seize the plants unsparingly, and when their hunger presses, to destroy them root and branch. Herbivorous animals cannot, like men, foresee that in the consumption of the means of subsistence the plants robbed of all their green organs must perish, that consequently in the following years for them and their descendants food will be wanting, and that 
in the destruction of their food-plants, their own existence is imperilled. If man removes a portion from the plants serving for his livelihood, a limit is always fixed to this consumption which prudent consideration and foresight never overstep. He always leaves as much as is necessary to the plant in order that it may maintain itself and multiply. Indeed, he even tries to assist and to further the nourishment, growth, and multiplication of the plants useful to him, and is at considerable trouble to protect and to save serviceable vegetation from the ravages of animals. This protection of man, however, is limited to a comparatively small section of plant species; all those from which he derives no benefit remain uncared for, and these would be surrendered to the overwhelming onslaughts of animals, and final destruction, if means were not at their disposal by which they could protect and maintain themselves. Of course these means are not adapted to offensive attacks upon the animal kingdom; and the attitude of the vegetable world towards animals must not be looked upon as one of war, but rather as an armed peace.

But if plants have only at their disposal means of defence, these are none the less dangerous to offenders, and not only equipments comparable to pointed weapons, but also poisons and corrosive fluids are abundantly turned to account.

First of all, with regard to poisons. It is to be pointed out that these are only developed in those parts and to an extent necessary in order to preserve at least the greater portion of the foliage, and then also the flowers and fruit. Moreover, it must be remembered that the same chemical compound does not act as a poison to an equal degree in all animals. The foliage of the Deadly Nightshade (Atropa Belladonna) is a poison to the larger grazing animals, and by them is left undisturbed; but the leaves of this plant are not only non-poisonous to a small beetle (Haltica Atropa), but form this animal's most important food. The larvæ of this beetle often eat numerous holes in the leaves, which, however, by no means prevent the development of the Deadly Nightshade. Accordingly these leaves are protected by the alkaloid contained in them only against wholesale extermination; limited portions of them can be surrendered and sacrificed with impunity. The same thing occurs in numerous other plants which contain poisonous alkaloids, or other materials harmful to large herbivorous animals. It is puzzling how grazing animals find out the materials in the leaf which are injurious to them. In many instances the plants in question possess characteristic odours which act offensively on the olfactory nerves of men at any rate, as, for example, the Thorn-apple (Datura Stramonium), the common Henbane (Hyoscyamus niger), the Hemlock (Conium maculatum), the common Birthwort (Aristolochia Clematitis), the Dwarf Elder (Sambucus Ebulus), and the Sabin (Juniperus Sabina); many other poisonous species, however, which are likewise avoided by grazing animals, bear leaves which to men are odourless as long as they are intact-as, for example, the numerous species of Monkshood (Aconitum), Black Hellebore (Helleborus niger), the White Hellebore (Veratrum album), the Meadow Saffron (Colchicum autumnale), the Mezereon (Daphne Mezereum), species of Spurge (Euphorbia) and Gentians (Gentiana), which are never disturbed by stags, roes, chamois, hares, and just as 
little by oxen, horses, and sheep, not even by the omnivorous goat. As long as the plants remain undisturbed in wood and meadow, their characteristic materials have no effect on the olfactory nerves of men, but they must make themselves known to the animals mentioned by the sense of smell, and this even before the plants have been bitten and injured. The fact that plants which contain no alkaloids, and generally are not poisonous to men, are at the same time carefully avoided by grazing animals makes it probable that to eat them would be in some way injurious to these animals. This remark applies particularly to mosses, ferns, succulent plants (Sempervivum and Sedum), many cresses (Lepidium Draba, perfoliatum, crassifolium), Toadflax (Linaria vulgaris), the Greater Plantain (Plantago major), and many oraches.

That horse-tails (Equisetum), the green leaves of the Crowberry and Bearberry (Empetrum and Arctostaphylos), the Rhododendron and Cowberry (Rhododendron and Vaccinium Vitis-Idcea), and numerous other low evergreen shrubs, which form a chief constituent of the vegetation of heaths and moors, as well as the declivities of high mountains; further, that the Proteaceæ and Epacrideæ which compose the bush of Australia and the Cape, are avoided by the animals seeking their food there, is indeed explained by the fact that the tissue of these plants is very difficult to digest in consequence of strongly developed and partially silicified cuticular strata. It is certain, therefore, that in the formation of a very thick, firm cuticle, and in the deposition of silica in the cell-wall, a protective measure is provided against the attacks of grazing animals; though, of course, it must not be supposed that this is the only function discharged by these structures.

In many plants water forms an excellent protection against grazing animals, including that which falls as rain and dew on the foliage-leaves, and then remains for days and even weeks collected in special hollows. In the morning when the plants are richly bedewed, the ruminants do not usually graze; they wait until the cold dewdrops and rain-drops which adhere to the leaves are evaporated; and later also, they leave on one side those plants on which the rain-drops still remain. In this respect the Lady's-mantle (Alchemilla vulgaris), known also in popular language by the name of Dew-cup (illustrated in fig. $52^{2}$ ), is a very striking instance. Rain and dew remain collected here at the bottom of the cupshaped leaves, when already, in the meadow round about, the surfaces of other plants have become quite dry. While these latter, if they are not protected in other ways, are devoured by the grazing animals, the Dew-cups remain undisturbed, and are evidently avoided. This is not caused, as in the ferns, by the possession of certain objectionable materials-since the leaves of an Alchemilla, from which the water has been shaken, are eagerly taken as food by the grazing animals, which must, therefore, in some way dislike to feed on leaves on which water is standing.

The most important rôle in the defence against food-seeking animals is performed by the organs terminating in strong, sharp, tapering points, which wound offenders, and may be called the weapons of plants. In botanical 
terminology they are known as spines and prickles. A structure which is mainly composed of wood, or whose interior is at least traversed by vascular bundles springing from the wood, and which, therefore, ends in a firm sharp point, is called a spine (spina). On the other hand, a prickle (aculeus) is a structure which proceeds from the epidermis or cortex of a plant member, contains no vascular bundle within, may be multi- or unicellular, but always terminates in a point which is capable of wounding the skin of the offender. This distinction is not always an easy one to make, and botanists have never laid much stress upon it.

Spines and prickles may arise from all the plant members and organs, and appear at all heights. They are observed most usually on or near the green tissues to be protected, but often even the road to the green organs, passing over the leafstalk, the stem, and occasionally also over aërial roots, is provided with prickles and spines in order that in this way the animals which feed on vegetables, particularly snails, which creep up from below, may be kept off. Thus very pronounced spines are seen, for example, on the aërial roots springing from the lower part of the stem in Trithrinax aculeata. The lower portions of the main axes up which these animals must climb in order to reach the green portions are armed with spines or prickles, in many Bombax and Pandanus, in Erythryneæ, gleditschias and roses, and in the fan-palms very abundantly on the leafstalks.

The size, direction, position, and distribution of the weapons depends generally upon the nature of the attack, on the form and size of the food-seeking animals. and on the nature of the implements at their disposal. The gigantic floating leaves of the Victoria regia are only armed with prickles on the under surface and on the turned-up margin, i.e. only where they are exposed to the attacks of planteating aquatic animals. It is also an interesting fact that many woody plants are only protected when young, i.e. while they are short and their foliage can be reached by ruminants, viz. by goats, sheep, and oxen; but on the boughs and branches removed beyond the reach of the mouths of these animals, no prickles and spines are developed. Young, low trees of the Wild Pear, only one or two metres in height, bristle with the spines into which the ends of the woody branches are transformed; while the branches of the crown of trees four or five metres high remain without spines. The same thing occurs in the Chinese Gleditschia (Gleditschia Chinensis), and in the Holly (Ilex Aquifolium). In the latter it can be seen that the leaves of the crown of tall trees have almost entire margins and are unarmed, while the margin of the leaves in shrubby specimens is drawn out into bristle-like, pointed teeth.

Plants armed with weapons for warding off the attacks of animals may be arranged together in two groups. One of these consists of those forms which protect their green tissue by structures actually developed on the organs in question, and the other group comprises those forms which have no such capacity of self-help, where, rather, one member protects another, and where division of labour has brought it about that certain plant-organs deprived of chlorophyll VOL. I. 
and metamorphosed into weapons assume the protection of the unarmed adjoining chlorophyll-bearing members.

To the first division belong chiefly most of those leafless plants which have developed green tissue in the cortex of their branches and twigs. Indeed, the green branches of these plants are, as a rule, so firm and rigid that one would imagine they would scarcely ever tempt animals to eat them. But "hunger is a hard master", and in cases of necessity, as shown by experience, even the stiff switch-like shrubs of the Mediterranean and other floral districts are attacked. In order that they may not succumb entirely to these assaults, many of the leafless green-branched plants are suitably armed by the possession of spines at the ends of their green branches, which confront the assailants. Many of these plants, indeed, are actually built up entirely of much-branched green spines, which fact, of course, gives them a very peculiar appearance. The spinose flora of Spain and of the opposite coast of Africa exhibits a whole series of these plants, but here only the Furze (Ulex nanus, Gallii, micranthus), and the spring Asparagus (Asparagus horridus, Broussonetia, and retrofractus) need be cited as examples. Also the green leaf-like branches of plants with flattened shoots, which are not protected by poisonous substances like those of Phyllanthus, run out into sharp points, as may be seen in the European Butcher's-broom (Ruscus aculeatus), illustrated in fig. 82, and in the South American Colletia cruciata, represented in fig. $83^{1}$.

The weapons developed on green leaves are far more complicated than the implements with which green stems are furnished. In some instances points which wound aggressors project from the ends of the ribs and veins which form the groundwork of the leaves, rising up like needles above the green tissue of the foliage; in other cases they consist of cells and groups of cells which originate from the epidermis of the green leaf, and are inserted, sometimes at the margin, sometimes on the surface, like little daggers. In the first instance the vascular bundles, which are seen traversing the leaf as ribs, are provided, at the point where they project beyond the green tissue and terminate as spines, with a covering of very hard cells; in the latter case the cells and cell-groups springing from the epidermis and rising up as prickles, bristles, and pointed hairs, exhibit thickened and stronglysilicified walls. The following equipment appears particularly often in several pines, many grasses, sedges, and rushes, in species of the genus Yucca, in several caryophyllaceous plants (Drypis and Acanthophyllum), in Acantholimon, belonging to the order Plumbagineæ, and in some saltworts and succulent plants (Umbilicus spinosus, Sempervivum acuminatum). The green leaves are numerous, usually crowded thickly together to form a tuft, and project from the axis in all the directions of the compass; they are rigid, undivided, linear, round or triangular in cross section, and terminate in a sharp, strong, piercing spine. This form of leaf may be termed acicular (or needle-shaped). In many cases, at all events, such leaves have exactly the form of needles, and in regions where the unaltered products of nature are still preferably used as tools and utensils, they actually serve as needles. That plants possessing leaves with these needle-like 


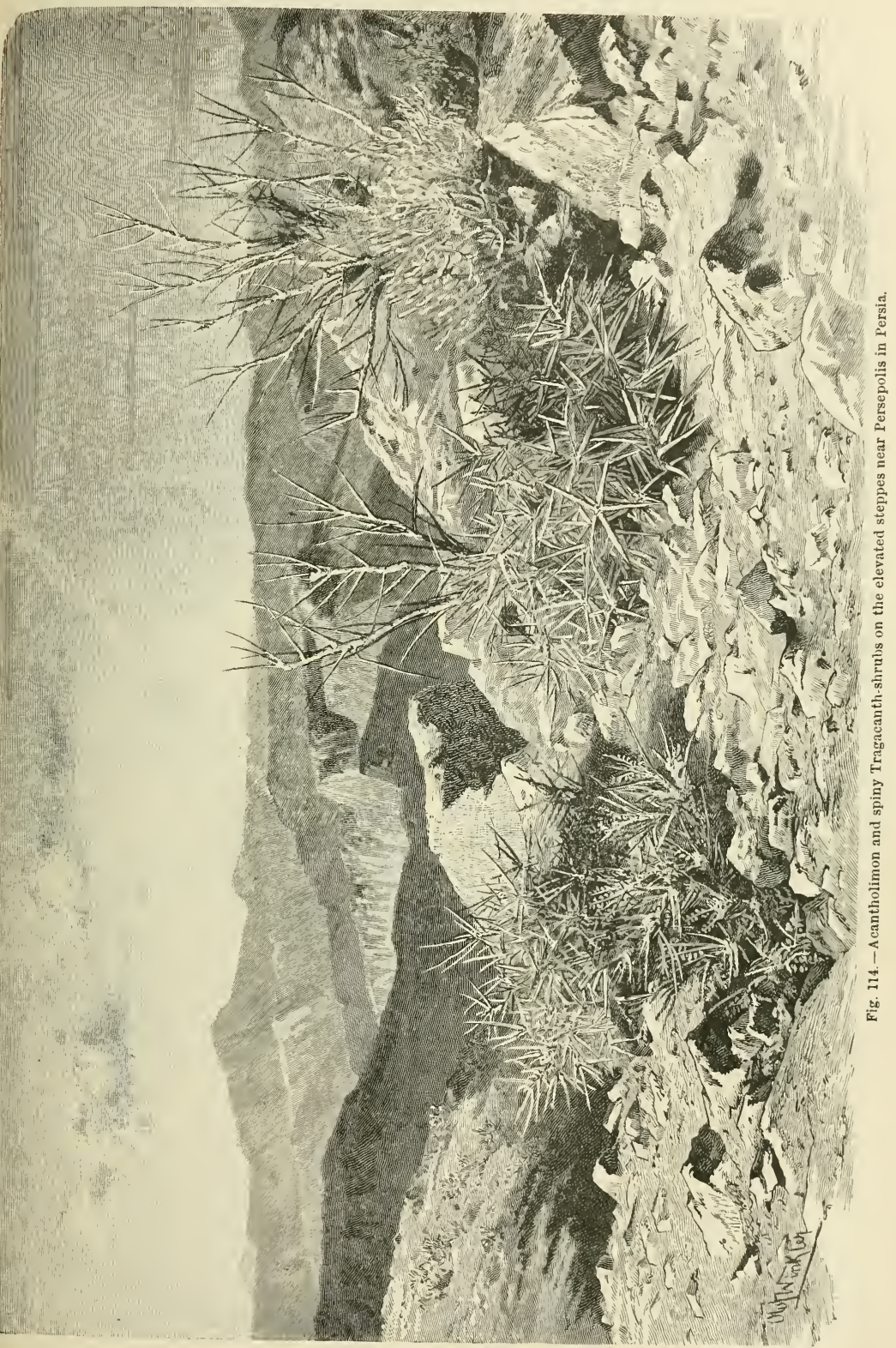


points are excellently protected against the attacks of animals, scarcely requires to be proved more in detail; however, it might be indicated by special mention of two interesting examples. In the Southern Alps, in the neighbourhood of Monte Baldo, and on the opposite mountains behind Vallarsa, a species of grass (Festuca alpestris, see fig. $86^{5}$ ), is found here and there, whose rigid leaves, projecting in all directions,

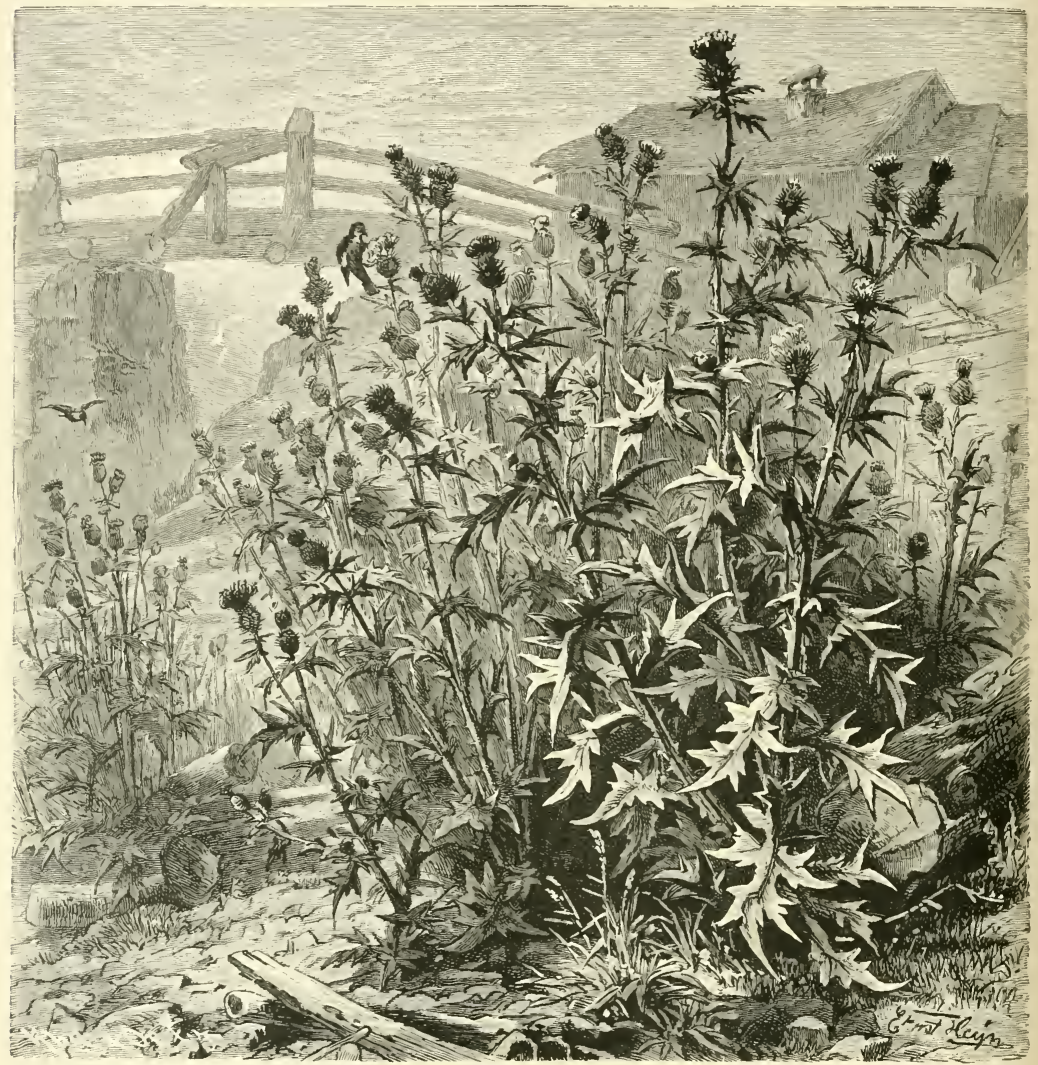

Fig. 115.-Group of Thistles (Cirsium nemorale).

terminate in needle-shaped points. This grass is the plant most detested in the whole district, and the shepherds try to destroy it by burning, wherever it appears in quantity, since the grazing animals, when seeking other plants growing between the patches of Festuca alpestris, cut their nostrils so severely that they often return from their grazing in a bleeding condition. It is remarkable that when these grasses can be easily uprooted, the grazing animals themselves bring about this destruction. The Mat-grass (Nardus stricta), when growing in the meadows, is seized low down 
between the teeth of the oxen, torn from the ground, and dropped, so that it forthwith dries up and perishes. I saw thousands of the tufts, which had been rooted up by oxen, lying, dried and bleached by the sun, on the meadows on the Almboden of Oberiss, in the Tyrolese Stubaithal. It must not be supposed that the animals accomplish this clearance of the meadow deliberately; but it may indeed be admitted that they root up the patches of Mat-grass in order thus to obtain the

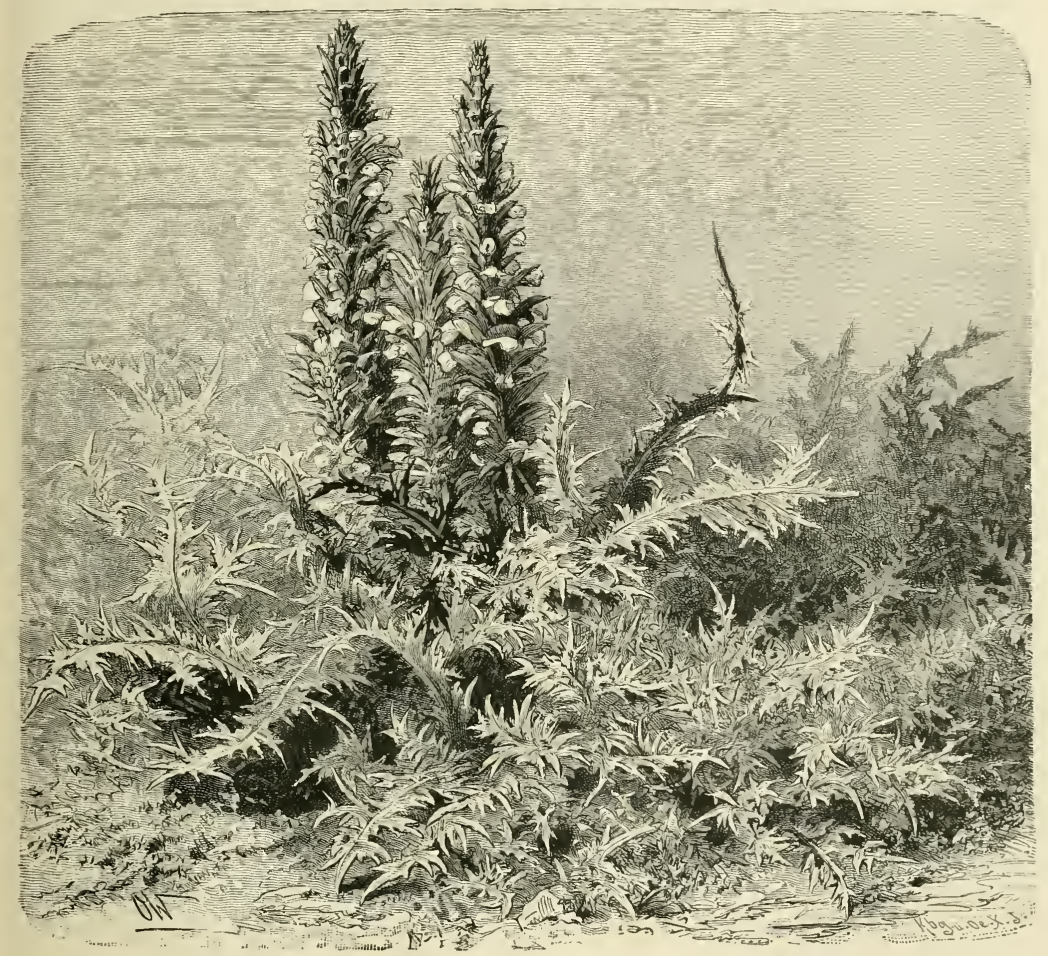

Fig. 116.-A canthus spinosissimus.

enjoyment of the other plants growing between them, and avoid the risk in doing so of wounding their mouths with the pointed Mat-grass leaves.

A considerable proportion of plants with sharp acicular leaves inhalit steppes specially distinguished by the great dryness of their summer, particularly the elevated steppes of Persia, where they form a remarkable feature of the landscape. This applies most of all to the numerous species of the genus Acantholimon, a group of which, intermixed with spiny Tragacanth bushes, drawn from nature by Stapf, is exhibited in fig. 114. Like gigantic sea-urchins, lying strewn in groups on the sea-bottom, these plants, growing in hemispherical patches, live on the stony 
soil of the elevated steppes, where they are so well protected by their needle-shaped leaves, projecting all round from the stem, that they are never eaten by grazing animals.

With the acicular form of foliage-leaves are ranked those which may be best compared to the process of the sword-fish. The outline of the leaves belonging to this form is linear, or linear-lanceolate, generally they are elongated, and often also slightly curved. Many of them are thickened and fleshy, but at the same time very hard and rigid on the outside. The points, produced by the terminations of the vascular bundles, spring from both margins of the leaf, and in the majority of instances stand at right angles to the margins; more rarely are they directed forwards. Each leaf either ends in a strong-pointed thorn, as in the agaves, or in a bundle of threads, as in Bonapartea and Dasylirion. The teeth on the leaves of the last-named plants remind one most in form, surface, and colour, of the scales of a shark, and can inflict frightful wounds on those who come too closely into contact with them. The table-land of Mexico is particularly rich in plants with leaves armed in this manner; that country is especially the habitat of agaves and Bromeliaceæ, of species of Dasylirion and Bonapartea. The Cape also is the home of a series of these forms, viz. those belonging to the genus Aloe. Species of Eryngium, with agave-like leaves (Eryngium bromeliafolium, pandanifolium, \&c.) belong to Mexico and South Brazil. It is worthy of note that several aquatic plants, such as Hydrilla, Naias, and the Water Soldier (Stratiotes aloides), have their leaves similarly armed, and are thus protected from the attacks of plant-eating aquatic animals.

A third form of foliage-leaf, armed with spines, is that of the thistle. The word thistle is here used in its widest sense, and is not restricted to species of the genus Carduus and Cirsium (see fig. 115). By the term thistle-leaves are indicated all those which are more or less lobed and divided, whilst the margins and the extremities of the lobes are provided with stiff, projecting spines. Such leaves are possessed by very many composites of the genera Carduus, Cirsium, Chamapeuce, Onopordon, Carlina, Echinops, Kentrophyllum, Carduncellus, especially also in many Umbelliferæ (e.g. Eryngium amethystinum, Echinophora spinosa, Cachrys spinosa), some nightshades (e.g. Solanum argenteum, pyracanthos, rigescens), several Cycadeæ (Zamia, Encephalartos), and are very strongly developed in Acanthus, of which a species, Acanthus spinosissimus, growing in the Mediterranean floral district, is illustrated in fig. 116.

Nowhere in the whole world is the thistle-leaf met with so abundantly and in such manifold varieties as in the Mediterranean flora; Spain and Greece, Crete and Algeria, are particularly rich in districts covered with thistles. Thistleleaves often appear divided into three, four, or five portions, and split up into numerous points, sections, and lobes. The ends of all the separate portions being transformed into stiff points, not much remains of the green tissue of the leaf; only a small narrow green lamina is seen, from which radiate out yellow and white spines on all sides, like lances of various lengths 
Prickle structures, which are not to be regarded as metamorphosed terminations of leaf-ribs, but which originate from the epidermis of the leaf, are sometimes multicellular, sometimes unicellular. The former are termed prickles (aculei), the latter bristles (setce). In this series of weapons, barbs are particularly worthy of notice. These are formed by obliquely directed conical cells, which project from the margin of the leaf, and terminate in a hard silicified, generally somewhat bent, apex (figs. $117^{7}$ and $117^{8}$ ). Leaves, whose margins are thickly beset with these cells, exhibit, under the microscope, a saw-like appearance. It is
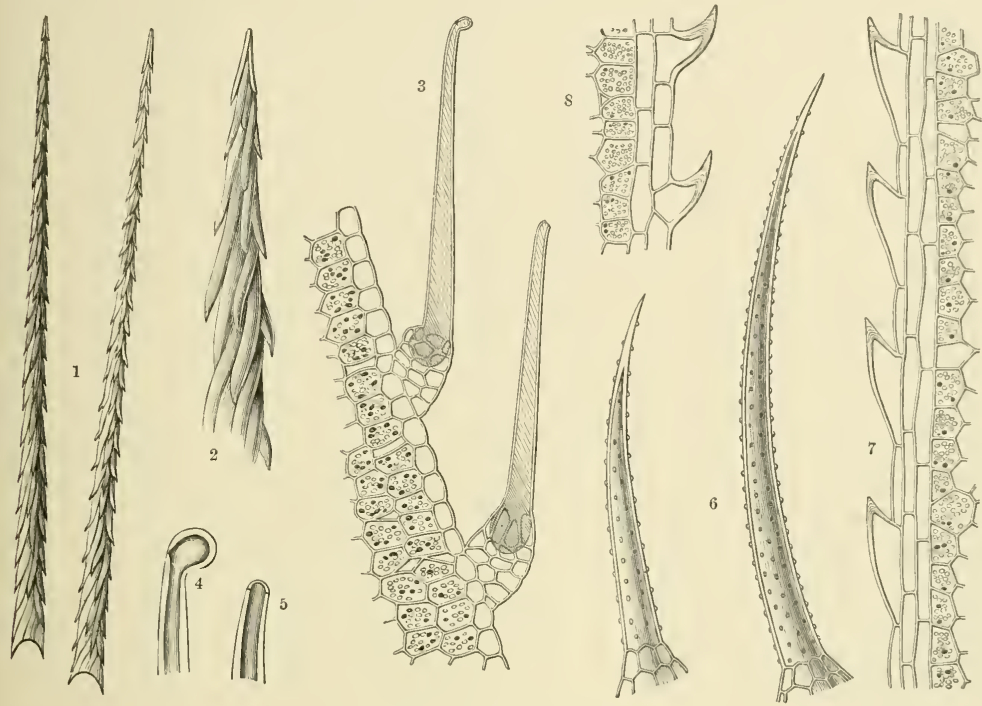

Fig. 117.-Weapons of Plants.

1 Barbed bristles of Opuntia Rafinesquii; $\times 25$. 2 Upper portion of this barbed bristle; $\times 180$. S Vertical section through a part of the leaf, based with stinging hairs, of the Stinging Nettle (Urtica dioica); $\times 85$. 4 Capitate termination of a stinging hair; $\times 150$. 5 The capitate termination broken off; $\times 150$. 6 Pointed bristles of Echium Italicum; $\times 40$. ${ }^{7}$ Margin of a scabrous leaf, beset with barbs, of a Sedge (Carex stricta); $\times 200.8$ Margin of a scabrous leaf, beset with barbs, of a Grass (Festuca arundinacea); $\times 180$.

to be noted that leaves of this kind can, under certain circumstances, really act as saws. If such leaves are very gently stroked in the direction opposite to that of the points, they do not, of course, immediately cut the hand, but they do not bend, and under increasing pressure, the lamina of the leaf becomes arched. Since the leaf is also well stiffened, a resistance is encountered which could scarcely have been expected from so fragile a leaf. If a surface on which portions of these leaves have been laid be shaken, the bits move in a direction opposed to that of the points of the barbs. Movement in the opposite direction is impossible, because it is opposed by these apices. When such leaf-portions get into the mouths of ruminants, they can easily move forward to a particular 
side, and in a particular manner, such as does not suit the purposes of the grazing animal, and is by no means welcome to it. By firmly stroking the margin of such a scabrous leaf, a wound is produced, the silicified points on the margin acting like the teeth of a fine saw. It is readily intelligible that grazing animals will shun such scabrous leaves; indeed, it is a matter of observation that they seldom (and then only when impelled by great hunger) eat sedges (e.g. Carex stricta and C. acuta), and those grasses which possess particularly sharp-edged leaves.

Still worse than the barbs of scabrous leaves are the barbed bristles (figs. $117^{1}$ and $117^{2}$ ), which, it is true, but rarely occur in plants; indeed, almost exclusively on the branches of opuntias. They are always found surrounding the buds, which rise like warts, with fine bristles above the green tissue in opuntias or prickly pears. If such a spot be ever so lightly touched, small stiff bristles will certainly remain sticking in the skin of the hand, and will produce a very unpleasant itching sensation. On trying to pull out these small brown bristles the matter is only made worse, for they then penetrate much deeper into the skin, and may produce violent pain and inflammation. The reason of all this is at once evident on examining one of the bristles under the microscope. Each bristle is composed of numerous rigid, fusiform cells, arranged in spiral rows; at the upper end each of these cells is wedged in between the others, but the very hard, backwardly-directed, pointed end is free, and thus the whole structure is set with barbs. When once the point of the bristle has penetrated the skin, it is held there by the barbed cells. With the slightest pressure they are easily moved forward in one direction, but on trying to produce a movement in the opposite direction, the free ends of the cells resist the attempt, and it is unavoidable that the forcible extraction of one of these bristles should injure a larger area of the skin than would have been thought possible from the small size of the structure.

Another form of weapon originating from the epidermal cells consists of stiff hairs or bristles, with hard silicified cell-wall and sharp apex, which prick and wound like needles, though only unicellular; they are called pointed bristles. They usually project from the surface of the green leaves, closely crowded together, and their points are turned in the direction from which an attack might be expected. They appear gigantic in comparison with barbs, for even the smallest are much longer than these, and the largest resemble pins with their heads imbedded in the leaf-blade. This comparison becomes the more fitting since the pointed bristles are surrounded at their base by very regularly arranged cells which rise above the surface like a cushion, or often like a short white cone. The bristle itself on the end of this pedestal is formed of a single cell, which, when fully developed, loses its protoplasm and becomes filled with air. The wall of this elongated cell is hardened by the deposition of silica, and is usually unequally thickened by small knobs (fig. $117^{6}$ ). Although pointed bristles are developed in numerous groups of the vegetable kingdom, one group is especially so armed. 
This is the family of the Boragineæ, which has been thus named, indeed, in consequence of its characteristic armour. Examples of the equipment described are furnished in abundance particularly by species of the Viper's Bugloss (Echium), from which the pointed bristles in fig. $117^{6}$ are taken, and of the genera Onosma, Comfrey (Symphytum), and Borage (Borago).

On the leaves on Nettles, Loasaceæ, Hydrophylleæ, and Euphorbiaceæ, occurs a very peculiar mode of protection against the attacks of large herbivorous animals, in the formation of stinging hairs or bristles. These stinging hairs are formed of single large cells like the pointed bristles of Boragineæ. They expand like a club at the lower end, and are much elongated above. Only in Wigandia urens, which belongs to the Hydrophylleæ, is the upper free end finely pointed; in the species of the genus Jatropha, in Loasaceæ, and in nettles, the extremity is swollen into a small head, which is bent to one side. At the kneeshaped bend the cell-wall of the stinging hair is extremely thin (figs. $117^{3,4,5}$ ), so that the slightest contact suffices to break off the head. As the head is broken off obliquely, a very sharp point is produced, and the opening formed by the rupture is not horizontal, but oblique, so that the broken end resembles the poison-tooth of a snake or the nozzle of a hypodermic syringe. The breaking, independently of the extreme thinness of the cell-wall below the head, is helped by the brittleness of the hairs, and this is caused by the silicification, sometimes by the calcification, and in Jatropha by the lignification, of the cell-wall. This modification of the cell-wall, however, is restricted to the upper part of the hairs. The cell-wall of the club-like swelling at the base of the stinging hair is neither silicified nor calcified, but consists of unaltered cellulose, and yields to an external pressure, so that by such a pressure the outflow of the cell-contents is assisted. By these means, also, the stinging hair is enabled to become turgid, which property certainly plays a very important part in the outflow or outspurt of the cell-contents from the silicified or calcified funnel-shaped apex after the head has been broken off. When by a pressure from above the brittle end of the hair is splintered, and the head broken off, the point formed at the place of rupture penetrates into the body causing the pressure, provided this is soft, as, for example, the skin of men and animals; and the contents are injected into the wound so formed. In the fluid contents of the stinging hair a substance occurs together with formic acid, resembling the unorganized ferments or enzymes, and it is this which produces the violent inflammation round the wound formed by the puncture. The painful sensation felt immediately after the puncture, which is popularly called "burning", on account of its resemblance to that produced by a burn, is indeed caused by the formic acid; but a series of other phenomena which are observed after the puncture, can only be placed to the account of the enzyme, which acts like a poison. When numerous stinging hairs penetrate the skin in close proximity, a wide area becomes reddened, and inflammatory swellings, with violent pain, are produced. Even the European nettles, viz. Urtica dioica and urens, give rise to unpleasant burning and 
itching, and very severe attacks, tetanus, \&c. are produced, as by snake-bites, by the Urtica stimulans of Java, the Urtica crenulata, which is a native of India, and the Urtica mentissima, growing in Timor. Generally, an analogy between stinging hairs and the hollow poison-fangs of snakes cannot fail to be recognized.

The mass of tissue in which the stinging hair is imbedded consists of chlorophyll-bearing cells, and is elastic and flexible; whenever a stinging hair is pressed on one side it lies close to the leaf-surface, so that the point does not penetrate the skin of the fingers pressing it, and does not form, or poison, a wound. When the pressure is removed, the hair becomes erect again in virtue of the elasticity of its knob-like support, and directs its brittle point outwards. Upon this fact depends the trick of stroking a nettle with the hand so as not to be stung. The lower, unarmed part of a leafy nettle, whose foliage is beset with innumerable projecting stinging hairs, is taken in one hand, and the other hand is then passed from below upwards over the foliage, and in this way the hairs touched are pressed on to the leaf surfaces and do not wound. But if the nettle is touched from above, the heads of the hairs are immediately broken off, the perforated points penetrate the skin and discharge their poisonous fluid into it. Grazing animals carefully avoid plants furnished with stinging hairs, and do not let their nostrils, nor the mucous membrane of their mouths, get poisoned by the corrosive fluid. The nettle is, therefore, well protected against larger animals. Their foliage is, indeed, eaten by the larvæ of Vanessa Urticce in spite of the stinging hairs, but this injury is restricted to only a portion of the leaves; they can always develop new leafy shoots from the intact stems and buds, and, at any rate, the nettle does not perish on account of the ravages of these larvæ.

This is also the most suitable place for the consideration of a form of planthair, whose cells, indeed, possess no stiff silicified walls, and which, therefore, do not prick and wound, but which, nevertheless, keep the plants they clothe from injury by grazing animals, and which thus far must also be regarded as agents for protecting the green tissue. These hair-structures have already been described when making clear the protection afforded to leaves against excessive transpiration. Such hairs, as we saw, are particularly well shown by many species of the genus Mullein (Verbascum). These branched, radiating hairs, reminding one of tiny fir-trees, are easily detached from the surface of the leaves from which they spring, and a very slight pressure of the hand is sufficient to lift off numerous flocks of this hair-felt. Although the cells which build up the hairs of the leaf-felt are not stiff and prickly, and do not penetrate into the skin, they very readily remain hanging to the smallest inequalities on the surface of the disturbing body. If grazing animals bring the mucous membrane of their mouths into contact with the leaves of the Mullein, this mucous membrane immediately become covered with flocks of the detached hair-felt, which establish themselves in the inequalities of the surface, and they certainly produce anything but a pleasant sensation. On the peculiar adhesion of the felt-hairs of the 
Mullein to the mucous membrane rests the necessity for the caution which people observe in the preparation of Mullein. The flowers of the Mullein (Verbascum thapsus) have been used from time immemorial in the preparation of a tea. When hot water is poured over the flowers, which are covered on the under side, just like the foliage-leaves, with a fine felt of hair, portions of the felt are detached, and remain floating in the infusion. If the decoction is not filtered through a piece of linen, some of the hairs may stick to the mucous membrane of the mouth, and there produce an intolerable irritation and itching. This unpleasant sensation is certainly much more powerful in animals when taking the leaves of Mullein in their mouths than with us when we drink unfiltered mullein tea, and it no doubt deters animals from eating the foliage of the plants in question.

The protective mechanisms just described are all borne directly by the particular organs needing protection. But there are many plants whose foliage is unequipped with armament of this sort, and in which adjacent parts of the plants afford the protection. One may instance all such plants as have soft, unarmed leaves, sheltered from attack by lateral shoots transformed into spines. The stem and branches of these plants are not clad with foliage entirely to their summits. The ends are usually leafless, and look as if their leaves had been stripped off. Generally speaking, if leaves are present on the summits of the branches, they are stunted, small, indicated only by scales and protuberances, and are anything but an attractive food. Consequently, the end of the woody branch appears tapering, and terminates in a stiff, sharp spine. In a bush whose branches project out in all directions with leafless apices, while their green foliage-leaves are collected behind the apex, a most efficient system of defence is produced, resting upon division of labour. The green leaves can carry on the work assigned to them undisturbed under the protection of the spines, and if it happens now and then that a large food-seeking animal, driven perhaps by greed or hunger, pushes his mouth carefully between the confronting spines, and knows how to procure some green leaves from behind the spines, the existence of such a bush is not seriously threatened. The Alhagi shrubs of steppes, as well as several brooms and Cytisus shrubs, viz. Alhagi Kirgisorum, Genista horrida, and Cytisus spinosus (fig. $118^{5}$ ), exhibit the protective mechanisms just described in a marked manner. In many other shrubs, such as sloes, sea buckthorns, and buckthorns (Prunus spinosa, Hippophaë rhamnoides, Rhamnus saxatilis), the same contrivance is indeed met with, but it only has the full significance while the foliageleaves are quite young. Only so long as the tender leaves, which have just emerged from the buds, are overtopped by the spiny branches are they protected from being devoured; afterwards when they have developed, those only are protected which clothe the base of the spiny branches. On the long axes of the Hawthorn, in the axils of the lower foliage-leaves, there are always developed, close together, a lung spine and a small bud, in the axils of the upper leaves a bud only. In the following year, reduced axes develop from the buds situated close to the 
long, shiny, brown spines, which often bear flowers; but from the buds on the upper half of the shoot, a long axis arises, which repeats the development just described. Spines, which on the American species of hawthorn, become in Cratcegus coccinea, $4 \mathrm{~cm}$., in C. rotundifolia, $6 \mathrm{~cm}$., and in C. Crus galli, 7-8 cm. long, resemble sentinels which have to protect these developing reduced axes. Most of these bushes develop horizontal projecting branches, and therefore extend as far transversely as vertically, and since the spines remain for many years, the leaves of all these axes, which, in later years, spring laterally from the branches, almost in the interior of the bush, behind the old spines, are protected by them. In several Brazilian mimosas, the spines situated on the branches do not indeed project beyond the outspread leaves, but as soon as animals disturb the leaves, they are revealed from their concealment behind the protective defence of spines, and the animals retreat before the sharp points now confronting them.

A very peculiar relation is observed between green leaves and spines in most of those semi-shrubs which Theophrastus in olden times grouped together under the name of "Phrygana". In these semi-shrubs, of which the Vella spinosa, represented in fig. $118^{8}$, may be selected as an example, each shoot growing out from the winter buds develops green foliage-leaves on the lower half and above these, and frequently also in the region of the inflorescence, green lateral twigs transformed into sharp-pointed spines. These spines, which, in many instances, as when they appear in the region of the inflorescence, may be considered as metamorphosed flower-stalks, are at first soft and succulent, contain green tissue in their cortex, and function at first exactly like the narrow foliage-leaves situated near them. In the first year they play no part as protective agents on account of their softness; in the autumn, the green leaves fall from the shoots; the spinous tips of the branches are also dead and withered, but they still remain, and do not fall off. During the summer, having become hard and stiff, they now wound anyone who seizes them roughly, and obviously protect the shoots which spring from the lateral buds in the following year behind their dried-up ends, in which the development just described is repeated. Thus arise, in time, bristling shrubs, from whose periphery radiate out a quantity of dried-up spiny branches, and which often look as if the branches had become frozen and shrivelled in the winter, and as if the whole plant were in a dying condition. This "Phrygian" underwood is not certainly an embellishment of that region in which it occurs in masses, but it forms a highly characteristic feature in certain floral districts. The Mediterranean area is particularly rich in these "Phrygian" bushes, and species belonging to the most diverse families develop in this form. To mention only a few examples, of Cruciferæ, Vella spinosa and Koniga spinosa, of Rosaceæ, Poterium spinosum, of papilionaceous plants, Genista Hispanica and Onobrychis cornuta, of Compositæ, Sonchus cervicornis, of Euphorbiaceæ, Euphorbia spinosa, of saltworts, Noёa spinosissima, and of Labiateæ, Teucrium subspinosum and Stachys spinosa, may be pointed out. The elevated steppes of South-west Asia also exhibit Phrygian forms, and indeed, chiefly, as isolated, 
prickly, and spine-stiffened, low bushes, growing together with thorns, and low Tragacanth-shrubs, in which the green foliage is protected differently. In northern regions not exposed to summer drought, where grazing animals find in summer enough green fodder, this form of plant is almost entirely atsent. It is only met with in the heaths and pine forests of Central and Western Europe, in some species of broom (Genista Germanica and Genista Anglica).

In these regions, certain shrubs and young trees, which do not possess the spine formation described above, acquire from the grazing inimals themselves a shape which strongly resembles the Phrygian form. It is brought about in the following manner. If young trees of beeches, oaks, and larches, or bushes of Ling (Calluna vulgaris), are accessible to goats, sheep, and oxen, these bite off the ends of the fresh shoots, together with the leaves attached to them. The remaining portion of the mutilated shoot in the neighbourhood of the wound dries up, but the part behind keeps alive, and the buds on it develop even more vigorously than would have been the case if the mutilation had not occurred. The shoots which in the following year arise from these buds, however, may suffer the same misfortune; they may again be pruned by grazing animals, and when this is repeated every year, the mutilated beeches and larches at length come to resemble the beeches and larches of old French gardens, which have assumed the shape of pyramids and obelisks in consequence of the continual clipping of the gardener's shears. The branches of these small mutilated trees become so thick, and the dry, hard twigs on the periphery of the crown are so crowded together, that even the greedy goats are prevented from breaking through the armour, and abstain from pulling out the green shoots from behind the dry stumps. Thus at length the unprotected plants obtain a defensive armour which is capable of saving them entirely from the further attacks of grazing animals. Many of these young mutilated and bitten trees, of course, never develop into strong lofty specimens; but in some species the rough treatment which they undergo in their youth does not result in lasting injury. This applies especially to larch-trees growing in Alpine valleys. The young trees gradually form thick branched bushes in their struggle with the goats, and a top cannot be definitely distinguished in them, since the central shoots, as long as they can be reached by the goats' mouths, are not spared. But, at last, after a number of years, the bushy larches attain to such a height and circumference, that the goats can no longer reach the upper shoots. And behold, a strong shoot arises from the middle of the much-branched bush, develops a whorl of lateral branches, elongates from year to year, and being no longer harassed by the grazing animals, grows up into a lofty larch-tree. For a long time, at the lowest portion of the tree, may be seen projecting the oldest lateral boughs, which have become abundantly branched in consequence of the mutilation, and which serve to protect and defend the developing central stem. But gradually these lower branches decay, and fall crumbling on the ground; thus the last reminiscence of their severe youth is obliterated. 
The contrivance for protecting the green tissues of the cactuses, depending upon a division of labour, is accomplished in a very peculiar way. Our conception of a plant is a stiff grey or brown stem bearing soft green leaves. In the cactus-like plants, however, the most important types of which we have already recognized in the Cactaceæ of the New World, and the columnar Euphorbiaceæ of Southern Asia and Africa, everything is reversed. Here the stem is green and succulent, and the leaves it supports are transformed into stiff grey or brown spines. Food is conducted to the green transpiring tissue in the cortax of the stem, in which, and not in the leaves, new organic materials are produced. The leaves which have been changed into spines, on the other hand, have to keep guard that the green tissue in the cortex of the columnar or flattened stem is touched no more than is necessary. This reversed state of things strikes us as most strange in the opuntias (Cactaceæ), because here the portions of the stem have the form of thick elliptical leaves, and consequently are usually held by non-botanists to be leaves. But the spines, or, strictly speaking, the leaves transformed into spines, occasionally attain to an extraordinary length in these opuntias. They are $3-5 \mathrm{~cm}$. long in Opuntia Tuna, decumana, and magacantha, and even $8 \mathrm{~cm}$. in Opuntia longispina. It has already been mentioned that the buds of opuntias are based with very small barbed bristles, and consequently these plants are armed with a twofold defence against possible attacks with large spines, visible from afar, and with these horrible small inconspicuous barbed bristles. In the cactus-like plants the variety of weapons is very great, and if all the various forms of long and short, thick and thin, knotty and smooth, straight-pointed and barbed, arched and wavy spines and bristles were placed together side by side, quite a goodly collection of arms would be the result. A single species often bears three or four kinds of weapons, and these are arranged and distributed in a great variety of ways, and in this respect a diversity is developed which has a fascinating effect on anyone who has an inborn taste for such changes of form, and we can understand how it is that so many lovers of flowers have devoted themselves to the study and culture of these curious representatives of the vegetable kingdom. Although it is impossible to show the connection between the kind of armour and the attacks to be warded off in each individual instance, even the most cursory glance shows us that the points of the spines, however these may be shaped and arranged, are always placed in front of that portion of the stem which is best furnished with green tissue. In the columnar euphorbias, e.g. in Euphorbia cœrulescens, the stems are furnished with shallow longitudinal grooves clothed with green tissue. On the ridges between the grooves are inserted pairs of divergent spines with their points in front of the grooves, and thus ward off every assault on the green tissue. Exactly the same thing is seen in the columnar Cereus, and in the coneshaped Echinocactus and Melocactus.

On looking at these columnar, flattened and spherical cactuses, the question arises whether it is necessary for them to be surrounded with such a complicated 
system of spines. According to the ordinary conception of the method of feeding of the herbivorous animals referred to, it would be thought that these green clumps, pillars, and balls, even without their terrible equipment, would form anything but choice food. But when they are seen in their original habitats, it is easily seen that they have every need to protect themselves and to defend their existence. While on the stony and sandy plains and slopes which form the habitat of cactuses, all other plants have long been withered, and a green leaf can no longer be seen for far and wide, when all the springs of water are dried up, and not a drop of rain has moistened the ground for months-then the cactuses still remain always fresh and green, and by the assistance of their central aqueous tissue, they are able to survive through the greatest drought and aridity which are ever observed on the earth. But at such periods of drought, every cactus-ball appears like a cordial to the hungry and thirsty animals, and frequently even as the only alternative to death. In spite of the frightful spines with which species of Melocactus are bristling, these are sought by the wild asses in the plains of South America at the periods of greatest drought, and are rooted up where possible by their hoofs in order to get at the juicy tissue of the unarmed lower parts; or the animals try to split the cacti with their hoofs, and in this way to get at the interior, in which proceeding it very often happens that the assailants injure themselves by the spines and receive dangerous wounds.

Next to the cactuses, the strangest spine-formations are exhibited by the low half-shrubby tragacanth bushes (Astragali) belonging to the group Tragacanthacei, which, in an inexhaustible variety of species have their habitat throughout Southern Europe, but chiefly in the east, on rocky mountains and elevated steppes. We will pick out one, viz. Astragalus Tragacantha, from the large number of species, and explain by words and picture the remarkable protective contrivance of its green foliage-leaves (fig. $118^{1}$ ). On observing this plant very early in the spring, a tuft of numerous long, dry, grey spines, whose points are directed upwards and outwards, is seen on the free extremity of each branch. In the centre of this tuft of spines lies a bud, which forms the top and termination of the branch in question. The warmth of spring causes this bud to develop, and the close-pressed pinnate leaflets become loosened, stretch out, and unfold; but weeks pass by, and the leaflets are always still surrounded by the bushy garland of spines. Their green colour can only be seen shining through from behind the long spines as from behind the grey lattice bars of a cage. When they are fully developed, and when they have also somewhat lengthened the branch they adorn, the uppermost leaflets at length project beyond the points of the spines. But see-the end leaflet which had been situated on the rachis of the pinnate leaf has already dropped off, and often a pair of the lower leaflets with it (fig. $118^{2}$ ), and all that now projects beyond last year's spines has also become changed into a spine. The rachis of the leaf at the point where the terminal leaflet was formerly inserted becomes hardened and transformed into a pricking point. Then comes autumn, the period of the leaf-fall. 
Most deciduous shrubs now throw off the leaves with which they have worked all the summer, by means of the formation of a layer of separation at the place where the leaf is inserted on the stem, as previously described. But this does not occur in the tragacanth bushes. Only a portion of the long grey spines by which this year's leaves were surrounded are cast off. The leaflets of the present year are now detached from the leaves; the strong midribs or axes whose ends had alrearly become changed into spines during the summer remain firmly joined to the stem and dry up, forming thus a new stiff tuft of spines which is as like the one thrown off as one egg is to another. Accordingly the dried-up remains of the leaves of one year, now changed into spines, become an apparatus for protecting the developing green leaves of the year following. Observation in the natural state shows that these projecting spines are capable of protecting the green leaves behind them from the attacks of grazing animals. One may see how grazing animals stop in front of the shrubs bristling with spines, and actually abstain from further attacks after the first attempt, although the foliage of the tragacanth named, like that of other papilionaceous plants, would furnish a very desirable meal.

Different from the Tragacanth is the Barberry (Berberis). On looking in summer time at a shoot in vigorous growth, it will be seen to be beset with two kinds of leaves: first, with leaves which have anything but the appearance of foliage, being transformed entirely into spines like those of the cactuses. These at the base of the shoot are drawn out into from five to seven, and further upwards into three, needle-shaped points, as shown in figs. $118^{6}$ and $118^{7}$. Short branches beset with ordinary green foliage-leaves arise simultaneously in the axils of these metamorphosed leaves. These short branches terminate in buds which develop early in the following year, and then form either flowers, or long branches. The foliage-leaves of the short branches, below these buds, fall off in autumn. The three-pronged spines at the bases of the short branches, i.e. of the buds which have passed through the winter, remain behind, and radiate out from the shoot with their three needles in three directions. Now, when in the following spring the buds at the end of the short branch swell, and young tender foliage-leaves burst from them, these are excellently protected against being devoured as long as the points of the three-pronged spine still project beyond them.

In Robinia Pseudacacia, popularly known by the name of Acacia, and also in numerous other robinias as well as in several Siberian caraganas (Caragana microphylla and pygmcea), the stipules are transformed into prickles, and not, as in Berberis, the whole leaf. In all Leguminosæ, structures arise right and left of the place of insertion of the leaf on the stem, known as stipules (stipulce), on account of their position. They are not leaf-like in the robinias and shrubs named, but are transformed into brown spines drawn out into a sharp point. When the foliage-leaf becomes detached and falls off in autumn, both the spinous stipules remain behind and persist even on into the following summer. In the axil of the two divergent spine-stipules is situated a bud which unfolds in the following spring. Here we have again repeated the same protective mechanism as was previously 
sketched in the case of the Barberry. As long as the young tender foliage-leaves remain in this situation between the two spiny stipules (fig. 118 $)$ they are avoided by animals; the protection is only at an end when they have grown beyond the points of the old spiny stipules.

Most of the last-described protective contrivances only defend the green foliage
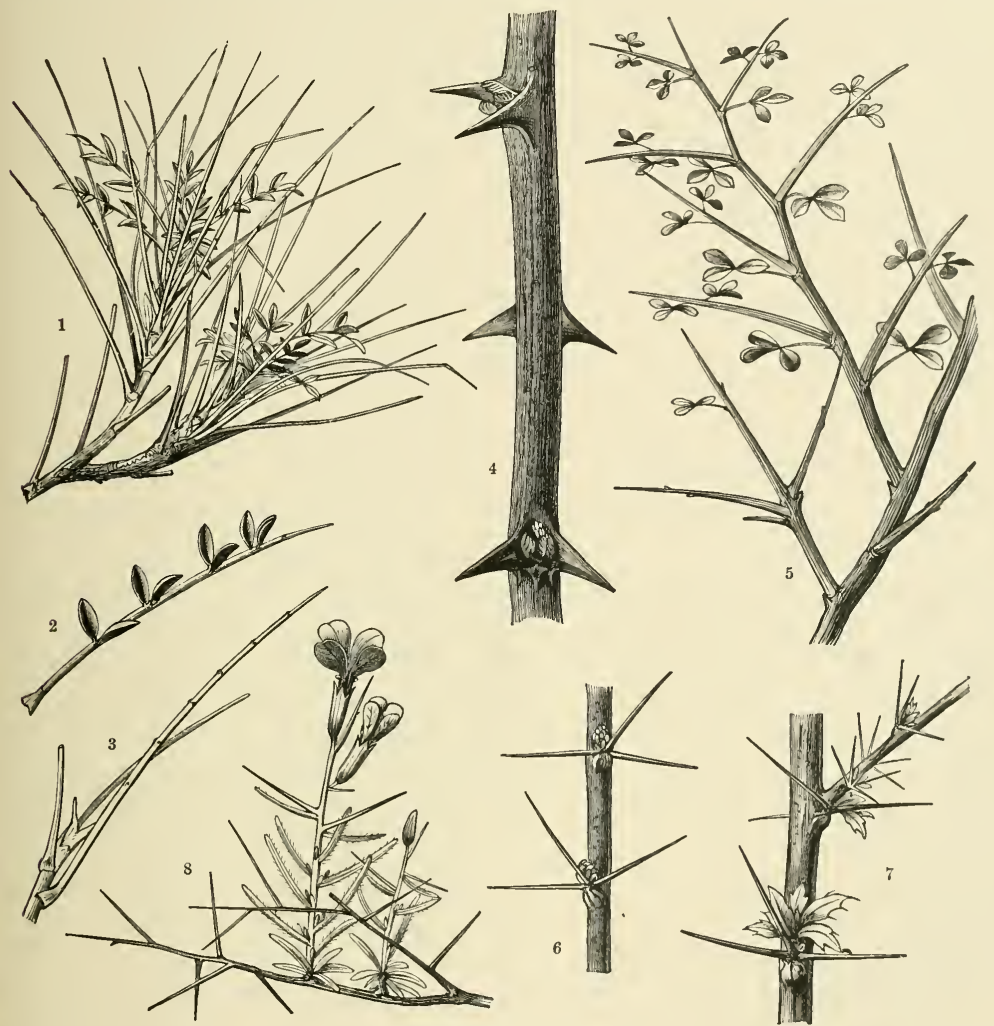

Fig. 118.-Weapons of Plants.

' Branch of the Tragacanth bush (Astragalus Tragacantha) in spring. 2 A single leaf of this Tragacanth from which the three upper leaflets have fallen. 3 Leaf-axis from which all the leaflets have fallen. 1 Portion of a shoot of Robinia Pseudacacia in spring. \$ The spiny Cytisus (Cytisus spinosus). 6, 7 Portions of branches of the Barberry (Berberis vulgaris) in spring. ${ }^{8}$ Vella spinosa; the end of last year's shoot is dried up; this year's shoot bears flowers.

whilst it is young. But this is exactly the time when protection is most needed. If, later on, isolated foliage-leaves, which have grown beyond the points of the prickles, are eaten, this does not so much matter, as part of the foliage still certainly remains, and this is really the important point.

From the fact that the protection of the young green leaves is secured by Vor. I. 
a portion of the old dead leaves, by dried-up structures of the previous year, in tragacanth bushes and also in many caraganas, and generally in numerous other plants, two things may be learned: first, that one and the same plant-member may in the course of a year change its function; and secondly, that dead, withered portions are often called upon to play an important part in the life of a plant. The same thing is frequently observed in flowers and fruits. It often happens, for example, that floral leaves, which originally served to allure insects and to protect the pollen from moisture, are of use later on when dried, in the dissemination of the fruits and seeds. In foliage-leaves, on the other hand, such a change of function is comparatively rare, and is hardly ever observed except in the plants of steppes and of the Mediterranean flora.

It would naturally be expected that the protective contrivances required by the green tissue against an excessive destruction by animals, would also exercise an influence on the gregarious growth of plants as well as on the dwelling together and distribution of plants and animals; and this is proved by numerous observations. Let us suppose ourselves in a country where plants of a hundred different kinds grow up side by side. The shrubs, bushes, and herbs, mixed together, contain the most diverse substances. Some abound in milky juices; others are as bitter as gall; whilst others again taste frightfully sour or contain in their sap alkaloids, the partaking of which would be deadly to many animals. Here is a plant armed with stinging hairs; there from a bush radiate out innumerable spines; and again in other places thistles rear their prickly leaves. The one prevents snails from eating the foliage, the other caterpillars or grasshoppers; a third, goats; a fourth, horses, \&c. Let it be supposed that the country producing this rich vegetation is temporarily quite shut off from everything which creeps or flies. But now arrives a numerous herd of some species of animal against whose attacks one portion of the plants is protected as completely as possible, a second portion only partly, a third not at all. What will be the consequence? The last will be wholly or partly devoured, while the first will remain uninjured. If this is often repeated, at last the one will vanish from the scene, while the other will develop in overwhelming quantity. But in this manner the peculiar composition of vegetation in places where grazing animals regularly appear is naturally explained.

It must strike everyone who visits the Alps that in the neighbourhood of the cow-chalets a luxuriant vegetation springs up from the richly-manured soil, very tempting in appearance, but nevertheless left unattacked by the grazing animals. The shepherds do not prevent the animals from eating of this luxuriant growth; it is not necessary, for instinctively they detest these plants. The bush consists entirely of species which are poisonous or disagreeable to the animals, or which when disturbed, wound them-viz. of Monkshood, Good King Henry, Nettle, and Fuller's Thistle (Aconitum Napellus, Chenopodium Bonus Henricus, Urtica dioica, Cirsium spinosissimum), which are found together here, and have developed so much the more vigorously, since the other species originally existing (which were innocuous and undefended) have been long ago destroyed by the grazing animals. 
On the forest pasture of the Lower Alps often all that is to be seen covering the ground are mosses and ferns, which are offensive to the animals, along with the bitter Gentiana asclepiadea and Aposeris foetida, abounding in a malodorous milk, detested by all ruminants. In some meadows in the Central Alps the fern Allosorus crispus, and with it the Mat-grass (Nardus stricta), are so prominent that scarcely any other species of plant are to be seen there. Again, in other places, the ground is overgrown with the Bracken fern (Pteris aquilina), detested by grazing oxen, and also with prickly juniper-bushes. On the cultivated grounds near Trieste the stiff, prickle-leaved and steel-blue Eryngium (Eryngium amethystinum) impresses one by its profusion. In the Hungarian uplands one may recognize the spots where cattle are kept by the abundant occurrence of Xanthium spinosum and Eryngium campestre, of tall thistles and of Mullein, of Thorn-apples and Henbane, and of several species of spurge, which are only eaten by the animals under the greatest stress. It is thus shown by a hundred examples that in tracts exposed to the pasturage of larger animals, those plants always obtain the upper hand which are not attacked by the animals, in consequence of their poisonous and disagrceable properties, or because of their defensive spines and prickles.

A phenomenon connected with the conditions here described deserves mention. This is the regular occurrence of defenceless plants under the protection of those which are provided with abundant means of defence. Thus certain wild vetches and Umbellifers (species of Vicia, Lathyrus, Anthriscus, Myrrhis, Egopodium, Chcerophyllum, \&c.), which would furnish very good fodder for grazing mammals, are regularly seen in the prickly hedges along the roads, and under spiny bushes, which form a belt around forests. The bushes defend not only their own foliage, but also that of the delicate vetches and Umbellifers which have established themselves under their protection. In neighbourhoods where the primeval character and distribution of the vegetation is almost entirely lost, the companionship of certain plants is so general that one might be tempted to regard it as a symbiosis. Here, however, this is certainly not the case, for the advantage is all on one side-that of the plants protected; while the bush, armed with spines against the assaults of animals, under whose branches the defenceless plants have grown up, receives no thanks, no profit, and no return from them, and certainly does not afford the protection intentionally. 


\title{
METABOLISM AND TRANSPORT OF MATERIALS.
}

\section{1.-THE ORGANIC COMPOUNDS IN PLANTS.}

\author{
Carbon Compounds.-Metabolism in Living Plants.
}

\section{CARBON COMPOUNDS.}

It is naturally to be expected, from analogous relations in the inorganic world, that the variety to be observed in the vegetable kingdom as to colour, taste, and smell, should depend upon the diversity of the materials manufactured in the individual species. Numerous characteristic materials have been shown by the researches of chemists to belong to certain species, and in the names given to many of these (as in the terms oxalic acid, benzoic acid, salicin, amygdalin, asparagin, nicotin, strychnin, atropin, cocain, \&c.) we recognize the names of well-known plants. But it would be erroneous to suppose that the series of substances belonging to the vegetable kingdom would be exhausted by the sugars, acids, salts, alkaloids, oils, ethers, and pigments which are already known to us by their varied effects on our nerves of taste, smell, and sight. What is accurately known, indeed, in this respect is apparently only a fraction of what actually exists. In the meantime we cannot venture on even an approximate estimate of all the substances produced by plants. Only this much can be affirmed with certainty, that their number is far greater than that of inorganic or mineral bodies. This is the more remarkable, since the elements of which the inorganic compounds are built up are comparatively so many, whilst the elements which serve as building materials for organic compounds are so few. The fact is thus explained, that carbon, an element whose chemical nature admits of its union with other elements in inexhaustible combinations, appears as the centre of all organic compounds in plants.

For the purpose of the following discussion it is fitting, first of all, to give here a brief sketch of this important property of carbon. Chemists call carbon a tetrad element, by which is meant that each atom of carbon enters into combination with four atoms of another element, and can form a mechanically inseparable group, i.e. a molecule. It can be shown that each atom of a tetrad element possesses four centres of attraction, i.e. four connection points, to which the atoms of other elements become attached, and where they are held fast. These points are called bonds of union, and are said to be saturated when other atoms have become 
annexed or united to them, or free when this is not the case. When, for example, four atoms of hydrogen unite with one atom of carbon (represented graphically in figure 119, with its four bonds of union), its four bonds are thereby saturated, and a molecule known as marsh gas is produced. Apart from its tetravalency, carbon also has this remarkable property, that its atoms can also combine with each other, and to a much higher degree than the atoms of any other element. Carbon atoms themselves, and not the atoms of other elements, saturate the separate, free bonds of union in such instances, and in this way are produced groups of atoms, each of which behaves like a

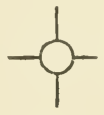

Fig. 119. chemical unit. Suppose that one of the four bonds of an atom of carbon has united with one of the four bonds of a second carbon atom; then a group of atoms like that shown in fig. 120 will be the result. Where the two carbon atoms have become connected their bonds of union are saturated; but in each atom there are still three unsatisfied bonds, and accordingly they can together annex six atoms of another element. The pair of carbon atoms may now be considered as hexavalent, and if they annex six atorns of hydrogen, a compound is produced which is called ethane. If three atoms of carbon combine together, so that one bond of each is united to a bond of the neighbouring atom, as represented graphically in fig. 121, four bonds are saturated and eight remain free. These free bonds may be satisfied with atoms of other elements, for example, again with

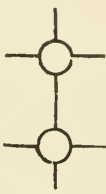

Fig. 120. hydrogen. Thus a compound arises which contains three atoms of carbon and eight of hydrogen, and which has been called propane. In like manner four, five, \&c. atoms of carbon may enter into combination together, in which case the remaining ten, twelve, \&c. bonds of union, which remain free, may be saturated with atoms of other elements. If we suppose that all the free bonds are satisfied by hydrogen, we then have a series of hydrocarbons whose successive members differ from their predecessors by the increment of one atom of carbon and two of hydrogen, but which must each be regarded as a chemical unit, i.e. as a chemical individual and as a particular substance with peculiar properties not possessed by the others.

Parallel with this series of hydrocarbons run two comparable series, whose members respectively contain two and four atoms of hydrogen less than the corresponding members of the main series;

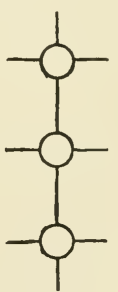

Fig. 121. and here the carbon atoms, from which the atoms of hydrogen have been removed, must have combined with one another by the bonds thus liberated.

The view that several atoms of carbon are only grouped in one direction in linear series, and that the neighbouring atoms are only mutually combined by means of one of their four bonds, as shown in the above graphic representations, is not always confirmed. In many instances we are obliged to suppose that the carbon atoms are distributed in several directions in space, and are combined into a net-work, or grouped in the form of a hexagon, perhaps in the manner illustrated 
in fig. 122. Here each of the six carbon atoms is always united to one of its neighbours by one, and to the other by two, bonds, and thus only six bonds remain free. When these are saturated by atoms of hydrogen, we have a molecule of that important compound called benzene. In all the special instances hitherto mentioned the free bonds of the carbon atoms have been satisfied by atoms of hydrogen, and these combinations have all been found actually realized in nature. It is an extremely important property of carbon, as regards the chemistry of vegetable substances, that all the free bonds of its groups of atoms, no matter how numerous these may be, can be satisfied with hydrogen. Whilst other elements can only form a very limited number of hydrogen compounds, we have a practically unlimited quantity of hydrocarbons. But this is not all. These hydrocarbons form the foundations of innumerable other compounds which are produced by the displace-

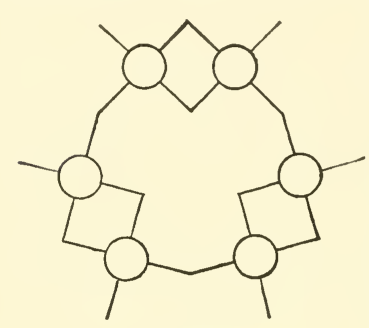

Fig. 122. ment, by atoms of other elements, of one or several atoms of hydrogen in each member of the hydrocarbon series. Many of the substances occurring in plants are hydrocarbons in which a part of the hydrogen has been displaced by oxygen; in others the hydrogen is partly replaced by nitrogen; or for the hydrogen may be substituted the so-called compound radicles (groups of atoms which play the part of an element in combination), as, for example, cyanogen, hydroxyl, \&c. If the number of compounds in which carbon is combined with nitrogen is indeed large, the number of compounds obtained from them by the partial replacement of the hydrogen by some other element, and known as derivatives of hydrocarbons, becomes almost beyond conception.

Finally, the astounding variety which one and the same compound can exhibit in its outward appearance, in form, colour, hardness, and transparency, in taste, and in smell, is due to the inexhaustible permutations in its percentage composition, which is shown by the hydrocarbons as well as by their derivatives. The same phenomenon is here repeated as is observed in pure carbon uncombined with any other element. It is known that carbon appears either ainorphous as charcoal, or crystalline as diamond, or as graphite-in the latter case, in crystals which belong to another system than those of the diamond, and differing from them in colour, hardness, and specific gravity. It is not easy to imagine a greater contrast as regards physical properties than that shown by these three substances, and yet it is beyond question, that, chemically, they are identical. The same thing happens in some of the compounds of carbon. Dextrin, starch, and cellulose all have, for example, the same percentage composition; each molecule contains six atoms of carbon, ten of hydrogen, and five of oxygen. And yet how different these bodies seem to our senses; how different is their behaviour to heat and light, to various solvents, and to other chemical compounds! We explain this remarkable phenomenon by the way in which the atoms are 
grouped, and imagine that the varied arrangement of the atoms building up a molecule finds expression in the whole mass of the substance in question. If six black, ten blue, and five red balls are placed close together in a frame, they can be grouped in the most diverse ways into beautiful symmetrical figures. They are always the same balls, they always take up the same space, and yet the effect of the figures produced by the different arrangements is wholly distinct. It may be imagined, similarly, that the appearance of the whole mass of a carbon compound becomes different in consequence of the arrangement of its atoms, and that not only the appearance, but even the physical properties undergo very striking alterations.

A glance back at the history of the development of carbon compounds, very briefly stated here, will render sufficiently clear how it becomes possible that many thousand different organic substances are compounded from carbon and a few other elements, viz., hydrogen, oxygen, and nitrogen; and how this almost infinite multiplicity of vegetable organic compounds is connected with the remarkable chemical nature of carbon. The materials of which these substances are formed are extremely simple, and the changes undergone by plant-substances depend entirely upon the insertion and rejection, on the grouping and arrangement, of the atoms of a few elements.

\section{METABOLISM IN LIVING PLANTS.}

In the living plant these combinations, decompositions, and rearrangements are accomplished with great ease, and multitudes of substances, which cannot be manufactured, either directly or indirectly, in a chemical laboratory, are produced in plant cells, with a hand's turn, so to speak. This applies principally to those organic materials already generally described in a previous section of this book, which have been formed from inorganic food, from carbonic acid and water. It is exactly these, however, which have the greatest claim upon our interest. They are of the utmost importance to everything which lives and moves on our earth; their formation is the adjustment of one of the greatest contrasts in nature, they form the bridge which connects the inorganic with the organic world, the dead with the living. As a matter of course, these primary organic substances, derived from carbonic acid and water, are the starting-points for all the other chemical compounds of which the bodies of plants and of animals are composed; or, in other words, they form the commencement of all these further chemical changes in living cells which are understood by the term Metabolism.

The process of formation of these primary organic compounds is, on the whole, easily comprehensible. It is known that carbon dioxide, i.e. carbonic acid, is absorbed by plants, and that oxygen is given out; it is also known that, when this process is carried on in a plant kept in a confined space, a volume of oxygen is given out which is equal to the amount of carbon dioxide taken 
up and consumed by the plant. In this way, without doubt, a reduction of the carbon of the carbon dioxide occurs, and hand in hand with this reduction a union of carbon with water must take place. Thus is formed some one of the compounds known as carbohydrates. The process has been interpreted in the following manner. The carbonic acid is reduced in the green cells, by the separation of oxygen, to carbon monoxide; this combines with hydrogen to form a body known by the name of formic aldehyde, and from this is produced, by the action of alkaline substances, a carbohydrate. This latter process is more easily understood, from the fact that it has been found possible to produce a sugar from the formic aldehyde (which consists of one atom of carbon, one of oxygen, and two of hydrogen) by means of lime. Thus a definite carbohydrate would be established as the first organic substance formed in a vegetable cell. It is scarcely probable, however, that this carbohydrate alone forms the startingpoint for the whole of the other organic compounds in all living plants. It is much more likely that in the large, fundamentally different series of plant-forms, in Fucaceæ, Florideæ, mosses, ferns, conifers, grasses, palms, \&c. different carbohydrates are produced as the first organic derivatives of carbon dioxide and water. It must not be forgotten that in this building process the protoplasm of the green tissue plays a very important part, that this is actually the builder, and that the structure and chemical composition of the constructor, or, in other words, the specific constitution of the protoplasm, will not be without influence on the arrangement of the atoms in the carbohydrate formed. The whole of this process has been termed assimilation, and by it is meant that the protoplasm in each plant forms materials from the inorganic food absorbed, resembling those of which the protoplasm itself is made up. Assimilating protoplasm thus continues to organize after its own type, and in this matter cannot pass beyond the bounds drawn for it by its own atomic construction. The assumption is now justified that in these formative processes assimilation takes effect from the beginning, and that protoplasm which exhibits a different constitution, and which is known to have the capacity of choosing between the mineral food-salts, will form different carbohydrates. However this may be, this much is certain, that the first organic compound arising in the green cells is a kind of sugar or some other dissolved, undemonstrable carbohydrate.

Under the influence, and by the means of living protoplasm, and in accordance with the requirements of the plant species in question, very diverse alterations and the most varied arrangements and connections, insertions and separations of the atoms are carried on in these primary carbohydrates, and as long as the plant is alive, a continuous transformation of the materials takes place. And this transformation is carried on in very many directions. First, compounds are formed indirectly or directly from the primary carbohydrates. They contribute to the extension of the protoplasm and the envelopes produced from it. Without them no increase in cells, or growth of the plant, would be possible. They may be fitly termed the building materials. 
Of the various sorts of building materials, the albumens must first be considered; they are to be reckoned as the most important constituents of living protoplasm. Although their chemical composition has not as yet been ascertained with complete certainty, it is known that, besides the carbohydrate constituents, albumens contain nitrogen and 0.8-1.7 per cent of sulphur; that carbon with

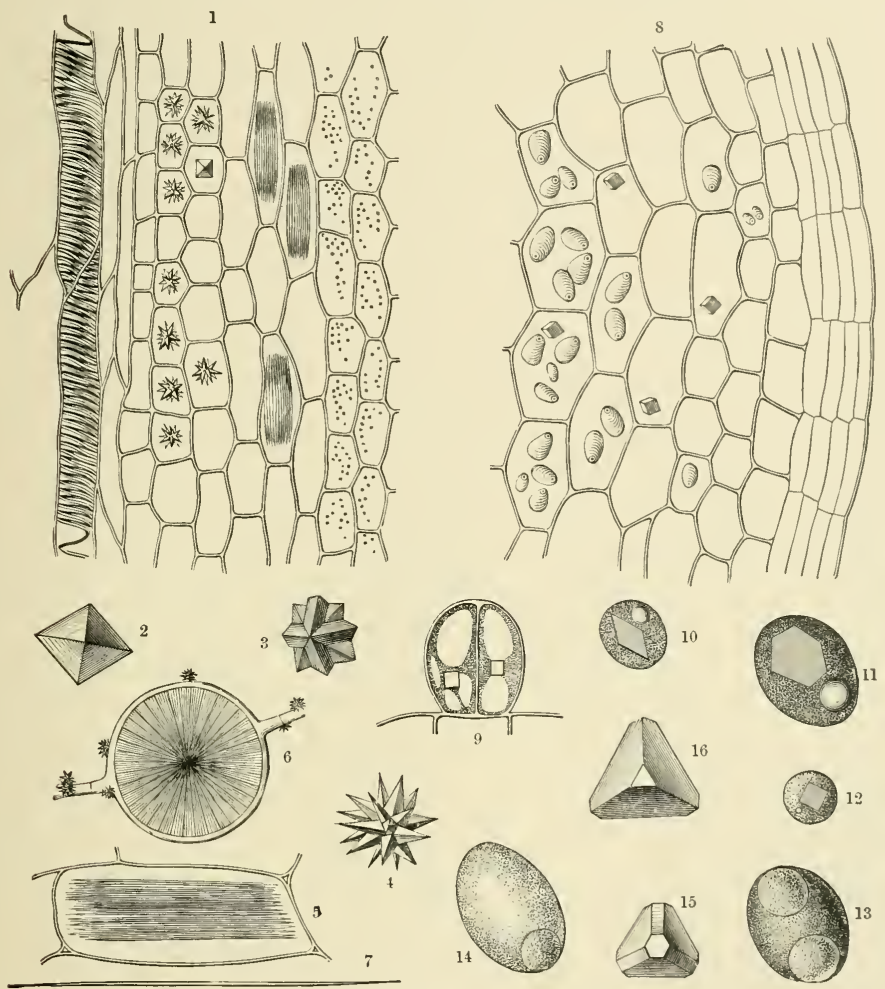

Fig. 123.-Crystals and Crystalloids.

I Vertical section through a fallen leaf of the Virginian Creeper (Ampelopsis hederacea). In some of the cells are clusteredcrystals, in others bundles of needle-shaped crystals (raphides); in one cell there is a single envelope-shaped crystal. 25 Solitary and clustered crystals and raphides of oxalate of lime; more highly magnified. 6 Sphere-crystals in the interior of a swollen bladder-like hypha, with small clustered crystals on the outer side of the hyphal thread; from Phallus caninus. 7 A single needle from a bundle of raphides. 8 Section of a portion of a Potato-tuber with crystalloids and starch-grains in its cells. 9 Crystalloids in the cells of a gland on a Potato-leaf. ${ }^{10-12}$ Crystalloids in aleurone grains. 13. 14 Globoids in aleurone grains 15, 16 Isolated crystalloids. 10-16 From the seed of Ricinus communis; very highly magnified.

many, perhaps with more than a hundred, atoms takes part in the construction of a molecule, and that consequently the molecules of albumen are very large. In order that a carbohydrate may become transformed into an albuminous body, nitrogen and sulphur, at any rate, must be drawn into the combination. Nitrogen 
is obtained from nitric acid and ammonia, and sundry of their compounds, especially calcium nitrate. These are absorbed by the plant and conveyed by the crude sap to the place of consumption. The nitric acid must, of course, be liberated from this salt, and this is brought about by the union of the calcium with oxalic acid, derived from a portion of the carbohydrates, the two thus forming insoluble crystals and crystalline masses of oxalate of lime (fig. 123). The liberated nitric acid is now reduced in a manner analogous to that of the carbonic acid in the formation of carbohydrates. It is supposed that the nitrogen of the nitric acid then combines with a hydrocarbon, forming an amide (asparagin, leucin, tyrosin), and that the albumen is formed by the union of this with a carbohydrate. Sulphur is derived from the calcium sulphate, or from some other sulphate, by the intervention of oxalic acid, in the same manner as just described for nitrogen. The oxalic acid forms an insoluble salt with the calcium or other base of the sulphate, which separates out in the cells in the form of small crystals. The liberated sulphuric acid must then, in some way, undergo a further reduction, in order that sulphur may enter into the molecule of the albumen. Among the vegetable albumens are to be distinguished albumin, casein, and fibrin. The glutin contained in corn, is a mixture of a casein and a fibrin. All these albumens appear in soluble and insoluble forms. Thus, for example, the conglutin contained in almonds is a soluble casein, and goes into solution when milk of almonds is made by adding water to the almonds; while the legumin contained in peas, beans, lentils, and other pulse seeds, is not soluble in water, and can only be dissolved by pepsin in the presence of an acid. Although all these albuminous compounds cannot be recognized by any definite form, the aleurone grains and the so-called crystalloids have perfectly definite shapes. The crystalloids are formed of albuminous substances, and have exactly the appearance of crystals (fig. $123^{8-12}$ ).

Next to the albuminous substances, the most important building material to be noticed is cellulose. This is a carbohydrate consisting of six atoms of carbon, ten of hydrogen, and five of oxygen, and is produced from the primary sugar-like carbohydrates. The transformation is effected by the living protoplasts, which form a layer of cellulose on their periphery, called the cell-wall. At first this cell-wall is mainly composed of pure cellulose; then, according to need, the carbohydrate is changed by the protoplasm, either wholly or partially, into some other carbohydrate, either into woody material (lignin) or cork (suberin), or the cellulose becomes mucilaginous, as, for example, in the seed-coat of the Quince. In the stems and branches of cherry, plum, almond, apricot, and peach trees, the cellulose is generally hardened into a sticky, shapeless, amber-coloured substance, which exudes from the fissures of the bark, and is known by the name of cherrygum (cerasin). In like manner gum-arabic (arabin) is formed from the cellulose in the stems of some acacias, and gum-tragacanth in several tragacanth shrubs (species of Astragalus).

Protoplasm forms cellulose from a portion of the primary sugar-like carbo- 
hydrate at certain points in the interior of its substance as well as at its periphery, in addition to another carbohydrate, the so-called granulose. Cellulose and granulose, very intimately intermixed, appear in the form of grains, and the mixture is called starch or amylum. Starch-grains are among the commonest of cell-contents. They appear regularly in chlorophyll-bodies and are conveyed from the places where they are first formed to all parts of the plant. This of course is only effected by
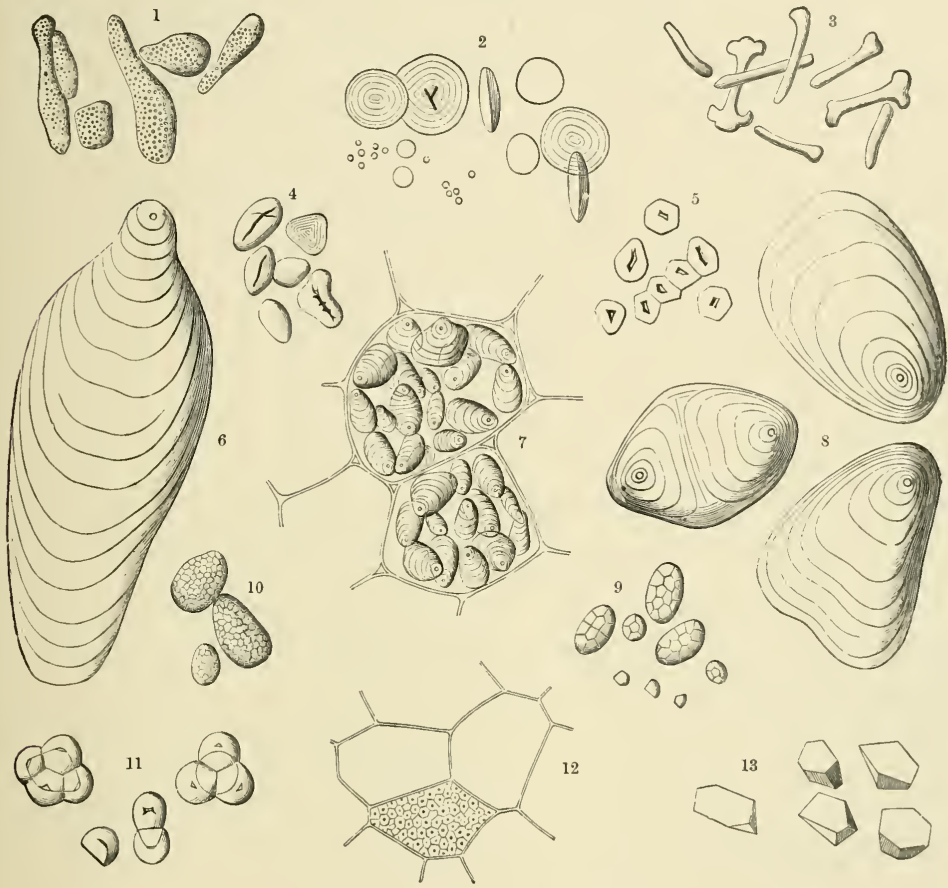

Fig. 124. - Various Forms of Starch-grains.

1 From the seeds of the Corn-cockle (Agrostemma Githago), 2 From a grain of Wheat. 8 From Spurge. 4 From a Bean seed. 5 From a grain of Mlaize. 6 From the root-stock of Canna. 7 From a Potato-tuber (inclosed in cells). ${ }^{8}$ From a Potato. tuber (isolated and very highly magnifled). 9 From a grain of Oats. 10 From the seed of Lolium temulentum. ${ }^{11}$ From the corm of the Meadow Saffron (Colchicum autumnale). 12 From a grain of Rice. ${ }^{13}$ From a grain of Millet. All highly magnifled.

the solid starch bodies being made fluid, as often as they pass from one cell to. another, by the help of an accessory substance, called diastase, which has yet to be described. In many tissues the starch-grains become so accumulated that the cells appear to be crammed with them (see fig. $124^{7}$ ). Starch is one of the most important of reserve materials, i.e. of those materials which are not consumed immediately after their formation, but are put away for a time in store-rooms or reservoirs, and then consumed as required in the places needing them. For example, they may 
remain in seeds unaltered for years, and as if dead; but if the seed germinates, and the seedling begins to develop, the starch is dissolved, that is to say, becomes changed into another carbohydrate, and finally is made use of in the construction of the cellwalls of the growing seedling by a fresh transformation. Starch-grains in various species of plants differ very much in size as well as in shape. The largest grains exhibit under the microscope alternating blue and red zones, which are accounted for by the difference in the amount of water contained in the several zones. The bluish zones contain less, and the red-tinted more water. Many starch-grains exhibit a "nucleus" or hilum which is rich in water, and which is situated excentrically in the grains of the Potato and of Canna (fig. $124^{6}$ ); centrally in those of the Wheat. A space may be present instead of the hilum, as in the starch-grains of beans and other pulses (fig. $124^{4}$ ), in consequence of the drying up of the substance of the hilum. In most plants the starch-grains have a rounded form; but those of the Corn-cockle (Agrostemma Githago) are fusiform and club-shaped (fig. $124^{1}$ ). Those of species of Euphorbia resemble tiny bones (fig. $124^{3}$ ), and others again are angular and cornered like crystalline figures (figs. $124^{5}$ and $124^{13}$ ). This last form is seen especially when the cells which serve as store-houses are densely crowded with starch-grains so that growth becomes checked, and a mutual flattening takes place. In the Oat and Rice many small angular starch-granules are cemented together to form ellipsoidal grains (figs. $124^{9}$ and $124^{10}$ ), and in the starch from the corm of the Meadow Saffron, regular groups of four rounded grains, each exhibiting a hollow hilum, are found (fig. $124^{11}$ ). Granulose forms the chief of the two carbohydrates which are intimately mixed to form starch. It is soluble in saliva, and is therefore extracted by it, while the cellulose remains behind insoluble, a fact which is of great importance with regard to the digestibility of the starch present in such abundance in flour and bread.

In close connection with these essential building materials are other substances which, though not themselves serving as building materials, take an active part in their production. These furnish the conditions under which the manufacture and transport of the building substances, and the growth and propagation of the plants can occur. They avert injurious influences, regulate light and heat, and are of use to the plant in a hundred minor directions.

To these substances, which may be termed accessory, belong, first of all, the pigments chlorophyll, phycoërythrin, and anthocyanin, which are so important on account of their relations to light and heat, and whose rôle has already been alluded to. Then we have those compounds whose function is to allure animals to the plants in order to bring about fertilization or the distribution of the seeds and spores, or whose significance lies in the fact that they frighten and ward off animals which might be injurious to the plants. In this connection are of course to be mentioned colouring-matters which are formed in flowers and fruits in order that these may be rendered visible at a distance to those animals whose visits benefit the plant: first of all, anthocyanin, which in the presence of acids is red, but otherwise appears violet or blue; and then anthoxanthin, to which most yellow flowers and 
fruits owe their colour. On the other hand must here be mentioned that scarlet-red colouring-matter, as yet little known, probably belonging to the anthracenes and allied to the madder-red, which perhaps serves to frighten animals, and which is so pronounced, for example, in the accrescent calyx surrounding the fruit of the Winter Cherry (Physalis Alkekengi).

Besides the colouring-matters, sweet-tasting substances, especially cane-sugar, and also mannite and dulcite, play an important rôle of a similar nature. Although their function can only be discussed in detail later on, it is nevertheless well to point out here that the distribution of the spores of Ergot of Rye (Claviceps purpurea), for example, is brought about by means of a sweet fluid excreted by the mycelium, which is eagerly sought for by ants and other insects. The insects in sucking and licking up this fluid carry off the spores of the Ergot, and then deposit them on other plants. Countless plants secrete sweet honey in certain parts of their flowers, which serves to attract bees, humble-bees, and butterflies, whose task is to carry the pollen from flower to flower. Certain animals, on the other hand, whose visits would be injurious to the flowers, are kept away, or still better, diverted from them by the honey secreted at the base of the foliage-leaves, which serves as a counter-attraction.

The numerous ethereal oils, resins, and balsams have a like significance for the life of the plant. The ethereal oils are principally hydrocarbons, only a few containing oxygen in addition; oils of lavender, cumin, and eucalyptus, oil of turpentine and camphor, and many others consist of ten atoms of carbon and sixteen of hydrogen. In spite of this similar percentage they differ very markedly in their optical properties, their boiling point, and particularly in their smell, as can indeed be observed from the few examples cited. There are some plants whose foliage, flowers, and fruit contain ethereal oils having different odours, as, for example, the Orange-tree, whose leaves yield "petit grain", the flowers neroli, and the fruit oil of orange. But since these three oils contain the same number of carbon and hydrogen atoms, it must be assumed that their difference depends upon the varying arrangement of the atoms in a molecule. The majority of these oils are transformed into resin by the addition of oxygen, or mixtures of volatile oils and resins are produced, which are called balsams. Volatile ethereal oils, which are perceived by the olfactory nerves even at a distance, function in part as means for alluring animals which benefit the plants in question by transferring the pollen or disseminating the fruits, seeds, or spores; but they also function in part as measures for protecting the plant against attacks from the animal kingdom. The latter is the case especially in foliage-leaves with powerful odours, and in resinous fruits, and these are not used by animals as food. Balsams, which cover foliage-leaves issuing from the buds like a varnish, form a protection against excessive transpiration, and also render material help in the absorption of water by the leaves, as has been already described. The viscous excretions, formed of a mixture of resin and mucilage on the stems and leaf-stalks, which are formed so abundantly in caryophyllaceous plants, keep off animals which try to climb up the stem to reach 
the flowers in order to obtain the honey, but which would not be welcome guests to the plant.

Fats take a part in the life of plants similar to that played by ethereal oils. Fats are combinations of fatty acids with glycerine, and may be divided into two groups; in one group the members dry up when exposed to the air by the separation of carbonic acid, as, for example, in poppy oil and linseed oil, which are used for this very reason in oil painting. In the other group, e.g. in almond and olive oils, the members remain fluid when exposed to the air, and give rise to malodorous fatty acids, and when this change occurs the body is said to become rancid. The most abundant production of fats takes place in fruits, seeds, and spores, where they are stored up as reserve materials, but in many instances they also function as attractive or protective agents. Nor must we forget the erystalline or amorphous fatty excretions formed on the epidermis of foliage-leaves, stems, and fruits, which are popularly known as "bloom". These are very like wax, and have a very manifold significance; they prevent hurtf -1 moistening by water, regulate transpiration under certain circumstances, and can also ward off the disadvantageous attacks of animals. The branches of many willows which bear honey-laden flower-catkins, as, for example, those of Salix pruinosa and daphnoides, are provided with these waxlike, extremely smooth and slippery coverings up which the unwelcome wingless ants, scenting the honey in the catkins, in vain try to climb.

Alkaloids and glucosides are developed principally as means for protecting the green tissues of the leaves and fruit, and the underground portions of the plants - the roots, rhizomes, tubers, and bulbs-against demolition and extinction by animals. The alkaloids are distinguished by the presence of nitrogen in them. Some of them contain no oxygen, and are volatile, as, for example, trimethylamine, which occurs in the leaves of many oraches, and in the flowers of Hawthorn and Pear, as well as in the American Pachysandra. Most, however, are non-volatile, and contain oxygen. To this latter class belong the well-known alkaloids-morphine, nicotine, atropine, and strychnine, which are poisonous to man and most mammals; also the well-known drugs-quinine, cocain, and many others. Leaves provided with these materials are rejected as food by grazing animals, and consequently they at least may be regarded as efficient in protecting the plants from being devoured. Only the volatile trimethylamine in flowers can serve to attract insects. Glucosides, of which more than a hundred are already known, have a use very similar to that of the alkaloids. Saponin is poisonous to man and mammals; amygdalin splits up into the poisonous prussic acid, oil of bitter almonds and sugar; and many others behave in exactly the same way. Tannin has an extremely bitter taste, and therefore protects branches, cortex, and fruits from being eaten. It is interesting to see, that in many fruits which are distributed by means of animals, the pericarp remains acid and unwholesome in consequence of bitter or poisonous glucosides, until the seeds hidden within have matured. As soon as they can germinate, the glucosides become changed; they are split up by means of ferments, which will be described later, 
or by the acids which are present in such abundance, into sugar and various other barmless materials, and the pericarp, which, until now had been sharp, acid, and unwholesome, becomes sweet and luscious. The same coat which formerly served as a protection, now forms an attraction. The ripe fruits, with the seeds they inclose, are now sought for and eaten as food, especially by birds; the sweet covering is digested, while the seeds, excellently protected against the action of digestive juices, are excreted with the waste materials of the food, and germinate in the places where they are deposited; thus the widest dissemination of the plants is brought about. All this will be discussed in detail later when distributing agents of plants are being considered; but it seems appropriate to mention these processes here in order to point out that the metabolism of materials in plants keeps pace with the requirements for the time being; that even when the division of labour in the plants is as much developed as in the cases just mentioned, the arrangements and displacements of the atoms, and the decomposition and formation of chemical compounds, are always carried on in the right place and at the right time, i.e. always where and when the plant is benefited thereby; and that frequently the reasons for all these changes only become intelligible when we consider the inter-relations of animals and plants.

The significance of salts of sulphuric and nitric acids, as well as the relations of these to oxalic acid, have already been discussed and explained - how by means of the latter the sulphuric and nitric acids are liberated, yielding sulphur and nitrogen for the construction of albuminous substances. If oxalic acid accordingly does not appear to be a necessary plastic constituent of the framework of the plant, it is nevertheless quite indispensable as an accessory to metabolism. The same thing applies to the other organic acids which exist in plants. They are only accessories in the transformations, or intermediate steps between the final stages of the compounds formed in the plants, viz. between the first carbohydrate on the one hand, and the completed substance used for building or further purposes on the other. Under these conditions, it is intelligible that the organic acids should be distributed through all parts of the plant, and that the juices in living plants almost universally have an acid reaction. It is also intelligible that the number of organic salts should be extremely large. Oxalic, tartaric, citric, and malic acids may be cited as examples, but more than two hundred such acids are known which have been detected in various plants. Many of them are also found in animal bodies, viz. isolated members of the series of the so-called fatty acids, which form fats when combined with glycerine, as, for example, butyric and formic acids, the latter, as already stated, being also contained in the stinging hairs of nettles. It has, moreover, been already pointed out that glucosides are decomposed by organic acids, and give rise to various kinds of sugar. It is interesting with respect to these sugars that they arise as the first organic products (which result from the assimilation of carbonic acid), and also again as the terminal members of a very long chain of transformations and decompositions of glucosides effected by the action of organic acids. An important 
rôle may be assigned to the organic acids of the type of oxalic and formic acids with regard to the turgescence of cells in living plants, since they suck up water with great energy to replace that lost by evaporation, and are thus able to maintain the turgidity.

An especial function is also assigned to the so-called amides, by which are understood asparagin, tyrosin, leucin, glutamin, \&c. These are produced by the splitting up of albumens, but at the same time they promote the reconstruction of these substances in the living protoplasm. When the carbohydrate, which is derived from the albumen, together with the amide, is used up, the amide again draws to itself a fresh carbohydrate (which has just been formed in the green cells), enters into combination with it, and in this way again forms an albumen. This process may be repeated indefinitely, and will be referred to in the discussion on respiration. Moreover, when albumens, which, in their usual condition, cannot pass through the cell-wall, are to be transmitted from one place to another, they are probably first transformed into asparagin, or a similar amide, which again becomes a complete albuminous compound by the union with a carbohydrate in the place where the albumens are to remain.

Finally, the group of enzymes or ferments comes under the head of accessories. These substances, so extremely important to the life of plants, have the remarkable property of being able to decompose other substances without themselves being split up, and in consequence a very small quantity of them can produce very marked results. They all contain nitrogen, and are widely distributed in plants, but since they are only formed in minute quantities in the places where they are required, their presence is not always easy to demonstrate. How they arise is still a problem; perhaps in the same way as the nitrogenous albumens. They are to be found wherever solid bodies are to be liquefied or dissolved; for example, when the stores of organic food, i.e. the so-called reserve materials, which have remained resting for a long time in the seeds, tubers, and roots, and have been, so to speak, put out of the way, are to be liquefied and again brought into action; further, when substances which cannot pass through the cell-walls are to be brought into a condition suited to this translation, in which case they act like the amides previously described; further still, as often as organic compounds are to be absorbed as food, insects and other animals to be digested by insectivorous plants, the dead bodies of plants to be broken up by saprophytes, or the organized portions of living plants to be consumed by parasites. When the sucking cells of the parasitic plants wish to obtain the juices of the host-plant; when the hyphæ issuing from the spores make their way through the epidermis into the interior of the plant on which they have fallen; or hyphal threads in the interior of a many-celled tissue wish to pass. from one chamber into another, they must dissolve the cell-walls, thus creating an open passage for themselves. Enzymes also appear to come into action wherever those remarkable processes are carried on which are known as fermentations, and which will be considered in the following pages. It is to be supposed that they 
form a constituent of the protoplasm of the fermentative organism, and themselves affect and decompose their environment through the cell-wall.

The most important enzymes are, first, pepsin, which peptonizes albumens in the presence of weak acids, i.e. changing them into a soluble condition, whereby they are enabled to pass through the partition-walls from one cell-chamber to another. The pepsin contained in plants does not, indeed, differ from that in the gastric juice of animals, so that the part performed in both cases is essentially the same. In t'ie stomach of animals it has to perform the important task of bringing the albumens taken in as food into a soluble form, so that they can then enter the blood. The presence of pepsin in insectivorous plants has already been alluded to. Another enzyme to be mentioned is diastase, which makes starch grains soluble, since it decomposes them into sugar and dextrin. It is found wherever starch-grains have been stored up when they are again to be utilized and to be assimilated. Emulsin and myrosin should also be pointed out. They decompose glucosides in the manner already described, and thereby give rise to sweet sugar, especially in fruits; but they can also effect various other decompositions, as, for example, the splitting up of the amygdalin contained in almonds into glucose, prussic acid, and oil of bitter almonds, which is effected by emulsin. Papain, occurring in the fruits of Carica Papaya, and invertin, which has been observed in Yeast, are to be regarded as enzymes. All substances which have a decomposing action on their environment, without at the same time undergoing any chemical change themselves, are called ferments, and, so far, all enzymes are to be considered ferments. It has been demonstrated, however, that under certain conditions, acids-for example, phosphoric acid-and even water at a high temperature, exhibit a ferment action, and for this reason the name enzyme has been chosen for the nitrogenous compounds detailed.

We have now enumerated the most important of those substances whose building up and breaking down, whose transformations and interactions constitute what we recognize as the life of plants.

\section{TRANSPORT OF SUBSTANCES IN LIVING PLANTS.}

Mechanisms for Conveyance to and fro.-Significance of Anthocyanin in the Transportations and Transformations of Materials.-Autumn Colouring of Foliage.

\section{MECHANISMS FOR CONVEYANCE TO AND FRO.}

It has already been explained that the decomposition of carbonic acid, and the formation of organic matter out of the absorbed gaseous and liquid inorganic food, can only occur in cells which contain chlorophyll-bodies. The shape and arrangement of the chlorophyll-corpuscles in individual cells, and further, the form and arrangement of these green cells themselves, have also been there vol. I. 
described. It has, moreover, been stated that numerous plants exist which consist only of single green cells; that others, which are multicellular, exhibit in all their cells the same shape and grouping of the chlorophyll-corpuscles; and finally, that in most seed plants, a division of labour has taken place in each plant, so that certain cells only are provided with chlorophyll, while others are always destitute of it. Many parasites are quite free from chlorophyll, and consequently are unable to decompose carbonic acid and to manufacture organic materials. They are obliged to suck these up from their hosts. Closely connected with these are cases of symbiosis, in which plants possessing, and plants devoid of, chlorophyll enter into partnership, and in which the latter receive in exchange certain freshly-manufactured organic substances from the former. The conclusion of this long series is formed by the saprophytes, devoid of chlorophyll, which derive their organic materials not from living green plants, but from dead animal or vegetable bodies, and from the lifeless organic substances arising out of plants or animals. In the green unicellular plants, as, for example, in the Desmidieæ, two species of which are illustrated in fig. $25 \mathrm{~A}, i, k$, all the various combinations, arrangements, and separations of the atoms which lead to the formation of sugar, starch, cellulose, chlorophyll, albumen, \&c. are accomplished within a single cell; and these minute plants furnish evidence that the manifold changes of the materials connected with growth and construction can occur side by side at the same time and in limited compass. In order to be able to demonstrate this, it must be assumed that each tiny protoplasmic mass, which forms the living body of the single cells, is made up of various portions to which are assigned different functions. One breaks up carbon and forms carbohydrates; another takes up these carbohydrates and forms albumen from them; and yet another transforms the sugar into cellulose and builds up the cell-wall.

With this assumption, however, is necessarily connected the further assumption of a transportation of materials. In unicellular Desmidieæ, the path which the sugar produced in the central chlorophyll-bodies has to travel in order to reach the periphery of the cells, is perhaps only two or three thousandths of a millimetre long; it is, however, a measurable distance, and therefore there is such a thing as conveyance and removal of sugar in cells of Desmidier. The transportation is without doubt again carried on by certain portions of the protoplasm, and perhaps the manifold strands which are observed in the substance of the protoplasm are associated with this. In multicellular plants the road which the materials have to follow, in order to reach their destinations, though frequently limited to the space of a single tiny cell, is often represented by a long row of cells. This is especially the case when certain functions are assigned to the different cells of a plant, as happens in many spore-bearing plants, but still oftener in seed-plants. The materials formed in the green leaves of a moss, if they are to be employed in the construction of the spore-capsule and in the production of spores, must be transported from cell to cell, to the archegonium situated on the moss stem-a road which varies according to the species from some millimetres 
to several centimetres. The materials which serve to promote the growth of the branches of an Aspen are manufactured in the long-stalked, green leaf-blades of this plant. That they may reach the growing branch, they must pass down the long leaf-stalk and travel along a road many thousand times exceeding the length of those cells in which they were formed. Let us glance at a palm, whose few large leaves, forming a plume, sway about at the summit of a slender stem. In order to reach the growing roots, the constructive materials formed in the green leaves have to travel over a road 20 or 30 metres long. The distance is still greater over which the sap prepared in the foliage of tropical vines is conveyed in order to reach the roots, where it serves as food to parasitic rafflesias growing thereon. It is naturally to be expected that in such instances the routes followed by the travelling materials, and also the starting and end stations, should exhibit characteristic features. What has been ascertained on this point may be here briefly set forth.

The green tissue which is developed in by far the larger number of cases in the cortex of green stem-structures, in foliage-leaves, young fruits, \&c., more rarely in floral leaves and roots, must be regarded as the first or departure station. In the green multicellular thallophytes and in mosses, the chlorophyll-containing cells also form the channels of removal for the materials which have been formed in the cells, and these are always extended lengthwise in accordance with the direction of the stream. In the leaves of mosses very frequently cell-rows and cell-bands arise which converge towards the base of the leaf, and in the vicinity of these points the cells are most elongated according to the direction of the current. The conducting cells in the stem are also much elongated in the direction of the current. But here no definite line can be drawn between the forms of the cells at the departure station, in the channel, and at the termination of the current.

It is different in those plants whose leaves and stem are traversed by vascular bundles. These cells devoid of chlorophyll, and peculiar tubes belonging to the bundles, take up the materials proceeding from the green tissues to conduct them to the places of consumption. Division of labour has been so far carried out in all these cases that a portion of the cells undertakes the decomposition of carbonic acid and the formation of the first organic compounds, and another the conveying away of these first products; but obviously this does not preclude the possibility that manifold changes may still take place during the transit. In such a division of labour it is important that the organic compounds which have been formed in the superficial green cells, under the influence of light, should be removed as quickly as possible from the places where they are produced, so that the important process of the decomposition of carbonic acid should suffer no kind of interruption. It is on account of this rapid removal by the shortest path that the green cells are elongated in the direction in which they transport their products, and that the neighbouring green cells are separated as much as possible from one another. However they may be arranged in other respects, the indicated direction and isolation are always observed by them under all circumstances. 
The isolation is brought about by the elongated cells, which lie parallel side by side, assuming a cylindrical form in consequence of which they merely touch one another, leaving large air-spaces between. An exchange of materials between these cylindrical cells, i.e. a passage of materials transversely across their elongated sides is wholly prevented, and the transport of the materials is effected only in that direction in which the cylindrical cell in question is elongated. The organs which convey the materials away from the green cells lie within the strands which form the veining of the leaf, which traverse the leaf-stalk and stem as thick bundles, and when densely aggregated, form the chief part of the trunks of woody plants. But it would be erroneous to suppose that these strands (i.e. the vascular bundles) are composed exclusively of structures for conveying away plastic materials. Adjoining these, and connected with them, are regularly found woody cells, tubes, and other vessels, which conduct the mineral food-substances absorbed by the roots, and the water in which these are dissolved, upwards to the transpiring tissues. Finally, elastic thread-like bast-cells are always added to these structures, which serve for the two kinds of transport, by which means the whole is given the necessary firmness and elasticity. In these strands, therefore, which are called vascular bundles, the most varied structures with widely-differing functions are found crowded together in a small space, and it happens that the cells and vessels which serve as the passage for the current of organic materials formed in the green tissues, only occupy a very moderate share of the space.

Four kinds of mechanisms for carrying on the work of removal have been discovered. First of all, there are groups of parenchymatous cells which adjoin the other elements of the vascular bundle, especially the water-conducting woody cells and vessels which they usually surround, forming an actual mantle round them, termed the vascular bundle sheath. These vascular bundle sheaths are particularly well developed in the foliage-leaves, and form there an important constituent of the leaf-ribs and veins traversing the green tissue (see fig. $126^{2}$ ). In the finest and most delicate veinlets, representing the ultimate terminations of the vascular bundles, the few water-conducting cells, stiffened by spiral thickenings, are surrounded by parenchymatous cells. These are continued on beyond the vascular bundle, and frequently the finest veinlets are formed to such a large extent of these parenchymatous cells that they have been distinguished as a particular form of tissue by the name of nerve-parenchyma.

Next to the vascular bundle sheaths, medullary rays are to be regarded as organs for conveying the formed materials from the green leaves. These consist also of parenchymatous cells with lignified walls which are elongated at right angles to the axis of the stem-structure to which they belong. They form layers of tissue which are situated between the vascular bundles, and connect the pith in the centre of the stem with the cortex. Besides these medullary rays, which are known as primary, quite similar layers are formed of parenchymatous cells in the body of the vascular bundles, which, however, are in no way connected with the pith in the rentre of the stem, and which are known as secondary medullary rays. On cutting 
across the trunk of a fir or of a leafy tree, it is seen that in most cases the vascular bundles in the cross section are so arranged that they form together a ring round the central pith. This ring appears interrupted by the tissues just described, which radiate out from the medulla; and thus is explained their name, medullary rays.

Soft bast is to be considered as a third form of conducting mechanism for the organic compounds formed in the green cells. It consists partly of thin-walled parenchymatous cells, and frequently also of long, narrow cells tapering at the ends (cambiform cells), which are elongated in the direction of the bundle or strand to

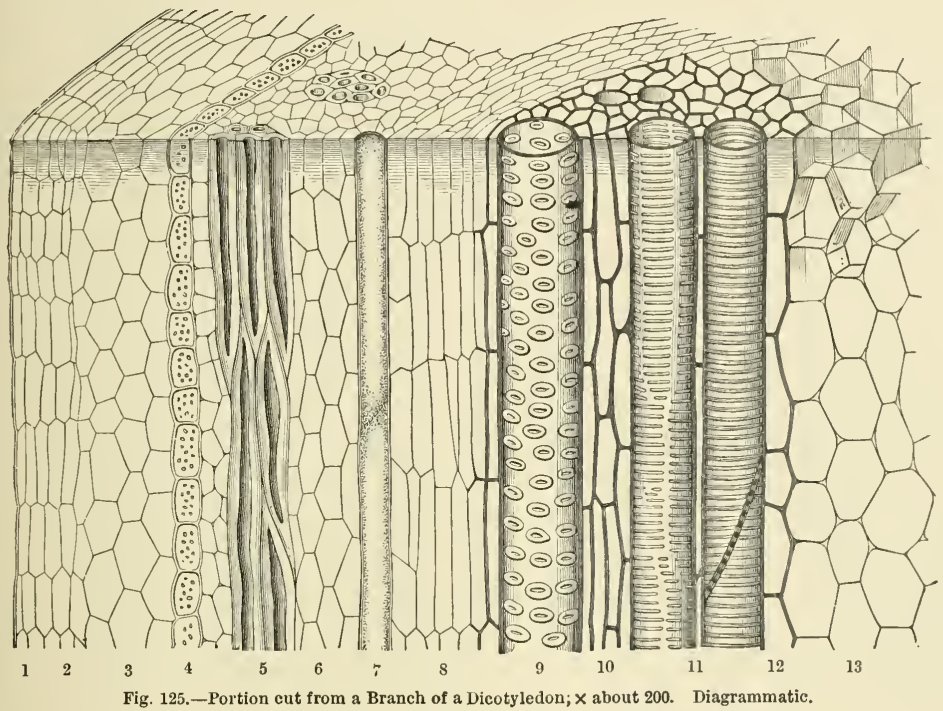

I Superficial coat (Epidermis). 2 Cork (Periderm). 3 Cortical parenchyma. 4 Vaseular bundle sheath. 5 Hard bast. 6 Bast parenchyma. 7 Sieve-tubes. 8 Cambium. ? Pitted vessel. 10 Wood-parenchyma. 11 Scalariform vessels. 12 Medullary sheath. 13 Medulla or pith.

which they belong, and form a tissue called the bast parenchyma (see fig. $125^{6}$ ). The other part of it consists of tubes which contain walls separated by comparatively large intervals, often measuring $2 \mathrm{~mm}$., usually placed horizontally, but often obliquely, which give the tubes a jointed appearance. Sharply-defined perforated areas appear on the interpolated horizontal walls as well as on the lateral walls of the tubes, they have a sieve-like aspect, and are called sieve plates, the tubes themselves being called sieve-tubes, bast-tubes, or bast-vessels (fig. 125 ${ }^{7}$ ). The soft bast but rarely forms isolated strands, as, for example, in some Melastomaceæ; as a rule, strands of firm, elastic, string-like, hard bast cells adjoin it, but these have nothing to do with the transportation of materials, and have merely a mechanical significance (see fig. $125^{5}$ ).

This fibrous or hard bast, together with the soft bast, forms in very many plants 
one-half of the vascular bundles, the so-called bast portion, while the other half, the so-called woody portion, consists of woody cells intermingled with lignified tubes, and other water-conducting elements (see figs. $125^{9,10,11}$ ).

Laticiferous tubes form a fourth mechanism for conducting away the products of the green cells (fig. $126^{1}$ ). These are thin-walled, much branched, frequently anastomizing, tubular structures which seem to penetrate all the parts of the plant, leaves, stem, and roots, without much regularity.

They may be divided, according to their development, into laticiferous vessels and laticiferous cells. The former are produced from rows of cells, whose partitionwalls have become obliterated, so that the rows of cells have become converted into tubes; the latter arise from isolated cells, at first very small, but which elongate enormously, become much branched, and whose branches penetrate between the cells of other tissues just as the hyphæ of parasitic fungi grow through the tissues of their host-plants. Laticiferous tubes are not to be found in all plants. They are particularly abundant in species of Spurge, some thousand species of Compositæ, for example, in the Salsify, Lettuce, and Dandelion; in the Oleander, many Asclepiadeæ, Papaveraceæ, and Artocarpeæ. In the gigantic trunks of tropical Figtrees, the latex often wells up in large quantities from rifts in the bark which have arisen spontaneously, and thickens into long strings and ropes of india-rubber hanging down like a mantle.

The Cow Tree of Venezuela (Galactodendron utile) is especially worth noticing herc; when pierced, a quantity of sweet, delicious milk pours out from it, also Collophora utilis of the Amazon, from which is obtained a viscous latex, used as a medium for colouring matters; finally the poppy (Papaver somniferum), whose dried latex is known as opium. In the majority of cases the latex is white, but in Papaveraceæ other colours are also to be found; thus the Celandine (Chelidonium majus) contains an orange, and the Bloodwort (Sanguinaria Canadensis) a bloodred latex. The milky Agarics (Lactarius) contain partly white, partly sulphuryellow, partly orange, and vermilion latex.

In the foliage-leaves the laticiferous tubes run with the vascular bundles, and occasionally replace the bundle sheath; at least, the bundle sheath is defective, and only very incompletely formed where the laticiferous tubes adjoin the vascular bundle. It has also been observed that in the stems of the Asclepiadeæ, where the laticiferous tubes are abundantly developed, the sieve-tubes are much reduced, and it is therefore supposed that the various mechanisms for conducting away materials are sometimes able to mutually replace one another. It must, moreover, be expressly noted here, that the laticiferous tubes do not serve exclusively to carry away the materials manufactured in the green cells; they are used, under certain conditions, and at certain times, as receptacles for reserve materials, exactly as the medullary rays, sieve-tubes, and bundle sheaths which in the winter, when the decomposition of carbonic acid, and the formation of carbohydrates in the green cells have ceased, and when generally there is nothing to remove, function as reservoirs, in which stores are deposited until the following spring. The parenchy- 
matous cells of the vascular bundle sheaths which, in summer, had been used for conducting away materials, are then crowded with starch-granules, the pores of the sieve-plates are closed up during the winter; the sieve-tubes, laticifcrous tubes, bundle sheaths, and medullary rays do not again commence their activity until the next period of vegetation, when everything becomes liquefied, and the green cells again form fresh carbohydrates. These structures then serve again, of course, chiefly as conducting organs.

With regard to the junction of the conducting organs with the green cells, we have a very great variety, but the many different contrivances may be grouped into
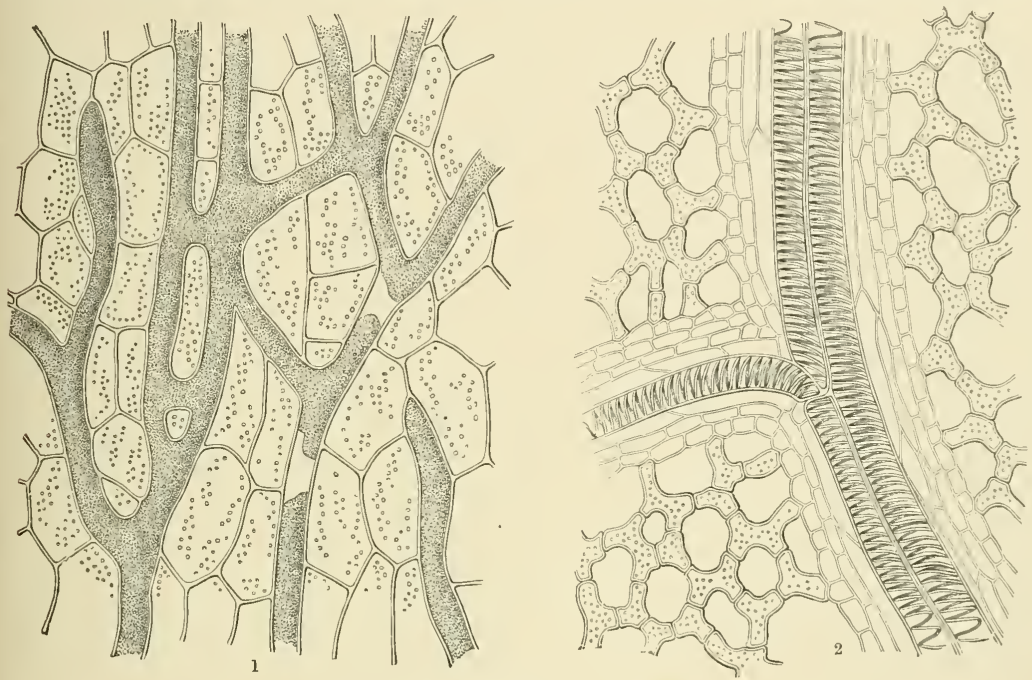

Fig. 126.-Organs for Removal of Substances.

${ }^{1}$ Laticiferous tubes from the leaf of Lactuca virosa; $\times 250$. 2 Vessels with spirally thickened walls, surrounded by the bundle sheath, from a leaf of Ricinus communis; $\times 210$.

two chief forms, viz. where the junction is direct, and where it is effected by means of specially interpolated cells.

In the first group, the switch shrubs are first to be noted, in which the foliage is entirely or almost entirely absent, and where the main portion of the green tissue is developed in the cortex of the rod-shaped branches, as, for example, in Cytisus radiatus and in the Broom (see figs. $69^{3}, 69^{4}, 81^{1}$, and $81^{2}$ ). Here the ring of vascular bundles forming the framework of the whole branch is surrounded by a common bundle sheath, and the cells of the green tissue in the cortex are connected on one side with the epidermis, and on the other with this bundle sheath, to which the organic materials produced are given up directly. In the foliage-leaves of many liliaceous plants, especially in the equitant leaves of irises, the green cells are elongated transversely, forming a kind of bridge stretched between the vascular 
bundles, which run almost parallel from the base to the apex of the leaf. Each of the bridge-like green cells is connected at either end with a vascular bundle, and delivers the materials produced to the conducting portions of these vascular bundles on both sides, i.e. to the vascular bundle sheaths. In other liliaceous plants, especially in the leaves and green stems of species of onion, the green cells are palisade-shaped, and their longer diameter is perpendicular to the surface of the part to which they belong. Here we have only a one-sided connection with the conducting cells of the vascular bundle, but the junction is again a direct one. Finally, the peculiar connection of laticiferous tubes with the palisade-cells in the leaves of species of spurge must be considered.

Although the laticiferous tubes appear to be very much branched wherever they occur in plants, the formation of branching tubes is nowhere else so abundant as in the vicinity of the palisade-cells. Many of the twigs directly adjoin these cells. It also happens that single terminations of the laticiferous tubes impinge upon the lower ends of several palisade-cells, which are inclined towards one another (fig. $25 \mathrm{~A}, s$ ), and that single laticiferous ramules push their way in between these cells. In all these examples the materials manufactured in the green tissue are taken up without further intervention by the ultimate terminations of the conducting laticiferous tubes.

Of the second group, which is characterized by the fact that the junction is brought about by specially intercalated cells, the first instance to be considered is that often observed in the leaves of grass-like plants, where the intermediate cells, which are also called conducting cells, are more or less extended transversely, and unbranched. The green cells lying under the epidermis are palisade-shaped, and at right angles to the leaf-surface; the longer diameters of the cells lying below these, which are much poorer in chlorophyll-corpuscles, are, on the other hand, placed obliquely to the leaf-surface, or even parallel to it, and apparently are directed towards the large cells of the bundle sheaths in the middle of the leaf. These cells, poor in chlorophyll, therefore connect the palisade-cells with the conducting cells of the bundle sheath, and serve as agents in the removal of the substances. But the commonest cases are those in which the conducting cells ire much branched. The foliage-leaves which possess these branched cells are differently constructed on the upper and under sides of the leaf. Under the epidermis of the upper side is seen the palisade-tissue, consisting of cylindrical or prismatic cells, whose long axis is directed perpendicularly to the plane of the leaf (see fig. $62^{1}$ and fig. $25 \mathrm{~A}, r$ ). Below these palisade-cells come the branched cells, which are connected with one another by their arm-like processes, forming a large-meshed tissue, the frequently-mentioned spongy parenchyma, interrupted by wide interstices. The spongy parenchyma is connected with the palisade-tissue by means of single processes bordering the lower, that is to say, the inner ends of the palisade-cells; very often a single process is connected with the inner ends of several palisade-cells, in which case these have a clustered arrangement. As with the palisade-cells, the branched cells of the spongy parenchyma are connected with 
the parenchyma sheaths of the veins. Thus the branched cells of the spongy parenchyma become agents in the transportation of the materials; with one branch they take up the organic substances manufactured in the palisade-cells, and with another they deliver these materials up to the bundle sheath for further translation to the places of consumption or storage.

That the cells of the spongy parenchyma serve not only for conduction, but have to perform several other functions, does not need to be confirmed in detail. It is enough to point out that they contain chlorophyll-corpuscles, and therefore are capable of decomposing carbonic acid and of forming carbohydrates, although to a much less extent than the palisade-cells, which are so richly supplied with chlorophyll. Moreover, the excretion of aqueous vapour occurs in the spongy parenchyma whose lacunæ and passages communicate with the outer world by the stomata, and where also a vigorous inflow and outpouring of other gases takes place. Then the part which the conducting structures play in the metabolism of the materials must not be overlooked. All these structures contain active living protoplasm, in all there is a protoplasmic cell-body, although very often it is only in the form of a delicate parietal layer, and in all, under the influence of this living protoplasm, we have not merely a movement, but also an inexhaustible and infinite changing of the materials, corresponding to the individuality of the species and to the requirements of the time being. These structures must then be regarded not only as simple channels for the fresh carbohydrates produced in the green cells, but also as regions for transformations, where the first organic compounds manufactured in the green cells are prepared for ultimate consumption at the end of the journey. It is precisely in this respect that they differ essentially from that conducting apparatus, whose task is to transmit water and mineral salts to the green tissues, and which, as already repeatedly remarked, is present in the same bundle as the cells and vessels which take away the organic materials. When once the water-conducting tubes and cells have attained their full dimensions, they no longer contain protoplasm, and no transformation of the transmitted raw food-sap is carried on in them; the water, with the mineral food-salts dissolved in it, is carried through them unaltered to the transpiring cells. To employ the simile, often used before, of the arrangements of a well-conducted household, the woody cells and vessels of a vascular bundle may be compared to an apparatus for delivering water and salts into the kitchen, so to speak; the green tissue forms the kitchen in which the raw materials are worked up and so prepared that they can be brought back by the removing cells to the places where they are required and consumed.

That these two fundamentally different kinds of conducting apparatus are so universally found united together in one and the same bundle is explained by the fact that the places which form the goal for one are at any rate to some extent the starting-point of the other; besides, of course, this combination economizes the building materials. All conducting apparatuses must be strengthened and protected in their position, and therefore it is beneficial and saves building materials if 
the different structures taking part in the conduction are mutually of use to one another, and are protected and saved from injurious external influences by the same arrangement.

The vessels and cells whose task is to conduct water and salts become lignified, and the massive bodies of wood which exist in the trunks of old woody plants form such a firm support that the thin-walled soft bast, when it clings to these and runs parallel with them, is excellently protected from breaking. In those organs which require to resist bending, however-in leaf-ribs and leaf-stalks, culms, and young branches,-hard bast is put in as an accompaniment of the cells and tubes which conduct up and down. These strands of thick-walled, but at the same time flexible and elastic, cells of hard bast prevent the organs which they adjoin from being broken and ruptured even under the influence of a considerable push and strain. Let us look at the haulms, stems, branches, and leaves in a meadow or in a wood during the sultry period which precedes the outburst of a storm. Not a leaflet stirs, even the supple haulms are still, and every part of the plant, that true child of light, assumes that position with regard to the light most beneficial to it. The storm bursts, the wind whistles over the meadows, the leaves tremble, sway, and flutter, the leaf-stalks shake, the culms bow and bend, the stems and branches are smitten and arched so that their tops almost touch the ground; the foliage is pelted with the rain, and shaken and displaced by every drop that falls on it. An hour later the storm is over; here and there perhaps may be still seen a group of stems and leaves prostrate under the weight of the rain-drops, and part of some herbaceous stem which has been shaken by the storm bent like a bow, but the rest stand again erect and motionless, as if they had never been disturbed by a breeze; finally, even the plants bent by the shock and so severely prostrated by the raindrops raise their branches and foliage, and everything again resumes the same conditions and position as before the outbreak of the tempest. But this is only rendered possible by the presence everywhere of the elastic flexible strands of hard bast accompanying the sieve-tubes and the other soft and delicate structures which take part in the preparation and transportation of the organic materials. It is indeed unavoidable that the cross section of the cells and vessels should become narrowed in consequence of the push and strain caused by the gusts of wind, and that, for example, the cross section of a cylindrical tube should become elliptical in consequence of the curvature; but since the prostrated stem or leaf again rebounds into the former position by reason of the elasticity of the hard bast, the alteration produced by the push and strain is only temporary, necessitates no interruption of function, is perhaps even beneficial to the movement of the materials, and, which is the main point, no rupturing and no permanent bending of the soft delicatewalled structures ensues.

These delicate-walled elements, especially those of the soft bast, are protected against harm from lateral pressure by the deposition of various tissues, especially cork, in front of them (fig. $125^{2}$ ), which, like the buffers of an engine, keep off, or considerably weaken, the lateral thrust and pressure. Remarkable contrivances for 
protection against lateral pressure are also found in creepers and climbing plants with perennial woody stems, and in those plants which are commonly called lianes. In order to comprehend these contrivances rightly, it is necessary first to get an idea of the position of the parts requiring protection in perennial woody plants,

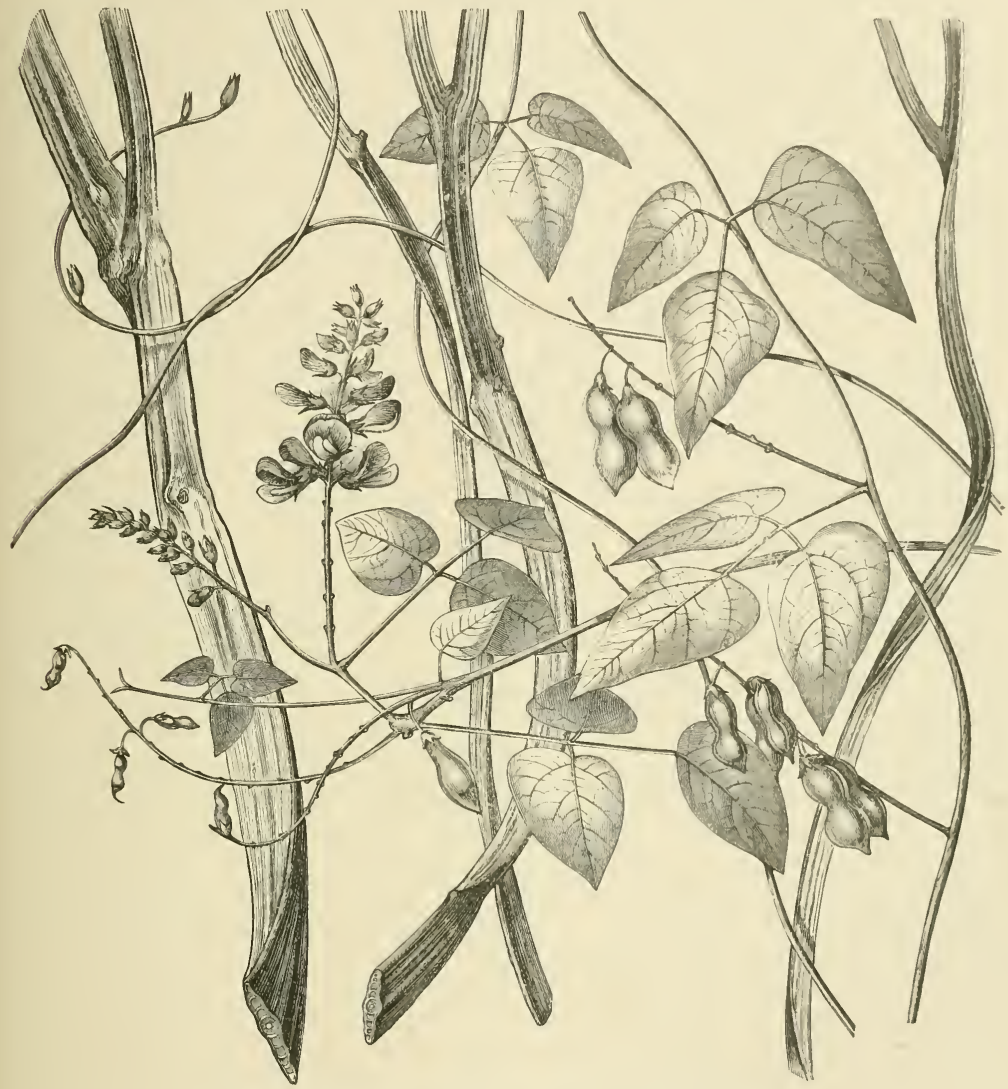

Fig. 127.-Rhynchosia phaseoloides, a Liane with ribbon-like Stems.

which neither climb nor creep, and which possess an erect, straight, column-like trunk. As previously stated, in these plants to which belong the firs, oaks, beeches, elms, limes, apple-trees, and, generally, the majority of leafy trees, the vascular bundles are arranged in a ring round the central pith, and consist essentially of the woody portion, serving to conduct the raw sap, and the bast portion, which is employed in the transmission and transformations of the organic substances formed in the green cells. These two portions are separated in the plants men- 
tioned by a layer of tissue in which a very vigorous formation of new cells is carried on, termed the cambium (fig. $125^{8}$ ). From this cambium, which appears as a ring in the circular cross section of erect stems, cells develop which on one side abut upon the wood already present in the interior, and on the other the existing bast portion of the vascular bundle to the exterior. In this way both portions, and in fact the whole stem, increase in dimensions; and in the wood, in particular, arise the annual rings which are visible in a cross section. The cambium ring also stretches; it becomes larger and larger, but always retains the same position and relation to the wood and bast of the vascular bundle, and keeps its ring-like form although the trunk in question may have become ever so old and thick, and may exhibit hundreds of annual rings. Here, therefore, the soft bast lies outside the cambium ring, and is screened towards the exterior by various tissues, by hard bast and corky tissue among others, and the latter may undergo considerable development in trunks of many years' growth; while the hard bast, on the contrary, diminishes in older trunks, because it is no longer required as a protection against bending. Accordingly the soft bast is situated fairly near the surface. Since a strong external lateral pressure is not to be feared in them, this position cannot be characterized as unfavourable. The cork and other external portions of the cortex comprehended under the term bark afford a sufficient protection against small pressures in old stems. In lianes it is very different, especially in those which make use of erect stems as supports. Apparatus for increasing the bearing capacity and elasticity in lianes would be superfluous, these tasks being performed by the support; on the other hand, a protection against lateral pressure is urgently required, for if the support up which the lianes climb, to which they are attached by adventitious roots, or which they encircle and entwine, increases in thickness, as is usually the case, then a lateral pressure on the adherent liane coils is unavoidable. And when, as a result of such pressure, the sieve-tubes and bast parencliyma become squashed over considerable distances, they are obviously unable to perform their functions satisfactorily, and nutrition will certainly be impaired. Lianes are protected by the most varied contrivances against this source of injury, and some of the most striking will be here briefly indicated.

In Rhynchosia phaseoloides, the young, green, twining stem is circular in cross section, and exhibits a structure which does not differ materially from that of young normal stems. In the centre is a pith, round which the vascular bundles form a ring-first wood, then soft bast, further out hard bast, then a layer of green cells, and, finally, the epidermis, which envelops the whole. It might be expected that in the second year, the newly-formed cells and tubes would deposit wood on wood and soft bast on soft bast, and that the cylindrical stem would increase regularly in circumference without altering its shape. But, strangely enough, this does not happen. New cambiums arise at two points near the periphery of the stem, below the green cells, by which the formation of wood proceeds in the direction of the first year's vascular bundle ring (i.e. inside), and soft bast accompanied by hard bast on the opposite side (i.e. outside). At 
the end of the second year the stem is no longer circular, as at the first; it has added two rings, as it were, and now appears elliptical in cross section; and since new portions are added in this way repeatedly from year to year, and new rings are always becoming annexed to those already existing, the stem gradually becomes ribbon-like, and exhibits a cross section like that shown in fig. $128^{2}$. The soft bast has thus received the most protected position imaginable, and lateral pressure is unable to interfere with its functions. When the supporting stem round which the Rhynchosia has twined grows enormously in thickness, the liane becomes stretched, and experiences a lateral strain, but the sap can, nevertheless, continue its journeys unhindered in the soft bast.

In the liane Thunbergia laurifolia, a cross section of whose stem is represented in fig. $128^{1}$, the protection is obtained in quite a different way. Here the green
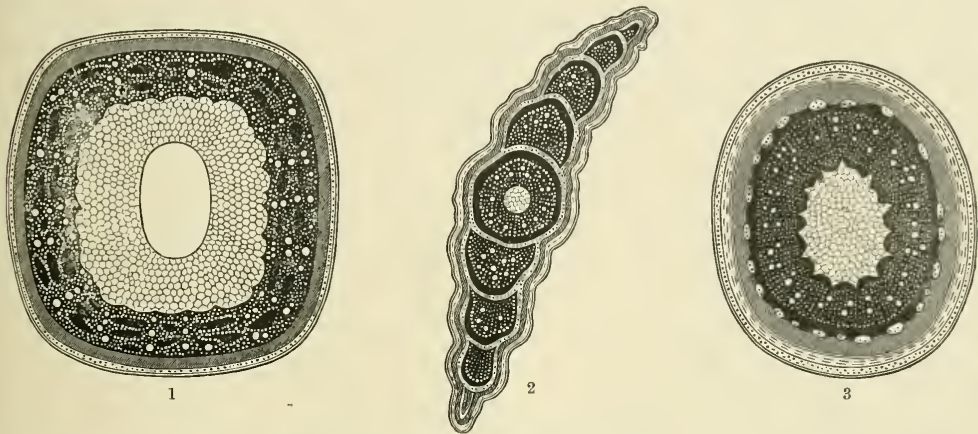

Fig. 128.-Transverse sections of liane Stems.

1 Thunbergia laurifolia. 2 Rhynchosia phaseoloides. 3 Tecoma radicans; $\times 30$. Diagrammatic. The various tissues are indicated in the following manner: Soft bast, entirely black; wood, larger and smaller white dots on a black ground; hard bast and other mechanical tissues, obliquely shaded; cork (periderm), short lines; pith, reticulated.

stem is hollow, and the cavity is surrounded by an enormous pith. In the vascular bundle ring which surrounds the pith, the wood and hard bast are not arranged from the first in successive concentric circles, as is usually the case, but are placed side by side. The cambium continues to form soft bast in some places, and wood in others, towards the interior. In consequence of this, the bundles of soft bast appear to be walled in by the wood ("bast-islands"), and are consequently well protected against pressure. The protection is increased by the fact that this liane is hollow in the centre, and can "give", an uncommon feature in twining plants.

Sometimes the delicate soft bast is protected against compression by its position in niches and grooves at the periphery of the hard wood; this is to be seen especially in several twining Asclepiadeæ and Apocynaceæ. One of the most remarkable protective arrangements is found in the climbing Tecoma radicans, which adheres to its substratum by tufts of aërial roots, and whose leafless branches are depicted in fig. 129. A cross section of the stem is shown 
in fig. $128^{3}$. The young branches rooted to the wall are elliptical in transverse section, being always somewhat compressed on two sides. The outer portion is composed of the epidermis, two layers of elastic parenchymatous cells below it, and a layer of green cells. Then comes the ring of soft bast, outside which bundles of hard bast are deposited; then the rings of cambium and wood, and in the centre a large pith, which sends out single- and double-rowed medullary rays through the wood ring. So far the arrangement of the various tissues exhibits nothing particularly noticeable, and coincides with that in the young branches of numerous woody plants. But tracts of cambium cells are subsequently formed in a remarkable manner on the inner side of the ring of wood adjoining the pith; these develop wood towards the exterior and soft bast on the interior. The constituents of the soft bast-sieve-tubes and bast parenchymaform quite conspicuous bundles which project into the pith, and being here excellently protected against lateral pressure, can perform their duties undisturbed. Should the conducting cells and sieve-tubes of the outer ring of bast not perform their duty, these inner ones still remain for the transmission of the plastic materials.

Thus the various arrangements of the constituents of the stem, and especially the position of the channels for the streams of materials formed in the green tissues, is in part accounted for by the protection gained against the injurious action of external pressures and strains, and these act in the most varied way on the exterior, according to the individual mode of life of the plant and the conditions of its habitat.

It is to the growing parts of plants, the extremities of roots and branches especially, that organic matter is conveyed; also to places where the cells already present become stimulated to fresh activity, where dead and dying cells are replaced by fresh ones, and where constructive materials in sufficient quantity must be at hand. Then again, the travelling substances are directed to those places where protective and attractive agents are necessary to contribute indirectly to the maintenance and multiplication of the species, and where a consumption of materials is connected with this protection or allurement. It is thus of importance, for example, that the honey excreted from certain parts of flowers, which serves as food to the insect guests which effect fertilization, should be always present in sufficient quantity, and that in case of its removal from the receptacles by bees or butterflies, it should be immediately replaced by fresh supplies. Care must also be taken that pepsin and other substances necessary for digesting prey should be abundantly present in the pitfalls and other mechanisms which serve for the capture of animals, and that a sufficient quantity of alkaloids and bitter substances, which prevent ruminants from devouring foliage, should be brought to the right places at the right time. In connection with the process of rejuvenescence and multiplication also, it is necessary that those cells and groups of cells, which become detached from the plant-shoot and journey out into the wide world as spores and seeds, should be equipped with 
a store of materials, so that they may be nourished until they can manufacture for themselves the necessary food from the air, water, and soil. The places where spores and seeds are produced, therefore, constitute an important destination for certain journeying materials. Finally, it also happens that in regions where a temporary standstill of the vital activity of the plants occurs, and where the succulent green foliage is liable to be dried up by the periodic drought, or frozen by the winter cold, all the useful substances are withdrawn from the threatened

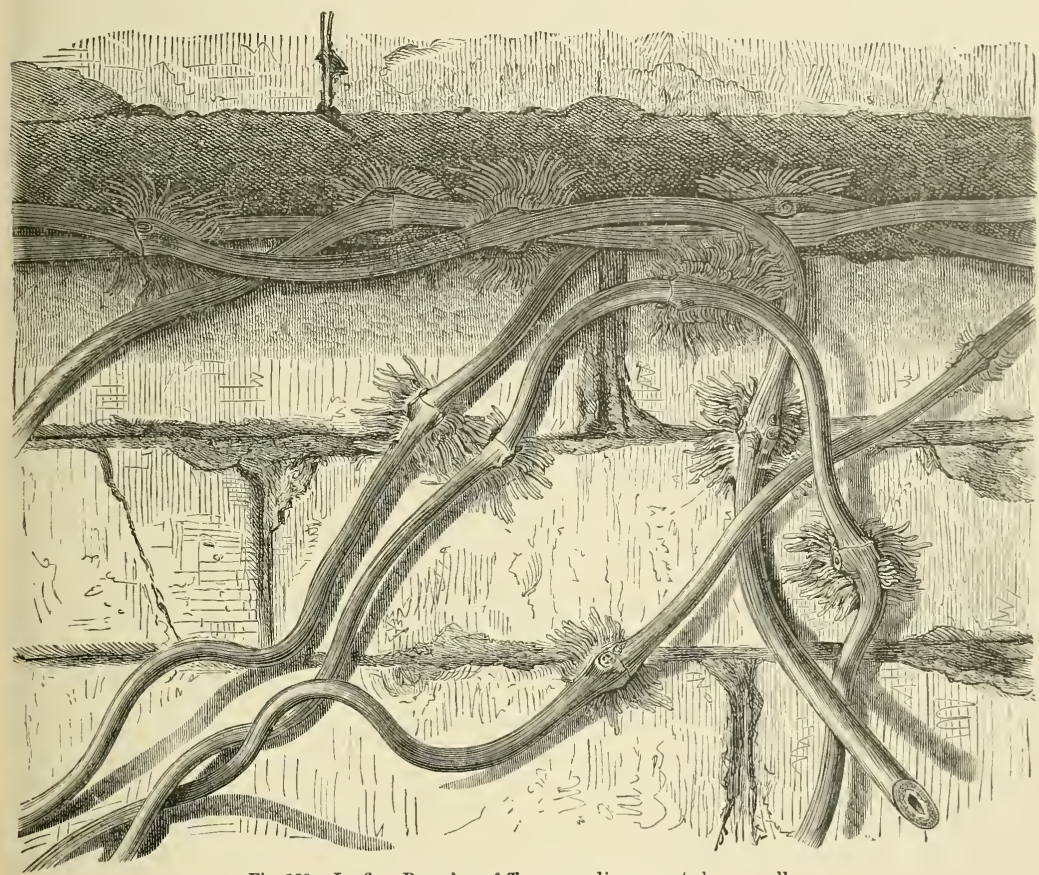

Fig. 129.-Leafless Branches of Tecoma radicans, rooted on a wall.

leaves, and are deposited in a suitable form in safe places, and stored up for employment later. In these instances, these safe places, these storehouses or reservoirs, form the destination of the transported materials.

Not only are there channels of distribution to the various destinations enumerated, but we find even distinct routes provided for the different substances transmitted. Investigations have shown that the conducting mechanisms divide the work to some extent between them. The medullary rays and wood parenchyma chiefly conduct carbohydrates, the former radially, and the latter longitudinally, in the stem. The vascular bundle sheaths of the leaf-veins are particularly rich in glucosides. Certain tracts of cells in the parenchyma accompanying the vascular bundles in the stem also conduct glucosides, while others conduct sugars 
(sugar sheaths), and ochers again are the route for the transmitted starch (starch sheaths). The sieve-tubes and bast parenchyma, on the other hand, convey principally albuminous substances which are employed as constructive materials for the growing and enlarging portions of the plant.

This important relation of the soft bast to the growing organs explains many remarkable phenomena, two of which must be briefly described here. First, the surprising increase of growth in certain places which gardeners produce by the operation of ringing. If two parallel circular cuts are made round a growing branch of a tree through the whole thickness of cortex down to the wood, and if the circular piece of cortex, together with the soft bast lying between the two cuts is removed, the sap current in the soft bast from the upper portions to the base of the branch is interrupted. The cut surfaces dry up; the route down the soft bast is therefore closed, and the part of the branch lying below the excision can no longer receive from the soft bast the materials necessary to its further growth. The same result is obtained by passing a cord tightly round the young leafy branch of a tree at some spot, say about half-way up. In this way all the soft tissues which lie outside the wood, including the soft bast, are compressed, the sieve-tubes and tracts of cells of the bast parenchyma tightly squeezed together, and the conduction of sap by them to the base is rendered impossible by the strangling cord. The ascending current of water and dissolved food-salts, in the deeper-lying firm wood, flows on unimpeded in either case. The green foliage-leaves can thus decompose carbonic acid and manufacture organic substances above the circular cut or ligature; these products are then conducted away; the albuminous substances enter the soft bast, but only travel as far as the place where the cut has been made or the ligature been tied. They can no longer pass these places, and consequently the plastic albuminous materials become dammed up above the "ring" or ligature, and a particularly luxuriant growth of all these parts results. Fruits which develop from the blocked-up region of the branch sometimes enlarge to an extraordinary degree, and become almost twice as heavy as they would have done had the operation not been performed.

The following phenomenon is also explained by the fact that the passage of plastic albumins takes place in the soft bast. If a branch of a willow, e.g. of Salix purpurea, be cut off and the entire cortex down to the wood be removed from the lower third of the branch, and the branch so treated be then plunged half-way into a vessel of water, after a time it will send out roots into the water. But these differ from one another very much according as to whether they arise from the lower stripped portion of the branch or from higher up where the cortex has not been removed. The roots developed from the stripped portion are few and remain very short; those springing from the upper thickening portion of the willow branch, where the cortex is intact, are, on the contrary, abundant and elongated, since they can utilize the plastic juices above the place where the cortex, together with the soft basst, has been removed. 
Both of the experiments described only exhibit the results mentioned when performed on plants, the whole of whose soft bast bundles lie outside the cambium ring, since interruption of the sap-current by ringing only takes place under these conditions. If plants are experimented on which possess internal bundles of soft bast in addition to those lying near the surface, as in Tecoma, Thunbergia, and many others, the ringing does not have the result described, because the inner bundles of soft bast (being protected by the hard wood) are not cut through by the knife, and cannot be compressed by the ligature. It should, however, be observed that even in woody plants, whose soft bast lies entirely outside the cambium ring, this result is restricted to the year in which the ringing or ligaturing was performed. In consequence of the absence of supplies of albuminous materials through the soft bast, the portion of the branch below the cutting or ligature becomes unhealthy, its cortex dries up and dies, and the disparity between the two portions lying above and below the ringed cut or the tight ligature usually occasions the death of the whole branch tampered with in the following year.

In the tubular conducting mechanisms, especially in the laticiferous tubes, which are entirely free from transverse walls, and also in sieve-tubes, in which perforated horizontal walls are inserted here and there, a transport of substances en masse may occur, but this is impossible in those conducting apparatuses consisting of rows of cells whose length is usually only three or four times their width. In these tracts of cells the numerous non-perforated partition-walls of the adjoining cellchambers are interposed, and must be passed through by the travelling materials. Whether this passage through the walls be regarded as a diosmosis or a filtration, it is at least certain that solid bodies of definite form cannot traverse the walls. Even starch-grains of the smallest diameter are always much larger than the interstices which we imagine to exist in every cell-wall between the groups of molecules; and therefore it follows that even the tiniest visible bodies must always remain behind, as on a filter, in one of the two adjoining cell-chambers, that is to say, on one side or the other of the dividing partition-wall. Accordingly, only fluid materials travel through such cell-tracts as serve for the conduction of substances in the soft bast, parenchyma, and in the bundle sheath. If solid substances take these routes, they must be first brought into a soluble condition. This applies especially to the starch-grains which play such an important part in the life of plants, and not only share in the formation of cellulose, chlorophyll-corpuscles, and fats, but are also heaped up in the storehouses of the plants as materials well suited for storage during the summer drought or through the winter, for use in the next period of vegetation. They are also given to the seeds which have to lead an independent existence, as the first food for the journey after leaving the parent plant. If starch-granules are to travel through the cells of the bundle sheath, composed of hundreds of single ceils, they must be dissolved a hundred times, and a hundred times reformed. It has been definitely proved that this transitory starch is not liquefied at the beginning of its journey and again formed into solid only when it has reached its destination, but that, as stated, a liquefaction, and after it has vor. I. 
passed through the dividing wall, a solidification, occurs in each of the succeeding members of a string of cells. This is a very laborious and wearisome process, and the question involuntarily arises, after observing these methods of transmission, why these numerous partition walls in the rows of cells are not done away with. The wood vessels have been produced from rows of cells by the solution of the dividing partition walls; why are the many transverse walls retained here to complicate and retard the transportation of the substances? It must be supposed that these cross walls, which break up the free channel, are in some way beneficial to the plant, since they occur so generally and with such regularity. It might be thought, first of all, that these walls keep open the road, and that thereby the delicate walls of the cells forming the channel are protected from collapse. Apart from the fact that the cells of bast parenchyma, imbedded in niches and grooves in the periphery of the hard wood, are prevented from collapsing by their sheltered position and nevertheless exhibit transverse walls, while the delicate-walled laticiferous tubes, which are anything but well-protected, possess none and yet do not collapse-apart from this, such a delicate wall would form but a bad stiffening agent, and the support would be obtained much better by band-like circular thickenings. It has also been surmised that the cross walls inserted in the channels might be of use in that they prevent an excessive accumulation of solid bodies at certain places on the road. Where the cells of a cell-row stand vertically above one another, as, for example, in erect stems, it is found that the small starch-granules sink to the bottom of the cells and lie on the lower transverse wall. If all the solid corpuscles contained in the sap of a long vertical tube were to sink to the bottom, of course an obstruction might arise which would be anything but beneficial. But the significance of the partition walls most probably lies in the transformations they produce in the substances. It may be safely assumed that those materials which must pass through not merely the cellulose transverse wall, but also the protoplasmic parietal layer of the cell-chamber, undergo an alteration thus under the influence of the living protoplasm; that the position of the atoms becomes different, or that new atoms enter into combination and others are displaced, in short, that re-arrangements and transformations occur from which it results that the transmitted materials arrive at their destinations prepared in the best possible way. With this, however, we return to the important theorem previously stated, that these rows of cells have not merely the significance of a road along which the materials, formed at the starting-points, are conducted unchanged to the terminal stations; but that they also form places for the .continuous transformation and alteration of these materials for subsequent use. 
SIGNIFICANCE OF ANTHOCYANIN IN THE TRANSPORTATIONS AND TRANSFORMATIONS OF MATERIALS. AUTUMNAL COLOURING OF FOLIAGE.

In connection with the foregoing results of investigations into the transmission of substances, the fact must be noted that those agents which take part in the transformations of carbohydrates and albuminous substances are to be found all along the road which these follow and not merely at the beginning and end of the journey. Diastase, for example, is to be found everywhere along the strands of cells forming the path of the transitory starch, and when these strands run near the surface that colouring-matter called anthocyanin, a somewhat detailed description of which must be given, is also present.

In many instances the route of the travelling substances can be recognized by the naked eye, since it is coloured blue, violet, or red. Whether all these tints actually originate from one colouring-matter, which is red, violet, or blue according to the presence or absence of acids, has not been ascertained. The chemical composition of colouring-matters is yet little known, and it is possible that at present a whole group of them is collected together under the name anthocyanin. It is a matter of indifference with regard to the question in hand, as also is the question as to the way in which anthocyanin originates in plants. It need only be mentioned here that the statement according to which anthocyanin arises from the chlorophyll-corpuscles present in young plant organs cannot be correct in all cases; since this pigment occurs regularly in parasites entirely devoid of chlorophyll, in the Balanophoreæ, Rafflesiaceæ, and Hydnoreæ, in the Toothwort, in Monotropa, and numerous other plants destitute of green colour. In green-leaved plants anthocyanin is most usually met with in places which have little chlorophyll, or which have never possessed any, in flowers and fruits, along the ribs of leaves, and principally in leaf-stalks and herbaceous stems. In hundreds of species belonging to widely-differing families the leaf-veins and ribs, leaf-stalks and leaf-sheaths are coloured violet, red, or blue, and this colouring is co-extensive with the vascular bundles beneath them.

It is difficult to say whether anthocyanin exercises a photochemical effect on the travelling substances in the given paths, or whether it is only of use in that it keeps back the light rays which would decompose the travelling materials. In support of the latter view we have the fact that anthocyanin is much more abundantly deposited in paths exposed to the light than in those which are shaded, and that in shaded organs the same changes and transmissions of materials occur as in those exposed to bright light, where the superficial cells are coloured with anthocyanin, and where consequently the paths of the transmitted substances below are to some extent screened. It is noticeable that plant organs which are very thickly covered with hairs scarcely ever develop anthocyanin. From all this it may be concluded that anthocyanin, when it appears in places directly illumined by light rays, serves principally as a screen, i.e. as a protective agent or awning against injurious light rays. 
Here another very remarkable phenomenon may be considered. If the colourless and scaly rhizome of Dentaria bulbifera be dug out of the dark forest soil, it appears beautifully white, as if carved out of ivory. If it is put in a glass vessel which is filled up with water and placed in the sun, so that the rhizome is illumined by the direct rays, the white scales in a very short time assume a slight violet tint, and in a few days the whole of the scaly rhizome becomes coloured a deep violet. The same thing happens with the rhizomes of several species of Cuckoo-flower, Violet, Toothwort, \&c., but in these it is a little longer before the violet colour appears. The tissues brought from the darkness into the bright light try to neutralize the influence of the light which is injurious to certain substances, and therefore anthocyanin must not be regarded merely as an agent for protecting chlorophyll alone, but other chemical compounds also. That a far wider significance in the life of plants is also assigned to it will be demonstrated in the following section.

Very often anthocyanin only appears temporarily, when the transmission of food substances occurs on a very large scale. When seeds are germinated, and their reserve materials are conducted into the rapidly sprouting seedlings, such as those produced from the starchy seeds of polygonums, oraches, palms, grasses, \&c., anthocyanin regularly appears, while later on it partly or wholly vanishes. When in spring the foliage-buds on subterranean root-stocks or branches begin to develop, and the materials stored in the stem structures travel into the young leaves, to be employed there in further construction, these leaves do not appear green in most cases, but reddish-violet or reddish-brown in colour. As instances of this may be mentioned the well-known Tree of Heaven (Ailanthus glandulosa), Walnut (Juglans regia), Pistacia (Pistacia Terebinthus), the Sumachs (Rhus Cotinus and Rhus Typhinum), the Judas Tree (Cercis Siliquastrum), Berberideæ (Mahonia, Podophyllum, Epimedium), Ampelideæ (Vitis, Cissus, Ampelopsis), the Trumpet Tree (Catalpa syringafolia), the red-berried Elder (Sambucus racemosa), Cherry (Prunus avium), Peony and Sea Lavender (Poonia and Statice), and Rhubarb and Dock (Rheum and Rumex). Later on, when the transmission is effected, when the foliage-leaves are developed and are able to act independently, the green chlorophyll appears; the leaves become green, and the anthocyanin either vanishes entirely or remains only in those places where it is required as a protection to the chlorophyll, or for another important purpose to be dealt with in the following section, viz. the transformation of light into heat.

In many plants, anthocyanin is again developed in great abundance when the leaves are obliged to stop their activity for a time on account of the commencing dryness of the soil, or still more, on account of cold and the consequent delay of supplies of crude sap. In order to describe this formation of anthocyanin and everything connected with it, it is necessary to go back a little, and to discuss, first of all, the metabolism and transport of materials connected with the stoppage of activity in the green leaves at the close of the vegetative period. These 
differ essentially according as the leaves of the plant continue active through one, or through several vegetative periods, i.e. according as the leaves are deciduous or lasting but one year, or evergreen, that is to say, lasting for two or more years. Evergreen leaves are so organized in all those regions whose climate necessitates a temporary suspension of vital activity, that they may be able to survive the periods of drought or frost of one or even of several years without injury. Before they enter upon their summer sleep in regions of summer drought, or their winter trance in regions with cold winters, alterations occur in their cells, which, in the main, terminate in the diminution of the water contents and the formation of substances which will not be altered by the prevailing frost or dryness. In regions where we have a winter sleep, the chlorophyllgranules assume a yellowish-brown or brownish-red colour, and adhere together in clumps, which withdraw as far as possible from the surface of the leaf, travelling down to the floor of the palisade-cells and occupying their lower ends. These alterations are only slightly apparent outwardly in perennial leaves preparing for their winter period of rest; the only thing one notices is that the leaves, which in summer are a vivid green, exhibit a darker green, or incline to brown or yellow; which change of colour is observed to the greatest extent in Thuja, Cryptomeria, Sequoia, Chamcecyparis, Libocedrus, and generally in most evergreen conifers.

The changes which are accomplished in leaves lasting only one year, at the onset of the summer drought or winter cold, are much deeper rooted and obvious. These leaves are not clad so as to be able to defy the drought or frost, and are therefore thrown off at the commencement of the unfavourable period. If these leaves were to fall without further ceremony, all the substances in the tissues of the leaves, whose production entailed a considerable amount of work, would be entirely lost. But it is part of the economy of plants that such a waste is carefully guarded against. Before the leaves are detached, the carbohydrates and albuminous materials, in general everything which is of use to the plant, is conveyed from the leaf-blades into the woody branches or subterranean root-stocks, and there deposited in places where they find a safe resting-place, and can survive the drought of summer or cold of winter unharmed. In this way the plant suffers only the slightest loss in the materials manufactured by it in the preceding vegetative period; for the leaves from which everything useful has been transported into the stem-structures now form nothing more than a dead framework, and their cell-chambers contain only small yellow granules, together with crystals of calcium oxalate, which cannot be employed further, and are of no more use (see fig. $123^{1}$ ). The shining yellow granules, which are found in the cells of fallen leaves, and to which is due the yellow colouring of autumn foliage, are to be regarded as the ultimate useless residue after the withdrawal of the transformed chlorophyll-corpuscles. The crystals of calcium oxalate have arisen in the formation of albumens by the decomposition of nitric and sulphuric acids. Both of them can be sacrificed. As a matter of 
fact, the rejection of these structures is no sacrifice in reality, since they are only superfluous ballast by which, under certain conditions, the plants may be hampered in their next year's work, and of which they therefore rid themselves most seasonably and suitably. The fall of the leaf may be looked upon, so far, as an excretion of superfluous matter, which, in deciduous plants, occurs only once every year, but is then carried out on a grand scale. To the benefits which this wholesome excretion of waste, formed in the metabolism, affords to individual plants must be added the fact that the fallen leaf reaches the ground with its abundance of lime, decays there, contributes to the formation of humus, which contains calcium nitrate, and so becomes of use to the vegetable kingdom as a whole, as already described in detail.

The emigration of the useful materials from the leaf-blades to the store-rooms in the interior of the branches and root-stocks must, as a rule, be accomplished fairly quickly; most rapidly, of course, where the period of vegetation during which the foliage-leaves can be active is short, when the leaves are obliged to make use of the favourable time to the utmost, and where the change of seasons occurs abruptly. The materials withdrawn travel by the same route as in general is taken by the substances normally manufactured in the leaves. The accessories by which the carbohydrates and albumens to be removed are prepared for emigration, might (one would think) be the same in every case. But, just as in one species one kind, and in another a different kind are developed when the leaves are most active, so in different species at the close of the vegetative period, when the great emigration takes place, we have again various accessories, and various despatching and protective agents. In many instances the accessories are colourless, and are not recognizable by the naked eye even when developed in great quantity. It can only be seen that the leaves lose their fresh green on account of the change experienced by the chlorophyll bodies for the purpose of emigration, and that a yellowish tint appears instead of the green colour, which is produced by the already-mentioned yellow granules remaining behind after the departure of the chlorophyll-corpuscles. In many leaves the number of these yellow granules is so small that even the yellow tint is hardly apparent, and these leaves then are a dirty yellowish-white, shrivel up very quickly, and become brown.

Anthocyanin, however, is produced in many plants during the emigration of the carbohydrates and albuminous materials, and to such an extent, that it becomes plainly visible on the exterior. It appears red in the cell-sap in the presence of acids which occur very regularly as metabolic accessories in the autumn leaves, blue when no acids are present, and violet when the amount of free acids is but small. If there is an abundance of yellow granules together with the acid, red anthocyanin, the leaf assumes an orange colour. Thus the green colour of the foliage changes at the period of the great autumal emigration, sometimes into yellow, or brown, or red, violet or orange, and in this way we have a play of colour exhibiting the greater variety the more numerous are the plant species 
growing associated together in the particular spot. If the leaves are thickly covered with silky or woolly hairs, or if the hairs are felted or peltate, anthocyanin is scarcely ever developed; but when the green tissue of these leaves becomes also changed in colour, the new tint can be seen as little as was the green previously, on account of the hairy coat over the coloured cells. Accordingly, such felted, silky, or scale-covered leaves remain grey or white even when they fall from the branches. If plants of this kind grow among others whose foliage is bare, their grey and white tints considerably increase the variety of the entire collection. But the greatest amount of colour is seen when the neighbourhood is sprinkled with plants having evergreen foliage; it may then happen that a relatively small space of meadow or wood appears decked in all the colours of the rainbow in the most manifold variety.

The splendour of colours exhibited by tropical forests, which is usually represented as much more magnificent than it really is, stands no comparison with that developed in autumn in the north temperate zone. The forests of firs and leafy trees on the mountain slopes along the Rhine and Danube in Europe, and on the shores of the Canadian lakes in North America at that season present a scene of entrancing beauty. The heights along the middle course of the Danube, for example, the region known as the Wachan, below the town of Melk, shows wide expanses of forests, in which beeches, hornbeams, evergreen oaks, common and Norway maples, birches, wild cherries and pears, mountain ashes and wild service-trees, aspens, limes, spruces, pines and firs take a share in the greatest variety. Bushes of Barberry (Berberis vulgaris), Dogwood (Cornus sanguinea), Cornel (Cornus mas), Spindle Tree (Euonymus Europceus and verrucosus), Dwarf Cherry (Prunus Chamcecerasus), Sloe (Prunus spinosa), Juniper (Juniperus communis), and many other low shrubs arise as undergrowth, and spring up on the margins of the forests. The mountain slopes abutting on the valleys are planted with vines, and near by grow peach and apricot trees in great abundance. In the meadows on the shore, and on the islands of the Danube, rise huge abeles and black poplars, elms, willows, alders, and also an abundant sprinkling of trees of the bird cherry (Prunus Padus). The nights are bitterly cold there; even in the middle of October, damp mists hover over the river, and hoar-frost covers the grassy regions at the bottom of the valley. But during the day it is still fairly warm, the morning mists are dispelled by the rays of the sun, a cloudless sky stretches over the landscape, and soft breezes, swaying the threads of the vagrant spiders, blow from the east through the river valley. The first frosts are the signal for the beginning of the vintage; all is busy in the vine-planted districts, and the call of the vine-dresser resounds from hill to hill. But it is also the signal for the forests on the mountain slopes and in the meadows to change their hues. What an abundance of colour is then unfolded! The crowns of the pines bluish-green, the slender summits of the firs dark green, the foliage of hornbeams, maples, and white-stemmed birches pale yellow, the oaks brownish-yellow, the broad tracts of forest stocked with 
beeches in all gradations from yellowish to brownish-red, the mountain ashes, cherries and barberry bushes scarlet, the bird cherry and wild service trees purple, the cornel and spindle-tree violet, aspens orange, abeles and silver willows white and grey, and alders a dull brownish-green. And all these colours are distributed in the most varied and charming manner. Here are dark patches traversed by broad light bands and narrow-twisted stripes; there the forest is symmetrically patterned; there again the Chinese fire of an isolated cherry-tree or the summit of a single birch, with its lustrous gold springing up among the pines, illuminates the green background. To be sure this splendour of colour lasts but a short time. At the end of October the first frosts set in, and when the north wind rages over the mountain tops, all the red, violet, yellow, and brown foliage is shaken from the branches, tossed in a gay whirl to the ground, and drifted together along the banks and hedges. After a few days the mantle of foliage on the ground takes on a uniform brown tint, and in a few more days is buried under the winter coat of snow.

The autumnal colouring of the foliage in those parts of the North American forest regions, whose vegetation presents the greatest analogy to that of the Old World just described (i.e. in the neighbourhood of the St. Lawrence and from the Canadian lakes to the Alleghany Mountains), lasts much longer than in the forest regions of Central Europe. There also evergreen conifers grow side by side with deciduous trees, and there again a rich underwood flourishes in the forest regions. To some extent we have exactly the same species composing the woods-pines and firs, beeches and hornbeams, oaks, ashes, limes, birches, alders, poplars, mapies, elms, hawthorn, guelder-rose, and dogwood; but the wealth of forms is far greater than in Central Europe. In the neighbourhood of the shores of Lake Erie, for instance, a district pre-eminent in respect of the glow of its autumnal tints, we have in addition to the trees enumerated the Rhus Toxicodendron and R. Typhinum, the Tulip-tree, Western Plane, several walnuts, robinias, Gymnocladus, Liquidamber, and especially some Ampelideæ which climb like lianes to the highest tree-top. This greater variety of species produces an even richer play of colour in autumn than in the central European districts. The change of colour of the deciduous trees begins in some species always at the commencement of September, and stretches over a whole month, so that the fall of the last leaves usually does not occur until about the middle of October. The American beech (Fagus ferruginea) changes colour exactly like the European; and the American birches (Betula nigra and B. papyracea) exhibit in their autumn foliage the same golden yellow as do their European allies; but the autumn foliage of oaks, which flourish with an extraordinary number of species south of the Canadian lakes, present every tint from yellow to orange and ruddy brown; the Red Maple (Acer rubrum) shrouds itself in dark red, the Tulip-tree exhibits the lightest yellow, the large-spined hawthorn bushes, the Sheep-berry (Viburnum Lentago) and the Rhus Toxicodendron become violet, the Sumach (Rhus Typhinum), and the wild vines (Vitis and Ampelopsis), climbing up the branches of the trees, clothe 
them in flaming scarlet. With this gay assemblage of vivid colours the Canadian firs mingle their deep, dark green, and the Weymouth Pines the dull bluish-green of their needle-leaved summits. Where such a wood is developed with all its wealth of species, and where there is an opportunity of seeing it pass slowly under view in the soft light of a September day, as, for example, in a journey along the southern shore of the Canadian lakes, the eye revels in the changing pictures of scenery and in a wealth of colour such as it meets with in no other forest country.

Of course the autumnal colouring is not limited to the deciduous foliage of the trees and shrubs enumerated, but includes the perennial low shrubs and herbs. In forest regions, however, only the large forms of the greater trees stand out, and the low bush only rarely forms a characteristic feature in the autumn landscape. But where lofty trees are absent, and where the clumps of low plants are the characteristic feature, as in the regions of the Arctic flora, and especially in the mountain slopes above the tree limit, the matter is quite different. Of these latter regions, however, there is scarcely one which can rival the Alps of Central Europe in respect of the autumnal change of colour of the vegetation. It is especially in those parts of the Central Alps characterized by the great variety of their flora and their wealth of Ericaceæ, where strata of slate and limestone alternate or lie side by side, that the spectacle here described passes with a splendour of which the ordinary summer visitor to the Alps can form no conception. The time of commencement of the display cannot be definitely given; it varies from year to year according to the prevailing conditions of temperature and moisture. If even at the end of August fresh-fallen snow remains for several days on the slopes above the tree limit, the colouring may make its appearance as early as this; but if, as is usually the case, the heights do not assume their white mantle of snow until the middle of September, after a storm, and if during the latter half of the month the fresh snow melts and a clear sky prevails over the mountain heights, then the autumnal change of colour is retarded so much longer. Below, in the depths of the valley, which lie for wide expanses already in the shade on account of the low position of the sun, the ground remains continuously whitened by the frost, while up above, on the southern slopes of the mountain heights, the night's frost vanishes with the first glimpses of the sun, and soft breezes blow over them throughout the day. Ptarmigans and swarms of birds of passage journeying over the Alpine passes, but stopping here for a short rest, are busy in picking off the berries from the low bushes which cover the slopes in great abundance; but the butterflies which were so active in the summer among the Alpine flowers have vanished; here and there isolated scabiouses and the dark spikes of the late-blooming Gnaphalium still linger, but everything else is in fruit, and the procession of the flowers is past. And yet the slopes have all the brightness of summer meadows, which are adorned with innumerable flowers. The deciduous foliage of the low shrubs and herbs, and especially that of the stunted thick-carpeting bushes (whose materials are conveyed into the woody branches and underground stem-structures) attains even in this short time red, violet, and yellow tints, which are in no wise inferior 
in glow and brilliancy to the most vivid colours of flowers. The deciduous whortleberries and a species of bearberry are most conspicuous. While the leaves of the Bilberry (Vaccinium uliginosum) assume a violet colour, the red Whortleberries ( $V$. vitis idcca) clothe themselves in deep red, and the Bearberry (Arctostaphylos alpina) in vivid scarlet. The autumnal leaves of these plants exhibit the most beautiful red observed in any autumnal foliage; it is much more fiery than in the North American vines and the sumach trees; and when the foliage of this Bearberry is illuminated by oblique sunbeams on a mountain slope, the observer below might fancy he saw flames of strontium forking up out of the ground. The leaves of many herbaceous plants also, such as Alpine geraniums and Alpine hawkweeds, become coloured with anthocyanin at the margins, and along the veins, or even over the whole surface, before withering; and seen from a distance, look like red, violet, and variegated flowers. The Alpine willows, however, especially the carpeting Salix retusa, and the low bush of Salix hastata and S. arbuscula, together with the red-fruited Sorbus Chamo-mespilus, take a golden yellow. The latter chiefly border the water-courses, and on looking down from above on the gullies and ravines through which the water pursues its tortuous way, interrupted by small cascades, these bushes are recognized as a twisted, golden fretwork, thrown up against the darker background. Between the low undergrowth of whortleberries and bilberries, but principally between the low-lying sprays of Alpine bearberries, spring up everywhere white and grey lichens, especially the Reindeer-moss and the Iceland-moss, and some rocky ridges and slopes are so exclusively covered with these structures that they look from a distance like white patches and stripes on red, violet, and yellow grounds. The display of colours in Alpine regions is materially heightened by the fact that broad patches of dark tints are not wanting. The number of evergreen plants is comparatively large, and some of those species which appear in clumps retain their green foliage under the long-continued winter coat of snow until the vegetative period of the next year. The groups of mountain pines (Pinus humilis, Mughus, and Pumilio), the rhododendron bushes (Rhododendron hirsutum and ferrugineum), the tufts of Crowberry (Empetrum nigrum), and the glistening carpet of the evergreen Bearberry (Arctostaphylos Uva-Ursi), with their dark-green tints, bring a certain calm into the gay confusion. The carpets of Azalea procumbens, which in the autumn becomes brownish-green in colour, in consequence of the collection of the chlorophyll-corpuscles of the green leaf-cells into balls, also moderate the glare of the picture in a harmonious manner.

The charming spectacle of the colouring of deciduous foliage in Alpine regions as a rule only lasts for about a fortnight. If the slopes still remain free from snow for a short time, all the red, violet, and yellow leaves become detached from the twigs and branches. Whatever useful materials were still present in the foliage have emigrated during this time to the stem-structures, where they are to pass the winter; and the fallen leaves become brown and blackened. Soon the wintry pall of snow is spread upon the mountains; and the ridges, slopes, and hollows, from 
which flamed so recently tints of red gold between the dark mountain pines, are now covered with dazzling white from the winter sky.

\section{3.-PROPELLING FORCES IN THE CONVERSION AND DISTRIBUTION OF MATERIALS.}

Respiration.-Development of Light and Heat.-Fermentation.

\section{RESPIRATION.}

One of the most remarkable things about metabolism in plants is that every species is its own model and type, that the compounds which are manufactured in various species always remain the same in successive generations, and that from the same soil, the same water, and the same air, under equal illumination and under the influence of the same temperature, the most different organic compounds are prepared by various species situated in close proximity. Within an area of a square metre spring up from the forest soil the poisonous Boletus sanguineus, the savoury Mushroom, and the latex-swollen Russula; and if the seeds of Mustard, Corn-cockle, and Poppy (Sinapis nigra, Agrostemma Githago, Papaver Rhoeas) are strewn on a garden bed of uniform soil, so that the plants germinated from these seeds grow simultaneously side by side, their seeds will indeed exhibit materials of the most varied composition, but every mustard seed, every seed of the corn-cockle, and every poppy seed will present exactly the same compounds as were possessed by the seeds sown, compounds which the seeds of these species have contained for thousands of years. This phenomenon can only be explained by the association of like to like always and everywhere in the plant, and by the supposition that every molecule of a certain material not only operates as a centre of attraction on its surroundings, but that the attracted atoms are grouped according to the special type, just as happens in the crystallization of mineral substances.

If the atoms in the colourless cells of a seed germinating in the darkness of the soil are attracted in the manner indicated, arranged in a certain way, and connected together to form a solid body, the chemical equilibrium in those cells must be disturbed. If the materials thus attracted were previously dissolved in the sap of these cells, the degree of concentration of their sap must have been diminished in consequence of their withdrawal, and will be less than that of the neighbouring cells. But this dissimilarity cannot be maintained, and therefore a compensating movement occurs, which spreads to more and more distant cells; or, in other words, the materials stream towards the places of consumption. We return to this process, already once described, in order to review the propelling forces which are concerned in the metamorphoses and distribution of the materials. 
The process of the combination of atoms into a solid body which we are now considering, for example, the formation of cellulose, is a performance of work combined with the fixation of sensible heat and with the transformation of kinetic into potential energy. But whence do the colourless cells derive their sensible heat and kinetic energy? When carbonic acid is decomposed and sugar or some other carbohydrate is formed in a green cell, a sunbeam becomes imprisoned and fixed. But this is not the case in cells devoid of chlorophyll, especially in those working in darkness under the ground. The protoplasm of these cells derives the sensible heat and kinetic energy which it consumes or renders latent from the sun, not directly, but by very indirect methods. It obtains them by a portion of the material conveyed to it becoming decomposed, by whose synthesis in the green cells above-ground the kinetic energy of the sun's ray has been changed into potential, and in this way the potential energy becomes again changed into kinetic, and the latent heat transformed into sensible heat. The materials which the green cells manufacture out of inorganic food would be merely an accumulated dead capital lying unused if they were to remain in the condition in which they had been formed. They must be turned to account, dissolved, transformed, and distributed; the impelling forces necessary for this are obtained by a portion of the material manufactured in the green cells undergoing a process which is exactly the opposite of that carried out in their formation. At the very time when carbonic acid is split up, oxygen given out, a carbohydrate formed, and heat rendered latent thereby, carbohydrates are being decomposed, oxygen taken up, carbonic acid excreted, and heat liberated. Of course this process of decomposition cannot extend to the whole mass of the materials manufactured by the green cells. It would indeed be absurd if in one part of the plant those materials became again disorganized and changed into air and water which in another part bad been compounded of these same elements. As a matter of fact, this process of decomposition is limited to but a part of the materials produced in the green cells, and the whole process may be most correctly represented thus: one portion of the materials formed from inorganic food in the green cells is employed in the further growth of the plant body; but this further growth only becomes possible if the other portion supplies the forces necessary for the carrying on of the building. The one process is therefore just as important as the other; they mutually supplement each other, and this supplementing is one of the most important life-processes of plants.

It has been stated that in order to obtain the necessary impelling forces oxygen is taken in, the molecules it attacks are decomposed, and carbon dioxide is liberated. This process is therefore an oxidation, a burning of organic material, and is to be placed in the same category as the burning of carbohydrates, which occurs in animal bodies in respiration. It is called respiration in plants also, although here we do not find special localized respiratory organs as is usually the case in animals. In plants all the living parts can breathe, and to them the atmospheric air, that is to say, the oxygen contained in it, obtains access- 
to roots and tubers, stems and foliage, fruits and seeds, green plants and parasites devoid of chlorophyll, plants with and without stomata, saprophytes and water plants. All these breathe as long as they are alive, and in plants no less than in animals breathing and living can be used as synonymous terms for all practical purposes. The first fundamental condition of respiration is naturally the presence of free atmospheric oxygen. When this is absent, plants, like animals, are suffocated and die. If plants are put under the receiver of an air-pump, from which the air is exhausted, or in a chamber filled with hydrogen, nitrogen, or coal-gas, the streaming movement of the protoplasm in the cells ceases in a short time, foliage and floral-leaves, if they exhibit phenomena of movement in the living plants, become rigid, and if kept for a longer period in the atmosphere without oxygen, the plants die. Even if again brought into air containing oxygen, they can no longer be resuscitated, but remain dead.

The parts of plants surrounded by atmospheric air are never in want of oxygen, but roots often get into an unfavourable position where the quantity of oxygen in the air of the soil is very small, or where the atmospheric air is replaced by other gases. This explains why plants do not prosper in so-called "dead" earth, and why the roots seek principally those loose places of the upper strata of soil which are porous and well-ventilated, and avoid the deeper-lying, badlyventilated, dead ground. The decay of trees which have been planted in towns and parks near gas-pipes, whose roots have been surrounded with coal-gas for some time owing to a leak in the pipes, is also explicable in this way.

Aquatic plants take up the oxygen of the atmospheric air absorbed by the water. Where there is none, vegetable life under water becomes impossible. If anyone, when sending off water plants, tightly corks up the bottle after filling with the necessary water, under the impression that the plants, being still in their element, will thus bear the journey well, he will be sadly undeceived. The small quantity of oxygen in the atmospheric air contained by the water is soon exhausted, and the aquatic plants are suffocated within twenty-four hours, or even in a much shorter time, just like fishes which have been conveyed in a tightlycorked bottle of water.

All plants do not breathe with the same energy, and in any plant a great difference can be noticed in the respiration of the various organs. The floralleaves, possessing no chlorophyll, respire much more vigorously than the green foliage-leaves; underground root-stocks, bulbs, and tubers, also without chlorophyll, to a much greater extent than the green stem. In the green organs of plants exposed to sunlight two processes are carried on, the formation and the splitting up of carbohydrates. The latter process, however, is so obscured by the former, that it can only be observed with difficulty. It has been estimated that in a laurel leaf the amount of carbohydrates formed in any given time is thirty times as great as of those decomposed, i.e. respired.

A great difference is also exhibited according to the stage of development of the individual plant organs. Roots, stems, and leaves breathe much more vigorously 
when young than when fully formed. When seeds are allowed to germinate in damp earth, respiration is at first quite inconsiderable, but when the parts of the seedling begin to elongate, and when the stores of materials furnished by the parent plant are dissolved and used up, respiration becomes very energetic. Later on, when the seedling has grown up so far that it can itself work with the help of its leaves, which have meanwhile become green, respiration again diminishes. The same thing occurs in the development of buds; there, too, the young leaves just emerging from the covering of the bud breathe to a much greater extent than the fully-formed green foliage. That organs which have attained their full size, and are apparently quite inactive, still respire, is shown from the observation that roots and tubers which have been dug up in the autumn and left in a cellar through the winter exhale carbonic acid without any visible outward change. In beet-roots which have been dug up a 1-per-cent decrease of sugar, and an exhalation of carbonic acid corresponding to this decrease, have been observed within two months, a proof that change of materials and respiration can occur even in structures which lie dormant during the winter.

According to what has just been stated about the significance of respiration to the life of plants, it is quite obvious that the energy of respiration which is reckoned by the amount of carbonic acid exhaled from a certain organized mass, or better, by the amount of oxygen absorbed, becomes greater the more vigorously the plant grows, and the quicker it builds up its body, just as a machine requires more fuel the greater the results required from it. If fuel is wanting or not present in sufficient quantity the machine stops, or does not perform as much work as it should be capable of doing. It is exactly the same in living plants. If the respiratory materials are absent, respiration is discontinued even in the presence of oxygen, and life becomes extinguished. If the supply of these materials is insufficient the plants only prolong their existence with difficulty, and their growth will be insignificant in consequence of the insufficiency of materials for carrying on the work. When shoots sprout from the "eyes" of a potato, it is at the cost of the carbohydrates and other materials stored up in the tuber. If this sprouting occurs in the open and the shoots grow up into the daylight, their leaves become green and manufacture new carbohydrates under the influence of the sun's rays; and of these a portion is at once employed as fuel for the further construction of the potato plants, that is to say, it is respired. If, on the other hand, the development of shoots from potatoes takes place in a dark cellar, their leaves cannot become green, and consequently no carbohydrates can be manufactured. The shoots then only continue to grow so long as the respiratory materials stored up in the tuber last; when these are exhausted, respiration comes to an end, and the shoots die off.

An approximate idea of the significance of respiration as an impelling force in those changes of materials whose end is the further growth of the plant may be obtained from a consideration of the following figures. A cubic centimetre of carbon dioxide contains 0.5376 milligramme of carbon, whose burning furnishes 
4677 units of heat. The mechanical equivalent of this is 1,987,725 grammemillimetres. When a carbohydrate is respired, for every cubic centimetre of carbon dioxide exhaled a store of energy is obtained which is equal to $1,987,725$ gramme-millimetres, and therefore by it a gramme weight can be raised to a height of 1987 metres. It has, however, been ascertained that seedlings of poppy (which, when subsequently dried, weighed 0.45 gramme) exhaled 55 cubic centimetres of carbon dioxide in 24, hours, and seedlings of mustard (which, when dried later, weighed 0.55 gramme) 32 cubic centimetres in the same length of time. It can therefore be easily imagined what a large store of energy is obtained by respiration, even although the result, in consequence of various interruptions and obstructions, should fall far behind this estimate.

In comparing the living plant to a machine heated by coal, and trying to measure the work performed by it numerically, we are justified by the analogy of the transactions in the two cases, which are obvious. The comparison suggests itself naturally from the fact that in both cases similar impelling forces come into play, and that in both the necessary store of vital force is obtained by the combustion of carbon. Yet, on the other hand, combustion in a machine and respiration in a living plant are widely different. The peculiarity of plant respiration lies in the fact that materials are combined with the oxygen of the atmospheric air which would not enter into combustion with it at ordinary temperatures outside the living plant. Neither carbohydrates, fats, nor albumins, which are either directly or indirectly affected in respiration by the process of combustion, undergo, outside the plant cell, the alterations and decompositions which are carried on within it, and it may be taken as an established fact that oxygen only operates on them when conveyed to them by means of the living protoplasm. The effect of the transmitted oxygen is also restricted by the living protoplasm to the carbohydrates and other non-nitrogenous compounds which it incloses. Nitrogenous compounds are not respired directly, and the quantity of nitrogen in breathing plants is not lessened. We can only imagine these remarkable correlations as occurring in the following manner. The starch grains and droplets of oil are first rendered soluble, and are then oxygenated by the oxygen brought by the protoplasm; the albumins, on the other hand, are first split up into asparagin and a carbohydrate. The latter alone becomes oxidized, for the nitrogenous asparagin is not only not burnt, but is reconstructed into albumin, with the co-operation of the sun's rays, by attracting the newly-formed carbohydrates of the green cells and combining with them.

If we adhere to this view, it at once becomes evident how important is the co-operation of respiration and the formation of fresh carbohydrates in the green cells. If, in a plant, the production of new carbohydrates should be suspended, the reconstruction of albumins cannot ensue. At first all the respirable materials which yet remain in the plant are used up for the continuance of action, but if the formation of fresh carbohydrates remains unaccomplished, and even the last reserves are consumed, then the plant becomes exhausted, and ceases to breathe 
and live. It has been estimated that a plant, in which the supplies of freshlyformed carbohydrates are lacking, can consume over 50 per cent of its substance by respiration before it perishes from exhaustion. This is the case, for example, in the potato-tubers mentioned, whose stems, developed in dark chambers, become overgrown, i.e. elongate exceedingly, while their rudimentary foliage-leaves remain very small and destitute of chlorophyll. Here, in the dark, no new formation of carbohydrates occurs, but respiration continues as long as any respirable materials yet remain. At length, when everything that can be made use of in this way is consumed, the shoots die off. Their dry weight, however, is only half as much as was that of the tuber from which they sprung; the other half has been completely burnt, i.e. changed into carbonic acid and water, which have rapidly evaporated.

Sunlight is not necessury to respiration, although without it the decomposition of carbonic acid and the formation of carbohydrates cannot take place. Breathing can be carried on in complete darkness. Underground organs: roots, tubers, bulbs, rhizomes, runners, likewise the mycelia and fruit-stalks of the plants classed together as fungi, as well as seeds buried in the earth-all these normally breathe in darkness. Respiration continues throughout the darkest night. That growth, the most important of all the processes stimulated by respiration, is restricted by the influence of light, will be discussed when describing growth; concentrated light produces a rapid oxidation and disorganization of the organ exposed, which, however, must not be looked upon as the respiration of the plant.

\section{DEVELOPMENT OF LIGHT AND HEAT.}

It is to be expected that respiration will be more vigorous in plants the higher the temperature, since the process of respiration is a combustion of carbon compounds, and all combustion is helped by a rise of temperature. As a matter of fact, it has been observed that the exhalation of carbonic acid (that is to say, respiration) also increases with rise of temperature. Of course this is true only up to a certain point. Respiration may commence even at $0^{\circ}$, and reaches a maximum between $15^{\circ}$ and $35^{\circ} \mathrm{C}$. according to the species, but beyond that it quickly diminishes. It entirely ceases at temperatures which produce coagulation of the proteids, and which are followed by the death of the living protoplasm.

When once respiration is started, the oxygen necessary for the combustion of carbohydrates is derived from the surrounding atmospheric air. But the first incitement to respiration does not proceed from this, or in other words, the absorbed oxygen does not furnish the first stimulus to respiration. Dead plants into which oxygen is made to enter do not breathe any more than do butterflies which have been suffocated by withdrawal of oxygen, and then subsequently brought into the fresh air. Oxygen cannot produce those movements of the atoms which are peculiar to life either in plants or animals which have been completely suffocated. Since only living plants can breathe, respiration must be brought about by a force which is liberated in the living protoplasm, by that 
specific force which must be designated as vital. The first movement, i.e. the first chemical process with which respiration commences, seems to be a splitting up of the albuminous molecules in the living protoplasm, the same process as that by which albumen is separated into asparagin and a carbohydrate, perhaps into asparagin, a carbohydrate, and carbon dioxide. The next thing, of course, would be a withdrawal of oxygen from the air, but it should be noted that this is only for the purpose of continuing the metabolic changes, which have originated spontaneously in the living protoplasm.

Heat likewise is liberated in all combination of oxygen with other substances, especially in every combustion of carbon compounds. This heat is not always easily demonstrable in the plant organ in which it is set free. The heating of the respiring tissue is counteracted by the evaporation of water and by radiation in organs above-ground, particularly in the flattened, outspread foliage-leaves. Carbon is also reduced in the green foliage during the day under the influence of sunlight, and this is a process which goes hand in hand with the fixing of sensible heat. Now, since this process masks the respiration in the green leaves to a certain extent, it is intelligible that the heat liberated by respiration in these organs is but seldom perceptible, and that as a rule green leaves actually feel cool. It is even probable that the pleasant coolness under a shady canopy of leaves is not solely due to the interception of the sun's rays, but that the imprisonment of these rays and the fixation of heat during the manufacture of the primary carbohydrates also shares in the cooling of the air surrounding the leaves. But where these conditions are out of the question, the heat liberated can be demonstrated in respiring vegetable organs just as in animal bodies; and if respiring green leaves could neither transpire, nor radiate heat, and if, moreover, a supply of carbohydrates were stored up in them, the heat liberated by respiration would make itself evident in the immediate neighbourhood. This applies still more to subterranean bulbs and tubers in which transpiration and radiation are not only partly or entirely absent, but which are incapable of manufacturing carbohydrates for themselves as they have no chlorophyll, and which, consequently, render no heat latent.

Germinating seeds, and seedlings without chlorophyll behave in the same way as these respiring underground organs, provided that they are protected against evaporation and radiation. Barley-corns which have begun to germinate and are respiring vigorously raise the temperature of their environment quite noticeably if they lie heaped together so that the heat liberated becomes thus concentrated. It is well known that malt is germinated barley, and in the preparation of malt heaped-up barley-corns are caused to germinate. In this process the temperature of the immediate neighbourhood rises $5-10^{\circ} \mathrm{C}$. above the temperature of the air which surrounds the piles of barley-corns outside. The liberation of heat in fungi is also very instructive. These derive the organic compounds from which they build up their mycelia and fruit bodies from other living organisms, or from the decaying remains of dead plants and animals. The receptacles often vol. I. 
develop very rapidly to a considerable size, and connected with this rapid growth there is always a rapid movement of the food absorbed by the mycelium, combined with an energetic respiration. Respiration is carried on chiefly at the periphery of the receptacle-in the mushrooms especially in the hymenial layer, which is very well protected from evaporation and radiation by its position on the lower side of the cap. Transmission of the food, and in particular of a large amount of water, takes place through the stalk which bears the eap. Numerous observations of fungi growing in their natural free condition, and rising but little above the soil, have invariably shown this result: the rise of temperature in the tissue of the cap is most pronounced where respiration is carried on most actively, i.e. in the hymenial layer. It is less in the central portion of the cap, and least in the stalk, through which the watery fluid travels at a temperature which differs but slightly from that of the surrounding earth. Respiration, of course, cannot be considerable here. For example, in Boletus edulis, from its size and shape particularly well suited for these investigations, the following results were obtained while the temperature of the surrounding earth was about $13^{\circ} \mathrm{C}$.: temperature of the stalk, $14 \cdot 2-15 \cdot 6^{\circ}$; temperature of the body of the cap, $15 \cdot 2-16 \cdot 8^{\circ}$; of the hymenial layer, $16 \cdot 7-18 \cdot 1^{\circ}$. Further developed (but still quite fresh) fructifications exhibit higher temperatures than younger ones which have just appeared above the ground. Observations on other fungi of the Hymenomycetes yield like results. When the temperature of the surrounding earth was $12 \cdot 2^{\circ}$ Lactarius scrobiculatus exhibited in its stalk a temperature of $14.8^{\circ}$, and in its cap of $16.0^{\circ}$; Agaricus muscarius in its stalk $14.2^{\circ}$, and in its cap $15 \cdot 2^{\circ}$, while the temperature of the surrounding soil was $13.0^{\circ}$; Hydnum imbricatum, $13.0^{\circ}$ in the stalk and $14.5^{\circ}$ in the cap, while the surrounding earth showed a temperature of $12 \cdot 2^{\circ}$. The peculiar shape of the cap in these last-named fungi is not well adapted to a separate measurement of the temperature in the body of the cap and in the hymenial layer, but it is probable that a slight difference exists between them, similar to that found in Boletus. The puff-balls belonging to the Gasteromycetes also exhibit a considerable rise of temperature above that of their surroundings in the respiring portions of their fructifications. Thus in Lycoperdon coelatum a temperature of $15.8^{\circ}$ was observed in the spherical receptacle shortly before dehiscence, while the temperature of the surrounding soil was only $12 \cdot 2^{\circ}$.

The liberation of heat appears especially noticeable, too, in respiring flower-buds and in the rapidly-growing stalks which bear them, as well as in opened flowers. If the flowers are small, and if there are but few of them at the end of the stem, or if only a single small flower is borne at the end of a delicate stalk, the heat liberated may easily escape observation; but under very favourable conditions it makes itself readily manifest, and gives rise to a phenomenon so strange and unintelligible that everyone on observing it for the first time is surprised and puzzled. I refer to the fact that small and delicate flowers grow buried beneath the snow, and obtain the space they require by melting the hardened snow. The Alpine Soldanella is a very marked instance in point. As the snow melts and the 
tricklings therefrom moisten the earth below, the Soldanella plants are aroused from their winter's rest. Their little arched flower-stalks begin to elongate and come into contact with the hard under surface of the snow, though the temperature here is zero. Growth is carried on at the expense of the supplies of materials obtained by the Soldanellas in the previous summer, which had been stored up partly in the evergreen leathery leaves lying flat on the ground, and partly in the short root-stock embedded in the soil. The reserves are employed as substances for building, and a portion of them is respired, in order that it may be possible to dissolve the rest, to bring them to the places where they are required, and to obtain the force necessary for the work. The heat liberated by this respiration melts the granular ice covering in the immediate neighbourhood of the flower-buds. In consequence of this a cavity is formed in the ice above each bud, or rather, each bud becomes overarched as if by a tiny dome of ice. But the stem continues to grow in height; and the flower-bud borne on it, which is respiring and giving out heat, is accordingly raised up in the dome-shaped hollow space and pushed into it. There it promotes afresh the melting of the ice and an extension of the cavity, and thus actually bores a path for itself upwards through the ice-strata. This goes on until at length the respiring and heat-producing Soldanella bud has melted an actual canal through the covering of hardened snow, and makes its appearance above, the stem looking as if it had been stuck into the snow. The flower-buds now open and the pretty violet bells sway about in the wind. Naturally the snow will be penetrated first where it is thinnest, i.e. near the margin, where also the melting from above proceeds most rapidly. Consequently it is the edge of the snow-field mainly which is riddled, the Soldanellas growing up through the holes. It is not at all uncommon to find places where 10-20 flowers appear on the border within a stretch a metre long. On looking closer and making cuttings through the ice, all the stages of development described may be seen side by side. Two other phenomena, however, are not a little surprising. Here and there are to be found single Soldanellas whose buds have already opened before they have emerged above the ice-covering. These Soldanellas actually blossom in a small eavity of the ice, and remind one of plantorgans or insects inclosed in amber or small coloured splinters which have been fused inside glass balls. This sub-glacial blossoming of the Soldanellas is not limited, strangely enough, to the opening of the corolla; the anthers actually dehisce and liberate their pollen.

What also surprises us very much on closer inspection is the fact that the holes in which the flower-stalks are situated narrow like a funnel towards the base, so that there the ice touches the stem, or, in other words, that the canal down below is completely filled by the stem. When it is remembered that the flower-bud which melted the ice and formed the canal had a diameter at least three times as large as that of the stem, it would be expected that the stem would be placed in the centre of a comparatively wide hole. But, as stated, this is not the case, and the phenomenon can only be explained by supposing that the porous granular ice forms a plastic mass, and that the granules displaced by the melting sink down in accordance 
with the law of gravitation, unite together where a boring has taken place, and again form a compact mass in consequence of the regelation of the lower strata. It has still to be mentioned that the green leaves of the Soldanellas, which lie flat below the snow and ice, becoming flaccid during the growth of the flowers, and that the reserve materials stored up in them are completely used up by the growing stem and flowers. The green leaves then become wrinkled and perish, while new leaves develop after the snow has melted. These provide themselves with reserve food in order that in the next period of vegetation the growing stem and flowers may be efficiently nourished.

Here and there with the flowers of Soldanellas are found the young, but nevertheless yellowish-red, foliage-leaves of Polygonum viviparum, which grow up from below into the ice, and occasionally melt holes in it close to the edge of the snowfield. The white flowers of Ranunculus alpestris growing in company with the soldanellas in the same habitat have, on the other hand, not attained to the capacity of growing through the ice, and need as an incitement to growth a temperature which is rather above $0^{\circ} \mathrm{C}$.; in consequence of which they always open their flowers first in places from which the snow has vanished a short time before.

The amount of the heat set free by the small flower-buds of soldanellas might be estimated by the quantity of ice melted, but so many sources of error enter into a calculation of this kind that the numbers obtained cannot lay claim to much accuracy, and we must be satisfied with the fact, even although it is not verified by figures based on a calorimetric experiment.

The melting of the ice by the heat liberated in the respiration of soldanellas is also of the greatest interest, since it furmishes a proof that single, small, extremely delicate flowers warm not only their own tissues but also their environment, and that the heat liberated in them does not become perceptible only because, as already remarked, it is counteracted by evaporation and radiation which are carried on at the same time, and because the respiring flowers are usually surrounded by atmospheric air, i.e. by a medium which is movable, fluctuating, and unstable. The air which in one moment is warmed by the respiring leaves is carried far away in the next instant, and is replaced by other air. This is the case especially in shallow flowers with recurved leaves, or in salver-shaped corollas widely opened above, round which there cannot be said to be any stagnation of air. But if the flower has the form of an inverted bell, as in the Foxglove, Gloxinias, and most campanulate flowers; if the floral-leaves bend upwards like a helmet, as in the Monkshood; if the flowers are tubular, inflated at the base like a flask, or pitcher-like as in Aristolochias, or form deep goblets as in the Cactaceæ and many gourds-then the air in the inclosed space is scarcely at all disturbed, there is stillness within the flower, the air there collected and warmed is retained almost unaltered in its quiet corner, and is not very easily replaced by other air.

On cool days a rise of temperature above that of the surrounding air can be usually perceived in the interior of such flowers, even when they stand quite alone. In an Alpine meadow the interior of a flower of Gentiana acaulis in the morning 
shortly before sunrise exhibited a temperature of $10.6^{\circ} \mathrm{C}$. when the temperature of the surrounding air was $8.4^{\circ}$. On a mountain meadow under a cloudy sky and in calm air the interior of a flower of Campanula barbata showed a temperature of $166^{\circ}$, and not far off on the borders of a forest the interior of the helmet-shaped sepal of Aconitum paniculatum, 14.6 $6^{\circ}$, while the temperature of the outside air in both instances did not exceed $13 \cdot 2^{\circ}$. The temperature of the air in the neighbourhood of a respiring plant shows a much greater rise if numerous, small, thicklycrowded flowers are inclosed in a common sheath, and especially when the space inclosed is undisturbed. In the same mountain meadow in which the temperature of the interior of the ball in the above-mentioned campanula (Campanula barbata) was tested, the Carline Thistle (Carlina acaulis) was also in full bloom. As the sky was cloudy, the capitula were closed, i.e. the apices of the stiff, involucral leaves were bent together, and formed a hollow inverted cone over the flowers. A thermometer placed between these bracts and pushed down as far as the flowers, showed a temperature of $20.4^{\circ}$, the temperature of the surrounding air being $13.2^{\circ}$, the difference, therefore, was more than $7^{\circ} \mathrm{C}$.

In palms, whose numerous small crowded flowers are covered by large floral sheaths or spathes, the air within these coverings exhibits a rise of temperature which is so noticeable that it can be felt by placing the bare hand inside. The same thing occurs in the aroids. Here numerous small flowers are united into a spike on a thick fleshy axis, forming the so-called spadix, and each spadix is surrounded by a bract which at first is twisted together like a conical paper bag, being often distended like a barrel or inflated like a bladder. It is soon formed into the characteristic shape, but always incloses a cavity whose air is hardly ever disturbed by the influence of other air currents. With care a thermometer may be introduced into this cavity, and the temperature shown by it may be compared with that of the surroundings. For example, it was found when the temperature of the outer air was $25^{\circ}$, that in the interior of the spathe of the Brazilian Tornelia fragrans was almost $38^{\circ}$. At the same air-temperature the interior of the spathe of Arum cordifolium, in the island of Bourbon, exhibited a temperature of 35-39 . But the highest temperature has been noticed in the Italian Arum (Arum Italicum). This plant is very common in the region of the Mediterranean flora, and is frequently to be met with in vineyards under bushes, and even in hedges and roadsides. Its spadices, surrounded by large pale-green spathes, push their way in the spring through the soil like inverted conical bags; the spathe begins to open between 4 and 6 o'clock in the afternoon, and at the same time a peculiar fragrance, like wine, becomes noticeable in the neighbourhood of the plant. If a thermometer is introduced into the cavity of this spathe, it is shown that while the temperature of the outside air is about $15^{\circ}$, that in the interior has risen to $40^{\circ}$, sometimes even to $44^{\circ}$. These Aroideæ therefore exhibit a temperature in the neighbourhood of their respiring flowers which exceeds that of blood-heat.

In proportion as the energy of respiration increases with the rising temperature of the surrounding air from morning till afternoon, the temperature in the interior 
of the flowers also rises, as shown by the following observations which were conducted in a place in the garden shaded from the direct influence of the sun's rays:

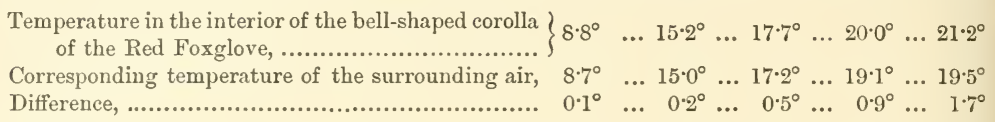

While the liberation of heat occurs in all living plants, and is a natural consequence of respiration, i.e. of the combustion of carbon compounds, the development of light, which in other respects appears to be in many ways connected with the processes of combustion, is observed in living plants but seldom. It is only recognized with certainty in the Hymenomycetes, a group of fungi in which the rise of temperature during respiration has already been described. But even of these Hymenomycetes only relatively few are luminous, and these few only in certain stages of development. Most frequently the luminosity occurs in the mycelium of mushroom-like forms (Agaricineoe), which permeate the wood of old tree-trunks and the creeping roots of trees on the surface of the damp forest ground. This mycelium forms thicker dark strands, frequently joined together by cross-connections, which penetrate principally between the wood and the cortex, and these form most characteristic nets and lattice-works; it also consists of very slender dark threads, which take up their position in the wood usually at right angles to the long axis of the trunk; and, finally, there are extremely delicate colourless threads which grow through the woody cells in the manner shown in fig. 32. These actually permeate the entire wood, and are only perceptible to the naked eye when they are woven into net-works, and then are seen as whitish fringes and membranes situated on the sides of the holes formed in the disorganized wood.

It is these fine threads and webs of the mycelium which exhibit the remarkable illumination. Where they completely invest the wood-cells, it looks as if the wood itself were luminous, and we commonly speak of luminous wood and the luminous decay of tree-trunks. There is no doubt that the luminosity is exhibited by the mycelia of various agarics, which destroy the wood of firs and other foliage-trees. Usually the Rhizomorph (Agaricus melleus) is alone pointed out as the cause of the luminosity in wood, since this species is widely distributed; and where it has established itself sends up every year many receptacles, so that there is no difficulty in determining the species. But since luminous wood is also observed in the pine forests of higher mountain districts where the Rhizomorph is no longer found, it must be concluded that the mycelia of various other agarics, whose species cannot be determined on account of the absence of fructifications, exhibit the same phenomenon. The light is best seen in the open, in midsummer and autumn, after many days of wet weather, when the wood permeated by the mycelium has been moistened by the rain. But the moisture absorbed by the wood must not exceed a certain amount. Too much saturation prevents the phenomenon of luminosity just as much as excessive dryness. If the wood is removed from the place where it shines so well, the luminosity rapidly diminishes, 
and ultimately entirely vanishes, although apparently the relations and conditions of life are exactly the same as before. I have repeatedly taken up luminous wood at night, and having brought it home, have tried to reproduce as far as possible the conditions under which the luminosity existed in the open; in the first night the light was unweakened, but after twenty-four hours it had usually disappeared entirely. If the luminous wood is placed in a closed space where the renewal of the air, i.e. of oxygen, is not carried on to a sufficient extent, the luminosity soon ceases. A rise of temperature is not favourable to its continuance, principally from the fact that a higher temperature brings about an alteration in the hygrometric condition of the wood. In pure oxygen the wood shows a decrease rather than an increase of light. In the depths of the forest the luminosity may be observed day after day for more than a week on the same trunk, if the conditions of humidity remain the same.

It is difficult to compare the light emitted from the mycelium with any other. It is not so green as that of glowworms, and has not the brilliancy of the phosphorescence of the sea; it is a dull white light. It most resembles that of pure phosphorus held under water. In the gloom of the forest it has a strange and therefore uncanny appearance, and the "will-o'-the-wisp" may, in part at any rate, be attributed to luminous wood. If a decayed tree-trunk penetrated by the light-giving mycelium is vigorously struck, so as to split it into hundreds of fragments, which fly out in all directions and fall scattered on the ground, each splinter becomes luminous, and the dark forest ground seems to be strewn with dots of light. The luminosity of these fragments, however, comes to an end before the next night.

The Rhizomorph and other allied agarics only exhibit the luminosity in their mycelium, their fructifications remaining dark under all circumstances. In a series of other agarics, viz. in the Brazilian Agaricus Gardneri, in Agaricus igneus, a native of Amboina, in Agaricus noctilucens, living in Manila, and in Agaricus olearius, which is widely distributed through the Mediterranean floral district, the actual fructifications emit light, usually from the hymenium developed on the under side of the cap, but more rarely the stipe also which bears the cap. The light produced by these fungi is like that from the mycelium of the agarics described previously, and the external conditions under which it occurs are also similar, except that the hygrometric state has not such a noticeable effect on it as on the luminous wood permeated by mycelial threads. At least in Agaricus olearius, a mushroom which grows among the roots of olive trees and forms its golden-yellow fructification in late autumn, the luminosity is to be seen equally well in dry and wet weather. As soon as the temperature falls below $+3^{\circ}$, the light immediately ceases; it is best at $8-10^{\circ}$, and under higher temperatures it does not increase but gradually diminishes. If oxygen be kept away or withdrawn from the air, the luminosity immediately vanishes, but as soon as the atmospheric air is again restored, the phenomenon reappears. Dying agarics become less and less luminous, and their light is extinguished at their death. It is to be noted that not only agaries with luminous hymenia, but also those with luminous mycelia, emit light both by day and night. 
On fine days in the open the light is not seen, but as soon as these structures are brought into a dark room, the phenomenon of light is to be seen, even during the day. The luminosity of the night is not increased by sun-illumination during the day, and consequently the phenomenon has nothing in common with that peculiar phosphorescence exhibited during the night by fluor-spar, which has previously been exposed to sunlight.

There are certain organic substances which shine in alkaline solutions when oxygen is present. It seems natural to suppose that such materials are formed in the agarics mentioned, and that oxygen is conveyed to them in respiration, thus producing the phenomenon of light. At any rate this would be the simplest way of explaining the luminosity. As to the advantage accruing to the plant itself, we can only form surmises. It seems most probable that the fungus-flies and beetles which deposit their eggs in the mycelia and fructifications of Hymenomycetes, and which are connected with the distribution of their spores in a manner to be described in detail later, are thereby guided to the fungi in the dark of night. Many of these flies and beetles only fly at night, and, like so many winged nocturnal animals, direct their path towards a luminous object. It may be, therefore, that the light proceeding from the agarics cited serves as an allurement and guide to the night-flying insects, just as the odour and brilliant colouring of other Hymenomycetes attracts the fungus-flies and beetles which swarm in broad daylight.

\section{FERMENTATION.}

About thirty years ago the difference between plants and animals was formulated as follows:-Plants transform kinetic into potential energy, and form organic compounds by the reduction of inorganic food, especially from carbonic acid, nitric acid, and water; animals transform potential into kinetic energy, and decompose and burn by respiration the organic compounds, formed by green plants, which serve them as food. This distinction, however, only holds good in part. On the one hand, plants devoid of chlorophyll are not taken into consideration, and on the other, it has been established that green plants also breathe, and therefore transform potential into kinetic energy. The respiration of plants does not differ either in its method or in its object and significance from that of animals. In both cases the living protoplasm withdraws oxygen from the air in order to convey it to certain expressly prepared carbon compounds which have been rendered combustible, and in both cases these carbon compounds are burnt in order that the necessary impelling forces may be obtained for further life and growth. But the analogy between plants and animals holds still further in this respect. When animals which are tenacious of life, e.g. frogs, are placed in an atmosphere containing no oxygen, they do not immediately perish, and do not at once cease to exhale carbon dioxide, consequently they still convert a certain amount of potential energy for a short time, by the combustion of carbon compounds in their bodies. They cannot derive the oxygen necessary for this from the surrounding air; there 
is nothing left for them except to obtain it from the organic compounds of their own bodies. This cannot be carried on permanently, and if the frog is kept for a long time in an atmosphere without oxygen, it will at length die. For a short period, however, it is able to prolong its life in the way indicated. Exactly the same thing is seen in plants. When placed in a chamber from which free oxygen is absent, they do not immediately die, but endeavour for a short time to retain their life by utilizing combined oxygen, by withdrawing it from nitrates which have been absorbed with food, or from the organic compounds of their own bodies, richly furnished with oxygen. The oxygen obtained in this way is able to replace that usually derived from the environment, and can also bring about a combustion of carbon compounds; it can therefore provide the kinetic energy necessary for the continuance of life. Carbon dioxide is then exhaled from plants, even in an atmosphere without oxygen, and heat is liberated just as in normal respiration. But this abnormal source of energy does not last very long. If free atmospheric oxygen continues lacking, the plants exposed to such unaccustomed conditions at length perish from exhaustion and suffocation.

But it is also possible that living plants may exist in a region which is indeed devoid of free oxygen, but in which combined oxygen is present. Let us suppose that a plant, hitherto surrounded by atmospheric air from which it obtained free oxygen for use in respiration, has been plunged into a sugar solution, in which, of course, free oxygen is absent, but which contains a large quantity in combination with carbon and hydrogen in the form of sugar. Would such a plant be able to wrest the oxygen from the sugar and to utilize it for itself? In most cases certainly not. But in a few instances the living protoplasm has the power of splitting up the fluid oxygen-containing compounds with which it comes into contact, and can so obtain the oxygen necessary for the continuance of its life. It can also make use of other materials liberated from combination in the decomposition. This process has the greatest resemblance to respiration, carbon compounds are actually burnt with the help of the derived oxygen; carbon dioxide is exhaled, and heat is liberated. The plant, the living protoplasm of which accomplishes all this, maintains itself alive, prospers, and even grows and multiplies in a surprising manner. This process, however, is not called respiration, but is known as fermentation.

Of course the plants producing fermentation must not be supposed to include large leafy structures. On the contrary, they are all very insignificant and belong exclusively to spore-plants which are devoid of chlorophyll, and which are generally classed together under the name of fungi. In particular there are the four allied families, Bacteria, Yeasts, Moulds, and Basidiomycetes, of which many species in certain stages of development are capable of inducing fermentation.

Bacteria, which are also called Fission-Fungi or Schizomycetes, are the smallest of all living organisms, and the question has repeatedly arisen as to whether they are to be regarded as independent organisms, or as organized portions of dead, decomposing protoplasm. The discussion of this question will be left to the second 
volume. Here it is sufficient to remark that bacteria appear as spherical, oval, or rod-like cells, which develop by repeated transverse division into chain-like or filamentous structures, very much resembling hyphal threads. These chains of cells break up, however, sooner or later, into their individual members, and then look as if they had been split into fragments, this appearance accounting for their name of "Fission-Fungi". In this way arise colonies of irregularly accumulated cells which are frequently embedded in a mucilaginous matrix. Many bacteria can live and multiply without taking free oxygen from the air. They obtain the materials necessary for this by setting up a fermentation in their immediate neighbourhood, i.e. a splitting up of carbohydrates and nitrogenous compounds. Fermentation gives rise to very different products, and makes itself evident in very different ways, according to the composition of the body attacked by the bacteria, and according to the species to which the bacteria, which are commencing their destructive activity, belong. In many instances pigments are produced, in consequence of the decomposition, which colour the attacked body yellow, red, violet, or blue; at another time, as, for example, in the souring of milk, a molecule of milksugar is decomposed into two molecules of lactic acid; or, by the ferment action of the Bacterium aceti, acetic acid is produced from alcohol; again in another instance, sugar is split up into dextrin, mannite, and carbonic acid, by a species of Bacterium, in the so-called viscous fermentations. One of the commonest fermentations is that to which albuminous compounds succumb, known as putrefaction. The albumens are decomposed by the action of one or perhaps several different species of bacteria into tyrosin, leucin, various amines, volatile fatty acids, ammonia, carbon dioxide, sulphuretted hydrogen, hydrogen, and water; and some of these make themselves evident by their offensive odour in a most unpleasant manner. To this class, too, belong the most notorious of all bacteria, which give rise to a decomposition of the liquids in living human and animal bodies, which deprive the blood of oxygen and bring about in it various other decompositions of organic compounds, and which are regarded as the cause of epidemic and endemic diseases. Contagions and miasmas are indeed for the greater part, if not wholly, bacterial, and the species which produce splenic fever in ruminants, diphtheria, small-pox, and cholera in man, are of such great interest that a whole section will be dedicated to them in the next volume.

The various species of yeast, which are called Saccharomyces, consist of spherical or ellipsoidal cells, which are much larger than the cells of bacteria, and also multiply in quite another way. They increase by sprouting, i.e. knoblike outgrowths arise on the surface of the multiplying-cells which rapidly enlarge, so that each outgrowth in a very short time is equal in size to the cell from which it originated. The daughter-cell thus formed is detached from the parent-cell, and may now itself produce daughter-cells by sprouting. Occasionally several successive buddings remain joined together, and then form colonies which somewhat resemble the prickly pears or opuntias in miniature. Yeast produces alcoholic fermentation. It causes grape-sugar to split up into alcohol and carbon 
dioxide, the process also giving rise to a small quantity of succinic acid and glycerine. This fermentation is never very noticeable in living plants in free nature; there it is at any rate only carried on to a small extent. It is very important in the extensive artificial production of alcoholic beverages, for example, of wine, cider, beer, brandy, "pulque", rum, and many more, from grapes and other fruit, and from grape-sugar, obtained from starchy seeds, tubers, and roots.

Moulds consist of colourless, elongated, thin-walled cells, which appear to the naked eye like extremely delicate threads. These divide up by the intercalation of transverse walls, but they do not separate into their individual elements like the bacteria. The threads multiply very rapidly, and frequently numerous threads are crossed and intertwined like the threads of a cobweb, forming a loose, white net-work. They generally dwell on damp or fluid substrata, and closely invest them with their crowded threads. They also penetrate into the interior of these substrata. The cells which make their way into sugary solutions assume another form; they remain short, and increase by sprouting. The bud-forms of the mould are often so like Yeast that they are mistaken for it. Only the parts of a mould which respire and are in contact with the oxygen of the air develop spores, these being usually distributed by currents of air; the parts submerged in a fluid to which the free oxygen of the air has no access do not form spores, but they multiply with incredible rapidity, just like Yeast and bacteria. This multiplication is carried on at the expense of the organic compounds contained in the liquids or succulent bodies attacked by the mould. The changes in the objects attacked are not limited to the acquirement by the mould of as many organic compounds as it requires for food, but the whole mass becomes decomposed and destroyed, and finally is wholly converted into carbon dioxide, water, sulphuretted hydrogen, ammonia, and other volatile substances-a process which has already been described. This decomposition brought about in the absence of oxygen must be termed fermentation. If the fluids and succulent bodies attacked by the moulds contain nitrogenous compounds, they make their presence known by the unpleasant odour they give off when undergoing fermentation, i.e. putrefaction. If, on the other hand, non-nitrogenous compounds are fermented by a mould, alcohol may be produced. In sweet, fresh fruit which has been attacked by moulds, the cells of the mould which permeate the succulent tissue produce a fermentation of the juices by which alcohol and ethereal oils first arise as products of decomposition, and by which the characteristic smell of putrescent fruit is produced. It is ascertained that one species of mould, Aspergillus niger, when on the surface of a tannin solution, consumes the tannin in the presence of atmospheric air, by which means carbon dioxide is formed. When this same species is submerged in the fluid, and has no free oxygen at disposal, it splits up the tannin completely into glucose and gallic acid. It has also been shown that mould cells which get into the blood of living men and animals cause it to decompose as do bacteria, i.e. they produce severe diseases, sometimes ending in death. Many species of mould not only bear the high temperature of the blood without injury, but 
even develop very luxuriantly there. The principal genera whose species cause fermentation are Mucor, Aspergillus, Penicillium, Botrytis, and Eurotium.

Finally, in addition to bacteria, yeasts, and moulds, the mycelia of those fungi, which are called Basidiomycetes (in reference to their characteristic reproduction, which will be described in the next volume), can induce fermentation. The thread-like cell-chains of these mycelia look like mould-structures; they grow through and permeate the dead bodies of plants and animals, dung and refuse, and black meadow-soil, the humus of the forest, and especially the trunks of dead trees. But living plants also, especially the wood of living trees, may be penetrated by these mycelia, and the tree ultimately killed in consequence. When the mycelial threads penetrate into the wood of a living or dead tree (see fig. $32^{3}$ ), they are not satisfied with merely piercing the cell-walls, and destroying those places only with which they come immediately into contact, and absorbing the results of the destruction as food; on the contrary, we have an extensive decomposition, with which is associated a liberation of carbon dioxide, water, and various volatile materials, not well known, which give rise to a peculiar musty smell. The wood loses weight, becomes rotten, and wholly transformed into a mass which on drying crumbles to powder, or into a fibrous asbestos-like substance. Finally, it disintegrates into dust. In popular language this fermentation produced by the mycelium is called "rotting". By many basidiomycetous mycelia the wood is not only changed into a powdery, but even into a liquid mass, as, for example, by the mycelium of the detested Dry-rot, or Wine-cask Fungus.

All these fermentations, whether caused by the mycelia of Basidiomycetes, the bud-forms of mould, by yeast, or by bacteria, have one thing in common, that they have been set up by ferment-causing cells, i.e. by the active living protoplasm within them without the excretion of any special chemically-active materials which would come directly into contact with their surroundings. The living protoplasm of the mycelia named, of bacteria, yeast, and mould, itself remains chemically unaltered; it acts most energetically in the immediate neighbourhood, less vigorously further off, and its effect diminishes with increasing distance. The effect proceeding from the ferment-cells might be compared with the concentric waves produced on the surface of water into which a stone has been thrown. A hypothesis has been formulated, according to which the groups of atoms in the ferment-protoplasm are supposed to be oscillating as long as it is alive, and it is imagined that these oscillations are propagated and conveyed to the environment after the manner of a wave-motion. Alterations in the construction of the shaken molecules, displacement of the atoms, and decomposition of the compounds in question, would thus result from the shaking so produced. It has even been estrmated that the vibrations which proceed from the living protoplasm of, e.g. Yeast cells, are propagated to a distance of $\frac{1}{50} \mathrm{~mm}$. from the surface of the cells, and that they shake and alter the arrangement of the molecules of sugar even at this distance. The shaking would of course vary according to the specific constitution of the protoplasm. It may be assumed that vibrations differing in quality 
proceed from different fermentative agents, and that consequently diverse decompositions are produced by different bacteria.

This much is certain, that in fermentation, as in respiration, a certain amount of kinetic energy is set free by the living protoplasm and transmitted to the environment, and that in this respect fermentation and respiration behave alike. Thus it also becomes evident that fermentation and respiration can replace and supplant one another. In many moulds, as, for example, in Mucor racemosus, this substitution is very noticeable. If the mycelial threads rise up from the liquid, which serves for its substratum, into the air, and if they can draw oxygen from the surrounding atmosphere, then respiration takes place; but if this mould is submerged in the liquid, so that it can no longer obtain free atmospheric oxygen, then the cells become altered, pass into the sprout form, and instead of respiration we observe in them the ferment-action described. Submersion may be regarded as an abnormal condition for these moulds, and perhaps for Yeast also, but for bacteria it is scarcely so, and for them respiration must be regarded rather as the abnormal condition.

I cannot close these speculations without again repeating that fermentation and respiration are only carried on by living protoplasm, that the movements which thus proceed from the protoplasm cease immediately life is extinguished, and that these movements must be assigned to that force of nature acting in the protoplasm for which I claim the old term "vital force". 


\section{GROWTH AND CONSTRUCTION OF PLANTS.}

\section{1.-THEORY OF GROWTH.}

Conditions and Mechanics of Growth.-Influence of Growing Cells on their Environment.

\section{CONDITIONS AND MECHANICS OF GROWTH.}

Whoever wishes to germinate seeds must moisten the earth selected as soil, or else must supply water to the seeds in some other way. The seeds absorb the water; the embryo bursts its covering, sends out rootlets into the ground, and its stem and leaves grow up towards the light. The young seedlings must now be diligently watered if they are to flourish and increase in bulk, for they require for their growth an astonishingly large amount of water. Other plant organs which it is desired should grow or be kept in vigorous development are like the seeds, and the suitable watering of cultivated land is, and always has been, one of the fundamental conditions of plant culture. In uncultivated districts the dependence of growth on the water supply appears no less remarkable. Where vegetative activity is brought to a standstill not by the cold of winter, but by heat, the commencement of the rainy season is, each year, the signal for the revival of growth. The amount and duration of the rainfall govern in a most striking way the whole progress of plant development. As soon as the first moisture soaks through the soil, after a long drought, the plants wake up from their lethargy, the dry, sunburnt landscape becomes adorned with vivid green, and the luxuriance of the shoots and leaves arising from the seeds and buds stands in strict proportion to the quantity of water daily supplied to the growing plants.

Why do plants require these quantities of water? The answer to this question has already been partly given in a previous section of this book, when it was shown how the absolutely necessary mineral food-salts were taken up by means of water; how the water in which the food-salts are dissolved is conveyed by root-pressure and by suction to the place of consumption. But this is certainly not the only significance water has for plants, for it would leave unexplained why growing seedlings which cannot yet absorb mineral food from the earth, and which do not even require it, still consume so much water. It must also be remembered that those chemical processes in vegetable cells in which mineral foodsalts are worked up do not yet themselves constitute growth, but only a preparation for growth. Mineral salts play an important rôle in the transformations going 
on in the living cells, and the manifold changes in the production of organic compounds of the food taken in from outside, and in the preparation of these compounds for building materials. But they are not directly concerned in the insertion and fixing of the building materials in the living cell-body, in the further growth of protoplasm, and in the increasing dimensions of the growing cells, which lastnamed processes alone may be looked upon as growth. Exactly how far water is concerned in growth will be described in the following lines.

Although only very little is known with regard to the minute structure of the protoplasm of the cell, yet this much is beyond question, that it consists of firmer and softer parts, which form an extremely complicated net-work, ever varying in structure from species to species, and with meshes filled with very many different substances, with water, fluid carbohydrates, albuminous compounds, dissolved salts, \&c. It may also be imagined that fluid substances can be interpolated in the network, resembling it in structure and in consistency at the moment of insertion; that is to say, which receive the same molecular arrangement, and so become an organized portion of the cell-body. The cell-wall also, at the periphery of the protoplasm, must possess a structure which renders it possible that between the already-formed firm portions fluid molecules can be inserted, which then assume the properties of those established portions. This insertion, however, presupposes an extension of the firm parts already present, a separation of the groups of molecules of the organized structures, and a place for the particles to be inserted, and, on the other hand, repelling and attractive forces which control the portions to be introduced.

We are now prepared to admit that here a very important part must be assigned to the turgidity of cells. As has been shown, the cell-sap of growing cells is acid, and the acids and acid salts contained in it attract water from their surroundings with considerable energy. The water thus brought into the vacuoles of the protoplasm exercises a strong pressure on the peripheral layer, and indeed on the cell-wall as well as on the protoplasm, which pressure first of all causes an extension of these layers beyond the normal cohesive limit. By the elasticity of the extended layers obviously a pressure is exercised on the fluid in the interior, and this condition of inutual tension is called turgidity. In order to explain the existence of this turgidity, it must be taken for granted that the water conveyed into the vacuoles of the protoplasm by the attraction of the acids and acid salts does not go back again, in spite of the pressure it exercises on the surrounding layers; that it rather is held fast by the molecules of sugar and albumin in the protoplasm. Experience confirms this supposition, and it is evident that water penetrates with great energy from the surroundings into the cells, that the cell swells, the peripheral cell-layers experience a tension, and that yet no water proceeds through them. When protoplasm forces out water in consequence of a stimulation, or when the strained layers are artificially punctured, only then does the fluid come out of the rent formed like a tiny spring. But this again only shows that the fluid in the interior is subject to a strong contra-pressure from the 
peripheral layers. This pressure is obviously stronger the more elastic and the firmer are the peripheral layers; and the elastic outermost parietal layer of the cell is of course adapted to exercise an especial reaction on the fluid in the interior of the cell. But that a pressure exists both towards the interior and in the reverse direction, in structures which have no cell-wall and consist only of protoplasm, is shown by the fact that if rents are made in the outermost layer of myxomycetous plasmodia, fluid substances immediately pour out.

It is indeed a matter of course that in a swollen, turgid cell the molecules of the peripheral extended layers will be separated beyond the usual limit of cohesion, and this is near to assuming that in the widened interstices so formed fluid materials are forced which become firm the moment they are deposited, and then resemble in every way the molecules they have driven apart. This intercalation and hardening of constructive materials, which indicates an accompanying increase in the bulk of the organized substances, is to be regarded as growth. We thus obtain a conception of the mechanism of growth, which, though only hypothetical, is in harmony with the external visible phenomena. We are led to it especially by the fact that only cells which are turgid grow, whilst cells stop growing, even although the necessary amount of fluid building material is present, as soon as their turgidity diminishes.

The turgidity of cells, that is, the presence in them of water necessary for their swelling, is, however, only one condition of growth; a second condition, no less important, is warmth. Without heat there is no growth. When in the temperate zones, where the year is divided into summer and autumn, winter and spring, the summer draws towards a close, and the days become shorter and shorter, when during the long nights the soil loses more warmth by radiation than it gains during the day, and when, too, plants become very much cooled, growth above-ground entirely ceases, and the whole energy of the plants is concentrated, as we have shown in previous sections, in changing itself into a chrysalis for the winter, in withdrawing from the deciduous foliage the materials which can be employed in the ensuing period of vegetation, and in lodging them in protected store-rooms. During the winter, then, the cooled portions, unprotected against frost, rest, and their growth is completely interrupted. At length winter is past, the last snow has vanished under the breath of mild spring breezes; the hard frozen earth is liberated from the bondage of the frost; everywhere new life stirs, buds swell, trees adorn themselves with flowers and fresh foliage, the meadows become green, seeds germinate, and the crops in the fields spring up vigorously, to the joy of the farmer. On warm, sunny spring days everything grows with astounding rapidity: on cool, dull days the increase is only small. If occasionally a relapse occurs, and the temperature again sinks low, then the growth is wholly arrested. It has been found that the increase of young herbaceous plants on two successive days had sunk in consequence of a sudden storm and visitation of cold from $8 \mathrm{~cm}$. to $\frac{1}{2} \mathrm{~cm}$. There is no doubt that such a decrease of growth stands in causal connection with the fall of temperature, and also that quick growth is to be laid to the account of 
a rapid increase of heat, provided, of course, that the other factor of growth previously indicated, viz. water, is present in sufficient quantity.

It has been shown in a previous section that the mineral salts, which plants require for the production of building materials, are brought by means of water to the place of need, and that this transporting water is raised from below by evaporation from the surface of organs exposed to the air and sun. This evaporation, however, requires much warmth, and there can be no doubt that the hastened or retarded development of vegetation is partly dependent on quickened or retarded transpiration; that is to say, on the greater or less amount of heat supplied. The conduction of food-salts by means of water from the soil is, however, not by any means growth; it is only a preparatory process, as also is the formation of organic materials in green cells, and the complicated transformations and distribution of materials which follow the elevation of the water from the ground. Warmth is an essential condition of that process which is being here discussed, that is of growth in its narrow sense, as well as of all these preparatory processes.

The part taken by heat in actual growth, that is, in the transformation of fluid building materials into firm, organized portions of the plant-body, and increase of bulk of the cells, cannot be essentially different from that which occurs in other molecular re-arrangements and chemical changes. Heat, according to prevalent theories, is the expression of vibration of ultimate particles. Those vibrations of ether which are known as free heat can induce a corresponding motility of the molecules in any ponderable body. Similarly, heat induces a state of motility amongst the molecules of living protoplasm. We must imagine that work is done upon the organic bodies which constitute the building materials of plants, that they are led in a fluid state to the regions where they are required, and there transformed into solid organized matter. In this way free heat is transformed into latent heat, and in this sense we may regard growth as a consumption of free heat. Accompanying this organizing action of heat there is an insertion of new molecules between the pre-existing ones. The separation of these latter is of course brought about, as already described, by turgidity. Thus, by the co-operation of heat and turgidity, fluid organic materials are changed into firm, solid, organized substances, and in this way the organized portions increase in bulk, in other words, they grow.

\section{EFFECTS OF GROWING CELLS ON ENVIRONMENT.}

Work is not only performed in the interior of cells, but pressures also come into action which operate on the surroundings with irresistible power. What the cells, apparently so delicate, are able to perform, borders almost on the incredible.

Where the filamentous hyphal threads of crustaceous lichens have penetrated vol. I. 
into the tiny crevices of stone, they crack and crumble the permeated substratum not only by lateral pressure, but they act also lever-wise, and vigorously press up the shattered particles. The absorbent cells or rhizoids of mosses and liverworts also exercise a like action on their substratum, and this is maintained, as in the lichens, essentially by the fact that substances are excreted from the growing cells by which the substratum is partially converted into soluble compounds. Moreover, the pressure which these delicate cells exert on the substratum by their growth may be demonstrated by experiment. If liverworts are laid upon damp folded filter-paper in a space saturated with vapour, in forty-eight hours they will send out rhizoids which grow through the paper. The holes in which the cells of the rhizoids are now seen certainly did not previously exist in the paper. The felt of threads in the filter-paper is so dense that starch-grains of maize, having a diameter of only about 2 thousandths of a millimetre, cannot find sufficient space to slip through, and thus still less can the rhizoids of liverworts penetrate the felt, as these have a diameter of from 10 to 35 thousandths. The holes must, therefore, be first formed by the growing cells of the rhizoids. The threads of the felt must be powerfully driven asunder, and this requires at any rate a comparatively large expenditure of force.

The hyphal threads of a mushroom, which unite to form dense fructifications and grow up from the subterranean mycelium in a comparatively short time, often raise considerable pieces of earth, and the cap-shaped fructifications of Lactarius scrobiculatus, Agaricus vellereus, and Hydnum repandum are indeed frequently thickly covered with larger and smaller fragments of earth, raised by them during their upward growth. An instance is also known in which a stone of $160 \mathrm{~kg}$. was raised and shifted by the growing fructification of a fungus of the mushroom tribe.

Nor is the pressure which the growing cells of flowering plants exert on their environment less considerable. The absorbent cells of roots embedded in the earth, which are called root-hairs, appear fairly straight, although the spaces between the particles of soil filled with air and water are certainly not rectilinear. It cannot be doubted, therefore, that the root-hairs in spite of their delicacy, nevertheless push the small particles of earth on one side, and in their growth follow, as nearly as possible, a straight course. The apices of the main roots of flowering plants, when they grow downwards, form actual channels by pressure on their environment, pushing the portions of soil powerfully asunder, and penetrating into the ground like a gimlet. And it would be a mistake to suppose that they are only drawn downwards by gravity. The roots of bean-seeds which have been germinated in a layer of water spread above quicksilver actually penetrate into the quicksilver. It has often been noticed that the roots of trees which have reached fissures in walls or clefts of rocks are able to shatter the walls and to crack the stone by their further thickening. One at least out of the great number of instances may here be noticed. On either side of the little Tyrolese Gschnitz-thal are terraces strewn with large blocks of stone, which are considered ancient diluvial moraines. The blocks 
of stone are composed for the most part of crystalline schist, especially of gneiss, in which mica is arranged in almost parallel streaks. On one of these blocks (represented in fig. 130), at a height of 2 metres, a larch has long ago established itself and rooted firmly, so that the strongest of its roots grow downwards in a cleft parallel to the direction of the mica streaks. By the thickening of this root the crevice became widened; half of the upper block was separated from the lower and was raised about $30 \mathrm{cms}$. It is estimated that the weight of this raised portion amounts to $1400 \mathrm{~kg}$., and the root which was able to raise this burden exhibits in

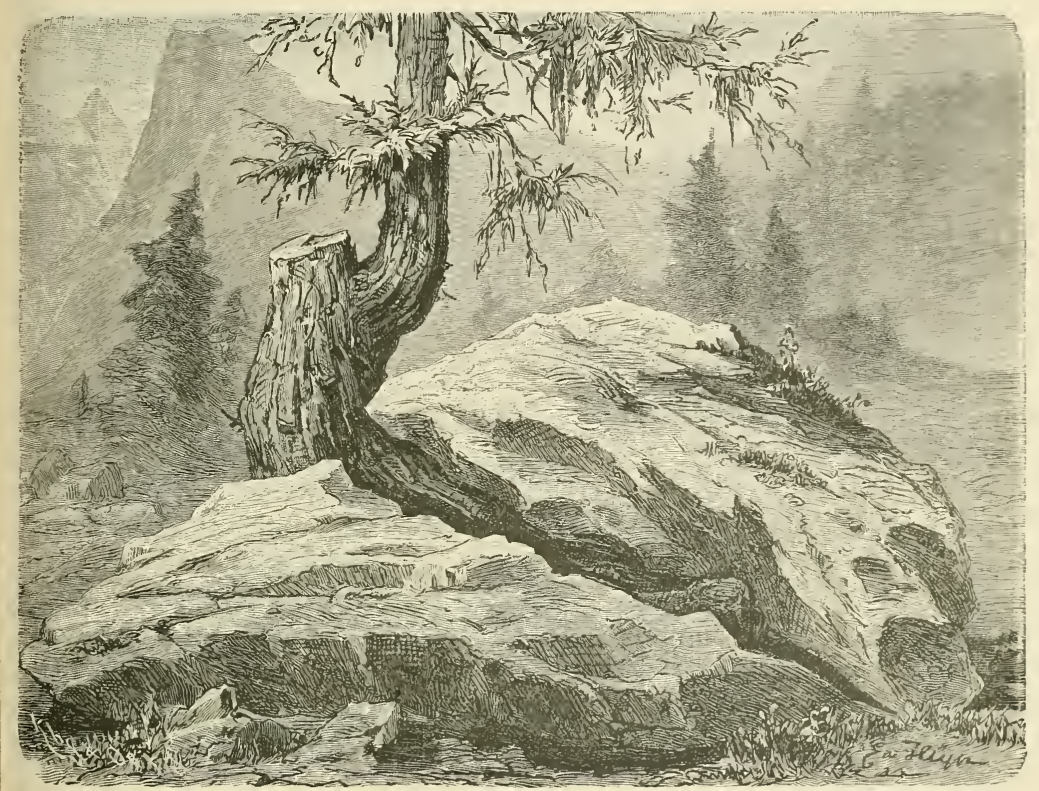

Fig. 130.-Elevation of a Block of Stone in consequence of the growth in thickness of a Larch Root.

its thickest part a diameter of only $30 \mathrm{~cm}$. Moreover, the burden overcome by this larch root is small in comparison with that raised by the roots of old trees. The large superficial roots which creep over the ground of the forest like gigantic snakes were not always situated in this position. As long as the trees were young their roots extended under the ground. Only with increasing thickness did these roots, pressing against the firmly compacted earth lying beneath them, become visible above ground, since they burst through the layer of earth situated above them. But with this must also be connected the elevation of the whole trunk with its boughs, which all bear upon the roots, and often weigh several thousand kilogrammes.

It is a matter of course that growing stem-structures also exercise a considerable pressure on their environment. Those underground stems which are called runners do not in this respect differ materially from roots, and are similarly able to shift 
and press asunder small stones and clumps of earth. In many plants the growing points of the runners are covered with hard scales, which produce exactly the same effect as the points of an auger. This applies especially to several grasses (e.g. Calamagrostis, Lasiagrostis, and Agropyrum). The runners of the common creeping Couch-grass (Agropyrum repens) bore through the roots of trees, and not only through old and rotten but also through young vigorous specimens. The runners of the Couch-grass are often found penetrating through the centre of potatotubers, and it has been confirmed experimentally that these runners in their growth are capable of even boring through dises of tin-foil. Very instructive also is the penetration of old tree-trunks by the stems of various small shrubs and shrubby trees whose growing points are comparatively delicate and soft in texture, and are not beset, like those of the Couch-grass, with hard pointed scales. Almost everywhere in our mountain regions are to be seen, in places where not very long before a forest has been cleared, dead stumps of fir-trees, rising perhaps a metre above the ground, and overgrown with cranberry and bilberry bushes. The surface where the saw has cut through the huge trunk is partly overgrown with the same plants as those growing in the soil round about, and it has a very peculiar appearance when on these decayed stumps, as if on the platform of the base of a pillar, small colonies of cranberry bushes are seen to flourish luxuriantly-a story higher than on the surrounding ground. Without closer investigation anyone would think that these bushes had germinated from seeds which had previously fallen into the cracks of the stem section, and it is not a little surprising therefore, on splitting such old tree-stumps, to find that this is not the case, but that rather the cranberry bushes of the surrounding forest-ground have sent out their runners into the lower portion of the tree-trunk, and that these have then grown up through the rotten wood of the stump-especially through the decayed part between the wood and bark-until they have again reached the daylight above on the exposed section, showing at any rate that they must have exerted a very considerable pressure on their surroundings. The thin stems, also, of plants growing on boulders have frequently to make a new pathway for themselves when their habitat has been covered by a torrent with sand and stones a span high, and thus have to push out of the way obstacles of comparatively large dimensions. On a forest soil covered with sand and boulders I saw indeed how the delicate thread-like stem of a wintergreen (Pyrola secunda) had grown up more than $60 \mathrm{~cm}$., and in doing so had pushed on one side stones of a gramme weight. If peas, beans, and other large seeds are buried in the earth and allowed to germinate, it may be seen how by the growth of the seedling small clods of earth and stones are raised, and the earth in which pine-seeds, oats, and beech-nuts have been embedded, looks when the seeds are germinating as if it had been rummaged and thrown up by mice. A fine example of external work done by growing stems must yet be instanced in the growth in height of the forest-trees which we have daily before our eyes, but only too easily overlook on account of its commonness. A young beech trunk $50 \mathrm{~cm}$. thick will raise each year a crown which has a weight of two thousand kilogrammes 
through a metre, and in still larger forest-trees the figures become even more impressive.

And all this is accomplished by the invisible atoms of the living protoplasm, which, set in motion by heat, alter their position, attract and repel one another, displace and travel between one another, assume new groupings, and in these new arrangements appear outwardly to our senses in altered form and increased volume.

On glancing over these effects of growing cells and groups of cells, one is reminded involuntarily of the analogous phenomena of ice crystallization. When ice is formed in a glass bottle filled with water, it bursts the vessel with irresistible force, and the splitting of masses of rock in high mountains and in all those regions where the temperature in winter sinks below freezing-point depends in no small degree on the freezing of the water which has penetrated into the smallest crevices and rocky clefts. And yet there is an essential difference between growth and crystallization. Crystals are formed spontaneously from fluid substances, and grow from the depositions of small atoms on their surface. Vegetable cells, on the other hand, never arise spontaneously from fluid materials, but always only by means of an already present organized and living mass of protoplasm. Thus all growth in living things is really only a further development of what already exists. The crystal can again be transformed into a formless fluid mass, can be reconstructed from this fluid, and this alternation may be repeated innumerable times. In plants, on the other hand, the passage from the formed, organized, to the formless, fluid condition is synonymous with death, and from the gases and fluids which are derived from the decomposition of a vegetable-cell, a plant-cell never again forms itself spontaneously, that is, without the interposition of a living agent. While, as above remarked, crystals grow by the deposition of small particles on their surface, growth of protoplasm takes place by the interpolation of new molecules between those already present; these are separated from one another, and only subsequently can parts of the cell increase by deposition brought about by living protoplasm.

\section{GROWTH AND HEAT.}

Sources of Heat.-Transformation of Light into Heat.-Influence of Heat on the Configuration and Distribution of Plants.-Measures which protect Growing Plauts from Loss of Heat.-Freezing and Burning.-Estimation of the Heat necessary for Growth.

\section{SOURCES OF HEAT. TRANSFORMATION OF LIGHT INTO HEAT.}

Whence do plants derive the heat necessary for their growth? With regard to this question one may first of all think of that heat which is liberated by the plant itself in respiration, and which can again find employment immediately after its release, not only in metabolism and transport of materials, but also in growth. Further, we may be reminded of that heat which is liberated by the breathing of 
animals and in various other instances of slow and quick combustion of organic, bodies, which the growing plants can now and then directly utilize. These, however, are only derived sources of heat. Heat which is liberated in respiration is really only the sun's rays which the plants have absorbed on a previous occasion, and ultimately, so far as it comes under consideration for the life of plants, all heat. is derived from the sun. The heat which is conducted to plants from the soil, from water, and from air, also takes its origin from the sun, which is therefore to be looked upon as the fountain-head of all the heat utilized by plants.

It has been found that the sun sends out three kinds of rays distinguished by their different periods of vibration, and known respectively as heat rays, light rays, and chemical rays. These three undulating movements of the ether interfere with each other in their course as little as the wave-circles which intersect on the surface of water. We recognize and measure them by their effects. As soon as they strike a body, work is performed by the active force of these ether waves which we picture to ourselves as movements of the molecules and atoms of the body affected; and this work appears either as heat, or light, or chemical change. But it is exceedingly remarkable that only that movement which we regard as heat can produce that transformation of building substances into organized materials, which is, in other words, growth. The vibrations which constitute light, and whose great importance in the formation of the constructive materials, and generally, of organic compounds from inorganic food, has been previously described in detail, are not able to cause such an effect, at least directly. There are even instances which justify the opinion that growth is actually restricted and hindered by light. This much is certain, that growth can proceed in the deepest gloom, if only the two earlier-mentioned factors-turgidity and heat-are undiminished. Seeds and the majority of spores germinate in darkness. The cells of underground stems and scale leaves, those of roots embedded deep under the soil, as well as the mycelia of fungi, grow in regions wholly deprived of light. Moreover, plant organs which are brought from the light into darkness continue to grow there, provided always that the necessary amount of moisture and heat be supplied to them.

Nevertheless very numerous experiments tend to prove that growth can be assisted by light. The following is one of the most remarkable. If plants are cultivated in two places, identical as to the amount of heat affecting them during growth, but differing in the intensity and duration of the flow of light, they will exhibit a quicker growth in the place where the light can act on them more powerfully and for a longer time. Thus plants grow much more quickly in the far north, where they are daily illuminated for twenty hours, than in southern latitudes where they are exposed to the light for only twelve hours, even although in the same space of time comparatively less heat reaches them in their northern habitat. From the small table inserted opposite, giving the commencements of the flowering periods best adapted for the comparison of a definite amount of growth in several widely-distributed plants, at Athens, Vienna, and Christiania, it may be seen that Athens is about forty-six days earlier than Vienna, but Vienna only 


\begin{tabular}{|c|c|c|c|}
\hline Commencement of Flowering. & $\begin{array}{l}\text { Athens. } \\
37^{\circ} 58^{\prime} \text { North Lat. }\end{array}$ & $\begin{array}{l}\text { Vienna. } \\
48^{\circ} 11^{\prime} \text { North Lat. }\end{array}$ & $\begin{array}{l}\text { Christiania. } \\
69^{\circ} 5^{\prime} \text { North Lat. }\end{array}$ \\
\hline $\begin{array}{l}\text { Hepatica (Hepatica triloba),... } \\
\text { Sloe (Prunus spinosa),............ } \\
\text { Gean (Prunus avium),.......... } \\
\text { Wild Pear (Pyrus communis),. } \\
\text { Barberry (Berberis vulgaris),... } \\
\text { Elder (Nambucus nigra),........ } \\
\text { Privet (Ligustrum vulgare),.... } \\
\text { White Lily (Lilium candidum), }\end{array}$ & $\begin{array}{l}\text { 22nd January } \\
\text { 5th February } \\
\text { 1st March } \\
\text { 20th March } \\
\text { 10th April } \\
\text { 15th April } \\
\text { 20th April } \\
\text { 1st May }\end{array}$ & $\begin{array}{l}\text { 11th March } \\
\text { 18th April } \\
\text { 19th April } \\
\text { 23rd April } \\
\text { 9th May } \\
\text { 26th May } \\
\text { 4th June } \\
\text { 24th June }\end{array}$ & $\begin{array}{l}\text { 2nd April } \\
\text { 18th May } \\
\text { 19th May } \\
\text { 22nd May } \\
\text { 6th June } \\
\text { 2nd July } \\
\text { 6th July } \\
\text { 16th July }\end{array}$ \\
\hline
\end{tabular}

about twenty-nine days before Christiania. And yet the difference of the geographical latitude between Athens and Vienna amounts to $10^{\circ} 13^{\prime}$, and that between Vienna and Christiania to $11^{\circ} 43^{\prime}$; from which it would be expected that Vienna would have a start of fifty-one days in advance of Christiania.

One is tempted to think at first, in explanation of this phenomenon, that growth depends upon the formation of constructive materials from inorganic food; that this latter process can only be accomplished under the influence of light; and that therefore light so far is important for growth. On the other hand, it is difficult to imagine that the light enjoyed by plants growing in Athens should not be sufficient for the formation of organic compounds in the green cells, and for the production of a sufficient quantity of building materials, since, as a matter of fact, the species in question do not appear in any worse condition in Athens than in Christiania, which, however, it must be supposed would be the case if there were a disparity between the food absorbed, metabolism, and growth. This phenomenon suggests rather that the light in the north is able. to take the place of heat. And herein lies also the solution of the problem. Not only is there compensation alone; but the light is changed into heat before it acts on the building materials. A portion of the light falling on the plants is reflected, another portion penetrates into the plants, and of these latter rays part bring about the transformation of carbonic acid into carbohydrates, and increases the store of chemical energy, while another portion is changed into heat. This applies particularly to those light-rays which are most vigorously absorbed by chlorophyll and anthocyanin, and which also cause the fluorescence of these colouring-matters; and among the tasks assigned to chlorophyll and anthocyanin, the transformation of light into heat is certainly not the least important.

But with this we come back once more to anthocyanin-that remarkable colouring-matter which has repeatedly been spoken of in detail. It has been mentioned that anthocyanin frequently occurs only on the under side of foliageleaves. This is observed especially among plants in the depths of shady forests, which, although belonging to widely-differing families, agree in a remarkable manner in this one point. One group of these plants has thick, almost leathery, evergreen leaves lying on the ground, which arise from subterranean tubers, or root-stocks, or from procumbent stems. The widely-distributed Cyclamen europceum may serve as a type of this group. A vertical section of a similar 
leaf is given in figure 25 $\mathrm{A}, q$. Amongst other species belonging to this group may be mentioned Cyclamen repandum and $C$. hederifolium, Cardamine trifolia, Soldanella montana, Hepatica triloba, and Saxifraga Geum and cuneifolia. Growing in habitats similar to these are to be met biennial, occasionally perennial, plants which in autumn form a rosette of leaves on their erect stems which survive the winter; these are always coloured violet on the side turned towards the ground, while the leaves which develop in the following warm summer on the elongated flower-stalks usually appear green below. To this group belong, especially, numerous Cruciferæ (e.g. Peltaria alliacea, Turritis glabra, Arabis brassicaformis); species of spurge (e.g. Euphorbia amygdaloides), bell-flowers (e.g. Campanula persicifolia), and hawkweeds (e.g. Hieracium tenuifolium). Finally, deciduous shrubs are to be found in the depths and on the margins of forests whose leaves do not survive the winter, but which produce on the stems developing in the summer flat leaves whose under side contains abundant anthocyanin, as, for example, Senecio nemorensis and nebrodensis, Valeriana montana and tripteris, Epilobium montanum, Lactuca muralis, and many others. Amongst non-European species may be noticed many Flowering Rushes, Tradescantias, Begonias, and Cypripediums, as well as the Japanese Saxifrages (Saxifraga sarmentosa and cortuscefolia), which are coloured deep violet on the lower side of the leaf with anthocyanin, and are only found in shady spots in forests.

Since anthocyanin has been already indicated as one of the means of protecting chlorophyll, the question must first of all be considered as to whether such a relation does not exist in the instances just enumerated. It might even be possible that the violet side of the foliage-leaves now turned earthwards was originally turned towards the incident rays of light, while the leaves were still very young, and that the anthocyanin remains in the position once assumed in consequence of the twisting of the leaves, without being assigned any particular function on that account. Opposed to this idea, however, are the facts that in the majority of the plants cited, anthocyanin is only first developed when the side of the leaf in question has already been turned towards the ground; that in many species the violet side is never turned upwards at any period of development; and especially that in all these plants which grow in the shade, no protection of chlorophyll against an over-abundance of light appears necessary; that, on the contrary, it is important for these shaded growths that the scanty light and heat should be appropriated and utilized to the utmost extent.

We cannot therefore assign to the anthocyanin on the under side of foliageleaves any protective influence upon chlorophyll. On the other hand, everything goes to show that the anthocyanin developed here absorbs light and changes it into heat. Light which, passing through the leaf, would reach fallen dead and dry foliage, or the ground itself in the depth of the forest, would be wasted and useless there. When absorbed by the anthocyanin and changed into heat, it becomes serviceable to the plants, and can exert a helpful influence on the growth of neighbouring cells, and to a less extent apparently also on the metabolism and 
transportations of the substances. In the evergreen leaves of those plants in the depths of the forest which are natives of inclement regions, this advantage is obtained from the layer of anthocyanin developed on the lower leaf-surface, that every sunbeam, even in the cooler seasons, can be utilized to the utmost. It is in harmony with this explanation that foliage-leaves of trees, shrubs, and high bushes which grow a considerable distance above the ground, and have below them other green foliage-leaves, are never violet-coloured on their earthward side, and that in richly-leaved bushes whose lowest leaves lie on the soil, these only are provided with anthocyanin. That portion of the light not turned to account in the highest green leaves, and which is allowed to pass through them, can still be utilized by the lower ones; only that light which would pass through the lowest leaves would be lost to the plants, and therefore we have a violet absorbent layer only on that side which lies on the ground.

That which occurs in plants of the forest shade occurs similarly in those marsh plants whose leaf-like stems or flat, disc-like leaves float on the surface of the water. The green discs of duckweeds (e.g. Lemna polyrrhiza), of the Frogbit (Hydrocharis morsus-rance), of the Villarsia (Villarsia nymphoides), of water lilies (Nymphoea Lotus and thermalis), and of the magnificent Victoria regia, are strikingly bi-coloured, being light-green above and deep violet below. Here again it cannot be said that the anthocyanin forms a protection for chlorophyll, but the violet colouring-matter can retain light in the cells on the lower surface of the leaf, and can change it into heat and so make it useful to the plants. The rays which penetrate the green leaf-dises and shine through the water would otherwise be lost to the plants in question, for none of the species enumerated have submerged leaves, but possess only these floating discs, green on the upper and violet on the lower side.

If anthocyanin were found, not only on the under but also on the upper side of the foliage-leaves, then indeed the significance would primarily be assigned to it of a means of protection for chlorophyll, and of assisting the metabolism and transport of materials; but obviously the blue colouring-matter would not, on the upper side of the leaf, behave essentially otherwise as regards its capacity of changing light into heat, than on the lower side. It is even probable that the importance of anthocyanin lies, not only in its retention of the rays injurious to metabolism, but also in the transformation of light waves into heat. In support of this view there is at least the fact that anthocyanin is also richly deposited on the upper side of the foliage-leaves at times when, and in places where, other sources of heat are deficient, and that generally leaves and stems of many plants growing in such places are entirely overspread with red or violet. A number of small annuals which grow very early in the spring at a low temperature (e.g. Saxifraga tridactylites, Hutchinsia petrcea, Veronica procox, and Androsace maxima) are usually coloured with anthocyanin on all sides of their growing organs. Moreover, seedlings which spring up from the earth at low temperatures, and above all high Alpine forms in the neighbourhood of the snow-line, are abundantly 
provided with anthocyanin on both leaf-surfaces. The leaflets and stem of the Alpine Sedum atratum, those of Bartsia alpina, and, above all, numerous species of Pedicularis (e.g. Pedicularis incarnata, rostrata, recutita) are coloured wholly purple or dark violet, and this in habitats where the colouring could not possibly be regarded as a protection for chlorophyll. It is also a very striking phenomenon that widely-distributed grasses (e.g. Aira caespitosa, Briza media, Festuca nigrescens, Milium effusum, Poa annua and nemoralis), which in the valley possess pale-green glumes, develop anthocyanin in them on lofty mountains, so that there the spikes and panicles exhibit a deep violet tint, and on this account the regions in which grasses of this kind grow in great quantities receive a peculiar dark colouring. Indeed, this tint becomes the more intense the nearer the habitat of the plants in question is to the snow-line, and the more intense the action of the sunlight becomes. In this case anthocyanin can certainly not be looked upon as a means of protecting chlorophyll, as the glumes generally contain but little of that substance, and take so little part in the formation of organic materials, that the few chlorophyll-grains might be entirely absent without the plant suffering any damage. On the other hand, it may be supposed that the intense light of the elevated region is changed into heat by the abundant anthocyanin of these glumes, that this heat reaches the germs hidden under the glumes, and there favourably influences the growth of the seeds as well as the transformations of materials. The same occurs in the numerous sedges and rushes growing in the Alps, which have dark-violet, almost black, scales covering the flowers (e.g. Carex nigra, atrata, aterrima, Juncus Jacquinii, trifidus, castaneus), and probably some of the varieties of tint observed in the corollas of Alpine plants are also to be explained in the manner indicated.

It is known that the floral-leaves of many plants growing on lofty mountains, and in the far north, are coloured blue or red by anthocyanin, whilst in the same species, growing in the warm lowlands and in southern districts, they appear white. Particularly noticeable in this respect are the Gypsophyllas (Gypsophylla repens), the Carline Thistle (Carlina acaulis), the large-flowered Bitter-cress (Cardamine amara), the Milfoil (Achillea Millefolium), and many of those Umbelliferæ which have a very wide distribution, and occur all the way from the lowlands up to a height of 2500 metres in the Alps, such as; Pimpinella magna, Libanotis montana, Chorophyllum Cicutaria, and Laserpitium latifolium. Since it has been proved that the colours of flowers are eminently important as a means of attracting insects, it might be thought that the above cases are in some way connected with insect-visits. Without wishing altogether to deny such a relation, the possibility, on the other hand, must not be excluded that anthocyanin plays the same part here in the flowers as in the glumes of grasses, and in the clothing scales of sedges and rushes; and that in the cold Alpine regions, that which is deficient in the amount of heat directly absorbed as such, is compensated for by such as is obtained from light-rays by means of anthocyanin. In support of this view there is also the phenomenon that many plants which develop white flowers in the warm 
summer, as, for example, Lamium album, produce late in the autumn, under a very low temperature (if they bloom a second time), corollas whose upper side is tinged with red; and that in the winter, and in frosty habitats, the ray-florets also of many Compositæ, as, for example, of the well-known Daisy (Bellis perennis), are coloured red on that side which is turned towards the sky when the capitulum is closed, and towards the ground when the capitulum is open.

\section{INFLUENCE OF HEAT ON THE CONFIGURATION AND DISTRIBUTION OF PLANTS.}

On high mountains near the snow-line, and generally in all those districts where the heat supplied to the plants is extremely scant, there occurs, together with a production of anthocyanin, a dwarf and tufted habit. Usually this phenomenon is explained by the large amount of snow, which must have a great effect in these frosty heights during the long winter, and it is believed that high Alpine plants are protected by this form and position of their stems and leaves from injury by the pressure of snow. It cannot indeed be denied that the pressure of the snow has some influence on the form and direction of the stem-structures, and this influence will be explained fully in the following pages in a particularly instructive example, viz. in the mountain pines. But this nestling on the ground of plants growing in the high Alps can only be partially referred to this cause.

It is a mistake to suppose that the annual snow-fall increases with the height. The amount of snow which falls attains a maximum at about 2500 metres above the sea-level. This height marks only the upper limit of mountain pines, dwarf junipers, alders, and rhododendrons. Above this the fall diminishes, and at a height of 3000 metres the snow is no deeper than far down in the valleys. Even where the maximum fall occurs trees are still met with; there are yet larches and Arolla pines, which, on account of the great elasticity of their branches and the downward direction of their older boughs, can bear very heavy weights of snow without becoming broken or crushed. The willows of mountain regions, characterized by the way in which their elongated stems and branches are pressed to the earth (Salix serpyllifolia, S. retusa, Jacquiniana, reticulata), and which are represented in fig. 131, grow, however, far above the tree limit, at a height above the sea where the depth of snow, already beginning to diminish, is in no case greater than in the valleys, where Purple and Sweet Willows, and other species of large-leaved willows raise their straight stems several metres high above the ground on the banks of streams. It must also be remembered that the woody growths close to the ground in high Alpine regions are very often established on steep places, where the snow could not easily lie, could in no instance be deeply piled up, and could not exert a pressure on the stems and branches. The delicate Thyme-leaved Willow (Salix serpyllifolia) nestles with an especial predilection to the surfaces of rocks, and covers them with an actual carpet, and the Buckthorn (Rhamnus pumila) is found exclusively on steep declivities, where it roots in the crevices of the narrow 
rock gulleys, and growing out from them overspreads like ivy the vertical rockfaces.

In all these cases it is certain that the weight of snow cannot have any determining influence upon the form of the plants, and some other explanation must be sought. May it not be perhaps that strong winds render it impossible for woody

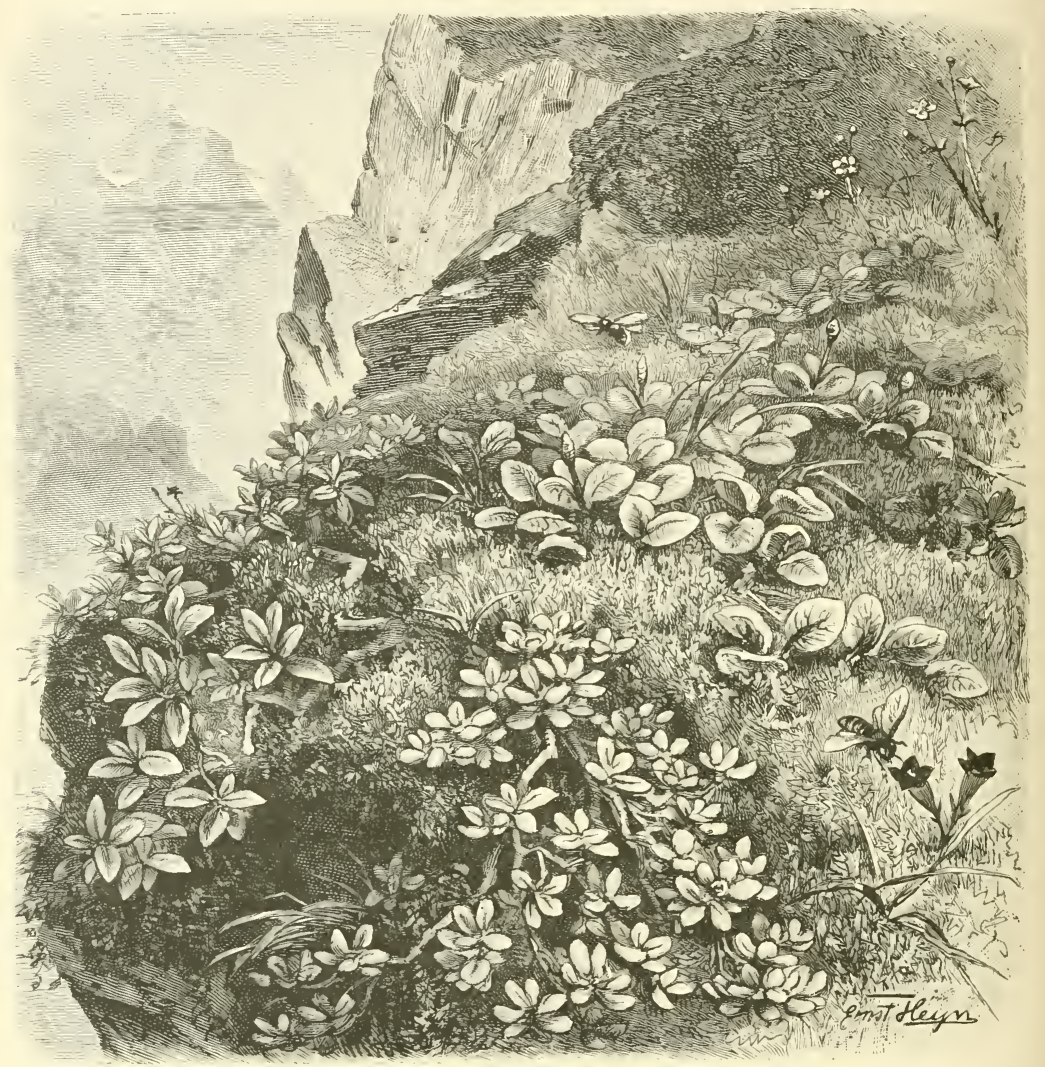

Fig. 131.-Alpine Willows with stems and branches clinging to the ground in the Tyrol

plants with erect stems to grow in high Alpine regions? Observing the mist and volumes of clouds rushing across the tops of the mountains, one gets some idea of the strength of the air currents which operate there, and whoever has experienced the effects of a storm on a high mountain ridge can estinate the force of the powerful gusts of wind. And yet it would be erroneous to suppose that the force of the storms on lofty mountain heights is greater than in mere hill regions. In the case of many winds it is even certain that they increase in violence as they rush down 
from the mountain ridge deeper into the valley. The Föhn-wind in the $\Lambda$ lps often appears on the heights as only a slight breeze, but accelerates its velocity as it enters the valley, and when it arrives there may be as destructive as a hurricane. Therefore if the woody plants on the slopes of high mountains were unable to exhibit erect growth on account of storms, then the neighbouring valleys must also be deprived of upright trees, which, however, is known not to be the case.

The clinging of woody plants to the ground in high Alpine regions must not be regarded either as an adaptation to snow pressure or to storms; it is due rather to the fact that in the high Alps the ground is relatively much warmer than the air, and that plants lying on the soil profit by this higher temperature. I have ascertained through numerous observations at different heights in the Central Tyrolese Alps that the mean temperature of the soil exceeds that of the air by the following amounts:-

$\begin{array}{cccc}\text { At a height of } 1000 \text { metres, about } 1 \cdot 5^{\circ} \mathrm{C} \text {. } \\ " \quad & 1300 & " & 1 \cdot 7^{\circ} \mathrm{C} \text {. } \\ " & 1600 & " & 2 \cdot 4^{\circ} \mathrm{C} \text {. } \\ " & 1900 & " & 3 \cdot 0^{\circ} \mathrm{C} \text {. } \\ " & 2200 & " & 3 \cdot 6^{\circ} \mathrm{C} \text {. }\end{array}$

Thus the soil, in comparison with the air, becomes warmer the higiner one ascends the mountain. Everywhere the earth absorbs the sun's rays to a much greater degree than the air does; but that the excess of the heat of the soil above that of the air increases so remarkably with the increasing altitude, is due to the fact that the intensity of the sun's rays increases as we ascend.

This is further explained by the fact that the layers of air which absorb the sun's rays are less dense the greater the elevation above the sea-level, or, to use a current expression, that the air is thinner on the heights than in the valleys. As is well known, the water vapour of the air also absorbs the sun's rays, and since this aqueous vapour diminishes rapidly with the height, as might be concluded from the lessening of the pressure, the intensity of the sun's rays consequently increases with the increasing altitude. It has been estimated that the force of the sun's rays on the top of Mont Blanc (4810 metres) is 26 per cent greater than at the level of Paris, and that at an altitude of 2600 metres the chemical activity of the sun's rays is 11 per cent greater than at the sea-level. Everything which is benefited by the sun has, in consequence, a relatively striking appearance in the higher regions of the mountains, and the illuminated soil especially exhibits a temperature of surprising height. On the Pic du Midi in the Pyrenees (2885 metres) the temperature of the illuminated soil rose on a clear September day to $33.8^{\circ} \mathrm{C}$., while the air only registered $10 \cdot 1^{\circ} \mathrm{C}$, and in point of fact the temperature of the soil on this summit was almost twice as great as at the Bagnères situated 2326 metres below. On the Diavolezza (Switzerland) the black bulb thermometer registered $59.5^{\circ}$ in the sun, and at the same time in the shade a temperature of $60^{\circ}$. In the Himalayas the blackened thermometer at a height of over 3000 metres showed in the sun $40^{\circ}-50^{\circ}$ above the temperature of the shade, and once stood at $55.5^{\circ}$ while the temperature on the snow, in the shade close by, amounted to only $-5 \cdot 6^{\circ}$. In Leh (Kashmir) at 
3517 metres, a blackened thermometer in vacuo rose to even $101.7^{\circ}$, that is almost $14^{\circ}$ higher than the boiling point of water, which at that height is only $88^{\circ} \mathrm{C}$.

It is readily intelligible that under such conditions growing plants which require heat should nestle to the ground in high mountain regions, or more correctly, that only such plants are capable of living at these heights which make the best possible use of the most abundant of all sources of heat; which, so to speak, seek a warm situation and settle themselves against the sunny stones and the black humus, occupying and covering the rocky crevices. Plants whose nature is to grow erect with their woody stems in the air would not succeed well in Alpine regions, and ultimately would be crowded out by species which thrive better by clinging to the relatively warm soil.

The increase in the excess of the ground temperature above that of the air with the increasing altitude is also manifested in another phenomenon which, though it has been frequently observed and discussed, has not always been correctly interpreted. The Ling (Calluna vulgaris), which extends from the lowlands at the foot of the Alps up to high Alpine regions, blossoms on the sea-coast in Istria usually at the end of July; in Alpine valleys, which lie 1000 metres above the sea-level, it opens its first flowers at the end of August, and therefore the retardation of flowering at 1000 metres amounts to something over a month. From this it might be expected that the Ling would first blossom at an altitude of 2000 metres at the end of September, but this is not so, for on mountains of the Central Alps at 2000 metres the Ling nestling on the ground is seen to be in full bloom before the middle of September. By comparing the time of blossoming of high Alpine plants cultivated in the botanic gardens at Innsbruck, with the time at which the same species open their flowers at various altitudes on the neighbouring mountains, it was shown that the retardation of the blossoming amounted to a mean of 25 days at an altitude of 500-1000 metres; an average of 18 days at 1500-2000 metres; and 14 days at 2500-3000 metres; and this can only be explained by the much greater intensity of the sun's rays in the high regions, and the consequent elevation of the temperature of the ground above that of the air. It must yet be mentioned, for the completion of the observations here detailed, that all plants in the valleys develop larger leaves and taller stems than those on lofty mountain sites. While the Ling forms considerable bushes with erect branches on the coast of Istria, plants of the same species on the slopes of high mountains 2000 metres above the sea, appear as dwarf shrubs, whose woody stems lie on the ground and are partially imbedded in the dark humus.

The great contrast which vegetation on a mountain exhibits in different parts of the world may be explained by the action of the sun's rays. On slopes illuminated directly by the sun, the temperature of the soil, and indirectly that of the layer of air in contact with it, rises far higher than on shady declivities, and in consequence of this very remarkable differences may occur even in the closest proximity. Observations of the temperature of the soil at a depth of 80 centimetres, spread over 
three years, at Innsbruck in the Tyrol, and in the eight points of the compass round an isolated conical sand-hill, have shown the following mean temperatures:-

$\begin{array}{cccccccc}\text { North. } & \text { North-east. } & \text { East. } & \text { South-east. } & \text { South. } & \text { South-west. } & \text { West. } & \text { North-west. } \\ 15 \cdot 3^{\circ} \text {. } & 17 \cdot 0^{\circ} \text {. } & 18 \cdot 7^{\circ} \text {. } & 200^{\circ} \text {. } & 193^{\circ} \text {. } & 18 \cdot 3^{\circ} \text {. } & 18 \cdot 5^{\circ} \text {. } & 150^{\circ} \text {. }\end{array}$

The difference between the south-east and north-west amounts, according to this, to not less than $5^{\circ}$, and it is probable that at higher altitudes it would show even a more marked increase. And herewith is connected the rising and falling of the upper limit of vegetation on the different sides of a mountain. On slopes long exposed to the sun the plants advance much further upwards than on the shaded sides of a mountain, or those which are warmed by the sun's rays during only a short time; and the difference of the upper limit on the north and south sides oscillates in high mountain regions between 200 and 300 metres. It frequently happens that species reach their upper limit on the north side at 2000 metres, while on the south side not until 2400 metres is reached. From this it strikes us that the contrast between the upper limit of plants on the north and south sides becomes greater the higher we climb up into the mountain. In this respect a comparison of beeches and firs is very interesting. Beech trees (Fagus sylvatica) find their upper limit in the Limestone Alps of the North Tyrol on an average at an altitude of 1430 metres; on the sunny side of the mountains the beech limit rises to 149 metres above this average, while on the shady side it falls short of the average by 112 metres; thus the difference between the sunny and shady side for beeches amounts to 261 metres. Norway spruces (Abies excelsa) find their upper limit in the same region, on an average, at an altitude of 1777 metres; on the sunny side of the mountain the spruce limit rises to 185 metres over, while on the shady side it remains 125 metres below the average, and thus the difference between the sunny and shady side amounts for spruce to 310 metres. Thus whilst in the zone stretching from 1300-1600 metres, the difference between the shady and sunny sides amounts only to 261 metres, it rises to 310 metres in a zone from 1600 1900 metres, which again can only be accounted for by the rising intensity of the sun's rays with the increasing altitude.

From all this it may be seen how vegetation adapts itself to the given heat conditions; how the smallest advantage offered in any spot is made use of; and how much the form of the plant depends upon the conditions of warmth in the habitat.

The above statements also demonstrate that the distribution of plants on the earth stands in the closest connection with the distribution of heat. In another volume of this work an opportunity will be taken of discussing this connection fully; here it is sufficient to mention that from the local conditions of warmth, viz., from the elevation of the temperature of the soil effected in circumscribed spots in mountainous districts by the sun's rays, the preservation of colonies of plants, from earlier, warmer periods is explained. The largest part of the central European uplands, especially the Northern Limestone Alps, exhibit colonies of plant-species on limited areas, which are entirely absent in the immediate neighbournood, and 
which now no longer spread beyond the narrow circle of their confined habitat, although they ripen seeds capable of germinating, and are met with again in great quantities one or two degrees farther south. We may conclude that these plants were first brought to their isolated habitats within the historical period by wind or other distributive agents, and everything tends to show that they represent the remnant of a vegetation which was distributed very widely over adjacent districts in ages long past, but have withdrawn thence in consequence of the severe climate which has intervened; that is to say, have died and been replaced by other vegetation. That such foundlings on isolated mountain slopes, often only a small steep ravine, or on a single rocky face, could maintain themselves even in the later cold periods, is explained by the fact that conditions of warmth can prevail over very restricted areas on the mountains which differ in toto from those of the environment, and are only found generally prevailing quite a degree further south. The southern slope of the Solstein range, between Hall and Zirl, produces in limited areas Hop-hornbeams and Bladder-senna (Ostrya carpinifolia and Colutea arborescens); from the boulders an umbellifer, the curious Tommasinia verticillaris, rises to the height of a man; the rock terraces are overgrown with Stipa pennata, Lasiagrostis Calamagrostis, Saponaria ocymoides, Dorycnium decumbens, and here and there one might imagine one's self a degree further south on the other side of the Alps. It is beyond question that the plant forms named on the warmest and most protected of the Solstein range are remnants from a primeval warmer period, and were formerly distributed generally over the adjoining mountain ranges. These cursory remarks should show that the accurate knowledge of the relation of heat to individual species of plants may render important help in speculations about the history of our vegetation.

\section{MEASURES FOR PROTECTING GROWING PLANTS FROM LOSS OF HEAT.}

Since certain developments in plants have assigned to them the task of utilizing external circumstances as far as possible so that heat may reach the growing organs to the extent actually necessary, it is naturally to be expected that contrivances will not be wanting to protect them from an excess of heat, and also that care will be taken that the heat once obtained is not again lost. It would not be in harmony with what we know of the economy of vegetation that a plant exposed to the sun should lose by radiation in the following night all the heat which it had gained during the day. It is known that growth is carried on during the night, and, indeed, that certain organs grow more in the night than in the day, and in these an excessive loss of heat would be most disadvantageous.

As a matter of fact arrangements exist for protecting plants from an excessive loss of heat. These contrivances coincide in great part with those which regulate transpiration, and have already been fully described in the discussion on that subject, to which therefore we may refer. But those developments which claim a particular interest as measures of protection against the danger of excessive lose 
of heat which are not at all connected with transpiration, or only to a slight degree, are brought together here in the form of a general sketch.

First of all in this respect are to be considered flowers of comparatively rapid growth, whose parts therefore require much warmth, but for which many of the contrivances suited to foliage-leaves are not well adapted as protective measures against loss of heat, since other functions might be encroached upon. And yet these flowers especially require an abundant protection against loss of heat, even more than other plants on account of their great sensitiveness. If in the spring a blossoming snowdrop, having already penetrated the soil, is surprised by a frost, the flower-stalk and the leaves sink down as if withered, while the flowers outwardly are not at all altered. Anyone observing this might think that the green stem and leaves had been injured, but that the flowers, on the contrary, had survived the catastrophe without harm. But exactly the opposite is the case. The stem and leaves become erect with returning warmth and continue to grow, but the pollen in the anthers of the flower is dead; also the ovules, styles, and stigmas are affected so that they wither and shrivel up: obviously the production of ripe seeds is then out of the question. It is also observed that the pollen in the anthers is best formed when the flower-buds in question are warmed through by the sun, and when the blossoming plants grow on a free open space which the sun's rays can reach. Moreover, the floral envelopes develop much better in such spots than in cool shady places; they become larger, exhibit brighter colours, and consequently are more often visited by insects than those which receive relatively little light and heat. But the danger that the flowers and flower-buds will again lose by radiation, through the night, the heat which they have gained during the day is most likely to be felt in open, unshaded habitats, that in consequence of the great loss of heat the formation of the pollen in the yet closed anthers will be injured, and finally, that the petals will also be disturbed in their growth and function. In order to avoid this, in many cases the flower-buds and also the open flowers are pendulous, bell-shaped, and tubular, or leaves become arched in the shape of a dome, cap, or umbrella above the stamens and pistils, in which case the inner portions of such flowers are hidden as in a niche or groove. In these hidden nooks they are comparatively well-protected against loss of heat, and at least no radiation of warmth towards the night sky proceeds from the anthers and stigmas. Only the coverings spread over the stamens and pistil, as a protecting roof, lose during the night a great part of the heat obtained in the day. These, however, are not so much endangered, since they have already obtained their normal size and have no need of heat for further growth; besides, they are usually clothed with air-containing, hairy structures, surrounded by dry membraneous edges or entirely transformed into dry parchment or paper-like scales, in which case they can suffer no further damage from loss of heat. The air in the pendulous bell-flowers is 1-2 degrees warmer, in the morning before sunrise, than the surrounding air; here, closed in, it remains practically unaltered during the night; and this of course is exceedingly useful to the warmth-loving anthers and stigmas there hidden. 
In many instances the flower-buds and young flowers only assume an inverted position periodically, i.e. only when a cold night is to be expected. Many umbelliferous plants are particularly noticeable in this respect, especially Falcaria Rivini and the Burnet Saxifrage (e.g. Pimpinella magna, and saxifraga) and Carrot (e.g. Daucus Carota and maximus). The sun has scarcely set when in all these species the stalks which bear young flower-umbels bend downwards, crooklike, so that the flower-buds, which during the day have been turned towards the sun, now face the earth, and the finely-divided involucral leaves spread out like an umbrella over the nodding umbel. These finely-divided coverings radiate out heat in the night without injury; the flower-buds below them, on the other hand, are protected in the manner described against the nocturnal radiation so fatal to them; whilst the heat they absorb during the day is thus in great measure, if not entirely, retained. With the next sunrise the young umbels rapidly become erect; their bent stalks rise up stiffly; and the flower-buds are again exposed to the sun, as may be seen in the illustration of the Common Carrot (Daucus Carota) inserted opposite (Fig. 132 1, 2). Later, when fertilization has taken place, and the young fruits are developing, the necessity for protecting the stamens and pistils from radiation no longer exists, and the periodic bending down of the umbel is discontinued. Young flower-heads of several scabiouses (e.g. Scabiosa lucida and Columbaria) behave like the umbelliferous plants named, as also do the single flowers of pansies (Viola tricolor), represented in fig. 132 3,4, in day and night position next the umbels of the carrot. In numerous Compositæ, Labiatæ, and plantains (e.g. Leontodon hastile, Mentha sylvestris, Plantago media, recurvata and maritima) there are no such regular periodic movements; in these the capitula and spikes are always pendulous while the flowers are still in bud, and they remain in this position as long as it is advantageous to them. Afterwards, when the nocturnal loss of heat can no longer be injurious to the anthers and stigmas, or if other protective measures have been developed meanwhile, the axis of the inflorescence becomes stiffly erect. In many Compositæ the involucres of the capitula or the peripheral ligulate florets, and in other families the sepals and petals, bend up after sunset over the stamens and pistils. They thus form a protecting roof under which the temperature of the air alters comparatively slowly, and the delicate anthers and stigmas are secured from radiation.

A very striking contrivance for protecting against loss of heat by nocturnal radiation is also observed in the seedlings of flowering plants, in those which possess two seed-leaves or cotyledons. As long as the embryo surrounded by protecting coats remains quiescent in the seed, the two seed-leaves are situated with their upper surfaces in contact; later, when germination has taken place when the radicle has penetrated into the earth and the seed-coat is thrown off, the two seed-leaves become separated, turn their upper sides towards the sky, so that the seedling above-ground resembles an open book. In this position the broad surfaces are exposed to the sun's rays; they are also illuminated and warmed as much as possible, and if they are coloured green, the formation of organic 
substances from inorganic food can be carried on in them. These cotyledons are frequently seen to increase in extent, and to grow and function exactly like foliageleaves. It would certainly be a great disadvantage to green cotyledons of this kind if they were obliged to give up either partially or perhaps entirely in the following night the heat received during the day. In neighbourhoods where the greater part of the seeds germinate at a low temperature, at the close of winter at a time when the nights are still long, warmth must be economized as far as practicable, and especially must the loss of heat from the cotyledons by nocturnal

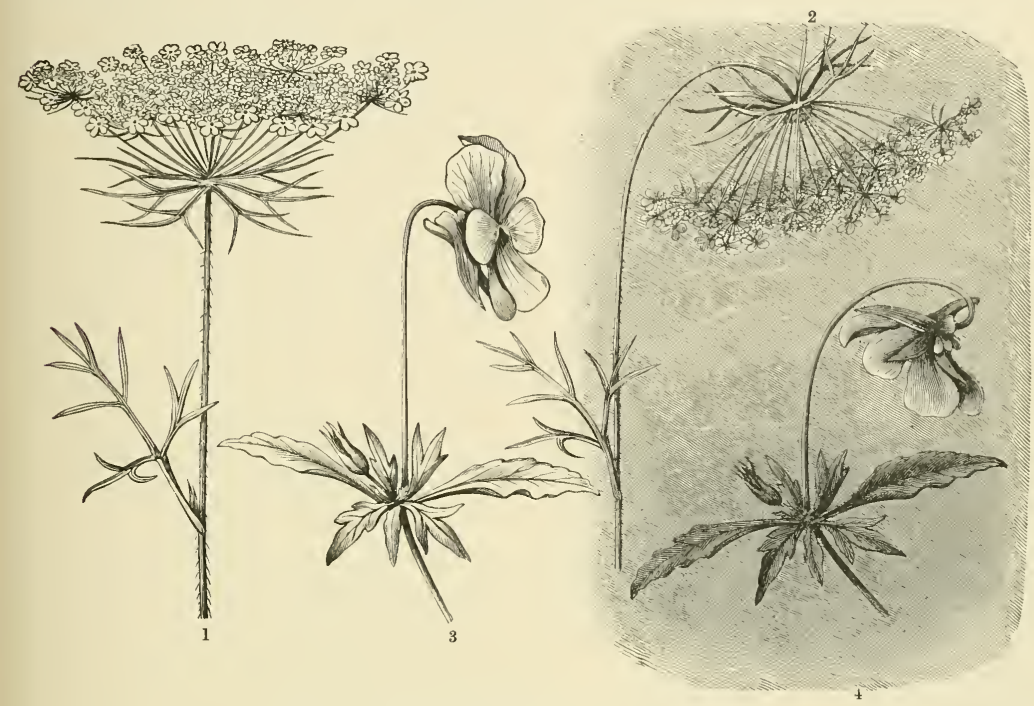

Fig. 132.-Periodic bending of Flowers and Inflorescences.

1 The umbel of the Carrot, day position. 2 The same umbel, night position. ${ }^{8}$ Flower of Pansy, day position. 4 The same flower, night position.

radiation be prevented. This is accomplished by the cotyledons, which are separated from one another like the leaves of a book, and whose broad surfaces are turned towards the sky, closing together when the sun sets, and again assuming that position which they occupied in the quiescent seed. In this way the two cotyledons now have their narrow edges turned skywards, and the loss of heat in the night is as much as possible minimized. This movement of the cotyledons, which on cloudless evenings and in exposed spaces occurs more quickly than under cloudy skies and in places which are overshaded by trees, has also the advantage that the small foliage-leaves, which are visible on the rudinents of the shoot between the cotyledons, are covered over through the night. When the morning breaks, and the danger of excessive loss of heat is passed, the cotyledons again open out in order to sun themselves afresh to their full. 
This opening and closing of the cotyledons is seen particularly well in species of clover and Bird's-foot Trefoil (Trifolium and Lotus), in all mimosas and bauhinias, and numerous other leguminous plants; also in species of wood sorrel (e.g. Oxalis Valdiviana, rosea, sensitiva), in the gourds, cucumbers, and melons, in the Sunflower (Helianthus annuus) and in the Tomato (Solanum Lycopersicum), in species of Mimulus and Mirabilis, the Corn-cockle (Agrostemma Githago), the Chickweed (Stellaria media), and many others.

By alterations of position, similar to those exhibited by cotyledons, the so-called compound leaves are also in many instances protected against nocturnal radiation. By compound leaves are understood those which bear either pinnate or radiating leaflets on a common stalk.

These compound leaves in some cases, which have already been alluded to, are spread out during the mild night, but are, on the contrary, folded together under the burning noonday sun. In by far the greater number of cases, however, especially in species whose habitat is exposed to great cooling in the night, the reverse is observed. In sunshine the surfaces of the leaflets are arranged more or less parallel to the ground, the upper side is turned to the sky, and is fully and completely flooded by the sun's rays. If this position were retained after sunset, the surfaces of the leaflets would be forced to give up much heat by radiation towards the night sky. In order to avoid this the leaflets fold together either upwards or downwards, and place themselves, so to speak, on edge. In this way their broad sides become vertical, in which position they are protected from radiation as much as possible.

There are provided for the accomplishment of these movements certain swollen cushions of succulent tissue at the bases of the several leaflets, and often at the base of the common petiole. These are known as pulvini and each consists of parenchymatous thin-walled cells surrounding a strand of compressed vascular bundles, which further up becomes the midrib of the leaflet, which is inserted on the pulvinus. The parts of this strand where surrounded by the pulvinus are supple and very flexible, but above the pulvinus they become stiff and firm, forming as it were the main support of the whole leaflet, so that indeed alterations of position of the midrib are participated in by the whole.

In order to represent clearly how a movement is brought about in the leaflet by means of its supporting pulvinus, let us imagine a straight rod which is only flexible at the base, and is there held fast between two springs. The pressures proceeding from the two springs is equally strong, and the rod is therefore maintained in an upright position. If the pressure of the spring relaxes on one side, the stick must bend over in the direction of the diminished pressure. If the pressure of the two springs be afterwards equalized, the rod will again assume its original erect position. If for the rod we now substitute a leaflet traversed by a rod-like midrib, i.e. by the vascular bundle-strand mentioned above, and imagine two opposed halves of a turgid cell-tissue instead of the two springs, then the leaflet will be kept upright by the equal tension of the pulvinus situated at the base of the strand; but as soon 
as the turgidity of the cells increases in one of the halves of the pulvinus, in consequence of an increased afflux of water, this half elongates, bulges out, becomes convex, and exerts a stronger pressure than the opposite half, so that the latter becomes concave and much contracted. The supple portion of the bundle-strand between the two halves of the cushion becomes bent, and the leaflet, whose stiff
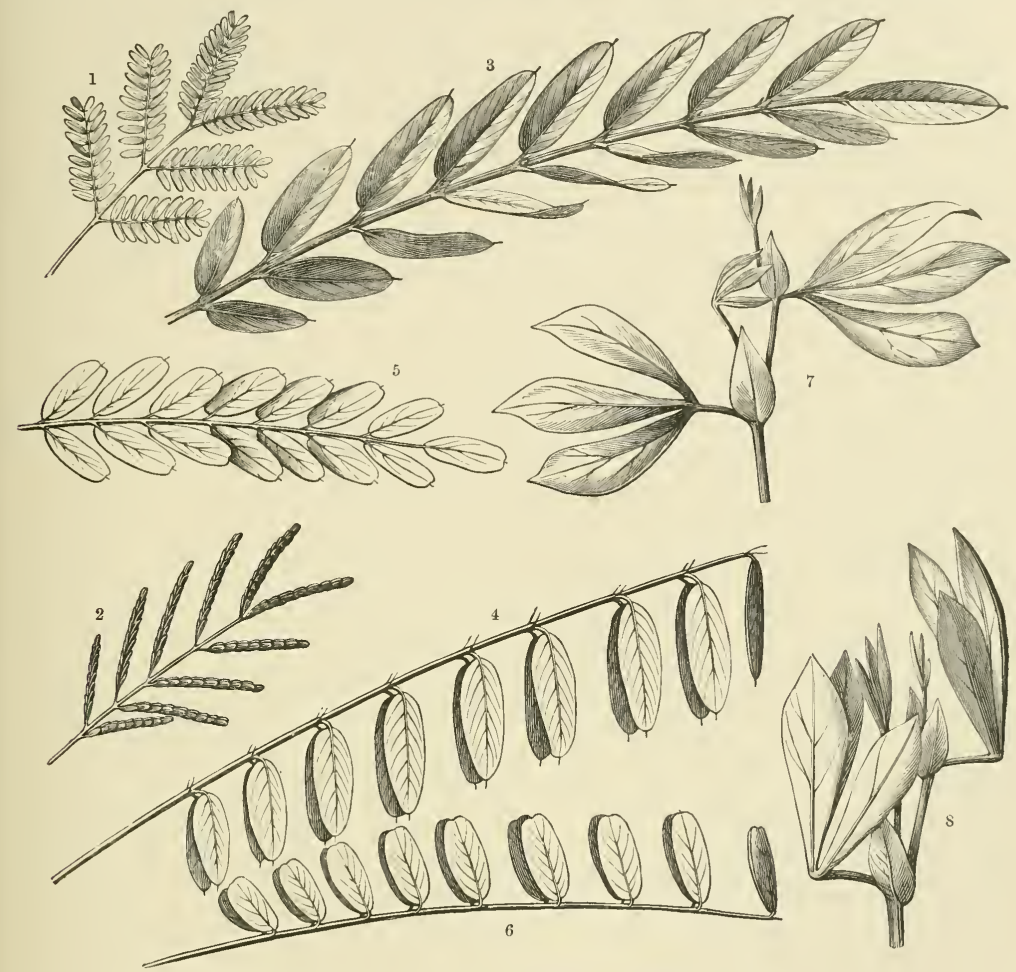

Fig. 133.-Alteration of Position of Leaflets in Compound Leaves.

1 Leaf of Mimosa Lindheimeri, seen from above, in day position. 2 The same in night position. 3 Leaf of Amorpha fruticosa in day position. 4 The same in night position. ${ }^{5}$ Leaf of Coronilla varia in day position. 6 The same in night position. 7 Leaf of Tetragonolobus siliquosus in day position. 8 The same in night position.

midrib is formed by the continuation of the bent bundle-strand, is inclined over in the direction of the concave half of the pulvinus. If the increase of turgidity occurs alternately first in the one and then in the other half of the pulvinus, the leaflet will also be bent now to the one, then to the other side; and if the leafsupport has a horizontal position, an alternate rising and sinking of the leaflet will occur. It is to be noticed here that the leaflet itself remains actually quite passive, and that the pressures which have come into play only operate in the pulvinus.

The commonest stimulation to periodic alteration of the turgidity in the pulvini 
is the diminution of light and heat after sunset, and since the rising and sinking of the leaflets effected thereby coincides with the nocturnal sleep of birds and other animals, the phenomenon described has been interpreted in this sense, and termed the sleep of plants.

The rapidity with which the movement of the leaflets is accomplished varies very much in different plants, and even in the same species is sometimes quicker, sometimes slower, according to external influences. All the circumstances which increase the turgidity of vegetable-cells have also an accelerating effect on these movements. It is still an unsolved problem how far light and darkness influence the turgidity of the pulvini. It is supposed that the darkening produces an increased afflux of water and an increase of turgidity in the whole pulvinus, but more rapidly in one half than in the other; while the protoplasm in one half of the cells of the pulvinus is stimulated by light to give up a portion of the watery sap, lying at the time within the sphere of its influence, to the surroundings-by which indeed not very much is explained.

In one section of plants whose leaflets assume a sleep position when darkness sets in after sunset, the leaflets provided at the base with pulvini move upwards, and in the other section downwards. The movement is upwards as a rule in ternate leaves, of which the clover may serve as a type. When the elevation has been accomplished, the leaflets are directed either all three almost at a right angle to the horizon, or the terminal leaflet is bent up rather more than the two lateral ones. A very pretty example of this is furnished by Tetragonolobus siliquosus, represented in figs. $133^{7}$ and $133^{8}$, and also by Desmodium penduliflorum as well as by various species of Lotus, Trifolium, Melilotus, Medicago. Pinnate leaves, whose leaflets rise up and arrange themselves next one another like the leaves of a closed book, are found on numerous small scrubby mimosa bushes of Peru, of which a species, viz. Mimosa Lindheimeri is represented in figs. $133^{1}$ and $133^{2}$, in the day and night positions. In the Australian Acacia lophantha and several other true acacias, in Schrankia aculeata and species of Eschynomene, in the American gleditschias, further in the Australian Clianthus Dampieri and in the widespread European Coronilla varia. In fig. $133^{6}$ is shown how the erect leaflets of the Coronilla lie against one another very regularly in pairs. Just as often, instances are observed in which the leaflets of the pinnate or digitate leaves fall downwards after sunset. An example of this group is afforded by the leaf of one of numerous American amorphas (Amorpha fruticosa), which is illustrated in figs. $133^{3}$ and $133^{4}$. These leaflets which droop at night are also very noticeable in the Indian Averrhoa Carambola, in various species of indigo and liquorice (Indigofera and Glycyrrhiza), in the sophoras (e.g. Sophora alopecuroides), in the American tree, Gymnocladus Canadensis, and in robinias, of which Robinia Pseudacacia (popularly called acacia) is planted everywhere as a decorative tree. In like manner in the widely-spread common Wood Sorrel (Oxalis Acetosella), cf. fig. $90^{8}$, in the Indian pinnate-leared Oxalis sensitiva, and in numerous American sorrels.

With respect to the radiation, it is immaterial whether the leaflets rise up or 
sink down; the main point is that they turn their profile towards the night sky, and this occurs in all the above-mentioned cases. But it should be noticed here that besides the protection against excessive loss of heat, other advantages are gained by the periodic alteration of the position of the leaflets, and in this respect it is anything but a matter of indifference whether the leaflets fold together above or below. Since the vertical position of the leaf-surfaces also furnishes an important protection against excessive transpiration, various conditions of the leaf construction connected with this protection are also significant. For example, the question whether the stomata are developed on the upper or under side of the leaflet is determined, inasmuch as the sides provided with stomata, as a rule, come in contact with one another. Finally, it must not be denied that bedewing also has an influence on the alteration of position of the delicate leaflet.

A great number of plants whose leaflets assume the sleep position at nightfall also exhibit this phenomenon on bright days when shaken or touched, and indeed more rapidly under these circumstances than at the on-coming of darkness. The slightest touch of the finger, even shaking by a moderate wind, suffices to cause the leaflets of these plants to fold together. In the Oxalis sensitiva of tropical India even the disturbances of the air caused by the approach of man is enough to cause the pinnate leaflets to fall together rapidly, and the same thing occurs in several papilionaceous plants (e.g. Smithia sensitiva and Eschynomene Indica), as well as in several mimosas. When we move away from the immediate vicinity of these plants, and complete stillness once more reigns in the air, the folded leaflets again spread out and turn their upper surfaces skyward. The phenomenon that the plants close up, frightened at the approach of man, as if they felt or in some way became aware of his approach, caused the older botanists to name them Sensitive Plants.

From a cursory examination it appears that the folding of the leaflets in these sensitive plants caused by shaking, and the assumption of the sleep position at the setting in of darkness, are the same processes; but closer investigation shows that there is an essential difference between them. Outwardly this difference is recognizable by the fact that in the sleep position, brought about by darkness, the pulvinus below a leaflet remains quite rigid, while in the folding of the leaflets produced by shaking a relaxation of one half of the pulvinus occurs. In sections through the pulvinus of sensitive plants, it is seen that numerous intercellular spaces are contained in that portion of the parenchyma which adjoins the flexible strand of vascular bundles. It is also seen in these sections that the thickness of the cellwalls in one half of the pulvinus is three times as great as in the opposed half, and that all these cells communicate with each other by extremely fine canals. If the thick-walled portion of a pulvinus is disturbed with a bristle, no alteration is produced; but as soon as that side characterized by its delicate cell-walls is touched ever so lightly, it changes colour. It now appears a darker green, due to the fact that watery sap has been pressed out from the cells into the intercellular spaces. The slightest pressure is felt, accordingly, as a stimulus by the protoplasm in those 
cells, and causes them to discharge a portion of their water into the adjacent spaces. By this means the turgescence in this part of the cushion is very much diminished, the tissue becomes flaccid, and in proportion as this flaccidity obtains the turgidity in the tissues of the opposite half of the lcaf-cushion increases. It seems that a portion of the water given up by the stimulated protoplasm is forced into the opposite tissue, and that thus the turgidity there is augmented. Such a contrast in the turgidity of the two halves of the pulvinus cannot be without influence on the strand of vascular bundles lying in its centre; it becomes bent in the direction of the diminished turgidity, and the leaflet, whose midrib is formed by a continuation of the said vascular bundle-strand, follows this movement.

In nature, of course, stimulation of the protoplasm by contact of a solid body only occurs exceptionally. There the process described above is brought about by currents of air, and principally by falling rain-drops. Few phenomena have such a peculiar appearance as the movements which occur in the foliage of the already mentioned Oxalis sensitiva when rain comes on. Not only do the leaflets on which the first rain-drops fall, fold together in a downward direction, but all the neighbouring ones perform the same movement, although they have not themselves been shaken by the impact of the falling drops, and one is involuntarily reminded of the children's game in which sloped cards are placed behind one another lengthwise in a long series, and the fall of the outermost card, produced by the touch of a finger, causes in a moment the collapse of all the others. But it is not enough that the opposite leaflets, until now flatly outspread, are depressed by the shaking. The movement is continued to the common leaf-stalk bearing the numerous pinnæ. This also bends towards the ground, and hangs down apparently prostrated, in consequence of the alteration of turgidity in the pulvinus at its base. The raindrops now slide over the bent leaf-stalk, whose point is turned towards the ground, and down over the depressed leaflets, and not a drop remains behind on their delicate surfaces.

The transmission of the stimulus, at first received only by a single leaflet, to the neighbouring leaflets and common leaf-stalk, and finally even to the whole plant, reminds one strongly of the like process in the leaves of the Sundew and of the Venus's Fly-trap. It also recalls the transmission of the stimulus in the protoplasm of lower animals, and is indeed to be explained in a similar manner. Probably the protoplasmic masses of the sensitive groups of cells in all pulvini are connected together by continuous delicate protoplasmic threads penetrating the cell-walls, and the molecular disturbance of the protoplasm, produced by the stimulus, although at first it comprehends only a single cell, is transmitted like an electric current in telegraph wires over the masses of protoplasm, strung together in close connection, and linked by the delicate plasma-threads; thus the same phenomenon is produced in all, viz. contraction of the cells and a forcing out of cell-sap into the intercellular spaces.

The other sensitive plants behave like the above-described Oxalis sensitiva, except that there is a difference in the direction in which the leaves fold together. 
Eschynomene Indica, an elegant herb-like plant with papilionaceous flowers and extremely delicate doubly-pinnate leaves, as well as the Indian Smithia sensitiva (which likewise belongs to the Papilionaceæ), fold their leaflets together above, and

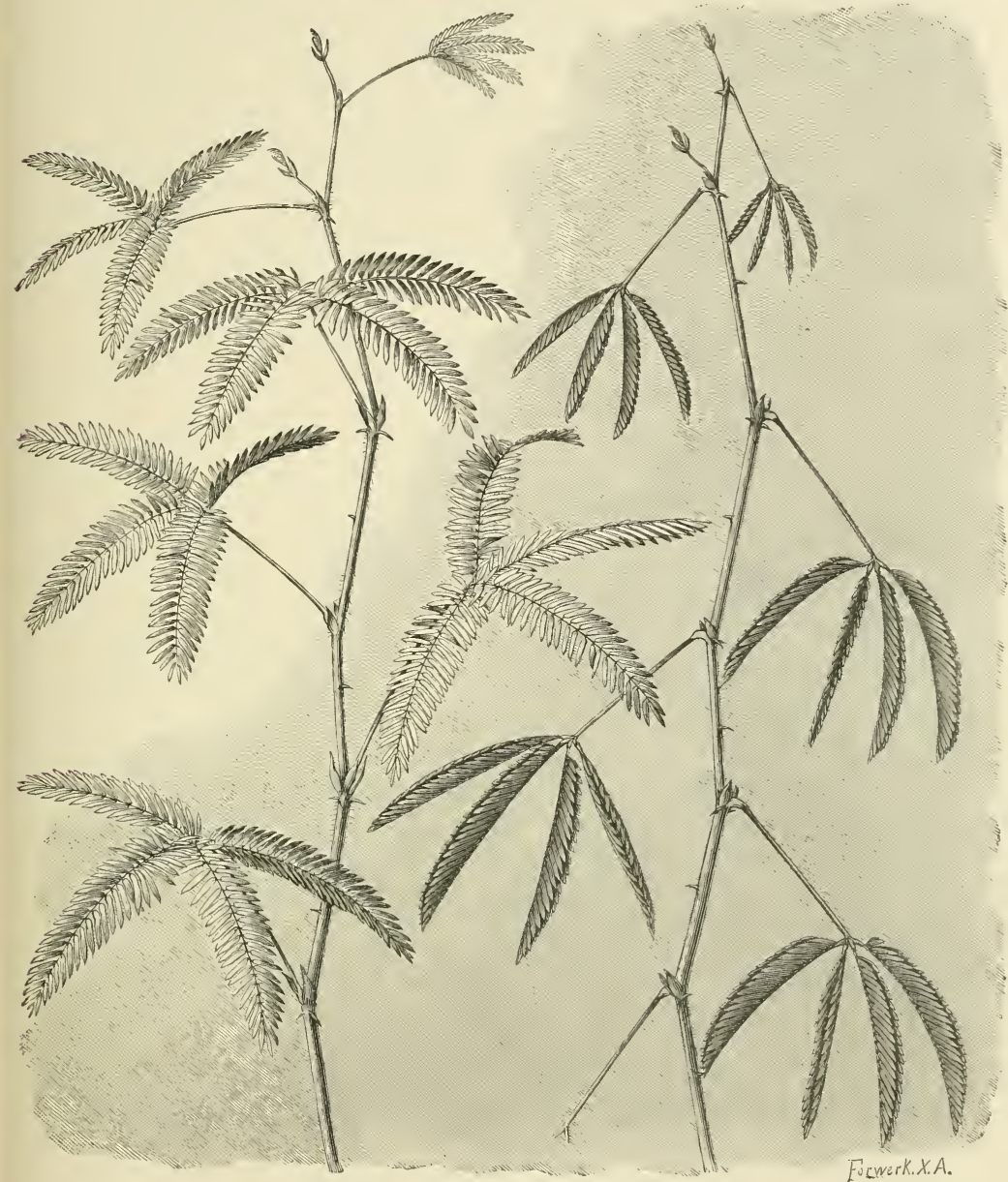

Fig. 134.-Mimosa pudica in day and night positions.

depress the common leaf-stalk directly the first rain-drop has produced a shaking. The same applies to several mimosas (Mimosa pudica, sensitiva, casta, dormiens, humilis, viva), of which the first, a species native in Brazil, is represented in fig. 134. In these mimosas there is really to be noted a threefold movement; first of ail the tiny leaflets fold together above, and at the same time direct themselves a 
little forward, so that each in front is partly covered over by the one immediately behind it; then the four ribs or axes, beset with the tiny leaflets, move towards one another like fingers which had been outspread and are now closed together; and thirdly, the common leaf-stalk, bearing in front the four axes, droops downwards. The leaflets of several species of wood sorrel which have clover-like or fan-like leaves, and not pinnate leaves like the above-mentioned Oxalis sensitiva, also fold their leaflets together when shaken by rain-drops. In these species of wood sorrel, however, we have again a way of diverting water, essentially differing from that above described. The common leaf-stalks do not bend towards the ground, but remain erect; on the other hand, the drooping leaflets fold slightly along the midrib, each of them forming a shallow groove, and the rain water trickles on to the delicate leaves, and then flows through these channels to the ground. (Cf. fig. $90^{8}$, the lowest leaf, whose three leaflets are beginning to droop and to fold.)

From the above it is indeed evident that one benefit which the sensitive plants obtain by the sudden folding together of their leaflets lies in the rapid diversion of the falling rain-drops thereby rendered possible. By this we do not imply that this is the only advantage which ensues from the movements described. It frequently happens that dry, gusty winds and drifting sand and extraordinary noon-tide heat cause the folding of the leaflets. In the last-mentioned instances it is rather the danger of excessive transpiration which causes the plants to place the broad surfaces of their leaflets vertically, and all observations go to show that the leaflets can escape very various dangers by the assumption of the so-called sleep position-in the clear night, the loss of heat by radiation towards the starry sky; in the hot mid-day, drying up in consequence of rapid evaporation; in rainy weather, the breaking up of the tender leaves and their inclination towards the ground, as well as the collapse of the whole plant under the weight of the falling drops in a sudden severe downpour of rain. It is possible that yet a fourth advantage is obtained by these movements. Grazing animals which sniff the delicate leaves of the sensitive plants and disturb them with their mouths are perhaps astonished and frightened at the sudden movements of the leaflets, and abstain from eating these uncanny plants, especially when between the descending leaflets pointed rigid spines become visible, as is especially the case in many mimosas.

It cannot be too often insisted that the same and similar contrivances, as well as the same and similar processes, may have a very different significance according as they occur in this or that plant, in this or that habitat, and under these or those climatic conditions; just as, on the other hand, several advantages can be simultaneously obtained by one and the same contrivance. Thus for instance, the position which the leaves emerging from the buds in spring assume is very instructive. When the vegetative activity is interrupted by a cold winter, and when, moreover, the temperature occasionally in clear spring nights sinks below zero, the surfaces of the leaves just escaping from the buds are regularly placed vertically (cf. fig. 90). During the day excessive transpiration from the still thin- 
walled tissues is prevented by this position, and during the night the vertical position of the young leaves has this advantage, that by it radiation, that is to say, excessive loss of heat, is hindered. The young not yet completely developed foliage is in both these respects very sensitive, much more so than adult foliage, and this depends upon the fact that the latter is comparatively poor in watery contents, and the composition of the protoplasm has become altered. It may happen that in the same plant, under the same conditions of habitat and like conditions of temperature of air and soil, while the young leaves perish after bright nights in consequence of too great loss of heat, the fully-developed leaves suffer no injury. This brings us to the question, Wherein the damage to plants caused by great loss of heat actually consists?

\section{FREEZING AND BURNING.}

Pancratius, Servatius, and Bonifacius, whose names stand in the calendar against the 12th, 13th, and 14th of May, have popularly been called "Eismänner" in southern Germany and Austria. They have received this nickname on account of the fall of temperature which takes place every year about the middle of May, the cause of which is not yet fully explained. Later in the summer such falls in temperature, connected with cooling of the atmosphere on a large scale, occur on certain days with some regularity; but these have not received so much notice because they are not so dangerous to field products, fruit and wine, as the relapses about the middle of the month of May. Moreover, although really cold days occur in June or July, they are never followed by a frost, while the three "Eismänner" of May usually bring with them severe frosts at night, even in the mildest regions of Central Europe, thus doing incalculable mischief to vegetation.

What first of all strikes us in a frozen plant-organ is that it has completely lost its elasticity. On bending and pressing back with the finger the frozen, stiffened foliage-leaf, a permanent fold is immediately produced; the leaf is broken along this fold, and can no longer resume its former position. At the time of breaking a noise is heard like the crushing of pounded ice, and as a matter of fact it is actually crystallized ice formed in the interior of the leaf which is broken by the pressure and causes this crunching to be heard. As the temperature rises during the day, the frozen plants become thawed, but most of them retain no longer the elasticity which they possessed before the frost. The leaves hang down flaccidly, are of a different green, and are more transparent than formerly. The surface is damp, and the epidermis is easily detached from the deeper tissue-layers. Gradually the languid leaves shrivel up, become dried, and assume a brown or black colour. They entirely resemble burnt or charred leaves, and the farmer says that the frost has burnt them.

What takes place in the interior of the plant on account of this freezing? The idea which botanists once held is as follows: the watery cell-sap of the plants stiffens to ice; but the ice takes up a larger space than was occupied by 
the fluid cell-sap, and consequently the walls of the cells are torn and burst like a glass bottle in which water has frozen. A tissue whose cells are rent can, however, no longer perform its functions. Moreover, although the ice melts by and by, the damage to the ruptured cell-walls is irreparable. Besides, the cell-sap streams from the cell-chambers of a thawing plant, and the leaves and stem which have thawed after freezing are seen to be not only blackened, soft, and pulpy, but are also covered over with a watery film which is never absorbed again into the interior.

Renewed investigation has shown that this idea of the freezing of plants needs revision. First, in that no rupturing and bursting of the cell-walls occurs by the pressure of the ice formed in the interior of the cells. In the tissues of plantorgans surrounded by air the formation of ice does not usually commence in the interior of the cells, but in the intercellular spaces; and the ice-crystals are first formed in the interior of the cells only in those aquatic plants in which intercellular spaces are absent.

If Nitella syncarpa, belonging to the Characeæ, which is met with in the clear water of lakes and pools in Central Europe, is exposed to a temperature of $0^{\circ} \mathrm{C}$., its vital activity is not disturbed. Even the streaming of the protoplasm in the cells is still very active, and even if by further cooling of the surrounding water to $-2^{\circ}$ needles of ice are formed, the streaming of the protoplasm may still be recognized. The cells are indeed somewhat compressed by the ice-needles, but the protoplasm is never killed, even at $-3^{\circ}$. It first begins to shrivel up between $-3^{\circ}$ and $-4^{\circ}$, gives up a portion of its water, shrinks away from its cell-wall, forms a folded, contracted sac in the middle of the cell, whilst the water excreted stiffens into ice between this sac and the cell-wall. If this Nitella be again exposed to a higher temperature the ice melts, the protoplasm expands, and lies close to the cell-wall; but it is incapable of again producing the streaming movement; it has ceased to live; its molecular constitution has evidently become so fundamentally altered by the separation of water from it that a reconstruction is no longer possible.

In the stems and leaves of plants surrounded by air, the ice always first arises, as remarked above, in the intercellular spaces. But since usually air, and not water, is contained in the intercellular spaces, the water stiffening into ice in them must have been first excreted from the neighbouring cells shortly before the freezing. And this is what actually happens. The structure of the ice-crystals plainly shows that the water has come from within through the cell-wall to the exterior, and that not once, but over and over again; for on the outer walls of the cells which face towards the intercellular spaces the ice is seen in the form of small discs placed above one another and combined into pillars, and these discs can have been only gradually formed one after the other. This observation, however, rasses the questions, What portions of the cell give up the water? and why does the water freeze in the intercellular spaces and not in those places which it occupied before the frost? In answer to these questions it must first 
e remarked that the water absorbed by the plants only partly enters into hemical combination with the materials of the cell-body and cell-wall; that unother part, which we have called the water of imbibition, is not chemically ombined. The cell-wall and cell-body are saturated with this latter, and the :ell-sap in the vacuoles of the protoplasm also contains a large quantity of such water. In the cell-sap it appears as the solvent of the acids, salts, and other naterials there present. The water by which the protoplasm and cell-walls are ;aturated, and which we must imagine filling the interstices, like capillary spaces, setween the groups of molecules, is indeed held fast by the molecules of the rotoplasm and cell-wall, and the water in the cell-sap by the molecules of acids ind salts, but yet certainly not so energetically as the chemically-combined water $\mathrm{n}$ the albuminous substances of the protoplasm.

What happens now in a body which holds fast the water in its smallest nterstices, like paste, for example, or in which the water appears as a solvent is in an alum solution, when warmth is withdrawn, and when it is cooled down ;o the freezing point of water? It is very remarkable that the water does not mmediately stiffen into ice as long as it is retained in the capillary spaces, or is a solvent, and many salt-solutions can be cooled down to $5^{\circ} \mathrm{C}$., some even to $10^{\circ}$, below zero without freezing. When at length under the influence of still ower temperatures a stiffening occurs, a separation has always taken place oreviously; the water has run together from the finest interstices of the paste nto its larger spaces; it is first changed into ice in these cavities, and the water of the salt solution has separated from the molecules of salt, and is then first shanged into ice-crystals.

The same thing occurs, however, with the water saturating the cell-wall and protoplasm, and serving as solvent of certain materials contained in the cells. The formation of ice occurs in a very few species only on cooling the plant-tissues down to $-1^{\circ}$; in most instances the temperature must sink to $-2^{\circ}$ or $-3^{\circ}$ in order that ice may be formed in the cooled tissue. And indeed the water here has sepacated from the molecules by which it was hitherto held fast before it congealed, and it does not freeze in the interior of the cells, but outside them in the intersellular spaces. In order that the water should get from the interior of the cell into the adjoining intercellular spaces, a pressing and squeezing is necessary, and this pressure can only proceed from the living protoplasm in the cell-chambers; sonsequently the process of freezing can be most correctly represented in this way, viz. that the protoplasm becomes stimulated and roused by the lowering of the temperature to transport a portion of the water from the interior to the exterior of the cell, by means of contraction and pressure. What happens there is accordingly not unlike the excretion of watery sap into the intercellular spaces in the stimulated pulvini on the leaf-stalk of Mimosa; but the advantage obtained by the excretion of water in the two cases is very different. In the cooled leaves the benefit of course is to be sought for in the fact that the living portion of the cells is protected from destruction as long as possible by the formation of ice-crystals in the inter- 
cellular spaces. If the water were forthwith frozen inside the cells, between the groups of molecules of the living cell-body and its wall by a few degrees of cold, fundamental displacements and disorganizations of the groups of molecules would be unavoidable. On the other hand, the ice-crystals on the exterior of the cells do not produce such destruction. In the intercellular spaces they can form large clusters, the spaces may be even enlarged by them, and the adjoining portions of tissue may be compressed and split, without a disorganization of the molecular structure of the living cells occurring simultaneously.

It is shown by numerous other phenomena that the excretion of water described does not connote the death of the living cells. It is also beyond doubt that the excreted water can be again received back subsequently under favourable conditions; and that by slow thawing of the ice formed in the intercellular spaces, the water again returns to the places it previously occupied within the cell. If, on the other hand, the cells are no longer able to take back the separated water, or if the cold becomes so severe that finally the water retained by the protoplasm and indispensably necessary to its existence, becomes changed into ice, then a disorganization of the molecular structure is the natural consequence; or, in other words, the protoplasm of the cells in question has been killed by the loss of heat. Then we say the plants are frozen dead.

Thus the difference between mere freezing and freezing to death is made clear; and at the same time the experience of gardeners is confirmed, that the former is not necessarily attended by the latter.

At what degree of cold freezing occurs, and at what freezing to death, depends first of all on the specific constitution of the protoplasm of the various species, but also, in each individual species, upon the stage of development arrived at by the organs exposed to the cold. Just as the water in various salt solutions becomes changed into ice at various temperatures, so the protoplasm of one species exhibits a different behaviour to that of another. It has been mentioned above that the hydrophyte Nitella syncarpa is frozen at a temperature of $-4^{\circ} \mathrm{C}$. Other aquatic plants bear a much greater degree of cold without their protoplasm being killed. Spharella nivalis, which produces the red colour of snow, is exposed in the winter for months to a temperature of $-20^{\circ} \mathrm{C}$. in Arctic regions, and is not destroyed thereby. This Spharella is also frequently exposed to very severe cold on the snow-fields of the Alps during winter nights, and the same remark applies to various species of the genera Epithemia and Navicula and to other Diatomaceæ which are to be found together with Spharella nivalis living on the glaciers. It may be mentioned here in passing that there are also animals which live with these unicellular plants in the ice regions, and are not killed although they remain frozen for months. As soon as they thaw, these Rotifers bring their cilia into action again; the black Poduras, known by the name of glacier-fleas, take their flying leaps, and the spotted spiders again stride with their long legs over the sun-illumined ice-fields; while, on the other hand, the insects driven by the wind to these glaciers are in a very short time killed by the frost 
The same thing occurs in land-plants and lithophytes as with animals and aquatic plants. Plants which closely resemble each other externally and show great similarity in their anatomy may yet behave quite differently in the matter of freezing. While the Stone Pine and the Shore Pine (Pinus Pinea and Halepensis) cannot bear the frost of winter, the Arolla Pine and Bhotan Pine (Pinus Cembra and excelsa) flourish in regions where the trunks and acicular leaves of all the trees are cooled down for weeks to $-20^{\circ}$. Rhododendron Ponticum freezes at $-2^{\circ}$, but Rhododendron Lapponicum survives the severest cold of the northern winter. If Echeverias are brought out of the greenhouse on a cold autumn night into an open place where the temperature falls to $-1^{\circ}$, they will be irretrievably lost; while most of the European succulent plants closely allied to the Echeverias, and agreeing with them in the structure of the fleshy leaves, endure the same degree of cold without injury-not only for a night but even for weeks. The northern Sedum Rhodiola and several Alpine species of house-leek growing on the narrow ledges of rock faces in the high Alps (e.g. Sempervivum montanum and Wulfenii) are exposed for weeks to a temperature of $-10^{\circ}$, and yet the protoplasm of their fleshy leaves does not freeze. There are also a number of biennial and perennial plants which cannot actually be called succulents, but which neverthelese form smooth, turgid leaves in the autumn arranged in rosettes lying on the ground, outwardly in no way protected against loss of heat. The leaves of these rosettes are exposed to the greatest cold in regions where the winter is severe, especially when little or no snow has fallen, and the temperature of the succulent tissue is often cooled down to $-20^{\circ}$, and yet the protoplasm is not killed. The Scurvy Grass (Cochlearia officinalis) is, in this matter, particularly worthy of notice. It would naturally be expected that its smooth, turgid, dark-green leaves would be killed with the first hoar-frost, while in reality they endure a very considerable cold without the slightest injury. There are few places on the earth where such a severe winter climate prevails as on the shores of Pitlekaj on the northern coast of Siberia, where the Vega expedition passed the winter of 1878-79. In November the mean temperature amounted to $-16.58^{\circ}$, in December to $-22.80^{\circ}$, in January to $-26.06^{\circ}$, in February to $-25.09^{\circ}$, in March to $-21 \cdot 65^{\circ}$, in April to $-18.93^{\circ}$. But these were only the averages; on many days the temperature fell to $-30^{\circ}$, and $-40^{\circ}$, and once the minimum even reached $-46^{\circ} \mathrm{C}$. On the summit of a fairly high sand-hill over which the icy north and north-east wind swept almost uninterruptedly, a plant of Scurvy Grass (Cochlearia fenestralis) was observed. This plant had begun to bloom in the summer of 1878, and had also partly developed fruit. When the winter began, however, this Cochlearia still possessed unripe fruits, flowers, and flower-buds as well as succulent green foliage-leaves; and it was to be expected that the delicate succulent tissue would be completely destroyed during the long winter under the influence of the continuous cold. But in the summer of 1879 the plant, whose tissue had undoubtedly been cooled down for a long time to $-30^{\circ}$, and frozen, began again to grow, and continued its growth where it had been interrupted at the beginning of winter. The leaves resumed 
their functions as in the previous summer, the flower-buds opened, and new inflorescences sprang from the axils of the leaves, proving that the protoplasm of this plant had not been killed even by a temperature of $-46^{\circ}$.

Myrtle and orange trees freeze dead from $-2^{\circ}$ to $-4^{\circ}$, cypresses and fig-trees from $-7^{\circ}$ to $-9^{\circ}$; vines at $-21^{\circ}$, oaks and beeches at $-25^{\circ}$, plums and cherries at $-31^{\circ}$, and apple and pear trees at $-33^{\circ}$, and this can only be explained by the specific constitution of the protoplasm. We are forced to assume that the cell-body is destroyed in one case by a certain temperature, and in another by a different temperature, in the manner already described.

It has been previously remarked that the temperature at which freezing takes place also depends upon the stage of development of the plants. It is generally known that woody trunks and branches, foliage, and flower-buds, and especially seeds bear quite extraordinary winter temperatures when they have been poor in water in the autumn. In Yakutsk and Werchojansk in Siberia, where the mean temperature in January amounts to $-42.8^{\circ}$ and $-49 \cdot 0^{\circ}$, and where $-62 \cdot 0^{\circ}$ and $-63 \cdot 2$ (the lowest temperature hitherto generally observed on the earth) were noticed, where for months the temperature in the shade does not rise above $-30^{\circ}$, numerous herbs and shrubs are found whose upper organs are exposed for weeks to a degree of cold at which mercury freezes; even birches and larches flourish there with the most vigorous growth, and there can be no doubt that the wood and buds of these trees are every year cooled down for a long time to $-30^{\circ}$, and yet are not frozen. Moreover, every winter the wood of the juniper and of spruce, of silver firs and arollas sinks down to $-10^{\circ}$ in inclement situations on the Central European mountains, and the evergreen leaves of these woody plants become cooled far below the freezing point of water without suffering the slightest damage. On this account the seeds inclosed in the berries and cones of the trees named bear the lowest temperatures without injury, which is so much the more remarkable since these seeds require two summers for ripening, and therefore must pass through the severe winter of the first year in a still unripe condition. The seeds of other plants also are exposed to great cold through the winter. Thus, for example, those of the Laburnum (Cytisus Laburnum) do not fall off as soon as they are ripe, but remain hanging to the sides of the dehisced pods, and as these are not detached from the branches until the following spring, the temperature of the seeds during the winter falls far below zero. They nevertheless maintain their germinating powers. Laburnum seeds, which had been during the winter for weeks under the influence of a temperature of $-15^{\circ}$, germinated in the following summer, and so had evidently suffered no injury from the cold. Other seeds, too, even from tropical regions, which had been experimentally subjected to temperatures of $-40^{\circ}$, were not found to have lost their germinating capacity, and consequently their protoplasm had not been killed even by this excessive temperature.

Since, on the other hand, it is known that the young fruits and seeds of the laburnum, and still more those of tropical plants, are already congealed by lowering the temperature to $-2^{\circ}$, it follows that portions of that same plant in various 
stages of development are affected differently by the lowering of their temperature below the freezing point. For the majority of plants the rule holds that death in consequence of frost occurs the sooner, the younger and richer the tissues in question are in water. The foliage of beeches, hornbeams, and deciduous oaks, which is not killed in the autumn, even after repeated frosts, withers, shrivels, and dries up when young if the temperature sinks below zero only for a single night in spring. Even many Alpine plants which bear very low temperatures without injury, when completely developed, may suffer harm if they are surprised by a frost at the period of most active growth. When on one occasion at the end of June the temperature sank to $-6^{\circ}$ on the mountains near Innsbruck, already free from snow, at an altitude of 2000 metres, the young foliage-leaves of the Rhododendron (Rhododendron hirsutum), which had just sprouted, and were not yet fully grown, were destroyed on all the plants. They became brown and dried up, while the old fully-formed green leaves, remaining on the same plants from the previous year, underwent no alteration from the frost.

Such phenomena can only be explained by the assumption that in young, undeveloped organs much water is present, and is not under the control of the living protoplasm. As such water we may consider that which is conducted from the roots to the green tissue, to be there liberated in the form of vapour; that which mounts through the vascular bundles of the stem, streams through the veins of the leaves, under certain circumstances is even forced into the intercellular spaces, and passes out by the water-pores in the form of drops. This water is not retained by molecular forces, nor protected against freezing, but turns into ice even at a temperature of $-1^{\circ}$. Since it is abundantly present in the young tissue when freezing takes place, extensive ruptures and mechanical injuries to the water-conducting tubes and rows of cells are unavoidable. But if the conduction of the crude foodsap is interrupted in a young plant-organ during its growth, transpiration in it can no longer occur properly, and the transpiring cells become withered and dried up even although their protoplasm should have suffered no direct harm from the frost.

Naturally connected with this discussion is the question whether a plant can freeze at a degree of temperature above that of the freezing-point of water. By the majority of gardeners this question would be answered in the affirmative, and their reply would be founded upon the fact that tropical Acanthaceæ, variegatedleaved Coleus, basils, melons, tobacco-plants, \&c., become withered, dry up and die if they are exposed for only a single night to a temperature of $+2^{\circ}$. In spite of the great outward resemblance between this phenomenon and freezing, it must nevertheless not be called freezing, for the most distinctive processes of the freezing of living protoplasm, viz. the excretion of water from the cell-body, the hardening of this water into ice, and its inability to return to the cell-body, do not occur in plants which are destroyed under the influence of temperatures above zero. It has been clearly shown that this so-called freezing of plants at temperatures above zero is really a drying-up in consequence of the disproportion between the transpiration from the leaves and the absorption of water by the roots. In consequence of the VOL. I. 
fall of temperature in the soil, the absorbent activity of the roots is so restricted that the loss of water by the leaves through evaporation can no longer be replaced. The leaves then become flaccid, shrivel and dry up, are blackened, and look exactly like leaf-structures which have been killed by frost. It can be demonstrated by a very simple experiment that the cause of death is only the fall of temperature in the soil. If on autumn nights, when the temperature falls to $+1^{\circ}$ or $+2^{\circ}$, "very sensitive" Coleus plants in pots are brought from the warm greenhouse into the open, the pots not being protected against cooling, these plants dry up even the next day. If, on the other hand, the plants are sunk in warm sawdust over which cotton-wool is strewn, and thus care is taken that the temperature of the earth in the pots does not sink below $+7^{\circ}$, then the Coleus does not dry up, and generally suffers no harm even although the temperature of the air and of the airsurrounded leaves should fall during the night to $+0.5^{\circ}$. Since the conduction of water to the transpiring leaves is sustained by the warmth of the soil, these leaves may be protected from the so-called "freezing" even when they cool down to $+0.5^{\circ}$.

Do means also exist by which plants may be protected from actual freezing? To this question the answer follows naturally from the above discussion about the real nature of freezing. If the plants in question can be hindered from assuming that temperature in which their protoplasm is killed, then, of course, a protection against freezing may be afforded. Usually bad heat-conductors are used as protective agents. The plant organs to be protected are clothed with dry straw and twigs, or covered with dried foliage. In regions with continental climates vines are protected against freezing by surrounding the lower portions of the stock with earth. Often plants are also protected by heaping up snow, and gardeners very generally use snow as an excellent protection against freezing. As a matter of experience numbers of plants perish with us during those winters in which no snow falls, while they survive without injury the coldest periods of winter when the snow is abundant. Many species of shrubs and low trees, of which only the lower half is snowed up, while the upper half rises above the surface are found after severe winters to be frozen from the apices of the branches down to the level to which the snow has reached. This happened, for example, in the Vienna Botanic Gardens (1880) with several young trees of the deodar (Cedrus Deodora), with the bushes of Fontanasia jasminoides, and with shrubs of many species of jasmine and indigo. But all these protective agents, twigs, straw, leaves, earth, and snow, fulfil their function only in neighbourhoods where the cold period is of comparatively short duration. In reality they ward off only the first onset of cold, and their principal use lies in the fact that the radiation of heat from the covered portions is retarded. In long and continuous cold the temperature of the coverings not only gradually sinks, but finally that of the covered bodies also, and in Yakutsk a plant whose protoplasm is killed at $-10^{\circ}$ can no longer be protected even by the thickest covering of straw, leaves, or earth.

Moreover, in nature we can only speak conditionally of a natural protection against freezing, and only in those regions where periods of great cold alternate 
with milder intervals during the winter, and where as a rule a warmer day succeeds the cold night, which is the case wherever the sun does not remain during the winter below the horizon for weeks or perhaps months. All coverings which protect from freezing in temperate zones are therefore entirely useless in Arctic regions. The snow which, as stated, is in the north temperate zone one of the best protective measures against severe temperatures, cannot in the Arctic regions at all hinder the penetration of the cold. Kane found the temperature in North-west Greenland at $63 \mathrm{~cm}$. under the snow to fall to $-21 \cdot 3^{\circ}$ and to $-16.3^{\circ}$ at $126 \mathrm{~cm}$. below. The observations which were undertaken during the wintering of the Swedish Polar Expedition in Mussel Bay on the north coast of Spitzbergen, showed that on the 14th February 1873, when the temperature of the air was $-35^{\circ}$, the snow had fallen to $-26^{\circ}$ at $26 \mathrm{~cm}$. below the surface, and at a depth of $35 \mathrm{~cm}$. to $-20^{\circ}$. On the $23 \mathrm{rd}$ February the snow at a depth of $30 \mathrm{~cm}$. showed a temperature of $-21^{\circ}$, while the temperature of the air was $-32^{\circ}$. On the North Siberian coast the snow at a depth of $30 \mathrm{~cm}$. was found by the Vega Expedition on the 22d March to be cooled down to $-16 \cdot 1^{\circ}$, and the earth below it to $-15 \cdot 1^{\circ}$, while the temperature of the air was -18.2 $2^{\circ}$ At the middle of March the sandy soil penetrated by the roots of the Northern Bent Grass (Elymus mollis) exhibited at a depth of $63 \mathrm{~cm}$. a temperature of $-20^{\circ}$.

It is quite different in the north temperate zone. When the sun shines on the snow, if only for a few hours of the day, it becomes warmed and usually melted at the surface. In the Alps, during the shortest days in December, when the temperature of the air in the shade is $-10^{\circ}$ to $-15^{\circ}$, melted drops may be seen in mid-day trickling down from the sun-illumined roofs of the hay chalets situated high up on the mountain slopes. Three Swiss, who had determined for the purpose of meteorological observations to pass the winter of $1865-66$ in the hut situated at an altitude of 3333 metres on the Matterhorn, observed on the 18th December, 1865, and on several other days, that in the sunshine the snow was melted. When the sun sets behind the mountains the melted water, of course, again freezes, but the next day the same process is repeated, while in Arctic regions, in the months of uninterrupted winter night, the fallen snow remains powdery. On mountains of the temperate zones, in consequence of melting under the influence of the sun's rays and the succeeding hardening during the nights, the superficial layer of snow forms a crust of ice which in time becomes so thick that wide stretches of snow-field may be traversed without breaking through it.

This alternation of thawing and freezing in the upper layers of the winter coat of snow has this important use, that in neighbourhoods where the sun shines in the winter the deeper layers of snow, and the solid earth bearing the snow, are never so much cooled as in the far north, where the cooling may continue for months, and where, as the above figures show, it actually does so continue. Minimum thermometers which were placed in the earth in the year 1869 on various mountain heights in the Tyrol, and at the end of the winter were dug up, showed the following temperatures: On the rocky summit of the Hafelekar, at Innsbruck. 
at an altitude of 2343 metres, and $40 \mathrm{~cm}$. below the surface, $-5.3^{\circ}$; on the north side of the summit of the Blaser, at Trins, at an altitude of 2239 metres, $40 \mathrm{~cm}$. below the surface, $-40^{\circ}$; on the northern slopes of the Patscherkofel, at Innsbruck, 1535 metres above the sea-level, $60 \mathrm{~cm}$. below the surface, $-2 \cdot 9^{\circ}$. The layer of snow lying on the ground at these three points was not a thick one, and varied from 30 to $60 \mathrm{~cm}$. Where the snow-layer was at least three times as thick, the minimum thermometer gave the following results: On the south side of the summit of the Blaser, at Trins, at an altitude of 2239 metres, $40 \mathrm{~cm}$. below the surface, $+0 \cdot 1^{\circ}$; rather lower on the same mountain, viz. at an altitude of 2086 metres, near the cottage of my experimental garden, under a snow-drift 3 metres thick, $+0.2^{\circ}$; on the Patscherkofel, at Innsbruck, 1921 metres in height, in the vicinity of Kreuzbrunnen, $65 \mathrm{~cm}$. below the surface of the ground, $+0 \cdot 1^{\circ}$; near the Heiligwasser, at Innsbruck, at, an altitude of 1261 metres, where the winter coat of snow had attained a thickness of almost 2 metres, $75 \mathrm{~cm}$. below the surface, $+1 \cdot 35^{\circ}$. These statements sufficiently show what a great significance is to be assigned to snow as a protective measure against cold in those regions which are not deprived of the sun in winter-time. While the temperature of the soil penetrated by roots of plants sank even under deep snow to $-20^{\circ}$ on the winter station of the Vega in Siberia, the soil occupied by the roots of plants on the Alpine heights of the Tyrol in places covered with abundant snow, was never once frozen, and where the snow layer was very thin, was cooled down only to $-5 \cdot 3^{\circ}$. Accordingly in the Alps, and generally in high mountains of the north temperate zone, a thick layer of snow plays the part of an excellent protective measure to the soil, and consequently to the plants rooted therein.

There are also plants in Alpine regions which are apparently dependent upon this protective measure, and whose structure makes it possible for them to survive through the severe winter hidden under thick masses of snow. To these belong, in the first place, numerous bush-like woody plants of which Pinus humilis represented opposite may serve as an example. The stems of these pines are not erect like those of other species, but assume a horizontal position even when they attain a considerable thickness. Stems of even $20 \mathrm{~cm}$. diameter, which would certainly be able to sustain the ample crown in an erect position, grow almost parallel to the ground without, however, directly resting upon it. In this respect it is very remarkable that on the slope of the mountain the growing end of the stem is always directed towards the valley, and it is also noticeable that this peculiar habit of growth occurs not only in the mountain pines growing wild in the Alps, but also in those raised from seed in the botanic gardens of towns, and must therefore be regarded as an inherent peculiarity. The boughs and twigs which curve upwards from the main stems are exceedingly elastic, and when pressed down stretch themselves out along the ground. Since all the boughs of the crown are turned upwards, we get here a considerable accumulation, so that in many old clumps of mountain pines the numerous boughs are so thickly crowded and so closely interwoven that progress through them is almost impossible. The extensive tracts 


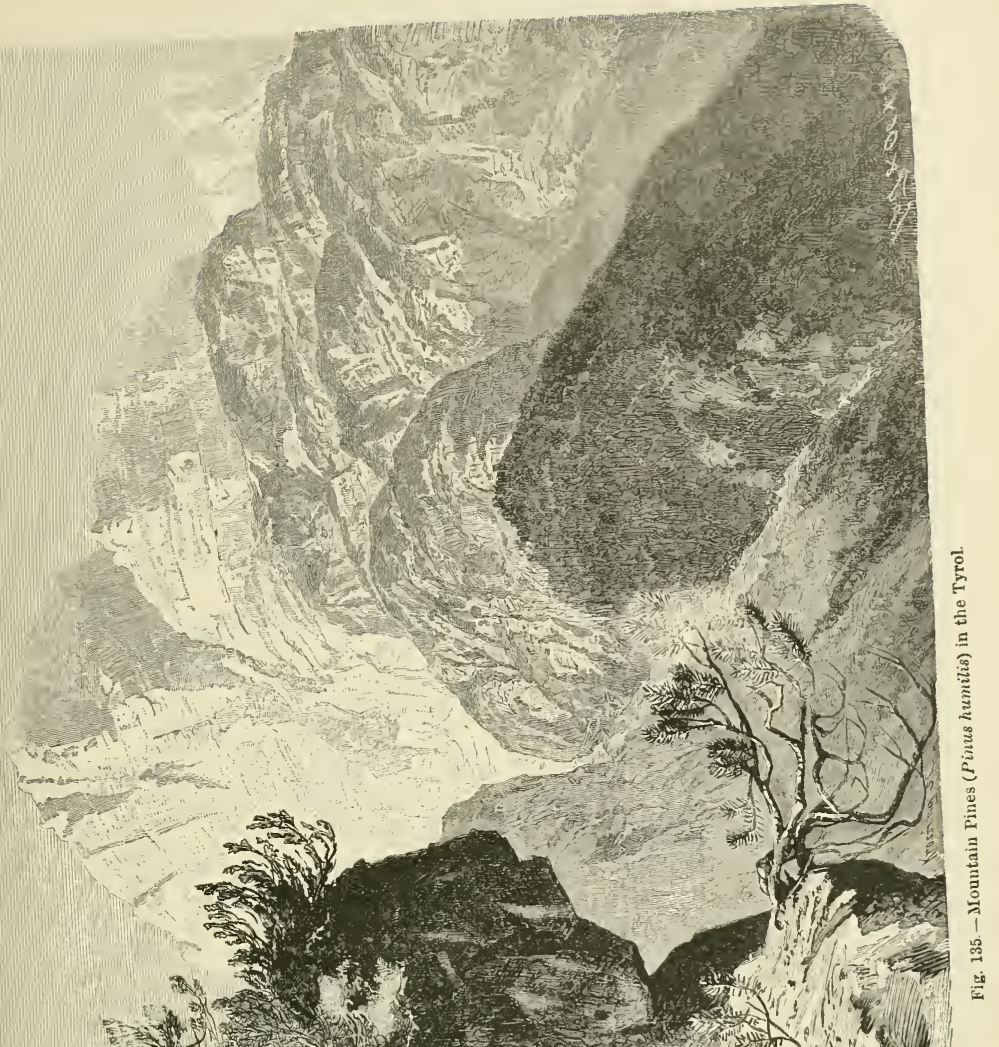
(1)

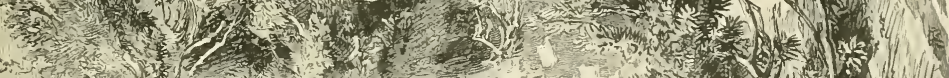

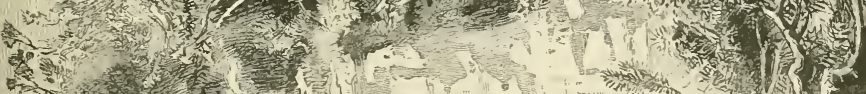
5.

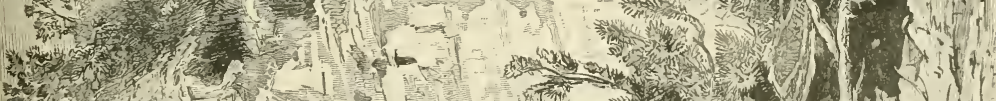

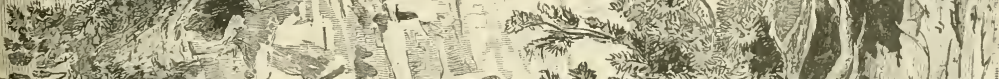

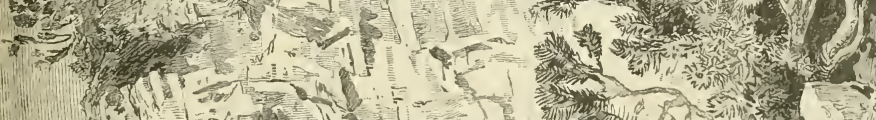

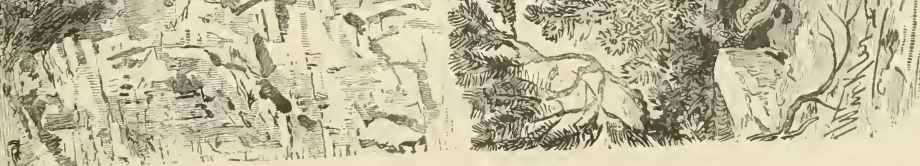


of mountain pines are therefore avoided and left alone, and many of them have never been penetrated by the foot of man during their whole existence. Woe to him who has the misfortune to lose his way in such a tangled wood! The difficulties one has to encounter in a tropical primeval forest beset with lianes are not greater than those with which one must struggle in attempting to press forward here. Frequently the mountain pines grow so high that one is considerably overtopped even when standing upright by the highest prickly branches. It is perhaps possible to make a little progress by climbing over the horizontal, arm-thick stems, but it is vain to endeavour to find one's way and to gain an outlook. If we mount on one of the curved ascending boughs in order to see above the highest branches, the bough bends down to the earth under our weight, along with the stem from which it arises, and we again sink despairingly into the sea of the dark-green crowns. Just such a down-bending occurs, however, under the burden of the winter snow. If then by chance the ordinary mantle of snow is added to by that from avalanches, the pressure increases so much that the branches are pressed down to the soil. This process may go on to such an extent that even many branches, which in the summer stand a metre above the ground, lie in the winter directly on the soil on account of the snow pressure. When the snow melts in the following spring, and the branches are gradually lightened, they rise up again in consequence of their extraordinary elasticity, and resume that position which they occupied in the preceding summer. The process which is here carried on automatically strongly reminds us of the manipulations of gardeners, who in the autumn bend down rose-trees to the earth and cover them with non-conductors, keep them in this position throughout the winter, and not till the next spring raise them again and fasten them to erect sticks. In the summer the old leaves on the ends of mountain pine branches, which wave above the ground more than a metre high, may be frequently seen plastered over with earth and small stones, and anyone knowing nothing of the processes above described would not easily understand how these small stones had come to be fixed in these situations. As a matter of fact the earth which lies on the branches through the winter, moistened by the snow-water, forms the adhesive agent, which is so efficient that stones more than $1 \mathrm{~cm}$. in diameter are attached by it to the old tufts of leaves. Many other Alpine shrubs behave like the mountain pines, as, for example, the Dwarf Juniper (Juniperus nana) and the Alpine Alder (Alnus viridis). In like manner the rhododendron bushes are also pressed to the ground by the snow, although not to such a great extent, and are thus protected against the great cold, and particularly against extreme radiation.

In forest regions the dry foliage, which falls from the trees and overspreads the ground and undergrowth to a greater or less thickness, appears also to be usually an excellent protective agent. This foliage layer is thickest in the beech forests of Central Europe, and the sheltered plants include the Woodruff, Lungwort, Hepatica, Asarabacca, Sanicle, and Waldsteinia (Asperula odorata, Pulmonaria officinalis, Hepatica triloba, Asarum Europoum, Sanicula Europcea, and Wald- 
steinia geoides) maintain themselves beneath it, unfrozen, even in very severe winters.

Other plants, again, appear to be protected against extreme cold by the fact that they retire underground during the winter. Large numbers of bulbous and tuberous plants manufacture organic compounds in their green leaves in the warm sunbeams of summer, at once transmitting them below to their subterranean portions. There, thick stems and tubers, fleshy scale-like leaves, and the rudiments of new foliage and flowers (which, however, do not appear above-ground

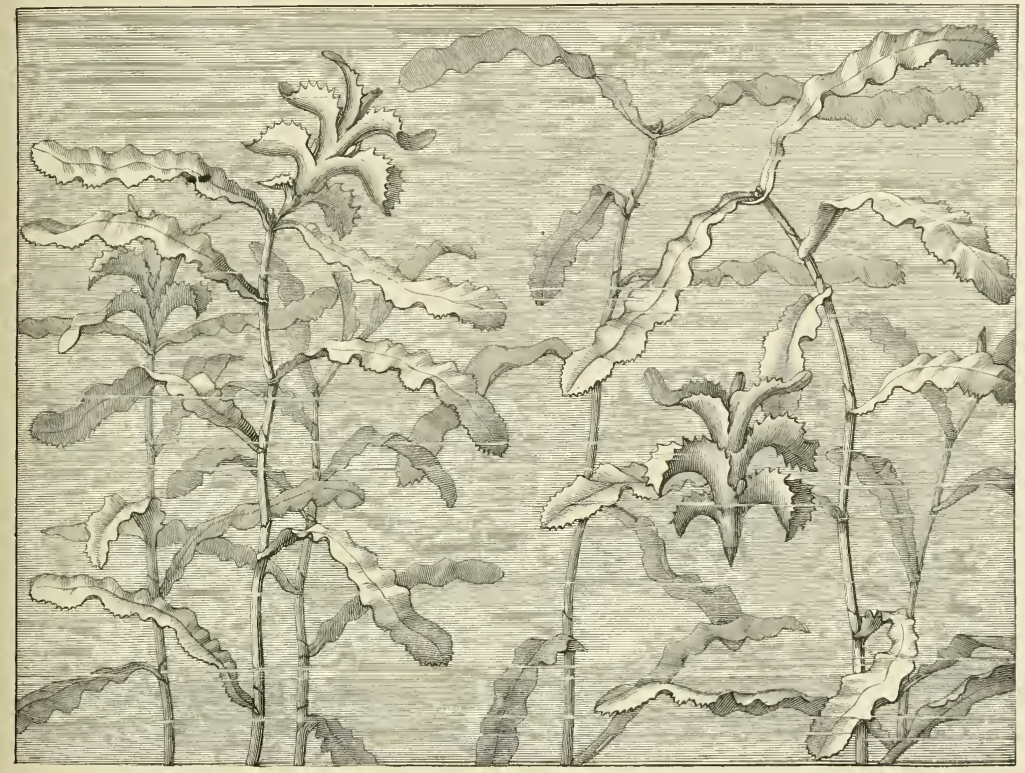

Fig. 136.-Detachment of special shoots of Potamogeton crispus, for hibernation under water.

the same year) are produced from the materials supplied. Throughout the winter these structures remain buried in the earth, and are there protected against excessive cold, just like roots. After the winter is over, the flower-stalks and foliage-leaves, commenced in the previous year, rise up in order to bloom and fructify, and to form anew, in the sunlight, organic materials for the subterranean bulbs, tubers, and root-stocks. It is very interesting to notice that bulbs and tubers bury themselves deeper in the earth the more exposed their habitat to radiation and cooling, the more they are threatened with the danger that the earth will be covered by only a thin mantle of snow. While, for example, the bulbs and tubers of Gagea lutea and Corydalis cava, when growing in the black humus of beech forests under withered foliage, lie only a few centimetres below the surface, in open 
meadows they are only to be found at a depth three or four times as great. The position of the tuberous roots of many orchids, and of the corms of the Meadow Saffron (Colchicum autumnale) may be actually used as marks to indicate how deeply in a given neighbourhood the ground is frozen, for these occur imbedded just at that depth to which the winter frost fails to penetrate.

The same thing is also observed in aquatic plants. In the still waters of lakes and ponds the plants bodily withdraw before the advancing cold of winter, and an actual retreat into the depths takes place. The Water Soldier (Stratiotes aloides) sinks down before the commencement of winter to the bottom of the lake, where it scarcely ever freezes; it passes the winter there, and does not rise again to the surface till the following spring. Potamogeton crispus, figured above, produces late in the autumn, near the surface of the water, shoots possessing' short leaves which are detached from the old stem before the uppermost layer of water is frozen. These sink into the depths, and bore their way into the mud by their pointed lower extremities. There, in their winter quarters, where there is never any formation of ice, these sprouts are excellently protected against injury from excessive cold.

Erect trees and shrubs, which rise up column-like above the earth, are little affected by the presence or absence of a covering of snow upon the ground. Generally the leaves have been already shed, after they have delivered up such substances as they contained of value. The leafless branches and the next year's buds indeed remain above the ground, being thus exposed to the winter cold, which they must be capable of bearing without injury. The branches are covered with a tough and compact investment; and it would seem as if such a covering would be able to protect the portion clothed by it against cold better than a mere epidermis. For a very short period of cold weather such may be the case, but for a longer period even the thickest coat would be unable to keep the cold from the covered portions, just as little in fact as the bark on old boughs and trunks. In longcontinued winters, with uninterrupted severe cold weather, the interior of the branches and trunks assumes the temperature of the environment, and it depends entirely upon the resisting capacity of the protoplasm whether the cooling is fatal or not. From various appearances it may be concluded that this resisting capacity is greater the better the opportunity afforded to the protoplasm of suitably preparing itself in the foregoing summer and autumn. If the summer was warm, and the autumn mild, if the advent of the first frost was much retarded, and the plant had time to become a chrysalis slowly, in preparation for the winter, then the branches do not freeze dead; but if the summer was cold and wet, and frosts appeared early in autumn, if the water of imbibition was not removed at the right time, and the wood, as gardeners say, is not "ripened", then a tolerably severe winter may result in the death of the branch, of the same branch, indeed, which perhaps in previous years survived without injury much greater cold.

Accordingly we always come back to this, that the freezing of a plant to death, or not, depends upon whether or not the condition of the protoplasm is such that its molecular constitution becomes permanently disorganized in consequence of the 
cooling, and that the most effective protection must be sought for in the constitution of the protoplasm itself. Since we do not know the constitution of the protoplasm, it is idle to puzzle ourselves in surmises about it; this only being certain, that the resisting capacity of protoplasm differs much from plant to plant, as well as at different times in one and the same plant.

The results which have been obtained by the study of the burning of plants are analogous to those afforded by researches into the nature of freezing.

When a plant organ loses its capacity of absorbing food, of breathing, and of further development, in consequence of the rise of temperature, we say then that it is burnt. The outwardly visible appearances of burnt plants resemble exactly those which have been observed in plants killed by freezing; the green tissue is discoloured, exhibits a darker tint, is more transparent, fades and dries up, and neither the supply of water nor the reduction of the temperature can reproduce the previous conditions. The protoplasm in the interior of the cells is massed into balls, and is detached from the cell-wall; water is excreted, which had stood hitherto in molecular combination with the protoplasm. These observations can be followed very easily in aquatic plants whose cell-walls are so transparent that they allow us to see into the interior of the cell-chambers. If the cells of the water-plant Elodea, illustrated in fig. $5^{3}$ (page 25), are examined under the microscope while the temperature of the surrounding water is $30^{\circ} \mathrm{C}$, the protoplasm will be seen to exhibit that active streaming movement described on p. 33 . If the temperature is raised to $40^{\circ}$, the streaming becomes slower, and at $41^{\circ}$ ceases entirely, although the protoplasm shows no other particular alteration. Even if the temperature is raised to $45^{\circ}$, and gradually to $50^{\circ}$, nothing is altered in appearance; not until $52^{\circ}$ does any very noticeable alteration occur. Then the starch-granules imbedded in the protoplasm split up; the protoplasm shrinks together and forms clump-like masses around the fractured starch-granules. The protoplasm now becomes rigid, the albuminous materials in it are curdled or coagulated. Subsequently, if the temperature again sinks to $30^{\circ}$, it does not become again living and active, and we must therefore assume that its molecular constitution has suffered at $52^{\circ}$ an irreparable alteration, in fact, that it has been killed.

In the main, therefore, burning depends upon the coagulation of the albuminous compounds, upon the destruction of the starch-granules, and the decomposition of the protoplasm. If the coagulation of the albuminous compounds and the alteration of the starch-granules were always brought about by one and the same temperature then probably all plants would be "burnt" at this same temperature. But such is not the case. The various albumens not only coagulate at different temperatures (viz. $60^{\circ}-80^{\circ}$ ), but the point of coagulation of the same albumen is materially affected by the watery contents, and by the presence of salts and acids. When, for example, many salts are present, coagulation may occur at $50^{\circ}$. Nor does the destruction of the starch-granules always occur at the same temperature; large starch-grains, swollen with water, at $55^{\circ}$, smaller ones not till $65^{\circ}$; 
and, in order that dry starch-grains may be destroyed, a still higher temperature is necessary. Under such conditions it is not to be wondered at that plants, whose protoplasm exhibits different coagulation points, should be "burnt" at very different temperatures. The processes which have been observed in the abovementioned Elodea at $30^{\circ}, 41^{\circ}$, and $52^{\circ}$, are seen to occur in other water-plants at other temperatures. In the cells of Vallisneria spiralis, represented in fig. $5^{2}$, the streaming of the protoplasm does not stop till $43^{\circ}$ has been reached, and the protoplasm is not formed into balls in consequence of the coagulation of the albumen till $53^{\circ}-54^{\circ}$. In the lattice-leaved Aponogeton fenestrale, growing in Madagascar, the coagulation and death of the protoplasm first occur at 55. Many algæ bear even still higher temperatures. In the channels through which the hot water of the Carlsbad spring flows, dusky oscillarias flourish even at a temperature of $55^{\circ}$ to $56^{\circ}$; in the springs of Abano, which reach a temperature of nearly $60^{\circ}$, Sphcerotilus thermalis is to be found, and in the Solfatara at Naples, the side-walls of the rocky clefts, from which vapour issues at a temperature of $55^{\circ}$ to $60^{\circ}$, are covered with a green film of algæ.

In plants which are not submerged in water, the watery contents as well as the specific constitution of the protoplasm have a material influence on the burning. If the exposed tissues are poor in water they can sustain much higher temperatures than when very turgid. The highest temperature which the turgid cells of lithophytes and land plants can endure without being burnt is in most cases $55^{\circ}$. In the sun, succulent plants can endure for a long time without injury temperatures of $50^{\circ}$ to $53^{\circ}$. The spores of moulds (Rhizopus nigricans and Penicillium glaucum) have been seen to germinate and develop at from $54^{\circ}$ to $55^{\circ}$. When dry, those cells and tissues which can be dried up without harm do not perish even under the influence of far higher temperatures. The crustaceous lichens adhering to the limestone rocks of the wild regions of the Karst of Istria and Dalmatia (Aspicilia calcarea, Verrucaria purpurascens, and V. calciseda) are regularly exposed on cloudless summer days to a temperature of $58^{\circ}$ to $60^{\circ}$ without injury, and the edible lichen (Lecanora esculenta), illustrated opposite, is often heated in the deserts, along with the stone on which it grows, to fully $70^{\circ}$, and yet is not destroyed. Moreover, seeds which are deposited on the desert sands, and survive in this position the long periods of drought, do certainly assume the temperature of their environment, and although at noon this often amounts to $60^{\circ}-70^{\circ}$, it does not injure these seeds; since, when the rainy season returns, they are roused from their summer sleep, and germinate in the cool and moistened soil. The highest temperature in the superficial layer of soil has been observed near the equator at Chinchoxo on the Loango coast. Here, in many cases it exceeds $75^{\circ}$, often attains $80^{\circ}$, and once attained to even $84.6^{\circ}$. Nor is this soil destitute of annuals during the rainy season, and without doubt the dry seeds of these plants have been lying for months in the sand, sometimes heated to over $80^{\circ}$, without losing their germinating power. It has been proved experimentally that seeds, which have been deprived by calcium chloride of as much water as 
possible, are not killed even at the boiling point of water. Of various seeds from which water has been withdrawn for fifty hours, and which have then been heated for three hours up to $100^{\circ}$, those of duckweeds ( 49 per cent of the seeds experimented upon) still germinated; of vetches, 50 per cent; of garlic, 60 per cent; of wheat, 75 per cent; of sweet marjoram, 78 per cent, and of melons 96 per cent. Even of seeds previously dried, which had been exposed for about fifteen months to a temperature of $110^{\circ}$ to $125^{\circ}$, a small percentage always germinated, and it is possible that there are species whose seeds bear without injury still higher temperatures.

From these experiments it is clearly shown that the albuminous substances in the protoplasm may give up with impunity much water, and that by this

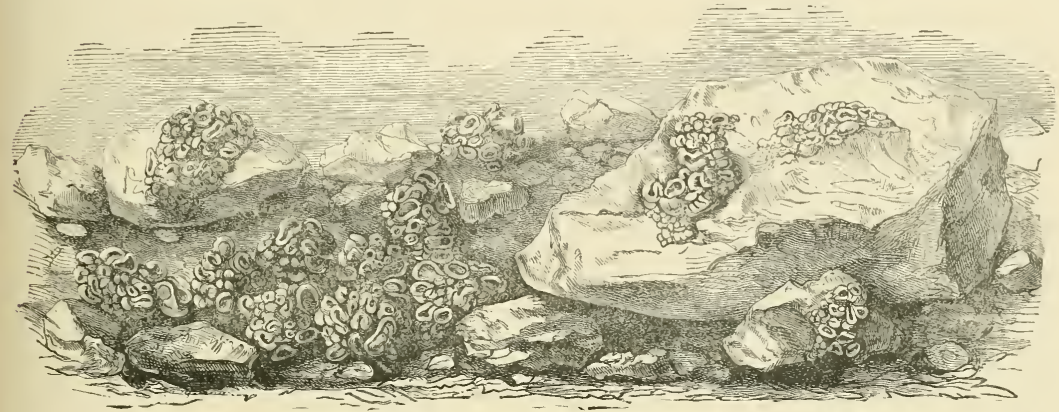

Fig. 137.-Edible Lichen (Lecanora esculenta) in the desert.

surrender a protection is obtained against coagulation and burning, up to a certain point.

In nature, most contrivances by which plants are protected against burning amount in reality to a periodic surrender of water. Lithophytes, especially crustaceous lichens, which are most threatened with the danger of being burnt, are so organized that they can give up a great deal of water in a very short time. They then become stiff and brittle, and can be rubbed into powder, and it appears scarcely credible that these dried-up structures can ever live again. In the rocklichens the same thing occurs. Also several Volvocineæ, Sphcerella pluvialis, and various other simply organized plants, living in shallow pools and ditches, dry up to dust along with the mud, after the evaporation of the water which had accumulated in their habitat, and are protected in this dried condition against burning. If the dust, which is warmed daily for several hours up to $60^{\circ}$ during the period of drought, becomes moistened later on, all the tiny plants wake up again from their trance, and, as should not be overlooked, the rotifers and various infusoria, which are present in the same heated dust, again bestir themselves, flourish their cilia, and give evidence that the surrender of water at the right moment affords the best protective measure against "burning" for animal proto- 
plasm also. In deserts and steppes, and in all regions where the earth is warmed up to $70^{\circ}$ in hot rainless seasons, there are, it is well known, a great many annuals. When the hot period commences, leaves, stems, and roots are already dead, and the plants have scattered their seeds. These seeds, however, possess very little water, and yet can give up a portion of that they contain without injury; they are thus protected in the best way possible against being burnt.

One portion of the perennial plants of these regions throws off its foliage at the close of the rainy period, and lives through the hot, dry period with leafless and apparently dried-up branches; others expose the whole of their organs above the ground to burning, maintaining themselves below the soil only, where the earth never acquires such a high temperature; these sleep through the hot period as resting tubers, bulbs, and root-stocks. It should also be remembered here that in regions where high temperatures are not combined with great dryness, the excessive heat can be diminished by the evaporation from the succulent tissue, since, as is well known, evaporating bodies always undergo a cooling. Finally, the fact is to be considered that many plants choose places for their settlement where they are not exposed to burning, even on the hottest days of the year. Under the protection of shade-giving rocks, and wherever the sun's rays are not able to operate directly and untempered, the soil, even at the equator, may not exceed those temperatures at which succulent plant-organs cannot be burnt, and still less could the normal warmth of the air in shady places bring about such an effect; for the highest temperatures hitherto observed in the shade rise scarcely above $40^{\circ}$ ( $42^{\circ}$ in Abu-Arisch, in Arabia; $43.1^{\circ}$ on the river Macquarie, in Australia), and at these temperatures the albuminous substances are never coagulated in any single plant.

The question now is how the results obtained from researches into the phenomena of freezing and burning can be brought into harmony with the earlier ascertained relations of heat to living plants, and especially with the theory of growth. We have conceived growth as a form of molecular work of living protoplasm, and we imagine the molecules and groups of molecules to be in a condition of heat-vibration of definite extent; or, in other words, that for all work, and especially for growth, a definite degree of heat is necessary. If the heat-vibrations exceed the fixed limit, the position and the mutual relations of the molecules in the protoplasm are completely altered, and disarrangements result which cannot subsequently be made good. The protoplasm has then lost the capacity of further maintaining itself and increasing-it is burnt and killed. The same happens if the intensity of the heat-vibration sinks below a certain degree. Then again changes are produced in the substance of the protoplasm which are irreparable, and are followed by death. Consequently, a superfluity as well as a want of heat can retard the molecular action of the living protoplasm which appears as growth, and can even completely stop it. And the interruption is brought about in the protoplasm of different species under the influence of different degrees of heat; just as water, alcohol, and mercury solidify at certain temperatures, and become vaporized at 
certain others, so there is a temperature for the protoplasm of every species at which it freezes, and another at which it is burnt. But this leads to the conclusion that the molecules and groups of molecules in all protoplasm vibrate definitely as to extent and intensity so long as the protoplasm is living, even if it is not exactly performing that work which appears to us as growth-in other words, that a definite amount of heat is necessary to the maintenance of life even in protoplasm apparently resting; and that consequently it is not correct to suppose that all the heat supplied to the plants is used up in growth.

\section{ESTIMATION OF THE HEAT NECESSARY TO GROWTH.}

According to the mechanical theory of heat, which gives the best explanations of numerous phenomena of life, all motion can be converted into heat, and measured as such. Should it not be possible to apply this principle to the vegetable kingdom, especially to the phenomena of growth? Ought we not to be able to estimate definitely how much heat is required for plants for each of their performances within a definite period, and therefore to determine their heat-requirement as a constant numerical quantity? This question has often been put, and experiments have not been wanting to supply the answer. It would not be only of theoretical but also of great practical value, to be able to tell how much heat our forest trees, our cereals and other economic plants, need for the accomplishment of their yearly cycle of life, to know how much heat is necessary for the germination of this or that cultivated plant, how much in order that the germinated plants may blossom, and what degree of heat they require to produce ripe seeds of full weight and capable of germination. If it were practicable to determine those quantities of heat, which might be called the thermal constants of vegetation, we should be able to estimate beforehand from the heat-conditions prevailing in any particular place, whether this or that plant species would thrive, whether it could produce ripe fruits, and whether or not its cultivation would be advantageous and worthy of encouragement.

Hitherto the results obtained in this direction leave very much to be desired, but are nevertheless so interesting that they cannot be passed over in silence here. First of all, it has been proved with regard to the earliest phases of growth, the germination of spores and seeds, that not a few species are able to germinate even at very low temperatures. The seeds of the White Mustard, of hemp, of wheat and rye, of the Norway Maple, and of the wild violet, germinate at a temperature very near freezing, between $0^{\circ}$ and $1^{\circ} \mathrm{C}$; ; others, such as the garden cress, flax, spinach, onions, poppy, beet-root, and the English rye-grass, germinate at temperatures between $1^{\circ}$ and $5^{\circ}$; French beans, sainfoin, millet, maize, sunflowers, at temperatures between $5^{\circ}$ and $11^{\circ}$; tomato, tobacco, and gourds at temperatures between $11^{\circ}$ and $16^{\circ}$; cucumbers, melons, and cocoa beans not until above $16^{\circ}$. That is to say, that melon seeds, if placed in damp soil whuse temperature lies below $15^{\circ}$, absorb, it is true, the moisture, and swell up, 
but that those alterations which really constitute growth are not produced in the cells of the embryo at this temperature. Not until the temperature of the soil rises above $15^{\circ}$ does the embryo elongate, and the radicle bore its way through the seed-coats. But all these figures would give by themselves a very incomplete idea of the heat-requirements of germinating seeds, were it not also ascertained how long the seed must be exposed to the given temperatures in order that its embryo should increase and develop. If a hen's egg is exposed for only two or three days to a temperature of $35^{\circ}$ to $40^{\circ}$, it will not be hatched; hatching can only take place if the egg remains for 20-21 days under the influence of this constant temperature. With seeds the case is the same. The following is a selection of the results obtained in this relation:-

\begin{tabular}{|c|c|c|c|c|c|}
\hline The seeds of & $\begin{array}{l}\text { germinated at a con- } \\
\text { stant temperature of }\end{array}$ & $\begin{array}{l}\text { in No. of } \\
\text { days. }\end{array}$ & The seeds of & $\begin{array}{l}\text { germinated at a con- } \\
\text { stant temperature of }\end{array}$ & $\begin{array}{l}\text { in No. of } \\
\text { days. }\end{array}$ \\
\hline $\left.\begin{array}{l}\text { Gold of Pleasure } \\
\text { Pea } \\
\text { Spinach } \\
\text { Poppy } \\
\text { Beetroot } \\
\text { Guinea grass } \\
\text { French Beans } \\
\text { Timothy-grass } \\
\text { Sainfoin }\end{array}\right\}$ & $10 \cdot 5^{\circ} \mathrm{C}$ & $\begin{array}{r}4 \\
5 \\
9 \\
10 \\
22 \\
24 \\
3 \\
6 \\
7\end{array}$ & $\begin{array}{l}\text { Pimpernel } \\
\text { Maize } \\
\text { Millet } \\
\text { Coriander } \\
\text { Sunflower } \\
\text { Tomato } \\
\text { Tobacco } \\
\text { Cucumber } \\
\text { Melons }\end{array}$ & $10 \cdot 5^{\circ}$ & $\begin{array}{r}10 \\
11 \\
13 \\
16 \\
25 \\
6 \\
9 \\
5 \\
17\end{array}$ \\
\hline
\end{tabular}

If the number of days is multiplied by the temperature, the product may be looked upon as an empirical formula for the heat necessary to the process of germination. It may be considered that this product is of regular amount, and it is regarded as a "thermal constant". Thus, for purposes of comparison, the thermal constants for the germination of the seeds of the Gold of Pleasure might be expressed as 18.4, of the Poppy $46^{\circ} 0$, of Maize $1155^{\circ}$, and so forth.

In these calculations, of course, only the constant temperatures of the soil not directly illumined by the sun's rays come under consideration. The matter becomes far more complicated when it is a question of determining the constants for other stages in the development of plants, such as the bursting of foliage from the buds, the opening of the first flowers, and the ripening of the first fruits. These phenomena of growth in the majority of plants in the open do not occur in the shade, but in the sun. Moreover, in the places under observation, the temperature is not constant, but changes from hour to hour, attaining its minimum shortly before sunrise, and its maximum in the first hours of the afternoon. Since experience has shown that the extent of growth is regulated according to the highest temperature in the sunshine, it follows that neither the shade temperature nor the mean temperature, but the readings of the maximum thermometer, exposed to the sun, must be used for the estimation of the constants in the above-mentioned phenomena of growth.

To obtain the thermal constants for foliage-production, flower-opening, and seed-ripening, of a plant growing in a situation illuminated by the sun, one must 
add together the daily maxima of sun-temperatures from the first of January until the event in question takes place.

A selection of constants obtained in this way from observations extending over many years in Central Germany (Giessen) may be suitably inserted here.

\section{Constants for the Issue of the Foliage-Leaves from the Buds.}

Gooseberry (Ribes Grossularia) $478^{\circ}$.

Hazel (Corylus Avellana) $1061^{\circ}$.

Beech (Fagus silvatica) $1439^{\circ}$.
Plane (Platanus acerifolia) $1503^{\circ}$.

Walnut (Juglans regia) $1584^{\circ}$.

\section{Constants for the Opening of the First Flowers.}

Hazel (Corylus Avellana) $226^{\circ}$.

Mezereon (Daphne Mezereum) $303^{\circ}$.

Snowdrop (Galanthus nivalis) $311^{\circ}$.

Sweet Violet (Viola odorata) $576^{\circ}$.

Cornel (Cornus mas) $576^{\circ}$.

Apricot (Prunus Armeniaca) $843^{\circ}$.

Corydalis (Corydalis cava) $863^{\circ}$.

Violet Willow (Salix Daphnoides) $968^{\circ}$.

Cowslip (Primula veris) $968^{\circ}$.

Norway Maple (Acer platanoides) $1100^{\circ}$.

Peach (Persica vulgaris) $1100^{\circ}$.

Gooseberry (Ribes Grossularia) $1138^{\circ}$.

Almond (Anygdalus communis) $1196^{\circ}$.

Gean (Prunus avium) $1265^{\circ}$.

Sloe (Prunus spinosa) $1265^{\circ}$.

Pear (Pirus communis) $1304^{\circ}$.

Bird Cherry (Prunus Padus) $1325^{\circ}$.

Apple (Pirus Malus) $1423^{\circ}$.

Plum (Prunus domestica) $1423^{\circ}$.

Alpine Woodbine (Lonicera alpigena) $1458^{\circ}$.

Oak (Quercus pedunculata) $1556^{\circ}$.

Lilac (Syringa vulgaris) $1556^{\circ}$.

Walnut (Juglans regia) $1584^{\circ}$.

Barberry (Berberis vulgaris) $1615^{\circ}$.

Poet's Narcissus (Narcissus poeticus) $1615^{\circ}$.

Hawthorn (Cratogus Oxyacantha) $1649^{\circ}$.

Lily of the Valley (Convallaria majalis) $1649^{\circ}$.

Horse Chestnut (Esculus Hippocastanum) 1708 .
Peony (Pconia officinalis) $1818^{\circ}$.

Laburnum (Cytisus Laburnum) $1818^{\circ}$.

Mountain Ash (Sorbus aucuparia) $1844^{\circ}$.

Norway Spruce (Abies excelsa) $1904^{\circ}$.

Plane (Platanus acerifolia) $2115^{\circ}$.

Elder (Sambucus nigra) $2313^{\circ}$.

Deadly Nightshade (Atropa Belladonna) $2346^{\circ}$.

Acacia (Robinia Pseudacacia) $2404^{\circ}$.

Scotch Pine (Pinus sylvestris) $2404^{\circ}$.

White Water Lily (Nymphaca alba) $2506^{\circ}$.

Arnica montana $2538^{\circ}$.

Tulip Tree (Liriodendron tulipifera) $2538^{\circ}$.

Rosa centifolia $2538^{\circ}$.

Fox-glove (Digitalis purpurea) $2640^{\circ}$.

Carthusian Pink(Dianthus Carthusianorum) $2640^{\circ}$

Vine (Vitis vinifera) $2878^{\circ}$.

Broad-leaved Lime (Tilia grandifolia) $3033^{\circ}$.

Small-leaved Lime (Tilia parvifolia) $3274^{\circ}$.

Oat (Avena sativa) $3444^{\circ}$.

White Lily (Lilium candidum) $3378^{\circ}$.

Chestnut (Castanea sativa) $3660^{\circ}$.

Immortelle (Helichrysum arenarium) $3918^{\circ}$.

Ling (Calluna vulgaris) $4164^{\circ}$.

Trumpet-tree (Catalpa syringrefolia) $4275^{\circ}$.

Blue Aster (Aster Anellus) $4874^{\circ}$.

Syrian Marsh-Mallow (Hibiscus Syriacus) $4986^{\circ}$.

Meadow Saffron (Colchicum autumnale) $5024^{\circ}$.

Ivy (Hedera Helix) $5910^{\circ}$.

\section{Constants for the Ripening of Fruit.}

Wild Strawberry (Fragaria vesca) $2671^{\circ}$.

Gean (Prunus avium) $2778^{\circ}$.

Mezereon (Daphne Mezereum) $2935^{\circ}$.

Red Currant (Ribes rubrum) $3069^{\circ}$.

Gooseberry (Ribes Grossularia) $3596^{\circ}$.

Alpine Woodbine (Lonicera alpigena) $4164^{\circ}$.

Mountain Ash (Sorbus aucuparia) $4339^{\circ}$.

Barley (Hordeum vulgare) $4403^{\circ}$.

Apricot (Prunus Armeniaca) $4435^{\circ}$.

Apple (Pirus Malus) $4730^{\circ}$.
Barberry (Berberis vulgaris) $4765^{\circ}$.

Carthusian Pink (Dianthus Carthusianorum) $4874^{\circ}$.

Elder (Sambucus nigra) $4913^{\circ}$.

Pear (Pirus communis) 5024.

Cornel (Cornus mas) 5416.

Plum (Prunus domestica) $5780^{\circ}$.

Vine (Vitis vinifera) $5780^{\circ}$.

Peach (Persica vulgaris) $6004^{\circ}$.

Horse Chestnut (Assculus Hippocastanum) 6034․

Oak (Quercus pedunculata) $6236^{\circ}$. 


\section{Constants for the Commencement of Leaf-Fall.}

Bird Cherry (Prunus Padus) 6179․

Small-leaved Lime (Titia parvifolia) $6644^{\circ}$. Elder (Sambucus nigra) $6644^{\circ}$.

Alpine Woodbine (Lonicera alpigena) $6759^{\circ}$. Pear (Pirus communis) $6788^{\circ}$.

Walnut (Juglans regia) $6816^{\circ}$.

Trumpet-tree (Catalpa syringafolia) $6816^{\circ}$.

$\nabla$ iolet Willow (Salix daphnoides) 6838 .

Horse Chestnut (Asculus Hippocastanum) $6863^{\circ}$.
Hazel (Corylus Avellana) 6884․ Gooseberry (Ribes Grossularia) 6884․ Beech (Fagus silvatica) $6884^{\circ}$.

Vine (Vitis vinifera) $6913^{\circ}$. Oak (Quercus pedunculata) $6979^{\circ}$. Apple (Pirus Malus) $6999^{\circ}$. Chestnut (Castanea sativa) $7023^{\circ}$. Gean (Prunus avium) $7023^{\circ}$.

Plane (Platanus acerifolia) $7145^{\circ}$.

Although the computations which have been made at different places and over several years, by way of trial, have given figures which do not differ materially from the above, and it seems as if these constants actually justified that term, yet confidence in them has been to some extent diminished by the following considerations.

With regard to the germination of seeds it is concluded from various phenomena that the heat liberated in respiration from the cells, as well as the temperature of the soil, has not a little influence also on the process of growth. Seeds in whose cells the protoplasm has once been set in action by an external impulse, perhaps by a minimum of radiated or conducted heat, respire with a fair amount of activity. In this way the reserve materials stored up in them are consumed, and so much heat is liberated that not only is the embryo able to develop, but heat may be even given up to the environment. Radicles of germinating maple and wheat seeds, which by chance were found in an ice cellar, were observed to grow down into the blocks of ice, and this could only have happened from the melting of the ice by the radicles, which push their way into the cavities formed, like the flowerbuds of the Soldanellas already described. In many cases of observed germination it may therefore be doubted whether the growth of the embryo alone is to be laid to the account of the measured heat, supplied to the seeds from their surroundings. On the other hand, it is doubtful whether the heat (registered by the thermometer), which reaches the plants from outside, is employed only in growth. One part may be used in order to maintain the plant-organ in question alive; another portion may be useful in the production, and in the transformation and transmission of constructive materials, and only a residual portion can then participate in growth. But this is not all. It is also doubtful whether the positive heat entering the plants from outside, can be always completely disposed of, within the given space of time, in the various chemical transformations and molecular arrangements carried on in the interior of the plant, and whether an unused surplus is not sometimes present which should be really withdrawn from the calculation. It is tacitly implied in the reckonings that if the plants are exposed to a constant temperature of $20^{\circ}$ for 12 hours, the total heat which was able to raise the mercury up to $20^{\circ}$ in that time would also be turned to account by the plants. But that this is not so, is shown by the following observations: 


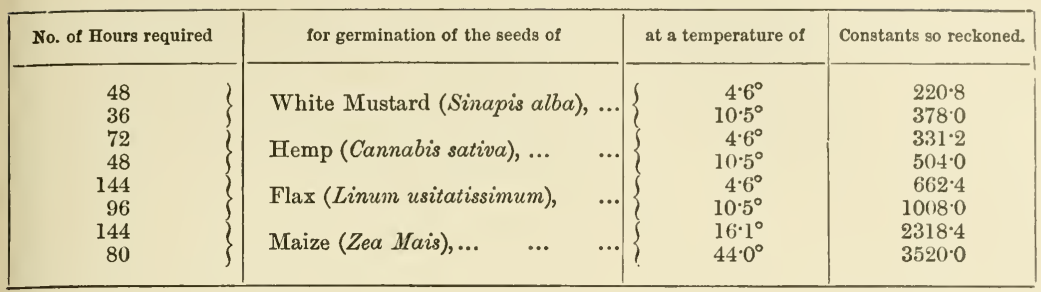

From these observations it is easily gathered that in those instances in which the seed was exposed to a higher temperature, only a portion of the heat supplied was actually employed in germination, and that, therefore, the constants calculated on the basis of these observations are much too high.

If the thermometer could tell us the amount of heat actually needed within a certain time by plants growing together, then only might the constants reckoned from these readings be regarded as accurate and become useful for comparison. But these conditions are not fulfilled. Usually here the conclusions are only post hoc propter hoc. Thermometric readings are brought into calculation which include the surplus of heat not used by the plants, and consequently the constants are not the correct expression of the quantity of heat actually consumed in growth.

The bases on which the calculations are founded, for growing organs directly under the influence of the sun's rays, are much more uncertain than those for seeds germinating in shaded ground. Besides, doubts must arise from the fact that the sun's rays have a widely differing effect on foliage, flowers, and fruits from that which they have on the mercury of the thermometer. This defect may indeed be removed by using the same instrument in all observations and employing suitable corrections; but it is a more serious matter that we have no means of ascertaining how much light is changed into heat in growing organs exposed to the sun's rays. With increasing altitudes the intensity of the light increases, and its significance for growth increases in a corresponding manner. But it is impossible to determine these relations numerically, more especially to determine them in plants and thermometers observed in the open.

Nor must it be forgotten that the absorption of heat also depends upon the individuality of the plant observed, and upon the constitution of the protoplasm of the particular species. The seeds of the White Mustard are incited to growth even by temperatures little removed from the freezing-point, while the seeds of melons do not germinate until they have been exposed to the influence of a temperature of $18.5^{\circ} \mathrm{C}$. for at least 17 days. This shows that every species has to a certain extent its own zero at which growth begins, and all calculations of the heat required for the growth of the stem and foliage of any particular species should always be reckoned only from this zero. Moreover, it is a matter of experience confirmed by all gardeners that higher temperatures are required for the development of flowers than for foliage, and that for the proper ripening of seeds higher temperatures still VoL. I. 
are necessary. Isolated species of course exhibit puzzling deviations in this respect. The Acacia (Robinia Pseudacacia) develops its flowers in Southern Italy before its leaves, and when the acacia trees are already in full bloom their foliage-leaves are still minute and unexpanded. North of the Alps the foliage-leaves everywhere unfold at the same time as the flowers; and yet we always reckon the heat indicated by the thermometer as if it were utilized in an identical manner by associated plants in all stages of development.

Finally, it must be pointed out that certain alterations which are carried on in the interior of a seed or plant during its apparent rest, and which have a great significance for those later phenomena of growth visible to the eye, are completely excluded from observation and registration. If potato-tubers are dug up in autumn and put in a cellar, it seems as if all movements and chemical transformations were entirely stopped in their individual cells. The potato-tuber lies tranquilly resting in the dark cellar, in which a constant temperature of $10^{\circ}$ prevails, throughout the winter. Spring arrives; above-ground everything germinates and sprouts from the sun-warmed soil, and we connect this phenomenon with the powerful heating caused by the rays of a more vertical sun. No heat-giving sunbeams reach the cellar, however. The temperature of the air, of the earth, and of the potatoes which have been lying there for months is always the same, $10^{\circ}$-even perhaps now a fraction lower, since according to experience the lowest temperature in the cellar is not reached until the end of the winter. Nevertheless the potato begins to grow and to send out a slender shoot from one of its buds, as if it knew that spring, the proper time for sprouting and growing, had arrived. Why does the growth not begin until now in March? Why did it not commence in December, since external influences, particularly the temperature of the environment, was not in any way different within the cellar then from what it is now in spring? There can be only one answer to the question, which is, that in December the potatoes were not yet equipped for growth. They were only apparently in absolute rest; in reality chemical transformations, the preparation and production of constructive materials, were being carried on in the cells, and in December, January, and February, these were not far enough advanced for the tubers to be able to produce stems, leaves, and roots. Not until now in March are the preparations for development completed, and not until now can that transformation of the constructive materials occur which is outwardly manifest as growth. The organic compounds, contained by the cells of the tubers in autumn, were not fit for the formation of stems, leaves, and roots, even under the influence of a temperature of $20^{\circ}$. All these processes require, therefore, a definite period of time, and this can neither be replaced nor sensibly shortened by a rise of temperature.

In the underground bulb of the Snowdrop (Galanthus nivalis) the rudiments of the leaves and blossoms of the following spring are already formed during the summer, and at the end of September all portions of the future flowers can be recognized between the enveloping sheaths and bulb-scales. It might be thought an easy matter to force this bulb by raising the temperature and 
moistening the surrounding soil, so that we might have the Snowdrop blossoming even in November. But very many experiments have shown that bulbs so treated, although they develop leaves and an inflorescence, do not properly develop their flowers, and always perish prematurely; while four months later the growth of the leaves and flowers takes place easily and quickly at temperatures which are not much above zero. And in many root-stocks, in most buds of branches above the ground, and in numerous seeds and spores, the same thing occurs as in tubers and bulbs of which the Potato and Snowdrop have been selected as well-known examples. How many plants there are which blossom early in the spring, ripen their fruits in the early summer, and whose seeds, being detached from the parent plant in the height of summer, lie scattered on the ground! Although the soil in which they are imbedded is damp and sufficiently warm, and although all the external conditions of germination are fulfilled, yet they do not germinate in the same year in which they have been produced. Not until the following spring do the embryos put forth their rootlets, and then usually under conditions apparently much less favourable than those of the previous summer and autumn. These seeds are not yet ripe, or rather, perhaps, they are not yet capable of germination when they fall from the parent plants. The materials contained in their cells must first pass through a process of transformation before they can promote the development of the embryo, and this transforming process can in no wise be hastened by increased supplies of heat and moisture. In many large seeds, as, for example, those of hazel, beech, and almond, this difference between seeds just fallen from the tree not yet capable of germination, and the seeds which have been mellowed and can germinate, may be easily perceived in their consistency, taste, and smell. The phenomenon here described is found in a specially remarkable manner in the fruits of the Water Chestnut (Trapa natans). If in autumn water-chestnuts just ripe be placed in water, and the temperature of the water be kept through the winter at $15^{\circ} \mathrm{C}$., the rootlets of the embryos will not emerge until the coming spring, and not then on account of a higher temperature, but at the same temperature to which they have been continuously $c$ : posed for six months. If the temperature of the water be raised to $20^{\circ}$ the growth of the rootlets is not accelerated, and the increased temperature cannot become effective as an incitement to growth until after the seeds have been suitably prepared for a period of six months. Gardeners say that such seeds must "mellow" and "ripen after gathering", and they have indeed hit the mark with this latter expression. Some spores also must mellow and ripen for a much longer time. Many, of course, germinate immediately after their detachment from the parent plant. The so-called resting spores, however, always pass through a quiescent period, whose duration usually can be determined with great accuracy and may be shortened a little by altered external influences. Very remarkable is the fact that in the seas of tropical regions, whose waters possess the same chemical composition, temperature, and illumination from one year's end to the other, certain species of red seaweeds develop in March, others in June, and others again in October. In these instances all grounds for an explanation are 
wanting. It can only be stated with certainty that the increase or diminution of lieat does not take any part in this remarkable periodicity. It would, however, be going too far to assert of all species that the resting period, normally observed by them, could not be shortened by external influences, especially by rise of temperature. Many seeds, such as those of cress, mustard, barley, and numerous so-called weeds, which appear as unwelcome guests on cultivated land, have no resting stage, germinate at any season if they are supplied with the necessary moisture; and the warmer the soil the more rapid is their development. It is also well enough known that there are plants which, to use the language of gardeners, may be "forced". Tulips, Lilies of the Valley, and Lilac, whose resting period lasts in Central Europe from the ripening time of the seed in summer until the spring of the next year, may be forced even late in autumn if planted in a greenhouse in warm, damp soil, soon after they have ripened their seeds and have begun to rest. Under these circumstances they produce their flowers even in January, and in these plants, consequently, the materials manufactured in the previous summer may be used as constructive materials for growth almost at once. I remember once drawing the shoot of a Clematis plant rooted in the open, after it had lost its foliage in the autumn, through a narrow crevice 3 metres above the soil into the interior of a neighbouring hothouse. Leafy shoots were developed from the buds of the upper portion thus warmed even in December; while the lower portion of the same plant, situated outside the hothouse and surrounded by cold air, was still frozen. In this plant also, the materials manufactured in the summer could be used as constructive materials as soon as ever they had been deposited in the reserve storehouses.

The same must indeed be the case in those plants which bloom normally in the spring, but yet often in years characterized by particularly mild autumns, burst open in October; the buds destined for the next spring thus sending out fresh leafy shoots and blossoms twice in the same year-for example, many apples and horsechestnuts, violets and strawberries, many primulas, gentians, and anemones.

Although it may be doubted whether the constants hitherto computed can be taken as an accurate expression of the heat consumed by plants for growth in their various stages of development, nevertheless their value must not be under-estimated. Comparisons of results obtained in various places by the same methods, with the same instruments, and on the same species, will without doubt yet lead to many interesting conclusions. The determination of the commencement of the various phenomena of development, the determination of the unfolding of the foliage and flowers, of the ripening of the fruit, and of the autumnal leaf-fall-at as many stations of observations as possible-is not only a highly attractive problem in itself, but is also of great scientific value; and this no less in its bearing upon the life of plants generally, than upon the geography of plants, since the barriers which confine plants in their distribution can be in great part explained by the fact that the species in question are unable to complete their annual cycle of development on the further side of the boundary. Finally, also for climatology, since the yearly process 
of development, in many cases, much more clearly exhibits the climate of a district than the readings of instruments erected in the places in question. The so-called phænological observations, that is, the determination of the awakening of nature at the close of the winter, or at the end of the summer drought, the ascertaining of the times at which growth and blossoming reach their maximum, and the fixing of the period at which the organism, on account of the unfavourable external conditions, falls into a winter or summer sleep, are consequently of interest even if we are unable to reckon the heat constants for the commencement of these phenomena. The results of such phænological observations have been already made use of repeatedly on pp. 519 and 525, and it has been there shown how valuable these may be in questions concerning the relations of heat to growth.

We cannot close this chapter without touching upon two valuable results of phænological observations, although only in passing. The following table gives first of all a view of the retardation of vegetative development with increasing latitudes in Europe in the spring.

Comparisons with Lesina in the Adriatic Sea, $43^{\circ} 11^{\prime}$ Nor. Lat., $16^{\circ} 40^{\prime}$ East Long.

\begin{tabular}{|c|c|c|c|c|c|c|}
\hline $\begin{array}{c}\text { North } \\
\text { Lat. }\end{array}$ & $\begin{array}{c}\text { Places between } \\
\text { o and 10 } \\
\text { Meridian. }\end{array}$ & $\begin{array}{c}\text { Retarda- } \\
\text { tion in } \\
\text { days. }\end{array}$ & $\begin{array}{c}\text { Places between } \\
10 \text { and } 30 \\
\text { Meridian. }\end{array}$ & $\begin{array}{c}\text { Retarda- } \\
\text { tion in } \\
\text { days. }\end{array}$ & $\begin{array}{c}\text { Places between } \\
\text { 30and45 } \\
\text { Meridian. }\end{array}$ & $\begin{array}{c}\text { Retarda- } \\
\text { tion in } \\
\text { days. }\end{array}$ \\
\hline $48-49^{\circ}$ & Paris & 43 & Pressburg & 58 & Sarepta & 66 \\
$50-51^{\circ}$ & Brussels & 50 & Prague & 59 & Kiew & 68 \\
$52-53^{\circ}$ & Osnabrïck & 63 & Warsaw & 65 & Orel & 79 \\
$59-60^{\circ}$ & Christiania & 86 & Pulkowa & 100 \\
\hline
\end{tabular}

As the starting-point in the comparison we choose the Island of Lesina off the Dalmatian coast, because there the climatic conditions lie midway between those of places situated in the same latitude in Western Oceanic and in Eastern Continental Europe. The stations of observation, situated not more than 300 metres above the sea-level, which are here compared with Lesina, have been arranged in three columns -a western between 0 and 10 meridian, a central between 10 and 30 , and an eastern between 30 and 45 . Reviewing the retardation due to the increasing latitude with regard to Lesina, we have the interesting result that the retardation in the column of the eastern continental stations is from two to three weeks more than in that of the western column. Thus, when in Paris many spring plants are in full bloom, vegetation on the Russian steppes (Sarepta), at the same latitude, is still deep in winter slumber, and does not reach the same stage until 23 days later.

In a second small table inserted on next page, very remarkable results are given with respect to the blossoming of the same species in Western Europe and in Eastern North America.

Here those American and European stations are placed side by side in which the blossoming of the same species occurs simultaneously, and hence the comparison shows that the geographical position of these places differs by about 8-10 degrees of latitude; so that, for example, in New York (which has the same latitude as 
Naples) plants blossom at the same time as in Marburg, situated $10^{\circ}$ further north.

Stations at which Spring Plants blossom simultaneously.

\begin{tabular}{|c|c|c|c|c|c|c|c|}
\hline \multicolumn{3}{|c|}{ North America. } & $\begin{array}{l}\text { Geograplical } \\
\text { Latitude. }\end{array}$ & \multicolumn{2}{|l|}{ Europe. } & $\begin{array}{l}\text { Geographical } \\
\text { Latitude. }\end{array}$ & $\begin{array}{l}\text { Difference of } \\
\text { Latitude. }\end{array}$ \\
\hline $\begin{array}{l}\text { New Albany } \\
\text { Sykesville } \\
\text { Belle Centre } \\
\text { New York } \\
\text { Germanstown } \\
\text { Baldwinville }\end{array}$ & $\begin{array}{l}\cdots \\
\cdots \\
\cdots \\
\cdots \\
\cdots \\
\cdots\end{array}$ & $\begin{array}{l}\cdots \\
\cdots \\
\cdots \\
\cdots \\
\cdots\end{array}$ & $\begin{array}{ll}38^{\circ} & 17^{\prime} \\
39^{\circ} & 23^{\prime} \\
40^{\circ} & 28^{\prime} \\
40^{\circ} & 42^{\prime} \\
42^{\circ} & 80^{\prime} \\
43^{\circ} & 40^{\prime}\end{array}$ & $\begin{array}{ll}\text { Dijon } & \ldots \\
\text { Kremsmünster ... } \\
\text { Heidelberg } & \ldots \\
\text { Marburg (Hesse) } \\
\text { Antwerp } & \ldots \\
\text { Utrecht ... } & \ldots\end{array}$ & $\begin{array}{l}\cdots \\
\cdots \\
\cdots \\
\cdots \\
\cdots\end{array}$ & $\begin{array}{ll}47^{\circ} & 19^{\prime} \\
48^{\circ} & 30^{\prime} \\
49^{\circ} & 28^{\prime} \\
50^{\circ} & 47^{\prime} \\
51^{\circ} & 13^{\prime} \\
52^{\circ} & 03^{\prime}\end{array}$ & $\begin{array}{rr}9^{\circ} & 20^{\prime} \\
9^{\circ} & 07^{\prime} \\
9^{\circ} & 00^{\prime} \\
10^{\circ} & 05^{\prime} \\
8^{\circ} & 33^{\prime} \\
8^{\circ} & 90^{\prime}\end{array}$ \\
\hline
\end{tabular}

\section{ULTIMATE STRUCTURE OF PLANTS.}

Hypotheses as to the Form and Size of the Smallest Particles employed in the Construction of Plants.-Visible Structural Activity of Protoplasm.

\section{HYPOTHESES AS TO THE FORM AND SIZE OF THE SMALLEST PARTICLES EMPLOYED IN THE CONSTRUCTION OF PLANTS.}

When anywhere within the limits of a flourishing town buildings are raised in great number and quick succession by the skilful hands of men, it is said that the houses grow up with astonishing rapidity from the ground. In the same way the growth of plants is appropriately compared by botanists with the erection of human habitations. In this book the latter comparison has already been made as occasion offered, and, even at the risk of repetition, I must again make use of the simile here in discussing the building up of plants.

As in the erection of human habitations, so in the production of the plant structure, it is a question of providing a home for living beings, of securing this home against injury by weather, and other dangers which might terminate the existence of the inhabitants. At the same time the inhabitants in these settlements must be able to take in food from the exterior, breathe, work up the foodstuffs, and extend themselves further. Where very numerous portions of protoplasm live, associated together in a plant in social union, and where corresponding to this a division of labour has occurred, the whole structure becomes naturally divided up into open spaces where there is no lack of air and light, into contrivances for ventilation, into mechanisms for conveying gas and water, and into chambers for storing up nourishment; in short, it is a question of varied mechanisms within and defences without, of the ensuring of strength throughout the whole structure, and of the necessary supporting framework for the individual parts. Each part occupies a position corresponding to the demand upon it; the light-requiring parts are exposed to the sun's rays; the mechanisms for conveying 
gas and water begin and end in the manner best adapted to the given conditions; while the pillars and beams are placed where something has to be protected, borne, or prevented from breaking down.

Such structures, just like the buildings produced by the hand of man, convey the idea of fitness of means to ends. Indeed, they often surpass mere human creations in the suitability of their arrangement. It can hardly be invariably said of man's designs that they are carried out in a way completely suited to the requirements of the case; while no plant lives and maintains itself which is not adapted to the given conditions of life in the most advantageous manner. The most remarkable thing about it is that this adaptation in plants is not produced directly by external influences, but that rather the individual portions assume the most suitable form and position, even in their first rudinents and very early stages of development; that is, at a time in which the forces acting outside the plant can have no considerable influence in directly moulding its form. Such an adaptation presupposes, however, a law of form; in other words, a plan of construction, a plan concerning the division of space best suited to the future division of labour, a plan of the most advantageous construction of the whole framework, the most suitable position of the conducting and ventilating mechanisms, and much besides, which will benefit the plant in the future.

This supposition being forced upon us, the question arises as to whether it is correct to speak of a constructive plan in plants. In the sense in which we speak of the constructive plan of a house, certainly not. Plants are not built according to a plan devised by themselves, but their organs receive their definite form, as if according to a prescribed law, from inward necessity, like the crystal whose shape is dependent upon and founded in the chemical composition of the fluid from which it is formed. But just as we can speak of the plan and elevation, of the symmetrical arrangement, even of the plan of construction, of a crystal, equally well can we speak of the plan of construction, or, if we prefer it, the law of form, of growing plants. The plan of construction is given and traced out for every plant by its specific constitution, and so far every species has its own plan quite independently of the external influences which it follows, indeed, is compelled to follow, as long as the constitution is not altered.

But by specific constitution we do not merely understand the chemical composition, the definite number of atoms, and their characteristic grouping into molecules. We understand further the union of molecules in definite groups of a higher order, which must be regulated in the plant body just as in the body of a crystal. This arrangement of the molecules is characteristic, we must suppose, for every species of plant; further, we must believe that the substance which is associated with the growth of the molecular groups, already present, is always subordinated to the laws of symmetry prevailing there, and that this grouping is not only specific, but also constant and invariable.

When we speak of crystals, we do not mean to say that the processes in question in them and in plants are identical. It is much more probable that there is 
a fundamental difference between the construction of crystalline bodies and plant bodies; that this very difference is bound up with the distinction between inanimate and living structures, and that especially are the organized parts of plants fitted by their characteristic structure to those movements which appear to us as life.

Molecules, united in the growth of crystals, admit of no further insertion of plastic substance, of no rearrangement and transformation, of no addition of new molecules between those already present, as is the case with the molecules of living organized bodies. When the molecules of water penetrate into a salt crystal, the molecules of salt separate from one another, and break away, so that we have a disintegration and solution of the crystal, and not its further development. The crystal, moreover, never shows at any time those displacements and movements of the smallest constructive particles which characterize the living organized parts of plants, which in the aggregate we call life. Crystals, therefore, cannot be considered as organized bodies; they are not directly concerned in the phenomena of life, and form no object susceptible to the influence of that specific natural force which we call vital force. They are not, and will never be living, just as they cannot die.

The analogy between the structure of crystals and of plants consists only in the fact that in both cases the grouping of the molecules cannot proceed irregularly, but must always follow definite laws of symmetry. In both cases the external visible form of the finished structure is the expression of a particular and specific grouping of the molecules, and of molecular aggregates known as micelloe.

Many attempts have been made to glean some idea of the actual shape of these groups of molecules or micellæ, the bricks--so to speak-of which the plant is constructed. That the hypotheses brought forward are very divergent is not surprising when we remember how few are the data of actual fact that have been observed, and how readily these data admit of varying interpretation, and how full a scope they offer to the imagination of the investigator.

Not long ago the idea found almost general acceptance that micellæ were crystalline in form. In many cell-walls, and especially in certain Desmidieæ, very regular systems of striæ were observed, which ran off into three dimensions of space, and strongly resembled the striæ connected with the cleavage planes of certain crystals (e.g. of calc-spar). Since these, and generally all cell-walls, light up the dark field in the polarizing microscope, that is to say, appear doubly refractive, the assumption was supposed warranted that the cell-walls and other organized substances consist of crystalline doubly refractive micellæ, which lie loosely but in regular arrangement next one another. It was imagined that every micella was surrounded, when moist, by an envelope of water, and that on drying, the micellæ came into mutual contact. But later researches have shown that the double refraction can be produced by pressure and strain in substances which do not normally exhibit this property, and that the refraction in the polarizing micro- 
scope is not always indicative of the crystalline nature of micellæ. The striation is brought about by dissimilar chemical constitution and unequal quantities of water in the successive strata of molecular groups, and may be present, equally well, where the groups of molecules do not possess crystalline form. Moreover, the results which have been obtained by the so-called carbonization or pulverization of the cell-walls goes against the assumption of crystal-like micellæ. By treating with sulphuric acid, heating up to $60^{\circ}-70^{\circ} \mathrm{C}$., and then operating with hydrochloric acid, the cell-wall is broken up into extraordinarily small fragments, exhibiting parallel striæ and frequent clefts; and these often subdivided into short, very fine filaments, which filaments break up by pressure into granules imbedded in a homogeneous gelatinous matrix. A definite geometrical crystalline form cannot be demonstrated in this ground-substance. Moreover, the granules are not bounded by plane surfaces and rectilineal edges, and have no resemblance to the smallest visible portions of crystals. All the observations obtained by this class of experiment tend rather to show that the granules are grouped into filaments, or lamellæ, or both, that they are joined together by extremely delicate protoplasmic threads, and that the cell-wall possesses a reticular structure. If these granules and filaments are not themselves the micellæ, but groupings, rather, of a higher order, still their outlines in no case suggest the forms of crystalline micellæ. The idea that the micellæ possess a reticular form was corrected much earlier. If the same rule which prevails in the grouping of the molecules into micellæ would also hold in the association of micellm into groups of high order, and ultimately into bodies which are in their outline recognizable by our senses, then one might hope to derive the form of the micellæ, and even the form of the molecules themselves, from the form of the smallest visible portions of the plant. This supposition would lead to the conception of reticular micellæ and reticular molecules in the organized parts of plants. It is, however, very noticeable that all researches concerning the form of the smallest visible elements of protoplasm point to a reticular structure. In the dry coating of the so-called plasmodia of myxomycetes, which contains no cellulose, but consists of protoplasm (in which are deposited crystals of calcium oxalate), for example, in the plasmodium of Leocarpus fragilis, it is seen that the entire papery skin consists of twisted threads extending in all directions, which anastomose in a reticular manner, and that the meshes of this net-work are filled with a highly refringent substance.

In the hyaline ectoplasm of the living protoplasm which inhabit the cellchamber, very fine threads have been observed lying side by side, and if this protoplasm is displaced and killed by alcohol, it can be ascertained by the aid of colouring matters that the whole cell-body is built up of very minute threads connected into a net-work, and that the meshes of this fine net-work are filled with a fluid substance. Within the threads are to be seen, however, corpuscles arranged in rows, which have received the name of microsomata.

The whole protoplasmic cell-body, including the cell-nucleus, appears generally to possess this same structure, for in the processes which lead up to the division 
of the cell we always see therein granules, rods, and shorter or longer, straight and curved tortuous threads, twisted in balls, and anastomosing into net-works, which undergo the most wonderful displacements, as will be described in the following pages.

All these observations at any rate do not contradict the supposition of reticular micellæ; and since the conception of molecules built up from atoms grouped in this manner has not been contradicted by chemists, the hypothesis should find support from this fact. Of course the hypothesis of the net-like form of the micelles is based upon an assumption, the accuracy of which is subject to many doubts. It is questionable whether the same rule always holds in all these groupings and connections. Just as pointed crystals often join up into spherical groups, whose construction follows other laws of symmetry than are observed by the molecules of which the individual crystals are composed, so it is always possible that the combination of the micellø into visible bodies follows other rules than the union of the molecules into the micellø.

This change in the relations of symmetry, occuring in minerals, gives rise to the idea of the possibility that micellæ may possess a spherical shape, that is to say, the highest degree of symmetry which can be imagined in a body. Some form of symmetry must exist under all conditions, and if the crystalline form of micellæ is excluded, then there remains the possibility of reticular and spherical micellæ.

Although our thirst for knowledge finds but little satisfaction in hypotheses of this kind, still they are not on this account to be held in contempt. The minutest structure of every substance, whose movements appear to the perception of our senses as life, is far too complicated for us to be able to bring it into the scope of our observations on the life of plants; and in order that we may be able to form a clear picture of all these matters, it is better at any rate to imagine the groups of molecules as net-works and spheres than to imagine nothing at all.

Though we may deny to the micellæ a crystalline nature, actual crystals can be produced by many organized portions of plants. Groups of crystals of calcium oxalate (see fig. $123^{4}$ ) are found very regularly deposited in the net-work which forms the pellicle of myxomycetes. Such groups of crystals are also to be found in the cell-membranes of many flowering plants (Cactaceæ, Nyctagineæ, Commelynaceæ, \&c.). The carbonate of lime excreted in the cell-walls of Lithothamnieæ, is likewise crystalline. In other cases these excretions and depositions of lime and of silica are not crystalline, but amorphous, which literally means without form. But we must be careful not to be misled by this expression. These substances cannot be conceived of without a definite shape governed by conditions of symmetry, only they are not composed according to the laws of symmetry governing crystals, and the word amorphous should therefore be interpreted here as non-crystalline. It does not lie within the scope of these remarks to enter into details about the hypotheses as to the shape of the molecules and groups of 
molecules of amorphous lime and amorphous silica; but this much must be said with regard to these depositions, that they cannot be looked upon as organized substances.

Here is the proper place to consider investigations as to the size of molecules. In these researches, especially for the ascertainment of the size of molecules of gas, very various physical facts offer themselves as data, such as the coefficients of condensation, the deviations from Boyle's law, the variability of the coefficients of expansion, the heat of evaporation, and, finally, the constants of dielectrics. The results differ considerably. For example, the estimates of sizes given for a certain gas by different methods differ from one another far more than those which have been obtained from different gases by one and the same method. But all calculations agree that the diameter of the hypothetically spherical molecules of gas must lie between the hundred-thousandth and the millionth part of a millimetre, and that these limits cannot be overstepped, either above or below, to any great extent even in the extremest cases. A cubic millimetre of gas would therefore contain about 866 billions of molecules, and if the gas were condensed into a fluid, the number in a millimetre would increase to a trillion.

The length of light-waves is of the smallest of measurable dimensions. If the diameter of a molecule is taken in round numbers at the millionth part of a millimetre, this is 700 times smaller than the wave-length of red light, and the diameter of a molecule bears about the same proportion to a millimetre, as a millimetre to a stretch of 2 kilometres. Particles of these dimensions are beyond the conception of our senses; even the highest powers of the microscope are unable to disclose them to us, as is shown by the following considerations. Sheets of gold-leaf are produced, whose thickness amounts to only a hundredth part of the wave-length of light, and which accordingly contain only 3-5 molecules of gold above one another. These gold-leaves are transparent to white light, and this may be regarded as a proof that rays of light penetrate through the chinks between the molecules. Nevertheless this leaf appears as a continuous mass under the best microscopes, and it is not possible to recognize the individual molecules composing it. Under the most favourable circumstances, our microscopes are able to render visible only particles which comprise perhaps two million molecules. Since there are no certain data to enable us to measure how great is the number of molecules from which micellæ are built up, and in what manner the molecules are grouped in them, it would be rash to attempt any conjectures as to the size of micellæ. The possibility of perceiving micellæ with the microscope in their outline and shape, especially those of albuminous bodies, whose molecules are composed of such a large number of atoms (see p. 456) is not to be wholly excluded, particularly since our microscopes are still capable of much improvement. Still, the probability is but a remote one, and as matters stand at present, all conclusions on this subject would be of the nature of theory, in which one uncertain hypothesis has to furnish the foundation for a second, still more doubtful. 


\section{VISIBLE CONSTRUCTIVE ACTIVITY IN PROTOPLASM.}

Though it is improbable that we shall ever succeed in seeing the micellæ of which the organized living portions of plants are built up, and though all attempts to form a picture of these tiny units are only founded upon conjecture and hypothesis, still we can follow with our eyes the general operations, the constructive and shaping activity of the protoplasm.

This formative activity can be most easily observed in the comparatively large protoplasmic bodies of myxomycetes, in their so-called plasmodia; therefore some of the most striking of these processes will now be briefly described.

The myxomycete Leocarpus fragilis, which commonly occurs on the bark of dry, fallen branches of the Pine, forms a viscous yellow mass, looking deceptively like the spilt yolk of an egg. The dead branch is covered by a thin layer of this substance, in which no particular projections can be recognized. Quite late in the evening Leocarpus can be seen in this plasmodial stage. During the night, however, it rises up in certain places into knobs and warts, and the whole mass then has a coarsely granular appearance. Towards morning, pear-shaped bodies, supported on thin stalks, are produced from these protuberances, which are now no longer viscous, but exhibit a thin dry pellicle. Within, they have become transformed into numerous hair-like threads, with black powdery spores lying between the threads. Leocarpus needs about 12 hours for this manifestation, and if one has the patience to observe the mass shaping itself throughout the night, one may actually see how it rises from the substratum, rounds itself off, forms a skin, and assumes the pear-shape form. Dictydium umbilicatum develops its plasmodia in the same way as Leocarpus. The light brown, irregular, flowing mass of protoplasm gathers itself up into a round cord, which becomes thickened in a clubshaped manner at its upper end, and then spreads out into a delicate net-work with spherical outline. Between the meshes of this net-work the protoplasm separates out into black powdery spores, which are at the mercy of the slightest breath of wind. The slimy protoplasm of Stemonitis fusca, on the other hand, rises up in the shape of numerous closely-compacted strands about $1 \frac{1}{2} \mathrm{~cm}$. long. Each individual strand is divided into a lower, stalk-like portion, and an upper, thick, cylindrical body. This is at first of slimy consistency, but soon becomes dry and divides into a central axis, from which proceed all round an endless number of very fine reticulating threads which break up into thousands of powdery spores, and at the periphery into a very delicate skin, which later on ruptures and allows the spores to fall out. This entire shaping of the protoplasm, with which is connected a change of colour from white to purple, is accomplished under the eye of the observer in about ten hours. The protoplasm of Chondrioderma difforme can scarcely be distinguished from that of Stemonitis fusca, and yet how very different is the form which it assumes as a plasmodium. First, it is massed into a round ball, and in this is separated out an enveloping skin of innumerable single slender 
threads, and a large quantity of dark spores which fill up the space inclosed by the skin. Soon after, the skin breaks up into stellate projecting lobes at the free apex of the spherical body, and now the dark spores can pour out of the open vesicle.

The protoplasm of Didymium shapes itself quite differently, and that of Clatroptychium differently again. If we were to exhaust the multiplicity of form which the protoplasm of this group of plants assumes, we should be obliged here, to actually describe the shapes of all myxomycetes. The above examples will suffice for the establishment of the fact that apparently quite similar protoplasm becomes, in each species, speedily transformed into a definite structure. It only remains to be noticed that the shape assumed by the specifically different protoplasm is quite independent of external conditions; and that in the same light, with the same degree of humidity, and at the same temperature, under the same glass shade, the pear-shaped Leocarpus, and the cylindrical strands of Stemonitis develop side by side (for illustrations of Myxomycetes ef. vol. II., fig. 355).

The pellicle which bounds the plasmodia of myxomycetes contains no deposited cellulose, and there is consequently in these plants generally no distinction between the pellicle and the body of the cell. The protoplasm of other plants, however, always provides itself, sooner or later, with an envelope in which cellulose can be demonstrated. Of course, cellulose is often present in the cell-wall only in small amount; thus, in yeast, as well as in the majority of fungi, the main part of the membrane is formed of nitrogenous compounds. Various phenomena lead to the conclusion that by the development of cellulose in the skin, advantages are obtained which are not enjoyed by myxomycetes, with their brittle pellicle built up of firm nitrogenous compounds. The soft protoplasm is better protected against injurious external influences by the cellulose wall, and the whole structure obtains that firmness and strength which are absolutely necessary, especially to plants composed of numerous cells.

Moreover, the cell-wall must not be conceived as always a rigid covering, as a chamber with immovable walls. In many instances it is rather to be compared to the skin of an animal, which adapts itself to each alteration in the shape of the body. In no case is the elasticity of the protoplasm hindered by the surrounding cell-wall. Frequently the cell-wall takes no share in the visible plastic processes of the protoplasm which it incloses, and it usually perishes when the transformations have been completed in the space it surrounds and protects. In many instances, on the other hand, the outline and shape of the cellwall alter in correspondence with the alteration of the protoplasm inclosed by it.

These remarks had first to be made in order to rightly understand the plastic processes to be described successively as Segregation, Gemmation, and Cell Division.

In the case of the Segregation associated with most of the previously described plasmodia, it is to be pointed out as characteristic that the protoplasm divides within a rigid, enveloping cell-wall into completely separate portions of identical shape, and develops no partitions continuous with the surrounding cell-wall. The inclosing cell-wall stands in no direct contact with the formed protoplasmic 
masses. Even when the wall remains, and is not ruptured nor disintegrated, it is separated from the protoplasmic masses by the new cell-walls, with which these have neanwhile surrounded themselves. For every species of plant the number, size, and shape of the bodies arising in the interior of a cell by division are quite definite, though they vary from species to species. In the cell-chambers of some species several thousand minute protoplasmic bodies arise. In others, again, the number is very limited. Frequently, indeed, the protoplasm only splits up into two similar halves. If the number is large, the individual masses are exceedingly small, and can only be recognized when very greatly magnified. If the number is limited, the divided portions are comparatively large. The shape of the structures is exceedingly various. Some are spherical, elliptical, or pearshaped; others elongated, fusiform, filamentous, or spatulate; some are straight, others are spirally twisted, and many are drawn out into a thread; others are provided over the whole surface with short cilia, others again with a crown of cilia at a particular spot, or with only a single pair of long cilia. The illustration on p. 29 represents the most widely differing forms, without, however, exhausting the wealth of configuration. In the majority of cases the small bodies exhibit active movements, and that even within the cell-covering which surrounds the dividing protoplasm; but sooner or later they come to rest, and then assume another shape, or fuse with another protoplasmic body.

With regard to the further changes experienced by the bodies formed by division, many events may be distinguished. In one, the cell in which the division of the protoplasm has taken place opens, the bodies formed glide out separately and swarm in the surrounding fluid. Often they are concerned in fertilization, and fuse with other protoplasmic bodies in a manner to be described later in detail. If not, they surround themselves with a cell-wall, but do not adhere together, or develop into a cell-colony.

In the Water-net (Hydrodictyon), described on p. 36 (cf. fig. 197, vol. II.), the parietal protoplasm of a cell divides up into 7000-20,000 minute clumps which exhibit the so-called swarming movement. At first a definite aim cannot be assigned to these movements, but after a short time the particles appear arranged very regularly in a net with hexagonal meshes. They assume the form of short rods, each of which joins at its poles with two others, being cemented to them by excreted cellulose. Instead of a protoplasmic parietal layer in the cell in question a miniature water-net is now seen to have arisen. This becomes free with the disintegration of the parent-cell; its cells grow and increase in all directions without, however, altering the shape once assumed. The process which is observed in Pediastrum (fig. 197, vol. II.), a very small water plant allied to the water-net, is very much the same. Here also the protoplasm of a cell which has isolated itself from the others divides up into small clumps which round themselves off, and swarm about for a short time. Gradually they come to rest, assume an angular form, and arrange themselves so as to form two concentric rings in one plane. Where they come into contact with each other, they excrete cellulose and 
thus become connected into a tiny disc. This disc consists of as many cells as there are connected clumps of protoplasm, and presents almost the appearance of a honeycomb. Out of this combination each cell can again separate itself from its companions, its protoplasm can divide up afresh, and generally the whole process described above may be repeated.

The Water-net and the discs of Pediastrum develop young nets and discs accordingly, from the divided protoplasm in the individual cells. These escape as small colonies of cells from the space in which they were formed, and here a definite isolation of the young cell-colony occurs. In Glococapsa, on the contrary, of which a species (Gloeocapsa sanguinea) is represented in figure $25 \mathrm{~A}, n$ and $o$, the young cell-groups remain joined together. Each cell always divides up, two and two, into protoplasmic clumps, which surround themselves immediately with a thick cell-wall. The old cell-wall, however, does not disintegrate nor rupture; it does not allow the young cell-colony to escape, but it stretches, and the young and old cell-walls are now seen layered one above another. If this process is repeated many times, protoplasmic balls arranged in pairs are to be seen inserted within a whole system of concentrically stratified cell-walls. A process similar to that just described is observed in the ovules of seed-plants, and has been called, though not very happily, "free cell-formation".

Gemmation is essentially different from the process just described. It is observed in plants both with and without chlorophyll, but is not really frequent in the vegetable kingdom. Its characteristic feature is that the protoplasm at a certain point of the circumference of a cell pushes outwards, and in this way a wart or bud-like elevation of the cell-wall, an actual protuberance, arises which, though at first not very prominent, soon increases in area, and in the end assumes the size and shape of the body from which it was produced. We may distinguish two kinds of gemmation. Either an open communication is maintained between the outgrowth and the structure from which it was produced, and no separation occurs at the place of origin; or, the parent cell is shut off from the outgrowth by a cellwall which subsequently splits, and the outgrowth is detached from the cell-body from which it arose. Very pretty examples of the first kind are exhibited by the Siphoneæ, especially in Vaucheria, illustrated in figure 25A, a. The tubular cells appear branched, each branch consisting of a tube ending blindly, and all these branched tubes are in free communication with one another. The entire Vaucheria is really only a single, much-branched cell-of course a cell which must be called gigantic in comparison with ordinary plant-cells. Species of the genus Bryopsis shape themselves similarly, but in these the outgrowths are much more regular than in Vaucheria, the whole cell, branched and thus pouched, almost resembling a moss with axes, leaves, and rhizoids. In the genus Caulerpa the cell also produces outgrowths, some of which resemble roots, whilst others imitate the shapes of leaves, reminding one, in some species, of small fern-fronds.

Of the second kind of gemmation yeast may be taken as a type. The shape of individual yeast-cells is ellipsoidal. When the yeast-cell grows, the elliptical 
form of the body is retained for a time, and the ellipsoid increases equally on all sides. When it has once attained a certain size, the protoplasm bulges out at a particular place, and a wart-like protuberance arises at the periphery, at first exceedingly small, but gradually increasing in extent, and at length reaching the size of the ellipsoid from which it was produced ( $c f$. vol. II. figs. $371^{3}$ and $371^{6}$ ). To say that the cell-wall of the yeast-cell protrudes or grows out, and that protoplasm immediately enters into the protuberance, is not a correct account of this process. The cell-wall here is only passive : it projects beyond the periphery of the ellipsoidal parent-cell only because it is itself the skin of the protoplasin pushing its way out at that point. From one yeast-cell two outgrowths may arise at different places, and each of them, when it has once reached a certain size, may again form protuberances. In this way the yeast shapes itself into a structure which strongly recalls to our mind the Prickly Pear in outline ( $c f$. vol. II. fig. $371^{3}$ ). When the protuberance has grown to an ellipsoid, equal in size to that from which it originated, the slightest pressure is sufficient to disconnect the two, and to separate the individual members of the irregular opuntia-like chain. Even without any external stimulus the cells separate, as may be well observed in brewers' yeast (Saccharomyces cerevisice), which of all the species of yeast has been most investigated.

The formation of yeast by the development of a cell-wall as a partition between two adjoining cells reminds one of the division of cells which has now to be described as the third formative process connected with growth. The division of the cells is always accomplished in the following manner:-The protoplasm, inclosed in its cell-wall, develops a partition in its interior by which it becomes divided into two halves, and the cell-space into two compartments or chambers. In some plants the sister-cells produced in division separate from one another, the partition-wall becoming completely split, but in most cases the neighbouring cells remain connected, and then in each of these the same process is repeated; in this way arise multicellular structures, that is, aggregates of cells.

A separation of the two cells arising from a division, due to the splitting of the intervening wall, is observed in the Desmidieæ, those small green aquatic plants, of which two species are represented in figure $25 \mathrm{~A}, i, k$. Although the Desmidieæ consist only of a single cell, their multiplicity of form is considerable. We have cylindrical, semilunar, tetrahedral, stellate, and disc-shaped forms in inexhaustible variety, often occurring in a restricted area, and forming a gay assemblage like the various herbs growing in a meadow. The cell of each species, however, adheres with wonderful tenacity to its plan of construction, and always develops only to a definite size. When once this size is attained, and after the cell has remained unaltered in form for a time, a noticeable change begins to take place. The central portion of the cell (which is constricted in all species) quickly elongates and expands. The protoplasm then develops a dividing-wall, and two cells are now produced from the one. These remain connected for only a little while; the intercalated cellulose wall splits; the two cells separate, and each forthwith assumes 
exactly the shape which the parent had possessed. These elegant desmids claim our interest because although their wall is composed principally of cellulose, and is comparatively thick, it has a determinate outline, and in this, and in its protuberances, and, generally, in its entire shape, it is governed by the living cell-body which has formed it. If such a desmid-cell extends in length or breadth, if it bulges out in one place and remains constricted in another, this is caused only by the activity of the protoplasm, which shapes and transforms the body in accordance with the constructive plan of the species.

The continued connection of the cell-couples produced by division, and the origination of extensive cell-aggregates by the repeated formation of partition walls, is much more usual than their separation. No less than five different modifications may be distinguished of this process, which is connected with the construction of so many plants.

In the green aquatic filaments, of which two species (Zygnema pectinatum and Spirogyra arcta) are illustrated in figure $25 \mathrm{~A}, m$ and $l$, a wall may be developed by the protoplasm of each cell, which is first formed as a ring-like band on the already existing cell-wall, and resembles the diaphragm in the tube of a microscope. Gradually from this circular band a completely closed partition-wall is produced, and the single cell becomes divided into two. In each of these cells this process may be repeated, and thus in a very short time may arise a row of four, eight, sixteen, \&c., cells. These remain connected with one another, and the whole row constitutes a cylindrical tube divided up by numerous transverse walls. If the single cells are much swollen at the sides, the row of cells has the appearance of a string of pearls. The intercalated partition-walls in these plants are all developed parallel to one another, and are placed at right angles to the axis of the cell-filament.

The fact of these intercalated partition-walls being parallel distinguishes this process from another, which is characterized by the fact that the insertion of partition-walls occurs in two dimensions of space. In this case neither partitioned tubes nor strings of pearls arise, but groups of cells arranged in one plane, which are plate-like in appearance, and, to the naked eye, look like membranes or leafy structures. This kind of structure is often shown by marine algæ which grow on stones. If all the cells adhere to the substratum, as in Hildenbrandtia, the outline of the plate is more or less circular, and green or red patches are to be seen on the stone, which continually increase in size without altering their general form. In this case there is no obstacle which could restrict the circular shape of the cellplate. If, on the other hand, only some of the cells adhere to the substratum, while the others rise up from the stone, so that the whole floats in the water as a thin film (only attached to the substratum at one point), then the further development is unsymmetrical. It is suppressed towards the substratum, and the whole layer usually has a fan-like appearance.

If the arrangement of partition-walls in a cell occurs in three dimensions of space, a tissue is then formed. The tissue-body developing most regularly in this Vol. I. 
manner is such as is exhibited by Sarcina ventriculi, a vegetable structure which will be presently treated of in detail. Here the eight daughter-cells produced from one cell appear so connected with one another, that they present, taken together, almost the form of a cube ( $c f$. vol. II. fig. $372^{10}$ ). One cell always comes to lie in each of the eight corners. Structures of such regularity are of course rare. Usually manifold variations take place. In the so-called pollinia of orchids hundreds of daughter-cells are developed by repeated division, grouped into small balls which again form a large, irregular, clumpy mass. It frequently happens that a group of cells, which increases at the periphery in three dimensions of space, in consequence of the intercalation of division-walls, does not exhibit, as would have been expected, a symmetrical growth on all sides, but increases chiefly in one of the three dimensions. This form, which is specially observed in stem structures, depends upon the development of a so-called apical-cell. By this is meant a cell which forms to some extent the apex of a cellular body constructed on a horizontal base. By the insertion of a partition-wall a chamber, a so-called segment, is formed from the lower half of the apical-cell. While fresh divisions are being accomplished in this segment the upper half of the apical-cell again grows up to the original size; and if one did not know that a segment had been cut off from it a short time before, it might be thought unaltered with regard to size, position, and shape. After a little time the segmentation just described is repeated and forthwith it again recovers from the loss, and attains to its original size. Thus the apical-cell cuts off one segment after another at the base, and builds a perestal on whose highest point it enthrones itself. The apical-cell comes in this way to be raised always higher and higher, as it were pushing its way through the surrounding air or water at the head of a group of cells; and to a certain extent the direction of growth, as well as the internal tissue of the groups of cells cut off from the apex, are ruled and ordered by the processes of division carried on within it.

This results from the fact that the position of the segments separated from the apical-cell (i.e. of the intercalated separation-walls), is always arranged in a definite manner. If the division-wall, which arises in the lower part of the apical-cell, parallel to the base and at the same time at right angles to the direction of growth of the cell, and if the further divisions arising in the repeatedly-divided segments occur in three dimensions of space, as is the case, for example, in the Characeæ, then the whole plant is built up in stories. The chambers of the lower story are produced from the first segment cut off from the apical-cell, those of the next higher story from the second, and so forth. The whole structure, however, is terminated above by the indefatigable apical-cell, which continues to divide in the same way as at the commencement of the edifice.

In other cases the separation-walls, which have been intercalated successively in the lower part of the apical cell, take up an essentially different position from that in the Characeæ. They are frequently placed obliquely to the direction of growth of the shoot-axis, and the base of the cell is either wedge-shaped or three-sided. It is wedge-shaped, for example, in some liverworts (Aneura and Metzgeria) as well 
as in Selaginella (belonging to the family of the Lycopodineæ). Here we have inclined walls formed alternately on the right and left, and thus arise two rows of segment-cells, arranged with regard to the axis of growth like the barbs of a feather on their axis. The base of the apical-cell is three-sided in the stems of horse-tails, most ferns, and mosses. Such an apical-cell may be best compared to a three-sided pyramid, whose sides are not flat but somewhat convex. The side of this cell, which corresponds to the base of the pyramid, forms the free end which is not bordered by other cells, but by the air, or earth, or water; the three other sides, directed towards the base of the growing plant-organ, converge at a point which lies in the axis of growth of the organ. The insertion of division-walls occurs parallel to these three slightly arched sides, and in a regular succession, so that the segments cut off appear arranged like the steps of a spiral staircase. The walls which are afterwards inserted in the segment-cells are partly parallel, partly at right angles to the first-formed walls. On the whole it cannot be questioned that in this building, as in the buildings of men, the walls are intercalated at right angles to one another in three dimensions of space.

In the root-tips of ferns and horse-tails, we also have a three-sided, pyramidal apical-cell, as just described, but the construction is to some extent complicated by the fact that division-walls also arise parallel to that side which corresponds to the base of the three-sided pyramid. The segments so cut off, which divide up again into many cells by radial walls, cover the apical-cell like a cap. This structure, which has been called the root-cap, serves to protect the apical-cell at the root-tip as it pushes its way into the earth, and would otherwise be exposed to inany dangers.

In some ferns, and in most flowering plants, two, or even a group of cells are to be found at the tips of the growing stems. Some trouble has been taken to reduce the arrangement of these to three types, but it does not lie within the scope of this work to describe these in detail. That the construction in these cases is extremely complicated, that in many cases it is very difficult, frequently even impossible, to follow and to establish with certainty the plastic processes, does not in the least alter our conviction that the construction of the growing organs in these plants is accomplished according to rule, and that a definite plan underlies the form of every species, which is indicated beforehand by the specific constitution of the protoplasm.

It must also be here remarked, to prevent misunderstanding, that in plants in which numerous organs are developed with various functions, all the growing parts are not formed in the same manner. This, however, is not opposed to the fact that in each species the same constructive plan is invariably adhered to. The directions of the septa inserted in the growing rhizoids, leaflets, and capsules of a species of moss may differ much among themselves, but in each species they are always the same in the various organs. In flowering plants, too, the processes in the formation of the root-cap, the stomata, the pollen-grains, and so forth, vary very much among themselves, but these processes are retained in each species of 
plant with great constancy. In the same species the root-cap, stomata, and pollengrains are always found to be constructed on the same plan. In poppy flowers which had developed two thousand years ago in Egyptian soil, and which were then placed in tombs as ornaments of the dead, the cells of the anthers and pollen are formed precisely as in poppy flowers which grow in our fields to-day. It is important to hold firmly to the fact of this constancy. On it is founded not only the possibility of distinguishing between species of plants, but, generally, the conception of kind or species, to which we shall repeatedly return.

The alteration of shape in the protoplasm and its walls, just described, refers in each case really only to the external contour. Obviously, definite displacements and arrangements in the interior of the living protoplasm lie at the foundation of these alterations, and it is reserved for further investigation to establish these latter as far as they are visible and recognizable. Hitherto the alterations occurring in cell-division in the substance of the protoplasm, especially in the so-called cell-nucleus, have alone been accurately observed, and what has been seen there has already been briefly stated on a previous occasion. This is the place to return to these remarkable phenomena, and to collect together the most important results in a brief review.

Let us look at a cell in which the protoplasm fills the whole interior. A large cell-nucleus is visible in the centre of the cell-body. The protoplasm exhibits when very highly magnified, granules, and fibrils, the latter long and short, curved and straight, knotted and twisted or rolled into balls, and anastomosing into a net-work. This structure appears most plainly, especially the filamentous formation, in large nuclei. The twisted threads there visible have been termed nuclear fibrils. In many instances there seems to be only a single much-twisted thread present in the nucleus. In other instances more are to be seen, and they appear to be distributed with some uniformity in the nucleus, as shown in fig. $138^{1}$. The change begins first of all with the division of the nuclear threads; from them are formed numerous short, twisted rod-like, or granular portions, which journey towards the centre of the nucleus, take up a position there corresponding to the equator of the cell-nucleus, which may be compared to a geographical globe (see fig. $138^{2}$ ), and arrange themselves into a plate which has been called the nuclear plate. Soon, however, a detachment again occurs of the constituents of this plate. They separate from each other, each fibril splitting into two, and seek the poles of the spindle (fig. $138^{3}$ ). As they do so the fibrils turn and bend themselves, usually so that those going one way have the form of a $u$, and in the other of $n$. Arrived at the region of the pole, the filamentous portions fuse, contract on every side into a dense skein (fig. $138^{4}$ ), and thus from one cell-nucleus two nuclei result.

A system of very delicate filaments also plays a part in these movements of the elements of the nuclear plate. These filaments, as may be seen in fig. $138^{2,3,4}$, form a spindle. This spindle arises, not from the nucleus, but from the surrounding protoplasm. The spindle appears to serve the nuclear fibrils for support and guidance in their movements, leading the fibrils to the poles, where they join 
together again into two fresh nuclei. After they have performed this function, these spindle filaments have a further and no less important part to play. Almost at the identical place where the nuclear plate was previously to be seen, an accumulation of exceedingly small granules arises, the repeatedly mentioned microsomata; and these are arranged to form a plate, the so-called cell-plate, which ultimately divides the whole cell into two compartments. Apparently these spindlethreads serve also as conductors to these microsomata, and many of the small granules are conveyed along them to the equator. But occasionally they arise there directly, and help to produce the cell-plate. The development of the cellplate does not seem to be always quite the same in different species, but it is established with certainty that in it cellulose micellæ are always formed, and that the partition-wall produced from them possesses the characteristics of a cellulose wall, that is to say, of a cell-wall. Already it has been mentioned (p. 44) that in

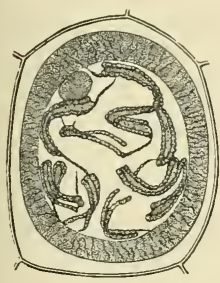

1

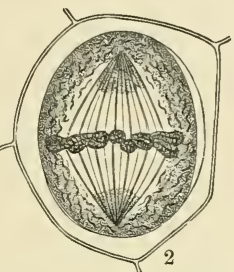

Fig. 138.-Changes in the Protoplasm of the Cell-nucleus during its Division.

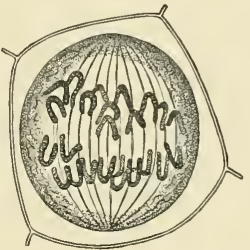

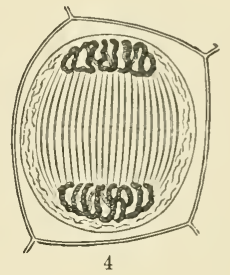

4

1 The nuclear fibrils distributed through the whole nucleus. 2 The broken-up nuclear flbrils arranged as the nuclear plate. 3 The elements of the plate separating from one another. 4 The same elements forming two skeins at the poles of the spindle. (After Guignard.)

this cell-wall, at least at first, albuminous portions of protoplasm are retained by means of which the intercalated membrane can undergo manifold metamorphoses, and that by them, if required, the communication is maintained between neighbouring masses of protoplasm.

In the cells of those green water-threads known as Spirogyra, Zygnema, and Cladophora, as well as in those of desmids and many other simple plants, the plants never seem to come to an end of this dividing. Each cell continues to grow until it has attained certain dimensions; it then divides into daughter-cells in the manner peculiar to it, and in these the process which has been performed in the parent cell is repeated afresh. This process continues perpetually under favourable external conditions, and an interruption occurs only when there is lack of necessary food, or when the living protoplasm is killed by unfavourable circumstances. In these plants, of which we can enumerate more than a thousand different species, there is thus no distinction into portions in course of formation and those which have been completed, and are no longer capable of development. It is otherwise in large plants in which a division of labour and a corresponding organization have taken place, in those plants whose different members perform different functions. In these, stability of some members is of the greatest advantage, and 
accordingly besides the cells which are still formative and promote growth, many others are present which undergo no further changes, whose size and shape is permanently retained, and which have therefore been termed permanent cells.

Organically-connected groups of permanent cells are called permanent tissue in opposition to the groups of constructive, dividing, and changing cells, the so-called meristematic tissue. All permanent tissue is obviously produced from meristematic tissue, and the meristem is ultimately derivable from a single cell capable of division.

The cells of meristems exhibit only very slight variations in form. It is impossible to recognize what forms the permanent tissues produced from them will in time assume. Of four exactly similar meristematic cells, the first may become the starting-point of several flattened epidermal cells devoid of chlorophyll; the second for the formation of a group of green palisade-cells; the third for a bundle of elongated, thick-walled bast-cells; the fourth for several delicate-walled, large parenchymatous cells. It is difficult to explain how this comes about, and we relinquish the attempt to give a full explanation here. Only this much may be remarked, that whilst the stimulus to these metamorphoses comes from outside, and external conditions have a determinative influence on the size of the developing permanent tissue, the shape, outline, and definite configuration which the individual cells in the permanent tissue assume, as well as the arrangement of the various cells in space, are independent of external influences. Just as in a plant the first division-walls assume a position defined beforehand in the dividing apical-cell, the further metamorphoses of the daughter-cells proceed within the limits settled by the specific constitution of the protoplasm, so the transformation of the cells of the meristem into cells of permanent tissue is governed according to a plan of construction peculiar to, and hereditary in, each species.

This law, derived from numerous facts, of the independence to external influences of the constructive plan and character of the cells, seems to be contradicted by the fact that alteration in the outline of individual cells can be produced by strain and pressure. Spherical cells with elastic, flexible walls may be changed by strain into ellipsoids; in consequence of all-round pressure a spherical cell may assume the form of a rhombic dodecahedron, or by lateral pressure, the form of a six-sided prism. In explanation of these conditions it has been pointed out that peas which are made to swell up in a cubical, thick-walled vessel, by pouring water over them, assume the shape of rhombic dodecahedra, because each individual pea is in this way allowed the greatest possible room together with the utmost economy of space. We are again reminded of the fact that the structure of slate-like stones is dependent upon the pressure acting upon the mass so far at least that the planes of cleavage and stratification are always at right angles to the direction of the pressure. But however valuable these facts are in the explanation of the condition of the form of inorganic bodies, they are of little significance to the question in hand. No one will deny that spherical cells on which an equal pressure operates from all sides may assume the shape of dodecahedra, but this form is not 
transmitted to the descendants. In the next generation of these same plants it is a group of spherical and not of dodecahedral cells, which arises at the particular place. The latter will, indeed, only reappear if the aforesaid pressures be again exerted.

How little, however, external influences define the form and grouping of permanent cells, is shown by the fact that from one and the same meristem, under the same pressure, the same temperature, and equal illumination, arise in the closest proximity the most different permanent cells; and that, on the other hand, the formation and grouping of these cells is not essentially different when the development of the meristem takes place under wholly different external pressure, or different temperature. We always come back to this important thesis:- the forces operating on plants from outside are only stimuli to the formative processes; these latter are accomplished independently of external influences in a manner established for each species, and founded in the specific construction of its living protoplasm. 


\title{
PLANT'FORMS AS COMPLETED STRUCTURES.
}

\author{
1.-PROGRESSIVE STAGES IN COMPLEXITY OF \\ STRUCTURE FROM UNICELLULAR PLAN'TS TO PLANT-BODIES.
}

Though all plants are mortal, they have the capacity of renewing themselves and rejuvenating, so that, in spite of their perishable nature and limited duration, the species now existing on the earth are in no danger of extinction. The rejuvenescence is always effected by means of the protoplasm of a single cell; i.e. by a small mass of slimy substance which can only be perceived by the naked eye in the rarest instances on account of its minute dimensions. The largest palm in its rejuvenescence must pass through this unicellular stage exactly in the same way as the smallest of mould-fungi. The only difference is that in large and usually long-lived plants a longer time elapses before this stage is reached, while in small plant-forms many generations may pass away and be replaced in the course of a single year. The protoplasm in the minute rejuvenating cell always grows at the expense of its surroundings, moulds itself in the manner peculiar to its species, and divides when it has attained to a certain size into two or more masses, which have inherited the capacity of dividing afresh.

Each one of these protoplasmic masses is to be regarded as an individual. When the adjacent masses of protoplasm, the result of continued division, remain in connection with one another, as indeed seems to be usually the case, each retains a certain degree of independence; nor, should a severance take place, are they necessarily abandoned to destruction. Under favourable conditions they may, although separated from their companions, enlarge, divide, and continue to grow. In not a few unicellular plants it is even customary for each mass of protoplasm immediately after its formation to separate itself entirely, and, for the future, to live independently. It is remarkable that in most of these unicellular plants a time arrives, i.e. the time of pairing, when they again seek each other with the view of uniting; but this period is of short duration compared with the length of the isolated life. Moreover, a definite bond of union has been recognized between the separate individuals produced from one cell. Just as caterpillars which creep out of the eggs laid by a butterfly are seen not to disperse, but to follow common paths and ways, so the swarm-spores of Spharella pluvialis swim together in groups from one place to another, and select a suitable spot for settling down. The 
single cells of diatoms and desmids form similar social groups living within restricted areas. We must suppose that here-exactly as in the young brood produced from the spawn of a fish, which swim in company through the water, or in midges hatched simultaneously, which dance up and down in the evening sun-here must be some kind of family feeling which binds the different individuals together, although we cannot fully comprehend these relations between the several organisms.

When the single genetically-connected masses of protoplasm, each retaining its own individuality, can transfer themselves in common from one place to another, like caterpillars, midges, grasshoppers, fishes and the like, the community is called a swarm; if, on the other hand, the isolated individuals settle quite close to one another on a substratum, and there take up a definite position for their lifetime, then we speak of a colony. The amœboid bodies of some myxomycetes, several unicellular Palmellaceæ, Desmidieæ, and Diatomaceæ, live in swarms; the numerous Siphoneae, on the other hand, as well as the species of the genera Synedra and Gomphonema belonging to the family of the Diatomaceæ, dwell in colonies ( $c f$. vol. II., fig. $369^{1}$ and $369^{14}$ ). These colonies often attain to considerable dimensions. The Acetabularias attached to stones and mussel-shells at the bottom of the sea, the swollen bladder-like Valonias, the moss-like forms of Bryopsis, and the dusky species of Codium, form, arranged in thousands side by side, very extensive colonies. The Vaucherias, dwelling on damp earth and in cold springs, appear as extensive cushions which cover the ground over a wide distance with a green felt. Besides the swarms and colonies, we have a third form of assemblage, the cell-union, in which the genetically-connected masses of protoplasm grow together in a body. This union, again, differs essentially according as to whether the individual masses of protoplasm forming it are devoid of, or are surrounded by, a cell-wall. In the former case they are fused into a mass in which the limits of the single individuals can no longer be recognized, as is the case, for example, in many myxomycetes. The expression "fusion" can here be employed figuratively with the utmost propriety, for indeed the process strongly resembles the fusing of fluid metallic globules into a larger mass, or the fusing of numerous drops of oil floating on the surface of water into a larger drop, in which the contours of the single fused portions are obliterated. It is indeed doubtful whether the fused masses of protoplasm do actually surrender their individuality. Certain phenomena tell rather against than for this view. Thus many myxomycetes form so-called sclerotia, i.e. they lose their mobility and pass into a temporary state of rest. The whole mass becomes rigid, assumes a wax-like consistency, and dries up, and the shapeless protoplasm divides into innumerable, clearly-defined, rounded or angular particles. When at the end of the resting period the stiffened mass is to be again transformed into the mobile condition, the individualized particles become fluid and a fresh fusion takes place. The phencmenon observed in the whole series of myxomycetes suggests the idea that the isolated corpuscles in 
the sclerotia correspond to the single masses of protoplasm from which the whole mass had previously been formed, and that these have not surrendered their individuality, although their boundaries cannot be recognized in the mass. The unions of fused masses of protoplasm devoid of cell-wall are inconsiderable in number in comparison with the enormous quantity of those combinations in which each portion of protoplasm is surrounded by a cell-wall, by means of which the cohesion of the whole is brought about. The latter are classed as cell-complexes or tissues and are for the sake of clearness divisible into four groups, which may be distinguished as rows, nets, plates, and masses.

Its name tells us what a filamentous cell-complex looks like. As regards -its production, it is to be noticed that the partition-walls, which are formed by the segmentation of its cells, always stand at right angles to the long axis of the cell-filament, and are therefore parallel to one another. The general appearance of this tissue is regulated according to the particular shape of the single cells. If the individual members of the row are spherical, chain-like strings of pearls are produced, such as are found in the Nostocaceæ; if the individual cells are cylindrical, long or short, then thread-like structures arise from their end-to-end arrangement, as may be frequently observed in the Zygnemeæ and the CEdogonieæ. If the cylindrical cells decrease in thickness as the filament increases in length in one direction, whip-like forms arise, as, for example, in the species of the genus Mastichonema. Occasionally the single members of the row are tabular, and the tablets are joined to one another by their narrow edges, in which case ribbon-like rows are produced, as in Odontidium; or the neighbouring tabular cells are only connected by their corners, in which case the row has a zigzag appearance, as in the genus Diatoma (c.f. vol. II. fig. $369^{15}$ ).

In the reticular cell-complexes the numerous cells are seen to be so arranged that they adjoin one another by comparatively small contiguous surfaces, joining together at two or three, more rarely four, angles of corresponding size. The partition-walls intercalated during the division are not all parallel with one another, but are arranged in more than one dimension of space. Nets may be distinguished as open and closed. In the former, which may be best compared with the net-work of rivers on a map, the cells only seldom form closed meshes, but start out from one another like the prongs of a fork. Open nets occur very often, especially in the mycelia of fungi, in species of the green Confervoidex living in water (Cladophora and Chaetophora) and in numerous red Florideæ. Much rarer are the closed nets with hexagonal meshes, as, for example, those of the Water-net (Hydrodictyon) described on p. 36, and the remarkable nets of Volvox globator, comparable to hollow spheres, which were considered on p. 37. Open reticular cell-complexes permeate the decayed trunks of trees, the mould of the forest-soil, and the humus of the meadowground. Here they exist as saprophytes, or on living plants and animals as parasites; or they are only attached by a few cells to the substratum, and the forked ramifications stretch out from these starting-points like fans and radiate 
forth into the surrounding water, as in most water-plants belonging to this category. The closed nets, on the other hand, are never joined to a substratum, but remain floating in the water from which they derive their nourishment.

The plate-like cell-complexes are composed of cells arranged in one plane, and adjoining one another so as to leave no intercellular spaces. The partitionwalls inserted in the separate chambers during the development of this form are arranged in two dimensions of space, and frequently intersect at right angles. These cell-complexes either form a thin coating on stones or other solid bodies, and then adapt themselves closely to all inequalities of the substratum, as e.g. in Protoderma viride, which covers the stones and old treetrunks in mountain-brooks; or they appear as membranes, ribbons, and delicate leaf-like structures, which are attached to the substratum only at one point, and for the rest float freely in the water. This is what occurs in the Sea Lettuce (Ulva), and in many Florideæ, as, for example, Porphyra. Sometimes the plate-shaped complexes are developed as quite independent, unattached tablets and discs, as in the genus Pediastrum (c.f. vol. II. fig. $197^{6}$ ). The leaf and ribbon-like forms which float in water are but seldom quite flat; usually they appear much bent, undulated, and pitted; the margin, also, is generally crinkled or slit, and divided into lobes and fringes, and these forms thus furnish transitional stages, half cell-plates, and half cell-nets. In the matter of size, all possible gradations are to be found, from the minute discs of Pediastrum, and the small membranes of Prasiola flourishing in glacier-streams, up to the Ulvas, living in the sea, many of which grow up into membranes a square metre in area.

Mass-like cell-complexes are those whose constituents adjoin one another in three dimensions of space. Both in transverse and longitudinal sections of their tissues we have at least two, but as a rule several stratified cell-layers. Usually the whole body is elongated much more in one direction than in the others; frequently it has the shape of a solid cylinder or prism, or it forms thick fibres, cords, and ropes. Many remind one of earth-worms, or they resemble the tentacles of polyps and sea-anemones. In many Floridex, and especially in the brown leathery sea-wracks, these cell-complexes are strapshaped, or they are contracted into a stalk below, where they are attached to the substratum, and above widen out into leaf-like structures, as, for example, in the Laminarias of the North Sea (see fig. 139), and in many other cases. These strap-like, ribbon-like, and leaf-like structures occasionally remind one of the similar plate-shaped cell-tissues of Ulvacex, previously mentioned, but are distinguished from them by the fact that they are always built up of two or more stratified layers of cells, so that a section taken at right angles to the leaf-like structure always exhibits two cell-layers at least. Cake-like and ballshaped tissues are rarer. As examples of the latter various species of Glcoocapsa may be instanced, one of which is illustrated in fig. $25 \mathrm{~A}, n$.

In most of these simple cell-complexes the bulk of the cells are shaped similarly 
Usually only the portions serving for reproduction exhibit differences of shape, and these are so subordinate in number and extent that the appearance of the whole is very little altered whether they are present or not. It is of more importance with regard to the general appearance, that most of the simple tissues enumerated,

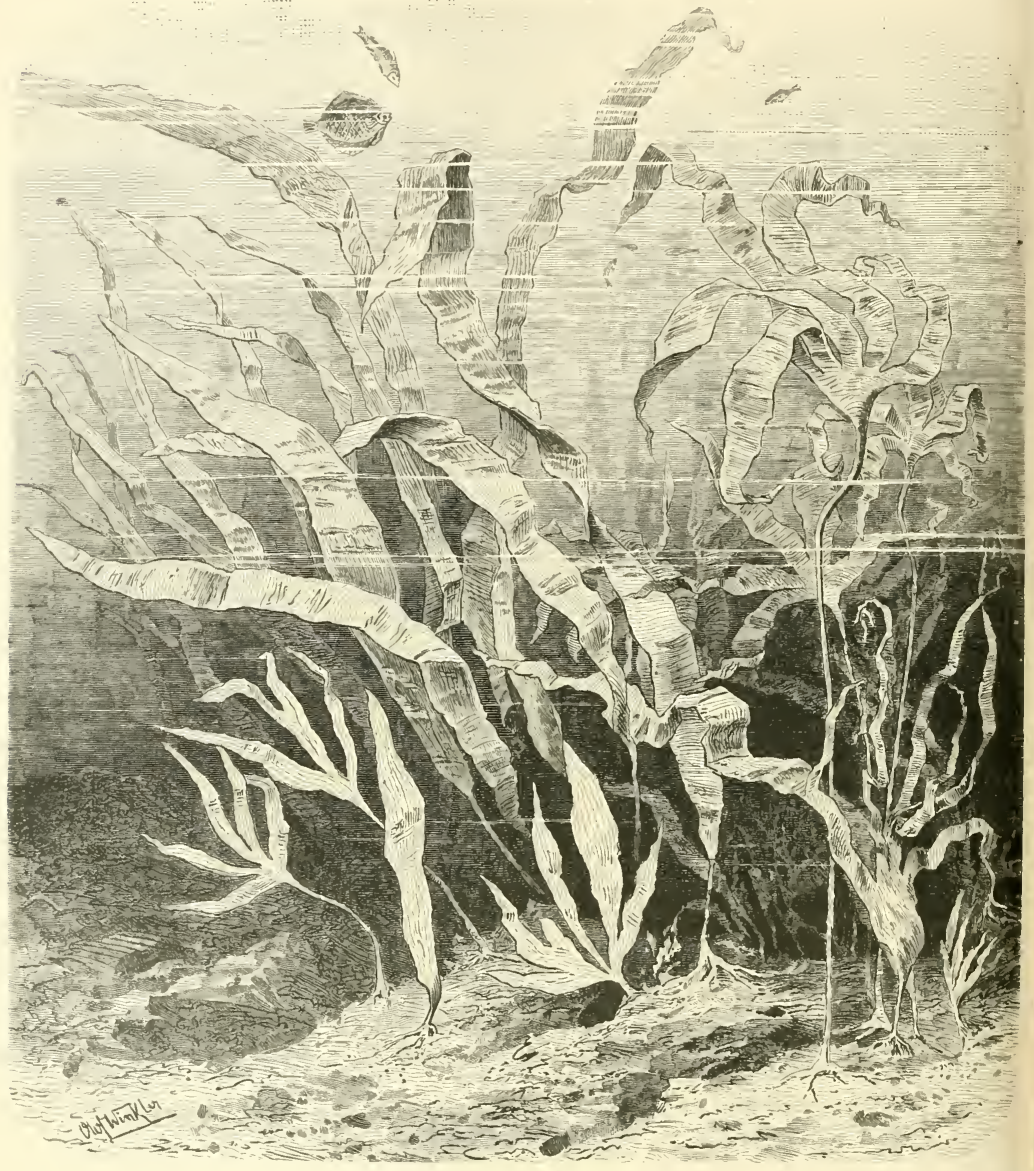

Fig. 139.-Laminarias in the North Sea.

multiply and divide without the portions thus produced becoming separate or detached. The nets of Hydrodictyon indeed multiply by the formation of daughter-nets within single cells, which then become detached from the parent plant. The disc-shaped plates of Pediastrum also multiply in a similar manner, and in this sort of plant whole swarms of cell-complexes are always developed, so that 
in the pools where these species grow hundreds and thousands of separate nets are to be found living together within a limited area. But the number of instances of swarm-forming cell-complexes is, however, utterly insignificant in comparison with the enormous number of those forms in which the tissues arising by rejuvenescence remain connected. We call these permanently-connected cell-tissues systems; and distinguish between systems of cell-rows, cell-nets, cell-plates, and cell-masses. The arrangement of the individual parts, and the fitting together of the systems is quite irregular, but is defined for each plant-species in the established manner, inherited from generation to generation. The simple celltissues which build up an extensive system can be distinguished as separate parts, and may be compared to the members of a body, and even called members of that system. There are, of course, systems which consist of very many simple celltissues, and therefore have a much-membered appearance; and others which exhibit only a slight organization, i.e. are built up of only a few simple tissues. Setting aside the question of greater or smaller, the kind and manner of connection must be taken into consideration in a general review of the forms of plants, and these systems can be comprehended under two divisions.

The first division comprises those whose members (i.e. cell-complexes) are all of similar shape, so that the whole plant-body consists only either of cell-filaments, or of cell-nets, or of cell-plates, or, finally, of cell-masses. These uniform systems are found more especially in plants growing under water, which reproduce themselves by spores, as well as in fungi, and the commonest forms to be noticed are as follows:-first, the clusters of tortuously twisted and intertwined rows of cells, like strings of pearls, such as occur in the Nostocaceæ, the bundles of elongated, straight filamentous rows of the Oscillatoriex, the flock-like Scytonema and other aquatic plants, and the dark cushions of whip-like rows grouped in bundles, as shown in the genera Euactis and Dasyactis. Among the series of complex systems a particular interest is claimed by those which are produced from the frequently-mentioned hyphæ. When the branched hyphæ, often knitted into meshes, and united into net-works, are crowded together in great numbers, plexuses and strands arise which have exactly the appearance of a cell-mass, but which may be distinguished therefrom by the fact that neighbouring cells, whose sides adjoin one another, are not produced by the intercalation of partition-walls. The fungal hyphæ have a common development and manner of growth; hundreds of hyphal threads which are joined together into a strand or plexus continue to grow at the apices with equal rapidity and in the same direction; they carry out in common the same curves and twistings, often divide into single threads, then reunite, and thus form the most peculiar shapes. The so-called Hercules-club (Coryne pistillaris), the strange forms of Clavaria, resembling pieces of coral, the Cap-fungi, divided into cap and stalk, the Helvellas and Morels, the very peculiar puff-balls and earth-stars, and many other forms are built up of hyphal strands and plexuses, which, as already stated, are nothing else but conglomerated cell-nets. Systems of cell-plates are more rarely to be met with. This construction is found most 
noticeably in the marine Padina Pavonia, older species of which are composed of superimposed, thin, leaf-like cell-plates. Systems of cell-masses are found in many Florideæ and especially in the large brown sea-wracks, Cystosira, Sargassum, and Fucus. The separate cell-complexes, which form a system in these plants, frequently assume the form of leaves, and these sea-wracks are occasionally ranked with leafy plants, which will be described later. Hydrurus and the Stonewort (Chara) are systems of cell-complexes; but while the individual complexes in Hydrurus are connected very irregularly, they exhibit in Chara an extremely regular, geometrical, whorled arrangement ( $c f$. vol. II., fig. $206^{1}$ ).

Following this first division of systems, which have a uniform construction, is the second, in which the body is built up of different kinds of cell-complexes. These are called mixed systems. Each member of such a mixed system, regarded by itself, exhibits a simple homogeneous cell-tissue; but the simple complexes are so combined that in one case cell-rows are carried by a cell-plate, while in another case a cell-mass forms the starting-point for several open cell-nets, and so forth. All possible combinations are realized in nature, but none more frequently than that in which a cylindrical cell-mass forms the centre or axis of the whole plantbody, whilst cell-plates or nets are laterally articulated. In many sorts of Batrachospermum open nets are seen which are borne on a central pillar of cell-masses; and in a liverwort (Jungermannia trichophylla) the same thing occurs, except that here there are cell-rows which proceed from the lower parts of the central mass (fig. $140^{6}$ ). Many mosses and liverworts (e.g. Hookeria splendens and Jungermannia polyanthos) exhibit a stem-like central tissue which does not carry cell-nets, but single-layered cell-plates. As shown in the illustration opposite, all possible stages are to be seen in moss vegetation between central supports provided with cell-nets, and those with cell-plates; this must be particularly noted here, in order to establish the fact that all classifications and distinctions based upon external forms are really only artificial, and that sharp limits between the various forms do not exist. Still it conduces to clearness, none the less, if we collect together and classify the various forms as well as we can. The mixed systems which are represented by the liverworts, illustrated here, claim an especial interest, inasmuch as they are to a certain extent the prototypes of plantbodies, i.e. of those complex forms which botanists in earlier times alone recognized when speaking of the configuration of plants; these alone were considered, for example, in Goethe's Theory of Metamorphosis.

The Plant-body is always membered, and each of its members is composed of cell-complexes of the most varied kinds. In this lies the distinction between plant-bodies and the previously-described forms. The members of a simple and of a mixed system are simple cell-complexes:-cell-rows, cell-plates, and the like. The members of a plant-body are, on the other hand, combinations of cell-rows, cell-nets, cell-plates, and cell-masses. The cell-complexes combined in a member of a plant-body are connected from their first origin. A single cell is always the starting-point for the particular member of the body; this divides; the compart- 
ments are again divided, and from the single compartments (i.e. cells) originate here plate-like, there mass-like complexes, in this place cell-rows, in that cell-nets; these, however, are not isolated, but remain joined and produce small, wonderfullyarranged structures. The result of this modelling process is therefore a plantmember composed of varied cell-complexes, with a definite internal structure, with definite external contour, and also with entirely definite functions in the economy of the plant. In spite of the variety of shape which plant-members, formed from various constituent cell-tissues, exhibit in the many thousands of plantspecies which develop into bodies, they can yet be referred to a few fundamental forms, viz., to the leaf, stem, and root. These members of the plant-body are in
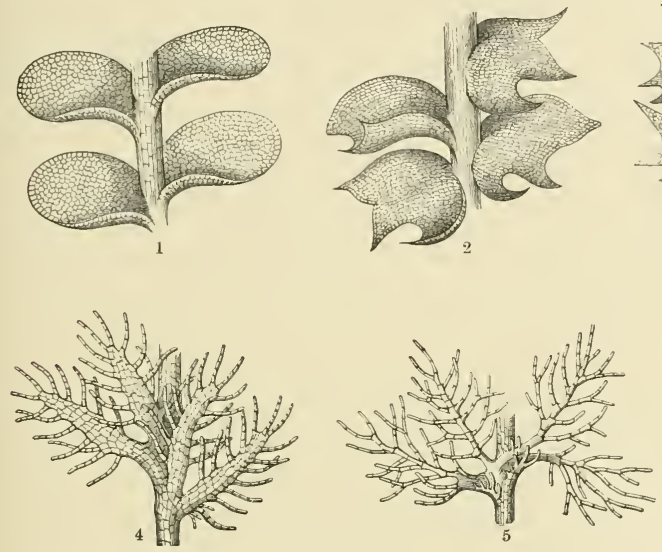

Fig. 140.-Liverworts with Cell-nets, Cell-plates and Cell-rows in various transitional forms.

I Jungermannia pumila. 2 Jungermannia quinquedentata. 3 Polyotus magellanicus. \& Ptilidium ciliare. 5 Trichocolea tomentella. 6 Jungermannia trichophylla. (All the figures magnified.)

most cases so arranged that a stem represents the starting-point and support of many leaves and roots. In the simplest form the plant-body appears as embryo and as bud. The latter consists of a very short stem, beset with leaves lying closely above one another, and grows later into a shoot which agrees in structure with the parent plant producing the bud, of which it actually forms a replica. If the young body remain connected with the old, it is called a branch; the branches may again form buds, and these, again, twigs; and in this way originate much-branched plant-structures which often attain to considerable dimensions, and must be regarded as compound. In rare instances the laterally-inserted buds are detached from the body producing them, before they develop further; and these buds, which are known as bulbils, give rise to an independent plant-body. This process reminds one of the swarm formation of cell-complexes which has been spoken of above.

This is also the place to notice the analogy' between vegetable and animal 
bodies. In polyp-colonies, the individual polyps formed by budding remain in connection with the parent-animal, and behave accordingly like the branches of a compound plant-body. Yet between the parts there exists this remarkable reciprocal relation, that the digestive cavities of individual polyps communicate with one another, and that the liquids acquired by the individuals are at the common service of the colony. This connection of the individual parts by communicating, sap-conducting channels also exists in plant-bodies. We call these conducting channels vascular bundles, and have already had repeated occasion to speak of them. They are a peculiarity of plant-bodies, and are absent in all other forms of cell-unions, even in mixed systems, many of which have a great resemblance to true plant-bodies, as, for example, the mosses. The difference existing in this respect was the reason for placing the plants in two great divisions in respect of their construction-into (1) the group of those in which vascular bundles are present as architectural elements in their bodies, and (2) that in which this form of cell-system is absent. The former, which are called vascular plants, form a natural group; the latter, which are called Thallophytes, are, on the other hand, classed quite unsuitably. By "thallus" we understand the most different vegetable structures which are devoid of vascular bundles, that is to say, not only all possible tissues and systems, but also the masses of myxomycetes, even the colonies and swarms of unicellular plants, structures which could not differ more widely in constitution.

It is a remarkable phenomenon that the majority of aquatic plants are aevoid of vascular bundles, and therefore, according to the older signification, belong to the Thallophytes; and, on the other hand, that those plants which have assumed the shape of plant-bodies with vascular bundles, belong almost entirely to land-plants. This difference can be more accurately formulated as follows:-plants which throughout their life, or at the time when they absorb nourishment, are surrounded by water, saprophytes which are wholly imbedded in humus, and parasites which are situated entirely within their hosts, absorb nourishment by the whole of their superficial cells. Such structures do not require common sap-conducting mechanisms, penetrating and connecting the several members. Those plants, on the other hand, whose shoots are surrounded by air; which derive their fluid food from the soil, and have to conduct it to the aërial organs, especially the leaves; which finally conduct to the growing parts in a fluid form the organic compounds manufactured in the green tissues in sunlight; such plants require special transmitting mechanisms, and as such, vascular bundles are developed in all land-plants. It is necessary for the stability of the conducting mechanisms that the cells and vessels in question should be lignified, or that so-called mechanical cells, i.e. hard bast, should be placed near or in contact with them. Thus it is again made evident that there is a difference between water-plants and land-plants in the matter of rigidity. The numerous submerged plants do not possess woody and bast cells, while these are always abundantly developed in land-plants, and to a greater extent the more the plant in question requires in its natural habitat to 
resist strains and bending pressures. Just as we distinguish soft, pulpy animals from such as are provided with skeletons, so also we distinguish soft plants, without wood and hard bast, from hard plants possessing these tissues. I would only point out this analogy in passing, and avoid entering into any further discussion upon it lest thereby misconceptions might arise. In discussing the hypotheses relating to the history of development of the whole vegetable kingdom in the second volume, I shall take the opportunity to return to these analogies, as well as to the relation of the habitat to the structure and form of plants. There the speculations about the evolution of plants on the ground of the comparison here only indicated will receive an impartial consideration. In this place, however, such discussions would be premature, and our remarks might share the same fate as the speculations of the Nature-Philosophers of which examples were quoted on p. 13 of the Introduction.

\title{
2. FORM OF LEAF-STRUCTURES.
}

Definition and classification of Leaves.-Cotyledons.-Scale-leaves, Foliage-leaves, Floral-leaves.

\section{DEFINITION AND CLASSIFICATION OF LEAVES.}

\author{
-'T is written-"In the Beginning was the Word."- \\ Already at a stand-and how proceed! \\ Who helps me? Is the Word to have such value, \\ Impossible-if by the spirit guided. \\ Once more- "In the Beginning was the Thought." \\ Consider the line first attentively, \\ Lest hurrying on the pen outrun the meaning. \\ Is it Thought that works in all, and that makes all? \\ -It should stand rather thus:- "IN The Beginning \\ WAS THE Power." - yet even as I am writing this \\ A something warns me we cannot rest there.
}

Of this speech-which Goethe puts into the mouth of Faust-the naturalist is involuntarily reminded when he attempts to explain terms which popular language from time immemorial has associated with certain ideas. These terms have later gained admission into scientific terminology, and here, once adopted, have gradually been employed to indicate things which no longer correspond to the original current notions. Whosoever introduced into common language the words "leaf", "stem", and "root", little suspected how difficult it would come to be, to say, shortly and exclusively, what botanists mean by these designations-to write down what the man of science understands by a leaf, a stem, a root; nor did he surmise that over the question as to whether or not certain plant-structures should be regarded as leaves, and should be so named, continuous eager strife would rage amongst the learned, and that the polemic writings on this matter, if carefully collected, would fill a book much more extensive than the present one, in which I am attempting to describe the life of the whole Vegetable World. 
When a botanist of the 16 th or 17 th century used the word "leaf" in describing plants, it was exclusively in the sense of the popular acceptation of that term. $\mathrm{He}$ understood by "leaf", a flattened outspread structure, such as appears on the branches of trees as a foliage-leaf, green in colour, or, as a floral-leaf, adorned with red, blue, and other colours. Not until the 18th century, and in great part through the influence of Goethe's Essay on Metamorphosis ( $c f$. p. 10), did botanists apply the term "leaf" also to the thick fleshy scales of bulbs, to the scales of winter-buds, to many spines and tendrils, to stamens, and to parts of the fruit-capsule. The causes of the movement in this direction were threefold. First, the wish to collect the extremely manifold phenomena synoptically; the struggle to find a simple general law of nature to which the shapes of innumerable single living organisms would conform; further, the similarity of origin-the agreement actually observed over and over again in the earliest stages of development of structures which afterwards become so different; and, finally, the circumstance that occasionally under abnormal external influences, viz., under the influence of mites, plant-lice, and other animals, green leaves are actually formed from the spines, tendrils, stamens, and fruitcapsules. Now, an original or fundamental type of leaf was imagined, of which naturally the shape of the ordinary green foliage-leaf became a standard of comparison. It was represented that the other structures enumerated, which do not agree in their shape, although they agree in their origin with the green leaves, had been produced from these by modification, and that they also must be regarded as leaves, of course as changed or metamorphosed leaves. According to this view, the bulb-scales, the stamens, and the parts of the fruit-capsule are metamorphosed leaves, although they do not correspond in their adult form to the idea of a leaf conceived by people who are not botanists.

The struggle after perfection, the gradual refinement of the sap conveyed to the leaves in their first stages, and many other things were at first supposed to be the causes of the transformations. In modern times this metamorphosis is associated with the division of labour, and with the change of function in the members of the plant-body. The green foliage-leaves effect the formation of organic materials from inorganic food in sunlight, but they are not suited at the same time for the protection of seeds or for the manufacture of pollen; nor would they be well adapted as underground storehouses for reserve materials. Consequently certain other leaves of the plant assume shapes better suited to these functions, or, in other words, they are metamorphosed to suit the particular function required of them. We, therefore, do not see green leaves, but stamens developed for the manufacture of the pollen; we do not have green, flattened, outspread foliage as a storehouse for reserve materials in the dark bosom of the earth, but thick, white, fleshy scales. The stamens manufacturing the pollen, the green leaf-blades preparing organic materials in sunlight, and organs, of one and the same plant, fitted to various other definite tasks, are so entirely similar in their origin and first stages, of development, that they are included under a common abstraction, and the word "leaf" has been employed to express it. As in a beehive the adult workers, 
the drones, and the queen are of different forms in accordance with their different tasks, as demanded by the division of labour-so the leaves, which agree in their first stages of development, exhibit, in their fully formed condition, another construction in accordance with the function assigned to them. Hence we come to this conclusion:- the variety of the tasks accomplished for the prosperity and maintenance of the whole plant, and the consequent division of labour, necessitate the metamorphosis of the leaves in each plant-body.

From what has been said it follows that a definition of the botanical leaf must be connected with the first stages of development. At the earliest stage each leaf appears as a lateral swelling or protuberance below the growing point of the stem. It arises from the peripheral portions of this region, which are still in a state of active growth. The growth of the leaf is limited, so that in respect of their development, we may say that leaves are laterally developed members of limited growth, which spring in geometrical succession from the outer layers of tissue below the growing point of the stem.

In many foliage-leaves we can plainly distinguish a plate-like, outspread, green portion, traversed by lighter veins, the blade (lamina), also a firm and stalk-like support for this blade, the leaf-stalk (petiolus), and, finally, that portion which connects the leaf-stalk with the part of the stem in question. In many plants this latter portion is widened, grooved, and occasionally provided with a membraneous border, so that the stem is then surrounded by this portion as the blade of a dagger is by the sheath. This part of the leaf has in fact been termed the sheath (vagina). Where the leaf projects from the stem there are frequently two outgrowths, one on the right, the other on the left of this sheathing portion. These have generally the form of membraneous scales (see fig. $92^{6}$ ). They are often dilated, as, for example, in the Tulip-tree (fig. 91), and usually fall off when the leaf at whose base they are inserted is fully developed. In other plants they have the form of small lobes or auricles, are coloured green, and are retained as long as the leaf remains connected with the stem. These structures are called stipules (stipulce).

Leaves in which the blade, leaf-stalk, sheath, and stipules are plainly developed, are on the whole less frequently met with than those from which one or other of these portions is absent. Often no trace is to be seen of the stipules. Sometimes only the leaf-sheath is present in the form of a concave, husk-like scale. In other instances the leaf-stalk is absent, and the blade is then situated directly on the stem (fig. 14); or it may happen that the green tissue of the blade surrounds the whole stem like a collar, so that it might be thought that the stem had been stuck through, or had grown through this leaf. If two or more of these leaves with sessile blades arise together, they may be fused into a bowl or cup, being partially or wholly united, and then again it looks as if the stem from which these leaves arise has been thrust through the middle of the united leaf-group (see fig. 56). Occasionally the green tissue of sessile leaf-blades is seen to be continued down the stem in the shape of two green bands or wings. For the forms here only very briefly described, the botanical terms are sessile leaves (folia sessilia), perfoliate 
leaves (folia perfoliata), connate leaves (folia connata), and decurrent leaves (folia decurrentia), of which terminology this explanation must be given, that in earlier, and indeed even in modern times, the leaf-blade-as the most noticeable part of the leaf-has been in describing plants, shortly called "the leaf" (folium).

The classification of leaves with regard to their point of origin from the stem is of particular importance, and in this connection we must first of all distinguish between seed-leaves and shoot-leaves. The former only occurs in the embryo, the latter in all those structures comprised under the term "shoot". The embryo which has developed from the fertilized egg-cell in the embryo-sac-in a manner which has yet to be described in detail-presents in many instances a tissue-body in which as yet no trace can be recognized of a differentiation into stem and leaf, or rather, the embryo, when it leaves the fruit-capsule, is like a stem in which all indication of leaves is absent, e.g. in several thousand orchids, the numerous Balanophoreæ and Rafflesiaceæ, species of Broom-rape (Orobanche), Winter-green (Pyrola), Bladderwort (Utricularia), Bird's-nest (Monotropa), Dodder (Cuscuta), and many other epiphytes, saprophytes, insectivorous plants and parasites, as well as many plants living together symbiotically. In the majority of instances, however, a distinct differentiation can be recognized in the embryo hidden in the seed, and one or two leaves may be seen issuing from the tissue-mass which forms the axis of the embryo. These are the seed-leaves or cotyledons. The short axis or stem-portion from which the seed-leaves originate, and which looks like the pedestal of the cotyledons, is called the hypocotyl. At one end of the hypocotyl a tissue-mass is developed, termed the radicle (radicula); at the opposite end a tissuemass named the plumule (plumula). (See figs. $141^{1}$ and $141^{2}$.) The plumule is situated above the place where the cotyledon, or pair of cotyledons, issue from the hypocotyl. It is the rudiment of a new portion of the stem, which is situated above the cotyledons, and is called the epicotyl. The epicotyl thus originates from the apex of the hypocotyl, and the boundary between these two portions of the stem is the place of origin of the cotyledon, or pair of cotyledons.

The epicotyl in the resting seed is frequently only a tiny knob or cone, on which no indications of leaves are yet to be seen. In the majority of instances, however, distinct, although as yet very small, leaflets may be found on it, and where this is not the case swellings sooner or later arise which are the leafrudiments. Each short stem-structure, with closely-crowded and overlapping leaves or leaf-rudiments, is called a bud (gemma); consequently the plumule is a bud, in fact it is the bud of the embryo, which arises from the apex of the hypocotyl. This bud, at the germination of the seed, elongates; its axis, hitherto very short, stretches; the overlapping leaflets are separated, new leaves arise under the growing-point, and so the bud develops into a structure termed a "shoot" (innovatio). The bud is accordingly the primary groundwork of a shoot, and when considering the form of a compound plant-body, special regard should always be paid to the places where the buds originate. The first bud, which is established in every plant-body at the commencement, is situated at the apex of the hypocotyl, 
close above the cotyledons. But later on buds are also developed on this primary shoot, and most usually close above the place where leaves arise from the axis of this shoot. Many of the buds elongate and themselves become shoots, and we then say the shoot has formed branches. Some of the buds, however, only undergo a slight extension, and we distinguish between long branches and short branches, to which we shall return subsequently.

Of special interest to us here are the leaves of these shoots, the whole of which are comprehended under the general term shoot-leaves. They exhibit much greater diversity in form than do cotyledons, and this is quite intelligible, since the tasks required from a shoot are much more numerous, and the allotment of various functions to the leaves inserted on the shoot at different heights necessitates a greater wealth of form. But the extraordinary abundance of shapes makes it necessary to group the shoot-leaves according to their origin, their mutual position, and their succession in time. We have long ago complied with this requirement, since we distinguish them as scale-leaves, foliage-leaves, and floral-leaves. Lowest on the shoot we see the scale-leaves. They are developed earliest, and their rudiments are frequently to be seen even in the bud from which the shoot is produced. They generally appear only as the sheathing portions of leaves-as scales devoid of chlorophyll, and exhibit relatively small dimensions. Following these scale-leaves further up the shoot are the foliage-leaves; these arise later, are larger in size, and generally developed with green laminæ directed towards the sun's rays as foliage. Finally, above these, the floral-leaves, which form the termination of the series of leaves growing on a shoot, and take part either directly or indirectly in the production and union of the sexual cells. One and the same shoot does not always bear the three kinds of leaf-structures one above the other at the same time. There are some plants whose shoots never bear foliage-leaves, and it is a very common occurrence for a compound plant-body to develop no floral-leaves on one shoot, and no foliage-leaves on another, while in the Lathrophytum Peckoltii, one of the Balanophoreæ described on p. 196, only floral-leaves are formed, and neither a foliage-leaf nor a scale-leaf has ever been seen on this plant.

The leaves, hitherto distinguished only with regard to their succession in age, relative position, and insertion on the stem, must now also be described in connection with the shapes which they assume and the functions which they fulfil. Touching this I cherish the conviction that the special form is always correlated with a special function, and that the recognition of the relation of shape to the performance of work is the highest problem of the science of plants. 


\section{COTYLEDONS.}

The cotyledons or seed-leaves are borne by the embryonic stem. Their function in the first instance is to provide this organ, as well as the rudiments of the radicle at the one end and the small bud at the other, with food. - These portions of the embryo as long as they are still surrounded by the skin-like envelope of the seed-the so-called "seed-coat"-and even still later, when they have burst through these envelopes, cannot at once absorb inorganic food from their environment, and still less can they transform this into organic materials. And yet they require these substances for growth, that is to say, they require materials for the building of the foundation of the plant-body which is to issue from the seed. Only when the radicle has penetrated into the soil and produced its root-hairs, and green leaves have forced their way to the sunlight from the little bud which formed the rudiment of the epicotyl, is the young, newly-settled plant placed on its own feet; henceforth it can nourish itself independently. But up to the moment of this independence it draws its food from a store which is deposited in the seed; it lives on materials derived from the mother-plant, i.e. on a supply of starch, fat, and proteid formed by the parent and deposited in special cells for the benefit of the embryo. A fully-equipped embryo is provided with food reservoirs in either of two ways. Sometimes the cotyledons themselves form the storehouse for the food to be consumed later on. In this case the reserve materials are deposited by the parent plant in the cell-chambers of the cotyledon, and when the suitable time arrives, and when the need for them has arisen, these materials are employed in the further construction of the hypocotyl, and of the radicle, springing from one end of it, and of the bud at the other end. In the second case a special storehouse is formed within the enveloping seed-coat beside the embryo. The cells of this storehouse are quite filled with fat and starch and proteid granules. The tissue of this particular store-chamber of the embryo is in most instances composed of cells which have arisen, together with the germ-cell, in the so-called embryo-sac (the large cell in which the egg is produced), and it is then termed endosperm. Less frequently this tissue is formed outside the embryo-sac, in the nucellus, and is then called perisperm. This distinction is without significance in the processes here to be discussed, and therefore in the following description, endosperm and perisperm are included under the term reservetissue.

When the cotyledons themselves form the reserve-tissue, the maintenance of the young plant is relatively simple. The transformation and transportation of the reserve-materials are carried on in the manner already described. In proportion as the radicle of the embryo develops into the root, and a leafy shoot is produced from the embryonic bud at the cost of the building materials conveyed to them, the cells of the cotyledons lose their store of food materials, 
and their rôle of nurse is at an end. Often, indeed, the cotyledons take on later another function, but they have ceased to be of importance as reservetissue for the use of the developing embryo. In those instances where the supply of food given by the parent plant is not stored up in the cotyledons,
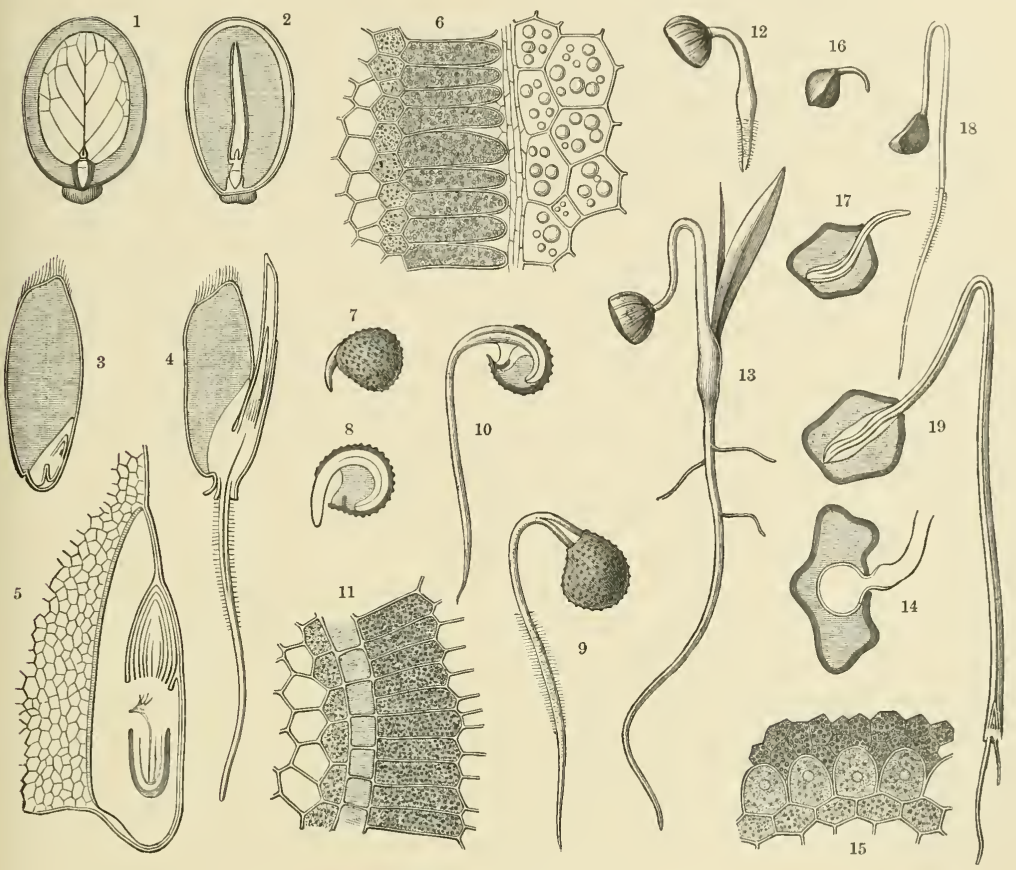

Fig. 141.-Cotyledons.

1 Longitudinal section of seed of Ricinus, parellel to the plane of the embryo. 2 Longitudinal section of the same seed, taken at right angles to the two parallel cotyledons. ' 3 Longitudinal section through a grain of Wheat (Triticum vulgare); $\times 4$. 4 Longitudinal section through a grain of Wheat after germination has taken place; $\times 4$. 5 The embryo with its scutellum in a grain of Wheat; $\times 80 .{ }^{6}$ Absorbent cells on the surface of the scutellum of a grain of Wheat; $\times 210.7$ Germinating seed of the Corn-cockle (Agrostemma Githago); slightly magnified. \& Longitudinal section of the same. 9 Seedling of the Corn-cockle in a later stage of development. 10 The same in longitudinal section. 11 Absorbent cells on the surface of the cotyledon adjoining the reserve-tissue in the seed of the Corn-cockle; $\times 210.12$ Germinating seed of Tradescantia Virginica; slightly magnified. 13 The same in a later stage of development. 14 Transverse section through the knoblike end of the cotyledon of Tradescantia Virginica embedded in the reserve-tissue; $\times 10 .{ }^{15}$ Absorbent cells on the surface of this knob-like end; $\times 180.16$ Germinating seed of the Onion (Allium Cepa); natural size. 17 The same cut in half; slightly magnified. ${ }^{18}$ Seedling of the Onion in a later stage of development; natural size. ${ }^{19}$ The same cut through longitudinally; slightly magnified.

but is deposited as a special reserve near the embryo, its nourishment is more complicated.

In this state of affairs the cotyledons have an essentially different function. They serve as agents, and their first task is to take up the building-materials. These have been liquefied in the reserve-tissue, either by changes in that tissue itself, or by the direct solvent action of the cotyledons. They are then conducted 
to the growing parts of the embryo. In order to do this, it is necessary that those cells of the cotyledon which adjoin the special reserve-tissue should have the power of absorbing organic compounds from it, and of leading them away. The cotyledons in this respect resemble the suckers of parasites, and, like these, are provided with absorbent cells. In many species, e.g. in the Corn-cockle (see fig. 141 ${ }^{11}$ ) they remain short, form a continuous cell-layer which borders on the special reserve-tissue, and remind one of the absorbent cells of the Bird's Nest (fig. $16^{2}$ ); in others, as, for example, in Tradescantia (see fig. $141^{15}$ ), they appear as papillæ, slightly separated from one another at the sides, and resembling the absorbent cells of a Gentian root ( $c f$. fig. $16^{1}$ ); again, in other instances, as, for example, in the Wheat (fig. $141^{6}$ ), they increase, at the time of absorption, to ten or twelve times their previous length, and then their side-walls separate from one another so that they are comparable to the absorbent cells of Cuscuta (fig. $35^{2}$ ). If the embryo is entirely embedded in the special reserve-tissue, it may happen that all its superficial cells in contact with the food-containing tissue, and not only those on the exterior of the cotyledons, act as absorbent cells. If, on the other hand, the embryo only adjoins the reserve-tissue on one side, the absorbent cells also are only developed on this side. The embryo of the Corn-cockle, which is bent like a horse-shoe around the special reserve-tissue (fig. $141^{8}$ ), exhibits, for example, absorbent cells only on the lower side of one of its two cotyledons, which is directed towards the middle of the seed. Frequently only a very small part of the cotyledon possesses absorbent cells adjoining the reserve-tissue, as, for example, in the Onion, where only the end of the cotyledon bears absorbent cells (figs. 141 17, 18, 19); or in Tradescantia, where the end of the cotyledon presents a knob-like absorbent tubercle (fig. $141^{14}$ ). It is worthy of notice that in many instances where the reserve-tissue is ample and the embryo very small, the extent of the absorbent surface of the cotyledon becomes enlarged during germination. As the reserve materials are absorbed, and the exhausted reserve-tissue shrinks, the absorbing portion of the cotyledon advances. The knob-like termination of the cotyledon of Tradescantia, originally of small dimensions, becomes larger in proportion as the reserve-tissue diminishes. The absorbent, hollow, conical or inflated end of the cotyledons of many palms, e.g. of the Date and Cocoa-nut Palms, increases in proportion as the reserve-tissue diminishes, presses forwards just as far as the tissue to be absorbed shrinks back, and occupies the space vacated by it (figs. $144^{9}$ and $\left.144^{10}\right)$. A similar relation is to be seen in rushes and sedges. In the embryo of Coffee and Ivy seeds, the cotyledons are at first very small, but grow further and further into the reserve-tissue during the process of germination, till they gradually fill up the whole space in the seed. The cotyledons of umbelliferous plants also behave in a very characteristic manner. The small embryo lies in the seed at the base of the reserve-tissue, and its minute cotyledons project into a space occupied by empty cells, which are however, surrounded by the well-filled cells of the reserve-tissue. Now when 
germination commences the two cotyledons grow in length, penetrate through this loose central cell-layer and attach themselves to the reserve-tissue which has to be absorbed.

On the whole it may be taken as correct that the surface of contact between the absorbent part and that which has to be absorbed is greater the quicker the absorption has to be accomplished, on account of the local climatic conditions. Starch is best suited for rapid liquefaction and absorption; fat takes much longer to become changed into a form adapted for absorption; and the transformation of layers of cellulose requires a still longer time. In accordance with this the embryo comes into contact with the reserve-tissue whose cells are crowded with starch (as, for example, in the seeds of pinks, oraches, polygonums, and grasses), presenting a broad surface, or else wrapped with its long cotyledons around the tissue either horse-shoe-wise or spirally. On the other hand, in plants whose special reserve-tissue is principally filled with fat, the surface in contact is much smaller, and the seeds of those plants whose reservefood consists chiefly of cellulose, e.g. those of the Date, usually exhibit only a very limited area of contact between the cotyledon and the reserve-tissue. But in these latter the liquefaction and absorption continue for months, while the same processes in the starchy seeds of grasses and oraches are completed in a few days.

In addition to this first task of the cotyledons, which we have just described, in many instances we have a second function, viz. the extrusion of the hypocotyl and its crowning bud from the interior of the seed-coats. After the formation of the embryo from the parent plant, it remains quiescent for a time, and during this period appears to be protected in the most diverse ways by coverings against the external dangers that might threaten its existence. When a special reserve-tissue is present, the embryo is frequently found embedded in the centre of it, or hidden in its folds. The reserve-tissue is often horn-like, or as hard as bone, as, for example, in the seeds of Date and Coffee, and therefore an excellent protection is provided by this tissue for the dormant embryo. In any case the embryo is surrounded by the seed-eoat, which may consist of a single or a double layer. In very many plants the seed is also walled in by a non-dehiscent pericarp (or fruit-covering) and occasionally by other structures. The seed-coat forms an envelope which allows of the entrance of moisture into the interior only by a very restricted opening. It is not flexible or extensible to a great degree, and consequently if the contents swell up and the growth of the embryo begins, then the portion of the embryo designed for further development must either find an exit through the abovementioned aperture or else it must burst through the husk; or both kinds of escape may occur together.

This process, in which the cotyledons take a very prominent part, is carried on in a manner defined for every species, but in different species by an incalculable variety of methods. Occasionally larger alliances of the vegetable kingdom 
exhibit a remarkable agreement, but it also happens that even closely related species of one and the same genus differ considerably with respect to the liberation of the embryonic plant from the bondage of the seed-coat. That some idea may be conveyed of the methods obtaining at germination, eight different cases will be described.

Let us begin with one of the most remarkable cases, viz. with the germination of mangroves, which grow in extensive forests on the tidal swamps of tropical coasts.

The species which I select as example, and whose whole process of development is clearly shown in the figures opposite, is called Rhizophora conjugata. A longitudinal section through the pendent flower of this species (figs. $142^{1,2,3,4}$ ) exhibits two compartments of equal size in the ovary, and in each compartment is discovered the commencement of a seed. After fertilization the corolla and stamens fall off; the calyx remains, and the much-enlarged ovary assumes the form of a stunted cone, whose apex bears two stigmas, now transformed into shrivelled points. If the ovary is cut through longitudinally at this stage of development, it may be seen that one compartment (fig. $142^{5}$ ) with its young seed is atrophied, and the other with its seed has widened and enlarged very much. Within the young seed (which is attached to one side of the originally central wall of the ovary) an embryo can now be plainly distinguished, surrounded by its reserve tissue. Together they fill the egg-shaped cavity, open below, formed by the thick seed-coat. The embryo consists of the hypocotyl, whose free end is directed downwards, that is, towards the point of the pendent ovary, and the cotyledon which forms the upper termination, tubular below, and above not unlike a Phrygian cap. The cotyledon covers like an inverted bell the embryonic bud, which is inserted upon the apex of the hypocotyl. In the lower tubular portion of the cotyledon are numerous vascular bundles which pass down into the hypocotyl and supply it with food. A true radicle is not developed at the lower end of the hypocotyl, and that which was formerly regarded as a root may be more accurately interpreted as the hypocotyl itself. Strangely enough, the fruits of mangroves do not become detached from the branches after the formation of the embryo; nor do they dehisce to allow the seeds to fall out. On the contrary, these germinate while still inclosed in the fruit hanging on the tree. The embryo develops within the seed-coat at the cost of the reserve-food in which it is embedded, absorbing this nourishment by means of the cotyledon. The whole of the exterior of the upper portion of the cotyledon is covered with absorbent cells, and the materials drawn by these cells from the surrounding slimy, gelatinous mass are conducted by the aforesaid vascular bundles to the hypocotyl. Since, in spite of this, the amount of the food stored up does not diminish, and since it is not proportioned to the size of the growing embryo, it may be safely concluded that whatever food is absorbed by the cotyledon, and employed for the growth of the hypocotyl, is continuously replaced by the parent plant.

When the hypocotyl has attained a length of 2 centimetres, the tubular portion 
of the cotyledon also extends and pushes the hypocotyl in front of it until the apex has bored its way through the wall of the fruit and come out into the daylight (see figs. $142^{3,4,5,6}$ ). The hypocotyl now elongates in a month to about $4 \mathrm{~cm}$., and in from 7 to 9 months attains a length of $30-50 \mathrm{~cm}$., and from $1-5 \mathrm{~cm}$.
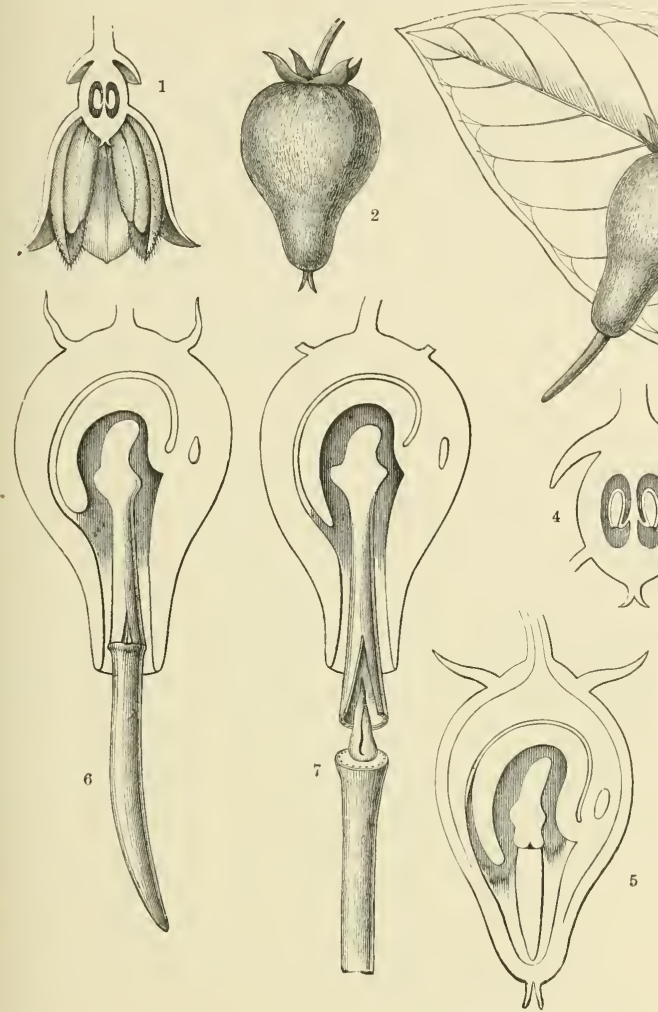

Fig. 142. - Rhizophora conjugata.

1 Flower, cut in half longitudinally. 2 Fruit. 3 Twig with two fruits, whose conical ends have been broken through by the pressure of the elongating hypocotyis. 4 Longitudinal section through the ovary; about twice the natural size. 5 Longitudinal section through a fruit; the cap-like cotyledon surrounded by reserve-tissue; the lower end of the hypocotyl having grown through the seed-coat has reached the lower hollow conical apex of the pericarp. ' Longitudinal section through a fruit two months later; the tubular sheath of the cotyledon has elongated and pushed the hypocotyl quite out of the pericarp. 7 Longitudinal section through a fruit eight months later. The hypocotyl is separating from the tubular portion of the cotyledon. 8 Part of the same; slightly magnifled. ${ }^{9}$ Upper end of the hypocotyl with the embryonic bud. The two lowest leaves of the bud are expanding, the two upper are still folded together.

in thickness. It is thickest in its lower third, and is there slightly curved. Its weight now amounts to almost 80 grammes. These long heavy hypocotyls projecting from the fruits sway to and fro with every breath of wind. At length the vascular bundles, by which the connection with the tubular portion of the cotyledon was retained, are ruptured (see figs. $142^{7}$ and $142^{8}$ ). The embryo falls away, and 
its lower end bores deeply into the mud. Even a layer of water half a metre deep is pierced by it with such force that it remains standing upright in the mud beneath. After a few days the pericarp, with the cotyledon inside, is also detached. At the upper end of the fallen hypocotyl the bud which was formerly covered over by the tubular cotyledon is now to be seen. The four small green scale-leaves of this bud only increase slightly in length; but immediately, from the shoot arising from it, large elliptical shiny green leaves are developed which become active as foliage; whilst from the lower end of the hypocotyl which has bored into the mud, as well as from the epicotyl itself, roots arise which are at once the means of fixing the plant in the muddy shore, and of conducting foodsalts to it. In the neighbourhood of old mangrove trees, dozens of these young plants may be seen, which have fallen and bored their way into the mud; and on the shoots produced from their upper ends sometimes only scale-leaves, and sometimes foliage-leaves are developed. The illustration opposite, taken from a sketch near Goa, on the coast of Bombay, drawn from nature by Ransonnet, shows all this very clearly.

The second form of cotyledon to be brought forward is that which occurs in grasses, and is called by botanists the scutellum. Although variously modified, it is in the main developed similarly in the many thousands of different species. The small embryo of the grass is in lateral contact with one end of the large starchy reserve-tissue, by means of its cotyledon, as shown in the grain of wheat chosen as type (see figs. $141^{4}$ and $141^{5}$ ). The free edges of the cotyledon arch over the embryo bud, sometimes actually curling round it, forming a sheath-like envelope. Below, the cotyledon is continued into a sac which incloses the radicle of the embryo. When the materials are conveyed from the reserve-tissue to the hypocotyl, radicle, and embryo-bud, by means of the absorbent cells of the cotyledon described on p. 600, these portions quickly increase in length. The radicle pierces the sac-like envelope, penetrates into the ground, and unites by abundant roothairs with the particles of the soil. The bud also elongates and the leaves grow up into the light from the sheath-like envelope of the cotyledon. The lower leaves are usually scale-leaves without green blades, but the leaves following these all exhibit large green laminæ which function as foliage. The starch of the reservoir is soon completely consumed in the rapid growth of the embryo. As soon as this has happened the cotyledon has no further task to fulfil, it shrivels and perishes, but the young grass-plant with its roots and its green foliage-leaves is now in a position to manufacture for itself the substances necessary for its further construction.

The third form of cotyledon is shown in the embryos of sedges and rushes, of irises, snowdrops, narcissus, aloes, and butcher's-broom, of flowering rushes, bananas, and palms, and numerous other plants belonging to the class of monocotyledons. In all these plants the embryo is embedded in the reserve-tissue of the seed, and the cotyledon proceeding from the hypocotyl forms a sheath surrounding the bud situated upon it. The cotyledon is provided with absorbent cells only at 
its apex, and is connected with the cells of the reserve-tissue at that point. In germination the cotyledon increases in length and pushes the hypocotyl with the embryonic bud and radicle out of the seed. The food absorbed from the reservetissue by the remaining portion of the cotyledon is conducted from the interior of the seed to the extruded embryo by the lengthened part of the cotyledon. With

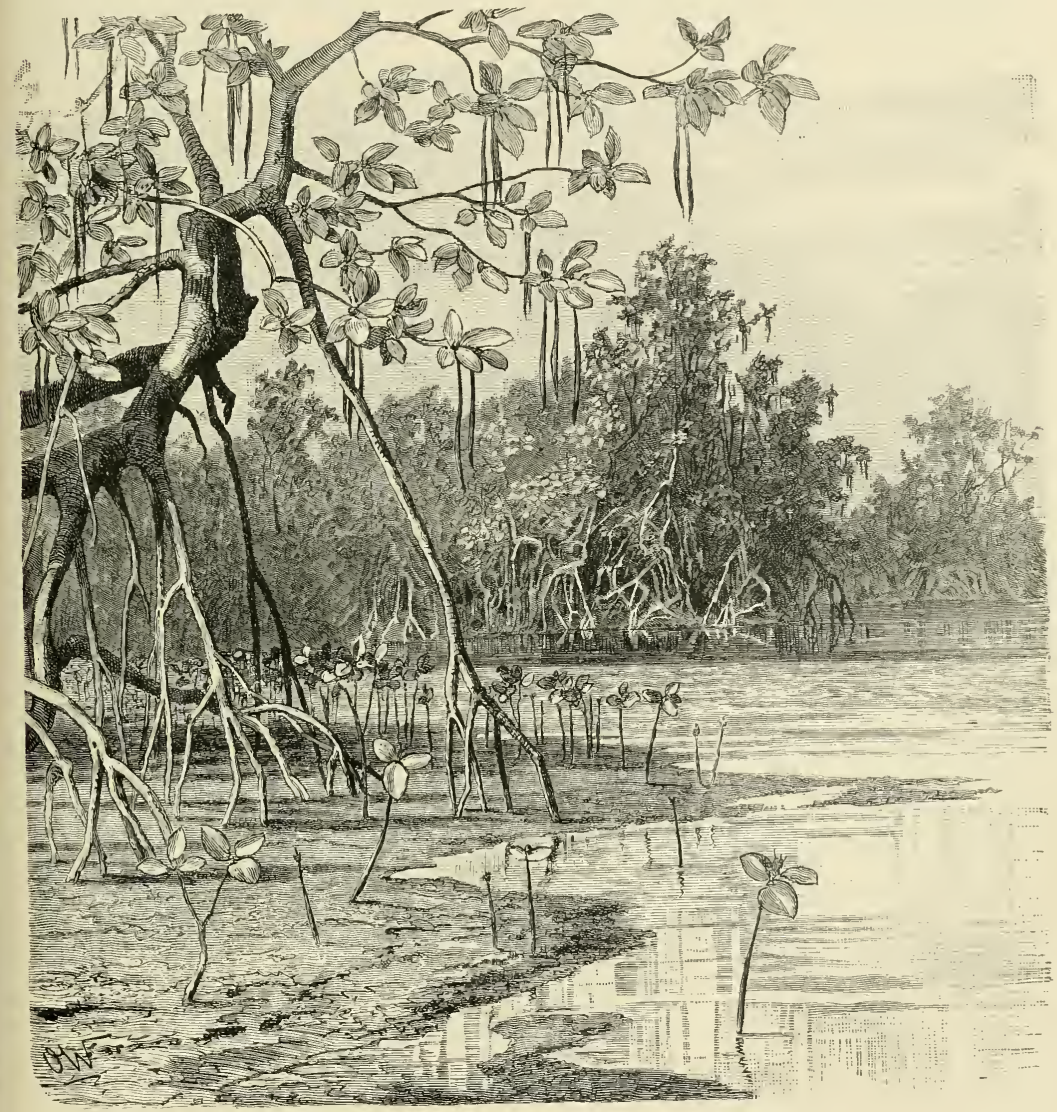

Fig. 143. - Mangroves near Goa on the West Coast of India at ebb-tide.

the help of food thus conveyed to it, the embryo is enabled to develop its radicle into an absorbent root penetrating into the ground, and also to develop its leafrudiments into green leaves. Numerous modifications of the process here only sketched quite generally may be distinguished, and these consist chiefly in the varying direction and length of the portions of the cotyledon thrust out from the seed. In sedges, rushes, and cyperuses germinating in marshy ground, or eveu 
in the mud under water, the extruded portion of the cotyledon surrounding the embryonic stem, with its bud and first shoot-leaves, becomes bent upwards after it has issued from the interior of the seed (see figs. $144^{14}$ and $144^{15}$ ), while in species of $Y u c c a$ and Tradescantia it grows downward in an arch (see fig. 14.19); and in cycads and palms, growing in soil exposed superficially to drought, it bends round immediately after its exit from the seed, and penetrates vertically into the deeper layers of earth which are always somewhat moist (see figs. 144, 9, 10.). In the Areca-palm and the slender Chamcedorea the sheath-like extruded portion of the cotyledon is very short, while in the Commelynacee it is much elongated, so much, indeed, that it looks as if the sheath-like portion surrounding the hypocotyl and the bud were connected by a long thread with the absorbent portion which remains behind in the seed. This central portion of the cotyledon is also much elongated in the Date palm and in the Cocoa-nut palm, as well as in the cycads Zamia, Ceratozamia, Encephalartos. The figs. 7, 8, 9, 10 of the illustration opposite show all the stages of development in the Date seedling. As long as the cotyledon has not pushed out from the interior of the seed, it forms a mantlelike envelope for the bud of the hypocotyl, and is continued into a sac-like covering for the radicle. At germination the cotyledon increases much in length; the free end is sheath-like, the middle portion forms a stalk-like, rolled-up structure, and the part remaining behind in the seed forms a hollow cone which becomes dilated like a vesicle where absorption of the reserve materials occurs (figs. $144^{9}$ and $144{ }^{10}$ ). In a still later stage the radicle develops into a root, and breaks through its sac-like covering, while the scale-leaves of the epicotyl stretch, and push their way out of the cotyledonary sheath (fig. $144^{8}$ ). Gardeners employ what they call a "dibble", a tool by the help of which the seeds and seedlings are planted in a suitable depth of earth. One is involuntarily reminded of these dibbles in observing how the tubular, rolled, stalk-like cotyledon-sheath-which grows out of the seed-not only pushes the embryo out of the interior, but presses it deeper and deeper into a layer of earth which by its depth is protected from drying up; there it is planted in a suitable place-actually in the most favourable position. In many palms the cotyledonary sheath is half a metre long, and many months pass before all the reserve-materials of the gigantic seed, often weighing as much as 8 kilograms, are conducted by this sheath to the embryo planted below.

Numerous species of Onion (Allium), and of Reed-mace (Typha) exhibit our fourth form of cotyledon. The extrusion of the embryo by the cotyledon is conducted in the same way as in the type just described, but there is this essential difference, that here the cotyledon, after it has absorbed the reservematerials of the seed by its apex, entirely vacates the cavity of the seed-coat, becomes green, and then acts like a foliage-leaf. In the seed of the Garlic (Allium sativum) the embryo is embedded in the centre of the reserve ( $c f$. fig. $141{ }^{17}$ ). As soon as germination begins, the cotyledon pushes its way out of the seed-coat, and grows first upwards, then bending round at an angle, so that the extruded end surrounding the hypocotyl and the bud, comes to lie below the 
level of the seed (figs. $141^{18}$ and $141^{19}$ ). Here long root-fibres develop from the radicle and from the base of the hypocotyl; these burst through the cotyledon, grow down into deeper layers of earth, and fix the young plants in the spot where the cotyledon has placed it. The apex of the cotyledon remains in the

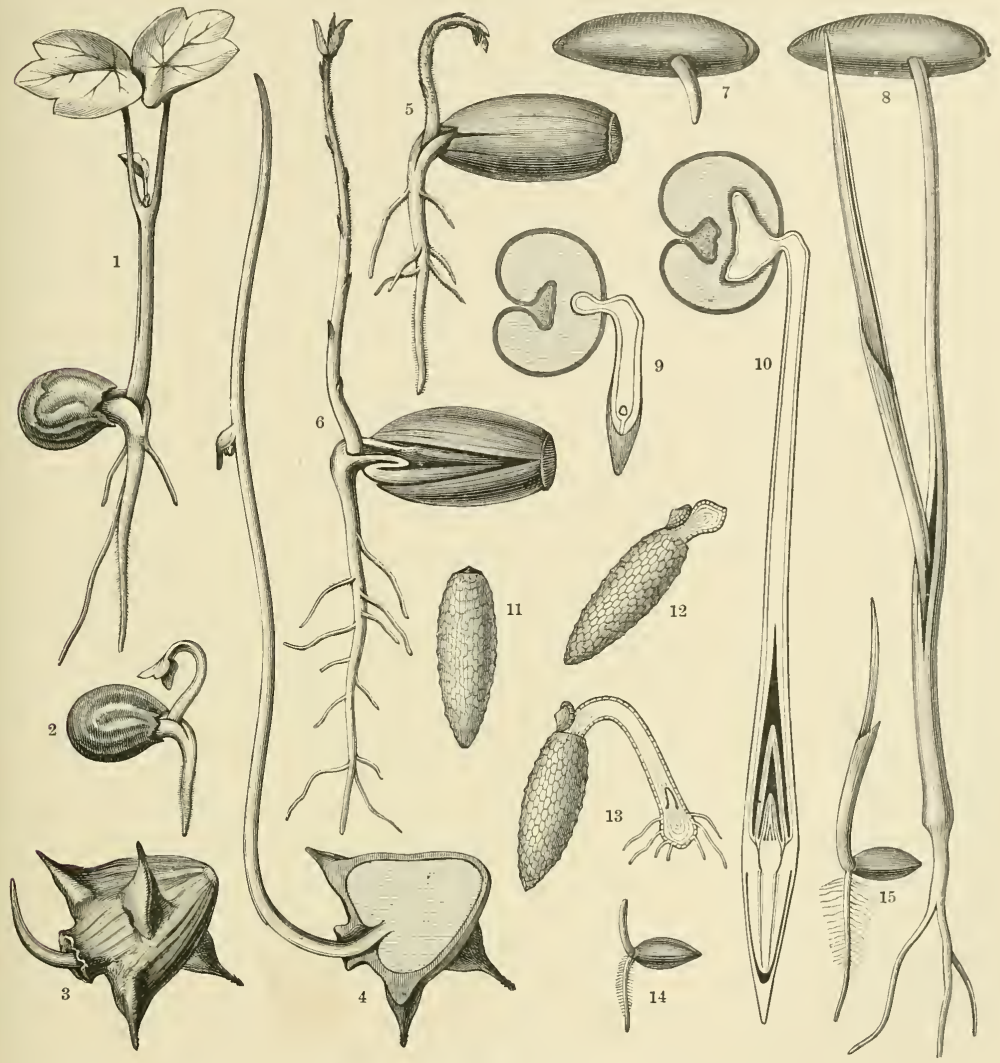

Fig. 144.-Germinating Seeds and Seedlings.

1 Seedling of the Nasturtium (Tropoeolum majus). 2 The same at an earlier stage of development. 8 Water Chestnut (Trapo natans), from which the embryo is emerging. 4 Later stage of development. ${ }^{6}$ Young seedling of the Austrian 0ak (Quercus A ustriaca). 6 The same, further developed. 7 Seed of the Date (Phonix dactylifera) from which the embryo is emerging. 8 The same eight weeks later, after the seedling has already developed root and scale-leaves. 9 Young Date in longitudinal section. 10 Older Date in longitudinal section. 11 Seed of the Reed-mace Typha Shuttleworthii. 12 The same with protruding embryo. 13 The same at a later stage of development. 14, 15 Seedling of the Sedge Carex vulgaris. Fig. $1-8$, natural size; $9,10, \times 8 ; 11-13, \times 4 ; 14,15, \times 6$.

seed, and here absorbs the last remnants of the reserve-materials. When these are at last exhausted, one limb of the bent cotyledon grows upwards, and its apex is drawn out from the emptied seed-coat. All this occurs underground. Now the cotyledon also has to reach the sunlight and become green. This is 
brought about by the knee of the upwardly-growing cotyledon acting like a wedge, and thus making a path upwards through the ground. This penetration of the ground is materially assisted by the presence of cells on the convex side of the knee which, unlike the other superficial cells of the cotyledon, are somewhat curved outwardly, and highly turgescent-a contrivance which will be described more in detail later on. When finally the free end of the cotyledon has been drawn out of the ground, the knee-shaped bend is obliterated as the green cotyledon straightens out.

The germination of the Reed-mace (Typha) is quite peculiar. The small fruits which are blown off the spike, fall on to the surface of the water and remain floating for some days. Then the pericarp opens and the seed sinks slowly down into the water. The husk of the seed is pointed at one end, and at the other is closed by an extremely pretty trap-door (cf. fig. $\left.144^{11}\right)$. While sinking through the water the pointed end is turned downwards, and the covered end upwards. At the bottom the seed lies in the position indicated and germination commences. The cotyledon grows in length, pushes open the trap-door, and makes its appearance at the mouth of the seed-coat (fig. 144 ${ }^{12}$ ). It now describes an arch and the end in which are concealed the hypocotyl and the bud reaches the mud. Scarcely has it done so, however, when its epidermal cells elongate and form long tubular structures which penetrate into the slime, and the free end of the cotyledon is thus firmly fixed (fig. $1444^{13}$ ). Later on rootlets make their appearance, which, proceeding from the hypocotyl, break through the unresisting cotyledon. Meanwhile the reserve food has been sucked up by the apex of the cotyledon which remained in the seed; this apex is now drawn out of the seed-coat, the cotyledon straightens itself, turns green, and functions as a foliage-leaf.

In the four cases just described the embryo only possesses one cotyledon, and each seed contains a reserve-tissue beside the embryo. In the fifth case now to be described, however, the embryo is equipped with two cotyledons, and the building materials which are at its disposal for the first period of growth are stored up in the embryo itself, almost entirely indeed in the cotyledons. To this class belong plants with stone-fruits as well as most species with seeds and fruits of nut-like appearance, and many the seeds of which have a softer, leathery covering. As examples may be named the Walnut and Hazel, the Oak, Chestnut and Horse-chestnut, the Almond, Cherry, Apricot, and Peach, the Laurel and Pistachio-nut; the Nasturtium (Tropcolum), Broad-bean, the Scammony (Cynanchum), and the Bastard-Balm (Melittis). The two leaves proceeding from the hypocotyl almost completely fill the space inclosed by the seedcoats in all these plants; and the small embryonic bud as well as the radicle are situated between the two cotyledons, just like a dried plant between the sheets of paper in a herbarium. The cotyledons are thick, swollen, and tense, of fleshy appearance in section, and always comparatively heavy. Many of them are wavy, and they rarely look leaf-like. Occasionally the two cotyledons are fused together 
by their adjacent surfaces, as, for example, in the Chestnut, Horse-chestnut, and Nasturtiums. Everything which one is generally accustomed to consider an attribute of a leaf is entirely wanting. When these seeds take up water from the environment and begin to germinate and grow, first of all the seedcoat bursts at one end of the seed, and the radicle together with the axis and also the thick stalks of the two cotyledons are extruded through the rupture. The cotyledons themselves, however, remain inside, lose weight in proportion as they give up materials to the growing parts, dwindle, and finally appear quite shrivelled and emptied. The extruded radicle has, on the contrary, visibly increased, it bends downwards, penetrates vertically into the ground, and produces lateral roots with root-hairs, which now absorb nourishment from the soil. The bud which was hemmed in between the short thick stalks of the two cotyledons has, on the other hand, curved upwards, elongated pretty quickly, and become a shoot which in the Nasturtium immediately develops green, lobed foliage-leaves. In other plants, e.g. in the Oak, first scale-leaves appear and then green foliageleaves above them. In fig. $144^{1,2,5,6}$ these conditions are depicted both in the Nasturtium and the Oak. The cotyledons have here a threefold part to play; first of all they function as storehouses for reserve materials, and at the same time as protecting envelopes for the small squeezed rudiment of the future plant; in addition they also have the task of thrusting the embryo out of the cavity of the seed so far that its members can elongate as required-some towards the light, and some into the dark ground. When they have performed these duties they die off and disintegrate so completely that at the place where they were connected with the hypocotyl, scarcely a trace of their insertion is to be recognized.

A peculiar condition of the cotyledons, the sixth in the series here described, is observed in the Water Chestnut (Trapa). One of the cotyledons is small and scale-like, containing no reserve materials; the other is very large, and so completely fills up the nut that it looks as if someone had poured wax into the interior of the fruit, and that it had there become hardened into a solid mass. The Water Chestnut germinates on the mud under water. As soon as germination commences, a white worm-like body is extruded from the aperture of the nut, and though many consider this to be the hypocotyl, it should, strictly speaking, be regarded as a root ( $c f$. fig. $144^{3}$ ). This structure elongates under the water and grows directly upwards. Of the two cotyledons only that one which was inserted as a tiny scale on the short hypocotyl, leaves the cavity of the nut and is connected by a long stalk with the hypocotyl. This long stalk, together with the very small hypocotyl and the root, pass so gradually into one another that they resemble a single unjointed white cord ( $c f$. fig. $144^{4}$ ). The reserve materials deposited in the large, fleshy cotyledon are conducted by the stalk-like connection to the growing parts of the embryo in the water; a process which occupies a considerable time. By the time that this cotyledon has yielded up the reserve food, the root has grown so strong that it is able to take up materials from its surroundings; it bends down towards the mud VoL. I. 
in which it fixes itself by numerous lateral fibres. The bud has meanwhile grown out and formed a shoot which develops scale-leaves below, with green foliage-leaves above these, and so grows up to the surface of the water. The exhausted cotyledon never leaves the interior of the nut, but gradually decays with it. Thus we have here the rare instance of one cotyledon being extruded from the cavity of the seed (that is to say, of the fruit) while the other remains behind.

In the seventh case the embryo exhibits two (only exceptionally more than two) cotyledons, which are drawn out of the seed-coat during germination, and spreading out in the sunlight, turn green and serve as foliage-leaves. These foliage-leaves first function as absorbent organs; that is to say, they adjoin a special reserve-tissue in the seed from which they derive the materials required for their first growth, and do not issue from the cavity of the seed until the storehouse is exhausted and emptied of food. This is the case, for example, in the repeatedly-mentioned Corn-cockle (Agrostemma Githago), whose two cotyledons, folded together, are bent like a horse-shoe round the reservetissue, but are withdrawn from the seed-coat after the consumption of this food, when they separate and become green $\left(c f .141^{7,8,9,10}\right)$. Much more rarely the seed-coat bursts at the beginning of germination, the large folded cotyledons together with the surrounding reserve-tissue are drawn out so that the absorption of the reserve-food does not take place till after vacating the seed-coat, after which follows the unfolding and colouring of the two cotyledons in the sunlight. The seeds of Ricinus ( $c f$. figs. $141^{1}$ and $141^{2}$ ) show this process of development, which on the whole is very uncommon. On the other hand, it frequently happens that no special reserve-tissue (endosperm) is present, that the small amount of reserve-food is deposited in the cotyledons themselves, and that immediately after germination has commenced the two cotyledons leave the cavity of the seed-coat and become green foliage-leaves. As an example of this the germination of the Gourd (Cucurbita Pepo) is given in fig. $145^{1}$.

The way in which cotyledons are withdrawn from the cavity of the seed-coat is very characteristic, and it is worth while to inspect the most remarkable contrivances of this kind somewhat more carefully. One of the most peculiar is observed in the seed and embryo of the Gourd, which is figured opposite in natural size. The seed of the Gourd is pretty large, flattened, oval in outline, rounded at one end, and somewhat tapering at the other, and here cut off short, and provided with a small aperture. If these seeds are disseminated they lie flat on the ground, and easily glue themselves to the soil, especially if their surface is covered with the adhesive juice of the fleshy fruit, as is always the case when the seeds are naturally distributed. Since the embryo inclosed by the seed-coat is straight, it has a position parallel to the surface of the ground. When germination begins the radicle is first of all pressed out through the small opening mentioned. It immediately arches and grows quickly downwards into the earth by the help of the food conveyed to it by the two cotyledons. It there develops lateral rootlets, and unites firmly with the 
particles of soil by means of abundant root-hairs. The hypocotyl also, into which the root merges, grows at first downwards into the earth, but of course only for a short time, for this is very soon altered; and growth then takes place in an opposite direction towards the light, and immediately after this alteration of direction the withdrawal of the cotyledons commences. It follows from what has been said that the hypocotyl is fixed both above and below-below by the root which has grown

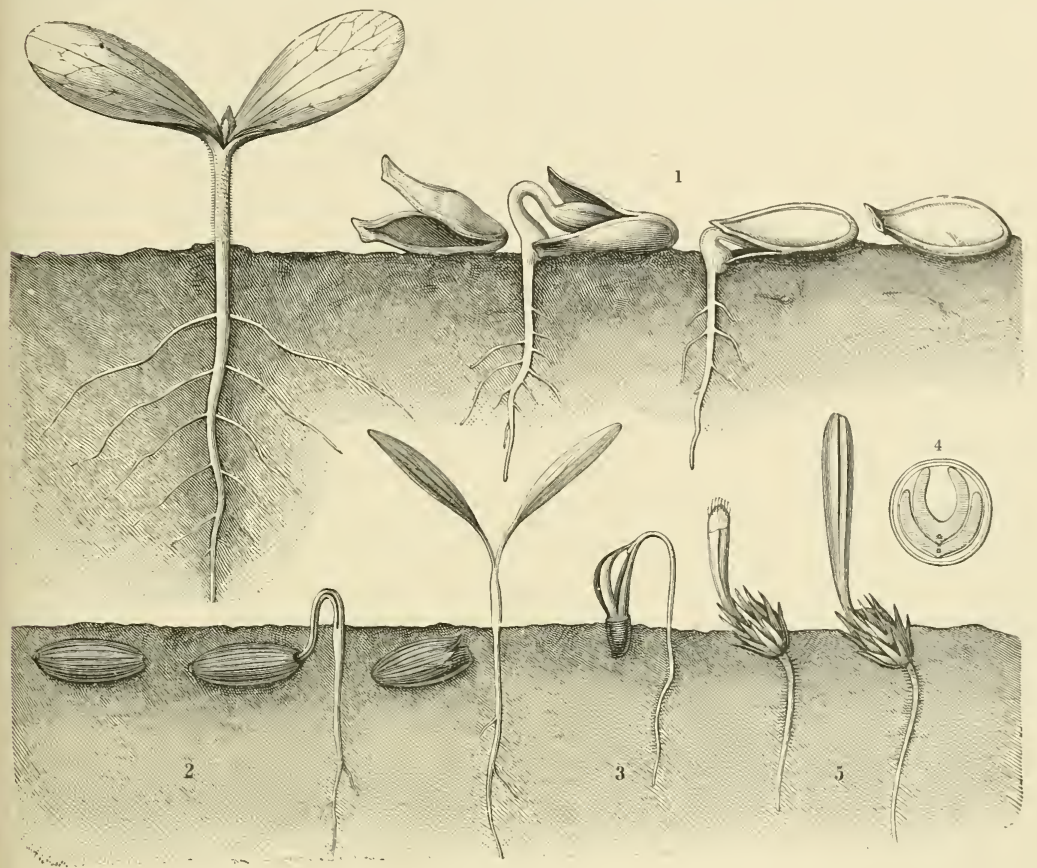

Fig. 145.--Liberation of the Cotyledons from the cavity of the seed or fruit husk.

1 Gourd (Cucurbita Pepo). 2 Asafœetida (Scorodosma Asa foetida). 3 Immortelle (Belichrysum annuum). 4 Cross-section through the cotyledons, showing them curled up in the pericarp of the Immortelle. 5 Cardopatium corymbosum (after Klebs). Fig. 1-3, natural size; fig. 4-5, somewhat enlarged.

firmly into the ground, above by the firmly glued seed-covering in which the cotyledons lie. As soon as it increases in length it forms a well-marked arch, frequently even a loop, with the convex side turned upwards ( $c f$. fig. $145^{1}$ ). Naturally it thus exercises a severe strain on both ends. The root, well planted in the earth, can no longer be disturbed from its position, but the effects of the tension make themselves felt on the cotyledons, which still lie in the seed. The coat of the Gourd seed bursts, the cotyledons are drawn out from the yawning cleft, the hypocotyl straightens itself, and the two cotyledons separating from each other turn their upper sides towards the light (fig. $145^{1}$, on the left). 
The splitting of the seed-coat and the withdrawal of the cotyledons in the Gourd are materially assisted by the development of a projecting lip at the union of the radicle and hypocotyl. This lip presses against the lower edge of the hard seedcoat, and holds it to the ground, so that after the bursting takes place the upper portion of the seed-coat is raised up lid-wise from the lower. A smaller projection is also developed on the hypocotyl in the embryo of the Sensitive Plant (Mimosa pudica), and in that of Cuphea, and here again it presses against the lower part of the seed-husk, and so assists both the bursting and the withdrawal. When the seeds are surrounded by a pericarp, sometimes bands and projecting corners are developed on it, sometimes projecting edges of the dried calyx and the like, which serve as a fulcrum to the lip of the hypocotyl. The presence of numerous structures formerly considered to be stunted organs useless to the plant thus receives its natural explanation.

Many plants, e.g. certain Umbelliferæ, develop a very short hypocotyl. This does not bend, and exercises only an insignificant strain, or perhaps none at all, on the cotyledons, and so would not be able to release them from the integument of the seed or fruit-husk. In all these plants the cotyledons have long stalks, and these assume the function of the hypocotyl, at least in so far as the withdrawal of the blades of the cotyledons is caused by them in the same way. This phenomenon is very noticeable in the germination of the Asafœetida (Scorodosma Asa fuetida), as is clearly shown in fig. $145^{2}$. The stalks of the cotyledons, arising from the very short hypocotyl, rapidly elongate, and exhibit the same s-shaped bend as that formed in the hypocotyl of the Gourd seedling. These stalks also produce a similar effect on the blades of the cotyledons still lying within the fruit-husk, and actually draw them out. As soon as this has happened, the stalks straighten, and the blades borne by them turn their upper surfaces towards the light.

Almost a third of all seed-bearing plants have cotyledons whose liberation from the bondage of the seed-coat or pericarp is effected in this manner, and this consequently is the form of cotyledons which has been most frequently observed and described. Much less frequently the two cotyledons make their appearance at one end of the pericarp or seed-coat, while the radicle grows out at the opposite end. In this case, which must be regarded as the eighth of the series here given, the embryo is straight, and the hypocotyl is short and bears two thick cotyledons whose apices, lying close together, form a truncated cone. When the radicle has been once pushed out, and has planted itself firmly in the ground, the hypocotyl at once elongates in the opposite direction without bending, pushes the folded cotyledons in front of it, and presses them out of the fruit-husk. The tissue of the fruit-husk lying above the cotyledonary cone must be pierced, and this is not difficult to do, since this tissue consists of thin-walled cells. When the radicle has grown out from one pole, and the pair of cotyledons from the other, the seedling is surrounded half-way up by the vacated fruit-husk, as though by a girdle (cf. fig. $145^{5}$ ). The apices of the cotyledons still folded cone-like together usually bore through the soil above the husk after they have left the cavity, and 
not until this has occurred do they unfold and become green. In this penetration of the earth the cotyledons are exposed to so many dangers that special arrangements are frequently to be found with a view to protecting their advancing points.

As the cotyledons push through the ground, a pressure is exercised upon the layers of soil above by the straightening of their stalks. The cotyledons raise portions of soil on their backs, so to speak, without actually bursting or boring through them. In this way the danger of injury is at any rate much diminished, and the supposition is fully justified that cotyledons which develop after the type of the Gourd or Asafœetida are those which occur most frequently. Plants whose straight embryo has to pierce through the fruit-husk and the layer of earth above it by means of their conically-folded cotyledon apices are, as already stated, less common. Fig. $145^{5}$ shows this rare form in Cardopatium corymbosum. It has also been observed in many other species allied to this composite, and in the Mediterranean Atractylis cancellata.

In all those cases where the cotyledons are withdrawn through a cleft or hole in the seed-coat it seems quite obvious that the aperture should have a diameter at least equal to that of the organs to be withdrawn. As a rule this is so; but occasionally the cotyledons are actually broader than the eleft, and one asks in astonishment how the withdrawal could have been accomplished without injury to their fabric. The feat is performed in the following way. Before the strain on the cotyledons comes, they are folded together, and are then drawn out as a long roll through the narrow opening of the integument. Scarcely have they been liberated ere they begin to unroll and spread themselves out flat. This is the case, for example, in the Immortelle (Helichrysum annuum) (see figs. $145^{3}$ and $145^{4}$ ), also in the umbellifer, Smyrnium Olusatrum, and in many others. In some plants, e.g. in the Beech (Fagus sylvatica), the cotyledons, as long as they remain within the husk, are folded together lengthwise like a fan, and in this position occupy but a limited space. They are also withdrawn from the nut through a comparatively small aperture, and then expand in a very short time after they are free (see figs. $148^{1,2,3}$ ). The two cotyledons of Pterocarya caucasica are each divided into four lobes, and each pair of lobes lying close to one another are imbedded in a special excavation in the seed. Altogether the fruit presents four compartments, in each one of which lies such a pair of narrow, closely-packed lobes. The aperture of the nut-like pericarp now affords sufficient space for each pair of folded lobes to be drawn out, nor does their withdrawal occur simultaneously, but rather so that the pairs of lobes emerge one after the other. The cotyledons of Schizopetalon Walkeri behave in a similar manner, each of them being divided into two long narrow lobes which are drawn out successively through the small aperture of the spherical seed. Moreover, in the embryos of Pinus there are five or more whorled linear cotyledons (see fig. $148^{6}$ ). These also leave the cavity of the seed one after the other. Speaking generally, it would seem that the dimensions and form of the cotyledons are correlated with the shape of the seed-coat or other investment, and with its manner of opening. 
The external form of the seed and the position which it consequently assumes on falling to the ground is by no means an unimportant item in this connection. If the seed comes to lie so that the axis of the hypocotyl is perpendicular to the surface and the tip of the radicle is directed downwards, we seem at first sight to have a very favourable position for germination; but it is not so in reality. In this position the hypocotyl would have to perform the most complicated curves in order to be able to withdraw the cotyledons from the seed. On the other hand, the most favourable condition is obtained when the axis of the hypocotyl and radicle lie parallel to the surface of the ground, as, for example, in the gourd seeds illustrated in fig. $145^{1}$. Here the radicle immediately after leaving the seedcoat can bend down at a right angle and grow into the earth, and the hypocotyl is able to withdraw the cotyledons very rapidly. When seeds of this sort are sown, they usually assume the last-mentioned position. Flat or compressed seeds lie with their broad surface on the ground; oval and elongated cylindrical seeds fall so that their longer axis is parallel to the substratum; whilst in spherical seeds the centre of gravity is so situated that the most favourable position possible for germination is obtained.

The importance of numerous developments on the exterior of the seedcoat or pericarp will at once become evident to anyone who observes attentively the process of withdrawal of cotyledons. It is manifest that the withdrawal only occurs without delay when the seed is in some way or other firmly fixed and when arrangements are present which prevent a favourable position being lost when once assumed. This would not be so were the seed the plaything of every gust of wind or current of water. Equipments for retaining fruits and seeds in the position of germination occur in great number and variety. Even the wing-like and hairy appendages, the curred, pointed, and barbed processes, and the various adhesive arrangements of fruits and seeds, which in the first instance have the function of agents for distributing the fruits, often afford this advantage, viz. that by their means the seeds are fixed where germination can successfully take place. If we look at the damp mud by a river's bank, towards the end of May, when the fluffy seeds of willows and poplars are escaping from the dehisced fruit-capsules and are carried along by the wind, we there see countless numbers of these seeds sticking by their hairs to the mud so tightly that they cannot readily be displaced. All such seeds (differing from the generality of seeds) germinate in a few days, while seed lying on the ground in loose flakes close by do not germinate. The hairy coat which first served as an agent for distributing the seed, now functions as an agent for fixing it in the germinating bed. This also applies to the tufts of silk adorning the small seeds of tropical tillandsias (Tillandsia usneoides and T. recurva) which grow as epiphytes on the bark of trees. These first serve as wings, and the tiny seeds are carried by the wind far away from the burst fruit-capsules. If these seeds are stranded on the bark of a treetrunk which is swept by the wind, the hairs cling firmly and bring the seeds 
intu contact with the substratum. Accordingly the weather side of the trunk is seen to be beset with large numbers of such seeds, and many of them, pressed into the crannies of the substratum, begin to germinate. A similar process is observed in the settling of the seeds of Anemone sylvestris and many composites. To cite yet another example, we may name the hooked fruits of Xanthium spinosum and Lappago racemosa. When detached from their place of origin by wandering animals, these seeds remain fixed by their barbed processes to the hairy coats of the animals, and thus are often removed considerable distances. Naturally the animals try to free themselves from these irritating appendages by rubbing themselves against the ground until they detach the fruits from their coats. In this way a part of the fruits are pressed into the soil, and are there anchored by their barbed spines. Only the embryos of the firmly-anchored fruits develop into vigorous plants; those seeds which lie more loosely on the ground, on the contrary, either do not germinate at all, or the seedlings whose cotyledons are imperfectly withdrawn from the pericarp soon perish.

Besides these outgrowths, which, as we see, possess a double function, there are also those which have no connection whatever with distribution, and have apparently no other use than to fasten the seeds to the germinating bed. In this connection we have first to notice adhesive materials which exude from the surface of the seed-husk, whereby the seeds are cemented to the soil. These make their appearance when the surface of the seed is moistened, as when water is sucked up by the seed from the soil of the germinating bed. Usually the slimy cement arises from the superficial cells, as, for example, in the many species of flax and plantain (Linum and Plantago), in the Cress and the Gold-of-pleasure (Lepidium sativum and Camelina sativa), in Teesdalia, Gilea, and Collomia, and in many other species of the most diverse genera. All, however, agree in this particular, that the seed-coat possesses a smooth, polished surface. In the Basil (Ocymum Basilicum) and in the numerous species of Salvia and Dracocephalum, the mucilaginous substance arises from the smooth surface of the pericarp. Frequently the adhesive mucilage is only developed in certain cells arranged in rows on the surface of the fruit or seed-husk, as in the New Zealand Selliera and in numerous Compositæ, of which the Wild Chamomile (Matricaria chamomilla) may be cited as the best-known example. In Oxybaphus there are five longitudinal ridges on the integument of the seed covered with small mucilage-organs. When the integument is moistened, five white slimy lines appear on it, and these bring about its adhesion to the germinating bed. In many Compositæ, e.g. in the common Groundsel (Senecio vulgaris) and in Euriops, Doria, Trichocline, and in many other genera, special hairs are developed on the fruit-husk which excrete adhesive mucilage. In other instances, again, as in many aroids, the cement is not developed by superficial cells, but a part of the fleshy pericarp, in which the seeds were inclosed, remains as a dried-up crust. If these seeds be subsequently moistened, the 
crust again becomes changed into a mucilaginous adhesive mass which glues them to the substratum. The whole succulent decaying pericarp often becomes the fixing agent of the seeds, as, for example, in gourd-like plants, and in many plants with berries and stone-fruits.

In numerous plants, e.g. in the Corn-cockle ( $c f$. figs. $1417,8,9,10$ ), and in Neslia paniculata which grow abundantly in loamy fields, the fixing of the fruits or seeds to the soil is not effected by mucilaginous cement-materials, but by inequalities on the surface of the integument. Here are developed extremely diversified warts, pegs, ridges, or net-works, and between them pit-like depressions into which the earth-particles penetrate, and when moistened become closely connected with the superficial cells. The adhesion is therefore very close, and if we try to cleanse these seeds or fruits, and to remove the clinging soil from all the small hollows, we shall not completely succeed even after a great deal of trouble. And here we must point out the interesting distinction between rugged seeds like these, and such as become slimy. Seeds with rough, wrinkled, and pitted surfaces never develop adhesive agents, since they are fixed to the soil by these inequalities of the seed-coat; on the other hand, seeds with smooth surface, which would otherwise be easily displaced, adhere by means of mucilage developed by their epidermal cells.

The Water-chestnut (Trapa), whose germination was described on p. 609, behaves in a very peculiar manner. Each of its large fruits exhibits two pairs of projecting spines arranged cross-wise, which have been formed from the sepals, and which protect it during ripening from the attacks of aquatic animals. These spines, as well as the whole fruit, are as hard as stone, but only in the interior. The outer cell-layers are soft, decompose quickly under water, and separate from the deeper tissue in irregular tatters and shreds. At the apex of the spines, after the detachment of the soft portion there remain not only the strong hard midrib, but also the commencements of some recurrent bundles of very firm elongated cells which spring from the midrib immediately behind the apex. These spines therefore have the appearance of anchors (see fig. 146), and indeed they function as anchors, adhering at the bottom of the lake by means of their barbed points to various vegetable remains which cover the mud, and are actually anchored there. The seedling arising from the nut does not consequently lift the pericarp with it, but this remains fixed in the place where it fell.

Peculiar contrivances for anchoring fruits to spots suited for germination are observed in many steppe-grasses, especially in the Feather-grass (Stipa) and in the Stork's-bill genus (Erodium). The feather-grasses are a striking feature of steppes; indeed, together with various Papilionaceæ-especially with tragacanth shrubs (Astragalus) - and with numerous Compositæ, pinks, and low irises, they compose the chief constituent of the vegetation. The appearance of the Feathergrass is appropriate to its name, consisting as it does of tufts of white feathery streamers swinging in the wind. This characteristic feature makes its appearance 
after the flowers have been pollinated, and is due to the awns which belong to the flowering glumes, of which one surrounds each ovary, undergoing a very remarkable elongation and hair-like branching, recalling a like behaviour in the styles of Clematis and some species of Anemone.

The glume, which is crowned with the feather-like awn, together with a second short glume, destitute of awn, incloses the small fruit. As soon as it is ripe, the fruit, wrapped in its glumes, becomes detached; the first breeze carries it off and blows it like down over the steppe. The long feathery awn arising from the glume has thus, in the first instance, the significance of a flying apparatus, like so many of the feathery or wing-shaped structures which beset or envelop seeds and fruits. It effects the distribution of the feather-grass in question over wide

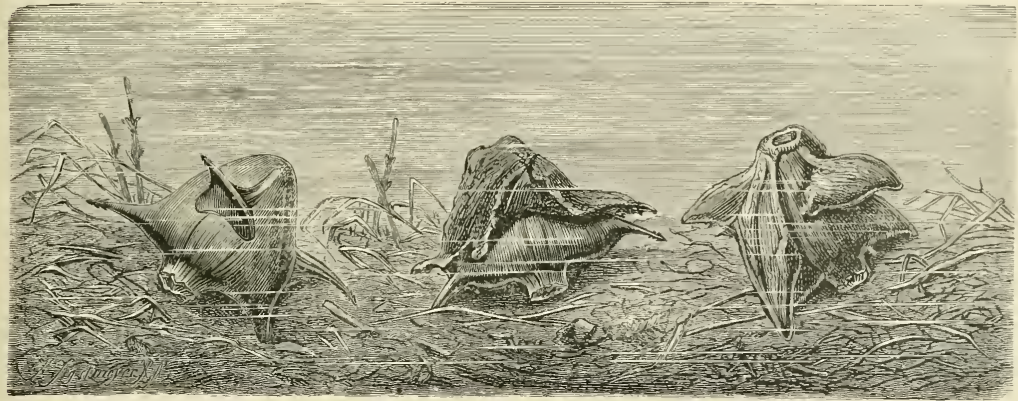

Fig. 146.-Anchoring of the Water-chestnut (Trapa).

tracts of country. But after the awn has become stranded somewhere on the soil of the steppes, it has yet another function to perform.

Let us suppose that a feather-grass fruit has fallen on to the bare earth, as in the illustration on p. 619; that part containing the fruit inclosed in the glume, as it is the heaviest, will obviously come first into contact with the ground; and since the tip of this portion is hard and sharply pointed, the stranded fruit often sticks in the ground immediately upon alighting (fig. $147^{1}$ ). Should it fall obliquely, the tip will penetrate into the ground by the continued twisting of the long feather waving in the air. This first penetration is materially favoured by the fact that the point is bent a little obliquely towards one side.

When once the point has penetrated into the soil, the other portions of the glume surrounding the fruit soon follow, owing to the action of the following contrivance. Close above the point of the enveloping glume are inserted upwardly-directed hairs which are at once elastic, flexible, and yet stiff. As long as these stiff hairs lie close they offer no resistance to the penetration of the glume into the soil, and some of them are actually embedded in the soil even at the first penetration of the point. Now if the fruit as it pierces the ground be inclined to one side, by some pressure operating ever so lightly from above, then the hairs on that side are pressed still more closely against the glume, while those 
on the other are made to stand off somewhat; these latter press against the particles of earth above them, and act as levers, by means of which the whole fruit at the moment of bending in the given direction becomes pressed down deeper into the ground. This action is continued every time the fruit wobbles from side to side, so that bit by bit it is buried. The only question is, how these sudden alterations in position of the fruit fixed in the ground can be brought about. A glance at fig. 147 shows that every wind, even though slight, which strikes the long feathery portion of the awn, must immediately be followed by an alteration in the position of the fruit. Just as a weather-cock on the top of a roof in a strong east wind does not point invariably towards the east, but generally makes short veerings to the north and south, so the plumed awns fluttering to and fro in the east wind swerve momentarily towards the north and south, and this change of direction causes the fruit sticking in the soil to incline to various sides. When the wind veers round of course an alteration occurs in the direction of the feathery awn, and consequently a more strongly-marked inclination of the fruit occurs, so that a see-sawing motion of the latter will be unavoidable. The wind, therefore, is an important factor in driving the fruit into the ground. The awns of the Feather-grass, however, have two other peculiar contrivances, viz., below the feathery portion they are bent twice like a knee, and they are also spirally twisted like a corkscrew. This bent and twisted part of the awn is exceedingly hygroscopic; in rainy weather the knee-shaped bend almost entirely disappears; the awn bristles and straightens itself, the spiral unwinds in damp weather, and twists up again in dry air. These movements are evidently conveyed to the glume, and produce alterations in its inclination, which again cause an advancement of the tip into deeper layers of earth.

These movements of the lower portion of the awn produced by the varying humidity of the air make themselves specially felt when the upper portion has in some way become entangled with the stems and leaves of the other steppe-plants, as frequently happens. When in dry weather the fruits of the Feather-grass become detached and are blown by the wind over the steppes, it is almost unavoidable that they should remain fastened by their knee-like bent portion to haulms, stems, and the like-that the feathery part should be hemmed in between two neighbouring stems of other plants, or occasionally even entangled with them ( $c f$. fig. $147^{2}$ ).

But as soon as the upper portion of the awn is fixed, and later on in damp weather the lower knee-shaped portion of the same awn has become straightened and the spiral twists uncoiled, the fruit is necessarily forced into the ground with a twisting movement, and is also pressed now to this side and now to that by the unequal straightening of the knee-shaped bend. Any backward movement of the fruit from a subsequent drying up of the awn is prevented by the above-named stiff hairs, in the manner already described. It is much more likely that one of the stems to which the awn has attached itself should be somewhat bent by this contraction of the awn, than that the glume already driven a certain depth into the ground and there anchored should be drawn out. 
The fruits of the Stork's-bill (Erodium) get planted in the same way as those of the Feather-grass. The five mericarps (or fruit segments) in this plant detach themselves in a very characteristic manner from their support, as may be seen in fig. $147^{3}$. First the lower thick end inclosing the seed splits off, and later

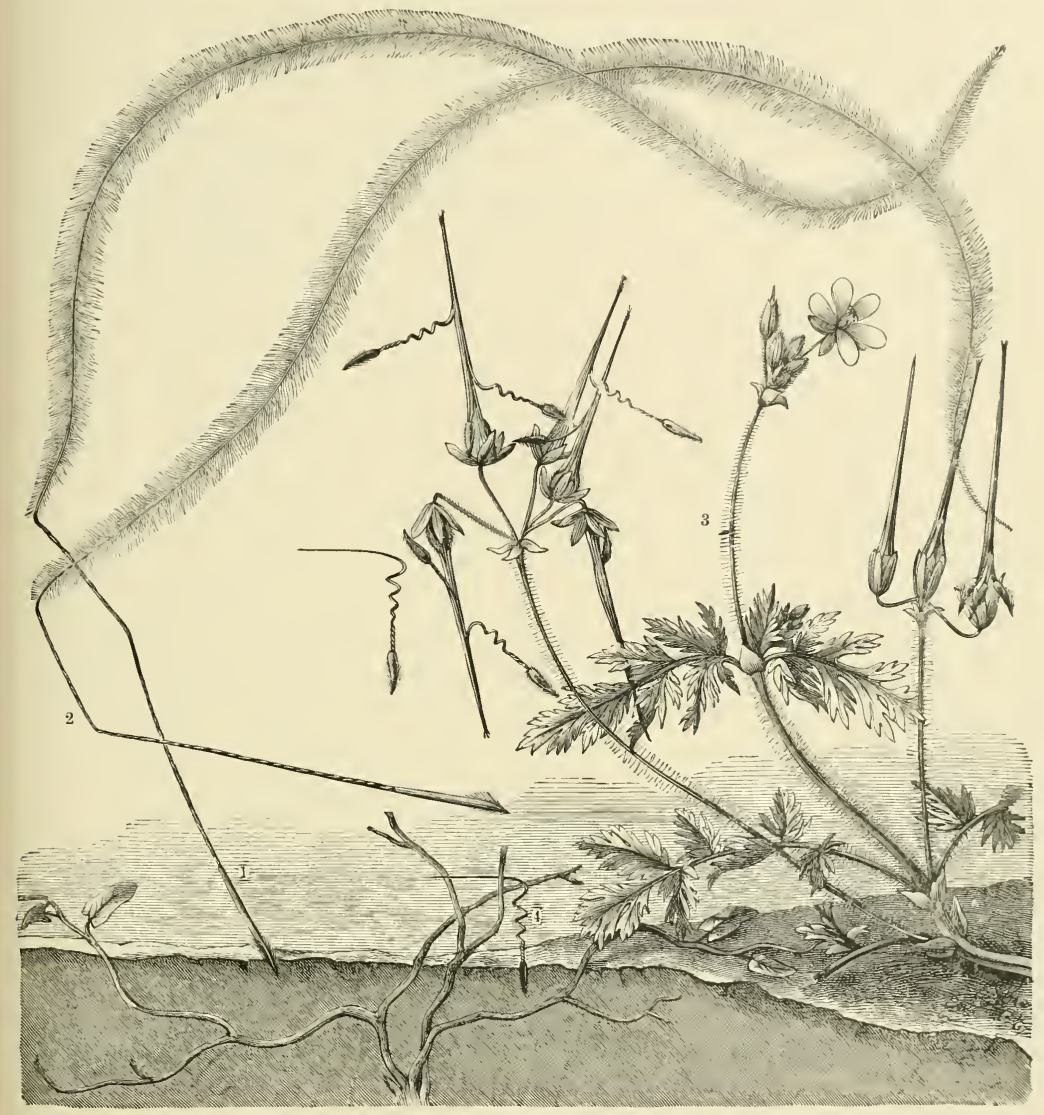

Fig. 147.-Showing the boring of fruits into the ground.

1, 2 Fruits of the Feather-grass (Stipa pennata). s, a Fruits of the Stork's-bill (Erodium Cicutarium).

also the long drawn-out point of the carpel. A part of the latter twists up spirally, and only its free end stretches out in a slight curve, like the hand of a watch. It is well known that this fallen fruit-segment is used as a hygrometer. It is placed with its lower thick end which, like the fruit-end of the Feather-grass. possesses a sharp point, on a board covered with paper, in the centre of a circle. Marks are made on the circumference of the circle corresponding to the position 
of the pointer-like end of the Stork's-bill fruit in very damp and in very dry weather respectively, and we can then draw conclusions from the position of the pointer as to the relative dampness of the air. In this application of the fruit we have an exhibition of the torsions which take place in the course of its penetration into the ground, and which are produced by the alterations in the humidity of the air. When such a fruit falls on the ground, however, it is not the lower thick end inclosing the seed which is fixed, as in the hygrometer, but the pointer-like process, and consequently in nature it is the seed-end and not the pointer which is set in motion by an alteration of humidity in the air. The fixing of the fruit in the ground is naturally effected thus: the point of the arm lies on the soil, and in consequence of the untwisting of the spirals in damp weather, the thicker seedcontaining end (which is provided with a sharp point) bores deeply into the ground. More frequently the fallen fruits hang between the entangled stems, \&c., of other plants lying on the ground, as shown in fig. $147^{4}$. Here again the arm is fixed, and the thicker, lower end is set in motion. The movement may be compared to that of an augur, although in consequence of the swaying and alteration in position of the beak, unavoidable in windy weather, see-sawing movements occur in the boring part, and these are apparently advantageous. Like the fruits of the Feather-grass those of the Stork's-bill are beset above the sharp point with erect, stiff hairs. These hairs also play the same part as in the Feather-grass. According to the species various divergencies are found in the fruits of Feather-grasses and Stork's-bills. The twisting of the lowest portion of the awn generally differs from that of the knee-like bent part; the bristles on the glumes are sometimes arranged in two longitudinal rows and sometimes they form a ring below and are continued upwards as a one-sided longitudinal stripe, and so forth. Many species of Stipa have no plume to the awn, and approximate closely in form to the fruit of the Stork's-bill. The same remark applies to fruits of the genera Aristida and Heteropogon, which are allied to Stipa. But in the main all these developments agree with one another. The aim and object of the wonderful mechanism just described is not so much the penetration of the pericarp or seedhusk to a great depth into the soil, as the fixing of it firmly in the germinating bed.

It still remains to be pointed out that the cotyledons are only withdrawn without delay from their investments when the latter are firmly cemented, anchored, or fixed in some way to the substratum. When this is not the case, it often happens that the fruit, or seed-coat, is raised up like a cap by the enlarging cotyledons. The pull, otherwise exerted by the elongating hypocotyl, cannot under these circumstances assist the cotyledons in their liberation. Often, indeed, the cotyledons throw off the seed-coat unaided, but this is not always the case. In many instances their apices remain squeezed up in the cavity of the husk, stunted and yellow, and this reacts injuriously on the seedling, often causing weakness and even death. It is therefore a mistake for gardeners to plant seeds in loose earth where no good hold is afforded, since then, on germination, the seed-coats are raised up by the only half-liberated cotyledons, whose apices are still imprisoned. 
With regard to the forms assumed by the cotyledons now withdrawn from the seed under favourable conditions, and which have become green in the sunlight, it is to be noticed that they present far fewer variations than those of ordinary foliageleaves. Usually their margins are entire, their form elliptical or linear, more rarely circular and obovate. Sometimes the cotyledons are indented in front, resembling a heart in outline; this is especially the case where the embryo is folded

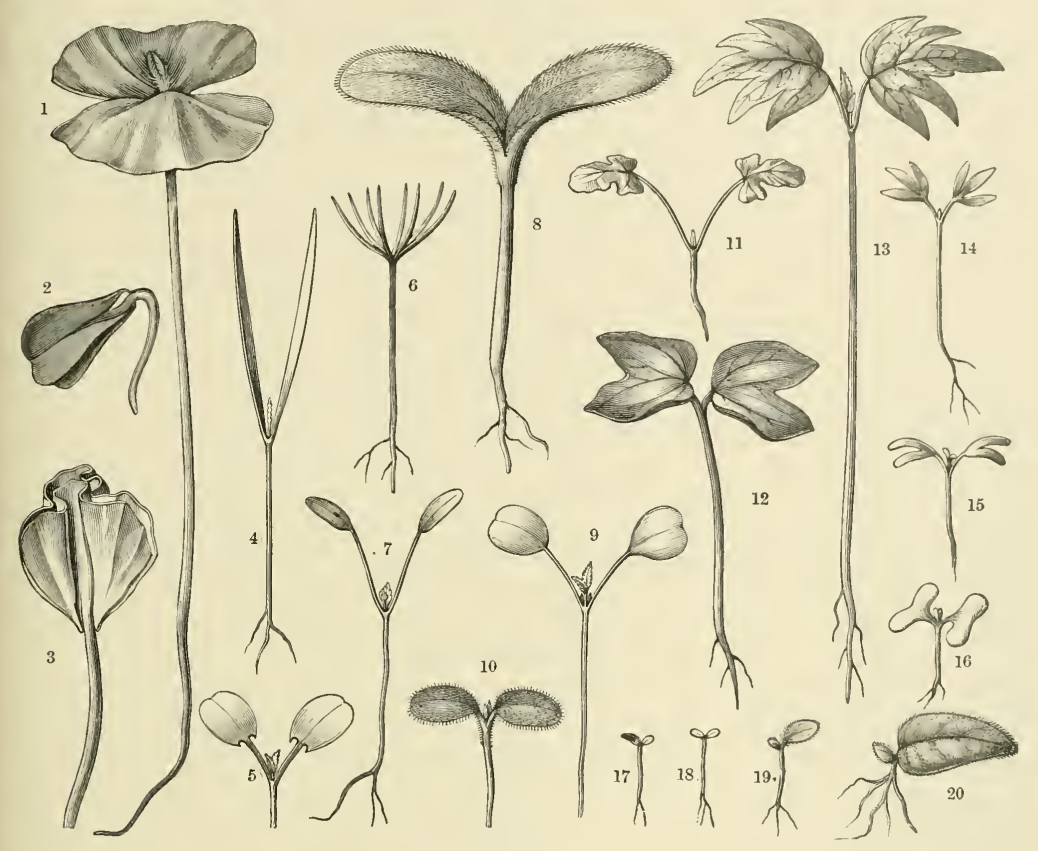

Fig. 148.-Cotyledons.

1, 2, 3 Fagus sylvatica. 4 Fumaria officinalis. 5 Galeopsis pubescens. 6 Abies orientalis. 7 Convolvulus arvensis. 8 Borago officinalis. 9 Senecio eruccefolius. ${ }^{10}$ Rosa canina. ${ }^{11}$ Erodium Cicutarium. 12 Quamoclit coccinea. 18 Tilia grandifolia. 14 Lepidium sativum. ${ }^{16}$ Eucalyptus orientalis. ${ }^{16}$ Eucalyptus coriaceus. 17-20 Streptocarpus Rexii.

in the seed, so that the radicle lies close to the outer margin of the cotyledons, and may be explained as an economy of the scanty space within the seed. Most rarely of all the cotyledons are two-lobed (Raphanus sativus), and bisected (Eucalyptus orientalis, Eschscholtzia Californica), three-lobed (Erodium Cicutarium), and trisected (Lepidium sativum), four-lobed (Pterocarya Caucasica), and five-lobed (Tilia). It is still to be mentioned that in all seedlings whose hypocotyl is short, the blade of the cotyledons has a long stalk, while in seedlings with elongated hypocotyls the blade is sessile. This is connected with the processes already mentioned, and also partly with the fact that it is of importance to seedlings that 
their green blades should be exposed as much as possible to the sun, and that so they should rise above other objects which might place them in the shade. The accompanying figure 148 shows the most noticeable forms of cotyledons after they have unfolded and spread out in the sunlight.

When two green cotyledons are present they are usually similar in shape and size, only that which has served in the seed as an absorbent organ is generally somewhat smaller in the adult condition, as, for example, in the Corn-cockle, Mustard, and Hemp. Frequently the limited character of the space within the seed makes it necessary that one of the cotyledons should give place to the radicle, or that it should only attain to an inconsiderable development, as, for example, in Petiveria and Abronia. In species of Streptocarpus belonging to the Gesneraceæ (see figs. $\left.148^{17}, 18,19,20.\right)$, the two cotyledons have the same shape and size in the seed, after they have left the seed-coat they are still entirely similar, but later on the growth of one is retarded, and it dies, while the other increases to an extraordinary degree, and develops into a green foliage-leaf lying on the ground, $22 \mathrm{~cm}$. long, and $12 \mathrm{~cm}$. broad. Strangely enough, many species of this genus, e.g. Streptocarpus polyanthus develop no other green leaves, but content themselves with the development of the one cotyledon into a gigantic foliage-leaf prostrate on the soil, with which later on the epicotyl appears to be united, and from whose thick midrib it rises up as a flowering axis.

It is without question that cotyledons which become green possess, in common with other green tissues, the property of manufacturing organic materials in the sunlight from the absorbed food-gases, salts, and water. As a rule chlorophyll does not appear until the cotyledons have issued from the seed-coat, and have spread out in the sunlight. It is, however, sometimes formed even while the cotyledons are still in the seed and shrouded in darkness, as, for example, in firs and pines, in maples, and some Cruciferæ, in Loranthus, Mistletoe, and the Japanese Sophora. Green cotyledons exhibit all the characteristics of foliage; their epidermis is provided with stomata, whilst palisade-cells and spongy parenchyma can usually be distinguished in the green tissue. Many plants, especially those which subsequently develop subterranean tubers, or tuberous roots, e.g. many species of Ranunculus, Monkshood, Corydalis, Eranthis, Leontice, Bunium, Smyrnium perfoliatum, and Chcerophyllum bulbosum, do not in the first year after germination go beyond the formation of green cotyledons; green shoot-leaves are not developed from the bud or plumule until the next year. Many plants, on the other hand, unfold green shoot-leaves almost simultaneously with the cotyledons, but the cotyledons function with them as foliage, and sometimes remain fresh and green until the time of flowering, or even until the ripening of the fruit. Examples of these are afforded by numerous quick-growing annual weeds in our fields and kitchen-gardens (e.g. Fumaria officinalis, Scandix Pecten-Veneris, Arnoseris pusilla, Urtica urens, Adonis cestivalis). The cotyledons, in rapidly-developing annuals, sometimes attain dimensions scarcely inferior to those of the green shoot-leaves. For example, the cotyledons of the Gourd are more than a decimeter long, and $4-5 \mathrm{~cm}$. broad. It is 
to be expected that these green cotyledons, whose function is precisely the same as that of the green leaves of the shoot, should also be protected in exactly the same way against external injurious influences, and as a matter of fact many of the protective contrivances are found on them which have been described in detail previously.

The cotyledons of many Boragineæ are beset with stiff bristles (e.g. Borago, Caccinia, Anchusa, Myosotis (see fig. $148^{8}$ ); those of roses are fringed with glandular hairs (see fig. $148^{10}$ ); and those of many nettles bear stinging hairs on their upper surface. It has been already pointed out on p. 350 that cotyledons protect themselves, and the young shoot-leaves hidden between them, against the injury which might happen from loss of heat on clear nights by folding together and assuming a vertical position.

\section{SCALE-LEAVES, FOLIAGE-LEAVES, FLORAL-LEAVES.}

When the leaves borne on the shoot were distinguished as scale-leaves, foliageleaves, and floral-leaves, it was not implied that these three kinds of leaf-structures were actually developed on all shoots. Scale-leaves are only found developed on perennial plants. In annual plants they are entirely absent. Even the bud which arises at the apex of the hypocotyl of an annual plant begins at once with green foliage-leaves, nor are traces of scale-leaves to be seen in the buds which are subsequently developed on the epicotyl. Now, what can be the cause of this difference between annual and perennial plants? Obviously annual plants require no scaleleaves. It is of great importance for them that they should develop fruits and seeds in the short period of a single summer; for this they must manufacture the building materials necessary by the help of their green foliage-leaves. A portion of the building materials is employed in the formation of the embryo in the seed; another part in the production of well-stocked food-reserves associated with the embryo. The seeds become detached and scattered, whilst the parent plant which has produced them shrivels up and dies. It leaves no buds behind to persist through the winter and sprout next year; consequently any provision for the maintenance of such buds would be superfluous. It is different in perennial plants, as the buds formed by them must be provided with the necessary reserve-food, and protected during the period of inactivity, throughout the winter sleep and summer rest, against extremities of cold and heat, from freezing, burning, and drying up. They must also be protected as well as possible against the attacks of animals, and these tasks are assigned to the scale-leaves, which serve on the one hand as storehouses for reserve food-materials, and on the other as protective envelopes covering the still short axis with its rudiments of foliage or floral-leaves. Of course no green leaf-blades, and, generally speaking, no green tissue is required for the fulfilment of these functions. The brown or colourless leaf-sheath is sutricient for the purpose; which explains why the scale-leaves appear on all shoots as pale, husky or scale-like structures without green blades. Even the first bud of the plant arising at the apex of the hypocotyl is provided in most perennial plants with pale scaly leaves, 
and this is the case not only in woody plants, e.g. in the Oak, as illustrated in fig. $144^{5}$ and $144^{6}$, but also in quite small herbaceous plants, as in the Moschatel (Adoxa Moschatellina), in which small scale-leaves without chlorophyll, followed by green foliage-leaves, are developed above the cotyledons on the epicotyl, and above these floral-leaves. All the shoots (that is to say, buds) developed later on in perennial plants start below with scale-leaves from which the green blade is absent, perhaps because it would be superfluous.

The scale-leaves which are developed on subterranean shoots, especially on bulbs, rhizomes, and turions, differ considerably from each other according to the various conditions of growth of these three kinds of shoot-structures. By bulb (bulbus) we understand an erect subterranean shoot, whose very short, thick axis is covered with relatively long, closely-packed, scale-leaves lying one above another. The resting bulb is really a bud, and its form is occasioned almost entirely by the shape of its scale-leaves. These are in most instances broad and concave, and they are arranged so that the inner ones are completely invested by the outer, as, for example, in tulips and species of onion; and they are elongated, ovate, or lanceolate, and lie on each other like the tiles of a roof, as in the lilies (Lilium Martagon, album, \&c.). The adjacent scale-leaves are sometimes united, as, for example, in the Crown Imperial (Fritillaria imperialis). Those of bulbs function chiefly as storage-organs. The shoot, whose base they cover, when it begins to develop, withdraws the necessary building materials from this storehouse until its foliageleaves become green and emerge above the ground; then the leaves are able to manufacture new organic materials in the sunlight. Bulbs are protected against the risk of drying up by the earth surrounding them, but it is very important for them that they should also be protected against the attacks of animals which live underground, and particularly from their nibbling. In addition to the poisonous materials for warding off these attacks, further protection is afforded chiefly by the fact that the exhausted and dead older scale-leaves do not entirely decay and disintegrate, but remain as a sheath. Sometimes they form a tough parchment-like investment, or their thick reticular and latticed strands remain as a sort of cage, within which the young and succulent bulbs are inclosed and protected, as may be particularly well seen in crocuses, gladioluses, and tulips.

The scale-leaves also perform the part of storage-tissues in subterranean, horizontally elongating shoots, called rhizomes or root-stocks (rhizoma). They also often serve as protecting envelopes, especially when they cover the apex of the stem as it pushes its way through the ground. In the latter case their cells are strongly turgescent, or more frequently very hard, almost horny, and are folded closely over the apex of the shoot, forming a stiff, pointed cone which is able to penetrate even clayey soil like a borer.

By turion (turio) is meant a bud originating laterally on underground stemstructures and developing in the summer into a shoot which rises above the ground. In the autumn its upper part dies off, whilst its lowermost, subterranean portion persists through the winter and originates new buds. Here 
the scale-leaves principally function as protecting envelopes for the foliageleaves. The young and still very delicate foliage-leaves, folded together within the bud, are entirely surrounded and over-arched by them. The sheath-like scales close together like a dome over the bud, and form an actual shield for it. Either hard, much-thickened cells, or more usually, strongly turgescent cells are present at the apex of each of these scale-leaves, and often these coverings are injured in penetrating the soil; but this is not of great importance because the scale-leaves become superfluous and perish when once the foliage-leaves have emerged and expanded above the ground. If earth is thrown up over the underground stock of such a plant as the Rhubarb, the scale-leaves of the turions increase in length in proportion to the thickness of the heaped-up stratum. The growth of the leaves keeps pace with the growth of the enveloped shoot; scarcely has the earth been penetrated when the scale-leaves stop growing, and the shoot-no longer requiring a protection against the ruggedness of the soil-rises up from its sheathing envelope and unfolds its young, green foliage-leaves in the sunlight. If the layer of earth which has been piled up above the subterranean stock is too thick, and if the store of building-materials for the lengthening of the sheathing scale-leaves is inadequate, then the young, green foliage-leaves are forced to leave their protecting envelopes even below the ground, and make their appearance above usually damaged, torn, and mutilated. Many fumitories (e.g. Corydalis fabacea) have only a single sheathing scale-leaf which surrounds that part of the shoot possessing green foliage-leaves. Here also it can be plainly seen that the scale-leaf affords protection only as long as it is necessary, i.e. the scale stretches up from the lowest portion of the shoot-axis until it has reached the surface of the ground, where the delicate, green foliage-leaves no longer require protection, and can unfold in the air. If the Corydalis is rooted only superficially in the earth, the scale-leaf is raised a very little, often scarcely a single centimeter, but if it is very deeply rooted, or if earth is heaped up over it either purposely or accidentally, then this lengthening of the lower portion of the stem amounts sometimes to more than 20 centimeters. In either case that portion of the stem by which the sheathing scale-leaf is raised stops growing as soon as the apex of the sheathing envelope has reached the surface of the soil, and it looks as if the Corydalis had deliberately adapted itself to the existing conditions.

Many plants have two kinds of underground scale-leaves. Firstly, those whose cells are filled with starch and other food-reserves. These are always thick and fleshy, and they do not continue to grow, but are absorbed by the growing shoots. Secondly, sheath-like ones, which elongate, inclose, and protect the green foliage- or floral-leaves, in their passage through the layers of earth as they grow up towards the light; these do not cease growing nor lose their turgescence until the delicate structures within reach the surface, when they are in no more danger, and require protection no longer. 
Scale-leaves situated above-ground are found on the buds of all woody plants, both on foliage and floral buds, i.e. both on the lowest portions of those rudimentary shoots which are destined to become leafy shoots, and in those which develop floral-leaves immediately above the scale-leaves. As a rule they present a hard, tough epidermis, are frequently covered externally with adhesive substances, hairs, and the like, and chiefly serve as a protection against the drying up of the little shoot within. When in springtime this axis begins to elongate, they are either immediately detached and thrown off, as in willows, or they may separate just sufficiently to permit the shoot to grow through, as in hoelveuteria paniculata. In many species they remain undisturbed and unaltered in their position; in others they separate and remain for some time at the base of the new shoot, as in the Walnut and Ash; whilst in others, again, they are turned back and soon fall off, as in the Mountain-ash (Sorbus Aucuparia), and in most species of the Horse-chestnut (Esculus). Esculus neglecta is especially noticeable in this respect, since its bud-scales, which are detached almost simultaneously, are large and red in colour, and when they fall off they cover the ground under the tree quite thickly as if with autumnal foliage. In most instances the scale-leaves on the buds of woody plants are brown and devoid of chlorophyll, and increase in size only slightly during their separation from one another; those of Gymnocladus, however, have a green colour, and increase in the spring to twice or even three times their former size.

On the buds of willows only a single scale-leaf is to be seen; limes have two, alders three, manna-ashes four, while in the beech, hornbeam, elm, and Celtis occidentalis there are very many bud-scales. If only a single scale exists, as in willows, it is deeply hollowed, and surrounds the part of the bud to be protected like a husk. If only a few scale-leaves are developed, as in Gymnocladus, they arch like a dome over the young green foliage-leaves; but if many, then they lie close above one another like the slates of a roof. It remains yet to be noticed that in all cases where the bud is protected by a single or only a few scale-leaves, their texture is always very tough and hard; but where many are present they are thin and membraneous. It has been previously mentioned that the stipules of many plants, as, for example, of figtrees, magnolias, and the tulip-tree (fig. 91), take the place of scale-leaves as protective coverings.

Foliage-leaves, unlike scale-leaves, exhibit an almost inexhaustible variety in their internal structure and external form, a fact partly due, no doubt, to the multifarious duties they have to discharge. The most important of all these functions is the manufacture of organic materials from inorganic foodon the efficient discharge of which the existence, not only of individual plants but of the whole organic world depends. This almost entirely devolves upon the foliage-leaves. Of course, in numerous instances cotyledons and floralleaves, the cortex of branches, and in some plants even aërial roots discharge this function; but all these are so subordinate that we may say that more 
than 90 per cent of the organic matter manufactured throughout the whole world every year should be reckoned to the account of the green foliageleaves.

That those members of the plant to which is allotted the manufacture of organic matter should exhibit such a marvellous diversity can hardly astonish us. For how infinitely varied are the conditions under which this function is performed in the different zones and regions of the globe! Even within the narrow confines of a restricted area, one may find habitats damp and dry, sunlit and shady, tranquil and tempest-tossed. Nor should we be surprised to find leaves of diverse shape at different heights on one and the same shoot, and that the foliage borne by any plant may exhibit variations in form in successive seasons of the year. And then we must remember that besides the most important function mentioned, foliage-leaves have often to provide for the irrigation of rain-water to the absorbent roots, to play the part of climbing organs, or to serve as weapons; more than this, they even act as organs for digesting imprisoned animals, with which last function is associated very curious metamorphoses of foliage-leaves. By the segmentation of the leaf into those parts, into the blade, leaf-stalk, and sheath with stipules, an allotment of these various functions becomes possible; but evidently, in consequence of this division of labour in one and the same leaf, the structure becomes much more complex and manifold.

A distinctive name has been given to each shape by botanists, who have endeavoured to define the different forms by descriptions. For foliage-leaves alone perhaps a hundred different expressions have been used to shortly designate the most remarkable varieties. Since these terms of botanical nomenclature can be combined and varied according to the actual facts, we are able to describe the many thousands of differently-shaped foliage-leaves, briefly and tersely, and-what is of especial value, and really the most important aim of these descriptions - another person is able from them to picture the object to himself.

First of all, let us describe the leaf-blade, the outline of which may exhibit every imaginable geometrical form: obovate, circular, elliptical, rhombic, rhomboidal, triangular, pentagonal, \&c. Very often, too, the leaf-blade is much elongated, and the margins are parallel to one another; this is known as linear. The free end of the blade is sometimes pointed, sometimes blunt, and sometimes drawn out into a long point; occasionally, again, it is truncate, pressed in, or cut out in the form of a heart. The base of the leaf-blade may be narrowed and attenuate towards the stem; or its outline may be kidneyshaped, arrow-shaped, lanceolate, ovate, spathulate, crescent-shaped, \&c. The blade is either undivided, when it is termed entire, or the margin is to a greater or less extent indented; if the indentations are but slight, the leafblade is said to be crenate, serrate, or dentate, but if they are considerable, the margin is said to be sinuous or incised; if, again, the indentations go more 
deeply into the green surface of the blade, the expressions lobed, cut, divided, or partite may be used. A partite leaf appears as if composed of several leaflets, and such leaves have also been termed compound leaves, especially when the already-described pulvini are present at the base of the individual leaflets.

The distribution of the strands traversing the green tissue is connected in the closest manner with the structure and shape of the leaf-blade. Expressions have been borrowed from the anatomy of the animal body to designate these strands, and they are called indifferently veins, ribs, and nerves. The term "vein" has some justification, since most of these strands contain cells and vessels which serve to conduct fluid materials to and fro; but since there are also strands which have nothing to do with this conduction, which are developed exclusively for the support of the whole blade, the name is unsuitable, and can only be used figuratively. The same may be said of the term "ribs". In many instances the strands in question, of course, do present the appearance of ribs, and the whole arrangement of them in a blade may be compared with a skeleton upon which the soft portions are attached. We even speak of "leaf-skeletons", an expression which seems justifiable, since by removing the soft portions a white framework is obtained exhibiting a great analogy to the bony skeleton of an animal body. Thus if the blades of green foliage-leaves are allowed to macerate for some time in water, the epidermis and thin-walled green tissues decay, while the tougher strands remain intact; if these leaves are now dried and brushed, all the soft disintegrated parts are removed, and only the skeleton of the leaf remains, in which, as in the skeleton of an animal, larger and smaller structures may be recognized, connected together in the most varied manner. But from the fact that most of the strands, together with those cells which serve to strengthen the whole blade, also contain conducting tubes; that many of them indeed consist only of conducting vessels, it is hardly permissible to speak of skeletons, or to apply the term "ribs" to the strands so beautifully interlaced. Finally, the name "nerves" is still more unfortunately chosen, for the strands of leaf-blades have no resemblance to animal nerves, either in structure or function. Consequently this designation must be also condemned, although it is the one most often employed by descriptive botanists.

It is simplest and most correct to call the structures in question what they really are, viz. strands, strands consisting of elongated and fibrous cells, which are combined in the most diverse ways with tubular and pipe-like vessels, and whose elements serve partly for the conduction of fluid materials to and from the green tissues, and partly to afford the necessary protection to the whole blade-protection against strain, pressure, and bending, according to the need of the moment.

In looking for the origin of the strands on a leaf-blade, we are always directed to the stem from which the leaf in question springs. In other words, the first trace of those strands, which traverse the leaf-blade as a richly-articu- 
lating system, is already found in the stem. From this they extend through the leaf-sheath and petiole to the base of the blade. This last is therefore in a manner the entrance-gate for the strands, and as soon as they have passed it a division takes place not unlike that of a stream which flows from a narrow valley into a plain, and there breaks up into numerous larger and smaller: branches; or they may perhaps be still better compared to an aqueduct whose main stream is inclosed and strengthened by masonry and embankments, but which branch out, at the confines of the town which has to be supplied with water, into several subordinate conduits which penetrate the different districts, and then again break up into numerous smaller water-pipes leading to the buildings and other places of consumption.

We may distinguish two kinds of distribution in respect of the course of these strands as they enter the leaf. In the one case there is only a single thick strand, the primary strand, which distributes itself and breaks up inside the narrow gate. In the other, three or more distinct main strands pass over, side by side, into the blade, each following a separate course. As a rule, these are connected by bridges and inter-networks. Thus we distinguish between leafblades with a single main strand and those with several.

Leaf-blades with one main strand may be sub-divided into two groups according to the form and course of the lateral strands which arise from the primary one. Either these lateral strands are all weaker than the main one, and originate from it successively, at intervals, like the ribs of a spinal column, or like the barbs on the axis of a feather, when we speak of a feather-like (or pinnate) arrangement of the lateral strands (see figs. 149 1, 2, 3,4, 5, 6, 7, 10, 13); or the lateral strands are almost as strong as the main one, arise from it directly at the base of the blade, and run out from this point like rays towards the margin of the lamina. This arrangement of the lateral strand is called radiating (see figs. $149^{8,9,11,12}$ ).

When the lateral strands are arranged like a feather, it generally happens that they are alike in the matter of strength, that they are distributed symmetrically over the whole blade, and originating at fairly equal intervals from the main strand, take, at least at first, a parallel course. More rarely it happens that stronger and weaker lateral strands alternate, and that they diverge from the primary one at unequal angles. In the Camphor Tree (Laurus Camphora, fig. $149^{4}$ ), the Cinnamon Tree (Cinnamomum), and many other plants related to the Bay Laurel, this peculiarity is found, viz. that two lateral strands which proceed from the lower third of the main one are stronger than the others, looking as though a three-pronged fork had been inserted in the leaf. In the Wall-Pellitory (Parietaria), whose leaves show a similar character, stronger and weaker lateral strands alternate, and, strangely enough, the stronger spring from the main strand at an acute and the weaker at a right angle. For the rest, the lateral strands with feather-like arrangement may be distinguished as reticulate, looped, arched, and undivided. 
Those lateral strands are termed reticulate (dictyodromous), which break up into a delicate net-work soon after their origin from the primary strand, or at least before they have reached the margin of the blade. The meshes of the net-work are of almost equal size, so that it is impossible to distinguish in the confusion of small strands near the margin of the blade any particular one more vigorous than the others. The leaf of the Wild Pear (Pyrus communis) is given as an example of this form in fig. $149^{1}$. The same distribution of strands, however, is found in very many other plants allied to pear-trees, as also in willows, rhododendrons, and species of barberry and sage.

The lateral strands called looped (brachydodromous) run fairly straight and distinct towards the margin, but before reaching it they bend round in a graceful sweeping curve, towards the apex, uniting with the next lateral strand above, and with it form a loop. Such loops ean always be seen standing plainly out from the delicate net-work of small strands, and the arrangement may be recognized at the first glance. It is observed in the leaves of the Mahaleb and common Cherry, in the Buckthorn (Rhamnus Frangula and Wulfenii, see fig. 1492), in myrtaceous plants (Myrtus, Metrosiderus, Eugenia, see fig. $149^{13}$ ), in many species of dock and nightshade, and especially in rough-leaved plants (Boraginaceæ). The net-work of fine strands inserted between the laterals is often so delicate that it is scarcely visible to the naked eye, and then only a series of bold loops, like arcades, is to be seen in each half of the leaf. In the Comfrey and Lungwort (Symphytum and Pulmonaria) these loops are developed at some little distance from the margin of the leafblade. In the cherry and buckthorn, on the other hand, the loops are quite close to the margin. The lateral strands are frequently very delicate, and extend in a straight line from the primary strand right up to the margin, when they bend suddenly round, like a knee, almost forming a right angle. The outer limb of this right angle then runs parallel to the margin, and unites with the knee of the next upper lateral strand. In this way we have a strand running parallel with the leaf-margin connected with the central primary strand by cross-ties. This looped form occurs very regularly in the Myrtaceæ, but many tropical Moreæ are also distinguished by it, and the leaves of the Forget-me-not (Myosotis) also exhibit this peculiar arrangement of lateral strands (see fig. $149^{10}$ ).

Arched strands (kamptodromous) are those which run out from their place of origin on the main strand towards the margin of the leaf, which, however, they never reach, but turn in an arch towards the leaf-apex, and there lose themselves without forming definite loops. As a rule, the places of origin are crowded together in the lower half of the main strand, and the two uppermost arched lateral strands then inclose an oval central area. The Cornel (Cornus mas), illustrated in fig. $149^{3}$, is chosen as an example of this form.

Those lateral strands are called undivided (craspedromous) which run in a straight line from the main strand to the margin and there terminate. They end 
either in the apices of the lobes or serrated teeth of the margin, as in hazels, oaks, chestnuts, hornbeams, and hop-hormbeams (see fig. $149^{7}$ ), or in the indentations

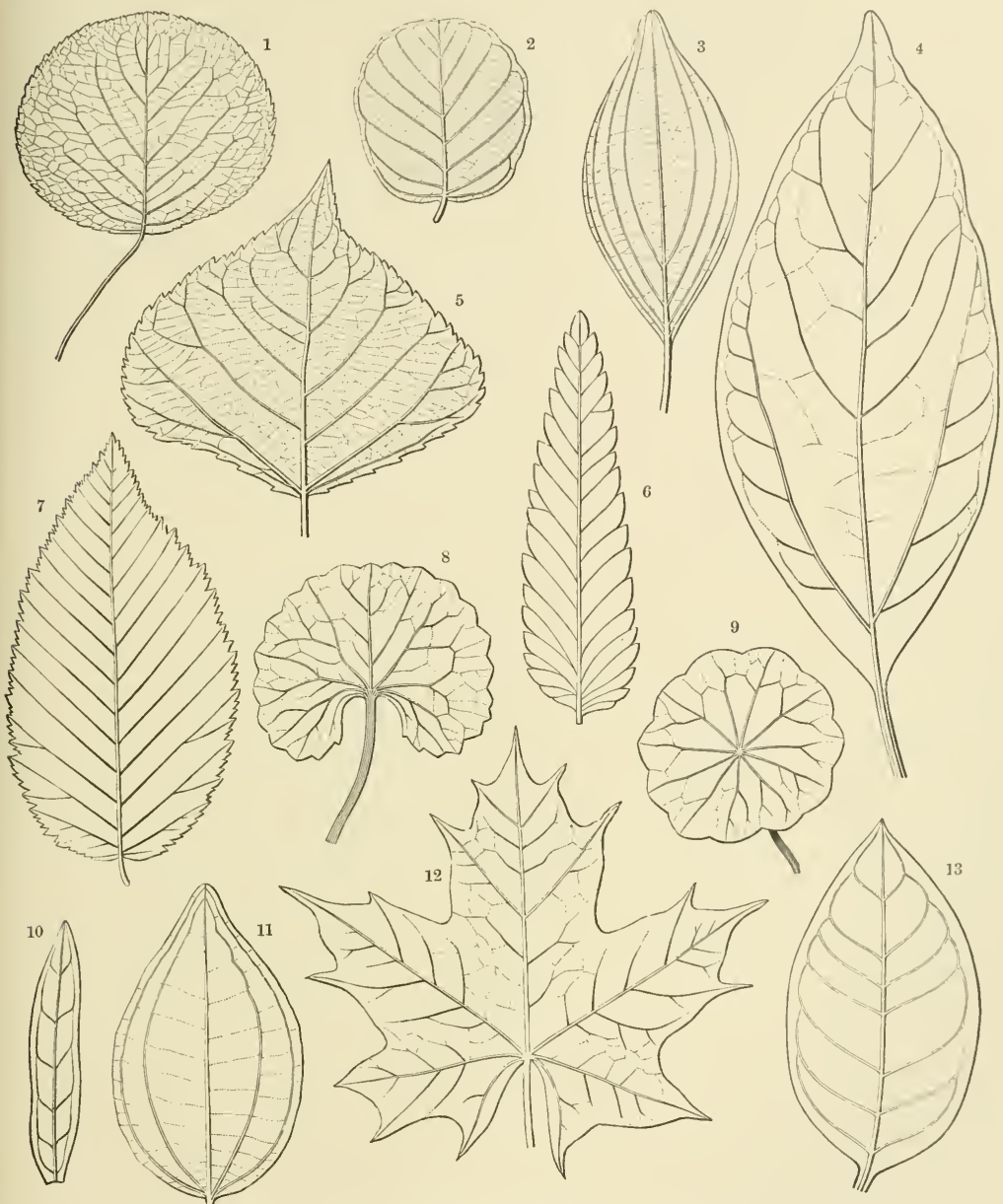

Fig. 149.-Arrangement of Strands in the blades of Foliage-leaves. Forms with one main strand.

1 Reticulate (Pyrus communis). 2 Looped (Rhamnus Wulfenii). 3 Arched (Cornus mas). 4 Arched; the two lowest lateral strands much stronger than the others (Laurus Camphora). ${ }^{5}$ Reticulate-pinnate (Populus pyramidalis). ${ }^{6}$ Undivided strands, ending in the incisions of the crenate leaf margin (Rhinanthus). ${ }^{7}$ Undivided strands, terminating in the projecting teeth of the margin (Ostrya). 8 Reticulate (Bydrocotyle asiatica). 9 Reticulate strands in the blade of a peltate leaf (Hydrocotyle vulgaris). 10 Looped (Hyosotis palustris). ${ }^{11}$ Arched (Phyllagathis rotundifolia). 12 Radiate and undivided (Acer platanoides). 13 Looped (Eugenia).

of the margin, as in Bartsia, Eyebright, and Yellow-rattle (Bartsia, Euphrasia. and Rhinanthus), and, generally speaking, in all Rhinanthaceæ (see fig. $149^{6}$ ). 
Lateral strands with radiate arrangement exhibit conditions quite similar to those with a feather-like course. Frequently they are reticulate as in geraniums and mallows, the Judas Tree (Cercis Siliquastrum), and many Umbelliferæ, as, for example, in the leaves of Hydrocotyle asiatica, illustrated in fig. $149^{8}$. In some water-lilies looped lateral strands are also observed, whilst arched lateral strands are very characteristic of Melastomaceæ. In these Melastomaceæ (see fig. $149^{11}$ ) the lateral strands originate from the main strand at the base of tl.e leaf-blade, and travel towards the apex of the leaf in elegant, sweeping arches parallel to the margin. Numerous cross strands, like ties, connect the lateral strands with one another and with the primary one, giving an extremely ornamental appearance to this class of leaf. The leaves of maples exhibit lateral strands radiating towards the margin; this is particularly well shown in the Norway Maple (Acer platanoides), the leaf of which is illustrated in fig. $149^{12}$. Planes (Platanus) also have lateral strands running towards the margin and terminating in the points of the leaf, but it is worthy of remark that in some species the branching of the lateral strands from the primary one does not take place immediately at the base of the blade, but somewhat above it. A peculiar modification of lateral strands with a radiating arrangement is observed in many so-called peltate leaves (see fig. $149^{9}$ ). In these leaves the blade is more or less circular, and is connected with the central stalk as the cover of an umbrella with its stick. The strands radiate out in all directions from the point of attachment of the stalk, and without close investigation of the relations between such a leaf and its petiole, it is often quite impossible to say which of the radiating strands is to be regarded as the main one. This arrangement is found in most species of Pennywort (Hydrocotyle, cf. fig. $149^{9}$ ), in Nasturtiums, Ricinus, and Nelumbium; the last-mentioned plant has also this peculiarity, that its peltate leaves are somewhat depressed in the centre like a bowl.

Leaf-blades with several main strands offer far less variety than those with only one. The margin is almost always entire, and they are generally elongated. The most noticeable variations consist in the number of the main strands which enter the base, in their varying thickness, and in the direction which they take in the blade. We have also to consider whether they divide like a fork, and whether the lateral nerves which they give off are developed as cross-connections, or as a fine-meshed net-work.

When the latter is the case, that is to say, when the main strands entering separately into the blade, and travelling towards the apex of the leaf are linked together by a net-work of lateral strands with angular meshes, they are then said to be apical (acrodromous). The numerous broad-leaved species of Plantain (Plantago), species of Hare's-ear (Bupleurum) belonging to the Umbelliferæ, the leaf of one species of which (Bupleurum falcatum) is represented in fig. $150^{1}$, show apical main strands. In the leaf of the Hare's-ear the main strands are crowded together in the narrow base of the blade, and the meshes 
of the net-work between these strands are principally formed from transversely-running lateral strands. In the Australian Leucopogon Cunninghami, one of the Epacrider (see fig. $150^{6}$ ), the very narrow meshes of the net-work are, on the other hand, formed by the longitudinally-running lateral strands. A very peculiar form of the apical arrangement of strands is that which the older botanists called pedate. Three distinct strands enter the base of the blade from the leaf-stalk; the central strand is relatively thin and passes

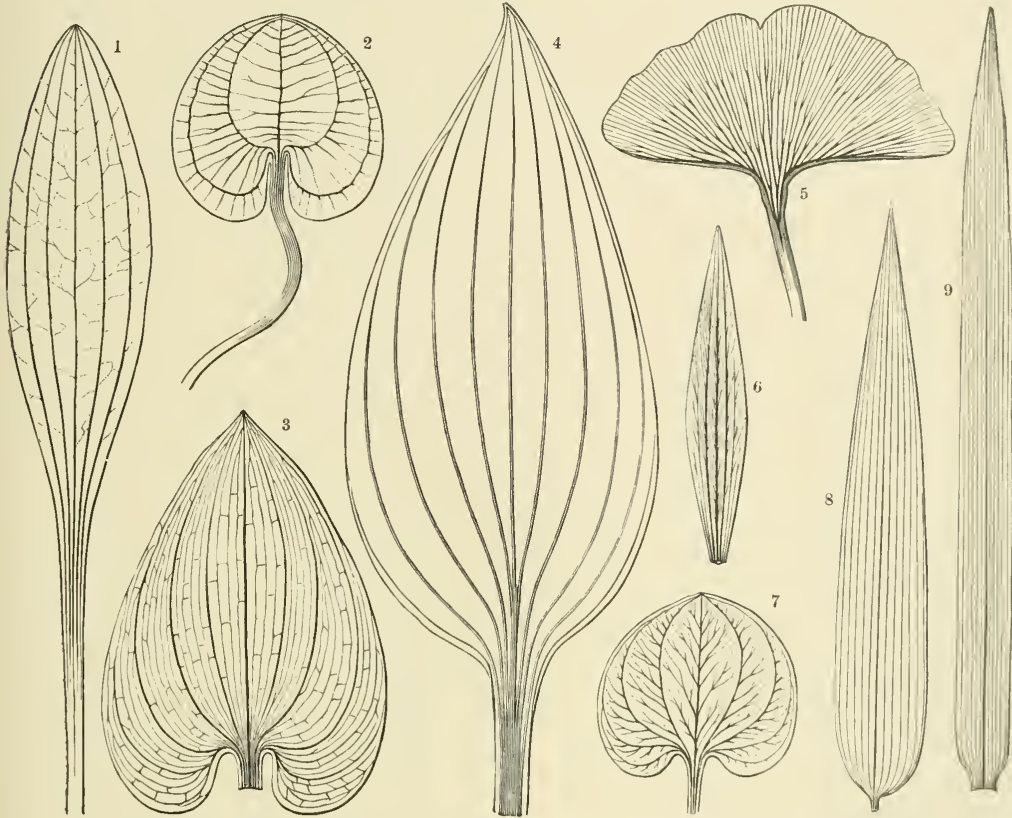

Fig. 150.-Distribution of Strands in the blades of Foliage-leaves: Forms with several main strands.

1 Apical or acrodromous (Bupleurum falcatum). 2 Curved or campylodromous (Hydrocharis Morsus-rance). 3 Curved (Maianthemum bifolium). $\quad$ Curved (Funkia). 5 Fan-like or diadromous (Ginkgo biloba). 6 Apical or acrodromous (Leucopogon Cunninghami). 7 Apical, "pedate" (Parnassia palustris). 8 Parallel (Bambusa). 9 Parallel (Oryza clandestina).

direct towards the leaf-apex; the two lateral are thick, bend round like an arch to the right and left as soon as they have entered the blade, and then send arched lateral strands toward the upper margin, which are almost equivalent to the central main strand, and may at first sight even be taken for main strands. This arrangement is found in the Birthwort and Asarabacca (Aristolochia Clematitis and Asarum Europaum), in numerous violets and Ranunculaceæ, and in the Grass of Parnassus (Parnassia palustris), the leaf of which is shown in fig. $150^{7}$.

Main strands which enter the blade in large numbers, but always separately, 
and of which the external ones travel towards the apex of the leaf in an arch parallel to the margin, are termed curved (campylodromous). The lateral strands which are usually so delicate that they cannot be recognized by the naked eye always form spans, connecting the adjacent main strands transversely. In the leaf of the May Lily (Maianthemum bifolium) depicted in fig. $150^{3}$, the number of main strands is very large and the span-like laterals are short. In the leaf of the Frogbit (Hydrocharis Morsus-rance, see fig. $150^{2}$ ) only five main strands traverse the leaf-blade, the connecting ties being remarkably long and distinct. In Bananas and Scitamineæ (Musa, Maranta, Zingiber, Canna) the curved main strands look like arched laterals branching off from a single central strand, but on looking eloser it becomes evident that the thick rib traversing the centre of the leaf, like a keel, is not a single main strand, but consists of many separate strands which are embedded in a large-celled mass of tissue. These main strands are inclined one above the other laterally away from the keel, travel towards the leaf-margin and there curve up towards the apex. In bananas this bundle of separate strands, surrounded by parenchyma, extends from the base to the apex; in species of the genus Funkia (see fig. $150^{4}$ ) only part way to the middle of the blade.

When several distinct main strands enter the blade from the leaf-sheath or leaf-stalk, running parallel to one another in a relatively wide area without dividing and not converging until the actual leaf-apex is reached, they are termed parallel (parallelodromous). Such an arrangement of strands is found in many liliaceous plants, in orchids, rushes, sedges, and especially in the thousands of different grasses. The strands enter the blade either from a broad sheath, as, for example, in Oryza clandestina (see fig. $150^{9}$ ), when their separate nature can be easily recognized even at the base of the blade; or they enter by a sort of stalk on which the blade is inserted, as in bamboo leaves (see fig. $150^{8}$ ), where the strands entering the base of the blade appear bent like a knee. Parallel strands are usually of unequal thickness, the central being almost always stronger and more vigorous than the lateral. But even among the lateral, thicker and thinner often alternate in a manner definite for each species. In the slender False Brome Grass (Brachypodium sylvaticum), for example, from two to five weaker strands always appear between every pair of stronger ones; the weaker are often so exceedingly delicate that they cannot be recognized by the naked eye. The unaided vision recognizes eleven almost equally thick strands in the leaf of Oryza clandestina, represented in natural size in fig. $150^{9}$; under a lens five more delicate strands are to be seen between every pair of these. When lateral strands are present, connecting the adjacent parallel main strands, they always take the form of transverse ties.

Finally, we have here to consider that remarkable arrangement of strands which is called fan-like (diadromous). A few separate main strands enter the leaf-blade, divide up repeatedly into forked, straight-running branches, and the ultimate twigs terminate at the upper margin of the leaf. This course of the 
strands goes with a very peculiar form of leaf, which may best be compared to an open fan. The Maiden-hair Tree (Ginkgo biloba, see fig. $150^{5}$ ) may serve as an example of this distribution of the strands, which, on the whole, is not common. It is also observed in several true ferns (e.g. Adiantum arcuatum, Acrostichum sphenophyllum, and Livingstonei). With regard to the Ginkgo, it should be mentioned that as a rule only four distinct strands enter the blade from the leaf-stalk; two central which are very delicate, and two lateral which are very strong, and from which arise a large number of fine, forking strands running upwards.

Besides the arrangements of strands in leaf-blades here described, there are many which cannot readily be brought under the limits defined; in the same way there are intermediate forms which may be placed just as well in one as in another of our artificial divisions, and which we try to describe clearly by connecting the technical terms together. For example, we find intermediate forms between arched and reticular strands which are described as arched-reticulate, and so forth.

The fact should be emphasized that the distribution and arrangement of the strands in any given species is remarkably constant. This, however, is by no means the case in genera and families. Of course there are plant-families the whole of whose members exhibit marked agreement in this respect, as, for example, the Rhinanthaceæ, Melastomaceæ, and Myrtaceæ; but, on the other hand, instances are not lacking where the reverse is the case. Thus the various genera of Primulaceæ present the widest varieties, and even the individual species of the genus Primula differ more from each other in the arrangement and course of the strands than perhaps the Myrtaceæ from the Boragineæ. Nevertheless the accurate determination and description of the distribution of these strands in the leaves is very important for that branch of botanical study, the object of which is to provide criteria for the discrimination of species. The careful investigation of the distribution of strands in leaves is, perhaps, of the greatest interest to the palæo-botanist, the investigator of pre-existing vegetation. Those parts of plants which have come down to us from earlier periods, embedded in geological formations, consist principally of single leaves or fragments of leaves, often of very insignificant appearance. In these fragments often we cannot even recognize plainly the edge, much less the whole contour of the blade; but the strands themselves, and the net-work which intervenes between the coarser strands, may be distinguished on the smallest fragments. Often enough the palæo-botanist has only such scanty remains to refer to when he seeks information about the species of plants with which our globe was covered in long-past ages. Consequently even the most insignificant-looking bit of leaf-network becomes of the highest importance. Just as an investigator, busy with the history of the human race, can draw certain inferences from the characters of a hardly decipherable papyrus roll about the state of the household, about the political institutions, the customs, manners, and civilization of the population settled more than two thousand years ago in the valley of the Nile, so can the botanist, 
investigating the history of plants and attempting to clear up the connection between past and present, recognize from fossil leaves the species living in periods long past, and read the condition of vegetation as it existed many thousands of years ago. Although the results of investigation hitherto obtained in this field are still imperfect, and although these results may receive manifold additions and corrections as more abundant materials come to hand, still the history of vegetation is already exposed in its principal features, and that which has been obtained in this respect during the comparatively short period of half a century is noteworthy in a period of remarkable additions to natural knowledge. In imagination we see replaced the woods and meadows which long agres ago adorned the continents of the Coal period; colonies of slender calamites, the rigid fronds of the cycads, and thickets of countless ferns rise up before us; we are able to sketch landscape pictures of the Jurassic and Cretaceous periods, and to see the banks of the rivers fringed with species of Cinnamomum, evergreen oaks, walnut-and tulip-trees. And all these pictures of the vegetation of the most remote periods would harlly have been possible except on the basis of the determination of species with the help of the minutest investigations into the arrangement and distribution of the strands in the fossil leaves.

When the leaves of fossil and living plants are compared, we notice that the strands in the former appear more distinct than in the fresh succulent green blades. This is in consequence of the fact that in living plants the strands are often embedded in parenchymatous tissue so that they cannot be seen on the surface, while in fossil plant-remains the parenchyma has been wholly destroyed and only the strands have been preserved. When the strands run in the interior of the substance of a leaf, and are not visible at the surface, they are hidden, or, to use the technical term, hyphodromous. Succulent leaves almost always have such hidden strands, which may be contrasted with those which project above the general level on either side of the leaf. On the whole this latter condition is rare, most usually the strands project on one side only, and that the lower surface. Often we find a plexus of ridges on the under side, and one of grooves on the upper side corresponding to the course of the strands. The enormous circular leaves of the Victoria regia, which float on the surface of the water, have very strong projecting ribs on the lower side. In leaves of submerged water-plants, however, the strands are insignificant; many are even destitute of vessels, and present only strands of elongated cells, as, for instance, the leaves of the celebrated Vallisneria. This is easily understood, as the need of resisting pressure and bending in submerged leaves is very slight. Nor do submerged plants require special conducting tubes for their food-salts. Numerous other striking relations existing between the inner structure of the leaf-blade and the peculiar conditions of the habitat of plants have alrearly been discussed, and we need here merely refer to the description of flattened, rolled, succulent, spiral, arched, hinged, and tubular leaves occurring in the section which begins on p. 209 of this volume.

The form of the leaf-stalks, stipules and leaf-sheaths, in their dependence on 
the peculiar conditions of the environment, has also been repeatedly diseussed, and it is enough to remember here that the prineipal duties of the leaf-stalk, as the support of the light-needing green blade, are to turn and twist it, to raise and lower it, to bring it always into a position where it will be properly illuminated; to keep it in that position in spite of storm and tempest. The chief function of stipules consists in screening young and tender leaves-not yet emerged from the bud-from excessive illumination, and protecting them from too much loss of heat on clear nights. The stipules in many cases actually serve as bud-scales, as may be seen in the fig-tree, where the tiny leaf-blades are rolled up together and inclosed in the spathe-like stipules. When this is the sole function of the stipules, they become detached after the unfolding of the leaves wrapped round by them. Consequently, shortly after the unfolding of the foliage of oaks, beeches and other trees, the floor of the forests formed by these trees is strewn with enormous quantities of fallen stipules. When the stipules persist at the sides of the leafstalk and become green, there can be no doubt but that they supplement the green leaf-blades in their function, and like them manufacture organic substances from inorganic food. In the Woodruff, Bed-straw, Madder (Asperula, Galium, Rubia) the stipules actually possess the same size, shape and colouring as the blades of the real foliage-leaves, and thus a star of green leaf-structures is formed, to which these plants owe their name of Stellatæ. In the Pansy (Viola tricolor) and numerous species of violet allied to it, the stipules are green and sometimes larger than the leaf-blade, at the base of which they occupy a subordinate position.

A peculiar formation is observed in the Yellow Vetch (Lathyrus Aphaca), a common weed in the fields of Southern Europe, though not so frequent in England. In this plant the leaves are completely transformed into tendrils which serve as climbing organs; the two stipules which stand at the base of the metamorphosed leaf have, on the other hand, assumed the function of leaf-blades; they are very large, provided with green tissue, of arrow-like or lanceolate contour, and at a cursory glance may be easily taken for leaf-blades. It has already been mentioned on p. 335 that a like modification of function occurs in many Australian acacias, the foliage-leaves of which are devoid of green blades whilst the leaf-stalks are developed as green, flattened, outspread organs, the so-called phyllodes.

In all these cases we have only treated of the most important function of foliage-leaves, that is, the formation of organic materials from inorganic food in sunlight. But as mentioned previously, the foliage-leaves of many plants are assigned other functions, which again require certain peculiar adaptations, and contribute not a little to the great variety in the form of this organ. One series of these metamorphoses, viz. the transformation of the leaf-blades and leaf-stalks into traps and digestive organs in insectivorous plants; the metamorphosis of blades, leaf-stalks, and stipules into weapons; and the development of furrows and channels on different parts of the foliage-leaves for the irrigation of rain-water; and finally the transformation of foliage-leaves into inere seales, as in the switeh plants, \&c.-all these have already been fully treated of in earlier chapters. But 
a further series of such transformations, especially the metamorphosis of parts of foliage-leaves into tendrils, hooks, and claws, with the help of which the stem is able to climb up firm supports towards the light, and the transformation of the leaf-sheaths into mechanisms for protecting flowers against unbidden guests; the consideration of these must be deferred till we deal with climbing contrivances and protections for flowers in general; here, there only remains to be considered the production of floating contrivances in certain marsh and water plants, and the development of special cells to assist those foliage-leaves which are unprovided with scale-leaves in breaking through the soil.

Floating arrangements occur in only a few species of plants, most noticeably in the Brazilian Pontederia crassipes, and in the few species of the water-chestnut (Trapa). In both instances the leaf-stalks are swollen up into floats, and remind one to some extent of the swollen utricular leaf-stalk of Cephalotus, Sarracenia, and of pitcher-plants. They are distinguished from these by the fact that the buoy-like swelling is quite closed, and that the partitioned interior neither contains digestive organs, nor is beset with spines, \&c., to hinder the exit of imprisoned animals. Pontederia crassipes is not fixed in the mud beneath the water by roots, but the plants float freely on the surface of the pond. It is of great importance to these plants that they should have a small specific gravity, and that their leaves, grouped in rosettes, which have been unfolded above the water, should offer a large surface to the air, while at the same time the illumination of the green portions should not be encroached upon. Both these requirements are met by the bladderlike leaf-stalk, and these strange floating plants are driven by the wind like ships hither and thither over the surface of the water.

The plants of water-chestnut are held fast to the muddy bottom under water by roots, and are not adapted to floating freely. The submerged leaves are finely divided like a comb, and have such a small specific gravity that when detached from the stem they immediately rise to the surface of the water. The uppermost leaves lying on the surface of the water, and grouped into rosettes, have rhomboidal, tough, almost leathery blades, and these also do not sink when they are isolated, and therefore it is difficult to see what advantage is afforded in this instance by the swollen leaf-stalk. But when in the height of summer large heavy fruits are seen to be produced from the flowers developed amongst the leaves of these floating rosettes, it then becomes evident that the floating capacity of the rosette-leaves must be maintained, lest they be drawn underneath by the weight of the nuts and placed in a position as unfavourable as could be imagined for the proper discharge of their functions.

In the subterranean buds of perennial plants the rudimentary foliage-leaves are usually surrounded by scales, which function as shields and screens, and in particular play the part of protective organs in the work of breaking through the ground. Most of these sheath-like scales, as already stated, grow up with the elongating buds until the soil has been pierced, and their points strengthened by turgid cells serve as actual earth-breakers. But in some plants which survive through 
the winter, with underground buds or bulbs, the young sprouting foliage-leaves do not have this assistance; they must carve their way through the soil unaided, and press above the surface without a sheathing envelope. Accordingly they have to bore through a more or less thick layer of earth, often a stiff clay; or one perhaps containing pointed stones and angular grains of sand. Now in order that the foliage-leaves traversing this rugged and uneven path may suffer no damage, they are variously folded and twisted together so as to form a cone; and most important of all, the apex of this cone, which operates like a ground-auger, and therefore exercises a strong pressure on the soil, is armed with special cells. These cells have a great resemblance to those at the apex of the sheath-like scale-leaves, and to those of the knee-shaped bent cotyledon of the onion (see p. 606). In many plants possessing lobed or deeply-divided leaf-blades, the boring apex of this cone is formed by a bend of the leaf-stalk, which is doubled over like a hook. Thus in the foliageleaves of the Yellow Monkshood (Aconitum Vulparia, Lycoctonum, \&c.) it is not the apex of the leaf which emerges first from the ground but the convex part of its bent and knee-like stalk. As long as the leaf is still occupied in boring, the delicate free apices of its lobes are directed inwards and downwards, and not until the hooked leaf-stalk has emerged above the surface of the soil does it straighten and draw the leaf-blade out of the ground. The free points of the leaf-blade, which were hitherto directed downwards, are inclined in the opposite direction when they arrive above the earth, and the whole leaf then unfolds into an expansion parallel to the surface of the ground. An exactly similar process is observed in large ferns with underground winter buds, e.g. in the common Male-fern (Aspidium Filix-mas). The fronds at the end of the root-stock are spirally rolled, their delicate segments are packed closely together, one above the other, and covered by the strong rachis of the leaf as by a thick hoop. Only the back of this rachis comes into contact with the forest soil as it is broken through; the rachis prises up the top layer of the soil in its gradual unrolling, and the delicate segments are only unfolded when the part of the axis in question has emerged and straightened itself.

The earth is broken through in a very peculiar manner by the peltate leafblades of Podophyllum peltatum. As long as the leaves of this plant are still small and below the ground, they resemble a closed umbrella; the folded blade is directed downwards, and nestles close to the thick stalk, which grows straight up. At the free end of the stalk, which would correspond in position to the ferule of an umbrella held upright, is found a group of thin-walled, turgid cells, without chlorophyll, situated like a white knob at the converging-point of the leaf-strands. When the leaf-stalk grows in height, it is this cell-group which presses on the layers of earth above it, and it is the first to appear at the surface. The leaf-blade, still furled to the stalk, is then raised up through the hole thus bored. Once above the surface, the blade expands just like an opening umbrella. The above-mentioned group of cells, having served as a buffer, now loses its turgescence, but remains visible as a white spot at the centre of the brownish-green expanded leaf-blade. In the species of the genera Acanthus and Hydrophyllum, which are characterized by divided leaves, 
the lobes of the blades whilst still under the ground are depressed as in Podophyllum, but here the penetration is accomplished by means of peculiar bumps and bladder-like protuberances on the uppermost lobes, which again consist of strongly turgid cells. In the Asarabacca (Asarum) it is the apex of the lower leaf folded together lengthwise which is composed of turgescent cells, and which growing upwards, presses the earth apart like a wedge. In the Broad-leaved Allium, Dog'stooth Violet (Allium ursinum and Erythronium Dens Canis), in the Star of Bethlehem and Hyacinth, and many other bulbous plants, also in numerous orchids of our meadows and woods whose buds pass the winter embedded in deep soil, the apex of the lowest leaf-blade is transformed into an actual ground-auger, usually shaped like a hood or folded cap-like over the apices of the other leaf-blades of the plant. A group of cells without chlorophyll is always found on the apex of that leaf which envelops the others, and this apex may be plainly distinguished by its white colour. In most of the plants examined the cells are thin-walled but very turgid; only a few present thickened walls, as, for example, the Broad-leaved Allium (Allium ursinum), where the whole leaf-apex is almost horn-like. This group of turgescent cells always forms the apex of the leaf-cone growing out from the subterranean bud; afterwards when this cone has grown up, and the leaves are spread out over the soil, the formerly tense cells of the leaf-apex collapse, dry up and present a withered appearance. In the Asarabacca and in many orchids the apices of the mature and lower leaves are regularly browned, and look as if burnt, even when they have not been actually injured in penetrating the ground.

The term floral-leaves comprehends all those which are directly or indirectly concerned in the processes of fertilization, and in the production of the embryo. First of all we have the leaf-structures within which the germ-cell is formed, that cell from which the embryo proceeds after fertilization. Then there are those in which arise the fertilizing cells known by the name of pollen-grains. Finally all those which are concerned in bringing about the union of the pollen-cells with the germ-cells, or whose task is to protect these two kinds of sexual cells during their development from injurious external influences. Since the processes only shortly indicated here will be fully described in the second volume of The Natural History of Plants, and since the forms of the floral leaves will be considered in these descriptions, we need not here give a detailed representation of these stractures. In the pages which follow they will only be treated of so far as is necessary for the comprehension of the architecture of the whole plant, and of a series of botanical terms.

With regard to the succession and arrangement of floral-leaves, it has to be noticed as one of their most characteristic features that the last and uppermost floral leaves are always very close together, and are usually developed as closely appressed whorls. These assemblages of floral-leaves together form the flower. The axis which bears the flower at its free end is termed the flower-stalk (pedunculus).

The axis which is terminated by the flower is only in rare instances, viz. in a few annual herbs, the direct continuation of the shoot which is produced from the 
first bud (plumule) situated above the hypocotyl (cf. fig. 2). In this case the floral leaves, collected together to form the flower, follow directly above the foliageleaves on the same shoot. Such a flower is called terminal. Much more frequently the flowering axis or peduncle is inserted laterally on an older shoot, and originates close above a leaf, called a subtending leaf; here we speak of lateral flowers. Usually several flowers are grouped in a definite way, and the term inflorescence (inflorescentia) has been introduced to distinguish these groupings. The subtending leaf (folium fulcrans) either agrees in general character with the lower foliageleaves, and is then said to be "leaf-like", or it differs in shape and size as well as in colouring, and is then spoken as a bract (bractea).

These leaves, differing from foliage-leaves, always have a special relation to the processes of fertilization; and are therefore to be reckoned with the floral-leaves. Frequently a whole inflorescence is surrounded and supported by a single enormous bract, and in such inflorescences, which are very characteristic of palms and aroids, the bracts at the base of the individual flower-stalks are usually undeveloped. This large common bract is called a spathe (spatha). The Climbing Palm (Desmoncus) illustrated in fig. $157^{3}$, has such a spathe beset with prickles. It sometimes happens that some of the flowers of the inflorescence do not develop, and that then bracts are to be seen without flowers. If such "empty bracts" are found crowded together at the base of the inflorescence arranged at one level, or are there grouped in very close spiral revolutions, we speak of an involucre (involucrum). Sometimes they are to be seen at the apex of the whole inflorescence, the group forming what we may call a crest. Minute, stiff, dry bracts, without chlorophyll, in the centre of thickly crowded inflorescences are called palece (palece).

In flowers we distinguish perianth-leaves, stamens, and carpels. The perianthleaves are arranged either spirally or in whorls. The former arrangement is observed most noticeably in the cacti, of which several species, including Cereus, Mamillaria, and the remarkable hedgehog-like Echinocactus capped by its flower, are illustrated at vol. II., p. 787. In the many-membered perianth inclosing the flowers of this plant more than a hundred perianth-leaves are so arranged at small vertical intervals along a spiral line that the smallest stand lowest, the largest uppermost, not unlike the leaves of the involucral cup around the capitulum of a composite. This spiral arrangement, however, is rare, at least in such a striking form. Much more frequently the perianth-leaves form two successive whorls. If the lower whorl consists of green leaves, which agree in texture and in general appearance with foliage-leaves, while the upper is composed of more delicate leaf-structures displaying all possible colours except green, the lower is called the calyx, and the upper the corolla. If all these perianth-leaves are shaped and coloured very much alike, so that there is no marked contrast between the whorls, we then speak of a perigone (perigonium). This may be either green like a calyx, or coloured like a corolla.

The stamens (stamina), the "attire" of the older botanists, are, like the perianth-leaves, usually whorled, or, more rarely, arranged in spirals. Each stamen VoL, I. 
consists of the anther (anthera), i.e. that part in which the pollen is developed, and of the support to this anther, which is usually threadlike, and bears the name of filament (filamentum). Filaments and anthers, in many instances, correspond to the sheathing-part and stalk of the leaf, and in these stamens the blade is wholly suppressed; in other instances the anther is to be regarded as the lower part of the blade, and then the apex of the blade appears as a scale-like appendage. The blade of the stamen sometimes resembles a perianth-leaf, and this is a case to which there will be frequent allusion.

The carpels (carpophylla) are arranged, like the perianth-leaves and stamens, sometimes in whorls and sometimes spirally. In one section of flowering plants they are scale-like, and present free margins not joined together. In another section they are rolled together and their margins fused, so that a capsule called the pistil (pistillum, ovarium) is formed. If many carpels are present in one flower, each of them may form a separate ovary, and then the more or less numerous one-leaved ovaries appear arranged either spirally or in a stellate manner as the termination of the shoot in the centre of the flower, e.g. in the Ranunculaceæ and Dryadeæ. In the Papilionaceæ and several others allied to these groups of plants there is only a single one-leaved pistil at the end of the flower-shoot; but usually several whorled carpels are united together to form a single ovary in the centre of the flower. A great number of different constructive plans of many-leaved pistils are distinguished according to the manner and extent of union, and these in particular afford excellent marks for characterizing the families and genera. The most striking differences are produced by the whorled carpels being at one time fused with one another along their whole length, while at another, the fusion is restricted only to the lower part; by the fact that frequently the rolled united margins of the adjoining carpels become partition-walls in the interior of the pistil, resulting in the formation of compartments, while in other cases this formation of septa does not occur, the carpels adjoining one another like the staves of a cask, and forming an unchambered capsule.

The pistil may be divided into the ovary (germen), style (stylus) and stigma (stigma). The ovary corresponds to the sheathing portion, the style to the stalk, and the stigma perhaps to the blade of the leaf. The ovary forms in most cases an expanded structure; its contour and surface offer little variety, especially when compared with the inexhaustible diversity of other parts of the flower. Usually its shape is ovate, ellipsoidal, spherical, or disc-like, more rarely elongated, ' cylindrical, or barrel-shaped; sometimes it is flattened from side to side, and has the form of a sword or sabre. Projecting knobs, cushions, angles, ridges, and bands are often found on its circumference in accordance with the number of the carpels of which it is composed, and three- or five-sided forms are met with very frequently. The hairs, bristles, spines, and wings appearing so noticeably on the ovary when it has been transformed into the fruit-capsule are usually so undeveloped at the time of flowering that perhaps not even the rudiments of these outgrowths can be recognized. 
The ovary contains structures which, from analogy with the eggs of animals, have been termed ovules (ovula). They are also called "seed-buds", as the seeds are produced from them after fertilization. Formerly the name "germ-buds" was frequently employed for these structures. Those botanists who endeavour to refer the infinitely manifold members of plants to a few fundamental forms, and especially to settle whether a certain structure is to be considered as a stem or a leaf, have fought very much over the ovules. First of all, ovules were regarded without exception as stem-structures, as parts, that is, of the axis, and the uppermost portion of the stem which bears the ovules-or from which the supports of the ovules branch off - were designated as fruit-axes. It was thought that these fruit-axes divided up in the most varied manner, and that they sometimes also became leaf-like, resembling flattened shoots, in which case the ovules would arise from the margins of the flattened expansion. It was also supposed that such fruit-axes might be united with the carpels, and the impression would then be given that the ovules were produced from the carpels. Later, the ovules of all plants were interpreted as leaf-structures, i.e. as parts of the carpels, and their direct origin from the axis, that is, from the stem, was denied. Even those ovules which are situated on the apex of the axis, projecting into the centre of the ovarian cavity, were regarded as outgrowths of the carpels, and it was supposed that a freely-ascending, ovule-bearing column projecting into the cavity of the ovary rose up from the base of the united carpels. Various other forced explanations have been given, but it is hardly suitable to consider them here.

These false interpretations are corrected when we no longer lay that stress on the difference between stem and leaf, which was asserted by the advocates of the two views quoted above, and when we remember that really all leaves are produced from a stem, and that it is by no means easy to settle where the stem ceases and the leaf begins. If we rigidly adhere to the history of development and to the actual fact rather than to those speculations on which is based the conception of an "ideal plant", and if at the same time we set ourselves against the attempt to refer all plans of construction to a single fundamental ground-plan, we arrive at this result, that in many cases the ovules proceed directly from the apex of the stem, and that even in the earliest stages of development they have no organic connection with the carpels. They stand in the same relation to the stem as carpels do, and there is no reason why they should not, like them, be regarded as peculiarly metamorphosed leaves. They form the last uppermost leaves originating from the axis, become subsequently a constituent of the fruit, and may also be looked upon, in consequence, as upper carpels. In such instances as these, two successive whorls of carpels are developed, one situated below, whose members develop no ovules, and one placed above, whose members are only formed of ovules and their supports. The lower carpels, without themselves developing ovules, form the capsule arching over the upper carpels which have been reduced to ovules. This view receives the more justification from the fact that similar conditions are observed in the stamens; that 
is to say, there are flowers in which the outer lower stamens are flat, leafy expinsions, whilst the upper are reduced to anthers and filamentous supports. Of course this is only a view which it would be unwise to insist upon after the foregoing strictures.

This supposition does not exclude the fact that in many instances a single whorl of carpels is developed, and that the carpels of this whorl not only form the capsule, but that at the same time ovules may arise from them. At one time the teeth of the margins of these carpels become ovules; at another, whole segments of a leaf are metamorphosed into ovules; again, in another instance, groups of cells have given rise to ovules over the midribs of the carpels; and lastly, innumerable ovules may have developed from the whole inner surface of the carpels.

The internal structure of the cavity of the ovary is still further complicated by the fact that the end of the axis in one case rises up like a hemisphere or truncated column in the centre of the capsule, while in other instances the end of the axis is hollowed into a pit, and sometimes even deeply excavated. In consequence of these manifold arrangements, very different relations between carpels and axis naturally follow, and the most various constructive plans result, which, however, will be more suitably discussed in the second volume when considering the individual families, especially the Primulaceæ and Onagraceæ.

In whatever way the ovules may be explained, they exhibit a great agreement in structure. In them may be distinguished the nucellus (nucleus), surrounded by two, or less frequently by only one coat (integumentum), and also the portion by which the ovule is connected with its substratum, the placenta. Usually this has the form of a stalk or filament (funiculus), and then the ovules appear, as it were, suspended in the interior of the ovary. When the ovule is straight, and is a direct continuation of the funicle, it is called orthotropous; if the straight ovule is hung on a thread-like support, but reversed, and more or less fused with the support, it is said to be inverted or anatropous; when it is curved, the designation campylotropous is used. The coats do not completely inclose the ovules, but at sne pole a spot which bears the name of micropyle is left uncovered.

As already remarked, the style corresponds to a leaf-stalk as regards its position and relation to the other portions of the pistil. In the one-leaved pistil its form frequently resembles a leaf-stalk, especially in papilionaceous plants. If the ovary of a one-leaved pistil be regarded as arising from the sheathing portion, and the style from the stalk of a leaf, it will be easily conceived that the style appears to be affixed to one side of the ovary. The lateral position of the style can be clearly understood if we imagine that the sheathing portion of the ovary is swollen up like a vesicle, as it is on the foliage-leaves of Umbelliferæ, or that it bears large stipules as in the Cinquefoil (Potentilla). In the one-leaved ovary of the cinquefoils the style in fact is not seen to spring from the apex of the ovary, but looks as if it had grown out laterally from its capsule. In pistils which are built up of many carpels arranged in a whorl, and having 
only their sheathing portions fused, as, for example, in the Meadow Saffron (Colchicum), or in the much-cultivated "Love in a Mist" (Nigella Damascena), the styles are separate and always fixed at one side of the compartment of the ovary corresponding to them. But when several whorled carpels are completely united with one another as far as the stigma, only a single style is to be seen. This style, which may be considered as a combination of several grooved leafstalks, then rises up above the centre of the many-chambered ovary. Just as the leaf-stalk may be frequently absent from foliage-leaves, so sometimes the pistil has no style, and the stigma is sessile or seated immediately upon the ovary.

The stigma corresponds to the blade portion of a leaf, but is expanded in only a few families of plants, amongst which the irises are the best known. It has to receive and hold the pollen-grains, and its form varies according as to whether these are carried by the wind as flower-dust or are brought to the flowers by insects in cohering masses. In the former case the stigmas are brush-like or feathery, often extended like a cobweb or spread out like a plume; in the latter case, projecting papillæ, knobs, ridges and bands are found on them, against which the insects knock off the pollen as they enter the flower.

If we consider now the functions of the various floral structures rather than the position and succession of the individual members, we arrive at the following result. Of all the structures known as floral-leaves the ovules and pollen-grains (i.e. those parts of the flower on which these structures are produced) alone are indispensable. These portions of the flower, however, must be protected not only during their development and at the moment of fertilization against possible external injurious influences, but the union of pollen-grains with ovules must be brought about by a suitability in the form of the floral-leaves in addition to the mere production of these bodies. In order to be able to fulfil these tasks the floral-leaves which develop ovules or pollen are themselves often suitably equipped and adapted, or a division of labour takes place, so that only one portion of the floral-leaves develops ovules or pollen, while the other exists for protection and as a means of ensuring fertilization. In many plants, for example, the carpels are not only the bearers of the ovules, but also at the same time their protectors, and by the peculiarity of their structure they conduct the pollen to the ovules they bear. In numerous other plants, on the contrary, a division of labour has occurred; the ovules spring from the axis as independent structures, and the carpels proper surround and protect them, and receive the pollen for them, as may be seen typically in the flowers of primulas. In the American Pachysandra, in the Persian Halimocnemis, and in many other plants, the stamens produce pollen in coherent masses, but some of them are also provided with allurements for those insects which carry the pollen from flower to flower, and distribute it to the suitable stigmas. A division of labour is met with in most of these plants which have coherent pollen, two, three, or more whorls of stamens are developed, the upper bear anthers and produce pollen, the lower are without pollen, but assume the function of attracting insects and of protecting the upper anther-bearing stamens. Regarded from this 
stand-point the perianth-leaves are, as it were, only antherless stamens, and this view is supported by the fact that in the so-called double flowers the anther-bearing stamens regularly change into antherless perianth-leaves. In the flowers of waterlilies as a rule no sharp limit can be drawn between stamens and perianth-leaves, but a gradual transition from one to the other may be plainly noted. The flowers of certain limes (Tilia Americana, alba, argentea), as well as those of the arrowgrass (Triglochin), of which an illustration is given below, are very instructive in this respect. In the Silver Lime (Tilia argentea, figs. 15 ${ }^{1}$ and $151^{2}$ ) a whorl of
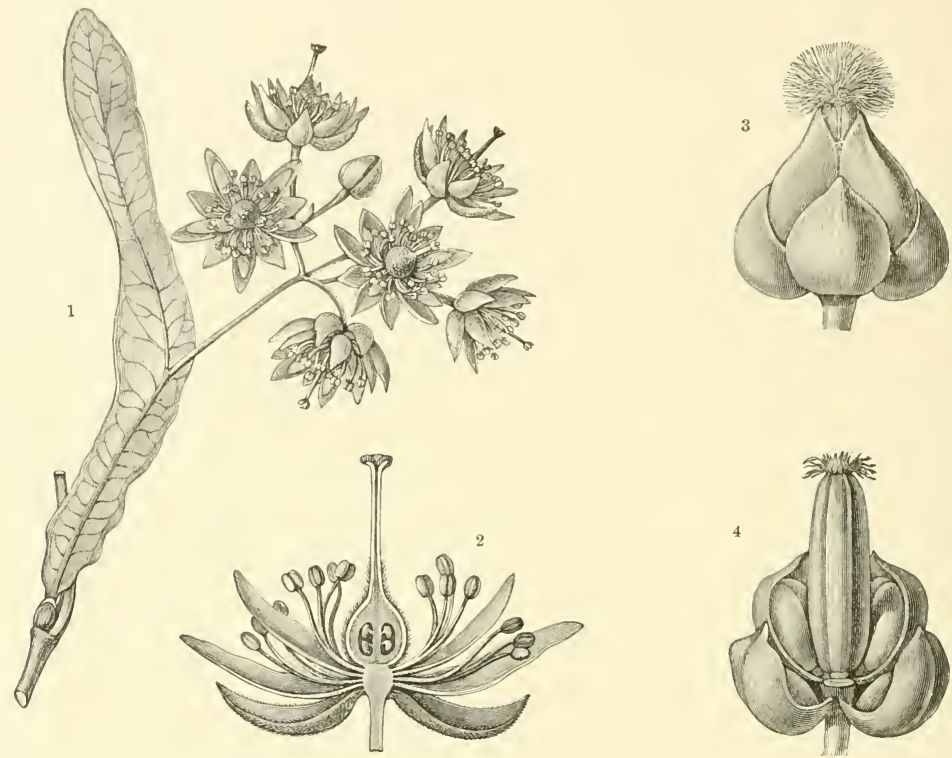

Fig. 151.-Flowers of the Silver Lime (Tilia argentea), and of a species of Arrow-grass (Triglochin Barellieri).

1 Inflorescence of the Silver Lime, natural size. ${ }^{2}$ Longitudinal section through a single flower. 3 Flower of the Arrow-grass, in the first stage of blossoming. The same flower in a later stage of development; one of the upper perianth-leaves cut away. 2,8 , and 4 are enlarged.

stamens with anthers is first formed below the pistil, followed by a whorl of leaves without anthers, which, however, secrete honey to allure insects; then again comes a whorl of leaves with, and below these again two whorls of leaves without anthers. The same is the case in Triglochin, whose flowers look as if they were composed of two stories standing one above the other, quite similarly arranged (see figs. $151^{3}$ and $151^{4}$ ). The flower commences below with a whorl of three hollowed antherless leaves; above these comes a whorl of three leaves with anthers, and the large anthers are surrounded and protected during development by the hollowed leaves as if by a hood; then again follows a whorl of three hollowed antherless leaves, and above these yet again a whorl of three stamens with large anthers, an 
arrangement resembling that of the lower story. When the powdery pollen falls from the anthers it is not immediately carried away by the wind, but falls first of all into the hollow cavities of the leaves below the anthers, where it remains deposited until the proper time has arrived for its transmission to the stigma of another flower. These hollowed leaves, although themselves antherless, are therefore filled with pollen for a time, and look like anthers which have just dehisced. They are of the greatest importance for the timely distribution of the pollen and for the accomplishment of fertilization, and may be regarded with respect to the part which they have to play as antherless stamens.

Usually all these leaf-structures of the flower originating from the axis below the pistil and bearing no anthers are designated as perianth-leaves-as calyx- and corolla-leaves, or, lastly, as staminodes. What descriptive botanists understand by perianth, calyx, and corolla has been already described on p. 641 ; with regard to the term staminode, it should be mentioned that it is applied to all such antherless leaves as are inserted between the whorls of perianth or corolla leaves on the one hand, and the carpels on the other; i.e. they occur where in most instances the anther-bearing stamens are placed. Staminodes resemble the stamens very much in shape, but are distinguished from them by the fact that they develop no pollen. They, however, make themselves useful in other ways. Thus in the transmission of the pollen, they secrete honey and allure insects; or they may serve as protective agents for their neighbours, the anther-bearing stamens, against various external injuries. A detailed description of the part performed in the process of fertilization by all these floral leaves which are so differently shaped and are arranged in such manifold ways with regard to one another, is reserved for the second volume of this work.

\section{FORMS OF STEM STRUCTURES.}

Definition and Classification of Stems. - The Hypocotyl. — Stems bearing Scale-leaves. - Stems bearing Foliage-leaves.-Procumbent and Floating Stems,_Climbing Stems.-Erect FoliageStems.-Resistance of Upright Stems to Strain, Pressure, and Bending.-Floral-Stems.

\section{DEFINITION AND CLASSIFICATION OF STEMS. THE HYPOCOTYL. STEMS BEARING SCALE-LEAVES.}

In certain seeds consisting of rounded or ellipsoidal masses of tissue, the embryo shows no obvious division into stem and leaf; nor can any distinction be recognized between the embryo and the surrounding seed-coat. When such seeds begin to germinate, as, for example, those of orchids, their cells become partitioned and multiply, and the whole tissue-body increases in size, but for a long time no trace is visible of a division into stem and leaf. It is shown by the development of the seed of Cuscuta, described and figured on p. 173, that the embryo, the seed-coat and the reserve tissue which nourishes the embryo for a time and provides it with the 
necessary building materials, may all be distinguished from each other; but the embryo itself shows no segmentation into axis and leaves. It looks to the naked eye like a filamentous, spirally-rolled structure, which breaks through the envelope of the seed-coat on germination, extends and elongates, then grows up straight and afterwards twists and winds and seeks for a resting-place from which it can derive nourishment. This thread may, without further discussion be considered as a stem although it bears no leaves, and indeed never presents even the rudiments of leaves. Not until later, when this thread-like stem has developed haustoria at the spots where it is in contact with the host-plant, and has grown still longer by the help of the absorbed nourishment, do small scales which must be interpreted as leaves arise below the growing point ( $c f$. fig. $35^{1}$ on p. 175). Projections are then developed above the scales which grow out into lateral shoots.

The fact that stems exist, which, in their young condition exhibit neither leaves nor even the rudiments of leaves, is specially emphasized here, because it has been repeatedly denied that the stem is a special member of the plant. This may of course seem strange to the uninitiated, and it will be asked, how then are we to regard the stem if it has not the value of an independent morphological member? Although this theme is so delicate, and its treatment so difficult for those who are not initiated into the details of the speculative science of form, yet I will try to briefly state the grounds which have led to the opinion stated above

At the free extremity of a growing leafy shoot a slight difference may indeed be recognized between the cells of the periphery and those of the interior, but no clear boundary can be fixed between these parts, and the end looks like an undifferentiated conical or hemispherical mass of tissue. On observing more narrowly the growth and further development of the mass, it will be noticed that cushions or protuberances arise on the periphery of the cone and form leaves, while the inner portion above these leaf-rudiments continues to elongate as an undifferentiated mass. Soon, however, fresh rudiments of leaves arise from it, and so as the process continues quite a large number of the cells are grouped together and form in their turn the starting-points of leaves. If we examine the tissue of a leaf as it arises thus below the tip of the shoot, we shall seek in vain for the place where the substance of the leaf ceases and that of the stem begins. It is on such grounds as these that the view has been formulated that the whole stem is really nothing else than a collection of leaves, standing one above the other, whose basal portions remain united, while the peripheral parts according to need rise up and project more or less. Against this view, of course, there is apparently the fact that not only leaves but also lateral shoots appear on the circumference of a growing shoot, from which it follows that the whole of the tissue is not employed in the formation of leaves, but that a part remains over from which the commencements of lateral stems are produced, and that it is this part which does not form leaves which represents the tissue of the main stem. It has also been proved that the rudiments of leaves arise on the growing shoot-cone from cells lying nearer the periphery than those from which the rudiments of lateral stems develop. This different origin has 
been used as a mark for distinguishing between leaf and stem, and the peripheral tissue has been explained as the basis of the leaves, and the tissue lying below it as that of the stem-structures. The outer layer of cells of the growing cone, called dermatogen, never forms the starting-point for lateral stems, although it inay occasionally give rise to leaves. The two or three outer layers of cells of the tissue below, called the periblem, usually form the leaves, but lateral stems often originate from the second to the fourth layers of the periblem. However, these possible differences of origin are insignificant, and no sharp limit can be drawn between the tissues from which originate the rudiments of leaves and lateral stems; consequently in this particular point there is no essential difference between leaf and stem.

In the stem the vascular bundles form a ring round the axis, but in the leafstalk, in other respects often very like the stem, they are grouped in a semicircle or in a plane. This, however, does not invariably occur. Leaf-stalks which bear peltate blades, as well as those which pass into blades with pinnate or palmate strands, as, for example, those of Solanum jasminoides, Anamirta Cocculus, Menispermum Carolinianum and of many other Menispermacex exhibit circles of vascular bundles and an actual ring of wood, so that they cannot in their internal structure be distinguished from stems. All other differences between leaf and stem which have been brought forward at different times and by different investigators apply indeed to a number, often to a very great number of plants, but unfortunately not to all. The following have been suggested as relatively the best marks of distinction, viz. that the leaf shows a limited growth, and that no new leaves spring directly from it, while the stem grows indefinitely and produces leaves laterally below its growing point. I say expressly the relatively best marks of distinction, because structures exist which cannot be forced into the limits of this definition. The flower-bearing as well as the flowerless phylloclades of the Smilacinere (which are really reduced axes) have always a limited growth; and, on the other hand, there are plants from whose leaves other leaves grow out. In the leaf-blades of the American twining plant Aristolochia Sipho, which is often met with in gardens as a covering for arbours and trellis-work, green projecting bands and lobes, which can indeed only be explained as leaf-structures, sometimes arise on the lower side of the blade, especially in those places where the finer strands form delicate anastomoses. This is a case where leaf-like structures actually spring directly from leaves, and the only difference is that the places of origin of the leaflets are not arranged in geometrical succession.

On reviewing the results of the developmental and morphological researches, here only briefly touched upon, we are forced to confess that it is very difficult to state absolute distinctions between leaf and stem, and that, moreover, the view already mentioned, viz. that the stem does not form an independent member of the plant, is not really contradicted. The single fact opposed to this view is the occurrence of stems without leaves; those, for example, which spring from the seeds of Cuscuta. But here also it may be objected that this stem in its further development forms small leaves below the growing-point, and that its tissue is nothing more 
than the continuation of the basal portion of these leaves. As in so many similar instances, the whole matter finally ends in an unfruitful strife of words where everyone is in the right. The simplest way is to regard as a stem every axis of a plant which, when developed, always bears geometrically-arranged leaves, and to avoid speculations as to whether this stem is to be considered as an independent structure apart from leaves, or as a combination of their basal portions.

Whatever theory we may hold of these relations, not only the form but also the function of the leaves borne by the part of the stem in question must be regarded as the predominating factor in the portrayal of the stem structureespecially when the peculiar construction of a given stem is to be explained by the special duties assigned to it.

There is no plant in which the stem is developed quite uniformly from the base to the apex. We can always distinguish in it stories following one above the other, each of which is fashioned in accordance with the work it has to perform. Just as in buildings the underground walls, which serve as the foundation of the whole and usually also as a store-room for food, \&c., exhibit quite a different kind of structure from the upper stories which are inhabited, and where kitchen, bed-rooms, airy parlours and passages are found, so, in one and the same plant, different plans of construction are realized according as to whether the part in question bears cotyledons, scale-leaves, foliage-leaves, or floral-leaves, the functions of which are so extremely various. It therefore seems most natural to classify stems as hypocotyls, scale-leaf stems, foliage-stems, and floral-stems.

There is not much to be said about the hypocotyl (fundamentum). The little that is of interest has been stated already in describing the cotyledons. After it has drawn the cotyledons from their envelope and has straightened itself, the hypocotyl undergoes no alterations worth mentioning and is only of importance in that the bud of the main shoot is developed from its apex, and the food absorbed by the radicle is conducted by its means to this bud.

The stem bearing scale-leaves (subex) is usually so short in its first stages that its leaves lie close packed above one another, the upper ones being wholly or for the most part covered by the lower. In many instances it remains very short throughout life, and is then termed a reduced axis or "short branch". In others it extends and elongates so that its leaves are separated, and it is then called a "long branch". It may happen that one of these scaly stems is at intervals sometimes a long and sometimes a reduced axis; it may then be compared to a string, in which knots have been tied at certain distances. In the case of a scaly stem passing over into a foliage-stem beset with green leaves, the former usually has the form of a reduced axis. It is then either flattened or disc-like, or it may be of a shortly cylindrical or conical form. If it is beset with large scale-leaves and is considerably thicker than the leafy foliage-stem into which it almost directly passes, we speak of it as an abbreviated stem. This, together with its large and hollowed scale-leaves, is termed a bulb (bulbus); it is almost always underground, and its axis is vertical, as, for example, in lilies, tulips, hyacinths, and stars of Bethlehem. 
A scale-leaf stem which remains short, which is clothed with membraneous scales and does not exceed in thickness the foliage or floral stem which often proceeds from it, is called a sucker (surculus). The sucker, beset with scale-leaves, appears as a bud (gemma) so long as the foliage or floral stem has not grown out from it; later it forms to some extent the basis of the foliage or floral stem, and is not very remarkable, especially after its hollowed scale-leaves, as is almost always the case, become detached and fall off. The scaly stem is but seldom developed at the base of the first shoot (plumule) arising between the cotyledons (e.g. in the Moschatel, Adoxa Moschatellina). On the other hand, it is scarcely ever absent from the base of the lateral shoots of woody plants, those bearing leaves as well as those which are terminated by flowers. In the subterranean buds of undershrubs the stem is occasionally very thick, and such buds have almost the appearance of bulbs. The subterranean buds, especially those of shrubs and trees, always possess, on the other hand, a short cylindrical or conical stem.

The tuber (tuber) seems to be to some extent a link between the reduced and the long axes formed by scale-leaf stems. It is always thicker than the shoots arising from it; its scale-leaves are situated so far apart that a clear space is visible between them, and they never cover and envelop one another. The scale-leaves of the tuber are insignificant; they only appear as narrow horizontal bands, or they are merely indicated by ridges and protuberances. In old tubers the scale-leaves are often scarcely recognizable externally. Most tubers are, moreover, very perishable structures; all those which appear as local thickenings of an underground shoot, of which the Potato (Solanum tuberosum) may serve as a type, grow very quickly, and have a resting-period of about half a year, but perish completely after they have developed shoots from their buds (the so-called "eyes") which unfold their green foliage above ground in the sunlight. Perennial tubers, whose lower half only is often embedded in the earth, or which are only covered with a thin layer of soil, are much less common. From these spring up every year a few shoots which, however, do not completely exhaust the tuber, but, on the contrary, supply it with materials manufactured by the green foliage in the sunlight, by which means the tissue of the tuber is actually enlarged. These perennial tubers frequently look like tuberous leafy stems, and the whole history of development must be known in order to be able to determine and prove that they really are scaly stems. Tubers are generally subterranean. More rarely they are formed above the soil in the axils of foliage-leaves, as, for example, in the Lesser Celandine (Ranunculus Ficaria), where those remarkable little tubers arise, which become detached after the withering of the plant; they afterwards lie on the ground, and have often, where they have been produced in great quantities, given rise to the myth of "potato rain".

Whilst some of the stems which bear scale-leaves are green, others are deroid of chlorophyll, and of these latter the following types may be distinguished:- first, the aërial, thread-like, twining and parasitic stems of the genus Cuscuta; second, the thin subterranean shoots of the Couch-grass (Triticum repens) and of numerous 
allied grasses, clothed with sheathing, membraneous scales; third, the erect and fleshy stems of the Balanophoreæ and Orobanchaceæ, covered with dry scales ( $c f$. figs. 41 and 42); fourth, the branched stems of Lathrcea, lying embedded in the earth, covered with large fleshy scales (cf. fig. 37 ); fifth, the coral-like scaly stems branching in all directions, which have no roots and are only covered with delicate scaleleaves, as shown by Epipogium (cf. p. 111) and by the Coral-root (Corallorhiza innata); sixth, the stems of the Tooth-cress (Dentaria), creeping underground with thick, fleshy scale-leaves and clearly defined roots; seventh and last, the cylindrical, subterranean stems with weak membraneous scale-leaves and many roots, as in Solomon's Seal (Convallaria Polygonatum), the Sweet Spurge (Euphorbia dulcis), and numerous other perennial undershrubs. Subterranean scale-leaf stems developed as elongated shoots are classed together in botanical terminology under the name "root-stock" or "rhizome" (rhizoma); the term "creeping stem" (soboles) is applied to the thin, branching scaly stems which often creep for a considerable distance under the ground.

In the forms belonging to the first, third, fourth, and fifth groups, just enumerated, the scaly stem passes directly into a floral stem, i.e. on the same stem below are to be seen scale-leaves which stand in no direct connection with the processes of fertilization, and above them perianth leaves, as in the Rafflesiaceæ ( $c f$. figs. 44 and 45 ), or bracts, as in the Broom-rape and Toothwort ( $c f$. fig. 37). In these plants no green foliage leaves are developed; they are unnecessary, because these plants are all parasites or saprophytes, and do not require to manufacture organic compounds for themselves, but derive the material necessary for their further growth from their host, or from the humus of the forest ground. In plants of the other groups, of which Dentaria, Couch-grass, and Solomon's Seal may be taken as types, two kinds of shoots are developed:- Shoots whose stem is beset only with scaleleaves without chlorophyll, and those which branch off from these grow up above the ground, and there unfold green foliage-leaves. Here, too, must be mentioned those strange plants whose perennial underground stems develop two kinds of shoots which appear above ground;-first, shoots whose stem is covered below with scale-leaves, but which bears flowers above and later on when these first shoots begin to wither, leafly, flowerless shoots whose green leaf-blades unfold in the sunlight. This remarkable division of labour is observed in many Alpine plants, in species of Butter-bur (Petasites), and in the widely-distributed and well-known Colt's-foot (Tussilago Farfara).

Green scaly stems which develop as elongated shoots are obviously all aërial, or rather, they grow above the ground and the cortex of their stems becomes green so far as the light can influence them. That part of the shoot which remains hidden in the dark earth does not become green, and many such shoots, e.g. those of Asparagus, are white and without chlorophyll in the lower half, their upper portions alone being green, viz. the small needle-shaped branches (phyllocladia) growing out from the axils of the small scale-leaves. Amongst the green scale-leaf stems must be included the cactiform plants, the switch plants, and the plants with 
flattened shoots, which have been fully described on p. 333. The Horsetails (Equisetaceæ) belong also to this group, and in one group of these (Equisetum arvense, E. Telmateja) the division of labour is similar to that in the Colt's-foot. The first pale shoots which emerge from the ground are terminated by a spike of sporangiabearing scales, and not until later, after the spores have been scattered by the wind and the pale primary shoots have withered, do the summer shoots appear whose stems develop green tissue in the cortex.

The inner structure of the green scaly stems, whose duty is to manufacture organic materials, agrees essentially with that of the foliage-stem. In these plants, indeed, the functions have only been transposed in this way, viz. that normal green leaves are not produced, but only small colourless scales, whilst the work usually allotted to the leaves has been assumed by the cortex. Green scaly stems are just as much exposed to wind and sunlight as leafy stems are; they must, like them, direct and establish themselves in accordance with the particular conditions of their habitat and offer the same resistance to the wind; they must be just as elastic and flexible, and consequently present a similar arrangement of their tissues rendering it possible for them to maintain the favourable position once assumed. The subterranean scaly stems have no need of such contrivances; no winds press against them and their tissue does not require to be strengthened against bending. The stems of Balanophoreæ require only a slight elasticity, the part which rises above the ground is relatively very thick and almost reminds one of the stalks of the cap-fungi. Many of these scale-leaf stems under the ground or rising only a little above it, are very brittle, and when stems of Dentaria, embedded in the humus of the forest soil, are dug up, the greatest care must be taken to prevent their breaking. The same is true of underground tubers and bulbs; they need none of those contrivances by means of which a definite position with regard to the light, or a great capacity of resistance to wind is obtained. Protective measures against excessive transpiration are likewise unnecessary, and this accounts for the lack of cuticle to the epidermal cells, and for the absence of hair-like structures and varnish-like coatings. When dry, tough scales occur as envelopes to bulbs, they are probably of a protective nature-not against transpiration or over-illumination, but against subterranean animals which might come and nibble them for their foodreserves.

These subterranean shoots excavate their own bed by the pressure which their turgescent tissues exert on the surrounding earth during growth. Growing bulbs and tubers in this way widen out a bed, often of considerable size, and the pressure exerted is so great that the loose earth in their neighbourhood becomes compressed, and sometimes transformed into hard cakes. It has already been mentioned that not only stiff soil but even bits of wood and other objects may be bored through by the stiff, pointed scale-leaves of the Couch-grass. A most important function falling to the lot of underground shoots, and especially to tubers and bulbs, is the storage of reserve materials. These are manufactured during the summer by the green tissues in the sunlight above ground and are then conducted down into the 
subterranean reservoirs. Here they remain quietly deposited during the winter and are not brought into requisition until the plant, at the beginning of the next vegetative period, sends up new shoots which manufacture organic materials afresh. It is in the production of these shoots which are to be sunned above the ground that material is always employed which was conducted down into the storehouses during the preceding year.

We cannot help surmising that this remarkable alternation between rest and vigorous activity, together with the temporary disappearance of all the aërial portions of the plant, is connected with the peculiar conditions of the habitat. This opinion is confirmed by the actual distribution of tuberous and bulbous plants. Most of these plants are found in those regions where all the succulent tissues exposed to the air would be liable to the danger of shrivelling up in consequence of months of drought, and where also the superficial layers of soil in which the tubers and bulbs are embedded dry up so much that they would not be able to replace the water evaporated from the leaves. But when the soil has lost all its water, it forms an excellent protection to the tubers and bulbs; the earth forms an actual crust round the succulent structure, and in many regions the clay soil, coloured red by iron oxide, is hardened into a mass which resembles brick. Embedded in this mass the tubers and bulbs can survive the dry period which lasts over seven or eight months with impunity. When the rainy season comes and the hard crust is moistened, a wonderful life stirs everywhere through it. Innumerable tuberous and bulbous plants spring from the softened clay and unfold their flowers and green foliage-leaves during the brief wet period. This is what occurs in the clay steppes of Central Asia, in the mountainous districts of Asia Minor, in Greece, and generally all the countries bordering the Mediterranean Sea. In especial degree is the Cape celebrated for its almost inexhaustible wealth of bulbous and tuberous plants ("cape bulbs"). In Central Europe, where the activity of vegetation is interrupted not by dryness but by frost, the number of these plants is strikingly less than in the districts previously enumerated, whilst the ground in which the few species occur exhibits quite different conditions. Here the soil is never exposed to severe drought, indeed, strangely enough, the majority of tuberous and bulbous plants in the depths of the Central European forests are found in loose and dampish earth, rich in humus. It is well known that in such places as these snowdrops and yellow Gagea, the Two-leaved Squill, the purple Martagon Lily, the Cuckoo-pint, the Broad-leaved Garlic, and the various species of Corydalis (Galanthus nivalis, Gagea lutea and G. minima, Scilla bifolia, Lilium martagon, Arum maculatum, Allium ursinum, Corydalis fabacea, C. solida, C. cava), flaunt themselves with a luxuriant and vigorous growth; and, what is especially worth noticing, their flowers blossom in the first part of the year, their green foliage unfolds early in spring and at midsummer is already yellow and withered, although, as stated, the necessary moisture would not be lacking at this season.

This peculiar phenomenon demands a reason, and we shall not be far wrong if we explain the preference of our early-flowering bulbous and tuberous plants for 
the ground of forests somewhat as follows. The leaf-covered forest floor, sheltered as it is by the trees, gives out but little heat, and the frost only penetrates it to a slight depth in the winter, thus the tubers and bulbs are far less exposed to the danger of freezing than in the open country. The early flowering and the quick fading of the leaves are caused by the fact that the light required for the activity of the green foliage can only penetrate to the forest ground while the crowns of the trees are bare. Later, when a leafy canopy and shady roof is spread out above, only a sunbeam here and there can steal through the chinks to reach the damp, cool soil of the forest ground. But this scanty light would no longer suffice for the work to be done by the green leaves of the bulbous plants, and they must therefore achieve this before the leafly roof has developed. The weak light is, however, quite sufficient for parasites and saprophytes, and it is worthy of notice that in the summer in place of the green leaves of bulbous and tuberous plants, which even in June have turned yellow and disappeared, the Monotropa without chlorophyll, the leafless Epipogium, and a host of pale fungi spring up from the deep humus in the gloom of the forest.

\section{STEMS BEARING FOLIAGE-LEAVES.}

The foliage-stem (stirps $s^{1}$ ) is characterized by the fact that the leaves borne upon it are provided with green blades, and realize the popular idea of leaves. This portion of the stem might indeed be called "foliage-leaf stem", and its essential characteristic would be expressed in the term, but since the cotyledons frequently assume the form of foliage-leaves, it is perhaps better, in order to avoid confusion, to keep to the term "foliage-stem". No part of the plant is so striking to the eye as the foliage-stem. The rhizomes, tubers, bulbs, and other forms of scale-bearing stems are hidden from view in the earth, just like roots. The flowers borne by the floral stems are ephemeral structures, the leafy stems alone retain their character during the whole vegetative period as the most important portion of the plant. When one attempts to reproduce the character of the vegetation of any region either in words or in the form of a picture, it is to the leafy portions of grasses, shrubs and trees that one confines oneself; these, blended in infinite variety, compose the carpet of the meadow, the bush, the thicket, the woods and forests. It is the style of architecture of the foliage-stem, so to speak, which expresses the style of the whole plant-body.

This peculiar style of architecture, and the habit of the whole plant subservient to it, depends primarily upon the size, length and thickness of the foliage-stem. It is evident that in this respect conditions obtain quite analogous to those in the

1 Agreement in matters of terminology is only partial among batanists. The older botanists used the term stirps as synonymous with "plant" (planta); later it was claimed for the stem in the wider sense. The entire main axis of flowering plants was called the "caudex" by Linnæus, and from it he distinguished the descending portion or root (radix), and the aseending part or stem (stirps). In modern times the term caudex has been enployed in a different sense from that of the Linnæan terminology for the stems of palms. Here the stem of a plant is spoken of as the cormus, it is divided into: (1) The hypocotyl (fundamentum); (2) the scale-leaf stem (subex); (3) the foliagestem (stirps); (4) the floral stem (thalamus). 
already-described scaly stems; here only are the differences in size more marked. Contrasts, like that between filamentous leafy stems, barely a centimetre long, and the giant trees of North America and Australia, have not their like in the whole vegetable kingdom. In those plants which germinate, grow, blossom, and fruit and, after the distribution of their seeds, perish, all in a single year-in these shortlived annuals - the foliage-stem seldom attains to a considerable diameter. In many small Cruciferæ, e.g. in the small-flowered Shepherd's Purse (Capsella pauciflora) and in the tiny Chaffireed (Centunculus minimus), the diameter of the stem often scarcely amounts to half a millimetre. The largest dimensions in annuals are found

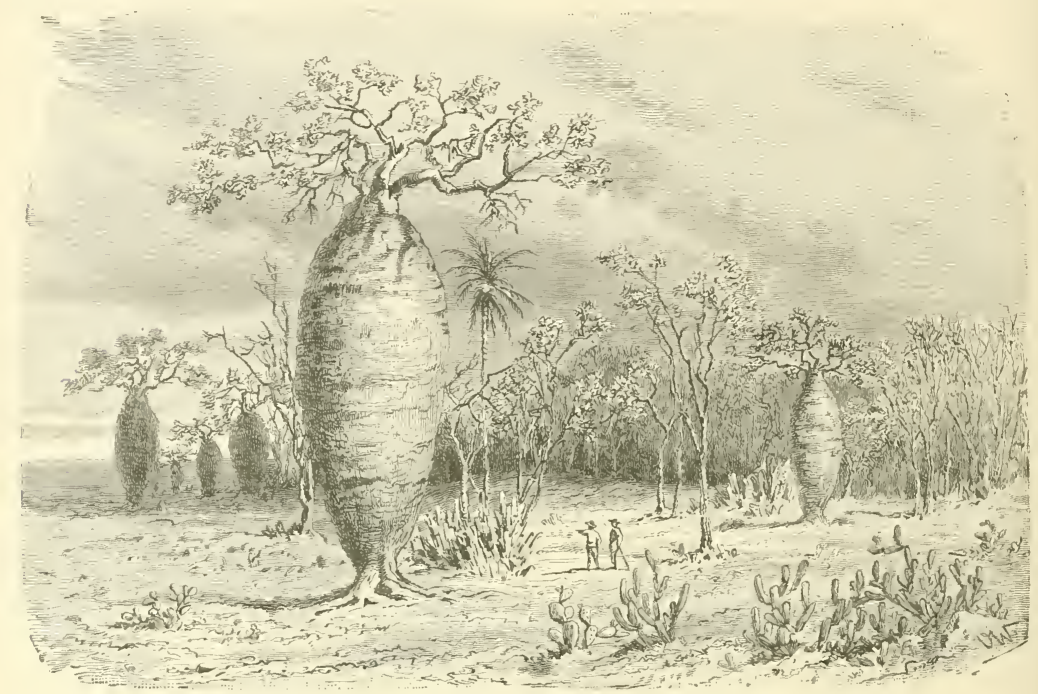

Fig. 152.-Cotton Trees (Cavanillesia tuberculata) of the Brazilian catingas. (After Martius.)

in the Castor-oil plant (Ricinus communis), many of the stems attaining to a diameter of 7 centimetres, and in the balsams of the Himalayas (Impatiens tricornis and glanduligera) which sometimes have a diameter of 4 centimetres. In these annual plants the stem which bears the leaves perishes with them every year. It is otherwise with plants whose stem remains alive for more than one period of vegetation, and which have been called perennial. When these throw off their foliage, they do not die, but fashioning themselves into supports for the leafy shoots which arise from their buds, attain a circumference in just proportion to the new burden to be borne. The structure of such foliage-stems then becomes altered. The stems of annuals and those of the young new shoots of perennial plants have a green succulent cortex with a peculiarly-developed epidermis; such a shoot we: call "herbaceous" (stirps herbacea). In the leafless stems of perennial plants, now transformed into columns, a dried crust or bark replaces the succulent green cortex 
whilst within masses of wood are continually formed and are deposited on the bundles of woody cells and vessels produced in the first year-thus increasing the circumference of the stem. Such a stem is said to be "woody" (stirps lignea). Woody stems which have been thickened continuously in this manner for centuries sometimes attain a circumference of 50 metres; that of the Mexican conifer

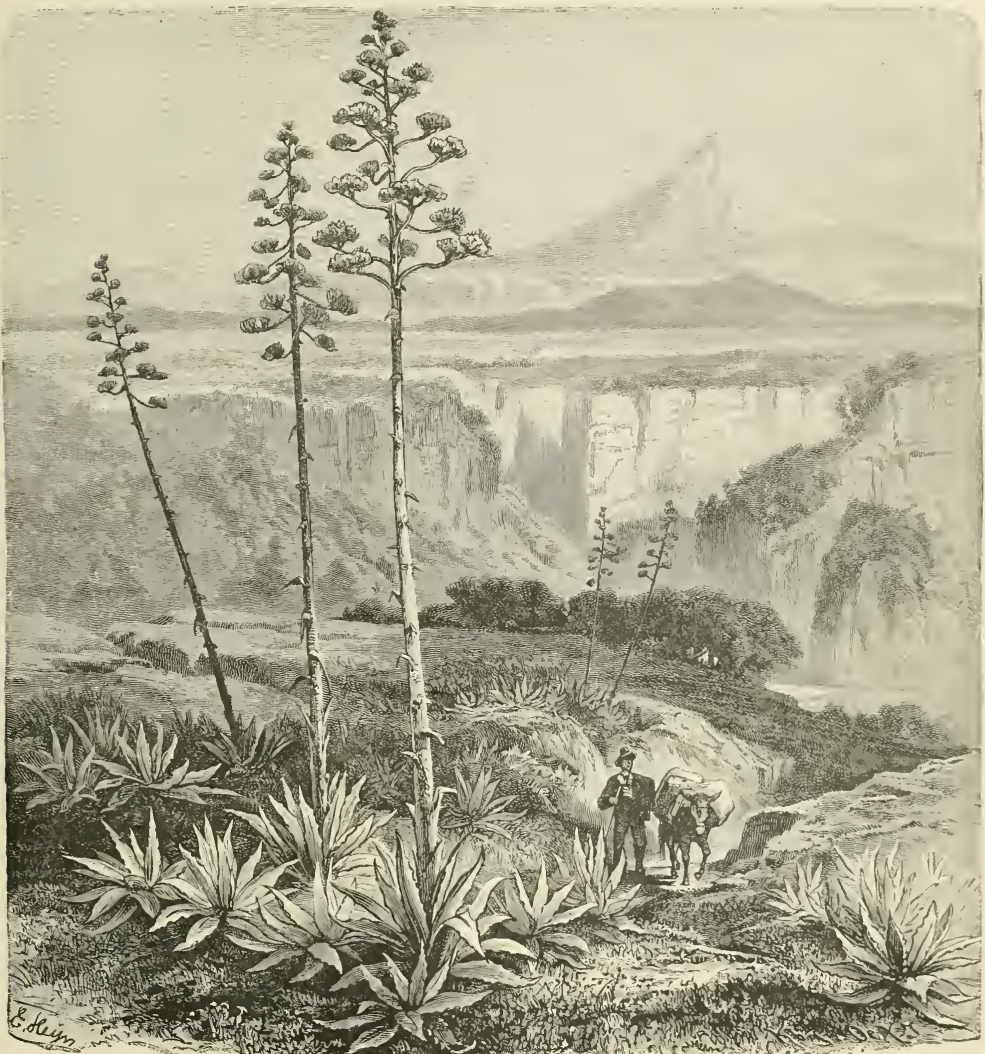

Fig. 153.-Agaves of the Mexican uplands (from a photograph).

(Taxodium mucronatum) has even been found with a girth of $51 \cdot 88$ metres; this circumference exceeds that of the above-mentioned stem of Centunculus more than a hundred thousand times. The thickness of the stem is in general greatest at the base and gradually tapers off above; only a few palms are thicker immediately below their crown of green leaves than at the base, and in the strange cotton-trees of the Brazilian catingas (Cavanillesia tuberculata) of which an illustration is inserted opposite, the stem forms a swollen, barrel-shaped mass attaining its maxi-
vor. I. 
mum about half-way up. Very often an unequal thickening may be observed in the foliage-stem; this is due to the fact that at the places where leaves arise from the stem knotty swellings are developed, while those portions of the stem which come between successive leaf-insertions (or nodes), and which are called internodes, are cylindrical or prismatic in form. A foliage-stem which has this peculiarity is said to be "nodose" (nodosus). Sometimes the internodes of such nodose stems adjoin one another at obtuse angles, and such a stem is then called in botanical terminology "zigzag” (flexuosus).

The fully-developed internodes of which the foliage-stem is built up, are only rarely, and then only for short distances, of precisely equal length. Sometimes longer and shorter internodes alternate, and quite as often it happens that a single much-elongated internode succeeds several short ones. If such an elongated internode passes over into the region of the flowers, it is known as a "scape" (scapus). As in the scaly stems, where short and long axes can be distinguished, so is it with the foliage-stem. The leaves are usually so crowded on these short axes that they form rosettes or fascicles which quite cover the stem which bears them. On the other hand, on many long axes the leaves are developed scantily and at long intervals and we are tempted at first glance to take such an elongated shoot for the leafless stem of a switch-plant. A large number of plants develop in one year only short axes with rosette-like radical foliage-leaves; in the following year the apex of the short axis grows up into a slender, elongated shoot which passes above into a floral stem. This is the case in most plants whose stem is said to be "biennial" (stirps biennis). Similar conditions are observed, however, in many perennial species of house-leek (Sempervivum), Aloe, and various other plants with fleshy, succulent leaves, only in these the alternation of long and short axes extends over several, often very many years. A very noticeable form of this kind is Agave Americana, known by the name of the "Century Plant", illustrated in fig. 153. Often 20, 30, even, it is alleged, 100 years pass by, during which long period the plant produces only a short stumpy axis beset with leaves grouped in a rosette. At length a long axis arises from the centre of the rosette and terminates in a voluminous inflorescence. As soon as the fruits have been produced from the flowers, and the seeds have escaped, not only the long axis, as in biennial plants, but also the short axis with its large, stiff and spiny rosetteleaves entirely dies away. In water-plants this type is also met with in the remarkable Water Soldier (Stratiotes aloides), to which allusion has been so frequently made. In this plant, as in the house-leeks and saxifrages, long axes which continue to grow until they have arrived beyond the circle of the whole rosette, arise from the axils of the lower leaves; when this has happened, the young horizontally-projecting shoot stops extending, and at its tip again forms $x$ short axis, i.e. a rosette, which, in the following year, sends up a fresh long axis. A similar alternation of long and short axes is also observable in numerous other plants, in the shrubby spiræas, and in roses, hawthorn, sea-buckthorn, barberry, and Astragalus, which we shall encounter later on as hedge-forming shrubs. Some- 
times long and sometimes short axes develop from the same shoot. Also in many conifers, e.g. cedars and larches, the branches proceeding from a shoot are for the most part short with needle-like leaves arranged in fascicles, and only a few of

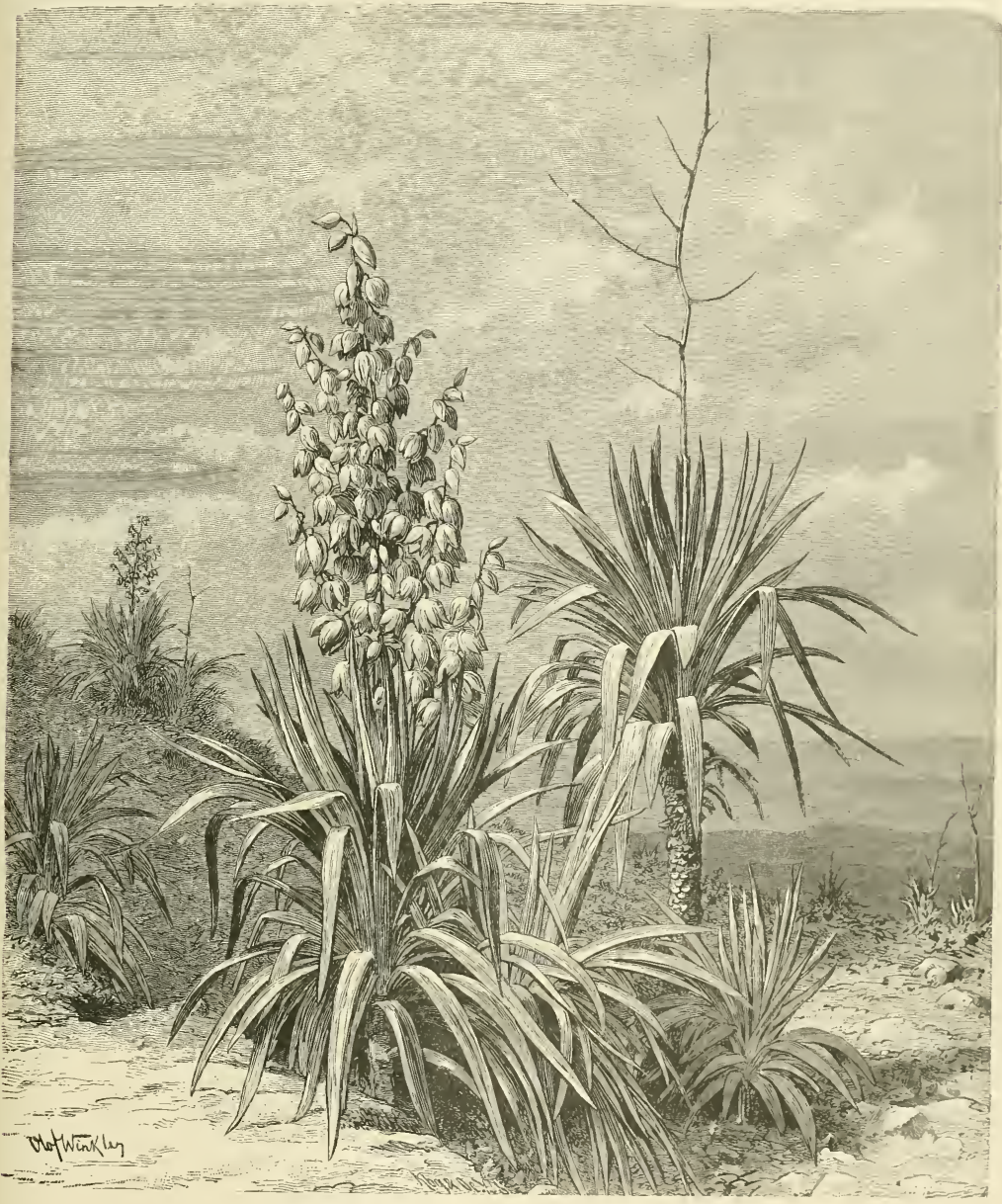

Fig. 154.-Yucca gloriosa (from a photograph).

them become long axes. In Pines, on the other hand, all the leaf-bearing twigs are short axes, and here we have also the remarkable circumstance that in several species, e.g. the Scotch Pine (Pinus sylvestris) a lateral twig bears only two such needle-leaves. Tree-ferns, Cycads, Pandaneæ, Grass-trees (X'Tuthorrhoa), many 
palms, dracænas, and species of Yucca, of which the Yucca gloriosa, illustrated in fig. 154, may serve as a type, exhibit a very peculiar structure. The yearly increase in length of the stem is comparatively small, the leaves which project all round from this portion of the stem are consequently crowded together and form a rosette which cannot be distinguished as regards the arrangement of the individual parts from the radical rosettes of Agaves and species of house-leek, and, like these, must be regarded as a short axis. In the following year the stem continues this curious, abbreviated growth, the foliagre-leaves of the previous year gradually die otf, and only the hardened remnants of their leaf-bases are left behind, thus the rosette or head of fresh green leaves is now seen borne by a naked columnar stem. This continues for many years, and the gigantic crown of leaves rises higher and higher above the ground. Plants with this manner of growth, moreover, never attain even in many years to anything like the height which is attained by foliagestems terminating in or branching out into long axes. Even the tallest palm terminating in a short axis is a dwarf in comparison with the rotangs or climbing palms, continually shooting out long axes. Rotang stems are known to extend to almost 200 metres. The length of 200 metres is perhaps the extreme limit reached by a foliage-stem, and if we again contrast the extreme cases, and compare with these climbing palms the stems of the minute Gentiana nana growing on the high Alps, it is seen that the shortest known of all foliage-stems is exceeded by the longest about twenty thousand times, in round numbers.

The ramification and facies of foliage-stems is in main part governed by the light-requirement of the leaves they bear. Necessarily the foliage-stem as the bearer of organs which have to prepare organic materials in the sunlight is chiefly influenced in its growth, and as to the position which its branches assume by the conditions of illumination. In order that all the green leaf-blades of a plant may be suitably illuminated, it is necessary that all these foliar axes should be grouped conformably, and should divide up the space most economically. Where foliage is chiefly borne on short branches, even under the most favourable conditions, only a relatively circumscribed space can be utilized. But when the reverse is the case and foliage is produced on long branches, the plant is much more favourably circumstanced. Such plants can unfold their leaves gradually above one another, and display them at appropriate intervals and distances to the sunlight. This elevation of the leafage above the ground is rendered possible either by the possession of a specially-contrived stem, or through the employment by the stem of some strong substratum or support up which it climbs to the light. Again, long axes, which have not the capacity of rising above the ground in either of these ways can elongate while embedded in the soil or extended on it, and, running out in all directions, can arrange their green leaves in a mosaic-like carpet. Lastly the foliage-stems can be sustainea in the position most suitable to their leaves by means of the surrounding water. According to the circumstances, foliage-stems may be broadly classed in four groups, viz. those which lie on the ground (stirpes procumbentes), those which float in water (stirpes fluctuantes), 
those which climb (stirpes scandentes), and the erect columnar stems (stirpes palares).

\section{PROCUMBENT AND FLOATING STEMS.}

If we review the plants whose characteristic appearance is chiefly due to their procumbent foliage-stem, we notice that most of them take root in turf, boggy ground, on the stony plateaus of hilly districts, in the rocky clefts of wind-swept mountain heights, or, lastly, in the sandy plains of the lowlands; in general they inhabit an infertile soil, on which the storm has free play, and where erect plants would find it difficult to maintain themselves. The leaves of such stems are usually undivided and small, and are present in large numbers on each year's growth. Where their number is small, and where correspondingly the internodes of the annual shoot are more elongated, the leaves are often divided, but then the individual segments are of the shape exhibited by the leaves of the short-membered shoots. The leaves always appear in two or three rows on the fully-formed procumbent stem, whether they are decussate or spirally arranged (cf. p. 417). Where no local insurmountable obstacles exist, the procumbent stems spread out in all directions from the spot where the plant first took root, and when the species in question are sociable weave a close carpet over the ground in a relatively short time. In the earliest stages of development the shoots are not extended over the ground, that is to say, the primary shoots, originating directly above the hypocotyl, are at first erect. Soon, however, as it elongates, the stem inclines to one side and nestles to the ground, or it arches over so that its free end reaches the soil. The apex of course is always more or less erect, and most young, procumbent shoots have the shape of an $\backsim$. As the stem elongates, the part immediately behind the growingpoint always nestles to the ground. In many instances these stems have not the strength to hold themselves erect; the soil on which they lie is their actual bed or support. If stems like these are held up above the ground, they hang limply down, as may be seen in the Periwinkle (Vinca), Strawberry (Fragaria), and in the Japanese Saxifrage (Saxifraga sarmentosa) so often grown in hanging baskets. But in all cases it is not their weight merely which causes many shoots to assume this manner of growth, in other words, that the shoots do not sink to the ground under the burden of their leaves, can be seen plainly enough in the procumbent stems of hawkweeds which produce runners (e.g. Hieracium Pilosella); these, when gathered and placed upright, remain quite stiff and straight, and do not show the slightest bending. When the stems of Globularia cordifolia or those of the Hairy Genista (Genista pilosa), growing on a rocky ledge, reach over the edge, they do not hang down vertically, as would be the case if their own weight were exclusively the cause of the direction taken, but they skirt along the face of the overhanging rock and remain closely pressed against it.

The first group of plants with procumbent foliage-stems is perennial; the growing-points of their stems advance over the substratum a little every year, and the new-fcrmed shoot is the continuation of the older portion of the already 
existing stem. At first the new portion of the stem is directed upwards, but after a year it lies flat on the ground or is actually pressed to it. It then sends out lateral branches which repeat the method of growth just described, but it always remains fresh and vigorous, serving for years after it has thrown off its leaves for the conduction of food from the ground and only dies off very gradually and slowly from behind.

In many forms belonging to this first group the older portions of the stem become lignified, and persist for a very long time. They may also increase in thickness, exhibiting numerous annual rings, as, for example, the stems of procumbent willows clinging to the rock terraces of the high Alps, as illustrated on p. 524. The elongating stems do not often throw out additional roots, as may easily be shown by raising the stems from their procumbent position. When such stems branch, and the branches have spread far and wide over the soil, they form an actual carpet, which can be raised from the ground or from the rock terrace as a coherent mass, as, for example, in the red Bearberry (Arctostaphylos Uva ursi) and the white Dryas (Dryas octopetala). Many members of this group possess evergreen foliage, as we see in the Trailing Azalea (Azalea procumbens) and Globularia cordifolia. The Cinquefoils with trailing woody stems (e.g. Potentilla nitida and Clusiana), Sibbaldia (Sibbaldia procumbens) and several valerians (e.g. Valeriana tripteris and montana), similarly provided, possess, however, no evergreen foliage, and may be distinguished from those named earlier by the fact that the annual increase of their stems is very slight, in consequence of which the older plants have usually a turf-like appearance. Many species of Thyme (Thymus) are, on the contrary, characterized by the fact that they every year develop fairly long and thin whip-like shoots which weave over the mossy substratum, or, like Dryas, form a carpet on the rocky bed. The stem of the forms hitherto brought forward is termed "prostrate" (stirps prostrata), from which is distinguished the "creeping" stem (stirps repens). Even when it has lost its leaves, the creeping stem is not lignified, but develops abundant root-fibres close behind the growing-point, which penetrate into the ground, and often draw the stem down into the soil or mud. The growths of former years do not here persist so long as in plants with woody prostrate stems; they usually die off after three or four years, and decay and vanish away altogether. Thus one might almost imagine the stem had been shifted en masse, that it had crept forward in the direction of the growing tip. Sometimes on the older portions of these stems, the situations where leaves were formerly inserted are marked by transverse scars and bands-reminding one very much of creeping worms and caterpillars. The umber stems of the Californian Saxifraga peltata which creep over damp rocks by the sides of streams are very striking in this respect. A likeness to worms crawling over the soil is also possessed by the stems of the European and American Asarabacca (Asarum Europacum and Canadense), by those of the marsh-inhabiting Buckbean (Menyanthes trifoliata), of the Snake-root (Calla palustris), of the purple Marsh Cinquefoil (Comarum palustre), and of several species of clover (e.g. Trifolium repens and fragiferum). 
In addition and in contrast to this first group of plants with procumbent foliagestems there is a second, characterized by the fact that only the buds arising on the new shoots remain throughout the year, strike root, and grow out into new plants, while the shoots themselves-the axes from which the buds have been developedsoon perish, thus severing the connection with the parent plant. These shoots are always thin, frequently quite thread-like. Little building-material is wasted upon them, since they are but ephemeral structures. Two distinct types of stem may be distinguished in this second group. These are known as the stolon and runner. By "stolon" (stolo) we understand a procumbent stem which dies off after a year, and is abundantly beset with leaves not very far apart. In the axils of many of these leaves no buds are produced, and often only at the ends of the stolons do buds arise from the axils of very minute leaves; these buds take root. This is especially the case in the arched stolons, as, for example, in the well-known Periwinkle (Vinca), and the purple Gromwell (Lithospermum purpureo-coeruleum). The shoots arising from old plants of these species form flat arches abundantly beset with pairs of leaves. Their free ends lie on the ground, swell and grow down into some dark chink or into the black humus itself, striking root and thus being drawn still deeper into the ground. The end of the stolon, thus embedded, finds itself next year, so to speak, on its own feet; it grows up into a new plant, while the arched or connecting portion dies off sooner or later, and in the following year, or the year after that, vanishes, leaving no trace. The stolons of the Pennywort (Lysimachia Nummularia) are similarly constructed, but in this plant the shoots lie flat on the soil, and the tip does not thicken, nor do the apices avoid the light, or become drawn far into the earth. Rooting buds arise in the axils of small leaves close to the up-bent apex of the stolon, and in the following year become starting-points for new plants. Several species of saxifrage and house-leek (Saxifraga and Sempervivum), the Common Bugle (Ajuga reptans), some hawkweeds (e.g. Hieracium Pilosella and Auricula), and numerous other plants develop richly-leaved stolons which produce at their free ends short axes which root. The leares on these short axes are grouped in rosettes; the short axes grow next year into new plants, the intervening stolon perishing. A peculiar modification of this method of growth is found in certain house-leeks (Sempervivum arenarium and Soboliferum). Here, as before, the tip of the thread-like stolon develops a short axis with leaves arranged in rosettes, but as soon as this is fully formed, the stolon withers, the spherical rosette becomes detached from it and rolls down over the steep ground where it had developed. Since these species of house-leek grow as a rule on the narrow ledges of precipitous rock-faces, it happens that the rosette thus detached falls from ledge to ledge, often to a depth of many metres, truly a remarkable method of distribution, which we shall allude to again in the second volume (ef. vol. II. fig. 425).

The "runner" (sarmentum) is distinguished from the stolon by the fact that its internodes are much elongated, and that leaves and buds which strike root and form the starting-points of new plants, are only formed at wide intervals 
on them. The long bare internodes are always thin and thread-like, and pernsn in the course of a year. One portion of the buds developing at the nodes of the runner forms short axes; another part may form even in the first year long axes which again assume the form of a runner. Since each plant sends out simultaneously several runners extended on the ground on every side, it comes to pass that in a very short time considerable areas are spun over in all directions with filamentous runners and a host of new plants produced. Well-known examples of this form of procumbent stem are furnished by the strawberries (e.g. Fragaria vesca, grandiflora, Indica), several cinquefoils (e.g. Potentilla reptans and Anserina), the Creeping Avens (Geum reptans), the Stone Blackberry (Rubus saxatilis), the Ground Ivy (Glechoma hederacea), and the Japanese Saxifrage (Saxifraga sarmentosa). A very peculiar appearance is presented by Androsace sarmentosa, which grows in the Himalayas. All its leaves are crowded together into a beautiful rosette on an erect short axis. From the axils of several of these rosette-leaves, long, thin runners, red in colour, radiate out during the summer; they extend themselves on the rocky soil, and each runner forms at its end only a single rooting bud or rosette. The red filaments perish in the second year, but by this time five or six freshly-rooted rosettes may generally be seen standing in a circle round the older one.

In a third group of plants the whole procumbent foliage-stem with all its branches dies off every year at the close of the vegetative period. The plants belonging to this group are either annuals and maintain themselves only by seeds, or they possess perennial subterranean scaly stems, in which ease each year new leafy stems arise. The foliage-stem of these plants is said to be "prostrate" (stirps humifusa). The following may serve as examples of such annual, prostrate shoots:-The Caltrops (Tribulus), the Strapwort (Corrigiola), Illecebrum, the Pimpernel (Anagallis), the Ivy-leaved Speedwell (Veronica hederifolia), the Portulaca (Portulaca oleracea), and numerous species of Polygonum, trefoil, and medick (Polygonum, Trifolium, Medicago); as examples of perennial prostrate plants - the Bird's - foot Trefoil (Lotus corniculatus), the variegated Coronilla (Coronilla varia), and several caryophyllaceous plants (e.g. Saponaria ocymoides, Telephium Imperati).

When the leafy shoot lies on the soil, it can easily dispense with the development of those cells which would otherwise be required to give to its stem strength for support and resistance to bending. Thus plants with procumbent stems have an advantage in this respect over such as stand erect, in that they can economize so much building material. On the other hand, however, the procumbent form has the disadvantage of being able to expose to the light relatively little green tissue; only those of its leaves can be well illumined which are arranged like a mosaic in a plane parallel to the substratum. The development of a second such layer of leaves higher up would be a decided disadvantage, for it would cause the lower stratum of foliage-leaves to turn yellow and pine away. Consequently, any upward extension of the green tissues in procumbent shoots is 
necessarily limited. Again, the earth offers an insuperable barrier to the development of foliage in a downward direction. In the dark bosom of the earth a green leaf would be quite useless, and, as a matter of fact, there is not a single plant whose green tissue is situated in the depths of the soil.

With water it is otherwise. In it green cells and tissues can function as far down as the light can penetrate. Since the water also maintains the stem and leaves in a definite position, and the plants consequently are spared the development of wood and bast and, generally, of masses of tissue for strength and resistance to bending, and since, finally, a saving of material and work is effected inasmuch as water-plants do not require to construct organs for conduction of water and for transpiration, it might be supposed that water would be an extremely favourable medium for green vegetation, and that, consequently, stretches of water all over the world would be quite crowded with green plants. That this is not the case is explained by the fact that light does not penetrate far enough into the water. In the deep gloom, 200 metres below the surface, green plant-life is as impossible in water as in the dark bosom of the earth, and the bottom of the ocean over an enormous area is a plantless waste shrouded in gloom. But as far as the water is illuminated, in all places where it fills shallow basins, and also in a comparatively narrow girdle around the coasts, an inexhaustible wealth of plants is to be found. Of course, spore-bearing plants, which are built up of rows, nets, and plates of cells, have the preponderance, whilst seed-plants are markedly in abeyance in relative number of species. But the latter species are just the ones which claim our interest in a special degree on account of the very peculiar conditions under which they live.

The floating stems of water and marsh plants, as already repeatedly stated, have no wood or bast, while, on the other hand, they are penetrated by remarkably large air-canals, and are, in consequence, exceedingly light and buoyant. If the erect stem of a water-plant growing at the bottom of a lake is cut through close above its roots, it rises immediately to the surface of the water, there assumes a horizontal position, and remains floating; under certain circumstances it may continue to grow and may perhaps take root should it drift to a shallow place. On the other hand, if a pond filled with Water Crowfoot, Myriophyllum, Elodea, \&c., be emptied, all these plants sink limp and withered on to the mud, as their stems have not the strength to hold themselves erect. The water in which they float supports and bears them, and in this respect they may be likened to climbing stems which also require a support to enable them to rise above the ground. The analogy between these plants is evident in so far as the need for "more light" influences the direction of growth in both cases - in the one case the stem grows out from the gloom of the forest floor up to the sunny tops of the trees, in the other, from the subdued light at the botton of the lake up to the surface of the water. In many cases, of course, the stem of water-plants remains so short that it scarcely rises above the mud at the bottom of the pond, but the leaves arising from it are shaped into long ribbons, whose 
freely floating ends ascend into the better illuminated upper layers of water, or leaves with large blades and elongated stalks spring from the short stems, and the stalks continue to grow until the plate-like blades have reached the surface, where, floating, they can enjoy the full sunlight. There are also some plants not fixed but swimming close to the surface. These sink down to the bottom only when the activity of their leaves is suspended and here for a time they pass a dormant period.

We mention here the most noticeable variations which are made use of for dividing the stem-forming water-plants into architectural groups. First of all is a group of plants, of which the Grass Wracks (Zostera) may be taken as a type. These have stems embedded in the mud, creeping, and anchored by root fibres. The leaves arising from these stems are erect, very long and narrow, looking like thin limp ribbons, which are only kept in their erect position by the water. The Zostera grows in large patches on the shore between tide-levels. Its leaves are collected and dried and under the name of Sea-grass are used as stuffing for cushions. To this group belongs also Vallisneria spiralis, which is figured opposite, and to the flowers of which we shall return in detail later on; lastly, we may mention certain species of Sparganium. In addition to this group is a second, as a representative of which may be named the curious Lattice-leaf plant (Aponogeton fenestrale or Ouvirandra fenestralis) inhabiting the waters of Madagascar. Its short stems are buried in the mud; the leaves have short stalks, and are not erect, but distributed in rosettes over the muddy bottom. The green colour of their chlorophyll is almost entirely obscured by a reddishbrown pigment; the parenchyma, which usually fills the meshes of the net-work of strands, is absent, and the strands forming the framework of the leaf-blade are covered only with a thin layer of chlorophyll-bearing cells, so that the whole structure reminds one of a leaf which has fallen from a tree in autumn and has been macerated under water, of which, after the falling away of the easily decomposed parenchyma, only the net-work of strands remains. The Water-lilies may serve for a type of the third group. Their stems are short, rooted in the mud, and send out leaves whose broad blades, often circular in outline, are borne on very long stalks. The disc-shaped leaf-blades lie with their under side on the surface of the water, while their upper surface is exposed to the air. The leaf-stalks thus traverse the whole depth of the water, and look like ropes by which the floating leaf-discs are anchored in the muddy bottom. The long scapes, terminating in floating flowers, serve a similar purpose. Here also must be included the aquatic fern-like plant-Marsilea. Its leaves remind one of those of the Wood Sorrel. The Frog-bit (Hydrocharis) and the Villarsia (Limnanthemum) form a fourth group, not unlike water-lilies on a small scale. Their leaves and flowers, however, do not arise directly from the main stem (as in the last group), but from long lateral shoots, quite bare of leaves, till just close to the surface ( $c f$. vol. II., fig. 419). Our fifth group includes forms transitional between the groups already described and the sixth and largest group. They include forms with 


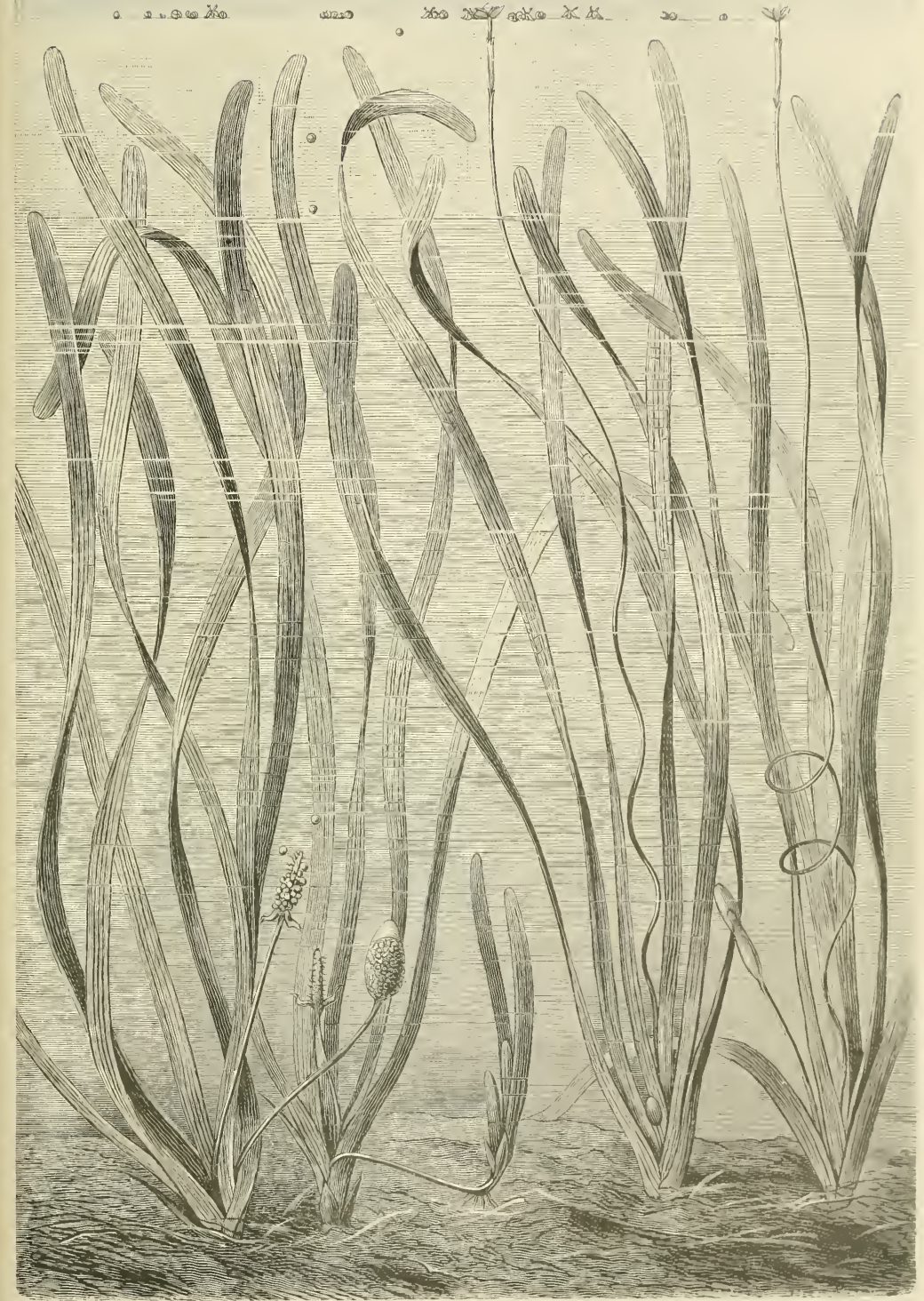

Fig. 155.-Vallisneria spiralis. 
finely-divided, submerged leaves, in addition to orbicular floating ones as in the water-lilies, \&c. Such plants are known as heterophyllous (plantoe heterophylla). Examples are furnished by several potamogetons (Potamogeton heterophyllus, rufescens, spathulatus), some water-crowfoots (Ranunculus aquatilis, Baudotii, hololeucus), the Cabomba (Cabomba aquatica) and the Water-chestnut (Trapa). In the sixth group the plants are firmly rooted in the mud like those of the former group, but the shoots rising from them bear only submerged, thin and limp leaves. These plants in descriptive botany are called "submerged" (plantre submersa). Their leaves - arising from the much-branched filamentous stems - exhibit an endless variety of form. They are sometimes decussate, sometimes spirally arranged, often broad and embracing the stem, and then again fall into the opposite extreme, and form long very narrow ribbons or threads. Frequently they are reduced to mere bristles; in other cases they are entire and undivided; again, in other instances, they have finely indented and sinuous margins (cf. fig. 136, p. 551). All these various forms of leaf are connected with the peculiarities of the habitat, with the attacks of animals to which they are liable, with the conditions of illurnination at different depths of water, but chiefly with the direction of the foliage-stem. The long thin stems can only maintain a vertical position in still water, and only in the calm inlets of lakes and in the deep pools where an active movement of the water is impossible are to be found species whose submerged leaves, arranged at definite intervals, exhibit a circular form. In running water, especially in quickly-flowing streams, the leaves are always long drawn out, ribbon-shaped, filamentous, or divided into thread-like lobes. They adapt themselves exactly to the current, and follow it in all its movements uninjured. These leaves of running water are always fairly tough; their cell-walls are correspondingly thickened; the stems from which they arise are protected against rupture by bundles of bast deposited in the cortex, and are strengthened against strains by various other contrivances to be presently described.

While the foliage stems of the water and marsh plants hitherto described are anchored fast by roots to the muddy bottoms of lakes, pools, and streams, those of the Aldrovandia, figured on p. 151, and also of the bladder-worts described and figured on pp. 120, 121, float in the water without a trace of root formation. Since the leaves require light, it is clear that they will take up their position near the surface. At any rate at the time when they are actively engaged in the manufacture of organic matter under the influence of light, they are obliged to seek such illuminated places. The bud-like tips of the shoots can, of course, in many species sink to the bottom for the winter rest, but at the commencement of the favourable season next spring, they again ascend and produce their flowering axes above the surface of the water. A horizontal, or obliquely ascending position is the most advantageous for the stems of these floating plants as regards the illumination of their leaves, and, as a matter of fact, this direction is observed in them. Running water would form a bad environment for such rootless, freely oscillating plants; they are found exclusively in the calm inlets of ponds and lakes and in pools and 
ditches amid reeds and rushes, where a great agitation of the water has never to be provided against.

In similar habitats other species of the last group of plants with floating stems are also found, viz. those known as "swimming" plants (planto natantes). They are distinguished from floating plants especially by the fact that their green foliage and in part their stems also lie on the surface of the water, and are in contact on the upper side with the air, or even rise above the water, when they are completely surrounded by air. The stem rests and moves on the surface of the water, and is never held fast in the muddy bottom-even when roots are present. Amongst the well-known forms belonging to this group are several Duckweeds (e.g. Lem na polyrrhiza, gibba, minor) with stems curiously flattened and leaf-like. Besides these, there are Salvinia and Azolla, belonging to the vascular cryptogams, and finally several species of Pistia, Pontederia, and Desmanthus, belonging to tropical waters. It has already been mentioned (p. 638) that the floating capacity of Pontederia crassipes is increased by the possession of a vesicular, air-containing tissue in its swollen leaf-stalks. Moreover, in Desmanthus natans an actual swimming apparatus is developed, not in the leaf-stalks, but in the stem itself. It takes the form of a large-celled, spongy, air-containing mantle, arising here below the epidermis of the internodes which renders sinking impossible. The mimosa-like foliage-leaves rise up from the nodes of these floating stems like masts with flags. When the leaves turn yellow, the stems rid themselves of their swimming organs which are no longer needed, and indeed it appears to be an advantage to the leafless stems to be able to sink down and to obtain a period of rest at the bottom.

Several species of the last group of plants with floating stems strongly remind us of plants with procumbent stems. At the stem-nodes they develop roots which sink into the depths, and green leaves which rise up to the sunlight, and the only difference consists in the fact that in the one case the water, and in the other the soil, forms the bed, and even this distinction is sometimes obliterated. When the level of the water sinks, the floating plants sink with it, till finally they lie on the mud, and then, as a matter of fact, they are scarcely distinguishable in habit from plants with procumbent stems which grow on the soil of the moor.

\section{CLIMBING STEMS.}

Often it happens that the name of a plant affects our imagination by its pleasing or harmonious sound. One associates with the name not merely the idea of the form of a certain plant, but more than this, its whole surroundings, framed in which it grows and flourishes. One conjures up a picture of a flowery meadow or scented wood with which the plant with pleasing name can only harmonize. It may be some far-back reminiscence is bound up with the pretty name, or we have read a vivid description in a book long ago. Thus idealized, one shrinks from approaching it with critical eye, from examining it with knife and microscope, and from classifying and describing it in the dry language of the specialist. 
I am thinking here especially of the word "liane". When this beautiful word is sounded a whole series of splendid pictures stand out in strong relief from the twilight of youthful recollections. I see a dense leafy canopy, lit by a stray sunbeam here and there, arching over the gigantic stems of the primeval forest-stems which rise up like the columns of a spacious hall. On the forest floor the scanty green of shade-loving ferns covers the remains of fallen trees. Further on a confused brown mass of tangled roots renders progress over the still dark ground almost impossible. In contrast to these gloomy depths how brilliant is the picture in the glades and on the margin of the primeval forest! Plant forms in indescribable confusion piled up into the thickest of hedges rise higher and higher to the very crowns of the giant-trees, so that it is impossible to obtain even a glimpse into the pillared hall of the interior of the forest. This is the true and proper home of the liane. Everything climbs, winds, and twines with everything else, and the eye in vain attempts to ascertain which stems, which foliage, which flowers and fruits, belong to which. Here the lianes weave and work green draperies and carpets in front of the stems of the forest border, there they appear as swaying garlands, or hanging down as ample curtains from the branches of the trees. In other places they stretch in luxuriant festoons from bough to bough and from tree to tree, forming suspension bridges, even actual arcades with pointed and rounded arches. Isolated tree-trunks are transformed into emerald pillars by the covering of woven lianes, or more frequently become the centres of green pyramids over the summit of which the crown spreads out in verdant plumes. Where the lianes have grown old with the trees on which they cling, and the older portions of their stems have been long stripped of foliage, they resemble ropes stretched between the ground and the tree-summits, and often assume peculiar and characteristic forms. Sometimes drawn out tightly, sometimes limp and swaying, they rise up from the undergrowth of the forest ground, and become entangled and lost far above among the boughs. Many are twisted like the strands of a cable, others are wound like a corkscrew; and others again are flattened like ribbons, hollowed in pits, or shaped into elegant steps - the celebrated monkey-ladders.

The green garlands, bowers, and festoons of lianes are adorned with the gayest flowers. Here a truss glows with flame-like brilliancy, there a large blue raceme sways in the sunshine, and here again is a dusky curtain studded with hundreds of bright star-like passion-flowers. And where flowers flaunt themselves and fruits ripen, guests are not wanting. The gay assemblage of butterflies and the joyous songsters of the wood regard the forest border interwoven with lianes as their favourite rendezvous.

From what has been hitherto said about lianes, one might think that this particular plant formation belonged only to the tropics. This would, however, be incorrect. In the neighbourhood of the Canadian lakes, and in the districts of the large central European rivers, the Danube and the Rhine, various species of Clematis, wild vines, climbing roses, honeysuckle, bramble, many Menispermaceæ, \&c., climb up to the summits of the trees; and even the woods of our lower Alps contain one 
of the most charming lianes, the Alpine Vine (Atragene alpina), adorned with large, blue, bell-shaped flowers. Of course the number of species increases immensely as we approach the torrid zone, and we shall not be far wrong if we estimate the number of lianes in the tropics at 2000, those in the temperate zones at 200 species. Lianes are foreign to the Arctic regions and to the treeless mountain heights; nor are they found on treeless steppes. It is remarkable that tropical America contains almost twice as many climbing plants as tropical Asia. Brazil and the Antilles exhibit the greatest wealth of these plants.

The sweet word "liane" originated in the French Antilles, and has now found its way into most languages. It seems strange that this word should never have been introduced into botanical terminology; we use the expression indeed in general descriptions of the vegetation of a district, but in that of individual species it is avoided. This is explained by the fact that we understand by lianes in the original sense of the word only climbing plants with woody perennial stems, and that there are many twining, creeping, and climbing plants possessing herbaceous stems to which the name liane is not properly applicable. On the other hand, the climbing plants are so much alike in their manner of life that they can only be treated together, and are therefore conveniently designated by a common name. We now name all inclusively "climbing plants", whether woody or herbaceous, and define the "climbing" stem (stirps scandens) as that which is able to obtain for its free end a resting position at a great height above the nourishing earth only by the aid of foreign supports. If, where climbing stems grow, there are no elevated objects which might serve for support, the earth itself is used by the free end as a restingplace; the stem then spreads its whole length upon the ground, or forms an arch, having at any rate its free end supported on the ground. Such a stem shows all the characteristics of a prostrate stem. In the earliest stages of its development, on the other hand, every climbing stem resembles an erect plant; it is difficult to name external characteristics by which young shoots of the one can be distinguished from those of the other. The shoots at first are erect and able to maintain themselves in a vertical position by their inner structure, and especially by the turgidity of certain groups of cells. Not until they have become older, and have reached a certain height does inclication of a climbing habit appear, when the shoot seeks to obtain a hold for its free end. It curves over foreign bodies in the vicinity, thrusts out horizontal branches over projecting edges of rocks or in the forks of boughs of trees which serve as supports; its tip revolves like the hand of a watch, and winds round an erect post, or it develops special organs, by which it becomes connected and entwined with adjacent objects. In respect of their varying behaviour, climbing stems may be divided into five groups, viz. weaving, lattice-forming, twining, creeping, and climbing, of which classification, of course, as in so many similar cases, it must be noted, that it is purely artificial, and is only used with the object of distinctness, and that intermediate and transitional forms between the several groups occur in abundance.

The weaving stem (stirps plectens) obtains a resting-place for its branches and 
foliage in the following manner:-As a young shoot it grows first of all vertically erect; it has as yet no lateral branches, and its leaves at the free-growing end are still small, furled, and crowded closely together into a cone. These young turgescent shoots readily pass through the forks of the boughs, even through narrow chinks and meshes of the net-work of twigs and branches in the thickets, without suffering injury. When its growth in length is terminated, the shoot unfolds its leaves and sends out lateral branches which project at right angles in all directions. These reflexed leaves and the lateral branches which have been produced above the gaps in the matted undergrowth, now get a good purchase on the rough boughs of the underwood; the slender upgrowing shoot is suspended by them as if by barbs, and it is frequently also actually woven into the underwood.

These forms of weaving stems may be distinguished according to the character of the support. First, that of the hedge-forming shrubs, of which Lycium may serve as type. It is astonishing how its long whip-like shoots, as they grow up from the ground on the edge of a wood, find their way between the spar-like branches of other growths, and then perhaps at the height of the lowest boughs of the crown of one of the trees, the free end projects as if from an opening in a roof. In the course of the summer the thin slender stem lignifies, and leafy lateral shoots spring from the axils of the upper leaves at about a right angle. These end in stiff spines. Meanwhile the highest portion of the shoot becomes bent over some bough, so that the whole shoot is so interwoven with the undergrowth, that in attempting to extricate it we tear innumerable supporting branches and twigs, and set the whole neighbourhood in motion. The lignified shoot of the first year survives the winter; next spring those portions of it which rest horizontally on the branches produce new shoots in pairs, close to the thorny lateral branches. Of these one usually remains small; the other, slender and vigorous, pushes up into the crown and repeats the method of growth of the former shoot. As this is repeated from year to year the whole crown of the tree becomes densely interwoven with the Lycium shoots. Often it happens that shoots are produced, which hang down from the tree-crown like branches of a weeping-willow draping the supporting tree as with a curtain, or forming an actual hedge in front of it.

The following well-known plants develop in accordance with this Lycium type:-Numerous roses (Rosa), brambles (Rubus), barberry (Berberis), spiræas (Spircea), sea-buckthorn (Hippophaë), jessamine (Jasminum), Celastrus scandens, and numerous other woody hedge-formers which grow preferably on the borders of forests. Many roses, as, for example, the Rosa sempervirens, abundant in the Mediterranean floral district, not only weave through the undergrowth, but often reach the tops of the highest oaks. Also many brambles (Rubus) reach far up into the boughs of the tree-crown, and then not unfrequently depend their long shoots in arching curves. I measured the length of a stem, $\frac{1}{2}$ centimetre thick in the middle, of a species of bramble (Rubus amcenus) which had interwoven with the tree-crown, and found it to be six and a half metres. The long whip-like shoots of Jasminum nudiflorum and Celastrus scandens also reach the tops of high trees 
in the same manner. If these hedge-shrubs have not the opportunity of interweaving in the branches of trees, \&c., they are obliged themselves to form a scaffolding. Their manner of growth resembles that already described, except that the shoots usually remain shorter, and the whole plant consequently appears more compressed. The erect shoots at first mounting vigorously upwards form, as they become lignified, flat arches, bent over so that their apices alınost trail upon the ground. The upper portions of these arches give rise next year to short flowering branches and to long vigorous shoots, which give rise to new arches. The free ends of the old arches dry up, and fresh arches come to lie above the dried remains. In the following year new arch-like shoots proceed from the last-formed ones. This being repeated year after year, an impenetrable natural hedge gradually rises, which grows continually higher and higher, since the stumps of the old, dried-up branches, whose ends have stopped growing, form supports for the younger shoots. It is also a very common occurrence for these hedge-shrubs, when they have become old, to develop suckers from their roots, which grow up, thin and slender, between the undergrowth formed of the old, dried-up arches, which they use as a support. This may be seen especially in the barberry, sea-buckthorn, mock-orange, roses, jessamine and the elm-leaved spiræas.

This property of forming hedges has long been familiar to agriculturalists, who are close observers of nature. Several such plants are used for the purpose of inclosing portions of land; thorny species are especially suited for the purpose, the so-called "quickset hedges". Gardeners, too, make use of this peculiarity of hedge-weaving shrubs when they plant species with beautiful flowers close against a trellis-work, which is soon quite overgrown with their vigorous shoots. The so-called climbing-roses in particular are used with the best results for covering trellis-work against the fronts of buildings, and it is remarkable how quickly they grow without assistance right up to the gables of the houses. Some climbing roses (e.g. Rosa setigera) have this remarkable peculiarity, that their new shoots at first seek the darkest places, turning their apices away from the bright sunshine, growing into the shaded nooks behind the trellis-work, and not inclining again towards the light until they are fully grown. In this way the advantage is obtained that the shoots originally turning from the light enter the gaps of the undergrowth and of the trellis-work, while later on, when lateral branches arise from them, they are excellently supported.

Generally resembling the woody stems observed in hedge-builders are those of several undershrubs which do not become lignified. The shoot growing up annually at the beginning of the vegetative period from the underground portion of the stern always dies off again in the autumn, whilst the dried remains still above the ground decay so quickly that only in rare cases can they be used as supports for the shoots which grow up fresh from the soil in the following year. As a type of wearing undershrubs the widely-distributed Marsh Crane's-bill (Geranium palustre) may be taken. The young shoots grow erectly among the bushes scattered over damp meadows or on the edge of a forest, but they do not become woody; their upper ends VoL. I. 
do not bend over the supporting branches, but, having once attained a certain height, develop stiff lateral branches, projecting like spars, and long-stalked leaves which push their way between the stiff, dry branches of the supporting bushes; in this way the whole shoot is held fast so that it cannot be displaced. When this same Crane's-bill grows in a meadow between low herbs which can afford it no support, the stem bends and the whole shoot lies with its lower internodes on the ground. The ends of the internodes are thickened, and a turgescent cell-tissue is formed at these places by means of which the younger parts of the shoot are brought again into an erect position, appearing at right angles to the older internodes lying on the ground. The advantage obtained by this arrangement, is that plants of Crane's-bill thus extended over the ground are able, should they encounter a firm shrubby undergrowth not too far removed from the place where they are rooted, to use it as a support and to weave themselves over it. As a matter of fact, plants of Geranium palustre are often seen with their lowest internodes lying on the ground, while the upper internodes as well as numerous lateral branches are interwoven into some bush growing in the meadow near by, and their red flowers are displayed more than a metre high above the soil from between the branches of the bush serving as a support. Several other species of crane's-bill resemble this one in habit (e.g. Geranium nodosum, divaricatum, \&c.), also several species of bedstraw and woodruff (e.g. Galium mollugo, Asperula aparine), the berry-forming Cucubalus (Cucubalus baccifer), and, finally, the remarkable Marsh Speedwell (Veronica scutellata). Here, too, belong several species of asparagus with projecting, spar-like branches and filamentous or needle-shaped phylloclades. The annual shoots of these asparaguses attain an astonishing length and push their way into the forkings of the boughs of erect-growing stems. In this respect the Asparagus acutifolius, very common in the region of the Mediterranean flora, is particularly worth mentioning, and also the Asparagus verticillatus growing in Asia Minor, the stems of which not infrequently attain a length of 3 metres, climbing up to the crowns of the lower oaks and there interweaving their horizontally disposed branches with the boughs.

The third group of plants with weaving-stems includes the rotangs, those peculiar palms celebrated for the fabulous length of their almost uniformly thickened stems. A species of rotang, drawn from nature in Java by Selleny, is given on the opposite page. The stem of all young rotang plants is erect, and the yet folded leaves grow vertically upwards in the same direction as the young axis. Later, when the leaves unfold and expand, they arch outwards and spread themselves over the confused mass of other growths, amongst which the rotang plant has germinated and grown up. If the vegetation in the immediate neighbourhood consists only of low herbs and bushes, the elongating rotang stem does not find a support sufficient to enable it to grow up in the original vertical direction. So it trails on the ground like a runner, often forming snake-like coils, as shown in fig. 156; still always bending up at the free end, and continually pushing up new leaves. If the rotang plant has developed amongst tall shrubs and trees, or if after 


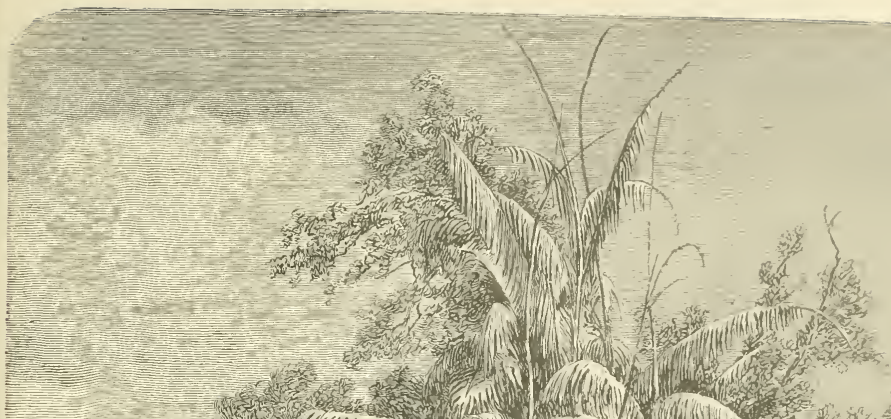

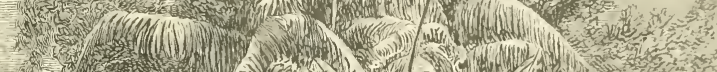

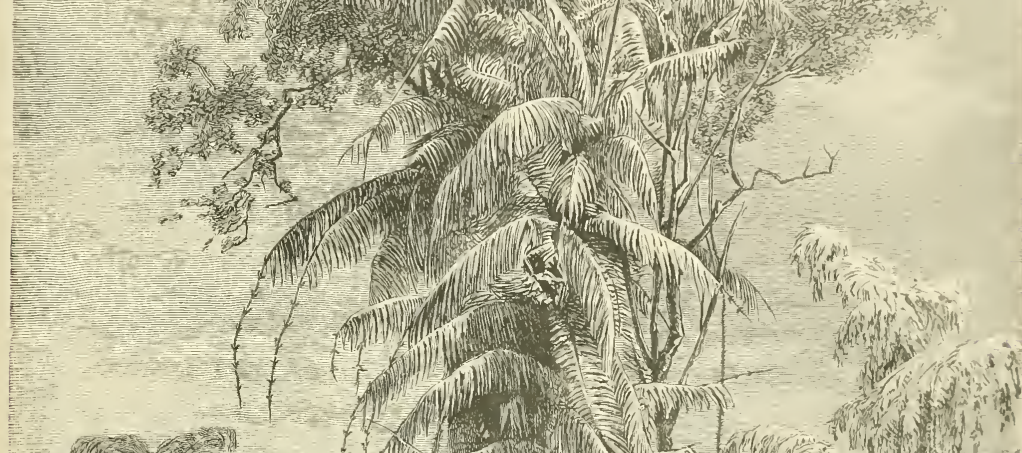

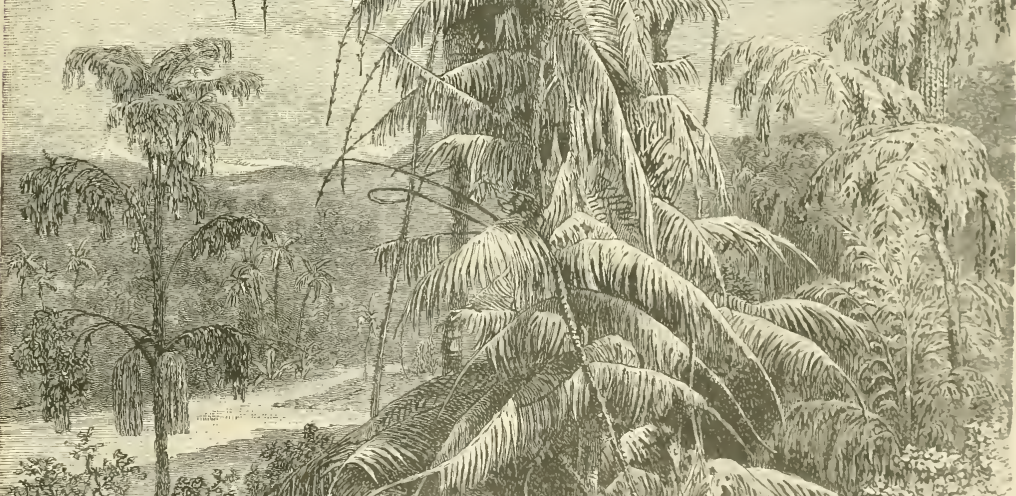

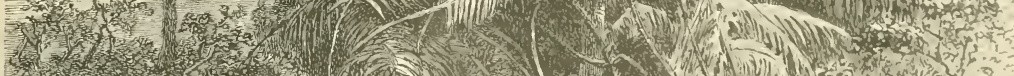
2.

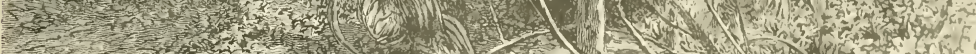

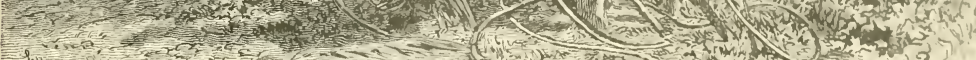

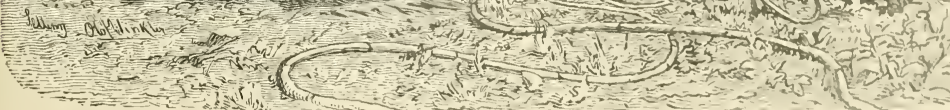

Fig. 156. - Rotangs in Java. (F rom a drawing by Selleny.) 
trailing, it has come within the range of a wood, it pushes its stiff, folded, spire-like leaves between the lower branches of the trees, and as these leaves unfold and bend outwards, they form strong supports or barbs by which the cord-like stem is anchored above in the branches of the tree ( $c f$. fig. 94, p. 363). Under favourable conditions the stem can grow up to the tops of the trees, its new leaves always anchoring thus in the branches above. Frequently the free end of a rotang shoot

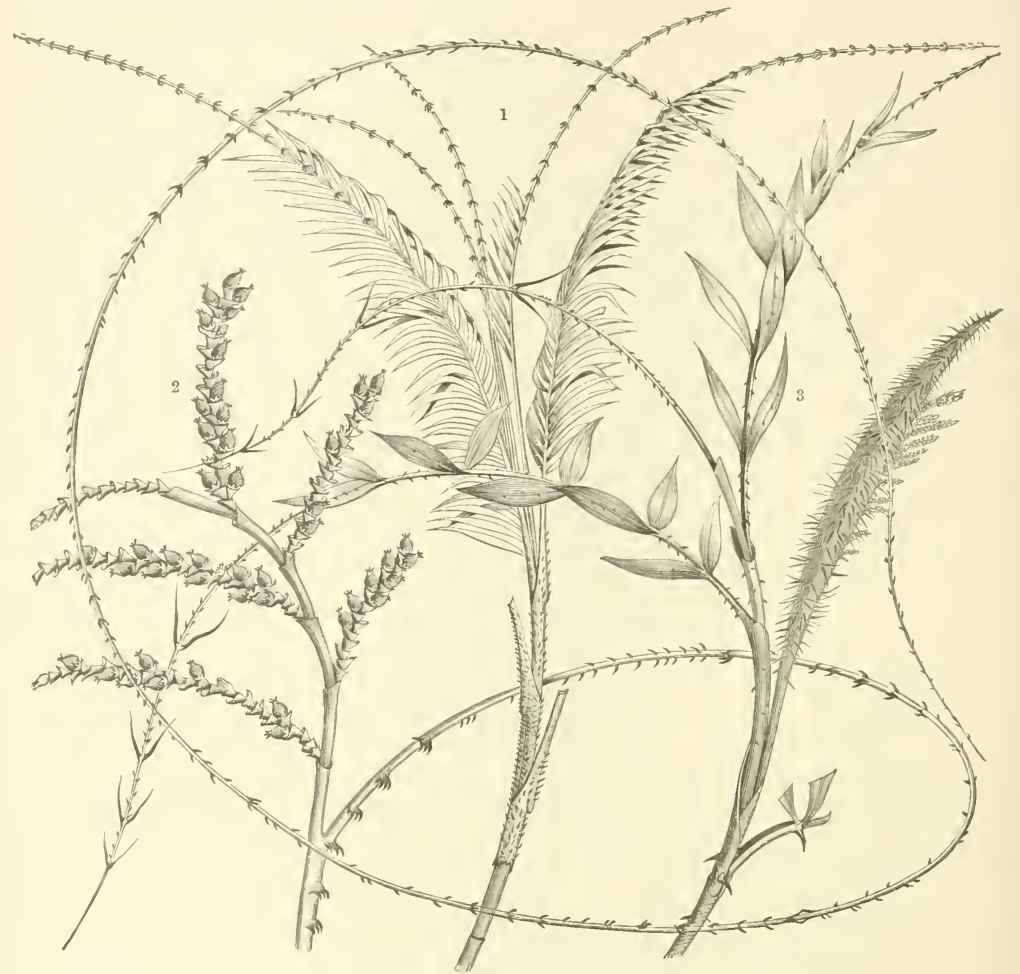

Fig. 157.-Shoot-apices of three species of Rotang.

1 Daemonorops hygrophilus. 2 Calamus extensus; with inflorescence. ${ }^{3}$ Desmoncus polyacanthus; much reduced.

grows from tree to tree-now ascending, now descending. It is shoots of this kind which attain to lengths unequalled by any other plant. There are credible statements according to which such rotang stems, with an almost uniform thickness of only $2-4 \mathrm{~cm}$., have reached a length of 200 metres.

We must not omit to mention that most, if not all, plants which weave into the thicket of other plants are equipped with barbed spines, prickles, and bristles, which assist them in maintaining themselves at the heights once reached. The goat's thorn is provided with horizontally-projecting spines; in the roses and brambles 
both the internodes and the ribs of the leaves are beset with sharp, backwardlydirected prickles; several bedstraws (e.g. Galium uliginosum and aparine) bear short, stiff, reversed bristles on the ridges of the stem and on the leaf-margins and ribs, whilst the midrib of the pinnate rotang leaves is continued beyond the blade as a long whip-like structure beset with barbs of the most varied description. The illustration of three species of rotangs inserted opposite shows the most striking forms of these peculiar leaves. In one species (fig. $157^{1}$ ) the leaf-rachis is beset at equal intervals with groups of small but very pointed barbs; in a second species (fig. $157^{2}$ ) the uppermost leaves are wholly devoid of green pinnæ, and bear only numerous claw-like barbs; while in the third (fig. $157^{3}$ ), very long, pointed, reversed spines are found on the foremost portion of the leaf, with little teeth between, so that this

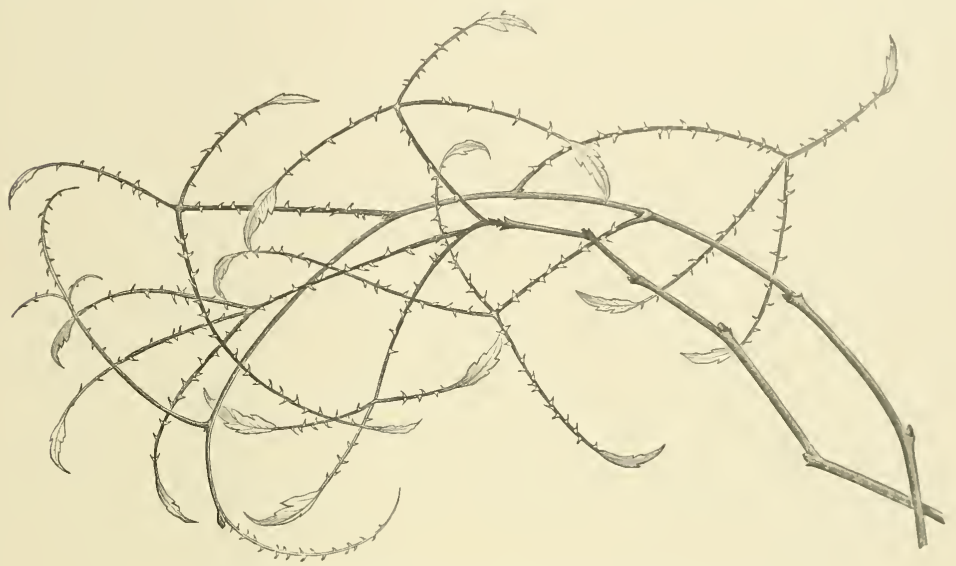

Fig. 158.-Branches of the New Zealand Bramble (Rubus squarrosus).

portion resembles a harpoon. When we look at these barbed structures and consider that the rotang leaves are exceedingly rough, we can understand how firmly the rotangs anchor themselves in the crowns of the tree-summits, and how difficult it must be to disentangle these climbers, fastened as they are with harpoons, from the trees they interweave.

A plant distinguished by its unusually rich development of barb-like spines, and deserving especial mention here, is the New Zealand bramble, Rubus squarrosus, illustrated in fig. 158. Each of its leaves is divided into three portions, each being provided with a tiny blade at its apex; these three portions as well as the leaf-stalk are green throughout their entire length and beset with yellow, pointed prickles which anchor so firmly in the intertwined bushes and shrubs that a wholly inextricable tangle is the result. Finally those plants still remain to be considered in which the support is afforded by the pointed teeth of the leaf-margin. To these belong especially several tropical Pandanacex, with long thin stems resembling rotangs, 
and also an insignificant little speedwell which grows in damp meadows in Great Britain, and rises above the ground by sprawling over its erect and stronger neighbours. This speedwell (Veronica scutellata) has long, narrow leaves which in section almost resemble those of the tropical Pandanus. Like these they are erect when young, and are inserted in pairs over the vertically-growing apex. By the further growth of the stem they are pushed in between the gaps in the confusion of herbage. By and by the leaves are reflexed and afford the plant useful support. While the serrated teeth of the leaf-margins in other species of speedwell have their apexes directed forwards, in this they are strangely directed backwards, i.e. downwards towards the ground; by this means the support which these leaves obtain is materially increased. In this speedwell the retro-serrate teeth of the leafmargin have undoubtedly no other significance than that of firm anchorage, though, in many of the other above-named instances, the pointed teeth, prickles, and spines have the additional task of protecting the foliage, and perhaps also the flowers and fruit, against animals which might climb up over the stem in their search for food.

The lattice-forming stem (stirps clathrans) does not twine, nor indeed has it any special climbing organs, and yet leaning against rock-faces or tree-trunks it gradually attains to heights which it would be unable to reach without these supports. It clothes its supports with branches, which, in the aggregate, constitute a solid lattice-work, reminding one of certain interweaving climbers, from which, however, it is distinguished by the fact that its elevation is achieved neither by lateral branches projecting like spars, nor by arched shoots, nor even by reflexed foliage-leaves. Lattice-forming stems occur comparatively seldom in the floras of the temperate zones; the most striking example in these regions is the small and dainty species of buckthorn known as Rhamnus pumila, whose lattice-work clothes the steep limestone rocks here and there in the outlying Alps between Switzerland and Styria, and in the Jura. Anyone looking from a little distance at a precipitous rocky face overgrown with this buckthorn, might think that it was ivy which had spread out its stems, climbing by means of clinging roots. The foliage shows, indeed, the same dark green and is about the same size as that of ivy, but it is easily recognized on a nearer view that the shape of the leaf, the distribution of the strands in the blade, and finally the character of the flowers and fruits are quite different, and, what is especially important here, that the much-branched woody stems adhering to the steep rocks have no clinging roots. It is also an interesting fact that the older stems are actually wedged into the crevices of the rock, and that the branches are exceeding brittle. With careless handling they break and fall to the ground, and only by proceeding very carefully can one succeed in detaching a complete stem with all its branches from the rocky face. We may conclude that this plant would necessarily perish without the supporting background, since its brittle branches would break off at the first violent burst of storm, and the bush would be mutilated by every tempest.

The peculiar structure and method of growth of this buckthorn explain all these striking phenonema. Here there are no strands of hard and fibrous bast deposited 
outside the soft bast, such as enable the young branches of other trees to resist flexion or to resume their position after bending by the wind. In the centre of the branch is seen a woody cylinder, surrounded by strands of soft bast; and beyond this a very voluminous parenchyma, but only a very few hard tenacious bast-fibres. It is evident, therefore, why the branches break away so readily. And that they split up most easily at their places of origin, i.e. where they arise from an older branch, is explained by the fact that the woody cylinder is weakest at these points. The method of growth of the branch is just as remarkable as its structure. When in the spring leafy shoots proceed from the foliage-buds, they do not grow towards the light, as in the greater number of plants, especially woody plants, but they turn away from the light and seek the darkness, and even curve round projecting angles into shady corners, growing into the dark crevices and clefts in the stone wall. If the face is not cracked for a wide distance, but is smooth and even, the longer, growing shoots always hug it closely, and take a straight course; but, as soon as a fissure is reached, the shoot immediately bends round into it, much in the manner characteristic of roots ( $c f$. p. 88 ). While in other shrubs the young, growing shoots arising from an old woody branch are directed upwards, it here frequently happens that a downward course is followed. The burden of the foliage unfolding on the shoots, and the consequent increase of weight cannot be regarded as the cause of this bending, for not infrequently from one and the same branch, as it runs horizontally over the rock wall, shoots arise side by side of similar shape, similarly leaved, and of about equal weight, some of which grow downwards and others upwards.

In this manner of growth it is unavoidable that the branches should sometimes cross one another, forming a lattice-work which adheres to the rock. I have never observed actual fusions of the intersecting branches in this buckthorn, but it often happens that the younger branches which lie across the older are so firmly attached to them that they still remain connected when large portions of the plant are removed from the rocky wall.

Such extensive lattice-branches have quite the appearance of a root-plexus which has extended over a boulder, and we are reminded of the remarkable latticed rootformation of certain tropical fig-trees, which will be discussed later on. There is also a temptation to take the older stems of Rhamnus pumila for roots, inasmuch as they are frequently seen embedded in the clefts and crevices of rocks, a phenomenon brought about in the following way. When the apex of the developing, light-avoiding shoot reaches a dark cleft, it continues to grow in a manner readily intelligible in the direction of the crevice, into which it nestles so far as its foliage permits. Later on it becomes lignified and loses its foliage; in the following year it sends up new shoots, but itself remains growing in diameter by the addition of wood and bast, till sooner or later it becomes so thick that it is jammed tight in the cleft, and resembles a root which has forced its way in.

The lattice formation in tropical Clusiaceæ, of which an illustration is given on page 681, is effected in a manner quite different from that obtaining in the buckthorn. The young stems of Clusiaceæ grow erect, and prefer to make use of 
tree-trunks as supports, particularly those of palms. At first they adhere very slightly, and lean on them only to a certain extent. All the shoots of these Clusiaceæ are thick and beset with opposite leathery leaves; they remain green for a very long time, and are still unlignified when they develop lateral shoots from the leaf-axils of their erect branches, and when the cortex is wounded, a thick adhesive juice like gamboge makes its appearance. The leaves are so heavy that the outstretched lateral branches are bowed under their burden, and sometimes even hang downwards. It is therefore unavoidable that many of these lateral branches should intersect and come into contact with each other, and that at the places of contact the epidermis should be wounded by the friction. But at such places an actual fusion of the branches occurs, and since this process is many times repeated, a lattice-work results, as shown in fig. 159. The individual portions of the latticed stem remain soft and pliant, and thus mutually supporting one another, the whole possesses a bearing capacity adequate to enable the erect shoots to rise higher and higher from this scaffolding. The lattice-work is additionally strengthened by the production of aërial roots from the older internodes. These, like the stems, fuse where they intersect. From their general external similarity it is often difficult to distinguish between the two sets of elements comprised in an old lattice-work. In cases where the inclosed stem increases in thickness, the latticed sheath becomes tightly stretched. Often, in Clusia, many of the branches die in consequence of this tension. Still, new shoots generally arise from the stumps, and repeating the alreadydescribed method of growth, become interlaced into a lattice-work. Sometimes the adherent stems become flattened and girdle-like, whilst aërial roots developing at many points become inextricably interwoven in the lattice-work till it is impossible to see the original palm stem. On the banks of the Rio Guama in Brazil, Martius saw whole groves of the Macaw tree (Acrocomia sclerocarpa) covered with Clusia alba. The Clusia formed an absolutely closed sheath bearing flowers and foliage, whilst 10 metres above the stately crown of the palm-tree projected.

The twining stem (stirps volubilis) is able to reach considerable heights by attaching itself to various objects and twisting spirally around them. In a state of nature, erect stems or even those of other climbing plants may serve as supports, whilst in gardens, sticks, strings and wires are utilized in this way when it is desired that twining plants shall cover walls, arbours, \&c. We find by experience that even very fine threads form excellent supports, while thick posts and bulky tree-trunks are less adapted for this purpose. In the case of many annual twining stems, props of even $20-25 \mathrm{~cm}$. diameter are too thick for the plants to twist around. Those perennial and lignifying stems called lianes are sometimes found round pillars of $30-40 \mathrm{~cm}$. diameter, e.g. those of Glycine Chinensis in the avenues of the park at Miramare, near Trieste. In the tropics, twining plants are seen embracing the trunks of trees as much as $40-50 \mathrm{~cm}$. thick, but in such instances it is probable that the trunk did not possess this thickness when it was first entwined, and only attained it later on. Of course this can only happen under particularly favourable circumstances, for most perennial, twining stems 
cannot stand the severe strain involved--a strain which must occur whenever the tree, around whose trunk a perennial twiner has wound, increases inuch in thickness. The twining stems of the Lonicera, figured on p. 160, certainly do not increase in length after lignifying, and must, therefore, act as constricting coils on the young actively-thickening tree-stems, which they often strangle to

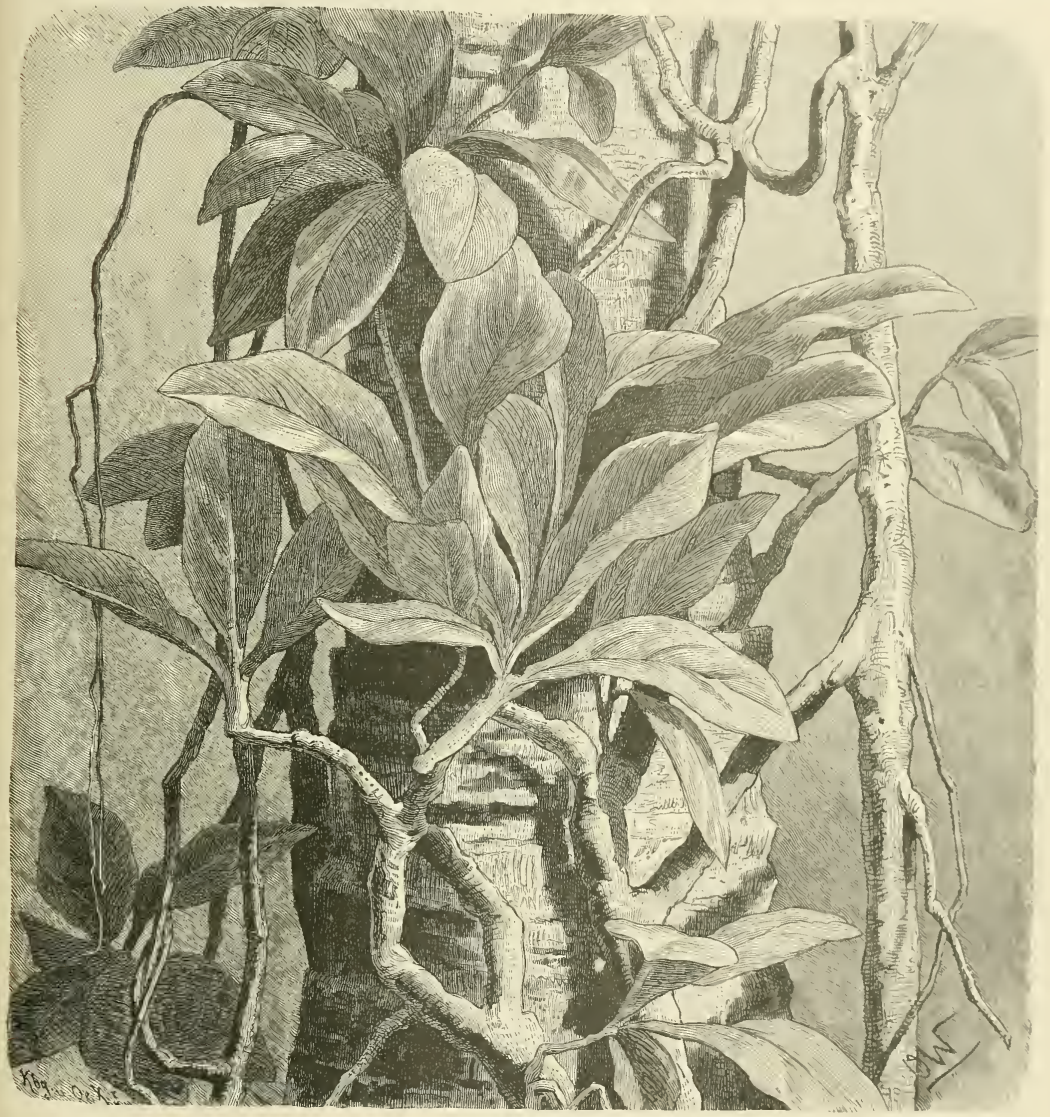

Fig. 159. -Palm-stem used as a support by the lattice-forming stems of one of the Clusiacea (Frayrcea obovuta).

death. Sometimes one finds the hard basal parts of a liane stem twisted and coiled apparently around nothing. This is due to the fact that the original support has been killed, and then slowly rotting into dust, has been denuded away by the wind and rain. Thus many a liane of the tropical forest seems to have made use, when young, of some living plant with a fairly thick erect stem as its first support, up which it has climbed into the crowns of higher trees. 
Subsequently, the first, lower prop has perished, while the branches supporting the upper portion of the liane remain still vigorous and afford a good hold. An erect corkscrew-like liane stem is then seen hanging from the upper branches; it has a very odd appearance, and is only surpassed in the peculiarity of its form by the twined stems of bauhinias and monkey ladders, to be described presently.

But if the erect, young stem is stronger and more vigorous than the twiner which encircles it, which has been used as a prop, it does not allow itself to be strangled; the twiner is destroyed when they both increase in thickness. The coils of the climber are gradually stretched tighter and tighter, and many are the contrivances which exist for preventing the tension from immediately acting injuriously on the movement of the sap in the interior of the twining liane stem. As this thickening continues, the pull on the coils becomes so great that the death of the liane results. With its decay, the coils of the liane offer no further resistance to the enlargement of the stem within; but become ruptured and unravelled. It is clear from this that it is not always advantageous for perennial and lignifying twining stems to make use of active stems as supports, and it is also obvious why old and very thick tree-trunks are never seeneven in tropical forests-encircled by twining stems. But those growths whose twining stems persist only through a single summer, and either perish entirely after the ripening of seed, like those of the twining Polygonum (Polygonum Convolvulus), or else die down to the ground like those of the Hop (Humulus Lupulus), would suffer no injury even if they were to twine round thick tree trunks. Such plants which have to develop stems and leaves in the course of a short summer, and to manufacture by the help of their green foliage the materials necessary for the formation of flowers and fruit, must spring up from the soil to the sunny heights as quickly as possible and by the most direct path. This they can best do by using a thin thread as a support, certainly not by twining round a thick tree-trunk. The path round a thick trunk would be much too long; the material necessary for the building up of such lengthy coils would be needlessly expended, and such a waste would be entirely opposed to the economy of plant-life. Of course this does not imply that twining plants have the capacity of seeking out the most suitable supports, or of selecting the most desirable from amongst many. The capacity of selection is at all times only apparent, and if hop stems never twine round props of more than $10 \mathrm{~cm}$. diameter, it is not because the hop shoots are able to recognize beforehand the unsuitability of large coils, but because with such extensive spirals they lose the power of firmly adhering to the stem. And with this we come to the description of the processes of adhesion and torsion of stems, so far as they are accessible to observation.

Like interweaving and lattice-forming stems, twining stems at first grow directly upwards. The lowest internodes still remain erect whatever may be the fate of those developing above them. After a sufficient number of successive 
internodes have been formed, the number varying according to the species, those uppermost bend over laterally, and the whole shoot now consists of a lower erect portion fixed in the soil, and an upper overhanging portion which ends freely. The lower part forms a firm and reliable support, the upper bent portion, waving in the air, undergoes movements the aim of which is to revolve the free end round in a circle or an ellipse. This movement of the nutating portion of the shoot has been compared to that of the hand of a clock; still better, it may be likened to the movement of a pliant switch or whip which is held in the hand above the head and its end swung round in a circle. The nutation of the climber is not so quick as that of the revolving part of the switch, but is accomplished with a rapidity which astonishes the observer. In warm weather the waving, revolving end of the Hop (Humulus Lupulus) makes a complete revoluiion on an average in 2 hours and 8 minutes; the French Bean (Phaseolus vulgaris) in 1 hour and 57 minutes; the Bindweed (Convolvulus) in 1 hour and 42 minutes; the Japanese Akebia quinata in 1 hour 38 minutes; and the Chilian climber, Grammatocarpus volubilis, in 1 hour and 17 minutes. Since these revolutions are performed by fairly long portions of the shoot, they may, like those of the clock-hand, be seen with the naked eye, especially when a collar of white paper is placed on the shoot in the sunlight below the overarched portion. The shadow of the moving part, like the hand on the dial-plate, is then seen slowly but plainly advancing over the surface of the paper. In other twining plants the motion is of course much slower, and many of them occupy 24 , or even 48 hours in a revolution.

Since in most twining stems a twisting of the extended fibrous bundles on the periphery of the stem occurs simultaneously with the circling of the free end, it was formerly supposed that this revolving movement was actually produced by this torsion of strands of fibres there situated. Very careful investigations in recent times have, however, demonstrated that this is not the case. The circling is produced independently of the torsion, and twining stems exist in which no torsion whatever of these fibrous bundles takes place.

We shall obtain the most accurate conception of the revolving movement of the tip of a shoot if we still retain our illustration of the movement of a switch swung round in a circle. When the switch, which may be best considered as a cylindrical body whose periphery is striped longitudinally with numerous straight lines running parallel to the axis of the cylinder, begins its motion, there is first of all an outward lateral bending. The side which becomes concave experiences a contraction, the convex side an elongation. Thus on the concave side a pressure, and on the convex a tension is set up. At any given moment these opposed strains are greatest along two opposite lines running along the periphery of the switch; in the next moment, however, the greatest strain passes over to the adjacent opposed lines, and since the greatest strain on the periphery of the switch moves in this way, and touches all the lines in succession, that remarkable circular movement of the free end of the switch results which 
entirely resembles a torsion, but which, however, as a matter of fact, is connected only with a successive bending to all the points of the compass, and with no actual spiral twisting whatever. This movement may also be seen on a switch fixed in the ground, and, generally, in any pliant shoot, by bending down the top in all directions successively, so that the point describes a circle; thus it can be easily demonstrated that no spiral torsion in the tissue of the shoot is caused by the successive bending on all sides. This movement has received the name of circumnutation.

We may now proceed to inquire into the series of changes within the stem which cause it thus to bend in all directions, what must go on in the cells along one line in this stem to cause it elongate, along another to make it contract, and to bring about this successive elongation and contraction in all the peripheral longitudinal rows. Here unilateral pressure from outside, which so often causes curvature in other cases, is shown to be just as little the reason as unilateral illumination, which it is known also produces a curving of leafy stems towards the incident sunlight. When we see that the young branches of beeches are overhanging under their burden of leaves, we may think of explaining the matter by gravity; but how can we thus explain the enigmatical advance of the inclination towards all the points of the compass, which is the point at issue here, and which has to be accounted for? The phenomenon has also been referred to growth, and it has been said that it was caused by the various longitudinal lines on the circumference of the shoot successively growing more actively than the sides opposite to them. But even supposing that the whole matter was only a phenomenon of growth (which is certainly not the case, since many shoots make these revolutions without showing the slightest increase in length), the question why it happens that the stronger growth is transferred from one longitudinal row to another, would still remain to be answered.

The first step in an attempt at explanation is to consider similar phenomena where the conditions are much simpler and where the investigation is hindered neither by simultaneous growth nor by simultaneous torsion. As such phenomena we may regard the rotating movements of protoplasmic threads in swimming swarm-spores, the circular movements of the threads of Oscillatorier composec of disc-shaped cells like rolls of coins, and the similar movements of the whiplike filaments of numerous species of Dasyactis and Euactis. Here we may disregard the question of the end to be attained by these movements. This much is certain (1) that in the one case protoplasmic threads, and in the other simple rows of cells, exhibit in their revolving movements those advancing, opposed strains which we have just noted in the rotating switch; (2) that the elongation on the one side and the contraction on the other in all these filamentous structures are not produced by a direct external stimulus. This elongation and contraction, this enigmatical advance of inclination towards all the points of the compass can therefore be caused only by internal forces, and we must suppose that the living protoplasm of the whip-like thread spontaneously elongates and contracts, bends 
and revolves in the manner described above. That which is performed by the naked protoplasm of a cilium may also be accomplished by the association of protoplasmic masses in the simple cell-filament of an oscillatoria-thread, and nothing contradicts the supposition that also in those extensive cell-aggregates which compose the shoot of a twining plant, the progressing, opposed strains, which appear as revolving movements in the shoot, occur in like manner. Why should not one portion of the masses of protoplasm, associated together and co-operating harmoniously for the welfare of the whole plant, perform that work which is accomplished in minute unicellular plant-organisms by an extended protoplasmic thread? Is it not simplest to suppose that the living protoplasm of certain rows of cells on the circumference of the shoot should effect the elongation and contraction, the advancing opposed strains above described, in a word, the twining movement of the whole shoot-apex? What it is that impels the protoplasm to this work is just as puzzling as the stimulus to the production of partition-walls in the interior of a cell, or the motive to those wonderful accumulative and divisional processes in the protoplasm of the Myxomycetes deseribed on p. 572. We know, indeed, that these processes, which depend on the displacement of the ultimate particles of the protoplasm, are possible only under certain external conditions, but it cannot be asserted that external conditions definitely shape and direct the work done by the protoplasm.

In a number of twining plants, e.g. the Hop, Honeysuckle, and the twining Polygonum (Humulus Lupulus, Lonicera caprifolium, Polygonum convolvulus), the shoots turn round from the east through the south towards the west, which is termed turning to the right (dextrorse or clockwise). Others, again, as, for example, the Scarlet-runner, the bindweeds, and various species of birthwort (Phaseolus multiflorus, Convolvulus sepium, Aristolochia sipho), turn round from the east through the north towards the west, and this is termed turning to the left (sinistrorse or counter-clockwise). External conditions have no influence on the maintenance of these directions. It is a matter of indifference to the direction of these movements whether we allow light, warmth, and humidity to operate on this side or that; the particular species always twists in the same direction, the Hop towards the right, the Scarlet-runner towards the left. More than this, even if the twining portion is continuously bound in an opposite direction, the result is all the same; the plant cannot be coerced into any other path, and will not depart from the direction peculiar to it. It continues to twist and twine according to an innate tendency inherited from generation to generation, and we can only refer the different directions of twisting to internal causes, to the peculiar constitution of the living protoplasm in each particular plant.

However puzzling the ultimate causes of this torsion may be, the end to be attained by these revolutions of growing shoots is patent enough. That it may twine upwards a shoot requires an erect support, with which it must come into contact almost at a right angle. If such a support exists in the immeliate neigh- 
bourhood, this contact occurs at the very beginning of circumnutation, but when there are no erect stems close by, the shoot in its search bends its apex to all the points of the compass, and describes wider and wider circles with its increasing length. If in the space so traversed it finds no suitable support, the lower portion of the shoot falls on the ground and becomes a procumbent stem; but the middle portion again rises up, and the free end twists round in a circle afresh. The place where the nutation now occurs is removed some distance away from the spot where it first began, and perhaps the revolving shoot in its new position may strike against something which may serve it as a support. But if here also no suitable support is encountered, a further migration may occur; thus a comparatively extensive area is explored by the circling shoot in its quest for something to climb around. The phenomena just detailed gave rise to the view formerly held, that twining plants possessed the power of searching for a support, indeed, the idea was favoured that the twining stem was positively attracted by such support. But such a notion is disposed of by the actual facts. The meeting of the nutating shoot with an erect stem must be held to be quite accidental, still it is certain that this meeting is facilitated by the movements described above, and the probability of an erect stem being encountered is obviously greater the more extensive the space traversed by the shoot-apex.

As soon as the revolving end of the shoot comes into contact with an erect support of suitable thickness, it embraces the support, and adhering to it, twists round it spirally and assumes the form of an elongated spiral wound around it. This process may be illustrated by comparing it with the movement of a rope swung in a circle coming in contact with a post, that is to say, when one swings a long rope or a long whip horizontally with the hands raised above the head, and at the same time approaches so near to an erect post that the revolving rope must reach it, then that portion of the rope beyond the point of contact twines spirally round the post.

It has been shown by manifold observations and experiments that erect props are most easily embraced by twining stems. When the inclination of the prop amounts to not less than $45^{\circ}$ with the horizon, the twining shoot still forms a spiral round it; but horizontal sticks are very seldom, though occasionally, entwined. It has also been ascertained that the revolutions made by the twining stem become both higher and steeper with increasing age. The coils formed by the youngest and uppermost portions of the shoot are often very close together and almost horizontal, but lower down the spiral appears more drawn out, and the newlyformed upper flat coils are gradually pushed passively upwards. Thus the advantage is obtained that the lower portion, as it assumes a steeper position, gets a better grip of the support. In most cases of twining the stem is to some extent twisted on itself, i.e. undergoes torsion. This torsion of the axis must not be confused with its twisting around the support. The two things are distinct. We can take two ropes, in one of which the strands are twisted, whilst in the other they are straight. Each of these may be wound round a support. The former (i.e. the twoisted rope) 
will have the best grip, as it is stiffer and its obliquely-running strands admit of a better hold. So it is with the climbing stem. By the torsion of its own axis it gets a better hold. The longitudinal ridges on its surface - due to its bundles - correspond to the strands of the rope. When these, by torsion, run obliquely, more purchase on the support is obtained.

Not infrequently the attachment of the twining stem is also strengthened by stiff, backwardly-directed bristles, and by barbs which are developed on the ridges, as is the case, for example, in the twining Polygonum, and in bean-plants. These reversed prickles are comparatively large in Ipomœa muricata, a species of bindweed. Hops also possess prickles of a remarkable form. In the Hop, as may be seen in fig. 160, they have the shape of an anvil; that is to say, a cell which is much extended in the longitudinal direction, and tapers to a point at either end, is developed on a peg-shaped or conical base. Its wall is silicified and very hard, and the points hook into softer tissue like claws. Such climbing hooks are found in regular rows on the six ridges of the twining hop stem, and are a great assistance in attaching it to the entwined support.

In Hoya carnosa, known for its waxen flowers, and often cultivated in greenhouses, the young twining stems are thickly beset with reversed hairs which under certain circumstances contribute materially to the adhesion to rugged substrata. Moreover, the stems of this plant, as soon as they have ceased to nutate, develop light-avoiding, climbing roots which nestle to the substratum and unite with it, thus adding to the security of the stem. The stems of Hoya, like those of Cassytha and Cuscuta, described on p. 171, are thus, in a way, intermediate between those of twining plants, in the strict sense, and climbing plants provided with clinging roots, which latter will be discussed presently.

When the nutating end of a twining stem has found no erect support in its neighbourhood, the older portions of its stem which no longer revolve take on, even without a support, a spiral twisting and a torsion of the axis. Just as a rope becomes more rigid when twisted, so the stiffness of these twisted stems, though they have no support, is increased in comparison with untwisted stems. Such a twisted stem may even rise a little above the ground, and in many instances the still nutating free end is enabled to reach some bough of a neighbouring tree or bush, and winding round it, to attain to the tree-crown. Many twining plants, as, for example, hops, frequently send up above the ground from their subterranean perennial portions several shoots. If these find no support in the ordinary way, they wind round one another, and a regular coil or cable is produced ( $f$. p. 36t). These cables often rise without any foreign support to a considerable height above the ground, and thus single nutating apices are afforded the possibility of grasping a support which otherwise might have been denied them.

Should all these methods prove of no avail the twisted stem takes up its position on the ground; its growth is retarded, and it has the appearance of a stunted, sickly plant. This fact is in so far interesting because it seems to indicate that the pressure experienced by a twining stem adhering to a supporting prop has 
a favourable influence on the growth of the shoot as a whole. This pressire must be regarded as a stimulus, just like the pressure which incites the tendrils, to be described below, to luxuriant growth. We may therefore conclude that twining
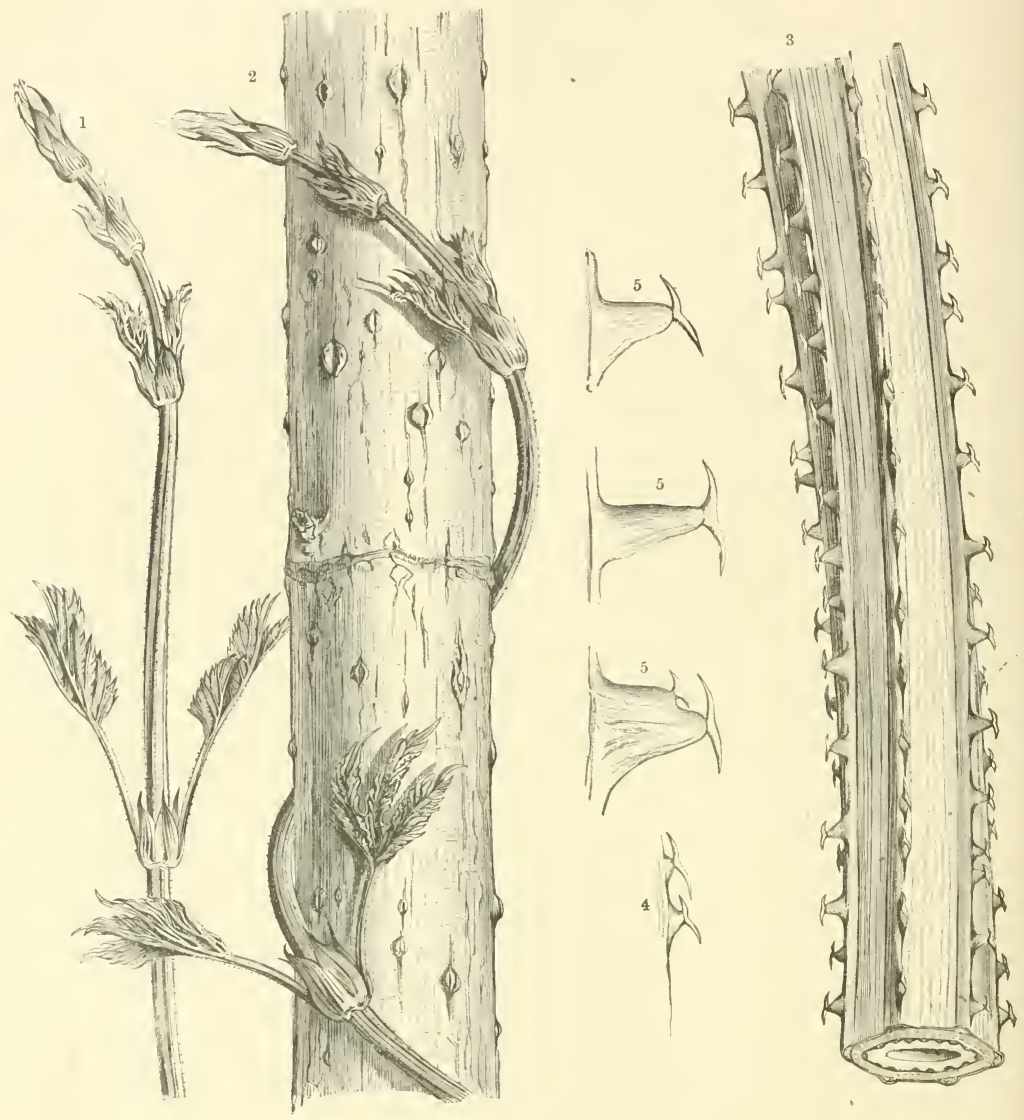

Fig. 160.-Twining Hop (Humulus Lupulus).

1 Free end of a shoot recently emerged above the ground. 2 Shoot of Hop twining round an elder-stem; natural size. $3 \mathrm{~A}$ portion of the Hop stem magnified. 4,5 Single, anvil-shaped climbing-hooks detached from the stem; more highly magnified.

stems are irritable, although the irritability in this case is not so conspicuous as in tendril-forming structures.

In the temperate zones the majority of twining stems have only a short life. The twining Polygonum is an annual; hops and bindweeds are indeed perennial, but their stems sent up fresh each year from the underground stock always perish 
in the following autumn. Only the Bitter-sweet (Solanum dulcamara) and several species of honeysuckle (e.g. Lonicera caprifolium and Periclymenum), which exist in comparatively inclement regions, possess twining stems which increase in thickness from year to year. But in many of these species the twining is not very conspicuous, and the Bitter-sweet forms, so to speak, a link between plants with twining and those with interweaving stems. In tropical regions, on the other hand, long-lived twining stems are by no means rare. Obviously the coils of a stem, firmly attached round a thin support and increasing in thickness, must approach one another very closely; thus arise those strange lianes which excite the astonishment of all visitors to tropical forests. Stems are quite common of a diameter of $4 \mathrm{~cm}$.,

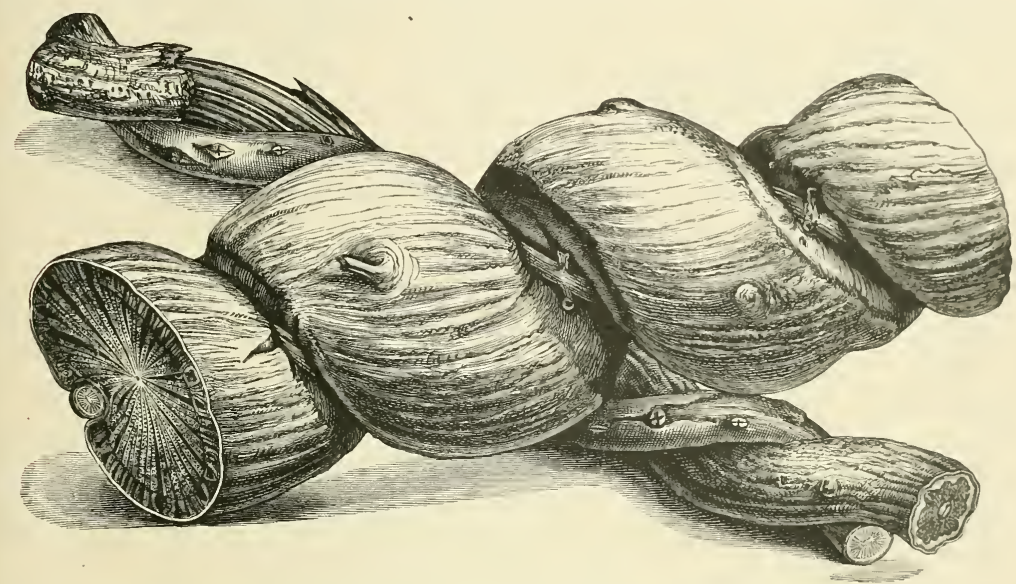

Fig: 161.-Portion of a Liane stem, twisted like a corkscrew, from a tropical forest; natural size

wound like a corkscrew round the thin stems of other lianes, and sometimes such structures-of which a small portion is represented in natural size in fig. 161-are seen stretching right up to the summits of the trees in hundreds of uniform twists, like a thick ship's cable many metres long.

The tendril-bearing stem (stirps cirrhosa) climbs up into the sunlight by the help of special organs known as tendrils. The tendrils are filamentous structures when young; sometimes of exceeding delicacy, sometimes thick and stiff. In some cases they are simple, in others forked, but always sensitive, and so constructed that they can grasp any body with which they come in contact, hold it fast, and use it as a support. Before the tendril adheres to a support it is straight, and extends in the direction in which there is the greatest probability of reaching a support. It also performs movements the aim of which is to strike against some firm object. If this end is attained, the support which it has encountered is firmly gripped by the tip of the tendril, whilst the part lying immediately behind the point of attachment contracts together spirally. By this spiral contraction the vol. 1, 
stem from which the tendril arose is drawn towards the support, and is, as it were, attached to it by a spiral spring.

Tendrils are always produced in numbers from the stem. Usually one, sometimes two tendrils arise from each of the upper nodes, and with the exception of the lowest portion, which is usually quite without them, the stem is very regularly

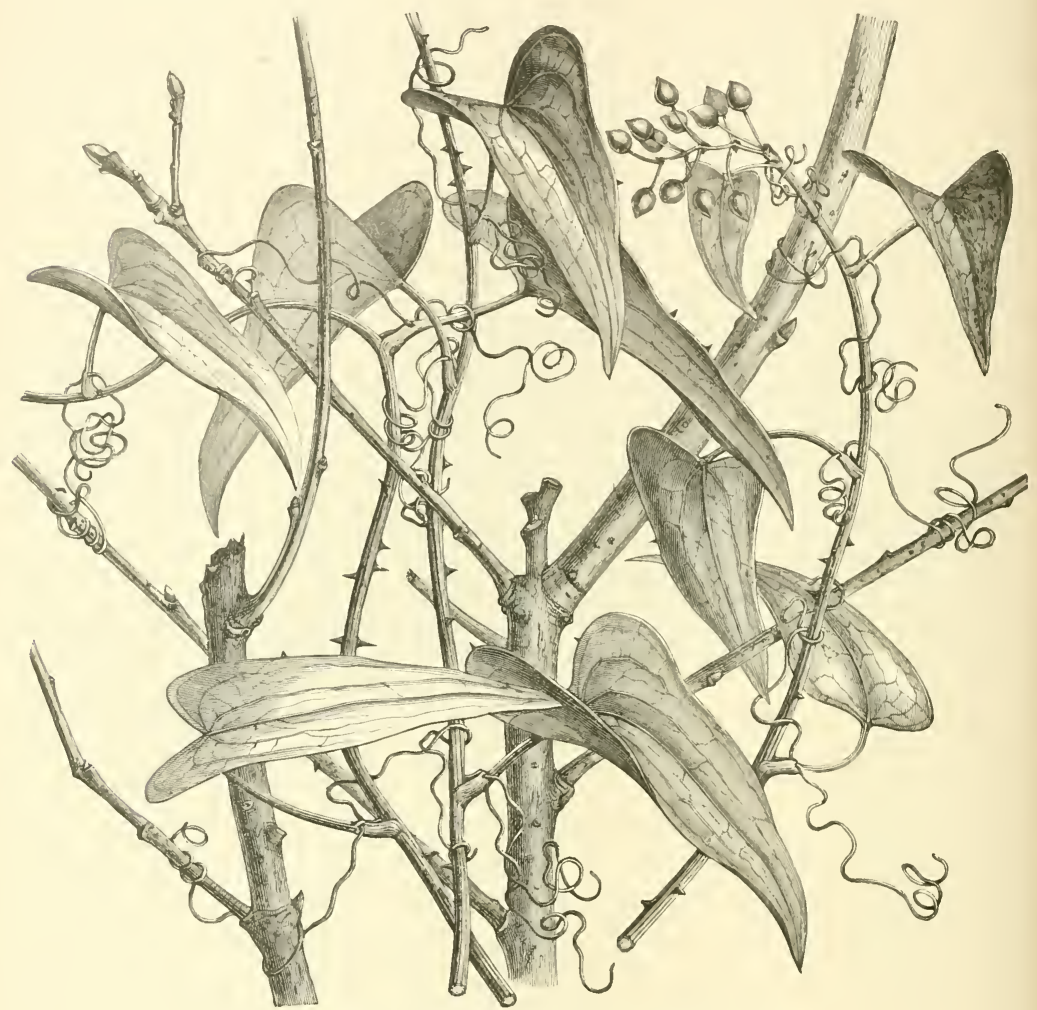

Fig. 162.-Stipular tendrits of the common Smilax (Smilax aspera).

beset with tendrils along its whole length. The advantage of this is that in case one tendril should fail or find no support, a neighbouring one can always take its place. Generally plants with tendril-bearing stems are at a decided advantage in comparison with all other forms of climbing growths, which explains the fact that their number is in considerable excess of the others. In climbing over a shattered rock-face or thick tree-trunk they have a great advantage over plants with twining stems. In some cases the tips of the tendrils fasten on even to the smoothest rocks by peculiar discs, or they grip and hold fast to small projecting portions 
of bark and the stumps of broken twigs, things which are impossible to twining stems. Tendrils preferably twine round horizontal twigs and leaf-stalks, and frequently round old tendril-bearing stems which have previously climbed up to the crown of a tree. When they have reached up to the branches, they can pass over from one bough to another, fasten themselves firmly above and below, and so gradually invest the whole of the crown. From the summit fresh shoots arise

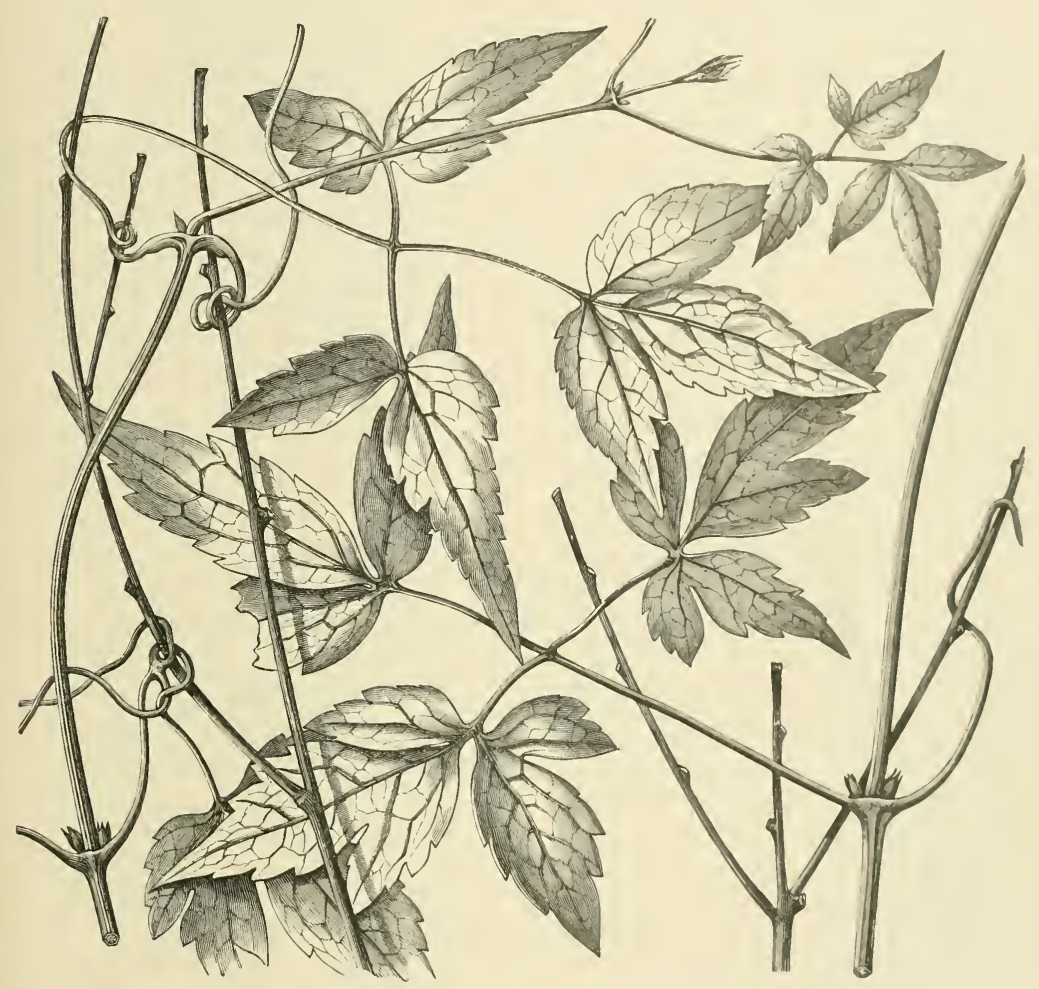

Fig. 163.-Leaf-stalk tendrils of Atragene alpina.

which curve downwards and are swayed by the lightest breath of wind; from them new tendrils project, like the tentacles of some sea-monster, and if one of them but touches a leaf-stalk or twig of a neighbouring tree, it curves round it and grasps it firmly. Very soon a second, third, and fourth tendril will similarly become attached, and, contracting spirally, will pull the pendent shoot up to the neighbouring tree-crown. The bridge so formed is again used as a means of transit by other climbing stems, and thus arise garlands and festoons, which hang from tree to tree; whilst not infrequently an actual arcade is formed whose roof, 
formed of tendril-bearing stems, is borne by two adjacent trees or thickets as though by two gigantic piers. Another advantage which tendril-bearing stems have over twiners consists in the fact that they can reach the same height above the ground with less expenditure of material. The twining stem of the Scarletrunner, which has climbed a metre above the ground, shows, when unrolled, a length of $1 \frac{1}{2}$ metres. The pea, which climbs with tendrils to the same height, is little more than a metre long. Of course in the production of tendrils building material is expended, but this bears but a small proportion to that which is required for the extra half-metre of stem.

Now as to the nature of tendrils, are they leaf, stem, or root? They may be each of these according to the species in question. A tendril may even be formed by metamorphosis from each of the different sections of a leaf independently, and the leaf-blade, the mid-rib, the leaf-stalk, even the stipules themselves may become tendrils. From the standpoint of development and with regard to the origin and mutual relation of individual plant-members, the exceedingly manifold tendrilstructures have been classed generally in the following groups. First of all the stipule-tendrils (cirrhus stipularis), of which species of smilax (Smilax) afford an excellent example. As may be seen in Smilax aspera (see fig. 162), so common in the region of the Mediterranean flora, the leaves are divided into lamina, leaf-stalk, sheath, and stipules, and the two stipules arising from the sheath are transformed into rather long tendrils which surround the branches of other plants, and even their own branches.

More common than this rather rare form is theleaf-stalk tendril(cirrlus petiolaris), which itself again shows numerous modifications according as to whether the whole leaf-stalk of an undivided leaf, or the stalks of single leaf-segments play the part of tendrils. The former is seen very beautifully in the numerous species of Nasturtium (Tropceolum) and in the tendril-bearing snap-dragon (Antirrhinum cirrhosum); the latter in many species of fumitory (Fumaria), in the Traveller's Joy (Clematis), and in the only liane of the European Alps, the Atragene alpina, illustrated on the last page (fig. 163). In pitcher-plants (Nepenthes) a portion of the leafrachis is transformed into a tendril, and by it the pitchers are suspended on the branches of supporting plants ( $c f$. fig. 24, p. 133). When the midrib of a foliageleaf projects far beyond the green tissue of the blade, as a filament which grasps and surrounds firm supports and attaches the whole plant to them, this structure is known as a midrib-tendril (cirrhus costalis). To this class belong the strange South American mutisias (e.g. Mutisia ilicifolia, hastata, subspinosa, decurrens), the Indian Flagellaria Indica and Gloriosa superba, and several fritillaries (Fritillaria cirrhosa, verticillata, and Ruthenica), attaching themselves to stiff culms and leaves of neighbouring grasses. The leaf-tendril (cirrhus foliaris) is also interpreted as the midrib of a leaf-blade or of a leaf-segment, but here none of the green tissue of the blade is developed, and only the midribs are seen to form filaments which curve and fasten as soon is they come into contact with a prop. This form of tendril is the commonest of all, and is found particularly in Papilion- 
aceæ in great variety. Sometimes the whole leaf-blade is metamorphosed into a single tendril, as in the Yellow Vetchling (Lathyrus A phaca); but usually tendrils are formed only in the place of the terminal leaflet and of the upper leaflets of tho pinnate leaves, as may be seen especially in vetches, peas, and lentils (Vicia, Pisum, Ervum). It should be mentioned here that in proportion as the green tissue of the leaf-blade is reduced in consequence of the formation of tendrils, the amomnt of

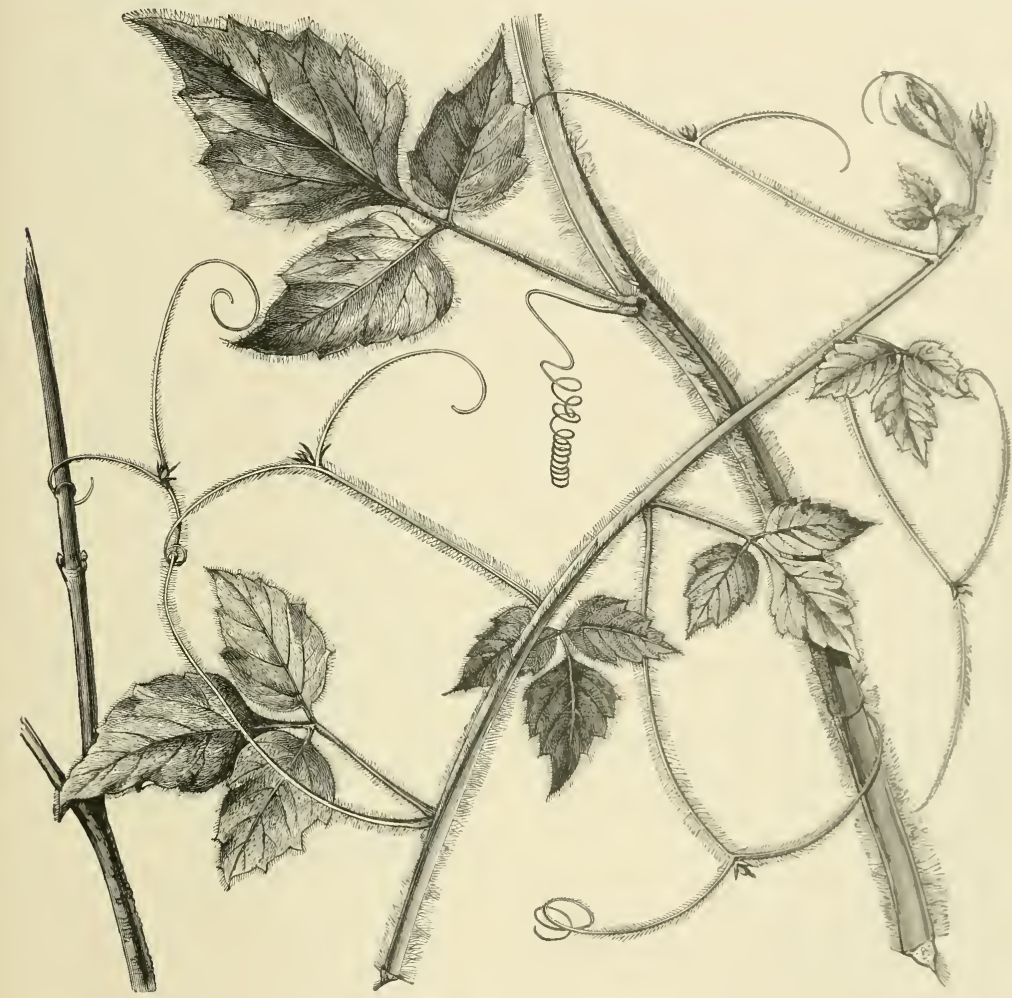

Fig. 184.-Branch-tendrils of Seriania gramatophora.

green tissue of the lowest leaflets, leaf-stalks, and stipules increases; in other words, that when tendrils appear in place of the upper leaflets, the lowest pair of leaflets and the stipules form large green laminæ. In many vetches even the stem and leaf-stalks are beset with green leaf-like bands and wings.

By a stem-tendril (cirrhus capreolus) is meant one which can be interpreterl as a stem-structure, and a distinction is drawn particularly between branch-tendrils (cirrhus rameaneus) and flower-stalk tendrils (cirrhus peduncularis) according as to whether the tendril is to be regarded as a metamorphosed flower-bearing or 
foliage-shoot. Flower-stalk tendrils are found in particular in grape-vines and in species of Cissus, in Passiflora cirrhiflora, in several species of the genera Paullinia and Cardiospermum; branch - tendrils in Fumaria claviculata, and in numerous gourd-like plants. These tendrils, of which the Serjania gramatophora ( $c f$. fig. 164) may be taken as an example, arise usually not from the true axil of a foliage-leaf, but are displaced, pushed to the side of or below the subtending leaf; frequently even opposed to leaves which one might think really subtended them. This displacement is particularly striking in vines and gourd-like plants, for which reason these tendrils were formerly explained not as stems but as leaf-tendrils. Finally we must consider here the root-tendrils (cirrhus radicalis), which really are roots arising from the foliage-stem, but in regard to their activity behave exactly like tendrils, and are chiefly observed in climbing, delicate-stemmed lycopodiums.

This classification of the manifold tendril-developments, useful for the speculative doctrine of form, and also to the descriptive botanist, has only a secondary value for the questions which are discussed in this book. It gives no conclusion concerning the significance which the different forms have with regard to the habitats of climbing plants, and it offers not the slightest assistance to our understanding how the stem is fastened to the support by the tendril arising from it. Tendril-bearing stems are extremely wonderful in this respect, and the different methods require a detailed description. For the purpose of this description we classify tendril-bearing stems into three groups, viz. into those with ringed tendrils, with nutating tendrils, and with light-avoiding tendrils.

Stems with ringed tendrils are especially adapted for climbing up between the erect and much-branched growth of dense hedges, copses, and low woods. Some of them are annual and do not rise far above the low underwood and shrubs, e.g. various species of fumitory and nasturtium (Fumaria and Tropceolum). Others, e.g. the Traveller's Joy and Atragene (Clematis and Atragene) are perennial; their stems become woody, often attaining to a considerable age, and the youngest branches of the old stems may climb up to the tops of trees. When one sees these plants hanging rope-like from the summits of tall, unbranched forest-trees, one may conclude that they first became fastened to them at a remote period, when the trees were still quite small, and that they have ever since kept pace with them in their growth. The young shoots of such climbers with their leaves still small, erect, and folded to the stem, appear capable of pushing through even very small gaps in the thickest undergrowth, thus reminding us strongly of the manner of growth of interweaving stems. They also agree with interweaving stems inasmuch as they form actual anchor-arms by extending and reflecting their leaves and leaf-stalks by whose help they suspend themselves on the horizontal branches of the supporting undergrowth. This is the case in Clematis and in the Atragene illustrated in fig. 163,- these plants having opposite leaves whose stalks project from the stem almost at a right angle. The stalks and blades of the leaflets, also, complete the semblance to the arms of an anchor, since the former sink down at an obtuse angle with the main 
stalk, whilst the latter, after the laminæo are unfolded, curve like an arch, forming an actual loop.

As already stated, no distinction can be recognized in the earlier stages between interweaving stems and those with ringed tendrils. The difference first appears $\Omega$. soon as the lower side of the leaf-stalks comes in contact with a branch of the undergrowth. This contact, if it is not of too transient duration, acts as a stimulus on the leaf-stalk, and the result is that it curves round the branch and grips it like a ring. The stalks always curve towards the side which has been touched, or pressed. Since the leaf-stalks are equally sensitive on all sides, the curvature may take place above or below, or laterally, according to whichever part has been stimulated. Even continuous contact with flower-stalks of hair-like delicacy is sufficient to produce the ring-formation, and it has been shown by experiment that the continued pressure of a thread weighted up to four milligrams is followed by a curvature. The stimulated leaf-stalk usually forms one or two, less often several annular coils on the branch embraced, as shown in fig. 163. It also frequently happens that neighbouring stems of the same plant are connected together by their tendrils and twined into inextricable knots. The conversion of the irritable leaf-stalk into an effective, gripping tendril in many of the plants in this group is materially assisted by the fact that the younger portions of the shoot revolve in circles like those of twining stems, though less regularly. Thus a much-increased number of suitable objects in the environment become possible as supports. These leaf-stalks, which become tendrils, do not, however, themselves nutate, consequently they are clearly distinguished from those of the following group, which are called nutating tendrils.

Stems with nutating tendrils have not the power of climbing up rocky walls or the bark of thick tree-trunks, and, like the foregoing, are only able to use as supports culms, leaves, and thin branches of other erect plants, to which they adhere and up which they are drawn by means of the spiral curvature of the attached tendrils. Plants equipped with this class of tendril require far more light than those with ringed tendrils, and they find their best and most favourable habitat in the open country dotted with isolated groups of trees, or on the edges of a forest bordered with bushes, and in sunny meadows studded with shrubs. They have not to interweave through the interlacing branches of an underwood; ringed tendrils are suitable there, but not tendrils with long nutating filaments which could either not accomplish their movements in the midst of the thick brushwood, or if they did, would not attain the desired end, viz. the subsequent elevation of the stem.

The lowest portions of the young shoot possess no tendrils, and they are kept erect solely by the turgescence of their tissues. In many species the stiff, spreading leaf-stalks, or the peculiar barbed leaf-blades help to support the young shoots on the neighbouring plants and to keep them erect. But these supports are but temporary measures, and the upper portion of the shoot soon develops tendrils. These elongate quickly and get to work. The filaments of these tendrils elongate with extraordinary rapidity, straighten out, and then project like tentacles far beyond the foliage-leaves. At their tips only do they exhibit a more or less hook-like 
curvature ( $c f$. fig. 165). When they have reached their full length they begin to move round in a circle just like the apices of twining stems. If by this movement they meet with an object suitable for a support, they grasp and embrace it by their hooked ends. That is to say, contact with a foreign body acts like a stimulus on the tendril; it loops itself over the object with which it is in contact, and then rolls up in a spiral, thus drawing the stem, which bears it, obliquely upwards. Now

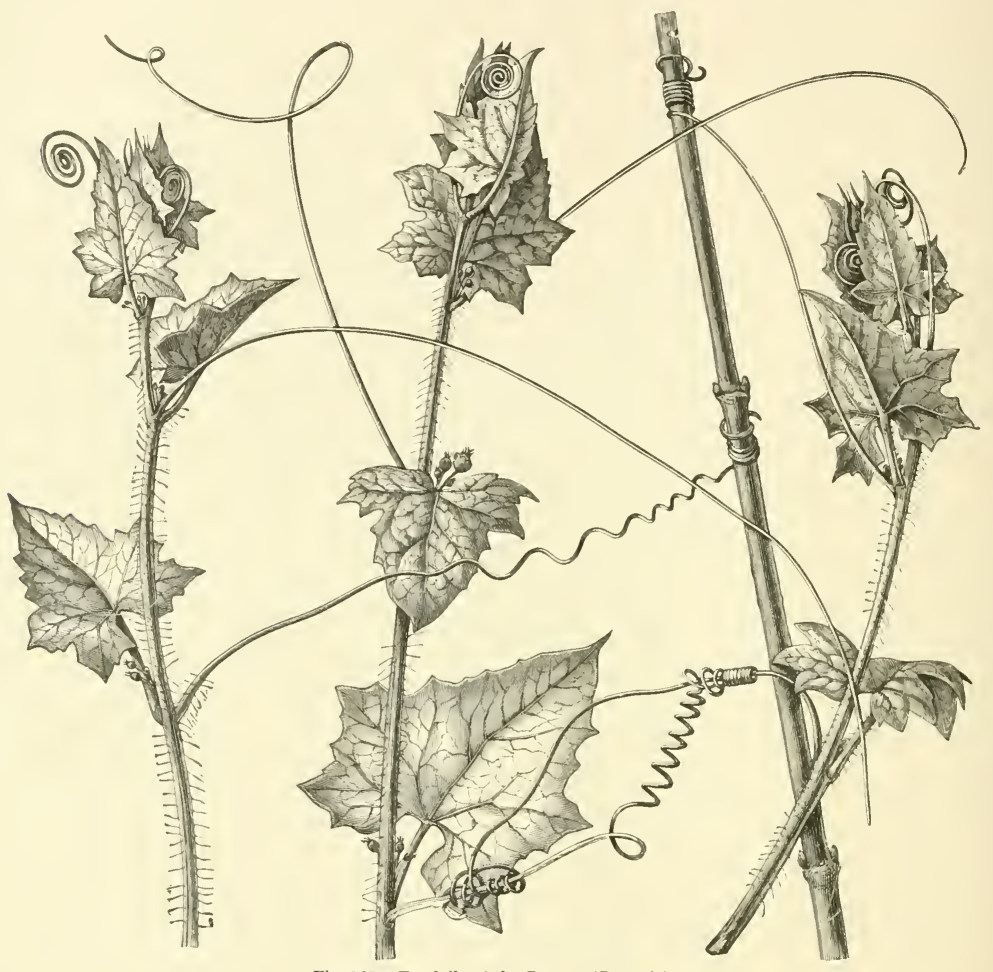

Fig. 165. - Tendrils of the Bryony (Bryonia).

comes the turn of the tendril inserted next above. This behaves exactly in the same way as the first, and in a very short time is succeeded by a third, fourth, \&c. It does not much matter if in its nutation one of these tendrils should have found no support, since the successive tendrils are placed so close to one another, and replace each other so quickly, that the shoot is still drawn up uniformly, and is prevented from falling. When whole series of tendrils find no places of attachment, the shoot of course falls down, under which circumstances possibly one of its tendrils may encounter a distant branch to which it can fasten, and which it can use as a support. If this should fail, the tip of the pendent shoot again rises up, sends out 
fresh, nutating tendrils, and so may still succeed in grasping some projecting twig in the neighbourhood upon which it can climb. The paths traversed by such tendril-bearing stems are therefore often wound oddly hither and thither, but the stem always follows the periphery of the bush or tree-crown which it has selected, and the inner branches of these supports are never interwoven by it. Plants whose tendril-bearing stems ramify strongly may invest the whole tree over which they grow with an actual mantle, and if the climber in question has large leaves, it may be quite impossible to determine from outside what species of plant has become thus enveloped.

The account given above deals only with such phenomena as are displayed by all tendril-climbers in common; but in individual cases there are innumerable special contrivances, which it would be impossible to describe in detail in the limits of this book, and I must therefore be content with enumerating some of the most striking that have been observed.

First, it has been pointed out that in many cases, for example, in the tropical passion-flowers, not only the young, extended tendrils, but also the whole shoot-apex revolve in circles thus widening the space traversed by the tendrils, and increasing the probability of meeting with a support. If the tendrils are forked, each of the two branches performs its particular oscillations, as can be seen, for instance, in the tendrils of the grape-vine. The period of revolution, taken by a nutating tendril, varies very much according to the species. Cobcea scandens takes only 25 minutes, Passiflora sicyoides $30-46$ minutes, and Vitis vinifera 67 minutes for a revolution. The rapidity with which the tendrils curve in consequence of the pressure exereised on them by a foreign body which acts as a stimulus also varies very much with the species. In Cyclanthera pedata the curvature commences 20 seconds after contact with a hard stick; in passion-flowers (e.g. Passiflora gracilis and $P$. sicyoides) after the lapse of about half a minute, in Cissus discolor after 4-5 minutes. If the stick with which the tendril is in contact is removed, the curved portion straightens out again. If it is left in contact, the curvature proceeds uniformly. In Cyclanthera pedata the first complete coil around the support is accomplished in 4 minutes, in others, on the other hand, it may take several hours, or even 1-2 days. Usually the tendril is not content with a single coil, but forms several of them. The coils are closely pressed to the prop, and in their growth adapt themselves like a plastic mass to all its projections and depressions; the substance of the tendril even penetrates into the small clefts and crevices, and when the tendril is detached from its substratum, an actual cast of all the inequalities of the support can be seen on its contact-surface. In many species, e.g. in Hanburya mexicana, peculiar callus-like growths arise here. The ends of the tendrils, as already stated, are curved like a hook so as to more easily grasp the object to which their circling movement brings them. In many species the tendrils terrninate in actual claws. The tendrils of Cobcea scandens, a native of Mexico, but frequently grown as a decorative plant in our conservatories, are specially elegant. They are leaf- or midrib-tendrils, and divide repeatedly in the most beautiful manner. Each of the ultimate branches 
bears a double claw whose points immediately fasten into any object at the slightest touch, and will even remain suspended in the skin of the hand. The three delicate branches of the tendril of Bignonia venusta also end in pointed claws which resemble those of insects' feet. The majority of tendrils are branched, whilst simple undivided filaments, as shown in the Bryonia (fig. 165), are comparatively rare. Passion-flowers and gourd-plants have the longest tendrils, those of the common gourd (Cucurbita pepo) often measuring more than $30 \mathrm{~cm}$. in length. The spiral contraction of the part of the tendril not wound round the support begins, according to the species, half a day, or one or two days after the apex has formed the first coil round the support, but it is very quickly accomplished when it has once begun. This torsion is sometimes towards the right, sometimes towards the left, and frequently it is accomplished partly in one direction and partly in the other, by the same tendril. The number of twists formed in this spiral contraction is very variable. In the long tendrils of some gourds as many as 30 or 40 are produced. By these spiral springs the fastening of the stem to the support is excellently accomplished; it is at once adequately attached to the support, but not pressed to it, consequently unnecessary friction is avoided. During a blast of wind there is a certain amount of "give", but directly the gust subsides, the climber-thanks to its elastic tendrils-resumes its former position. This spiral twisting occurs also in tendrils which have not succeeded in grasping a support, but strangely enough, they become stunted, shrivel, and wither, sometimes becoming detached from the stem like autumn leaves. Those tendrils, on the other hand, which have embraced a support become much stronger and thicker, and also undergo a series of changes in their inner structure which adapt them excellently to the part they have to perform.

Stems with light-avoiding tendrils remind us of the light-avoiding interweaving and lattice-forming stems, and, like these, are found in plants which have to climb up steep rock faces and over the bark of large trees. In these cases the more or less plane surface of the rock or tree-trunk is the only support afforded for climbing. The stem on such a substratum would extend its tendrils in vain on the side where there is only the air to be met with; here there is no resting-place or support which can be reached by circling movements. The best the tendril can do under the circumstances is to seek out the solid wall along which the stem has to climb as quickly as possible. In such cases the desired support is on the side turned away from the light, and as a matter of fact, the tendrils of these plants turn towards this side with great persistence. According to the position of the point at which the tendril springs from the stem, it curves at an angle of $90-180^{\circ}$ in less than 24 hours, and grows towards the background without digression and without wasting its energy in revolving movements. The leaves of the same plant, for exposure to light and air, are extended in a direction away from the wall, and try to assume the position most favourable for this purpose. The path it has taken soon brings the tendril in direct contact with the wall, with which it now has to obtain a firm hold. This it does either by peculiar adhesive dises, or by wedging itself 
into the dark clefts and crevices existing in the supporting wall. Several species of the genera Cissus, Vitis, and Ampelopsis develop adhesive discs. In the Vitis inconstans, a native of Japan and China, and known among gardeners by the name of Cissus Veitchii (figured on the right-hand side of fig. 166), as soon as the tips of the tendrils, which are provided with tiny knobs, come in contact with a hard wall, they spread out, just like the toes of a tree-frog. In a very short time disc-like pads are formed from the knobs, and these become cemented to the substratum by means of a sticky fluid mass secreted from the cells of the disc. This cement now holds so fast that on trying to separate the tendril from the substratum it is much

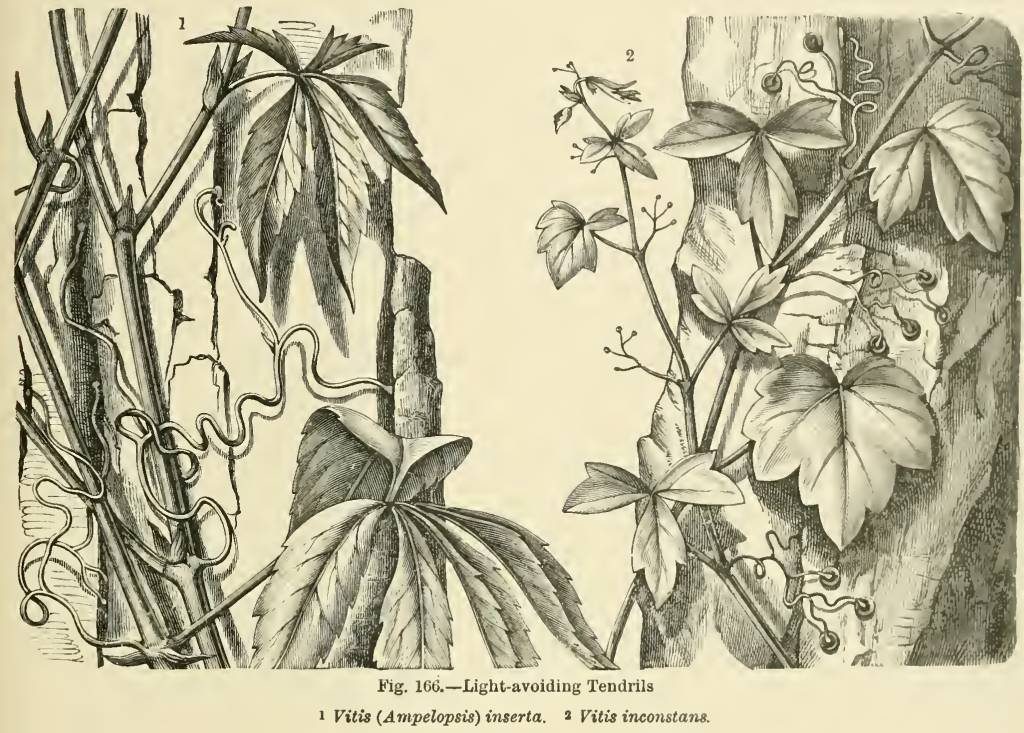

more likely that the tendril-filament will be torn than that the disc will be detached. Vitis Royleana and Ampelopsis hederacea (the Virginian Creeper) also develop these adhesive discs, but here they are not prefigured by knobs on the branches of the tendrils as in Cissus Veitchii; the ends are curved like hooks, and are barely thickened. As soon as they reach the hard wall the tendril-branches diverge, spread out on it laterally, and arrange themselves at definite intervals in the most advantageous manner. Within two days the curved apices thicken and turn crimson, and in another two days the discs are complete, and the tendrils are cemented by them to the wall. These plants can climb up smooth walls, and even planed wood, glass, and smooth, polished iron are not rejected as substrata.

Bignonia capreolata, and Vitis (Ampelopsis) inserta (whose tendrils are represented in fig. $166^{1}$ ) behave differently from the three tendril-plants just mentioned. Here the curved tips of the tendrils, growing towards the wall, seek the crevices 
and crannies of stone or bark and actually creep into them, or when only shallow grooves are to be found in the substratum, bury themselves in them. Smooth surfaces are avoided as far as possible since they afford no suitable hold to this class of tendril. When established in the chinks and crevices, the ends, which until now have been hooked, swell out like a club or ball, and in a short time thicken so much that they occupy the entire crack. It looks as if melted wax had been poured into the crevice and had then solidified and fitted itself to all its inequalities. The growth of the tissue extends, according to the depth of the crack and the nature of the contact-surface, over a sometimes larger, sometimes smaller portion of the embedded part of the tendril, and sometimes a callus-thickening is seen to arise even behind the apex, at places where the tendril has adhered closely to a small projection of stone. The thickened end of the tendril clings so firmly to the depression into which it has wedged itself, that it is very difficult to remove it; and here also the attachment seems to be completed by means of a secreted cement. It is seen on examining the parts of the adhesive disc or of the wedged callus-thickening which adhere firmly to the substratum, that the epidermis in particular has undergone a remarkable change. The epidermal cells are enlarged, drawn out as wartlike protuberances or conical projections, and adapt themselves to all the elevations and depressions of the substratum, grasping even microscopic projections, so that the contact-surface, after being detached by chemical agents, resembles sealing-wax against which a seal has been pressed while it was in a plastic condition.

It is remarkable that these adhesive discs and growths of callus are only developed after contact with a solid body. If from any cause the tendril is prevented from coming in contact with a solid substratum, the growth of tissue, the development of papillæ on the epidermis and the secretion of a cement-substance do not occur, and the end of the tendril dries up and dies. This process reminds us strongly of the formation of weals on human skin, and, like this, is dependent upon stimulus, friction, and pressure.

A spiral torsion occurs in the light-shunning tendril as soon as it has become attached in one way or the other to the substratum. The attached tendrils now become much stronger, and always much more vigorous than those whose apices have not found a resting-place. The stem is now fastened by the elastic tendril to the steep rock face or fissured back of an old tree-trunk. Strong winds may drag the stem somewhat away from the wall, but when they subside it again resumes its normal position, as in the cases previously described, by means of the elastic tendrils. If the stem subsequently grows in thickness the spiral springs holding it are drawn out just as far as is required. Very old stems no longer need their clinging organs; they stand, as strong erect stems, in front of the wall up which they had years ago clambered as young shoots, although their tendrils have now been long dried up; the young shoots alone, always striving higher and higher, still continue to climb up the substratum in the manner described.

The climbing stem in the restricted sense (stirps radicans) holds itself in the normal position attained by growth by means of climbing roots, and uses as supports 
the trunks of old trees, steep walls of rock, and under cultivation often wooden planks and palings. All these climbing stems have two kinds of roots-absorbent roots, by means of which they suck up fluid food, and climbing roots, which serve to maintain them on their supports. In most instances the functions of these two kinds of roots are kept distinct, so that a climbing stem soon withers and dies when it is cut across above the absorbent roots, although affixed to a rock or to the bark of a tree by a thousand climbing roots. But in a few cases the climbing roots will under these circumstances begin to absorb, provided, of course, that the substratum to which they adhere is able to afford them the necessary food.

In many respects climbing stems agree with the group of tendril-bearing stems just described, especially in the fact that the organs which furnish the adhesion to the support turn from the light, and also inasmuch as the adhesion to the support is brought about by a viscous substance either secreted by the cells in contact or produced by the breaking down into mucilage of the outer layers of the walls of these cells. The avoidance of light by all climbing roots is an extremely interesting fact. Whether the stem which forms climbing roots nestles close to its substratum, or some spans distant from it, whether it grows upwards along a stone wall or is deflected to one side by some obstacle-in all cases the first rudiments of the climbing roots make their appearance on the side of the stem turned away from the light. And when these small cushions develop into root-fibres, the direction assumed by their growth is aiways away from the light and towards the dark background. The darker the place, the more vigorous do the root-fibres become. When the climbing roots developed by Tecoma radicans (figured on p. 479) in the darkest places under a projecting ridge are compared with those which have been formed in less shaded places below, it is seen that the former are always much more luxuriant and longer than the latter. If by chance a shoot which has already begun to develop climbing roots is moved from its position so that the hitherto shaded side is exposed to the light, it twists round until the side with the rudiments of aërial roots is again turned from the light. If obstacles lie in the way of this torsion, the young climbing roots thus exposed remain undeveloped and grow no further; ultimately they wither and dry up.

As soon as the climbing roots originating from the shady side of the stem come into contact with the substratum below them their growth is noticeably increased, and in a very short time they become firmly united to it. Not only do the rootlets grow into all the crevices of the substratum and adapt themselves most accurately to its larger inequalities, but each single epidermal cell of the growing rootlet exhibits a like behaviour, fits itself to the smallest projections and depressions, and spreads out on entirely smooth surfaces like a plastic mass. Sometimes the epidermal cells are drawn out like tubes and form so-called root-hairs, these penetrate into the smallest clefts of the substratum and spread out like a hand whose palm and outspread fingers press against the soil. These epidermal cells of clinbingr roots also unite with the supports against which they have placed themselves like the absorbent cells described on p. 87, and the union is so firm that the roots ar 
much more likely to break off at the base than the united surfaces to separate when the roots are forcibly displaced.

The following types of climbing roots may be distinguished according to their shapes. First densely crowded, simply or only shortly branched, filamentous roots, arising in groups, but each separately from the stem; these are increased in number by the production of new batches as the lignifying stem becomes older and thicker, and they sometimes grow together and border the stem, adhering to the substratum in irregular, membrane-like rows. On older stems the climbing roots are usually for the most part dried up, and those which have not united with the support then project from the sides, often forming shaggy beards, and giving a very odd appearance to the stem. The Ivy (Hedera Helix, of which old stems are shown growing up on an oak in fig. 167) may be taken as an example of this type.

The second form presents a wholly different aspect; as the type we may select the Tecoma radicans, a native of the southern United States, often used for covering garden-walls. The climbing roots here are strictly localized. At each node of the shoot below the point of insertion of the leaves a paired cushion-like structure arises, and from each of these cushions several rows of protuberances, which grow out into as many rows of unbranched or shortly-branched, fringing fibres, 1-5 cm. long (see fig. on p. 479). The epidermal cells of this fringe, which come into contact with a firm substratum, elongate and form root-hairs, that is, papillæ and tubes, which in a very short time fasten to the support; after this they turn brown and die, thus never functioning as absorbent organs.

A form materially differing from these is shown by the climbing roots of the cactus Cereus nycticalus, known as the "Queen of the Night", a native of Mexico and the West Indies, and also of several tropical Bignoniacer, and especially in Ficus stipulata, so often used in greenhouses for covering the walls. In the last-named plant the climbing roots arise in fascicles in the shade of the green leaves; they are filamentous and terminate in many hair-like, spreading rootlets. They adhere by root-hairs to the substratum, and thus fasten to it the tender, pliant stems. These roots are not very long and soon dry up, but close behind them much stronger roots arise from the stem, which has meanwhile become thicker, and these traverse the walls like cords, repeatedly branching and intersecting, and form actual net-works, often becoming several metres long. These latter roots do not help much in fastening the stem to the supporting wall; they are absorbent roots, and take up the atmospheric water, with its abundance of food materials, which has condensed or trickled down the bark of trees and rock walls.

The clasping roots borne by the stems of Wightia, a genus of Scrophulariaceæ growing in the mountainous regions of the Himalayas, and of several species of fig in the same district, may be regarded as a fourth type. The attachment of the young shoots is brought about here as in the form just described by the finelybranched but not much elongated roots, which soon dry up. But when the climbing stem begins to thicken much stronger roots arise which surround the supporting tree-trunk like clamps and actually engirdle it. These girdle-like clasping roots 

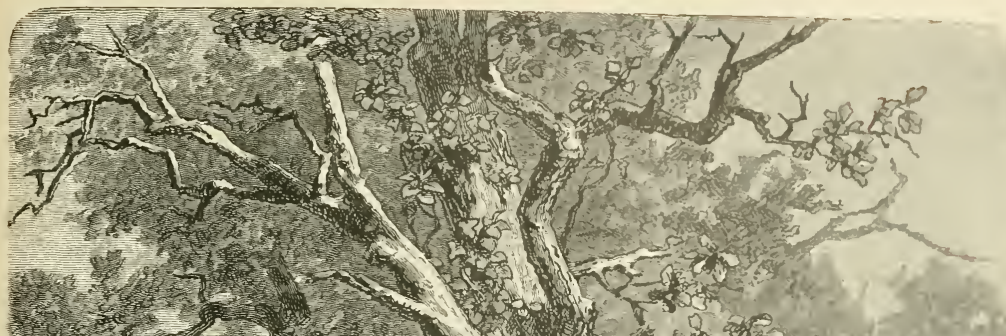

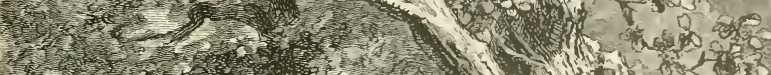

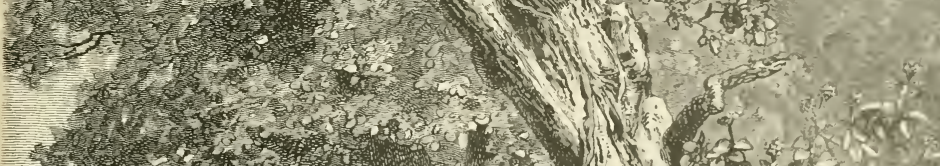

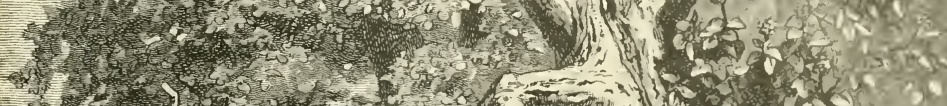

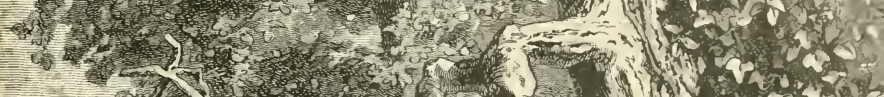

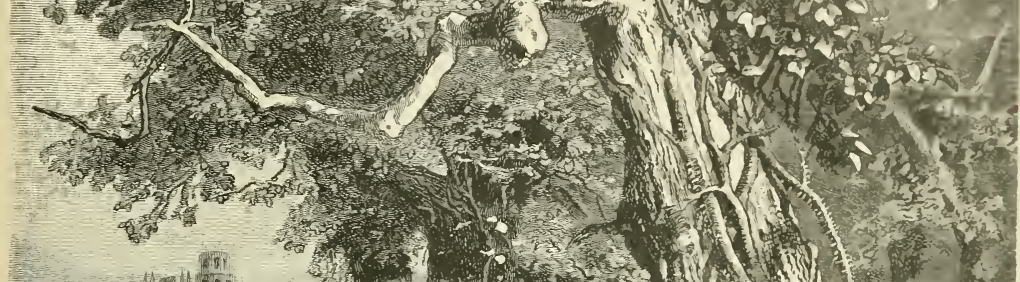

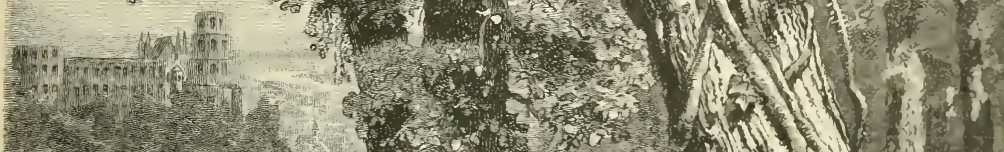

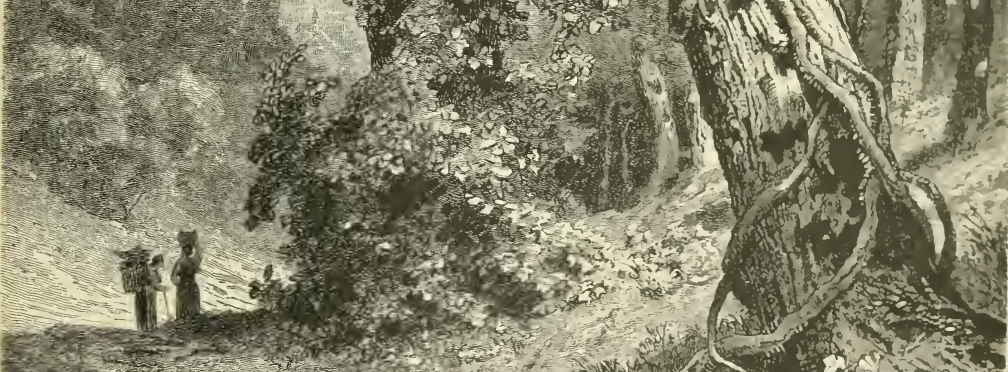

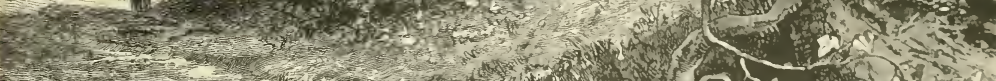

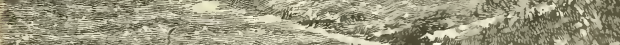
1.

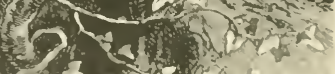

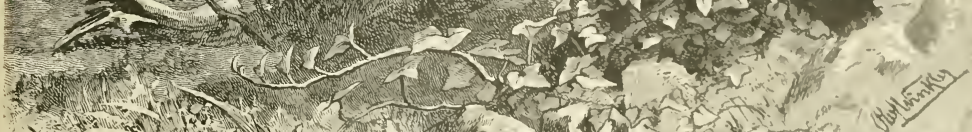

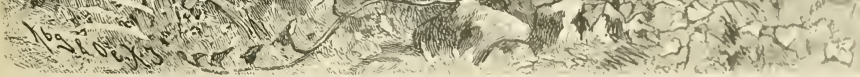

Fig. 167.-Ivy (Hedera Helix) fastened by climbing roots to the trunk of an Oak growing in the woods near Heidelberg. 
often fuse at the places where they adjoin one another and increase in circumference, frequently becoming as thick as a man's arm. The illustration on the next page (fig. 168), taken from a photograph at Darjeeling in the Himalayas, shows these stems, which look as if they had been actually tied on to the smooth trunks of tall trees, and which bend away somewhat from the support, and then ramify and develop abundant leafy branches.

Many tropical species of fig, which may serve as representatives of a fifth type, exhibit the following peculiarities:- their climbing roots, nestling to the substratum, flatten and spread out like a doughy plastic mass; the adjacent roots fuse together, and in this way irregular lattice-works, or incrusting mantles, only interrupted here and there by gaps, are formed, which lie on the supporting trunks and are firmly fastened and cemented to them without fusing with it or deriving nourishment from it. Frequently not the trunk only but the branches of a tree serving as support are incrusted with the flattened clamping roots of the climber. Sometimes the climbing Ficus sends columnar aërial roots down to the ground, whilst its leafy branches intersect those of the supporting tree; so complete is the entanglement that at first sight it is hardly possible to distinguish what belongs to the support and what to the climbing plant. Fig. 169 is a faithful reproduction of a sketch by Selleny drawn at Kondul, one of the small Nicobar Islands, showing one of these remarkable climbers with flattened roots incrusting the support, i.e. Ficus Benjamina on a supporting myrtaceous tree, the latter obviously suffering under the burden of its oppressor, and already in a dying condition.

These "tree constrictors", as one might call them, although they do not absorb materials from their supports, as was formerly supposed, are certainly not indifferent to them, and may injure and even kill them like the constricting, twining stems described and figured on pp. 159 and 160. The entwined tree decays and its wood disintegrates, perhaps termites assist in carrying away the remains of the dead trunk, but the climbing stem with the flattened, climbing roots remains still vigorous. It has meanwhile created a sufficient support for itself by its prop-like aërial roots, and these prevent it from falling. As Hooker says in his Himalayan Journals:- "We found great scandent trees twisting around the trunks of others and strangling them: the latter gradually decay, leaving the sheath of climbers as one of the most remarkable vegetable phenomena of these mountains". When at length the climber, deprived of its original support, also dies, its roots and stem-structures become bleached, and its curious forms, in which to speak with Martius, "the excited imagination fancies it recognizes fantastic spectres and gigantic voracious monsters", rise up weirdly against the dusky background of the primeval tropical forest.

The manner in which climbing roots become fixed upon their supports is not less varied than their manifold structural modifications. It has already been stated that the climbing roots are light-avoiding, and that their growing points are directed towards the rocky faces and boughless tree-trunks upon which they climb Should the distance between the stem and the wall be not great, the 

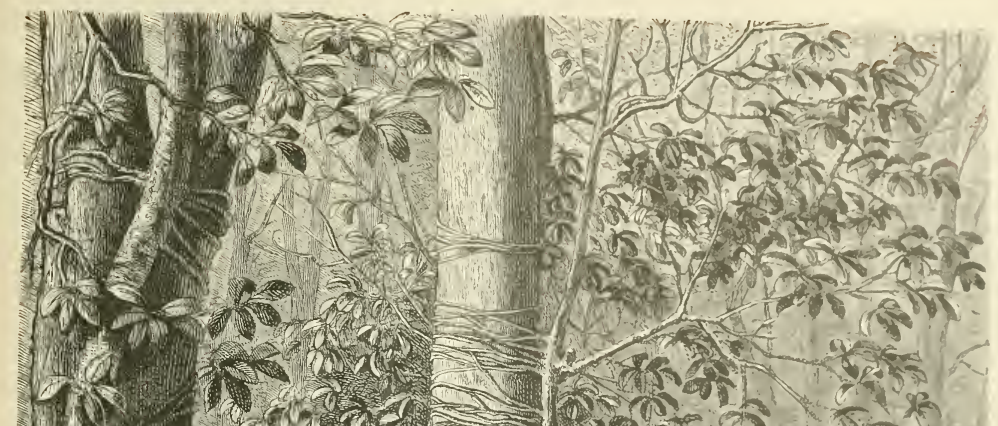

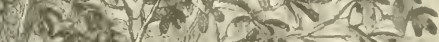
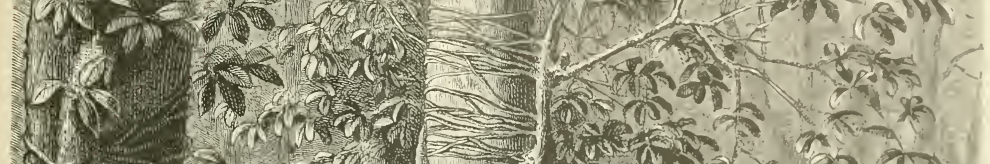

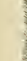

1)

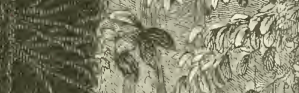

(1)
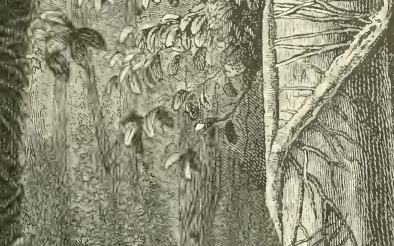

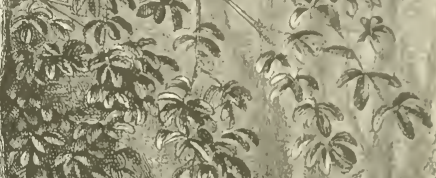

西

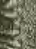

3.

X.:

Fis

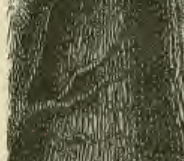

f.
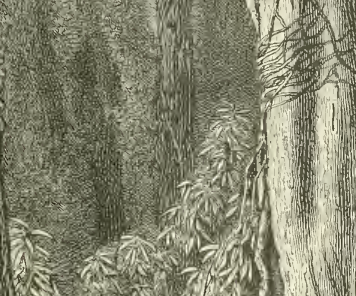

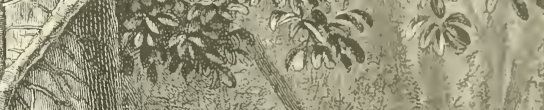

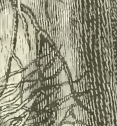

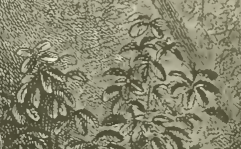

1. 210
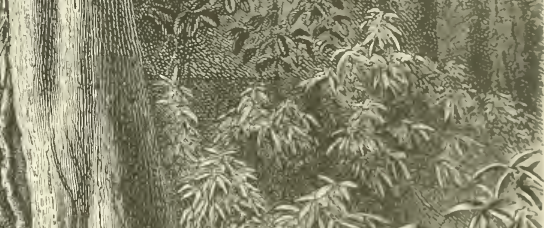

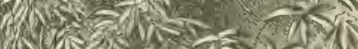

9 -

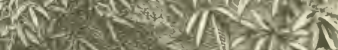

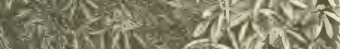

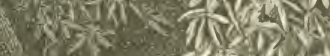

mandent

a
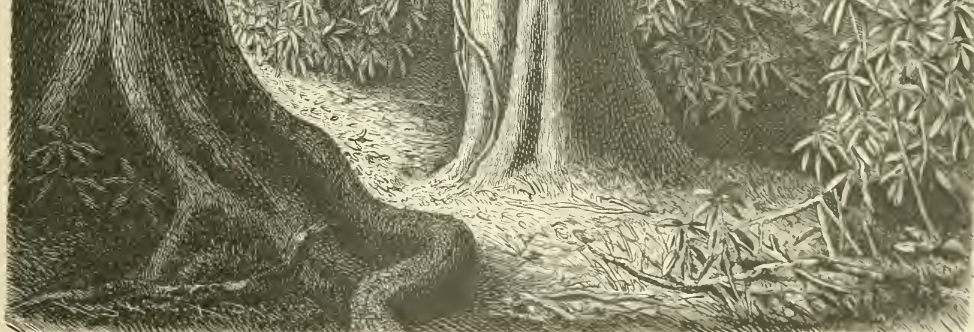

Fig. 168.-Ficus with girdle-like clasping roots, at Darjeeling in the Sikkim Himalayns. (From a photograph.)

climbing roots grow out directly to the wall. This is usually the ease with climbing plants. Several Aroidex and species of Ficus, and especially our ivy (Hedera Helix), the shoots of which occur anywhere at the foot of a tree-trunk or Vol. 1. 
of a rocky wall, develop climbing roots close below the growing shoot-apex. These roots speedily reach the wall and fasten the portion of the stem from which they arise to it. This continues slowly, the shoot-apex continually creeping higher up over the substratum. This is the simplest way in which climbing stems become attached. The process by which those of the often-cited Tecoma radicans are affixed is much more complex. These stems shun the light in a marked degree. If Tecoma radicans is planted in front of a wall covered with trellis-work, the actively growing shoots turn away from the light, slip behind the trellis-work, and adhere closely to the wall by those portions of the stem at which climbing roots appear. So soon as they come in contact with the hard substratum the small pale rootlets grow out from the cushions as a fringe of threads which cling very firmly to the wall. The growing shoot never leaves the wall, but keeps close to it, always seeking the darkest places under projecting tiles, ledges, and cornices, attaching itself at intervals by fresh clamp-roots.

The most remarkable method by which shoots destined for climbing reach a wall which will afford them a support is observed in several tropical Bignoniacex related to Bignonia unguis, one of which, Bignonia argyro-violacea, growing by the Rio Negro in Brazil, is represented in fig. 170. This plant bears two kinds of leaves: simple leaves, the blades of which attain to a considerable size, and others which, like the leaves of the vetch (Lathyrus), bear two opposite leaflets on one stalk and end in a structure which divides into three limbs with pointed hooked claws, and which is not unlike the foot of a bird of prey.

The development of this clawed, grasping organ always precedes that of the leaflets, so that in the youngest stages the green leaflets only appear as minute scales. Leaves ending in claws are only found on stems which, so to speak, have to seek a firm, safe support for the flowering and fruit-bearing shoots to be developed later on. These stems, however, are thin, much elongated, and are always pushing out new internodes. They hang down as long threads from the tree, whose bark is already quite covered, and which offers no space for a new settlement, and are easily set in motion by the action of the wind. At the end of each thread can be seen two young leaves placed opposite one another, on each of which only the three hooked limbs are at first developed, appearing to be extended for prehensile purposes, just as in a bird of prey. If the shoot oscillating in the air fails to encounter an object which it can seize with its claws, the latter fold back, and the leaflets are developed. Before the following day the filamentous stem has produced a new portion similarly equipped. These fresh claws are again extended, and the supple stem again sways in the wind, in the hope of being able to catch hold of a firm object. The same thing is repeated day after day until some suitable anchorage comes within reach of the elongating shoot. Now is the time for the development of the clamp-roots, which have to fix the stem to the substratum still more firmly than the claws could do. The climbing roots are really already present at each node of the filamentous stem as small protuberances, but they remain quite short until such time as the swaying shoot effects its attachment. Then 


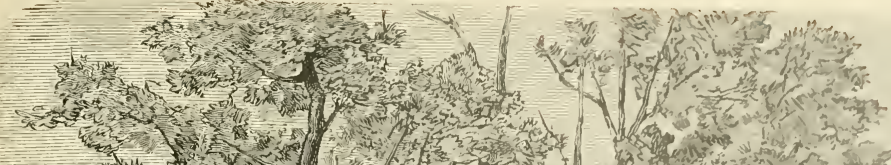

$4^{5}$

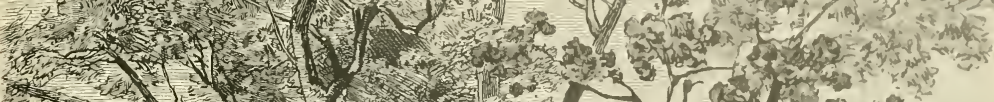

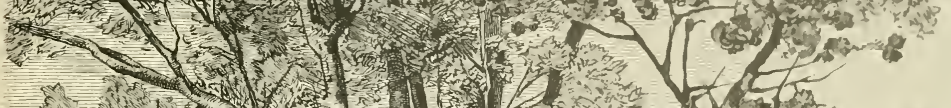

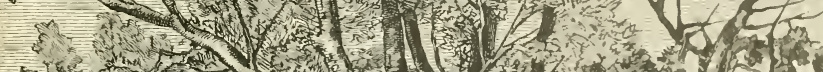

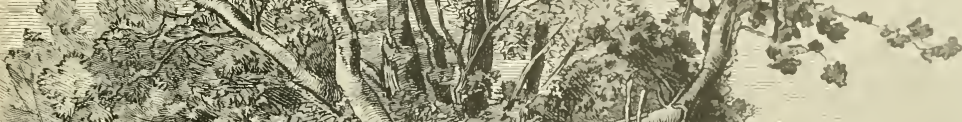

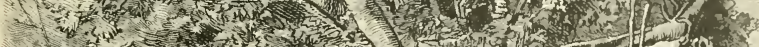

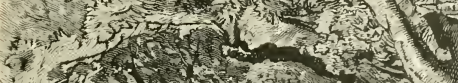

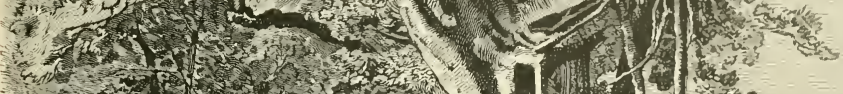

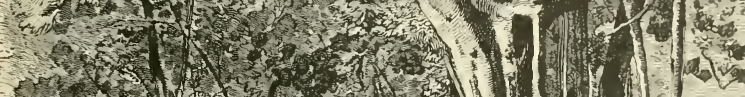

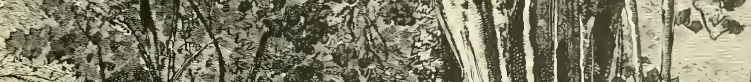
5.

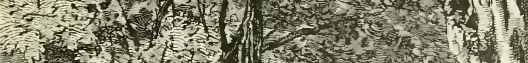
M. (1) (1) N1W

Fig. 169.-Ficus Benjamina with incrusting climbing roots. (After a drawing from naturo, by Selleny.) 
they grow out, elongate, and produce lateral branches, as may be seen in fig. 170 . Under favourable conditions, i.e. when these swaying shoots reach an unoccupied support and become permanently attached there by their scandent roots, these anchoring shoots exhibit a marked change of habit. They give rise to vigorous and compact shoots with simple leaves destitute of claws, and may unfold flowers and ripen fruit. In due time, when the space has become occupied, pendent shoots are again produced which explore the neighbourhood for a new anchorage in the manner already described.

The group of root-climbers as a whole undoubtedly presents many points of resemblance to forms with stems prostrate on the ground. The climbing stems of Ivy remind one of the stems of Periwinkle, the climbing stems of species of Pothos of the creeping stems of the Snake-root (Calla palustris), the climbing stems of Tecoma radicans of the runners of strawberry plants. The only real difference is that in one case the substratum is the surface of the soil, while in that of climbing stems it is the abruptly-ascending surface of rocks and treetrunks. And this distinction is wanting in the Ivy. Ivy stems which grow over stony ground, fix on to the horizontal blocks of stone by climbing roots exactly as on vertical walls of rock. If mould is present in the crevices of these stone blocks, the climbing roots become true absorbent roots, not only fastening the stem to its substratum, but also taking up food. But ivy stems climbing up steep rocky walls also behave in this way. The roots which proceed from the portions of the stem growing over the bare stone wall are climbing roots, but as soon as the stem in its growth comes to a crevice filled with earth, the roots developing at that point become absorbent like those which it produces when creeping on the ground.

Thus it is clearly impossible to draw a sharp line between climbing and creeping stems. Similarly, on the other hand, there are some climbing stems transitional between this condition and an erect habit. Ivy, Tecoma radicans, the climbing species of Ficus, even several tropical aroids, and the Brazilian Marcgravia umbellata, exhibit this peculiarity. In the last-named, so soon as it has climbed up a tree-trunk or steep rocky wall into an illuminated place, it alters its growth completely. The shoots now formed up above no longer avoid the light; they no longer develop climbing roots for attachment to the substratum, their wood becomes more extensive, the hard bast surrounding the wood is much more strongly developed, the shoots now not only stand erect without support, but are even able to withstand flexion, and develop flowers with abundant honey and ripe fruits. The erect shoots of Ivy and of the climbing species of Ficus, bathed in sunshine, also unfold foliage-leaves, which are strikingly different from those of the climbing shoots in size and shape, and even in their internal structure. Anyone knowing only the long filamentous shoots of Ficus stipulata, used for covering the walls in green-houses, happening to see the vigorous shoots of this plant with large leaves and figs, would think it impossible that both should belong to one and the same plant. 
The erect stems of the Ivy, adorned with cordate shining foliage-leaves when treated as slips or cuttings, send absorbent roots into the ground and ramify; but, strangely enough, the shoots which they develop, although they now spring close upon the ground, do not become climbing stems, but exhibit exactly the same structure, the same erect position, and the same foliage as the shoots on the top of a wall or on the summit of a tree. Anyone seeing for the first time

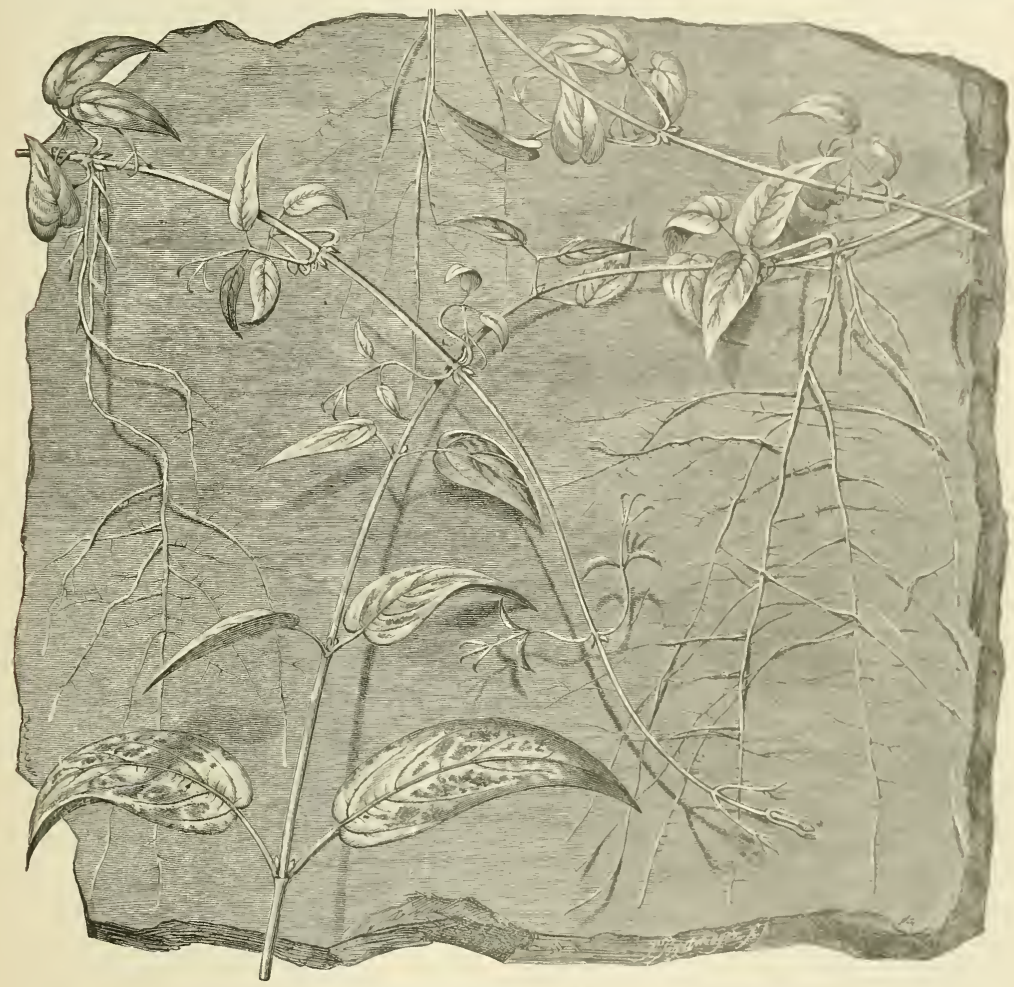

Fig. 170.-Bignonia argyro-violacea, from the banks of the Rio Negro in Brazil.

such Ivy grown in pots, is tempted to mistake it for some erect tropical A raliu, and even experienced gardeners and botanists may be misled by these plants. We are involuntarily reminded by these successive shoot-structures, which differ so much in their outer form and internal construction, of the alternation of generations occurring in Vascular Cryptogams, and so much the more since the climbing shoots which precede the erect flowering shoots do not develop fluwers and fruits, and thus to some extent resemble an asexual generation.

Several Indian species of fig, the stems of which climb up rocky walls and 
adhere to them by girdle-shaped, flattened, and in part lattice-forming roots, send up an erect stem with large foliage when they have reached the top of the wall or the summit of the block of stone. By this time the leaves of the climbing parts of the stem have fallen away. Generally, this climbing stem, the first stage, as it were, ean no longer be recognized as such; only the clamp-roots which proceeded from it, which have meanwhile become much thickened and wide-meshed, appear in a most remarkable manner like a lattice-work spread out over the stone. Any one not knowing the history of development of these species of fig, would think that the stems rising erectly from the top of a block of stone or in the cleft of a rocky wall, had germinated in the place where they rise up into the air, and had sent down from thence a net-work of aërial roots enveloping the stone. This idea, which at first occurs to everyone who looks at the two fig-trees faithfully represented on the left-hand side of fig. 171, does not, however, correspond with the actual process of development. The lattice-forming roots adhering to the stone are not sent out by the small trees rising above them, but have been developed by the climbing stem which had mounted up by their help, and then became transformed into an erect stem growing freely up into the air. We must also guard against generalizing and regarding all root-structures of this kind as climbing roots. In the tropics there are plants whose erect stems do send down aërial roots which continually ramify, and then look deceptively like lattice-forming climbing roots.

[For further details as to climbing plants the reader is referred to H. Schenck's masterly Beiträge zur Biologie und Anatomie der Lianen. Jena, 1892. ED.]

\section{ERECT FOLIAGE STEMS.}

Plants with procumbent and subterranean stems preponderate in high mountain and in arctic regions, whilst in these places the majority of woody stems cling closely to the substratum, or are embedded in the soil. Lateral shoots rising erect from the ground, of course, often spring from these main stems, but they bear no foliage, or possess green leaves only at the base, and terminate in flowers. They are essentially of the nature of flower-stalks or scapes, and are for the most part to be regarded as floral-stems. The few flowerless, erect foliage-stems which are met with in these frosty districts are all very short, usually closely crowded together into a carpet, or have the form of numerous erect branchlets; they seldom rise more than a span-high from the ground. The only noticeable erect stems besides the type of low, woody shrubs are the culm and the herbaceous stem. On passing from elevated regions down into the valley, and from the arctic zone southwards, besides these forms, we meet with reeds, high shrubs and trees, and still nearer the equator we se the erect stems of cactiform plants, bamboos, and palms.

In this connection the terms caudex, culm, stalk, and trunk are used to indicate the forms of erect foliage-stems standing out in the landscape, terms 


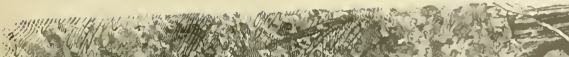

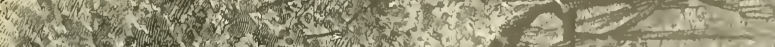

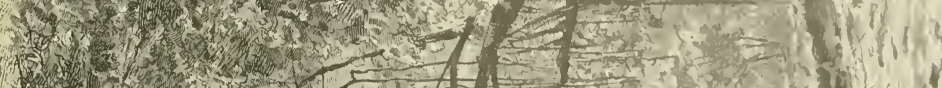

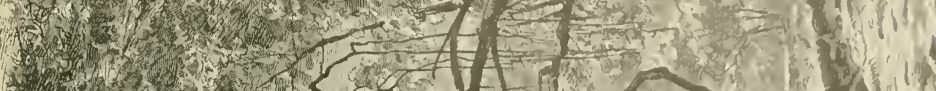

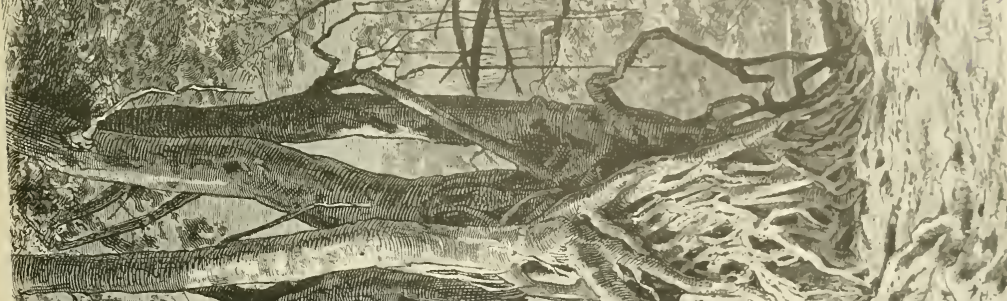

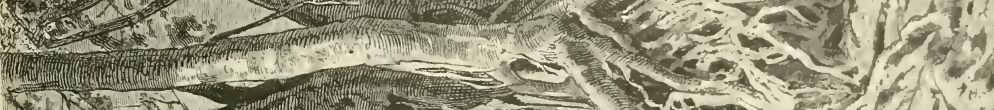

(1)
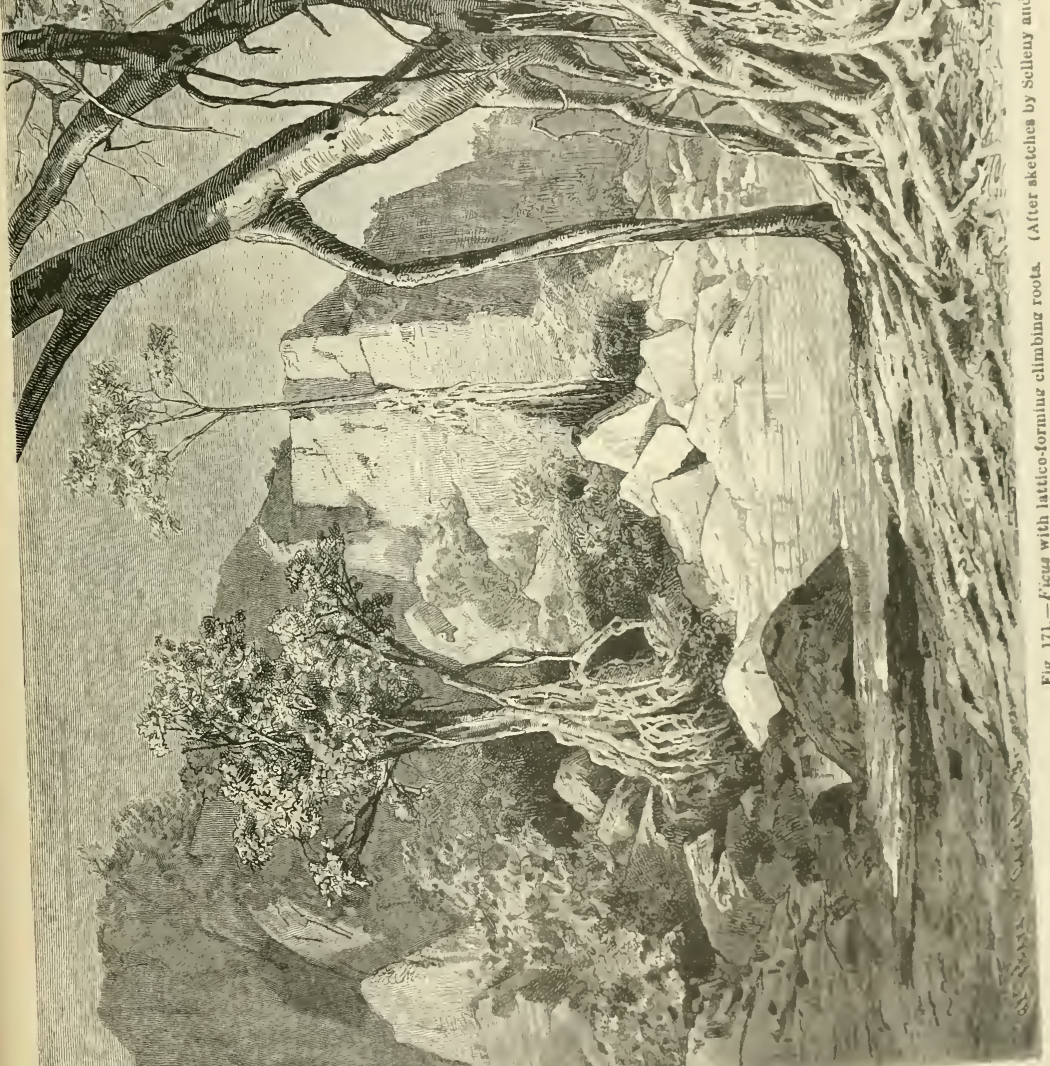
which have arisen in the popular tongue, and of which everyone thinks he knows the meaning; these words have also been admitted into scientific terminology, although, when more closely examined, they are seen to be ill-adapted for the nomenclature of erect stems. Thus there are procumbent culms, procumbent caules, and procumbent tree-trunks, and it is therefore not correct to use these terms for erect forms only. It has been proposed to designate the erect stem, which may be compared to a post, a standard-stem (stirps palaris), prefixing the word "standard" to the names of the various sorts of erect stem. The names resulting from this combination would prevent any confusion, but, unfortunately, they are cumbrous and unusual, and on the whole unsuited to this book. For these reasons the current expressions will be still employed, with, of course, the proviso that in this case they refer only to standard stems.

The cactiform stem, especially those gigantic specimens which are natives of the Mexican plains, and attain to a height of some 15 metres, might have been taken as a type of a standard-stem. In their erect habit, together with their lack of branches, they look like posts which have been driven into the ground to form the foundation for a scaffolding. But since these stems have no foliage-leaves, or rather, since their leaves have been transformed into spines, so that the formation of organic materials, which is usually performed by foliage, has to be done by the green cortex, they cannot really be reckoned as foliage-stems, and can only be mentioned here incidentally.

The caudex (cauloma, caudex) has the greatest claim of all the series of erect foliage-bearing stems to be compared to a standard. The form seen in slender palms, to which the term Caudex columnaris has been applied, stands foremost in this respect. The Palmyra Palm, one of the most beautiful of all palms, and so common a feature along portions of the coast-line of the island of Ceylon, gives a clear idea of this form of caudex. As a rule, the height of palms is much exaggerated; there is a great temptation, especially in the case of isolated stems, to estimate them as much higher than they really are. This is on account of an optical illusion which comes into play just as in the cstımation of the heights of mountains. An isolated mountain peak rising up abruptly is, at first sight, always thought to be higher than a continuous ridge which gradually ascends in gentle slopes, although both may have exactly the same elevation; and the same thing occurs in estimating the height of stems. An isolated Palmyra Palm rising from among low shrubs appears to be much higher than one which is actually taller, but which grows in the midst of a group of trees and whose summit only rises a little above the other tree-crowns. The highest columnar caudex is shown by Ceroxylon andicola, a palm growing in the Andes, of which stems are known 57 metres in length. The caudex of the Cocoa-nut Palm (Cocos nucifera) attains a height of 32 metres, and that of the Palmyra Palm (Borassus flabelliformis), not far behind the last, 30 metres. Most other palms are lower than this, the great majority never exceeding 30 metres. The so-called Dwarf Palm (Chamarops humilis) is 


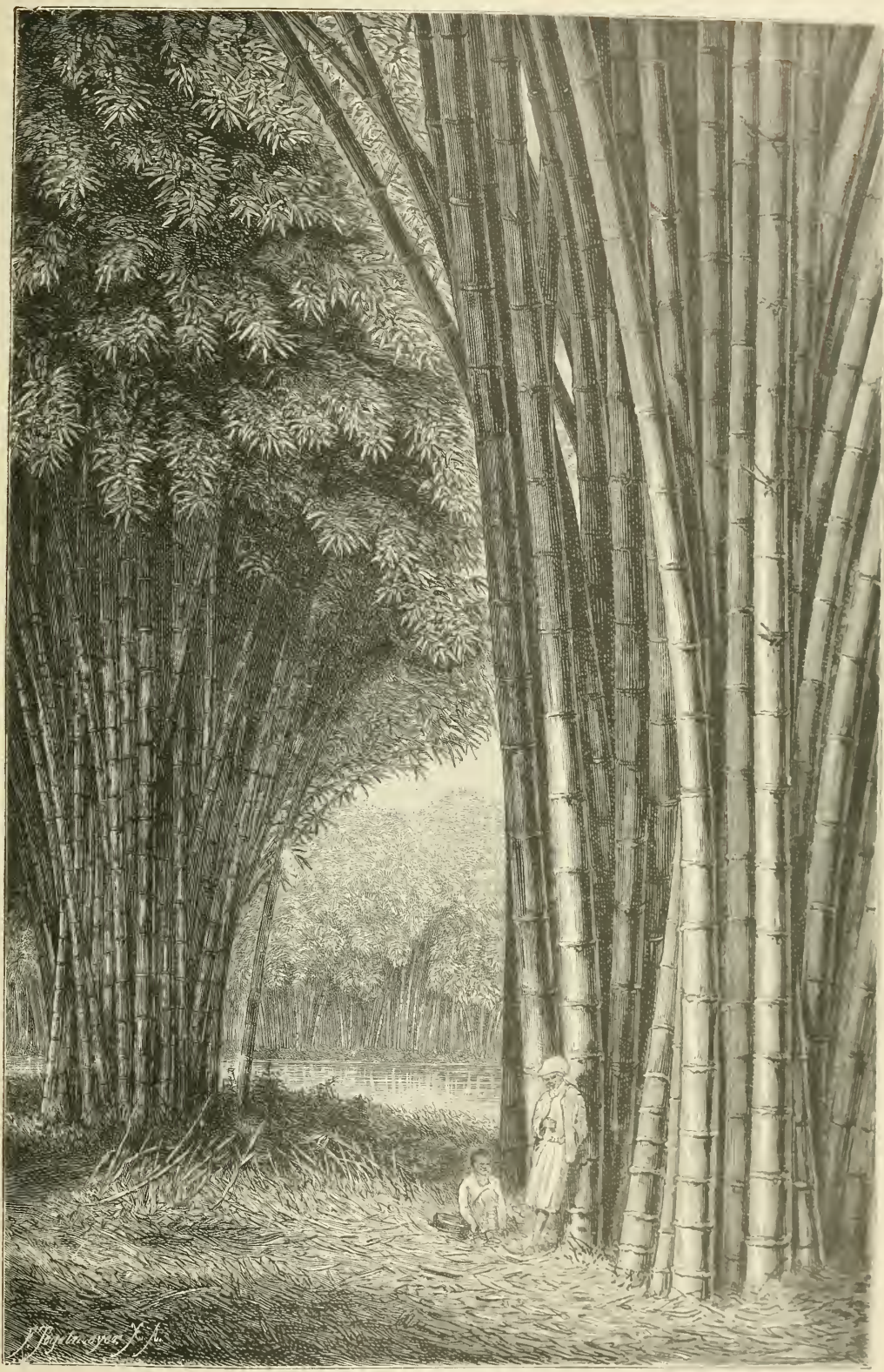

Fig. 172,-Bamboos in Java. (From a photograph.) 
only 4 metres high, and there even exist palms whose caudex barely rises above the ground.

The caudex of tree-ferns and cycads also remains comparatively short. When travellers speak of the gigantic trunks of tree-ferns, they only mean gigantic in comparison with the stems of the ferns growing in our European forests, which either never appear above the ground, or like those of the ostrich fern (Struthiopteris germanica), only $10 \mathrm{~cm}$. above the soil. The New Zealand tree-fern Dicksonia antarctica, with a diameter of $40 \mathrm{~cm}$. reaches a height of 15 metres, and the caudex of Alsophila excelsa, with a thickness of $60 \mathrm{~cm}$., is 22 metres high. The cycads scarcely ever reach this height, nor do the various other flowering plants possessing a caudex, such as the species of the genera Yucca, Dracana, Urania, Pandanus, Aloë, and Xanthorrhoea. The celebrated Dragon-tree (Draccena Draco) of Orotava, whose age is estimated to be 6000 years, has a circumference of 14, and a height of 22 metres.

The caudex is in most cases simple, but several Pandaneæ and dragon-trees, and among the palms, the Doum Palm (Hyphane thebaica) growing in the Nile valley and Hyphcene coriacea, fork and develop a few short branches when their main caudex has attained a great age. Many caudices, e.g. those of the tree-ferns Dicksonia antarctica and Todea barbata are completely covered with short aërial roots, in consequence of which their surface has a peculiar bristling appearance. Many caudices are also abundantly provided with thorns. For the appearance of most of them it is of importance whether the dead leaves break off above the base, so that the leaf-sheaths persist, or whether the leaf-sheaths are detached with them, only a scar being left on the caudex. In the former case the stem is clothed sometimes with ridges or scales, sometimes with a fibrous integument, or even with dry stumps. In the latter case it is covered with circular or shield-like scars. The caudex of Caryota ( $c f$. fig. 74, p. 311) becomes quite smooth after the leaves have fallen off, and looks like a gigantic culm; indeed, it forms a link between the caudex and that kind of stem which is termed a culm.

The stem-structures which are comprehended under the name culm (culmus) differ in size even more than does the caudex. They may be classified in the following groups, which, of course, are not sharply marked off from one another. First, the culm in the narrow sense of the word, which embraces those forms whose stem does not exceed a diameter of $\frac{1}{2} \mathrm{~cm}$; then the reed, which is not branched, whose internodes are always surrounded by long sheaths, and whose stem has a diameter of $\frac{1}{2}-5 \mathrm{~cm}$; : and, further, the bamboo, which divides into numerous branches, having short leaf-sheaths and a very peculiar anatomical structure; this will again be referred to in the next chapter. The culm exhibits its highest proportions in bamboos, especially in the species represented in fig. 172, which attains to a height of 25 metres and a thickness of more than half a metre. From this extreme, on the one hand, to the delicate little culm 2-3 cm. long, of many annual grasses of the Mediterranean flora, there exists an unbroken series about the middle of which comes the Southern Reed (Arundo Donax) with a height of $4 \mathrm{~m}$. and a diameter of $5 \mathrm{~cm}$. 
The stalk (caulis) does not become woody, but remains green; it persists only for a single period of vegetation, and then dies down. The stem of annual and biennial plants classed as herbs (herbor) is called a "herbaceous" stem (caulis herbarrus), and that of perennial plants a "suffruticose" stem (cuulis suffruticosus). By" the term "undershrub" (suffrutex) we understand especially those peremial plants whose underground stem annually sends up shoots which do not become wooly, but which die off at the beginning of the winter, e.g. the Dwarf Elder (Sambucus ebulus), the common Avens (Geum urbanum), and the Meadow Sage (Salvia pratensis). Whilst the caudex and culm are generally circular in cross-seetion, the caulis is usually 3-, 4-, and 5-ribbed. Longitudinal furrows traverse its exterior, and the relation of these to the nearest leaves will be described more in detail subsequently. The extreme limits of size of the caulis have already been discussed on $\mathrm{p} .656$.

The woody stem (truncus) either remains without branches until it has attained a considerable height, when it is called "arborescent" (truncus arborescens), or it is very short, and its branches spring from near the ground, in which case it is called "shrubby" (truncus frutescens). A distinction is also drawn in descriptive botany with regard to size between the "tree" (arbor) in the narrower sense, and the "small tree" (arbuscula), the "shrub" (frutex) and the "small shrub" (fruticulus). The expression "semi-shrub" (semifrutex) may be employed to denote shrubs whose yearly shoots only become woody at the base before the next period of vegetation, and which wither and die off above this. These form a transition to the undershrubs mentioned above.

Of all these forms of woody stem the tree, especially prominent on account of its mass, naturally claims most interest. Nor is this interest limited to the botanist; it is shared with him by the landscape-painter, forester, gardener, indeer?, by every lover of nature, each from his own particular point of view. Among all the forms of vegetation trees are the best known; they have received a special name in all languages, different nations have chosen certain species of their country as favourites, and have extolled them as national trees in their songs, and even in the religious observances and customs of ancient and modern times trees played and yet play a prominent part. Many persons who have never occupied themselves with botany, and have never observed plants closely, but who have a strongly-developed sense of form, are able to distinguish and recognize the various kinds of trees at the first glance and at considerable distances. How is this possible? The explanation is very simple. The aspect of every tree, like the face of every man, presents certain features which are peculiar to it alone; these features imprint themselves almost unconsciously on the memory of anyone who is occupied much in the open, and the species is recognized again by him, even at a distance, like an old friend one meets in the street. To the landscape-painter these features, which, taken together, form what has been termed the habit of the tree, are particularly important, for he has to seize them and give them artistic expression. Our task here, however, is to detail and to interpret these features in the appearance of the trees, or let us put it, to give a scientific basis to the "habit". 


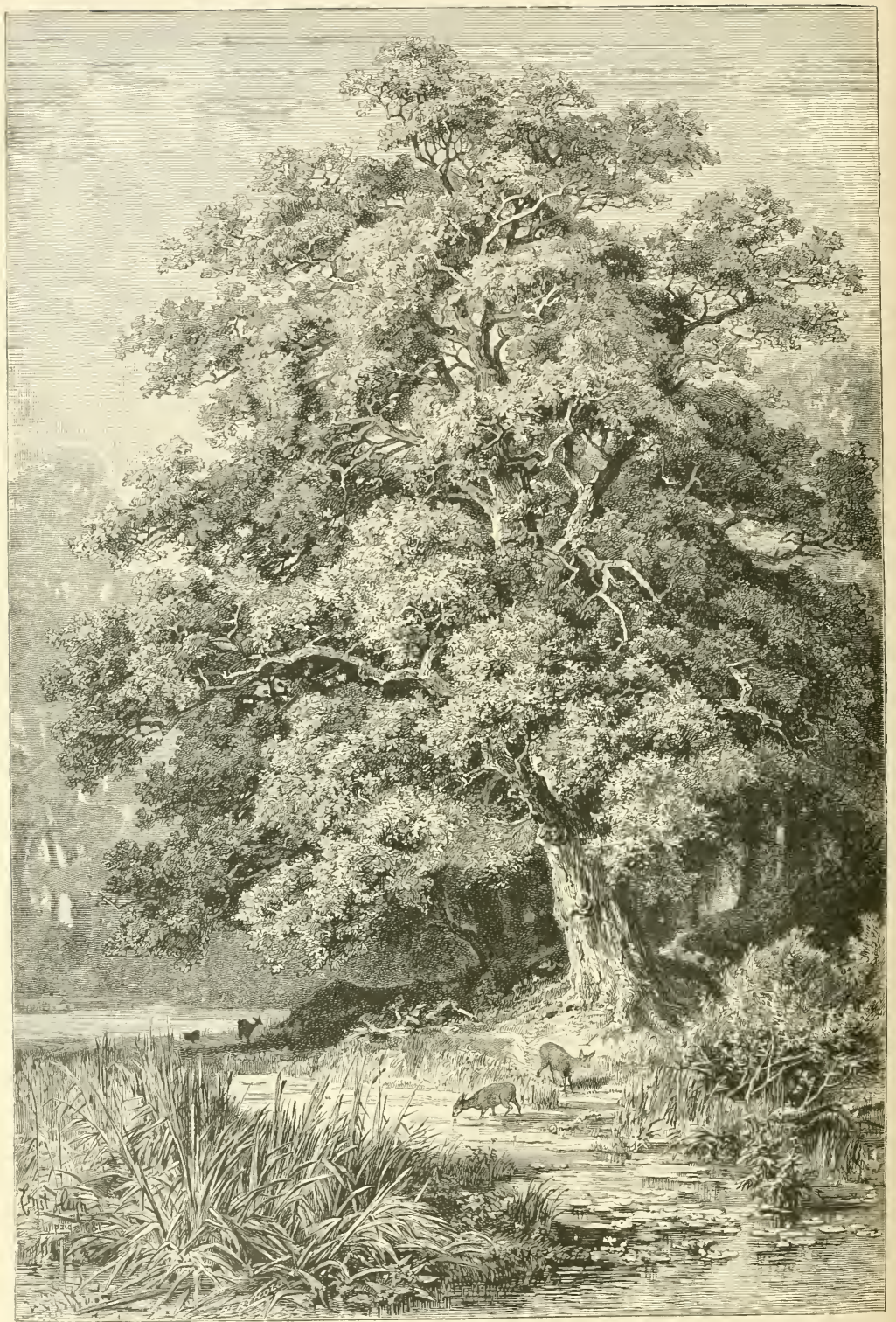

Fig. 173.-The Oak. 


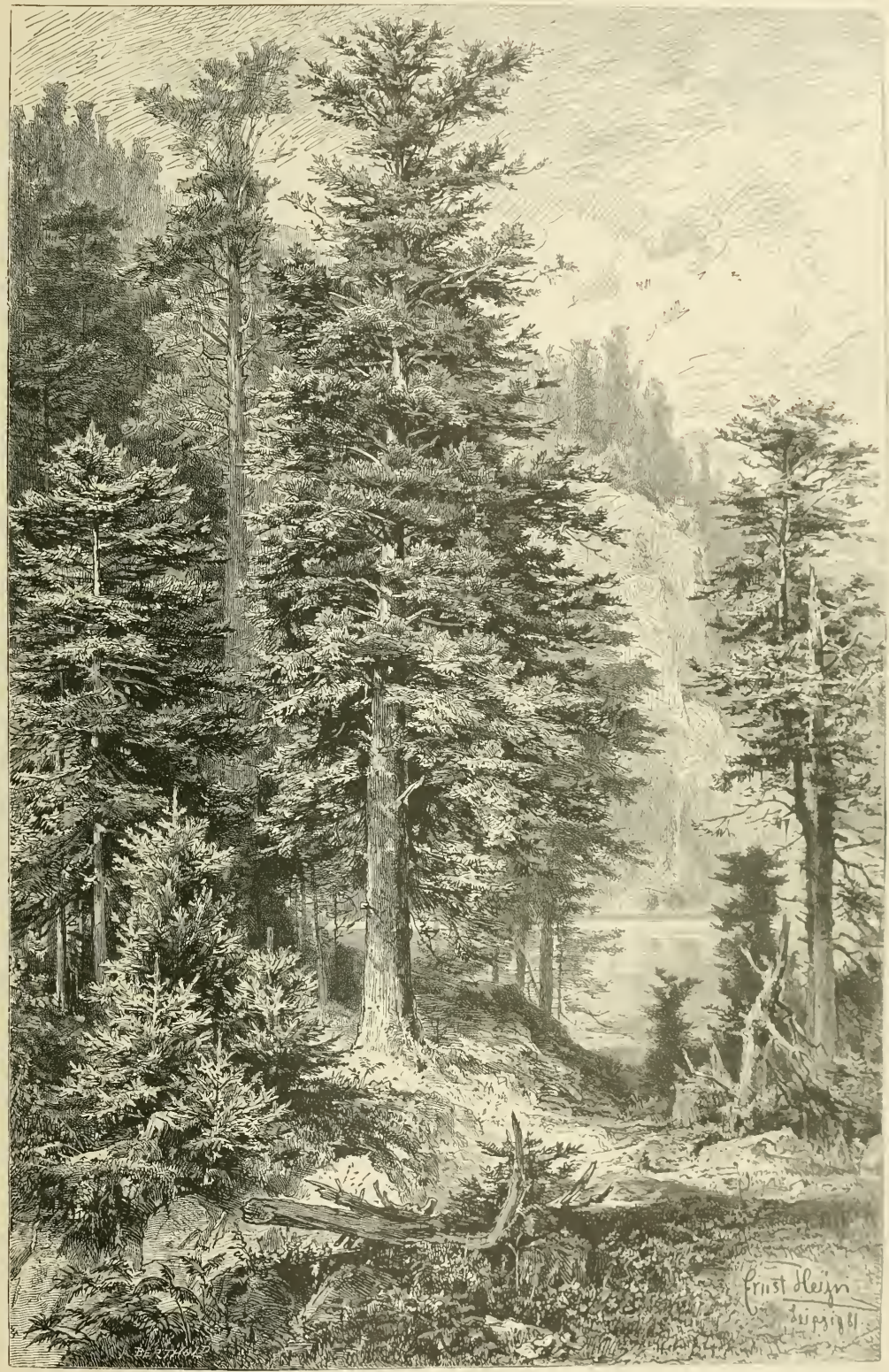

Fig. 174.-The Silver Fir. 
The limits of this book do not allow me to treat this theme as fully as my inclination and predilection for the relations between art and science would prompt me to do, but as a tree may be sketched on a wall with a few strokes, so I will endeavour to represent the principles of the "habit" in a few words.

In every tree the position of the buds depends upon the position of the foliageleaves, and it is evident that the distribution of the lateral twigs proceeding from a branch is also dependent upon the position of the leaves. The correlation between the arrangements of leaves and of branches is therefore the first which has to be considered in explaining the "habit". Like leaves, the branches are either whorled or decussate, or arranged along a spiral line, and it may therefore be said that the branches also exhibit the definite geometrical relations which were described in detail for leaves on pp. 396-407; even this fact gives a characteristic stamp to every tree. How very different are maples and ashes with their decussating branches, in comparison with elms, limes, and alders, with leaves arranged on the one-half and one-third system, and with beeches, oaks, and poplars characterized by the two-fifths and three-eighths arrangement; they differ not only in detail, but also in the grosser features of the whole tree-crown. Not only are the bare trees in winter-time readily recognizable at a distance by their ramification, but every portion of the leafy crown derives its particular contour in consequence of this branching. Then, again, the size and shape of the foliage-leaves have to be considered in interpreting the habit. This does not imply that the painter should represent the individual leaves, so that they could be recognized, for that would in a picture be undesirable. The significance of the configuration of the single leaves lies rather in the fact that they regulate the form of the whole tree. The boughs and branches of trees with narrow, linear, or needle-shaped leaves have far less to support than those which are adorned with large, flat, extended leaf-blades. Trees of the former class are characterized by their height, of the latter by their width, a difference which appears in the trees of all parts of the world. For example, the difference in the architecture of slender, narrow-leaved eucalyptuses and willows, and the broad-leaved paulownias, catalpas, and planes, with their widespreading boughs, is very striking. If we compare the illustrations of oak and fir placed opposite one another on the preceding pages, we notice that the needlebearing boughs and branches borne by the slender stems of the fir-tree scarcely occupy a third of the space of that taken up by the thick, heavy trunk of the oak, the leaves of which are so much broader.

A third point which comes under consideration is the light required by the leaves on the lower boughs of older trees. The thicker and more abundant the foliage on the summit or top of the crown, the deeper becomes the shade around the lower part of the main trunk. If the lower boughs are not able to elongate continually by means of new additions they die, together with their shaded foliage, withering up and breaking off either wholly or in part at the first opportunity and falling to the ground; but if they have this capacity of elongating, they push and extend their leafy branches as far as possible out of the circle of the shadow into the 
sunlight, and frequently curve up towards the light, as may be well seen in ash and chestnut-trees, and also in the spruce firs represented on p. 415 .

The lower portion of the stem which has lost its boughs increases in crrcumference as the burden it has to bear becomes greater, and its thickness and strength in every species bears a definite relation to the weight of the crown. The increas. of circumference is brought about by the addition each year of new masses of wood to those already present. In very young stems the wood appears in the form of strands, symmetrically arranged round the central pith, closely adjoining one another and forming a cylinder which is only interrupted by the medullary rays. The annual increments of wood, deposited on the periphery of this prinary ring, also have the form of rings in cross section; each is known as an annual ring. The age of a felled tree can be reckoned from the number of these annual rings, and obviously the girth of the stem increases with their increasing number. The enlargement of the circumference is, however, not without its effect on the external appearance of the stem. While still quite young, the stem possesses a covering skin (epidermis) which closely surrounds the green tissue of the cortex. This epidermis, however, only keeps pace with the development of the interior of the stem as long as this particular part continues to grow in length. When it stops, and increase in thickness commences, the first skin perishes, and is replaced by a second, the so-called periderm. This usually begins to develop even at the end of the first period of vegetation. The most important constituent of periderm is cork, a tissue of cells impervious to water and almost impervious to air, and therefore excellently fitted as a covering for the inner sap-conducting portions of the stem. Whatever lies outside this cork, or is secreted through it from the inner sap-containing portions, dries up and dies. If the periderm is developed immediately beneath the epidermis, this alone perishes; but if the periderm arises in the deeper layers of the cortex, a considerable thickness of cortex also dies and remains outside the cork as a dead dry crust. This inner periderm with the dead adhering parts of the cortex is called the bark.

The development of the periderm keeps pace with the development of the stem. As soon as the wood of the stem becomes thicker, by the intercalation of a new annual ring, the mantle of periderm stretches, and consequently the whole envelupe of bark. In many trees this bark remains year after year on the periphery of the stem; it becomes fissured by the continuous increase in thickness, but new lrark is as continuously produced from within closing up the fissures. In other instances a part of the bark falls off on to the ground in consequence of the thickening of the stem, and is again replaced by new bark from within.

Since every kind of tree has its own special bark, the texture and colour of this structure contributes not a little to the appearance of the whole tree, it forms one of the characteristic features which must not be overlookel when describing the habit of the tree. The following are the most important forms of bark. First the scale bark, which is detached annually in the form of shielils and plates, to be seen especially well in the stems of planes, almomi willuws, 
and many species of Australian eucalyptus. Then the membraneous bark, which separates as dry films and ribbons; this form of bark is shown in the Common Birch (Betula alba), illustrated opposite. Many species of the Australian genus Melaleuca exhibit a bark which, when stripped from the stem, looks deceptively like a thin silky material. A third form is the ringed bark, which is detached from the stem in the form of thin, irregularly-fissured tubes, and is especially developed in the Mock Orange (Philadelphus). A fourth form, of which the Vine (Vitis vinifer'a) may serve as an example, is the fibrous bark which is detached as numerous stiff threads. Finally there is the fissured bark, which is produced on the stems of the oak, lime, ash, and numerous other leafy trees. In this form the bark is not detached in large pieces, but is ruptured by the increasing thickness of the stem, causing longitudinal fissures with a sinuous or zigzag course, by which in one case only narrow ridges and grooves, and in other cases broad angular patches are outlined. Epiphytes, especially mosses and lichens, prefer to settle on fissured bark, and older stems with this kind of bark are in temperate regions usually overgrown with cushions of moss, in the tropics with ferns, bromeliads, and orchids. Such a colonization would be impossible in bark which falls off annually, and the stems of plane trees are not only free from epiphytes, but always look as if they had been scraped or peeled.

The form of the bark is so characteristic that by it alone the species of the tree can be recognized; it therefore constitutes an important feature in the picture of a tree, nor can it be altered according to fancy. It is inadmissible that artists should combine the studies they have made of various trees as they please, perhaps putting the crown of an oak on the trunk of a plane. That the colour of the bark is as important in the habit as the tint of the foliage goes without saying, and it is evident that the relative sizes of the various trees round about must also be considered.

The height and age of trees cannot be represented in definite figures, but this much is certain, that every species of tree, just like every species of animal, is limited to a certain size and age which is but rarely exceeded. The records of age which have come down to us are for the most part too great. When trees of primeval forests are said to be a thousand years old, the estimates are based upon conjecture, and only in rare cases on actual measurements. The celebrated Baobab (Adansonia digitata) was reckoned by Adanson on the ground of the thickness of the annual growth to be about 5000 years old, but whether a miscalculation has not crept in must remain uncertain. The age of the celebrated Dragon Tree of Orotava, already mentioned once before, has even been estimated at 6000 years; the Plane of Bujukdere, on the Bosphorus, at 4000; and the so-called Mexican Cedar (Taxodium Mexicanum) was estimated by Humboldt at 4000 years. I would not like to stand security for these numbers either. On the other hand, the following extreme limits of age are calculated with fair accuracy:-For the Cypress (Cupressus fastigiata), 3000 years; the Yew (Taxus baccata), 3000; the Chestnut (Castanea vulgaris), 2000, the Oak (Quercus pedunculata), 2000; the 


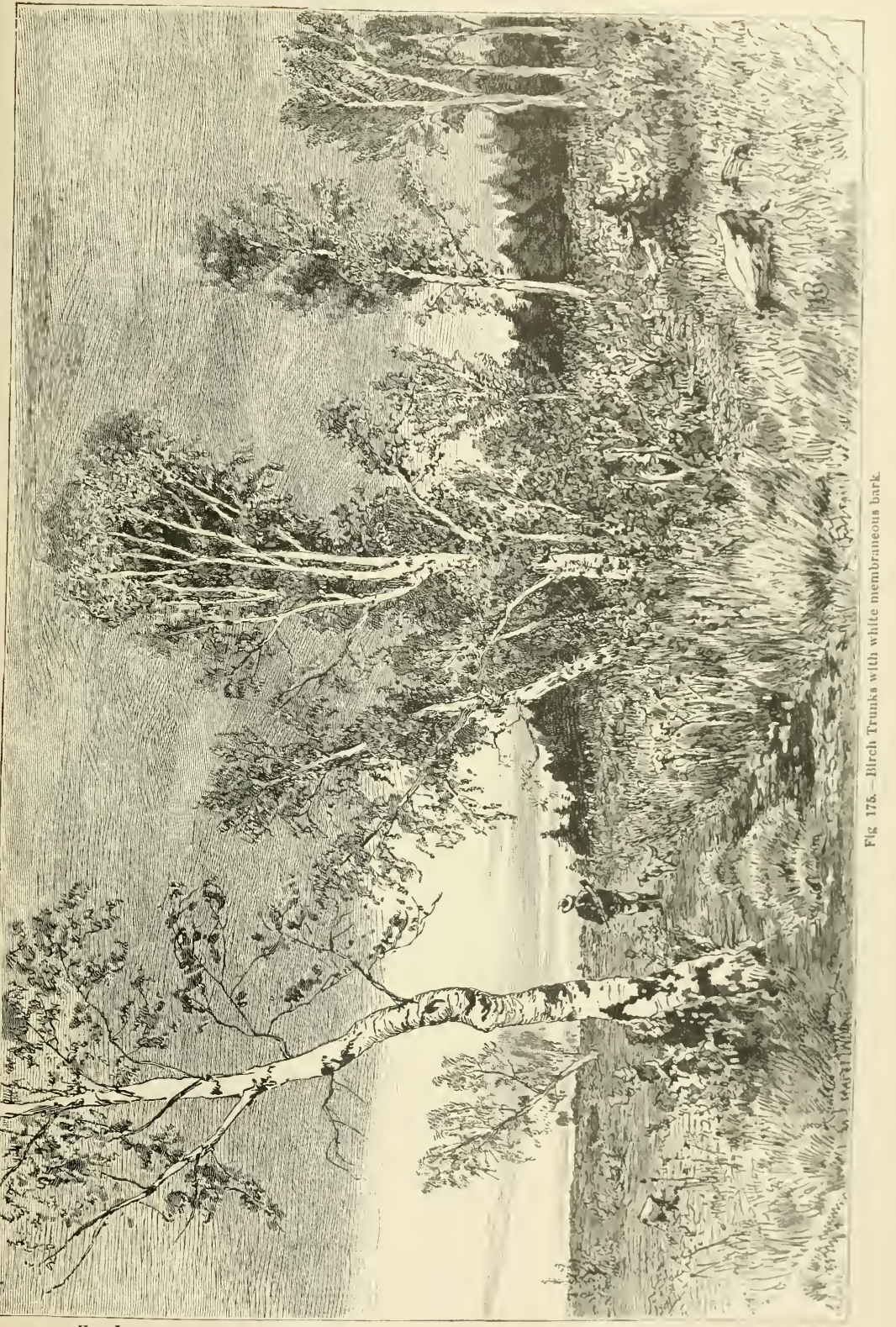

VoL. I. 
Cedar of Lebanon (Cedrus Libani), 2000; the Spruce Fir (Abies excelsa), 1200; the Broad-leaved Lime (Tilia grandifolia), 1000; the Arolla Pine (Pinus Cembra), 500-700; the Larch (Larix Europoea), 600; the Scotch Pine (Pinus sylvestris), 570; the Abele (Populus alba), 500; the Beech (Fagus sylvatica), 300; the Ash (Fraxinus excelsior), 200-300; the Hornbeam (Carpinus Betulus), 150 years.

The certified estimates of the heights of trees are of such general interest that they are included below in the following table:-

\begin{tabular}{|c|c|c|c|}
\hline Name. & $\begin{array}{l}\text { Height in } \\
\text { metres. }\end{array}$ & Name. & $\begin{array}{l}\text { Height in } \\
\text { metres. }\end{array}$ \\
\hline 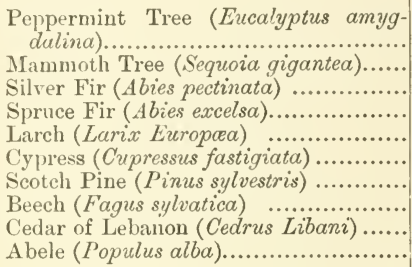 & $\begin{array}{l}100-130 . \\
79-142 . \\
75 . \\
60 . \\
53 \cdot 7 . \\
52 . \\
48 . \\
44 . \\
40 . \\
40 .\end{array}$ & $\begin{array}{l}\text { Mexican Cedar (Taxodium Mexicanum) } \\
\text { Durmast }(\text { Quercus sessiliflora) ............ } \\
\text { Plane (Platanus Orientalis) ............... } \\
\text { Ash (Fraxinus excelsior) ................. } \\
\text { Baobab (Adansonia digitata) ............ } \\
\text { Arolla Pine (Pinus Cembra) .............. } \\
\text { Tree of Heaven (Ailanthus glandulosa) } \\
\text { Oak (Quercus pedunculata) ............... } \\
\text { Hornbeam (Carpinus Betulus)........... } \\
\text { Yew (Taxus baccata) }\end{array}$ & $\begin{array}{l}38 \cdot 7 . \\
35 . \\
30 \\
30 \\
23 \cdot 1 \\
22 \cdot 7 . \\
22 . \\
20 \\
20 . \\
15 .\end{array}$ \\
\hline
\end{tabular}

Eucalyptus amygdalina (represented in fig. 176, after a drawing by Selleny), is amongst the giants of the vegetable kingdom. The highest of these stems placed beside St. Paul's Cathedral would tower about 20 metres above the cross, and would be only a little lower than Cologne Cathedral.

That the height and girth of trees do not increase proportionately will be seen by comparing the following table with the previous one:-

\begin{tabular}{|c|c|c|c|}
\hline Name. & $\begin{array}{c}\text { Diameter } \\
\text { of trunk in } \\
\text { metres. }\end{array}$ & Name. & $\begin{array}{l}\text { Diameter } \\
\text { of trunk in } \\
\text { metres. }\end{array}$ \\
\hline 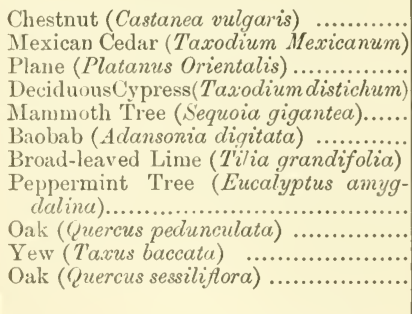 & $\begin{array}{l}20 . \\
16 \cdot 5 \text {. } \\
15 \cdot 4 . \\
11 \cdot 9 . \\
11 . \\
9 \cdot 5 . \\
9 . \\
8 . \\
7 . \\
4: 9 \\
4 \cdot 2 .\end{array}$ & 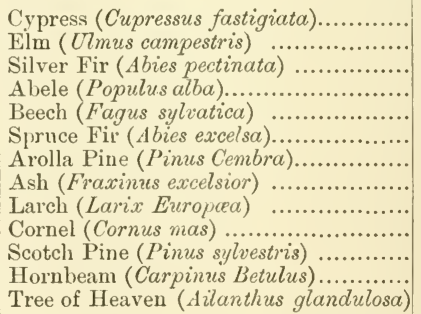 & $\begin{array}{l}3 \cdot 2 . \\
3 . \\
3 . \\
2 \cdot 8 . \\
2 . \\
2 . \\
1 \cdot 7 . \\
1.7 . \\
1 \cdot 6 . \\
1 \cdot 4 . \\
1 . \\
1 . \\
0.9 .\end{array}$ \\
\hline
\end{tabular}

According to these certified estimates there actually exist plants whose stems attain a diameter of 20 metres, and others whose stems rise to a height of $1 \pm 2$ metres above the ground. 


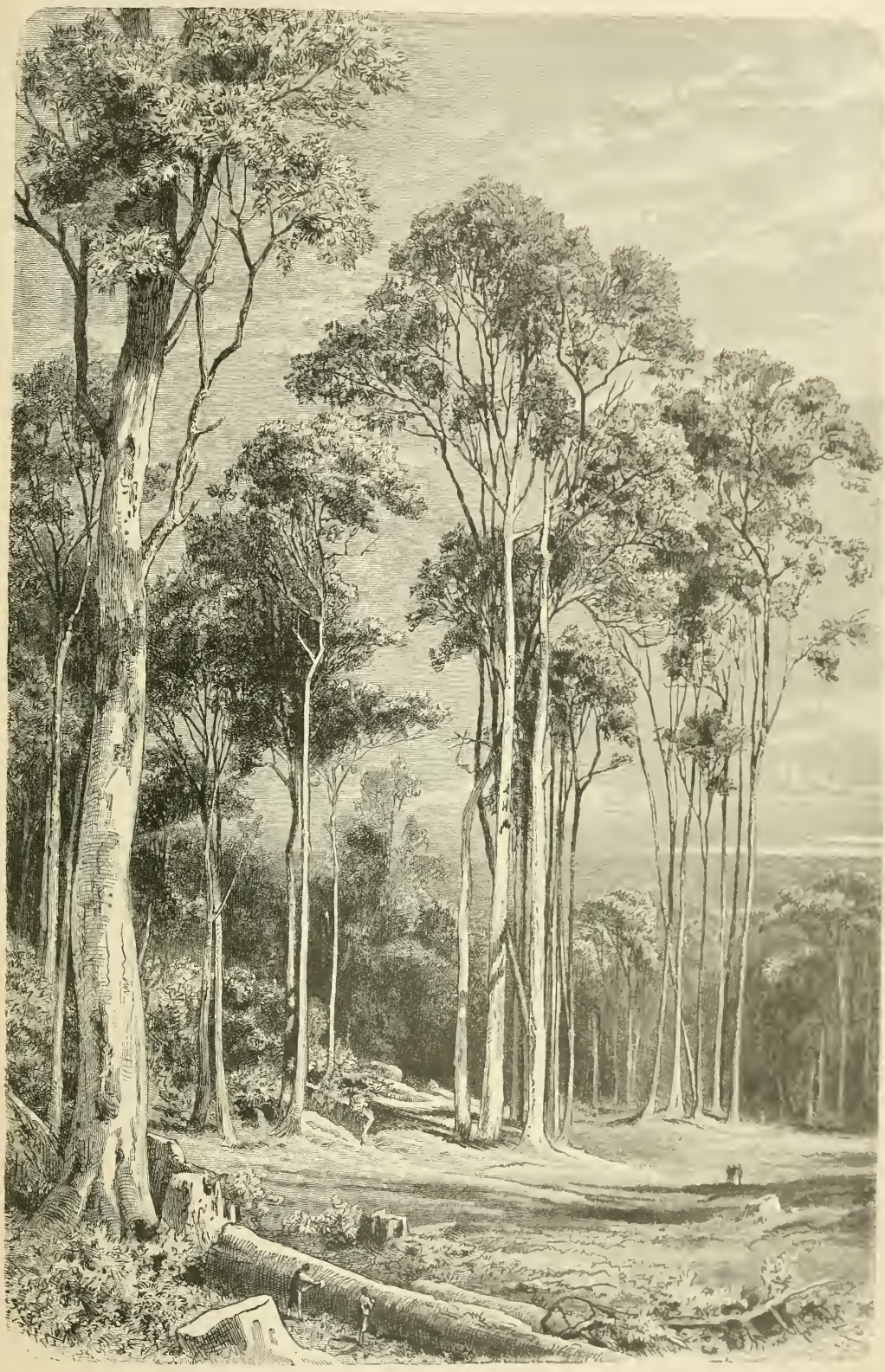

Fig. 176.-Eucalyptus trees in Australia. (After a drawing by Selleny.) 


\section{RESISTANCE OF FOLIAGE-STEMS TO STRAIN, PRESSURE, AND BENDING.}

When the weight of the individual parts of these huge trees is considered, it is difficult to understand how their comparatively slender main stems are able to support a crown weighing many thousand kilogrammes, and how it is that the boughs extending far out from the trunk horizontally do not crack and break under the weight of the branches and leaves they carry. The culms of grasses and the stems of bushes and herbs are also so loaded as to astonish us, and we cannot help asking how it is they are able to keep erect, and how, when their equilibrium is disturbed, they can resume their normal resting position almost at once. If we wish to investigate the mechanisms which make it possible for these plants to maintain their stems in this position without assistance, we must in the first place consider the lowest portion of the erect main stem, since it is that part which would naturally have the heaviest burden to carry. Given that the pressure caused by the loading operates in the direction of the axis, the main stem must exhibit contrivances enabling it to resist the vertical pressure; in other words, it must possess what is known as columnar strength. With the exception of some palms whose erect stems rise up like pillars from the ground, and whose leaves project equally in all directions, such a pressure, acting exactly in the direction of the axis of the stem, is but rarely found. As a rule some inequality in the stem or crown, although perhaps but slight, causes the pressure to be diverted from the central axis; the stem is bent by the one-sided burden, and has need not only of columnar strength, but of resistance to flexion as well. Winds also will effect a bending, not only by direct impact, but also inasmuch as they displace the centre of gravity of the load sustained by the lower part of the stem. Observation shows us that this bending is only rarely followed by the fracture of the stem. Not only grasses and reed culms, but also the thinner erect branches of trees, shrubs, and bushes, and even palm caudices may be bent down a considerable distance, but, when the wind subsides, quickly return to their erect position without having suffered the least harm.

Formerly but little attention was given to these phenomena, perhaps because they were so common and frequent, or perhaps because it was thought to be impossible to give a scientific explanation and reason for the swaying of branches in the wind. It was reserved for modern times to explain the mechanisms underlying the returning of bent stems to a definite position of rest, and the contrivances which permit such stems to bend but not break, even when considerably loaded and under strong pressure. Investigations into this subject have demonstrated that the bearing capacity and power of resisting bending moment in plant stems are obtained by structures exactly similar to those used by man in spanning a river with bridges, in fixing the supports of a roof, of wooden partitions, \&c. Further that the principle so important to every builder, the obtaining of the greatest strength possible with the smallest outlay 
of material, also finds expression in the construction of the stem. In one case we are reminded of the system of tubular bridges, in other cases of that of latticebridges; here of a massive pillar-like structure with architrave and flattened top, there of a Gothic building with pointed arches, buttresses, and steep gables; but the special conditions of the habitat are always taken into consideration, and the whole structure for this reason always exhibits the greatest adaptability of the means to the end.

The framework which gives the desired strength to the whole structure is made up of parts which would be called by builders "constructive pieces", and these are in turn made up of special cells, termed mechanical cells. Mechanical cells have already been alluded to in the description of the conducting-apparatus (cf. p. 474), although only very briefly. It was pointed out that the tubes and cells which serve for the transport of fluid materials up and down the plant are usually united into a bundle, the so-called vascular or conducting bundle, and that when the constituent parts of this bundle occur in organs which are exposed to the danger of being broken, mechanical cells always make their appearance alongside the conducting cells and vessels. The delicate vascular bundles then usually lie embedded in a channel of hard bast, or are protected laterally by a strand of hard bast, or more rarely they are interposed between two bands of this tissue. These strands and bands of hard bast are frequently of merely local importance for the vascular bundle, and may be likened to the strengthening appliances of gas and water-pipes in human dwellings, which are very important in their special use, but do not help to strengthen the whole house. Very often, however, these special supporting agents of vascular bundles are absent, and then the conducting tissues are affixed to the groups of mechanical cells which form the foundation-framework of the whole structure.

The hard bast is the mechanical tissue most often employed in both cases. To the naked eye the cells of hard bast look like tiny threads. They are elongated, fusiform, pointed at both ends, and interlaced and dovetailed with one another as shown in fig. $125^{5}$ (p. 469). They are generally about 1-2 mm. long, but in certain cases attain a much greater length; those of the Hemp are 10, those of Flax 20-40, of the Nettle 77, and of Boehmeria nivea even $220 \mathrm{~mm}$. long. The walls of hard bast cells are always very much thickened, and the cell-cavity is very narrow, often being reduced to an exceedingly fine canal, in some cases, e.g. in the cells of hard bast of Corchorus olitorius (known as Jute), the canal here and there is quite obliterated, so that the cell is transformed into a solid fibre. It is concluded from the direction of pores which sometimes appear in the walls that the micellie which build up the walls of these thick bast-cells are arranged in left-handed spiral lines, and this spiral torsion is supposed to be connected with the strength of the whole hard bast cell. It is known that bundles of straight threads are not as strong as bundles twisted into a string, and we are justified in supposing that this is also the case with the rows of micellæ forming the extremely fine fibrillie in the walls of the hard bast cell. When a cell of hard bast is fully developed, the living proto- 
plasm disappears from its interior, and the narrow space of the cell-cavity becomes filled with air, or less often with a watery fluid. The cell can then no longer continue its growth, neither can it serve to take up and conduct food nor to manufacture organic compounds; it cannot be employed in transformations and transmission of materials, and has exclusively an architectural significance. It is excellently adapted, however, to the task thus assigned to it. Its strength and elasticity are indeed extraordinary. It has been estimated that the bearing capacity of hard bast amounts to between 15 and $20 \mathrm{~kg}$. to the sq. mm. in cross section, and is therefore equal to that of wrought iron; indeed the bearing capacity of many species of plants is even equal to that of steel. Hard bast has this advantage over iron, that it is far more extensible and consequently less subject to breaking. From the consideration of all these properties it becomes evident why the hard bast of many plants has been used by man to such advantage in the manufacture of fabrics, string, ropes, and the like, since very remote times.

Woody fibres, also known as libriform cells, differ very little from hard bast cells. Whilst hard bast forms one of the most important constituents of the cortex, the woody fibres form an essential element in the wood of those stems which annually add a new layer to the already existing wood, thus increasing in circumference and exhibiting annual rings in cross section. Their length varies between 0.3 and $1.3 \mathrm{~mm}$., so that they are somewhat shorter than the fibres of the bast. Their walls are as a rule strongly lignified, but in other respects it is impossible to draw a sharp line between the two forms of cells. When a woody stem has grown in thickness and has developed bark on its periphery, the rôle played by the hard bast in the cortex is evidently at an end; the woody fibres then assume the tasks which in the young shoots are allotted to the hard bast, and they might therefore be called the hard bast cells of the wood.

In many plants a special form of mechanical cell-tissue is developed, known as collenchyma. The cells which compose it are elongated and connected with one another just like hard bast cells, but they differ from these and from the woody fibres in the fact that their walls are unequally thickened. Where three or four of these cells adjoin one another by their long sides the walls are very thick, but in places the wall common to two neighbouring cells remains thin; the whole of the tissue may be compared to a building in which thick main walls alternate with thin partitions which are strengthened here and there with quartering, and attain a great supporting capacity. A further distinction from hard bast cells and woody fibres consists in the fact that living protoplasm remains in the interior of collenchymatous cells in which ehlorophyll-corpuscles are often embedded; moreover, this protoplasm can draw some of the materials necessary for growth through the thin places in the walls from the surronding tissue, and can employ these as building materials;-in a word, the collonchymi is capable of further growth. This explains the advantage of collenchyma over hard bast cells, and woody fibres or libriform cells. The hard bast and libriform cells when once fully formed lose their capacity of further development, and would therefore be of little use as 
architectural elements in a still growing portion of the stem; they would wither prevent the lengthening of the other tissues, or would be ruptured by the force of the elongating cells, and in both instances would be injurious. The collenchy matous cells, on the contrary, are able to continue developing, they cau elongate and grow with the other tissues, and may be compared with the scaffolding of a several-storied building, which is constantly being raised as the work promresces. The collenchyma, of course, has this disadvantage when compared with the liard bast and libriform fibres, that its absolute strength is somewhat less; its bearing capacity is only 10-12 kg. to the sq. mm. in cross-section. The limits of elasticity of the collenchyma are also considerably less, but where hard bast or libriform cells would be unsuitable, from the reasons stated above, collenchyma replaces it. It cannot be said that hard bast and libriform fibres are more important than collenchyma; each in its own way has an especial architectural value, and sometimes the one, sometimes the other, is the more advantageous.

The hard bast, libriform cells, and collenchyma which are comprehended under the common term mechanical tissue are usually arranged in strands running parallel to the long axis of the stem. If they were confined to the centre it would be anything but a suitable arrangement, for an erect stem; they would contribute almost nothing to the resistance to flexion as will be seen from the following considerations. Let us imagine a horizontal, cylindrical stem resting on solid supports at either end and loaded in the middle; it will bend downwarels in proportion to the load laid on it, and in doing so the concave side will he shortened and the convex side lengthened; the shortened side will be subjected to compression and the elongated side to tension. These forces will be greatest at the periphery, on the upper and under limiting surfaces, of the bent stem. The opposed forces diminish towards the middle of the stem, and completely vanish at the centre, therefore, in order that the stem should resist bending as much as possible, it is obvious that the strengthening material is best applied when wholly used in the form of flat plates where the forces are greatest. These particular constructive pieces are known technically as flanges, and a flange is fixed at either side of a beam which requires to be strengthened against flexion. The mass lying between the two flanges is called the web, and the whole berm so constructed is termed a girder. Fig. $177^{1}$ gives a diagrammatic representation of such a girder in cross-section. The web may be composed of much softer material than the flanges; it may consist of a lattice- or merely of a frame-work. Where these girders are developed in plants, the web consists of vascular bundles or of parenchymatous cells, while the flanges are always built up of mechinicnl tissue. In flat, extended foliage-leaves the girders are fitted in so that their flunges are parallel to the upper and lower surfaces of the leaf, but these leaves only resist bending in one plane. This construction, which can be seen in the leaf-sections given in figs. $86^{1}$ and $87^{3}$ (pp. 342-343), would be ill-adapted to stems. An erect stem which is struck by the wind, sometimes from one side and sometimes from another, must be strengthened indifferently on every side, and in accordance 
with this demand the most different kinds of combinations of girders are seen developed in it. Usually several, at least two, but often very many girders are so combined that they traverse the axis in common, as shown in the diagrammatic cross-sections in figs. $177^{2,3,4}$. In this case all the flanges are on the periphery of the stem, and every pair-diametrically opposite one anothermust be regarded as belonging to the same girder. In many stems all the flanges have a parallel course; in other cases they are bent in and out, and so connected together as to form a lattice-work of the most complicated kind. In other cases
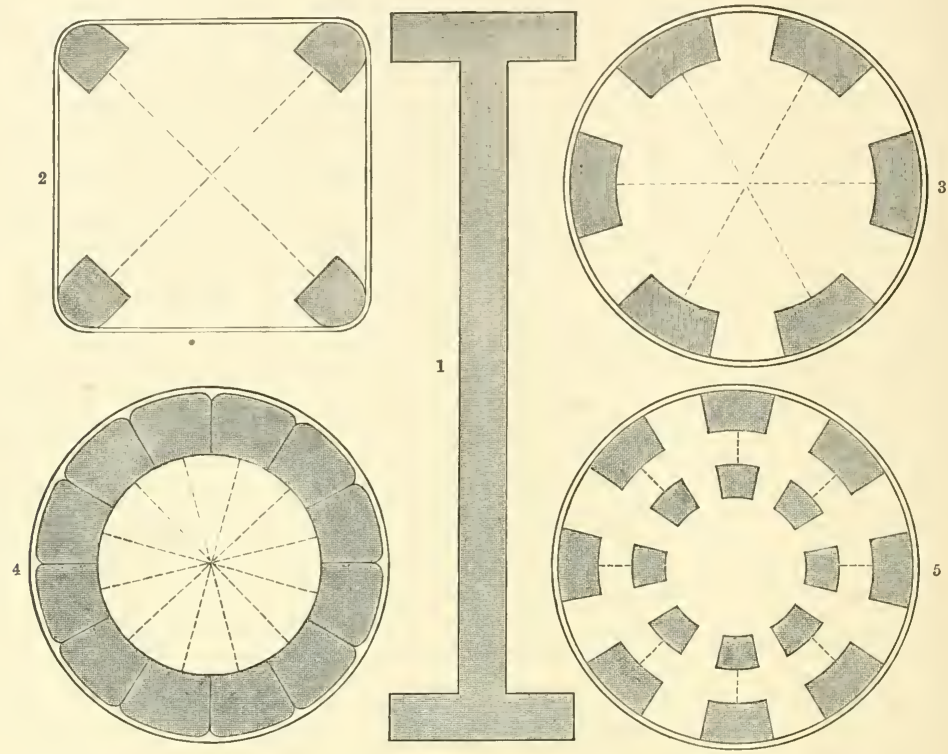

Fig. 177.-Diagrammatic representation of various combined girders.

1 A simple I (or double T) girder. 2 Two combined girders, arranged crosswise. 8 Three combined girders. 4 Six combined girders; the flanges are laterally in contact to form a cylindrical tube. ${ }^{5}$ Four combined girders; their flanges are formed of secondary girders. In Figs, $2-4$ the web of the girders is indicated by dotted lines.

all the flanges lying near the periphery of the stem are fused together (fig. $177^{4}$ ) so as to form a cylindrical tube, in which case the web is not required and the stem is either hollow inside, or is filled only with a loose pith. Sometimes each separate flange is itself transformed into a girder, and in this way the flanges of the chief girder become secondary girders, as represented in fig. $177^{5}$. There is almost as great a variety in this matter as there is in the arrangement of the strands of leaves, but since researches into the course and grouping of the strands of mechanical tissue in stems are still not far enough advanced for us to be able to place the various forms in well-arranged series, we must content ourselves with sketching the most noticeable cases. 
First we will give a general idea of the distribution of mechanical tissue, in as far as it enables erect stems to resist bending. We can distinguish three grouns of forms in this respect. The first group includes forms with simple girders whose flanges of hard bast are placed as near the periphery as possible, but are not fused together into a cylindrical tube. The line connecting every pair of flanges passes through the axis of the stem. To this group belong almost all young stems of woody plants, e.g. those of willows, oaks, elms, maples, and limes (cf. fig. $178^{1}$ ). Special emphasis must be laid on the words "young stems", since in the older stems of these trees-when the wood has become thickened-the hard bast on the outer side of the cambium-ring, and therefore outside the vascular bundle, has finisher its task, and its functions are transferred to the wood, more especially to the woody fibres (libriform cells) (cf. p. 726).

In the erect stems of undershrubs belonging to this group the simple girders are
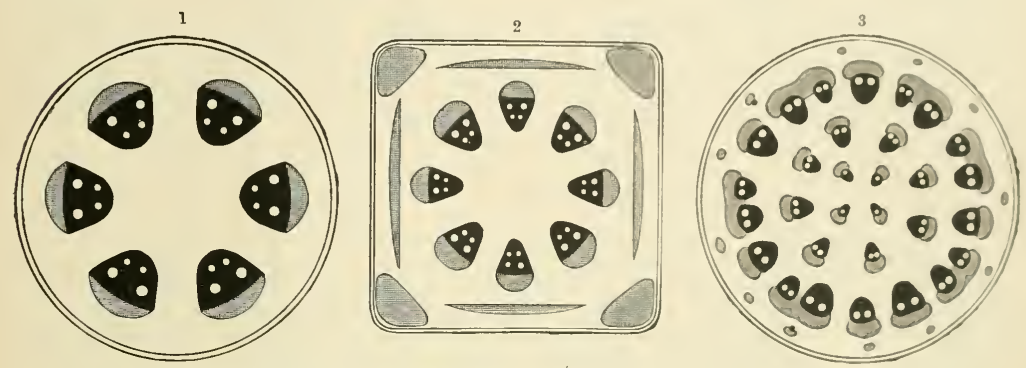

Fig. 178. -Transverse sections of erect foliage-stems with simple girders not fused together into a tube.

1 One-year-old branch of the Broad-leaved Lime (Tilia grandifolia). 2 White Dead-nettle (Lamium album). 3 Date Palm (Phonix dactylifera). In these diagrammatic figures the mechanical tissue is grey and the vascular bundles black with white spots.

very often assisted by collenchymatous strands which lie close to the periphery of the stem, and are arranged so that each strand appears to strengthen the bundle of hard bast forming a flange. Fig. $178^{2}$ shows a transverse section of a stem of an undershrub belonging to this group, the White Dead-nettle (Lamium album), in which the further peculiarity is noticeable, that the strengthening, collenchymatous strands in the corners of the four-sided stem are thick and pillar-like, while those at the sides of the stem are broad and flattened. This condition is not an uncommon one. In palms, of which the diagrammatic cross section of the Date Palm (Phœnix dactylifera, fig. $178^{3}$ ) may serve as a type, the strands accessory to the simple girders are in the form of numerous bundles of hard bast developed on the periphery of the stem, but not exactly in front of the flanges of the girders. These bundles of hard bast are always in pairs opposite one another, and may be regarded as the flanges of special girders. In these cases the number of girlers is always very large, and the flanges appear in two, three, or even more circles in a cross section of the stem. Sometimes also two or three adjoining flanges are fuser 
laterally with one another, and form what may be regarded as a link connecting this with the following group.

The second group comprises all stems in which the flanges of numerous simple girders are fused laterally so as to form a cylindrical tube. This tube lies as near to the periphery as possible, and consists of hard bast developed from the bast portions of the originally distinct vascular bundles. In consequence of this the vascular bundle is always in connection with the hard bast tube. The various methods of connection, and the presence or absence of accessories to this bast tube
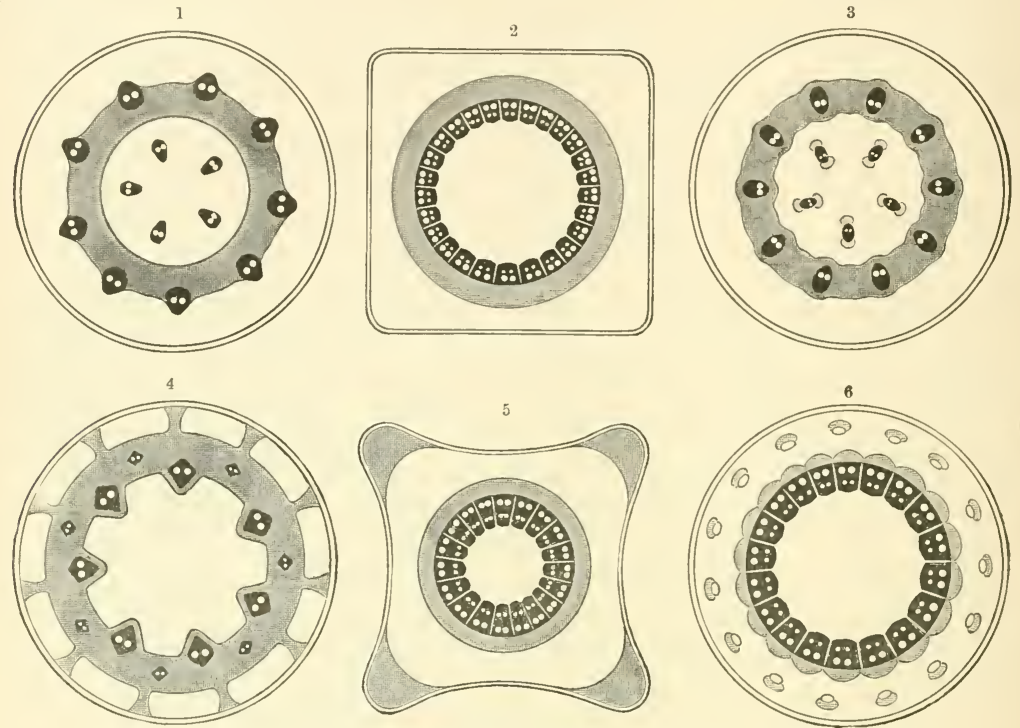

Fig. 179.-Transverse sections of erect foliage-stems with simple girders fused into cylindrical tubes.

1 Crow Garlic (Allium vineale). 2 Carnation (Dianthus Caryophyllus). 3 Polygonatum verticillatum. 4 Purple Molinia (Molinia cerulea. ${ }^{5}$ Woodruff (Asperula odorata). ${ }^{6}$ Sumbul (Euryangium Sumbul). In these diagrams the mechanical tissue is represented grey, and the vascular bundles black with white spots.

in its resistance to flexion, give rise in this group to a great multiplicity of structure. Some of the most interesting forms are represented in fig. 179. In fig. $179^{2}$, the cross section of the stem of a Carnation (Dianthus Caryophyllus), the vascular bundles are situated on the inner side of the bast ring; in fig. $179^{1}$, a transverse section of the stem of a species of garlic (Allium vineale), the bundles are partially embedded in the outer part of the bast ring, and in $179^{3}$, a transverse section of the stem of a species of Solomon's Seal (Polygonatum verticillatum), the bundles are wholly embedded in the ring of bast. The first case is by far the most common, and may be regarded as characteristic of most dicotyledonous herbs and undershrubs; the second case obtains in many bulbous plants; whilst the third, the rarest of all, is only found in a few monocotyledons. The accessory parts occur either as band-like 
projections from the bast tube, e.g. in the grass Molinia corrulea (fig. $179^{4}$ ), or as independent collenchymatous strands in the corners of the angular stem, as in the Woodruff (Asperula odorata, fig. $179^{5}$ ), or, again, a circle of independent bumlles of hard bast appears outside the bast tube, as in the stately umbellifer liuryangium Sumbul (fig. $179^{6}$ ). In this plant the strengthening accessories are combined into independent simple girders and a canal filled with air is situated on the inner sirle of each of the flanges ( $c f$. fig. $\left.179^{6}\right)$.

The third group consists of all stems in which the flanges are developerl as
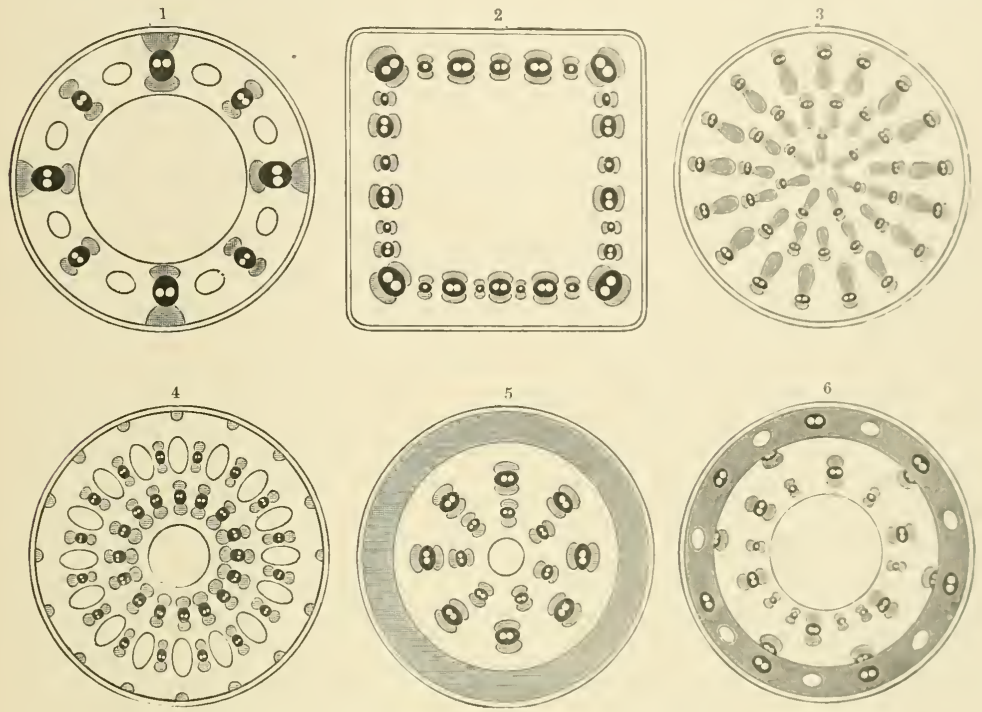

Fig. 180. -Transverse sections of erect foliage-stems with flanges developed as secondary girders.

1 Tufted Scirpus (Scirpus caspitosus). 2 Perfoliate Silphium (Silphium perfoliatum). \& Black-stemmed Bamboo (Bambuma nigra). ${ }^{4}$ Hard Rush (Juncus glaucus). ${ }^{5}$ Common Reed (Phragmites communis). $\quad$ 'Sugar-cane (Saccharum offina rum). In these diagrammatic figures the mechanical tissue is represented grey, and the vascular bundles black with white wrots.

secondary girders. The web in these secondary girders always consists of vascular bundles, and the flanges themselves of hard bast. Sometimes the secondary girders are arranged in a single circle, but in most instances they form several concentric rings. In fig. 180 some of the most striking forms of this group are given diagrammatically. Fig. $180^{1}$ represents a transverse section of a stem of Scirpus cuspitosus, in which the secondary girders - arranged in a single circle-alternate with large air-spaces; fig. $180^{2}$ shows a similar section of the stem of the composite illustrated on p. 239 (Silphium perfoliatum), with its numerous series of secondary girders parallel to the four sides; and fig. $180^{3}$ is the transverse section of a bamloo (Bambusa nigra) in which the secondary girders are grouped in several concentric rings. Here, as in the first and second groups, accessory structures are present, 
usually in the shape of tubes of hard bast, or as collenchymatous strands at the circumference of the stem. In the common Reed (Phragmites communis) this tube is quite uninterrupted and intact (fig. $180^{5}$ ); whilst in the Sugar-cane (Saccharum officinarum, fig. $180^{6}$ ) air-canals and vascular bundles are embedded in it. Much less frequently the strengthening is produced by bundles of bast which lie close under the epidermis of the stem and are not fused into a tube, as, for example, in the Hard Rush (Juncus glaucus), the transverse section of whose stem is shown in fig. $180^{4}$. This rush is also characterized by the insertion of large air-spaces between the accessory strands which form the outer circle. Some of the erect stems here cited which resist bending are hollow within, whilst others are filled with a loose pith. In the diagrammatic figures the central cavity has been marked off by a circular line.

We should naturally expect to find that stems which are not able to rise from the ground without external support (including those numerous forms which are comprehended under lianes), would exhibit a structure different from that of erect stems. In climbing plants the young shoots alone require to resist bending; stems which have found a support can dispense with this property, and consequently with contrivances designed for this purpose. On the other hand, these plants, especially when perennial and lignified, must be protected against strains which are unavoidable in consequence of alterations occurring in their supports. Rocky walls and old battlements overgrown with climbing plants, of course, do not alter sufficiently to materially affect the stems attached to them; but it is otherwise where the climber is supported by a thickening stem. This class of support continues to grow, its stem increases in volume, the extent of the boughs and branches differs from year to year, and displacements and alterations in position occur which cannot but influence the plants climbing over them. Suppose a twining plant has embraced and twined around the stem of a young tree or the branch of a young shrub; years pass by and the stem of the tree has meanwhile increased a hundredfold in diameter, and the entwined branch of the shrub has been shifted about a metre; this cannot be without effect on the twining stem, and it requires no further explanation to see that it will exert a pull and lateral pressure. Perennial twining plants must therefore be so organized that their stem will bear tension and lateral pressure without injury, in other words, that their skin must be constructed to resist tension and compression. Resistance to strain is obtained in twining and interweaving stems in very different ways; in many cases, such as in the Rotang or Climbing Palm, by ample depositions of hard bast in the vascular bundles lying next to the axis of the stem; in other cases, e.g. in Tamus and Dioscorea, by a considerable thickening of the cells of the pith, and in others, again, e.g. in many species of Pepper, by the development of a ring of mechanical cells within the peripheral circle of vascular bundles. It is, of course, an advantage to the twining stem which requires protection against strain if the tissues lying next its centre possess a corresponding firmness. Thus we find unmistakable differences between these and erect stems; correlated with this is the fact that the pith, or the medullary cavity, in twining stems 
is very much reduced, and that hollow twining stems, e.g. that of Thunbergia laurifolia (cf. fig. $128^{1}$, p. 477) are very rare. Perennial twining stems are usually: protected from lateral pressure by a layer of collenchyma surrounding the conducting tissues like a mantle. Sometimes the collenchyma is also conneeted with bundles of bast, and there is no doubt that the same mechanical cells which strengthen the young twining stem protect it later on against lateral pressure.

Perennial climbers which have clambered up growing woody plants are exposed to the same dangers as described in the case of twining and interweaving plants, but in them tendrils as a rule afford a protection against tearing, and tissues providing a resistance to strain are absent from the stems themselves. In such plants it is the tendrils especially which are constructed to resist tension, as, for example, in the Atragene (Atragene alpina, the stem of which is shown in cross section in fig. 181). Tendrils, therefore, are evidently of complex structure. First, they must have a great capacity of resisting strain, but since they also have other functions to perform, and since these functions are different before and after the attachment to the support, very remarkable alterations in their inner structure must occur during development. At first they are required to resist flexion, for which purpose mechanical tissue is developed round the periphery; later on they have to resist tension which renders it necessary that mechanical tissue should be developed nearer the axis. An abundant development of mechanical tissue is also required on the convex side of the tendril bending round the support so as to increase

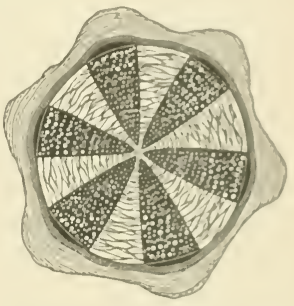

Fig. 181. - Transverse section of the climbing stem of the Atragene (Atragene alpina). The tissues are represented in the following way:-Soft bast, entirely black; wood, larger and smaller whito dots on a black ground; the me. chanical tissues, obllquely shaded: the cork (periderm), stratifled: the loose reticular tissue, whito with dark reticulations. the resistance to strain at that part, as also to prevent its unrolling from the support; such a development is actually to be seen in all tendrils.

Older lignified stems of climbing and twining plants often exhibit a longitudinal splitting in the wood. Before they obtain their split appearance the narrow vascular bundles, which consist for the main part of wood, are isolated by a loose, wide-meshed tissue, and there is no central pith. In transverse section the narrow vascular bundles of such a stem resemble the spokes of a wheel, the weakly-developed mechanical tissue, which had served to protect against bending in the one-year-old stem, together with the cork (periderm), forming to some extent the rim of the wheel (cf. fig. 181).

When lateral pressure is brought to bear on these old stems, the cork and hard bast become ruptured at the places acted on, but only above the dead, largemeshed tissue; above the narrow vascular bundles they remain uninjured. The loose, dead tissue also ruptures and crumbles, and falls out of the grooves between the vascular bundles. These bundles, which now resemble plates or lamellæ of wood, lie above one another like the leav's of a book on the side where the pressure is felt. The wood looks as if it had been diviled or split longi- 
tudinally in this way. These proceedings have no disturbing influence on the functions of the vascular bundles, on the conducting power of the wood, or on that of the soft bast, though by the compression of the woody plates the shape of the cross section of the stem is altered. The lateral pressure exerted on the broad side of the plate-shaped vascular bundle is now harmless, and interrupts the transport of the sap neither in the wood nor in the soft bast.

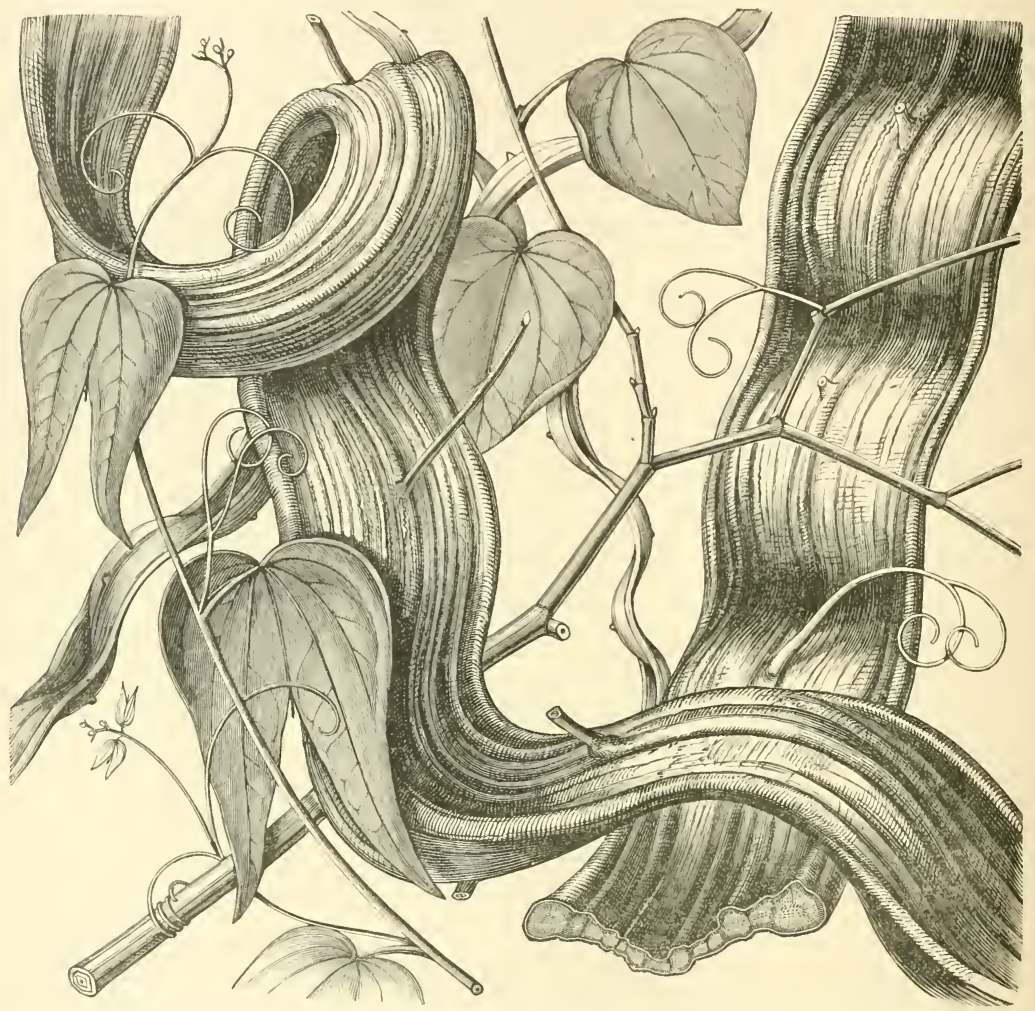

Fig. 182.- Undulations of old ribbon-shaped liane stems (Bauhinia anguina) from an Indian jungle.

It has already been shown on p. 477 in one example (Rhynchosia phaseoloides) that injuries due to lateral pressure in the conducting tissues, especially in the soft bast, are also prevented in twining or climbing plants by the development of ribbon-shaped stems, and it need only be added here that with this flattening and ribbon-like shaping of the wood, and with the development of these wings, there is combined an economy of building materials. If the stem were cylindrical, an abundant mechanical tissue would have to be developed for the protection of the 
soft bast against lateral pressure. The ribbon-shaped stem, however, can do very well without this, for the pressure along its edge is scarcely worth considering, and the soft bast is excellently protected against pressure on the broal side by the wood, which is broken up into a number of detached masses with the soft bast between.

There is no doubt that the spiral torsion of ribbon-like lianes (which is plainly shown in the illustration of Rhynchosia phaseoloides, fig. 127, on p. 475) increases the resistance to strain, a matter of some importance in all cases where growing trees or shrubs serve as supports, and where straining of the lianes clinging to then is unavoidable.

The undulations of ribbon-shaped liane stems in tropical forests may also be regarded as a protection for the sap-conducting tissues against strain. They vccur in many bauhinias and in the peculiar species of $\mathrm{Cau}$ lotretus known as monkeyladders. The central part of the ribbon-shaped stem is alone strongly undulated, as may be seen in the portions of a Bauhinia represented in fig. 182; the two edges are much less curved and are often quite straight, forming a framework for the sinuous middle part. In the case of a longitudinal
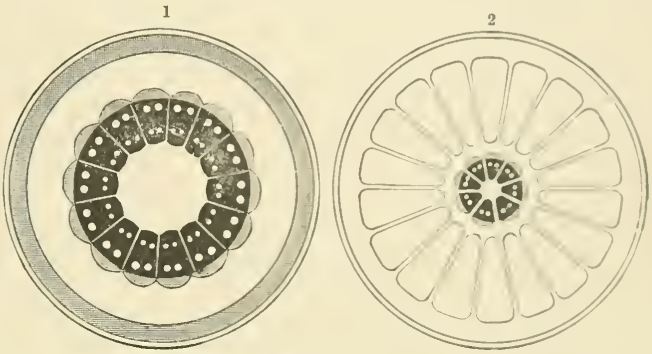

Fig. 183.

1 Transverse section of a runner of the Garden Strawberty (Fragaria grandiflora) which lies on the ground. 2 Transverse section of the stem of the Water Milfoil (Myriophyllum spicatum). In these diagrammatic figures the mechani. cal tissue is represented grey, and the vascular bundles black with whice spots. tension, at first only the

frame is affected, the tissues in the centre can still uninterruptedly conduct the sap to and from the branches which arise from its broad surface.

Stems of water-plants as well as those embedded in the ground, and the stemstructures which lie on the surface of the ground, have, like climbing plants, little need for resisting flexion, but, on the other hand, require a greater resistance to pressure and strain. The soil or the surrounding water forms the immediate support for all these stems, and the arrangement of tissues suited to erect aërial stems would be useless here.

As a matter of fact they do not possess the peripheral strands of hard bast and collenchyma so characteristic of erect stem-structures; the vascular bundles are placed together near the centre of the stem, as is most advantageous for orguns which have to resist strain, and the bast strands belonging to these bumlles are relatively far removed from the circumference of the stem. The central pith is much reduced and is often completely absent ( $c f$. the diagrammatic sections of a runner of the Garden Strawberry, Fragaria grandiflora, and of a hydrophyte, Myriophyllum spicatum, in the above figure). 
The stems here considered are protected against the lateral pressure by a layer of thick-walled parenchyma $\left(183^{1}\right)$, or by the strands of tissue crossing the larger air-canals which run longitudinally outside the circle of vascular bundles in the stem $\left(183^{2}\right)$. In the underground stems of the Grass of Parnassus (Parnassia palustris), and of several other herbaceous plants, there is no pith, they exhibit a central strand of compressed vascular bundles and their structure is very similar to that of roots growing in the ground.

From this general account it is sufficiently evident that the arrangement of the tissues in stems does not so much depend upon whether the part in question belongs to a scaly stem, a foliage stem, or a floral stem, but rather upon its relations with the outer world, and in particular upon the influences exercised by the surroundings serving as a support or substratum. The stem, as the bearer of the foliage and flowers, must be so constructed that the organs named may be raised into the air, sunned, exposed to the wind and to the visits of flying insects and birds, and retained in the most advantageous posture in spite of all opposing influences of the environment. In such a stem are comprehended the various organs of food-conduction, the conducting capacity of which must not be impaired by pressure, flexion, or strain. All the functions of the stem are influenced and governed in a variety of ways by the varying circumstances of the habitat, and by the forms of foliage and flowers peculiar to each species. These functions are wonderfully correlated, and the different arrangement of the tissues in the stem in each individual case is nothing but the expression of the relation of the form to the conditions under which the plant lives.

\section{THE FLORAL STEM.}

The portion of the stem from which floral leaves proceed is called the floral stem (thalamus). It has the form of an axis, from the upper part of which project the carpels and stamens, and below these the perianth leaves. The floral stem, like every other, is built up of internodes whose number corresponds to the number of leaves on its circumference, standing vertically above one another; but since the vertical intervals are usually very small, the articulation of the stem is but seldom plainly visible to the naked eye. Below the perianth leaves only the floral stem appears more or less extended, and this portion is distinguished as the "flowerstalk" from the part which bears the perianth leaves, which is termed the "floral receptacle".

The flower-stalk (pedunculus) originates only in a few Rafflesiaceæ immediately from the tissue which represents the scaly stem. It is also of comparatively rare occurrence (restricted to a few annuals) that the stem proceeding from the bud of the hypocotyl (i.e. the main axis of the whole plant) passes directly into the flower-stalk and terminates in a floral receptacle. The flower-stalk often springs as a lateral shoot from the main axis of the plant, and generally it proceeds as a lateral axis from a stem structure which is itself only a lateral axis of the main 
stem. The flower-stalk may originate from all three regions of the stem. In many parasites and saprophytes without chlorophyll it arises from the axil of a scale-leaf; arvensis and Veronica hederifolia), it springs from the axil of a green foliagre-leaf more frequently, however, it is developed in the axil of a so-called bract, which is to be regarded as a floral-leaf.

The flowers are seldom isolated; in most instances they are associated in clusters, each cluster being termed an inflorescence (inflorescentia). For deseriptive purposes it was found necessary to apply short names to the different inflorescences, and a special terminology was ereated by the older botanists which was most excellent, but which in modern times has become very cumbrous owing to the introduction and substitution of a host of Greek names which sound very learned, but are quite superfluous. It does not lie within the scope of this book to follow this terminology in detail. It is enough to bring forward the most prominent forms of inflorescence. I shall also touch as shortly as possible on the significance of these various associations and groups of flowers to the life of the plant, since this subject will be fully discussed in the second volume when describing the processes of fertilization, and especially the crossing of neighbouring flowers.

In describing inflorescences we shall frequently make use of the words "m min axis" and "lateral axis", and in order to prevent misapprehension, it is as well to point out here that the main axis of the inflorescence, i.e. that part of the stem from which the flower-stalks branch off, is only in rare cases the direet continuation of the stem which proceeds from the bud of the hypocotyl (i.e. the real main axis of the whole plant). Even in the Hyacinth the green scape which rises from the ground, and branches off into a wealth of flower-stalks in its upper part, is not the original main axis, but a side axis springing from the axil of a bulbscale. We are accustomed, however, to call that stem the main one which takes the lead in a certain region of the plant, forming buds which become lateral shoots in the axils of its leaves. The term "main axis" is therefore only relative; with respect to its lateral shoots it is a main axis, but with regard to the stem from which it originates, it itself must be looked upon as a lateral axis. In order to simplify the account and to shorten the descriptions of inflorescences, it is better to call the main axis-round which the individual flower-stalks are grouped as round a common centre, or which has conspicuously taken the lead in the whole system of axes-the " rachis".

Inflorescences have been classified into two groups, the centrifugal and centripetal. In centrifugal inflorescences the rachis terminates with a flower, but is retarded in growth and is outstripped by two, more rarely by three, lateral axes springing from the rachis below the first-formed flower-bud just mentioned. Secondary lateral axes may again spring from each of these lateral shoots, and their relative main axes may be again overtopped in the manner described. The flower-bud by which the rachis is terminated always opens first; then the flowerbuds on the first series of lateral axes, then those on the second series of lateral vol. I. 
axes, and so on throughout the entire series. The unfolding of the flower-buds therefore proceeds always from the centre towards the circumference of the inflorescence in accordance with the succession of age, and consequently such an inflorescence may be termed centrifugal. The simplest form, the type of all centrifugal inflorescences, is the simple cyme (cyma). This presents only three flower-stalks, a central older one (the rachis) and two younger lateral ones. Since the latter spring at the same level from the rachis, the simple cyme has the appearance of a three-pronged fork. It often happens that the flower-bud on the rachis becomes stunted or does not develop at all, and then the inflorescence looks like a two-pronged fork (e.g. in many species of Lonicera). If the lateral axes arising from the rachis serve as starting-points for secondary lateral axes, and if the arrangement just described is repeated in them, a compound cyme (cyma composita) results. The flower-stalks may be arranged either as two prongs or three prongs in the compound cyme, and this branching may be repeated almost indefinitely, as is the case, for example, in Gypsophila paniculata. When one of the opposite flower-stalks, or lateral axes of a cyme, does not develop, while the other, on the contrary, becomes very vigorous and projects beyond the rachis, this lateral looks like the main axis, and at first sight the rachis is mistaken for a lateral shoot. Similarly on this vigorous lateral axis, one of the secondary lateral shoots does not develop, while the other continues to grow the more strongly. If this happens continuously, the form of cymose inflorescence called scorpioid (cincinnus) is formed, numerous modifications of which have been distinguished. If the flower-stalks of a compound cyme are all plainly visible and the whole inflorescence bulky and diffuse, it is termed a panicle (panicula); if the flower-stalks are much shortened and the flowers consequently crowded thickly together, the inflorescence is called a fascicle (fasciculus). Caryophyllaceæ, Labiateæ, and Boragineæ exhibit an almost inexhaustible variety of cymose inflorescences.

Centripetal inflorescences may be recognized by the fact that the rachis terminates in a bud which is the youngest structure of the whole inflorescence, the flower-stalks which spring from the base of the rachis being the oldest lateral axes. Looking down from above on such an inflorescence, or observing the points of insertion of the individual flower-stalks in horizontal projection, the lowest, and at the same time the oldest flower-stalks, are seen to stand at the periphery, the youngest at the centre of the inflorescence. The flowers on the oldest flower-stalks unfold first, those of the youngest last; the blossoming therefore proceeds in a centripetal direction. The rachis is terminated as a rule by a stunted bud which does not complete its development; occasionally, however, this bud does develop; it assumes the form of a foliage-bud from which later on is formed a leafy shoot, as can be seen especially in several Australian Myrtales from the section of the Leptospermex (Callistemon, Metrosideros, Melaleuca), and also in many Bromeliaceæ (e.g. the Pine-apple, Ananassa sativa). Among centripetal inflorescences may be distinguished the raceme (raccmus) with elongated 
rachis and evident flower-stalks; the spike (spica) with elongated rachis and extremely reduced flower-stalks; the umbel (umbella) with an extrennely ruducud rachis and elongated flower-stalks; and the capitulum (cupitulum) with a very short thick rachis and exceedingly reduced flower-stalks. All these inflorescences are connected together by intermediate forms, of which the corymb (corymbus) especially characteristic of Cruciferæ-forming a link between the umbel and the raceme, deserves special mention. The capitulum exhibits the greatest variety, but this is produced less by the different forms of the floral stem than by the shape of the floral leaves, especially of the numerous crowded bracts which collectively surround the flowers as a cup-like envelope. $\Lambda$ form of spike with thickened rachis, called a spadix (spadix), is also worthy of note, and also the spike known by the name of catkin (amentum), the flowers of which are devoid of perianth-leaves, and spring from the axils of scale-like bracts. The whole catkin falls off after flowering, or after the ripening of the fruit, a separation of the tissue and a detachment of the cells having previously occurred at the base of the rachis.

When spikes are themselves arranged in a spicate manner, the whole inflorescence is called a compound spike (spica composita); racemes grouped into larger racemes form a compound raceme (racemus compositus); and umbels when arranged in larger umbels form a compound umbel (umbella compositu). The first two occur very often in grasses, the last in umbelliferous plants. The term panicle is also often applied-rather loosely-to any compound raceme.

Various combinations of the above simple inflorescences have been distinguished, particularly combinations of centripetal with centrifugal inflorescences. Capitula and compound umbels which are arrangeu in cymes, and cymes which succeed one another in a spicate or racemose manner are of very common occurrence. In these inflorescences the order of blossoming becomes altered. Of the many umbels which are grouped together in an extensive cyme, the central umbel is the first of the series, but it is the flowers on its periphery and not the central flowers which open first. If cymes are arranged like a spike, the lowest, i.e. those on the periphery of the whole inflorescence blossom first, though in each individual cyme the central flowers are always the first to open.

The order of blossoming, which is determinate for the flowers of every given species, is related to the transmission of the flower-dust or pollen to the stigma, and therefore with the processes of fertilization. When in one and the sume llower the organs in which the pollen and those in which the ovules are developed stand closely side by side, it might be thought that the pollen would be certain to reach the adjoining stigma. But this opinion is not confirmed by experience. It has been demonstrated, on the other hand, that it is of advantage to the plant that the pollen of one flower should reach the stigma of another, indeed of the flower of quite another plant often some distance away; thus we find that cross-fertilization is aimed at, at any rate at the commencement of the flowering periorl. I purposely say "aimed at", and avoid saying that crossing of diffirent plants 
always takes place, because very often the crossing is prevented from some cause or another. The event of failure is also actually provided for; in case the crossing of different plants does not succeed, care is taken that in the second stage of flowering the pollen should reach the stigmas of the neighbouring flowers of the same plant. In most plants only when this plan also fails, and at the last moment, so to speak, does the pollen developed in the stamens of a flower reach the stigma of the same flower which hitherto has remained intact although placed in the closest proximity. The wonderful and extremely complicated contrivances which are met with for the attainment of this threefold aim will be considered fully in the second volume; but they must here be mentioned cursorily, because the peculiar grouping and the remarkable order of the opening of the flowers represent contrivances which render possible the crossing of neighbouring flowers, and because the shape of the inflorescence can only be comprehended in connection with these contrivances.

In thousands of different species it can be seen that in the event of failure of crossing between flowers of different plants, a cross-fertilization between neighbouring flowers is brought about by elongations, shortenings, depressions, and various other alterations of position, sometimes of the style, sometimes of the stamens, of the floral receptacle, or of the flower-stalks. In the racemose inflorescences of Eremurus (a liliaceous plant) the long styles of the lower flowers, which are directed towards the rachis, bend upwards, towards the end of the flowering period, in order to obtain pollen from the younger flowers above; and the same thing occurs in the floral fascicles of a Woodruff (Asperula taurina), in which the styles bend down laterally to the neighbouring flowers in order to come into contact with their pollen-laden anthers. The stamens of the Wayfaring Tree (Viburnum lantana) curve down towards the neighbouring flowers so that the pollen, falling from their anthers, must alight on the stigmas of these neighbouring flowers. The same thing happens in Hacquetia, Chorophyllum hirsutum, Siler trilobum, and various other umbelliferous plants. In these we find that the stamens of the flowers in the centre of the umbel stretch out so far that their pollen-laden anthers are situated above the stigmas of the neighbouring older flowers, already deprived of stamens, at the periphery of the umbel. In Anthriscus sylvestris the younger umbels are placed above the older so that the pollen falling from the former must necessarily reach the latter standing below them.

In numerous composites, especially in asters and Golden-rod (Aster and Solidago), as well as in species of Cacalia, Senecio, and Arnica, the tubular florets are so arranged in the centre of the capitulum that the pollen expelled from the younger, inner flowers necessarily falls on the stigmas of the adjacent, outer flowers without the aid of any special elongation or curvature. In those composites, on the other hand, of which the Chamomile (Matricaria chamomilla) may be taken as a type, the stigmas of the older, peripheral flowers are brought under the pollen falling from the inner, younger flowers by an elongation of the arched or conical rachis, and by the slight raising or displacement of the flowers of the capitulum so pro- 
duced. Very many composites with ligulate florets, e.g. the species of salsify and Hawkweed (Tragopogon and Hieracium), periodically open anil close their cupitula, i.e. the ligulate portions of their flowers curve for a time outwards, so that the uprer side is turned towards the sky; they then again become erect, curve inwaris, and at length close tightly together. In this closing of the capitulum the stigmas of the peripheral flowers become pressed against the pollen of the central ones, and in this way a crossing is necessarily brought about between neighbouring tlorets. All these crossings, however, could not occur if the flowers of a plant were developed at great distances from each other and all unfolded at the same time, and there is no doubt that the formation of capitula, umbels, close racemes, spikes, and cymrs, ranks as an important contrivance for accomplishing the cross-fertilization of the flowers.

Another advantage obtained by the close grouping of the flowers consists in the fact that certain portions of one flower serve as temporary resting-places for the pollen falling from an adjoining flower, which at the moment of dehiscence is not yet ready for dispersion in the air. In order to clearly explain this contrivance, which may be observed in catkins, I will take the case of the flowers of the Walnut (Juglans regia) figured on the next page. As long as the male inflorescence is immature, the flowers are crowded together in a short, thick spike, the free end of the rachis being directed upwards. Simultaneously with the development of the pollen in the anthers, however, very remarkable changes are brought about in a short time in the whole inflorescence. Within a few days the rachis elongates to three or four times its former length, and becomes limp and pendent; the llowers are in this way somewhat separated and brought into an inverted position, so that now the open side of each flower is directed downwards, and the lower side upwards. When the wind is still, the anthers, hanging on thin short filaments, open, and the pollen rolls out of them as a powdery mass. It does not fall directly into the air, but, first of all, drops on to the under side of a neighbouring flower which previously, in the erect spike, stood above the anthers in question, but now that the spikes have become pendent, is situated below them. This under side is plainly excavated as a depression, and the pollen of the flower above is deposited in it for a time, as shown in the illustration over page (fig. $184^{2}$ ). The pollen has to reach the stigmas of flowers developed a long distance from the catkins, often on other branches up above. It would be highly disadvantageous, if, after the dehiscence of the anthers, the pollen should fall immediately to the ground; it would then be lost and wasted, and neither favourable winds nor lightly hovering insects would be able to carry it from the earth to the stigmatic flowers on the branches of the tree. But in the depressions on the under sides of the flowers, as if in a waiting-room, it occupies the most favourable position conceivable. While there is no wind, the tassel-like spikus are undisturbed, and the pollen remains quietly in its temporary resting-places, but as soon as a gust of wind comes, the spikes oscillate, swinging to and fro like pendulums, and the pollen, emptied and blown out of the pit-like cavities, is carried to the neighbouring branches and whirled round the tree-crown on to the stigmus, in 
the form of small clouds of dust. In this instance the pollen is not only prevented from being wasted by the spikate arrangement of the flowers, but this further advantage is obtained, that each flower shelters the pollen of the neighbouring flower in a safe harbour until it can be transmitted by a favourable wind to its desired goal.

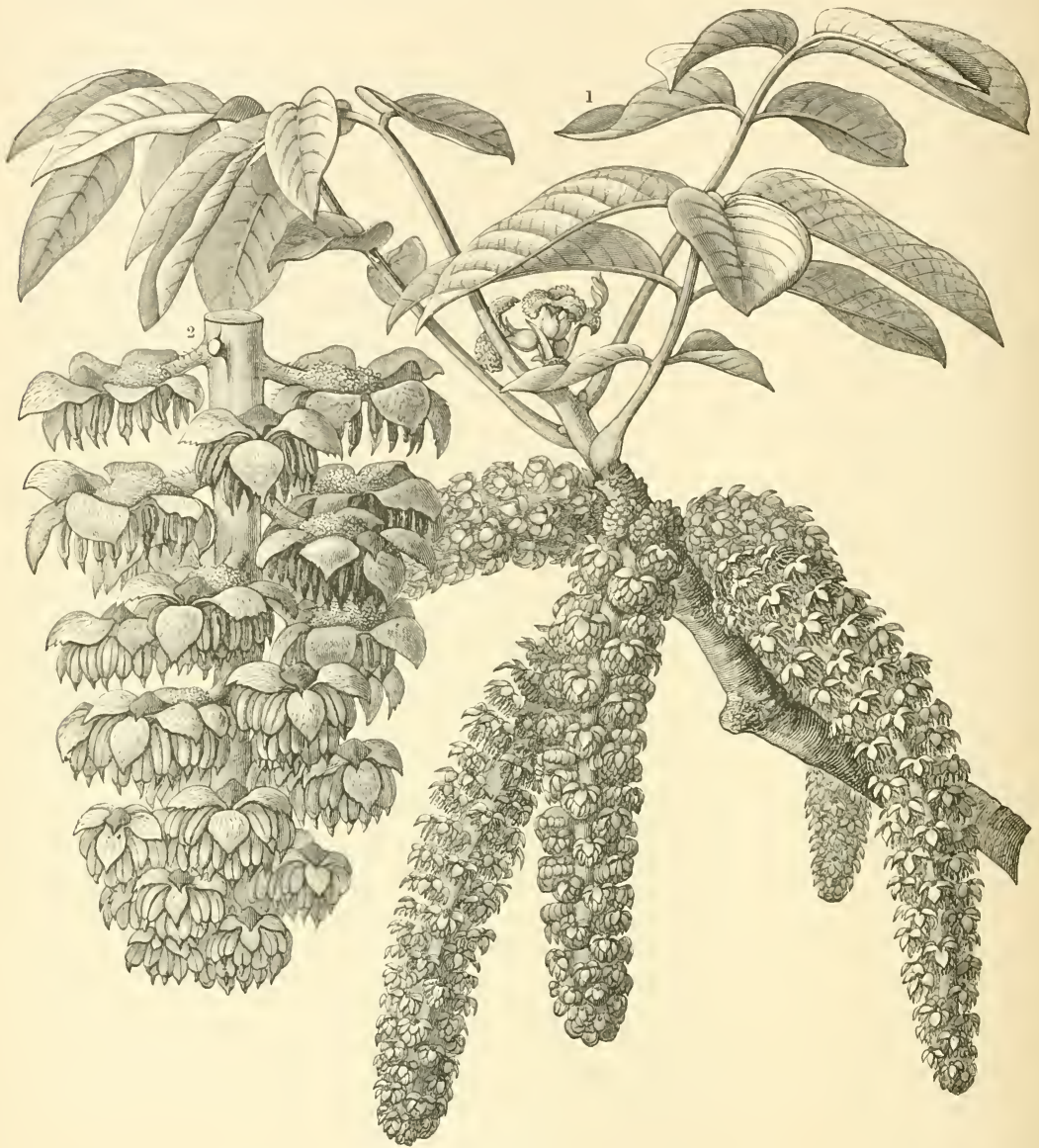

Fig. 184 .

1 Branch of the Walnut-tree (Juglans regia) with hanging male catkins, and a small cluster of female flowers; natural size 2 The tip of a male catkin; enlarged.

The grouping together of the flowers also offers numerous advantages with regard to flower-visiting insects. Flies, bees, and humble-bees do not content themselves when seeking honey with taking it from single flowers, but climb from one flower to another, from below up to the highest points of the spikes and racemes, 
or walk from one fascicle and umbel to a neighbouring one as if over a flower-strewn surface, thus moving the pollen from place to place and effecting innumerable cross ings which would not take place so easily if the flowers were isolated and not collected into inflorescences with a definite order of blossoming. The likelihood of a crossing between different flowers is of course increased with their greater number, and consequently plants with grouped inflorescences have so far an advantage over those whose flowers unfold singly at greater distances. Isolated flowers, it is true, possess large, brilliantly-coloured perianth-leaves which serve to allure honeyseeking animals on the wing; but, on the other hand, the same effect is produced by the accumulation of many small flowers, and an attraction is also afforded by the development of so-called ray florets on capitula and umbels, as well as by the brightly-coloured bracts forming a tuft on the top of cymose and spiked inflorescences, which is no less effective than the largest corolla. This explains why 90 per cent of plants visited by winged insects bear inflorescences and not isolated flowers. Large isolated flowers only serve the purpose of larger honey-seeking animals, of such butterflies and moths, humming and other honey-seeking birds, which would not be able to obtain the honey from small, conglomerated flowers. But it is a wellascertained fact îhat the number of small flies, bees, wasps, and humble-bees which visit flowers greatly exceeds that of larger animals, and this explains why clusters of small flowers occur much more frequently than large single flowers.

Remarkable correlations with the animal world also exist in other regions of the plant, but in no other part of the stem do they appear so striking and so manifold as in the floral region. Nowhere else can the harmonious co-operation of the members, the practical division of labour, and the mutual aid for the attainment of an end, be seen so plainly and convincingly as in the inflorescence. In many capitula and umbels one portion of the flowers forms the pollen; another develops the ovules; a third allures insects; and a fourth prevents the depredations of unwelcome visitors. Most remarkable of all, this practical division of labour within a single inflorescence does not terminate even with the fading of the flowers, but is still continued in the same parts during its subsequent passage into a fruiting state. Many processes give us the impression that the flowers collected in a raceme, umbel, or cyme mutually understand one another; thus, for example, in the Crucifera it often happens that older flowers, whose stigmas have already withered, and which have also entirely lost their pollen, allure insects to the adjoining younger flowers, since now, instead of falling, the petals enlarge and adorn themselves with conspicuous colours, visible at a distance. It also frequently happens that older flowers, whose time is over, vacate the most advantageous position for blossoming in favour of neighbouring younger flowers. When the flower of a nasturtium (Tropoolum) fades, its flower-stalk bends downwards, contracts in a spiral, and hides under the green peltate foliage-leaves, while a new bud pushes into the place where the older flower formerly stood; this bud opens next day and awaits insect visits, and hasty observers might think that the same flower had remained there for more than a week. The same thing occurs in Linaria cymbalaria, Leclum 
palustre, and numerous species of clover. In the Alsike Clover (Trifolium hybridum) growing abundantly in marshy meadows, the older flowers not only sink down in order to give the place best adapted to insect visits to the younger ones, but their corollas turn a beautiful red colour, contrasting vividly with the white of the younger flowers. The contrast is visible at a great distance and serves to attract insects. In the curled inflorescences of the Comfrey, Forget-me-not, and Viper's Bugloss (Symphytum, Myosotis, Echium), and many other Boragineæ, the inflorescence may be seen to unfold and fix itself, so that the flowers in turn are placed in the position in which they are best seen by and most accessible to flying insects; meanwhile the older flowers, whose time is over, and to which insect-visits are of no further use, move out of the way of those which have just opened, and always choose their position so as not to obstruct the entrance to the new flowers of the same inflorescence. In this process not only the flower-stalk but the rachia of the whole inflorescence takes part, and it is interesting to observe how even widely distant parts of the stem are sympathetically affected, so to speak, and how all the different parts of the system of axes are extended, raised, depressed, and curved exactly as required for the purpose of affording the most favourable position to each flower in turn.

The most remarkable thing of all, however, is that under certain conditions, which only occur exceptionally, the most favourable position for the flowers is striven after and obtained by means of curvatures produced in the stem in places where, in the ordinary course of things, such changes would not have occurred. When the Wood Forget-me-not, Larkspur, Monkshood, Adenostyles, the Willow Herb (Myostis silvatica, Delphinium elatum, A conitum variegatum, Adenostyles alpina, Epilobium angustifolium), and numerous other undershrubs whose stiff, erect stems are terminated by a group of brilliantly-coloured flowers adapted to insect-visits, are pressed down and extended on the ground shortly before the unfolding of the flowers by some unusual occurrence, so that the normally erect inflorescence lies on the soil, the stem will be seen to form a bend below the inflorescence, as it is no longer capable of raising up the whole length, and the portion bearing the flowers will be elevated until it again becomes erect, and its flowers are again placed in a position favourable to insect-visits. This curvature is no ordinary phenomenon of growth, for the portion of the stem forming the bend has already ceased growing, and the curvature does not extend to the rachis of the inflorescence, but takes place below it, being strictly localized there; the rachis itself, which is raised up, always remaining straight. Finally, no kind of stimulus can be shown to affect the internodes in which the bend is formed. Contact with the soil and illumination from above act just in the same way on it as on the internodes above and below it. No external causes whatever can be assigned to this knee-shaped bending, and only this much is certain-the bending could not take place at a spot better suited to the purpose of restoring the flowers once more to a favourable position.

The flowers of more than an eighth part of all flowering plants are grouped 
together into capitula, this inflorescence being the commonest of all. Next to it comes the cyme with its numerous modifications, and then the umbel, the raceme, and the spike. Of all plants, perennial undershrubs exhibit the most extensive inflorescences, in comparison with the size of the whole plant. Many of them only send up an axis every year which bears a few large foliage-leaves at the base, is beset with scale-like bracts further up, and terminates in numerous umbels, racemes, or cymes, forming a single gigantic inflorescence. As an example of this form found in the East, especially in the steppes of Persia and Turkestan, may be instanced Euryangium Sumbul. This umbelliferous plant, abundant near Pentschakend, south of Samarkand in Southern Turkestan, develops at the beginning of the vegetative period some five radical foliage-leaves divided into innumerable lobes, and having a musky odour; these leaves only retain their fresh green for a few weeks and then wither and become bleached, turning a pale violet colour comparatively early. As soon as these radical leaves have begun to change colour a leafless, blue-tinted, asparagus-like, slim shoot, 4-5 cm. thick, rises above the ground and branches repeatedly in its upper third into numerous umbels. $A$ whole series of oriental Umbelliferæ behave like this strange Sumbul plant, especially those of the genus Ferula, as also the Scorodosma Asa foetida, yielding the notorious asafcetida, and several Cruciferæ. One of these cruciferous hushes, Crambe cordifolia, develops within a few weeks an inflorescence with long branches, projecting like spars, about 2 metres high and almost as much across. The Agure Americana, known as the Century Plant (illustrated on p. 657), is also similar to these plants. The stem, 5-7 metres high and 6-12 $\mathrm{cm}$. thick, which rises above the rosette of thick fleshy foliage-leaves with spinous margins, is covered only with dry, scale-like leaves, without chlorophyll, and forms the rachis of an inflorescence which is one of the largest in the whole vegetable kingdom.

In contrast to the undershrubs, whose rapidly-growing stems, terminated by large inflorescences, remain herbaceous and wither and die down to the ground without lignifying after the maturing of the fruit and seeds, woody plants, especially trees, produce as a rule only small inflorescences. It is true that their number is correspondingly large. The perianth-leaves are frequently green, and the inconspicuous inflorescences distributed between the foliage-leaves are then entirely invisible at a little distance. Often, however, numerous small but brightlycoloured inflorescences are crowded so close together as to be inseparable; in cases where the flowers unfold before the green foliage, as, for example, in Almond and Cherry trees, each tree from a distance resembles a gigantic bouquet of flowers.

Only a few inflorescences are found in palms, but they are usually very larire and many-flowered. Generally speaking palm inflorescences are the largest of all. Those of the Doum Palm (Hyphone thebaica) and of several species of Phanix are more than a metre, those of Raffia Ruffii and of Plectocomia elongata 2 metres long, and the Talipot Palm (Corypha unbraculifera), illustrated on $\mathrm{p}$. 2s., is celebrated as possessing the largest inflorescence of all plants. This remarkable diccious palm grows comparatively slowly: its caudex often takes 30 - 40 years 
before attaining a height of 20 metres, and during this period flowers never appear. Not until the caudex has attained its full size of 22 metres does the inflorescence spring from its apex, the rachis reaching an additional height of 14 metres. Twelve or thirteen rounded branches are given off from this rachis, the longest of which becomes 6 metres long. All the branches terminate in numerous branchlets and twigs, and are richly covered with flowers. The whole inflorescence when fully grown exhibits the fabulous height of 14 metres, with a breadth of 12 metres. As soon as the flowers open, the fan-like foliage-leaves below begin to fade and often all fall off during the flowering period, so that the shaft alone remains, bearing the inflorescence at its apex. The flowering period lasts for 3-4 weeks. As soon as it is over and the fruits matured, the whole plant dies down, as in Agave Americana. Each of these palms therefore only blossoms once in its life.

With this, the largest inflorescence, may be contrasted that which is regarded as the smallest of all, viz. the capitulum of Nananthea, only 2-3 millimetres in diameter, found growing on the mountains of Corsica.

The size of the inflorescence, and that of the flowers composing it, do not vary proportionately. Extensive inflorescences usually have very small flowers, and vice versa, but a universal rule cannot be laid down in this matter. The inflorescence of Puulownia imperialis has 100 large flowers, and that of Spircea Aruncus, equal in extent, 10,000 small ones. The Talipot Palm is said to bear about 100,000 flowers in its gigantic bouquet. In simple cymes it often happens that the central flower is not developed, and the whole then consists of a pair of flowers, usually curiously united, as can be seen in many species of the genus Honeysuckle (Lonicera Xylosteum, nigra, corulea, alpigena). In many Acanthaceæ, bindweeds, and labiate flowers, on the other hand, it is observed that the two lateral flowers of the three of a simple cyme are suppressed, and that only the central one attains development, in which case the whole inflorescence is represented by a single flower.

The floral receptacle (podium, also torus), i.e. that part of the floral stem from which the perianth leaves spring, is always somewhat thickened in comparison with the flower-stalk, and may be either conical or disc-shaped. The conical receptacle (conopodium) has the form of a cone, being sometimes elongated and peg-shaped, but often short and but slightly curved; it is always narrowed from its base, the thickest part, up to the apex. Unlike the very simply-constructed conical receptacle, the disc-shaped receptacle (discopodium) presents a great variety of form. The apex of the floral axis is retarded in growth, the tissue round it thickens and becomes flattened, or surrounds the apex with a circular cushion or rampart often rising so much above the apex that the whole receptacle has a craterlike or cup-shaped appearance. In the first case, viz. when a circular wall has been formed, it surrounds the pistil, developed in the centre above the apex, without overtopping it, as, for example, in the flowers of Orange and Lemon trees. The stamens and perianth-leaves usually arise outside, less frequently within the ring, and most rarely of all from the edge of the ring itself. When a cup-shaped 
receptacle has been formed, the end of the axis is overtopped by the edge of the cup, and the actual apex of the receptacle must be sought at the bottom of the cup. The stamens and perianth-leaves then spring in most cases from the edges of the cup. In many instances the carpels also arise from the edges and cover over the crater-like depression of the receptacle. More frequently the carpels are developed at the bottom or on the inner walls of the cup, and then either a single carpel is to be seen in the depression, as, for example, in cherry flowers, or several carpels, as, for example, in the rose. Sometimes the pistil developed at the bottom of the cup-shaped receptacle is fused with the inner wall of the cup, as, for example, in the flowers of apple and pear trees.

The disc-shaped receptacle is not, as in the examples selected, always developed symmetrically all round. In flowers which project laterally from an erect rachis, the circular wall is often interrupted, or instead of the circular disc a one-sided projecting ridge or cushion is seen. The ring is often replaced by a circle of protuberances or papillæ, or the receptacle is drawn out on one side, taking the form of a peg, a tongue, or a scale.

Honey is usually secreted from that tissue of the disc-shaped receptacle which does not pass over into perianth-leaves, but which projects and is inserted between the whorls of perianth-leaves, stamens, and carpels in the form of knots, warts, cushions and rings; this serves to attract insects whose visits are of use to the flowers in effecting fertilization. The part of the receptacle which is developed as the under-structure or envelope of the carpels, on the other hand, very often becomes a part of the fruit. In most cases, however, the significance assigned to the various developments of the receptacle in respect to the life and welfare of plants is not yet rendered sufficiently evident. That the relations between receptacle and fruit-formation are of the greatest importance is the only thing that can be affirmed with certainty, but why in one instance this and in another that form of receptacle is produced remains entirely enigmatical. The opinion has been repeatedly stated that all the architectural conditions of plants are not necessarily beneficial, and that the forms in which the individual organs and plant members appear fall into two groups-those whose use to the species in question is obvious, and those in which this is not the case. The former were said to be variable, the latter invariable. This hypothesis was forthwith raised to a dogma, and it was further concluded that only structures whose significance in the life of plants cannot be explained are of use in the limitation and systematic determination of species and groups of species. I cannot justify this notion, and maintain rather that nothing is ever formed in a plant which is not beneficial, which is not even indispensable to it. Those organs which are so often termed "reduced" are not without importance in the life of plants; they are rather developed in this only apparently-reduced form for the welfare of the whole, and cannot be dispensed with without injury. If they were unnecessary, they would also be absent. The plant builds up nothing superfluous, and no hair, no cell even, is developed without some purpose. It is hazardous and unwarranted to say that 
this or that structure is useless, and to be interpreted only as the remnant of an organ which was developed more fully a long time ago in some ancestral species to which it was indispensable. When we cannot immediately see the advantage of any structure, we are not justified in saying that in its particular form it is worthless or indifferent to the plant. The saying dies diem docet is perhaps nowhere more applicable than to questions concerning the significance of forms. How many structures which were enigmatical a century ago are now recognized as essential members of very various contrivances, and explained in all their details; their recognition being regarded as an incontestable scientific thesis! The tendency of our age, indeed, is not merely to regard and describe forms as mute puzzles of nature, but to comprehend their value as parts of a living entity. Therefore I doubt not that sooner or later the importance of the different forms of floral receptacles will find interpretation and explanation in the individual species to which they belong.

A peculiarity which distinguishes the floral receptacle from all other stemstructures, which has to be considered here in conclusion, is its limited growth. As long as the receptacle forms floral-leaves on its periphery it always continues to elongate to some extent, although the increase in length is inconsiderable; but after the production of the highest floral-leaf no further divisions take place in the cells of the apex, and the elongation of the axis is at an end, not temporarily, but permanently. This fact is of importance inasmuch as one of the few differences which have been established between stem and leaf undergoes a material restriction thereby. But the limited growth of the floral receptacle has also a special significance in regard to the architecture of the whole plant. The portion of the stem which forms the floral receptacle separates usually with the flowerstalk, and not infrequently even with the whole rachis of the inflorescence from the floral stem below, as soon as the leaf-structures proceeding from the receptacle have fulfilled their function; or, in other words, the flower and fruit-stalks become detached as soon as the perianth-leaves have withered, the stamens emptied, and the fruits matured-a process which reminds us of the detachment of those foliage-leaves which are no longer able to fulfil their allotted tasks. Just as a scar arises, or a withered stump remains behind where once a leaf existed, so a healing tissue is formed at the place where a portion of the floral stem has separated off, and at this spot no further stem growth takes place. If the shoot terminates with a single flower, or an entire inflorescence, it can no longer elongate in a straight line after the fruit has fallen; its apical growth is terminated for ever. Lateral shoots, on the contrary, may spring from the axils of lower foliage-leaves and may grow up beyond the scarred places, a fact which of course materially influences the type of branching and the architecture of the whole stem. This influence is very noticeable, especially in tall woody shrubs and trees. Where for instance the scarred apex of a branch is overtopped by two lateral branches springing close under the scar, a more or less regularly two-pronged fork results; and when the process is repeated on the prongs of this fork, a very 
ornamental form of branching is produced which may even be recognized on the older boughs, and gives a characteristic habit to the shrub or tree. Although the annual growth in height in woody plants branching in this fashion is only slight, the crown grows in breadth to a striking degree, and the older, leafless boughs have usually the appearance of horns or of an interwoven lattice-work spreading out above, as may be seen in a remarkable manner in the Stag's-horn sumach (Rhus typhina), and also in several species of Horse Chestnut (e.g. Esculus flava and discolor). In the Oleander (Nerium Oleander), and frequently in the Mistletoe (Viscum album, c.f. fig. 46, p. 206), the scarred apex of the main shoot is overtopped by a whorl of three lateral shoots, which produces another characteristic modification of this form of branching.

The internal structure of the floral stem, especially the arrangement of the mechanical tissue, is always adapted to the tasks naturally assigned to the bearer of the flowers and fruits. When the floral portions and the fruits proceeding from them are to be maintained in an erect position, the stalks and also the rachis in question are constructed so as to resist flexion. The stalk and rachis of pendent flowers, and especially of heavy pendent fruits, are, on the other hand, constructed to resist tension; in both cases they are provided with mechanical tissue suitably strengthened and arranged. Frequently the same bast cylinder which afforded the resistance to flexion in the erect flower-stalk at the time of the opening of the flowers has subsequently to provide a resistance to strain, as when a pendent fruit is produced from an erect flower. The converse also happens, and not infrequently erect fruit-stalks, very resistant to bending, which take part in the dispersion of the seeds, are developed from pendent flower-stalks with the capacity of resisting strain. For the rest, in all these alterations of position, the turgescence of the parenchymatous tissue on the periphery of the flower-stalk plays a prominent part.

\section{FORMS OF ROOTS.}

Relation of external and internal structure to function.-Definition of the root.

-Remarkable properties of roots.

\section{RELATION OF EXTERNAL AND INTERNAL STRUCTURE TO FUNCTION}

Every seed is provided by the parent plant with as much starch, fat, sugar, and other materials as are necessary for its further independent development. The germinating seed respires, provides itself with water, dissolves the materials stored up in its cells, augments the number of its cells, and increases in size. The food-substances of the soil at first take little or no part in these processes. But as the seed germinates its aim is to develop organs capable of laying the foodsubstances contained in the soil and air under contribution, and of manufacturing 
fresh building-materials as those with which it was provided by the parent become exhausted. The tissues of the young seedling always contain cells for the absorption of the dissolved food-salts and gases; these enter immediately into a close union with the substratum, whether it consists of inorganic earth, of decaying organic matter, or of a living host-plant.

There are plants in whose seeds no differentiation into various parts, no separation into embryo and food-stores, can be recognized, and in the seeds of many thousand species we cannot even distinguish an embryo with cotyledons, in which case the whole of the group of cells forming the seed must be regarded as embryo. This group of cells first grows up, at the expense of its own materials, into a structure having the form of a small tubercle, which on one side joins with the substratum by absorbent cells, and on the other sends out a shoot, but develops no system of tissues which could be called a root. This occurs, for example, in Monotropa and in the Coral-root (Corallorhiza), described on p. 111, which are usually termed rootless plants. In other examples of this group, in which the undifferentiated embryo grows up directly into a small tubercle or stem, warts, papillæ, pegs and vermiform structures, equipped with absorbent cells, develop on this tubercle, and join with the substratum; these are, therefore, of the nature of roots. These structures always originate in great numbers from the tubercle, i.e. from the enlarged developing embryo; in many orchids living epiphytically on the bark of trees they are formed on the side turned towards the tree; in parasitic Orobanchaceæ around the thickened lower end of the tissue-body ( $c f$. figs. $34^{11}$ and $34^{12}$ on p. 173), and in species of Cuscuta and Cassytha laterally on the thread-like embryo where it has attached itself to a host-plant.

In plants whose seed contains an embryo differentiated into stem and leaf, only a single, wart-like or conical body arises at one end of the hypocotyl, opposite the bud of the epicotyl; it grows at germination into a cylindrical root provided with absorbent cells, and later appears as a straight, downwardly-directed continuation of the hypocotyl.

Neither the abundant roots proceeding from the undifferentiated embryo, nor still less the single root springing from the membered embryo, suffice for the requirements of the shoot arising from it. In proportion as this increases in size, forming one internode above another, developing leaves with buds in their axils which grow out into lateral shoots, the need of water and food-salts becomes greater and greater. Fresh sources must be obtained for these materials, and new conducting mechanisms must be established-in a word, new roots must be formed. When only a single primary root is present in the embryo, the new roots frequently spring from this as lateral branches, and it is customary to say that the primary or main root has become branched, that it has formed lateral roots. Of course each branch can again ramify, and indeed the branching is often repeated beyond measure. The branched root (radix ramosa) is to be seen especially in annual land-plants with erect leafy stems. Almost as often it happens 
that the root proceeding from the embryo perishes as soon as it has emerged from the seed, and that then many new roots originate from the hypocotyl, close to the place from which sprang the dead primary root; or that roots are developed on the lower end of the epicotyl embedded in the ground-in which case they stand closely crowded together in great numbers forming a cluster, and are then known botanically as fascicled roots (radix fasciculata). But many roots also arise further up on the shoot-axis, not only in the region of the scale-leaves, but also, if required, in the foliage portion of procumbent, erect, and climbing stems, and under certain conditions even on the foliage-leaves. These structures which may originate from the stem at all stages of age and height, and even from the leaves, are called adventitious roots (radices adventicice).

When roots are developed on a leafy stem, it is noticed that their places of origin are near the points of insertion of the leaves. In epiphytes, especially in aroids and orchids living on the bark of trees, they are sometimes seen to be so distributed that a single root, a pair of roots, or a whole fascicle of roots, arises at exactly defined places on the stem. Each internode in these plants has its own roots, and is therefore almost independent of neighbouring internodes, so that, supposing one or both the adjoining internodes should die from some cause or other, it can maintain itself independently ( $c f$. fig. 51, p. 224). In stems which lie on the ground, as in runners, the roots always originate only at the nodes, i.e. at the commencement of an internode. In the underground stems known as rhizomes, the roots are distributed in the same way. When the older internodes of these runners and rhizomes die off behind, the next youngest are not injured, for they are already provided with roots of their own, by whose help their requirements of water and food-salts are supplied, and by which they are firmly fixed in the ground. The general symmetry and geometrical distribution of the places of origin, as shown in leaves, is, however, absent in the majority of roots, the arrangement being frequently quite irregular, especially in underground, muchbranched roots where influences operate on them which will be spoken of later.

The functions assigned to the root are: first, the absorption and transport of water and of food-salts dissolved in water, and second, the fixing of the whole plant in the substratum. In most cases this twofold function is performed by the same root, but occasionally a division of labour occurs, so that one portion of the root-system serves only for the absorption of food, and another for the fixing in the substratum. For instance, the repeatedly mentioned Tecoma radicans has two kinds of roots; the first underground, absorbing water and food-salts from the soil, and the second the clinging roots (figured on p. 479), by which the lightavoiding shoots are attached to places from which no fluid nourishment could possibly be absorbed. When one of these shoots is cut across below the place at which it is fixed by roots to a wall or rock-face, the part above the section forthwith dries up, cven although these roots and the substratum are kept continually moistened and damp.

Roots of biennial and perennial plants, in regions where the vegetative activity 
is temporarily interrupted by drought or cold, frequently have a third function to perform, viz. that of storing up starch, fat, sugar, and other reserve foodmaterials. Obviously the parts concealed in the ground are protected in a high degree against aridity and frost in countries with long-continued summer drought or with severe winters, and therefore the underground root-structures principally, together with underground parts of stems and the scale-leaves arising from them, can be used most advantageously as reservoirs for the materials formed in the green organs above ground during the short period of vegetation.

The variety of functions assigned to roots, the diversity of the substrata, and the peculiar conditions of the habitat and climate render necessary a large number of different forms, the most noticeable of which bear special names in botanical terminology, and will be briefly enumerated here. According to the substratum into which the roots penetrate, and from which they derive water and food, we may distinguish between subterranean, aquatic, aërial, and parasitic roots.

Subterranean roots (radices hypogaece) push their ends, which are beset with root-hairs, into the ground with great energy, and are entirely covered over with soil, or at any rate in so far as their absorbent portions are concerned. Roots proceeding from the radicle of the embryo are chiefly subterranean. The roots springing from the different forms of scaly stem are almost all subterranean, and we shall not be far wrong if we estimate the roots of 70 per cent of all existing phanerogams as subterranean.

Aquatic or floating roots (radices natantes) spring laterally from floating stems and are generally arranged in clusters, more rarely singly, and are to a slight extent spirally twisted. They are developed both from stems whose foliage-leaves lie flat on the surface of the water, and also from the floating, leafless stemstructures which have been metamorphosed into phylloclades (e.g. in Lemna polyrrhiza, gibba, minor). In these plants the root-tips are also surrounded by water. If they come to lie on the slimy bottom in consequence of a fall in the waterlevel, they do not penetrate into it, nor do they enter into relations with the particles of mud. Marsh-plants, on the contrary, send their first roots right down into the mud, whilst those developed subsequently from the higher internodes are allowed to float in the water. The primary root produced from the seed of the Water Soldier (Stratiotes aloides) is embedded in mud, and is therefore really a subterranean root; after it has died off the whole plant rises up, remains oscillating below the surface of the water, and develops floating roots from its abbreviated, leafy stem; later the plant again sinks down, and the floating roots again become subterranean ( $c f$. the account on p. 76). Conversely it often happens that subterranean roots become transformed into aquatic roots. In alders, willows, and elms growing on the sides of streams, extensive net-works of roots are often to be seen which have grown out from the slope of the bank and float in the water. Indeed, many terrestrial roots, strangely enough, exhibit a much more luxuriant growth in flowing water than in the ground, and it is well known that the roots of the above-named trees, when they have effected an 
entrance into water-pipes, grow so extensively that in a short time the pipes are entirely blocked up and the water-flow in them interrupted. The net-works of roots taken out of these pipes resemble plexuses of hair, so abundantly are their tresses developed. Hyacinths and many other bulbous plants, and even various foliagetrees, as, for example, maples and horse-chestnuts, whose roots usually grow in the ground, can be reared with the best results if their roots are allowed to grow in water, provided that the water contains the necessary food-salts in adequate amounts.

Aërial roots (radices aërece) are developed on the periphery of the erect caudices of tree-ferns, and in great profusion on the stems of epiphytes, especially of aroids and orchids. In species of the tree-ferns Todea and Diclisonia the aërial roots are all very short but so numerous and crowded together that they form an actual mantle round the caudex. In orchids growing on the bark of old trees the aërial roots also arise in great numbers close together, but are always elongated and filamentous, and form manes, as shown, for example, in Oncidium, figured on p. 221. In other orchids, however, they may occur singly, and are then usually much thicker, fairly stiff, and curved sinuously in and out or spirally twisted as in the Sarcanthus rostratus, illustrated on p. 107. As already stated, in many aroids and orchids they appear arranged with great regularity, either singly or in pairs, opposite the points of insertion of the leaves on the stem. All these aërial roots are excellently adapted by their structure (described on p. 222) not only for the absorption of water and solutions of food-materials, but also for the condensation of aqueous vapour from the surrounding air. In most instances they are enveloped by a papery covering; more rarely they are thickly beset with so-called root-hairs, and then have a velvety appearance. Most of those with root-hairs are rusty brown in colour, whilst the others are white in dry air, and greenish in wet weather -in consequence of the abundant chlorophyll contained in the tissue below their papery envelope.

We must carefully distinguish these condensing aërial roots from such as, whilst springing from epigeal stems and surrounded by air, are unable to condense aqueous vapour or to absorb atmospheric water. These, on the other hand, grow down to the ground and must penetrate into it in order to obtain the water and food-salts they require. These root-structures are especially observed in climbing plants in which the lowest internodes have died, and which then no longer stand in direct connection with the soil. Their large foliage-leaves nevertheless require a much greater amount of water than could be obtained from the tree-trunks on which they support themselves. The large-leaved aroids illustrated on p. 365 , whose cord-like roots, from 4 to 6 metres in length, descend to the ground, may be regarded as typical of this class. These forms are indeed called aërial roots, but if we adhere to the distinction given above, they would be more accurately regarded as a special class of subterranean roots. But since it has been repeatedly observed that the aërial roots of some orchids, when they come in contact with the ground, penetrate into it and assume the character of subterranean roots, the boundary VoI. I 
between subterranean and aërial roots vanishes, and, as in other similar cases, it becomes evident that all these classifications are but artificial.

Parasitic roots (radices parasitica) grow down into the living tissue of the host-plant and absorb from it the materials needed by them, and by the plant to which they belong, for further development. They are sometimes called haustoria. They are either wart-like, disc-shaped, or spherical in outline, or assume the form of sinkers; occasionally they remind one of a hyphal net-work. Sometimes they spring laterally from an epigeal, sometimes from an underground stem. They also frequently proceed as lateral members from underground roots. Their structure and various developments were so fully described on pp. 173-213 that we need only now refer to what was there stated.

Roots, whose especial province it is to maintain a plant in the position it has once assumed, may be distinguished as clinging and as supporting roots. Clinging roots (radices adligantes) really comprise all roots whose absorbent cells are so closely united with the substratum that a displacement can only be brought about by the exertion of considerable force. Even floating roots, inasmuch as they adhere to the water and so give a certain amount of stability to the whole plant, may be regarded as clinging roots. The duckweeds (Lemna minor, polyrrhiza, gibba), whose long, spirally - twisted, fascicled roots grow down into the water, are not easily moved by wind from the position they have taken up. Plants are of course still better fixed in the substratum by subterranean roots which adhere to the solid particles of the soil by means of their root-hairs. By this union of roots and earth-particles we get a compact mass, difficult to break up, and it is well enough known that loose soil may be solidified by plants possessing much-branched, wide-spreading roots, and that certain grasses are made use of to bind shifting sands together. When clinging roots are mentioned in plant descriptions, those in particular are referred to which firmly connect epigeal portions of stems to any support, as, for example, the short, climbing roots of the Ivy, or of Tecoma radicans, the much-branched roots which cover stones and the bark of trees with their net-works, the adherent roots of numerous species of Bignonia and Cereus, and the ribbon-like roots of certain tropical orchids which have fastened to the bark of trees,-especially those of Phalanopsis Schilleriana, described on p. 108; and finally the girdle-like roots of Ficus and Wightia, figured on p. 705.

Supporting roots, as their name implies, have the task of supporting the stems to which they belong. They are always visible above-ground, and assume the form of butcresses when they spring from erect trunks, of pillars when they belong to the projecting lateral branches of a stem. They may be conveniently divided into tabular, stilt-like, and columnar roots. Tabular roots (radices parietiformes) proceed from the lower part of an erect trunk, and have the form of flanges placed on end. They may also be compared to massive planks of wood used for fencing in roads. They radiate out in all directions and give to the approaches to the main trunk the appearance of short precipitous valleys which become gradually narrowed and terminate blindly in an acute angle. The tabular roots frequently resemble narrow 

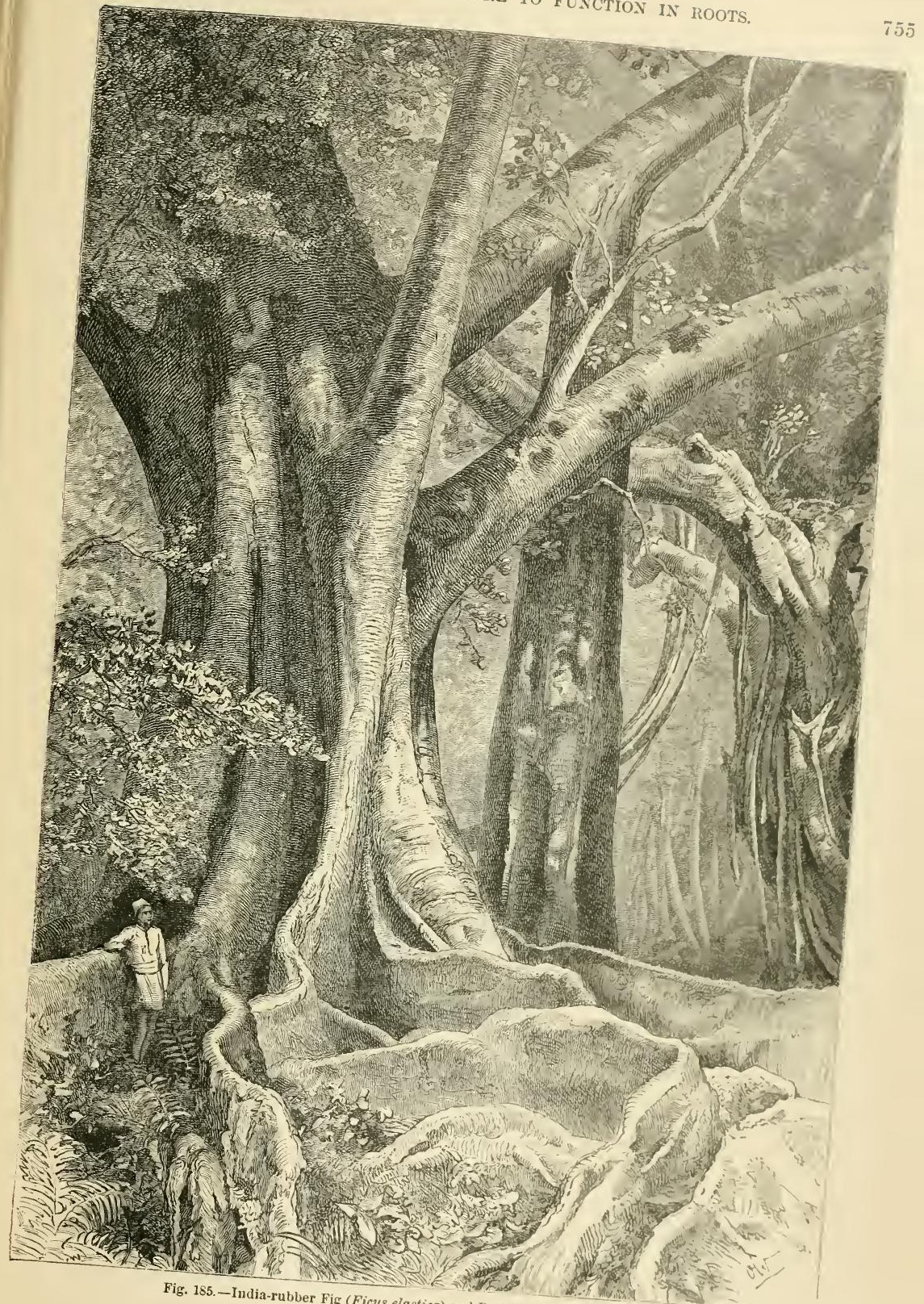
buttresses, with regular radiating arrangement around the trunk, inclosing small niches, much sought after as hiding-places by various animals, and offering very acceptable holes to foxes, for instance. In point of fact, these roots are often called "buttress-roots". Tabular roots are a peculiarity of tropical trees with huge, heavy' crowns. A particularly well-defined form is exhibited by the West Indian Cotton Tree (Eriodendron caribcum) and by the India-rubber Fig (Ficus elastica) belonging to tropical Asia, and yielding caoutchouc. The picture of this tree, drawn from nature by Ransonnet, fig. 185), gives us a very clear idea of these tabular or buttressroots; the same figure, in the background to the right, also shows another species of Ficus, viz. the celebrated Banyan-tree (Ficus Indica), which will be described presently.

Stilt-like roots (radices fulcrantes) also arise in the same way from the erect or oblique main trunk, but they are cylindrical, and have the form of oblique props. Sometimes the oldest, lowest portion of the erect trunk thus supported dies away, or the disintegration may be continued some little distance up, so that only the upper part of the stem remains fresh and living. The first roots of mangrove seedlings (illustrated on p. 605), which penetrate the mud, have also the power of raising the trunk to which they belong up above the mire by their growth in length. These trunks then look as if they were on stilts, and are only connected with the ground by means of the roots. On page 758 we have a figure of the Screw Pine (Pandanus), and in fig. 187, of a species of mangrove, in both of which these odd root-structures are seen. They are also to be found in many other plants of the tropics, viz. in palms, Clusiaceæ, and fig-trees. In some clusias the stilt-roots are thicker than the stem they support, and in the mangroves, growing in crowded forests on the sea-shore, where they are exposed to the ebb and flow of the tide, they branch and fork continually, forming a tangled confusion, the strange appearance being heightened by the fact that all the root-branches and stems, up to the level of the water at high tide, are covered with an armoured coat of various molluses and crustaceans.

Columnar roots (radices columnares) originate from horizontal or obliquely ascending branches of trees, and grow vertically down until they reach the ground. They then penetrate into it, unite with the soil, and thus form pillars on which the widely projecting boughs of the tree are supported. Trees whose erect trunks are supported by tabular roots and those which are provided with stilt-roots may at the same time develop columnar roots from their branches. One of the oblique branches of the India-rubber Fig, illustrated in the foreground of fig. 185, is seen to be supported by a huge pillar, which gets thicker towards the base, whilst the mangroves figured on pp. 605 and 759 also exhibit long, supporting roots passing down from the lower horizontal branches of the crown, which push in between the stilt-roots, and grow down into the mud. Not long ago these mangrove roots were thought to grow out of the fruits while these were yet hanging on the trees, and to grow lower and lower until finally they reached the swampy ground. It is, of course, true that the embryo grows out from the fruits while they are hanging 
on the branches; but they become detached, as described on p. 603, as soon as they are from 30 to $50 \mathrm{cms}$. long, and falling with considerable velocity, bore into the mud by their lower thickened end. It never happens that one of these embryos grows down to the ground from the branch, and there is no doult that the long roots extending from the crown of the tree down to the mud originate from the lower horizontal branches of the mangroves just like other columnar roots. Columnar roots are distinguished from the flexible, cord-like, aërial roots of aroids and other epiphytes ( $c f$. p. 365$)$ by their great resistance to bending and by their possession of a characteristic mechanical tissue, in consequence of which they have a totally different internal structure, which, however, will be described later on.

Perhaps the most imposing cases of development of columnar roots are exhibited by the Indian banyans (Ficus nitida, Tsiela, and many others), which are usually comprehended under the name Ficus Indica, one of which is illustrated in the background of fig. 185. To these also belongs the celebrated Asvhatta, the sacred Fig-tree of the Hindoo (Ficus religiosa), beneath the shade of which Buddha is said to have learned the vanity of existence and the mystery of the universe. In proportion as the boughs which project almost horizontally from the main trunk of this tree become stronger, and give rise to branches and increase in weight, they send out cylindrical roots which grow down to the ground, penetrate into the soil, strengthen themselves by lateral roots, and serve as supports for the branches in question. These columnar roots, which continue to grow in thickness, resemble erect stems, develop leafy branches, and not only function as supports, but also serve for the absorption and transmission of water and dissolved food-salts from the ground. Below the crown of one of these banyan-trees we might imagine ourselves in a spacious hall of which the roof is supported on pillars; and since the leafy covering of the crown is almost impervious to rain and sun, a weird twilight always pervades these halls even during the daytime. Tradition states that an army of 5000 men have encamped in the halls of a single banyan-tice. Near the village of Dena Pitya, in Ceylon, there stands an Asvhatta under whose shade a village of a hundred huts is established, and in a single banyan-tree :350 large and 3000 smaller columnar aërial roots have been counted. When left entirely to themselves the banyan-trees scarcely ever assume such gigantic proportions, because the ground under the crown is so dry and hard that the supporting props which grow down often fail to penetrate it and are unable to take root there; but in the trees held sacred by the Buddhists the rooting is assisted by conducting the roots descending from the branches through long bamboo tubes, and by breaking up and moistening the soil where they would penetrate into the ground.

The shape of roots differs materially according as to whether the plants to which they belong are annual, biennial, or perennial. Annual plants produce as many seeds as possible in the short period of vegetation allowed them, and provide the embryos within the seeds, which have to travel about the world, with the 


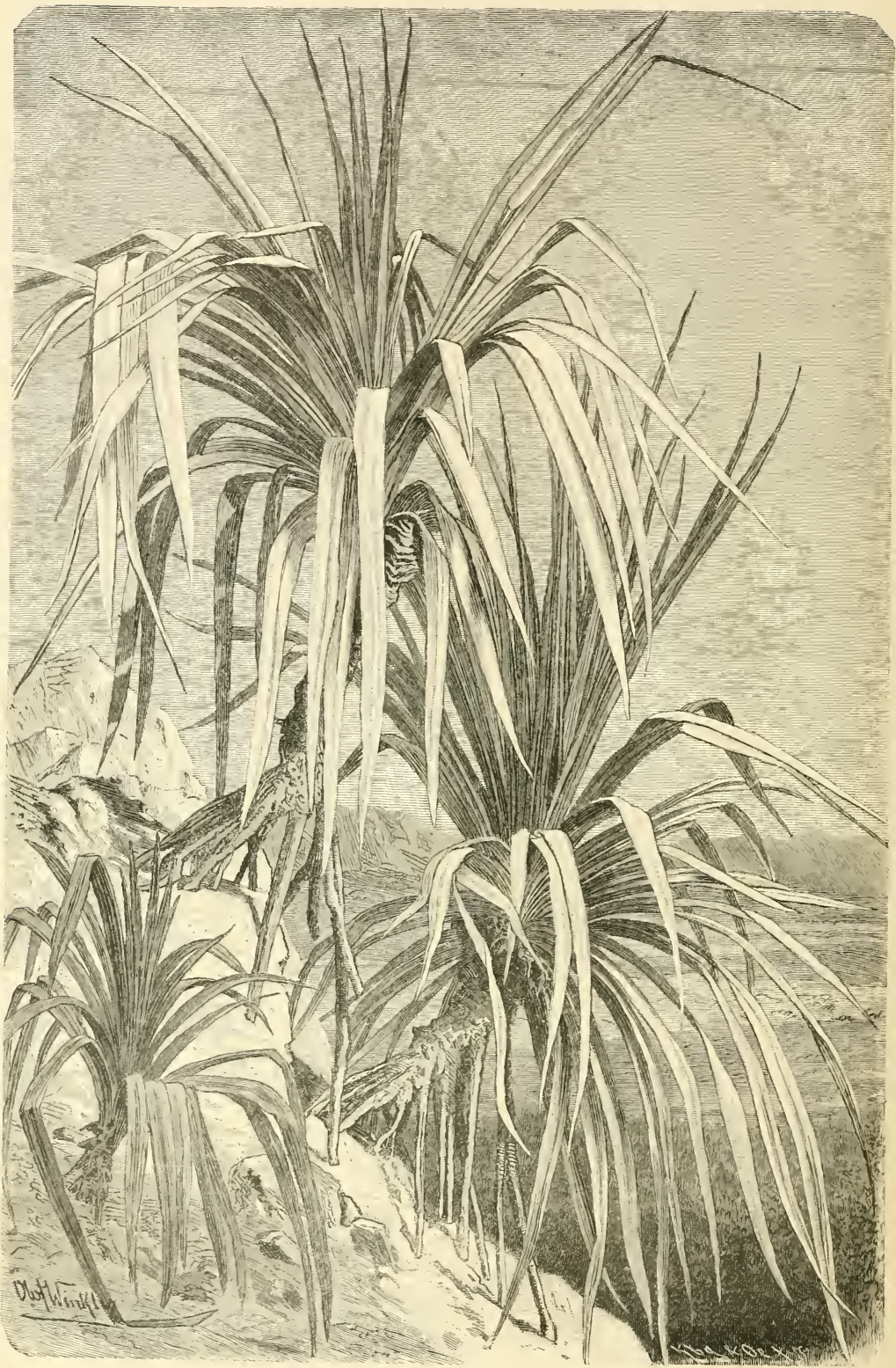

Fig. 186. - The Screw Pine (Pandanus utilis). From a photograph. 


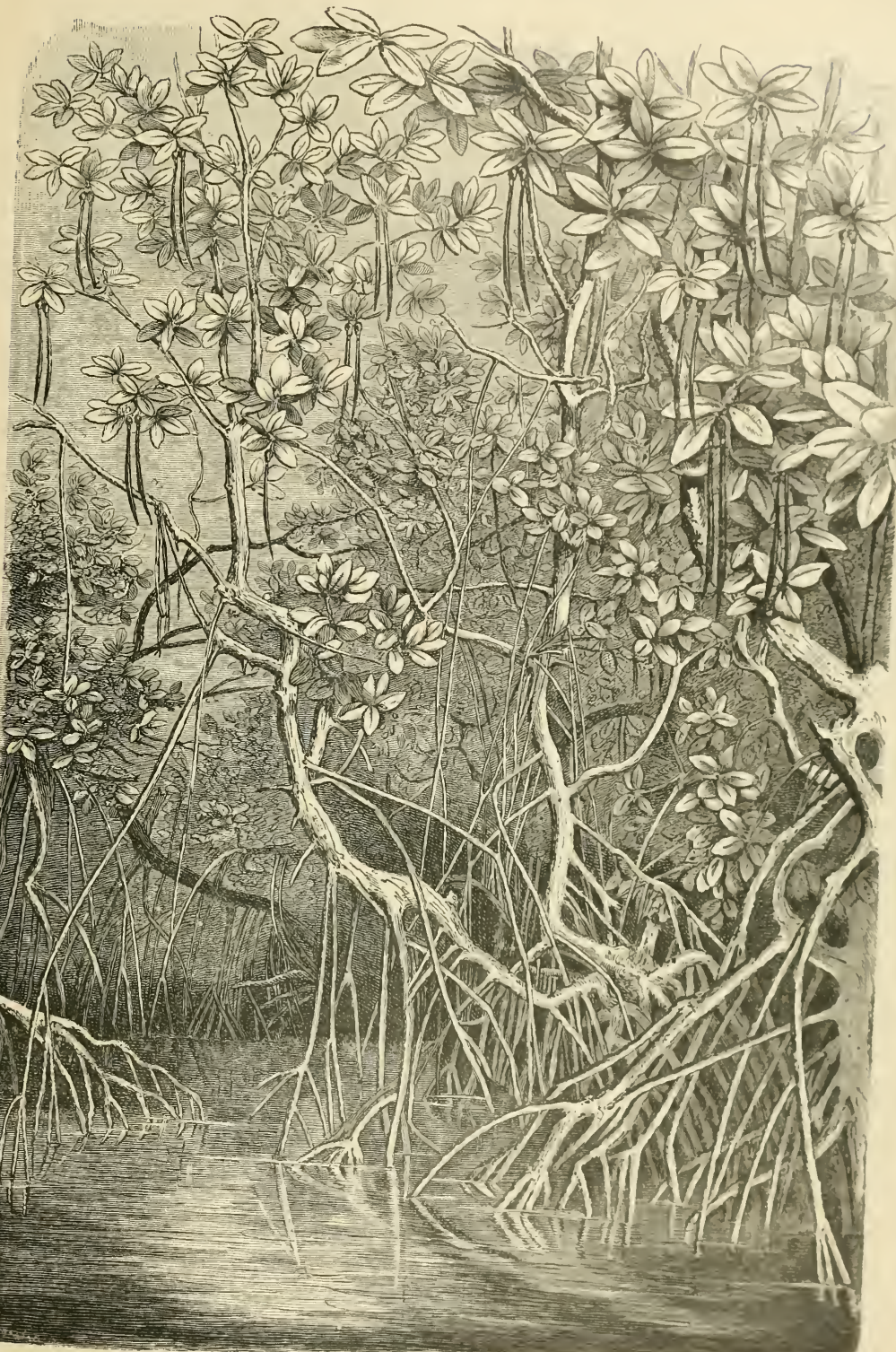

Fir. 187.-Stilt-roots of Mangroves (Rhizophora conjugala) 
reserves of food necessary for the founding of a new establishment. It would be of no use, and contrary to the economy of plants, if reserve materials were deposited in any other parts, say in the stem or roots, since these parts shrivel and dry up as soon as the seeds are dispersed, and the energy expended in the manufacture and storage of starch, fat, sugar, and other reserve food would be expended in vain. The roots of annual plants are therefore satisfied with delivering the necessary water and the required amount of food-salts to the plant during its short period of vegetation, and with providing a suitable attachment to the substratum; they waste no energy in founding subterranean reservoirs. In biennial and perennial plants it is quite otherwise. Biennial plants-as well-known examples of which may be taken the various roots used as vegetables, the Carrot (Daucus Carota), the Turnip (Brassica Rapa rapacea), and the Beet-root (Beta vulgaris rapacea)-develop during the first year a very short stem with foliage-leaves crowded in a rosette, and a thick, fleshy tap-root (radix palaris), or turnip-shaped root (radix napiformis). When vegetative activity recommences in the second year, an erect shoot with foliage and flowers is constructed at the expense or at any rate with the help of the materials stored up in the thickened root; fruits are produced from the flowers, and after the ripening of the seells the whole shoot dies off together with the exhausted roots. In perennial plants the roots, when they serve for the reception of abundant reserve-materials, are usually considerably thickened; but in these plants it is the clustered root-fibres springing from the lower end of the underground part of the stem, after the primary zuot has died off; which undergo this development. When the thickening is symmetrical and fusiform, as in the Orpine (Sedum Teleplium) and in the white-flowered Orobus Pannonicus, the roots are called fusiform (radices grumosce); when they are swollen at intervals into knots, as in the Dropwort (Spircea Filipendula), and in the yellow Day-lily (Hemerocallis flava), they are termed nodose (radices nodose). Iany of our terrestrial orchids have two kinds of roots united in a fascicle, long cylindrical vermiform roots and short thick roots filled with reserve-materials which look very like tubers, and are called tuberous roots (radices tuberosce). The IIediterranean flora and that of steppes, where in midsummer the vital activity of plants is much reduced, are particularly rich in plants whose roots are developed as storehouses for reserve materials. Plants of widely different families (e.g. Ranunculus Neapolitanus, Centaurea napuligera, Valeriana tuberosa, Rumex tuberosus, Asphodelus albus) there form thickened fascicled roots crowded with reserve-materials which pass through the dry season unharmed underground, and in the next period of vegetation supply the materials for the rapid construction of epigeal foliage and flowering shoots. These thickened bundles of roots are characteristic of the perennial, parasitic species of the genus Pedicularis. They serve for the storage of reserve foods, for the fixing of the plant, and for the absorption of nourishment, but the latter function is here carried on by means of suckers, which are developed at the end of the thickened fusiform fibres, and which attach themselves to the roots of the host plants in the manner described on p. 179. 
It would naturally be expected that in accordance with the various tasks assigned to roots there should be a difference in the arrangement of cells and tissues, and that, especially, supporting roots which exhibit the greatest analogy with erect stems, and subterranean roots, which have so much in common with procumbent and subterranean stem-structures, should resemble them in internal structure. Columnar roots cannot really be distinguished from upright stems in their inner construction, and stilt-roots also present an arrangement of cells and vessels which often agrees much better with that of erect stems than of underground rhizomes. In Fragrcea obovata, belonging to the Clusiacere, the cellular structure of the erect stem is only distinguishable from that of its supporting roots by the somewhat stronger development of the medulla and woody portion of the vascular bundles, but otherwise there is no sort of difference. The stilt-roots of the mangrove figured on p. 759 (Rhizophora conjugata) likewise show a stem-like internal structure. In the centre is a thick pith surrounded by numerous, conducting bundles, which together form a hollow cylinder, and are accompanied by mechanical tissue; further outwards come the cork, hypoderm, and a stronglycuticularized epidermis-exactly the same arrangement required in an erect stem as a protection against bending. In these mangroves the strength is even increased by a peculiar tissue, viz. by so-called trichoblasts, peculiarly interlaced fusiform cells with very thick walls, which are so hard that even the sharpest knife will scarcely cut through them. Though these adult roots are structurally indistinguishable from stems, this is not usually true of them at early stages in their development. When young, and as yet unthickened, these roots, as a rule, possess an internal structure characteristic of roots in general.

In mangroves and in the earlier mentioned Clusiacer, the supporting roots are thick and widely spread, and form extensive foundations which entirely replace the comparatively weak trunk, so far as fixing on the substratum is concerned; they need especially to be protected against bending. A resistance to tension scarcely comes under consideration in these plants. It is quite otherwise in plants whose stilt-roots have to support a stem bearing an extensive and richly-leaved crown. The Pandanus figured on p. 758 may serve as a type of these. As soon as wind sways the massive crown and slender stem bearing it, the roots supporting the stem on every side have alternately to resist bending and strain. If the wind blows from the north, the supporting roots springing from the south side experience a longitudinal pressure as the stem inclines to the south, and are pressed and curved down like an arch, while the supporting roots springing from the north side are at the same time subjected to a powerful strain. When the wind sinks, the stem is again brought into its erect normal position by the elasticity of the south roots. The reverse is the case when the wind attacks the crown and stem from the south. This form of stilt-root must therefore be constructed so as to resist strain as well as bending, and accordingly in the aërial roots of Panclumus are formed two cylinders of mechanical tissue, an outer one which is formed by the hard bast of a peripheral ring of vascular bundles, resembling the arrangement 
occurring in the majority of dicotyledons, and an inner which is formed of the hard bast of a ring of vascular bundles lying near the centre of the root. By the former the supporting roots are afforded the necessary resistance to bending, and by the latter the corresponding resistance to strain.

The stilt-roots springing from the lowest nodes of maize-plants are adapted to this double function just as are those of Pandanus. Here also are two cylinders of mechanical tissue. The outer one, situated in the cortex, consists merely of hard bast and provides a resistance to bending, while the inner, in connection with the conducting bundles, furnishes a resistance to strain. In the stilt-roots at the base of the maize-stem there is, however, a central pith or wide medullary cavity which is wanting in the roots of Pandanus.

Clinging roots adhering to the bark of trees, stones, or some other hard substratum, as well as the many forms of subterranean roots, are not required to resist bending, and in them there is none of the mechanical tissue which would be necessary for this resistance. On the other hand, these roots are unavoidably subject to a severe strain from the pulling exerted by the stem and branches as they sway to and fro. For a cylindrical body which has to resist a powerful longitudinal strain there is no better contrivance than the fusion of the resisting elements into a compact mass in the axis of the cylinder, and this arrangement is actually met with in clinging and subterranean roots. The conducting bundles and the adjoining mechanical tissue form a single central strand in the cylindrical root, and the typical form of a subterranean root is a cylindrical body of tissue which has no central pith and no hard bast cylinder near the circumference, but whose vascular bundles are so crowded towards the axis that they form there a single, thick strand or "central cylinder".

Roots embedded in the ground are of course exposed to a lateral pressure from their surroundings, and care must be taken that the functions of the conducting bundles are not disturbed by this pressure, that the transmission is not interrupted or even entirely stopped. This is effected by padding the central strand just described, that is, by surrounding it with a mantle of parenchymatous cells. The thickness of this coat varies according to the extent of the lateral pressure, and when the roots are subjected to very great pressure, the walls of the parenchymatous cells are even thickened in a corresponding degree.

Reserve-materials may also be deposited in this parenchymatous mantle. In biennial and perennial roots the tissue surrounding the sap-conducting and strainresisting strand is not only thickened so as to give the necessary support against pressure, but also provides a place for starch, fat, sugar and other supplies which are to be consumed in the next period of vegetation.

Naturally these soft tissues, often filled with reserve-food, are an attraction to diverse animals living underground, and the establishment of such a storehouse renders a corresponding protection against the attacks of mice and insect-larvæ necessary. Though the protective agents and weapons by which the green foliage, and flowers, and fruit are preserved from the ravages of animals would not serve 
here, still, by the development of poisonous and disagreeable substances, the subterranean, burrowing insects are, as far as possible, kept away. It is well known that roots are particularly rich in poisonous alkaloids, in resins which are repulsive to animals, in bitter substances and the like; these parts of planty are well known as providing many drugs of the pharmacopeia. These do not indeed afford an infallible protection against all attreks from animals, but that a partial safeguard at least is obtained by the storing up of certain materials seems very probable by the following observations. The field-mice in a garden at Innsbruck once caused great havoc under the winter coat of snow, and rarious roots were gnawed by them; but the roots and root-stock of the Soapwort (Saponaria officinalis), containing quantities of poisonous saponin, were alway: left untouched by them. The bitter roots of gentians (Gentiana punctata, luten, Pannonica), which are very rich in reserve-foods, and which grow in deep alpine meadows riddled by mice, were never seen to be attacked by a single animal. This was also the case with the thick tap-roots of the poisonous monkshood, the massive roots of rhubarb-plants and of many Umbelliferæ, which are all abundantly supplied with starch and other food-stuffs, and therefore would afford an excellent food for herbivorous animals under stress of hunger.

When the parenchymatous tissue surrounding the central strand of the conducting bundles in subterranean roots serves not only as an agent for protectine against lateral pressure, but also for the storing up of food-materials, and in addition possesses contrivances for warding off voracious animals, the structure of the roots is much more complicated than in cases where it affords protection against lateral pressure alone. There are also very many different developments of parenchymatous tissue on the periphery of subterranean roots in accordance with the various demands necessitated by the conditions of the habitat and the peculiar mode of life of the species. In aquatic roots the need for abundant ventilation has also to be considered, and the storage of reserve-foods in these organs must be avoided since the increase in weight, due to the massing of reserve-food, might draw the floating water-plant down into the water at an unsuitable time.

A storage of food-materials in the special tissue developed at the growing roottip, and known as the root-cap, would also be unsuitable. In subterranean roots the root-cap only protects the delicate dividing and multiplying cells at the growing end. The pressure to which these continually dividing cells are exposed in their penetration into the ground is much greater than that operating on the fully formed parts behind the root-tip. The growing point of the root has to push on one side hard grains of sand and other particles of earth, and to make a hole like a ground-auger in which later on the fully developed root can take up its position. The root-cap may be compared to a shield which is formed by the growing and therefore advancing cells in the direction required, these eonstantly pushing it in front of them. This shield is always being supplemented and renewerl by the growing tissue. The half of the root-cap adjoining the growing tissue consists of angular, closely-fitting cells; the outer half, directed towards the soil, consists of 
rounded, loosely-fitting cells, and on this outer side of the root-cap the cells are also seen to be partially separated and torn off. As the outer cell-layers are rubbed away by the advance of the root, and by unavoidable contact with the surrounding soil, new cells are always being pushed forward from within, and in this way the loss is made good, and the shield continually repaired.

Obviously, aquatic roots do not require a shield of this kind at their apex, and in aërial roots, at least in the form described, it would likewise be superfluous. Even roots which penetrate into mud do not require it. Accordingly many waterplants and the marsh-inhabiting mangroves do not develop a cap at their root-tip. The root-cap is also entirely absent in parasitic plants which it would only hinder from penetrating into the tissue of the host-plants.

\section{DEFINITION OF THE ROOT.}

In the preceding pages we have continually spoken of roots, although we have not yet defined technically what a root is, and now, contrary to the usual custom in scientific works, the definition of this organ has to come not at the beginning but in the middle of the chapter. This alteration in position has been caused by the necessity of establishing the definition on some peculiarities in the external and internal structure of roots, with which we could not suppose all readers to be familiar, and which therefore had to be described beforehand as far as required.

But many readers will ask if any definition is required, if everyone does not know without it what the root of a plant is, and how it can be distinguished from a stem and leaves? The case is exactly parallel with that of the leaf. Every one who is not a botanist thinks he knows what is meant when he hears the word "leaf", and cannot conceal his astonishment or possibly his smile when he is informed that scientific men are not agreed about such a simple question, and that they write violent polemics upon this question. To the impartial reader debates as to whether a certain part of a plant is to be regarded as a root or not doubtless appear hypercritical and a pedantic splitting of hairs, and with regard to many of the discussions I would hardly venture to deny the justice of his position. The savant who constructs for himself the picture of an ideal or primitive plant from a sometimes larger, sometimes smaller number of single observations, who finds out how the individual parts were situated in their succession as to time, and in their mutual relations in space, and who distinguishes and defines the various parts accordingly, is indeed very easily tempted to take the abstract ideal he has created as a standard for the whole vegetable kingdom. From his point of view, obtained by the consideration and comparison of so many individual cases, all forms are arranged and explained, everything must fit into the now firmly established groundwork, and where it will not coincide, he talks of exceptions, forgetting that in such a case exceptions are not permissible, but are rather a proof of inadequate generalization from the single cases observed.

In the comprehension of the results of general comparative studies of this kind 
into the configuration of plants, it is a matter of great import how the definitiuns of the individual parts and members of the plant are formulated, and whether the author lays particular stress on this or that characteristic. Suppose that some observer holds the opinion that the presence or absence of the root-cap aftiords an important distinction between a root and stem; then he would speak of the supports of mangrove-trunks as lateral stems which grow downwards; another, who lay's particular weight on the fact that roots produce no leaves behind their growing points, would, on the contrary, declare the supports of the mangrove-trunk to be roots devoid of root-caps. It would be the same with the contradictory explanations and different appellations which would be given to the supports of Clusiaceit and figs, to the fixing and absorbent apparatus of Mistletoe which penetrate into the host-plant, and to so many other hypogeal and epigeal parts of the plant-borly.

These examples will suffice to show how a conflict may arise over such an apparently simple thing, how easily the investigators into the region of the speculative science of form may become one-sided, what great difficulties are to be encountered in formulating a definition, and how in particular a hasty generalization must be avo:ded about characteristics which it is not at all certain are really to be met witl universally. Every definition is dependent upon the extent of our knowledge at the time; it may not hold good as our experience widens, and therefore has only a relative value.

From the standpoint of our present knowledge, however, the following may lue taken relatively as the best definition:-A root is a body of tissue provided with vascular bundles, which originates from an older, previously-existing part of the plant; its growth is not limited, and it never directly gives rise to leaves.

In connection with this definition, some remarks may be made here by which many relations between the root and other parts of the plant will be elucidated. First it should be noted that in the above definition the youngest developmental stage, viz. the embryo, is included under the term "plant". It has further to be explained why the characteristic which is first thought of in non-botanical circles, when speaking of roots, viz. their power of deriving fluid nourishment from another body, has not been mentioned in the above definition. It is perfectly correct to say that an absorption of fluids is generally observed in roots, but in reality it is only the root-hairs proceeding from the roots which perform this task, and these absorbent cells are known to be also developed on stems and leaves. The cotyledon extended from the seed of the Bulrush (Typha), penetrates into the soil with absorbent cells. The cavities of the green leaf-structures in insectivorous plants are also abundantly provided with them, and special absorbent cells are developed on the green leaves of many saxifrages, tamarisks, and so forth; whilst in those marsh-plants, the leaves of which float partly on the surface of the water and are partly submerged, the epidermal cells also function as absorbent cells. In mau aquatic plants (e.g. Hottonia, Ceratophyllum, Naias) absory tion is only carrical on by means of the epidermal cells of the foliage-leaves, and no trace of roots is to be found in them. Their foliage-leaves, however, remind one very much of root- 
structures, and in a floating water fern (Salvinia natans, cf. vol. ii. fig. 380 ) the submerged leaves have the greatest resemblance to roots in shape and colour. In such cases, though we can say that the leaves are metamorphosed into absorbent organs, we cannot assert that they have become roots. This applies also to plants whose underground stems are provided with absorbent cells (e.g. Bartsia, Epipogium, Corallorn/iza), or whose stem-structures, submerged in water, are furnished with epidermal cells functioning as root-hairs (e.g. Lemna trisulca). In these plants the stem-structures are indeed metamorphosed into absorbent organs, but they are never transformed into roots.

We are accustomed to think of the roots of plants as organs with white, yellow, red, brown, or black, but never green, colour, because as a matter of fact by far the greater number are devoid of chlorophyll. But there are plants whose roots do contain chlorophyll, e.g. those of Lemna minor, and various aroids and orchids. In orchids with aërial roots but no green foliage-leaves, the green roots must take on the formation of organic compounds from food-gases in sunlight, that is, the function which is performed by the foliage-leaves in so many other cases. We should, therefore, be as little justified in bringing forward the absence of chlorophyll as a characteristic feature of roots as in saying that the roots had become changed into green leaves. The roots of the orchids mentioned have indeed become transformed into assimilating organs, but they remain roots nevertheless.

It was formerly thought that roots and stems could be distinguished, the former by their inability to develop buds, and the latter by their power of forming them. But although this difference is actually observed in most instances, it cannot be applied universally. The roots in many plants develop buds which unfold into leafy shoots, and not merely lateral, but terminal buds also. When this happens it looks as if the root were continued directly into a leafy shoot, and this occurrence has led to the mistaken idea that the root-tip may become metamorphosed into a leafy stem.

Finally we have to consider the difference in the mode of origin of roots and stems. It cannot be denied that the points of origin of stem-structures are usually arranged geometrically, while roots only exhibit such an arrangement in rare cases. But we must again insert the words "usually" and "rare", for here too a universal distinction does not exist. The stem-structures springing from the underground roots of the Aspen (Populus tremula), and from old trunks of the Black Poplar (Populus nigra) make their appearance quite irregularly, whilst, on the other hand, the roots of many aroids originate with the same regularity as leaves and the lateral shoots arising from the axils. In most cases the root proceeds from a group of cells in the interior of a stem or older root, and it used to be thought that this constituted a difference between roots, and stems and leaves, since the latter arise from cells near the surface of the tissue-body which bears them. But aquatic roots, e.g. those of Ruppia and Zannichellia, also proceed from cells near the surface of the stem, and in the same way roots arise from the epidermal cells of the leaves of the Cuckoo Flower (Cardamine pratensis), and from 
the parenchyma lying immediately below the epidermis, so that this agrain does not furnish a universal distinction.

But although all those characters, which have been used in turn to characterize the root, cannot be thus employed because they have not a universal value, yet one distinguishing feature always remains, viz. that leaves are never produced from root-tissues, and the greatest stress is to be laid on this point. After weighing everything carefully we come to the conclusion that the plant, and even its youngest developmental stage, the embryo, begins with a stem, which develops leaves and roots. Stems, leaves, and roots may perform widely different functions, may shape themselves accordingly, and may be metamorphosed into widely different organs. A plant is comparable to a crustacean which is divided into a body and appendages. The appendages in most cases serve as organs for locomotion, grasping, and clinging, but are sometimes also metamorphosed into respiratory organs, egg-carriers, \&c.

\section{REMARKABLE PROPERTIES OF ROOTS.}

The small stem-structures which proceed from germinating orchid-seeds behave very differently according to the nature of their germinating bed. From the small tubercles of species growing as epiphytes on the bark of trees arise, first of all, hair-like absorbent cells which adhere to the substratum; then roots make their appearance, which also unite firmly with the bark, though their superficial cells are not able to penetrate into it. The small tubercles of the terrestrial orchids, which inhabit the meadows and the humus of the forest soil, develop roots which grow down into the ground and direct their growing tips towards the centre of the earth. In this way they draw the stem-structure from which they originate down with them, and thus the tuberous stem in two years' time comes to lie 6-10 cm. below that point in the meadow where the seed actually germinated. The same thing happens with the embryos of many biennial and perennial plants, especially of those whose underground roots and stems are subsequently used as storehouses for reserve materials, e.g. in Carrots, Evening Primroses, in the Monkshood, Meadow Clover, Vincetoxicum, Dog's Mercury, Martagon Lily, Bulbous Crowfoot (Daucus, Enothera, Aconitum, Trifolium pratense, Cynanchum Irincetoxicum, Mercurialis perennis, Lilium Martagon, Ranunculus bulbosus), and many others. In these plants also the embryonic stem is drawn more or less deeply under the ground, and the scarred point of insertion of the cotyledons is not infrequently found to be several centimetres lower down that it was at the time of their withdrawal from the integument of the seed.

Many roots arising later on from procumbent and from erect or twining and climbing leafy stems have the power of exercising a pull on their stem. The roots springing from the stem nodes of runners, e.g. from those of strawberry plants, draw the nodes a centimetre below the ground. This is also the case with the long roots proceeding from the stems of perennial primulas. When these primulas 
settle in the clefts and crevices of perpendicular rock faces, a phenomenon is produced by this down-drawing which surprises anyone noticing it for the first time, appearing at first quite inexplicable. The thick stems of these primulas (e.g. Primula Auricula, Clusiana, hirsuta) are terminated by a rosette of foliageleaves; these turn yellow, and dry up in the autumn, and a new rosette is laid down in the axil of one of them for the next year. Although the leaves of the rosettes stand close above one another, the portion of the stem clothed by them is quite a centimetre long, and the annual increase undergone by the stem which grows towards the light is also a centimetre. The increase during ten years would amount to $10 \mathrm{~cm}$., and it would be expected that the rosette of the tenth year would be about $10 \mathrm{~cm}$. above the level where stood the first year's rosette. But, strange to say, the rosettes of all the succeeding years always remain at the same place, that is, they always cling to the rocky edges of the crevice or cleft in which the stock is rooted. The explanation of the phenomenon is that the roots springing from the rosette-bearing stem draw it down every year about a centimetre into the soil or crevice filled with humus. But naturally this can only occur if the lower end of the stem annually dies off and decays to a corresponding extent, and this is what actually happens. In rocky clefts which are not well adapted to this process the primulas grow badly, and their stems project above the edges of the crevice; ultimately the entire plant falls into a slow decline and no longer blossoms, but perishes in a few years. The knowledge of their peculiar mode of growth is therefore of some importance in the cultivation of these primulas, since care can be taken to plant them so that the stems can be annualiy drawn a certain amount into the soil by the roots. It is of course needless to mention that many other plants beside primulas, rooted in crevices of rock, behave in the same way, e.g. Phyteuma comosum, Gentiana Clusiana, Campanula Zoisii, Paederota Ageria.

The ends of branches of many species of bramble are drawn under the ground in a very peculiar way. One of these species, Rubus bifrons, is represented in fig. 188, where the roots and the ends of the branches drawn under the soil by them are rendered evident, the earth in the foreground being removed as if dug away by a spade. Rubus bifrons develops strong five-ridged shoots beset with reversed prickles; they at first grow directly upwards, but towards autumn hang in broad curves, so that their tips approach the ground. Before they have reached the soil, however, small scale-like protuberances, looking like stunted leaves, are to be noticed arising near the tip; these are the commencements of roots. When the apex of the branch at length trails on the ground the protuberances, now in contact with the soil, elongate into roots which penetrate the ground. They lengthen very rapidly, sending out numerous lateral roots, and in a short time an extensive subterranean root-system is the result. But the apex of the brancl. which serves as a starting-point for these roots, and which is now considerably thickened, has also come under the ground. It has been drawn down by the roots, and is now embedded in the soil. In the following spring, sometimes even 
in the autumn in which the rooting has taken place, this branch apex, numrisherl by its roots, grows up into a shoot; which agrin appears above the ground. The

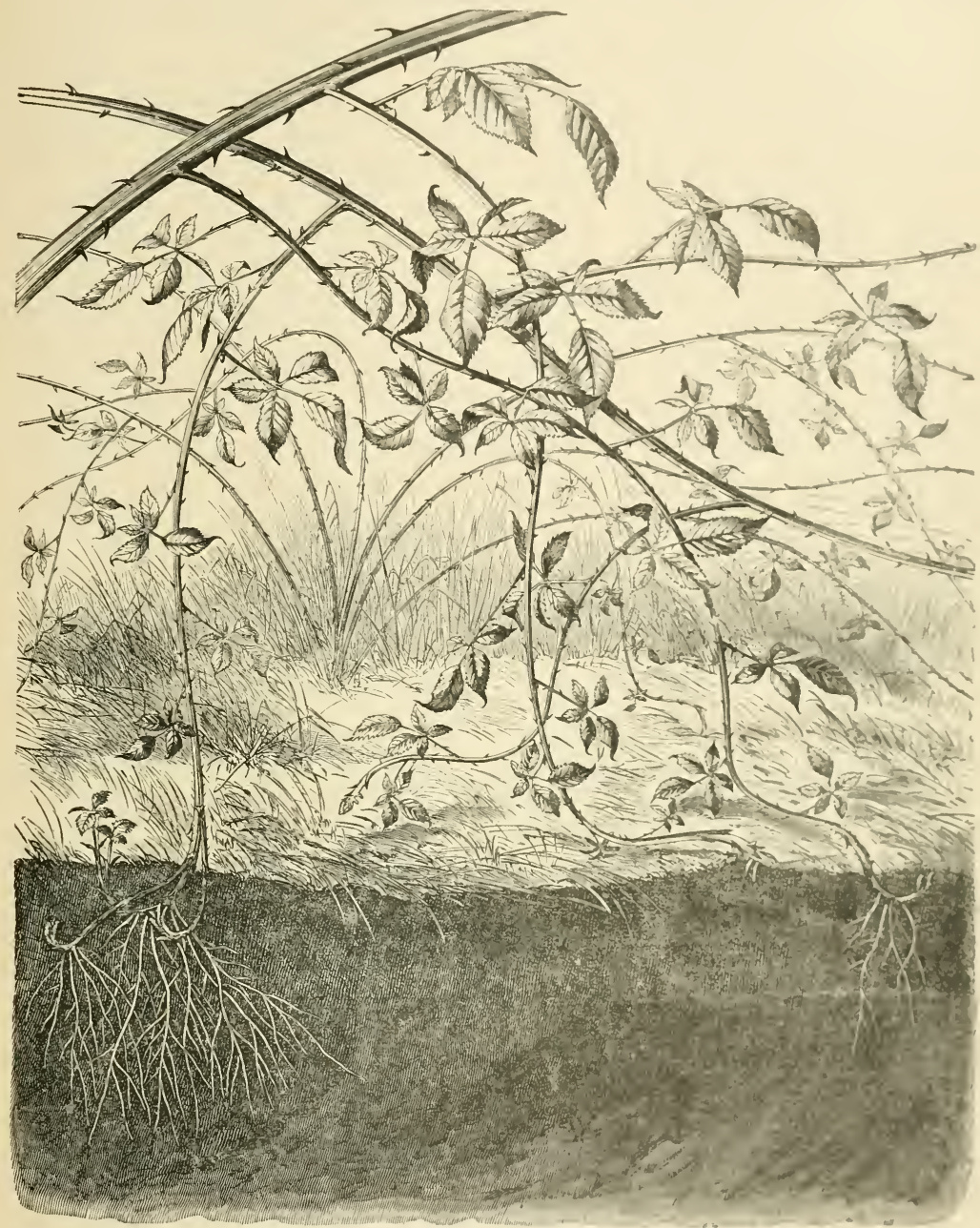

Fig. 188.-Bramble-bush in which the branches have taken root.

old branch, however, which had arched down to the soil, and whose apex hall been drawn into the ground by the roots, dies off sooner or later, consequently. a new, independent plant results from this action. VoL. I. 
It has been shown that where stems are drawn into the ground, it is by means of the roots. After the growth in length of a root is completed, it shortens, in some instances only 2-3 per cent, in other cases as much as 20-30 per cent, i.e. almost a third of its entire length. The shortening depends upon alterations in the turgidity of the cells connected with an absorption of water. While the cells of that portion of the root which is still growing elongate by increased turgescence, those of the fully-formed root become shorter and broader in consequence of the increase in their turgidity. The parenchymatous cells in fullyformed roots become broader at the expense of their length in consequence of the increased turgescence produced by the absorption of water, and the natural result is a shortening of the whole tissue-body. This contraction of the mature root-portion exerts a tension on both ends. At the lower end of the fully-formed part of the root is the still immature portion growing downwards, whilst at the upper end that part of the stem from which the root originated. Above the downwardly-directed point the immature part of the root is equipped with hairlike absorbent cells, and these are closely united to the surrounding soil. In this way a resistance is afforded which the strain of the contracting part of the root cannot overcome. And since, as already stated, the cells at the growing end of the root are lengthened by turgescence, the tissue is extended, and the root-tip, in spite of the strain operating from above, continues to penetrate into the ground. The strain, therefore, has no effect in this direction. But it is otherwise with the pull exercised on the stem by this contracting of the root. There is no powerful resistance here to be overcome, and consequently the part of the stem in question, whether the hypocotyl of the embryo, the end of the epicotyl, or a node from the middle or end of the leafy foliage-stem, is drawn down into the soil.

This remarkable planting of course only occurs where the roots grow down vertically into the ground, and as remarked, it is most noticeably observed in species whose subterranean stem and root-structures store up reserve materials. Roots which run horizontally below the surface of the ground are not adapted to influence the stem in the manner indicated. On the contrary, in certain circumstances, these are able to effect an elevation of the stem. This happens especially in trees with thick woody roots, e.g. in pines and firs, oaks and chestnuts, and is to be explained in the following simple way. The first embryonic root growing down vertically into the ground soon dies off, or its development, especially its increase in length, is greatly retarded, and much more vigorous roots develop from it, or from the lowest part of the erect hypocotyl. These spread out in a horizontal direction under the surface of the ground. They usually radiate out in all directions forming a whorl at the base of the erect stem, as can be plainly seen in pines uprooted by a devastating storm. These horizontal roots at first have only a slight thickness, but their diameter increases with age, and the successive layers of wood in them form annual rings, just as in stems. Now these subterranean roots, in addition to resisting the pressure of the surrounding soil, actually exercise a considerable lateral pressure 
by their growth in thickness. In consequence of this the soil below the cylindrical, horizontal root becomes compressed, but that above it is raised and burst open. The thick, woody root gradually becomes visible on the surface, and is entirely stripped of earth on the upper side. The axis of the horizontal root never again assumes the position of earlier years; then the roots were only a few millimetres thick, but now they have attained to a diameter of 20-30 centimetres, and the root-axis has been shifted upwards through almost half its diameter, i.e. through 10-15 centimetres. The erect trunk, which is firmly united to the horizontal roots in the way just described, is, of course, raised up to the same extent. In this manner may be explained the peculiar appearance so frequently to be seen in our pine and oak forests-the appearance of hugre tree-trunks with thick woody roots springing from their base which are quite devoid of earth on their upper sides, and run, half underground, in snake-like coils into the forest ground.

The elevation of stems by means of roots is more striking in tropical mangroves even than in our native trees. After the seedling has fallen from the tree and bored its way into the mud, protuberances arise on the circumference of its lower third which develop into roots directed obliquely downwards. Even in a few months the buried plant is raised up a little above the mud by the lengthening of these roots, so as now to look as if it were supported on stilts.

It has been repeatedly mentioned that the primary roots of the embryo originate from places on the hypocotyl which have been determined beforehand. So also does the origin of roots on many rhizomes, runners, and on climbing stems seem to be precisely determined, and to be quite independent of external influences. For example, the primary root of mustard and numerous other plants is developed under all circumstances from one pole of the hypocotyl. The runners of strawberry plants and of the Creeping Crowfoot (Fragaria vesca and Ranunculus repens) develop, without any external stimulus, a group of from two to five root-protuberances on the stem-nodes, and the bramble branches, described above, curve like arches to the ground, forming several root-prominences at definite spots near the apex before they have reached it, in order that they may take root in the soil. In many epiphytic aroids and orchids the places of origin of the roots are arranged as symmetrically round the stem as are those of leares, and many other examples might be cited from which it follows that the position of part of the roots is definitely fixed beforehand, being based upon the specific constitution of the protoplasm of the species in question. But as well as the roots developing in the manner indicated in definite positions, others are formed which require for their development a special stimulus from outside, whose place of origin is not determined beforehand, but is first fixed by some external agent. To this category belong the roots arising from the nodes of shrubs which have been battered down on the ground, and from stems coming in contact with damp objects, as well as those which proceed from foliage-leaves, and, finally, the wart-like roots of parasites known as haustoria. When shrubs 
with erect stems and thick stem-nodes, e.g. the various species of Galeopsis or Polygonum, are extended flat on the ground from some accidental cause, only a part of the stem rises up again after a time by a right-angled bend at one of the nodes, the part next the free apex rising up, while the part nearest the attachment remains prostrate on the ground. Contact with the soil acts as a stimulus to the formation of roots on this latter portion, and they are produced abundantly near the node from the knee-shaped bent portion, and penetrate into the ground, functioning as absorbent and fixing organs. These shrubby plants would not have developed any roots on their stem-nodes had they not met with the accident and so been stretched on the ground.

Cut branches of willow placed in water, wet sand, moistened soil, or moss, develop roots in about a week at the place where they are in contact with the water or damp objects mentioned; roots which are equally useful either as absorbent or fixing organs. If the branches had not been eut off or treated in this way, no roots would have been formed on them. These willow branches may be taken as a type of the shoots of a great number of plants which all readily develop roots from the stem when placed in damp surroundings. The propagation of plants by cuttings, so often performed by gardeners, depends upon the fact that when branches are cut off from a plant and placed in damp sand they "strike root" in it, i.e. they send out roots from the part of the stem situated in the sandy soil. Contact with damp earth operates as an incitement to the formation of roots in the aërial, cord-like roots of the aroids figured on p. 365 , just as in these cuttings. The aërial roots descending from the stems of these aroids do not develop alusorbent, lateral roots until they reach the soil; but they have scarcely come into contact with it when numbers of lateral roots arise which penetrate into the ground where they can suck up fluid nourishment. In the root-forming leaves of species of pepper, of begonias, and of the cuckoo flower, contact with damp soil stimulates the production of roots-in places, too, where no roots would have been formed without this contact. If a pepper or hegonia leaf is cut in pieces, and each piece laid on damp sand and so pressed down that the veins projecting from the lower side are embedded in the sand, roots will grow out of the parenchyma adjoining the veins, and turn downwards, while above they develop a tissue-body which turns upwards and becomes a leafy shoot, being provided with food by the roots. Long roots arise from the cellular tissue at the base of the stalk of rank ivy-leaves placed in wet sand or in water, which is never known to happen when the ivyleaves are growing freely in the air. We must not omit to mention here the roots of parasitic plants which attach themselves to the living tissue of other plants as so-ealled haustoria; these only arise in the parts of the parasite which come directly into contact with the succulent roots of the living host-plants.

The benefit which plants derive from the formation of these roots is easily perceived. In the stems of the prostrated shrubs the conduction of fluid fool from the ground is, no doubt, restricted and imperilled, and therefore it is important that the part of the shoot again rising from the ground should be provided with special 
roots at the node where the knee-shaped bending takes place, in order to concluct the absorbed nourishment directly to the foliage-leaves on the upper part of the shoot. The actual existence of the part of the plant in question depends upon the formation of such roots in the other cases enumerated above. The cut branches of willows, the cut-up foliage of begonias, the ivy-leaves torn from their stem, \&c., would all die if they did not provide themselves with roots. But although it is easy enough to perceive the benefit ensuing from this kind of root-formation, it is very difficult to explain how the mechanical impulse brings about this new production. It has been shown in all these instances cited that contact with a foreign body is an important factor, but it is very puzzling to understand how the deeper cell-layers are stimulated to develop roots by the contact of the epidermis with damp earth, water, and the like, and we must content ourselves with saying that the contact acts as a stimulus, which, when transmitted to the deeper layers of cells, stirs them up to construct roots as a deliverance from death. The explanation is still more difficult in cases where the cut parts of the plant develop roots to preserve their life, even without contact with a foreign body. Such a case has been considered earlier (on p. 89), when it was shown that on cut shoots of various species of stonecrop (e.g. Sedum reflexum, Boloniense, elegans), which are hung in the air by a thread, roots will develop from the internodes between the foliage-leaves in places where no roots would normally have arisen. They grow down and elongate until their tips come in contact with some solid body. Here no stimulus could have acted on the epidermis; the pendent shoots stand in no relation to the surrounding air other than obtained whilst they were still united to the rooted plant, i.e. before they were cut off. The stimulus to rootformation must, therefore, be referred to the separation of the shoot from the plant. We must not, however, imagine the action to be merely mechanical, but must be content with stating that the living shoot hanging in the air can only save itself from death by developing these roots.

To the most remarkable vital phenomena of plants belong also the various bendings, curvatures, and other movements performed by growing roots. Apparently every root tries to reach a definite goal, towards which it directs its way, endeavouring to obtain the advantages offered by it with as little expenditure as possible. The goal which growing roots strive after is the same for all, viz. the place in the nourishing substratum best adapted to them. The primary roots of plants settled on the bark of trees as epiphytes or parasites dircet their tips towards the axis of the branch of the tree in question, land plants on the other hand, towards the centre of the earth, and the primary roots proceeding from seeds lying at the bottom of still water sometimes direct themselves upwards and grow at the commencement of their development towards the surface of the water. The road to be traversed by the succeeding roots, from whatever part of the plant they may have sprung, is apparently not so clearly determined; but on a closer examination it is found that they too strive to attain to places where fluid nourishment abounds, and where they can obtain a firm hold. 'The 
soil is made up of alternating places containing a larger and a smaller amount of food-salts, and places which either retain water badly or well. In one place are situated nests of humus, in another sharp-edged stones, and it is only natural that these inequalities and obstacles in the path pursued by the roots should not be without effect on them. As a matter of fact manifold contrivances are met with for preventing the roots from, so to speak, blindly passing by favourable places in the soil without making proper use of them. The fact that the tips of many roots describe oscillations or nutations, not unlike those which are observed in twining stems and in certain creepers, is an instance of such adaptation. Roots growing in soil are of course much more restricted in their movements by the pressure of their environment than are the structures which circle round in the air, but in the main the principle is the same in both cases. The path travelled by the point of the growing root is most accurately depicted by a spiral line, and the most important advantage obtained by following such a path lies in the contact of the growing root with as large a portion of the soil as possible. A root growing in a straight line would not touch half as many points as that following a spiral, and since the likelihood that all the favourable spots will not be left on one side increases with the number of points of contact, the spiral movement of the roots may without hesitation be regarded as a contrivance for discovering the best sources of food in the soil. We must, of course, not undervalue various other advantages which are also obtained in this way, in particular, the greater ease with which roots following a spiral line can bore their way into the soil, and the better hold they obtain.

Although the root follows a spiral line in its growth, it may nevertheless maintain a straight direction on the whole; this is actually the case in water and in a homogeneous and uniformly moistened soil. In soil differently constituted and unequally moistened, however, a diversion takes place away from the side where the conditions are unfavourable to the root. This swerving may be caused by cold, dryness, by chemical conditions of the soil, and by pressure and injuries.

It is well known that in the far north the ground remains always frozen below a slight depth. During the short summer only the superficial strata are thawed, but below this the "perpetual ice" stretches to an immeasurable extent. A relatively abundant vegetation develops on the thawed strata under the warm rays of the sun, and in North America not only shrubs and low bushes but also colonies of huge fir-trees grow up. The roots of these plants penetrate straight downwards and grow towards the centre of the earth; but as soon as they come into the neighbourhood of the ice they bend aside, curve round, and continue their path only in the thawed stratum. The diversion is usually so striking that the diverted portion is sometimes actually at right angles to the older part which grew vertically downwards.

The same thing happens when the soil is moist in some parts and dry in others. Here again the growing roots seem to be repelled by the dry, inhospitable layers of soil, and turn towards the neighbouring moister region. This phenomenon has been 
termed hydrotropism. It frequently happens in mountainous districts that after heavy downpours of rain the overflowing streams tear deep furrows in the adjoining steep forest lands, and root up the ground, throwing everything into confusion and depositing below on the valley-floor a mass of detritus or rubbish. Usually numerous organic bodies, blocks of wood, pieces of turf, leaves, fir-cones, and the like are torn away by these turbulent streams with the stones and sand, and the deposit is therefore studded with nests and strips of humus which owe their origin to the organic fragments mentioned. Seeds of various plants from the neighbouring forest are swept into the rubbish heap, and among them those which only flourish well in the damp humus of forest soil. These seeds germinate, anil their roots penetrate downwards; many perish at once in the inhospitable soil, but others grow excellently, sending up a vigorous stem and unfolding foliage and flowers. When these well-grown plants are dug up in order to see the relation of their roots to their immediate environment, it at once becomes evident that the roots in their downward progress have curved towards the nests and veins of humus. They exhibit the most wonderful twists and bends, and look as if they had actually been attracted by the humus deposits. Without quite excluding the possibility of a chemical attraction, we must regard the aversion of the roots to dryness as the chief cause of the bending. The masses of humus embedded in sand and rubbish retain moisture like a sponge, and when the adjoining sand-strata have been for long dried up the dark nests and strips still retain their saturated condition. When a root shunning the dryness turns away from the sand, and in continuing its growth comes to a deposit of humus rich in water, it finds there no inducement to continue bending, and so grows straight through the region of the damp layer. When in its further growth it emerges from the ball of humus and enters the dry sand, it of course again bends and curves round the ball of humus, or wheels round in a half circle so as to return to the moist dark clump which is situated like an oasis in the dry desert of sand.

It is obvious that larger pebbles which cannot be displaced by growing roots must cause a swerving; the root whose tip is in contact with the hard stone bends sideways and evades the insurmountable obstacle lying in its path. A very noticeable bend ensues when the growing root is injured on one side of its tip, or is so fastened to some foreign object that the cells at the place of contact are damaged. It then bends away from the injured or attached side and assumes a divergent course.

In many cases it might be thought that the roots were not repelled by the unfavourable places in the soil, but were attracted by the favourable places, and, as already stated, the possibility of an attraction, a.mutual action of the sap of the root and the materials contained in the places in question in the soil, which might find expression in a movement of the growing root-end, is not entirely excluded, although it has not hitherto been demonstrated with certainty.

The circling, that is, the spiral movement of the growing root, has been explained in various ways. One view was that the cylindrical body of the root may be 
divided up into longitudinal strips, all of which were supposed not to grow at the same time or to an equal extent, but rather that the wave of stronger growth continually passes from one strip to the next one. This movement, however, like that of twining stems is probably an alternate bending towards the different radii of a circle drawn round the root, and since it is combined with an elongation of the part of the root in question, the growing root-end describes a spiral line.

The bend caused by the diversion of the root is either produced by a one-sided contraction, or by a one-sided elongation. Since the bend occurs in the growing portion of the root, a more rigorous growth on one side may be regarded as the inmediate cause of the bend, and every impetus which would promote such unilateral growth would also cause a bending. The bending of roots which shun dry places may perhaps be referred to a withdrawal of water from one side of the root-tip. Thus if the root lies imbedded between a damp and a dry layer, that side which abuts on the dry stratum will transpire more actively than the other, and it has been suggested that this active transpiration in some way promotes an increased growth in length in that half, and in consequence of this unilateral elongation on one side, the other half, adjoining the damp layer, will become concave.

The idea that the curvature is not produced directly in the place where the external stimulus operates, but in the growing region lying behind the stimulated root-tip, is much more interesting than these purely mechanical explanations. According to this view the stimulus is transmitted as in the leaves of the Sundew, Fly-trap, Aldrovanda, sensitive plants, and many other cases. The active stimuli may be afforded by pressure, cold, dryness, and probably chemical conditions also. Gravity, too, may be looked upon as a stimulus, indeed as one which influences the direction of growth. It is believed that gravity acts on the root-tip as a stimulus to growth and that this stimulus is conveyed to the growing region behind, and that in consequence the primary roots grow down towards the centre of the earth. But as primary roots are able to penetrate into mercury, and to bore through paper, in their downward growth, something more than mere weight operates, since this would not be the case if the roots were influenced by gravity alone.

The part of the growing root most sensitive to stimuli is-so far as experimental evidence points - the tip, and the phenomena which are exhibited in consequence of its great sensitiveness are so astounding that Darwin compared the root-tip to the brain of lower animals. He writes, "it is hardly an exaggeration to say that the tip of the radicle thus endowed, and having the power of directing the movements of the adjoining parts, acts like the brain of one of the lower animals; the brain being seated within the anterior end of the body, receiving impressions from the sense-organs, and directing the several movements".

Remarkable and interesting as are these vital phenomena observed in roots, there is still much to be wished for in the matter of their explanation and clear comprehension. Here, as in so many similar cases, a phrase, a technical term, a word, is introduced to designate the process observed, and not infrequently 
those who use it ultimately come to think they have given an explanation of the process, while really they have only stated it. This is especially the case with the term "stimulus". What is a stimulus? From the present state of our knowledge we cannot yet give a concise answer to this question, consequently explanations in which this word is inserted, are, as explanations, incomplete.

In these remarks there is no desire to depreciate the results obtained by the combined efforts of so many indefatigable investigators of past and modern times; on the contrary, we may regard the wealth of careful observations and sagacious inferences which form the present platform of our knowledge, and which have been generally reviewed in the preceding pages, with just pride and satisfaction. But this pride must not blind us to the recognition of the fact that most questions concerning the life of plants are as yet only at the commencement of their solution. Much has been done, but much is still reserved for the future.

"Manchen Flug wagt menschliches Wissen das doch Kaum ein blatt aufschlägt in dem Buch des Weltalls."

\section{PROPERTY OF
MSM COLLEG LIBRARY.}

END OF VOL. I. 



